NIST GCR 17-917-45

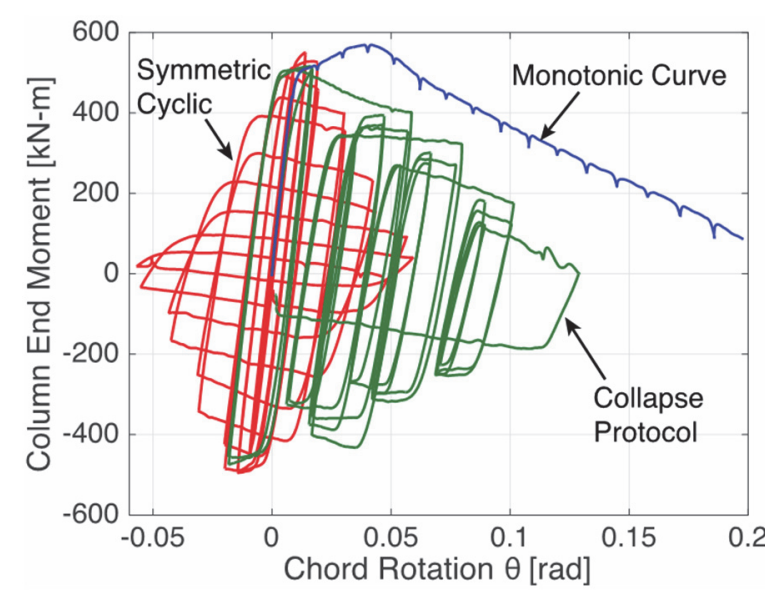

\title{
Recommended Modeling Parameters and Acceptance Criteria for Nonlinear Analysis in Support of Seismic Evaluation, Retrofit, and Design
}

Applied Technology Council

This publication is available free of charge from: https://doi.org/10.6028/NIST.GCR.17-917-45
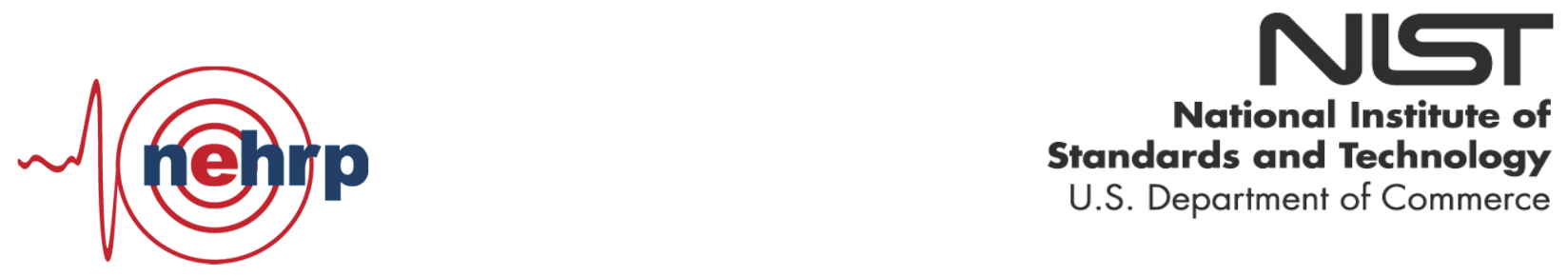


\section{Disclaimer}

This report was prepared for the Engineering Laboratory of the National Institute of Standards and Technology (NIST) under Contract SB1341-13-CQ-0009, Task Order 13-497. The contents of this publication do not necessarily reflect the views and policies of NIST or the U.S. Government.

This report was produced by the Applied Technology Council (ATC). While endeavoring to provide practical and accurate information, the Applied Technology Council, the authors, and the reviewers assume no liability for, nor express or imply any warranty with regard to, the information contained herein. Users of information contained in this report assume all liability arising from such use.

Unless otherwise noted, photos, figures, and data presented in this report have been developed or provided by ATC staff or consultants engaged under contract to provide information as works for hire. Any similarity with other published information is coincidental. Photos and figures cited from outside sources have been reproduced in this report with permission. Any other use requires additional permission from the copyright holders.

Certain commercial software, equipment, instruments, or materials may have been used in the preparation of information contributing to this report. Identification in this report is not intended to imply recommendation or endorsement by NIST, nor is it intended to imply that such software, equipment, instruments, or materials are necessarily the best available for the purpose.

NIST policy is to use the International System of Units (metric units) in all its publications. In this report, however, information is presented in U.S. Customary Units (inch-pound), as this is the preferred system of units in the U.S. engineering industry.

Cover image - Steel moment frame assembly subjected to alternative loading protocols (Suzuki and Lignos, 2015). 
NIST GCR 17-917-45

\section{Recommended Modeling Parameters and Acceptance Criteria for Nonlinear Analysis in Support of Seismic Evaluation, Retrofit, and Design}

Prepared for

U.S. Department of Commerce Engineering Laboratory National Institute of Standards and Technology Gaithersburg, MD 20899-8600

Applied Technology Council 201 Redwood Shores Parkway, Suite 240 Redwood City, CA 94065

This publication is available free of charge from: https://doi.org/10.6028/NIST.GCR.17-917-45

April 2017

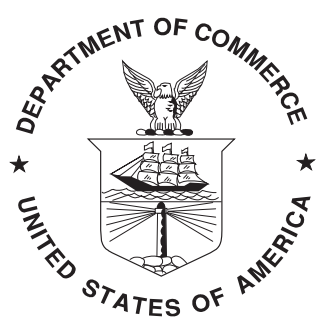

U.S. Department of Commerce Wilbur L. Ross, Jr., Secretary

National Institute of Standards and Technology Kent Rochford, Acting NIST Director and Under Secretary of Commerce for Standards and Technology 



\title{
NIST GCR 17-917-45
}

\section{Participants}

\author{
National Institute of Standards and Technology \\ Steven L. McCabe, Research Structural Engineer and Group Leader \\ John (Jay) Harris III, Research Structural Engineer \\ Siamak Sattar, Research Structural Engineer \\ Matthew S. Speicher, Research Structural Engineer \\ Kevin K.F. Wong, Research Structural Engineer \\ Earthquake Engineering Group, Materials and Structural Systems Division, Engineering \\ Laboratory \\ www.NEHRP.gov
}

\section{Applied Technology Council}

201 Redwood Shores Parkway, Suite 240

Redwood City, California 94065

www.ATCouncil.org

\section{Program Management}

Jon A. Heintz (Program Manager)

Ayse Hortacsu (Associate Program Manager)

Veronica Cedillos (Associate Project Manager)

\section{Project Technical Committee}

Ronald O. Hamburger (Project Director)

Gregory G. Deierlein

Dawn E. Lehman

Dimitrios G. Lignos

Laura N. Lowes

Robert Pekelnicky

P. Benson Shing

Peter Somers

John W. van de Lindt
Program Committee on Seismic Engineering

Jon A. Heintz (Chair)

Michael Cochran

James R. Harris

James Jirsa

Roberto Leon

Stephen Mahin

James O. Malley

Donald Scott

Andrew Whittaker

\section{Project Review Panel}

Martin Button

Charlie J. Carter

Kelly E. Cobeen

Philip Line

Justin D. Marshall

Lawrence C. Novak

Graham Powell

Michael P. Schuller

William Tremayne

Kent Yu (ATC Board Contact) 

In September 2014, the Applied Technology Council (ATC) commenced a task order project under National Institute of Standards and Technology (NIST) Contract SB1341-13-CQ-0009 to conduct comprehensive review of the generalized component models published in the current ASCE/SEI standard and relevant research, and develop recommendations for improvement (ATC-114 Project). The need for defining parameters for nonlinear force-deformation models for components, elements, or assemblies is identified as a high-priority research and development topic in NIST GCR 14-917-27 report, Nonlinear Analysis Study and Development Program for Performance-Based Seismic Engineering, (NIST, 2013b) which outlines a research and development program for addressing the gap between state-of-the-art academic research and state-of-practice engineering applications for nonlinear structural analysis, analytical structural modeling, and computer simulation in support of performance-based seismic engineering.

The current standard, ASCE/SEI 41-13, Seismic Evaluation and Retrofit of Existing Buildings (ASCE, 2014), is widely used by designers for evaluating and upgrading existing buildings. The component models in the current standard were developed for use in existing building analysis, but they have also become widely employed in new building analysis. The purpose of this report is to recommend broad improvements to seismic nonlinear modeling and acceptance criteria requirements for different structural systems.

This report was developed by the members of the ATC-114 Phase 1 project team. ATC is indebted to the leadership of Ron Hamburger, who served as Project Director. The Project Technical Committee, consisting of Greg Deierlein, Dawn Lehman, Dimitrios Lignos, Laura Lowes, Robert Pekelnicky, Benson Shing, Peter Somers, and John van de Lindt monitored and guided the technical efforts of the Project Working Groups, which included Jianyu Cheng, Ahmed Elkady, Siamak Epackachi, Alexander Hartloper, Maria Koliou, Daniel Sloat, and Andrew Whittaker. The Project Review Panel, consisting of Martin Button, Charlie Carter, Kelly Cobeen, Philip Line, Justin Marshall, Lawrence Novak, Graham Powell, Michael Schuller, Wiliam Tremayne, and Kent Yu (ATC Board Contact), provided technical advice and consultation over the duration of the work. The report also greatly benefited from the work of the ATC-114 Phase 2 and 3 projects, completed under the direction of Greg Deierlein and Curt Haselton. The names and affiliations of all who contributed to this report are provided in the list of Project Participants. 
ATC also gratefully acknowledges Steven L. McCabe (Contracting Officer's Representative), Jay Harris, Siamak Sattar, Matthew Speicher, and Kevin K.F. Wong for their input and guidance throughout the project development process. ATC staff members Veronica Cedillos and Carrie Perna provided project management support and report production services, respectively.

Ayse Hortacsu

Associate Program Manager
Jon Heintz

Program Manager 


\section{Table of Contents}

Preface ............................................................................................................................... iii

List of Figures.................................................................................................................... $\mathrm{xi}$

List of Tables ............................................................................................... xxvii

Executive Summary ....................................................................................................... xxxiii

1. Introduction ........................................................................................................ 1-1

$1.1 \quad$ Background ......................................................................... 1-1

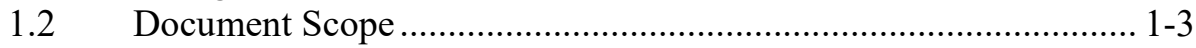

$1.3 \quad$ Intended Audience ………………………………………….... 1-3

1.4 Document Organization .............................................................. 1-3

2. General......................................................................................... 2-1

$2.1 \quad$ Use of Nonlinear Analysis .......................................................... 2-1

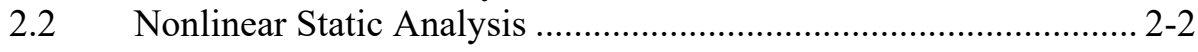

2.3 Nonlinear Dynamic Analysis........................................................ 2-5

2.4 General Hysteretic Model ............................................................ 2-8

$2.5 \quad$ Variability and Uncertainty.................................................... 2-12

3. Acceptance Criteria ................................................................................... 3-1

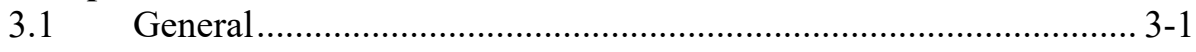

3.2 FEMA 273/274 …………………………………………... 3 -1

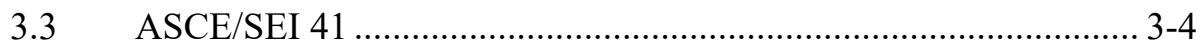

3.4 PEER Tall Building Guidelines .................................................... 3-4

3.5 ASCE/SEI 7-16 ................................................................. 3-6

3.6 Adaptive Model Response ........................................................... 3-8

3.7 Recommended Acceptance Criteria................................................ 3-8

3.7.1 Nonlinear Dynamic Analysis ........................................... 3-8

3.7.2 Nonlinear Static Analysis ............................................... 3-10

3.7.3 Linear Analysis ................................................................ 3-11

$4 \quad$ Steel Moment Frame Systems........................................................................ 4-1

4.1 Overview of Geometry and Behavior .......................................... 4-1

4.2 Summary of Design Code Provisions .............................................. 4-7

4.3 Performance and Damage Progression ........................................... 4-9

4.3.1 Beams as Part of Fully-Restrained Beam-to-Column

Connections ................................................................... 4-10

4.3.2 Beams as Part of Partially-Restrained Beam-to-Column

Connections with Intermediate Stiffness .......................... 4-15

4.3.3 Beam-Columns ............................................................. 4-17

4.3.4 Beam-to-Column Joint Panel Zones ................................ 4-25 
4.3.5 Column Splices

4.3.6 Column Base Connections ...............................................4-30

4.4 Recommendations for Modeling ............................................... 4-34

4.5 Force-Deformation Envelopes ...................................................4-35

4.5.1 Hinge Model for Beams as Part of Fully-Restrained

Beam-to-Column Moment Connections .......................... 4-38

4.5.2 Beams with Pre-Northridge WUF-B Connecti ................ 4-44

4.5.3 Non-Conforming Beams as part of Fully-Restrained

Beam-to-Column Connections .........................................4-46

4.5.4 Column Hinge Model...................................................4-46

4.5.5 Beam-to-Column Joint Panel Zone Model....................... 4-54

4.5.6 Hinge Model for Beams as part of Partially-Restrained

Connections with Intermediate Stiffness...........................4-56

4.5.7 Steel Column Splice Hinge Model.................................... 4-59

4.5.8 Column Base Connection Hinge Model............................4-61

4.6 Summary Findings of Application Case Study .............................4-69

5. Steel Concentrically Braced Frames......................................................5-1

5.1 Overview of Geometry and Behavior ..........................................5-1

5.2 Summary of Design Code Provisions ..........................................5-2

5.3 Performance and Damage Progression.........................................5-4

$5.4 \quad$ Recommendations for Modeling ……........................................

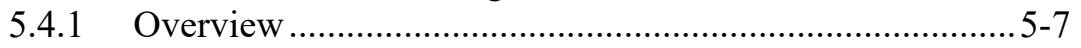

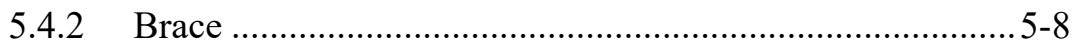

5.4.3 Gusset Plate Connections ................................................. 5-9

5.5 Cyclic Backbone …............................................................... 5-12

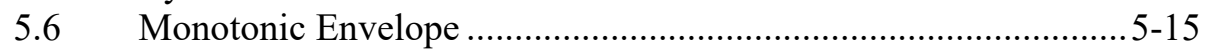

6. Reinforced Concrete Moment Frame Systems ......................................6-1

6.1 Overview of Geometry and Behavior ............................................. 6-1

6.2 Summary of Design Code Provisions ...........................................6-2

6.3 Performance and Damage Progression............................................. 6-2

6.4 Recommendations for Modeling ................................................ 6-3

6.5 Concentrated Hinge Model Parameters.......................................... 6-7

6.5.1 Column Model Parameters................................................ 6-7

6.5.2 Beam Model Parameters .............................................. 6-15

6.5.3 Modeling of Beam-Column Joints ................................. 6-16

6.5.4 Modeling of Gravity System Connections ....................... 6-18

6.6 Fiber-Type Component Models ...............................................6-21

6.6.1 Fiber-Section Modeling ............................................... 6-21

6.6.2 Fiber Material Modeling ............................................. 6-23

6.6.3 Modeling Bond-Slip and Shear Deformations ................ 6-29

7. Flexure-Controlled Reinforced Concrete Walls ........................................7-1

7.1 Overview of Geometry and Behavior .......................................... 7-1

7.1.1 Geometry and Reinforcement Layout ................................ 7-1

7.1.2 Behavior under Lateral and Gravity Loading...................... 7-4

7.1.3 Compilation and Evaluation of Flexural Wall Database.... 7-6

7.2 Summary of Design Code Provisions ............................................ 7-8

7.2.1 Vertical Reinforcement .................................................

7.2.2 Horizontal Reinforcement ........................................... 


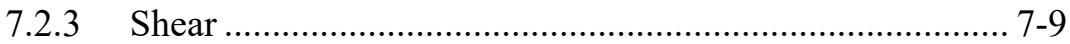

7.2.4 Boundary Elements........................................................ 7-10

7.3 Performance and Damage Progression ....................................... 7-12

7.4 Recommendations for Modeling................................................ 7-14

7.4.1 Constitutive Modeling …............................................. 7-16

7.4.2 Fiber-Type Beam-Column Elements .............................. 7-21

7.4.3 Zero-Length Hinge (or Plastic-Hinge) Models ................ 7-27

7.4.4 Fiber-Type Shell Elements .......................................... 7-28

7.5 Deformation Capacity ...................................................... 7-28

8. Shear-Controlled Reinforced Concrete Walls............................................ 8-1

8.1 Overview of Geometry and Behavior .......................................... 8-1

8.1.1 Classification of Shear Walls by Geometry in Plan and

Elevation ........................................................................... 8-1

8.1.2 Hysteretic Response of Low Aspect Ratio Shear Walls .... 8-2

8.2 Summary of Design Code Provisions ............................................ 8-3

8.2.1 Modeling Parameters in ASCE/SEI 41-13 ...................... 8-3

8.3 Database Development ......................................................... 8-4

$8.4 \quad$ Peak Shear Strength .................................................................. 8-5

8.4.1 Chapter 11 of ACI 318-14 …...................................... 8-6

8.4.2 Chapter 18 of ACI 318-14 ................................................ 8-6

8.4.3 Barda et al. (1977) ............................................................ 8-7

8.4.4 Evaluation of Results and Recommendations................... 8-7

8.5 Cyclic Backbone Curves............................................................... 8-9

8.5.1 Constructing a Cyclic Backbone Curve............................. 8-9

8.5.2 Control Points for Cyclic Backbone Curves.................... 8-11

8.5.3 Recommendations for Modeling and Control Points....... 8-39

8.6 Cyclic Hysteretic Models........................................................... 8-41

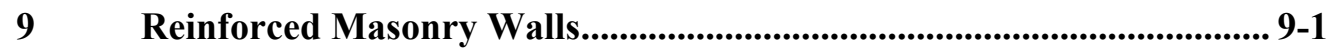

9.1 Overview of Geometry and Behavior ............................................. 9-1

9.1.1 Wall Configurations in Buildings ...................................... 9-1

9.1.2 Behavior of Wall Systems ............................................... 9-3

9.1.3 Behavior of Wall Components .................................... 9-5

9.2 Summary of Design Code Provisions ............................................. 9-9

9.3 Recommendations for Modeling.............................................. 9-10

9.4 Modeling of Wall Components with Beam-Column Elements .... 9-12

9.4.1 Types of Beam-Column Elements ................................. 9-12

9.4.2 Material Models for Masonry and Steel ......................... 9-14

9.4.3 Effective Plastic-Hinge Length....................................... 9-19

9.4.4 Shear Deformation....................................................... 9-20

9.4.5 Evaluation of Beam-Column Model with Test Data ....... 9-21

9.4.6 Influence of Lap Splices .............................................. 9-24

9.4.7 Influence of Loading History ......................................... 9-25

9.5 Backbone Force-Displacement Curves for Flexure-Dominated

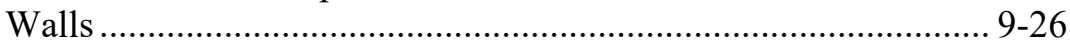

9.5.1 Construction of Idealized Backbone Curves.................... 9-26

9.5.2 Nondimensionalized Moment-Curvature Relations for Fully Grouted Rectangular Wall Sections ....................... 9-29

9.5.3 Comparison of Backbone Curves with Test Data for Fully Grouted Walls........................................................ 9-35 
9.5.4 Additional Remarks

9.6 Backbone Force-Displacement Curves for Shear-Dominated

Walls ................................................................................... 9-38

9.6.1 Idealized Backbone Curves for Fully Grouted Walls ......9-38

9.6.2 Comparison of Backbone Curves with Test Data for Fully Grouted Walls

9.6.3 Idealized Backbone Curves for Partially Grouted Walls $9-40$

10. Wood Shear Wall Systems. 10-1

10.1 Overview of Geometry and Behavior ........................................ 10-1

10.2 Summary of Design Code Provisions ........................................... 10-3

10.3 Performance and Damage Progression........................................ 10-4

10.3.1 Seismic Force-Resisting Systems................................... 10-4

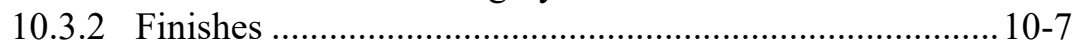

10.4 Recommendations for Modeling .............................................. 10-8

10.4.1 Introduction and Challenges..........................................10-8

10.4.2 Development of Backbone Curve Data Synthesis ......... 10-10

10.4.3 Numerical Modeling Recommendations........................10-13

10.5 Illustrative Example Summary .............................................. 10-21

Appendix A: Steel Moment-Frame Systems - Application Case Study ............ A-1

A.1 Steel Beams in Fully-Restrained Beam-to-Column Moment Connections ................................................................................... A-1

A.1.1 Composite Steel Beams in Fully Restrained Beam-toColumn Moment Connections .....

A.2 Beams with Pre-Northridge WUF-B Connections ........................ A-4

A.3 Steel Wide-Flange Beam-Columns .............................................. A-5

A.4 Hollow Structural Steel Beam-Columns ................................... A-10

A.5 Case Studies ........................................................................... A-13

A.5.1 Description of Archetype Buildings............................ A-13

A.5.2 Ground Motion Selection and Scaling .......................... A-14

A.5.3 Nonlinear Building Models ......................................... A-14

A.5.4 Results of the Nonlinear Static Analysis ......................... A-15

A.5.5 Results of the Nonlinear Response History Analysis...... A-19

Appendix B: Shear-Controlled Reinforced Concrete Walls - Database........... B-1

B.1 Loading Properties of Reinforced Concrete Walls........................ B-1

B.2 Statistical Evaluation of the Measured Data ................................ B-48

B.3 Calculated Parameters for Cyclic Hysteresis Models .................. B-54

Appendix C: Shear-Controlled Reinforced Concrete Walls - Data Analysis ....................................................................................................... C-1

Appendix D: Shear-Controlled Reinforced Concrete Walls - Comparison

Studies ............................................................................................................... D-1

Appendix E: Reinforced Masonry Walls - Database.............................................. E-1

E.1 Flexure-Dominated Walls .................................................... E-1

E.1.1 Cyclic Analysis for Flexure-Dominated Walls ................. E-2

E.1.2 Backbone Curves for Flexure-Dominated Walls ............ E-12 
E.2 Shear-Dominated Walls ..........................................................E-23

E.2.1 Backbone Curves for Shear-Dominated Walls .................E-24

Appendix F：Wood Shear Wall Systems - Database ...........................................F-1

Appendix G: Wood Shear Wall Systems - Application Case Study .................. G-1

G.1 Overview and Introduction .................................................... G-1

G.2 Structural System and Design Considerations ............................. G-1

G.3 Case Study Description and Results ........................................ G-3

G.3.1 Component Level Nonlinear Model Development and Structural Analysis........................................................ G-3

G.3.2 System Level Nonlinear Model Development and Structural Analysis.......................................................... G-5

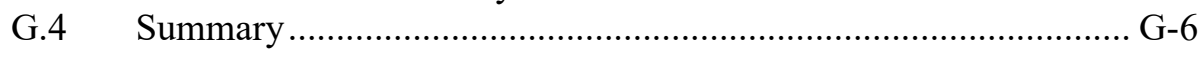

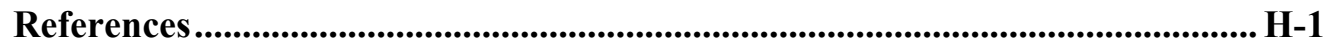

Project Participants .......................................................................................... 



\section{List of Figures}

Figure ES-1 General cyclic backbone and hysteretic envelope .................... xxxiv

Figure ES-2 Steel moment frame assembly subjected to alternative loading protocols.................................................................................

Figure ES-3 Typical steel moment frame cyclic hysteresis .......................... xxxvi

Figure ES-4 Typical brace hysteresis .............................................................. xli

Figure ES-5 Typical hysteretic behavior and modeling of reinforced concrete special moment frame beam-column assembly ............................ xliii

Figure ES-6 Typical hysteretic flexural response of rectangular reinforced concrete wall with superior detailing of reinforcement .................. xlv

Figure ES-7 Wall flexural failure mode as a function of shear stress and aspect ratio ...................................................................... xlvi

Figure ES-8 Regularization of concrete and steel constitutive properties ........ xlvii

Figure ES-9 Representative cyclic hysteresis of shear-controlled reinforced concrete walls ....................................................................... xlviii

Figure ES-10 Cyclic backbone for shear nonlinear in concrete walls................xlviii

Figure ES-11 Typical cyclic hysteresis, flexurally yielding solid grouted wall....... li

Figure ES-12 Typical cyclic hysteresis, shear yielding solid grouted wall............. li

Figure ES-13 Cyclic moment-curvature backbone control points for solidgrouted reinforced masonry walls............................................... li

Figure ES-14 Cyclic backbone for fully grouted reinforced masonry walls in

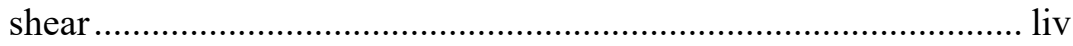

Figure ES-15 Typical hysteretic response of cyclically loaded wood shear

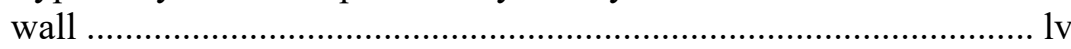

Figure ES-16 Hysteretic backbone for light framed wood walls ........................ lv

Figure 2-1 Pushover curve for hypothetical structure ….............................. 2-3

Figure 2-2 Typical backbone hysteretic relationships used in nonlinear

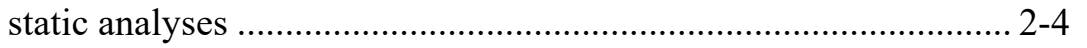

Figure 2-3 Alternative hysteretic models ................................................. 2-6 
Figure 2-4 Derivation of first and second cycle backbones from cyclic test

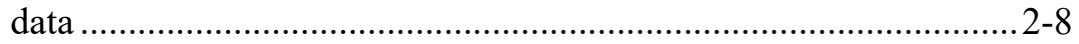

Figure 2-5 Representative cyclic and monotonic backbone curves ...............2-10

Figure 2-6 Standard cyclic and monotonic backbones with control points ....2-11

Figure 3-1 FEMA 273/274 acceptance criteria ...............................................3-3

Figure 4-1 Typical steel moment-resisting frame including its main structural components ............................................................ 4-2

Figure 4-2 Schematic representation of lateral load response of a steel moment-resisting frame under earthquake loading...

Figure 4-3 Dynamic response of a 4-story steel frame building up to "collapse

Figure 4-4 Effect of loading history on cyclic behavior of steel columns ........ 4-7

Figure 4-5 Typical RBS moment connection geometry ...............................4-11

Figure 4-6 Deduced moment-rotation relation for bare beam with RBS ........4-12

Figure 4-7 Typical damage progression for bare beam with RBS .................4-12

Figure 4-8 Deduced moment-chord rotation and typical damage progression for composite beams with RBS

Figure 4-9 Typical connection detail for pre-Northridge beam-to-column connection (WUF-B) and deduced moment-rotation relation of a steel beam as part of a Pre-Northridge beam-to-column connection

Figure 4-10 Typical failure modes observed in steel beams as part of pre-Northridge Welded-flange-bolted-web beam-to-column connections.

Figure 4-11 Typical single plate shear-tab beam-to-column connection

Figure 4-12 Typical deduced beam moment-chord rotation relations of single plate shear-tab beam-to-column connections including damage progression.

Figure 4-13 Typical deduced moment rotation relation of stocky wide flange columns including damage progression

Figure 4-14 Deduced column end moment-chord rotation relation for deep and slender wide flange steel columns under constant compressive axial load ratios

Figure 4-15 Typical damage progression in deep slender wide flange steel beam-columns under cyclic loading 
Figure 4-16 Failure modes associated with large deformations in steel wide flange steel columns: (a) loss of column axial load carrying capacity; (b) and (c) fracture due to high distortion in the k-area

Figure 4-17 Deduced column end moment-chord rotation relation for deep and slender wide flange steel columns under constant compressive axial load ratios coupled with unidirectional and bidirectional symmetric lateral loading protocols.

Figure 4-18 Deduced column end moment-chord rotation relation for wide flange steel columns under constant compressive axial load ratio and varying axial load coupled with symmetric lateral loading protocols.

Figure 4-19 Typical deduced column end moment-chord rotation relation of HSS steel beam-columns including damage progression ............. 4-24

Figure 4-20 Elephant foot local buckling mechanism ................................... 4-25

Figure 4-21 Interior beam-column joint panel zone connection detail............ 4-26

Figure 4-22 Typical hysteretic response of beam-to-column joint panel zone......

Figure 4-23 Typical hysteretic response of beam-to-column joint panel zone. $4-27$

Figure 4-24 Typical complete joint penetration welded splice detail $4-28$

Figure 4-25 Typical moment curvature relation for complete joint penetration welded splice.

Figure 4-26 Weld fracture in welded column splice 4-29

Figure 4-27 Typical detail, moment-curvature relation, and damage progression for pre-Northridge column splice connections

Figure 4-28 Column base connections and force transfer mechanisms............ 4-31

Figure 4-29 Hysteretic response of exposed column base connections............ 4-32

Figure 4-30 Typical failure modes observed in exposed column base connections

Figure 4-31 Moment drift relations for embedded type column base connections 4-33

Figure 4-32 Typical failure modes observed in embedded column base connections 4-33

Figure 4-33 Idealized models of beam-column elements 4-35

Figure 4-34 Monotonic and first-cycle envelope curves for various steel components 4-36 
Figure 4-35 Fitted idealized multi-linear models to represent the first-cycle envelope curve for a steel component

Figure 4-36 Composite effects on the hysteretic behavior of steel beams. $4-43$

Figure 4-37 Flexural strength with respect to steel beam unbraced length.......4-46

Figure 4-38 Comparisons of proposed modeling recommendations with ASCE/SEI 41-13 for steel wide-flange beam-columns under bidirectional loading.

Figure 4-39 Comparisons of proposed modeling recommendations with ASCE/SEI 41-13 for end beam-columns as part of steel moment frame systems.

Figure 4-40 Beam-to-column joint panel zone model and its trilinear model idealization

Figure 4-41 Boundary forces acting on interior composite panel zone and panel zone hysteretic material model....

Figure 4-42 Idealized moment-rotation relation for hinge model for beams as part of partially-restrained connections with intermediate stiffness

Figure 4-43 Effective depth for calculation of moment capacity.

Figure 4-44 Modified envelope curve for composite shear tab connections including flexural strength stiffening as a result of beam binding; illustration with experimental data.

Figure 4-45 Typical column splice with partial penetration flange welds ........4-60

Figure 4-46 Stress distributions assumed in current U.S. design practice for low and high-eccentricity conditions .... 4-61

Figure 4-47 Assumed deformation mode and contribution of various components

Figure 4-48 Idealized moment-rotation relation for hinge model for exposed column base connections. 4-64

Figure 4-40 Load-resisting mechanisms for embedded column bases

Figure 4-50 Comparison of experimental data and proposed first-cycle envelope curves for embedded column base connections......

Figure 5-1 Typical CBF components ........................................................... 5-2

Figure 5-2 Typical CBF configurations …….............................................. 5-2

Figure 5-3 Representative CBF connections in older construction..................5-4

Figure 5-4 Idealized response of CBFs ....................................................... 5-4

Figure 5-5 Cyclic response of single and paired braces................................5-5 
Figure 5-6 Damage to buckling brace under reverse loading. $5-6$

Figure 5-7 Yield mechanism and failure modes ......................................... 5-6

Figure 5-8 Comparison of SCBFs designed to comply with AISC and BDP .. 5-7

Figure 5-9 Comparison of brace model and AISC and Euler-buckling curves for HSS $5 \times 5 \times 3 / 8$ brace and various out-of-plane offsets. $5-8$

Figure 5-10 Schematic layout of fibers for HSS and wide flange cross sections

Figure 5-11 Brace clearance for corner and midspan connections $5-9$

Figure 5-12 Rotational connection model of corner connections... $5-10$

Figure 5-13 Models for SCBFs: pinned, restrained, and nonlinear spring connection.

Figure 5-14 Simplified cyclic backbone curves for CBFs for brace response in tension and compression... $5-12$

Figure 5-15 Combined backbone curve.................................................... 5-13

Figure 5-16 Drift capacity as a function of normalized slenderness ratio ........ 5-14

Figure 5-17 Comparison of recommended envelope and measured response for selected tests................................................................ 5-15

Figure 6-1 Idealized cantilever model of reinforced concrete beam-column with concentrated hinge at member end ......................................... 6-4

Figure 6-2 Representative force-deformation plot for cantilever beam-column

Figure 6-3 Experimental test data and calibrated model predictions for five different loading protocols .......................................................... 6-6

Figure 6-4 Illustration of reinforced Concrete P-M-M surfaces from PERFORM-3D.

Figure 6-5 Comparison of first-cycle envelope parameters to those specified in ACI 369

Figure 6-6 Recommended element configurations for modeling stiffness adjustment of beam and column offsets. 6-17

Figure 6-7 Recommended element configurations for modeling joint center rotational spring in a planar frame ............................................ 6-18

Figure 6-8 Idealized slab-column joint model............................................ 6-19

Figure 6-9 Cyclic stress-strain model for steel ......................................... 6-24

Figure 6-10 Stress-strain model for confined and unconfined concrete, with simplified bilinear and trilinear approximations .......................... 6-26 
Figure 6-11 Confined core for a rectangular hoop reinforced section

Figure 6-12 Confined strength determination from lateral confining stresses for rectangular section.

Figure 6-13 Concrete tension material models with and without tension stiffening

Figure 7-1 Common vertical wall-system configurations.

Figure 7-2 Common wall cross-sectional configurations .

Figure 7-3 Core vs. distributed planar walls. 7-3

Figure 7-4 Demands on individual wall or pier

Figure 7-5 Reinforcement configuration of wall cross sections $7-4$

Figure 7-6 Response of and damage to flexural walls

Figure 7-7 Selected damage states of reinforced concrete slender walls

(a) cover spalling; (b) exposed longitudinal reinforcement;

(c) bar buckling; (d) boundary element core crushing; (e) web core crushing; and (f) bar fracture.

Figure 7-8 Damage progression for C-shaped flanged wall exhibiting buckling-rupture flexural failure.

Figure 7-9 Idealizations of models used for simulating the nonlinear response of concrete walls..

Figure 7-10 Nonlinear 1D cyclic material models

Figure 7-11 Impact of mesh size on models without material regularization ... 7-18

Figure 7-12 Localization of damage and non-uniqueness of stress-strain response.

Figure 7-13 Regularization of concrete and steel material response using mesh dependent length

Figure 7-14 Measured and simulated response histories for planar wall WSH4 tested by Dazio et al.

Figure 7-15 Measured and simulated base shear versus drift response histories for planar wall RW1 tested by Thomsen and Wallace ... 7-23

Figure 7-16 Fiber discretization of planar reinforced concrete wall.

Figure 7-17 Linear and nonlinear shear response models for use with fiber-type beam-column elements.

Figure 7-18 Planar wall design criteria triggering compression-shear versus flexural only failure modes

Figure 7-19 Simulated response for a slender wall exhibiting compression-shear failure per Figure 7-14 
Figure 8-1 Hysteretic response of a low aspect ratio shear wall $8-2$

Figure 8-2 Load-deformation relationship for members controlled by shear... 8-4

Figure 8-3 Predicted and measured normalized lateral strength of reinforced concrete shear walls ............................................................... 8-8

Figure 8-4 Measured and idealized cyclic backbone curves ........................ 8-10

Figure 8-5 Describing post-peak response ........................................... 8-10

Figure 8-6 Effect of design variables on drift ratio at cracking, $\delta_{A} \ldots \ldots \ldots \ldots \ldots . . .18-12$

Figure 8-7 Effect of design variables on normalized force at cracking,

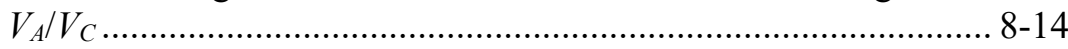

Figure 8-8 Effect of design variables on drift ratio at yield, $\delta_{B} \ldots \ldots \ldots \ldots \ldots \ldots . . . . .18-17$

Figure 8-9 Effect of design variables on drift ratio at peak strength, $\delta_{C} \ldots \ldots . . .8$ 8-19

Figure 8-10 Effect of design variables on normalized peak lateral strength,

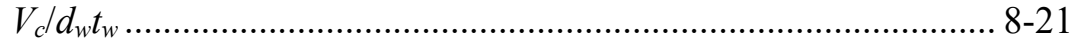

Figure 8-11 Effect of design variables on drift ratio at zero residual strength,

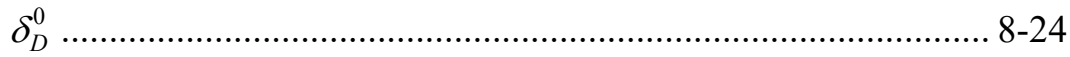

Figure 8-12 Effect of design variables on drift ratio at 20\% residual strength, $\delta_{D}^{20}$ $8-26$

Figure 8-13 Effect of design variables on drift ratio at $40 \%$ residual strength, $\delta_{D}^{40}$

Figure 8-14 Effect of design variables on drift ratio at $80 \%$ residual strength, $\delta_{D}^{80}$ 8-30

Figure 8-15 Effect of design variables on normalized initial stiffness, $K_{e} / K_{t}$ $8-33$

Figure 8-16 Effect of design variables on normalized yield stiffness, $K_{y} / K_{t} \ldots .8$ 8-35

Figure 8-17 Effect of design variables on normalized post-peak stiffness, $K_{p} / K_{t}$

Figure 8-18

IKP model $8-41$

Figure 8-19 IKP deterioration models

Figure 8-20 Modified backbone curve and hysteretic force-displacement relationship of the IKP model

Figure 8-21 Comparison of (a) test data, (b) MIKP prediction, and (c) proposed-model prediction for Alexander et al. (1973) panel 4 ... 8-46

Figure 9-1 Configurations of reinforced masonry wall systems. $9-2$ 
Figure 9-2 Shear failure of reinforced masonry walls in a three-story building tested on a shake table.....

Figure 9-3 Shear failure of reinforced masonry piers in a two-story building tested on a shake table.

Figure 9-4 In-plane lateral load-vs.-displacement curves for a flexure-dominated wall

Figure 9-5 Crushing and bar buckling in a flexure-dominated wall ..... $9-6$

Figure 9-6 In-plane lateral load vs. displacement curves for diagonal shear-dominated walls.

Figure 9-7 Failure of a shear-dominated wall $9-8$

Figure 9-8 In-plane lateral load vs. displacement curves for a wall dominated by shear sliding.

Figure 9-9 Frame models of cantilever and coupled walls $9-11$

Figure 9-10 Frame models of perforated walls.

Figure 9-11 Baseline stress-strain curve for the compression behavior of fully grouted masonry .

Figure 9-12 Stress-strain relation for steel considering bar buckling and fracture

Figure 9-13 Tensile strain at bar fracture as a function of steel quantity and axial compressive load for cyclic analysis

Figure 9-14 Comparison of numerical results with experimental data for flexure-dominated walls with varying amounts of vertical steel...9-23

Figure 9-15 Comparison of numerical results with experimental data for flexure-dominated walls with varying amounts of axial load.

Figure 9-16 Comparison of numerical results with experimental data for flexure-dominated walls with varying aspect ratio

Figure 9-17 Comparison of numerical results with experimental data for flexure-dominated walls for influence of lap splices

Figure 9-18 Influence of loading history on flexure-dominated wall behavior.

Figure 9-19 Backbone load-displacement curve for flexure-dominated reinforced masonry walls

Figure 9-20 Moment-curvature relation for a wall section

Figure 9-21

Cantilever wall

Figure 9-22 Fully grouted symmetric rectangular wall section subjected to compression and bending..... 
Figure 9-23 Influence of $a=l_{e} / l_{w}$ for walls with $\alpha=0.05$ and $l_{b} / l_{w}=0.25 \ldots \ldots .9-32$

Figure 9-24 Influence of bar spacing for walls with $\alpha=0.05$ and $l_{w}=72$ in ... 9-32

Figure 9-25 Comparison of proposed backbone curves with experimental data for flexure-dominated walls with an aspect ratio of one and zero axial load

Figure 9-26 Comparison of proposed backbone curves with experimental data for flexure-dominated walls with an aspect ratio of one and zero axial load. 9-36

Figure 9-27 Comparison of proposed backbone curves with experimental data for flexure-dominated walls with an axial load ratio of $5 \%$ and $\rho_{v}=0.72 \%$ 9-36

Figure 9-28 Backbone curves for wall WSU-W-2B. 9-37

Figure 9-29 Backbone lateral load-vs.-story drift ratio curve for shear-dominated reinforced masonry walls 9-38

Figure 9-30 Comparison of proposed backbone curves with experimental data for shear-dominated reinforced masonry walls

Figure 10-1 Typical components of a wood shear wall with conventional hold downs

Figure 10-2 Illustration of a stacked wood shear wall system........................ 10-2

Figure 10-3 Typical wood shear wall in a wall line $10-5$

Figure 10-4 Corner nail pulling through and tearing out of the bottom edge of a 15/32" OSB sheathing panel due to excessive drift during a shear wall test.. $10-6$

Figure 10-5 Sheathing rotating off resulting in complete loss of shear strength for the wood shear wall..... $10-6$

Figure 10-6 A $1 / 2$ " wide sill plate split along a garage wall during a full scale building shake table test performed by Filiatrault et al. (2010) .... 10-6

Figure 10-7 CUREE hysteretic model force-displacement response of wood shear walls under cyclic loading.....

Figure 10-8 Force-displacement response of wood shear walls for varying material combinations. $10-11$

Figure 10-9 Shear strength ratio-displacement response of wood structural panels with aspect ratio $1: 1$ $10-12$

Figure 10-10 10-Parameter CUREE hysteresis model fit to data varying material wall combinations..... $10-14$

Figure 10-11 Monotonic backbone curve described in Equation 10-1 ............ 10-16

Figure 10-12 Proposed monotonic envelope curve 10-17 
Figure 10-13 Proposed monotonic envelope compared to the average synthesis data for each wall combination

Figure 10-14 Proposed monotonic envelope compared to the average synthesis data for each wall combination for $8 \times 8 \mathrm{WSP}$

Figure 10-15 Proposed monotonic envelope curve envelope compared to the average synthesis data for each wall combination for $8 \times 4$ WSP

Figure A-1 Comparisons of proposed modeling recommendations with ASCE 41-13 for bare steel beams

Figure A-2 Comparisons of proposed modeling recommendations with ASCE 41-13 for composite steel beams.

Figure A-3 Comparisons of proposed modeling recommendations with ASCE 41-13 for steel beams as part of pre-Northridge beam-tocolumn connections

Figure A-4 Comparisons of proposed modeling recommendations with ASCE 41-13 for steel wide-flange beam-columns.

Figure A-5 Trends of pre- and post-peak plastic deformations with respect to the cross-section web local slenderness ratio for modeling the monotonic backbone curve of steel wide-flange columns

Figure A-6 Trends of pre- and post-peak plastic deformations with respect to the cross-section web local slenderness ratio for modeling the first-cycle envelope curve of steel wide-flange columns

Figure A-7 Illustration of proposed modeling recommendations for HSS beam-columns

Figure A-8 Trends of pre- and post-peak plastic deformations with respect to the cross-section local slenderness ratio for modeling the monotonic backbone curve of HSS beam-columns

Figure A-9 Plan view of archetype buildings from NIST

Figure A-10 Schematic representation of the analytical model representations of the beam-to-column joint panel zones

Figure A-11 Global pushover curves based on single-mode nonlinear static analysis procedure and various component-modeling options

Figure A-12 Prediction of the nonlinear static response of the first story interior beam-to-column panel zone joint of the 12-story steel SMF based on first-mode NSP

Figure A-13 Predicted peak story drift ratios along the height of the 12-story archetype based on NSP, first-mode lateral load pattern 
Figure A-14 NSP to NRHA comparison of predicted story-based engineering demand parameters, 12-story archetype; left DBE seismic intensity; right MCE seismic intensity..................................... A-21

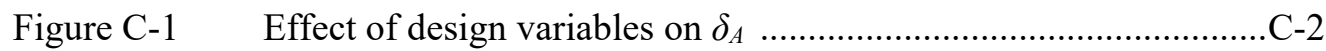

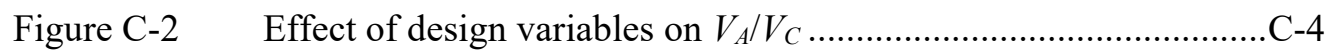

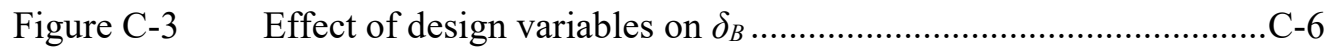

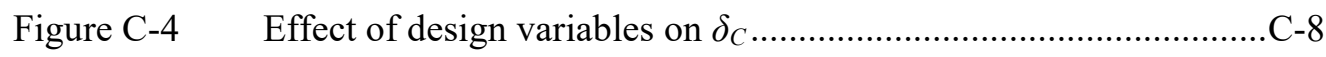

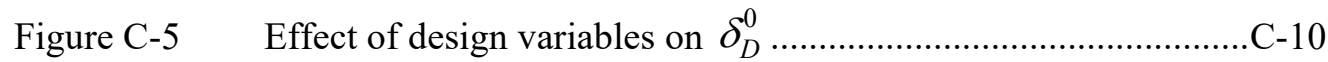

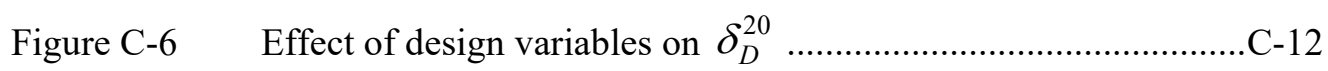

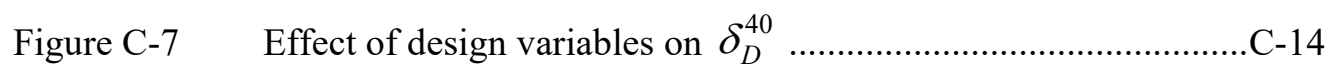

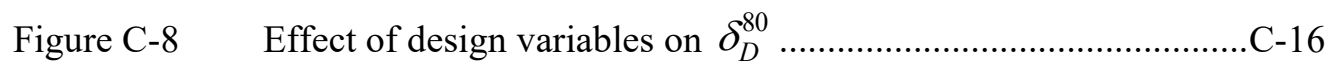

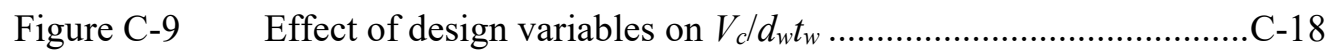

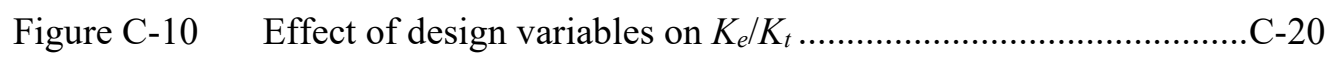

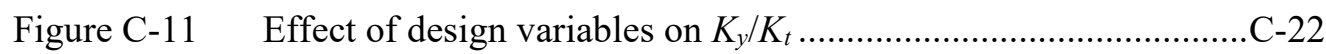

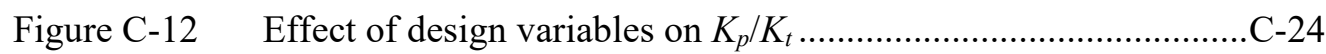

Figure D-1 Comparison of (a) test data, (b) MIKP prediction, and

(c) proposed-model prediction for Alexander et al. (1973) panel 4

Figure D-2 Comparison of (a) test data, (b) MIKP prediction, and

(c) proposed-model prediction for Saatcioglu (1991)

Specimen 1

D-2

Figure D-3 Comparison of (a) test data, (b) MIKP prediction, and

(c) proposed model prediction for Saatcioglu (1991)

Specimen 4

Figure D-4 Comparison of (a) test data, (b) MIKP prediction, and

(c) proposed-model prediction for Saatcioglu (1991)

Specimen 6

Figure D-5 Comparison of (a) test data, (b) MIKP prediction, and

(c) proposed-model prediction for Pilakoutas and Elnashai

(1995) Specimen SW4 D-5

Figure D-6 Comparison of (a) test data, (b) MIKP prediction, and

(c) proposed-model prediction for Pilakoutas and Elnashai (1995) Specimen SW5 D-6 
Figure D-7 Comparison of (a) test data, (b) MIKP prediction, and (c) proposed-model prediction for Pilakoutas and Elnashai (1995) Specimen SW6

Figure D-8 Comparison of (a) test data, (b) MIKP prediction, and

(c) proposed-model prediction for Pilakoutas and Elnashai

(1995) Specimen SW7 D-8

Figure D-9 Comparison of (a) test data, (b) MIKP prediction, and (c) proposed-model prediction for Pilakoutas and Elnashai (1995) Specimen SW8

Figure D-10 Comparison of (a) test data, (b) MIKP prediction, and

(c) proposed-model prediction for Pilakoutas and Elnashai (1995) Specimen SW9.

Figure D-11 Comparison of (a) test data, (b) MIKP prediction, and

(c) proposed-model prediction for Salonikios et al. (1999)

Specimen LSW1

Figure D-12 Comparison of (a) test data, (b) MIKP prediction, and

(c) proposed-model prediction for Salonikios et al. (1999)

Specimen LSW2.

Figure D-13 Comparison of (a) test data, (b) MIKP prediction, and

(c) proposed-model prediction for Salonikios et al. (1999)

Specimen LSW3.

Figure D-14 Comparison of (a) test data, (b) MIKP prediction, and

(c) proposed-model prediction for Salonikios et al. (1999) Specimen MSW1

Figure D-15 Comparison of (a) test data, (b) MIKP prediction, and (c) proposed-model prediction for Salonikios et al. (1999) Specimen MSW2

Figure D-16 Comparison of (a) test data, (b) MIKP prediction, and (c) proposed-model prediction for Salonikios et al. (1999) Specimen MSW3

Figure D-17 Comparison of (a) test data, (b) MIKP prediction, and (c) proposed-model prediction for Hidalgo et al. (2002) Specimen 1 D-17

Figure D-18 Comparison of (a) test data, (b) MIKP prediction, and (c) proposed-model prediction for Hidalgo et al. (2002) Specimen 2 D-18

Figure D-19 Comparison of (a) test data, (b) MIKP prediction, and (c) proposed-model prediction for Hidalgo et al. (2002) Specimen 4 D-19 
Figure D-20 Comparison of (a) test data, (b) MIKP prediction, and

(c) proposed-model prediction for Hidalgo et al. (2002)

Specimen 6.

Figure D-21 Comparison of (a) test data, (b) MIKP prediction, and

(c) proposed-model prediction for Hidalgo et al. (2002)

Specimen 7

D-21

Figure D-22 Comparison of (a) test data, (b) MIKP prediction, and

(c) proposed-model prediction for Hidalgo et al. (2002)

Specimen 8 .

D-22

Figure D-23 Comparison of (a) test data, (b) MIKP prediction, and

(c) proposed-model prediction for Hidalgo et al. (2002)

Specimen 9

D-23

Figure D-24 Comparison of (a) test data, (b) MIKP prediction, and

(c) proposed-model prediction for Hidalgo et al. (2002)

Specimen 10

Figure D-25 Comparison of (a) test data, (b) MIKP prediction, and

(c) proposed-model prediction for Hidalgo et al. (2002)

Specimen 11

D-25

Figure D-26 Comparison of (a) test data, (b) MIKP prediction, and

(c) proposed-model prediction for Hidalgo et al. (2002)

Specimen 12

D-26

Figure D-27 Comparison of (a) test data, (b) MIKP prediction, and

(c) proposed-model prediction for Hidalgo et al. (2002)

Specimen 13

Figure D-28 Comparison of (a) test data, (b) MIKP prediction, and

(c) proposed-model prediction for Hidalgo et al. (2002)

Specimen 14 .

D-28

Figure D-29 Comparison of (a) test data, (b) MIKP prediction, and

(c) proposed-model prediction for Hidalgo et al. (2002)

Specimen 15

Figure D-30 Comparison of (a) test data, (b) MIKP prediction, and

(c) proposed-model prediction for Hidalgo et al. (2002)

Specimen 16

D-30

Figure D-31 Comparison of (a) test data, (b) MIKP prediction, and

(c) proposed-model prediction for Luna et al. (2015)

Specimen SW1.....

D-31

Figure D-32 Comparison of (a) test data, (b) MIKP prediction, and

(c) proposed-model prediction for Luna et al. (2015)

Specimen SW2.

D-32 
Figure D-33 Comparison of (a) test data, (b) MIKP prediction, and

(c) proposed-model prediction for Luna et al. (2015)

Specimen SW3.

D-33

Figure D-34 Comparison of (a) test data, (b) MIKP prediction, and

(c) proposed-model prediction for Luna et al. (2015)

Specimen SW4.

D-34

Figure D-35 Comparison of (a) test data, (b) MIKP prediction, and

(c) proposed-model prediction for Luna et al. (2015)

Specimen SW5

D-35

Figure D-36 Comparison of (a) test data, (b) MIKP prediction, and

(c) proposed-model prediction for Luna et al. (2015)

Specimen SW6

D-36

Figure D-37 Comparison of (a) test data, (b) MIKP prediction, and

(c) proposed-model prediction for Luna et al. (2015)

Specimen SW7

D-37

Figure D-38 Comparison of (a) test data, (b) MIKP prediction, and

(c) proposed-model prediction for Luna et al. (2015)

Specimen SW8.

Figure D-39 Comparison of (a) test data, (b) MIKP prediction, and

(c) proposed-model prediction for Luna et al. (2015)

Specimen SW9

Figure D-40 Comparison of (a) test data, (b) MIKP prediction, and

(c) proposed-model prediction for Luna et al. (2015)

Specimen SW10.

Figure D-41 Comparison of (a) test data, (b) MIKP prediction, and

(c) proposed-model prediction for Luna et al. (2015)

Specimen SW11

D-41

Figure D-42 Comparison of (a) test data, (b) MIKP prediction, and

(c) proposed-model prediction for Luna et al. (2015)

Specimen SW12

Figure D-43 Comparison of (a) test data, (b) MIKP prediction, and

(c) proposed-model prediction for Mansour and Hsu (2005)

Specimen W2

Figure D-44 Comparison of (a) test data, (b) MIKP prediction, and

(c) proposed-model prediction for Mansour and Hsu (2005)

Specimen W3

Figure D-45 Comparison of (a) test data, (b) MIKP prediction, and

(c) proposed-model prediction for Mansour and Hsu (2005)

Specimen W4 
Figure D-46 Comparison of (a) test data, (b) MIKP prediction, and

(c) proposed-model prediction for Mansour and Hsu (2005)

Specimen W5

Figure D-47 Comparison of (a) test data, (b) MIKP prediction, and

(c) proposed-model prediction for Sato et al. (1989) Specimen

36-M8-30

D-47

Figure D-48 Comparison of (a) test data, (b) MIKP prediction, and

(c) proposed-model prediction for Sato et al. (1989)

Specimen 36-M8-50

Figure D-49 Comparison of (a) test data, (b) MIKP prediction, and

(c) proposed-model prediction for Tomii et al. (1988)

Specimen H2-W87.5-N50

D-49

Figure D-50 Comparison of (a) test data, (b) MIKP prediction, and

(c) proposed-model prediction for Tomii et al. (1988)

Specimen T1-W87.5-N50

Figure D-51 Comparison of (a) test data, (b) MIKP prediction, and

(c) proposed-model prediction for Tomii et al. (1988)

Specimen T2-W87.5-N50.

D-51

Figure D-52 Comparison of (a) test data, (b) MIKP prediction, and

(c) proposed-model prediction for Sittupunt et al. (2001)

Specimen WALL1

Figure D-53 Comparison of (a) test data, (b) MIKP prediction, and

(c) proposed-model prediction for Effendy et al. (2006)

Specimen SMAC

D-53

Figure D-54 Comparison of (a) test data, (b) MIKP prediction, and

(c) proposed-model prediction for Effendy et al. (2006)

Specimen SMAS

D-54

Figure D-55 Comparison of (a) test data, (b) MIKP prediction, and

(c) proposed-model prediction for Effendy et al. (2006)

Specimen SMAM

D-55

Figure D-56 Comparison of (a) test data, (b) MIKP prediction, and

(c) proposed-model prediction for Li et al. (2006) Specimen

LW1

D-56

Figure D-57 Comparison of (a) test data, (b) MIKP prediction, and

(c) proposed-model prediction for Li et al. (2006) Specimen

LW2

D-57

Figure D-58 Comparison of (a) test data, (b) MIKP prediction, and

(c) proposed-model prediction for Li et al. (2006) Specimen

LW3

D-58 
Figure D-59 Comparison of (a) test data, (b) MIKP prediction, and

(c) proposed-model prediction for Li et al. (2006) Specimen

LW4.....

Figure D-60 Comparison of (a) test data, (b) MIKP prediction, and

(c) proposed-model prediction for Li et al. (2006) Specimen

LW5

Figure D-61 Comparison of (a) test data, (b) MIKP prediction, and

(c) proposed-model prediction for Li et al. (2006) Specimen

MW1

D-61

Figure D-62 Comparison of (a) test data, (b) MIKP prediction, and

(c) proposed-model prediction for Li et al. (2006) Specimen

MW2

Figure D-63 Comparison of (a) test data, (b) MIKP prediction, and

(c) proposed-model prediction for Li et al. (2006) Specimen

MW3

Figure E-1 Cyclic analysis of flexure-dominated walls

E-1

Figure E-2 Backbone load-displacement curves for flexure-dominated walls

Figure E-3 Backbone lateral load-vs.-story drift ratio curves for shear-dominated walls

Figure G-1 Building elevation and typical floor plan

Figure G-2 Proposed backbone curve envelope

Figure G-3 Individual force-displacement curves for wood structural panels, gypsum wallboard and stucco panels used to synthesize the numerical model.

Figure G-4 Fragility curves for a typical exterior wood frame wall system for various performance levels

Figure G-5 Fragility curves for the building archetype for various performance levels 


\section{List of Tables}

Table ES-1 Backbone Parameters for Non-Composite Wide Flange Steel Beams meeting AISC 341 Criteria for Special SMFs and Fully Restrained Connections ........................................................... xxxvii

Table ES-2 Backbone Parameters for Wide Flange Steel Beam-Columns.... xxxix

Table ES-3 Backbone Parameters for Hollow Structural Section Steel Beam-Columns ......................................................................... $\mathrm{xl}$

Table ES-4 Cyclic Backbone Parameters for Braces ......................................... xlii

Table ES-5 Hysteretic Backbone Control Points for Reinforced Concrete Moment Frame Beams and Columns.......................................... xliv

Table ES-6 Cyclic Backbone Control Points for Reinforced Concrete Walls Controlled by Shear ................................................................... xlix

Table ES-7 Cyclic Moment Curvature Parameters for Rectangular Reinforced Masonry Walls ........................................................................... 1 ii

Table ES-8 Monotonic Moment Curvature Parameters for Rectangular Reinforced Masonry Walls lii

Table ES-9 Bias and Uncertainty Associated with Moment Curvature Control Points .................................................................................... liii

Table ES-10 Backbone Parameters Light Framed Wood Walls .......................... lvi

Table 3-1 Load Factor, $\gamma$, as a Function of Confidence and Demand Uncertainty, Assumed Capacity Dispersion Approximately $15 \%$ 3-10

Table 4-1 Summary of Design Requirements for Steel Moment Frame Systems 4-10

Table 5-1 Statistics for Ratios of Recommended-to-Measured Model Parameters $5-14$

Table 6-1 Behavioral Effects to Consider in Nonlinear Analysis $6-3$

Table 6-2 Prediction Uncertainties and Bias in Proposed Equations ............ 6-15

Table 6-3 Steel Reinforcing Hardening Onset Strain $\varepsilon_{s h}$ and Modulus $E_{s h} \ldots$ 6-24 
Table 7-1 Distribution of Parameters in Flexural Wall Database and Their Impact on Drift Capacity ........................................................... 7-8

Table 7-2 Boundary Element Requirements............................................ 7-12

Table 7-3 Comparison of Wall Modeling Methods...................................... 7-15

Table 7-4 Deformation Demands at Onset of Strength Loss for Walls Exhibiting Different Failure Modes

Table 8-1 Modeling Parameters and Acceptance Criteria for Nonlinear Procedures-R/C Shear Walls and Wall Segments Controlled by Shear.

Table 8-2 Data Bins for Developing Updated Guidance

Table 8-3 Cyclic Backbone Control Points for Reinforced Concrete Walls Controlled by Shear. $8-45$

Table 8-4 Calculated Pinching and Deterioration Parameters. $8-44$

Table 9-1 Nondimensionalized Moment-Curvature Backbone Curve Values for Fully Grouted Rectangular Wall Sections Subjected to Cyclic Loading.....

Table 9-2 Nondimensionalized Moment-Curvature Envelope Curve Values for Fully Grouted Rectangular Wall Sections Subjected to Monotonically Increasing Loading

Table 9-3 Statistical Data on Ratios of Calculated Values to Corresponding Experimental Values for Control Points on Backbone Curves.

Table 10-1 Damage Progression for a Typical Modern Wood-Frame Building Based on Full-Scale Experiment....

Table 10-2 Definition of Hysteretic Parameters of CUREE Hysteretic Model

Table 10-3 CUREE Hysteretic Parameters for the Wood Wall Material Combinations Considered in this Chapter

Table 10-4 Common Wood Shear Wall Fastener Statistics for the CUREE Model

Table 10-5 Parameters for Proposed Envelope Curve for Wood Wall Material Combinations Considered.

Table A-1 Modeling Parameters for Various Steel Column Sizes Based on Regression Equations

Table A-2 Modeling Parameters for Various Steel Column Sizes Based on Regression Equations 
Table A-3 Modeling Parameters for Various Steel Column Sizes Based on Regression Equations........................................................ A-10

Table A-4 Modeling Parameters for Various HSS Column Sizes Based on Regression Equations............................................................... A-12

Table A-5 Modeling Parameters for Various HSS Column Sizes Based on Regression Equations........................................................... A-12

Table A-6 Modeling Parameters for Various HSS Column Sizes Based on Regression Equations............................................................ A-13

Table A-7 Model Periods and Static Overstrength for Nonlinear Static Analysis

Table B-1 Loading Properties of the RC Walls with Rectangular CrossSection

Table B-2 Geometric Properties of the RC Walls with Rectangular CrossSection B-6

Table B-3 Reinforcement Properties of the RC Walls with Rectangular Cross-Section

Table B-4 Material Properties of the RC Walls with Rectangular CrossSection

Table B-5 Test Results of the RC Walls with Rectangular Cross-Section in the First Quadrant

Table B-6 Test Results of the RC Walls with Rectangular Cross-Section in the Third Quadrant.

Table B-7 Loading Properties of the RC Walls with Barbell and Flanged Cross-Section

Table B-8 Geometric Properties of the RC Walls with Barbell and Flanged Cross-Section

Table B-9 Reinforcement Properties of the RC Walls with Barbell and Flanged Cross-Section

Table B-10 Material Properties of the RC Walls with Barbell and Flanged Cross-Section

Table B-11 Test Results of the RC Walls with Barbell and Flanged CrossSection in the First Quadrant

Table B-12 Test Results of the RC Walls with Barbell and Flanged CrossSection in the Third Quadrant ......................................................

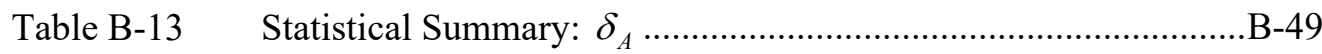

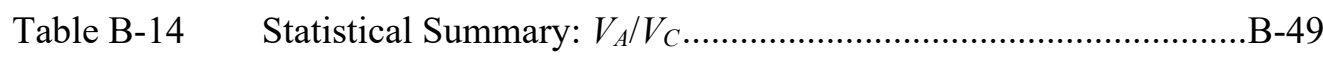




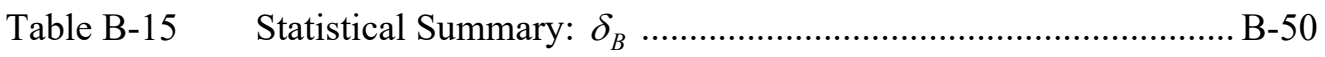

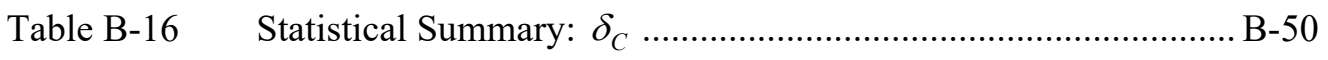

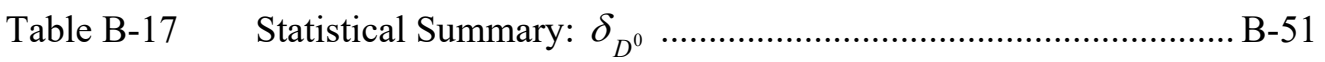

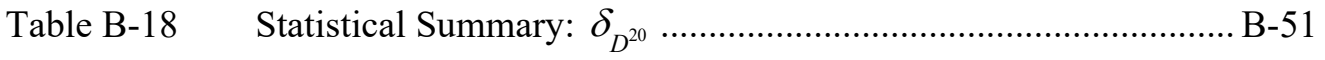

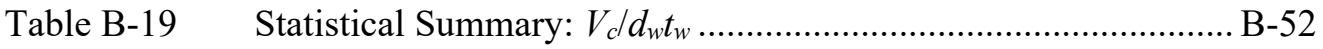

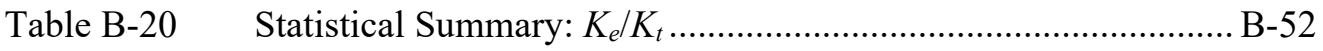

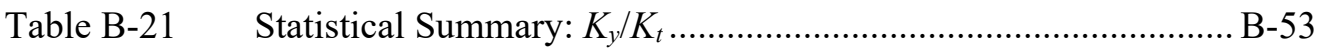

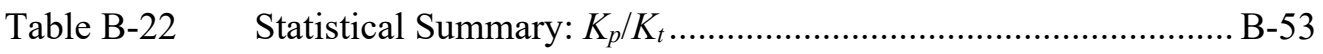

Table B-23 Calculated Pinching and Deterioration Parameters ..................... B-54

Table C-1 Statistical Summary of Design Variables................................... C-26

Table C-2 Ratio of the Mean ACI-Predicted to Mean Measured Peak Strength ............................................................................ C-29

Table E-2 Flexure-Dominated Wall Specimens Analyzed ……….................. E-1

Table E-2 Shear-Dominated Wall Specimens............................................ E-23

Table F-1 Gypsum Wallboard Sheathing Shear Capacities ............................F-1

Table F-2 Stucco Sheathing Shear Capacities ….........................................F-2

Table F-3 Stucco and Gypsum Sheathing Shear Capacities ...........................F-2

Table F-4 Horizontal Wood Siding Sheathing Shear Capacities ......................F-2

Table F-5 Wood Structural Panels ( $\left.8^{\prime} \times 8^{\prime}\right)$ Sheathing Shear Capacities and ASD design values ...................................................................

Table F-6 Wood Structural Panels (8'×4') Sheathing Shear Capacities and ASD design values ..................................................................... -4

Table F-7 Statistical Values for Varying Drift Ratio Values for Gypsum

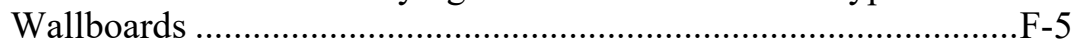

Table F-8 Statistical Values for Varying Drift Ratio Values for Stucco

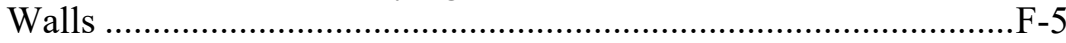

Table F-9 Statistical Values for Varying Drift Ratio Values for Stucco and Gypsum Wallboard Combination..................................................F-6

Table F-10 Statistical Values for Varying Drift Ratio Values for Wood Horizontal Siding Wall Elements.................................................F-6 
Table F-11 Statistical Values for Varying Drift Ratio Values for Wood Structural Panels $(8 ' \times 8$ ') ...............................................................

Table F-12 Statistical Values for Varying Drift Ratio Values for Wood Structural Panels $\left(8{ }^{\prime} \times 4\right.$ ') ........................................................... F-7

Table G-1 Weights Considered in the Design and Analysis .......................... G-2

Table G-2 Wood Frame Shear Wall Nailing Schedule .................................. G-3

Table G-3 Parameters for the Fragility Curves of the Wood-Frame Shear

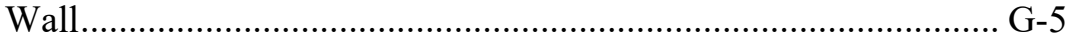

Table G-4 Parameters for the Fragility Curves of the 5-Story Wood-Frame Shear Wall............................................................................... G-6

Table G-5 Parameters for the Fragility Curves of the 5-Story Wood-Frame Shear Wall.............................................................................. G-7 



\section{Executive Summary}

This report presents recommended hysteretic relationships for use in nonlinear seismic analysis in support of performance-based seismic design and evaluation projects. The primary intended audience for this report are committee members engaged in the development of building code requirements for the use of nonlinear analysis in design, though individual engineers engaged in performance-based engineering projects may find the information presented herein useful.

Nonlinear static and dynamic analysis are commonly used by engineers to assess the probable performance of existing buildings and to design major new buildings. The most comprehensive guidelines presently available for performing nonlinear seismic analysis are contained in ASCE/SEI 41-13, Seismic Evaluation and Retrofit of Existing Buildings (ASCE, 2014). This guidance includes recommended backbone shapes and control points that describe the envelope of hysteretic response of various structural elements when subjected to ramped, fully-reversed, cyclic loading protocols. Most of these backbone recommendations are based on data available in the mid-1990s. Substantial additional research has been conducted since that time, enabling the development of updated backbones and control points for steel braced frame and moment frame, reinforced concrete shear wall and moment frame, and masonry and wood shear wall structures, presented herein. These recommendations are based on laboratory data and analytical studies benchmarked against these data. When possible, both cyclic backbone and monotonic envelope data are presented to facilitate the development and implementation of adaptive hysteretic elements that have the capability to reasonably simulate force-deformation behavior for any loading protocol. Figure ES-1 presents the general hysteretic backbone and cyclic envelope curves and the control points that define these curves.

Although ASCE/SEI 41-13 presents recommended acceptance criteria for evaluating the performance level associated with the many different elements covered, this report does not recommend specific criteria for each structural system and element type. Instead, this standard recommends a general protocol that includes global acceptance criteria and element action-level acceptance criteria, that can be used with the data presented herein to develop element-specific acceptance values. 


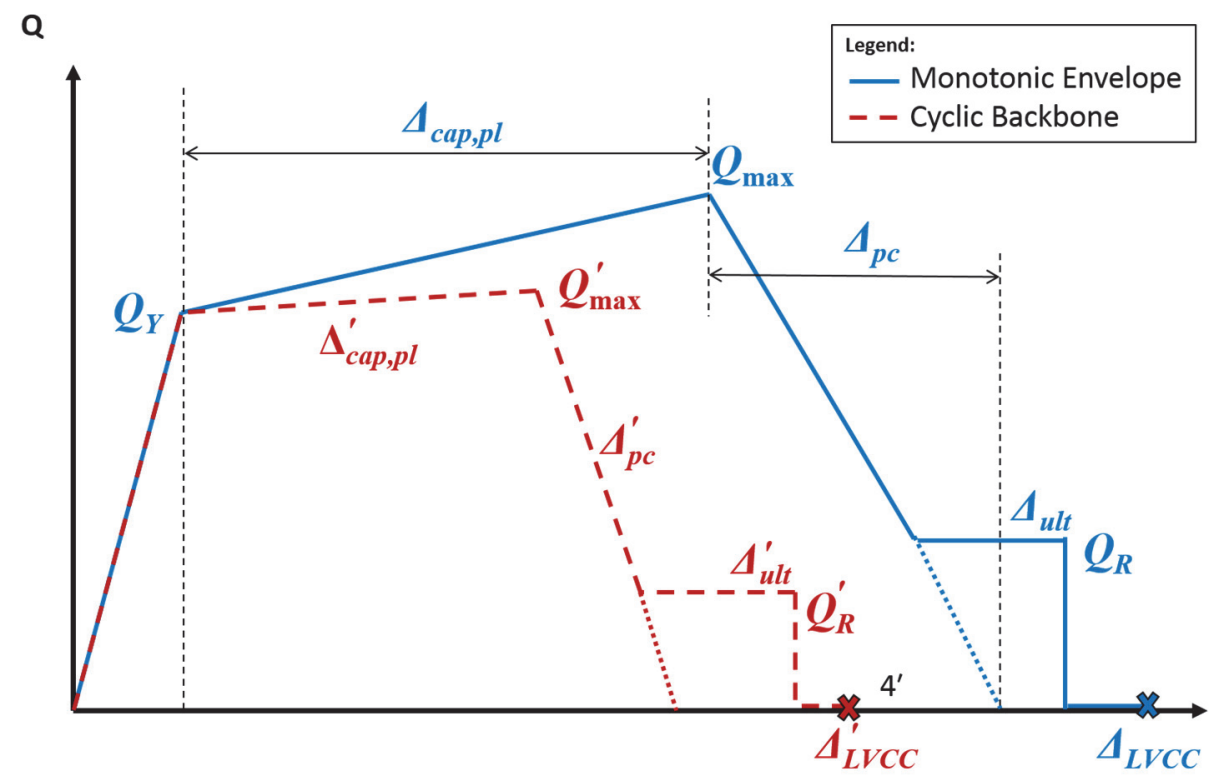

Figure ES-1 General cyclic backbone and hysteretic envelope.

For each of steel moment frame systems, steel concentrically braced frames, reinforced concrete moment frame systems, flexure- and shear-controlled reinforced concrete walls, reinforced masonry walls, and wood shear wall systems, a Chapter in this report presents information on:

- Configuration and behavior of the structural system

- Appropriate analytical models

- Historical development of code regulations governing design

- Recommended control points (force and deformation) for element backbones and envelopes

- Representative cyclic hysteretic plots

This Executive Summary presents a brief overview of these recommendations. More detailed information, and the basis for these recommendations may be found in the individual chapters, as well as a series of appendices.

\section{ES.1 Steel Moment Frame Systems}

Steel moment-resisting frames are assemblies of beams and columns, joined together with connections that restrain angular rotation between the beams and columns, thereby inducing flexure in both the beams and columns when the frames are subjected to lateral forces or displacements. These systems are inherently flexible and P-delta effects are significant to their performance. Inelastic behavior in steel moment frames designed for superior inelastic response is dominated by flexural yielding of the beams adjacent to the beam-column connections, flexural yielding of 
columns at their base, and limited yielding within column panel zones. Achieving such behavior requires a capacity design approach in which columns and connections are proportioned to develop the expected flexural strength of connected beams. Beams and columns are proportioned to have compact properties and are laterally braced such that the sections can develop their full plastic flexural capacities without developing lateral instability. To the extent that frames are not designed in this manner, inelastic response capability can be substantially reduced and limited by a number of behaviors including: lateral torsional buckling of beams or columns; local crippling of sections; fracturing of welded connections and failure of bolts.

ANSI/AISC 341, Seismic Provisions for Structural Steel Buildings, is the modern U.S design specification for steel moment frames and classifies these systems as Special, Intermediate, Ordinary, or not-detailed for seismic resistance, depending on the extent to which capacity design principles are applied in their design.

Inelastic behavior of steel moment frames proportioned for substantial inelastic response is highly dependent on loading history. Flexural hinging of beams and columns beyond rotations at which flange buckling initiates results in substantial strength and stiffness degradation with these effects accentuated during repeated cycles. Figure ES-2 illustrates representative moment-rotation plots for steel special moment frame beam-column connections subjected to three different loading histories. Steel moment frames are among the most ductile seismic force-resisting elements with cyclic response typically represented by broad hysteretic loops, such as those illustrated in Figure ES-3. Steel moment frame behavior can also be substantially influenced by the presence of concrete slabs. Slabs strengthen the beams, stiffen the frame, delay onset of beam top flange buckling, and provide lateral bracing. Axial compressive loading of steel flexural elements reduces available ductility.

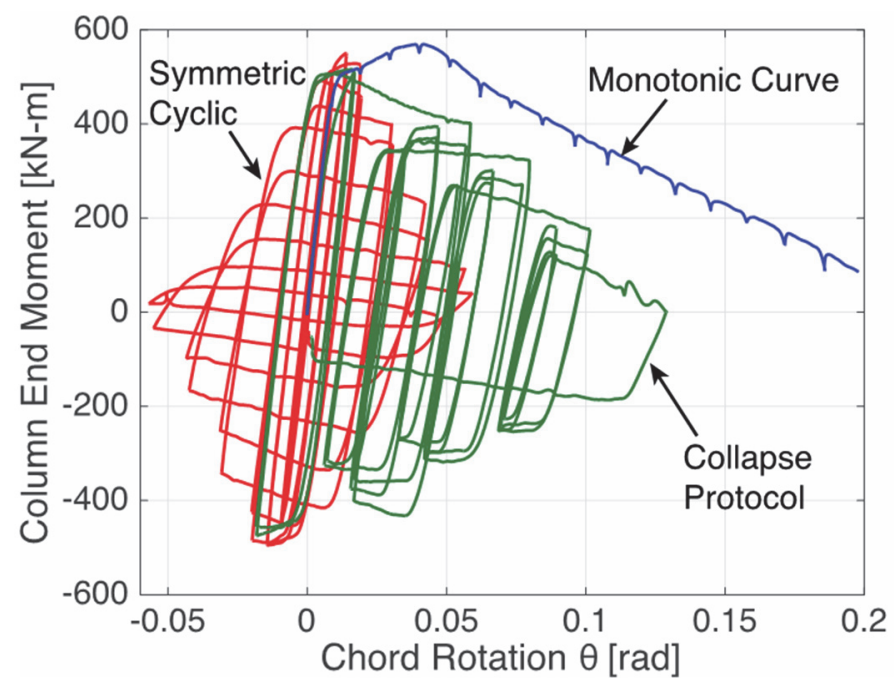

Figure ES-2 Steel moment frame assembly subjected to alternative loading protocols (Suzuki and Lignos, 2015). 


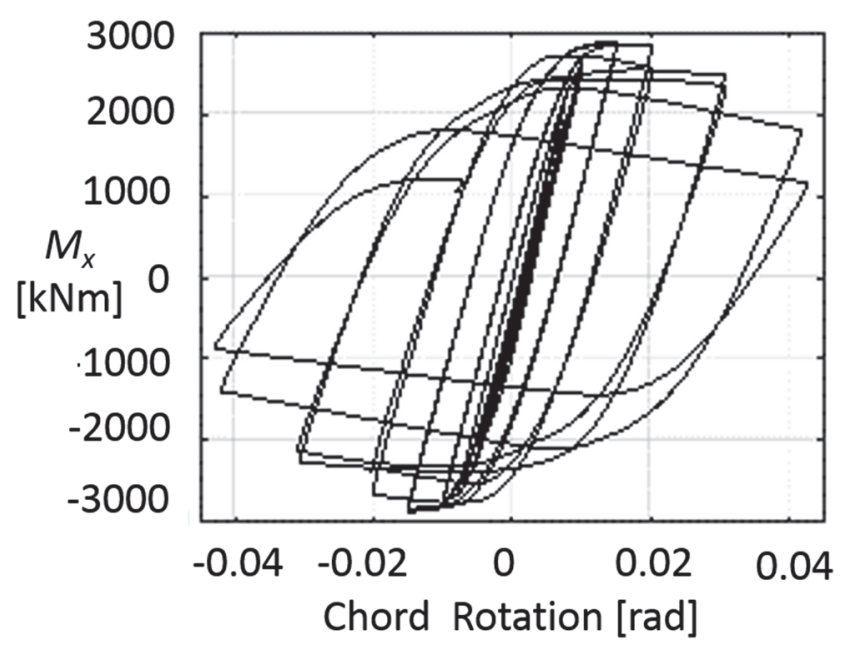

Figure ES-3 Typical steel moment frame cyclic hysteresis (FEMA, 2000d).

Hysteretic behavior of steel moment frames can be modeled using fiber elements or elements with discrete hinges to represent beams and columns and multi-node scissor type elements to model panel zones. Table ES-1 presents recommended cyclic and monotonic backbone parameters for steel moment frame beams proportioned and braced as Special steel moment frame per ANSI/AISC 341. In the table, $Q$ represents moment and $\theta$ chord angle rotation, which in most cases can be taken as equal to story drift ratio. Table ES-2 presents recommended parameters for wide flange beam-columns and Table ES-3, hollow structural section beam-columns.

In Table ES- $1, h / t_{w}$ and $b_{f} / 2 t_{f}$ are the beam web height to thickness and beam flange length to thickness ratios, $L / d$ is the beam shear span to depth ratio, $L_{b} / r_{y}$ is the ratio of the beam's unbraced length to minor axis radius of gyration, and $d$ is the beam depth.

In Table ES-2, $P_{g}$ is the axial load in the beam-column due to gravity effects; $P_{y e}$ is the expected yield strength in tension given as $R_{y} F_{Y} A_{g}$.

In Table ES-3, $D / t$ is the largest width to thickness ratio of the tubular section and $F_{y e}$ is the expected yield strength of the material.

Procedures for modeling and evaluating panel zones, partially restrained connections, column bases and column splices are provided. 


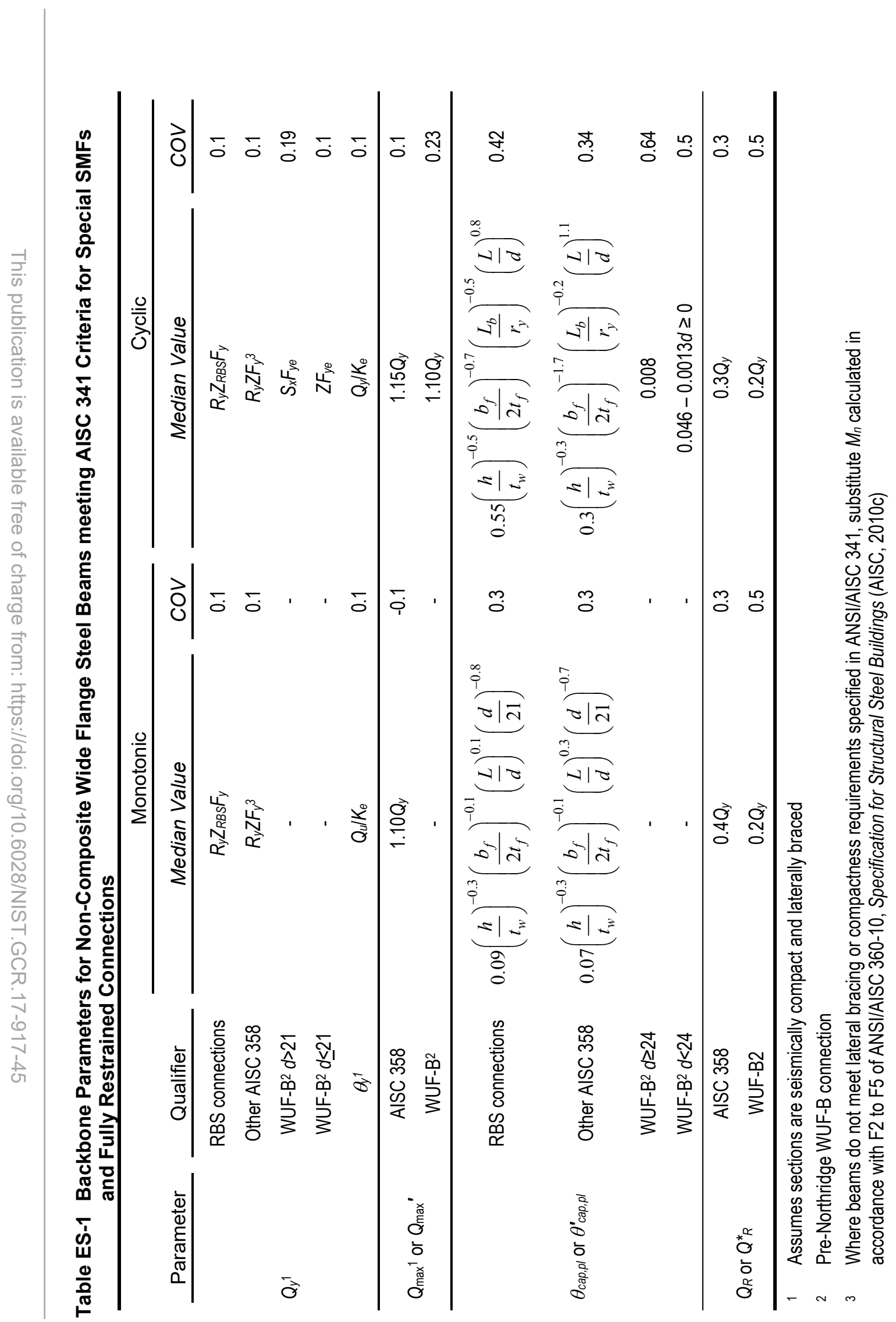




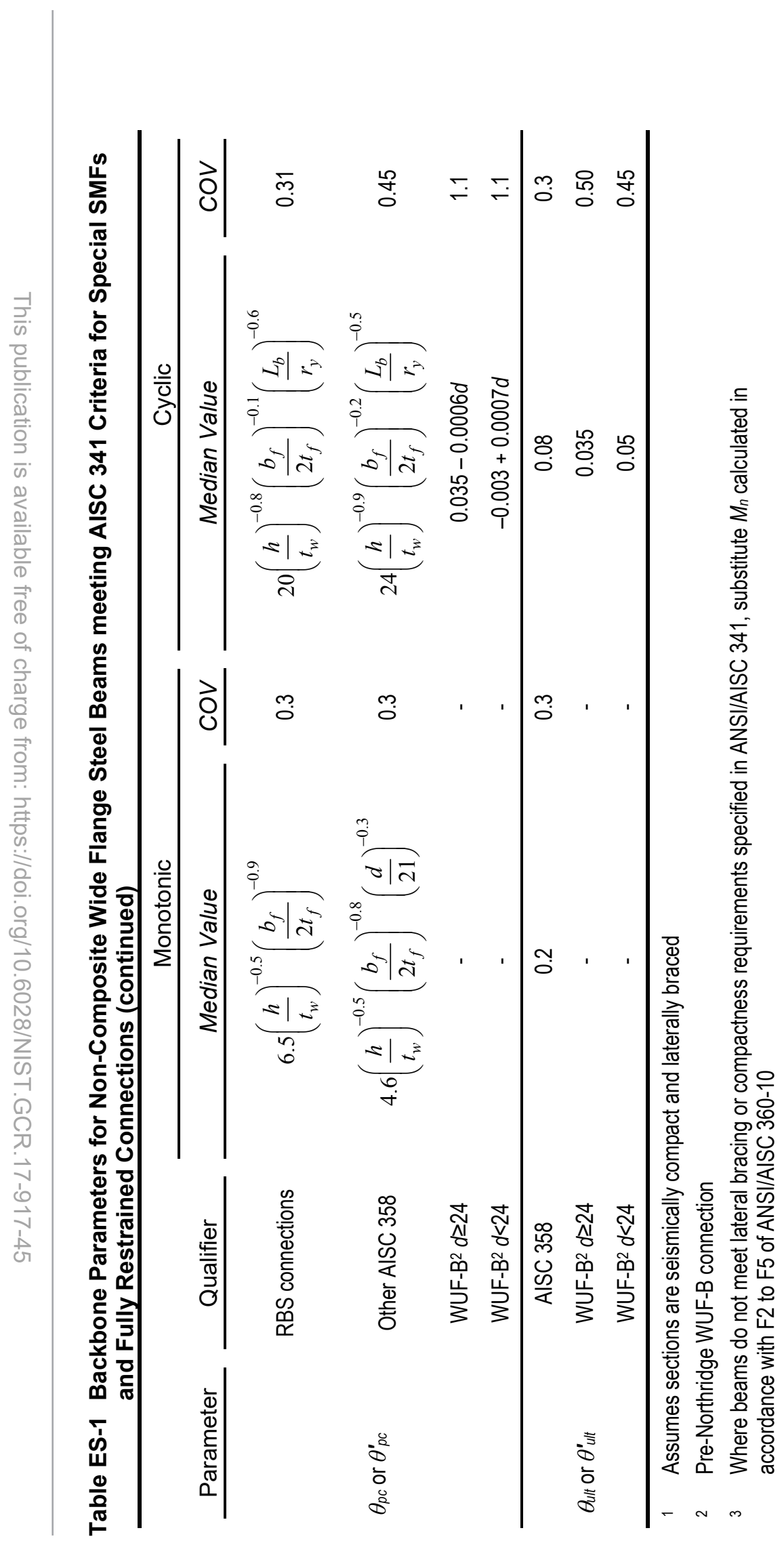




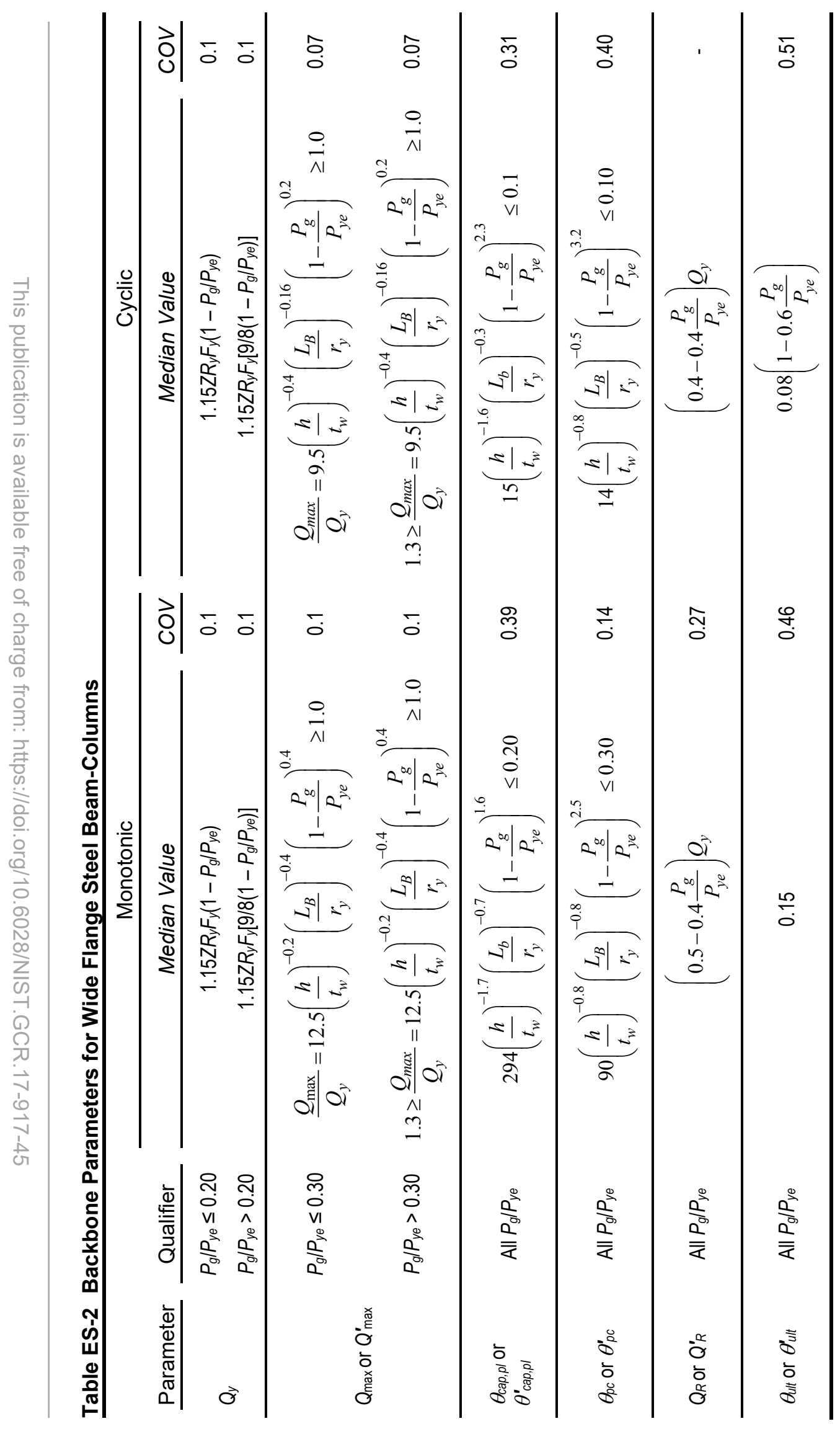



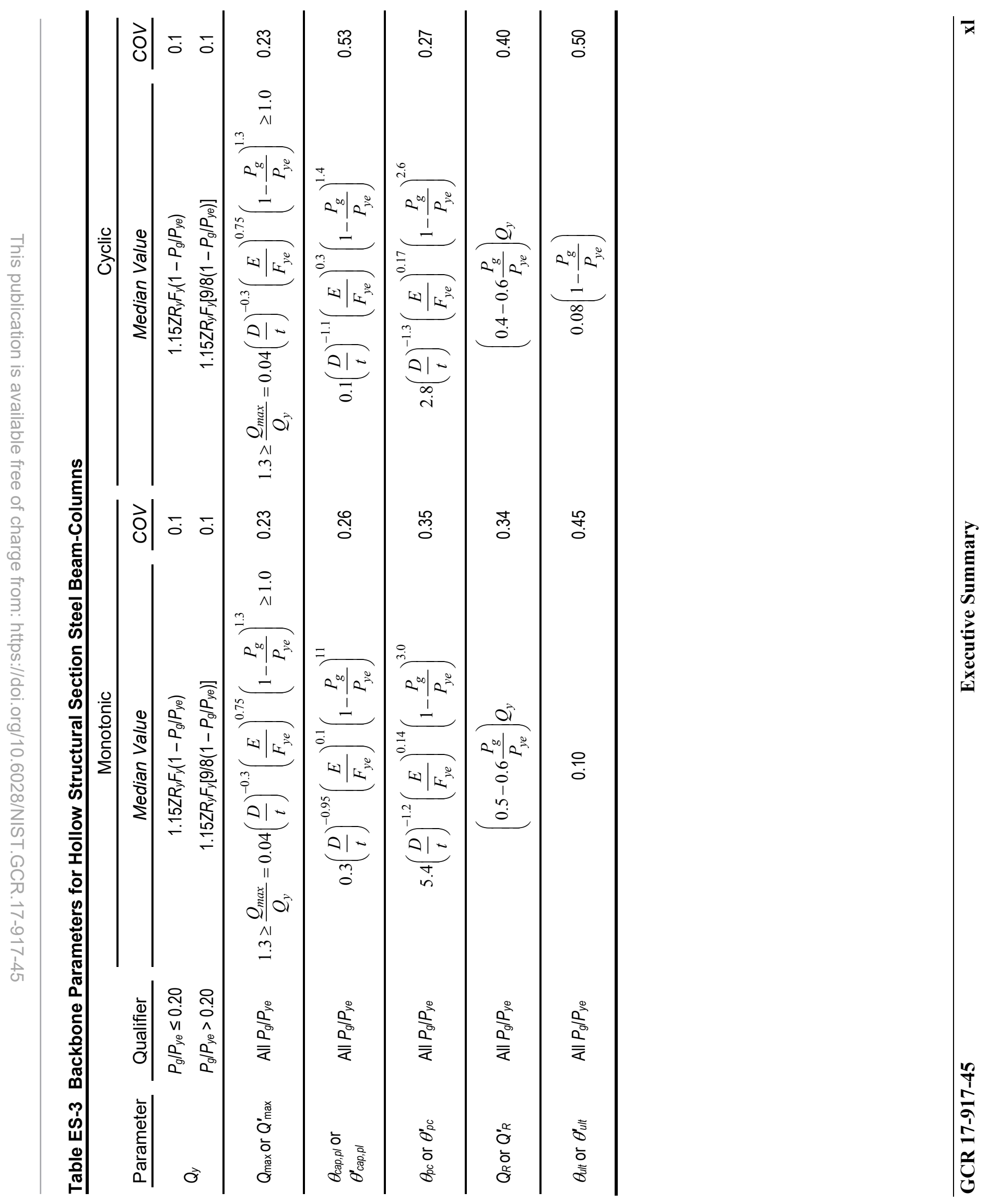


\section{ES.2 Steel Concentrically Braced Steel Frames}

Steel concentric braced frames (CBFs) consist of beams, columns, and braces joined by gusset plates at single concentric points. Assuming that gusset plates, and the associated bolts and welds are designed sufficiently strong to develop brace axial strength, nonlinear behavior of these frames at low levels of deformation is dominated by buckling and tensile yielding of braces, accompanied by yielding of the gussets. Following this nonlinear behavior, the assembly of beams and columns provide further linear and nonlinear resistance in the form of a reserve moment frame, whether designed in this manner or not.

Figure ES-4 illustrates typical hysteretic behavior of braces, controlled by buckling in compression, yielding in tension, and ultimately, fracture, typically initiating at the point of plastic hinging at brace mid-length that accompanies buckling. This hysteresis can be accurately modeled by representing each brace as an assemblage of fiber elements (a minimum of 4 are recommended) with initial out-of-plane imperfection at mid-span of $L / 500$. Gussets should be modeled as an element with axial stiffness, approximating that of the gusset, coupled with a rotational spring.

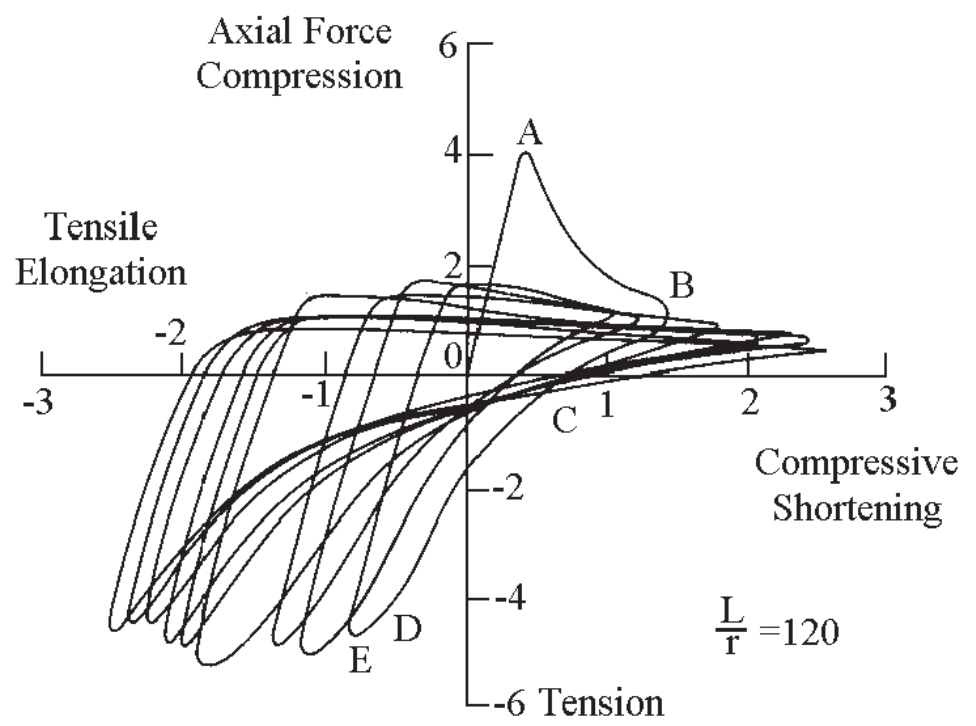

Figure ES-4 Typical brace hysteresis (Roeder and Popov,1977).

Table ES-4 presents recommended cyclic backbone control points for braces. In the table, $Q$ is the horizontal component of the brace force and $\Delta$ represents the horizontal story drift ratio, in \%; $R_{y}$ is the ratio of the expected yield stress to specified yield stress, per ANSI/AISC 341, Seismic Provisions for Structural Steel Buildings; $A_{g}$ is the brace gross cross sectional area, $\theta$ is the angle between the brace and horizontal; $F_{y}$ is the specified yield stress and $F_{c r}$ is the critical compressive stress per ANSI/AISC 360, Specification for Structural Steel Buildings, $L_{e}$ is the effective 
length of the brace, $\lambda$ is the critical $b / t$ or $h / d$ ratio for the brace and $\lambda_{h d}$ is the limiting value for this factor for highly ductile behavior, per ANSI/AISC 341.

For monotonic loading, all parameters are the same as shown for cyclic loading except that $\Delta_{c a p, p l}$ for tension and compression should be computed as the point at which ultimate tensile strain of the brace is reached and the value of $Q_{\text {max }}^{\prime}$ in compression should be taken as 0 .

Table ES-4 Cyclic Backbone Parameters for Braces

\begin{tabular}{|c|c|c|c|c|}
\hline \multirow[b]{2}{*}{ Parameter } & \multicolumn{2}{|l|}{ Tension } & \multicolumn{2}{|c|}{ Compression } \\
\hline & Median Value & COV & Median Value & COV \\
\hline$Q_{y}$ & $R_{y} F_{y} A_{g} \cos (\theta)$ & $R_{y}$ & $R_{y} F_{c r} A_{g} \cos (\theta)$ & $R_{y}$ \\
\hline$\Delta y$ & $100 \frac{Q_{y}}{A_{g} E} \frac{L_{e}}{h} \cos (\theta)$ & 0.05 & $100 \frac{Q_{y}}{A_{g} E} \frac{L_{e}}{h} \cos (\theta)$ & 0.05 \\
\hline$Q_{\max }^{\prime}$ & $R_{y} F_{y} A_{g} \cos (\theta)$ & $R_{y}$ & $0.73 R_{y} F_{c r} A_{g} \cos (\theta)$ & 0.04 \\
\hline$\Delta_{c a p, p l}^{\prime}$ & $2.5\left(\frac{\lambda}{\lambda_{h d}}\right)^{-1.53}-\Delta_{y}$ & 0.065 & $2.5\left(\frac{\lambda}{\lambda_{h d}}\right)^{-1.53}-\Delta_{y}$ & 0.065 \\
\hline$Q_{R}$ & 0 & - & 0 & - \\
\hline$\Delta_{\text {ult }}^{\prime}$ & 0 & - & 0 & - \\
\hline
\end{tabular}

\section{ES.3 Reinforced Concrete Moment-Frame Systems}

Reinforced concrete moment comprise either monolithic cast-in-place or precast concrete beam and column, or beam and slab assemblies. The relatively rigid joint between horizontal and vertical assemblies enable the frames to resist lateral forces primarily through flexural deformation in the beams, slabs or columns, with limited shear deformation in the joints. Precast assemblies joined such that they can emulate monolithic assemblies have similar behavior.

Reliable nonlinear behavior can occur through ductile flexural hinging of beams and limited ductile flexural hinging of columns accompanied by bar slip at joints. Limits on ductile behavior include buckling, and under repeated cycles, fracturing of longitudinal reinforcing steel; crushing and spalling of cover concrete; and at extreme deformations, fracture of confining reinforcement followed by crushing and spalling of member cores. Undesirable, non-ductile failure modes that prevent significant nonlinear frame response include member and joint shear failure; reinforcing splice failure; reinforcing pull-out at joints; and slab column punching shear failure.

Starting in the mid-1960s, researchers began to develop design procedures, presently embodied in the requirements for Special Moment Frames (SMF) in the ACI 318, Building Code Requirements for Structural Concrete and Commentary. Building codes have required such ductile design and detailing practices in higher seismic 
zones or design categories, since the mid-1970s. Figure ES-5 illustrates typical cyclic hysteretic response of beam column assemblies meeting modern SMF design and detailing criteria, together with commonly applied analytical hysteretic models. As shown in the figure cyclic response include modest pinching, a stable plastic range followed by strength degradation.

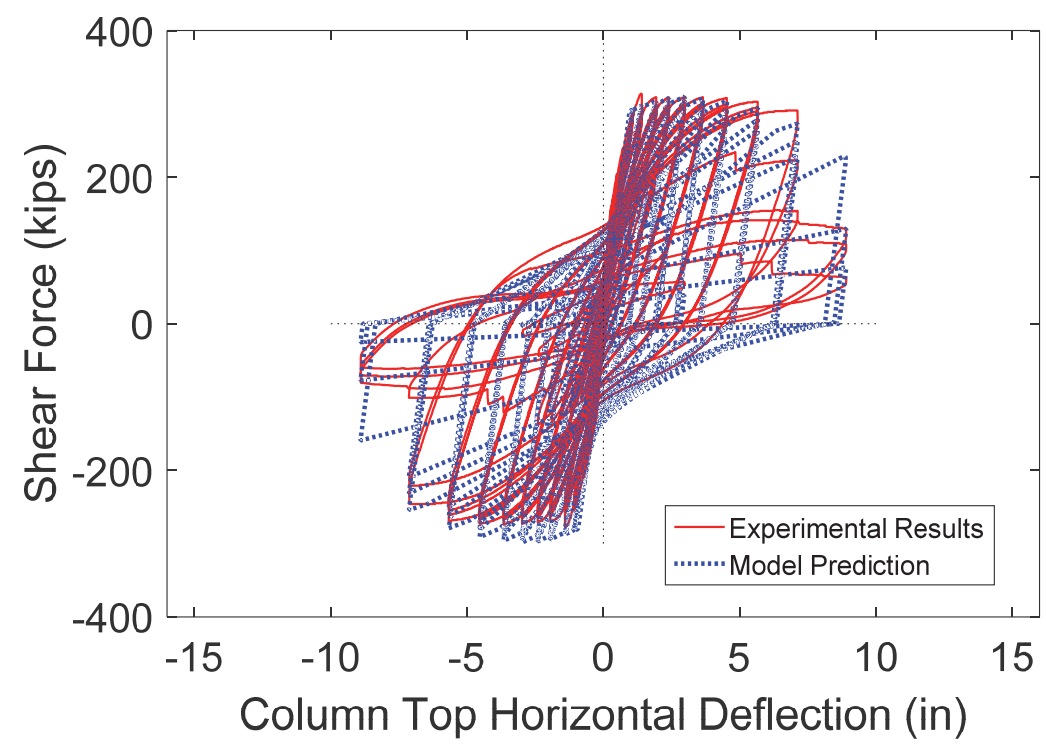

Figure ES-5 Typical hysteretic behavior and modeling of reinforced concrete special moment frame beamcolumn assembly (Nojavan et al., 2014).

The cyclic behavior in Figure ES-5 is applicable to reinforced concrete SMF beams and columns. Table ES-5 provides values for recommended control points (as defined in Figure ES-1). In the table, $M_{n}$ is the nominal moment, computed using expected material properties, and the procedures of ACI 318 considering the concurrent axial loading $P ; A_{g}$ is the gross cross sectional area; $E_{c}$ is the modulus of elasticity of concrete; $f_{c}^{\prime}$ is the expected compressive strength of the concrete; $I_{\text {eff }}$ is the effective moment of inertia, considering cracking; $I_{g}$ is the gross section moment of inertia; $v$ is the axial load ratio $P / A_{g} f_{c}^{\prime} ; \rho_{\text {sh }}$ is the hoop reinforcement ratio; $c_{\text {units }}$ is a units conversion, taken as 1.0 when $f^{\prime}{ }_{c}$ is in MPa and 6.9 when in ksi.

\section{ES.4 Flexure-Controlled Reinforced Concrete Walls}

Reinforced concrete walls have been used as lateral force-resisting elements in buildings for more than 100 years, often also serving as part of the primary vertical load-carrying system. Thin walls, less than 8 inches thick typically have a single curtain of horizontal and vertical reinforcing steel while thicker walls will typically have a curtain on each face. Walls can be arranged with single rectangular plan configurations, single rectangles with expanded boundary elements at the ends, or be constructed in L, C, and rectangular box configurations. In elevation, walls are often punched by openings for doors and windows. 

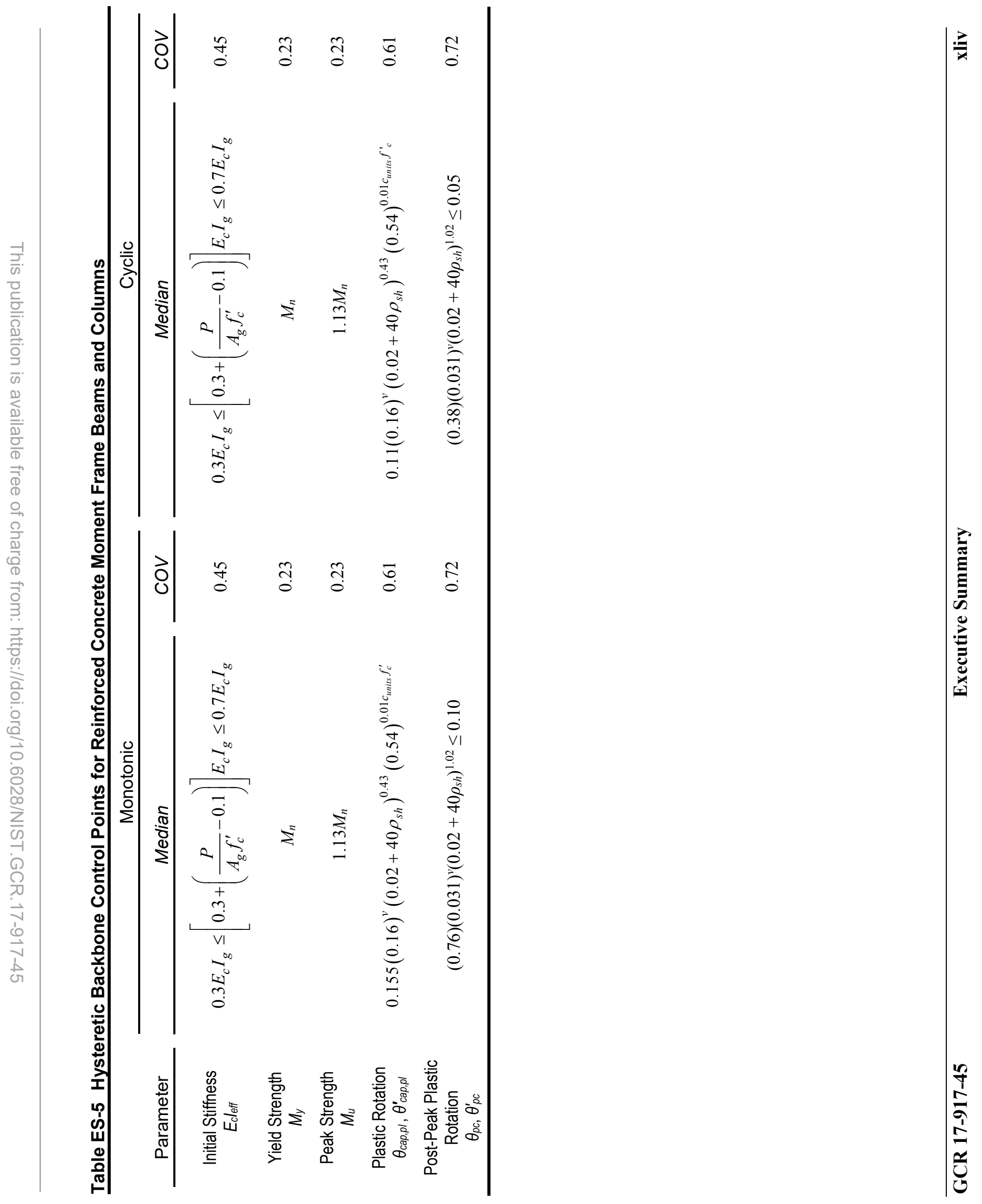
Depending on the configuration in plan and elevation, nonlinear behavior may be controlled either by flexural or shear yielding. Since the 1970s building codes have encouraged, but not required, design of walls that yield in the flexural mode. Concentrated boundary elements provided with transverse confining reinforcement around the vertical steel may be present depending on the era of design, the wall's configuration, and the computed demands during design. In the period 1979 to 1997, slender walls were often designed considering only the steel in boundary elements resisting overturning demands. Regardless, determination of flexural strength should consider all vertical steel that is present.

Flexural nonlinear behavior of high aspect ratio walls $(l / w \geq 2)$ is accommodated through flexural/tensile cracking of concrete followed by yielding of the steel reinforcement, bond slip at the wall base, spalling of compressive cover, buckling of reinforcing bars, crushing of concrete at boundaries, and fracture of reinforcing steel in tension following buckling. The ability of a wall to develop all these behaviors depends on the detailing of steel reinforcement as well as the axial load ratio. Walls detailed with confinement of concrete and reinforcing at ones of high strain can exhibit considerable flexural ductility with some pinching due to opening and closing of cracks and strength degradation occurring at later stages resulting from buckling of reinforcing and spalling of concrete. Figure ES-6 presents typical cyclic hysteretic response of well-detailed walls.

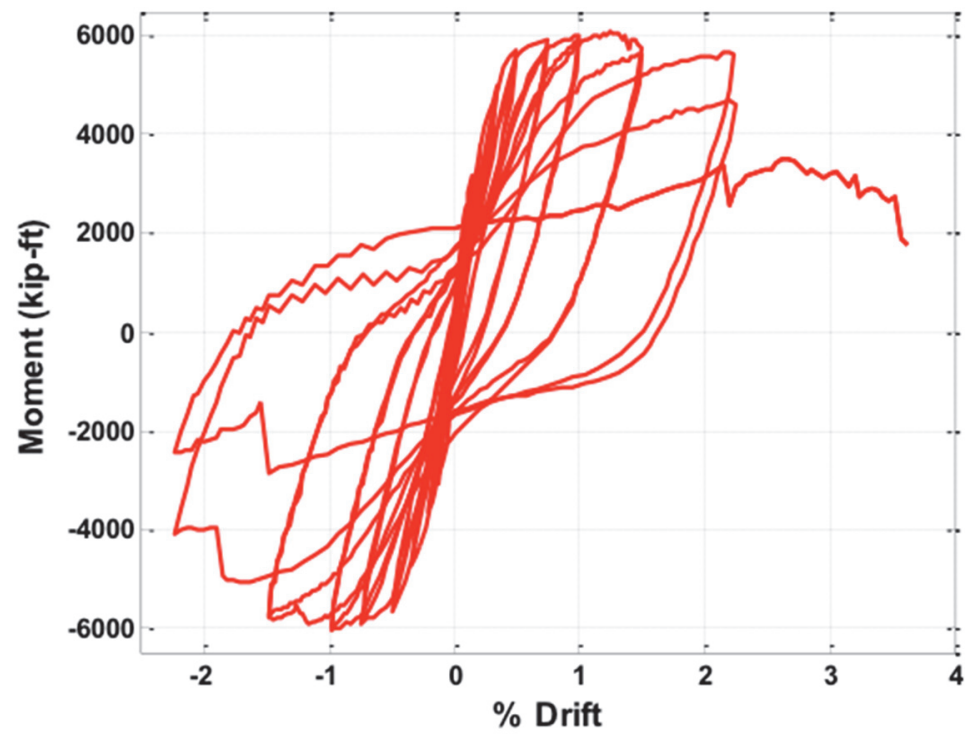

Figure ES-6 Typical hysteretic flexural response of rectangular reinforced concrete wall with superior detailing of reinforcement (Behrouzi et al., 2015).

Although other model types are available in research software, for practical office use, modeling of walls with inelastic behavior dominated by flexural yielding typically will employ either two-dimensional line elements with discrete hinges or 
fiber type shell elements, capable of simulating spread of yielding and plastic hinging. The former element type is not capable of capturing flexure-shear interaction or the dependence of flexural capacity on varying axial load, and cannot accurately capture onset of degradation or post-degradation response. The valid range of modeling using line elements with discrete hinges should be taken as the deformation associated with development of peak resistance, as determined by moment curvature analysis, not to exceed a hinge rotation of 0.016 radians.

For walls in which failure results from compressive crushing or buckling in flexure and/or buckling and fracture of longitudinal reinforcing steel, modeling to simulate behavior beyond development of peak strength using fiber type shell element is possible, as discussed below. This procedure is not applicable to walls that fail in combined compression-shear failure. Figure ES-7 indicates the probable failure mode as a function of peak shear stress demand, expressed as a factor of $\sqrt{f_{c}^{\prime}}$ where $f_{c}^{\prime}$ is expressed in units of psi.

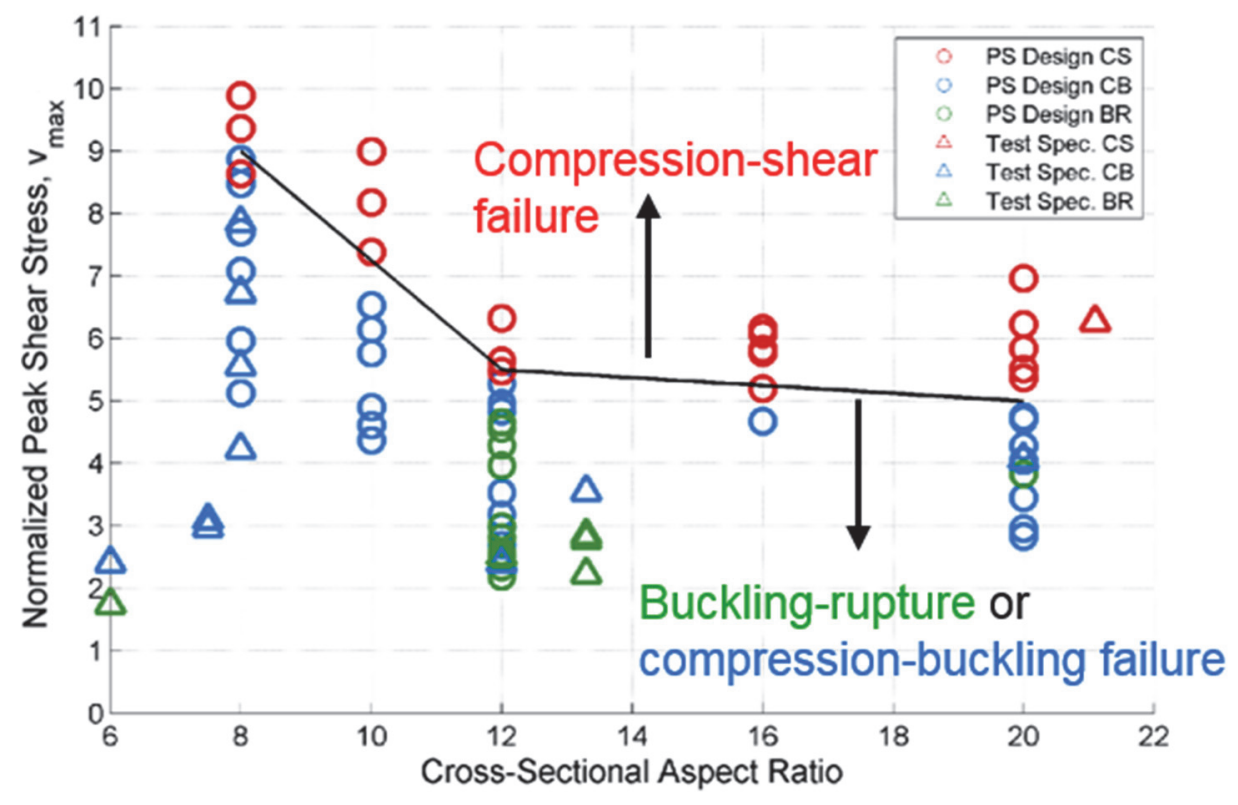

Figure ES-7 Wall flexural failure mode as a function of shear stress and aspect ratio.

For walls indicated to fail in the "buckling-rupture/compression-buckling modes" in Figure ES-7, modeling beyond development of peak strength is possible if regularization of constitutive properties, a process of matching the properties based on dissipated energy, is performed. Regularization of concrete material response is most easily accomplished by defining concrete strain capacity as a function of a mesh-dependent characteristic length and the concrete crushing energy, where crushing energy describes the energy dissipated as the concrete goes from the point of maximum strength to the point of residual compressive strength. Regularization of 
reinforcing steel is performed by defining strain capacity as a function of a meshdependent characteristic length and a steel yielding energy. Figure ES-8 illustrates these concepts.

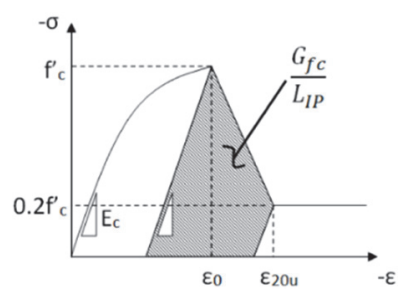

(a) unconfined concrete compression envelope

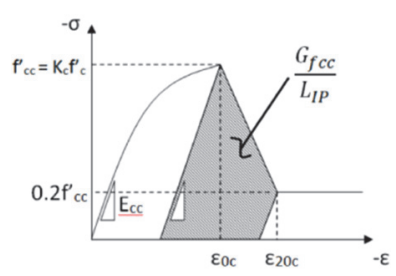

(b) confined concrete compression envelope

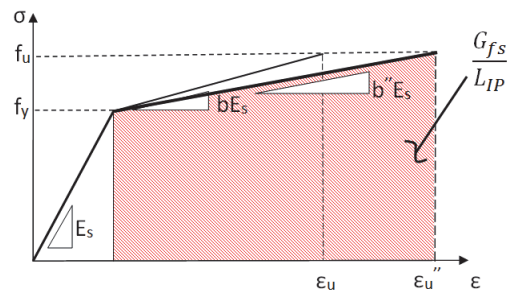

(c) reinforcing steel response envelope for tension and compression

Figure ES-8 Regularization of concrete and steel constitutive properties.

In the figure, $G_{f c}$ and $G_{f c c}$ are respectively the characteristic strain energy of unconfined and confined concrete; $L_{I P}$ is the height of the fiber wall element; $\varepsilon_{0}$ and $\varepsilon_{0 c}$ are respectively the concrete strain at which peak strength is obtained for unconfined and confined concrete respectively; $\varepsilon_{20}$ and $\varepsilon_{20 c}$ are the strains at which concrete strength degrades to $20 \%$ of the peak value; $G_{f s}$ is the characteristic energy for steel reinforcement; and $E_{c}$ is the modulus of elasticity of the concrete.

For rectangular wall elements, the strain energy quantities may be taken as $G_{f_{c}}=0.079 f_{c}^{\prime}$ for unconfined concrete and $G_{f c}=0.13 f_{c}^{\prime}$ for fully confined concrete and $8\left(\left(f_{c c}^{\prime} / f_{c}^{\prime}\right)-1\right)$ for concrete with intermediate confinement. The limiting regularized concrete strains for concrete may be taken as:

$$
\varepsilon_{20 u}=\frac{G_{f_{c}}}{0.6 f_{c}^{\prime}}-\frac{0.8 f_{c}^{\prime}}{E_{c}}
$$

The value of the strain energy for steel may be taken as:

$$
G_{s}=4\left(\varepsilon_{u^{\prime \prime}}-\varepsilon_{y}\right)\left(f_{u}+f_{y}\right)
$$

which, for For ASTM A706, Grade 60 steel, $G_{s}$ may be taken as $11 \mathrm{kips} / \mathrm{in}^{2}$. The value of the limiting regularized steel strain should be taken as:

$$
\varepsilon_{u}^{\prime \prime}=0.5\left(\varepsilon_{y}+\frac{L_{\text {gage }}}{L_{I P}}\left(\varepsilon_{u}-\varepsilon_{y}\right)\right)
$$

where $L_{\text {gage }}$ will typically have a value of 8 inches, and for ASTM A706, Grade 60 steel, $\varepsilon_{u}^{\prime \prime}$ may be taken as $\varepsilon_{u}^{\prime \prime}=0.07 L_{\text {gage }} / L_{I P}$. The valid range of modeling should be taken as that deformation producing strains of $\varepsilon_{20 u}$ or $\varepsilon_{u}^{\prime \prime}$, whichever is less.

\section{ES.5 Shear-Controlled Reinforced Concrete Walls}

Cyclic nonlinear shear behavior of low aspect ratio $\left(h / l_{w} \leq 1\right)$ walls tends to exhibit limited ductility with significant pinching of hysteretic loops and rapid post-peak degradation as illustrated in Figure ES-9. 


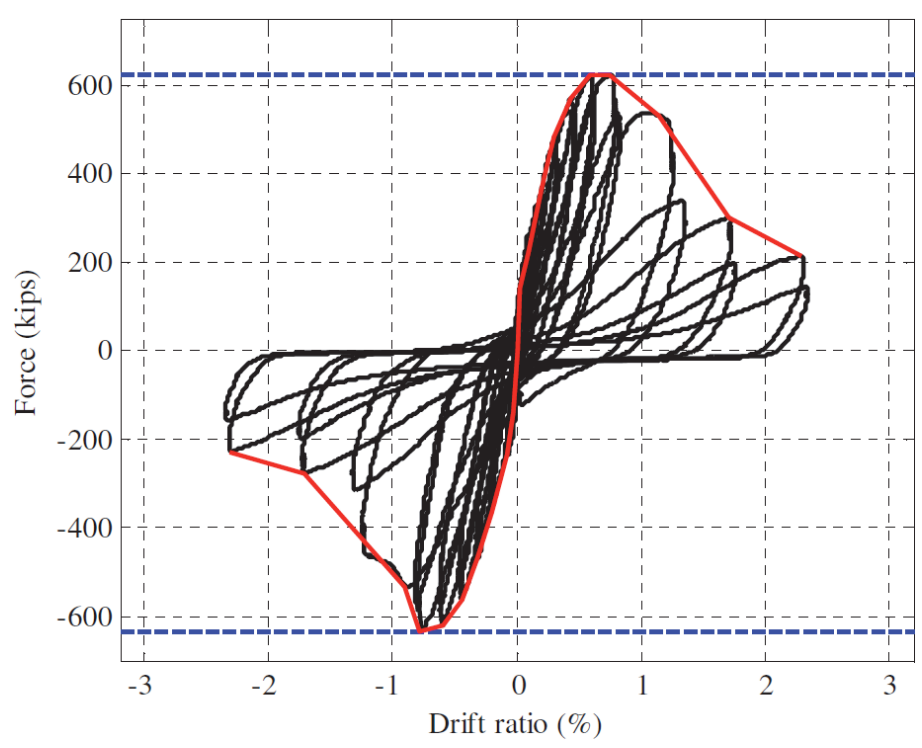

Figure ES-9 Representative cyclic hysteresis of shearcontrolled reinforced concrete walls (Luna et al., 2015).

Figure ES-10 presents the recommended cyclic backbone for shear walls controlled by shear behavior. The control points are: A, initiation of cracking; B, development of peak strength; $\mathrm{C}$, onset of degradation; and, points $\mathrm{D}^{80}, \mathrm{D}^{40}$ and $\mathrm{D}^{20}$ respectively the points at degradation to $80 \%, 40 \%$ and $20 \%$ of peak shear strength respectively. In sufficient data is available to permit characterization of monotonic response, different from cyclic response.

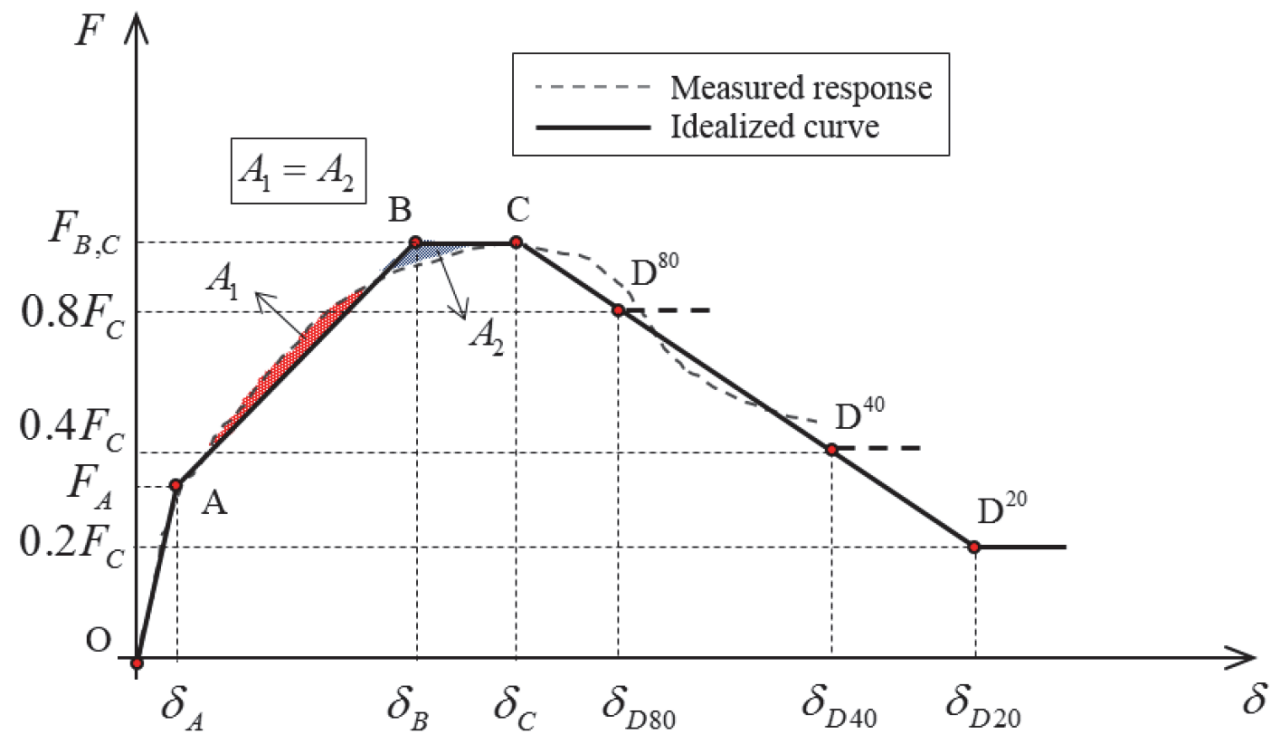

Figure ES-10 Cyclic backbone for shear nonlinear in concrete walls.

Median estimates of the peak shear strength for rectangular walls are best obtained using the criteria of Section 11.5.4.3 of ACI 318-14 for $V_{n}$, but using expected 
properties of concrete and steel reinforcement. Median shear strength for walls with enlarged boundary elements or return walls can be calculated using the expression:

$$
V_{n}=\left(8 \sqrt{f_{c}^{\prime}}-2.5 \sqrt{f_{c}^{\prime}} \frac{h_{w}}{l_{w}}+\frac{N_{u}}{4 l_{w} t_{w}}+\rho_{l} f_{y}\right)
$$

where, $f_{c}^{\prime}$ is the expected concrete compressive strength, $f_{y}$ is the longitudinal reinforcement yield strength, $h_{w} l_{w}$ and $t_{w}$ are the wall height, length and thickness respectively, $\rho$ is the reinforcement ratio for horizontal steel and $N_{u}$ is the axial force on the wall. When the wall is in net tension, only the steel portion of the expression should be used. Table ES-6 presents the values of the control points with strength given as fractions of the median strength, $V_{n}$.

\section{Table ES-6 Cyclic Backbone Control Points for Reinforced Concrete Walls Controlled by Shear}

\begin{tabular}{|c|c|c|}
\hline Control Point & $V$ & $\delta$ \\
\hline A initiation of cracking & $0.5 V_{n}$ & $0.1 \%$ \\
\hline B- Peak strength & $V_{n}$ & $0.5 \%$ \\
\hline C- Initiation of Degradation & $V_{n}$ & \\
\hline Rectangular walls & & $1.0 \%$ \\
\hline Flanged walls, $h_{w} / l_{w}<1$ & & $0.7 \%$ \\
\hline Flanged walls $1<h_{w} / l_{w}<2$ & & $0.9 \%$ \\
\hline$D^{80}$ & $0.8 V_{n}$ & Linear interpolation \\
\hline$D^{40}$ & $0.4 V_{n}$ & Linear interpolation \\
\hline$D^{20}-20 \% V_{n}$ & $0.2 V_{n}$ & \\
\hline Rectangular walls $h_{w} / l_{w}<1$ & & $2.1 \%$ \\
\hline Rectangular walls $1<h_{w} / l_{w}<2$ & & $2.5 \%$ \\
\hline $\begin{array}{l}\text { Flanged walls } h_{w} / l_{w}<1 \text { and } \\
P I P_{n}<0.05\end{array}$ & & $1.8 \%$ \\
\hline $\begin{array}{l}\text { Flanged walls } h_{w} / l_{w}<1 \text { and } \\
0.05<P / P_{n}<0.2\end{array}$ & & $1.4 \%$ \\
\hline $\begin{array}{l}\text { Flanged walls } 1<h_{w} / l_{w}<2 \text { and } \\
P I P_{n}<0.05\end{array}$ & & $2.6 \%$ \\
\hline $\begin{array}{l}\text { Flanged walls } h_{w} / l_{w}<1 \text { and } \\
0.05<P / P_{n}<0.2\end{array}$ & & $1.6 \%$ \\
\hline
\end{tabular}

In addition, a modified version of the Ibarra Krawinkler pinching model for simulating nonlinear dynamic response of such walls has been developed and compared against data from available tests. Parameters for implementing this model are presented. 


\section{ES.6 Reinforced Masonry Walls}

Reinforced masonry walls are of three basic types: cavity walls, in which reinforcing and grout is placed within a central cavity between two wythes of solid masonry; partially grouted walls comprising a single wythe of hollow concrete or clay masonry units, with reinforcing placed in selected vertical and horizontal cells, which cells are grouted, and sometimes, in horizontal mortar joints; and solid-grouted walls, similar to partially grouted walls except that all cells are filled with grout and horizontal reinforcing is placed in hollow bond beam units, rather than in joints. To qualify as Special Reinforced Masonry, walls must be solid grouted with reinforcing in each direction spaced not greater than 48 inches on center. There is extensive test data available for special reinforced masonry walls, limited data available for partially reinforced walls and negligible data for cavity walls, which are not covered in this report.

Nonlinear behavior of reinforced masonry walls may be dominated by flexural or shear behavior. Flexural modes include flexural cracking, reinforcement yielding, bond slip, buckling and fracture, with spalling and crushing of masonry in compressive zones and potentially buckling of compressive zones. Behavior is generally ductile and exhibits hysteresis typified by that illustrated in Figure ES-11. Shear behavior is dominated by diagonal cracking, sliding at bed joints and at base connection to foundations, slip and buckling of reinforcing. Behavior is generally less ductile than that exhibited by walls dominated by nonlinear flexural behavior and is typified by pinched, degrading hysteretic loops, such as those illustrated in Figure ES-12.

Modeling of masonry walls typically is performed using standard two-dimensional frame elements with rigid links to represent the large panel zones at intersections of coupling beams and wall piers, discrete hinges representing nonlinear flexural behavior and nonlinear springs in series with the wall or beam elements to represent shear nonlinearity. Figure ES-13 illustrates the recommended moment-curvature backbone for a solid grouted reinforced masonry wall controlled by inelastic flexural behavior. Table ES-7 provides recommended cyclic control points and Table ES-8 monotonic control points respectively for rectangular walls with different reinforcing and axial compression ratios. It is recommended that $\Delta_{u l t}$ computed for masonry piers using these inelastic relations not exceed $4 \%$ of pier height. 


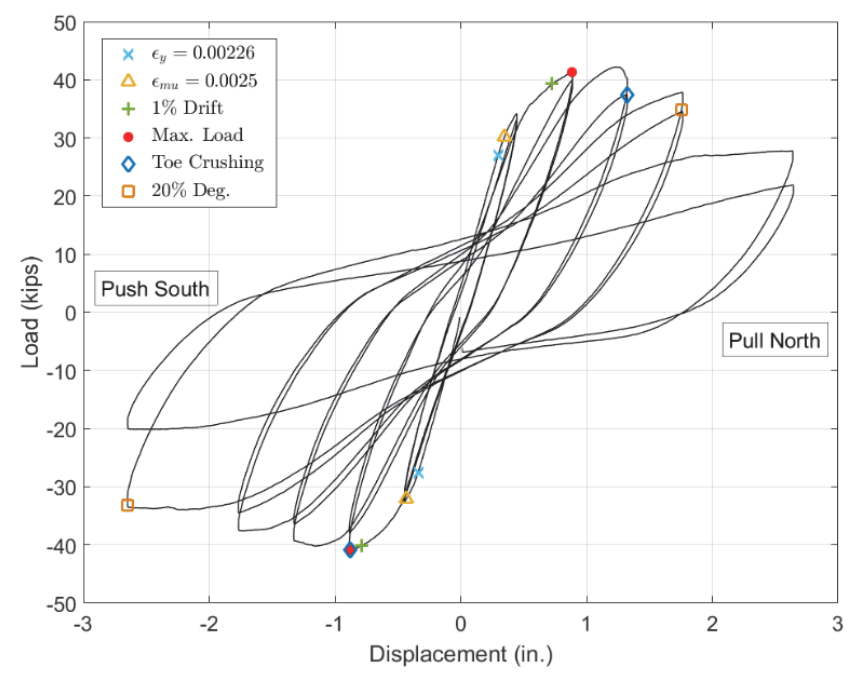

Figure ES-11 Typical cyclic hysteresis, flexurally yielding solid grouted wall (Sherman, 2011).

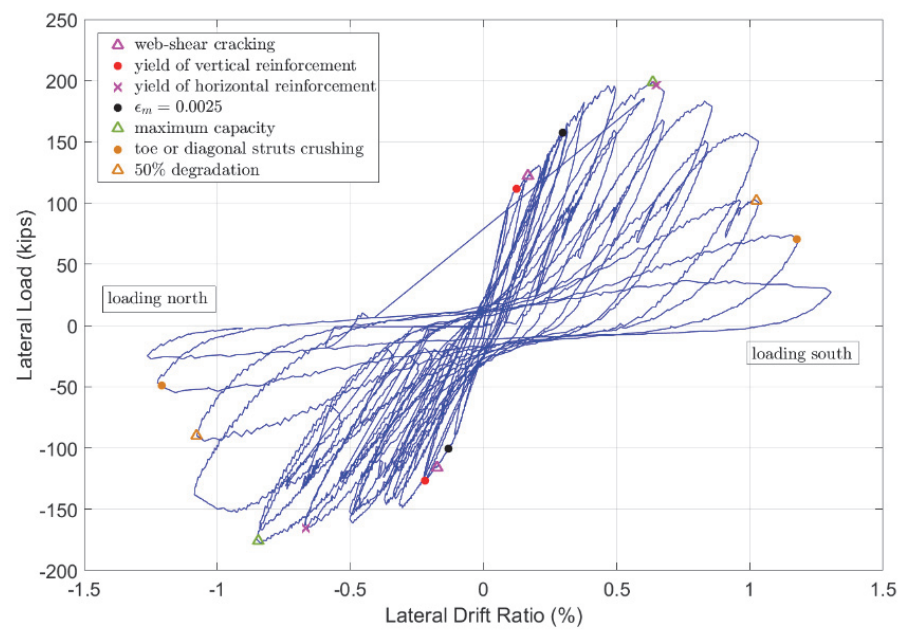

Figure ES-12 Typical cyclic hysteresis, shear yielding solid grouted wall (Ahmadi, 2012).

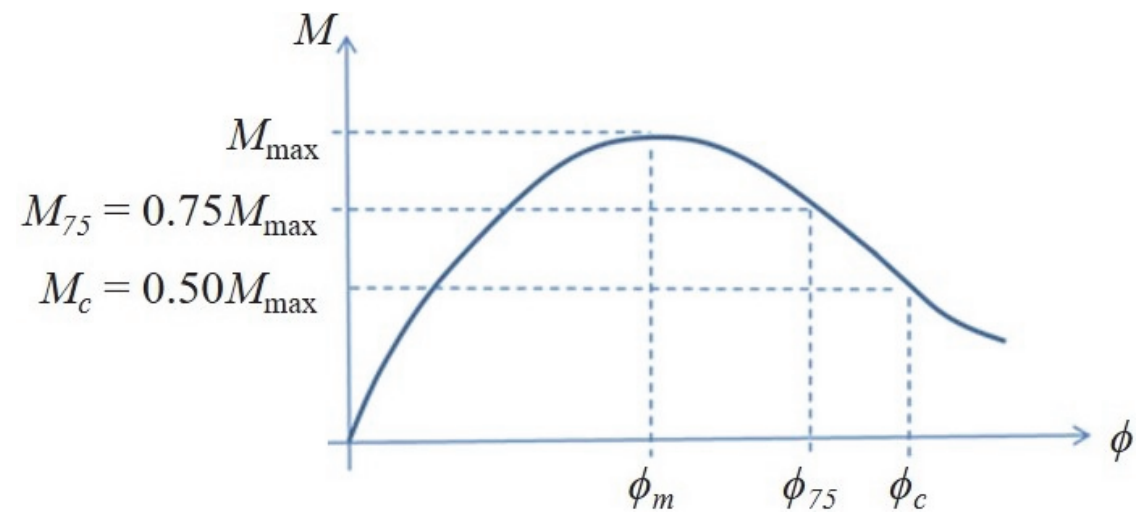

Figure ES-13 Cyclic moment-curvature backbone control points for solid-grouted reinforced masonry walls. 
Table ES-7 Cyclic Moment Curvature Parameters for Rectangular Reinforced Masonry Walls

\begin{tabular}{|c|c|c|c|c|c|}
\hline$\alpha=\left(f_{y} / f_{m}^{\prime}\right) \rho_{v}$ & $\beta=P /\left(f^{\prime} m A_{n}\right)$ & $\phi_{m} l_{w}$ & $\phi_{75 / w}$ & $\phi_{c} / w$ & $M_{\max } /\left(f_{m}^{\prime} A_{n} l_{w}\right)$ \\
\hline \multirow{4}{*}{0.01} & 0 & 0.0782 & 0.1002 & 0.1210 & 0.0060 \\
\hline & 0.05 & 0.0481 & 0.1290 & 0.2099 & 0.0285 \\
\hline & 0.1 & 0.0224 & 0.0505 & 0.0785 & 0.0487 \\
\hline & 0.15 & 0.0151 & 0.0289 & 0.0435 & 0.0662 \\
\hline \multirow{4}{*}{0.05} & 0 & 0.0681 & 0.0904 & 0.1073 & 0.0285 \\
\hline & 0.05 & 0.0362 & 0.0631 & 0.1132 & 0.0472 \\
\hline & 0.1 & 0.0201 & 0.0415 & 0.0947 & 0.0653 \\
\hline & 0.15 & 0.0139 & 0.0271 & 0.0408 & 0.0810 \\
\hline \multirow{4}{*}{0.10} & 0 & 0.0355 & 0.0540 & 0.0673 & 0.0490 \\
\hline & 0.05 & 0.0244 & 0.0535 & 0.0849 & 0.0677 \\
\hline & 0.1 & 0.0171 & 0.0376 & 0.0767 & 0.0833 \\
\hline & 0.15 & 0.0125 & 0.0260 & 0.0383 & 0.0978 \\
\hline \multirow{4}{*}{0.15} & 0 & 0.0328 & 0.0567 & 0.0752 & 0.0712 \\
\hline & 0.05 & 0.0206 & 0.0498 & 0.0841 & 0.0870 \\
\hline & 0.1 & 0.0151 & 0.0350 & 0.0685 & 0.1012 \\
\hline & 0.15 & 0.0115 & 0.0248 & 0.0363 & 0.1147 \\
\hline \multirow{4}{*}{0.20} & 0 & 0.0232 & 0.0529 & 0.0763 & 0.0904 \\
\hline & 0.05 & 0.0179 & 0.0478 & 0.0872 & 0.1042 \\
\hline & 0.1 & 0.0136 & 0.0324 & 0.0616 & 0.1178 \\
\hline & 0.15 & 0.0121 & 0.0238 & 0.0350 & 0.1302 \\
\hline
\end{tabular}

Table ES-8 Monotonic Moment Curvature Parameters for Rectangular Reinforced Masonry Walls

\begin{tabular}{|c|c|c|c|c|c|}
\hline$\alpha=\left(f_{y} / f_{m}^{\prime}\right) \rho_{v}$ & $\underline{\beta}=P /\left(f_{m}^{\prime} A_{n}\right)$ & $\Phi_{m} l_{w}$ & $\phi_{75} / w$ & $\phi_{c} l_{w}$ & $M_{\max } /\left(f_{m}^{\prime} A_{n} l_{w}\right)$ \\
\hline \multirow{4}{*}{0.01} & 0 & 0.1083 & 0.1446 & 0.1731 & 0.0066 \\
\hline & 0.05 & 0.0488 & 0.2028 & very large & 0.0285 \\
\hline & 0.1 & 0.0225 & 0.0509 & very large & 0.0487 \\
\hline & 0.15 & 0.0151 & 0.0289 & 0.0435 & 0.0663 \\
\hline \multirow{4}{*}{0.05} & 0 & 0.1172 & 0.1582 & 0.1901 & 0.0304 \\
\hline & 0.05 & 0.0361 & 0.1728 & 0.3226 & 0.0476 \\
\hline & 0.1 & 0.0203 & 0.0416 & 0.2789 & 0.0655 \\
\hline & 0.15 & 0.0139 & 0.0271 & 0.0409 & 0.0811 \\
\hline
\end{tabular}


Table ES-8 Monotonic Moment Curvature Parameters for Rectangular Reinforced Masonry Walls (continued)

\begin{tabular}{|c|c|c|c|c|c|}
\hline$\alpha=\left(f_{y} / f_{m}^{\prime}\right) \rho_{v}$ & $\beta=P /\left(f^{\prime} m A_{n}\right)$ & $\Phi_{m} /_{w}$ & $\phi_{75} / w$ & $\phi_{c} l_{w}$ & $M_{\max } /\left(f_{m}^{\prime} A_{n} / w\right)$ \\
\hline \multirow{4}{*}{0.10} & 0 & 0.0511 & 0.1736 & 0.2249 & 0.0516 \\
\hline & 0.05 & 0.0250 & 0.0638 & 0.2933 & 0.0682 \\
\hline & 0.1 & 0.0173 & 0.0378 & very large & 0.0836 \\
\hline & 0.15 & 0.0127 & 0.0261 & 0.0384 & 0.0979 \\
\hline \multirow{4}{*}{0.15} & 0 & 0.0331 & very large & very large & 0.0723 \\
\hline & 0.05 & 0.0209 & 0.0545 & 0.2859 & 0.0875 \\
\hline & 0.1 & 0.0152 & 0.0350 & very large & 0.1015 \\
\hline & 0.15 & 0.0116 & 0.0248 & 0.0364 & 0.1148 \\
\hline \multirow{4}{*}{0.20} & 0 & 0.0236 & 0.1072 & very large & 0.0913 \\
\hline & 0.05 & 0.0182 & 0.0476 & 0.2840 & 0.1048 \\
\hline & 0.1 & 0.0137 & 0.0325 & 0.0619 & 0.1181 \\
\hline & 0.15 & 0.0121 & 0.0239 & 0.0352 & 0.1304 \\
\hline
\end{tabular}

Table ES-9 presents statistics on the bias and variability observed in comparisons of analytical predictions of backbone behavior obtained using the data in Tables ES-7 and ES- 8 when compared with available laboratory data. The bias is presented as the ratio of the calculated value to the laboratory-measured value.

Table ES-9 Bias and Uncertainty Associated with Moment Curvature Control Points

\begin{tabular}{|c|c|c|c|c|}
\hline Parameter & $Q_{\max }$ & $\Delta_{m}$ & $\Delta_{75}$ & $\Delta_{c}$ \\
\hline Bias & 1.02 & 1.06 & 0.94 & 1.20 \\
\hline Coefficient of Variation & 0.11 & 0.46 & 0.33 & 0.30 \\
\hline
\end{tabular}

Figure ES-14 presents the recommended cyclic backbone for shear resistance of solid grouted reinforced masonry walls. $Q_{\max }$ should be computed using the applicable formula in TMS 402 using expected material properties. The contribution of the masonry to shear strength should be neglected where walls are in net tension. The residual strength, $Q_{R}$ should be taken as the contribution to shear strength provided by the reinforcement alone. For partially grouted walls, it is recommended that $Q_{\max }$ and $Q_{R}$ be taken as $75 \%$ of the values for fully grouted walls. The drift at the peak strength $Q_{\max }$ should be reduced from $0.5 \%$ to $0.2 \%$, and that corresponding to $Q_{r}$ be reduced from $1 \%$ to $0.4 \%$. The maximum allowable drift is to be capped at $0.8 \%$ rather than $2 \%$. 


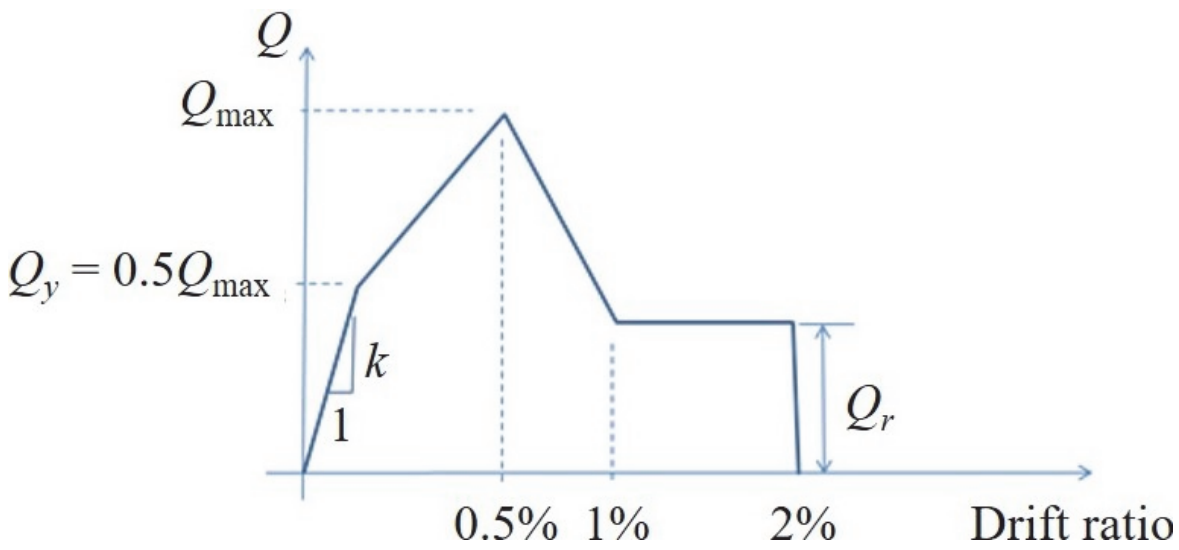

Figure ES-14 Cyclic backbone for fully grouted reinforced masonry walls in shear.

\section{ES.7 Wood Shear Wall Systems}

This structural element type consists of vertical wood studs, commonly of $2 \times 4$ or $2 \times 6$ framing, toe- or face-nailed to top and bottom flat wood framing, termed sill and top plates. Modern walls intended to provide shear resistance are sheathed on one or two sides with plywood or oriented strand board panels, commonly in sizes of 4 feet by 8 feet, and with one or more types of finish materials including gypsum board or cement plaster. Archaic walls and walls not intended to be part of a structure's lateral force-resisting system may be sheathed with gypsum board, plaster on wood or metal lath, or straight or diagonal timber sheathing with a finish of gypsum or plaster.

Damage under cyclic loading includes cracking of gypsum and plaster sheathing materials; loosening and withdrawal of fasteners attaching sheathing to walls, longitudinal splitting of wood sill plates at anchor bolts, horizontal splitting of wood sill plates neat corners at fasteners of sheathing to the plates, and splitting of studs. At extreme drifts, entire sheathing panels can shear off the walls. Regardless of sheathing material, hysteretic behavior is highly nonlinear, without a definable elastic ranges, and under reversed cyclic loading, hysteresis is severely pinched (Figure ES-15). The strength of the panels is largely dependent on the type, size and spacing of sheathing fasteners and the deformation capacity on the aspect ratio and sheathing material. 


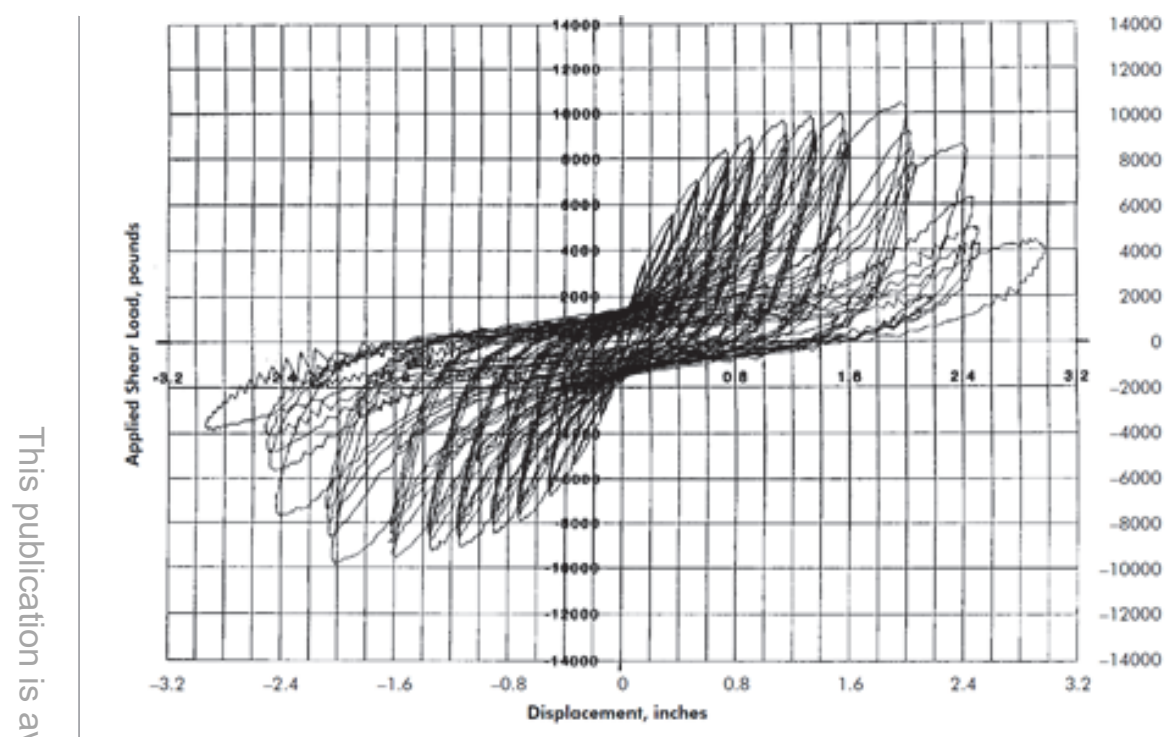

Figure ES15 Typical hysteretic response of cyclically loaded wood shear wall.

A 10-parameter hysteretic model developed by Folz and Filiatrualt, under the CUREE wood frame project is thought to best capture the nonlinear cyclic behavior of wood walls sheathed with a variety of materials. Typical values for walls of common configuration and sheathing are provided. However, any hysteretic model that approximates an appropriate backbone shape and displays significant pinching as illustrated in Figure ES-15 can be used. Figure ES-16 is a generalized backbone that can be used for this purpose. Table ES-10 provides recommended values for control points illustrated in Figure ES-16.

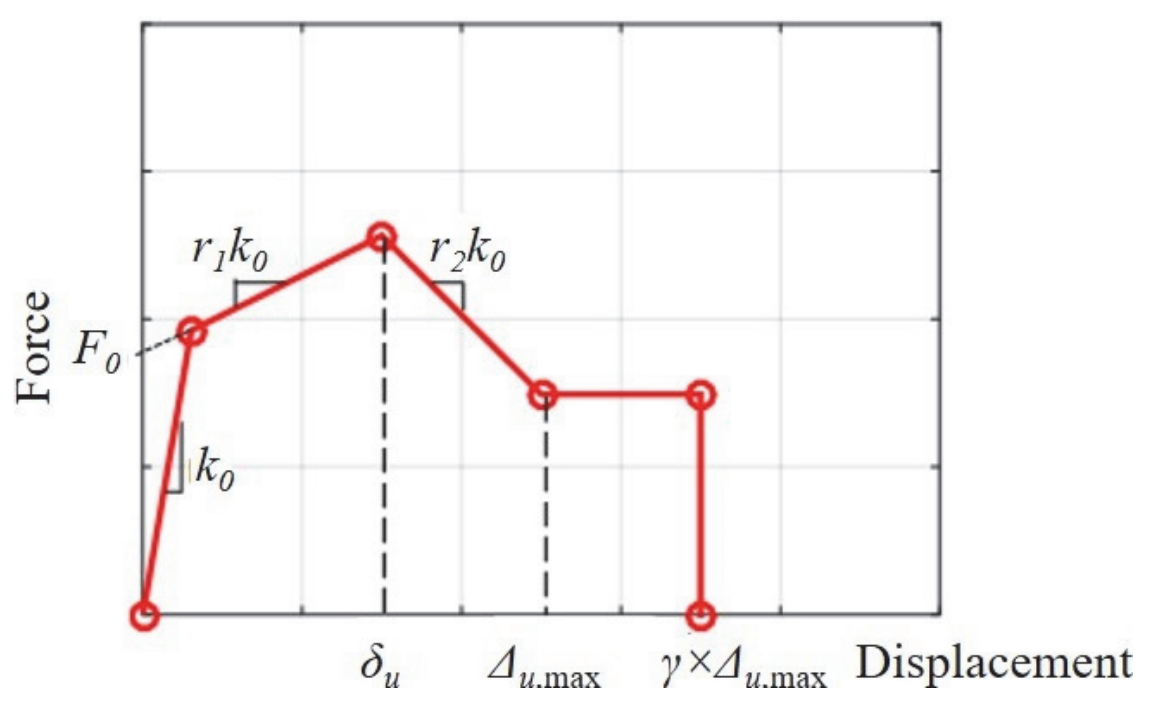

Figure ES-16 Hysteretic backbone for light framed wood walls. 
Table ES-10Backbone Parameters Light Framed Wood Walls

\begin{tabular}{|c|c|c|c|c|c|c|c|}
\hline Combination & $K_{0}[\mathrm{lb} / \mathrm{in} / \mathrm{ft}]$ & $\begin{array}{c}F_{0} \\
{[\mathrm{lb} / \mathrm{ft}]}\end{array}$ & $r_{1}$ & $r_{2}$ & $\begin{array}{c}\delta_{u} \\
{[\mathrm{in} / \mathrm{in}]}\end{array}$ & $\begin{array}{l}\Delta \text {,umax } \\
{[\mathrm{in} / \mathrm{in}]}\end{array}$ & V \\
\hline Gypsum wallboard & 430 & 126 & 0.10 & -0.060 & 0.71 & 5.0 & 1.4 \\
\hline Stucco & 800 & 280 & 0.10 & -0.082 & 1.13 & 5.0 & 1.4 \\
\hline $\begin{array}{l}\text { Stucco \& gypsum } \\
\text { wallboard }\end{array}$ & 1550 & 890 & 0.10 & -0.075 & 1.13 & 5.0 & 1.4 \\
\hline $\begin{array}{l}\text { Horizontal wood } \\
\text { siding }\end{array}$ & 110 & 50 & 0.13 & -0.050 & 8.00 & 9.0 & 1.2 \\
\hline 8×8 WSP - 6d@6" & 712 & 389 & 0.12 & -0.16 & 2.00 & 5.0 & 1.5 \\
\hline 8×8 WSP - 6d@2" & 1812 & 849 & 0.15 & -0.45 & 2.50 & 3.0 & 1.5 \\
\hline 8×8 WSP - 8d@6" & 769 & 422 & 0.15 & -0.24 & 1.50 & 4.0 & 1.5 \\
\hline 8×8 WSP - 8d@2" & 1911 & 1345 & 0.15 & -0.24 & 2.25 & 5.0 & 1.5 \\
\hline 8×8 WSP - 10d@6" & 1088 & 649 & 0.08 & -0.16 & 2.00 & 4.0 & 1.5 \\
\hline 8×8 WSP - 10d@2" & 2645 & 1240 & 0.15 & -0.20 & 2.50 & 5.0 & 1.5 \\
\hline 8×4 WSP -6d@6" & 712 & 423 & 0.18 & -0.12 & 2.00 & 4.0 & 1.5 \\
\hline 8×4 WSP - 6d@2" & 1812 & 580 & 0.14 & -0.20 & 3.50 & 4.0 & 1.5 \\
\hline 8×4 WSP - 8d@6" & 768 & 1776 & 0.20 & -0.12 & 2.00 & 4.0 & 1.5 \\
\hline 8×4 WSP - 8d@2" & 2195 & 915 & 0.15 & -0.38 & 3.00 & 5.0 & 1.5 \\
\hline 8×4 WSP - 10d@6" & 1095 & 629 & 0.22 & -0.08 & 2.00 & 4.0 & 1.5 \\
\hline 8×4 WSP - 10d@2" & 1497 & 1393 & 0.17 & -0.15 & 2.50 & 4.0 & 1.5 \\
\hline
\end{tabular}




\section{Chapter 1}

\section{Introduction}

The primary purpose of this document is to recommend broad improvements to seismic nonlinear modeling and acceptance criteria requirements contained in ASCE/SEI 41-13, Seismic Evaluation and Retrofit of Existing Buildings (ASCE, 2014), and other design standards such as ASCE/SEI 7, Minimum Design Loads for Buildings and other Structures (ASCE, 2010). These recommendations include specific modeling criteria guidance for structural components commonly found in structural steel and reinforced concrete moment frames, concentrically braced steel frames, reinforced concrete and masonry walls, and wood shear wall systems.

\subsection{Background}

In 1997, the Applied Technology Council, acting in partnership with the Building Seismic Safety Council and the American Society of Civil Engineers (ASCE) concluded a 5-year effort and published FEMA 273 and FEMA 274, Guidelines and Commentary for Seismic Rehabilitation of Buildings (FEMA, 1997a; 1997b), funded under the Federal Emergency Management Agency's (FEMA) Existing Buildings program. These guidelines provided comprehensive performance-based seismic evaluation and upgraded design criteria for existing buildings of all types and introduced a number of important concepts into the practice of performance-based seismic design including:

- The direct use of nonlinear analysis to predict seismic demands for the purpose of design.

- A series of standardized performance levels (Immediate Occupancy, Life Safety, and Collapse Prevention) characterized by the amount of strength demand or nonlinear deformation demand predicted to occur by analysis.

- General procedures to derive hysteretic backbone curves from laboratory test data for use in nonlinear analysis.

- General procedures to derive acceptance criteria from laboratory test data, comprising permissible strength and inelastic deformation demands appropriate to achieving each of the standard performance levels.

- Recommended hysteretic backbones and acceptance criteria for the many different types of structural elements commonly found in existing concrete, masonry, steel, and wood building construction. 
FEMA 273 and FEMA 274 were enthusiastically received by practicing structural engineers, and in fact, were used as the basis for evaluation and upgrade designs before formal publication. Following their publication, software developers brought computer applications based on the Guidelines to market, enabling practical implementation of nonlinear analysis in the design office.

Shortly following publication of FEMA 273 and FEMA 274, FEMA and ASCE collaborated to convert the guidelines into FEMA 356, Prestandard and Commentary for the Seismic Rehabilitation of Buildings (FEMA, 2000a). Following this, acting on its own initiative, and considering input received from practicing structural engineers based on their use of FEMA 356, ASCE published ASCE/SEI 41-06, Seismic Rehabilitation of Buildings (ASCE, 2007). In this form, the procedures and recommendations originally developed and published in the FEMA 273/274 Guidelines and Commentary could be, and were, referenced by the building codes. Though not intended for that purpose, structural engineers began to use ASCE/SEI 41-06 for performance-based seismic design of new buildings, as well as seismic upgrade of existing buildings. In 2014, ASCE improved and republished the standard as ASCE/SEI 41-13, more clearly differentiating between performance objectives intended for new and existing buildings, facilitating the use of the standard for both purposes, although the intent of the "new building" performance objectives was to enable retrofit of existing buildings with the expectation they would perform as well as a new building. Design of new buildings remains outside the intended scope of ASCE/SEI 41.

During the development of FEMA 356, ASCE significantly improved the hysteretic backbone curves and acceptance criteria for steel moment-resisting frame structures based on extensive laboratory testing conducted under the FEMA-funded SAC Joint Venture, a partnership of the Structural Engineers Association of California (SEAOC), Applied Technology Council (ATC), and California Universities for Research in Earthquake Engineering (CUREe). Later, in development of Supplement No. 1 to ASCE/SEI 41-06, ASCE incorporated similar updated information for some concrete elements, based on updated research. However, much of the ASCE/SEI 41-13 requirements for nonlinear analysis and associated acceptance criteria are still based on the limited body of research available during the mid-1990s, when the original FEMA 273/274 Guidelines and Commentary were developed. In the time since, researchers have considerably extended the body of available laboratory testing for structural elements of all four basic construction materials. Further, analytical research has yielded improved understanding of the hysteretic characteristics important to predicting seismic response as well as new elements capable of representing these characteristics. The purpose of this publication is to provide recommendations for improvement of the requirements associated with nonlinear analysis and associated acceptance criteria in ASCE/SEI 41-13, and other design 
standards so that these standards, and design based on these standards, can take advantage of the substantial body of research that has become available in the last 20 years.

\subsection{Document Scope}

At the time this document was developed, the American Iron and Steel Institute was engaged in extensive testing of light frame walls of cold formed steel construction, however, this data were not available for inclusion in these recommendations. Recommendations for other systems, such as unreinforced masonry are not included because the body of available research data has not improved substantially since the original publication of FEMA 273/274. Recommendations associated with proprietary systems and products, such as buckling-restrained braced frames and proprietary moment-resisting connections, are not included because they are the property of the license holders. Because it is necessary to discuss overall system modeling approaches to provide context to specific recommendations for hysteretic relationships and acceptance criteria, this document does discuss these issues to some extent. However, this document is not intended to be directly useful as a guideline on performance of nonlinear analysis of buildings having specific structural systems. Other publications fulfill this need.

\subsection{Intended Audience}

The intended audience for this document is the ASCE/SEI 41 development committee. It may also be useful for other committees and individuals engaged in development of structural analysis and design standards and guidelines including ASCE/SEI 7, Guidelines for Performance-Based Seismic Design of Tall Buildings (PEER, 2010), and similar documents. It is important to note that the recommendations presented herein are intended for use in seismic analysis and design rather than blast evaluation or evaluation of structures subjected to other loading conditions. Individual engineers engaged in performing nonlinear analysis, either as part of building evaluation, design or upgrade projects either for seismic or other loadings may find information presented herein useful; however, this is not the primary intent of this document.

\subsection{Document Organization}

Chapter 2 provides a general overall discussion of the current use of nonlinear analysis in structural engineering practice, as background information for the recommendations presented later in the document. Chapter 3 discusses acceptance procedures embedded in contemporary seismic design standards and guideline documents including ASCE/SEI 41-13, ASCE/SEI 7-16 and the Guidelines for Performance-Based Design of Tall Buildings. Chapters 4 through 10 present detailed recommendations for hysteretic modeling and acceptance criteria applicable to steel 
moment frame systems, steel concentrically braced frames, reinforced concrete moment frame systems, flexure- and shear-controlled reinforced concrete walls, reinforced masonry walls, and wood shear wall systems. In addition, these chapters present a brief overview of the typical behaviors of these systems during inelastic response; how design criteria for the system have evolved over the years, where this affects the likely behavior; and, also, discussions of the most effective modeling techniques presently available to capture the system's nonlinear behavior. More detailed discussions of these topics as well as summaries of research data upon which the hysteretic recommendations are based are contained in Appendices to this report. 


\section{Chapter 2}

General

\subsection{Use of Nonlinear Analysis}

Today, nonlinear analysis is widely used by the structural engineering profession as an aid to seismic and blast evaluation of buildings and non-building structures. However, this was not always the case. Prior to publication of the ATC-40, Seismic Evaluation and Retrofit of Concrete Buildings, (ATC, 1996) and FEMA 273, NEHRP Guidelines for the Seismic Rehabilitation of Buildings, (FEMA, 1997a) and FEMA 274, NEHRP Commentary on the Guidelines for the Seismic Rehabilitation of Buildings, (FEMA, 1997b) documents in the late 1990s, nonlinear analysis was rarely used by structural engineers, except as a research tool. Exceptions to this included:

- Design and evaluation of steel jacket structures used to support offshore petroleum production platforms, where nonlinear static (pushover) analysis was commonly used to evaluate structural stability under extreme wave loading.

- Design of seismic isolation systems for buildings, using simple analysis tools with nonlinearity limited to macro-elements representing the isolation system.

- Design of structures incorporating active damping systems in which the dampers were the only nonlinear elements.

There were a number of reasons for the sparse use of nonlinear analysis at that time including:

- Building codes and other design standards did not provide guidance for the use of nonlinear analysis, except as applied to structures incorporating seismic isolation or seismic energy dissipation

- Software available to assist in nonlinear analysis was limited in capability and could not support the wide range of structural types considered by engineers or structures with many nonlinear elements

- Most engineers had little familiarity with nonlinear analysis and did not have the necessary knowledge to implement it in practice

Following publication of the ATC-40 and FEMA 273/274 documents, this changed rapidly. These documents provided design criteria associated with the use of nonlinear analysis that could be directly used to evaluate the broad range of existing buildings present in the United States and to design retrofits for them. Shortly after the publication of these documents, the ASCE/SEI 7 standard also adopted criteria 
for the use of nonlinear analysis in design, making this available as a general tool for the design of new buildings as well. Structural applications software vendors then rapidly brought practical analysis applications with substantially improved analysis capability to market, making the use of this technique increasingly practicable. Universities began to train more students in the use of nonlinear analysis, providing industry with a cadre of engineers who were familiar with the approach and less apprehensive to employ it on real projects. Finally, as design offices began to use the ATC-40 and FEMA 273/274 guidelines, they found that the use of nonlinear analysis could be beneficial, enabling more reliable assessment of building performance and also more economical design, encouraging further use.

There are two types of nonlinear seismic analysis: static and dynamic. The ATC-40 publication only considered static analysis. FEMA 273/274 considered both static and dynamic analysis but discouraged the use of dynamic analysis as being complex and primarily useful as a research tool rather than a design aid, limited in practicality by the available software and hysteretic models, limited computing capability, and being highly sensitive to modeling assumptions, especially hysteretic properties, for which there was only limited consensus.

Following publication of ATC-40 and FEMA 273/274 many engineers began to use nonlinear static analysis in their evaluation and design work. Then, as engineers became comfortable performing nonlinear analysis and more capable software became available, many of these engineers transitioned to the use of nonlinear dynamic analysis.

Today, nonlinear analysis, particularly nonlinear dynamic analysis, is frequently used for seismic performance evaluation, and also for retrofit design. It is also commonly used for seismic design of new tall buildings and is increasingly seeing use in seismic design of other buildings types as well as some non-building structures.

\subsection{Nonlinear Static Analysis}

Nonlinear static analysis, or pushover analysis as it is commonly called, consists of a series of sequential linear static analyses in which the stiffness matrix, and sometimes the loading vector as well, are adapted to represent the effects of damage onset and deflected (modal) shape as load and deformation response is increased in a stepwise manner. The process starts with a linear model that represents the undamaged, elastic stiffness of the model. A loading vector is applied to this model to represent the inertial forces associated with earthquake response. The loading vector shape may be taken to approximate first mode elastic response, higher mode response, approximate combined modal response, or the effective deflected shape considering that damage has occurred. The loading vector and resulting structural deformations and forces are scaled to a level that brings the element with the largest demand to capacity ratio 
(DCR) to a point of incipient nonlinearity, e.g., yielding and buckling. The force demands on all elements and structural drifts at all levels corresponding to that loading are recorded, as is the total applied load in the form of a base shear force, $V$. Then the structural model is modified, to place a release in the element with the highest DCR in the degree of freedom in which nonlinearity will occur. A load vector is again applied to the model and a static elastic solution performed to derive the incremental forces in all elements. This second analysis is scaled such that when the forces from this second increment of loading are added to those from the first analysis, the element with the next highest DCR in the previous analysis will reach incipient nonlinearity. The element forces and structural deformations and base shear from this load increment are added to those obtained from the first analysis. This procedure is repeated until such time as: (1) a target displacement is reached; (2) structural instability occurs; or (3) critical failure is predicted in one or more of the structure's elements.

Typically, upon completion of the pushover analysis, a pushover curve is drawn. The pushover curve is a plot of the total applied lateral shear force, $V$, at each increment of loading as the ordinate against the lateral displacement $\Delta$, at a reference location, typically taken as the center of mass at the structure's highest level, as the abscissa. Figure 2-1 is a representative pushover curve for a hypothetical structure. Each point at which the multilinear pushover plot changes slope represents a point at which new nonlinearity is occurring.

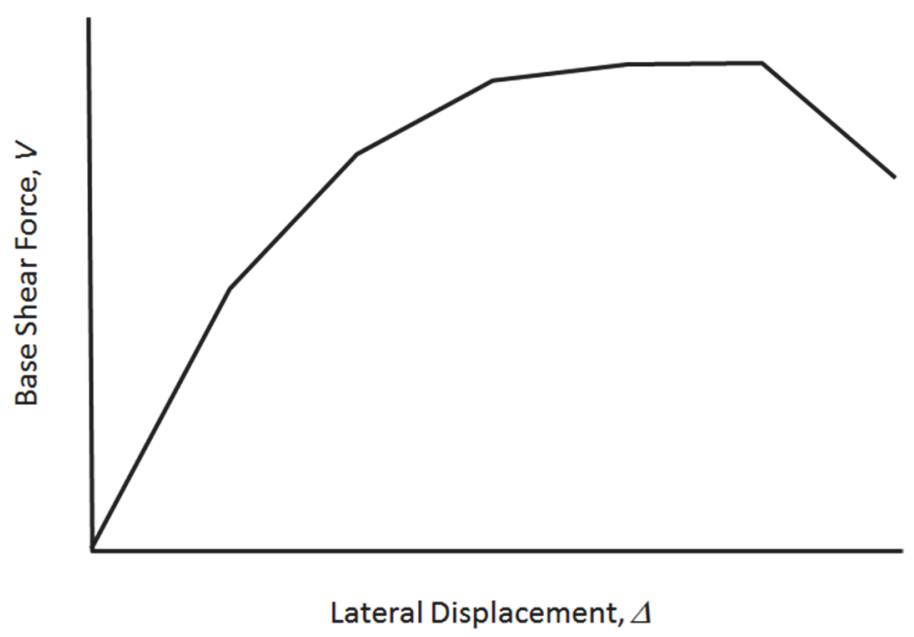

Figure 2-1 Pushover curve for hypothetical structure.

The pushover curve by itself says nothing about how much demand a particular earthquake will produce in a structure, but rather is a representation of global force versus deformation behavior through a range of possible earthquake intensities. In order to determine the demand produced by a particular intensity of earthquake shaking, it is necessary to select an elastic acceleration response spectrum representing that earthquake intensity. The ASCE/SEI 41 procedures use the 
structure's effective inelastic properties derived from the pushover curve, and approximate relationships that relate elastic spectral demand to inelastic spectral demand, to determine a target displacement, representative of a median estimate of the amount of demand produced in the structure by the earthquake of interest. In the ATC-40 methodology, target displacement is obtained using a graphical procedure known as the capacity-spectrum method. Interested readers should refer to the ASCE/SEI 41 and ATC-40 documents for more information on this aspect of nonlinear static analysis.

Key inputs required to perform a pushover analysis are the structure's geometry, interconnectivity, boundary conditions, as well as representation of each element's force-deformation behavior throughout a range of deformations. Because the loading function used in pushover analysis is monotonically increased, the force-deformation behavior for elements need only be represented in the form of a so-called "backbone curve" which portrays the force-deformation envelope for the element independent of such considerations as unloading and reversed loading. Figure 2-2 portrays the typical form of such backbone relationships used to portray element behaviors in pushover analyses. In this figure and following figures, $Q$ represents a generalized force quantity, such as axial load, moment, shear, or stress, while $\Delta$ represents a generalized displacement quantity, such as elongation, shear deformation, rotation, curvature or strain.

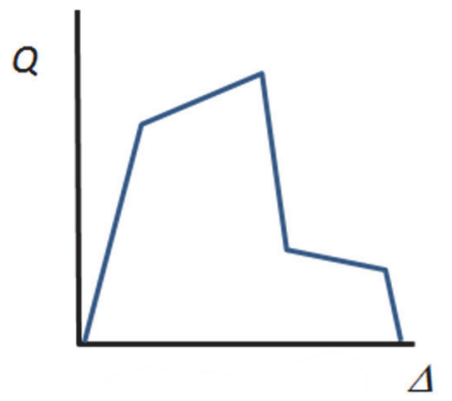

(a) Highly ductile

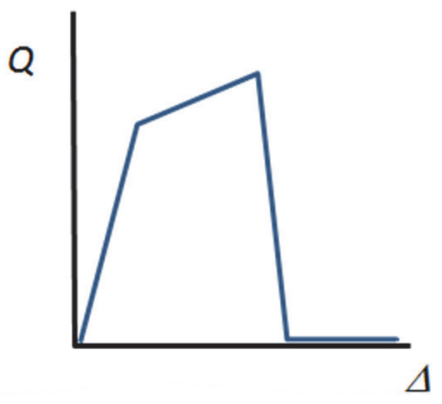

(b) Ductile

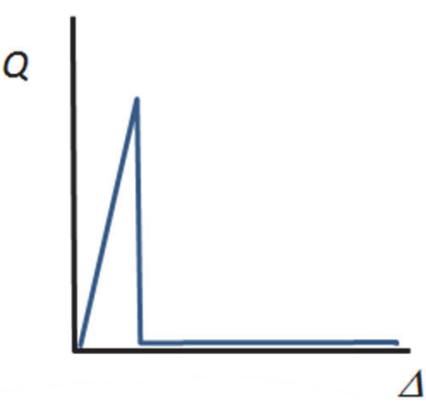

(c) Brittle

Figure 2-2 Typical backbone hysteretic relationships used in nonlinear static analyses.

Because nonlinear static analysis neither directly simulates dynamic behavior nor reversed loading, the effects of cyclic degradation that many real structural elements undergo when subjected to large cyclic inelastic demands must be approximated through selection of backbone relationships that account for an assumed amount of such degradation.

The principal advantages of pushover analysis are that it is conceptually simple to perform; does not require sophisticated software; allows easy visualization of a structure's response state at different levels of demand, presents a solution in which the structure is always in static equilibrium; and, presents a unique solution for structural response at a given earthquake intensity level. Disadvantages of the 
pushover technique are that the solution for target displacement is at best approximate; the procedure cannot easily be used to represent the results of higher mode effects or cyclic degradation; the unique response characteristics associated with individual ground motions, sometimes called record to record variability is lost; and, the structural solution can become unstable once the slope of the pushover curve becomes negative (declining branch in Figure 2-1).

\subsection{Nonlinear Dynamic Analysis}

Nonlinear dynamic analysis, also frequently called nonlinear time history or nonlinear response history analysis is a more sophisticated technique than nonlinear static analysis. Nonlinear dynamic analysis is performed using a process of stepwise solution of the basic equation of motion:

$$
M(\ddot{X}(t)+\ddot{u}(t))+C \dot{X}(t)+K X(t)=0
$$

where $M, C$, and $K$ are respectively the mass, damping and stiffness matrices for the structure, $\ddot{u}(t)$ is the ground acceleration as a function of time $(t)$ and $\ddot{X}(t), \dot{X}(t)$, and $X(t)$ are the instantaneous acceleration, velocity, and displacement vectors for the structure at time $t$, relative to the ground, and are unknowns. Most software used to perform this analysis type solve for the displacement vector $X(t)$ as a function of time through inversion of the stiffness matrix, which is adaptively modified based on the computed deformation and strength demands on each of the elements. Once the displacement vector is known, the velocity and acceleration can be determined using finite difference or similar numerical methods. Alternatively, some software applications solve for the acceleration vector directly, in a so called explicit solution technique and then use numerical integration to determine the velocity and displacement vectors.

In addition to the key inputs required to perform nonlinear static analysis, nonlinear dynamic analysis also requires appropriate ground acceleration histories and complete definition of the hysteretic behavior of elements, including unloading and cyclic behaviors. A number of different hysteretic models are available to represent these nonlinear cyclic behaviors. Figure 2-3 illustrates several common hysteretic models. These include:

- Elastic-Perfectly-Plastic. An idealized behavior in which initial loading produces deformation at a constant stiffness rate until the applied force equals the element's yield strength at which point the element will continue to deform plastically, under this constant force, at zero stiffness. When the applied force is reduced, the element will recover deformation at the same stiffness rate experienced in the initial loading until such time as the force reverses and again reaches the yield level, at which time reversed plastic deformation under constant force will occur. The elastic loading stiffness and yield strength remain constant 
regardless of the number of cycles of motion or magnitude of imposed deformation.

- Strain Hardening. A behavior similar to elastic-perfectly plastic with the exception that when yielding initiates further deformation occurs at positive, nonzero stiffness. Under successive cycles of loading yielding does not re-initiate until the applied force exceeds the prior peak applied force in the given direction. This form of hysteresis is representative of the behavior of some steel elements prior to the onset of buckling or fracture.

- Stiffness Degrading. A behavior similar to strain hardening behavior with the exception that upon reloading deformation will occur at a reduced stiffness. The reduced stiffness is a result of damage that has occurred, such as cracking in concrete or masonry walls, or withdrawal of fasteners between sheathing and studs in a light-frame wall.

- Strength Degrading. A behavior similar to stiffness degrading behavior with the exception that each successive cycle of motion initiates yielding at a lower force level. Like the stiffness reductions, the reduced yield strength can be attributed to damage, such as local crushing or spalling of concrete or masonry.

- Cyclic Degrading. A behavior similar to strength degrading behavior with the exception that in successive cycles of yielding, an increasingly negative postyield stiffness occurs. This type of behavior is commonly associated with buckling of steel elements or reinforcing.

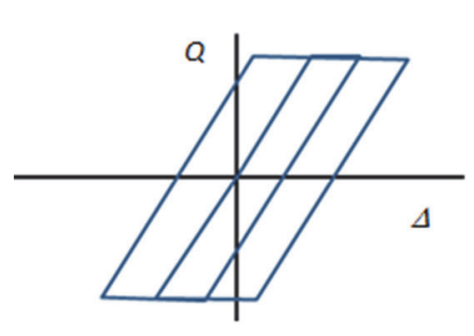

(a) Elastic perfectly plastic

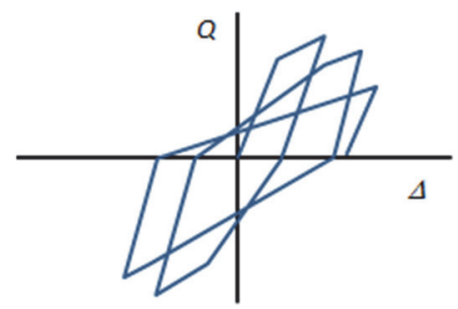

(d) Strength degrading

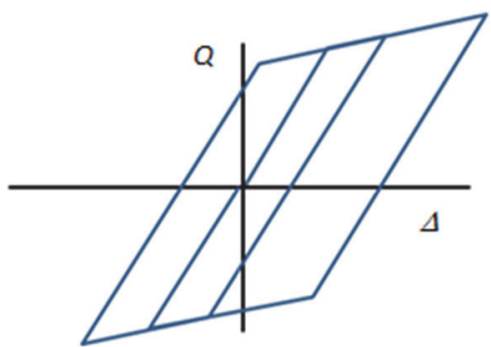

(b) Strain hardening

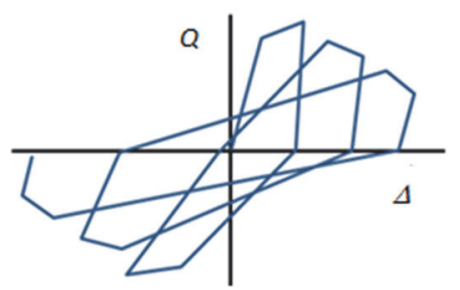

(e) Cyclic degrading

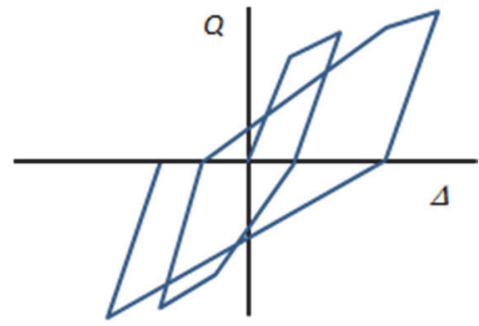

(c) Stiffness degrading

Figure 2-3 Alternative hysteretic models.

These and other types of nonlinear behavior can be modeled in several ways including discrete hinges, distributed plasticity models, and phenomenological methods. Elements incorporating discrete hinges are a convenient means of 
representing nonlinear behavior and are ideal for modeling behaviors such as formation of plastic hinges in beams. In this approach, the element stiffness formulation includes direct mathematical coding of the hysteretic relationship in the form of a macro moment-rotation, or similar relationship. Such elements will provide reasonable representation of the effect of the nonlinearity on the structure as a whole, but will not provide any information on the length of the element involved in the nonlinear behavior. If information on the length of element involved in the nonlinear behavior is desired, distributed plasticity models, such as fiber elements can be used. These elements are capable of monitoring strains and modifying the stiffness of individual fibers and can simulate progressive strain hardening and plasticity in an element. Such models are commonly used for deep beams and shear walls. Phenomenological models use detailed finite element meshes with geometric and stiffness nonlinearity that are capable not only of capturing yielding but also buckling, fracture and similar nonlinearities. Such models can directly simulate the actual damage that occurs, but result in very large models, long solution times and very large storage space for the results.

The appropriate hysteretic model to use depends on the actual characteristics of the type of element being represented and the type of information desired. Often the selection of software used to model and perform the analysis will limit the available hysteretic/element models. The choice of hysteretic/element model used can significantly affect the resulting predicted response. Further discussion of this is contained in individual materials chapters, later in this report.

The acceleration time history used as input to the model to represent the ground motion also can significantly affect the predicted response and even ground motion records that appear to have similar spectral shape and amplitude can produce significantly different predictions of response.

The principal advantage of nonlinear dynamic analysis relative to nonlinear static analysis is that approximations associated with selection of loading pattern and solution for target displacement are avoided and higher mode effects and cyclic degradation can be directly accounted for in the analysis. However, response predictions obtained by dynamic analysis are not necessarily more accurate than those obtained from static analysis depending on the extent to which appropriate hysteretic elements and ground motions are used. Further, because there can be significant variability in the results obtained even with the same hysteretic model, but using different input ground motion records, it is typically necessary to use a large number of records as input, and to determine demands considering the statistical variation obtained from these various records. This results in having to manipulate, store and review large amounts of data, as well as deal with significantly longer solution times. Nevertheless, nonlinear dynamic analysis is gaining in popularity significantly relative to nonlinear static analysis, due to the perceived enhanced 
accuracy of the results obtained as well as the greater availability of the software and computing power necessary to make this analysis type practical.

\subsection{General Hysteretic Model}

Figure 2-2 presents the general hysteretic models defined in ASCE/SEI 41, which refers to these as Type 1, 2 and 3 behaviors, respectively. These models were originally conceived in FEMA 273/274 as being derived from test data obtained using a progressively increased reversed cyclic loading protocol, similar to that specified in ATC-24, Guidelines for Cyclic Seismic Testing of Components of Steel Structures, (ATC, 1992). Specifically, FEMA 273/274 specifies use of the so-called second cycle backbone curve. As illustrated in Figure 2-4, the second cycle backbone curve is derived as a series of secants connecting the intersections of the first cycle curve for the $(i)$ th deformation step with the second cycle curve for the (i-1)th deformation step. FEMA 273/274 selected the second-cycle backbone curve to capture, in an approximate way, the strength degradation that occurs in some structural elements under reversed cyclic loading. It was felt important to do this because strength degradation can result in increased building response, relative to non-degrading hysteretic behaviors, is both an indicator of impending element failure and also, can lead to development of global instability. Further, since nonlinear static analysis, the principal tool considered by FEMA 273/274, is unable to directly capture cyclic degradation, it was felt that the second cycle backbone was a reasonable approximation to the average response of elements in a structure responding to strong motion and experiencing significant nonlinearity.

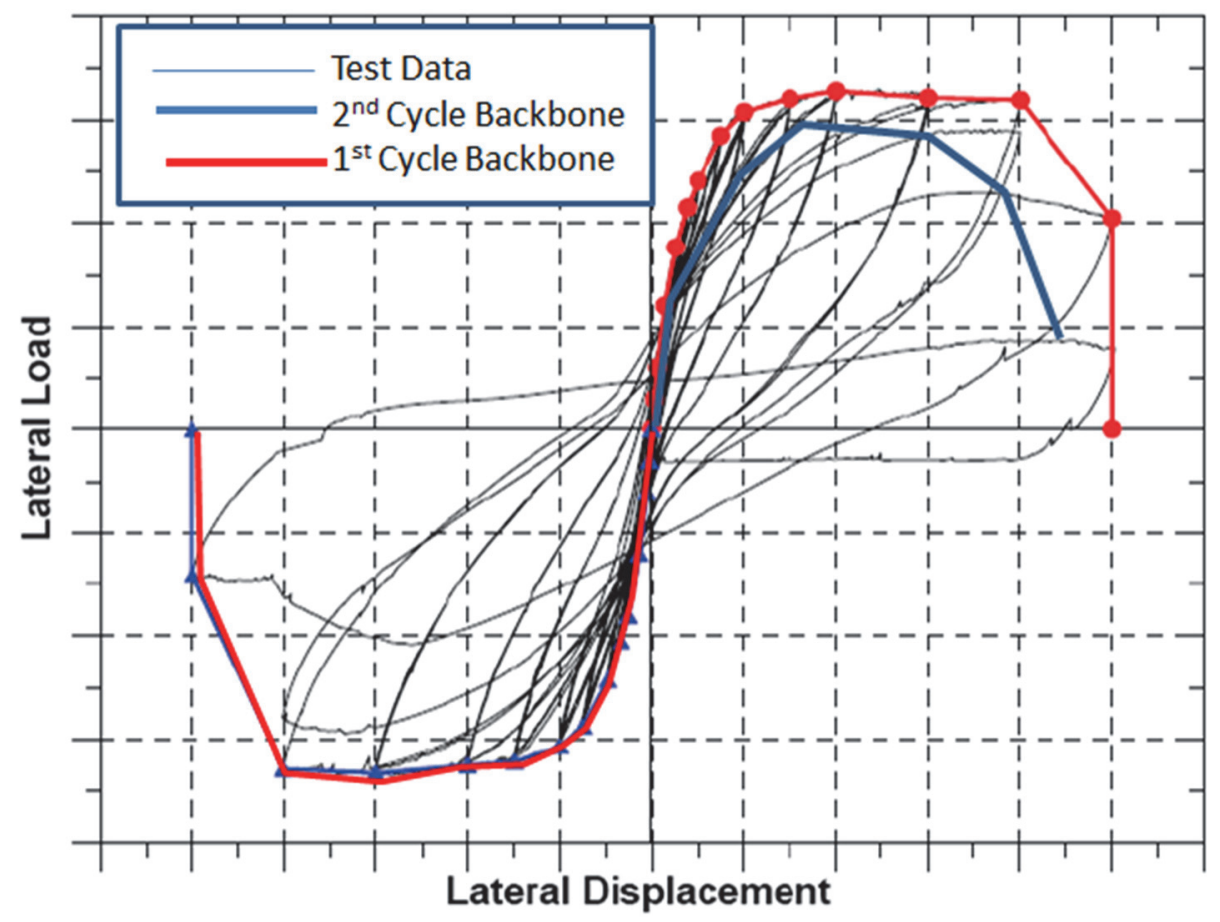

Figure 2-4 Derivation of first and second cycle backbones from cyclic test data. 
More recently, the ASCE/SEI 41 committee decided that the extent of degradation incorporated in the second-cycle backbone curve is excessive and revised the specified derivation for the hysteretic backbone curves to be first cycle backbone curves. The first cycle backbone curves are constructed as the secant envelope drawn through the peak response points for each first cycle test to a given displacement level. As can be seen in Figure 2-4, which plots both first cycle and second cycle backbone curves for a hypothetical set of cyclic element test data, the first cycle will generally show higher strength at a given deformation level and delayed initiation of degradation as compared with the second cycle backbone. Regardless, most of the tabulated control points in ASCE/SEI 41 have never been updated from the values originally presented in FEMA 273/274 that were derived to represent second cycle backbone curves. Finally, it is important to note that only in rare cases will the backbone curves derived from test data actually match the shape of the standard ASCE/SEI 41 representations illustrated in Figure 2-2. Thus, at best, the hysteretic control points specified by ASCE/SEI 41 based on the idealized backbone curves are approximate.

It is important to note that the ASCE/SEI 41 general hysteresis models were derived to be useful for nonlinear static rather than nonlinear dynamic behavior. The backbone curves do not provide any direct information on the shape of individual hysteretic loops or the amount of pinching or in-cycle strength degradation that may or may not be present, both of which should be considered when performing nonlinear dynamic analysis of structures where these effects will be significant.

For many types of structural elements, the shape of the backbone curve derived from test data will be highly dependent on the loading protocol used in the test. For these element types, loading protocols with a large number of cycles tend to produce more degradation and lower ultimate deformation capacity than do protocols with fewer such cycles. Regardless, real earthquakes never produce perfectly reversed cyclic loading, as occurs in these test protocols. Therefore, regardless of the test protocol used, the actual hysteresis exhibited by an element in a structure responding to earthquake shaking is likely to be different than that obtained in laboratory testing. Ideally, elements used in forming nonlinear models should be capable of replicating the hysteretic behavior obtained in any protocol, or for that matter, when subjected to actual loading as one part of a larger structural system. Researchers are presently developing some elements, herein called adaptive models, which have this capability. However, for the present, most elements do not have this capability and are tuned to hysteretic data obtained from tests using specific loading protocols. Given that most seismic testing conducted over the past 20 years has used reversed cyclic loading protocols, most such elements, presently, are tuned to such protocols.

Figure 2-5 schematically illustrates the difference in backbone curves that would be obtained for many types of structural elements when subjected to (1) a fully reversed 
cyclic loading protocol and (2) a monotonic loading protocol. As can be seen, the monotonic envelope curve has higher peak strength, less degradation, and greater ductility than does the cyclic backbone curve. For a given ground motion, analytical models based on cyclic backbone curves will generally predict greater peak and residual displacement than will models based on monotonic curves and also will have greater propensity to exhibit instability. However, use of models conditioned on cyclic backbone curves will not always yield conservative results. Nonlinear behavior of some elements or actions in a structure will limit the amount of strength demand experienced by other elements. As an example, the axial forces in columns in a moment frame structure are limited by the shear in beams framing into the columns. These beam shears are in turn limited by either the shear or flexural strength of the beams. In models of such a structure, if the hysteretic model for the beams uses overly pessimistic assumptions as to the amount of degradation that occurs or understates the peak strength of the beams, the analysis may underpredict the amount of axial load that can be delivered to the columns. Similar unconservative results can be obtained for braced frame and wall structures and for elements other than columns.

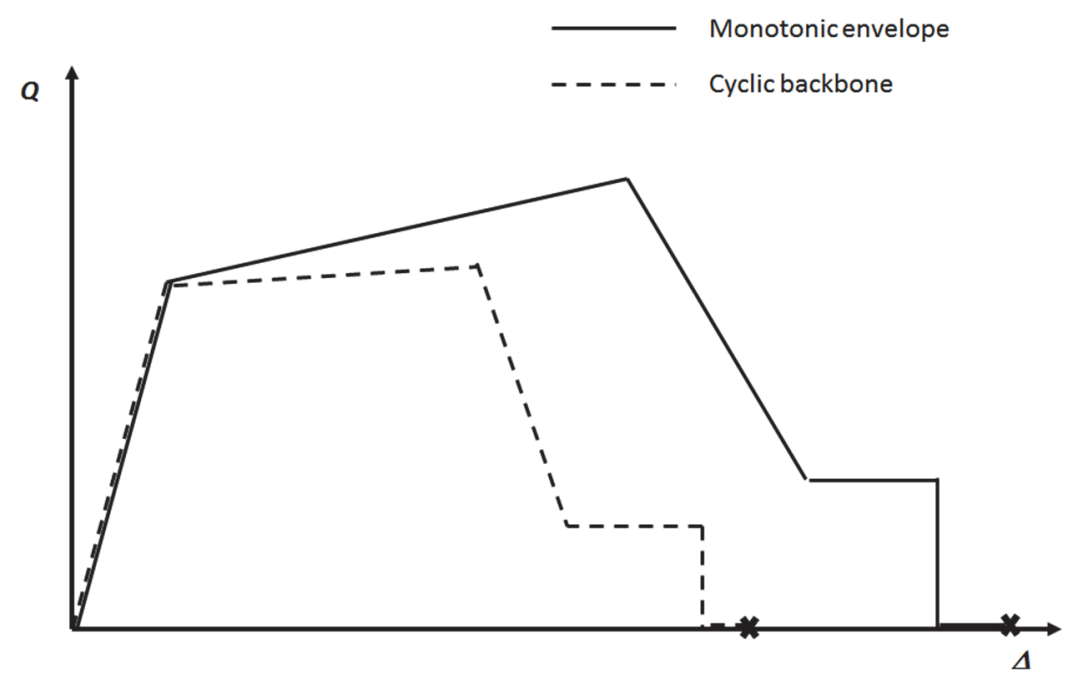

Figure 2-5 Representative cyclic and monotonic backbone curves.

As noted previously, ideally, hysteretic relationships in nonlinear elements should be capable of capturing the likely force-deformation behavior, considering the true loading history applied to the element. To promote the development of such adaptive elements in the future, where possible, this report presents recommended backbone curves for both cyclic and monotonic cases. Throughout the balance of this report, to differentiate between cyclic and monotonic behaviors, the term backbone is used when referring to cyclic behavior and envelope is used to describe monotonic behavior. Figure 2-6 illustrates the terminology used for key control points in this report for backbones and envelopes throughout this report. The figure is a generalized case but is not applicable to all materials and systems. Where needed, 
individual chapters on specific structural framing types have used somewhat different backbone shapes and nomenclature.

Q

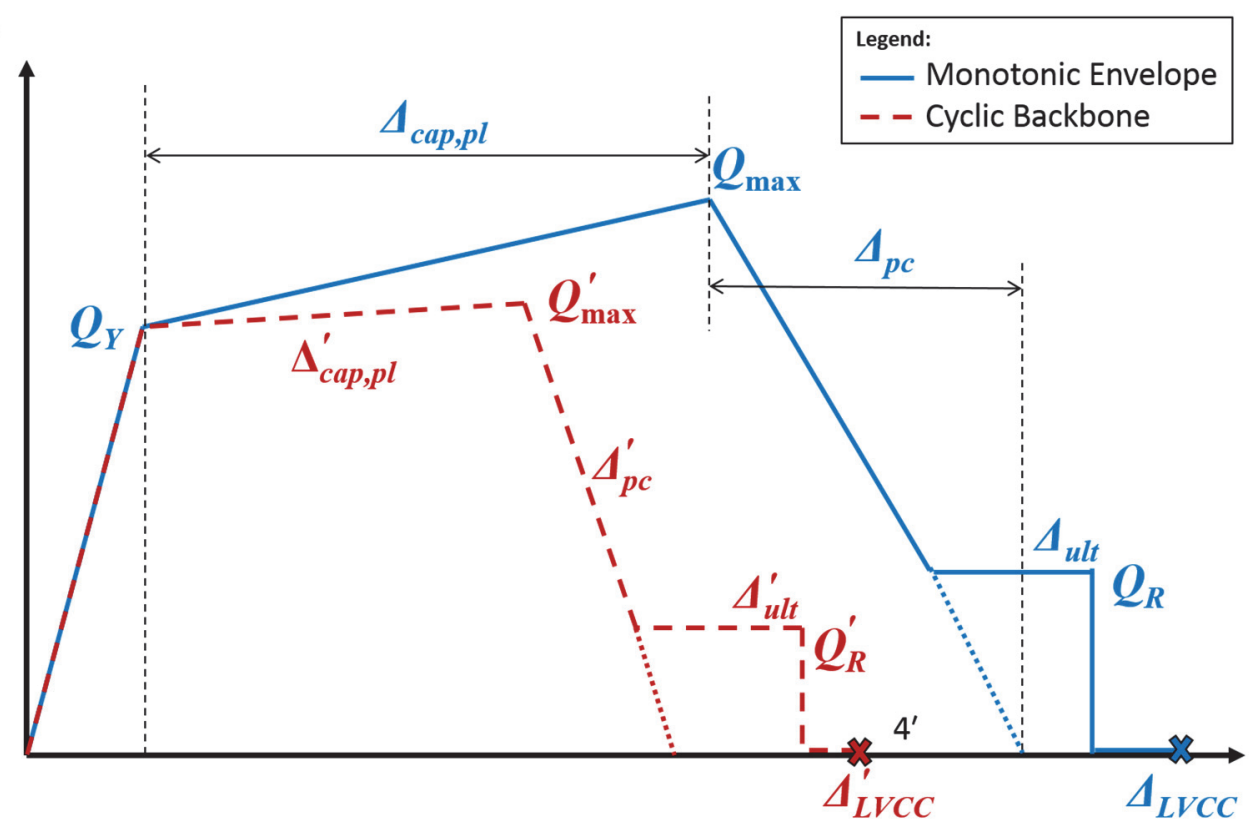

Figure 2-6 Standard cyclic and monotonic backbones with control points.

In the figure, the following notation applies:

$Q_{y} \quad=$ element yield strength

$Q_{\max } \quad=$ element peak strength, monotonic loading

$Q_{\max }^{\prime} \quad=$ element peak strength, cyclic loading

$Q_{R} \quad=$ element residual strength, monotonic loading

$Q_{R}^{\prime} \quad=$ element residual strength, cyclic loading

$\Delta_{\text {cap } p l}=$ plastic deformation, monotonic loading

$\Delta_{c a p, p l}^{\prime}=$ plastic deformation, cyclic loading

$\Delta_{p c} \quad=$ effective post-peak deformation, monotonic loading

$\Delta_{p c}^{\prime} \quad=$ effective post-peak deformation, cyclic loading

$\Delta_{u l t} \quad=$ ultimate deformation, monotonic loading

$\Delta^{\prime} u l t \quad=$ ultimate deformation, cyclic loading

$\Delta_{L V C C}=$ deformation at loss of vertical load carrying capacity, monotonic loading

$\Delta_{L V C C}^{\prime}=$ deformation at loss of vertical load carrying capacity, cyclic loading 
It should be noted that the displacement associated with loss of vertical load carrying capacity is rarely, if ever, determined in laboratory testing. This is because the specimen failure that occurs at loading to this failure level can result in damage to laboratory equipment as well as safety hazards to laboratory personnel. Therefore, this quantity must typically be determined either through judgment, analytical approaches, or a combination of these.

Adaptive element models are the preferred type for nonlinear dynamic analysis, however, they should not be used for nonlinear static analysis since nonlinear static analysis is unable to trigger the degradation in strength that would occur under the shaking associated with response to a specific ground motion. Instead, nonlinear static analysis should continue to use cyclic backbones as the basis for element nonlinear behavior.

\subsection{Variability and Uncertainty}

Even for a single defined loading protocol and backbone definition, the values of the hysteretic parameters illustrated in Figure 2-6 for multiple elements of the same type and design will be somewhat different owing to differences in material strength, homogeneity, and construction quality. It is seldom possible to precisely define what the actual values of these parameters will be for the many elements that form a real structure, even when extensive material testing is performed. Therefore, it is necessary to use estimates of these properties when developing analytical models, considering the probability that the real values may be somewhat different than those assumed in analysis.

Alternatives for accounting for these uncertainties include:

- Constructing alternative models using upper and lower bounds, respectively, of the probable values in order to bound the likely structural response

- Constructing a large series of models in which the values of these properties are treated as random variables in order to produce, through Monte Carlo analysis or other statistical techniques, such as latin hypercubes, an estimate of the potential range of response and the probable distribution of response

- Constructing a single model using best estimate (mean or median) values to provide an approximation of expected response

The first of these approaches, in which bounded properties are used, is specified both by the ASCE/SEI-7 standard and ASCE/SEI-41 for design of seismic isolation and passive damping systems. In this approach, the entire isolation or damping system is assumed to have either lower bound or upper bound properties in an attempt to find the most sever results on displacement (of the isolation system or dampers) and force on the structure. ASCE/SEI 41 specifies a similar approach for representation of 
foundation and soil nonlinearity. In general, other elements of the structures are modeled using either nominal specified or best-estimate properties.

The bounding technique is seldom applied to every element in a model for several reasons. First, each of the bounding conditions (high stiffness with high strength, high stiffness with low strength, low stiffness with low strength and low stiffness with high strength) is unlikely to exist uniformly across all elements of a real structure. In complex structures, with many elements, the actual values of strength, stiffness, and other hysteretic properties will be distributed with many elements having properties at or near the central values and others having properties that are either higher or lower than these central values with relatively few having properties represented by the extreme values. Also, one of the most significant potential effects of property variability are concentrations of stiffness and strength, owing to fortuitous circumstances, that result in formation of soft story, weak story or torsional irregularities in a structure that is otherwise uniform. Such effects cannot be determined by modeling all elements at the bounds.

The second approach, in which properties are treated as random variables and statistical analyses are performed to explore the distribution of possible response states overcomes these limitations but requires extensive effort beyond the resources of most engineering projects.

Except for the case of seismic isolation, energy dissipation systems, and foundations, both ASCE/SEI 41 and ASCE/SEI 7 recommend the use of best estimate properties for structural elements in models with acceptance criteria adjusted to attempt to account for uncertainty in approximate ways. ASCE/SEI 41-13 constructs acceptance criteria for force-controlled, i.e., brittle behaviors, using lower bound properties in an attempt to reduce the potential for undesirable brittle element failure, while basing acceptance criteria for deformation-controlled (ductile) behaviors on best estimate, or expected values. ASCE/SEI 7-16 however, attempts to select acceptance criteria for both force-controlled and deformation-controlled behaviors considering the statistical uncertainty in both demand prediction and capacities. Chapter 3 presents further discussion of these approaches to acceptance criteria development.

Although it is not the purpose of this report to recommend specific ways to account for the variability in real element properties when performing analysis, the report does present information on the likely variability of element hysteretic properties in the form of estimated median values and coefficients of variation. This will permit others to select appropriate modeling rules and acceptance criteria using any of the three previously described approaches. 



\section{Chapter 3}

\section{Acceptance Criteria}

\subsection{General}

Acceptance criteria, sometimes also called capacities, are limiting values of strength and/or deformation demands, as predicted by analysis, that are used to determine either a structure's probable performance or its conformance with design requirements. This chapter describes the approach adopted by several important structural engineering guidelines and standards to evaluate structural acceptance and recommends an approach for future development of such standards.

\subsection{FEMA 273/274}

FEMA 273, NEHRP Guidelines for the Seismic Rehabilitation of Buildings, (FEMA, 1997a) and FEMA 274, NEHRP Commentary on the Guidelines for the Seismic Rehabilitation of Buildings, (FEMA, 1997b) define three global structural performance levels: Immediate Occupancy, Life Safety, and Collapse Prevention, each representing a progressively more severe state of damage. The Immediate Occupancy level is a state of minimal damage in which the structure, as a whole, retains nearly all of its pre-earthquake resistance and is essentially as safe to occupy after the earthquake as before. Structures meeting the Immediate Occupancy performance level are intended to be safe for occupancy, without the need to first conduct repairs or provide stabilization. In addition, structures meeting the Immediate Occupancy performance level are expected to have sustained such little visible damage that the average person would not be concerned about their safety when using the building. The Collapse Prevention level is intended to be a state of extreme damage, just short of collapse. While structures meeting the Collapse Prevention level have not collapsed, they are anticipated to have little remaining earthquake resistance and if subjected to strong aftershocks could collapse. It is envisioned that substantial repair would be necessary before structures meeting the Collapse Prevention level would be fit for occupancy and it is recognized that required repairs may be so extensive as to be economically impractical. The Life Safety level is intermediate to the Immediate Occupancy and Collapse Prevention levels. Structures conforming to the Life Safety level have sustained significant damage and impairment of their seismic force-resisting capacity, but retain some margin against collapse. As with the Collapse Prevention level, structures meeting the Life Safety level are not suitable for reoccupancy until repaired or stabilized. 
However, unlike the Collapse Prevention level, it is expected that repair of buildings meeting the Life Safety could practically be accomplished.

Although the FEMA 273/274 guidelines define these performance levels based on global consideration of the anticipated impact of the sustained damage on the building's safety and fitness for post-earthquake occupancy, actual evaluation of a building's ability to conform to these performance levels is conducted at the component action level. A component action is defined as the force or deformation associated with an individual, element-level degree of freedom such as axial force, shear deformation, or flexural rotation. A structure's ability to meet a performance level for a given earthquake shaking level is evaluated by comparing the demands predicted by analysis, against acceptance criteria for various component actions.

The FEMA 273/274 guidelines developers recognized that performing acceptance evaluations at the individual component action level is conservative. If this approach is used with even only a few components that are predicted to have demands that exceed their capacities for a particular performance level, the structure as a whole would be predicted to not meet that performance level. This is in contrast with the observation that most structures are able to tolerate the failure of one, or even a few components, without collapsing or presenting an appreciable collapse risk. To moderate the conservatism inherent in evaluating a structure's global performance based on individual component actions, FEMA 273 introduced the concept of primary and secondary components.

Primary components are those that are deemed essential to a structure's performance in that they provide a substantial portion of the structure's lateral resistance and strength. Secondary components are not by themselves essential to a structure's lateral resistance. In theory, the failure of a few secondary component actions would not result in unacceptable performance, while the failure of a few primary components would. Recognizing that the failure of many such elements could compromise the structure's stability, the procedures also limit the number of elements designated as secondary such that in aggregate they do not comprise more than $25 \%$ of a structure's lateral stiffness or strength.

FEMA 273/274 derived acceptance criteria from the hysteretic backbone curves based on two primary considerations: (1) the ability of the component to undergo inelastic response while continuing to carry load; and (2) the criticality of the element to overall structural performance. Element acceptance evaluations are performed at the "action" level, where each such action is designated either as deformationcontrolled or force controlled. Deformation-controlled actions are those for which the component has measurable ductility and is able to maintain load under inelastic deformation. Force-controlled actions do not have such ductility and the component will lose load-carrying capacity once the elastic limit is exceeded. As implied by 
their nominative designations, the acceptability of computed demands for deformation-controlled actions is judged based on the value of a deformation quantity, such as plastic rotation, drift distortion, or similar measure. The acceptability of demands on force-controlled elements is determined using strength demands, including axial force, shear force, and moment.

Figure 3-1 shows the type 1 hysteretic backbone referenced in FEMA 273/274 as well as ASCE/SEI 41. For secondary deformation-controlled actions, collapse prevention performance is deemed to occur at the deformation associated with point $\mathrm{E}$, designated $\Delta_{C P-S}$ in the figure. Behavior at this point was assumed to include loss of all strength including both the strength to support gravity forces as well as to participate in lateral force-resistance. For primary actions, the acceptable deformation for collapse prevention performance was taken as that at point $\mathrm{B}$ in the figure, also labeled $\Delta_{C P-P}$. This point was selected to provide some reserve deformation capacity, before true failure occurred, and also because it was felt that analysis itself may be unreliable if carried to deformations at which important actions were strained beyond point $\mathrm{B}$ on the backbone.

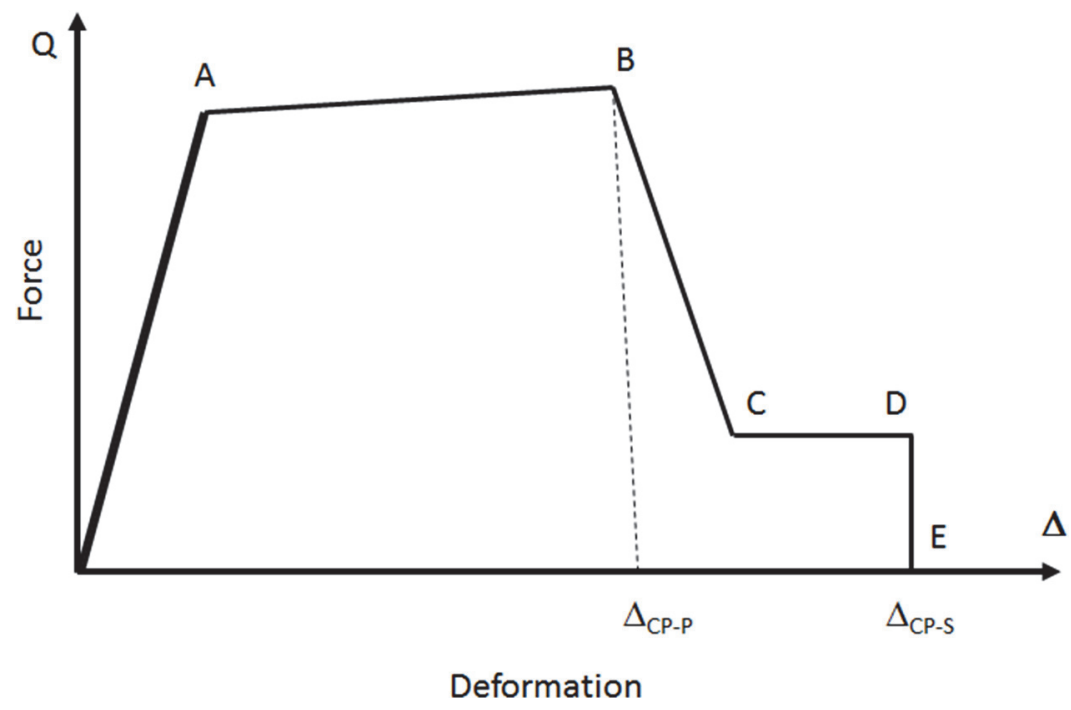

Figure 3-1 FEMA 273/274 acceptance criteria.

The Life Safety acceptance criteria for deformation-controlled actions is taken at $75 \%$ of the acceptance criteria for Collapse Prevention with the exception that for primary actions, this could not be taken at a deformation greater than that at point B. FEMA 273/274 did limit specific points on the backbone for Immediate Occupancy performance; however, it generally occurs between points A and B and usually closer to A.

Acceptance criteria for force-controlled actions are all at deformation levels less than or equal to that at point A. Collapse Prevention acceptance deformation for primary or secondary elements is taken at point A. Life Safety acceptance is taken at $75 \%$ of 
the deformation at point A. Immediate Occupancy performance is taken the same as Life Safety for force-controlled components.

\subsection{ASCE/SEI 41}

The acceptance criteria under ASCE/SEI 41 are similar to those of FEMA 273/274 as previously described with the exception that there is no differentiation between the acceptance criteria for primary and secondary deformation-controlled components when nonlinear analysis capable of accounting for the effects of strength degradation is performed.

\subsection{PEER Tall Building Guidelines}

In 2010, the Pacific Earthquake Engineering Research Center (PEER) published a guideline for performance-based design of tall buildings (Guidelines for

Performance-Based Seismic Design of Tall Buildings) that relies extensively on the use of nonlinear analysis for performance verification. PEER published an update to this guideline in 2017. The PEER TBI Guidelines address two performance levels: Serviceability and Collapse Prevention. The Serviceability level is similar to the Immediate Occupancy level of FEMA 273/274 and ASCE/SEI 41. Evaluation for this performance levels is typically performed using linear analysis and so, acceptance criteria for this level are not discussed here.

The Collapse Prevention level in the PEER TBI Guidelines is similar to that in FEMA 273/274 and ASCE/SEI 41. The PEER TBI Guidelines separately evaluate global and component level acceptance. Global acceptance checks include peak transient drifts, residual drifts and loss of story strength. Under the PEER TBI Guidelines, the average value of peak transient drift ratios at any story for the suite of analyses cannot exceed 3\% while the peak transient drift ratio in any story for any one analysis cannot exceed $4.5 \%$. The $3 \%$ value was selected to be approximately equivalent to the limiting drift level inherent in the building code for MCE shaking. The $4.5 \%$ drift limit for any one analysis was selected because it was felt that present analysis techniques may not provide meaningful predictions of response when drifts exceed that level.

The 2010 edition of the PEER TBI Guidelines also limits loss of story strength in any story to $20 \%$ of the initial strength. This criterion was placed in the Guidelines to guard against having tall buildings damaged in earthquakes, not collapsing, but presenting an imminent hazard to the surrounding community. It should be noted that procedures to determine the loss in story strength were not specified in the 2010 publication and it is not clear how an engineer would actually do this. In recognition of this, the 2017 edition of the guidelines deleted this criterion. 
For element-level acceptance, the PEER TBI Guidelines, like ASCE/SEI 41, differentiate between deformation-controlled and force-controlled behaviors. For deformation-controlled actions, there are no specific limiting values of deformation for Collapse Prevention performance other than that response must be within the valid range of modeling. The assumption behind this is that as long as the analysis does not predict collapse, performance is acceptable, presuming that elements are not modeled as continuing to provide structural resistance at deformation levels beyond those at which they will actually be able to do this. In essence, if element models used are capable of simulating strength degradation, the valid range of modeling will be similar to that at point E in Figure 3-1. If the element models are not capable of such simulation strength degradation, the valid range of modeling is point $\mathrm{B}$ in Figure. In no case would the valid range of modeling exceed deformation levels at which laboratory testing demonstrates behavior.

For force-controlled actions, the PEER TBI Guidelines differentiate between critical elements and non-critical elements. Critical elements are those, the failure of which, would lead to structural instability or potential life-threatening damage. Noncritical force-controlled elements are those the failure of which does not lead to these conditions. In the 2010 edition of the guidelines, both critical and noncritical forcecontrolled elements are evaluated using the relationship:

$$
F_{u}<\phi F_{n, e}
$$

where $F_{u}$ is the strength demand taken as the forces due to gravity and seismic loading, evaluated at a mean plus one standard deviation level, $\phi$ is the resistance factor obtained from the applicable material standard, e.g., ACI 318, Building Code Requirements for Structural Concrete and Commentary, and $F_{n, e}$ is the nominal strength capacity of the element evaluated using expected material strengths. For non-critical elements the resistance factor may be assigned a value of 1.0 and $F_{u}$ is taken as the mean demand predicted by the suite of analyses. For critical elements $F_{u}$ is taken as an approximation of one sigma response. The intent is to accept mean probability of failure at MCE shaking for non-critical elements and approximately a $16 \%$ chance of failure for critical elements.

In the 2017 update to the guidelines acceptability of force-controlled actions is performed using evaluated using separate criteria depending on whether the demand on the force-controlled action is limited by formation of a ductile yield mechanism in the structure. Where this occurs, acceptance is evaluated by the formula:

$$
\begin{gathered}
1.2 D+0.5 L \pm 0.2 S_{M S} D+E_{M} \leq \phi_{s} R_{n} \\
0.9 D \pm 0.2 S_{M S} D+E_{M} \leq \phi_{s} R_{n}
\end{gathered}
$$

In Equations 3-2 and 3-3, $D$ represents dead load effects, $L$ is taken as the live load effect under $80 \%$ of the unreduced live loads that exceed 100 pounds per square foot 
and $40 \%$ of other unreduced live loads. $S_{M S}$ is the site-adjusted $\mathrm{MCE}_{\mathrm{R}}$ spectral acceleration at short period from ASCE/SEI 7; $E_{m}$ is the expected value of the earthquake force assuming development of the ductile yield mechanism; $\phi_{s}$ and $R_{n}$ are respectively, the resistance factors and nominal strength specified the applicable materials standard.

Where the force on an action is not limited by a ductile yield mechanism, acceptance is evaluated using the formula:

$$
\begin{gathered}
1.2 D+0.5 L \pm 0.2 S_{M S} D+1.3 I_{e}\left(Q_{T}-Q_{n s}\right) \leq \phi_{s} B R_{n} \\
0.9 D \pm 0.2 S_{M S} D+1.3 I_{e}\left(Q_{T}-Q_{n s}\right) \leq \phi_{s} B R_{n}
\end{gathered}
$$

In these formula, all terms have the same meaning as above with $Q_{T}$ representing the mean value of the total strength demand, $Q_{N S}$ representing the non-seismic portion of $Q_{T}$ and $B$ is a factor to account for conservatism inherent in the material standard's formulation for nominal resistance, if any. These modifications were made for greater compatibility with the guidelines and industry standards.

\subsection{ASCE/SEI 7-16}

Chapter 16 of ASCE/SEI 7-16, Minimum Design Loads and Associated Criteria for Buildings and Other Structures, (ASCE, 2017) specifies procedures for design using nonlinear dynamic analysis. The intent of the ASCE/SEI 7-16 criteria is to produce structures that have less than a defined probability of collapse, given exposure to $\mathrm{MCE}_{\mathrm{R}}$ shaking, when evaluated using procedures described in FEMA P-695, Quantification of Building Seismic Performance Factors (FEMA, 2009b). For Risk Category II, III, and IV structures respectively, the acceptable probability of collapse is taken as $10 \%, 6 \%$, and $3 \%$.

ASCE/SEI 7-16 requires analysis of a suite of not less than 11 ground motions to assure reasonable approximation of mean values of response quantities, and also to provide some confidence that Risk Category II structures are likely to meet the $10 \%$ probability of collapse goal. Like the TBI Guidelines, under ASCE/SEI 7-16, nonlinear analysis results are evaluated against a series of global and local acceptance criteria.

Global acceptance criteria include:

- For Risk Category II structures, not more than one motion in the suite of $11 \mathrm{can}$ produce unacceptable response. Unacceptable response is defined as development of numerical instability in the analysis, or prediction of response beyond valid ranges of modeling. Valid ranges of modeling for deformation controlled elements are as described under the PEER TBI Guidelines. Valid range of modeling for demands on force-controlled elements are limited to the element's failure capacities. For both element types the valid range of modeling 
is extended if the effect of element failure on the structure's response is captured in the analysis. For Risk Category III and IV structures, all analyses in the suite of 11 must produce acceptable response.

- Drift limits cannot exceed twice the acceptable values specified by ASCE/SEI 7 Chapter 12 for design level shaking.

With regard to the first of these criteria, one unacceptable response prediction out of a suite of 11 is not statistically significant with regard to the $10 \%$ probability of collapse goal. However, if a structure's actual collapse probability is $10 \%$ or less, it is highly unlikely that more than one unacceptable response will occur in a suite of 11 analyses. The second criterion is intended to provide reasonable parity with structures designed using linear procedures, recognizing that nonlinear analysis is more likely to provide meaningful predictions of nonlinear deformation than linear analyses.

As with FEMA 273/274, ASCE/SEI 41 and the PEER TBI Guidelines, local acceptance is judged by separately evaluating force-controlled and deformationcontrolled elements. Force-controlled actions must satisfy the formula:

$$
\gamma I_{e}\left(Q_{u}-Q_{n s}\right)+Q_{n s} \leq Q_{e}
$$

where $I_{e}$ is an risk category-related importance factor intended to account for differences in the acceptable probability of collapse for different risk categories; $Q_{u}$ is the mean demand from the suite of analyses; $Q_{n s}$ is the demand due to loads other than seismic; $Q_{e}$ is the expected component strength; and $\gamma$ is a load factor taken as 2.0 for critical force-controlled components, 1.5 for ordinary force-controlled components and 1.0 for non-critical components. This acceptance check is intended to provide the target probabilities of collapse avoidance given $M \mathrm{MEE}_{\mathrm{R}}$ shaking. Critical components are those the failure of which would lead to structural instability or collapse of a large portion of the structure. Ordinary components are those the failure of which would lead to only limited collapse. The failure of non-critical components would not lead to collapse

For critical and ordinary deformation-controlled actions, the mean inelastic deformation, $Q_{u}$, is not permitted to exceed $\phi_{S} Q_{n e}$ where $\phi_{S}$ is taken as $0.3 / I_{e}$ for critical elements and $0.5 / I_{e}$ for ordinary elements. In this evaluation, $Q_{n e}$ is intended to be the deformation at which loss of gravity load carrying capacity occurs. Recognizing that data as to the magnitude of $Q_{n e}$ are not generally available, it is permitted to use the acceptance check for deformation-controlled behaviors in ASCE/SEI 41 in lieu of this formulation. 


\subsection{Adaptive Model Response}

ASCE/SEI 41-13 notionally derives acceptance criteria based on first cycle backbone curves as noted in Chapter 2. As also discussed in Chapter 2, most real earthquakes do not typically provide the extent of cyclic degradation in many structural elements commonly observed in reversed cyclic testing. To the extent this is true, the acceptance criteria specified by ASCE/SEI 41-13 for deformation-controlled behaviors are conservative in that likely, when subjected to real earthquake shaking, most elements would be capable of sustaining these levels of deformation without failure. Chapter 2 also described adaptive modeling procedures in which hysteretic elements capable of tracking the expected force-deformation response of real structural elements, subject to specific motions, would be used. It is not clear how acceptance criteria for such elements would be determined given that if the element does not fail in a given ground motion, it is not generally known how much more shaking input the element could have sustained without failure. Therefore, if pathdependent hysteretic models are adopted, it may be necessary either to continue to use acceptance criteria like those in ASCE/SEI 41-13 or to adopt the PEER TBI Guidelines approach of not specifically evaluating deformation-controlled behavior for acceptance as long as response remains within the valid modeling range.

\subsection{Recommended Acceptance Criteria}

This section recommends a basis for development of acceptance criteria for incorporation into future editions of the ASCE/SEI 41 standard that is consistent with the use of adaptive models and also the achievement of target levels of reliability. Separate recommendations are provided for nonlinear dynamic analysis, nonlinear static analysis, and linear analysis approaches.

\subsubsection{Nonlinear Dynamic Analysis}

Acceptability should be judged similar to the PEER TBI Guidelines and ASCE/SEI 7-16 considering global and local response. Assuming a 10\% probability of failure given the occurrence of design shaking is acceptable, like ASCE/SEI 7-16, a suite of not less than 11 motions should be evaluated and not more than one of the motions should be permitted to result in unacceptable response. Unacceptable response should be defined as it is in ASCE/SEI 7-16, as any analysis that produces demands exceeding the valid range of modeling, including prediction of demands on elements that produces local failure, unless the model is capable of simulating the effect of such failure on global response. If the acceptable failure probability is less than $10 \%$, than a larger suite of motions will be required.

For Immediate Occupancy performance, the global criteria should be conditioned on the occurrence of permanent, or residual, drift that exceeds a level that would be deemed acceptable for occupancy. Although there is no consensus on how large such 
drift should be, FEMA P-58, Seismic Performance Assessment of Buildings, (FEMA, 2012a) suggests that a residual drift of $1 \%$ has a $50 \%$ probability of encouraging placement of an unsafe placard, with a dispersion, or uncertainty on that value of $30 \%$. Given this, at a $90 \%$ confidence level, the limiting residual drift would $0.6 \%$. Therefore, if the median residual drift predicted by a suite of analyses is $0.6 \%$ or less, this should produce $90 \%$ confidence that the building would not be placarded unsafe, on the basis of residual drift.

Like ASCE/SEI 41 and ASCE/SEI 7-16, acceptance should also be judged on the basis of individual element evaluations. The basis for these criteria is the assumption that the uncertainty in both demand and capacity can be considered to be represented by lognormal distributions with respective medians of $\hat{D}$ and $Q_{e}$ with respective dispersions of $\beta_{D}$ and $\beta_{C}$. The demand uncertainty, $\beta_{D}$, is a function of record-torecord variability in response to different ground motions, and errors in estimating the mean demand, due to modeling errors and the use of a limited number of ground motions. The uncertainty in capacity, $\beta_{C}$, is a function of material variability and construction tolerance and quality. Given this, it can be shown that the ratio of demand to capacity is also lognormally distributed having a median value equal to $\hat{D}$ divided by $Q_{e}$ and having a dispersion equal to $\sqrt{\beta_{D}^{2}+\beta_{C}^{2}}$. Given this, if the median demand to capacity ratio determined from a suite of analyses is known, the probability of failure (demand greater than capacity) can be solved directly from the lognormal distribution. This is the basis of Load and Resistance Factor Design (LFRD) procedures.

For force-controlled behaviors, the ASCE/SEI 7-16 approach is recommended for Collapse Prevention performance, where acceptance is evaluated on the equation:

$$
\gamma\left(Q_{u}-Q_{n s}\right)+Q_{n s} \leq Q_{e}
$$

where $Q_{u}$ is the mean demand obtained from the suite of analyses; $Q_{n s}$ is the nonseismic demand; $Q_{e}$ is the expected strength capacity computed using the procedures in ACI 318, AISC 360 and similar industry standards; and $\gamma$ is a reliability based load factor intended to provide the desired reliability, considering uncertainty in demand prediction, and uncertainty and bias inherent in the predictive equation for $Q_{e}$. Table 3-1 indicates the recommended value of $\gamma$ as a function of the desired confidence level in meeting targeted performance. These values of $\gamma$ have been computed assuming a dispersion in capacity of approximately $15 \%$, a bias in capacity of 1.0 and several values of dispersion in mean demand. Note that the values of $\gamma$ can be directly reduced by the value of any inherent bias in the predictive equations for capacity. The uncertainty in mean demand is a function of the number of ground motions evaluated in the prediction of $Q_{u}$. The values of $\gamma$ are relatively insensitive to the dispersion in capacity within the likely range of such uncertainties. 
Table 3-1 Load Factor, $\gamma$, as a Function of Confidence and Demand Uncertainty, Assumed Capacity Dispersion Approximately $15 \%$

\begin{tabular}{|c|c|c|c|c|c|}
\hline \multirow[b]{2}{*}{ Confidence Level } & \multicolumn{5}{|c|}{ Demand Uncertainty } \\
\hline & $10 \%$ & $20 \%$ & $30 \%$ & $40 \%$ & $50 \%$ \\
\hline $85 \%$ & 1.2 & 1.3 & 1.4 & 1.6 & 1.7 \\
\hline $90 \%$ & 1.3 & 1.4 & 1.5 & 1.7 & 2.0 \\
\hline $95 \%$ & 1.4 & 1.5 & 1.7 & 2.0 & 2.4 \\
\hline
\end{tabular}

In the table, demand uncertainty of $20 \%$ might exist when the demand is limited by a well-defined yield mechanism, such as exists in the columns of a moment-frame and when the yield capacity of the mechanism is well constrained when demands are clearly forcing the structure into full yield. Dispersion on the order of 40 to $50 \%$ would exist as an upper bound when strength demands are not so limited.

For Life Safety and Immediate Occupancy performance these load factors can be increased to provide the desired increase in confidence that collapse will not occur.

For deformation-controlled behavior, and Collapse Prevention performance, adequacy of response should be obtained simply by assuring that in each of the suite of analyses, no deformation controlled elements is deformed beyond the valid range of modeling, except as specifically permitted for unacceptable runs. For Life Safety performance, the same criteria should be used; however, a larger suite of ground motions should be used, or the desired confidence of non-collapse should be increased.

For Immediate Occupancy performance, deformation demands on deformationcontrolled elements should be limited such that there is $90 \%$ confidence that demands will not exceed point B on the cyclic backbone of Figure 3-1. This can be achieved by evaluating the equation:

$$
4_{u} \leq \frac{1}{\gamma} 4_{C P-P}
$$

where $\Delta_{u}$ is the mean deformation demand on the element; $\Delta_{C P-P}$ is the deflection at point B in Figure 3-1; and $\gamma$ is the load factor from Table 3-1. A dispersion of 0.45 is recommended when selecting values of $\gamma$.

\subsubsection{Nonlinear Static Analysis}

Nonlinear static analysis should use the same procedures recommended for nonlinear dynamic analysis with the following differences:

- Since only one analysis is performed, rather than a suite of motions, global criteria as to unacceptable response apply only with regard to the single static analysis. 
- Evaluation of both deformation and force-controlled elements should be performed using the acceptance criteria of Equation 3-7, except that the total dispersion (uncertainty) should be taken as not less than 0.6, except when evaluating force-controlled behaviors for demands that are limited by a welldefined plastic mechanism and the demand value is taken as the value associated with development of that mechanism. In those cases, dispersion may be taken as having a value of 0.2 .

\subsubsection{Linear Analysis}

Acceptance criteria for linear procedures should remain the same as described in ASCE/SEI 41-13, using cyclic backbones with the exception that the backbones should be updated based on the data presented herein. 



\section{Chapter 4}

\section{Steel Moment Frame Systems}

\subsection{Overview of Geometry and Behavior}

This chapter discusses the general behavior of steel moment frame systems under earthquake loading including a brief summary of code provision changes associated with their seismic design in North America since 1970s. The performance including damage progression of main structural steel components in steel moment frame systems under cyclic loading is discussed in detail. Recommendations for nonlinear modeling of steel moment frames are developed based on available experimental data conducted over the past 40 years around the world. Such recommendations include target force-deformation envelopes per structural steel component.

Figure 4-1a illustrates a typical steel moment-resisting frame. Its main structural components that provide lateral load stiffness and resistance consist of the steel beams and columns, the beam-to-column joint panel zones, the floor deck and foundation systems. Some of these components can be identified in Figure 4-1a. In North America, a common practice in steel moment frame systems is the use of wide flange steel sections for the design of the main framing system. However, steel hollow structural sections may be an alternative option especially for steel columns. Figure 4-1b illustrates schematically the aforementioned structural components of a steel moment frame system. The floor deck system shown schematically in Figure 4-1c consists of girders (i.e., primary beams) and beams (i.e., secondary beams) connected with typical shear tab connections with the main girders and a concrete slab on a ribbed steel deck.

Since 1980, a common design practice in North America is the use of fewer momentresistant connections in planar frames typically located in the perimeter of a building plan view (FEMA, 2000d). According to this practice, deeper and often times heavier wide flange steel members are likely to be employed in order to satisfy the lateral drift requirements imposed by the seismic design provisions. The interior framing utilizes partially restrained beam-to-column connections such as conventional single plate shear tab connections. The effects of the gravity framing on the overall behavior of steel moment frame systems under earthquake loading are typically ignored in today's practice. Considering the impact of the gravity framing on the overall steel frame system lateral load resistance and stiffness, this assumption is typically fairly conservative given that the gravity force supported by the gravity system contributes to the destabilizing P-delta effects (Gupta and Krawinkler, 1999; 
Flores et al., 2014; Elkady and Lignos, 2015a). In most cases, the steel frame building is stronger and stiffer than the assumed "lateral-only" steel frame building. The estimated period is longer than it actually is in reality (Elkady and Lignos, 2015a); therefore, the strength demands on the lateral load-resisting system may be typically larger than those assumed. However, in most cases this is not a controlling design consideration for moment-resisting frames because they are drift-controlled.

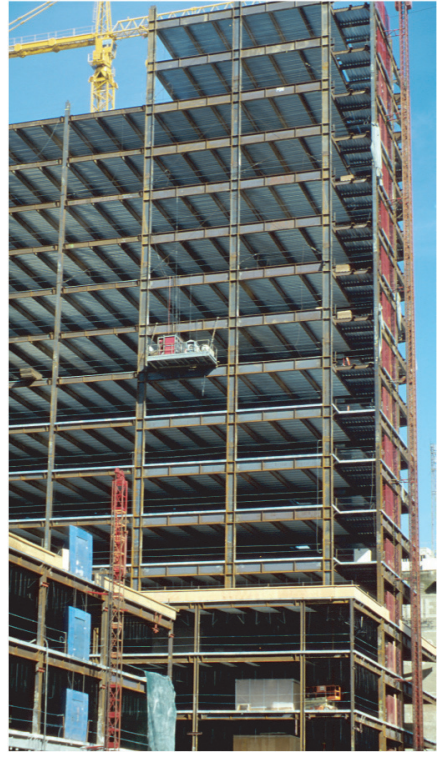

(a)

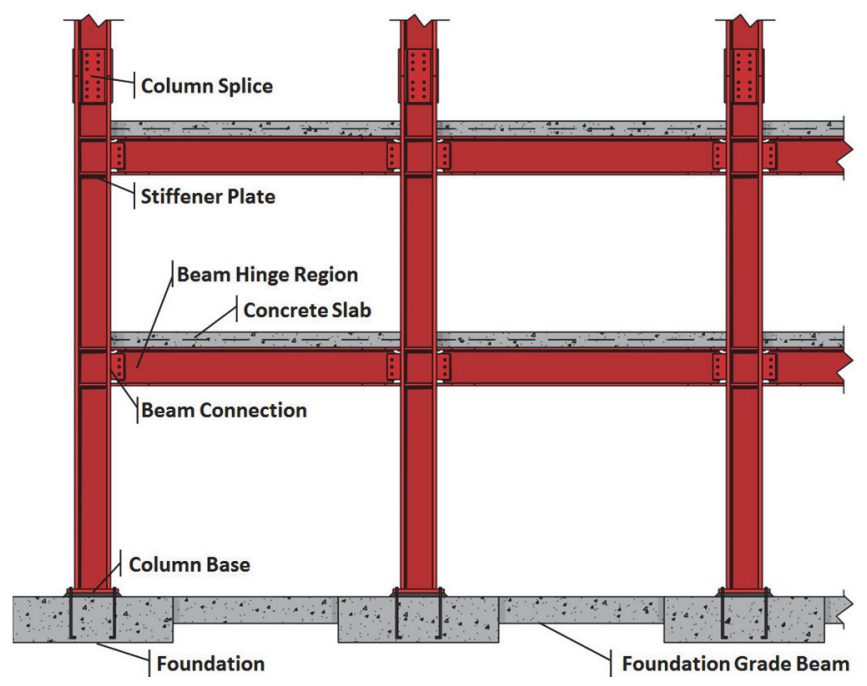

(b)

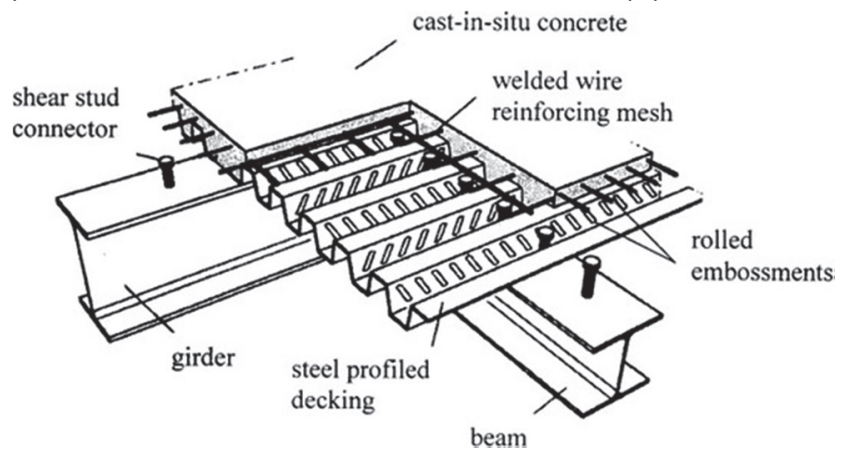

(c)

Figure 4-1 Typical steel moment-resisting frame including its main structural components (image 4-1a courtesy of Michael Engelhardt, image 4-1b from NIST (2016), image 8-1c, Ganesh et al. (2006)).

Steel moment frame systems are organized in seven main design categories per ANSI/AISC 341-10, Seismic Provisions for Structural Steel Buildings (AISC, 2010a) and ASCE/SEI 7-10, Minimum Designs Loads for Buildings and Other Structures (ASCE, 2010), design provisions. These categories are summarized as follows:

- Steel Ordinary Moment Frame (OMF). The members and connections of an OMF are designed without any specific requirements for stability and limitations on width-to-thickness ratios of the respective steel members. Such members and 
connections are expected to provide minimal inelastic deformation capacity during an earthquake.

- Steel Intermediate Moment Frame (IMF). This seismic load-resisting system is expected to provide inelastic deformation capacity through flexural yielding of the beams and columns as well as shear yielding of the column panel zones. Beams are typically braced to satisfy the requirements for moderate ductility members per ANSI/AISC 341-10.

- Steel Special Moment Frame (SMF). Such a system is expected to provide significant inelastic deformation capacity through flexural hinging of the steel beams and controlled panel zone shear yielding. Beams are braced to satisfy the requirements for highly ductile members. Column flexural yielding is possible. Column flexural yielding is only allowed at the base of the first story columns of the steel SMF. Columns are designed to be stronger than the fully yielded and strain-hardened girders (i.e., Moment Ratio requirements) except at roof levels. Note that steel beams are typically connected to columns using various prequalified beam-to-column connections specified in ANSI/AISC-358-10, Prequalified Connections for Special and Intermediate Steel Moment Frames for Seismic Applications (AISC, 2010b).

- Special Truss Moment Frame (STMF). Such a system is expected to provide significant inelastic deformation capacity within a special segment of the truss and it is limited to span lengths between columns not to exceed 65 feet and overall depth not to exceed 6 feet.

- Ordinary Cantilever Column System (OCCS). In this case, minimal inelastic deformation capacity is provided through flexural yielding of the columns, which are not braced with any specific stability requirements.

- Special Cantilever Column System (SCCS). Limited inelastic deformation capacity is provided through flexural yielding of the columns, which should be braced to satisfy the requirements for moderately ductile members.

- An additional category includes moment frames designed according to ANSI/AISC 360-10, Specification for Structural Steel Buildings (AISC, 2010c), without any seismic detailing (i.e., $R=3$ systems).

Steel moment frame systems that employ seismic capacity design requirements per ANSI/AISC 341-10 and ANSI/AISC 360-10 utilize steel beams that are typically designed as bare components (i.e., the composite action is ignored). However, during an earthquake a steel beam would typically act together with the slab system. This depends on the level of the composite action indicated by the number of the installed shear studs on the steel beam as shown schematically in Figure 4-1c. This has an effect on the lateral stiffness of a steel moment frame system and on the hysteretic 
behavior of steel beam-to-column connections under cyclic loading (FEMA, 2000d; Elkady and Lignos, 2014). Column splices and column bases (exposed and/or embedded) are supposed to remain elastic during earthquake excitations.

Figure 4-2 shows schematically alternative lateral load responses of a steel moment frame under earthquake loading. Steel beams and columns typically deform in double curvature with the exception of the first story columns. In this case, depending on the level of fixity at the column base, the inflection point may be located closer to the column top (i.e., fully-fixed column base) or closer to the column base (i.e., partially-fixed column base). Figure 4-2a shows the desired behavior where steel beams and the column base yield in flexure leading to a collapse mechanism. When inelastic deformations concentrate in beam-to-column joint panel zones a collapse mechanism similar to the one shown in Figure 4-2b may form. Even though the hysteretic response of a beam-to-column joint panel zone is generally stable, excessive panel zone yielding due to shear distortion may allow deformations that cause bottom flange fractures of steel beams intersecting steel columns (Krawinkler et al., 1971) when conventional steel construction materials are employed. Structural engineering design packages have adopted modeling strategies to capture the elastic deformation of the panel zone region. In the past, it was common to consider the finite dimensions of a beam-to-column panel zone joint through a rigid offset zone. However, this is not a good practice. In IMFs and OMFs, it is common that columns yield in flexure. Such plastic hinges may be part of a story collapse mechanism of a steel moment frame as shown in Figure 4-2c. This is undesirable, as large inelastic deformations will accumulate in the single story, rendering the structure susceptible to P-delta collapse at reduced inelastic demand levels.

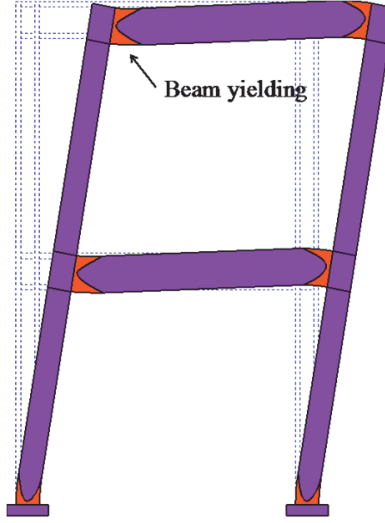

(a)

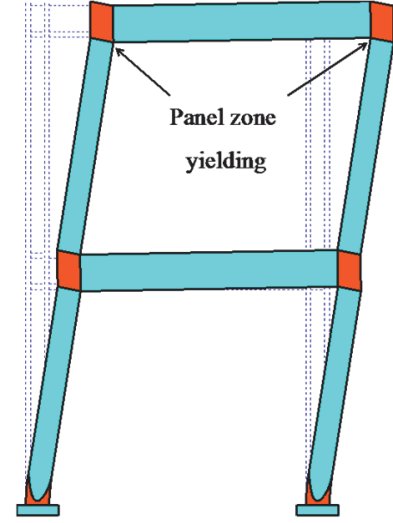

(b)

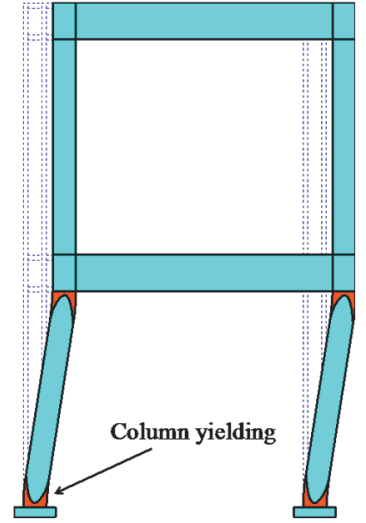

(c)

Figure 4-2 Schematic representation of lateral load response of a steel moment-resisting frame under earthquake loading (images courtesy of Michael Engelhardt).

Ideally, flexural yielding dominates the steel moment frame inelastic deformation. However, depending on the magnitude of the earthquake forces, cyclic and/or in- 
cycle component deterioration in strength and stiffness may be evident due to geometric instabilities associated with local, global, or lateral torsional buckling. In addition, ductile tearing due to steel deterioration may be evident. In steel moment frames designed prior to the 1994 Northridge earthquake brittle fracture of welded beam to column connections may be another deteriorating mode (FEMA, 1997c). When a story or a number of stories of a steel moment frame system displaces laterally sufficiently, P-delta effects accelerated by cyclic or in-cycle strength and stiffness deterioration of steel components can offset to zero the first order story shear resistance leading to dynamic instability. If the moment frame system does not collapse it is likely to have excessive residual story drift ratios along its height in the aftermath of an earthquake (Ramirez and Miranda, 2012).

In brief, steel beams and columns may buckle locally at their flanges and/or web. This deteriorating mechanism mostly depends on the cross-sectional slenderness, and to a lesser extent to the steel material. Floor decks may affect degrading response of beams due to the neutral axis shifting within the composite cross section compared to the bare cross section. Furthermore, concrete slab cracking may affect the flexural strength deterioration of a steel composite beam under cyclic loading. Depending on member slenderness, steel beams and columns may buckle globally and/or twist due to lateral torsional buckling. Ductile tearing typically occurs at locations that develop large plastic strains. These could typically be regions that have buckled excessively under cyclic loading (see Sections 4.3.1 and 4.3.3). In such cases the crack is stable unless the applied stresses are increased. Axial shortening as well as out-of-plane instabilities of steel columns are another common deteriorating mechanism (see Section 4.3.3).

Depending on the level of shear distortion, beam-to-column joint panel zones may buckle in shear after the formation of shear yielding. Conventional single plate shear tab beam-to-column connections may deteriorate in flexural strength due to crushing of concrete of floor slab bearing against the steel column and net section fracture of shear tab or web of the steel beam or bolt fracture due to shear. However, the aforementioned failure modes occur at large inelastic rotations. Extended shear tab beam-to-column connections (i.e., the distance between the support face and the first vertical row of bolts is larger than $3-1 / 2$ inches) may also deteriorate in strength due to shear tab buckling (Sherman and Ghorbanpoor, 2002; Hertz et al., 2015).

Crack formation, propagation, and ultimately, fracture is a common deterioration mode that depends on the ability of the steel material to undergo plastic deformations. Such deterioration modes may occur in column splices, welds at column bases, and at fully restrained beam-to-column connections. In preNorthridge welded beam-to-column connections brittle fractures typically initiate in beam flange groove welds due to low fracture toughness of the weld metal, poor weld quality, notch effects from weld backing bars, and discontinuities in weld runoff 
regions at weld shear tabs (FEMA, 2000d). Other contributing factors included weld access hole geometry, beam web attachment detail and panel zone geometry (Ricles et al., 2000b; 2000c). Extended column base plates and end plate connections may deteriorate in flexural strength due to plate bending and anchor rod or bolt yielding or fracture. Plate bending is associated with the amplification of tensile forces into anchor rods or bolts due to prying action.

The extent of cycle-to-cycle and in-cycle deterioration in strength and stiffness of various steel components depends on the employed loading history. If component behavior close to collapse is of primary interest, then in many cases a typical component response-history will be very different from the one that the same component experiences under a routinely used symmetric loading protocol similar to the one specified by Chapter K of ANSI/AISC 341-05 (AISC, 2005a) (Krawinkler, 2009; FEMA, 2009a; Lignos et al., 2011a). Figure 4-3 illustrates the first story drift history (see Figure 4-3a) and first story column deduced moment-rotation relations at the base (see Figure 4-3b) and top (see Figure 4-3c) locations of a 4-story steel frame building with moment-resisting frames thatwas tested at full-scale through collapse in Japan (Suita et al., 2008; Lignos et al., 2013). As seen from these figures, the importance of in-cycle deterioration in member flexural strength diminishes due to "ratcheting" behavior of the 4-story building, where lateral deformations (i.e., story drift ratios) increase in one direction and inelastic load reversals become insignificant.

The effect of loading history on member response is further illustrated in Figure 4-4, where the moment-rotation relations of three identical steel wide flange columns tested under three different lateral loading histories is superimposed. In particular, based on the monotonic curve (noted with blue color) the steel column reached zero flexural strength at about 0.2 radians. When a symmetric loading protocol is employed (i.e., noted with red color) the same column reached to a zero flexural strength at about 0.05 radians. The steel column lost its axial load carrying capacity at about the same lateral deformation level. In Figure 4-4, the same column deteriorates in strength and stiffness much slower when a "collapse" loading protocol is employed (noted with green color) that represents the "ratcheting" behavior of steel moment frame systems prior to collapse (Suzuki and Lignos, 2014; 2015). Note the difference between the first-cycle backbones between the two cyclic loading protocols compared to the monotonic envelope. In Figure 4-4 it is understood that the cyclic backbone is influenced by the employed loading protocol but the monotonic envelope can be considered as a characteristic property of the steel column. 


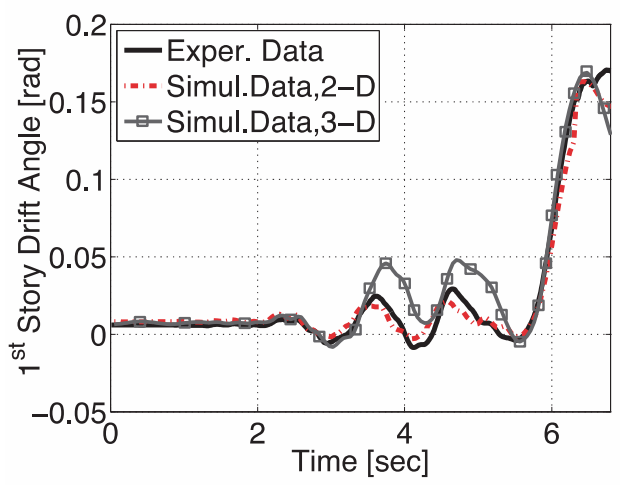

(a) First story drift history

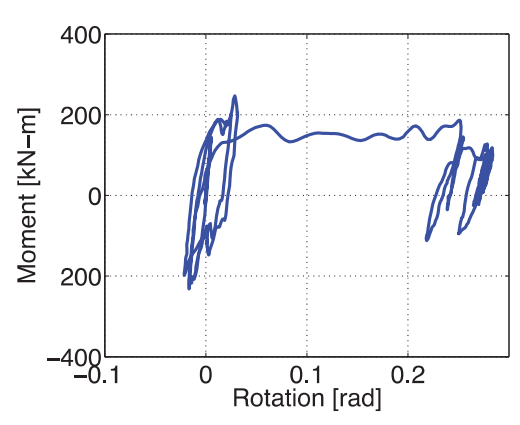

(b) Base location

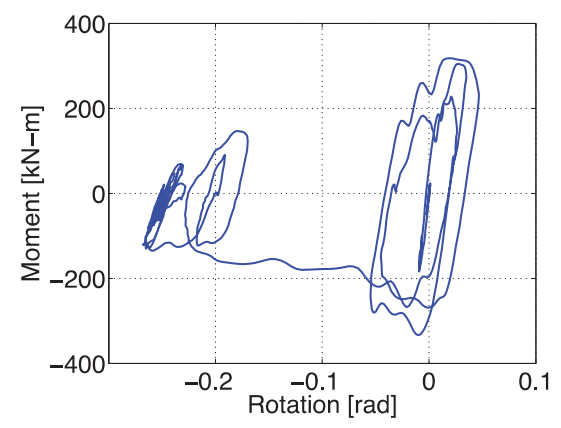

(c) Top location

Figure 4-3 Dynamic response of a 4-story steel frame building up to collapse (Lignos et al., 2013).

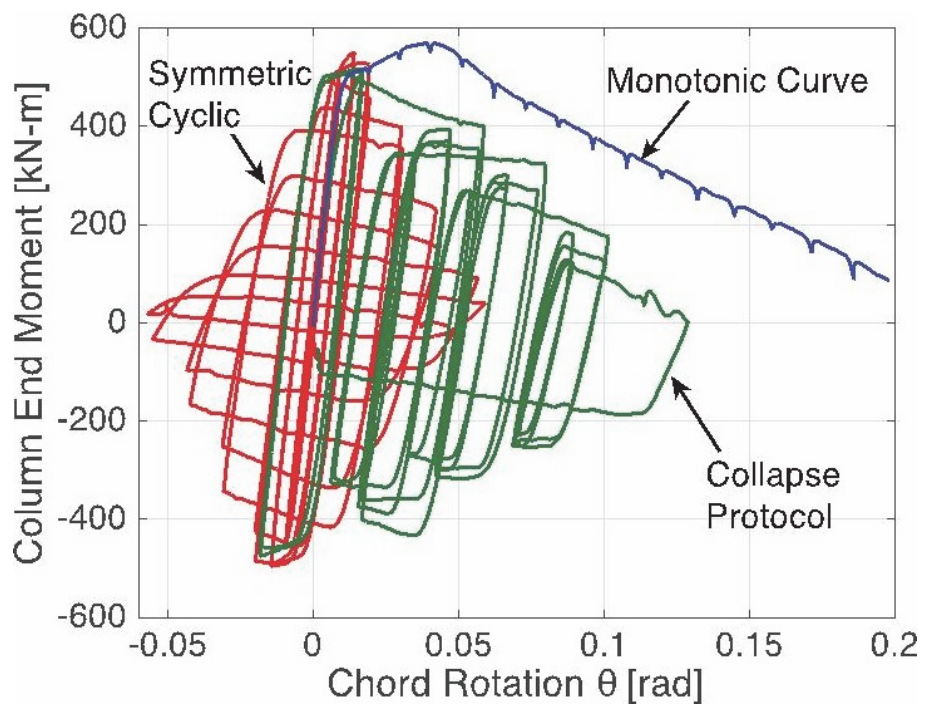

Figure 4-4 Effect of loading history on cyclic behavior of steel columns (Suzuki and Lignos, 2015).

\subsection{Summary of Design Code Provisions}

This section provides a brief summary of the development of U.S. code requirements for steel moment frame design, since the mid-1970s. In this era, in higher seismic zones buildings exceeding 160 feet in height were required to incorporate moment- 
resisting space frames, either concrete or steel, with the capacity to resist at least $25 \%$ of the specified seismic design forces. Specified design forces were computed using a system quality factor, $K$, of 0.67 if the frame provided $100 \%$ of the resistance, or 0.8 if less.

Until the 1994 Northridge earthquake the Uniform Building Code (UBC, 1994) prequalified only the welded flange-bolted web wide flange column connection (i.e., WUF-B) for steel moment frames. Use of other connection types were permitted only upon demonstration that they could provide adequate performance. With the incorporation of such connections, believed to be capable of ductile behavior in design practice, in higher seismic zones emphasis was placed on the inelastic behavior of ductile, later special, steel moment frame systems. Beams were designed to develop their full plastic capacity. Members expected to experience inelastic deformations were required to comply with the requirement for "plastic design sections" (UBC, 1973). Welded connections with tension butt welds had to be tested by nondestructive methods for compliance with UBC Standard No. 27-6. In lower seismic zones there were few special requirements, similar present criteria for frames designed with a Response Modification Coefficient, $R=3$. The UBC (1985) imposed few new requirements related to steel ductile moment-resisting space frames designed in Seismic Zones 3 and 4 related to ultrasonic testing of partial penetration groove welds when used in column splices. In addition, ultrasonic inspection requirements were imposed for through thickness welds in steel components that utilized base metals thicker than $1-1 / 2$ inches.

Around 1988, the UBC seismic provisions made no distinction between frames that utilized strong-column-weak-beam (SCWB) and strong-beam-weak-column (SBWC) frames in the determination of the $R$ factor and the seismic design forces (Roeder et al., 1993). Seismic design provisions at that time (UBC, 1988) allowed the limited use of strong beam-weak column framing if the column axial forces did not exceed $40 \%$ of the column yield axial strength and if the story shear resistance was more than $50 \%$ greater than the story above it.

In an effort to ensure large plastic deformation capacities in steel moment frame systems, UBC (1988) employed special compactness limits as well as global slenderness requirements for lateral support for beams. In addition, around the same time steel frame buildings in the U.S. became lighter and more flexible than before. In an effort to minimize the economic cost due to welding, designers tended to concentrate moment-resistant connections in fewer frames that were typically concentrated in the perimeter of the building plan view. Such design resulted in deeper members in order to accommodate the lateral drift requirements imposed by the design provisions. In 1988, the UBC incorporated provisions that encouraged beam-to-column joint panel zone yielding (FEMA, 2000d; Nakashima et al., 2000) as well as limitations associated with the flexural strength of columns be at least 1.25 
times the moment that corresponded to the panel zone shear strength. These requirements were further refined in UBC (1991) that included design details for doubler plates to reduce panel zone shear stress as well as explicit flexural strength ratio checks for steel beams and columns at any moment frame joint.

After the 1994 Northridge and 1995 Kobe earthquakes, steel moment frame systems suffered significant damage consisting of cracking at welded beam-to-column connections (Mahin, 1998; Nakashima et al., 2000; FEMA, 1997c; 2000d).

Based on extensive research conducted as part of the SAC Joint Venture project, improved seismic performance can be achieved if an improved connection geometry is used, that includes improved weld access hole details and web attachments (Ricles et al., 2000b; 2000c) use of weld filler metals with notch-toughness; backing bar removal, backgouging, and reinforcement of complete joint penetration (CJP) welds. However, even WUF-B connections employing these details, now permitted for Ordinary Frames, have inadequate behavior for use in Special Moment Frames. The 1997 UBC and later building codes required that all connections be demonstrated by testing and analysis to be capable of sustaining the required inelastic rotation demands without excessive deterioration leading to publication ANSI/AISC 358-10. A variety of connection technologies have now been prequalified. Other important considerations to achieve improved seismic performance is to keep the span-to-depth ratio in steel beams to appropriate proportions; and to employ local and global member slenderness requirements depending on the steel moment frame system category discussed in Section 4.1. These requirements that are explicitly summarized in FEMA 355D, State of the Art Report on Connection Performance, (FEMA, 2000d) that formed the basis of the current ANSI/AISC 341-10. A summary of recommended seismic design criteria for steel moment frame buildings is included in ANSI/AISC-358-10. These criteria have been refined compared to what was originally included in FEMA 350, Recommended Seismic Design Criteria for New Steel Moment Frame Buildings (FEMA, 2000b). Table 4-1 summarizes in brief the current design requirements for steel moment frame systems.

\subsection{Performance and Damage Progression}

This section characterizes the seismic response of the primary structural steel components of steel moment frame systems. The section is organized in categories based on the individual steel component types. Each category includes a basic drawing of the respective component, a force-deformation relation that describes its characteristic hysteretic behavior under cyclic loading and images that illustrate key failure modes. Emphasis is placed on failure modes associated with cyclic, i.e., cycle-to-cycle, and/or in-cycle deterioration in strength and stiffness. For consistency, the force-deformation relations presented are primarily based on a symmetric loading protocol as discussed in the Chapter K of ANSI/AISC 341-10. 
This is intentionally done because the target force-deformation envelopes to be developed for nonlinear modeling of steel components (see Section 4.5) are mostly based on first-cycle envelopes based on symmetric loading histories.

Table 4-1 Summary of Design Requirements for Steel Moment Frame Systems

\begin{tabular}{|c|c|c|c|c|}
\hline Component & $\begin{array}{c}\text { Not Detailed for } \\
\text { Seismic Resistance } \\
R=3, \Omega_{0}=3, C_{d}=3\end{array}$ & $\begin{array}{c}\text { Ordinary } \\
\text { Moment Frame } \\
R=3.5, \Omega_{0}=3, C_{d}=3\end{array}$ & $\begin{array}{c}\text { Intermediate } \\
\text { Moment Frame } \\
R=4.5, \Omega_{0}=3, C_{d}=4\end{array}$ & $\begin{array}{c}\text { Special } \\
\text { Moment Frame } \\
R=8, \Omega_{0}=3, C_{d}=5.5\end{array}$ \\
\hline Beam & No requirements & No requirements & $\begin{array}{l}K L / r_{y}, b_{f} / 2 t f, h / t_{w} \text { for } \\
\text { moderately ductile } \\
\text { members }\end{array}$ & $\begin{array}{l}K L / r_{y}, b_{f} / 2 t_{f}, h / t_{w} \text { for highly } \\
\text { ductile members }\end{array}$ \\
\hline Column & No requirements & No requirements & $\begin{array}{l}K L / r_{y}, b_{f} / 2 t_{f}, h / t_{w} \text { for } \\
\text { moderately ductile } \\
\text { members }\end{array}$ & $\begin{array}{l}S C W B>1.0, K L / r_{y}, b_{f} / 2 t_{f}, h / t_{w} \\
\text { for highly ductile members, } \\
P_{u c} \text { at end columns amplified } \\
\text { based on } \Omega\end{array}$ \\
\hline \multirow{2}{*}{$\begin{array}{l}\text { Beam-to- } \\
\text { Column } \\
\text { Connection }\end{array}$} & No requirements & FR with CJP & FR with CJP & FR with CJP \\
\hline & & $\begin{array}{l}\text { groove welds } \\
\text { or PR }\end{array}$ & $\begin{array}{l}\text { groove welds, story drift } \\
\text { angle }>0.02 \text { rad \& } \\
\text { measured flexural } \\
\text { resistance }>0.80 M_{p, \text { beam }}\end{array}$ & $\begin{array}{l}\text { groove welds, story drift } \\
\text { angle }>0.04 \text { rad \& measured } \\
\text { flexural resistance }> \\
0.80 M_{p, \text { beam }}\end{array}$ \\
\hline \multirow[t]{2}{*}{ Panel Zone } & No requirements & No requirements & No requirements & $\begin{array}{l}\text { If } P_{r} \leq 0.75 P_{c}, R_{n}> \\
0.60 F_{y} d_{c} t_{w}\left(1+3 b_{c f} t^{2}{ }_{c} / d_{b} d_{c} t_{w}\right) ; \\
\text { else } R_{n}> \\
0.60 F_{y} d_{c} t_{w}\left(1+3 b_{c t} t^{2}{ }_{c} / d_{b} d_{c} t_{w}\right)(1 . \\
\left.9-1.2 P_{r} / P_{c}\right)\end{array}$ \\
\hline & & & & Continuity plate requirements \\
\hline Column Splice & No requirements & No requirements & $\begin{array}{l}\text { CJP groove welds or } \\
\text { bolted with } M_{r, s}>R_{y} F_{y} Z_{x} \\
\text { and } V_{r, s}>\sum M_{p c} / H\end{array}$ & $\begin{array}{l}\text { CJP groove welds or bolted } \\
\text { with } M_{r, s}>R_{y} F_{y} Z_{x} \text { and } V_{r, s}> \\
\Sigma M_{p c} / H\end{array}$ \\
\hline Column Base & No requirements & No requirements & $\begin{array}{l}\text { Demand critical groove } \\
\text { welds }\end{array}$ & Demand critical groove welds \\
\hline $\begin{array}{l}\text { Protected } \\
\text { Zones }\end{array}$ & $\begin{array}{l}\text { No designated } \\
\text { protected zones }\end{array}$ & $\begin{array}{l}\text { No designated } \\
\text { protected zones }\end{array}$ & End of beams & End of beams \\
\hline \multicolumn{5}{|c|}{ FR = Fully-Restrained moment connections per ANSI/AISC 358-10 } \\
\hline \multicolumn{5}{|c|}{$\begin{aligned} \text { CJP = } & \text { Complete Joint Penetration welds: for beam flanges to columns per Section A3.4b and I2.3 designated as demand } \\
& \text { critical welds; for column splices per section D2.5 }\end{aligned}$} \\
\hline \multicolumn{5}{|c|}{ PR = Partially-Restrained moment connections } \\
\hline \multicolumn{5}{|c|}{$P_{u c}=$ Required compressive strength including the amplified seismic load } \\
\hline \multicolumn{5}{|c|}{ SCWB = Strong-Column-Weak-Beam Ratio (i.e., Moment Ratio per ANSI/AISC 341-10) } \\
\hline \multicolumn{5}{|c|}{$P_{c} \quad=P_{y}($ based on LRFD) } \\
\hline \multicolumn{5}{|c|}{ Note $=$ Strength equations are all based on LRFD } \\
\hline
\end{tabular}

\subsubsection{Beams as Part of Fully-Restrained Beam-to-Column Connections}

Typical post-Northridge fully restrained beam-to-column connections per

ANSI/AISC 358-10 mostly concentrate plastic deformations in the beam region 
outside the column face. Controlled panel zone yielding due to shear deformations is also possible as discussed in Section 4.3.4. Figure 4-5 illustrates a typical bare beam (i.e., composite action is neglected) with a reduced beam section (RBS). From the deduced moment-chord rotation relation shown in Figure 4-6 its hysteretic behavior is symmetric. Flexural yielding is developed in the RBS region (i.e., point 1 in the hysteresis). Due to cyclic hardening the flexural resistance of the same beam increases up to about 1.4 times its expected yield moment. This increase depends on the steel material that the beam is fabricated from. Typical values for such increase can be found in Lignos and Krawinkler (2011; 2012a; 2012b). In fully restrained steel beam-to-column connections the onset of flexural strength deterioration due to cyclic loading is mostly attributed to web and flange local buckling as shown in Figure 4-7a. This point is identified in the $M-\theta$ relation of Figure 4-6 for the beam with RBS (i.e., point 2). From this point on, local buckles grow with increased loading amplitudes. This causes in-cycle deterioration in flexural strength of the beam as shown in Figure 4-6.

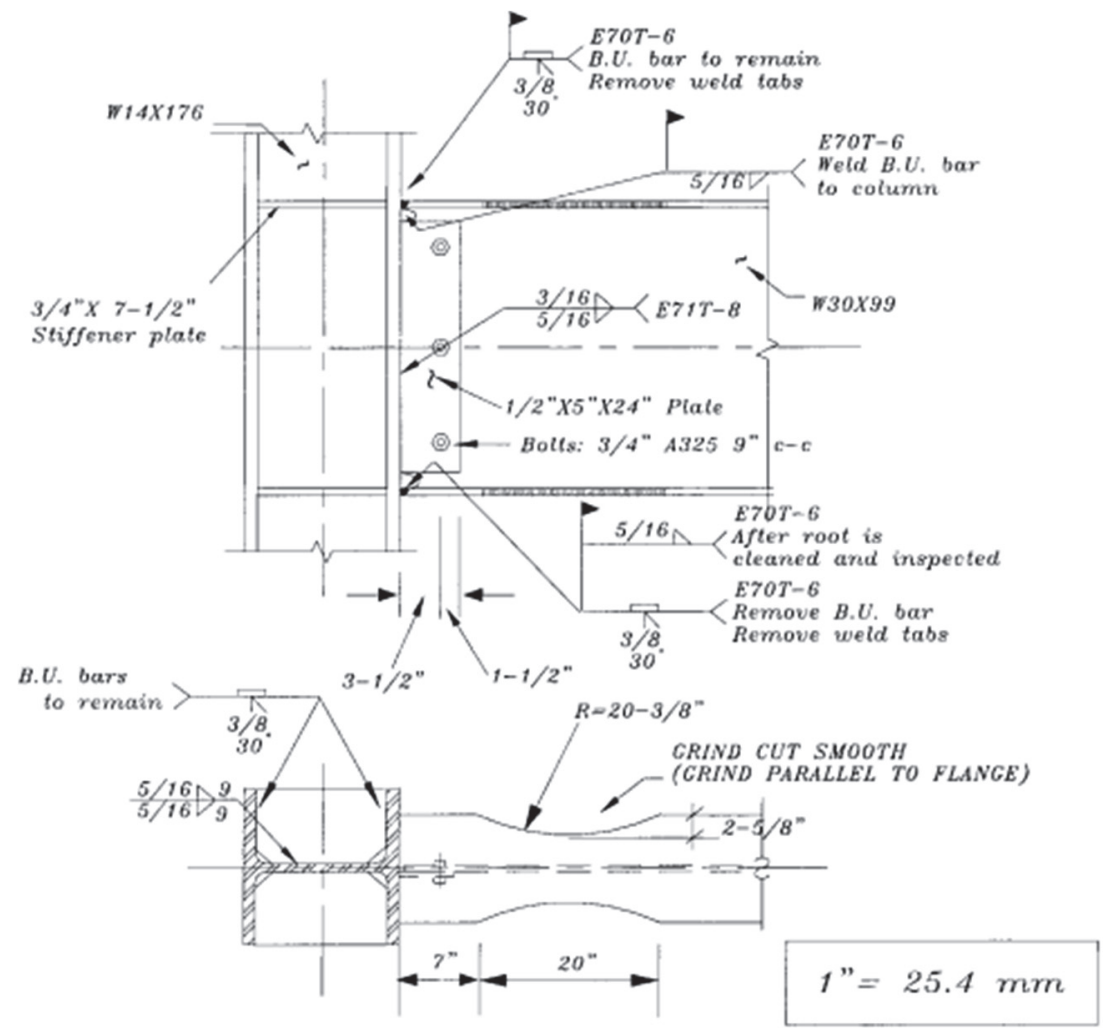

Figure 4-5 Typical RBS moment connection geometry (Uang and Fan, 2001).

Depending on the lateral bracing of the beam, at increased loading amplitudes, cyclic degradation in beam flexural stiffness is likely to occur as shown in Figure 4-7b. This is a very common failure mode in non-conforming beams. Ductile tearing may occur at regions within a steel beam that have developed large local buckles. Typically, large plastic strains develop within these regions. Ductile tearing is a 
stable deteriorating mechanism. Figure 4-7c illustrates an example of ductile tearing within an RBS region based on a testing program by Zhang and Ricles (2006).

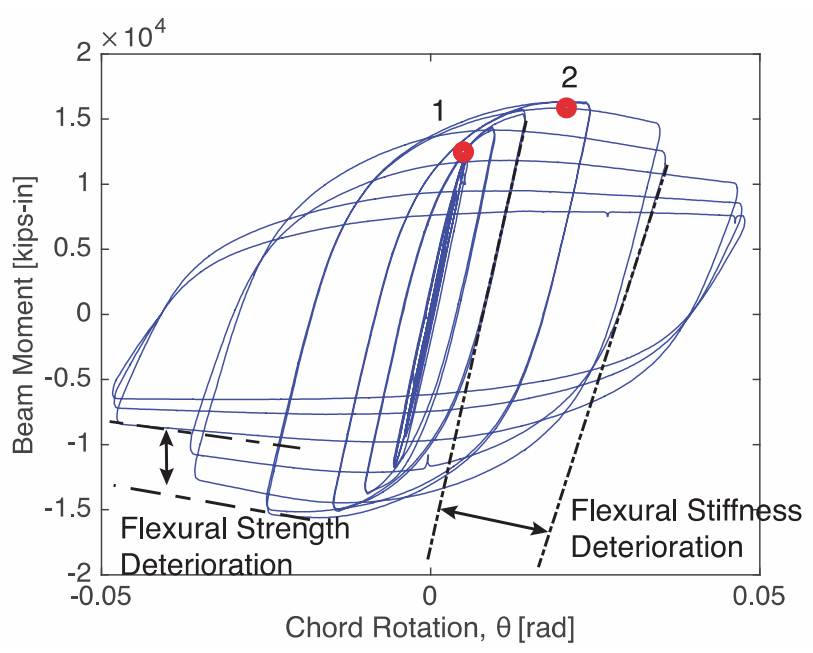

Figure 4-6 Deduced moment-rotation relation for bare beam with RBS (FEMA, 2000d).

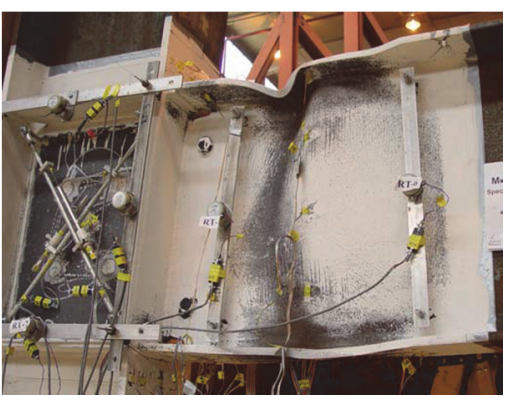

(a) Local buckling

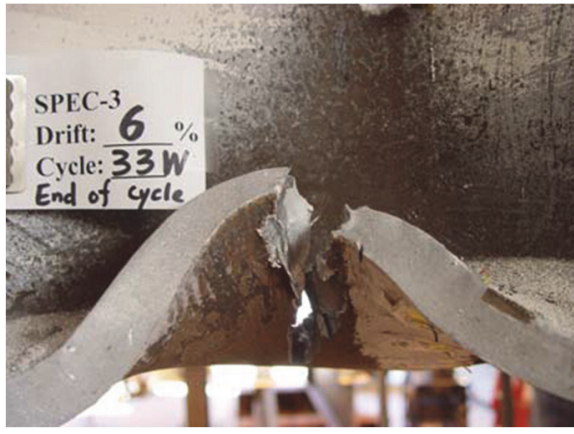

(c) Ductile tearing

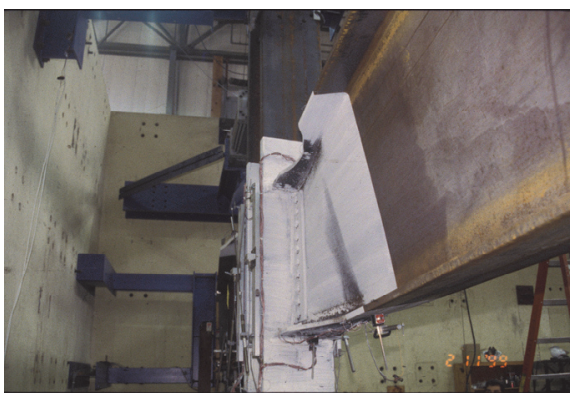

(b) Lateral torsional buckling

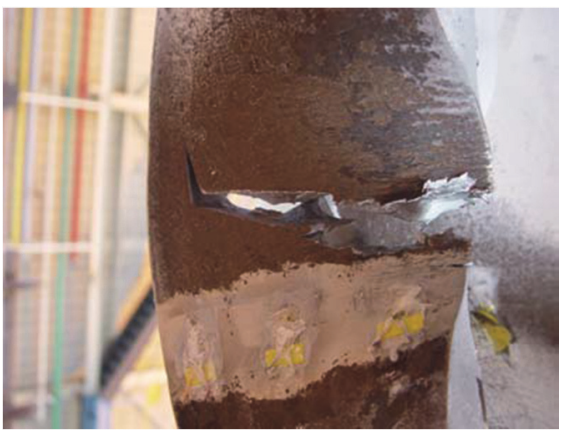

Figure 4-7 Typical damage progression for bare beam with RBS (FEMA, 2000d; Zhang and Ricles, 2006).

Beams in steel moment frame systems typically develop composite action due to the presence of a concrete floor slab. The observed deteriorating mechanisms in this case are typically as those observed in bare steel beams. However, the presence of the floor slab typically results in non-symmetric hysteretic response. For interior beam-to-column subassemblies, when the floor slab is in compression the flexural 
strength of the composite steel beam is typically higher than that of the same beam when the slab is in tension. This is illustrated from the moment-chord rotation relation of a composite steel beam with RBS shown in Figure 4-8a. The onset of local buckling at the top flange of the beam is delayed compared to a bare beam due to the lateral restraint provided by the slab to the top flange. On the other hand, the lower flange of the composite beam is more susceptible to local and lateral torsional buckling due to the beam neutral axis location shift; thus cyclic degradation in both flexural strength and stiffness in such case is non-symmetric as illustrated in Figure 4-8a. Steel deck local buckling (see Figure 4-8b) is a secondary reason for cyclic strength deterioration. The composite action is completely lost once crushing of concrete due to bearing on the column face occurs as shown in Figure 4-8c. This would typically occur at a chord rotation of $4 \%$ radians.

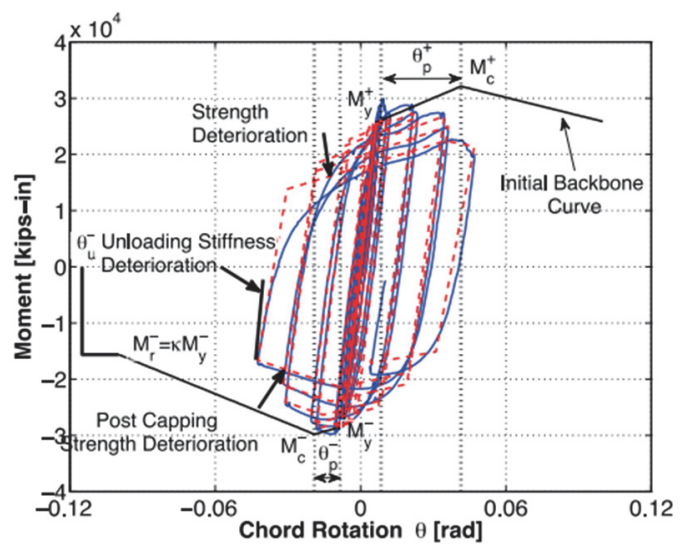

(a) Deduced moment-rotation relation of composite beam with RBS

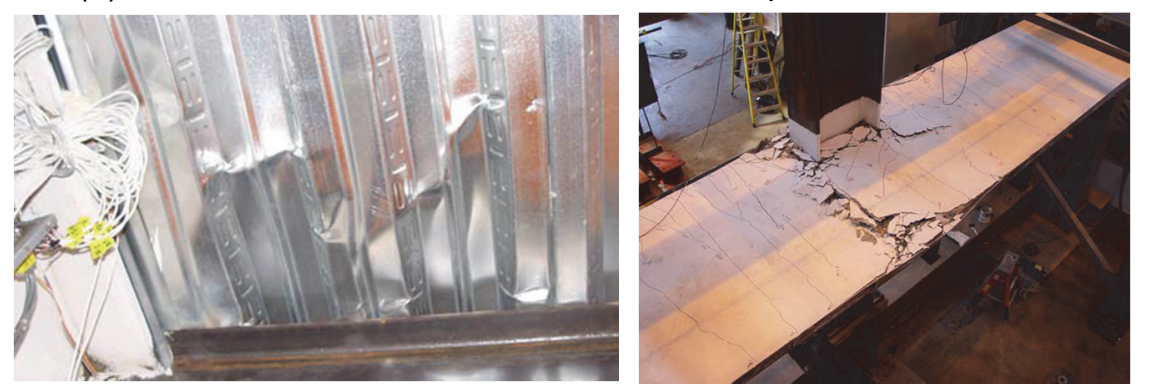

(b) Deck local buckling

(c) Concrete slab cracking and crushing

Figure 4-8 Deduced moment-chord rotation and typical damage progression for composite beams with RBS (Elkady and Lignos, 2014; FEMA, 2000d; Zhang and Ricles, 2006).

Experimental evidence suggests composite effects may not be as significant for exterior framing with a slab only on one side of the beam or in cases where a gap between the concrete slab and the column face exists (Tremblay et al., 1997). Such cases are discussed in detail in Lignos et al. (2011b).

Typical pre-Northridge welded beam-to-column connections (see Figure 4-9a) would normally fracture at the bottom flange to column flange joint at fairly small chord 
rotations in a brittle manner. Shown in Figure $4-9 b$ is the deduced moment-chord rotation relation of a steel beam as part of a welded unreinforced flange (WUF) beam-to-column connection with bolted shear tab. Once beam flexural yielding occurred the steel beam fractured at the weld of the top beam flange to the steel column face. Figure 4-10b illustrates another typical failure mode associated with fracture in the heat-affected zone in the bottom flange of steel beams as part of typical pre-Northridge beam-to-column connections.

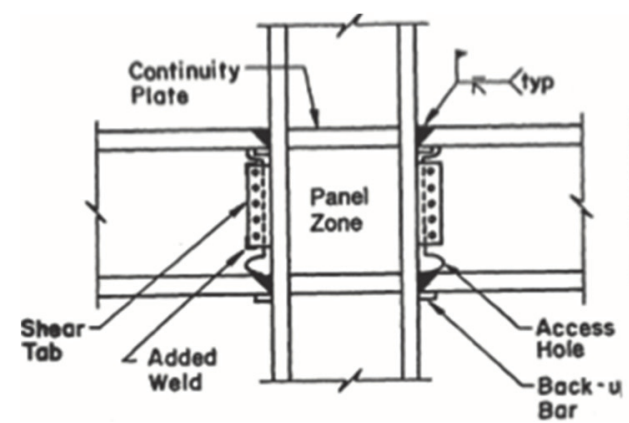

(a)

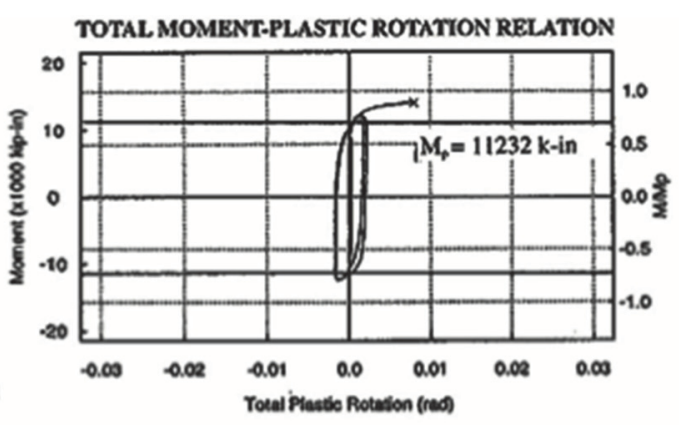

(b)

Figure 4-9 $\quad$ (a) Typical connection detail for pre-Northridge beam-to-column connection (WUF-B) and (b) deduced moment-rotation relation of a steel beam as part of a Pre-Northridge beam-to-column connection (Lu et al., 2000; FEMA, 1997c).

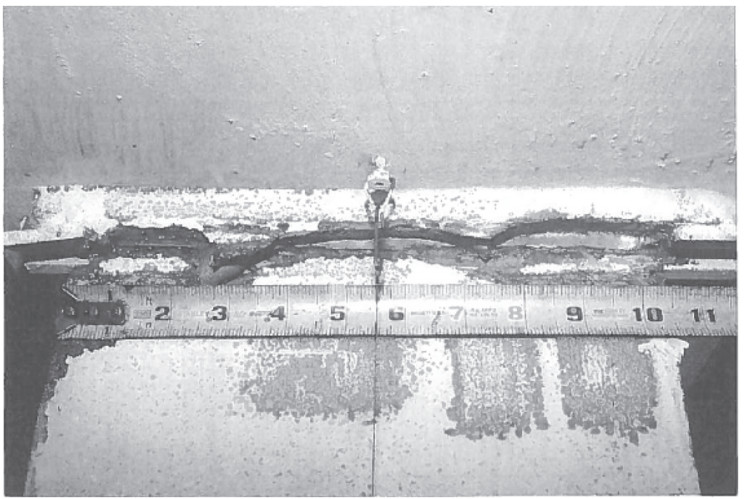

(a) Fracture across welded beam top flange

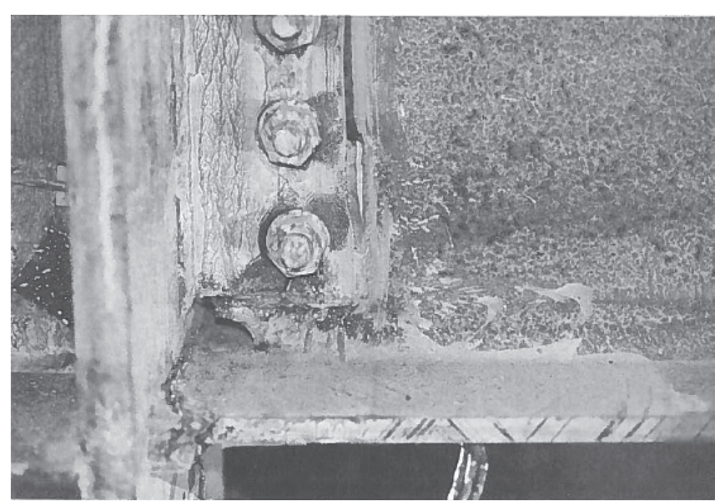

(b) Fracture in heat affected zone

Figure 4-10 Typical failure modes observed in steel beams as part of pre-Northridge Welded-flange-bolted-web beam-to-column connections (FEMA ,1997a).

Failure modes in pre-Northridge connections were fairly diverse including fractures at the beam weld access hole or fracture initiated at the weld and progressing into the column. Other failure modes included fracture or excessive plastic deformation in the column web or flange due to inadequate continuity plates (FEMA, 2000d). In some cases, the fracture developed into a crack of the column flange material behind the CJP weld and a portion of the column flange remained bonded to the beam flange, but pulled free from the remainder of the column. This fracture pattern is commonly known as divot or nugget failure. Once such fractures have occurred the steel beam has experienced a significant loss of its flexural strength and stiffness as 
shown in Figure 4-9b. The bolted web shear tab was also subject to failure including weld fractures of the shear plate to the column or fractures through the weak section of shear plate aligning with the bolt holes. In case that supplemental welds were used to the beam web, fracturing of these welds was also a common failure mode.

\subsubsection{Beams as Part of Partially-Restrained Beam-to-Column Connections with Intermediate Stiffness}

Partially restrained (PR) connections may be partial stiffness connections, partial strength connections or both. Steel beams with web-angle connections do not normally develop a rotational resistance and may be considered to have a pin boundary (FEMA, 2000d). The damage progression observed in steel beams with stiff PR connections, such as end plate connections, is not very different than steel beams with fully restrained connections. This is not true when failure modes concentrate in bolts and/or plates as part of bolted-flange plate beam-to-column connections (see FEMA, 2000d).

This section characterizes the damage progression in steel beams with PR beam-tocolumn connections with intermediate flexural stiffness. These may include T-stub connections, double-flange angle connections, and conventional single plate sheartab beam-to-column connections as shown in Figure 4-11. The latter is used to facilitate the discussion in this section because it is typically used as part of gravity framing connections in North America.

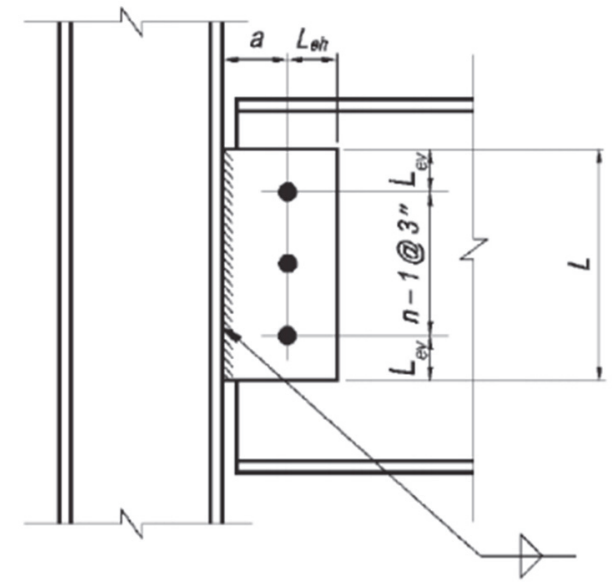

Figure 4-11 Typical single plate shear-tab beam-tocolumn connection (FEMA, 2000d).

A pinched moment-chord rotation relation typically characterizes the hysteretic behavior of steel beams with conventional single plate shear-tab connections under cyclic loading. This can be seen in Figure 4-12 for bare and composite beams with such connections. In this figure, bare single plate shear tab beam-to-column connections would typically behave in a symmetric manner up to a certain deformation amplitude. The non-symmetric hysteretic behavior of a bare steel beam 
as part of a single-plate shear tab beam-to-column connection is attributed to the initial moment in the connection due to gravity load that makes the beam and subsequently the connection weaker in one loading direction and the binding that occurs when the steel beam bottom flange touches the column face.

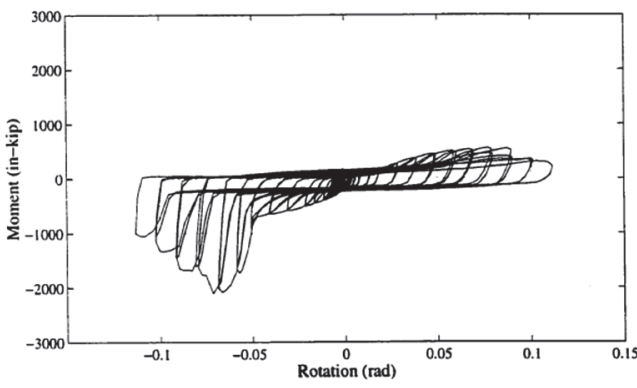

(a) bare steel beam

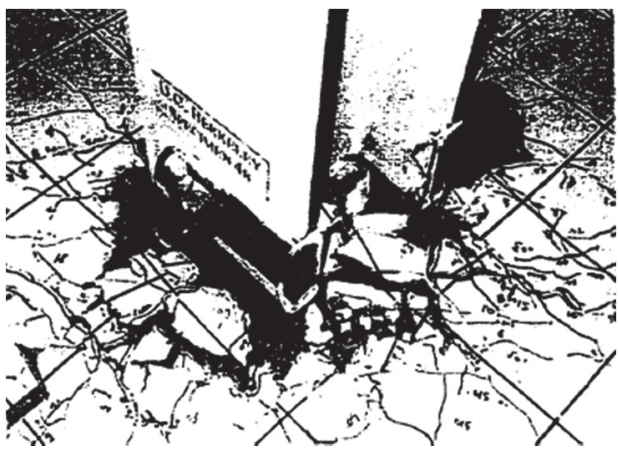

(c) crushing of concrete slab

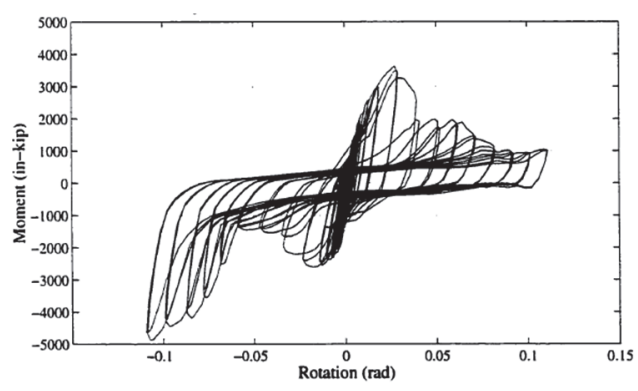

(b) composite steel beam

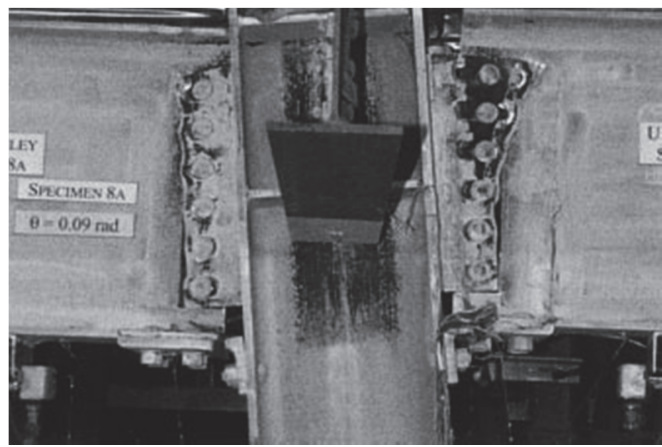

(d) net section fracture

Figure 4-12 Typical deduced beam moment-chord rotation relations of single plate shear-tab beam-to-column connections including damage progression (Liu, 2000; Liu and Astaneh, 2004).

The flexural resistance of bare steel beams with single plate shear tab beam-tocolumn connections is not insignificant compared to that of steel beams in fully restrained beam-to-column connections (see Figure 4-12a). Particularly in the presence of a composite slab, the composite steel beam would typically exhibit flexural capacities on the order of 30 to $45 \%$ of its respective beam plastic moment or roughly 2.5 times the plastic capacity of the shear tab. This can be seen in Figure $4-12 b$ that illustrates the deduced beam moment-chord rotation relation of a composite beam as part of a single plate shear tab beam-to-column connection.

The cyclic behavior of conventional single plate shear-tab beam-to-column connections is controlled by yielding of the gross area of the shear tab or web of the steel beam, bearing-edge distance failure of bolt holes on the shear tab or web of the beam, crushing of concrete of floor slab bearing against the steel column, net section fracture of shear tab or web of the beam, fracture of bolts and fracture of welds (Liu, 2000; Liu and Astaneh, 2004). Due to crushing of the concrete slab the composite action is lost at around 4\% radians. This failure mode is illustrated in Figure 4-12c. After this point, the cyclic behavior of the connections reverts to that of a bare steel 
beam as part of the same connection. Depending on the "a" distance of the bolts and the column face (see Figure 4-11) other failure mechanisms may be observed such as shear plate buckling and shear yielding (Hertz et al., 2015). Beam binding between the bottom flange of the beam and the column face may occur depending on the distance of the two structural steel components. This failure mode is accompanied with an increase in flexural strength of the respective beam as shown in Figures $4-12 \mathrm{a}$ and $4-12 \mathrm{~b}$. This additional flexural strength comes only at very large chord rotations (i.e., larger than 0.06 radians) and it depends on the gap between the beam flange and column face as shown in Figure 4-11. The additional increase in flexural strength can cause bolt shear and/or block shear as shown in Figure 4-12d. In Figure 4-12, such deteriorating modes are typically observed at large inelastic rotations.

Similar to fully restrained beam-to-column moment connections, in the presence of a floor slab, the hysteretic response of a composite steel beam as part of a single plate shear tab beam-to-column connection becomes highly non-symmetric in both loading directions. This can be seen in Figure 4-12b, which shows a deduced beam momentchord rotation relation of a typical single plate shear tab connection including a slab. It should be pointed out that in the presence of a floor slab, in-cycle deterioration of beam flexural strength due to concrete slab crushing is also evident as shown in Figure 4-12c.

\subsubsection{Beam-Columns}

Wide flange steel beam-columns are typically subjected to axial load coupled with lateral drift demands during an earthquake. The applied compressive axial load on interior steel columns in moment frame systems remains more-or-less constant during the building response history. Based on a number of analytical studies of the seismic behavior of modern steel moment frames (Gupta and Krawinkler, 2000b; 2000c; FEMA, 2000c; NIST, 2010a; Elkady and Lignos, 2014; 2015a) the expected applied compressive axial load ratio on these columns ranges from $P_{g} / P_{y e}=0.10$ (i.e., low rise steel moment frame systems) to $P_{g} / P_{y e}=0.30$ (i.e., midand high-rise steel SMFs) depending on the number of stories, where, $P_{g}$ is the gravity load demand on the column; $P_{y e}$ is the column axial yield strength based on expected material properties. However, end columns in steel frame buildings may experience large axial load variations due to dynamic overturning effects ranging from $P_{g} / P_{y e}=-0.2$ in tension to about 0.50 in compression (Suzuki and Lignos, 2014; 2015). Note that steel columns in existing steel frame buildings in the West-Coast of the United States often experience gravity loads in the range of $P_{g} / P_{y e}=0.6$ (Bech et al., 2015).

In North America, current practice mostly uses wide-flange steel columns in planar moment frame systems. An alternative but less common option may be the use of hollow square steel columns. In particular, the use of such members was common in 
late 1970s in the United States (e.g., Bech et al., 2015). This section discusses the damage progression for both alternatives based on available results from recent fullscale experimentation.

\subsubsection{Wide Flange Steel Beam-Columns}

The inelastic behavior of wide flange steel beam-columns under monotonic and cyclic loading is typically characterized by their global and local slenderness, boundary conditions, direction of lateral loading (unidirectional versus bidirectional) and the applied axial load ratio (constant versus varying due to transient loading). Wide flange steel beam-columns that utilize thick web and flanges (i.e., stocky members) tend to have a fairly stable hysteretic behavior even at large inelastic deformations coupled with high axial load. This can be seen in Figure 4-13a that illustrates the deduced column end moment-story drift relation of a W14 $\times 176$ beamcolumn under varying axial load [range of $P / P_{y}=-0.20$ (tension) to 0.75 (compression)] coupled with a symmetric lateral loading protocol. Such members develop plastic hinges due to flexural yielding as shown in Figures 4-13b and 4-13c. However, cyclic degradation in flexural strength and stiffness for this member is minimal and is only attributed to flange local buckling at story drift ratios larger than 0.10 radians (see Figure 4-13c). Due to the relatively small global slenderness ratio $L / r_{y}$ and the large warping and torsional properties of these members, global geometric instabilities associated with lateral torsional buckling are unlikely to occur even at high axial load demands. This can also be seen in Figure 4-13b that shows a global view of the $\mathrm{W} 14 \times 176$ steel column at $10 \%$ lateral drift ratio.

On the other hand, steel beam-columns that utilize deep and slender wide flange steel members with width-to-thickness ratios close to the seismic compactness limits for highly ductile members per ANSI/AISC 360-10 tend to deteriorate cyclically in flexural strength much more rapidly. Figure 4-14 illustrates the deduced column end moment-chord rotation relations for a W24×146 steel beam-column subjected to symmetric cyclic lateral loading protocol coupled with various levels of constant compressive axial load. In this figure, steel wide-flange beam-columns with web and flange local slenderness ratios close to the current compactness criteria for highly ductile members per ANSI/AISC 360-10 are susceptible to severe flange and web local buckling as shown in Figures 4-15a and 4-15b. Depending on the member global slenderness as well as warping and torsional properties of the cross-section this may be coupled with global geometric instabilities associated with lateral torsional and/or global buckling as shown in Figures 4-15b and 4-15c. This may occur at fairly small chord rotations (i.e., in the range of 0.02 radians or less) depending on the applied compressive axial load ratio and member end boundary conditions. 


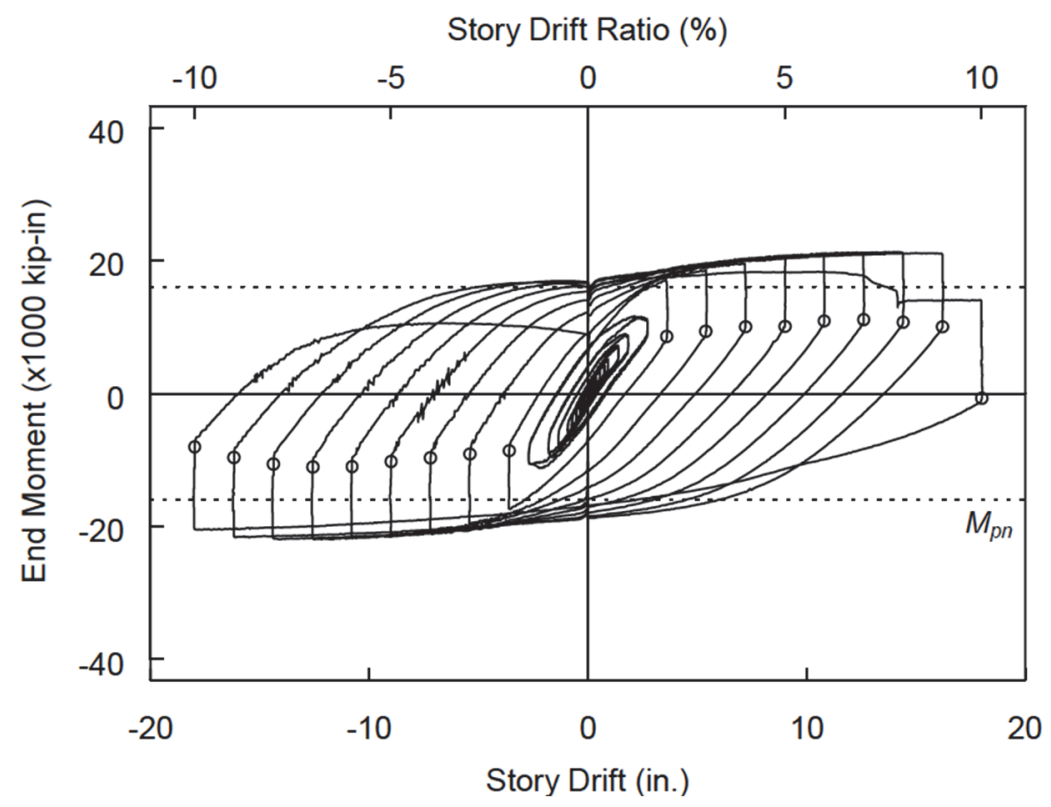

(a) Deduced column end moment-story drift relation for $\mathrm{W} 14 \times 176$

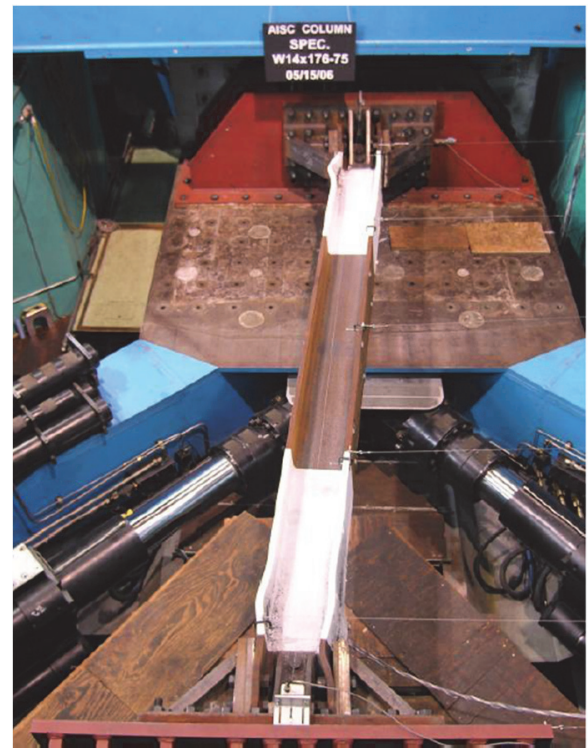

(b) Local buckling - global view

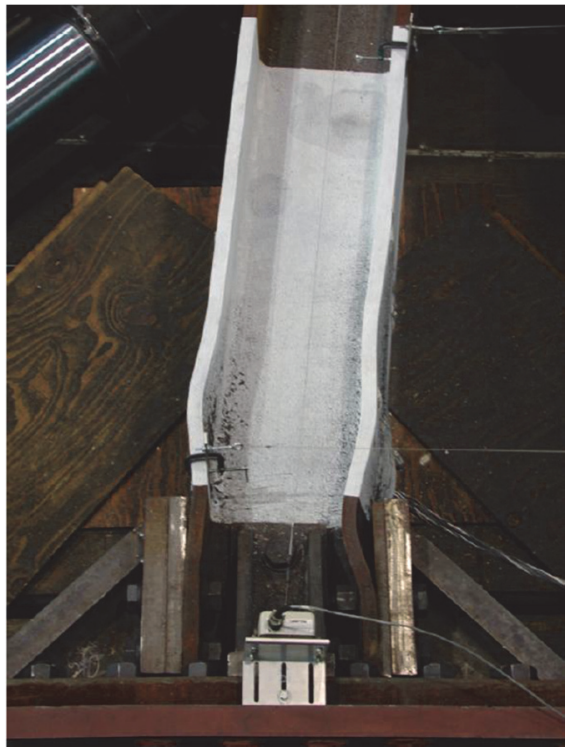

(c) Local buckling - local view

Figure 4-13 Typical deduced moment rotation relation of stocky wide flange columns including damage progression (Newell and Uang, 2006a).

The plastic hinge region in steel beam-columns typically shifts away from the column ends with the increase of the applied compressive axial load due to second order moments. Lateral torsional and/or global buckling typically causes flexural stiffness deterioration as shown in Figure 4-14. 


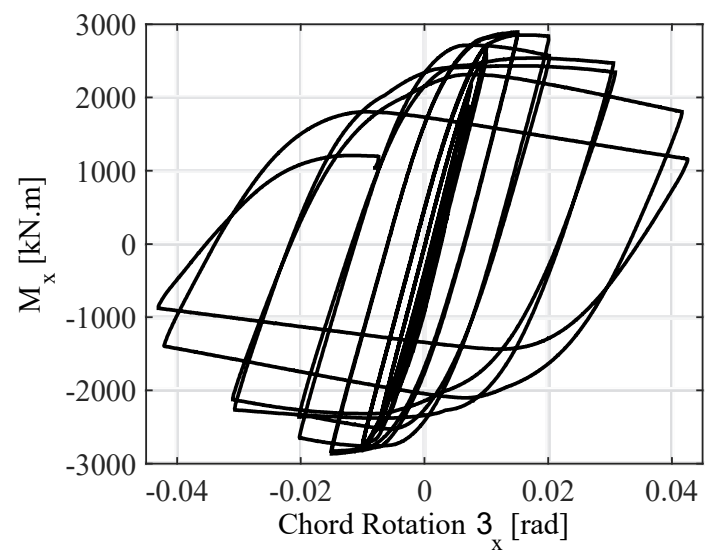

(a) $\mathrm{W} 24 \times 146-P / P_{y}=0.20, L / r_{y}=50$

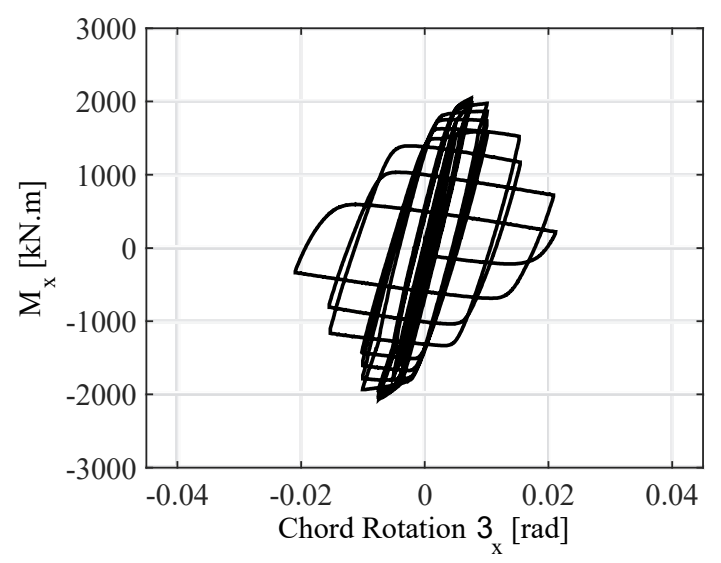

(b) $\mathrm{W} 24 \times 146-P / P_{y}=0.50, L / r_{y}=50$

Figure 4-14 Deduced column end moment-chord rotation relation for deep and slender wide flange steel columns under constant compressive axial load ratios (data from Elkady and Lignos, 2016).

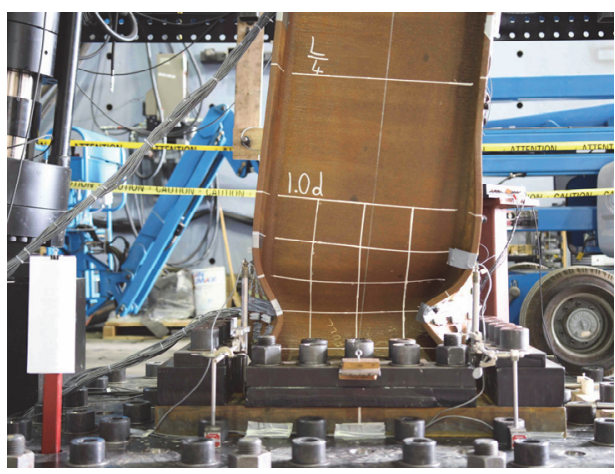

(a) Local buckling

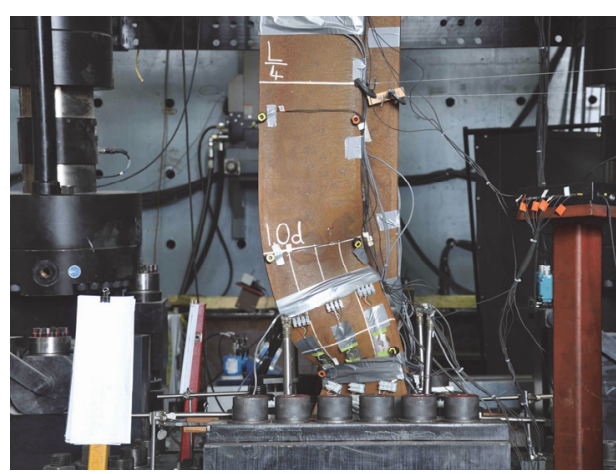

(b) Local buckling coupled with lateral torsional buckling

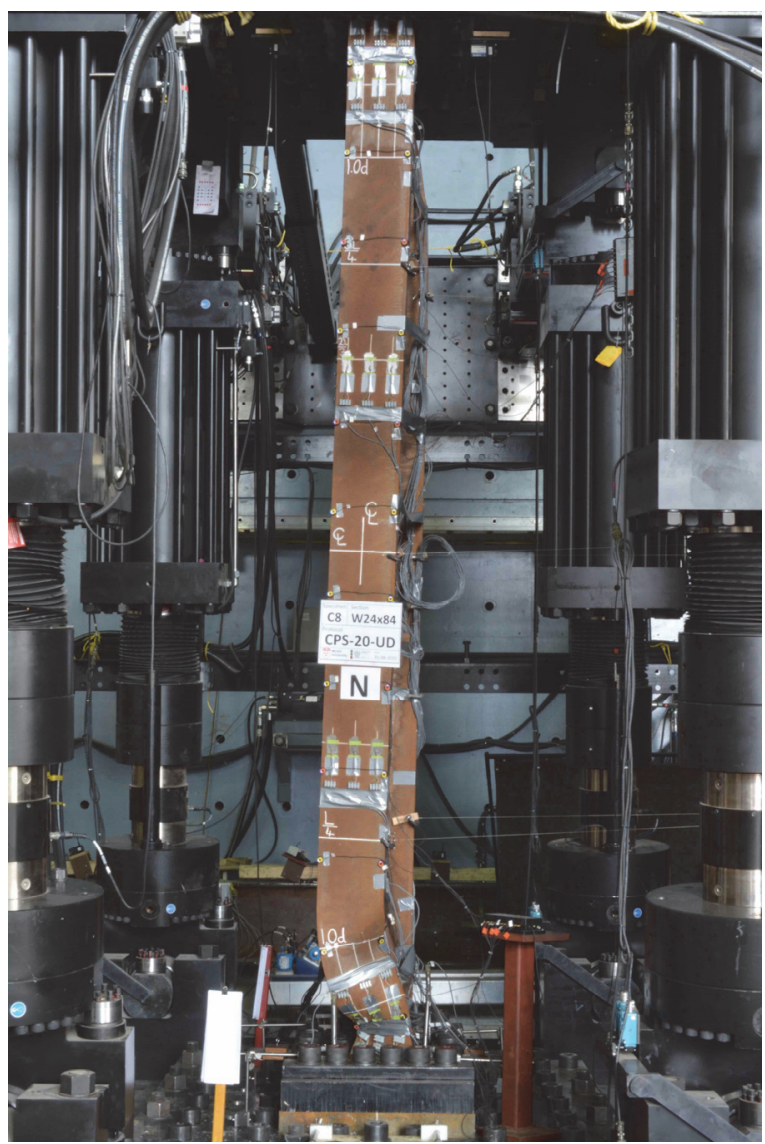

(c) Lateral torsional buckling for W24×84 $-P / P_{y}=20 \%, L / r_{y}=80$

Figure 4-15 Typical damage progression in deep slender wide flange steel beamcolumns under cyclic loading (Elkady and Lignos, 2016). 
In slender wide-flange steel beam-columns, flexural hinging at the column ends is normally coupled with member axial shortening due to web local buckling. This deterioration mode, which can be seen in Figure 4-16a, causes axial strength deterioration to the steel beam-column and ultimately loss of its axial load carrying capacity. Depending on the local slenderness of the respective steel beam-column, fracture due to high distortion may initiate near the k-area, i.e., area defined from the outside of the flange to the web where the cross-section radius ends, of the wideflange at the local buckling region after severe axial shortening takes place. This is shown in Figures 4-16b and 4-16c.

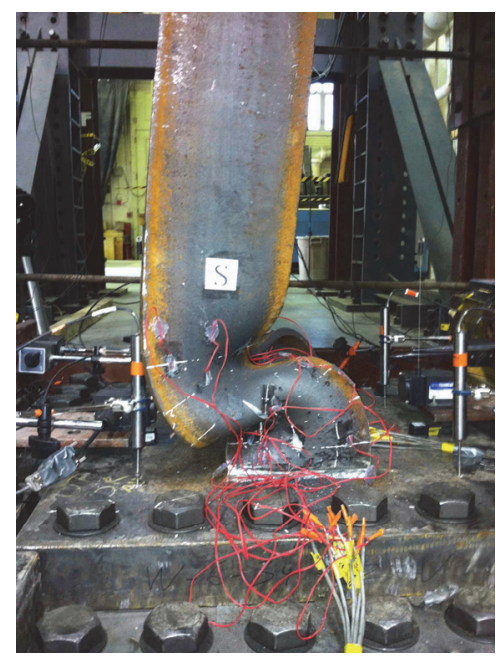

(a) severe column axial shortening

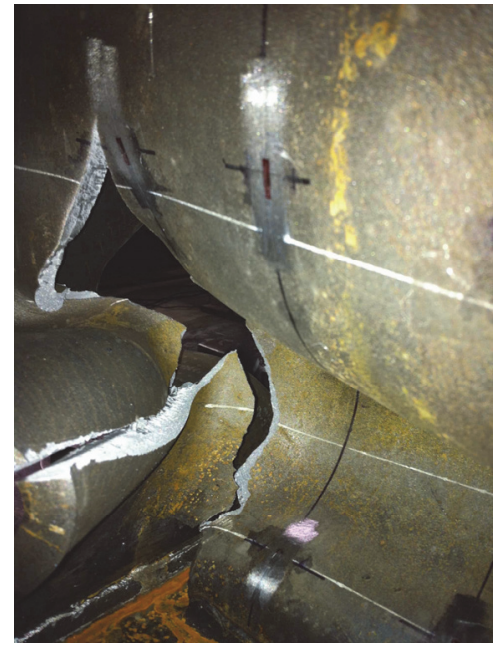

(b) k-area and flange fracture

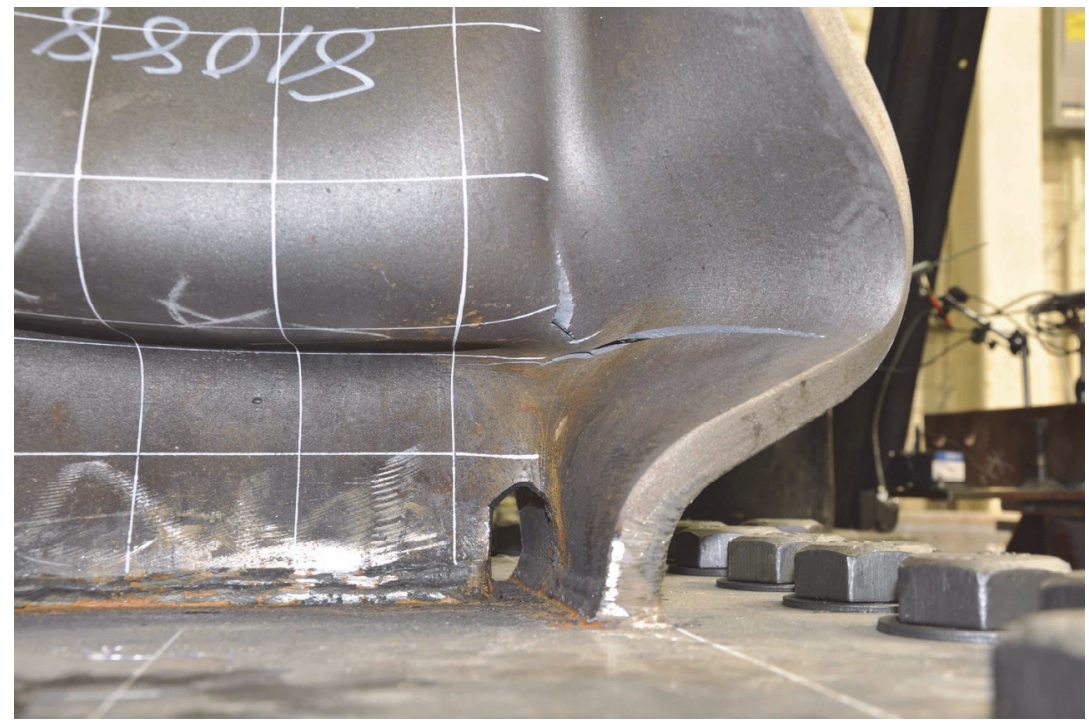

(c) k-area fracture inititation

Figure 4-16 Failure modes associated with large deformations in steel wide flange steel columns: (a) loss of column axial load carrying capacity; (b) and (c) fracture due to high distortion in the k-area (Suzuki and Lignos, 2015). 


\section{Beam-Columns under Bidirectional Loading}

Experimental data on nominally identical steel columns subjected to unidirectional and bidirectional symmetric lateral loading suggest that the plastic deformation capacity of a beam-column is practically not sensitive to the type of loading (Elkady and Lignos, 2016). This observation holds true regardless of the cross-sectional slenderness. The rate of in-cycle deterioration in flexural strength of a beam-column is slightly larger under bidirectional lateral loading compared to that from unidirectional lateral loading. This is attributed to the additional flexural demands due to weak-axis bending of the beam-column. The aforementioned observations can be seen in Figure 4-17 that shows the moment-rotation relation of a W24 $\times 146$ and a W24 $\times 84$ column subjected to unidirectional and bidirectional symmetric lateral loading protocols. In this figure, the column end moment has been normalized with respect to the plastic bending moment of the cross-section for comparison purposes. Same observations hold true for the amount of axial shortening with respect to the inelastic cumulative damage that a beam-column experiences under unidirectional and bidirectional loading.

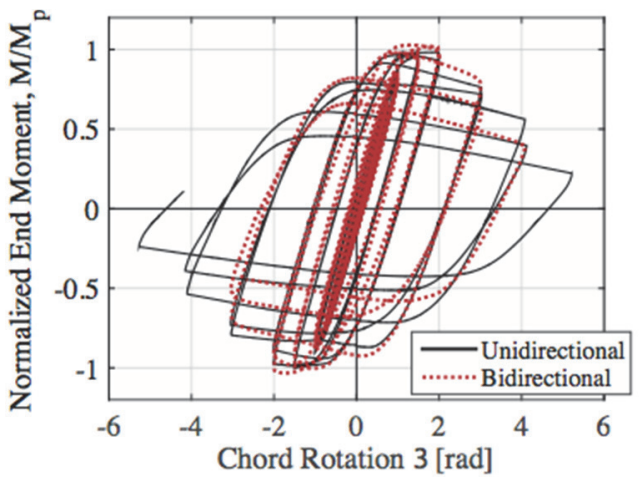

(a) $\mathrm{W} 24 \times 146-P / P_{y}=0.20, L / r_{y}=47$

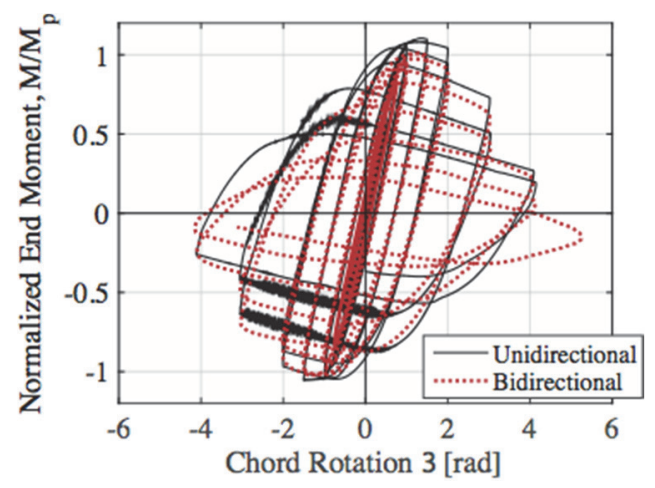

(b) $\mathrm{W} 24 \times 84-P / P_{y}=0.20, L / r_{y}=80$

Figure 4-17 Deduced column end moment-chord rotation relation for deep and slender wide flange steel columns under constant compressive axial load ratios coupled with unidirectional and bidirectional symmetric lateral loading protocols (data from Elkady and Lignos, 2016).

\section{Wide Flange Beam-Columns under Varying Axial Load}

End columns of moment frame systems may experience large axial load variation due to dynamic overturning effects in addition to lateral drift demands. Figure 4-18 illustrates the column end moment-chord rotation relation of two nominally identical beam-columns that were tested under a symmetric lateral loading protocol coupled with a constant compressive axial load ratio $P_{g} / P_{y e}=0.30$ (see Figure 4-18a, Suzuki and Lignos, 2015) and varying axial load demands ranging from $-0.15 P_{y e}$ in tension to $0.75 P_{y e}$ in compression (see Figure 4-18b, Lignos et al., 2016). For comparison purposes, the initial gravity offset in this case was also $P_{g} / P_{y e}=0.30$. The former represents an interior column in a steel moment frame; the latter represents an end 
column in a steel moment frame. It is assumed that both columns have the same tributary area (i.e., gravity offset is the same).

In Figure 4-18a, the interior steel column lost its axial load carrying capacity during the second excursion of the $4 \%$ lateral drift amplitude due to severe axial shortening that triggered fracture at the k-area of the member near its base (see Figure 4-16).

In Figure 4-18b, the hysteretic behavior of end columns is non-symmetric due to the variation of the applied axial load ratio. In particular, end columns maintain their flexural strength once the axial load is in tension. This is due to the fact that flange and web local buckling are straightened when the axial load varies from compression to tension. The post-peak slope of the moment-rotation relation of an end column becomes fairly steep when the axial load increases from the gravity offset to a higher axial compressive load. However, the in-cycle flexural strength deterioration of an end column is fairly small compared to that of an interior column. Due to the application of the constant axial load ratio in the case of interior columns the axial shortening is much larger than that observed in end columns for the same lateral drift amplitude. These differences are more pronounced when non-symmetric lateral loading histories are employed (Suzuki and Lignos, 2015) or relatively stocky crosssections are used in column members (Newell and Uang, 2006a).

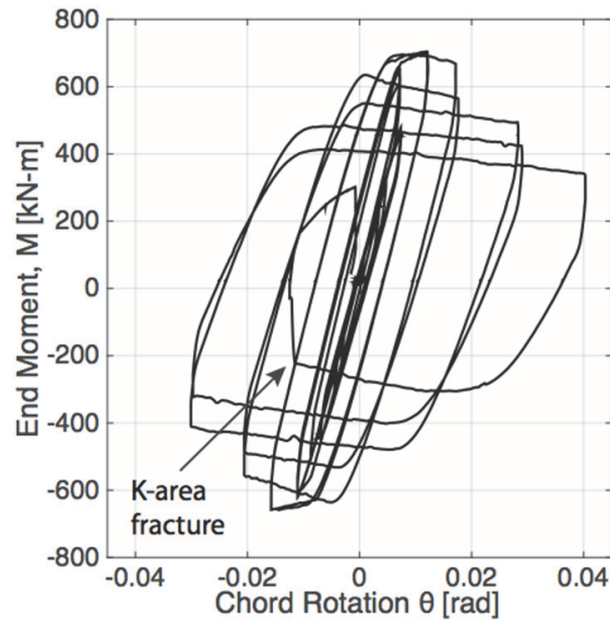

(a) W14×61: $P_{g} / P_{y e}=0.30$

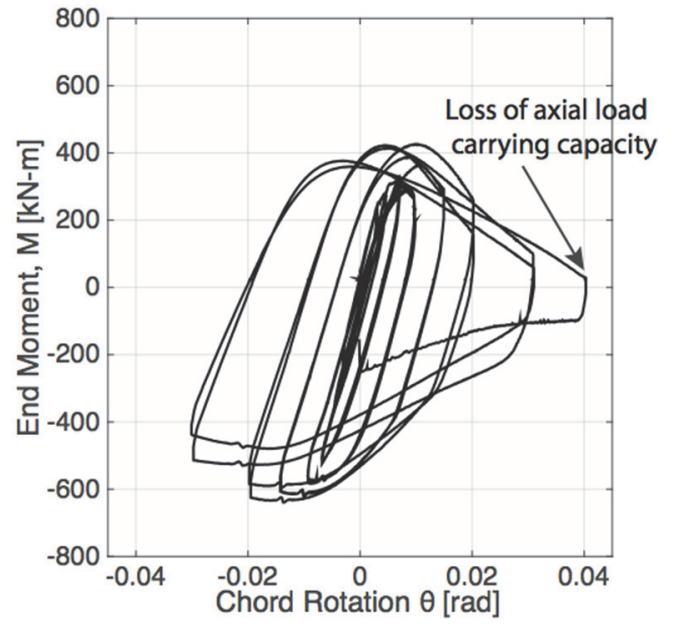

(b) W14×61: $P / P_{y}=-0.15-0.75, P_{g} / P_{y e}=0.30$

Figure 4-18 Deduced column end moment-chord rotation relation for wide flange steel columns under constant compressive axial load ratio and varying axial load coupled with symmetric lateral loading protocols (data from Suzuki and Lignos, 2015; Lignos et al., 2016).

\subsubsection{Hollow Structural Steel Beam-Columns}

The cyclic behavior of hollow square steel beam-columns subjected to compressive axial load and lateral drift demands is fairly similar to that of wide flange steel columns except for the fact that deterioration mechanisms associated with lateraltorsional instabilities do not occur. Figure 4-19a illustrates the deduced moment- 
chord rotation relation of an HSS10" $\times 10^{\prime \prime} \times 3 / 8$ " steel beam-column subjected to constant compressive axial load coupled with a symmetric lateral loading protocol. The onset of local buckling occurs at about 0.015 radians and becomes more evident with increased lateral loading amplitude because the local buckle grows as shown in Figures $4-19 b$ and $4-19 \mathrm{c}$ for the lateral loading excursions of $3 \%$ and $4 \%$ radians, respectively. This results in rapid in-cycle deterioration of the flexural strength of the HSS beam-column (see Figure 4-19a). In addition, column axial shortening is evident. It is often common to observe fracture near the corner part of the HSS member due to high distortion because of local buckling as shown in Figure 4-19d once the steel column loses its axial load carrying capacity. Hollow square steel beam-columns with fairly small local slenderness ratios ( $D / t$ ratios less than 20$)$ may often develop an "elephant-foot" local buckling failure mechanism as shown in Figure 4-20. However, for all practical purposes this does not change the damage progression of an HSS steel beam-column under cyclic loading.

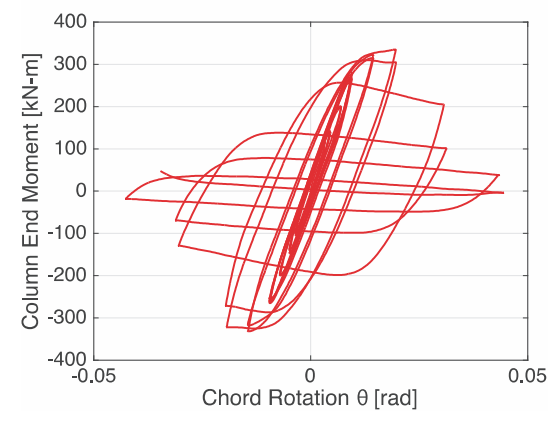

(a) HSS10" $\times 10 " \times 3 / 8 "-P / P_{y}=0.30$

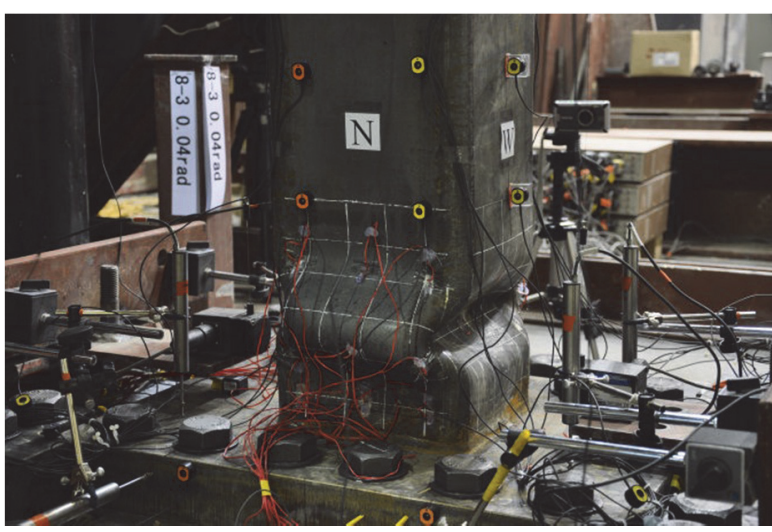

(c) Local buckling progression

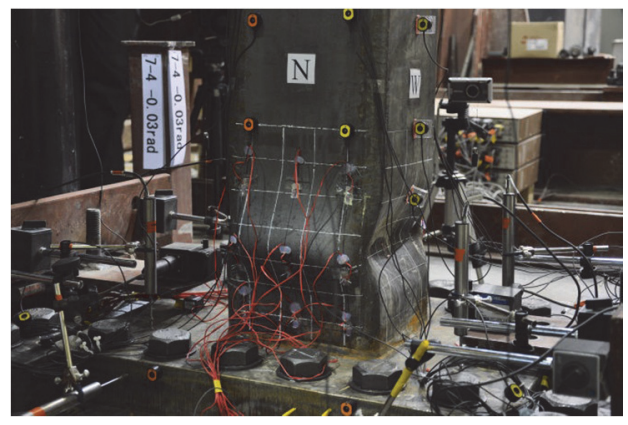

(b) Onset of local buckling

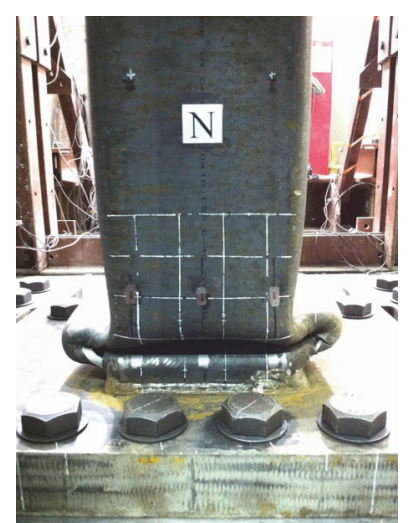

(d) Severe column axial shortening

Figure 4-19 Typical deduced column end moment-chord rotation relation of HSS steel beam-columns including damage progression (Suzuki and Lignos, 2015). 


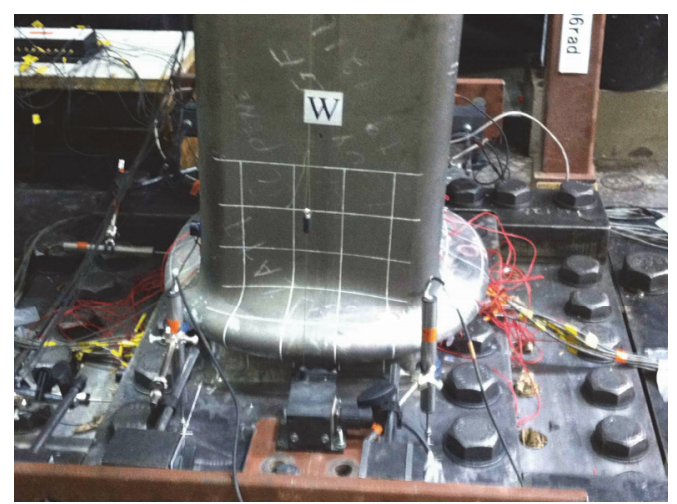

\section{Figure 4-20 Elephant foot local buckling mechanism (Suzuki and Lignos, 2015).}

\subsubsection{Beam-to-Column Joint Panel Zones}

Figure 4-21 shows a typical interior beam-to-column joint panel zone as part of a deep W33 $\times 263$ steel column intersecting two W30 $\times 108$ steel beams. This joint has been designed such that it violates the current AISC panel zone shear strength requirements. When beam-to-column joint panel zones yield in shear, their hysteretic response in terms of panel zone shear force, $V_{p z}$, versus shear distortion, $\gamma$, is fairly stable even at large amplitudes of panel zone shear distortion as shown in Figure 4-22. The same figure shows that the post-yield region of the beam-to-column joint panel zone additional shear strength is provided due to the contribution of column flanges and continuity plates (if any). The full panel zone shear resistance, $V_{p}$, is reached at a shear distortion angle equal to approximately four times the panel zone yield distortion, $\gamma_{y}$ (Krawinkler et al., 1971; Krawinkler, 1978). At this point the panel zone shear stiffness is decreased. This failure mode is illustrated in Figure 4-23a. Minor shear strength deterioration due to shear web buckling may also occur as shown in Figure 4-22b. This deteriorating mechanism is shown in Figure 4-23b.

Bare steel beam-column joint panel zones have a symmetric hysteretic response when they are subjected to a symmetric cyclic lateral loading protocol as shown in Figure 4-20. However, past experimental research has demonstrated that the hysteretic behavior of steel beam-column joint panel zones is affected by the presence of the floor slab. In particular, the hysteretic response of a beam-to-column joint panel zone becomes non-symmetric in this case. This is attributed to the different effective depths of the panel zone in both loading directions of interest due to the presence of the floor slab. In the negative loading direction, the slab is in tension and the effective depth is practically equal to that of the bare steel joint panel zone. In the positive loading direction, the slab is in compression and the effective depth becomes larger than that of the bare steel panel zone. 


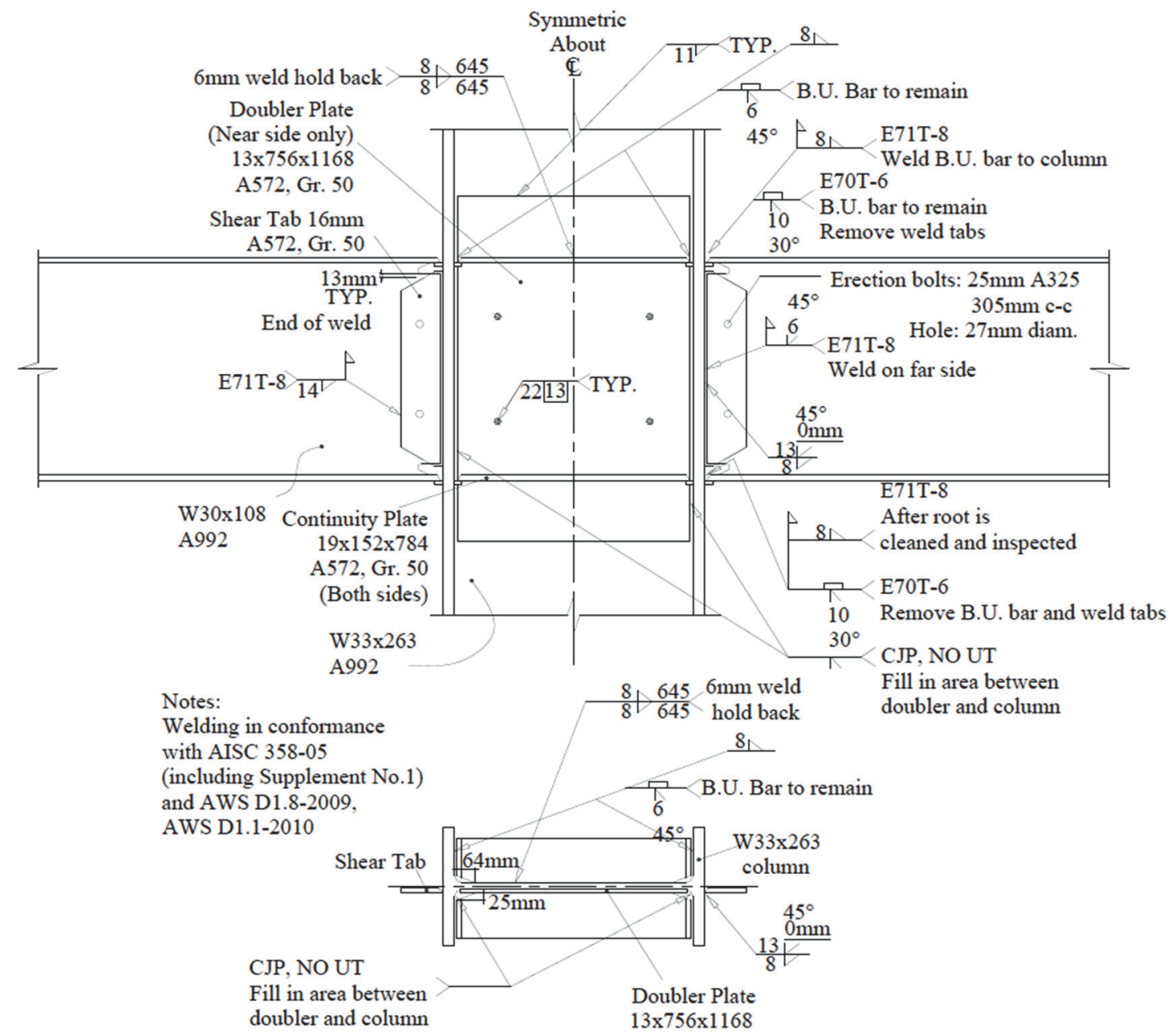

Figure 4-21 Interior beam-column joint panel zone connection detail (Shin and Engelhardt, 2013).

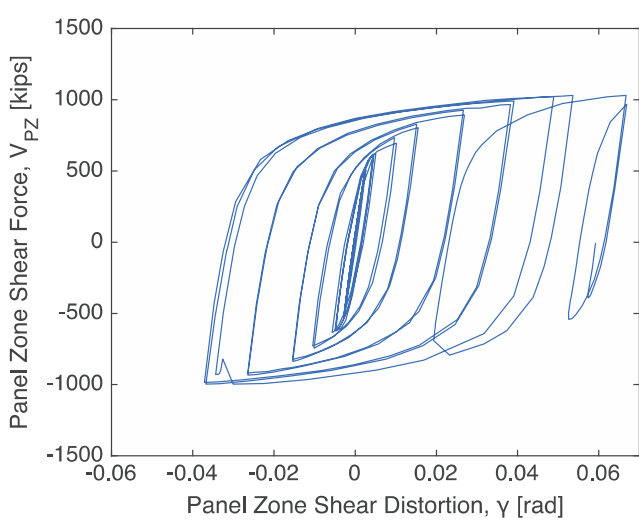

(a)

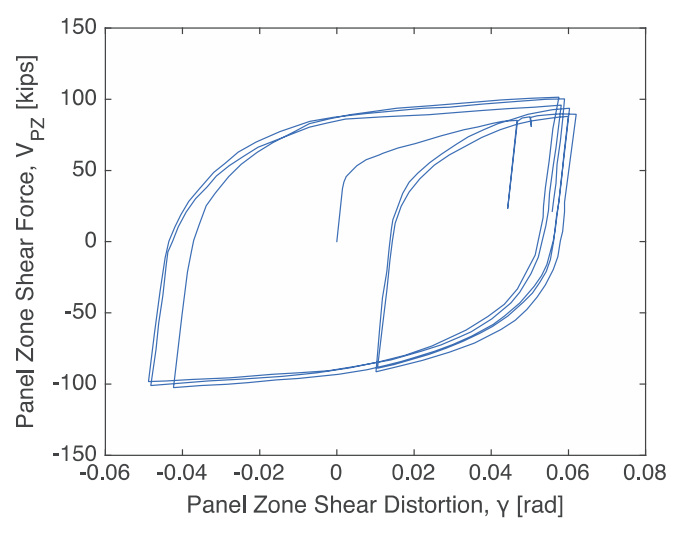

(b)

Figure 4-22 Typical hysteretic response of beam-to-column joint panel zone (Engelhardt et al., 2000; Krawinkler et al., 1971). 


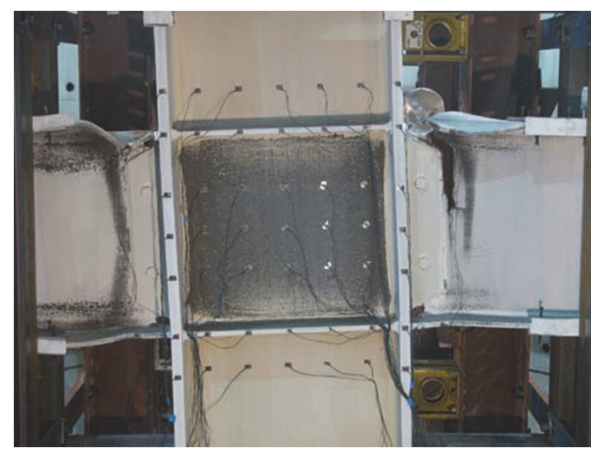

(a) Panel zone shear yielding

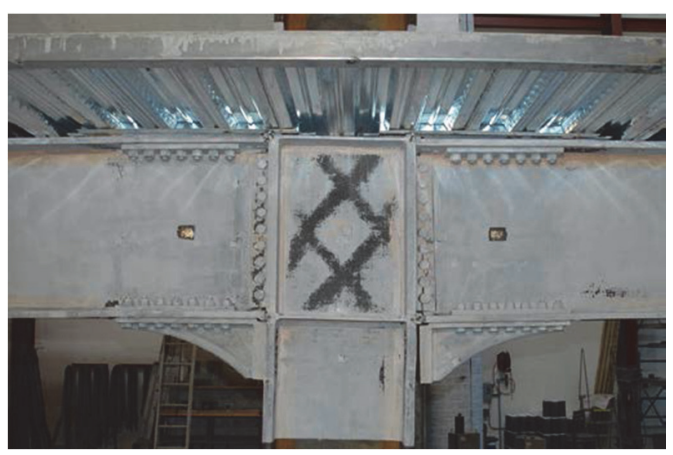

(b) Panel zone shear buckling

Figure 4-23 Typical hysteretic response of beam-to-column joint panel zone (Engelhardt et al., 2000; Newell and Uang, 2006b).

Excessive beam-to-column joint panel zone yielding may lead to local kinks in the column flanges, which can contribute to premature fracture at the beam-to-column interface (Krawinkler et al., 1971). However, the undesirability of excessive panel zone yielding in question is based on recent experimental data on full-scale prequalified beam-to-column connections (Zhang and Ricles, 2006; Shin and Engelhardt, 2013).

\subsubsection{Column Splices}

Column splice connections are typically located approximately four feet above floor levels in steel moment frame systems. They can be either bolted or welded with butt splices. For welded splices, additional stiffeners may be added for shear transfer in the splice region. In special moment frames or intermediate moment frames the use of CJP groove welds is recommended (see Table 4-1) as shown in Figure 4-24 in place of partial joint penetration (PJP) groove welds in order to minimize the stress concentration at the root of the joint. However, recent tests on column splices have shown that the use of PJP welds with toughness-rated weld filler materials and modern welding practices may offer acceptable performance under seismic loads (Shaw, 2013).

Due to column flexural hinging and in-cycle deterioration of beam flexural strength, force redistributions within a steel moment frame system during an earthquake may be such that a column splice may be subjected to inelastic flexural demands. Experimental testing that characterizes the nonlinear behavior of column splices is fairly limited and is typically based on pure reversed bending tests (Bruneau and Mahin, 1987; 1991; Shaw, 2013). 


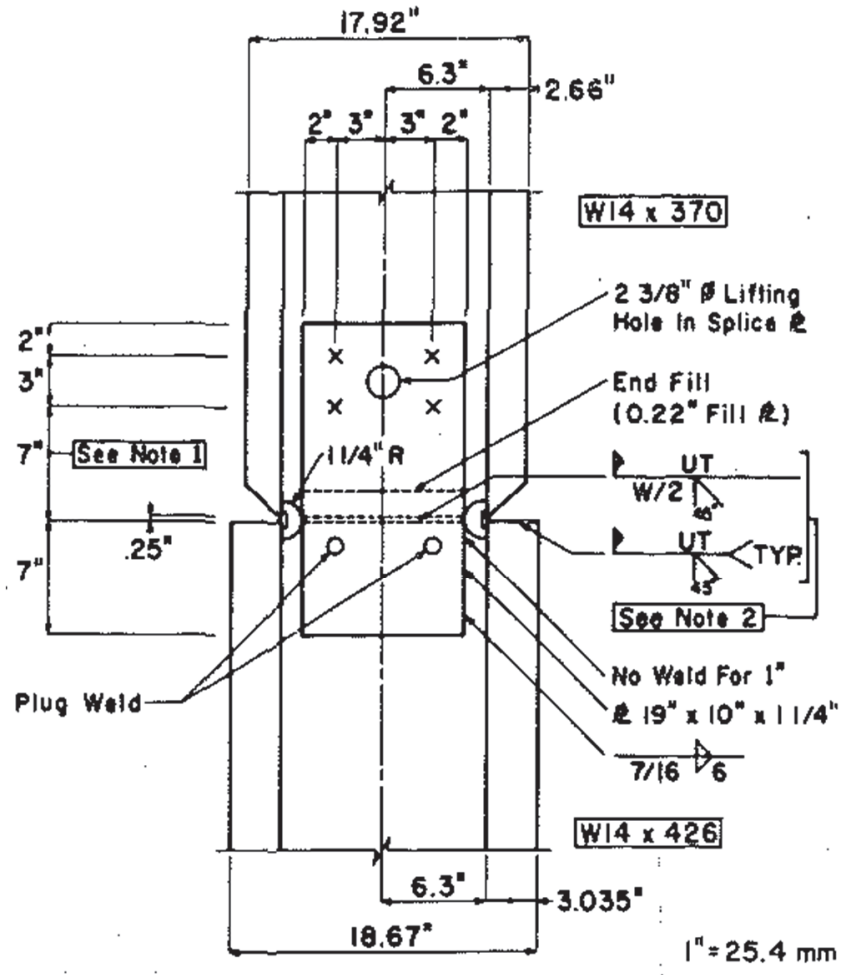

NOTES:

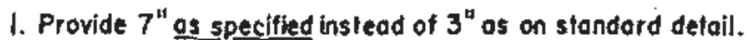

2. A certified inspector ts to be present during construction and perform ulfrosonic testing of the wetd.

Figure 4-24 Typical complete joint penetration welded splice detail (Bruneau and Mahin, 1991).

Figure 4-25 shows a typical moment-curvature relation for the steel column splice shown in Figure 4-24. From this diagram, it is fairly common that significant nonlinearity occurs at a fraction of the flexural yield strength (i.e., typically $40-60 \%$ of $M_{y}$ ) of the steel column member itself due to residual stresses from cutting and welding. Due to cyclic hardening, inelastic flexural demands of column splices may be $20 \%$ to $40 \%$ higher than the actual plastic moment, $M_{p}$, of the corresponding column cross section; however, this value depends on the level of the applied axial load within the steel column. The behavior of column splices under reversed bending is fairly stable up to large inelastic rotations. Depending on the cross sectional size and story shear demands, flexural yielding typically spreads far beyond the splice location within the column (Bruneau and Mahin, 1987; 1991; Shaw, 2013). A failure mode that can cause rapid flexural strength deterioration in welded column splices is column flange weld fracture as shown in Figure 4-26. Fracture may propagate into the column web as shown in the same figure. 


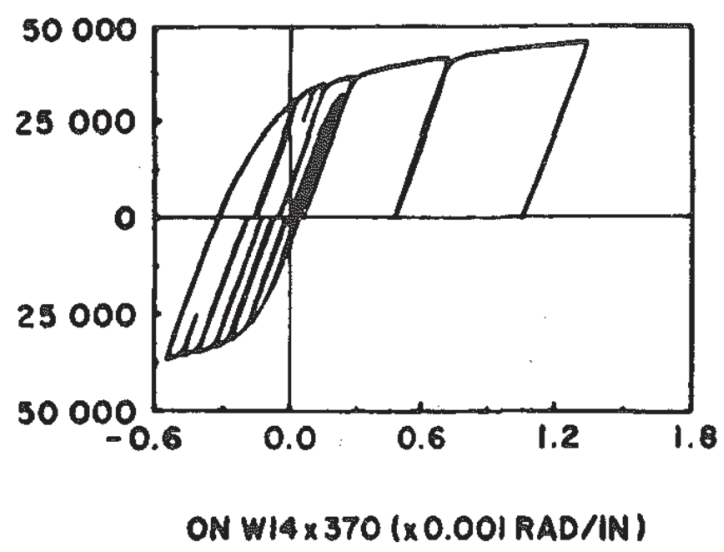

Figure 4-25 Typical moment curvature relation for complete joint penetration welded splice (Bruneau and Mahin, 1991).

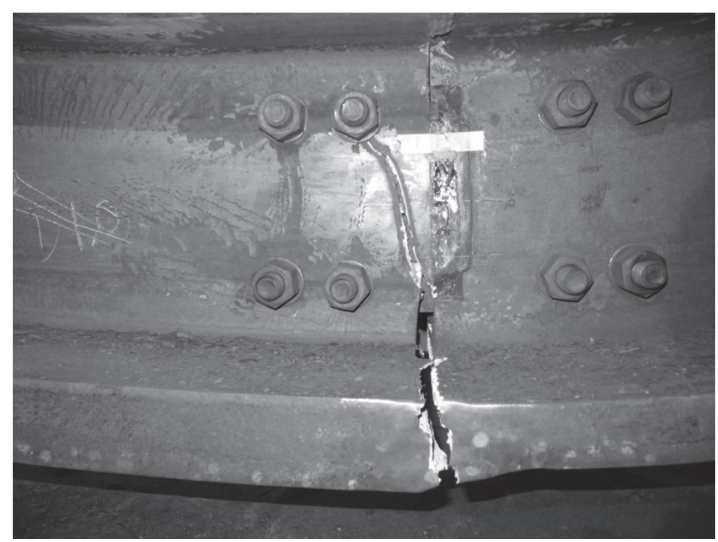

Figure 4-26 Weld fracture in welded column splice (Shaw, 2013).

Pre-Northridge column splices consist of PJP welds of roughly half the thickness of the smaller column section being connected. A typical detail for such a connection is shown in Figure 4-27a. Experimental findings from the early 1990s (Bruneau and Mahin, 1991) suggest that when such connections are subjected to tensile forces due to flexural demands and/or axial loading during the steel moment frame system earthquake response, they can only carry a small portion of the column flexural and/or axial tension capacity of the smallest column section being connected. This can be seen in Figure 4-27b that illustrates a moment-curvature relation for a typical pre-Northridge column splice that utilized heavy steel wide-flange sections with PJP welds. In this figure, the moment-curvature relation is practically up to the point that brittle fracture occurs through the weld (see Figure 4-27c). In these connections, once a crack develops in the tension flange of the column splice, it progresses through the whole section almost instantaneously. Based on experimental observations of Bruneau and Mahin (1991) the erection plate does not normally help restrain or stabilize the failure once initiated. 

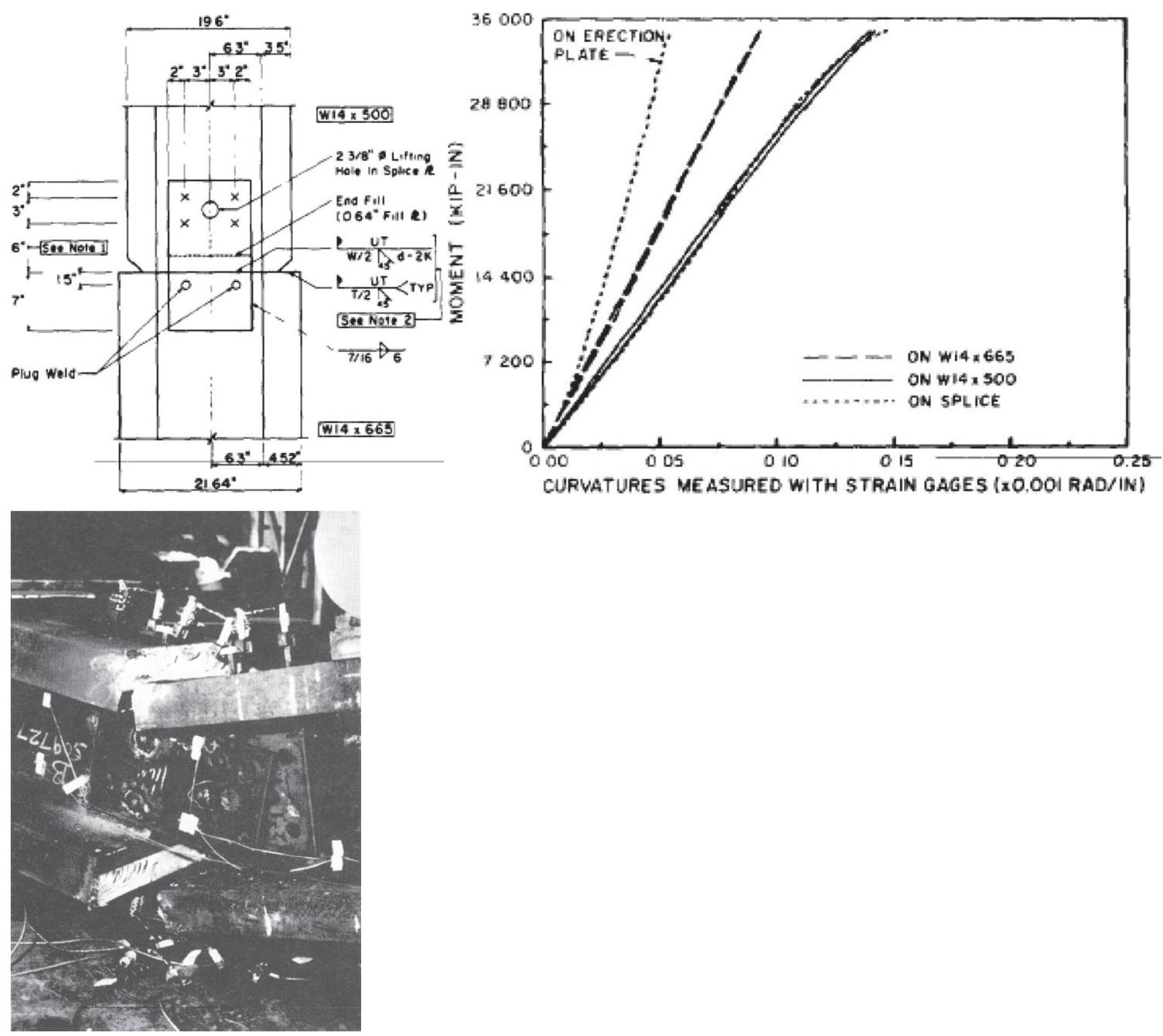

Figure 4-27 Typical detail, moment-curvature relation, and damage progression for preNorthridge column splice connections (Bruneau and Mahin, 1991).

\subsubsection{Column Base Connections}

Column base connections are categorized as exposed or embedded. Exposed column bases are typically used in low-rise steel moment frame systems. A steel base plate is welded to the steel column. This plate is then anchored to a concrete footing with anchor rods. Figure 4-28a illustrates a schematic representation of a typical exposed column base connection. For mid- and high-rise steel moment frame systems embedded column base connections are considered representative of a fixed support condition. Otherwise, a large number of anchor rods and very thick base plates would be required to "guarantee" a fixed condition. In embedded type column base connections moment resistance is provided through the direct bearing between the steel column and the concrete footing. Figure $4-28 \mathrm{~b}$ illustrates a schematic representation of an embedded column base connection including its force transfer mechanisms. 


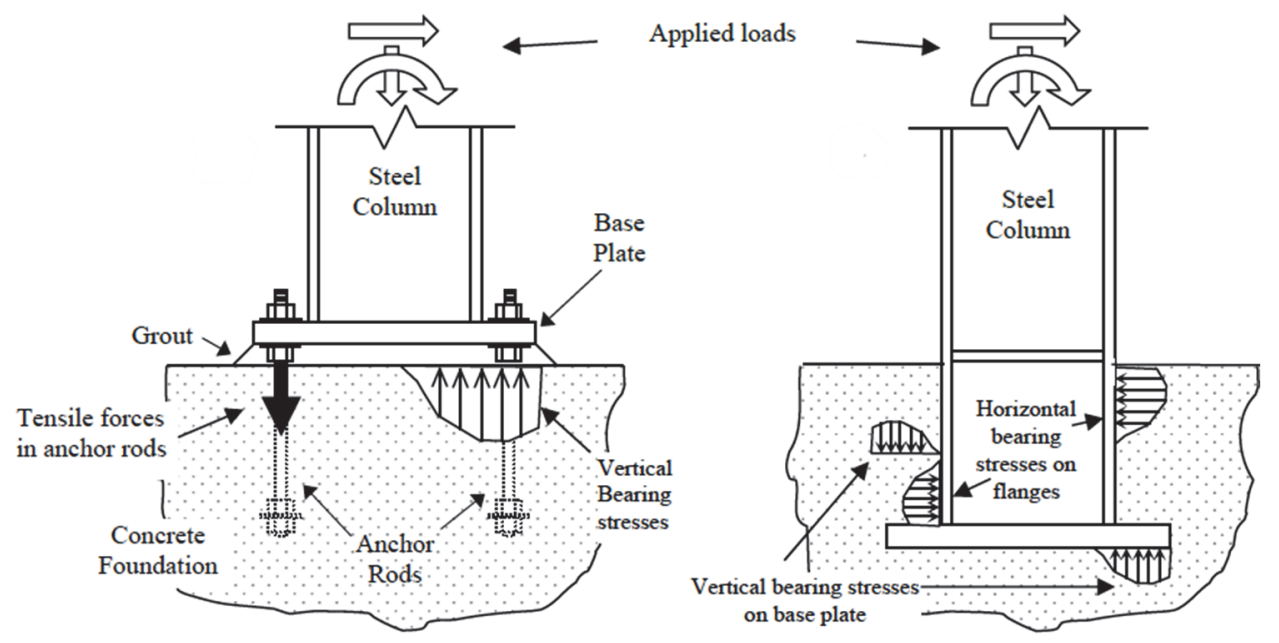

Figure 4-28 Column base connections and force transfer mechanisms (Grilli and Kanvinde, 2015).

\subsubsection{Exposed Column Base Connection}

Figure 4-29 illustrates a typical hysteretic behavior of an exposed column base in terms of deduced column base moment-column drift relation. In this figure, the hysteretic response of exposed column base connections is pinched with minor strength deterioration due to cyclic loading. Pinching is attributed to the interaction of the anchor rods with the respective base plate. In particular, for a column base under cyclic loading the anchor rods that are subjected to tension engage the column base plate through bearing contact of the rod nut with the base plate top. Grout damage may initiate at lateral deformations associated with design basis earthquakes (i.e., around 0.02 radians). At larger lateral deformations, the base plates yield in flexure. The anchor rods yield axially. This results in permanent rod elongation and disengagement from the base plate, as the connection is unloaded. This results in loss of flexural stiffness of the column base.

At higher lateral deformations the anchor rods are reengaged, and an increase in the flexural resistance of the exposed column base connection is observed as shown in Figure 4-29a. A typical deterioration mode that causes sudden loss of flexural strength is anchor rod fracture. Grout damage at this stage includes crushing at the base plate edges. Both of these failure modes are shown in Figure 4-30. Depending on the level of the applied compressive axial load the initial stiffness of the column base connection will vary. In addition, the hysteretic response of an exposed column base becomes similar to that of a self-centering connection as shown in Figure 4-29b. Under high axial loads, the primary failure mode occurs when the concrete foundation exceeds its ultimate bearing capacity. 


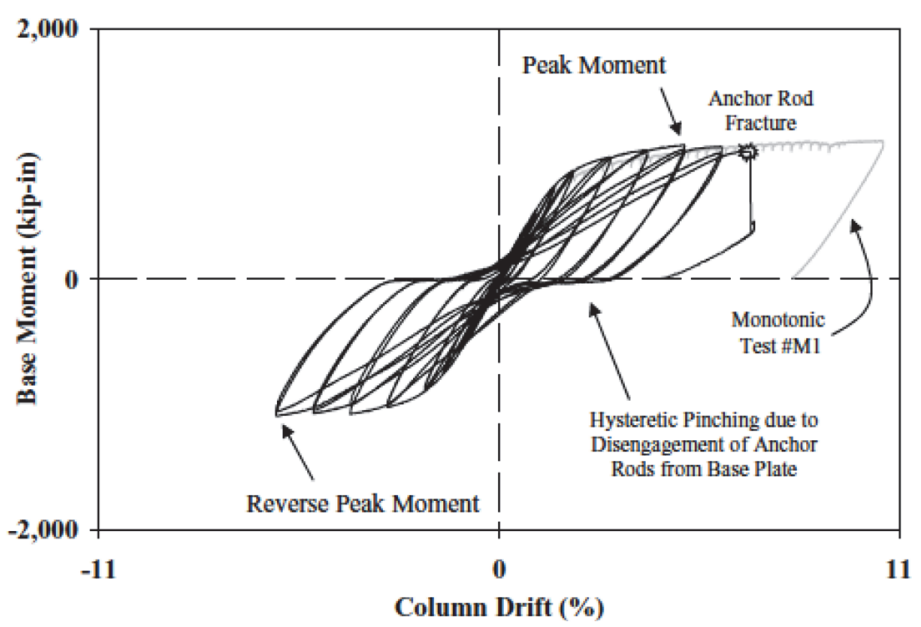

(a) Exposed column base connection $-P=0$

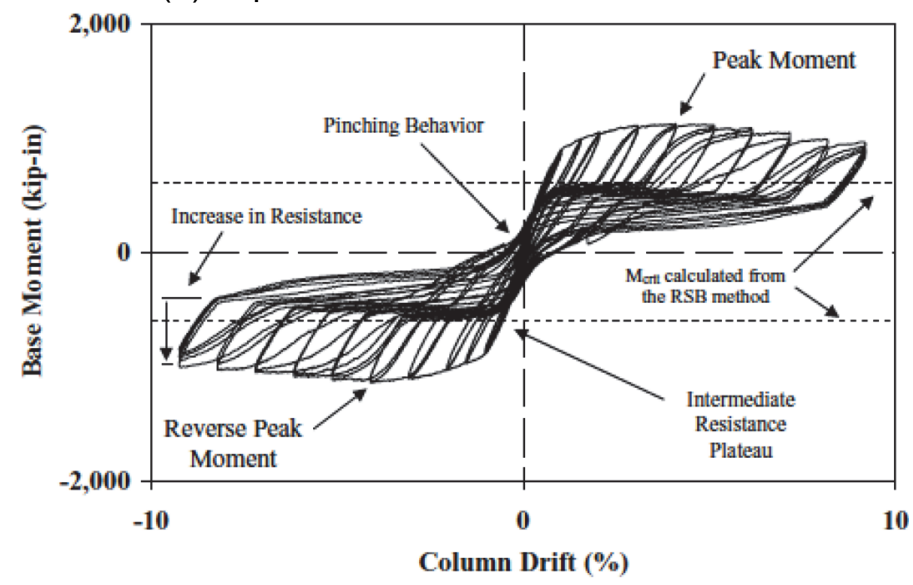

(b) Exposed column base connection $-P=92.5 \mathrm{kips}$

Figure 4-29 Hysteretic response of exposed column base connections (Gomez, 2010).

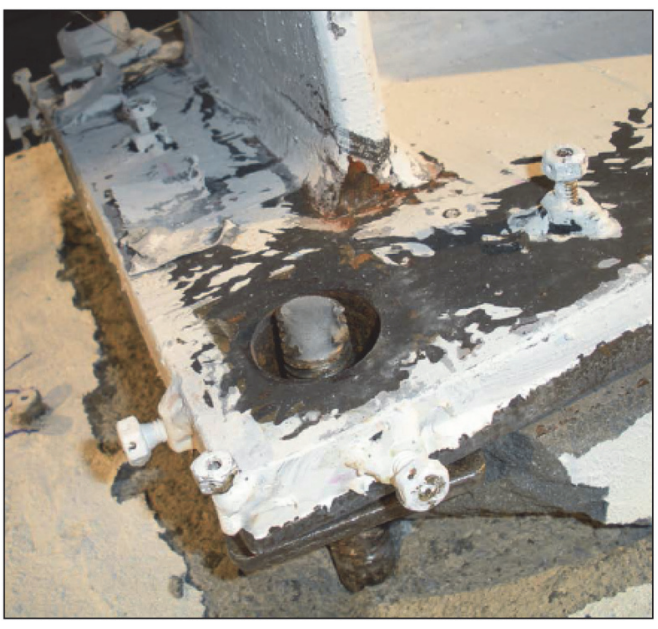

(a) Anchor rod fracture

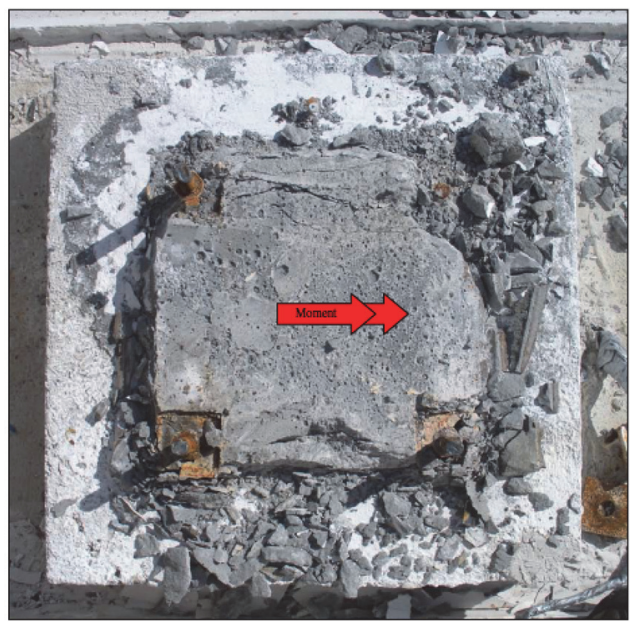

(b) Grout damage

Figure 4-30 Typical failure modes observed in exposed column base connections (Gomez, 2010). 


\subsubsection{Embedded Column Base Connection}

Figure 4-31 illustrates deduced moment-drift relations for typical embedded type column base connections under constant axial load coupled with lateral deformations. The fact that during the elastic range of the loading protocol the behavior of the embedded column base is not linear, is attributed to the small cracks that typically form near the corners of the steel column. At larger lateral deformations a gap adjacent to the tension flange opens. Diagonal cracks near the steel column corners are also apparent. Upward bulging in the bearing zone between these cracks is also observed. Due to the gap opening, the hysteretic response of the embedded column base connection is typically pinched. Eventually, flexural cracks develop on the sides and top of the concrete footing on the tension side of the connection. Shear cracks also develop in the concrete panel. These failure mechanisms are shown schematically in Figure 4-32a. With the increased lateral loading amplitude, the flexural strength of the connection deteriorates due to the reduction in the moment resisted by bearing ahead of the column flanges. This is shown in Figure 4-31.
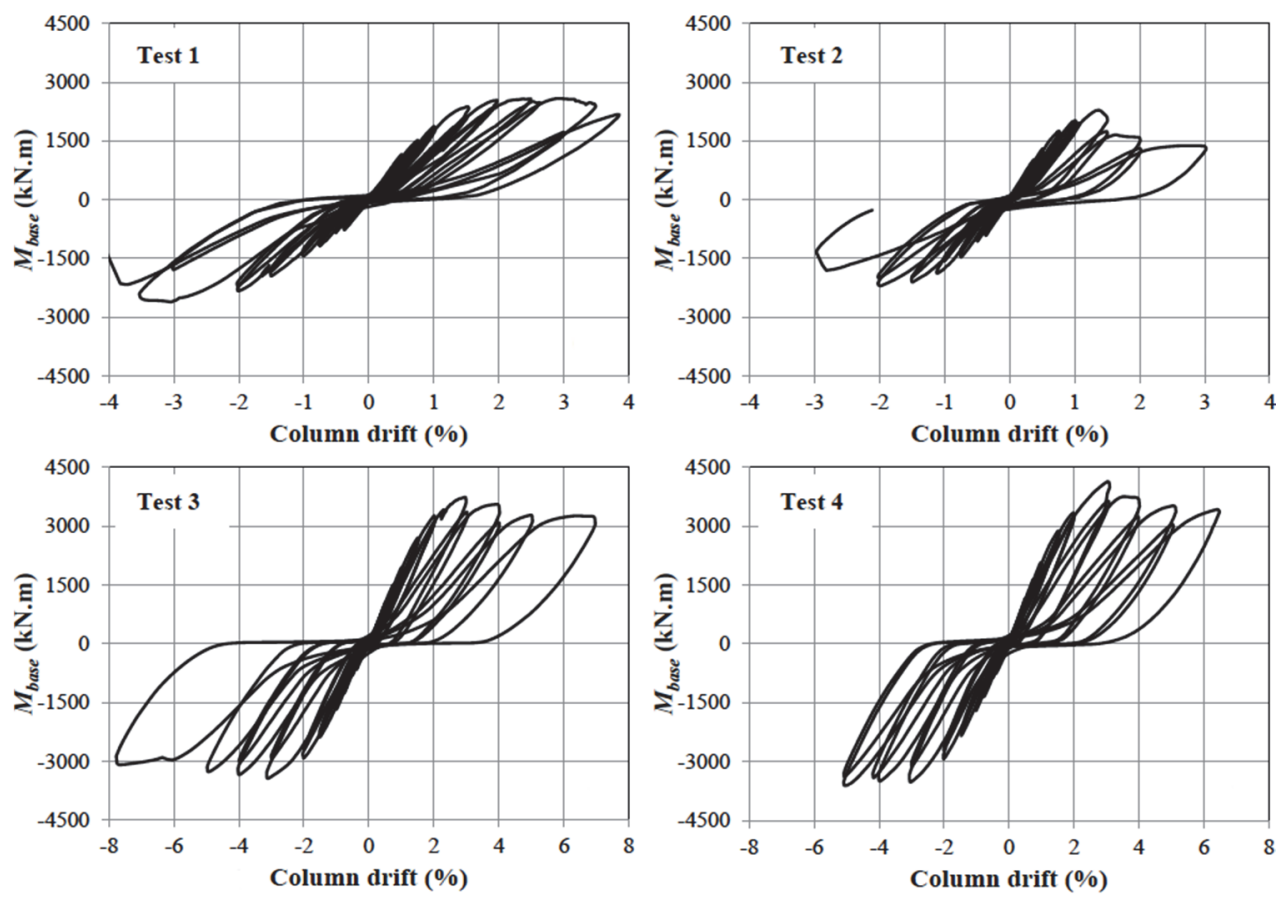

Figure 4-31 Moment drift relations for embedded type column base connections (Grilli and Kanvinde, 2015). 


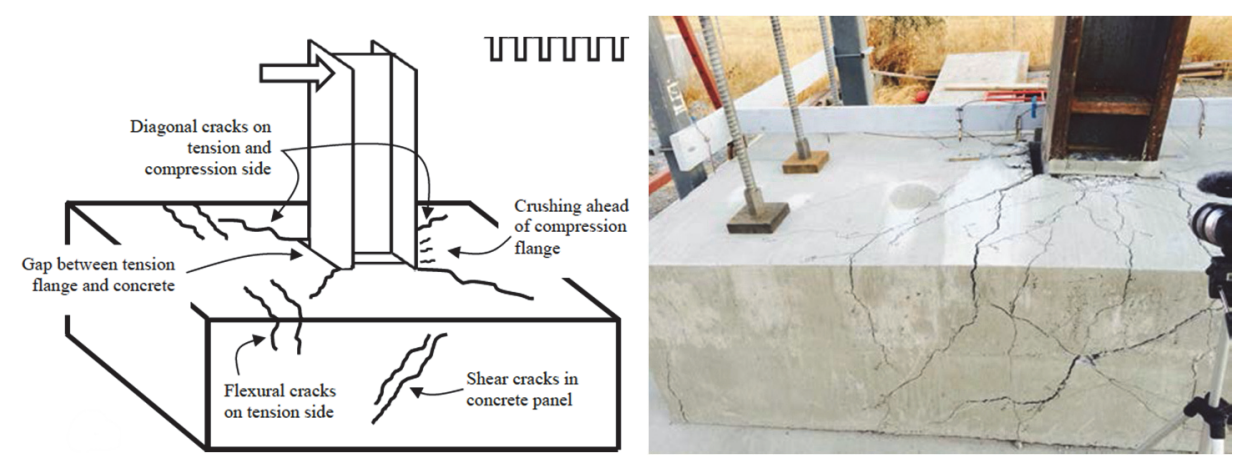

Figure 4-32

Typical failure modes observed in embedded column base connections (Grilli and Kanvinde, 2015).

\subsection{Recommendations for Modeling}

In order to realistically simulate the behavior of steel components in steel momentresisting frames a concentrated or distributed plasticity approach can be employed as shown in Figure 4-33. Based on the concentrated plasticity approach, the inelastic behavior of the respective steel component is simulated with the use of multi-linear nonlinear springs. These springs idealize the force-deformation relation (normally moment-chord rotation for beams and columns in steel moment frames) of a steel component. The main advantage of using such elements is typically the simplicity and computational efficiency needed for the simulation of the seismic response of steel moment frames. However, concentrated plasticity models typically require large sets of experimental data such that the input model parameters can be reliably estimated (e.g., Lignos and Krawinkler, 2012a; 2012b). Other limitations may arise from the fact that in steel columns, column splices, and column base connections the axial force-bending interaction is typically ignored. This may be a reasonable assumption for interior columns where the axial force applied to the columns does not typically vary during an earthquake due to the dynamic overturning effects. However, this is not the case for end columns and such variations may be considerable (Suzuki and Lignos, 2014). From Section 4.3.3, axial shortening may be an important failure mode that is not typically captured by concentrated plasticity models. In that sense, a fiber-based approach may be more suitable. However, this requires the use of equivalent stress-strain relations that include softening in order to implicitly model local instabilities associated with local buckling of the cross section. In this case, the selected fiber discretization becomes critical (Kostic and Filippou, 2012) but approaches are available that overcome issues with non-objective simulated response due to softening (Scott and Fenves, 2006). However, this approach may be suitable for HSS columns and wide-flange columns with fairly small $L / r_{y}$ ratios where lateral torsional buckling is not a controlling failure mode. Else, a continuum finite element approach may be more suitable (see Figure 4-33). The main challenges of this approach are to rationally calibrate material models that capture the combined isotropic and kinematic hardening behavior of typical steel 
materials due to input material parameter non-uniqueness (see Cooke and Kanvinde, 2015); the residual stresses due to manufacturing and/or welding-induced stresses as well as the magnitude of local and global imperfections within the cross-section and along the member, respectively, to trigger local and/or global instabilities associated with local and lateral torsional buckling. Strategies to address these issues are discussed in detail in Elkady and Lignos (2015b) for steel beam-columns. It is noted that the targeted force-deformation envelopes for monotonic and first-cycle idealizations as proposed and discussed in Section 4.5 are not dependent on the employed modelling approach that is schematically illustrated in Figure 4-33.
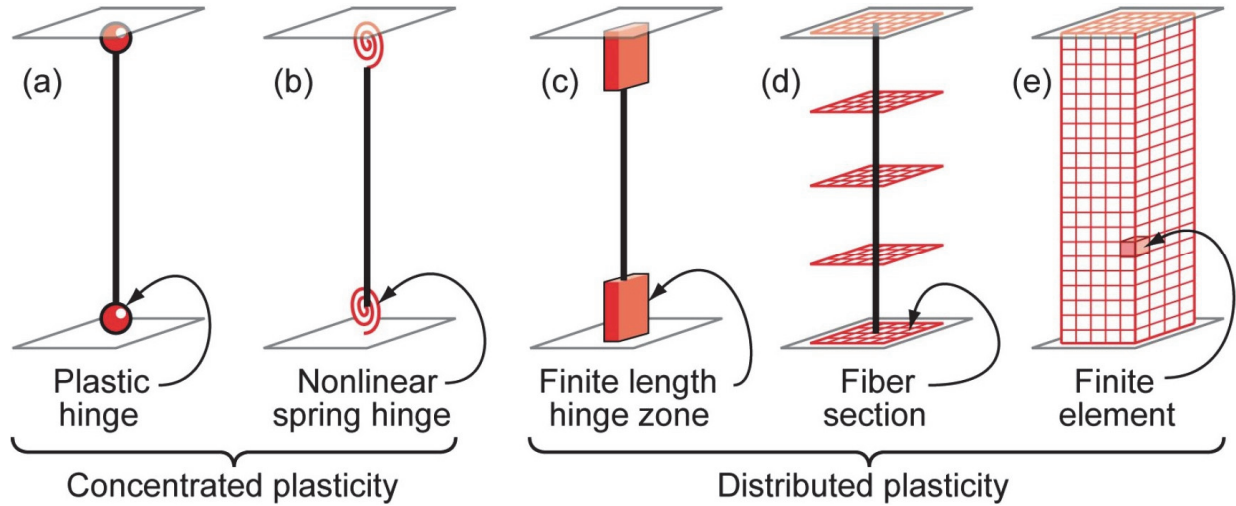

Figure 4-33 Idealized models of beam-column elements (NIST, 2010c).

\subsection{Force-Deformation Envelopes}

This section provides detailed recommendations on how to construct target forcedeformation envelopes for nonlinear modeling of steel components as part of steel moment frame systems discussed in Section 4.3. In particular, detailed information is provided to construct a monotonic envelope curve, which is considered as a characteristic property of a steel component because it is loading-history independent. This curve is shown in Figures 4-34a and 4-34b for a steel beamcolumn and a steel beam with RBS, respectively. Figure 2-6 shows an idealized monotonic curve that can be used for a steel component. It is noted that for steel components there is no residual strength for monotonic loading, thus the strength degradation portion of Figure 2-6 for monotonic loading is represented by a single line segment ranging from peak strength to zero strength. In this figure, the quantity $Q$ represents a force (e.g., shear or moment) quantity and the parameter $\Delta$ can be a displacement, elongation, rotation or curvature. The monotonic curve should be used in nonlinear response-history analysis of steel moment frame systems when the employed simulation platform for nonlinear response-history analysis offers modeling capabilities that capture explicitly the cyclic and in-cycle strength and stiffness deterioration of various steel components. 


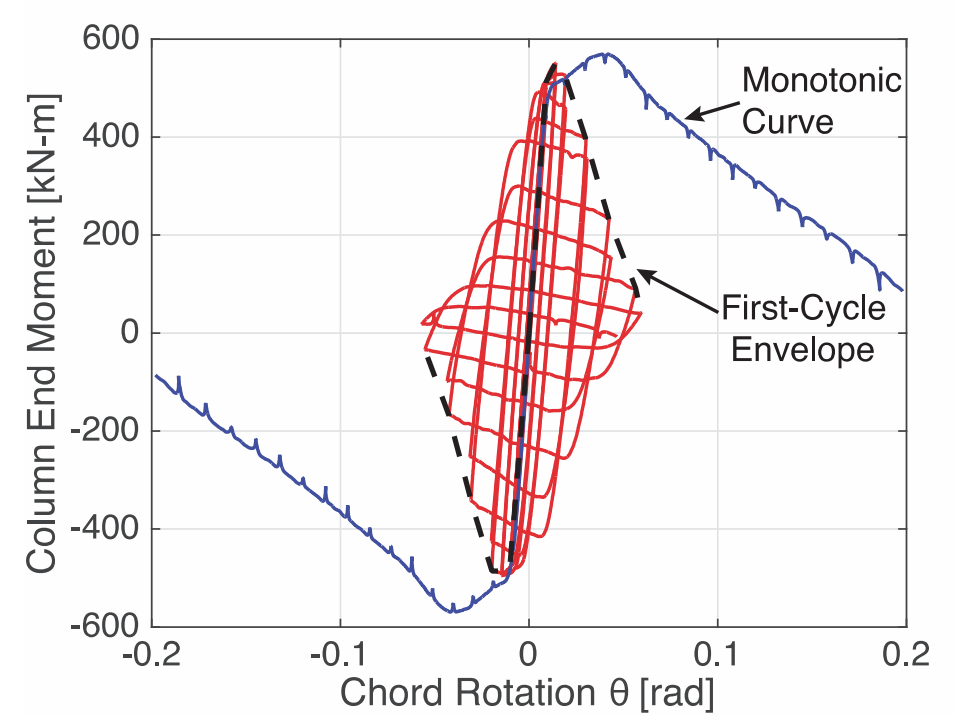

(a) Wide flange steel beam-column under constant axial load

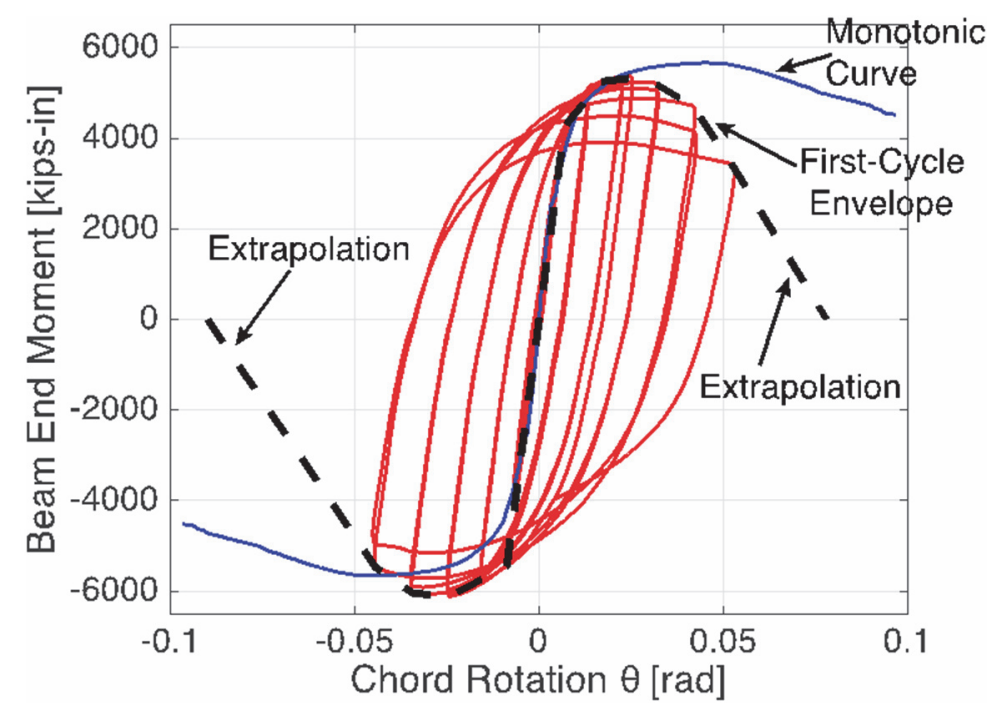

(b) Steel beam with RBS as part of fully restrained beam-to-column connections

Figure 4-34 Monotonic and first-cycle envelope curves for various steel components (Suzuki and Lignos, 2015; Tremblay et al., 1997).

When nonlinear analysis software does not offer capabilities for modeling in-cycle deterioration in strength and stiffness, the use of the cyclic backbone is recommended as discussed in ASCE/SEI 41-13, Seismic Evaluation and Retrofit of Existing Buildings, (ASCE, 2014) recommendations. This curve inherently captures the cyclic and/or in-cycle strength deterioration that occurs at a given deformation level under reverse cyclic loading and under increasing deformations within one loading excursion of a cycle, respectively. The first-cycle envelope curve is loading-history dependent (see Section 4.1). This curve is typically based on standardized loading protocols such as the symmetric cyclic protocol. Figure 4-34 shows the hysteretic 
response of typical steel components subjected to a symmetric loading protocol. Superimposed in the same figure is the first-cycle envelope for each component.

The development of modeling recommendations for the definition of the two idealized curves is based on available test data on various steel components conducted over the past 30 years. Where test data were sufficient, the input model parameters are related to geometric and material properties of the respective component through a multivariate regression model. If this is not possible, then a median value including a coefficient of variation (COV) of each model parameter is provided.

For steel beam-columns detailed finite element analysis results from parametric studies of Elkady and Lignos (2015b) are mixed with available experimental data. The finite element models have been properly calibrated with full-scale experimental data and are able to capture the failure modes observed in steel beam-columns (see Section 4.4) under cyclic loading based on the modeling procedures discussed in Section 4.4.

Figure 4-35 illustrates the deduced moment-chord rotation of a steel component under reversed cyclic loading. Superimposed in the same figure is the first-cycle envelope curve including three alternatives that represent the multi-linear idealizations of the first-cycle envelope curve depending on the capping (peak) flexural strength that is used. The modeling recommendations discussed below are based on the idealized curve that caps at the maximum moment, $M_{\max }$ as measured from each test (i.e., green curve shown in Figure 4-35). This is a conservative assumption because the use of $M_{\max }$ results in higher strain-hardening ratio compared to the use of $90 \% M_{\max }$ or $80 \% M_{\max }$. In this case, engineers analyzing steel SMFs with a strong-column-weak-beam ratio close to unity should have a better chance to trace weak-story mechanism development. In addition, with the use of the idealized curve based on $M_{\max }$, the post-peak plastic deformation, $\Delta_{p c}^{*}$, of the first-cycle envelope curve is always smaller than the post-peak plastic deformation, $\Delta_{p c}$, of the monotonic curve as one would expect. This is consistent with prior studies and the PEER/ATC-72-1, Modeling and Acceptance Criteria for Seismic Design and Analysis of Tall Buildings, (PEER/ATC, 2010) nonlinear modeling recommendations for steel components under cyclic loading. 


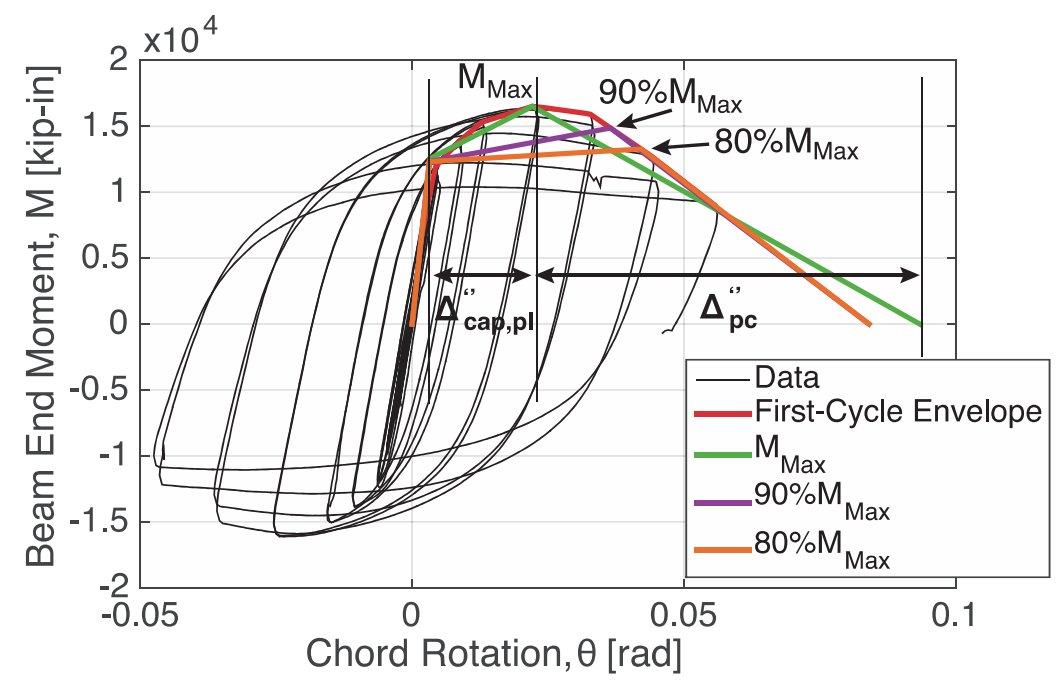

Figure 4-35 Fitted idealized multi-linear models to represent the first-cycle envelope curve for a steel component (FEMA, 2000d).

\subsubsection{Hinge Model for Beams as Part of Fully-Restrained Beam-to- Column Moment Connections}

Steel beams that generally conform to AISC seismic design criteria in terms of lateral bracing, cross sectional compactness, and are connected to columns such that the primary failure mode is beam flange and web local buckling followed by ductile tearing (i.e., no brittle fractures) can be modeled based on modeling parameter equations included in PEER/ATC-72-1 (PEER/ATC, 2010). These equations, which are based on work of Lignos and Krawinkler (2011; 2012a; 2012b), relate geometric and material properties of the respective bare steel beam with the input model parameters of the monotonic envelope shown in Figure 4-35. The equations have been developed based on test data for beams up to 36 inches deep. The effective flexural yield strength of the steel beam accounts for the effects of cyclic hardening through an adjustment factor that is based on test data. The input model parameters are statistically calibrated to median values. The variability in the same parameters is described in terms of a COV.

\subsubsection{Monotonic Envelope}

The input model parameters for conforming beams as part of fully-restrained beamto-column connections are summarized as follows:

Elastic Stiffness, $\boldsymbol{K}_{e}$. The beam hinge may be modeled with a finite elastic flexural stiffness or as rigid-plastic (i.e., infinite flexural stiffness). In the first case:

$$
K_{e}=a_{e} E I / L
$$


In which, $E I$ is the cross section stiffness of the steel beam; $L$ is the assumed beam length and $a_{e}$ is the stiffness coefficient. Commercial structural analysis software assume that the structural members are "infinitely" rigid. For practical purposes, it is suggested to make the hinge stiffness 10 times stiffer than that of a steel beam in double curvature (i.e., $6 E I / L$ ); therefore, $a_{e}=60 I$ in this case (Ibarra and Krawinkler, 2005). Assuming the characteristic beam stiffness is the moment-rotation relation in double curvature, the effective beam flexural stiffness is as follows (from Ibarra and Krawinkler (2005)):

$$
E I^{*}=E I /\left(1-\frac{6}{a_{e}}\right)
$$

Effective Yield Strength, $\boldsymbol{Q}_{\boldsymbol{y}}$. For steel beams this quantity refers to the effective flexural yield strength $M_{y}{ }^{*}$ and is computed as follows:

$$
M_{y}^{*}=\beta M_{p e}
$$

where $\beta$ is factor that represents an adjustment to the effective flexural yield strength to capture cyclic hardening effects and should be equal to $\beta=1.1$ for beams with RBS, $\beta=1.2$ for beams other than RBS (Lignos and Krawinkler, 2011); $M_{p e}$ is the plastic flexural strength of the steel beam computed with the expected yield stress of the steel material based on Tables 9-1, 9-2 and 9-3 of ASCE/SEI 41-13. For new steel frame buildings, $M_{p e}$ can be computed as $M_{p e}=R_{y} \times Z \times F_{y}$; in which, $R_{y}$ is the yield ratio coefficient depending on the steel material type and can be computed based on Table A3.1 of ANSI/AISC-341-10; $Z$ is the plastic modulus of the beam cross section at the assumed location of the beam plastic hinge; $F_{y}$ is the nominal yield stress of the respective steel material. For steel beams with RBS the plastic hinge is assumed at the center of the RBS region, $M_{y}^{*}$ should be computed based on the plastic modulus of the reduced section, $Z_{R B S}$.

Peak (Capping) Strength, $\boldsymbol{Q}_{\max }$. This quantity is the peak flexural strength of the steel beam, $M_{\max }$, under monotonic loading adjusted for the effects of cyclic hardening. It is computed based on a factored value of the effective yield strength, $M_{y}^{*}$ as follows:

$$
M_{\max }=1.1 M_{y}^{*} \quad(\mathrm{COV}=0.1)
$$

Plastic Deformation up to the Peak Strength of the Monotonic Envelope, $\Delta_{\text {cap,pl }}$. This quantity refers to the pre-peak plastic rotation, $\theta_{p}$, for flexural hinges and for standard (non-RBS) beam sections this can be computed as follows:

$$
\theta_{p}=0.07\left(\frac{h}{t_{w}}\right)^{-0.3}\left(\frac{b_{f}}{2 t_{f}}\right)^{-0.1}\left(\frac{L}{d}\right)^{0.3}\left(\frac{c_{\text {unit }} d}{533}\right)^{-0.7} \quad(\mathrm{COV}=0.3)
$$


For beams with RBS the following expression should be used:

$$
\theta_{p}=0.09\left(\frac{h}{t_{w}}\right)^{-0.3}\left(\frac{b_{f}}{2 t_{f}}\right)^{-0.1}\left(\frac{L}{d}\right)^{0.1}\left(\frac{c_{u n i t} d}{533}\right)^{-0.8} \quad(\mathrm{COV}=0.3)
$$

where $h / t_{w}$ and $b_{f} / 2 t_{f}$ are the web and flange depth-to-thickness ratios, respectively; $L / d$ is the shear span-to-depth ratio of the steel beam; $d$ is the beam depth; $c_{\text {unit }}=1$ if $d$ is defined in millimeters; else, $c_{u n i t}=25.4$ if $d$ is defined in inches.

Plastic Deformation of the Descending Portion of the Monotonic Envelope, $\Delta_{p c}$. This quantity refers to the post-peak plastic rotation, $\theta_{p c}$, for flexural hinges. It indicates the post-buckling behavior of a steel beam. For standard (non-RBS) beam sections, $\theta_{p c}$, can be computed as follows:

$$
\theta_{p c}=4.6\left(\frac{h}{t_{w}}\right)^{-0.5}\left(\frac{b_{f}}{2 t_{f}}\right)^{-0.8}\left(\frac{c_{u n i t} d}{533}\right)^{-0.3} \quad(\mathrm{COV}=0.3)
$$

Alternatively, for beams with RBS:

$$
\theta_{p c}=6.5\left(\frac{h}{t_{w}}\right)^{-0.5}\left(\frac{b_{f}}{2 t_{f}}\right)^{-0.9} \quad(\mathrm{COV}=0.3)
$$

Residual strength, $\boldsymbol{Q}_{\boldsymbol{R}}$. The residual flexural strength, $M_{r}$, for steel beams due to stabilization of the buckle size can be assumed as, $M_{r}=0.4 M_{y}^{*}$. This value is based on a relatively small set of data points from which an average of $M_{r} / M_{y}^{*}$ could be made with confidence regardless of the steel beam-to-column moment connection type (Lignos and Krawinkler, 2011).

Ultimate deformation, $\boldsymbol{\Delta}_{\text {ult }}$. For steel beams with flexural hinging this quantity represents the ultimate rotation capacity that is likely to be controlled by ductile tearing due to cracks that may develop in the steel base material or the shear capacity of the connection. With reference to Figure 4-35, it is recommended to assume $\theta_{u l t}=0.2$ radians with a $\mathrm{COV}=0.30$ regardless of the beam-to-column moment connection type.

\subsubsection{First-Cycle Envelope Curve}

Referring to the first-cycle envelope curve, shown in Figure 4-35, the input model parameters for conforming beams in fully restrained beam-to-column connections should be modified to account for cyclic and in-cycle strength and stiffness deterioration effects. The following recommendations should be employed to construct such a curve:

The effective flexural stiffness, $K_{e}$ and effective flexural yield strength, $M_{y}^{*}$ of the steel beam may be computed based on Equations 4-1 and 4-3, respectively. 
Capping (Peak) Strength for First-Cycle Envelope, $\boldsymbol{Q}_{\text {max }}^{\prime}$. This quantity refers to the peak flexural strength of a steel beam, $M_{\max }^{*}$, under cyclic loading. It includes the effects of cyclic hardening and it is computed based on a factored value of the effective yield strength, $M_{y}^{*}$ as follows:

$$
M_{\max }^{*}=1.15 M_{y}^{*} \quad(\mathrm{COV}=0.10)
$$

Plastic Deformation up to Peak Strength of the First Cycle Envelope, $\boldsymbol{\Delta}_{\text {cap,pl }}$. This quantity refers to the pre-peak plastic rotation, $\theta_{p}^{*}$ for flexural hinges adjusted for in-cycle deterioration in strength and stiffness. For standard (non-RBS) beam sections can be computed as follows:

$$
\theta_{p}^{*}=0.3\left(\frac{h}{t_{w}}\right)^{-0.3}\left(\frac{b_{f}}{2 t_{f}}\right)^{-1.7}\left(\frac{L_{b}}{r_{y}}\right)^{-0.2}\left(\frac{L}{d}\right)^{1.1} \quad(\mathrm{COV}=0.34)
$$

$L_{b} / r_{y}$ is the laterally unbraced length of the steel beam divided by the radius of gyration, $r_{y}$ of the bare steel cross section. For beams with RBS the following expression may be used:

$$
\theta_{p}^{*}=0.55\left(\frac{h}{t_{w}}\right)^{-0.5}\left(\frac{b_{f}}{2 t_{f}}\right)^{-0.7}\left(\frac{L_{b}}{r_{y}}\right)^{-0.5}\left(\frac{L}{d}\right)^{0.8} \quad(\mathrm{COV}=0.42)
$$

Plastic Deformation of the Descending Portion of the First Cycle Envelope Curve, $\boldsymbol{\Delta}_{p c}{ }_{p c}$. This quantity refers to the post-peak plastic rotation, $\theta_{p c}^{*}$ for flexural hinges adjusted for in-cycle deterioration in strength and stiffness. For standard (nonRBS) beam sections, $\theta_{p c}^{*}$, can be computed as follows:

$$
\theta_{p c}^{*}=24\left(\frac{h}{t_{w}}\right)^{-0.9}\left(\frac{b_{f}}{2 t_{f}}\right)^{-0.2}\left(\frac{L_{b}}{r_{y}}\right)^{-0.5} \quad(\mathrm{COV}=0.45)
$$

Alternatively, for beams with RBS:

$$
\theta_{p c}^{*}=20\left(\frac{h}{t_{w}}\right)^{-0.8}\left(\frac{b_{f}}{2 t_{f}}\right)^{-0.1}\left(\frac{L_{b}}{r_{y}}\right)^{-0.6} \quad(\mathrm{COV}=0.31)
$$

Residual Strength, $\boldsymbol{Q}_{\boldsymbol{R}}^{\prime}$. The residual flexural strength, $M_{r}^{*}$, for steel beams due to stabilization of the buckle size can be assumed to be, $M_{r}^{*},=0.3 M_{y}^{*}$, regardless of the beam-to-column moment connection type. This value is based on reversed cyclic tests according to a standard symmetric cyclic loading protocol.

Ultimate Deformation, $\boldsymbol{\Delta}^{\prime}{ }^{\prime}{ }_{l t}$. For steel beams with flexural hinging the ultimate rotation capacity with reference to Figure 4-35 that includes the cyclic and in-cycle deterioration effects can be assumed, $\theta_{u l t}^{*}=0.08$ radians with a $\mathrm{COV}=0.30$, regardless of the beam-to-column moment connection type. 
Equations 4-1 to 4-13 were derived for steel beams with the following range of parameters:

$$
\begin{aligned}
& 20 \leq h / t_{w} \leq 55 \text { for non-RBS; } 21 \leq h / t_{w} \leq 55 \text { for RBS; } \\
& 4 \leq b_{f} / 2 t_{f} \leq 8 \text { for non-RBS; } 4.5 \leq b_{f} / 2 t_{f} \leq 7.5 \text { for RBS; } \\
& 20 \leq L_{b} / r_{y} \leq 80 \text { for non-RBS; } 20 \leq L_{b} / r_{y} \leq 65 \text { for RBS; } \\
& 2.5 \leq L / d \leq 7 \text { for non-RBS; } 2.3 \leq L / d \leq 6.3 \text { for RBS; } \\
& 4 \leq d \leq 36 \text { in. for non-RBS; } 21 \leq d \leq 36 \text { in. for RBS }
\end{aligned}
$$

Most of the experimental data are for beams that were fabricated from A992 Grade 50 or A572 Grade 50 steel. For other types of steel materials (e.g., A36), the more complex equation forms discussed in Lignos and Krawinkler (2011) should be employed. Appendix A includes comparisons of the proposed equations with current ASCE/SEI 41-13 nonlinear modeling recommendations for steel beams in fully restrained beam-to-column connections.

\subsubsection{Composite Steel Beams}

The beam hinge model discussed in the previous section does not account for the non-symmetric hysteretic behavior of composite steel beams due to the presence of the floor slab. Therefore, the component modeling approach for composite steel beams must be modified to account for the presence of the slab. The recommendations summarized herein are based on work of Elkady and Lignos (2014) that assessed the effect of the composite action on steel moment frames under earthquake loading. This was based on available test data from full-scale cyclic tests on composite fully restrained beam-to-column connections. Figure 4-36a shows a typical calibration of the input model parameters of a widely used point plastic hinge deterioration model (Ibarra et al., 2005) with test data from full-scale beams with RBS conducted by Ricles et al. (2004). Based on these calibrations, the monotonic and first-cycle envelope curves for bare steel beams may be adjusted to include the composite effects due to the presence of the floor slab. Figure 4-36b illustrates schematic representations of the adjusted monotonic envelope and cyclic backbone curves for a composite beam in comparison with those for a bare steel beam. The adjusted monotonic envelope and first-cycle backbone curves are non-symmetric. The following scaling factor adjustments should be considered to adjust the monotonic and/or first-cycle envelope curves of bare steel beams to include composite effects. 


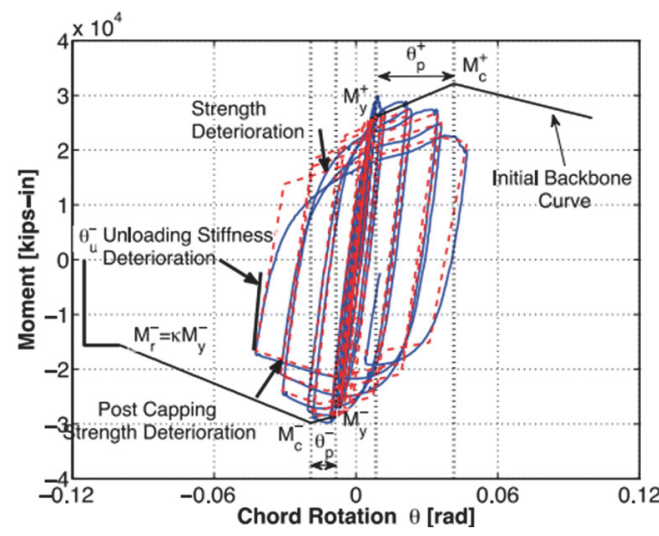

(a) Composite steel beam with RBS

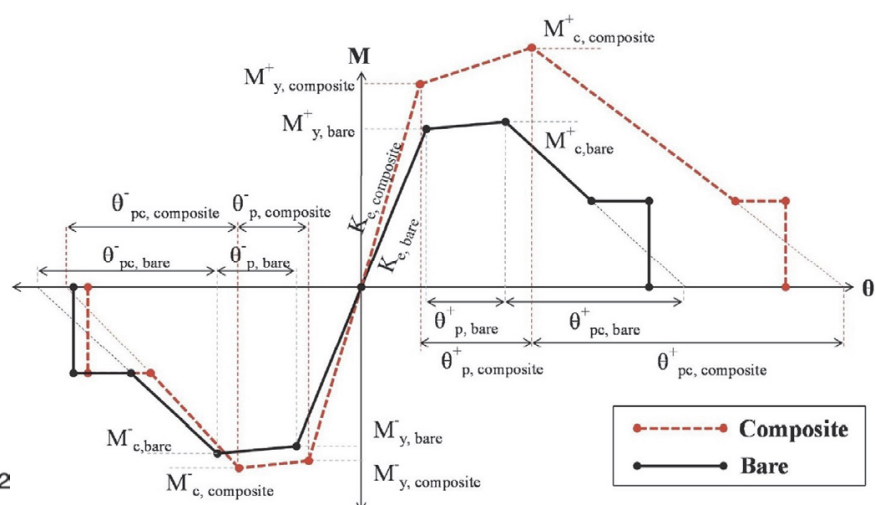

(b) Adjusted monotonic curve for composite beam effects

Figure 4-36 Composite effects on the hysteretic behavior of steel beams (Elkady and Lignos, 2013; 2014; experimental data from Ricles et al., 2004).

Effective Moment of Inertia, $\boldsymbol{I}_{\boldsymbol{c}, \boldsymbol{b}}$. This is the moment of inertia of the composite cross section, $I_{c, b}$, averaged for the positive and negative loading directions. The effective moment of inertia may be computed as $I_{c, b}=1.4 I_{b}(\mathrm{COV}=0.12)$. This adjustment is a conservative assumption given that the employed test data consisted of cruciform configurations wherethe frame continuity is neglected. Prior experimental studies that utilized the frame action including composite effects (Cordoba and Deierlein, 2005; Nakashima et al., 2007; Nam and Kasai, 2012) have shown that this factor may be in the order of two times the respective moment of inertia of the bare beam.

Effective Yield Strength, $\boldsymbol{Q}_{\boldsymbol{y}}$. This parameter should be separately defined for both the positive and negative loading directions for a composite steel beam as shown in Figure 4-36b. In particular, the flexural strength, $M_{y}^{*_{+}}$of the composite beam when the floor slab is in compression may be computed based Section I3 of ANSI/AISC $360-10$ as follows:

- Assume full composite action between the concrete slab and the steel beam.

- The effective width, $b_{\text {eff, }}$ of the composite beam should be calculated based on Section I 3.1a of ANSI/AISC 360-10 [i.e., $b_{\text {eff }}=\min \{$ One-eighth of the girder span center-to-center of supports, One-half the distance to the centerline of the adjacent girder, Distance to the edge of the slab\}].

- The effective stress before concrete crushing is taken as 0.85 of the specified concrete stress $f_{c}^{\prime}$.

Referring to the force-deformation relation shown in Figure 4-36b, the effective flexural yield strength $M^{*}{ }_{y}{ }^{-}$of the composite beam when the slab is in tension may be increased by 1.10 times the effective flexural yield strength of the bare steel beam (see Equation 4-3). This increase is attributed to the slab steel reinforcement. 
Capping (Peak) Strength, $\boldsymbol{Q}_{\max }$. This parameter should be defined for both the positive and negative loading directions. In particular, capping flexural strength, $M_{\max }^{+}$of the composite steel beam when the slab is in compression should be computed based on a factored value of the effective strength, $M_{y}^{*}$ of the bare steel cross section as follows:

$$
M_{\text {max }}^{+}=1.30 M_{y}^{*} \quad(\mathrm{COV}=0.1)
$$

The capping flexural strength of the composite beam in the negative loading direction, $M_{\max }^{-}$may be computed in accordance with Equation 4-9.

Pre-Peak Plastic Deformation, $\boldsymbol{\Delta}_{\text {cap,pl }}$. This quantity in the positive loading direction should be computed based on a factored value of the $\theta_{p}\left(\theta_{p}^{*}\right)$ given by Equations 4-5 for the monotonic envelope (or Equation 4-10 for the first cycle envelope) and 4-6 (or Equation 4-11) for standard bare non-RBS beams and beams with RBS, respectively, as follows:

$$
\theta_{p}^{+}=1.80 \theta_{p} \quad(\mathrm{COV}=0.20)
$$

Post-Peak Plastic Deformation, $\boldsymbol{\Delta}_{p c}$. This quantity in the positive loading direction should be computed based on factored values of the $\theta_{p c}\left(\theta_{p c}^{*}\right)$ given by Equations 4-7 (or Equation 4-12) and 4-8 (or Equation 4-13) for bare standard non-RBS beams and beams with RBS, respectively, as follows:

$$
\theta_{p c}^{+}=1.35 \theta_{p c} \quad(\mathrm{COV}=0.15)
$$

For all practical purposes the residual strength, ultimate rotation and deformation input model parameters for a composite steel beam under negative bending (i.e., negative loading direction) should be considered to be the same with the equivalent bare beam cross section as discussed in the previous section. Appendix A includes comparisons of the proposed equations with current ASCE/SEI 41-13 nonlinear modeling recommendations for steel composite beams.

\subsubsection{Beams with Pre-Northridge WUF-B Connections}

A set of more than 30 tests was collected from experiments that were mostly conducted as part of the SAC program on steel beam-to-column connections (FEMA, 1997c; 2000d). Modeling recommendations are provided only for a first-cycle envelope curve as there is no monotonic test data available for pre-Northridge WUF$\mathrm{B}$ beam-to-column connections. In addition, beams in this data set fractured very early in their loading history (FEMA, 2000d), such that little cyclic degradation occurred. The variability in the input model parameters of the first-cycle envelope curve for beams in pre-Northridge beam-to-column connections is described in terms of a COV value, if the available data are sufficient. 
Referring to the first-cycle envelope curve, shown in Figure 4-35, the input model parameters for beams with pre-Northridge WUF-B connections are summarized as follows:

The effective flexural stiffness, $K_{e}$ of the steel beam may be computed based on Equations 4-1 and 4-2. The effective yield strength, $M_{y}^{*}$ can be calculated as follows:

$$
M_{y}^{*}=S_{x} F_{y e} \quad(\mathrm{COV}=0.19)
$$

where $S_{x}$ is the section modulus of the steel beam and $F_{y e}$ is the expected yield stress of the steel material based on Tables 9-1, 9-2 and 9-3 of ASCE/SEI 41-13. In cases that the beam depth, $d<21$ " then an upper bound analysis should also be employed where $M_{y}^{*}$ is computed based on Equation 4-3. This is due to the fact that steel beams with fairly small depths in pre-Northridge beam-to-column connections seem to achieve an appreciable plastic deformation capacity prior to connection fractures (see Appendix A).

Peak (Capping) Strength, $\boldsymbol{Q}^{\prime}{ }_{\max }$. The peak flexural strength of the steel beam under cyclic loading is computed using a factored value of the expected flexural strength, $M_{p e}$ as follows:

$$
M_{\text {max }}^{*}=1.1 M_{p e} \quad(\mathrm{COV}=0.23)
$$

Pre-Peak Plastic Deformation, $\boldsymbol{\Delta}^{\prime}{ }^{\prime}$ ap,pl . The pre-peak plastic rotation, $\theta_{p}^{*}$ for beam flexural hinges can be computed as follows:

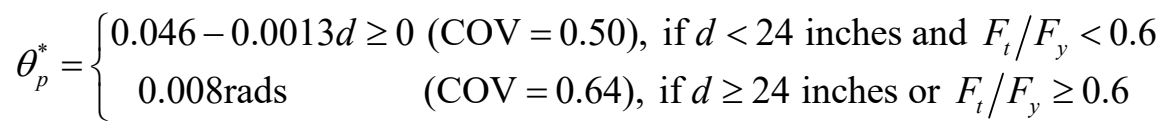

in which, $d$ is the steel beam depth; $F_{t}$ and $F_{y}$ are the nominal tensile and yield stress of the steel material, respectively.

Post-Peak Plastic Deformation, $\boldsymbol{\Delta}^{\prime}{ }_{p c}$. The post-peak plastic rotation, $\theta_{p c}^{*}$ for beams as with pre-Northridge WUF-B connections is given as follows:

$$
\theta_{p c}^{*}=\left\{\begin{array}{c}
-0.003+0.0007 d \geq 0(\mathrm{COV}=1.1), \text { if } d<24 \text { inches and } F_{t} / F_{y}<0.6 \\
0.035-0.0006 d \quad(\mathrm{COV}=1.1), \text { if } d \geq 24 \text { inches or } F_{t} / F_{y} \geq 0.6
\end{array}\right.
$$

Residual Strength, $\boldsymbol{Q}_{\boldsymbol{R}}$. The residual flexural strength, $M_{r}^{*}$ for steel beams with pre-Northridge WUF-B connections at loss of the gravity capacity, $M_{r}^{*}=0.2 M_{y}^{*}$ $(\mathrm{COV}=0.50)$.

Ultimate Deformation, $\boldsymbol{\Delta}_{\text {ult }}$. Steel beams as part of pre-Northridge connections indicate a relatively steep post-peak plastic deformation, $\theta_{p c}^{*}$. The ultimate rotation 
capacity of steel beams in pre-Northridge beam-to-column connections can be assumed as follows:

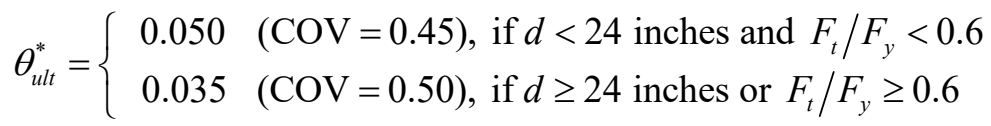

Appendix A includes illustrative examples and comparisons with current ASCE/SEI 41-13 nonlinear modeling recommendations for steel beams in pre-Northridge beamto-column connections (see Section C.3).

\subsubsection{Non-Conforming Beams as part of Fully-Restrained Beam-to- Column Connections}

Steel beams that do not meet the requirements for lateral bracing and cross sectional slenderness for highly ductile members per ANSI/AISC 360-10 may be modeled based on the recommendations discussed in Section 4.5.1. However, the effective yield flexural strength, $M_{y}^{*}$, of a non-conforming steel beam should be adjusted based on the beam-unbraced length, $L_{b}$ such that the effects of lateral torsional buckling on the beam flexural yield strength should be explicitly captured. This should be done in accordance with Sections F2 to F5 of ANSI/AISC 360-10 provisions for doubly symmetric compact wide flange members bent about their strong axis and based on their web and flange depth-to-thickness ratios. Shown in Figure 4-37 schematically is the adjusted effective yield flexural strength, $M_{y}^{*}$ of a steel beam with respect to its unbraced length.

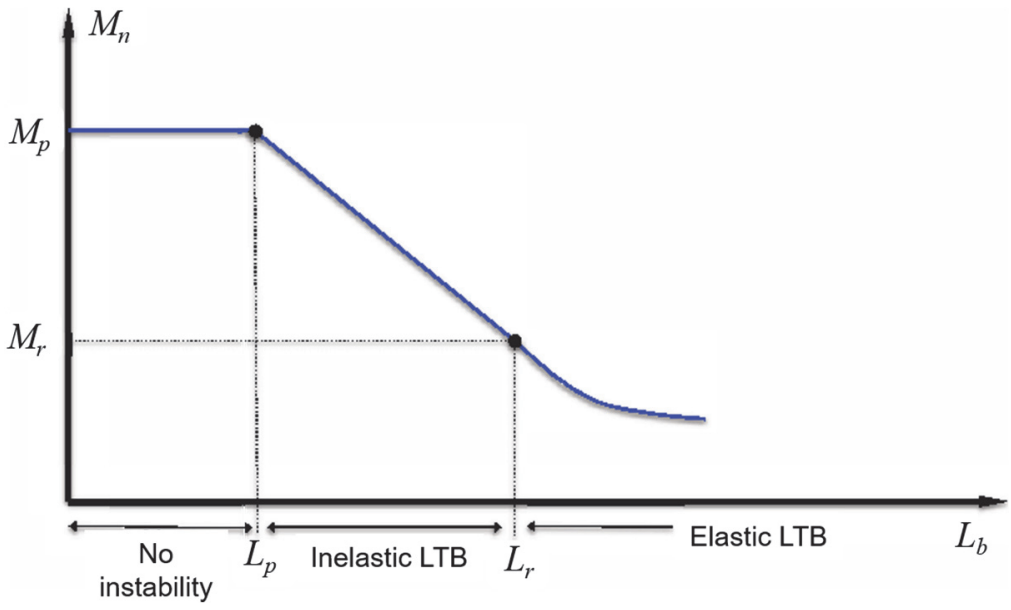

Figure 4-37 Flexural strength with respect to steel beam unbraced length.

\subsubsection{Column Hinge Model}

Steel columns that generally conform to AISC seismic provisions for moderately ductile and highly ductile members can be modeled based on the recommendations discussed herein that are based on work of Elkady and Lignos (2015b; 2016) and 
Hartloper and Lignos $(2016 ; 2017)$. The proposed equations have been developed based on test data from wide flange steel columns were tested at either large- or fullscale. The range of cross-sections includes W8 to W24. Data from detailed finite element studies on wide flange steel columns ranging from W8 up to 36 inches deep have been utilized as well (Elkady and Lignos, 2015b). Referring to Figure 4-35, the input model parameters to compute the monotonic and first-cycle envelope curves are statistically calibrated to median values. The variability in the same parameters is described in terms of a COV.

A similar approach was taken for modeling the two curves shown in Figure 4-35 for HSS columns subjected to axial load and lateral deformations based on prior work of Lignos and Krawinkler (2010; 2012b). Experimental data with constant compressive and varying axial load on nominally identical steel beam-columns (MacRae et al., 1990; Newell and Uang, 2006a; Suzuki and Lignos, 2015; Lignos et al., 2016) indicate that the post-buckling behavior of steel beam-columns is influenced more by the constant compressive axial load (i.e., gravity load, $P_{g}$ ) rather than the transient component. The former is common in end columns as part of steel moment frame systems due to dynamic overturning effects during an earthquake. Therefore, the predictive equations for the model parameters discussed below are based on the gravity axial load ratio, $P_{g} / P_{y e}$, where $P_{y e}$ is the expected axial yield strength of the steel column. The proposed column hinge parameters do not include any reduction due to P-delta effects. The experimental and analytical data that were utilized were processed such that the deduced moment reflected the actual moment within the column hinge.

\subsubsection{Steel Wide-Flange Beam-Columns}

Referring to the two curves, shown in Figure 4-35 the input model parameters for steel wide flange beam-columns can be computed as follows:

Elastic Stiffness, $\boldsymbol{K}_{e}$. The column hinge may be modeled with a finite elastic stiffness that considers both flexural and shear contributions using the equations to estimate the flexural stiffness of link beams in eccentrically braced frames per ASCE/SEI 41-13 (Bech et al., 2015) or as rigid-plastic. In particular, $K_{e}=$ $L^{2} K_{s} K_{b} /\left[2\left(K_{s}+K_{b}\right)\right]$ where the shear and flexural stiffness are $K_{s}=G A_{w} / L$ and $K_{b}=12 E I / L^{3}$, respectively; $G$ is the shear modulus of the steel material, $A_{w}$ is the area of the web of the wide-flange cross-section. Note that based on experimental findings from Suzuki and Lignos (2015) and Elkady and Lignos (2015b; 2016) the assumed plastic hinge length $L_{h}=1.4 d_{c}$; where $d_{c}$ is the steel column depth. This agrees with the seismic design criteria for steel columns according to the New Zealand seismic code (NZS, 1997). 
Effective Yield Strength, $\boldsymbol{Q}_{\boldsymbol{y}}$. For a steel beam-column this quantity refers to its effective flexural yield strength, $M_{y}^{*}$ reduced by the applied compressive axial load. The effective flexural yield strength is computed based on the AISC P-M interaction equations as follows:

$$
\begin{aligned}
& \text { If } P_{g} / P_{y e} \leq 0.20, \quad M_{y}^{*}=1.15 Z R_{y} F_{y}\left(1-P_{g} / P_{y e}\right) \\
& \text { If } P_{g} / P_{y e}>0.20, \quad M_{y}^{*}=1.15 Z R_{y} F_{y}\left[\frac{9}{8}\left(1-P_{g} / P_{y e}\right)\right]
\end{aligned}
$$

The 1.15 factor accounts for the effects of cyclic hardening on the hysteretic behavior of wide flange beam-columns $(\mathrm{COV}=0.10)$.

Peak (Capping) Strength, $\boldsymbol{Q}_{\max }$ (or $\boldsymbol{Q}_{\text {max }}^{\prime}$ ). This quantity refers to the peak flexural strength of a steel beam-column $M_{\max }\left(M_{\max }^{*}\right)$ under monotonic or cyclic loading adjusted for the effects of cyclic hardening. The capping strength is computed based on a factored value of the effective flexural yield strength of the steel column, $M_{y}^{*}$ as follows:

$$
M_{\max }=a M_{y}^{*}
$$

Where $a$ is a coefficient that defines the hardening ratio $M_{\max }^{*} / M_{y}^{*}$. This coefficient depends on the web local slenderness, $h / t_{w}$ and global slenderness of the member, $L_{b} / r_{y}$ ( $L_{b}$ is the unbraced length of the column; $r_{y}$ is the radius of gyration of the column cross-section with respect to the weak-axis) and the applied axial load ratio, $P_{g} / P_{y e}$ :

$$
a=12.5\left(\frac{h}{t_{w}}\right)^{-0.2}\left(\frac{L_{b}}{r_{y}}\right)^{-0.4}\left(1-\frac{P_{g}}{P_{y e}}\right)^{0.4} \geq 1.0 \quad(\mathrm{COV}=0.10)
$$

with reference to the first-cycle envelope curve in Figure 4-35 the hardening ratio coefficient $a^{*}$ is given as follows:

$$
a^{*}=9.5\left(\frac{h}{t_{w}}\right)^{-0.4}\left(\frac{L_{b}}{r_{y}}\right)^{-0.16}\left(1-\frac{P_{g}}{P_{y e}}\right)^{0.2} \geq 1.0 \quad(\mathrm{COV}=0.07)
$$

if $P_{g} / P_{y e}>0.30$ or $h / t_{w}<15$ then the above ratios should always be less than 1.3.

Pre-Peak Plastic Deformation, $\boldsymbol{\Delta}_{c a p, p l}\left(\right.$ or $\left.\boldsymbol{\Delta}_{c a p, p l}^{\prime}\right)$. This quantity refers to the prepeak plastic rotation, $\theta_{p}$ for beam-column flexural hinges and it can be computed as follows:

$$
\theta_{p}=294\left(\frac{h}{t_{w}}\right)^{-1.7}\left(\frac{L_{b}}{r_{y}}\right)^{-0.7}\left(1-\frac{P_{g}}{P_{y e}}\right)^{1.6} \leq 0.20 \quad(\mathrm{COV}=0.39)
$$

with reference to the first-cycle envelope curve in Figure 4-35 the pre-peak plastic deformation, $\theta_{p}^{*}$ for a steel wide flange beam-column is given as follows: 


$$
\theta_{p}^{*}=15\left(\frac{h}{t_{w}}\right)^{-1.6}\left(\frac{L_{b}}{r_{y}}\right)^{-0.3}\left(1-\frac{P_{g}}{P_{y e}}\right)^{2.3} \leq 0.10 \quad(\mathrm{COV}=0.31)
$$

Post-Peak Plastic Deformation, $\boldsymbol{\Delta}_{p c}\left(\right.$ or $\boldsymbol{\Delta}_{p c}{ }_{p c}$ ). This quantity refers to the post-peak plastic rotation, $\theta_{p c}$ for beam-column flexural hinges. It indicates the post-buckling behavior of a steel beam-column. For monotonic loading, $\theta_{p c}$, can be computed as follows:

$$
\theta_{p c}=90\left(\frac{h}{t_{w}}\right)^{-0.8}\left(\frac{L_{b}}{r_{y}}\right)^{-0.8}\left(1-\frac{P_{g}}{P_{y e}}\right)^{2.5} \leq 0.30 \quad(\mathrm{COV}=0.14)
$$

with reference to the first-cycle envelope curve in Figure 4-35 the post-peak plastic deformation, $\theta_{p c}^{*}$ is given as follows:

$$
\theta_{p c}^{*}=14\left(\frac{h}{t_{w}}\right)^{-0.8}\left(\frac{L_{b}}{r_{y}}\right)^{-0.5}\left(1-\frac{P_{g}}{P_{y e}}\right)^{3.2} \leq 0.10 \quad(\mathrm{COV}=0.40)
$$

Residual Strength, $\boldsymbol{Q}_{\boldsymbol{R}}$ (or $\boldsymbol{Q}_{\boldsymbol{R}}^{\prime}$ ). The residual flexural strength, $M_{r}$, for steel beamcolumns under monotonic loading can be computed based on the following equation:

$$
M_{r}=\left(0.5-0.4 \frac{P_{g}}{P_{y e}}\right) M_{y}^{*} \quad(\mathrm{COV}=0.27)
$$

The same quantity for the first-cycle envelope curve may be computed as follows:

$$
M_{r}^{*}=\left(0.4-0.4 \frac{P_{g}}{P_{y e}}\right) M_{y}^{*}
$$

These values are based on experimental and analytical data from Suzuki and Lignos (2015), Elkady and Lignos (2015b; 2016) and Lignos et al. (2016).

Ultimate Deformation, $\boldsymbol{\Delta}_{\text {ult }}$. For steel beam-columns with flexural hinging, this quantity represents the ultimate rotation capacity that is likely to be controlled by loss of the column axial carrying capacity. With reference to the monotonic curve (see Figure 4-33) it is recommended to assume $\theta_{u l t}=0.15$ radians with a $\mathrm{COV}=0.46$. For the first-cycle envelope curve:

$$
\theta_{u l t}^{*}=0.08\left(1-0.6 \frac{P_{g}}{P_{y e}}\right) \quad(\mathrm{COV}=0.51)
$$

Equations 4-22 to 4-32 were derived based on the following range of parameters:

- $\quad 3.71 \leq h / t_{w} \leq 57.5$

- $\quad 1.82 \leq b_{f} / 2 t_{f} \leq 8.52$ 
- $38.4 \leq L / r_{y} \leq 120$

- $0 \leq P_{g} / P_{y e} \leq 0.60$

Steel wide flange beam-columns under compressive axial load ratios $P_{g} / P_{y e}>0.60$ that have $h / t_{w}>43$ and $K L / r_{y}>120$ should be treated as force-controlled elements as per ASCE/SEI 41-13. Steel beam-columns utilizing stocky cross-sections that experience gravity loads, $P_{g}$, larger than $0.60 P_{y e}$ have a finite plastic deformation capacity, which is not zero. However, beam-columns with gravity loads over $0.6 P_{y e}$ may be under designed for gravity loads (Bech et al., 2015). Therefore, column yielding should be restricted.

Because most of the available data from experiments and finite element studies that were utilized to develop the above equations are based on Grade 50 steel (A992 or equivalent), the deformation parameters for steel wide-flange beam-columns are not a function of the yield stress, $F_{y}$, of the respective steel material. Appendix A includes illustrative examples and comparisons with current ASCE/SEI 41-13 nonlinear modeling recommendations for steel wide-flange beam-columns under monotonic and reversed cyclic loading (see Section C.4).

\subsubsection{Wide-Flange Beam-Columns under Bidirectional Loading}

Figure 4-38 shows a comparison of the first-cycle envelope curves of nominally identical steel column specimens (i.e., W24×146 and W24×84) that were subjected to a constant compressive axial load coupled with symmetric unidirectional and bidirectional lateral loading as discussed in Section 4.3.3 (see Figure 4-17). In this figure, the pre- and post-peak plastic deformation of these columns were nearly identical in both cases. Therefore, the proposed modeling recommendations for nonlinear modeling of beam-columns subjected to unidirectional loading may be used to assess the nonlinear behavior of wide-flange beam-columns subjected to bidirectional lateral loading.

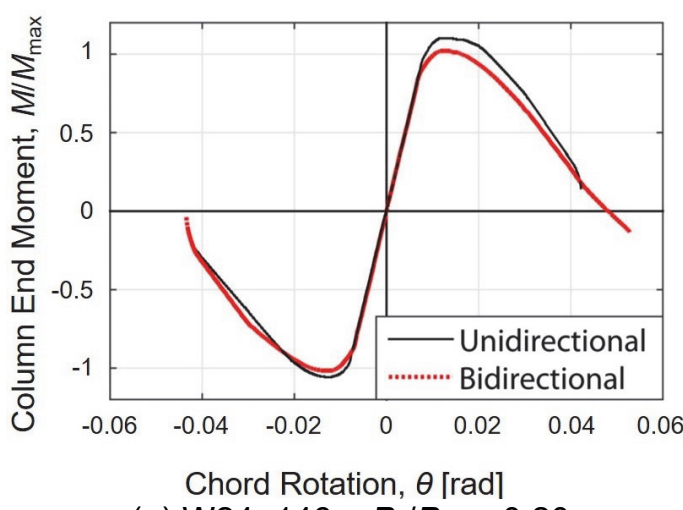

(a) $\mathrm{W} 24 \times 146-P_{g} / P_{y e}=0.20$ 1 st cycle envelope

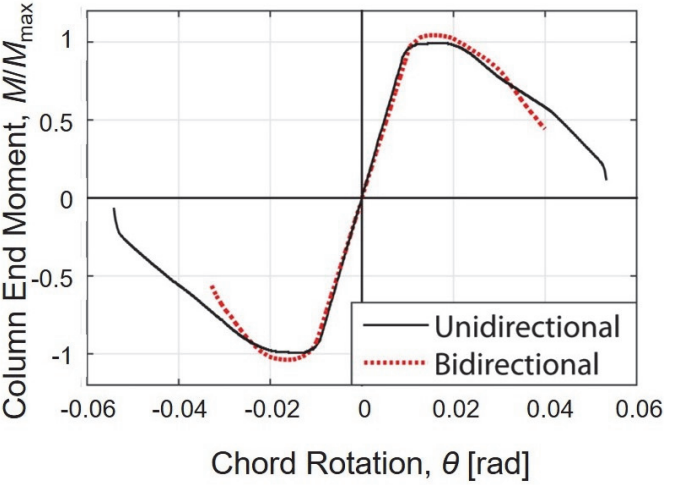

(b) $\mathrm{W} 24 \times 84-P_{g} / P_{y e}=0.20$ 1 st cycle envelope

Figure 4-38 Comparisons of proposed modeling recommendations with ASCE/SEI 41-13 for steel wide-flange beam-columns under bidirectional loading (Elkady and Lignos, 2016). 


\subsubsection{End Columns}

End columns in steel moment frame systems may experience large variations in their axial load demands due to the transient component coming from the dynamic overturning moment effects. Figure 4-39 shows the experimental data in terms of average first-cycle envelope curves from full-scale tests on steel columns subjected to varying axial load in addition to lateral drift demands. Figure 4-39a refers to a steel column that utilized a W14 $\times 233$ cross section (data from Newell and Uang, 2006a). The initially applied axial load ratio due to gravity in this case was $P_{g} / P_{y e}=0.15$; however, the applied axial load ratio varied from -0.15 to 0.75 during the test. Similarly, Figure $4-39 \mathrm{~b}$ refers to a steel column that utilized a W16 $\times 89$ cross section. The initial gravity offset was $P_{g} / P_{y e}=0.50$. This load ratio varied from 0.25 to 0.75 througout the lateral loading history (data from Lignos et al., 2016).

Superimposed in the same figure are the predicted first-cycle envelope curves based on the nonlinear modeling recommendations discussed above by using the gravity offset $P_{g} / P_{y e}$ as a reference axial load ratio in the predictive equations summarized in Section 4.5.4.1 and not the transient component as suggested by the ASCE/SEI 41-13 recommendations. This is also consistent with the recommendations discussed in Bech et al. (2015). In Figure 4-39, the proposed equations predict the first-cycle envelope of end columns fairly well regardless of the cross-sectional compactness ratio.

The effective and peak flexural strength of the column utilizing the $\mathrm{W} 14 \times 233$ crosssection are underpredicted by about $25 \%$ as shown in Figure $4-39 \mathrm{a}$. This is due to the fact that this cross-section is fairly stocky (i.e., $h / t_{w}=10.7, b_{f} / 2 t_{f}=4.62$ ) and it did not buckle locally during the lateral loading history. Therefore, cyclic hardening was signficant in this case. However, for more slender but still seismically compact cross-sections such as the one in Figure 4-39b (i.e., W16 $\times 89: h / t_{w}=25.9, b_{f} / 2 t_{f}=$ 5.92) the predicted effective yield and peak strength of the steel column is fairly close to the one measured from the test. In this case, the formation of local buckling near the member base occurred early in the lateral loading history; therefore, the member did not harden cyclically by much.

In Figure 4-39, the ASCE/SEI 41-13 component model treats both members as forcecontrolled elements because the transient component of the axial load demand exceeds the $P / P_{c r}=0.50$ limit. In the same figure, the same members can be treated as deformation-controlled elements even though they experience high axial load demands. 


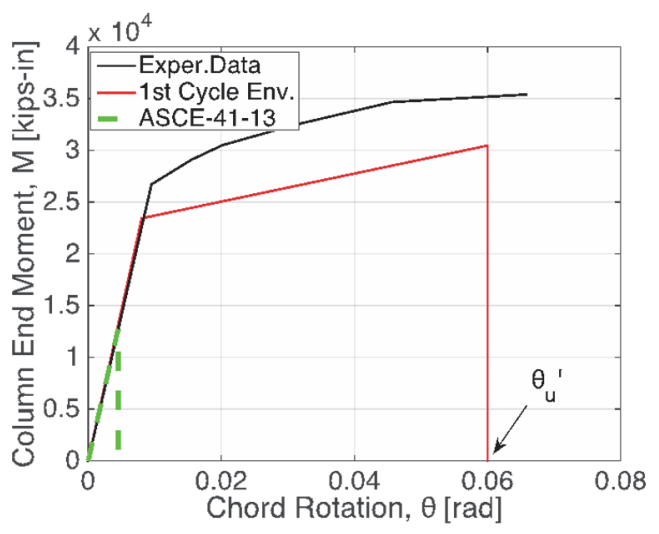

(a) $\mathrm{W} 14 \times 233-55, P_{g} / P_{y}=0.15$, $P / P_{y} \sim-0.15-0.75$

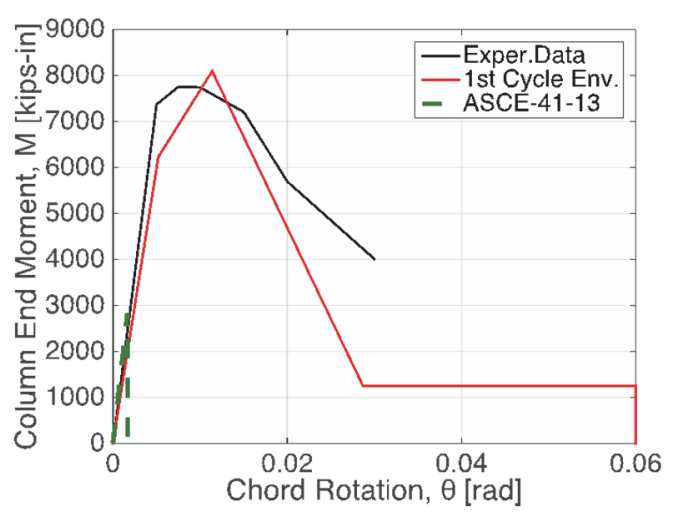

(b) $\mathrm{W} 16 \times 89, P_{g} / P_{y}=0.50$, $P / P_{y} \sim 0.25-0.75$

Figure 4-39 Comparisons of proposed modeling recommendations with ASCE/SEI 41-13 for end beam-columns as part of steel moment frame systems (Newell and Uang, 2006a; Lignos et al., 2016).

\subsubsection{Hollow Structural Steel Beam-Columns}

With reference to the two curves shown in Figure 4-35 the elastic stiffness $K_{e}$, the effective yield strength $M_{y}{ }^{*}$, and capping flexural strength $M_{\max }$ of HSS steel columns may be computed based on Equations 4-1, 4-22 and 4-23, respectively. The coefficient $a$ that defines the hardening ratio $M_{\max } / M_{y}{ }^{*}$ for HSS steel beam-columns should be defined as follows for both the monotonic and first-cycle envelope curves:

$$
a=0.04\left(\frac{D}{t}\right)^{-0.3}\left(1-\frac{P_{g}}{P_{y e}}\right)^{1.3}\left(\frac{E}{F_{y e}}\right)^{0.75} \geq 1.0 \quad(\mathrm{COV}=0.23)
$$

The hardening ratio, $a$ should always be $a \leq 1.3$.

Pre-Peak Plastic Deformation, $\boldsymbol{\Delta}_{\text {cap }, \boldsymbol{p l}}$. The pre-peak plastic rotation, $\theta_{p}$, for flexural hinges in HSS beam-columns under monotonic loading can be computed as follows:

$$
\theta_{p}=0.3\left(\frac{D}{t}\right)^{-0.95}\left(1-\frac{P_{g}}{P_{y e}}\right)^{1.1}\left(\frac{E}{F_{y e}}\right)^{0.1} \quad(\mathrm{COV}=0.26)
$$

with reference to the first-cycle envelope curve in Figure 4-35 the pre-peak plastic deformation, $\theta_{p}^{*}$ for HSS beam-columns is given as follows:

$$
\theta_{p}^{*}=0.1\left(\frac{D}{t}\right)^{-1.1}\left(1-\frac{P_{g}}{P_{y e}}\right)^{1.4}\left(\frac{E}{F_{y e}}\right)^{0.3} \quad(\mathrm{COV}=0.53)
$$

Post-Peak Plastic Deformation, $\boldsymbol{\Delta}_{\boldsymbol{p}}$. This quantity refers to the post-peak plastic rotation, $\theta_{p c}$ for flexural hinges in HSS steel beam-columns under monotonic loading and can be computed as follows: 


$$
\theta_{p c}=5.4\left(\frac{D}{t}\right)^{-1.2}\left(1-\frac{P_{g}}{P_{y e}}\right)^{3.0}\left(\frac{E}{F_{y e}}\right)^{0.14} \quad(\mathrm{COV}=0.35)
$$

with reference to the first-cycle envelope curve in Figure 4-35 the post-peak plastic deformation, $\theta_{p c}^{*}$ is given as follows:

$$
\theta_{p c}^{*}=2.8\left(\frac{D}{t}\right)^{-1.3}\left(1-\frac{P_{g}}{P_{y e}}\right)^{2.6}\left(\frac{E}{F_{y e}}\right)^{0.17} \quad(\mathrm{COV}=0.27)
$$

Residual Strength, $\boldsymbol{Q}_{\boldsymbol{R}}$. The residual flexural strength, $M_{r}$, for HSS steel beamcolumns under monotonic loading can be assumed to be:

$$
M_{r}=\left(0.5-0.6 \frac{P_{g}}{P_{y e}}\right) M_{y}^{*} \geq 0 \quad(\mathrm{COV}=0.34)
$$

The same quantity for the first-cycle envelope curve may be computed as follows:

$$
M_{r}^{*}=\left(0.4-0.6 \frac{P_{g}}{P_{y e}}\right) M_{y}^{*} \geq 0 \quad(\mathrm{COV}=0.40)
$$

These values are based on a steel database for HSS steel beam-columns (Lignos and Krawinkler, 2010; 2012b).

Ultimate Deformation, $\boldsymbol{\Delta}_{\boldsymbol{u l t}}$. For HSS steel beam-columns with flexural hinging under monotonic loading, the ultimate rotation capacity that the column losses its axial carrying capacity is recommended to be, $\theta_{u l t}=0.10$ radians with a $\mathrm{COV}=0.45$. For the first-cycle envelope curve:

$$
\theta_{u l t}^{*}=0.08\left(1-\frac{P_{g}}{P_{y e}}\right) \quad(\mathrm{COV}=0.50)
$$

The range of applicability of the above equations for HSS steel beam-columns is indicated as follows:

- $20 \leq D / t \leq 40$

- $0 \leq P_{g} / P_{y e} \leq 0.60$

- $40 \leq F_{y} \leq 72.5 \mathrm{ksi}$

Hollow structural steel beam-columns under compressive axial load ratios, $P_{g} / P_{y e}>0.60$ should be treated as force-controlled elements per ASCE/SEI 41-13. Appendix A includes illustrative examples for steel HSS beam-columns under reversed cyclic loading. 


\subsubsection{Beam-to-Column Joint Panel Zone Model}

Mathematical models to describe the behavior of the beam-to-column joint panel zones have been proposed by many researchers (Krawinkler et al., 1971; Krawinkler 1978; Lu et al., 1988; Kim and Engelhardt, 2002). These models agree more-or-less in terms of the representation of the elastic shear stiffness and the yield shear strength of the beam-to-column joint panel zone. However, most of these models differ in the way that the inelastic behavior of the panel zone is captured. In this section, the Krawinkler shear panel zone model as discussed in Gupta and Krawinkler (1999) is recommended for modeling the nonlinear behavior of beam-to-column joint panel zones. Other options may be available (Charney and Downs, 2004). Figure 4-40a illustrates the Krawinkler model. It utilizes rigid beam-column elements to form a parallelogram of dimensions, $d_{b}$ (i.e., depth of the beam) by $d_{c}$ (i.e., of the column). A trilinear rotational spring is utilized to capture the hysteretic behavior of the beamto-column joint panel zone as shown in Figure 4-40b. The other three springs placed at the corners of the panel zone are assumed to be flexural hinges. The idealized trilinear curve is constructed for a bare beam-to-column joint panel zone (i.e., composite effects are neglected) as discussed in Gupta and Krawinkler (1999). This curve is then adjusted to capture the composite action effects on the hysteretic behavior of the panel zone based on work of Kim and Engelhardt (2002). The difference is primarily attributed to the effective depth that is utilized to compute the flexural strength of the panel zone as shown in Figure 4-41a.

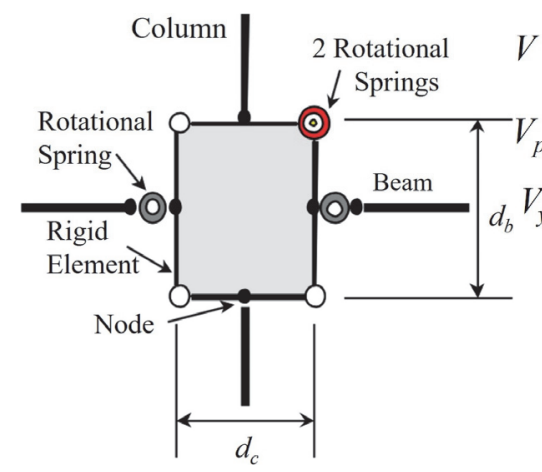

(a)

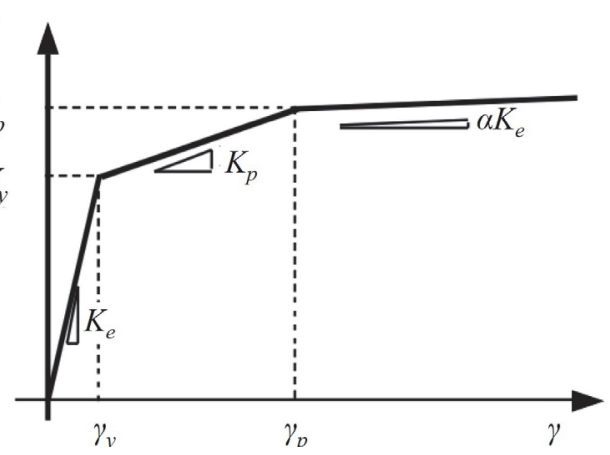

(b)

Figure 4-40 Beam-to-column joint panel zone model and its trilinear model idealization (Gupta and Krawinkler, 1999). 


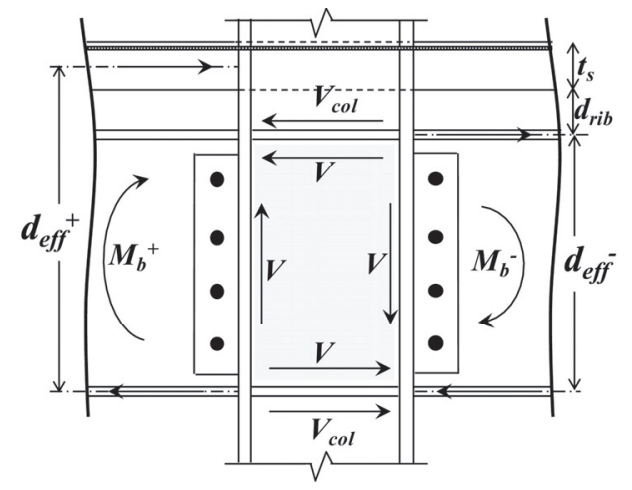

(a)

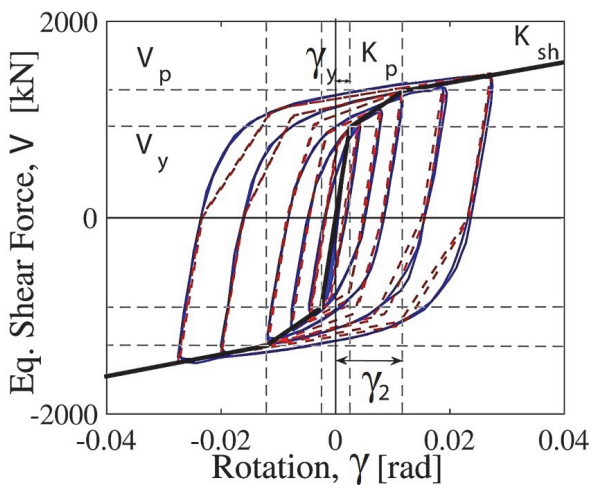

(b)

Figure 4-41 (a) Boundary forces acting on interior composite panel zone and (b) panel zone hysteretic material model (images from Elkady and Lignos, 2014; experimental data from Engelhardt et al., 2000).

\subsubsection{Bare Steel Beam-column Joint Panel Zone}

The flexural yield strength of the idealized trilinear relation is given as follows:

$$
M_{y}=V_{y} d_{b}
$$

in which, $d_{b}$ is the beam depth, $V_{y}$ is the yield shear strength of the panel zone given as follows:

$$
V_{y}=0.60 F_{y} d_{c} t_{p}
$$

the corresponding yield shear distortion $\gamma_{y}$ of the panel zone is given as follows:

$$
\gamma_{y}=\frac{F_{y}}{\sqrt{3} G}
$$

The full plastic moment of the idealized trilinear relation is given as follows:

$$
M_{p}=V_{p} d_{b}
$$

where:

for $P_{r} \leq 0.75 P_{c}, \quad V_{p}=0.60 F_{y} d_{c} t_{p}\left(1+\frac{3 b_{c} t_{c f}^{2}}{d_{b} d_{c} t_{p}}\right)$

for $P_{r}>0.75 P_{c}, \quad V_{p}=0.60 F_{y} d_{c} t_{p}\left(1+\frac{3 b_{c} t_{c f}^{2}}{d_{b} d_{c} t_{p}}\right)\left(1.9-\frac{1.2 P_{r}}{P_{c}}\right)$

Shear distortion, $\gamma_{p}$, is typically assumed to be $4 \gamma_{y}$ (Gupta and Krawinkler, 1999).

Figure 4-41b illustrates an example of the idealized trilinear relation of a panel zone compared to experimental data from Engelhardt et al. (2000). 


\subsubsection{Composite Beam-to-Column Joint Panel Zone}

For beam-to-column joint panel zones in the presence of a floor slab, the effective depth depends on the loading direction as shown in Figure 4-41a. In the negative loading direction (i.e., slab is in tension), the effective depth is similar to that of the bare steel panel zone; hence, the negative yield moment, $M_{y}^{-}$, of the panel zone should be calculated based on Equation 4-41. In the positive loading direction, the slab is in compression; therefore, the effective depth becomes larger than that of the bare steel panel zone. In order to compute the positive bending moment, $M_{y}^{+}$, of the composite panel zone the procedure proposed by Kim and Engelhardt (2002) is adopted as discussed in Elkady and Lignos (2014):

$$
M_{y}^{+}=V_{y} d_{e f f}^{+}=V_{y}\left(d_{b}-d_{r i b}+0.5 t_{s}-0.5 t_{f}\right)
$$

where $d_{r i b}$ is the depth of the ribbed section of the steel deck and $t_{s}$ is the thickness of the slab. For interior composite panel zones, the backbone curve is symmetric due to the effectiveness of the slab in both loading directions (see Figure 4-41a). However, for exterior composite beam-to-column joint panel zones, the backbone curve becomes non-symmetric.

\subsubsection{Hinge Model for Beams as part of Partially-Restrained Connections with Intermediate Stiffness}

If modeling of the gravity framing system is of particular interest the deduced beam moment-rotation relation of typical gravity connections may be modeled based on work of Liu and Astaneh (2000; 2004). Figure 4-42 illustrates the idealized momentrotation relation for steel beams as part of partially restrained connections with intermediate flexural stiffness.

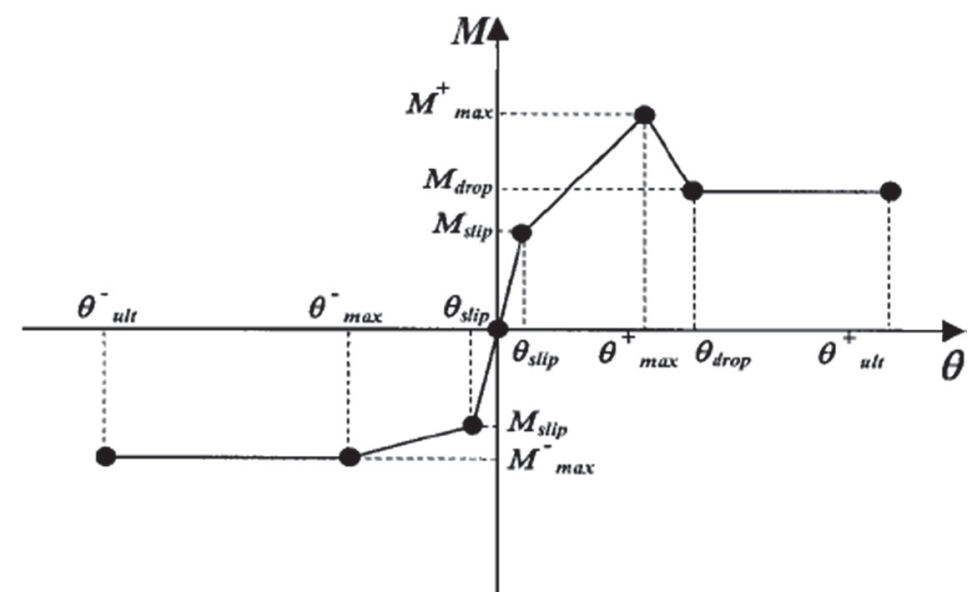

Figure 4-42 Idealized moment-rotation relation for hinge model for beams as part of partially-restrained connections with intermediate stiffness (image adopted from Liu and Astaneh, 2004). 
Based on the discussion in Section 4.3.2 the following points should be identified to fully define the idealized beam moment-rotation relation of a gravity connection.

Maximum Positive Moment Capacity, $\boldsymbol{M}_{\max }$. This moment reflects the flexural strength and stiffness provided by a moment couple formed between the tensile forces resisted by the bolts within the shear tab connection and the compression force developed in the concrete slab (if available). To compute $M_{\max }^{+}$the following procedure should be employed:

- Use the governing limit state for a bolt in shear and first determine how many bolts should be allocated to carry the design shear load. These bolts should be assigned as shear elements, beginning with the top bolt.

- Assume that the remaining bolt elements resist the bending moment. Based on the appropriate stress distribution, calculate the capacity, $T$, of the remaining bolt elements in tension for the governing failure mode.

- Compute the capacity of the concrete slab in compression, $C$ as follows,

$$
C=0.85 f_{c}^{\prime} b_{e f f} a
$$

where $b_{\text {eff }}$ is the width of the column face bearing against the concrete (depending on the column orientation may be the column flange or web); $a=r$ for the deck parallel to the shear tab and beam (i.e., strong axis); $a=0.6 r$ for the deck perpendicular to the shear tab and beam (weak axis); and $r=$ depth of slab above the deck ribs as shown in Figure 4-43.

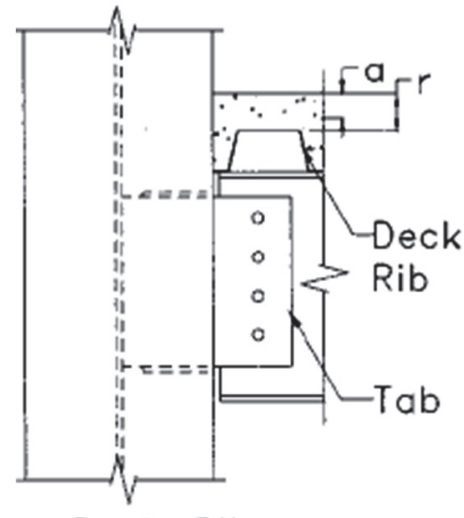

Deck Ribs

Perpendicular to Tab

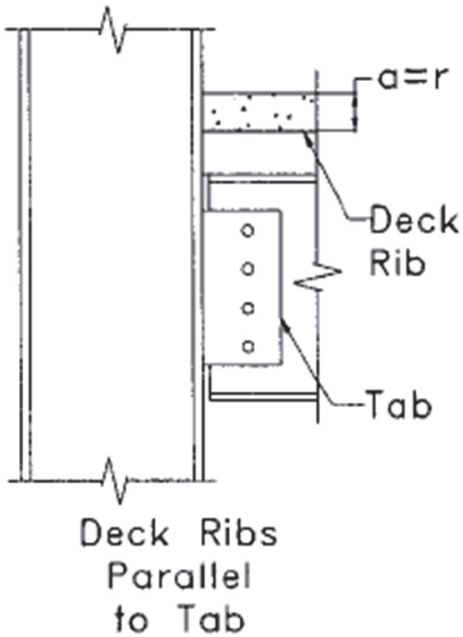

Figure 4-43 Effective depth for calculation of moment capacity (image adopted from Liu and Astaneh, 2004).

- The smaller of $C$ or $T$ governs. By axial equilibrium, $C$ equals $T$. From this, find either the new $a$ of concrete in compression or new number of bolts in tension required to satisfy the force equilibrium. The resulting forces and distributions should be used to calculate $M_{\max }^{+}$. 
To compute the negative bending capacity of a simple connection with composite slab, $M_{\max }^{-}$, conservatively a bare steel connection should be assumed. Similar to $M_{\max }^{+}$, a number of bolt-elements required to compute the gravity shear is calculated. The main difference compared to the computation of $M_{\max }^{+}$is that the bolts in the middle section of the shear tab are now the ones carrying the shear.

Flexural Strength, $\boldsymbol{M}_{\text {slip }}$. This is the flexural strength at which the partial restrained connection experiences bolt slip. This quantity is typically equal to $0.25 M_{\max }^{+}$(Liu and Astaneh, 2004). For bare connections the slip moment should be in considered as $0.55 M_{\max }^{+}$.

Residual Strength, $\boldsymbol{M}_{\text {drop }}$. This is the remaining flexural strength of the partially restrained moment connection with intermediate stiffness once concrete crushing occurs. For composite connections, this is normally computed as a factored value of the $M_{\max }^{+}$. In particular, $M_{d r o p}=0.55 M_{\max }^{+}$. For bare connections there is no associated drop in their moment-rotation relation.

Pre-Peak Plastic Rotation, $\boldsymbol{\theta}_{\max }$. This is the chord rotation associated with the peak flexural strength of the connection. In the presence of a concrete slab the following values should considered:

$$
\begin{array}{ll}
\theta_{\max }^{+}=0.03 \text { radians } & (\mathrm{COV}=0.20) \\
\theta_{\max }^{-}=0.02 \text { radians } & (\mathrm{COV}=0.20)
\end{array}
$$

For bare beams as part of gravity framing connections:

$$
\theta_{\max }^{+}=\theta_{\max }^{-}=0.05 \text { radians } \quad(\mathrm{COV}=0.35)
$$

Rotation at Bolt Slip, $\boldsymbol{\theta}_{\text {slip }}$. This is the rotation associated with bolt slip. Its value is based on averaging of backbone curves of moment rotation relations for typical shear tab connections. Normally, $\theta_{\text {slip }}=0.0042$ radians $(\mathrm{COV}=0.30)$.

In many cases, beam failure occurs at larger rotations than the ones at which beam binding occurs. In this case, a stiffening effect takes place (see Figure 4-44) where a considerable increase in both the flexural strength and stiffness of a steel beam as part of a shear tab connection is observed (i.e., 2.35 $M_{\max }^{-}$, where $M_{\max }^{-}$is the maximum flexural strength of the steel beam in the negative loading direction). This typically occurs at a chord rotation of $1.5 \%$ radians after beam binding occurs and it is sustained for about $4 \%$ radians till connection failure occurs; therefore, $\theta_{u}=\theta_{\text {bind }}+$ 0.04 . This effect can be simulated with two nonlinear spring elements combined in series as discussed in Elkady and Lignos (2015a) and shown in Figure 4-44a. The first spring utilizes the envelope curve discussed above. The second one utilizes a gap element. 


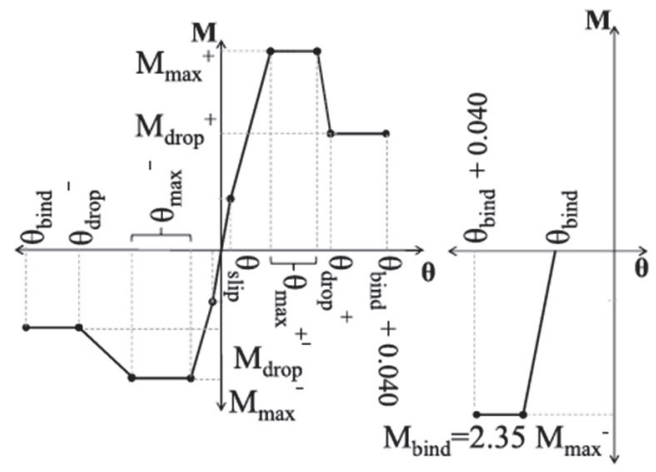

(a)

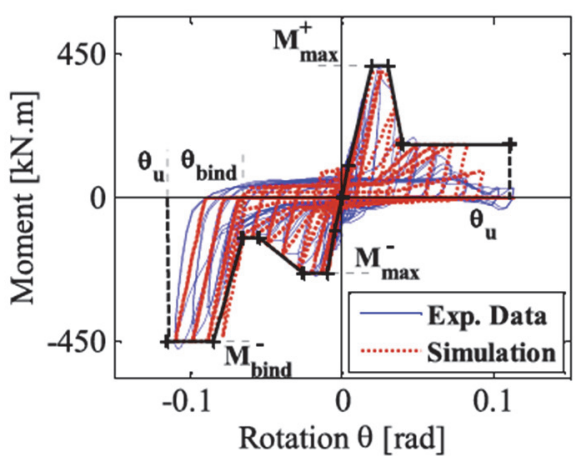

(b)

Figure 4-44 (a) Modified envelope curve for composite shear tab connections including flexural strength stiffening as a result of beam binding; (b) illustration with experimental data (image from Elkady and Lignos, 2015a, test data from Liu and Astaneh, 2004).

\subsubsection{Steel Column Splice Hinge Model}

This section summarizes the modeling recommendations for pre- and post-Northridge column splice connections. The recommendations only cover the first-cycle envelope curve as there is no monotonic data available for both types of connections.

\subsubsection{Pre-Northridge Column Splice Connections}

The modeling recommendations are based on work summarized in NIST (2017) and refer to typical configurations of column splices shown in Figure 4-45 where the compressive stresses are carried through bearing and the PJP welds and tensile forces due to combined flexural and axial force demands are carried through the PJP welds. Based on experimental data from Bruneau and Mahin (1987; 1991), a pre-Northridge column splice with PJP welds of low weld toughness is typically controlled by a stress-limit that is likely to be less than the yield stress of the connection due to lowtoughness materials and sharper discontinuities of the splice itself. The critical stress can be computed as follows:

$$
\sigma_{c r}=\frac{K_{I C}}{F\left(\frac{a_{o}}{t_{f, u}}\right) \sqrt{\pi a_{o}}} \leq F_{u, \mathrm{e}}\left(1-\frac{a_{o}}{t_{f, u}}\right) \leq F_{y, \mathrm{e}}
$$

where:

$$
F\left(\frac{a_{o}}{t_{f, u}}\right)=\left(2.3-1.6 \frac{a_{o}}{t_{f, u}}\right)\left(4.6 \frac{a_{o}}{t_{f, u}}\right)
$$

in which, $t_{f, l}$ and $t_{f, u}$ are the thickness of the lower and upper column flanges, $a_{o}$ is the internal flaw created by the unfused portion of the flange thickness, $F_{u, e}$ is the lesser 
of the weld or base metal ultimate strength; and $F_{y, e}$ is the expected yield strength of the upper column flange. Where $a_{0} / t_{f, u}=0.5$ and $K_{I C}=107 \mathrm{ksi} \sqrt{\text { in. }}$

The critical fracture stress should be compared to the maximum tensile stress in the column above the splice due to the combination of peak axial load, $P$, and peak biaxial bending moment demands. For end columns, $P$ should take into consideration the dynamic overturning effects. If nonlinear response-history analysis is employed, then the maximum tensile stress should be computed based on the instantaneous member forces at each time step. Because splices fail suddenly with rapid strength deterioration, it is recommended that once the computed stress demand due to combined actions exceed $\sigma_{c r}$ then the splice is treated as a force-controlled element.
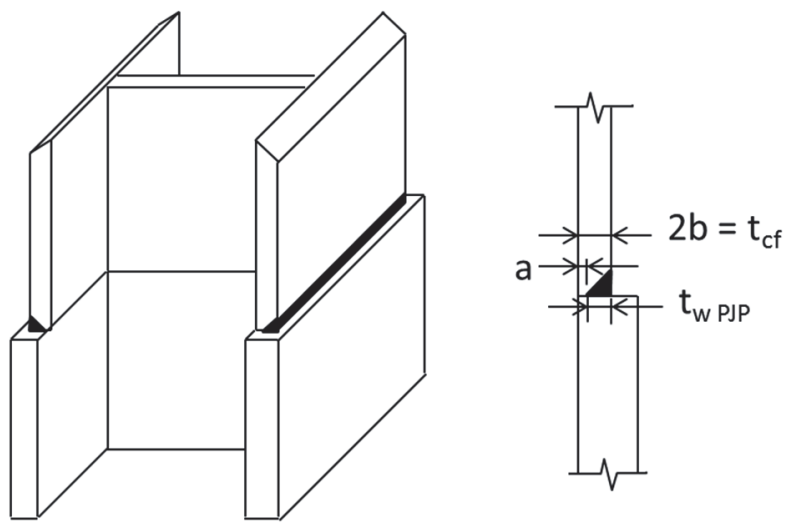

\section{Single-Edge Crack}

Figure 4-45 Typical column splice with partial penetration flange welds (NIST, 2017).

\subsubsection{Post-Northridge Column Splice Connections}

Column splices utilizing CJP welds in modern seismically-designed steel moment frame systems (e.g., IMFs and SMFs) that are constructed of weld metal with high fracture toughness should have sufficient toughness to develop their full expected yield strength as per ANSI/AISC 341-10. Because they are designed as forcecontrolled elements, inelastic deformations are not anticipated in the column splices. In this case, column splices may not be explicitly modeled. However, splices should always be checked if the coupled axial force and flexural demands exceed the expected splice strength.

Recent research by Shaw (2013) has demonstrated that under certain design and detailing conditions, PJP welds can be used to develop ductility commensurate with CJP welds; however, this is not generally the case. 


\subsubsection{Column Base Connection Hinge Model}

This section discusses how a first-cycle envelope curve can be constructed for column base connections as part of steel moment frame systems. The recommendations discussed herein that are based on work of Gomez (2010), Kanvinde et al. (2012), Grilli and Kanvinde (2015), Trautner et al. (2015). The proposed recommendations have been developed based on data from large- and fullscale experiments on exposed and embedded column base connections. Currently there are no available monotonic tests on column base connections; therefore, the input model parameters to compute a first-cycle envelope curve (according to Figure 4-35) are only provided. These parameters are statistically calibrated to median values. The variability in the same parameters is described in terms of a COV, if available.

\subsubsection{Exposed Column Bases}

The input model parameters for exposed column bases can be computed based on the process outlined below.

First Yield Moment, $\boldsymbol{M}_{\boldsymbol{y}}^{*}$. Use the rectangular stress-block (RSB) approach as suggested in Design Guide 1 (Fisher and Kloiber, 2006) and Kanvinde et al. (2012) to characterize the internal force distribution of the exposed column base. If the load eccentricity is low, the applied loading is resisted solely through the development of compressive bearing stresses under the base plate as shown in Figure 4-46a. For high eccentricities, the base plate will tend to uplift. In this case, bearing is resisted through a combination of bearing stresses on the compression side of the connection and tensile forces in the anchor rods (see Figure 4-46b). Assuming that the base plate width and length are $B$ and $N$, respectively, the critical load eccentricity $e_{c r i t}$ is computed as follows:

$$
e_{c r i t}=\frac{N}{2}-\frac{P}{2 B f_{\max }}
$$

where $P$ is the applied axial compressive load; $f_{\max }$ is the maximum bearing stress.
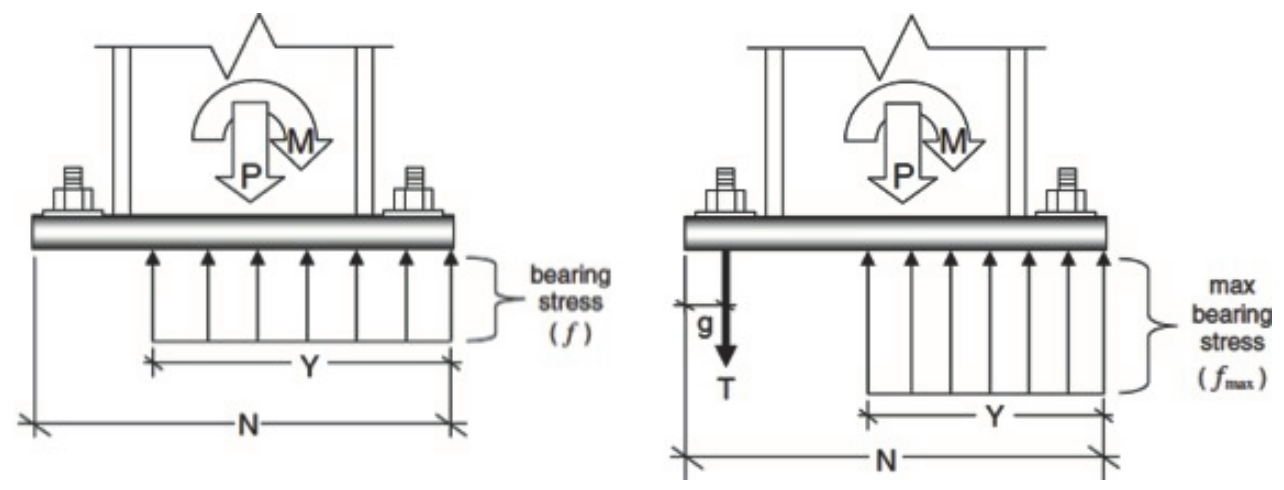

Figure 4-46 Stress distributions assumed in current U.S. design practice for (a) low; and (b) high-eccentricity conditions (Kanvinde et al., 2012). 
This stress can be calculated as follows:

$$
f_{\max }=0.85 f_{c}^{\prime}\left(\sqrt{\frac{A_{2}}{A_{1}}}\right) \leq 1.7 f_{c}^{\prime}
$$

where $f_{c}^{\prime}$ is the compressive strength of the concrete; $A_{1}$ is the plate bearing area; $A_{2}$ is the effective concrete area. For $e<e_{\text {crit }}$, the only possible failure mode is flexural yielding of the base plate on the compression side of the column base connection. For $e>e_{c r i t}$ three failure modes are possible: (a) flexural yielding of the base plate on the compression side (i.e., $M_{y}^{p l, c}$ ); (b) flexural yielding of the base plate on the tension side (i.e., $M_{y}^{p l, t}$ ); (3) tensile yielding of the anchor rods themselves (i.e., $\left.M_{y}^{r o d}\right)$. The first yield moment should be:

$$
M_{y}^{*}=\min \left(M_{y}^{p l, c}, M_{y}^{p l, t}, M_{y}^{r o d}\right)
$$

Rotation at First Yield of the Plate, $\boldsymbol{\theta}_{\boldsymbol{y}}$. In order to estimate the rotational stiffness $K_{e}$ of an exposed column base the deformations of the three main components of connection flexibility and its contributions to the overall rotation of the connection should be characterized. In particular, the deflection at the top of an anchor rod may be calculated as follows:

$$
\Delta_{r o d}=\frac{T_{r o d} L_{\text {total }}^{r o d}}{A_{\text {rod }} E_{r o d}}
$$

where:

$$
T_{\text {rod }}=\frac{T}{2}=\frac{1}{2} f_{\max } B\left((N-g)-\sqrt{(N-g)^{2}}-\frac{2\left[M+P\left(\frac{N}{2}-g\right)\right]}{f_{\max } B}\right)-P
$$

In Equation 4-57, $A_{\text {rod }}$ is the gross area of the anchor rod, which is typically threaded over a small length (i.e., 2 inches) in the vicinity of the nuts at the top and the bottom; $L_{\text {total }}^{\text {rod }}$ is the total length of the corresponding rod from the top of the base plate, to the nut-washer assembly at the bottom; $E_{\text {rod }}$ is the Young's modulus of the steel rod.

Figure 4-47 shows the column base connection. The deflection on the tension side of the column base connection, as shown in Figure 4-47, can be computed as follows:

$$
\Delta_{\text {plate }}^{\text {Tension }}=\left(T L_{\text {tension }}^{3} / 3 E_{\text {Plate }} I_{\text {Plate }}\right)+\left(T L_{\text {tension }} / A_{\text {plate }}^{S} G_{\text {plate }}\right)
$$

where $T$ is the force in both the anchor rods; $L_{\text {tension }}$ is the distance between the edge of the column flange and the centerline of the anchor rod as shown in Figure 4-47; $I_{\text {plate }}=B t_{p}^{3} / 12 ; A_{\text {plate }}^{s}=(5 / 6) B t_{p} ; E_{\text {plate }}$ and $G_{\text {plate }}$ are the Young's and shear modulus of the steel plate material, respectively. 


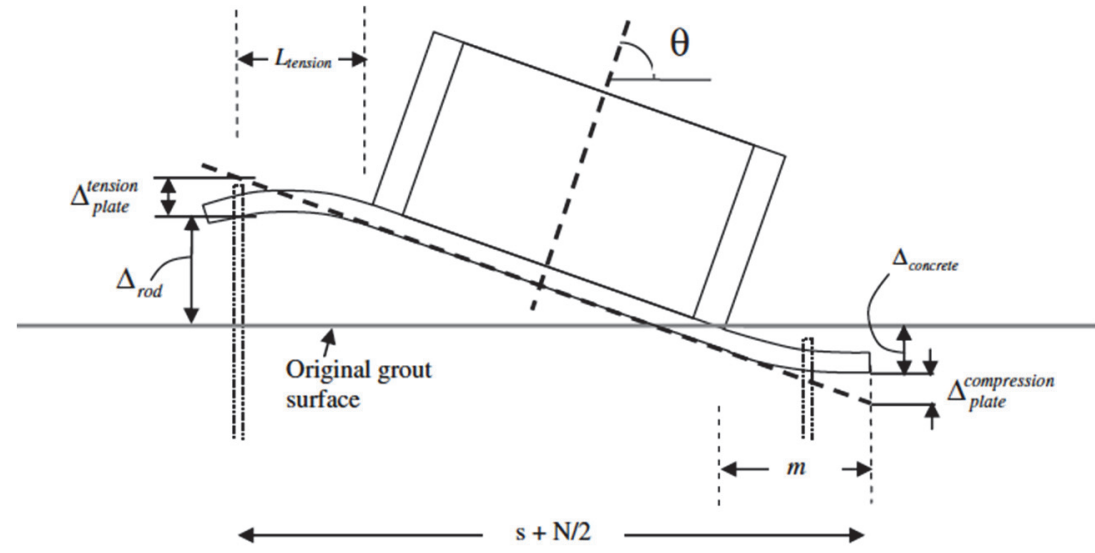

Figure 4-47 Assumed deformation mode and contribution of various components (Kanvinde et al., 2012).

Similarly, the deflection on the compression side (see Figure 4-47) may be calculated as follows:

$$
\Delta_{\text {plate }}^{\text {compssion }}=\left\{\begin{array}{l}
f_{\max } B\left(\frac{m^{4}}{8 E_{\text {plate }} I_{\text {plate }}}+\frac{m^{2}}{2 A_{\text {plate }}^{S} G_{\text {plate }}}\right), Y \geq m \\
\frac{f_{\text {max }} B}{8 E_{\text {plate }} I_{\text {plate }}}\left(m^{4}-\frac{1}{3}(m-Y)^{3}(3 m+Y)\right) \\
+\frac{f_{\max } B Y}{A_{\text {plate }}^{S} G_{\text {plate }}}\left(m-Y+Y^{2} / 2\right), Y<m
\end{array}\right.
$$

where $m$ is the flap length as indicated in Figure 4-47; $Y$ is the length of a stress block length shown in Figure 4-46. This length can be computed as follows:

$$
Y= \begin{cases}N-\frac{2 M}{P}, & e \leq e_{c r i t} \\ (N-g)-\sqrt{(N-g)^{2}-\frac{2\left[M+P\left(\frac{N}{2}-g\right)\right]}{f_{\max } B},} & e>e_{\text {crit }}\end{cases}
$$

where $g$ is the base plate edge distance, $N$ is the length of the base plate, $M$ is the moment demand on the base plate.

The concrete deformations can be computed as follows:

$$
\Delta_{\text {concrete }}=\frac{f_{\max }}{E_{\text {concrete }}} d_{\text {footing }}
$$

where $E_{\text {concrete }}=4,700 \sqrt{f_{c}^{\prime}} ; d_{\text {footing }}$ is the depth of concrete footing. Therefore, the exposed column base connection rotation can be calculated as follows:

$$
\theta_{y}=\left(\Delta_{\text {rod }}+\Delta_{\text {plate }}^{\text {tension }}+\Delta_{\text {plate }}^{\text {compression }}+\Delta_{\text {concrete }}\right) /(s+N / 2)
$$


where $s$ is the base plate base plate edge distance; $N$ is the length of base plate. Therefore, the elastic flexural stiffness, $K_{e}$, of an exposed column base connection can be calculated as follows:

$$
K_{e}=M_{y}^{*}(s+N / 2) /\left(\Delta_{\text {rod }}+\Delta_{\text {plate }}^{\text {tension }}+\Delta_{\text {plate }}^{\text {compression }}+\Delta_{\text {concrete }}\right)
$$

Peak (Capping) Strength, $\boldsymbol{Q}^{\prime}{ }_{\max }$. This quantity refers to the peak flexural strength of an exposed column base connection, under cyclic loading. This parameter depends on the first-yield mode of the base plate. In particular, if the first yield, $M_{y}^{*}$ is a result of anchor rod yielding or tension side of the base plate there will be no increase in the flexural strength capacity of the connection. Therefore, $M_{m}^{*}=M_{y}^{*}$. If the first yield, $M_{y}^{*}$ is a result of base plate yielding in compression there will be an increase in the flexural strength capacity of the connection, in particular:

$$
M_{\max }^{*}=\min \left(M_{y}^{\text {rod }}, M_{y}^{\text {plate,ten }}\right)
$$

Note that the COV of this method to estimate the peak flexural strength of the exposed column base connection is 0.03 (Gomez, 2010).

Pre-Peak Plastic Deformation, $\boldsymbol{\Delta}^{\prime}{ }_{\text {cap,pl }}$. This value is based on test data conducted by Gomez (2010). In particular, $\theta_{p}^{*}=0.065$ rads, $\mathrm{COV}=0.42$.

Figure 4-48 illustrates a comparison of the end moment-chord rotation relation of an exposed column base and the proposed first-cycle envelope curve. From this figure, the estimated flexibility of the exposed column base is captured fairly well compared to the experimental data. In addition, the effective yield strength of the column base is reasonably close to the experimental data.

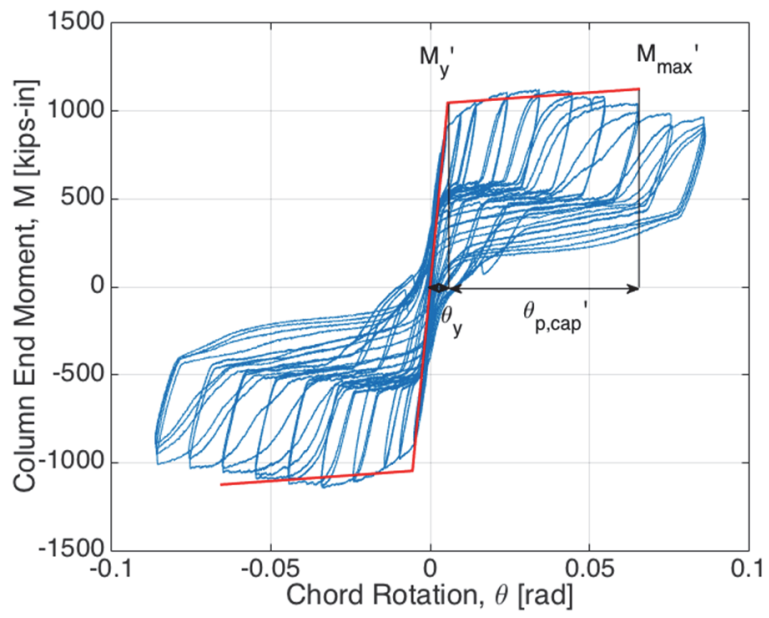

Figure 4-48 Idealized moment-rotation relation for hinge model for exposed column base connections (Gomez, 2010).

More recently, Rodas et al. (2016) proposed a hysteretic model formulation for simulating the cyclic behavior of exposed column base connections. This 
formulation is consistent with the approach outlined above to construct a first-cycle envelope for an exposed column base connection.

\subsubsection{Embedded Column Bases}

The input model parameters for embedded column bases similar to the ones discussed in Section 4.3.6.2 were estimated based on experimental data that were conducted by Grilli and Kanvinde (2015). These parameters can be estimated as follows:

Effective Yield Moment, $\boldsymbol{M}_{\boldsymbol{y}}^{*}$. The effective flexural yield strength of embedded column bases was found to be:

$$
M_{y}^{*}=0.7 M_{\max }^{*} \quad(\mathrm{COV}=0.10)
$$

in which, $M_{\max }^{*}$ is the maximum column base flexural strength. This value depends on the failure mode of the column base and can be estimated based on the procedure summarized in Grilli and Kanvinde (2015). The parameters that need to be defined for this purpose are the column embedment depth, $d_{\text {embed }}$, the column section, axial force $P$, base plate dimensions $t_{\text {plate }}, B, N$, and the shear-to-moment ratio, such that for a given base moment, $M_{\text {base }}$, the corresponding column shear, $V_{\text {column }}$, may be determined as a fraction of it. The maximum column base flexural strength, $M_{\max }^{*}$, is equal to the moment due to horizontal bearing stresses, $M_{H B}$, and a moment due to vertical bearing stresses, $M_{V B}$. In order to compute those the following steps should be employed:

$$
M_{H B}=\min \left\{M_{H B}^{\text {bearing }}, M_{H B}^{\text {shear }}\right\}
$$

in which, $M_{H B}^{\text {bearing }}$ is the moment corresponding to bearing failure (see Figures 4-31, 4-32, 4-50a) and $M_{H B}^{\text {shear }}$ is the moment corresponding to shear failure of the joint panel (see Figures 4-32 and 4-50b). The moment corresponding to bearing failure can be calculated as follows:

$$
M_{H B}^{\text {bearing }}=\beta \beta_{1} f_{c}^{\prime} b_{j}\left[d_{L} d_{\text {effective }}-\frac{\left(d_{L}^{2}+d_{U}^{2}\right)}{2}\right]
$$

in the above equation the bearing stress is represented by the term $\beta \beta_{1} f_{c}^{\prime}$ in which, $\beta=2.0$ simulates the effect of confinement, and the factor $\beta_{1}=0.85$ is used to establish equivalence between a rectangular stress block, and the idealized parabolic stress distribution which has a peak stress $2 f_{c}^{\prime}$ (Mattock and Gaafar, 1982; Deierlein et al., 1989; ASCE, 1994). In Equation, 4-66, $b_{j}$ represents the width of the joint, $b_{j}=\left(b_{f}+B\right) / 2 ; b_{f}$ is the effective joint width and $B$ is the base plate width; $d_{\text {efective }}$ is related to the embedment and should be calculated as follows: 


$$
d_{\text {effective }}=d_{\text {ref }} \leq d_{\text {embed }}, d_{\text {ref }}=\frac{C}{\rho}=C\left(\frac{E_{\text {concrete }}}{4 \cdot E_{\text {steel }} I_{\text {col }}}\right)^{-1 / 4}
$$

where $C$ is a composite factor and can be assumed as $C=1.77$ based on calibrations with the available experimental data; $E_{\text {concrete }}$ and $E_{\text {steel }}$ are the moduli of elasticity of concrete and steel, respectively; and $I_{c o l}$ is the moment of inertia of the steel column. In Equation 4-68, $d_{L}$ and $d_{U}$ represent the bearing dimensions shown in Figure 4-49c. The moment corresponding to bearing failure occurs if the bearing dimensions attain a critical value that can be assumed to be $60 \%$ of the distance between the free surface for $d_{U}$ and the distance $d_{\text {effective }}$ to $d_{L}$ and the line that bisects the stress-free region between the two bearing blocks as shown in Figure 4-49c.

The moment corresponding to shear failure of the joint panel can be computed as the sum of the moment strengths associated with various components in the steel web, the concrete strut and the concrete outer joint associated with the panel zone, as follows:

$$
M_{H B}^{\text {shear }}=\left(V_{\text {steel }} d_{w}\right)+\left(V_{\text {strut }, \text { inner }} h\right)+\left(1.25 V_{\text {strut,outer }} h\right)
$$

Where:

$$
V_{\text {steel }}=0.6 F_{y e}^{\text {column }} t_{w}\left(d_{\text {effective }}-\frac{d_{U}+d_{L}}{2}\right)
$$

where $F_{y e}^{\text {column }}$ is the expected yield stress of the steel material corresponding to the column; $t_{w}$ is the web thickness of the column.

$$
V_{\text {strut,inner }}=1.7 \sqrt{f_{c}^{\prime}} b_{f}\left(d_{\text {effective }}-\frac{d_{U}+d_{L}}{2}\right) \leq 0.5 f_{c}^{\prime} b_{f} h
$$

And:

$$
V_{\text {strut,outer }}=1.7 \sqrt{f_{c}^{\prime}} b_{0}\left(d_{\text {effective }}-\frac{d_{U}+d_{L}}{2}\right)
$$

where $f_{c}^{\prime}$ is the concrete compressive strength; $b_{0}$ is the outer joint panel zone (see Figure 4-49a). 


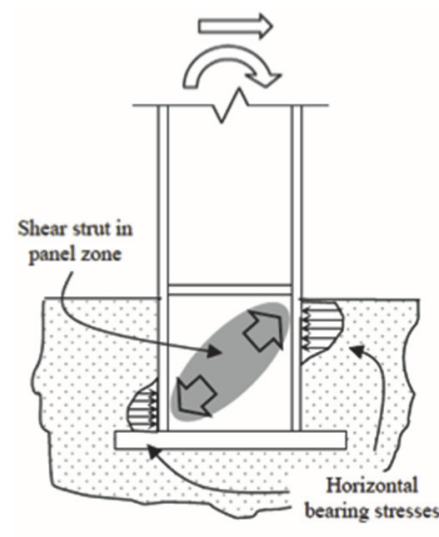

(a)

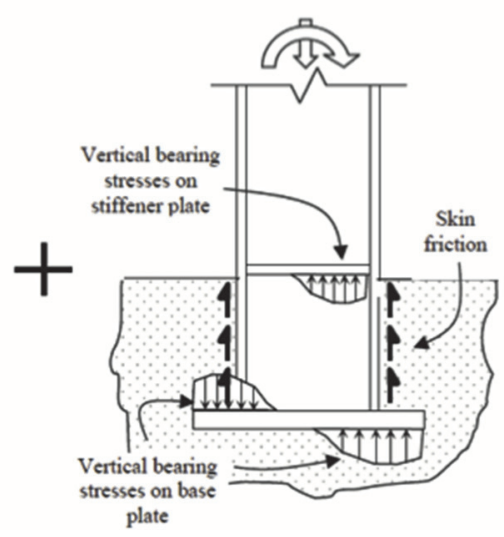

(b)

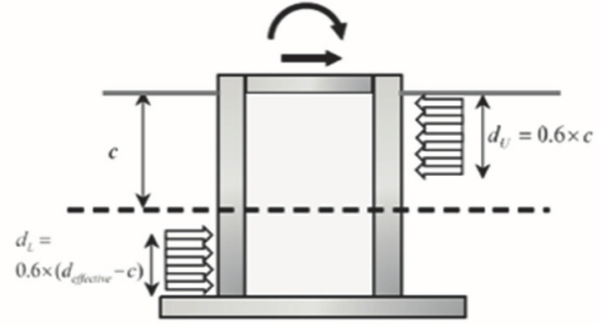

(c)

Figure 4-49 Load-resisting mechanisms for embedded column bases (a) horizontal bearing and panel zone accompanied by (b) vertical bearing and skin friction]; (c) critical condition for horizontal bearing failure; in the case shown deffective = dembed (Grilli and Kanvinde, 2015).

The moment due to bearing stresses can be controlled by one of four failure modes associated with bearing failure of the concrete above and/or below the base plate, $M_{V B}^{b}$; concrete breakout under the compression toe of the base plate, $M_{V B}^{c}$; concrete breakout above the tension side flap of the base plate, $M_{V B}^{t}$; yielding of the base plate on the tension or compression side, $M_{V B}^{c}$; it can be expressed as:

$$
M_{V B}=\min =\left\{M_{V B}^{b}, M_{V B}^{c}, M_{V B}^{t}, M_{V B}^{y}\right\}
$$

Bearing failure of concrete will occur when the stresses in the stress block reach the bearing strength of concrete considering the confinement. In this case:

$$
M_{V B}^{b}=\left(f_{V B}^{b}-\frac{P}{N B}\right) 0.21 B N^{2}=\left(1.7 f_{c}^{\prime}-\frac{P}{N B}\right) 0.21 B N^{2}
$$

The moment due to concrete breakout under the compression toe of the base plate or above the tension side flap of the base plate can be computed as follows:

$$
M_{V B}^{t, c}=\left(\eta \frac{40}{9} \times \frac{1}{\sqrt{d_{\text {cover }}}} \sqrt{f_{c}^{\prime}} A_{35}+0.3 P\right) 0.7 N
$$

where length of the flap of the base plate, $\eta$, is 1.5 based on calibration from the experimental data; $d_{\text {cover }}$ is the thickness of the material that must be ruptured for breakout and can be assumed to be $d_{\text {cover }}=d_{\text {embed }} ; A_{35}$ is the projected area of a failure cone emanating from the edges of the stress blocks being considered such that the angle between the cone surface and the horizontal is 35 degrees. 
The moment due to base plate yielding on the tension or compression side can be computed as follows:

$$
M_{V B}^{y}=\left(f_{V B}^{y}-\frac{P}{B N}\right) 0.21 B N^{2}=\left(\frac{t_{\text {plate }}^{2} F_{y}^{\text {plate }}}{2 \eta^{2}}-\frac{P}{B N}\right) 0.21 B N^{2}
$$

in the above equation, $\eta$ is the length of the flap of the base plate; $t_{p l a t e}$ is the thickness of the steel plate; $F_{y}^{\text {plate }}$ is the minimum specified yield stress of the base plate.

Compute $a=1-d_{\text {embed }} / d_{r e f} \geq 0 ; d_{r e f}$ is computed from Equation 4-69; if $M_{V B} / a \leq$ $M_{H B} /(1-a)$ then failure due to vertical bearing occurs before failure due to horizontal bearing. In this case:

$$
M_{\text {peak }}^{\prime}=\left\{\begin{array}{c}
M_{V B}^{t} / a, \text { if concrete breakout on the tension side } \\
M_{H B}, \text { if concrete breakout on the compression side } \\
M_{H B}+M_{V B}^{b} \text { or } M_{H B}+M_{V B}^{t}, \text { if concrete crushing or bas plate yielding }
\end{array}\right.
$$

if $M_{V B} / a>M_{H B} /(1-a)$ then failure due to horizontal bearing occurs before failure due to vertical bearing. In this case:

$$
M_{\text {peak }}^{\prime}=\max \left\{\kappa M_{H B}, M_{V B}, \frac{M_{H B}}{1-a}\right\}
$$

where $\kappa$ is a degradation factor for horizontal bearing and can be computed as follows:

$$
\kappa=\left(\frac{M_{V B}(1-a)}{M_{H B} a}\right)^{-2} \leq 1
$$

Pre-Peak Plastic Deformation, $\boldsymbol{\Delta}_{\text {cap,pl. }}{ }$ This value is based on calibrations from test data by Grilli and Kanvinde (2015). In particular, $\theta_{p}^{*}=0.015$ rads $(\mathrm{COV}=0.48)$.

Post-Peak Plastic Deformation, $\boldsymbol{\Delta}_{\boldsymbol{p} \boldsymbol{} \boldsymbol{} \text {. }}$. This quantity refers to the post-peak plastic rotation, $\theta_{p c}^{*}$ for embedded column bases under cyclic loading. According to Grilli and Kanvinde (2015), embedded column bases can fail in a ductile or brittle manner. The brittle failure modes were identified as tension breakout of the top of the concrete or joint shear failure of the steel column. In this case, $\theta_{p c}^{*}=0.03$ rads; the ductile failure modes observed in embedded column bases were identified as compression break-out of the bottom of the concrete, concrete crushing/plate yielding, or horizontal bearing failure of the concrete; for these modes, $\theta_{p c}^{*}=0.09$ rads.

Residual Strength, $\boldsymbol{Q}_{\boldsymbol{R}}^{\prime}$. The residual flexural strength, $M_{r}^{*}$, for embedded column base connections cannot be identified based on experimental evidence. At this point, it is recommended that a zero residual strength be considered. 
Ultimate Deformation, $\boldsymbol{\Delta}^{\prime}{ }_{u l t}$. For embedded column bases that fail in a brittle manner, this value should be assumed equal to 0.03 radians. Embedded column bases that fail in a ductile manner, $\theta_{p c}^{*}=0.06$ radians may be used.

Figure 4-50 shows illustrative examples on embedded column base moment versus rotation relations from test data by Grilli and Kanvinde (2015). Superimposed in the same figure are the first-cycle envelope curves based on the approach outlined above. Based on the comparisons, the following observations can be made:

- The column base flexibility as well as peak strength can be estimated reasonably well based on the approach discussed in Grilli and Kanvinde (2015).

- The effective yield strength of an embedded column base is reasonably close to $70 \% M_{\max }^{*}$ provided that the dominant failure mode can be clearly identified.
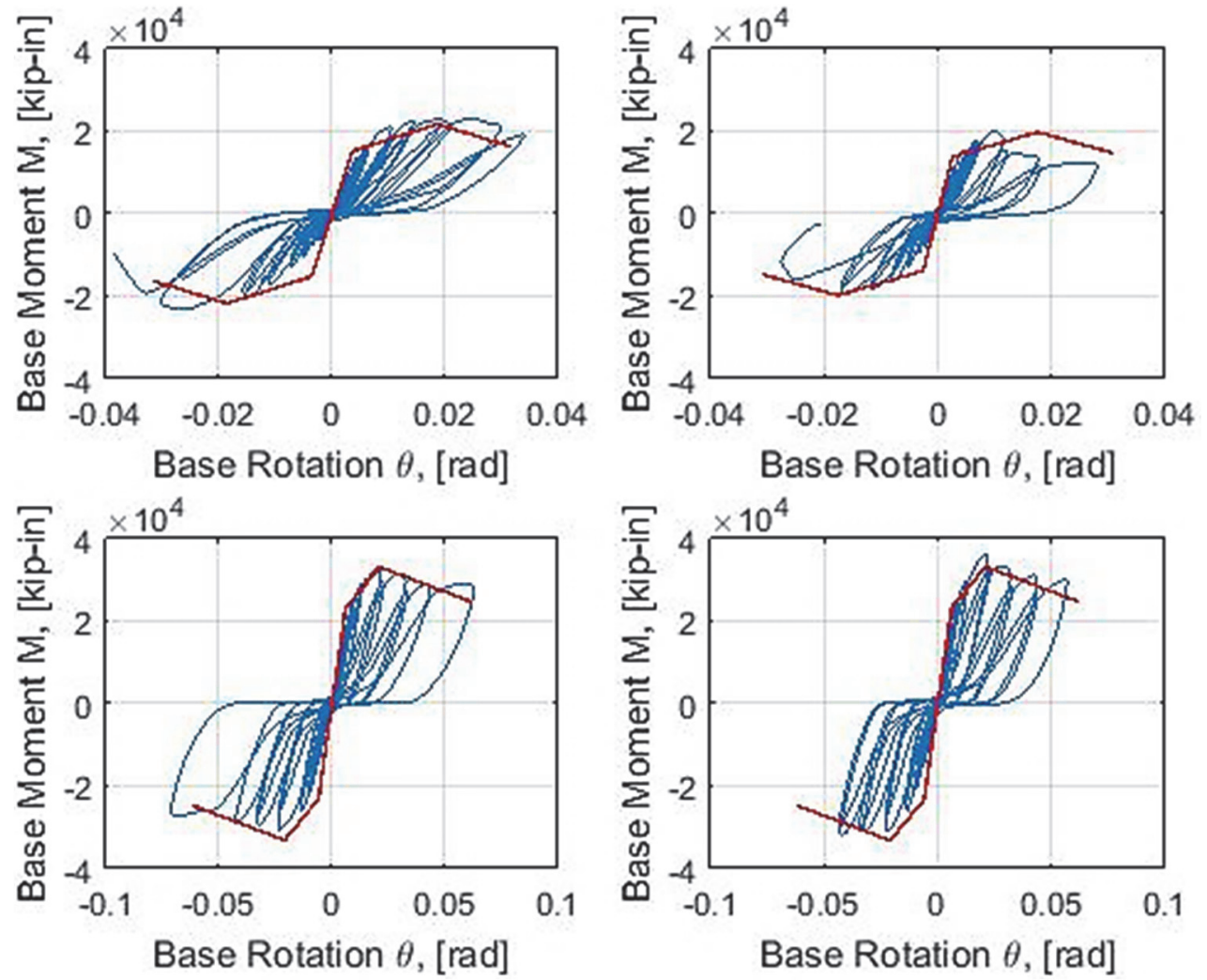

Figure 4-50 Comparison of experimental data and proposed first-cycle envelope curves for embedded column base connections (Grilli and Kanvinde, 2015).

\subsection{Summary Findings of Application Case Study}

This chapter proposes updated nonlinear modeling recommendations for steel components as part of new and existing steel moment frame systems. These recommendations have been thoroughly compared with the current ASCE/SEI 41-13 
nonlinear modeling provisions (see Appendix A). The main findings from these application case studies are summarized as follows:

- The post-yield hardening behavior of steel components is better described by the $M_{\text {max }}^{*} / M_{y}^{*}$ ratio rather than a constant $3 \%$ strain hardening ratio that has been historically used in ASCE/SEI 41-13. For steel beam-columns, this ratio is a function of the member and cross-section slenderness as well as the gravity induced compressive axial load ratio, $P_{g} / P_{y e}$.

- The pre- and post-peak plastic deformations $\theta_{p}^{*}$ (i.e., " $a$ " value in ASCE/SEI 41-13) and $\theta_{p c}^{*}$, respectively, of steel beams and columns are strongly dependent on the cross-section local slenderness ratios. This is not currently considered in the ASCE/SEI 41-13 modeling provisions. In the case of steel columns, member slenderness, $L_{b} / r_{y}$, and applied compressive axial load ratio, $P_{g} / P_{y e}$, strongly influence the aforementioned plastic deformation parameters.

- In steel beams as part of pre-Northridge beam-to-column connections, the corresponding $\theta_{p}^{*}$ value is primarily influenced by the beam depth, $d$. The ASCE/SEI 41-13 component model seems to underestimate the pre-peak plastic rotation $\theta_{p}^{*}$ (i.e., parameter " $a$ ") of steel beams with $d>30$ in. by $50 \%$, on average.

- The flexural strength of steel components that do not experience unanticipated brittle failure modes deteriorates gradually while the lateral drift demands increase. This is reflected well in the post-peak plastic deformation, $\theta_{p c}^{*}$, as computed from the proposed modeling recommendations. The large discontinuity in flexural strength of steel components shown in the ASCE/SEI 41-13 component model is not reflected in the available experimental data.

- Steel columns that utilize cross sections within the limits of highly ductile members as per ANSI/AISC 341-10 and subjected to $P_{g} / P_{y e}=0.50$ (i.e., $\left.P / P_{c r}>0.50\right)$ have an appreciable plastic deformation capacity and should not be treated as force-controlled elements as per ASCE/SEI 41-13. Instead, steel wide flange beam-columns under compressive axial load ratios $P_{g} / P_{y e}>0.60$ that have $h / t_{w}>43$ and $K L / r_{y}>120$ should be treated as force-controlled elements as per ASCE/SEI 41-13.

- Steel beam-columns utilizing stocky cross-sections (i.e., $h / t_{w}<15$ ) that experience gravity loads $P_{g}>0.60 P_{y e}$ have a finite plastic deformation capacity, which is not zero. However, beam-columns with gravity loads over $0.6 P_{y e}$ may be under designed for gravity loads. Therefore, column yielding should be restricted.

- Results from system level studies in code-conforming steel moment frame systems designed in highly seismic regions suggest that the consideration of composite beam effects in the nonlinear building model can shift the global 
collapse mechanism of the respective archetype at lateral drift demands associated with low-probability of occurrence earthquakes. For moderate seismic intensities associated with a design basis earthquake the use of centerline nonlinear building models seems reasonable. 



\section{Chapter 5}

\section{Steel Concentrically Braced Frames}

\subsection{Overview of Geometry and Behavior}

Steel concentrically braced frames (CBFs) are composed of braces, gusset plates, beams, and columns. The centerlines of the braces, beams, and columns join at a single, concentric, or nearly concentric point. This chapter addresses conventional CBFs in which braces placed into compression are permitted to buckle. Some modern CBFs, known as buckling-restrained braced (BRB) frames, employ proprietary braces in which tensile yielding, rather than compressive buckling, is the limiting, primary yield mechanism. These proprietary systems are not addressed here. Also, it is important to note, as explained later, that in addition to the braces, seismic response of CBFs also includes significant participation of the beams and columns through moment-resisting framing action, whether or not the frame has been designed to be moment-resistant. This chapter focuses on the behavior of the braces, and to a lesser extent, the gussets that attach braces to the frame. Moment-resistant frame behaviors are covered in other chapters of this report.

Steel concentrically braced frames have been used for many years in buildings of structural steel and wood construction, and have also been used as retrofit elements in buildings of concrete, masonry, steel, and wood. Key CBF components include: diagonal braces, gusset plates, as well as columns and beams (referred to herein as framing elements). A wide variety of cross sectional shapes have historically been used for braces including W, WT, single and double angle, HSS and pipe sections. Welded or bolted connections are used to connect the brace to the gusset plate and the gusset plate to the beams and columns. Figure 5-1 shows a typical braced frame with these key components highlighted.

Many bracing configurations exist, particularly in older buildings. Figure 5-2 illustrates typical configurations including single diagonal braced bay, opposing, paired braces in single story $\mathrm{X}$, multi-story $\mathrm{X}$, and chevron (or V or inverted $\mathrm{V}$ ). 


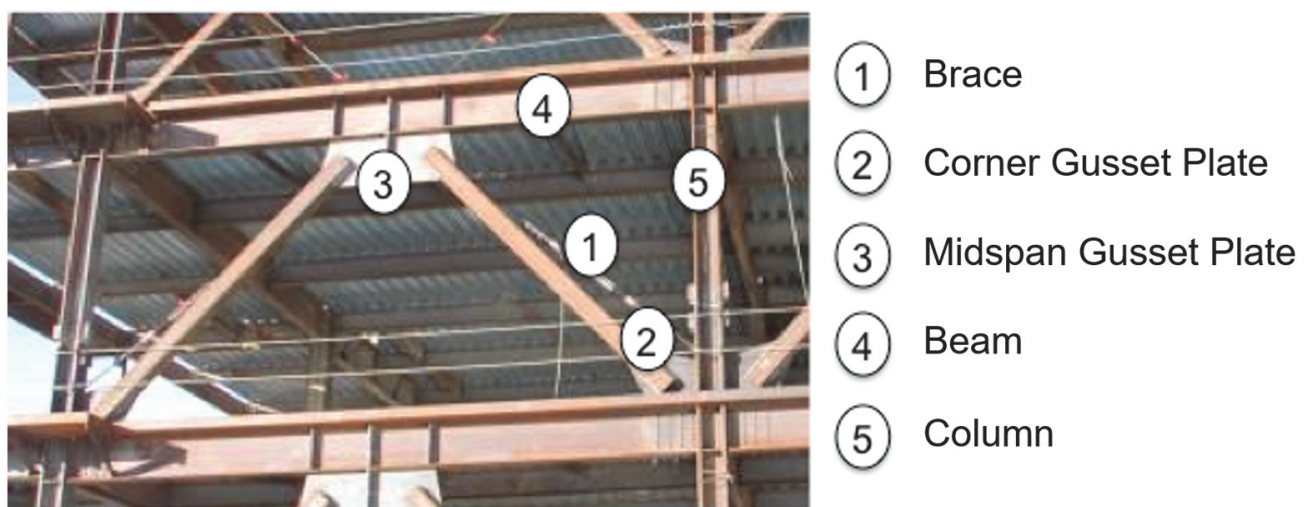

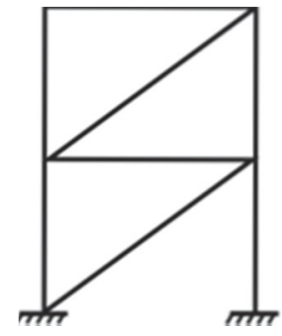

(a) Diagonal bracing

Figure 5-1 Typical CBF components.

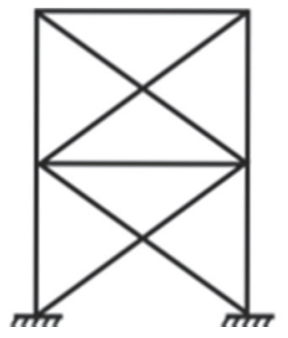

(b) X-bracing

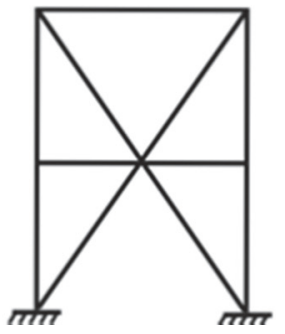

(c) Multistory

X-bracing

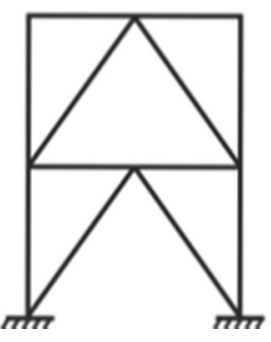

(d) Inverted C-bracing (Chevron)

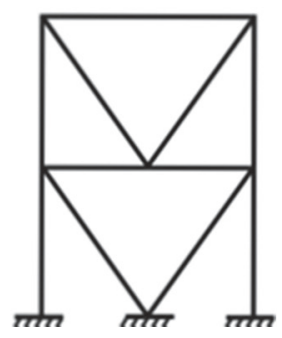

(e) V-bracing

Figure 5-2

Typical CBF configurations (NIST, 2013a).

\subsection{Summary of Design Code Provisions}

Prior to publication of the 1988 Uniform Building Code (UBC) (ICBO, 1988), building codes specified that braces and other framing elements be designed for a specified lateral force level, without consideration of failure hierarchy or mode.

Nonlinear behavior of many of these frames will be controlled by failure of the brace to gusset plate, or gusset plate to frame connection. The 1988 UBC introduced significant changes in seismic design, including capacity design principles intended to provide sufficient strength in other CBF components such that braces could yield in tension and buckle in compression without failure of other framing elements. These capacity design requirements continued to evolve over the period between 1988 to 1994, when the concept of special CBFs were first introduced as different from other CBFs, classified as "ordinary." Today the building code recognizes three types of CBF: Special, Ordinary, and Systems not Specifically Detailed for Seismic Resistance. This latter system, permitted only in Seismic Design Category B and C structures is designed like pre-1988 frames, without consideration of capacity design principles. Failure can occur through any of several modes. Ordinary CBFs are designed considering some capacity design principles, but do not control the design of gusset plates so as to permit ductile hinging at the ends of buckling braces. SCBFs are designed with full consideration of capacity-design principles. 
Important components of these capacity design principles include:

- Limiting bracing configurations to complete concentric systems

- Where Chevron pattern braces are used, ensuring that beams at the apex of the braces are capable of resisting the unbalance loading that occurs after compressive brace buckling

- Ensuring that columns in CBFs have adequate axial capacity to resist the imposed forces from the braces

- Ensuring that the portion of lateral resistance provided by braces in compression is similar to that provided by braces in tension

- Limiting member and cross-sectional slenderness ratios for braces and framing members to ensure ductile behavior

- Requiring connections to develop the expected brace capacity and to accommodate end rotation of the brace

- Requiring demand critical welds for yielding elements

One of the more important requirements for post-elastic $\mathrm{CBF}$ behavior is ensuring that end connections of braces to frames can develop the strength of the brace in compression and tension. This condition is commonly not satisfied in braced frames that do not conform to modern criteria for SCBF and OCBF systems. The hysteretic recommendations presented herein assume, as a minimum, that end connections of braces are capable of developing the brace strength, and also accommodating rotations associated with brace compressive buckling.

Figure 5-3 shows some gusset plate connection configurations that have historically been used. Each of the connections shown in Figure 5-3 provides different degrees of fixity at the beam-column interface and different restraint against out-of-plane brace end rotation. While some of these configurations can develop the brace strength and accommodate rotations associated with brace buckling, this is not guaranteed without detailed evaluation of the plate configuration, thickness and sizing of bolts and welds. 


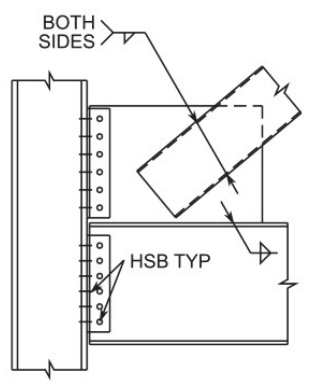

(a) Split double angle [S2L]

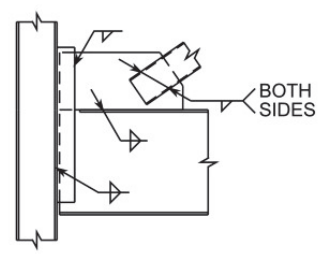

(b) Welded continuous shear plate [CSP-W]

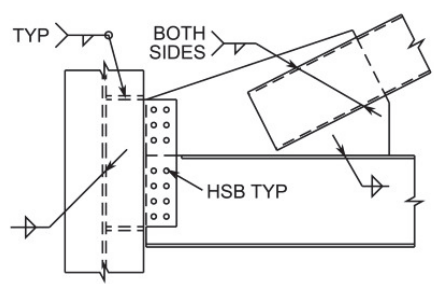

(c) Bolted continuous shear plate [CSP-B]

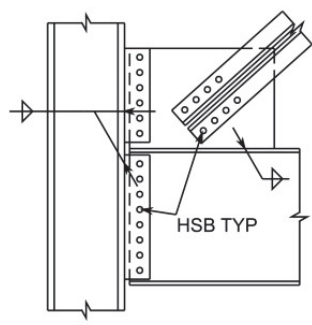

(d) Bolted split shear plate [SSP-B]

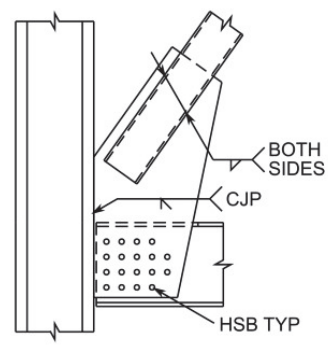

(e) Integrated gusset-shear plate [INT]

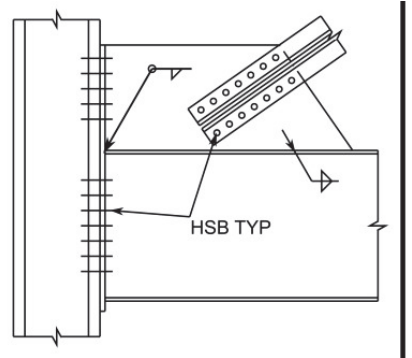

(f) Bolted end plate [END]

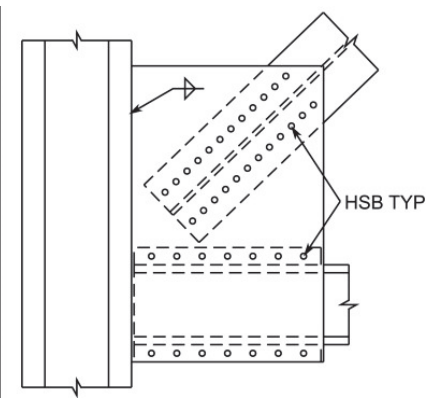

(g) Double integrated gusset-shear plate

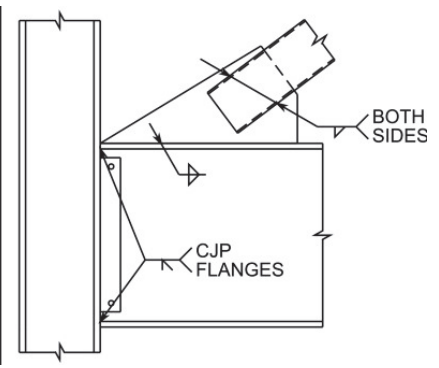

(h) Gusset-to-beam only

Figure 5-3 Representative CBF connections in older construction (from Sen et al., 2016).

\subsection{Performance and Damage Progression}

Braces without sufficiently strong connections to the frame will typically be capable only of elastic behavior, limited by loading that causes connection failure. Once the brace connection fails, inelastic behavior is limited to the residual moment-resisting capacity of the frame.

In CBFs with sufficiently strong brace connections, braces loaded inelastically in compression will buckle and lose strength rapidly. When tension and compression braces are paired as illustrated in Figure 5-4, and the connections and balance of the frame are designed incorporating the requirements for SCBFs that are included in modern building codes, more ductile response is possible.

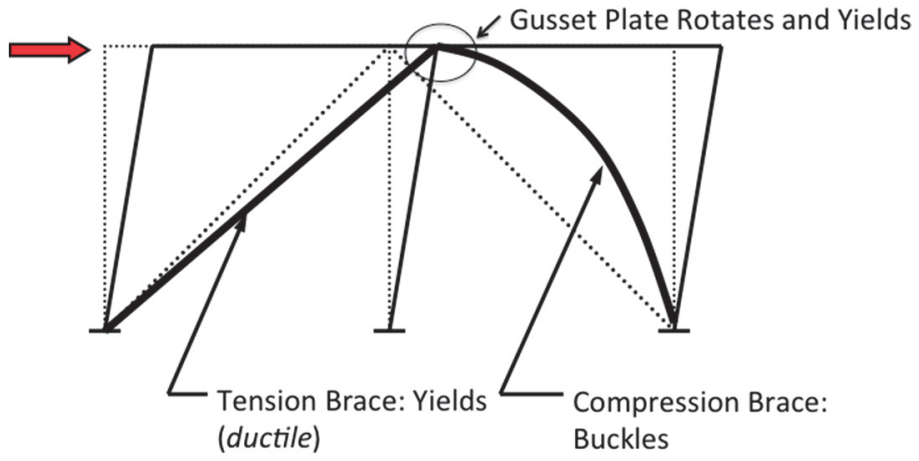

Columns and beams: "essentially" elastic

Figure 5-4 Idealized response of CBFs. 
As shown in Figure 5-4, brace buckling and yielding are the primary inelastic mechanisms. The columns and beams are intended to remain essentially elastic. After brace buckling/yielding, substantial stiffening of the beam-column connections by the gusset plates and elastic portions of the bracing system, provides the framing elements with non-negligible moment-resisting frame behavior which can add substantial post-elastic strain hardening and deformation capacity to the CBF system.

Figure 5-5a and Figure 5-5b show the typical hysteretic behavior of a single brace with strong connections. Under compressive loading, the brace buckles and a "plastic hinge" forms at the center of the brace. If the gussets provide substantive strength and stiffness, hinges will also form at the brace ends, or within the gussets. As the buckled brace is subjected to tension forces, the brace straightens and yields. As Figure 5-5a indicates, for most braces the tensile and compressive capacities are different. Compressive buckling is accompanied by rapid strength loss and pinching. Tensile behavior is more similar to elastic-plastic behavior. Placing the braces in pairs, with one brace acting in tension and the other in compression, results in symmetric behavior with substantially improved hysteretic characteristics, including limited strength degradation, and moderate pinching as shown in Figure 5-5c. The moment-resisting frame action of beams and columns will add further strength and post-yield hardening potential to the hysteresis shown in Figure 5-5c.

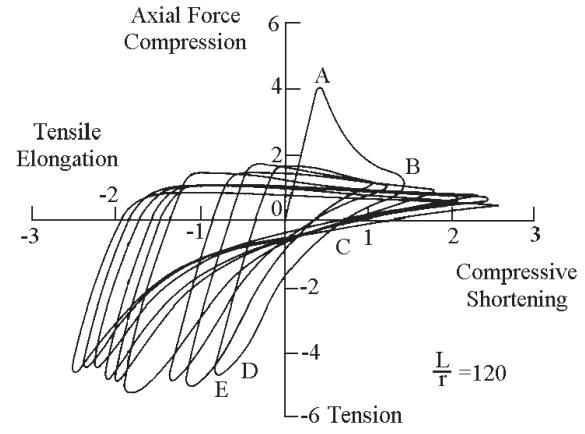

(a) Brace axial force-deflection hysteresis

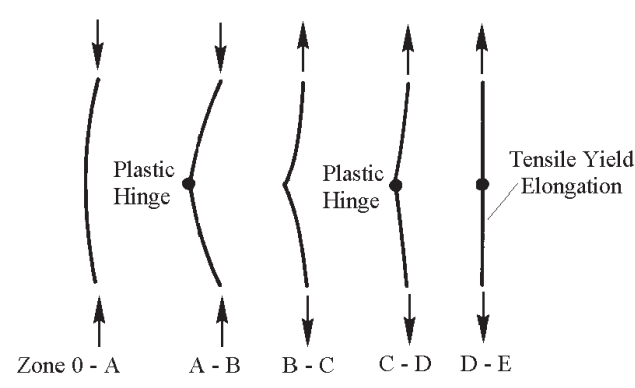

(b) Zones of behavior

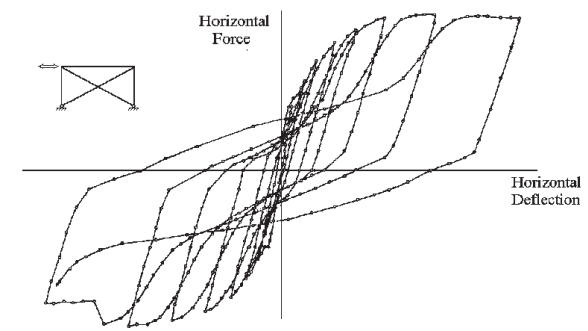

(c) Resulting frame lateral load-deflection hysteresis

Figure 5-5 Cyclic response of single and paired braces (Roeder and Popov, 1977).

Figure 5-6 illustrates typical cyclic failure of braces in SCBFs designed to conform to recent code requirements. Out-of-plane movement of the brace results in hinge 
formation. Local deformation at the hinge produces high strains and ultimately, low cycle fatigue at the brace plastic hinge. Tearing typically initiates at corners (for an HSS section) or edges (for a rolled section), and eventually leads to brace fracture (Figure 5-6c).

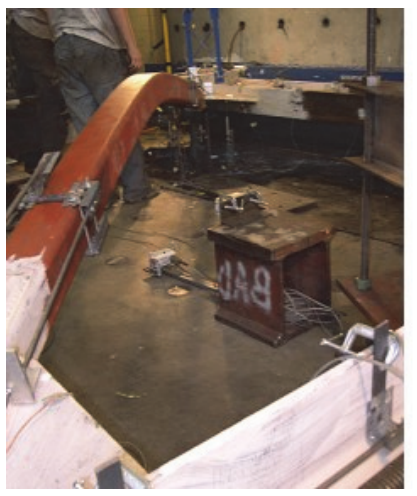

(a) OOP movement

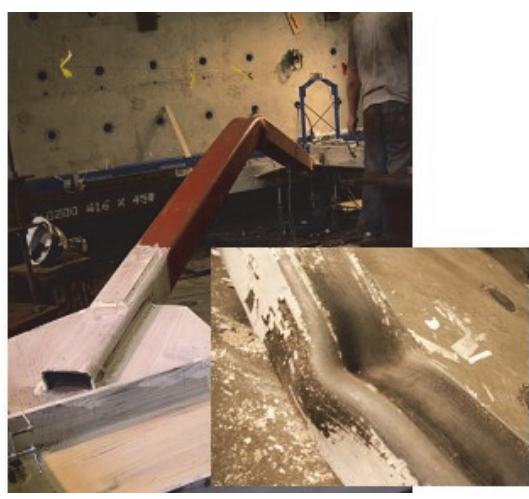

(b) Cupping at apex

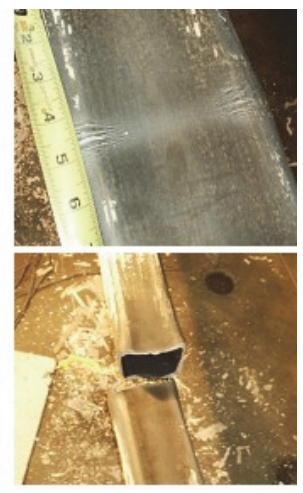

(c) Initial tearing and fracture

Figure 5-6 Damage to buckling brace under reverse loading.

Although brace buckling and yielding are the primary yield mechanisms and brace fracture is the preferred failure mode, other yield mechanism and failure modes are possible, as illustrated in Figure 5-7, particularly for braced systems that are not designed as modern SCBFs. These less desirable limit states include gusset plate or brace net section fracture, block shear failure, bolt and weld failure.

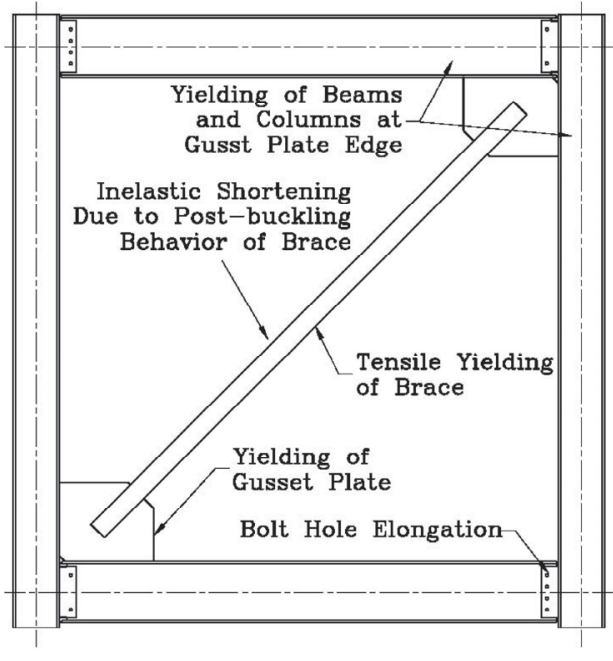

(a) Yield mechanisms

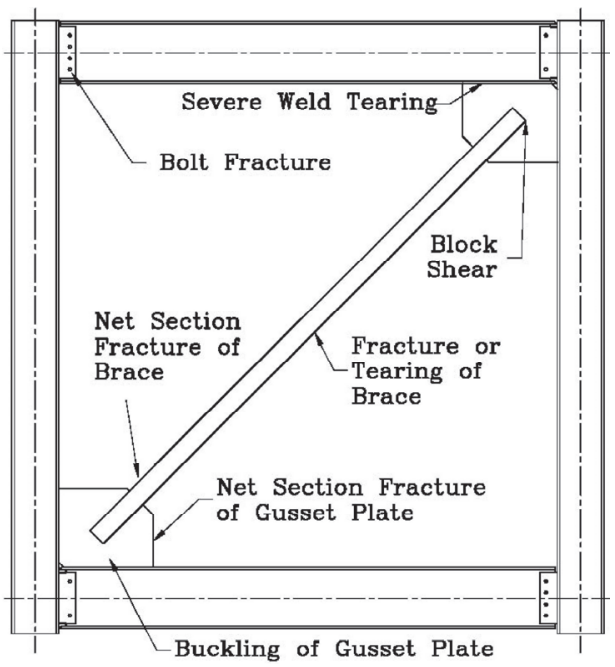

(b) Failure modes

Figure 5-7 Yield mechanism and failure modes (Lehman et al., 2006).

Development of a yielding hierarchy (multiple yield mechanisms balanced to achieve distributed yielding) before failure is key to sustaining cyclic deformation demands.

Figure 5-8 demonstrates the increased yielding provided by the establishment of a yielding hierarchy, as represented by the balanced design procedure. The primary 
difference between the two design philosophies is that current capacity design approach in ANSI/AISC 341-10, Seismic Provisions for Structural Steel Buildings (AISC, 2010a), relies on a single yield mechanism of braced yielding to resist the seismic deformation demands. In contrast, the balanced design procedure (BDP) designs the $\mathrm{CBF}$ to sustain sequential yield mechanisms before brace fracture, most commonly gusset plate yielding follows brace yielding. As shown in the figure, this sequential yielding increases the drift capacity. In part, this is because the secondary yield mechanism reduces the strain demand in the brace at a given drift demand, thereby increasing the total, global drift capacity corresponding to brace fracture.
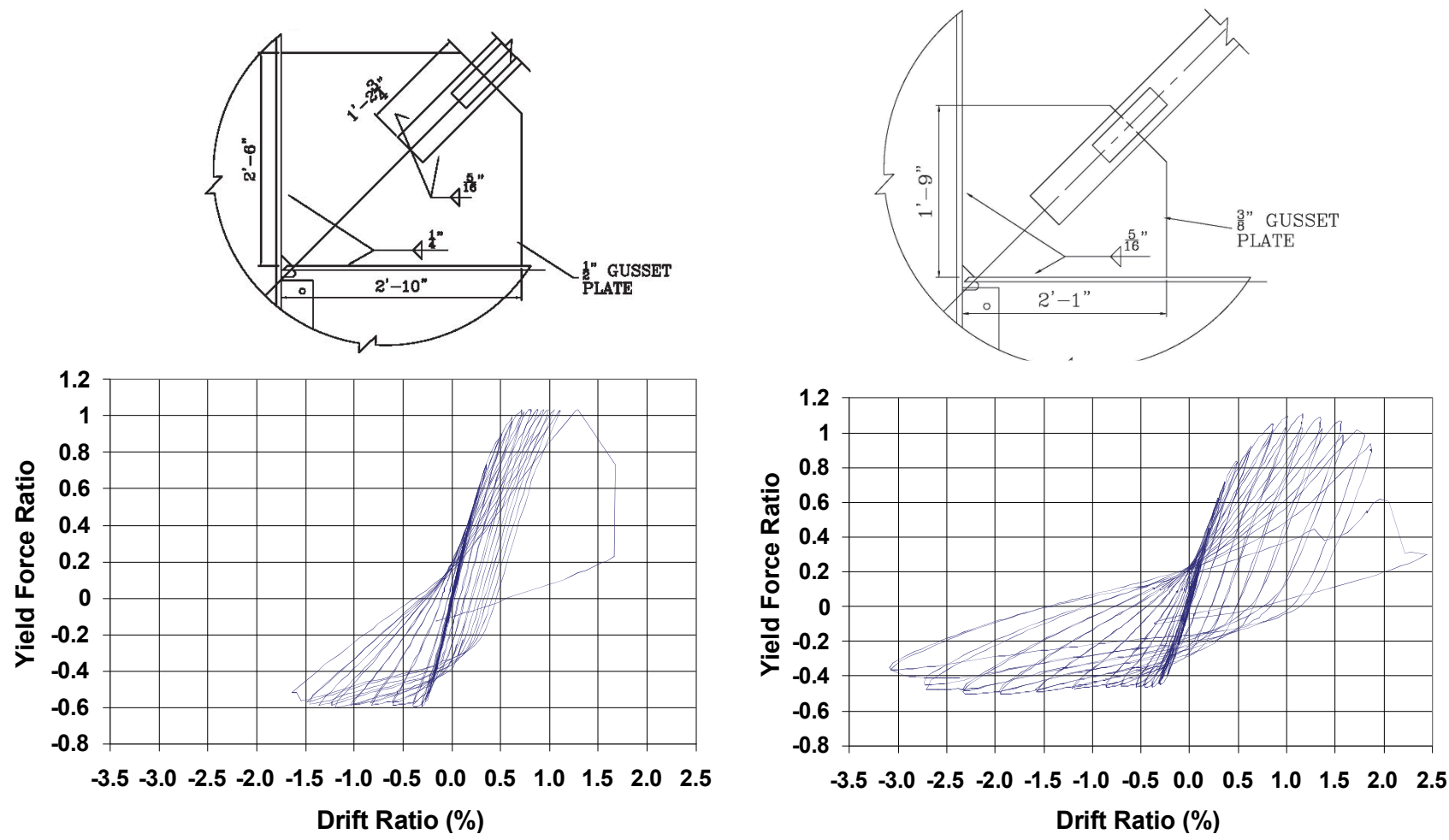

Figure 5-8 Comparison of SCBFs designed to comply with AISC (left) and BDP (right). The AISC frame relies on a single yield mechanism (brace yielding) where the BPD permits multiple yield mechanisms (brace yielding and gusset plate yielding) prior to brace fracture.

\subsection{Recommendations for Modeling}

\subsubsection{Overview}

CBF response depends on the brace, connection of the brace to the frame, and the frame itself. Although behavior of the braces dominates elastic behavior, post fracture or failure of the brace or connection results in residual strength and stiffness of the remaining "moment frame," which includes the beams, columns, beam-column connection, and the restraint of the gusset plate connections. The focus of this section is on modeling of the braces and gussets. Hsiao et al. (2012) provides additional information on such models. Modeling of the remaining of the "moment 
frame" is beyond the scope of this Chapter. The modeling approach described below assumes that the gussets have sufficient strength and deformability to enable the braces to develop their strength then buckle.

\subsubsection{Brace}

Nonlinear response of brace buckling can be accurately captured using a series of inline fiber beam-column elements configured with a small out-of-plane initial displacement. The following recommendations were validated using a wide range of brace tests and matched the AISC buckling curves (Gunnarsson, 2004; Hsiao et al., 2012).

- Each brace should be modeled using at least 4 beam-column elements along the brace length and an initial displaced secant shape approximating a sine function with out-of-plane amplitude of $L / 500$, where $L$ is the clear brace length. Figure 5-9 indicates the accuracy that can be obtained from such a model relative to the AISC curve. The figure compares the impact of the out-of-plane imperfection amplitude on predicted behavior.

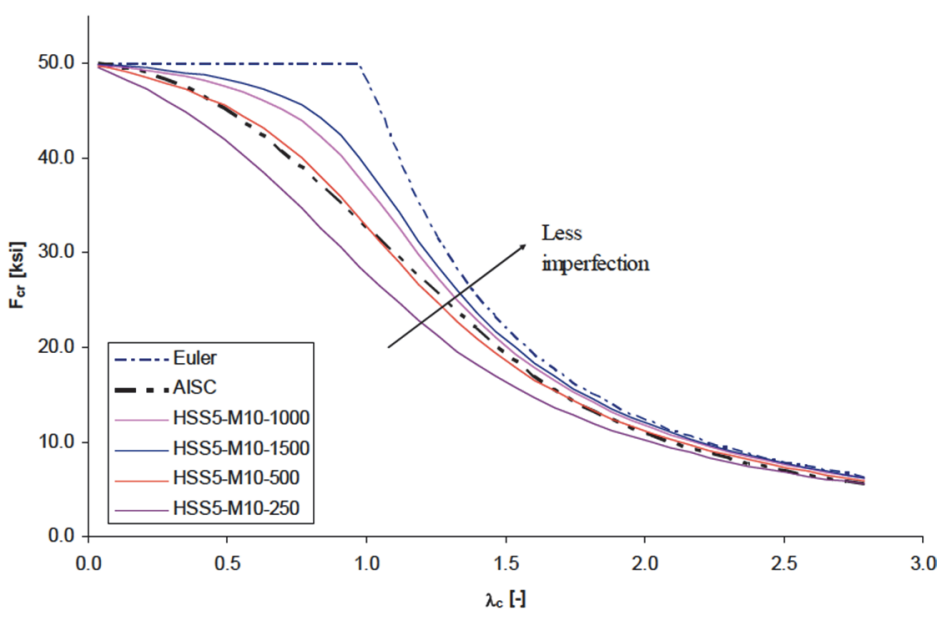

Figure 5-9 Comparison of brace model and AISC and Euler-buckling curves for HSS $5 \times 5 \times 3 / 8$ brace and various out-of-plane offsets.

- Research suggests that HSS braces should be modeled using four (4) fibers along each side of the cross section (12 total fibers) although fewer fibers might be acceptable if local deformation demands are not of interest. Wide flange brace cross sections should be modeled using 2 rows (thickness direction) by 10 rows (flange or web length direction) fiber discretization to capture local deformations of the brace. Figure 5-10 illustrates these modeling recommendations.

- The constitutive model should use expected material strengths and a strainhardening ratio of 0.01 for A500 and A992 steel. This value should be verified for other steel. 


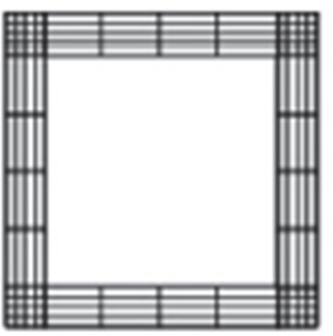

(a) HSS tube

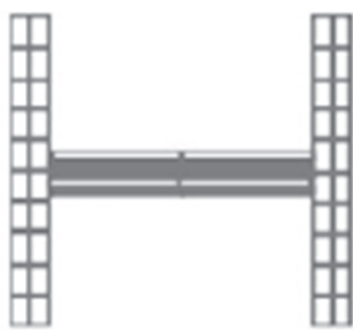

(b) Wide-flange

Figure 5-10 Schematic layout of fibers for HSS and wide flange cross sections.

- The brace is stiffer and stronger at the connection to the gusset plate and the addition of net section reinforcement. In the model, it is recommended to simulate this using a stiffer element at the end of the brace, or a rigid offset. If a stiffer element is used, it is recommended that the modulus and yield stress of the steel be magnified by a factor of 3 . This ensures this portion of the brace model will remain elastic.

\subsubsection{Gusset Plate Connections}

The connection model is critical. For SCBF systems, out-of-plane movement of the gusset plate is permitted through either linear or elliptical yield surfaces in the plate, as shown in Figure 5-11; yielding of the gusset plate in tension may follow brace yielding, if the design is based on a balanced yield hierarchy. Using this design approach can result in a SCBF drift capacity that is twice the drift capacity of an SCBF designed with this secondary yield mechanism (Roeder et al., 2011).

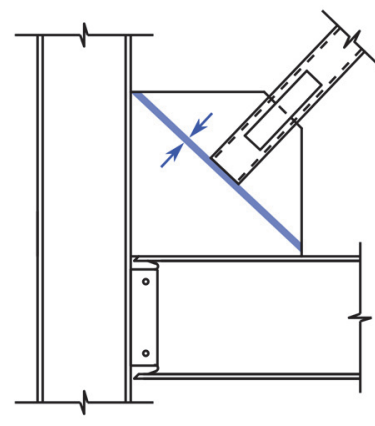

(a) Linear $2 t_{p}$

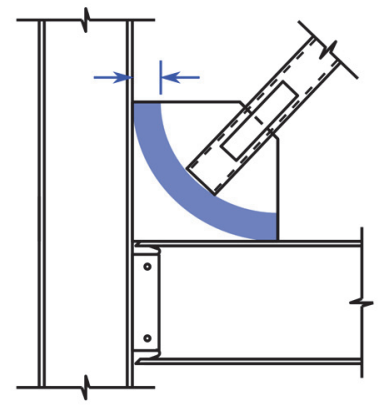

(b) Elliptical $8 t_{p}$ offset

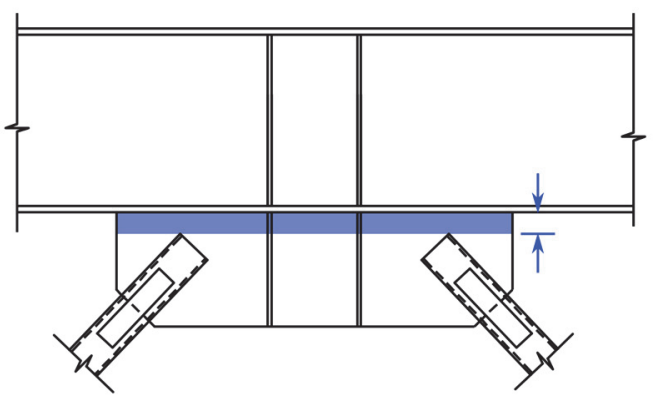

(c) Midspan $6 t_{p}$ parallel offset

Figure 5-11 Brace clearance for corner and midspan connections.

To simulate these nonlinear response modes, the connection model illustrated in Figure 5-12 (Hsiao et al., 2012) can be used. The brace is modeled as indicated previously with rigid end zones included to simulate the additional stiffness of the components provided by the gusset plate. As shown in the figure, from the concentric point joining the centerlines of the brace, beam and column, the rigid end zone of the column is the vertical dimension of the gusset ( $b$ in the figure) plus half the beam depth, the rigid end zone of the beam is $75 \%$ of the horizontal dimension of 
the gusset ( $a$ in the figure) plus half the column depth and the rigid end zone of the brace extends from the physical end of the brace to the concentric point. For the frames studied, the gusset plate thickness typically exceeded the thickness of the beam web, and therefore there was additional flexibility in the beam-to-gusset connection, which was approximated with a coefficient of 0.75 , rather than 1.0 , for the rigid end zone of the beam. For beams with web thicknesses that are greater than the gusset plate, a 1.0 coefficient is recommended. For beam web-to-gusset plate ratios between 0.75 and 1.0, the coefficient can be equal to the thickness ratio (e.g., if the thickness ratio is 0.85 , a coefficient of 0.85 can be used in place of the coefficient of 0.75$)$.

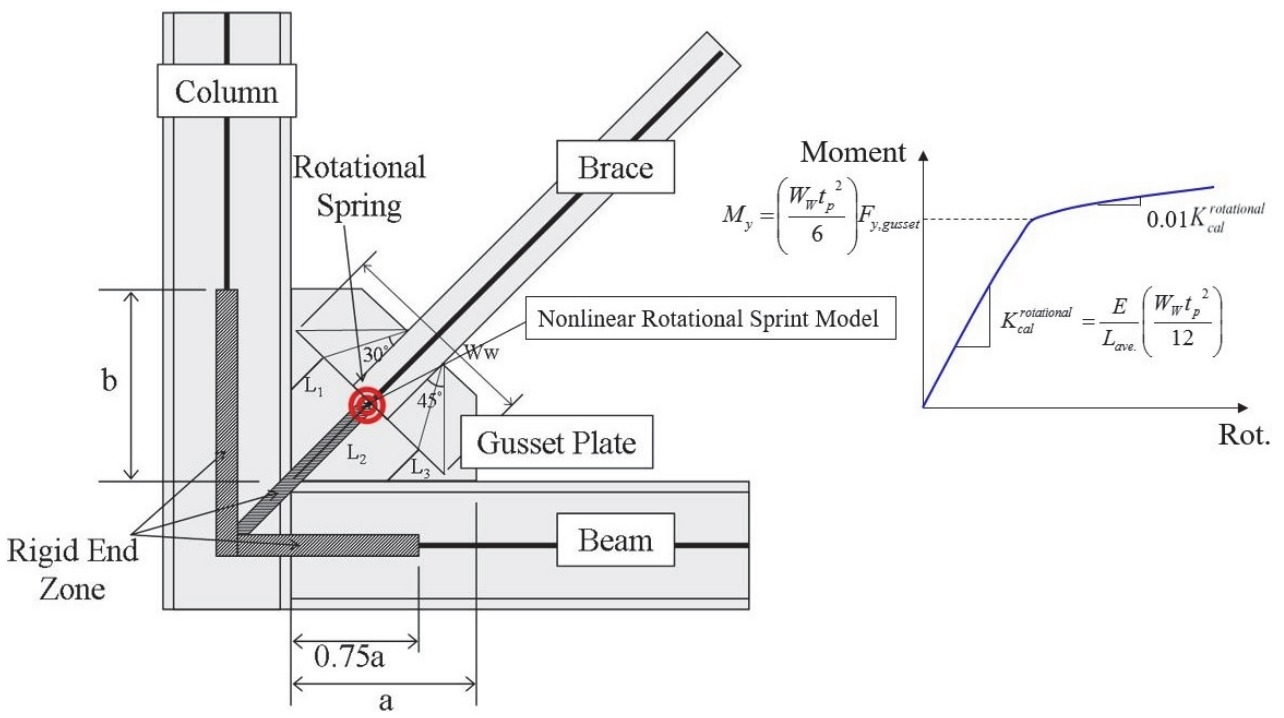

Figure 5-12 Rotational connection model of corner connections (Hsiao et al., 2012).

The gusset plate is modeled as a concentrated rotational spring with a nonlinear response, as shown in Figure 5-12. The flexural strength of the spring is a function of the gusset plate thickness, yield strength, $F_{\text {ygusset }}$, and the Whitmore width (per ANSI/AISC 341); the post-yield stiffness is $10 \%$ of the initial stiffness, which is also a function of the gusset plate dimensions. It is noted that the spring requires calculation of an effective length of the gusset plate, which depends on the clearance used.

This effective length is calculated as follows:

$$
L_{\text {ave }}=\frac{1}{3}\left(L_{1}+L_{2}+L_{3}\right)
$$

where $L_{1}$ and $L_{3}$ are the lengths from the end of the Whitmore width to the intersection with the column and beam flanges, respectively, and $L_{2}$ is the distance from the end of the brace to the beam or column flange, as indicated in the Figure 5-12. 
The impact of this specified connection response was compared with simulations using fiber beam-column elements for the beams, columns, and braces with simplified assumptions for the gusset plate connection, either fully pinned or fully restrained. The comparison is shown in Figure 5-13. The results of the comparison analyses are shown in the columns where the figure at the top of the column indicates the assumption used for the gusset plate connection (leftmost is fully pinned, middle is fully restrained, and the rightmost used a nonlinear, concentrated spring model). These modeling approaches were compared with test results of 1-story SCBF specimens. HSS1 simulated a SCBF with a $2 t_{p}$ linear offset; HSS5 simulated an SCBF designed to meet the balanced design procedure.

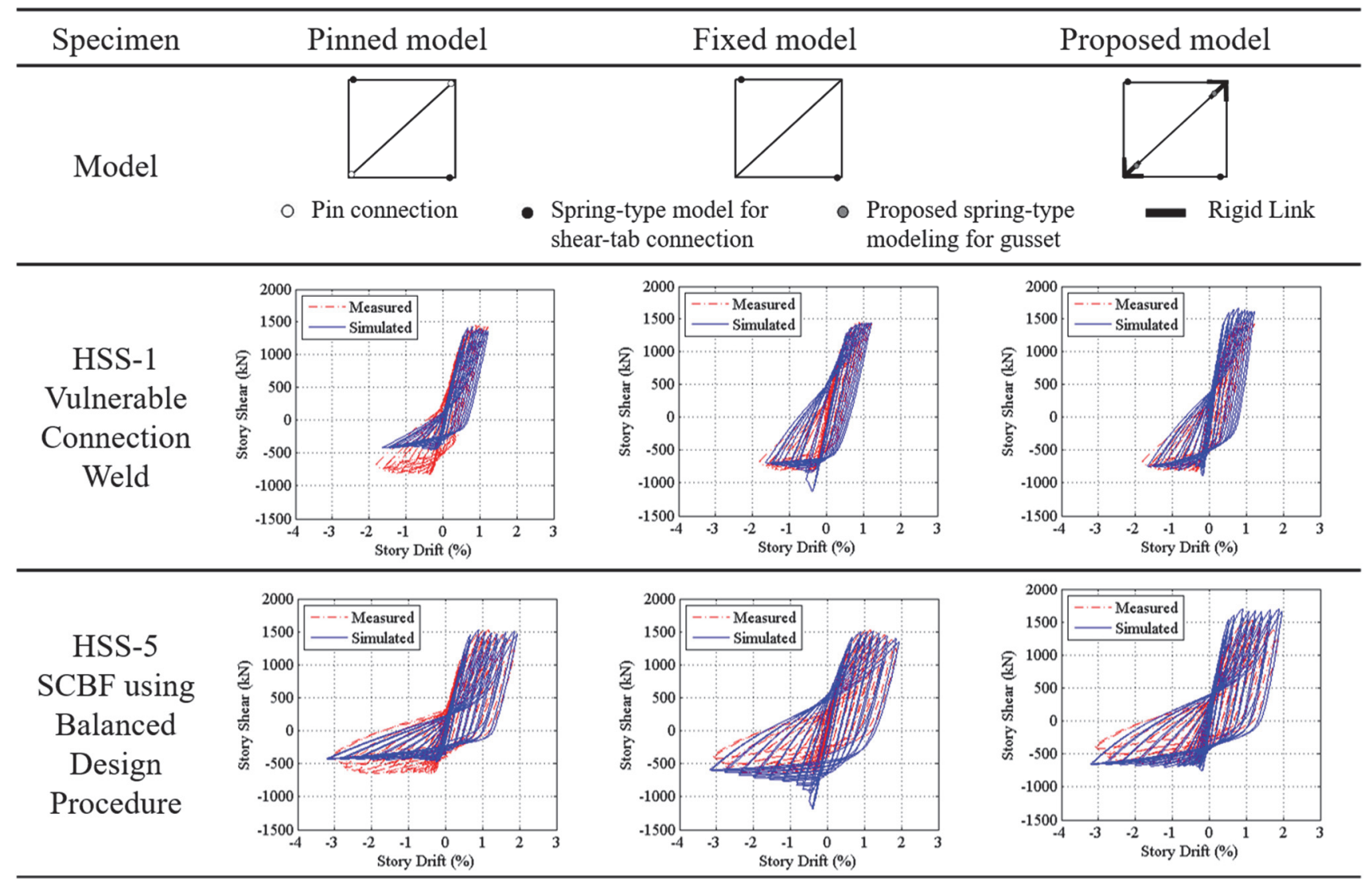

Figure 5-13 Models for SCBFs: pinned, restrained, and nonlinear spring connection.

The results show that models using pinned connections fail to simulate the compressive response; the capacity is underestimated. Restrained connections are an improvement relative to modeling SCBFs using pinned connections; however, these models predict a larger compressive capacity than is measured in the experiment. The rotational spring model predicts the fully hysteretic response of both the SCBF specimens designed using the ANSI/AISC 341 and balanced design procedures. Therefore, SCBF simulation should include a nonlinear rotational spring for the connection; in lieu of this approach, a fully restrained connection model is recommended. 


\subsection{Cyclic Backbone}

Figure 5-14 shows the form of the recommended simplified, multi-linear cyclic backbone curves. Since CBFs have very different responses in tension and compression, the deformation capacity, $d$, of the envelope is an average of the combined behavior associated with a pair of similar but opposed braces, where one acts in tension and the other in compression, Figures 5-14a and b, respectively.

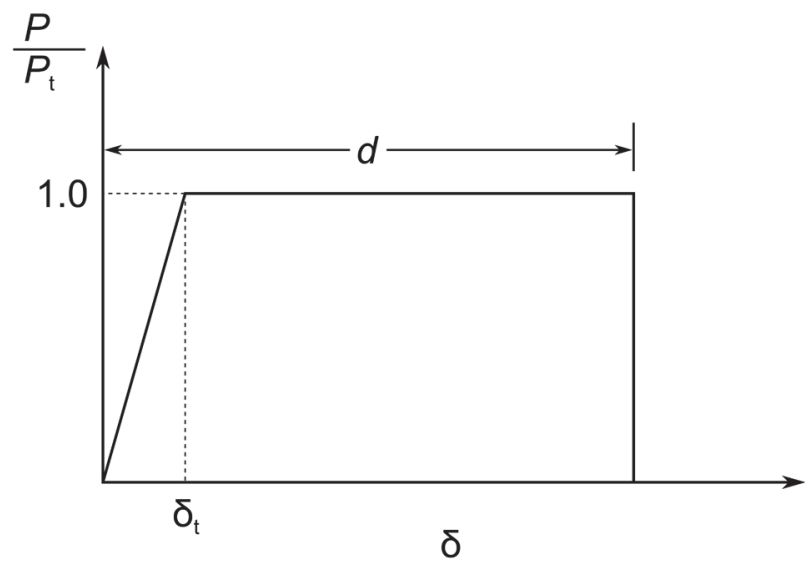

(a)

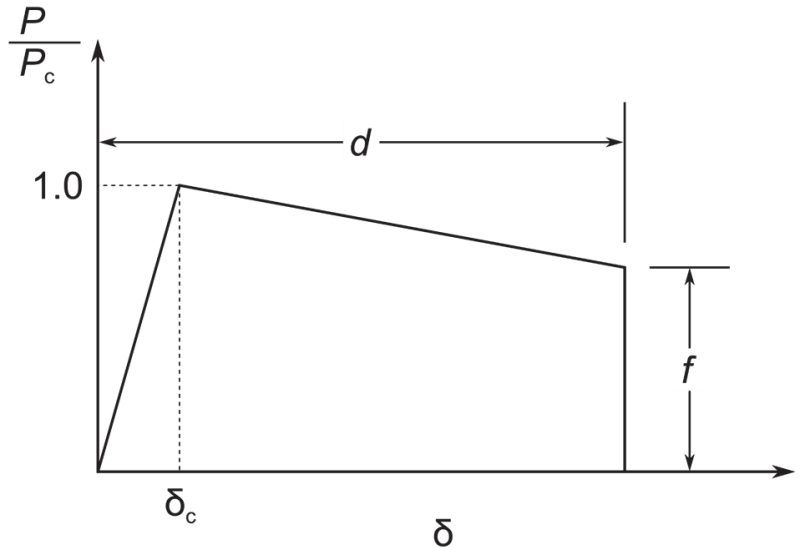

(b)

Figure 5-14 Simplified cyclic backbone curves for CBFs for brace response in: (a) tension, and (b) compression.

Note that these backbones can either represent the brace alone or the braced bay response. For the brace alone modeling approach, there is no capacity after brace fracture. For the braced-bay modeling approach, the post brace-fracture model capacity is the capacity of the residual moment frame where moment frame action results from the gusset plate. For the latter modeling approach, the residual moment frame capacity should be modeled to drop to zero at an average drift range of $4 \%$ (e.g., 4\% drift in each direction of loading). Neither modeling approach accounts for other failure modes such as beam-to-gusset weld tearing or bolt fracture. Additional information on those failure modes can be found in (Sen et al., 2016). If the model includes only the brace, the stiffness associated with the frame is not included.

Figure 5-14 follows the nomenclature in ASCE/SEI 41-13, Seismic Evaluation and Retrofit of Existing Buildings (ASCE, 2014), and is presented with the horizontal component of the brace axial force as the ordinate and story drift ratio as the abscissa. Figure 5-15 presents a single curve based on the tension and compression backbones. 


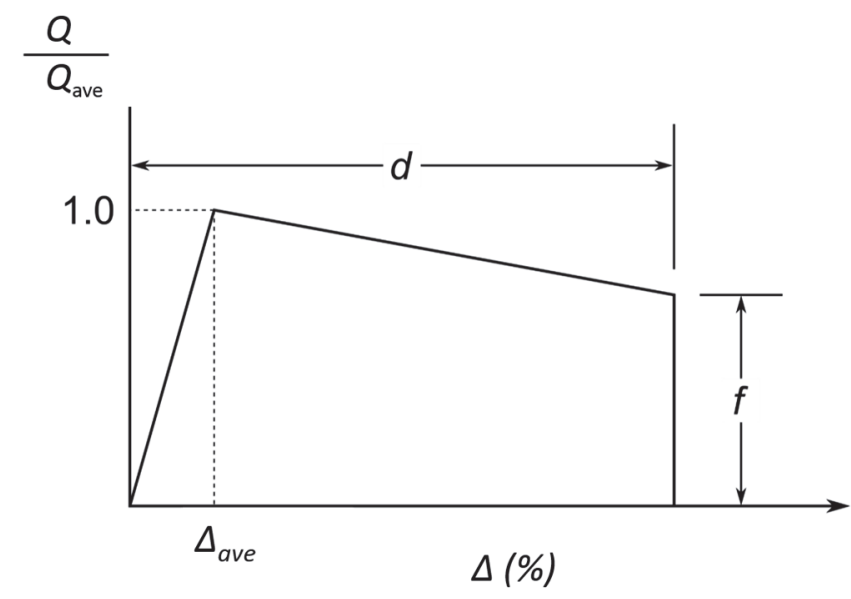

Figure 5-15 Combined backbone curve.

The following key parameters are depicted in Figures 5-14 and 5-15:

$Q \quad=$ lateral component of brace force

$Q_{a v e} \quad=$ average of lateral (horizontal) component of brace yield, $Q_{y}$, and buckling force, $Q_{c}$, where the brace axial capacities are determined using ANSI/AISC 341, Seismic Provisions for Structural Steel Buildings, where $P_{y}=R_{y} F_{y} A_{g}$ and $P_{c}=R_{y} F_{c r} A_{g}$ and the horizontal component of those capacities is determined from the braced frame geometry

$\Delta \quad=$ story $\operatorname{drift}(\%)$

$\Delta_{\text {ave }} \quad=$ average of brace yield and buckling drifts (\%)

$d \quad=$ drift capacity of brace $(\%)$

$f \quad=$ ratio of average brace force at incipient fracture

With the exception of the ultimate brace deformation capacity $d$ and the average brace force at incipient fracture, each of the above parameters must be obtained from the detailed brace model, described in Section 5.4.

Testing of braced frame specimens reported in Lehman et al. (2008) and Sen et al. (2016) indicate that the ultimate deformation capacity, $d$, most closely relates to the local slenderness ratio for the brace, $\lambda / \lambda_{h d}$, where $\lambda$ is the critical $b / t$ or $h / d$ ratio for the cross section and $\lambda_{h d}$ is the limiting value for highly ductile behavior per ANSI/AISC 341. Figure 5-16 provides median values for $d$ as a function of $\lambda / \lambda_{h d}$. The COV from the data to the expression is 0.065 . 


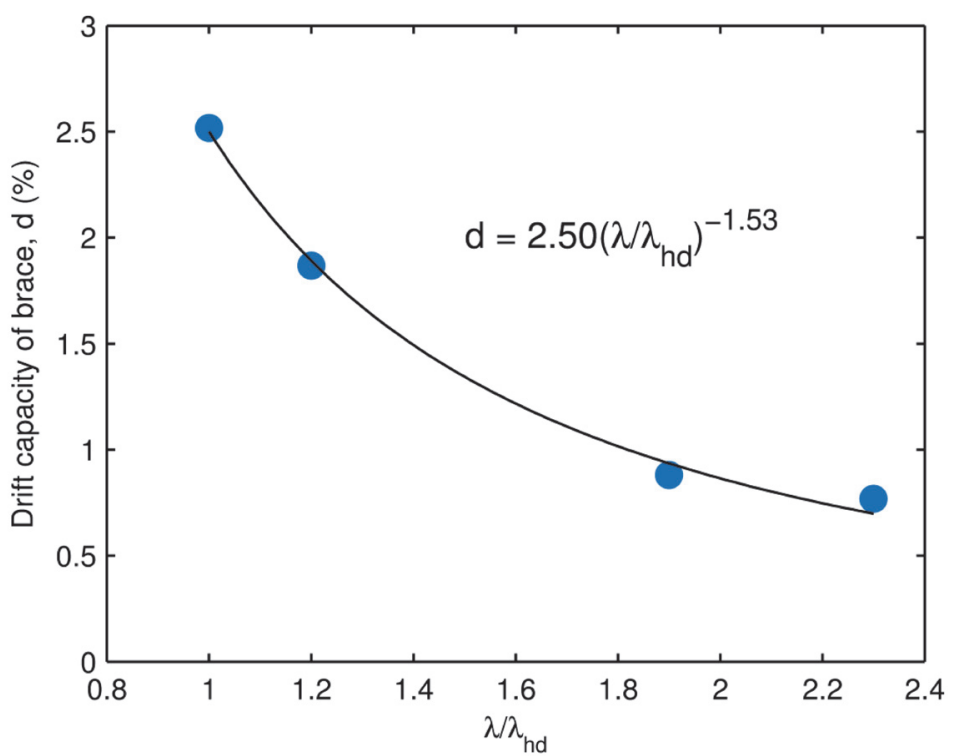

Figure 5-16 Drift capacity as a function of normalized slenderness ratio.

The average normalized brace force, $f_{c}$, at incipient fracture has a median value of $0.73 Q_{\text {ave }}$ on the compression side with a COV of 0.04 for the test data presented here and summarized in Sen et al. (2016). (It is noted that this force can also be determined using the fracture model described in Hsaio et al.) There is no degradation in strength on the tensile side, and therefore $f_{t}=1.0 Q_{a v e}$.

Figure 5-17 provides a visual comparison of the recommended backbone parameters and the measured response as a function of $\lambda / \lambda_{h d}$. It is noted that the backbone provides average drift range values, which explains part of the difference in the proposed backbone and the measured response. In addition, some specimens sustain connection failure or difference in the response due to the connection; these are not captured by the current proposed modeling approach. Table 5-1 provides the statistics on the ratio of the recommended ("fit") and measured model parameters using the data set.

Modeling recommendations for the framing members (beams, columns, and their connections) can be found in other chapters. With CBFs, the framing action depends heavily on the gusset plate, which provides the moment restraint. Since those chapters do not provide a limit on that type of connection, it is recommended that a maximum average drift ratio corresponding to failure of the post-fracture residual moment frame be limited to $4 \%$. This limit is based on over 50 tests of CBF systems. 

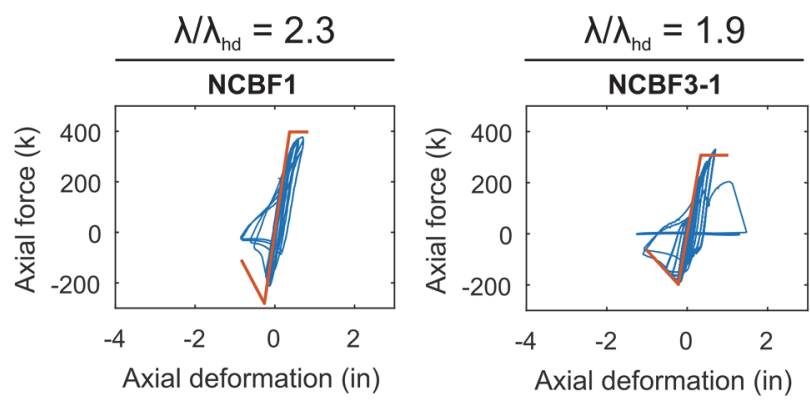

- Experimental hysteresis

- Proposed envelope

* Connection fracture prior to brace tearing or fracture
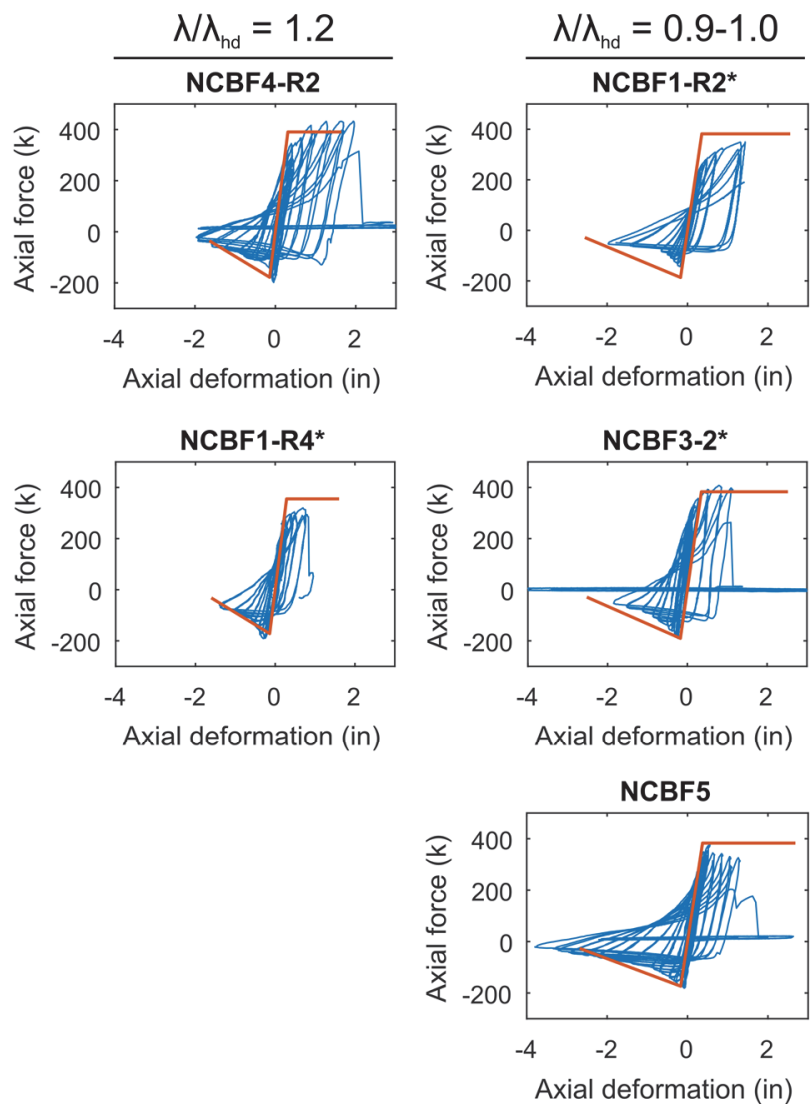

Figure 5-17 Comparison of recommended envelope and measured response for selected tests.

Table 5-1 Statistics for Ratios of Recommended-to-Measured Model Parameters

\begin{tabular}{|c|c|c|c|c|c|c|}
\hline \multirow{3}{*}{ Brace Only } & $\begin{array}{c}\text { Model } \\
\text { Parameter }\end{array}$ & Mean & $\begin{array}{l}\text { Standard } \\
\text { Deviation }\end{array}$ & $\begin{array}{l}\text { Coefficient } \\
\text { of Variation }\end{array}$ & Max. & Min. \\
\hline & $d_{\text {fit }} / d_{\text {meas }}$ & 1.03 & 0.100 & 0.097 & 1.15 & 0.90 \\
\hline & $f_{\text {fit }} / f_{\text {meas }}$ & 2.15 & 2.03 & 0.95 & 6.04 & 0.39 \\
\hline \multirow{2}{*}{ Braced Frame } & $d_{\text {fit }} / d_{\text {meas }}$ & 0.99 & 0.064 & 0.065 & 1.06 & 0.91 \\
\hline & $f_{\text {fit }} / f_{\text {meas }}$ & 1.00 & 0.049 & 0.049 & 1.08 & 0.95 \\
\hline
\end{tabular}

\subsection{Monotonic Envelope}

Differences between the monotonic response curve and cyclic backbone include: (1) peak strength; (2) post-yielding (or buckling) stiffness; and (3) deformation capacity. For steel braces, cyclic demands do not have a significant impact on the first two engineering characteristics. However, the monotonic envelope for braces has similar shape to that shown for cyclic in the previous section, with the exception that $d$, which as provided in Figure 5-16 is a function of low-cycle fatigue, is larger. In this case, $d$, the axial deformation capacity in the direction of tensile yielding of 
the brace should be based on the ultimate tensile fracture strain of the brace, or frame instability, whichever controls. 


\section{Chapter 6}

\section{Reinforced Concrete Moment Frame Systems}

\subsection{Overview of Geometry and Behavior}

Reinforced concrete moment frames include monolithic, cast-in-place, two- and three-dimensional beam and column assemblies, precast beam and column assemblies, and cast-in-place slab column assemblies. The frames are either proportioned and detailed for ductile nonlinear seismic response, herein termed seismic moment frames, or have not been designed in this manner. This chapter focuses on seismic moment frames as the nonlinear response of other moment frames is generally limited and cannot be relied upon. The chapter also focuses on monolithic beam-column assemblies. This chapter is based on NIST GCR 17-917-46v3, Guidelines for Nonlinear Structural Analysis and Design of Buildings, Part IIb - Reinforced Concrete Moment Frames (NIST, 2017c).

In seismic moment frames, capacity design is employed to concentrate inelastic flexural deformations to the end hinge regions of beams, while avoiding shear failure in beams, columns, and joints, and limiting inelastic deformations in columns, joints, and foundations. Columns are proportioned with sufficient strength to limit inelastic deformations in columns and the tendency to form single-story mechanisms. These so-called strong-column weak-beam design criteria do not, however, entirely preclude inelastic deformations in columns, as higher mode effects and inelastic moment redistribution can lead to hinging of columns. Moreover, in frames with stiff foundations, columns will inevitably develop inelastic hinges near their base under strong ground motions. Seismic detailing and capacity design principles are also intended to ensure ductile flexural yielding and preclude less desirable failure modes such as anchorage failures. Seismically detailed frame members are intended to have sufficient ductility to avoid significant strength deterioration under the story drifts expected to occur in response to maximum considered earthquake ground motions.

When capacity design measures are not employed, non-ductile failure modes may occur that cause significant strength losses and structural instability at low to moderate deformations. The nonlinear dynamic response of such frames is both limited and more difficult to reliably model. Possible deterioration and failure modes in non-seismically detailed frames include: story mechanisms, anchorage failures of 
longitudinal bars, lap-splice failures, shear failures, interface shear failures, premature buckling of longitudinal bars, and crushing of the concrete cores.

\subsection{Summary of Design Code Provisions}

In the 1960s, substantial improvements in the seismic design methodologies of concrete structures were introduced into the building code, based on early work by John Blume and others. However, use of these seismic design practices were not required by the code until the occurrence of dramatic concrete frame failures in the 1971 San Fernando earthquake. Even following the 1971 earthquake, the building codes required seismic design practices only in structures in regions of higher seismicity, designated in those codes as zones 3 and 4 . In other zones, the building codes continued to permit use of structures designed without these practices. Further continued improvement in the code requirements occurred throughout the 1980s, 1990s, and later codes. Structures designed and built prior to the late 1970s in zones of higher seismicity typically do not have sufficient strength or adequate seismic detailing to limit seismically-induced deformations and ensure sufficient deformation capacity.

\subsection{Performance and Damage Progression}

ACI 318-14, Building Code Requirements for Structural Concrete and Commentary (ACI, 2014), identifies three different seismic design levels for moment-resisting frames: Ordinary Moment Frames (OMF), Intermediate Moment Frames (IMF), and Special Moment Frames (SMF); OMFs are designed with limited detailing and capacity design requirements; IMF have additional detailing and capacity design requirements over OMF; and, SMF are designed to achieve ductile responses up to relatively large design drift levels (typically in the range of 3 to $4 \%$ of a story height).

Table 6-1 indicates the likelihood of occurrence of phenomena causing strength degradation for frames whose characteristics fall into the OMF, IMF, and SMF categories. These associations are approximate and only intended to provide some guidance about what type of response is likely to be encountered. Ultimately, the specific characteristics of each structure must be evaluated to determine which modes of behavior are likely to occur and should be modeled.

NIST GCR 17-917-46v3 provides detailed discussion on the expected behavior and failures modes that can occur in concrete frame components with emphasis on ones that are designed and detailed in general accordance with the ACI-318 requirements. ACI 369 provides expanded information for components that have non-ductile (nonconforming) details. 
Table 6-1 Behavioral Effects to Consider in Nonlinear Analysis (from NIST, 2017c)

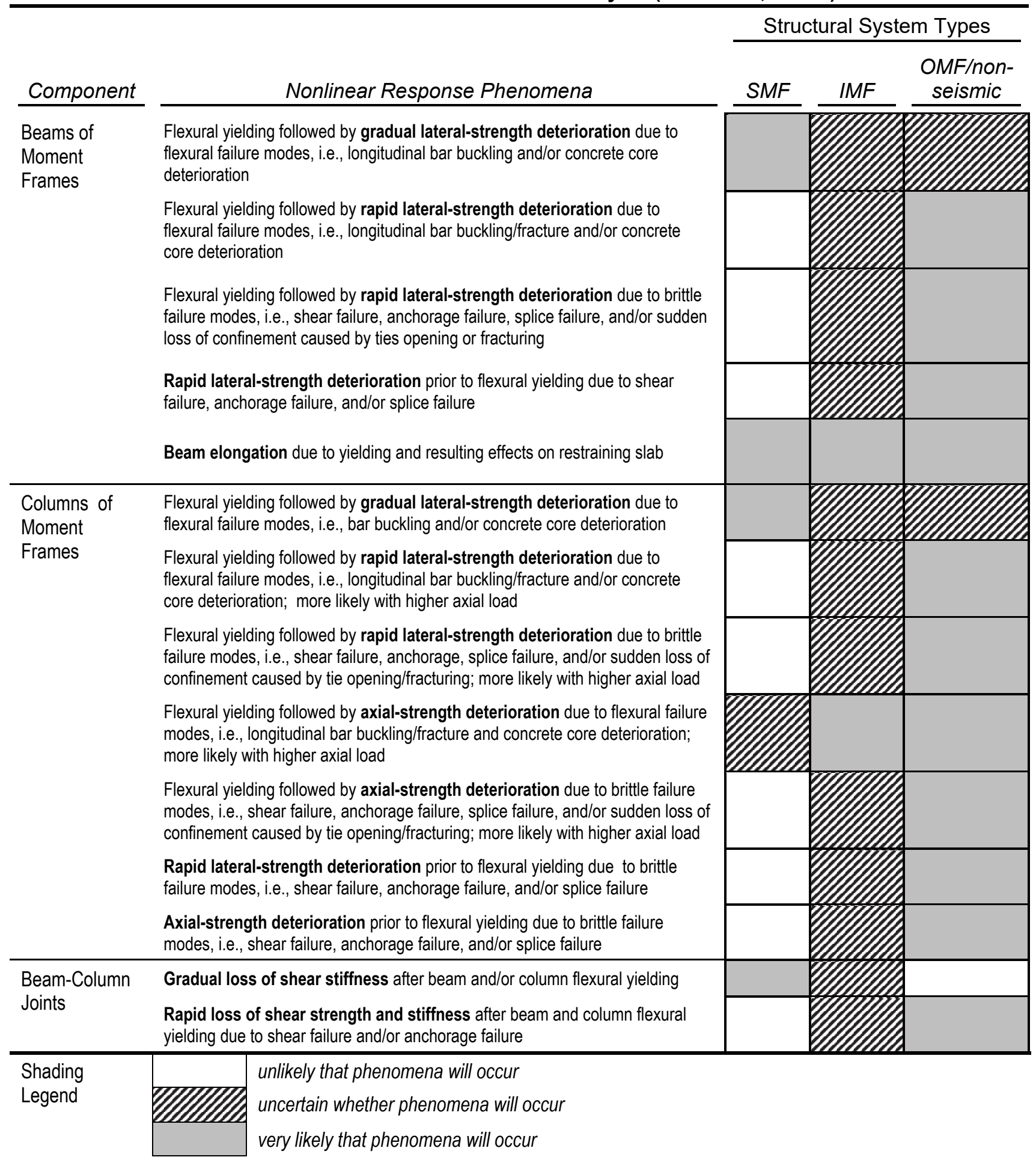

\subsection{Recommendations for Modeling}

Figure 6-1 defines the forces and associated deformations for a cantilevered concrete column (or beam), where response is described in terms of an applied shear force and tip deflection. Deflection can be distinguished into elastic and inelastic components, 
and the response parameters could equally be described in terms of local moment versus chord rotation of an idealized concentrated hinge.

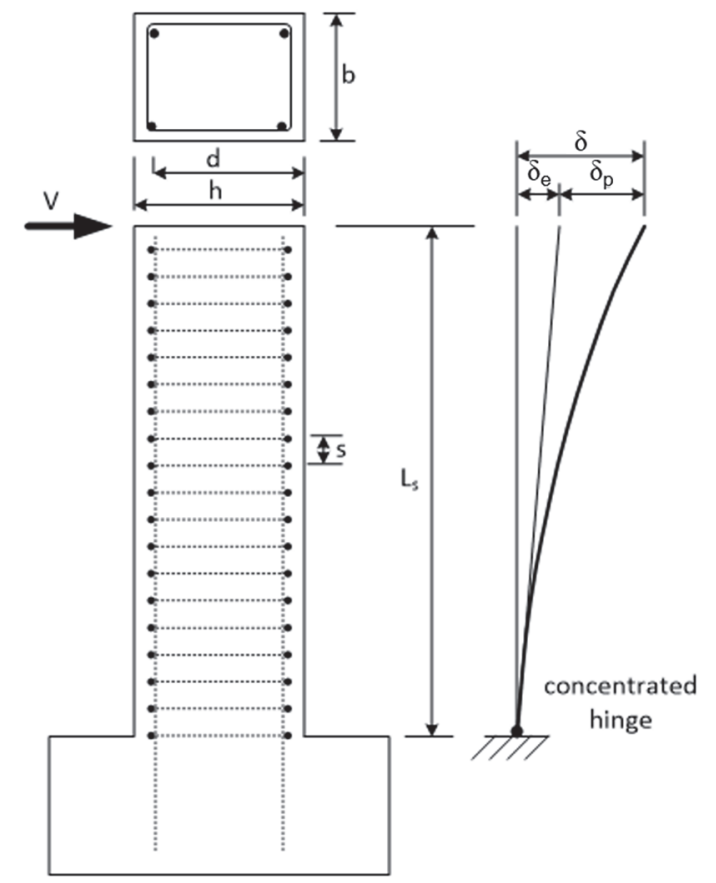

Figure 6-1 Idealized cantilever model of reinforced concrete beam-column with concentrated hinge at member end. Adapted from Haselton et al., 2016.

Figure 6-2 shows a typical monotonic force-deformation response for such a component. The initial effective stiffness of concrete components is usually characterized by a secant stiffness that is evaluated based on the expected force and deformation levels to be experienced by the structure. For example, under servicelevel (pre-yield) response, the effective stiffness might be evaluated at about half the yield strength, whereas when the structure is expected to reach or exceed yielding, the effective initial stiffness may be evaluated at the yield point. The main deformation components that need to be accounted for in structural analyses of beams and columns are flexural, shear, and bar-slip deformations. Typically, flexural deformations are the largest. However, bar-slip deformation can reach up to $40 \%$ of the total lateral deformations (Sezen and Moehle, 2006; Elwood and Eberhard, 2009; Panagiotakos and Fardis, 2001). Shear deformations typically account for 5\% to $10 \%$ of the total lateral deformations (Elwood and Eberhard, 2009), but can reach $15 \%$ in frame members with shear stresses close to the ACI 318-14 shear stress limit (Sokoli and Ghannoum, 2016). Although some nonlinear analysis methods (e.g., fiber beam-column elements) can directly model cracking due to induced loads, there are several complicating factors (such as initial micro-cracking due to concrete 
shrinkage and creep, localized bar anchorage and bond slip) that necessitate calibration of the initial effective stiffness.

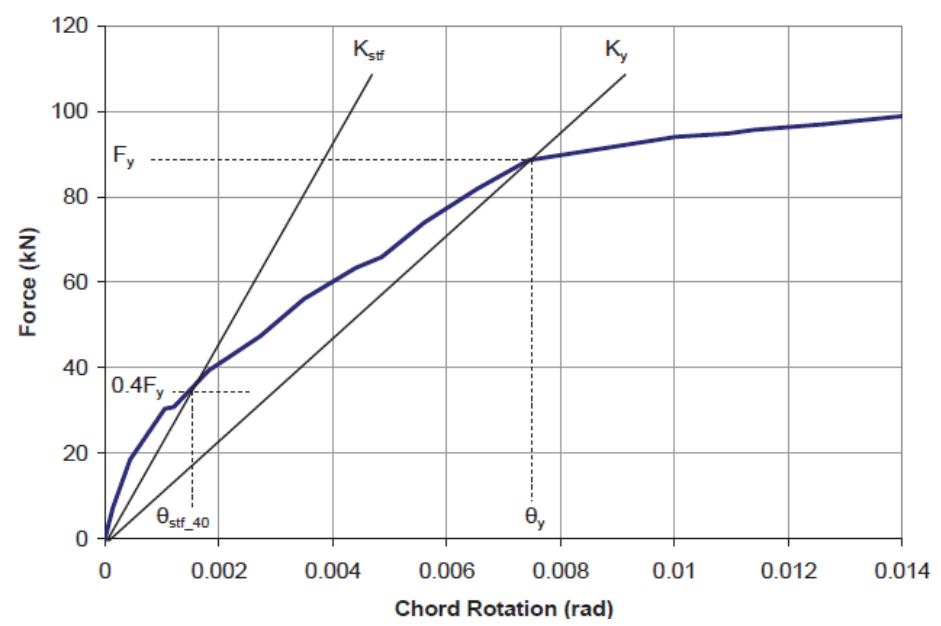

Figure 6-2 Representative force-deformation plot for cantilever beam-column (data from Ingham 2001 and figure from Haselton et al. 2008).

In nonlinear analysis, post-yield response is typically modeled explicitly, using concentrated hinge, fiber-type, or continuum finite element models. In such cases, the yield strength of the structural components is usually modeled directly or determined based on mechanics-based models that are validated against test data. Post-yield response is considerably more challenging to model, due the combined effects of concrete cracking, reinforcing bar yielding, diminished bond and anchorage of reinforcing bars, concrete crushing, and localization of these effects.

Stiffness and strength degradation in the post-yield region is highly dependent on loading history. Figure 6-3 shows response plots for five nominally identical concrete beam-columns that were subjected to different loading histories. The symmetric cyclic history shown in Figure 6-3b is the typical one used to calibrate the first-cycle envelope used in ASCE/SEI 41-13, Seismic Evaluation and Retrofit of Existing Buildings (ASCE, 2014), ACI 369, Guide for Seismic Rehabilitation of Existing Concrete Frame Buildings and Commentary (ACI, 2011), and related documents. The cyclic backbone represents a lower-bound response under most likely earthquake loading histories.

NIST GCR 17-917-46v3 provides a detailed discussion of idealized concentrated hinge and fiber-type analysis models for reinforced concrete frame systems, as well as modeling of column splices, beam-column joint panel zones, and slab-column connections. 


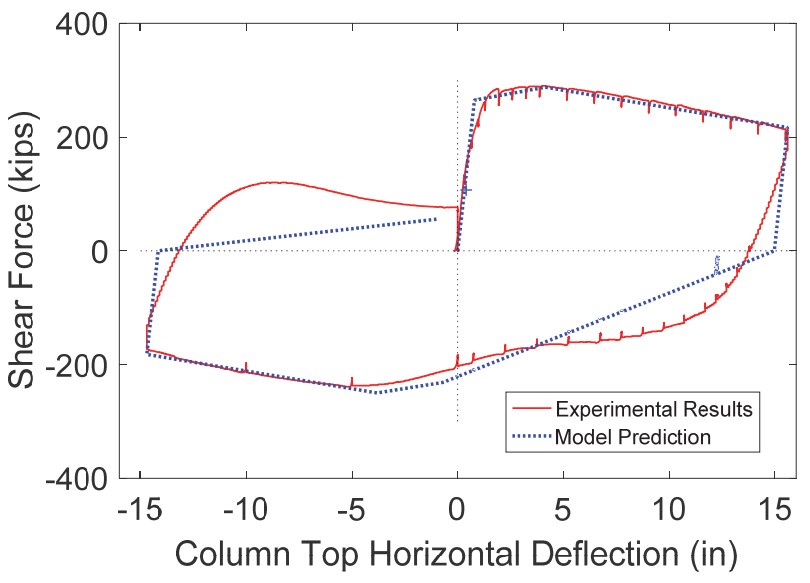

(a)

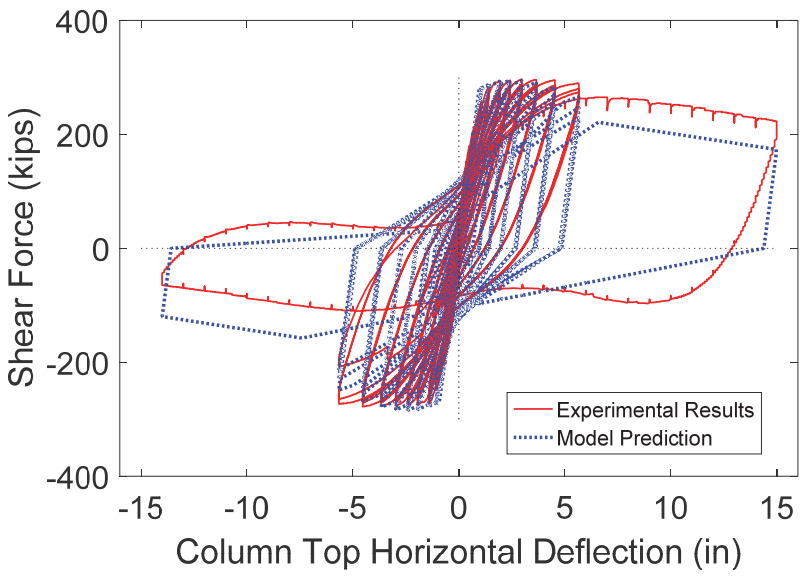

(c)

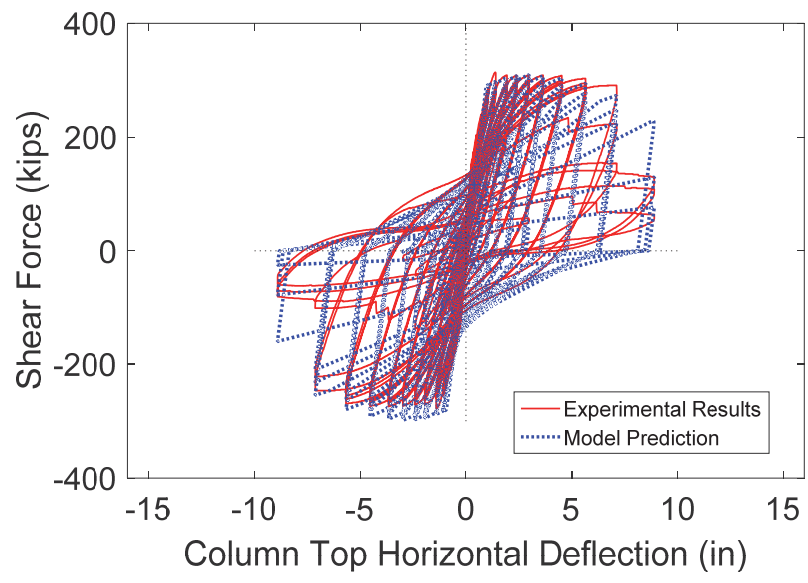

(b)

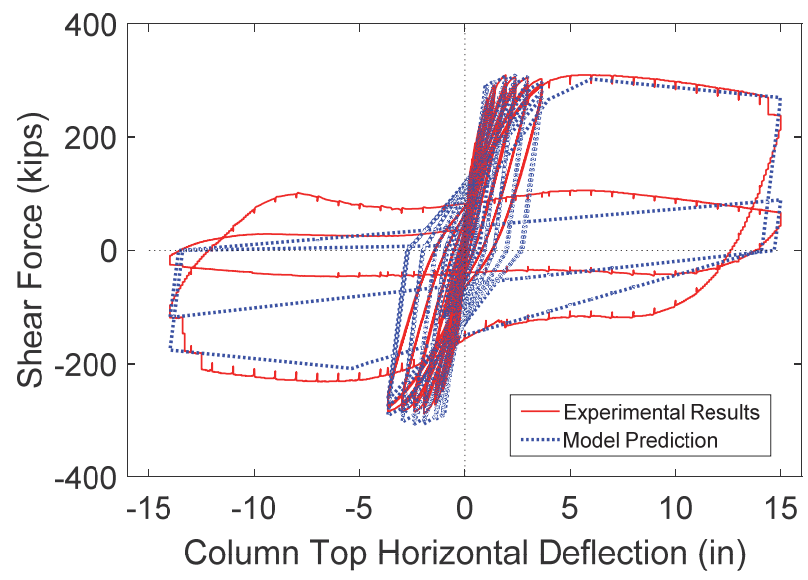

(d)

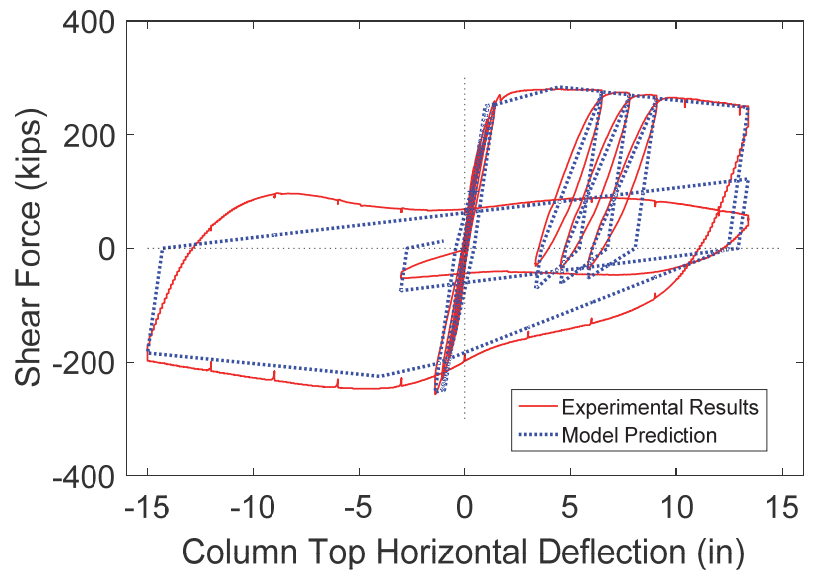

(e)

Figure 6-3 Experimental test data and calibrated model predictions for five different loading protocols: (a) monotonic in each direction; (b) ACl 374 (ACl, 2005) symmetric cyclic; (c) ACl 374 symmetric cyclic followed by monotonic push \# 1; (d) ACI 374 symmetric cyclic followed by monotonic push \# 2; (e) and a near-collapse loading protocol (after Nojavan et al. 2014; 2016). 


\subsection{Concentrated Hinge Model Parameters}

Figure 2-6 of this document shows an idealized monotonic curve that can be used for a steel component. For reinforced concrete moment frames, the moment-rotation relation attributed to the zero-length rotational springs can be idealized as illustrated in Figure 2-6, where the force demand $Q$ is the moment in the element, $M$, and the deformation quantity $\Delta$, is the beam end rotation, $\theta$. A monotonic curve is defined for spring elements including specification of effective initial member stiffness, $K_{e}$, yield strength, $M_{y}$, peak strength and rotation, $M_{u}\left(Q_{\max }\right)$ and $\theta_{p}\left(\Delta_{\text {cap }, p l}\right)$ and post-peak rotation, $\theta_{p c}\left(\Delta_{p c}\right)$. The rotational spring behavior would follow the envelope curve if it were pushed monotonically. However, when concrete frame members are cycled laterally, degradation in strength and deformation capacities occur in the inelastic deformation range. To simulate those degradations, the envelope relation can be adjusted during analyses based on cumulative dissipated energy or cumulative deformations. The first-cyclic envelope can be defined by modified (reduced) values of the peak strength, $M_{u}{ }^{*}\left(Q^{\prime}{ }_{\max }\right)$ inelastic rotation capacities, $\theta_{p}{ }^{*}\left(\Delta^{\prime}{ }_{c a p, p l}\right)$ and $\theta_{p c}{ }^{*}$ $\left(\Delta_{p c}^{\prime}\right)$, and a residual strength, $M_{r}{ }^{*}\left(Q_{R}^{\prime}\right)$. Finally, an ultimate rotation capacity is used to represent loss of any resistance, $\theta_{u l t}{ }^{*}\left(\Delta^{\prime}{ }_{u l t}\right)$. Unless the nonlinear analysis can simulate the complete loss in resistance, it is generally recommended to limit the applicability of the analysis to rotation demands less than $\theta_{u l t}{ }^{*}$.

The objective of modeling is to reliably capture the response that would occur under any generalized loading, such as those shown in Figure 6-3. In such an approach, the model would follow either the monotonic envelope when subjected to a monotonically increasing load, the first-cycle backbone if subjected to a cyclic loading with many symmetric cycles of loading, or an intermediate curve when subjected to a random loading, such as may occur under earthquakes.

\subsubsection{Column Model Parameters}

Effective Stiffness. Simplified empirical equations are typically used to determine the flexural stiffness of line elements in concentrated hinge models. The following recommended equations account for the component deformations coming from flexure, bar-slip, and shear. It is noteworthy that this approach only accounts for the linear portion of the bar-slip behavior, leaving inelastic bar-slip softening to be accounted for in the concentrated hinge element.

Elwood et al. (2007) proposed the following equation for column effective flexural rigidity that accounts for the stiffening effect of compressive axial load, $P$, based on an extensive column test database (Berry et al., 2004):

$$
\frac{E_{c} I_{y}}{E_{c} I_{g}}=0.3+\left(\frac{P}{A_{g} f_{c}^{\prime}}-0.1\right)
$$


where:

$$
0.3 \leq \frac{E_{c} I_{y}}{E_{c} I_{g}} \leq 0.7
$$

This equation is based on the secant stiffness at first yield ( $K_{y}$ in Figure 6-2) and is the basis of the effective stiffness values in ACI 369 and ASCE/SEI 41-13. Being calibrated to the secant stiffness at first yield, it does not capture the substantial additional stiffness that occurs for pre-yield levels of loading, nor does it differentiate between other parameters known to contribute to stiffness, such as the longitudinal reinforcement ratio. Kwon (2016) developed a more detailed equation for effective stiffness based on moment-curvature analyses, a database of over 200 column tests, and shake table test data. As per the following equations, the effective stiffness is a function of the characteristic lateral drift ratio (DR), the compressive axial load ratio, and the longitudinal tension-steel reinforcement ratio, $\rho_{T}$ :

$$
\frac{E_{c} I_{e f f}}{E_{c} I_{g}}=0.003 \mathrm{DR}^{-0.65}+\gamma \leq 0.8 \quad \mathrm{DR} \leq 0.012
$$

where:

$$
\gamma=\left(-50 \rho_{T}+2.5\right)\left(\frac{P}{A_{g} f_{c}^{\prime}}\right)^{\left(-20 \rho_{T}+2.15\right)}+\left(15 \rho_{T}+0.05\right)
$$

To estimate the secant stiffness at first yield ( $K_{y}$ in Figure 6-2), the recommended assumed drift ratio is $\mathrm{DR}=0.008$. A lower drift ratio value may be used in members at higher floor levels that experience limited forces or deformations even during high intensity ground motions. For service load levels, a value of $\mathrm{DR}=0.002$ is recommended for the purpose of evaluating the effective secant stiffness. The effective stiffness equations (6-1 to 6-3) provide mean estimates for member secant stiffness. A coefficient of variation of 0.45 is suggested for either stiffness equation based on statistical comparisons to test data (Kwon, 2016).

The effective flexural stiffness (EI) equations have been calibrated to include flexural deformations and bar slip deformations, prior to yielding of the reinforcement. In special moment frame beams and columns, where capacity design limits the maximum shear stresses to less than the shear strength, the shear deformations typically represent about $5 \%$ to $10 \%$ of the total displacements (Elwood and Eberhard, 2009), but may increase up to about $15 \%$ of the total when the shear stresses approach the strength limit close to that given by ACI 318 (Sokoli and Ghannoum, 2016). Assuming shear cracking is limited along most of the member, effective shear stiffness is generally taken as $0.4 E_{c} A_{w}$, where $0.4 E_{c}$ represents the elastic shear modulus of concrete and $A_{w}$ is the effective shear area. 
Column Cross Section Strength. The nominal flexural strength of column and beam members can be computed using the strain compatibility approach (assuming sections remain plane), and an equivalent rectangular compressive stress distribution under ultimate loads with a concrete crushing strain of 0.003 (ACI 318-14). When expected steel yield strength and concrete strength are used, this stress block approach results in a flexural strength close to the measured flexural strength from column tests, with a median ratio of calculated to measured strength of 1.02 and $\sigma_{L N}$ of 0.23 (Haselton et al., 2016).

To account for coupled axial and moment behavior, column section strengths can be defined as a P-M or P-M-M surface. As illustrated in Figure 6-4, a typical approach is to specify a parametric yield surface function that is defined by a few calculated anchor points.

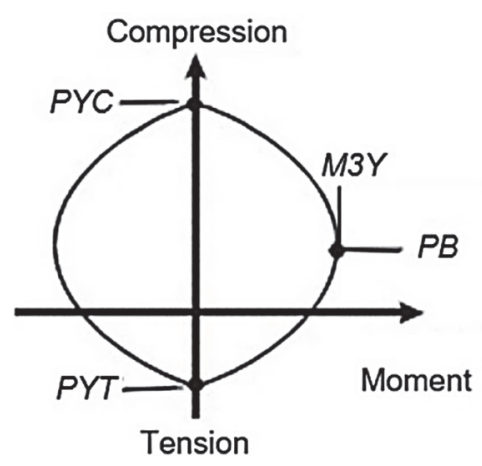

(a) P-M interaction at $M 2 Y=0$

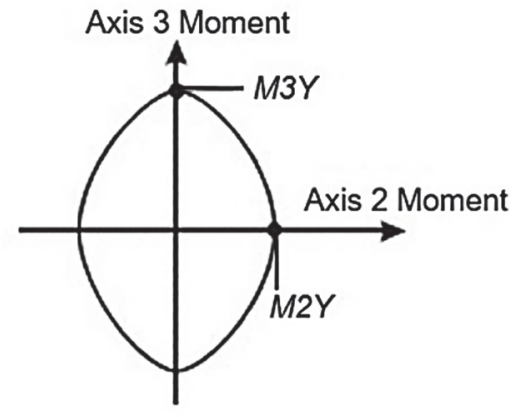

(b) M-M interaction at $P=P B$

Figure 6-4 Illustration of reinforced Concrete P-M-M surfaces from PERFORM-3D (CSI, 2016).

Post-Peak Moment-Rotation Response. Although defining the basic yield surface is relatively straightforward, accurately capturing how the shape of $M-\theta$ backbone curve changes under variable moments and axial load is more challenging. Fiberbased hinge models are generally recommended for direct modeling of P-M-M interaction. Otherwise, simpler $M-\theta$ backbone curves can be defined based on a constant value of axial load. The simpler $M$ - $\theta$ spring models can be used directly in the analysis when column axial forces are dominated by gravity loads and do not vary much due to earthquake overturning effects. Alternatively, the empirical $M-\theta$ spring models can be used to help validate the post-peak (softening) response of fiber-based models. The shape of $M-\theta$ backbone curve varies considerably depending on the level of axial load and biaxial bending on the member. For example, a section with high compressive axial load will most likely lose strength due to concrete crushing, resulting in a $M-\theta$ backbone curve that drops off steeply past the peak point. This contrasts with more gradual degradation for a column with light axial load. 
This section includes recommended nonlinear modeling parameters, based on calibration to a dataset of 255 rectangular column tests (Haselton et al., 2016). The column test data were obtained from the database compiled by Berry and Eberhard (2004); and an expanded version of the database is available online by Ghannoum and Sivaramakrishnan (2012a and b). Properties of the dataset are as follows:

- Square and rectangular columns failing in a flexural mode (220 tests) or in a combined flexure-shear mode (35 tests). Columns failing in a brittle shear mode were excluded

- Cross-section width, $b$, ranging from 150 to $550 \mathrm{~mm}$ (6 to $22 \mathrm{in})$; cross-section height, $h$, ranging from 150 to $610 \mathrm{~mm}$ ( 6 to $24 \mathrm{in}$ )

- Concrete compressive strength, $f_{c}^{\prime}$, ranging from 20 to $120 \mathrm{MPa}$ (3 to $17 \mathrm{ksi}$ )

- Steel yield strength, $f_{y}$, ranging from 340 to $520 \mathrm{MPa}$ (49 to $75 \mathrm{ksi}$ ))

- Compressive axial load ratio, $P / A_{g} f_{c}^{\prime}$ ranging from 0.0 to 0.7 , where $P$ is the axial load, and $A_{g}$ the gross concrete cross-sectional area

- Shear span ratio, $L_{s} / h$, of 1.5 to 6.0

- Longitudinal reinforcement is generally placed symmetrically in the cross sections, with steel ratio, $\rho$, (longitudinal steel area to gross concrete area, $A_{s} / A_{g}$ ) ranging from 0.015 to 0.043

- Spacing of transverse reinforcement, $s / d$, ranging from 0.1 to 0.6 , where $s$ is the tie spacing and $d$ is the cross-section bending depth (measured from the centroid of tensile reinforcement to the extreme compression fiber)

- Transverse reinforcement ratio, $\rho_{s h}$, ranging from 0.002 to 0.02 , where $\rho_{s h}$ is the ratio of transverse steel to concrete areas, $A_{s h} / s b$

Note that the proposed modeling parameters are generally median $\left(50^{\text {th }}\right.$ percentile $)$ estimates

\subsubsection{Post-Yield Hardening Stiffness}

Referring to Figure 2-6, the following median hardening ratio of $M_{u} / M_{y}$ is recommended for both the monotonic backbone and first-cycle envelope curves:

$$
M_{u} / M_{y}=1.13
$$

A single value is recommended for both, because the limited number of monotonic tests did not allow for distinguishing between the monotonic and cyclic envelop values. Because Equation 6-4 was derived directly from column global forcedeformation relations that include bar-slip deformations, it accounts for the inelastic bar-slip softening effects. 


\subsubsection{Plastic Rotation Parameter}

In contrast to the effective stiffness and strength equations, where there are some underlying relationships based on mechanics, the equation to determine rotation capacity to the peak point, $\theta_{p}$, is largely based on empirical evidence. The monotonic-curve rotation parameter (measured in radians) between yield and the peak moment resistance is given by the following:

$$
\theta_{p}=0.155(0.16)^{v}\left(0.02+40 \rho_{s h}\right)^{0.43}(0.54)^{0.01 c_{\text {unis }} f_{c}^{\prime}}
$$

where $v$ is the axial load ratio, $P / A_{c} f_{c}^{\prime}, \rho_{s h}$ is the hoop reinforcement ratio, and $c_{\text {units }}$ is a unit conversion ratio (1.0 when $f_{c}^{\prime}$ is in $\mathrm{MPa}$ and 6.9 when in ksi). The experimental data used to develop Equation 6-5 are limited to tests of columns with symmetrical reinforcement. To model members with non-symmetric reinforcement, Equation 6-5 can be multiplied by the following correction term (Fardis and Biskinis, 2003), based on the ratio of the normalized reinforcement ratios of compressive, $\rho$, and tensile, $\rho$, steel:

$$
\theta_{p(\text { non-symmetric })}=\left[\frac{\max \left(0.01, \frac{\rho^{\prime} f_{y}}{f_{c}^{\prime}}\right)}{\max \left(0.01, \frac{\rho f_{y}}{f_{c}^{\prime}}\right)}\right]^{0.225} \theta_{p(\text { symmetric })}
$$

\subsubsection{Post-Peak Rotation Parameter}

The parameters considered in the development of an equation for predicting postpeak rotation parameter, $\theta_{p c}$, are axial load ratio, transverse steel ratio, rebar buckling coefficient, stirrup spacing, concrete strength, and longitudinal steel ratio. In contrast to the calibration of other parameters where data from over 250 tests were used, there are only 15 tests that were loaded to large enough deformations to provide data to characterize the post-peak descending portion of response. Considering the trends of the limited data, the proposed equation is based on the axial load ratio, $v$, and transverse steel ratio, $\rho_{s h}$ :

$$
\theta_{p c}=(0.76)(0.031)^{v}\left(0.02+40 \rho_{s h}\right)^{1.02} \leq 0.10
$$

As this equation was created based on limited test data, 0.10 was selected as a conservative upper bound for $\theta_{p c}$. More recent research on large-scale ductile columns shows that this 0.10 upper bound is conservative (Nojavan et al., 2016).

\subsubsection{Cyclic Behavior and Energy Dissipation}

Once the envelope monotonic moment-rotation curve is defined in a concentrated hinge model (Figure 6-1), the cyclic behavior and degradation parameters need to be specified for nonlinear dynamic analyses. The nonlinear modeling parameters discussed here were calibrated using modeling parameters extracted from an 
experimental database. However, as most tests in the database were conducted using cyclic loading protocols, monotonic envelope parameters were inferred by projecting from the experimental data using the Ibarra et al. (2005) peak-oriented analytical model, which is implemented in the open source analytical software OpenSees (McKenna et al., 2000).

The calibrated monotonic envelope parameters proposed by Haselton et al. (2016) are therefore best used with the damage accumulation algorithms of the Ibarra et al. analytical model to achieve strength and stiffness degradation due to cyclic damage progression. Cyclic energy dissipation can be quantified using a reference energy dissipation capacity based on the yield moment, $M_{y}$, and the plastic rotation parameter, $\theta_{p}$ (Ibarra et al., 2005):

$$
E_{t}=\lambda^{\prime} M_{y} \theta_{p}
$$

Past research has shown that cyclic degradation is highly dependent on the axial load level with cyclic energy dissipation capacity decreasing with increased axial load (CEB, 1996). The following equation reflects this trend

$$
\lambda^{\prime}=(30)(0.3)^{v}
$$

The energy dissipation capacity was calibrated using the Ibarra et al. (2005) peakoriented analytical model (Haselton et al., 2016), including an energy-based degradation parameter that is applied to the strength, post-peak strength, unloading stiffness, and reloading stiffness hysteretic parameters. The Ibarra et al. damage algorithms use a damage parameter, $\beta_{i}$, by which these hysteretic parameters are adjusted at each drift excursion, $i$ :

$$
\beta_{i}=\left(\frac{E_{i}}{E_{t}-\sum_{j=1}^{i} E_{j}}\right)^{c}
$$

where following Ibarra et al.'s formulation, $c$ can be taken in the range of 1.0 to 2.0. Haselton et al. used a value of $c=1.0$ for calibrating the modeling parameters. Each time the element crosses the point of zero load, the Ibarra et al. model reduces the strength, post-peak strength, and unloading stiffness parameters by the factor $\left(1-\beta_{i}\right)$. The model also increases the reloading stiffness parameter by the factor $\left(1+\beta_{i}\right)$.

The cyclic degradation relations presented in this section can be used with any software possessing a peak-oriented material model with consistent energy-based damage accumulation rules. Otherwise, the cyclic degradation parameters of the chosen model should be calibrated against representative test data. 


\subsubsection{Relationships between the Monotonic and Cyclic Backbone Curves}

The rotation capacities provided by Equations 6-5 through 6-7 pertain to the monotonic backbone curve. Where the analysis model does not directly simulate cyclic degradation, the analysis model should be based on the first-cycle envelope, which is analogous with the generalized response curve that has traditionally been used in ASCE/SEI 41-13. As described in PEER/ATC 72-1 (PEER/ATC, 2010) it is proposed to define the first-cycle envelope model parameters by modifying the monotonic backbone parameters as follows:

$$
\begin{aligned}
\theta_{p}{ }^{*} & =0.7 \theta_{p} \\
\theta_{p c}{ }^{*} & =0.5 \theta_{p c}
\end{aligned}
$$

\subsubsection{Comparison of Proposed Model Parameters to Test Data}

Nonlinear analysis model simulations using the proposed model parameters were compared to the full-scale reinforced concrete columns shown previously in Figure 6-3 (data and figures are after Nojavan et al., 2014; 2016). Five different loading protocols were applied in the testing to represent the range of behavior from monotonic loading to cyclic loading with many cycles. Overall, the good agreement between the measured and calculated cyclic responses demonstrate the reliability of the proposed effective stiffnesss and monotonic backbone model parameters (Equations 6-2 to 6-7), combined with the cyclic degradation model and parameters (Equations 6-8 to 6-10) as implemented in the Ibarra et al. formulation.

\subsubsection{Comparison of Proposed Model to ACI 369 Model}

The latest update to ACI 369 (ACI, 2017) includes proposed revisions to the generalized moment-rotation parameters "a" and "b," which represent the first-cycle envelope response. The revised parameters are given in equation form, rather than the traditional tabular form of ASCE/SEI 41. Similar to the equations proposed in this report, the new ACI 369 parameters are related to the column axial load ratio and confining hoop steel ratio. In addition, the "a" parameter is a function of the shear demand in the hinge region.

The $\theta_{p}{ }^{*}$ parameter (given by Equations 6-5 and 6-11) is compared to the ACI 369 "a" parameter in Figure 6-5a. The values plotted as a function of axial load and compared for two hoop steel ratios $\left(\rho_{s h}=0.002\right.$ and 0.006$)$. The ACI 369 parameters are further distinguished between low and high shear demands $\left(V_{y e} / V_{C o l O E}=0.3\right.$ and 0.6). In general, the values compare well up to axial load ratios of about 0.5 , beyond which the ACI 369 values are smaller than the values proposed in this report. Part of the difference lies in the functional form of the parametric equations. Further, the ACI 369 values distinguish between shear demands, which is not reflected in the proposed equations. The ultimate rotation, given as the sum of $\theta_{p}{ }^{*}+\theta_{p c}{ }^{*}$ (given by 
Equations 6-5 through 6-11) and the ACI 369 " $b$ " parameter, are compared in Figure 6-5b.

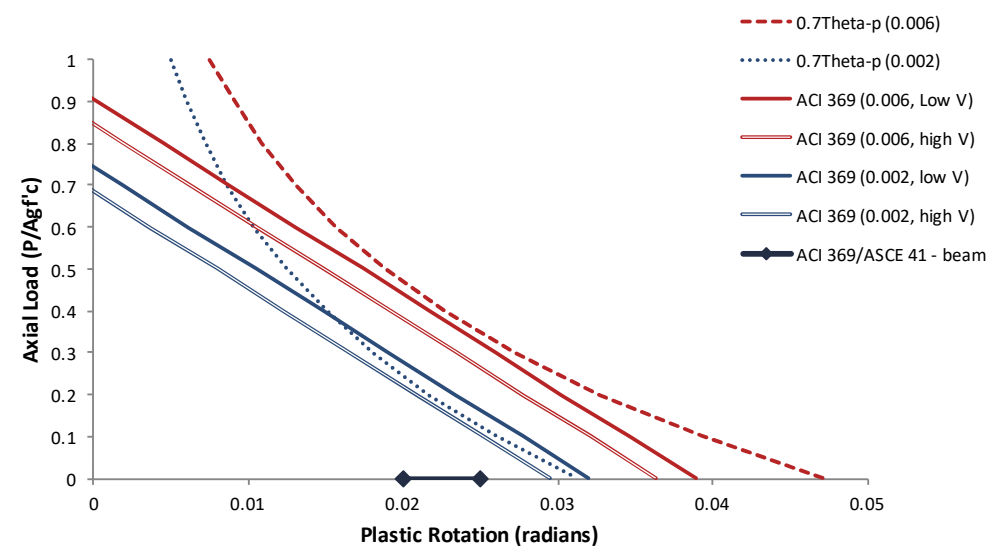

(a)

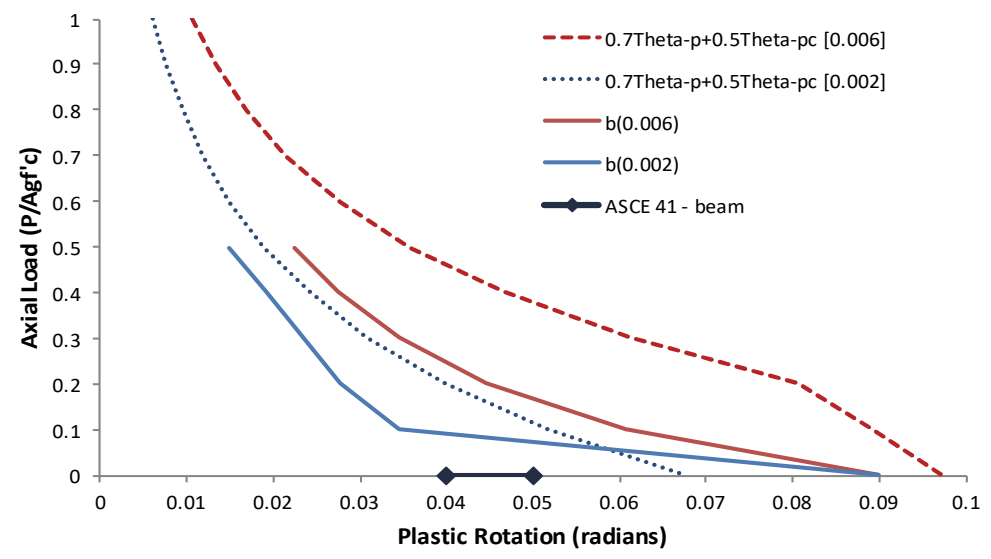

(b)

Figure 6-5 Comparison of first-cycle envelope parameters to those specified in ACI 369 (2017) for: (a) cyclic plastic rotation, $\theta_{p}{ }^{*}$; and (b) cyclic total rotation to zero resistance $\left(\theta_{p}{ }^{*}+\theta_{p c}{ }^{*}\right)$.

Note that the ACI 369 parameters are only defined for axial load ratios below 0.5 . In this case the differences are considerably larger, as compared to the data in Figure 6-5a. For columns with high confinement steel $\left(\rho_{s h}=0.006\right)$ and axial load ratios up to 0.5 , the proposed equations give ultimate rotations that are about 1.5 times larger than the ACI 369 counterparts. The differences are less for the case with lower confinement steel $\left(\rho_{s h}=0.006\right)$. In large part, this may be due to the difference in how the parameters were inferred from the test data.

\subsubsection{Uncertainties in the Component Modeling Parameters}

The relations for the modeling parameters proposed by Haselton et al. (2016) were derived empirically based on a compiled database of experiments. The median and logarithmic standard deviation values for the ratio of predicted response parameters 
to the experimentally derived parameters are presented in Table 6-2. The logarithmic standard deviation, $\sigma_{L N}$, is analogous to the coefficient of variation (COV).

Table 6-2 Prediction Uncertainties and Bias in Proposed Equations (Haselton et al., 2016)

\begin{tabular}{lcccc}
\hline \multicolumn{1}{c}{ Equation } & & $\begin{array}{c}\text { Median (predicted / } \\
\text { observed) }\end{array}$ & & $\sigma_{L N}$ \\
\cline { 1 - 2 } Post-yield Hardening Stiffness: $M_{d} / M_{y}$ (Eq. 6-4) & & 1.01 & 0.10 \\
Plastic Rotation Parameter: $\theta_{p}$ (Eq. 6-5) & 1.02 & & 0.61 \\
Post-peak Rotation Capacity: $\theta_{p c}$ (Eq. 6-7) & 1.00 & & 0.72 \\
Cyclic Energy Dissipation Capacity: $\lambda^{\prime}$ (Eq. 6-9) & & 1.08 & & 0.60 \\
\hline
\end{tabular}

In general, the variability in the nonlinear parameters is large, owing to the complexity of the inelastic response and the empirical nature of the calibration process. The values in Table 6-2 are not needed for most nonlinear analysis because the focus of the modeling is to have a mean-based model. However, these values are useful (1) to recognize the large inherent variability in nonlinear response modeling; and (2) as the basis for conducting probabilistic analyses of structural response or a sensitivity study.

\subsubsection{Beam Model Parameters}

In general, model parameters for beams are similar to those for columns. However, the composite action between beams and slabs in flexure needs to be considered, along with non-symmetry in the top and bottom beam reinforcement.

In general, it is recommended to evaluate the effective width of the slab using longstanding guidelines from ACI 318 that are also specified in ASCE/SEI 41 and ACI 369. These specify that the effective width of slab on each side of the beam web (or effective flange width) for strength and stiffness calculations be taken equal to the smallest of the provided flange width, eight times the flange (or slab) thickness, half the distance to the next web, and one-fifth the beam span length.

Once the effective width of the slab acting compositely with a beam is determined, beam modeling parameters can be determined based on column parameters but using a T-section as follows:

- Elastic stiffness. The moment-curvature approach as well as the empirical stiffness reduction relations can be used to estimate beam elastic stiffness. Because the response of T-beams is not symmetric with respect to bending direction, the average of the negative and positive bending flexural stiffnesses can be used to define the effective stiffness of the member between the hinges. 
- Moment strengths. Yield and peak moment strengths can be determined as for columns, but should include the slab steel within the effective flange width.

- Plastic rotation parameter. Beam plastic rotation parameter can be determined using Equation 6-5 with adjustments made based on Equation 6-6 for nonsymmetric reinforcement layouts. The slab steel within the effective flange width should be included in the reinforcement ratios when using Equation 6-6.

- Post-peak rotation capacity and hysteretic strength degradation. In the absence of experimental evidence to determine these properties for beams, provided that the beam hinge regions have seismically conforming hoop confinement steel, it is suggested to use the same formulae as for columns.

\subsubsection{Modeling of Beam-Column Joints}

Two approaches are considered in this report because they are numerically robust and allow for computationally efficient modeling. The approaches are: (1) adjusting the stiffness of beam and column offsets within the joint panel regions; and (2) introducing a rotational hinge at the beam-column intersection.

\subsubsection{Stiffness Adjustment of Beam and Column Offsets}

Birely et al. (2012) evaluated the accuracy of several commonly prescribed jointoffset stiffness recommendations, including those from ASCE/SEI 41 and ACI 369. The evaluations were performed considering test data from 45 interior beam-column joint sub-assemblages, and as such, coupled beam, column, and joint stiffness models. Birely et al. observed reasonably accurate sub-assemblage stiffnesses when the stiffness provisions of ASCE/SEI 41-13 were used. For special moment frames, in which column flexural strength exceeds that of beams by at least $20 \%$ at joints, ASCE/SEI 41-13 stipulates that column offsets within the joint panel should be modeled as essentially rigid, while those of the beams should be modeled as having the same elastic stiffness as the beams (Figure 6-6). These joint modeling recommendations do not account for softening effects due the slip of beam and column longitudinal bars within the joint region. Therefore, they should be used in conjunction with the ASCE/SEI 41 beam and column stiffness provisions or other frame member models that include the effects of bar-slip within the joint-panel region (Equation 6-1, described previously). 


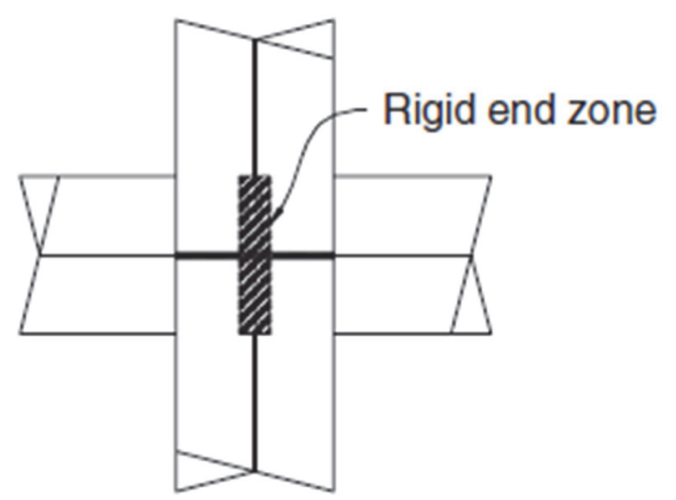

Figure 6-6

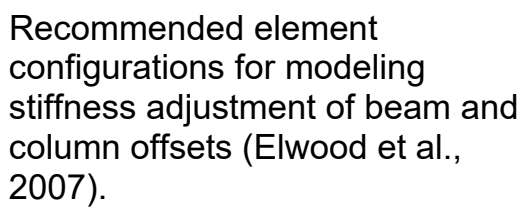

Birely et al. (2012) also considered beam and column member stiffnesses that are generally lower than those of ASCE/SEI 41-13 and more consistent with stiffness recommendations proposed by Kwon (2016). If the beam and column stiffness provisions proposed by Kwon (2016) are used (Equations 6-2 and 6-3), it is recommended to model both column and beam offsets within joints as essentially rigid. In this case as well, the softening effect of bar-slip within the joint panel region is modeled implicitly through the reduced effective stiffness of the connected beams and columns.

\subsubsection{Rotational Hinge at the Beam-Column Intersection}

This type of modeling approach includes rigid offset links to model the geometry of the joint and locations of the interface between the joint and the beams and columns. A rotational hinge is provided at the center of the joint model to account for the shear behavior of the joint (Figure 6-7). Spring models exist for both modern and older, non-ductile joints, though the research has focused more on older joints. A summary of available joint models is provided in Jeon et al. (2014) and several of the models define a joint shear stress versus strain response that can be used for modeling (e.g., Altoontash, 2004). Celik and Ellingwood (2008) provide guidance on transforming a joint shear stress versus strain relationship to a joint moment versus rotation relationship for use with the rotational hinge and element configuration shown in Figure 6-7.

However, given the reasonable accuracy obtained by adjusting beam and column offsets, and the added complexity of implementing and calibrating the hinge-type models, the simplified effective end offset approach is recommended unless significant joint damage is anticipated and a more detailed modeling approach is warranted. 


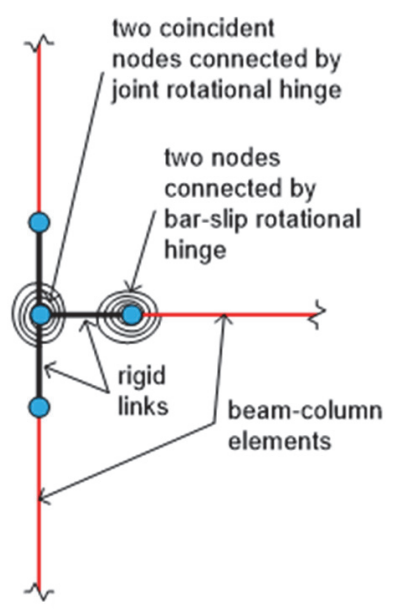

Figure 6-7 Recommended element configurations for modeling joint center rotational spring in a planar frame (Celik and Ellingwood, 2008).

\subsubsection{Modeling of Gravity System Connections}

As noted previously, although gravity framing systems comprised of flat slabs on concrete columns may not be considered part of the lateral system for design, the stiffness and strength these systems provide may be significant and should be considered in analysis. From a positive standpoint, including the slab-column frame action may reduce the calculated drift demands. On the other hand, the slab-column frame action may induce overturning axial forces into gravity columns that could potentially be unconservative. This section provides guidance for modeling the slabcolumn connection using a concentrated hinge modeling approach.

\subsubsection{Slab-Beam Strength}

If the slab-beam flexural strength is lower than the connection punching shear strength, inelastic response is assumed to concentrate at the hinges located at the ends of the slab-beam elements. The flexural strength of the hinges at slab-beam ends is estimated assuming that only the reinforcement within the column strip, as defined in ACI 318-14, is effective in resisting moments caused by lateral loads. The analysis should account for the effects of moments caused by gravity within the column strip by applying gravity loads before lateral demands are applied. A good reference is not available for the post-yield moment-strength hardening ratio $\left(M_{u} / M_{y}\right)$, so in the absence of other data, the value of $M_{u} / M_{y}=1.13$, as specified for beams and columns (Equation 6-4), is also recommended for slab-beams.

The slab-column connection punching-shear behavior is modeled by a rotational spring that can transfer moment between the slab and the column, as shown in Figure 6-8. 


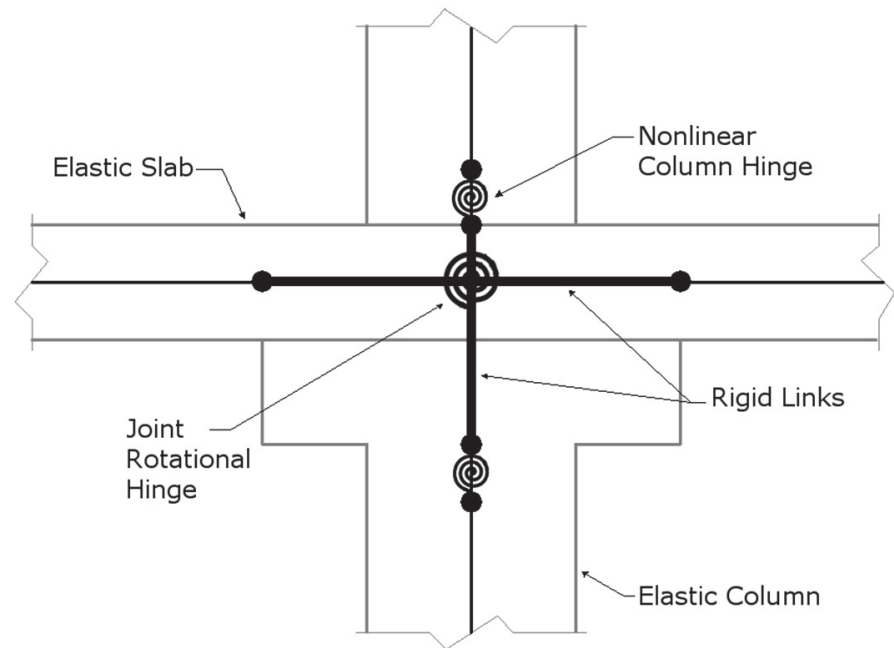

Figure 6-8 Idealized slab-column joint model.

This spring can be idealized as a rigid-plastic spring of strength $M_{f} / \gamma_{f}$.

$$
\gamma_{f}=\frac{1}{1+\left(\frac{2}{3}\right) \sqrt{\frac{b_{1}}{b_{2}}}}
$$

where $M_{f}$ is capacity of the connection to transfer moments through flexure (and should be computed using expected material properties) and $\gamma_{f}$ is the fraction of the moment assumed to be transferred through flexure in conventional design. The value of $\gamma_{f}$ can be computed as follows using Equation 8.4.2.3.2 of ACI 318-14, where $b_{1}$ is the column dimension in the direction of loading and $b_{2}$ is the column dimension in the orthogonal direction.

For interior slab-column connections, $M_{f}$ is limited by the strength of reinforcement (top and/or bottom) within $2.5 \mathrm{~h}$ from column faces perpendicular to the direction of moment $M_{f}$, where $h$ is the slab thickness. For exterior slab-column connections Moehle (2015) recommends assuming $\gamma=1.0$ and calculating $M_{f}$ for a yield line "along the inside face of the column and extending at $45^{\circ}$ angle to the slab edge." This yield line is not to extend beyond $1.5 \mathrm{~h}$ from column faces perpendicular to the direction of $M_{f}$.

\subsubsection{Slab-Beam Stiffness}

Estimates of the stiffness of the slab column system are obtained with assumptions different from assumptions used to estimate strength. To estimate stiffness, the effective width of the slab is recommended by Hwang and Moehle (2000) as follows:

$$
\text { effective width }=2 c+\frac{L}{3}
$$


where $L$ is the slab span perpendicular to the direction of bending and effective width and $c$ is the column cross-sectional dimension in the direction of $L$.

For exterior columns and for bending moment parallel to the slab edge, this effective width should be reduced by $50 \%$. These estimates of effective width were obtained assuming the slab-column joint is rigid.

Slab bending stiffness should be reduced to account for cracking. Bending stiffness of reinforced concrete slabs supported by square columns and having spans of similar length in both directions (with $f_{c}^{\prime}=\sim 4 \mathrm{ksi}$ and $f_{y}=60 \mathrm{ksi}$ ) may be assumed to be a fraction $\beta$ of the bending stiffness of the gross section (Hwang and Moehle, 2000) as follows:

$$
\beta=4 \frac{c}{L} \geq \frac{1}{3}
$$

Prestressed slabs should be expected to have reduced cracking and larger stiffness. ASCE/SEI 41-13 recommends $\beta=0.5$ for post-tensioned slabs (Kang and Wallace, 2005).

\subsubsection{Connection Plastic Rotation at Punching-Shear Strength Loss}

ASCE/SEI 41-13 provides modeling parameters for the plastic rotation at punching shear failure, which can be used in the torsional spring of the slab-column connection. The modeling parameters are provided in tabular from in ASCE/SEI 41-13 for prestressed and non-prestressed slabs. The table in ASCE/SEI 41-13 also distinguishes between slabs with and without continuity steel through a column connection, while the provided modeling parameters deliver estimates of mean response for slabs with continuity steel and mean minus one standard deviation for slabs without continuity steel. Elwood et al. (2007) discusses the development of the modeling parameters for slab-column connections.

Once punching shear occurs, there is an associated strength loss, with the postpunching behavior depending on whether the slab-column connection has bottom continuity reinforcing through the connection. The scope of this chapter is for modern buildings (which would have bottom continuity steel going through the joint), so a punching shear failure should result in strength loss but not a vertical instability problem for the building; therefore, provided that the strength loss is modeled, the occurrence of punching shear would not pose a safety risk. If continuity steel were not present, and the possibility of vertical instability existed, then the drift at vertical instability would need to be estimated and a deformationcontrolled acceptance criterion would need to be enforced. 


\subsection{Fiber-Type Component Models}

Fiber-type components can be used to provide more explicit modeling of cross section response and P-M-M interaction. In this approach, a cross section is discretized into fibers describing the section geometry, each having a uniaxial nonlinear material model. Fiber sections may be assigned to one or more sections (integration points) along the length of the frame element. At each section, the stresses are integrated over the fibers resulting in the overall forces acting on the cross section. Two general approaches to combining the fiber cross section models with the frame elements are through either fixed-length fiber-hinge formulations or distributed plasticity formulations that employ either displacement or force (moment) interpolation functions.

Although the fiber-based formulation relies on first principles and is well suited to modeling the effects of concrete cracking/crushing and reinforcing bar yielding due to section axial and bending actions, the fiber models cannot directly model other phenomena, such as reinforcing bar slip, buckling, or fracture. Although steel stressstrain models can be empirically calibrated to account for slip, buckling, and fracture behavior in fiber models, this often leads to numerical issues due to strain localization. If high levels of nonlinear degradation are being modeled, including modes like rebar buckling and fracture, then the concentrated hinge model may be preferred.

In concept, fiber models have the capability to simulate axial member lengthening due to concrete cracking, reinforcing bar yielding, and the resulting shift in the cross section neutral axis. However, intentional or accidental constraint to axial lengthening can lead to inaccurate results, such as when fiber models are connected to floor diaphragms that are modeled with rigid kinematic constraints. Therefore, care should be taken to not over constrain the axial deformations of a fiber beam fiber beam-column model.

\subsubsection{Fiber-Section Modeling}

With fully distributed plasticity element models, the fiber section locations and weights are usually selected to match optimal integration algorithms, and the user may not have the ability to control the integration weights at key locations, such as at the ends of the element. This may result in effective plastic hinge length for the end sections that is driven by the weight of the integration algorithm and the number of integration points, rather than by physical data. Alternatively, using an element with a finite length hinge zone (plastic hinge model) can partially resolve this limitation, since the user is able to provide the actual plastic hinge length over which the curvature and axial strain of the fiber section are integrated, resulting in better consistency between the global and local deformations. When using such techniques, 
it is recommended to calibrate the plastic hinge assumption, and monitor the hinge rotations during the analysis.

A simple rule for determining the plastic hinge length is to assume $l_{p}=0.5 h$, where $h$ is the depth of the section, which is reasonably accurate for moment frames with typical dimensions. Other more detailed expressions (e.g., Priestley and Park, 1987; Berry and Eberhard, 2008) have been proposed based on analyzing databases of reinforced concrete column test data. Their proposed expressions are functions of multiple parameters including member length, $l$, bar diameter, $d_{b}$, steel yield strength, $f_{y}$, and concrete strength, $f_{c}^{\prime}$. One such proposed expression by Berry and Eberhard (2008) is as follows (psi units):

$$
l_{p}=0.05 l+0.008 d_{b} f_{y} / \sqrt{f_{c}^{\prime}}
$$

When the plastic hinge length is unknown or difficult to estimate, fiber-section models with distributed plasticity can be used to estimate the extent of yielding along the element. This can occur, for example, in members with distributed large gravity loads or in members where the member strength varies along its length. Distributed plasticity models generally work well for hardening components, but if softening is experienced at the section level, numerical localization can occur and the estimated length of the yielding can become highly dependent on the integration scheme (number and weights of integration points). When distributed plasticity is used and the plastic hinge length is known, one possible modeling approach is to specify end elements with a specified length and reduced number of integration points (one or two) to explicitly control plastic hinge length, which can be used as an alternative to the plastic hinge model implementation discussed above. Alternatively, the number of integration points can be set such that the integration weights of the end fiber sections approximately match the plastic hinge length to element length ratio (Hachem, 2003). This typically requires the use of three to five integration points depending on the member slenderness.

Most distributed plasticity elements use displacement-based formulations that can represent a linear-curvature distribution along the element. When deformations localize in plastic hinge regions, multiple elements along the member's length may be warranted to better estimate the curvature distribution and improve accuracy. For a typical moment-frame beam, using three elements, two at each end (where plastic hinges typically form) and one for the rest of the beam is recommended as the minimum required mesh refinement.

The accuracy of the section response depends on the number of fibers used to discretize the section. In practice, the optimal number of fibers typically is on the order of 10 fibers within each direction of the section. The size and distribution of the fibers may be varied to obtain a higher accuracy with a smaller number of fibers, 
i.e., by using smaller fibers near the outer edges of the member where the strains are highest, and larger fibers used near the middle. Steel bars are typically represented using one fiber per bar.

\subsubsection{Fiber Material Modeling}

In typical reinforced concrete section, three uniaxial material models are usually used to model steel reinforcement, confined concrete, and unconfined concrete.

\subsubsection{Steel Reinforcement}

Typically, the steel stress-strain curve should have the following:

- Initial modulus representing elastic response. This modulus is also used for unloading from a plastic state.

- Modeling of the post-yield plateau and/or strain hardening modulus.

In addition, it is helpful to capture the following effects, which generally require empirical calibration of the effective stress-strain model of the reinforcing steel to phenomenologically capture the observed member response behavior:

- Bauschinger effect, where the plastic deformation of the steel material in one direction affects the subsequent response in the opposite direction. This can affect the cyclic response of the member and help with accurate simulation of pinching and unloading behavior.

- Cyclic hardening of the steel material through a combination of isotropic and kinematic hardening.

- Bond-slip behavior by adjustment of the steel stress-strain behavior in the fiber model to capture bond-slip and strain penetration of reinforcing bars into anchorage zones, such as beam-column joints and foundations. This is usually best implemented in special fiber end-sections.

- Bar buckling and fracture behavior, which is a function of the longitudinal bar diameter, tie spacing, and concrete crushing. Note that bar buckling is usually not a concern in seismically detailed special moment frame members at building drifts of $3 \%$ to $4 \%$ normally encountered for design. However, bar buckling and fracture may lead to degradation at larger drifts.

- Reduction of ultimate (fracture) strain due to low-cycle fatigue.

Figure 6-9 shows a commonly used steel stress-strain model by Menegetto and Pinto (1973) that includes the Bauschinger effect. Some structural analysis software support such highly nonlinear models, while other software offer simple bilinear models with kinematic or isotropic hardening, or a combination of the two. The basic input parameters for such models are usually the yield stress, $f_{y}$, and modulus of 
elasticity, $E_{s}$. Some models require the strain at onset of hardening, $\varepsilon_{s h}$, which can range from 0.005 to 0.015 with a typical value of 0.01 , strain hardening modulus, $E_{s h}$, which can range from $0.021 \times E_{s}$ for $f_{y}=60 \mathrm{ksi}$ to $0.042 \times E_{s}$ for $f_{y} \geq 90 \mathrm{ksi}$ (ACI 408R-03, 2003). Where required, ultimate strain at fracture, $\varepsilon_{s u}$, can be taken as 0.12 for \#10 bars and smaller and 0.09 for \#11 bars and larger, but is recommended to reduce these to 0.09 and 0.06 , respectively, to capture the likelihood of early fracture due to buckling or low-cycle fatigue (Caltrans, 2006). The values for $\varepsilon_{s h}, E_{s h}$, and $\varepsilon_{s u}$ can vary with the grade and size of the streel reinforcement.

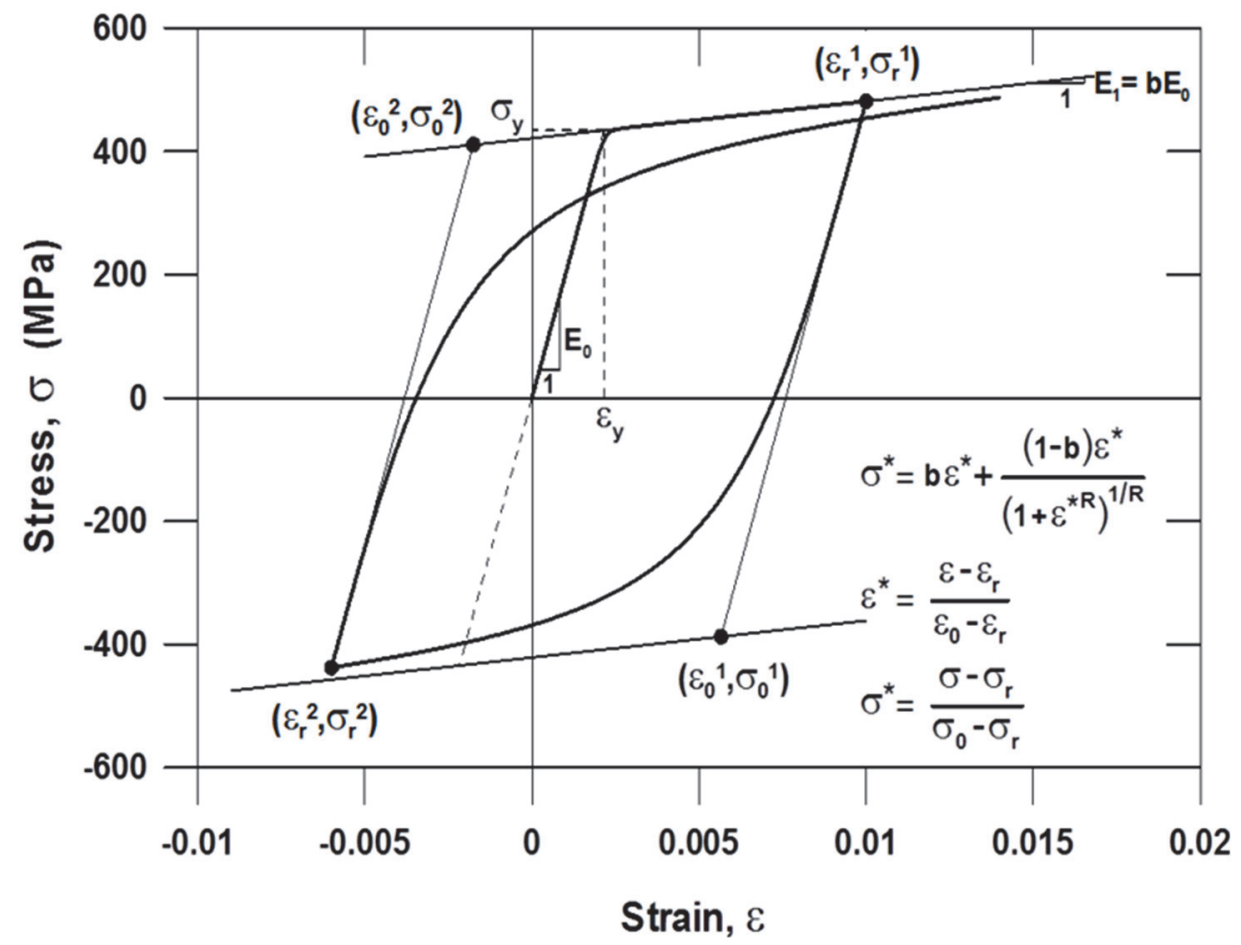

Figure 6-9 Cyclic stress-strain model for steel (Menegetto and Pinto, 1973).

Table 6-3 provides a simple set of recommendations for how $\varepsilon_{s h}$ and $E_{s h}$ vary with steel yield strength. The modeling of strain hardening is important for predicting monotonic and cyclic post-yield strength gains, and its omission can be unconservative since it can result in an underestimation of force demands on forcecontrolled member actions.

Table 6-3 Steel Reinforcing Hardening Onset Strain $\varepsilon_{s h}$ and Modulus $E_{s h}(\mathrm{ACl}$ 408R-03)

\begin{tabular}{cccc}
\hline$f_{y}(\mathrm{ksi})$ & $\varepsilon_{\mathrm{sh}}$ & $E_{\mathrm{sh}}$ \\
& 0.0086 & $0.021 E_{s}$ \\
75 & 0.0035 & $0.025 E_{s}$ \\
$\geq 90$ & 0.0035 & $0.042 E_{s}$ \\
\hline
\end{tabular}

Note: $E_{s}=29,000 \mathrm{ksi}$ 


\subsubsection{Confined and Unconfined Concrete - Compressive Stresses}

The concrete stress-strain curve typically exhibits a hardening stress-strain segment followed by a softening segment. The negative slope can be steep for unconfined concrete, whereas confined concrete can exhibit very stable response up to relatively large strains. There are several models that can be used for modeling concrete uniaxial behavior (e.g., Mander et al., 1988; Chang, 1994; and Popovics, 1973). The following is a summary of important stress-strain model parameters:

- Definition of maximum compressive strength and corresponding strain

- Nonlinear ascending branch to capture change in effective modulus due to concrete micro-cracking under load

- Post-peak descending branch whose slope (rate of degradation) reflects the level of transverse confinement

- Definition of maximum tensile strength and corresponding strain, possibly including strain softening response

- Reliable modeling of cyclic behavior through loading and unloading rules

The unconfined concrete strength, used to determine the concrete stress-strain model, should be taken as the expected concrete compressive strength. Mander et al. (1988) and other studies (e.g., Park el al., 1982; Sheikh and Uzumeri, 1982; Ahmad and Shah, 1982; Kappos, 1991; Saatcioglu and Razvi 1992) provide recommended values and equations for estimating key parameters of confined and unconfined concrete stress-strain curves including the peak confined concrete stress, $f_{c c}^{\prime}$, peak unconfined and confined strains, $\varepsilon_{c o}$ and $\varepsilon_{c c}$, spalling strain, $\varepsilon_{s p}$, and ultimate confined strain, $\varepsilon_{c u}$. Typical $\varepsilon_{c o}$ values for unconfined concrete range from 0.002 to 0.003 , and is usually taken as 0.002 . The spalling strain, $\varepsilon_{s p}$, is usually taken as $2 \varepsilon_{c o}$. The values for $\varepsilon_{c c}$ and $\varepsilon_{c u}$ depend on the level of confinement. Typical values of $\varepsilon_{c c}$ generally range between 0.004 and 0.01 , but can be as high as 0.05 for highly confined columns.

Figure 6-10 shows a stress strain model for confined and unconfined concrete for monotonic stress-strain in compression (Mander et al., 1988), where the model parameters are defined by Equations 6-17 through 6-26. A full implementation for response history analysis requires additional details about tension behavior (discuss in the next section), in addition to loading/unloading and cyclic degradation rules for cyclic behavior. Common structural analysis software typically do not have the capability to model exactly the backbone shape, and as illustrated in Figure 6-10, the response is usually approximated using an approximate bilinear or trilinear backbone curve. 


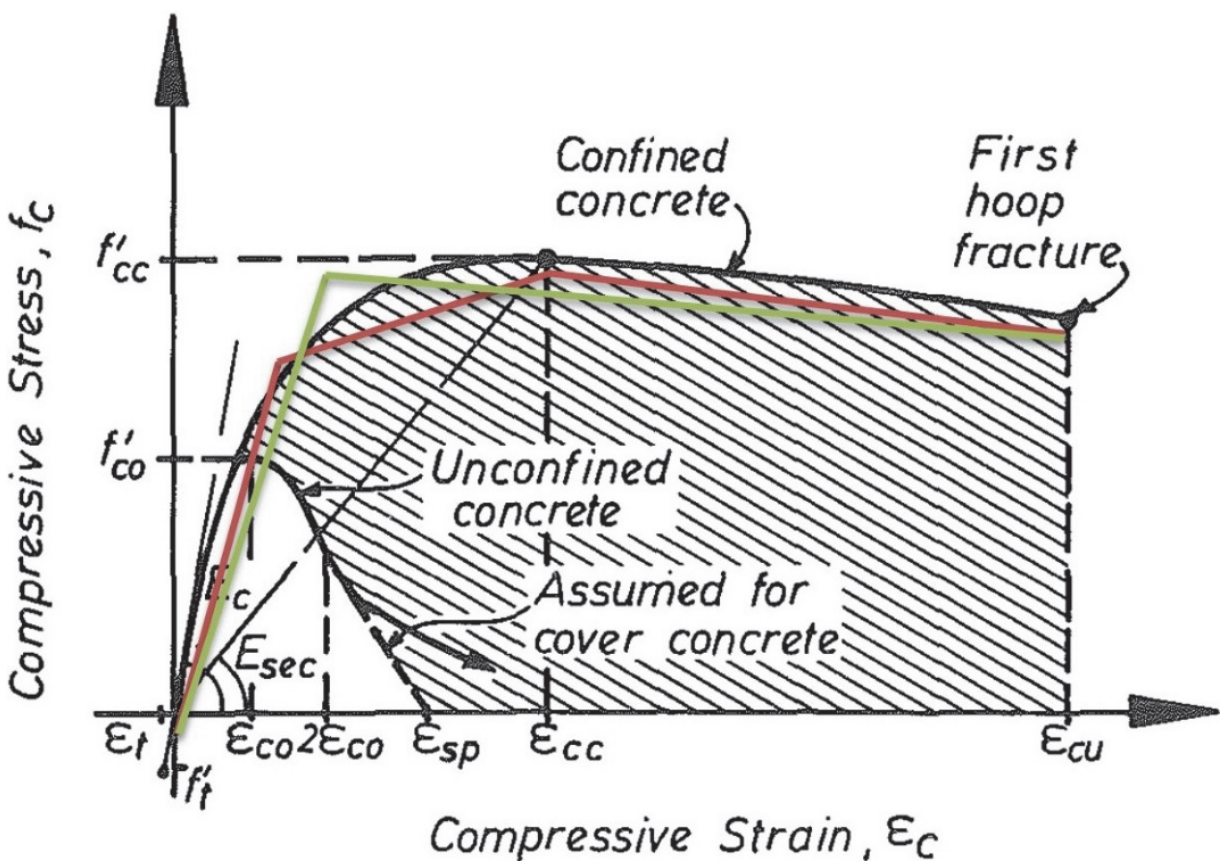

Figure 6-10 Stress-strain model for confined and unconfined concrete (Mander et al. 1988), with simplified bilinear and trilinear approximations.

$$
\begin{gathered}
f_{c}=\frac{f_{c c}^{\prime} x r}{r-1+x^{r}} \\
x=\frac{\varepsilon_{c}}{\varepsilon_{c c}} \\
r=\frac{E_{c}}{E_{c}-E_{\mathrm{sec}}} \\
E_{c}=57,000 \sqrt{f_{c o}^{\prime}}(\mathrm{psi}) \\
E_{\mathrm{sec}}=\frac{f_{c c}^{\prime}}{\varepsilon_{c c}} \\
\varepsilon_{c c}=\varepsilon_{c o}\left[1+5\left(\frac{f_{c c}^{\prime}}{f_{c o}^{\prime}}-1\right)\right] \\
f_{l}^{\prime}=\frac{1}{2} k_{e} \rho_{s} f_{y h}
\end{gathered}
$$

where $f_{c c}^{\prime}$ and $\varepsilon_{c c}$ are the maximum confined concrete stress and corresponding strain, respectively, $f_{c o}^{\prime}$ and $\varepsilon_{c o}$ are the unconfined concrete strength and corresponding strain, $\varepsilon_{c o}$ may be taken as 0.002 , and $f_{l}^{\prime}$ is the effective lateral confining stress on the concrete, and $\rho_{s}$ and $f_{y h}$ are the transverse reinforcement ratio and yield strength, respectively, and the confinement effectiveness coefficient, $k_{e}$, is defined below. Note that the confining stress, defined in Equation 6-23, is specified along a given 
direction, where it is possible to have different confining stresses $f_{l x}^{\prime}$ and $f_{l y}^{\prime}$ along the $\mathrm{x}$ and y directions of a section, respectively.

The confinement effectiveness coefficient, $k_{e}$, is defined by the following equation, where expressions for general and circular sections are provided in Mander et al.

(1988):

$$
k_{e}=\frac{\left(1-\sum_{i-1}^{n} \frac{\left(w_{i}^{\prime}\right)^{2}}{6 b_{c} d_{c}}\right)\left(1-\frac{s^{\prime}}{2 b_{c}}\right)\left(1-\frac{s^{\prime}}{2 d_{c}}\right)}{1-\rho_{c c}}
$$

where $\rho_{c c}$ is the ratio of the area of longitudinal reinforcement to area of the core of the section, and parameters $w_{i}^{\prime}, b_{c}, d_{c}$ and $s^{\prime}$ are defined in Figure 6-11.

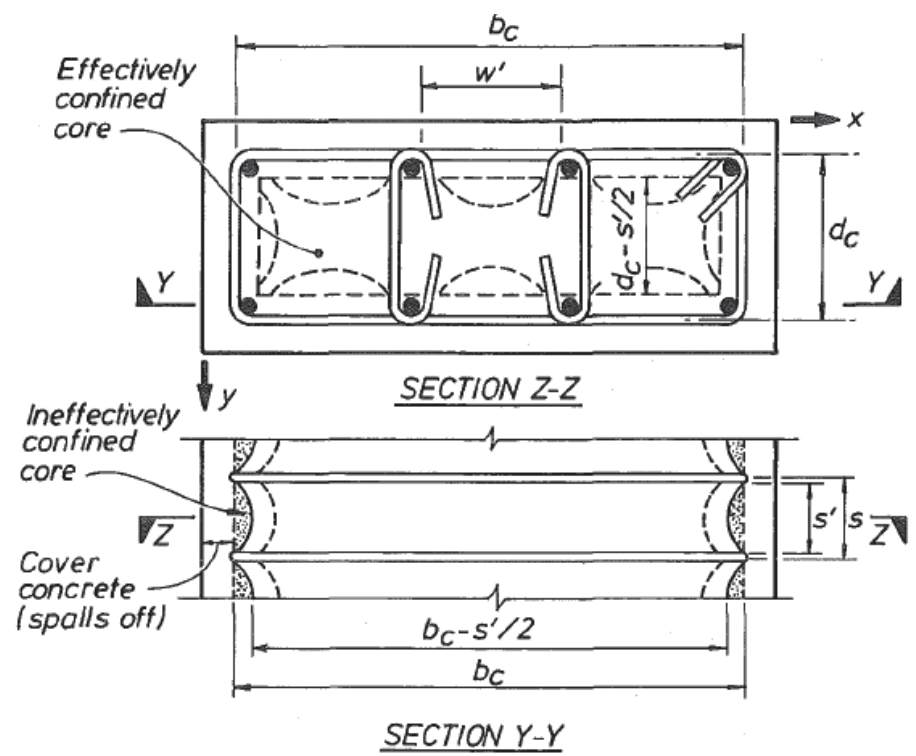

Figure 6-11 Confined core for a rectangular hoop reinforced section (Mander et al., 1988).

The peak strength of the confined concrete, $f_{c c}^{\prime}$, can be computed from the following equation where $f_{c o}^{\prime}, f_{l x}^{\prime}$ and $f_{l y}^{\prime}$ are evaluated using Figure 6-12, where $f_{l x}^{\prime}=f_{l y}^{\prime}$ :

$$
f_{c c}^{\prime}=f_{c o}^{\prime}\left(-1.254+2.254 \sqrt{1+\frac{7.94 f_{l}^{\prime}}{f_{c o}^{\prime}}}-2 \frac{f^{\prime}}{f_{c o}^{\prime}}\right)
$$

Mander et al. propose a fairly complex method for computing $\varepsilon_{c u}$, which relies on solving an energy balance equation. In contrast, Paulay and Priestley (1992) propose the following equation as a simpler and conservative estimate of the ultimate confined concrete strain, where $\varepsilon_{s u}$ is the ultimate tensile strain of the transverse steel:

$$
\varepsilon_{c u}=0.004+1.4 \rho_{s} f_{y h} \varepsilon_{s u} / f_{c c}^{\prime}
$$




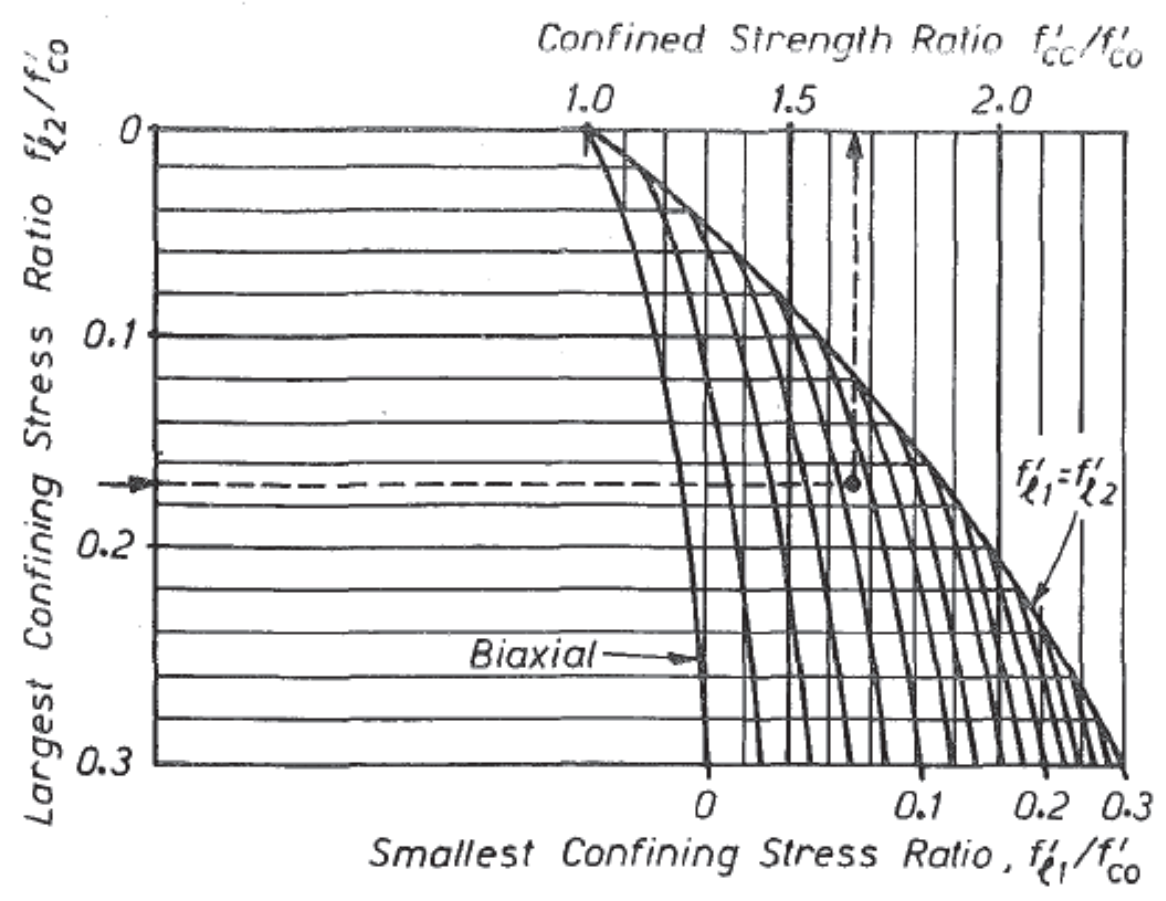

Figure 6-12 Confined strength determination from lateral confining stresses for rectangular section (Mander et al., 1988).

\subsubsection{Confined and Unconfined Concrete -Tensile Stresses}

The tension portion of the concrete stress-strain curve is typically modeled either as pre-cracked (i.e., no tensile capacity) or as linear-elastic up to the tensile cracking stress, after which the concrete suddenly loses all its capacity. The concrete tension strength generally does not have a significant impact on section strength, but it can be important for determining the uncracked stiffness, although, assuming the concrete as uncracked can overestimate the initial stiffness of the structure since it ignores cracking due to service loads, wind loads, or shrinkage. After cracking, the concrete between the cracks continues to bond to the reinforcement, which increases the flexural and axial stiffness of the member through a phenomenon known as "tension stiffening." Although the effect generally reduces with additional cracking, it can be important for estimating the effective stiffness at small deformations. The "tension stiffening" effect can be simulated by using a gradual descending branch for the concrete tensile stress-strain response. Figure 6-13 shows a simplified model which uses two parameters: $\alpha_{1}$, which describes the immediate stress loss after cracking and ranges from 0.4 to 0.7 , and $\alpha_{2}$ which is dependent on the reinforcement ratio and ranges from 12 to 22. Kaklauska and Ghaboussi (2001) and Torres el al. (2004) provide recommendations for $\alpha_{1}$ and $\alpha_{2}$. Alternatively, values of $\alpha_{1}=0.5$ and $\alpha_{2}=14$ may be assumed. 


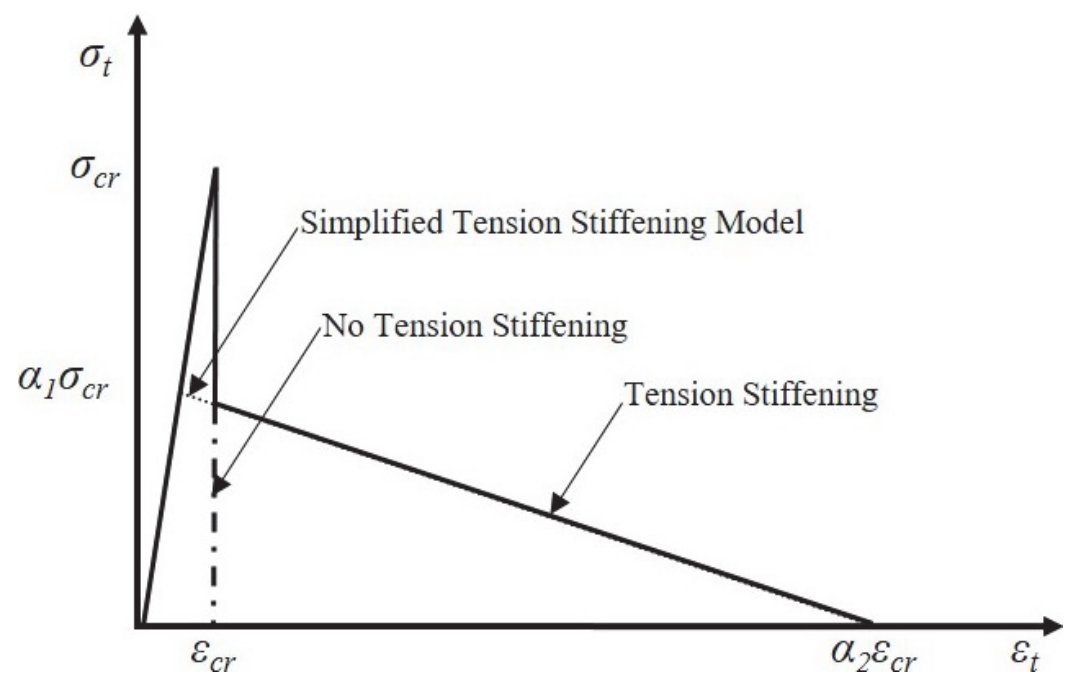

Figure 6-13 Concrete tension material models with and without tension stiffening.

\subsubsection{Modeling Bond-Slip and Shear Deformations}

The conventional formulation of fiber-section elements model flexural deformations by integrating moment-curvature relations over member length, which does not consider bond-slip or shear deformations. As noted previously, bond-slip at the anchorage of longitudinal reinforcement into adjacent beam-column joints or column footings can add significantly to deformations. Shear deformations are less significant, but should also be considered.

It should be noted that rotation capacities, such as those in the concentrated hinge models presented previously or in ASCE/SEI 41 and ACI 369, typically include total deformations and rotations. Therefore, deformation demands determined by any analysis should incorporate deformations due to flexure, shear, and bar slip to be consistent with deformation acceptance criteria.

\subsubsection{Bond-Slip Deformations}

Bar-slip deformations can be modeled by zero-length fiber sections that have the same geometry and discretization as the fiber sections of the adjacent element. All material model parameters of the zero-length sections should be identical to those of the adjacent frame elements, but with strain values multiplied by the scaling factor, $r_{y}=\left(S_{y} / \varepsilon_{y}\right)$; where $S_{y}=$ bar slip at yield, as given by the following equation where $\varepsilon_{y}=$ longitudinal bar yield strain (Ghannoum and Moehle, 2012):

$$
S_{y}=\frac{l_{s p} \varepsilon_{y}}{2}
$$

Although anchorage conditions and bar properties can influence the bond stresses developed and the resulting bar-slip component of deformation, past studies have shown that assuming a uniform bond stress in the anchorage regions of $9.6 \sqrt{f_{c}^{\prime}}$ (psi), 
$\left(0.8 \sqrt{f_{c}^{\prime}}(\mathrm{MPa})\right.$ reproduces the bar-slip flexibility at member ends with reasonable for anchorage in footings and beam-column joints (Elwood and Eberhard, 2009; FIB, 2010; Kwon, 2016). This value is generally recommended so long as sufficient anchorage length is provided to prevent severe bond deterioration and pullout. This value is applicable for all traditional anchorage details, i.e., straight-bar, hooked-bar and headed-bar anchorage. Using this uniform bond stress, the effective strain penetration depth $l_{s p}$ at bar yield is given by the following equation:

$$
l_{s p}=\frac{1}{38.4(3.2)} \frac{f_{y}}{\sqrt{f_{c}^{\prime}}} d_{b} \text { psi }
$$

Alternatively, instead of zero-length fiber sections, discrete elastic rotational springs can be added at the ends of beam and column fiber-section elements to simulate barslip rotations. A yielding bond-slip spring in series with a fiber element will cause inelastic deformations to localize in only one of the two elements. Therefore, it is recommended to only introduce elastic bond-slip springs with a rotational stiffness, $K_{S E}$, as specified in the following equation:

$$
K_{S E}=\frac{2 M_{y}}{\phi_{y} l_{s p}}
$$

where $M_{y}$ is the yield moment of the section, $\phi_{y}$ is the corresponding yield curvature, and $l_{s p}$ is the yield penetration length as specified in Equation 6-29. By keeping the bond-slip spring elastic, this leaves the inelastic bar-slip softening to be accounted for in the fiber elements through softening the steel-fiber hardening slope.

\subsubsection{Shear Deformations}

Shear deformations in concrete beams and columns are typically small and account for 5 to $10 \%$ of the total deformations. In fiber-section implementations, elastic shear stiffness is typically specified directly at the element level. For well detailed beams and columns that are flexurally dominated, a shear stiffness of $0.4 E_{c} A_{w}$ may be used. If the element implementation does not account for shear deformation, or if shear nonlinearity is expected, shear deformations can be introduced through shear springs within the element or at element ends with a lateral stiffness corresponding to the equivalent elastic shear rigidity along the element length. 


\section{Chapter 7}

\section{Flexure-Controlled Reinforced Concrete Walls}

\subsection{Overview of Geometry and Behavior}

\subsubsection{Geometry and Reinforcement Layout}

Reinforced concrete walls are used commonly to resist lateral forces from earthquakes and wind. They provide high stiffness and strength under service-level loading, can be detailed to provide ductility under design-level earthquake loading, and can take on many configurations to accommodate architectural requirements. Figure 7-1 shows continuous vertical configurations for walls, and Figure 7-2 shows common cross-sectional configurations. Note that vertical irregularities may lead to concentrated damage and collapse; discussion of system deficiencies including vertical irregularities and torsional eccentricities are beyond the scope of this chapter.

Wall cross section is largely a function of architectural layout. Mid- to high-rise buildings typically have walls located around a central service/elevator core as illustrated in Figure 7-3. Typically, the core walls are arranged to form a closed box, with $\mathrm{C}$, $\mathrm{L}$, or rectangular piers coupled with coupling beams extending over wall openings (Figure 7-2d). In lower rise buildings or other buildings without a central elevator core, it is more common to have walls distributed throughout the floor plate; the location and shape of the walls is typically constrained by the architectural layout. In many cases, asymmetric wall shapes are used (the most common asymmetric section is in the shape of an "L") as well as planar walls (Figure 7-3b).

In the case of isolated walls, wall demands include shear, bending and axial; these are idealized in Figure 7-4. In current design, wall section geometry is determined to meet strength and stiffness requirements. Typically, wall thickness is determined to meet shear capacity limits and wall length is determined to meet flexural strength requirements or lateral stiffness requirements.

Design of flexural concrete walls used as the primary seismic load-resisting system includes:

- Determination of longitudinal reinforcement required for flexural demand. Often much of this reinforcement is concentrated at the ends of the walls as this is the optimal location for flexural resistance and these regions referred to as boundary 
elements in ACI 318-14, Building Code Requirements for Structural Concrete and Commentary (ACI, 2014).

- Sizing and detailing of confinement reinforcement.

- Design of the horizontal reinforcement to meet the shear demand: ACI 318-14 allows use of a larger strength-reduction factor to calculate shear strength if the shear demand corresponds to plastic hinging.

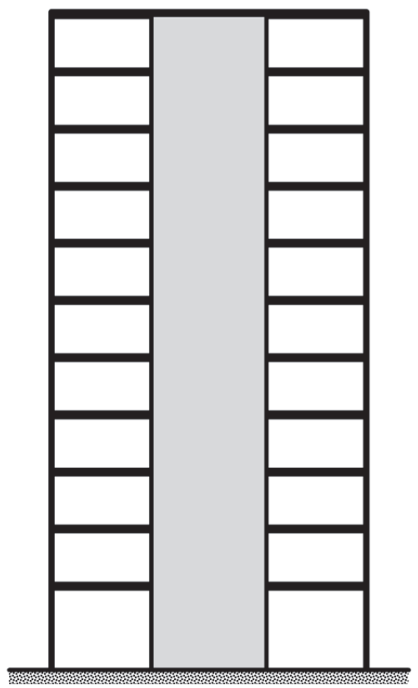

(a) Cantilever wall

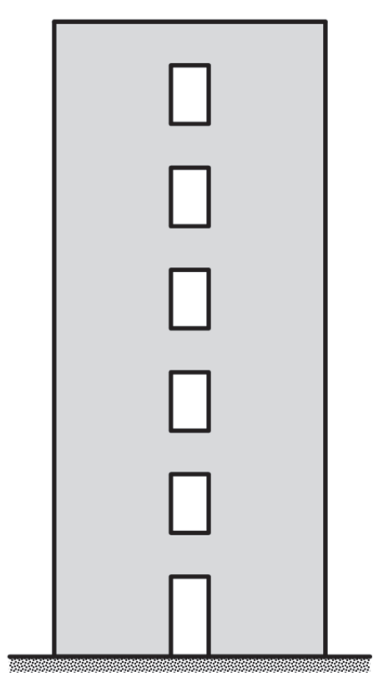

(b) Coupled wall

Figure 7-1 Common vertical wall-system configurations (Moehle et al., 2011).

(a) Rectangular shape

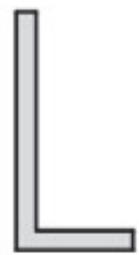

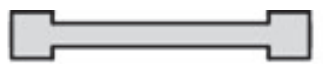

(b) "Bar bell" shape

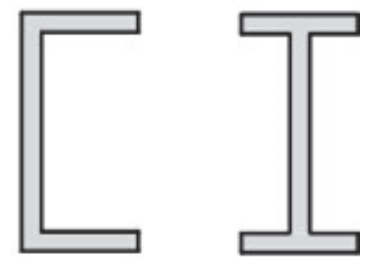

(c) Flanged walls in common T, L, C, and I shapes

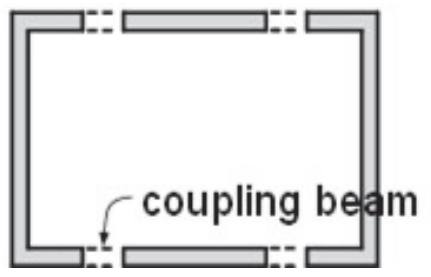

(d) Possible configuration of a core-wall

Figure 7-2 Common wall cross-sectional configurations (Moehle et al., 2011).

In regions of high seismicity, large volumes of confining reinforcement are required in "boundary element" regions that are expected to experience high compression 
demands under earthquake loading (Figure 7-5). For planar walls and some flanged walls, an efficient design for flexural strength is placement of a large volume of longitudinal reinforcement in the boundary elements of the wall. For nonplanar walls that resist loading in orthogonal directions, longitudinal reinforcement may be uniformly distributed; walls with uniformly distributed reinforcement typically require heavily confined boundary elements but may require confinement of the entire cross section. Since splicing reinforcement at the base of the wall facilitates construction, splices are common at this location and ACI 318-14 allows longitudinal reinforcement to be spliced at the base of the wall where the maximum flexural demand typically occurs.

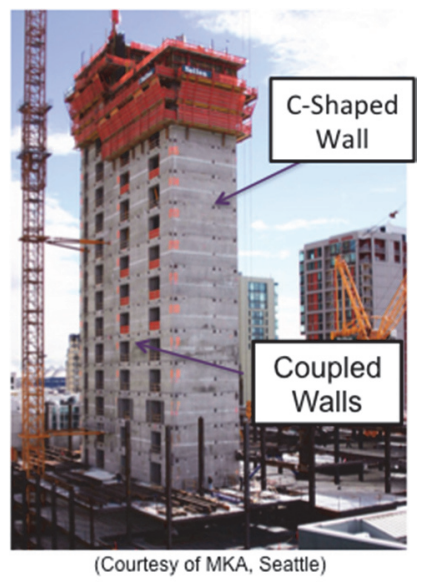

(a) Core wall

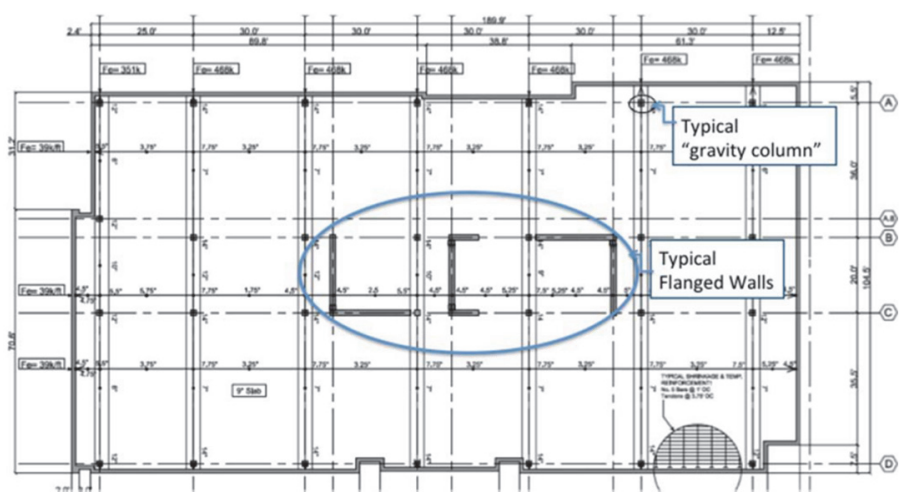

(b) Distributed planar and flanged walls

Figure 7-3 Core vs. distributed planar walls.

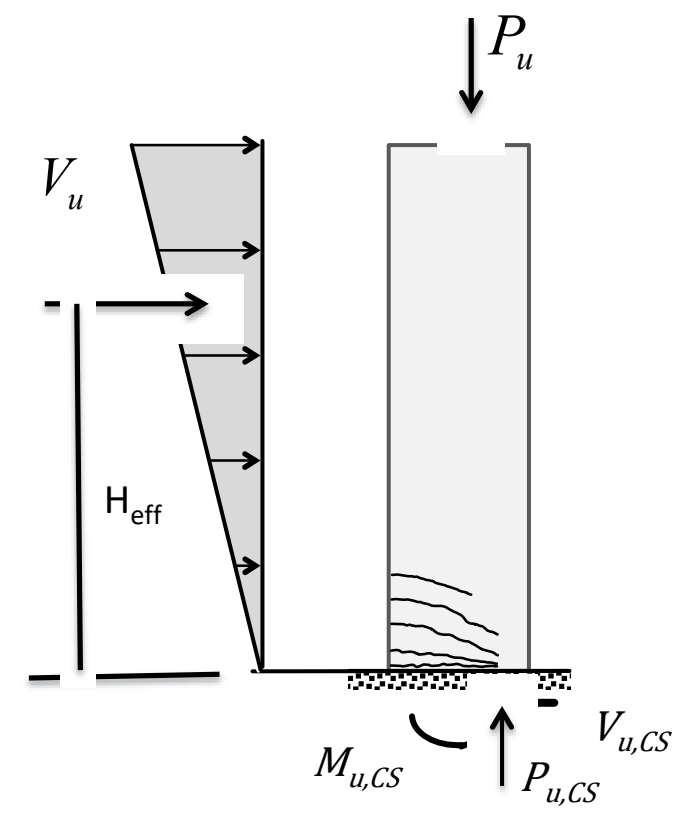

(a) Equivalent lateral forces (ELF)

(b) Wall elevation with reactions

Figure 7-4 Demands on individual wall or pier (Moehle et al., 2011). 


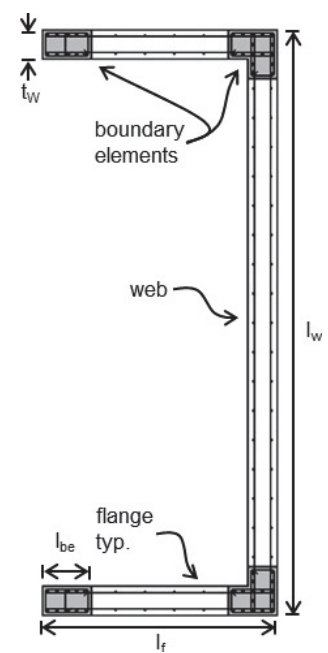

(a) Flanged wall

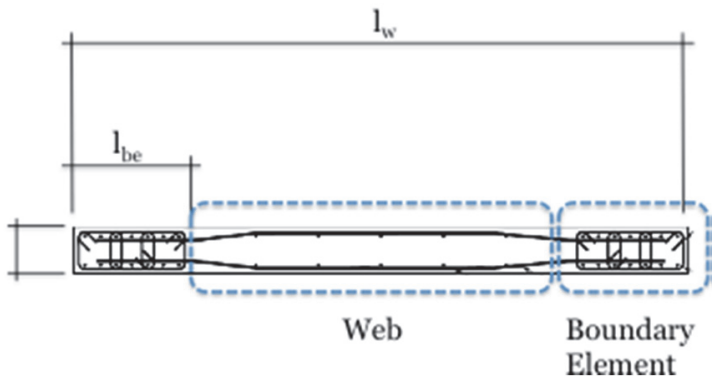

(b) Planar wall

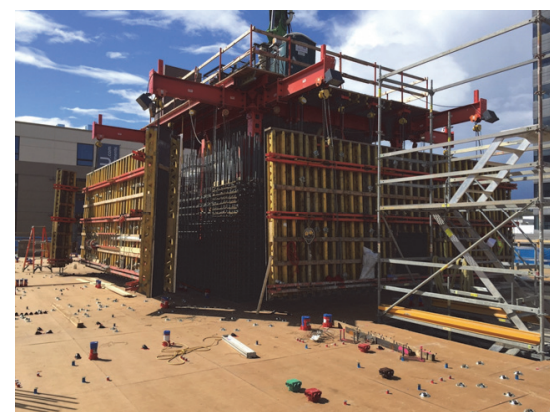

(c) Wall reinforcement

Figure 7-5 Reinforcement configuration of wall cross sections: (a) from Behrouzi (2016); (b) from Welt (2015); and (c) courtesy of KPFF Engineers, Seattle.

In contrast to the boundary elements, the interior of the wall web or flange, typically referred to as the wall web, are lightly reinforced with reinforcement ratios equal to or just larger than $0.25 \%$ common (Section 7.3 ). This interior section of the wall is expected to sustain large shear strains through diagonal cracking and straining of the interior steel. In flanged walls, it is not uncommon for wall webs to sustain significant spalling and crushing of the concrete; however, most walls are not confined in this region.

\subsubsection{Behavior under Lateral and Gravity Loading}

Typically, flexure-controlled walls have shear demands that do not exceed shear capacity as defined by ACI 318-14 and are slender with height-to-length aspect ratio that exceed 1.5. This chapter focuses on reinforced concrete walls with height-tolength aspect ratios that exceed 1.5, that sustain shear demands less than the defined shear stress limit in ACI 318-14, and therefore exhibit flexure-controlled response. It should be noted that due to higher mode effects, some tall, slender walls, though designed to be limited by flexural capacity, can sustain shear forces that exceed the design shear demand, even if calculated from plastic hinging. If this is the case, it is possible for the shear demand to exceed the design shear capacity.

Figure 7-6 provides typical response and damage state of selected types of flexural walls. The measured response indicates the following characteristics: (1) a flexural strength that is accurately estimated using standard methods (e.g., plane-section analysis); (2) minimal hardening under increasing drift demand (i.e., final strength is approximately the nominal moment strength, $M_{n}$, computed using expected strengths); (3) minimal strength deterioration under multiple cycles to the same drift demand; and (4) relatively rapid strength loss with increasing drift demand after development of a failure mode. 

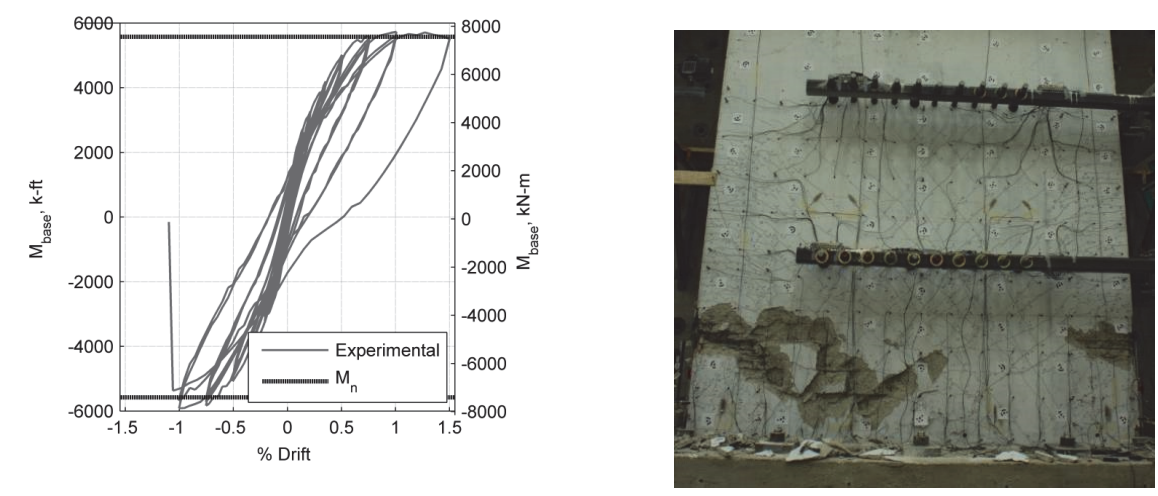

(a) Planar Wall (Lowes et al., 2012)
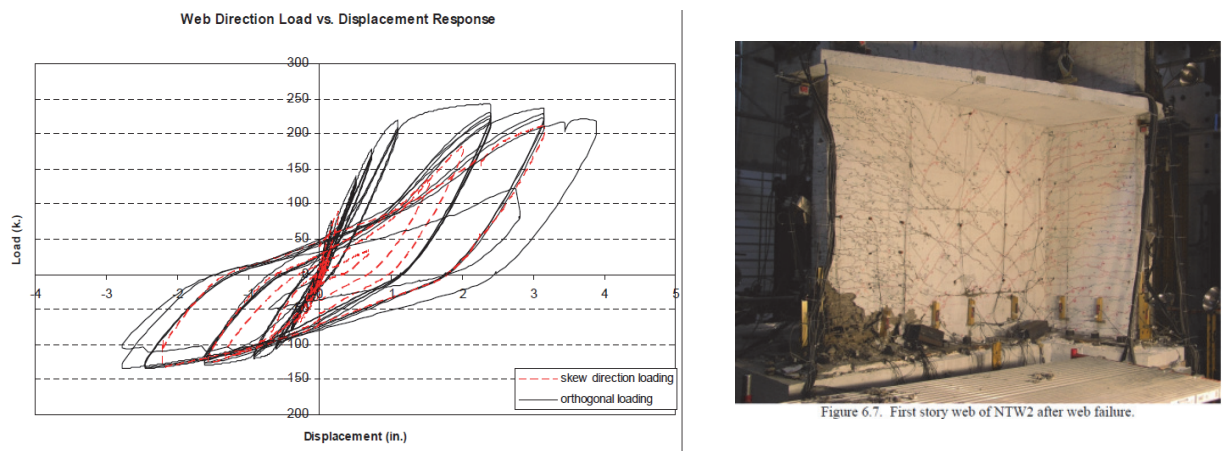

(b) Asymmetric Flanged Wall (Brueggen, 2009)
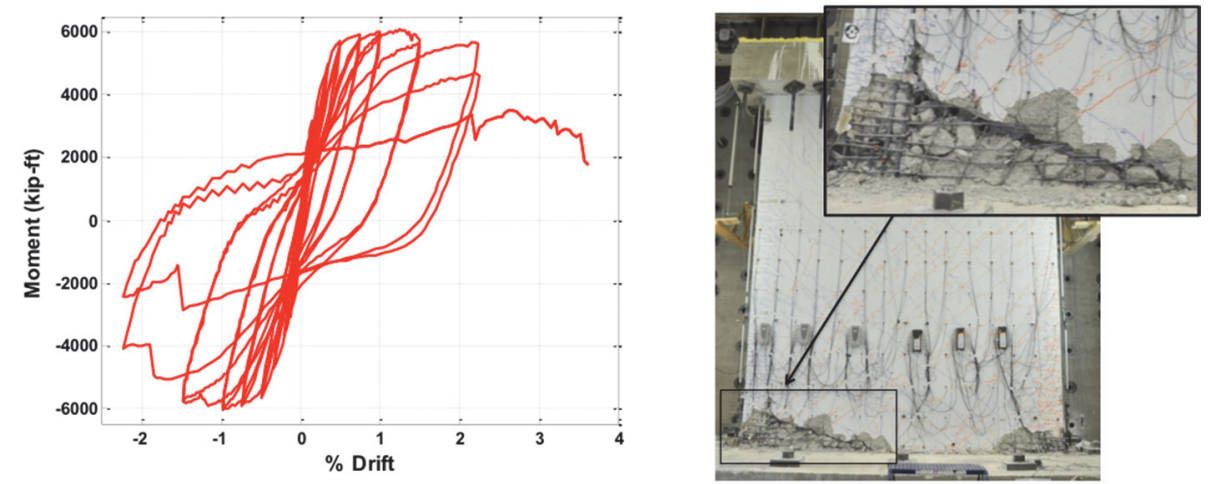

(c) Symmetric Flanged Wall (Behrouzi et al., 2015)
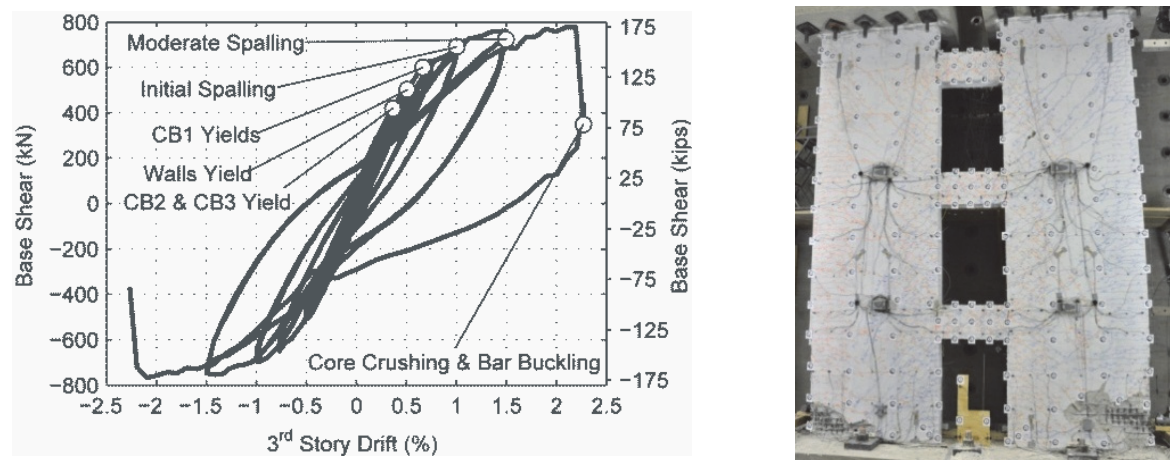

(d) Coupled Wall (Lehman et al., 2013)

Figure 7-6 Response of and damage to flexural walls. 
As show in the images in Figure 7-6, wall failure mechanisms (Birely, 2013; Whitman, 2015) results from either: (1) simultaneous concrete crushing and buckling of longitudinal reinforcement in the extreme compression region of the wall (compression-buckling failure); (2) rupture of previously buckled longitudinal reinforcement (buckling-rupture failure); or (3) concrete crushing across a large portion of the compression-region due to the combined compression demands resulting from flexural and shear loading. As shown in Figure 7-6, failure typically results in severe damage to the web and boundary element concrete, which can result in failure of the wall.

Additional information on wall performance is discussed in Section 7.3. Whitman (2015) presents an extended experimental data set characterizing the hysteretic response of planar walls and Behrouzi et al. (2015) summarize large-scale testing of flanged walls.

\subsubsection{Compilation and Evaluation of Flexural Wall Database}

The behavior of flexural walls is determined by several key design parameters. Figure 7-6 suggests that two of these parameters are cross-sectional shape and wall configuration. Other key parameters include: (1) cross sectional aspect ratio; (2) shear stress demand (normalized by $\sqrt{f_{c}^{\prime}} A_{\text {shear }}$ for $f_{c}^{\prime}$ in psi); (3) axial load ratio; and (4) boundary element confinement ratio. Table 7-1 provides histograms to describe the distribution of these design variables in an experimental test space that includes 21 planar wall tests conducted between 1976 and 2012 (Birely, 2013; Pugh, 2012). The accompanying plots indicate design variable value as a function of drift capacity, where drift capacity corresponds to $20 \%$ loss in peak strength on the backbone curve of the cyclic response. Specimens in the test space have an average effective height to length ratio of 2.4 with a coefficient of variation of $21 \%$, such that drift capacity can be considered a consistent measure across the experimental specimen space. The following observations are made for each design parameter:

- Cross Sectional Aspect Ratio. The cross sectional aspect ratio (CSAR) is easily quantified for planar walls as the ratio of the length of the wall, $l_{w}$, to the thickness of the wall, $t_{w}$. The histogram in Table 7-1 indicates that the majority of wall tests have CSAR values of 12 or less. For a wall spanning between column lines, approximated as a 25 foot span, a CSAR of 12 results in a wall thickness of $25 \mathrm{in}$. For most low- to mid-rise buildings, this thickness is much larger than that expected in an actual building, where wall thickness more typically ranges from 10 to 18 inches. The CSAR vs. drift plot indicates that walls with larger CSAR values have lesser drift capacity. Two recent analytical studies using nonlinear line element modeling (Welt, 2015) and continuum analyses (Whitman, 2015), have confirmed this relation. Additional information is provided in Sections 7.4 and 7.5. 
- Normalized Shear Stress Demand. The walls included in the database exhibited a flexural response and failure mode. However, the response and drift capacity are influenced by the normalized shear demand. The histogram indicates that most tests were subjected to normalized shear stress demands of 6 or less and walls that failed in a flexural failure mode had a maximum shear stress demand of 7. For shear stress demand less than 7, there is not an apparent relation between shear stress demand and drift. However, analytical studies that have evaluated shear stress demands larger than 6 indicate that these values can diminish the drift capacity, in particular for walls with higher CSAR values (Whitman, 2015), making shear stress demand an important variable when paired with CSAR.

- Axial Load Ratio. The majority of the specimens were subjected to axial load ratios, $P / A_{g} f_{c}^{\prime}$, of 0.1 or less. At these low values, there is little impact of the axial load ratio on the drift capacity, however wall tests that are not subjected to an axial load (i.e., the axial load ratio is 0 ) have larger drift capacities. These walls do not simulate walls in a building since all walls, even seismic walls, are subjected to a non-negligible axial load ratio. Using the drift capacities of wall tests without an axial load will result in an overestimate of deformability and ductility.

- Boundary Element Confinement Ratio. The confining reinforcement ratio varied from $0 \%$ to a high of $6 \%$ although only tests with a maximum ratio of $3.5 \%$ are included in the confinement ratio-drift plot. The data indicate that large amounts of confinement are required to increase the drift capacity beyond approximately $2.5 \%$. Most ACI 318-14 compliant and older walls will not meet this level and therefore the impact of the confinement is less significant than expected.

Table 7-1 presents distribution of parameters in the flexural wall database and their impact on drift capacity, where the red markers identify bar fracture and blue markers identify a compression failure. The table offers two important observations:

(1) many wall tests do not simulate the conditions or geometry of walls in the existing and modern infrastructure; and (2) the impact of axial load ratio and confinement on the drift capacity of flexural walls is much less than the impact of CSAR and normalized shear stress demand. The experimental data set used to generate the plots in Table 7-1 was used by Whitman (2015) to validate a finite element model. Simulation results were used to expand the data set, and simulation and experimental data were used to develop and validate the modeling procedures presented in Sections 7.4 and 7.5. 
Table 7-1 Distribution of Parameters in Flexural Wall Database and Their Impact on Drift Capacity

Distribution of Parameter in Database
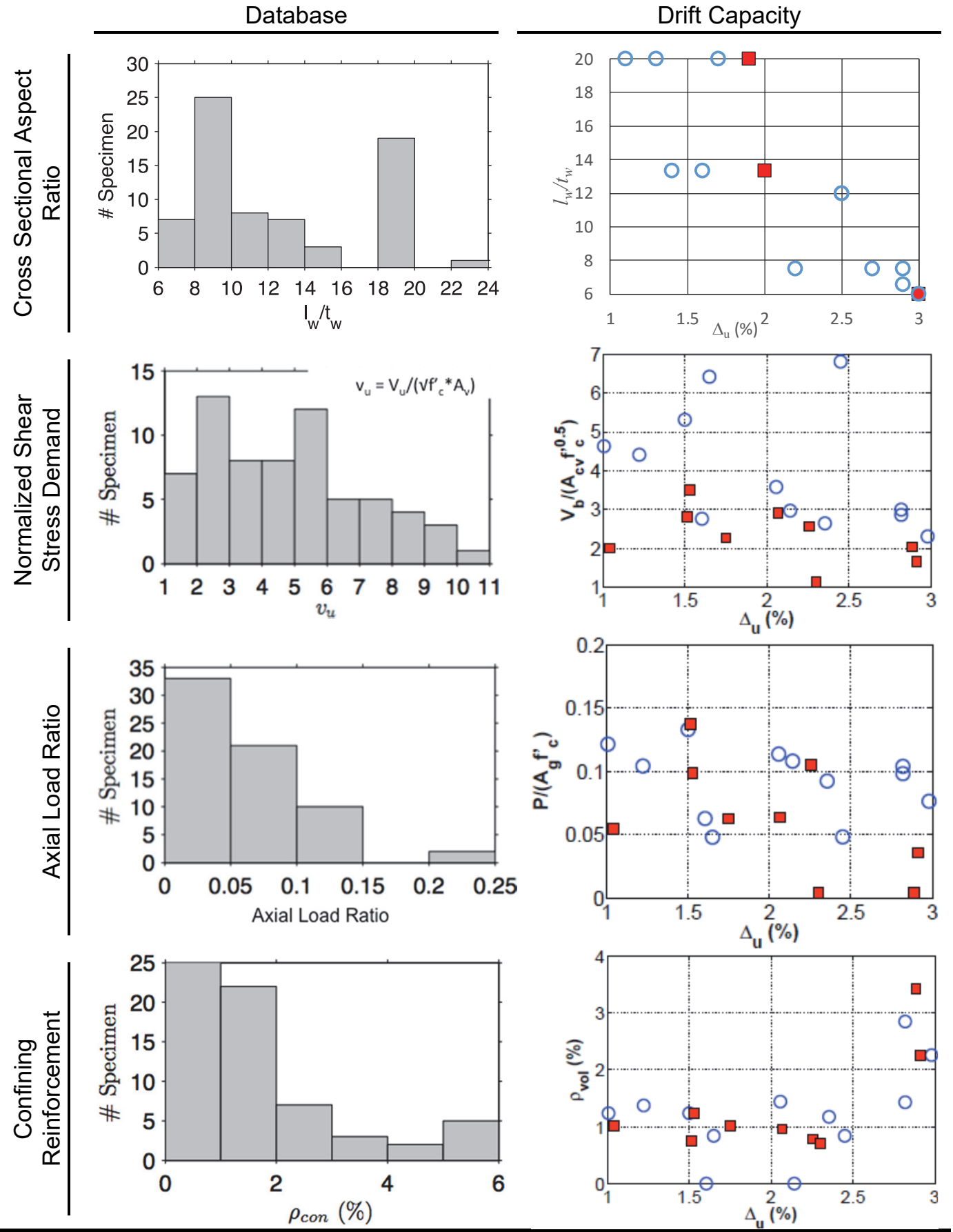

\subsection{Summary of Design Code Provisions}

The ACI 318 has addressed the seismic design of walls since the 1971 edition. In broad generalities, codified wall design provides design expressions to calculate the design strength, which includes a resistance factor, $\phi$. The flexural strength, $M_{n}$, is 
computed accounting for the axial load demand, $P_{u}$. The shear strength, $V_{n}$, is the summation of the shear strength attributed to the concrete, $V_{c}$, and the shear strength attributed to the steel, $V_{s}$. In addition, the total shear strength is limited as a function

of the concrete strength, typically expressed as $\sqrt{f_{c}^{\prime}}$. The section numbers (§) refer to the edition of the code in which the change/requirement initially appeared; these numbers do not necessary correlate to the current section numbers in ACI 318.

Fundamental changes in the seismic design of concrete structures were implemented in ACI 318 1989. The primary changes included: (1) use of a smaller strength reduction factor for shear design if shear demand is determined from lateral load analysis not from plastic analysis assuming flexural yielding at the base of the wall; (2) required material properties (i.e., A706 steel); and (3) detailing requirement of the boundary elements to increase their compressive strength and strain capacity. The following provides an overview of the primary changes including the provision number in the referenced year. Any remark marked with an asterisk (*) has been in ACI 318 since 1971 (earliest code reviewed).

\subsubsection{Vertical Reinforcement}

- Vertical reinforcement is designed to meet the flexural demands

- $\quad$ ASTM A706 steel required since 1989 (\$21.2.5.1)

- The minimum ratio, $\rho_{v}{ }^{*}$, is $0.25 \%$

- Maximum spacing limit of 18 in., introduced in 1983

- Two curtains of steel are required for walls thicker than 10 in.*

\subsubsection{Horizontal Reinforcement}

- Minimum reinforcement ratios in web, $\rho_{h}: 0.25 \% *$

- Two curtains of steel are required for walls thicker than 10 in.*

- $\quad \rho_{v} \geq \rho_{h}$ for $h_{w} / l_{w}<2$ was implemented in $1989(\$ 21.7 .3 .5)$

\subsubsection{Shear}

Historically, the shear demand, $V_{u}$, was calculated from the elastic equivalent lateral force (ELF) analysis. In 1983, the provisions permitted the designer to either calculate the shear demand resulting from plastic hinging in the wall, or, in lieu of a plastic analysis, to calculate $V_{u}$ from an elastic lateral load analysis and use a smaller strength reduction factor. The limit on maximum shear stress, $v_{n}, 8$ or $10 \sqrt{f_{c}^{\prime}}(\mathrm{psi})$, for single and multiple piers in a plane, respectively, was introduced in 1989

(§21.7.3.6 and $\S 21.7 .3 .7)$. 


\subsubsection{Boundary Elements}

Boundary elements are typically used in walls to maximize the flexural strength and to optimize steel placement. As a result, boundary elements can sustain large compressive stresses, and their seismic performance is improved with confinement sized and spaced to maximize their compressive strength and strain capacity. There are four primary parameters that are of interest in the design of boundary elements including (1) the limits by which they are required; (2) geometry (length and height) of the boundary element; (3) amount of confining steel; (4) the vertical spacing of the confining steel; and (5) the horizontal spacing of the confining steel. The history of these aspects of boundary element design is briefly presented.

- Boundary Elements Required. In codes prior to 1983, boundary elements were required if the axial load ratio was high, specifically, if $P_{e}>0.4 P_{b}{ }^{*}$, where $P_{e}$ is the axial force resulting from the earthquake load combination and $P_{b}$ is the balance load (balance axial load corresponding to yielding of the extreme tension bar and spalling of the extreme compression fiber or a strain of 0.003). In 1983 (and later versions of ACI 318) this requirement was replaced, and rather than determining the need for boundary element on the axial force, they were determine by estimating the extreme compressive stress from an elastic analysis, that is, they were required if the compressive stress in the extreme fiber exceeds $20 \%$ of the compressive strength, or $f_{c}>0.2 f_{c}^{\prime}$. ( $f_{c}$ is the stress in the extreme fiber, determined from the factored loads and gross section properties). In 1999, an additional limit was established (\$21.6.6.2) (note the compressive stress limit was retained) based on the ratio of the compression region to the wall length, or, if $c / l_{w}>1 /(600 \Delta)$, where $\Delta=\delta_{u} / h_{w}$ is the total drift of the wall, and cannot be less than $0.7 \%$.

- Boundary Element Height $\left(\boldsymbol{H}_{\boldsymbol{B} E}\right)$. In 1983, a provision was introduced permitting the termination of boundary elements. Wall for which boundary elements are required, the boundary element can be terminated if $f_{c}<0.15 f_{c}^{\prime}$ where $f_{c}$ is the stress in the extreme fiber, determined from the factored loads and gross section properties.

- Boundary Element Length $\left(\boldsymbol{l}_{\boldsymbol{B} E}\right)$. The requirement of the boundary element length (horizontal direction) was included in 1983, requiring a distance of at least the greater of $\left(c-0.1 l_{w}, c / 2\right)$ where $c$ is the neutral axis depth.

- Area. Originally (1971) the minimum area of the transverse reinforcement in a boundary element was:

$$
A_{s h}=\frac{h_{c}\left(0.12 f_{c}^{\prime} / f_{y}\right) s_{h}}{2}
$$


In 1983, the following minimum requirements were introduced (referencing the transverse reinforcement requirements in columns):

$$
\begin{gathered}
A_{s h}=\frac{0.3\left(s h_{c} f_{c}^{\prime} / f_{y}\right)\left(A_{g} / A_{c h}-1\right)}{2} \\
A_{s h}=\frac{0.9 f_{c}^{\prime}}{f_{y} s h_{c}}
\end{gathered}
$$

- Vertical Spacing. In ACI 318 1971, the maximum vertical spacing was 4 inches. In 1983 (and 1989) the vertical spacing was limited to 4 inches or $1 / 4$ of the minimum member dimension. In 1999, the vertical spacing requirements for wall boundary elements reference back to seismic column requirements (§21.4.4.2); required spacing was at least 4 inches and not greater than 6 inches, $6 d_{b}$ where $d_{b}$ is the diameter of the longitudinal reinforcing bar or $1 / 4$ of the minimum member dimension.

- Horizontal Spacing. Prior to 1983, the code did not limit this spacing. In 1983, the limit was $14 \mathrm{in}$.

In 2014, several changes were implemented following the damage to walls in the 2011 Chile earthquake. Primary changes in the confinement requirements are highlighted in bold in Table 7-2.

\begin{tabular}{|c|c|c|c|c|c|}
\hline \multirow[b]{2}{*}{$\begin{array}{l}\text { B.E. } \\
\text { Class }\end{array}$} & \multirow[b]{2}{*}{$\begin{array}{c}\text { Design } \\
\text { Par. }\end{array}$} & \multicolumn{2}{|c|}{$\mathrm{ACl} 318-08 / \mathrm{ACl} 318-11$} & \multicolumn{2}{|c|}{$\mathrm{ACI} 318-14$} \\
\hline & & $\begin{array}{c}\text { Code } \\
\text { Section }\end{array}$ & Provision & $\begin{array}{c}\text { Code } \\
\text { Section }\end{array}$ & Provision \\
\hline \multirow{6}{*}{ OBE } & Ibe,min & 21.9.6.4a & $\max \left\{\begin{array}{l}c-0.1 l_{w} \\
c / 2\end{array}\right.$ & 18.10.6.5 & $\max \left\{\begin{array}{l}c-0.1 l_{w} \\
c / 2\end{array}\right.$ \\
\hline & $b_{\min }$ & - & - & 18.10.6.4a,b & $\max \left\{\begin{array}{l}h_{u} / 16 \\
12^{\prime \prime}\end{array}\right.$ \\
\hline & $h_{\max }$ & 21.6.4.2 & $14 "$ & 18.7.5.2e & $\min \left\{\begin{array}{l}\mathbf{2 b} / \mathbf{3} \\
14 "\end{array}\right.$ \\
\hline & $S_{\max }$ & 21.6.4.3 & $8 "$ & 18.10.6.5 & $\min \left\{\begin{array}{l}6 d_{b} \\
6 "\end{array}\right.$ \\
\hline & $A_{\text {sh, min }}$ & 21.6.4.4 & No Requirement & - & No Requirement \\
\hline & $\begin{array}{l}\text { Crosstie } \\
\text { Hook Ext,'s }\end{array}$ & $2.2(7.1 .4)$ & $\max \left\{\begin{array}{l}6 d_{b} \\
3^{\prime \prime}\end{array}\right.$ & 25.3 .2 & $\max \left\{\begin{array}{l}6 d_{b} \\
3^{\prime \prime}\end{array}\right.$ \\
\hline
\end{tabular}

\section{Table 7-2 Boundary Element Requirements}




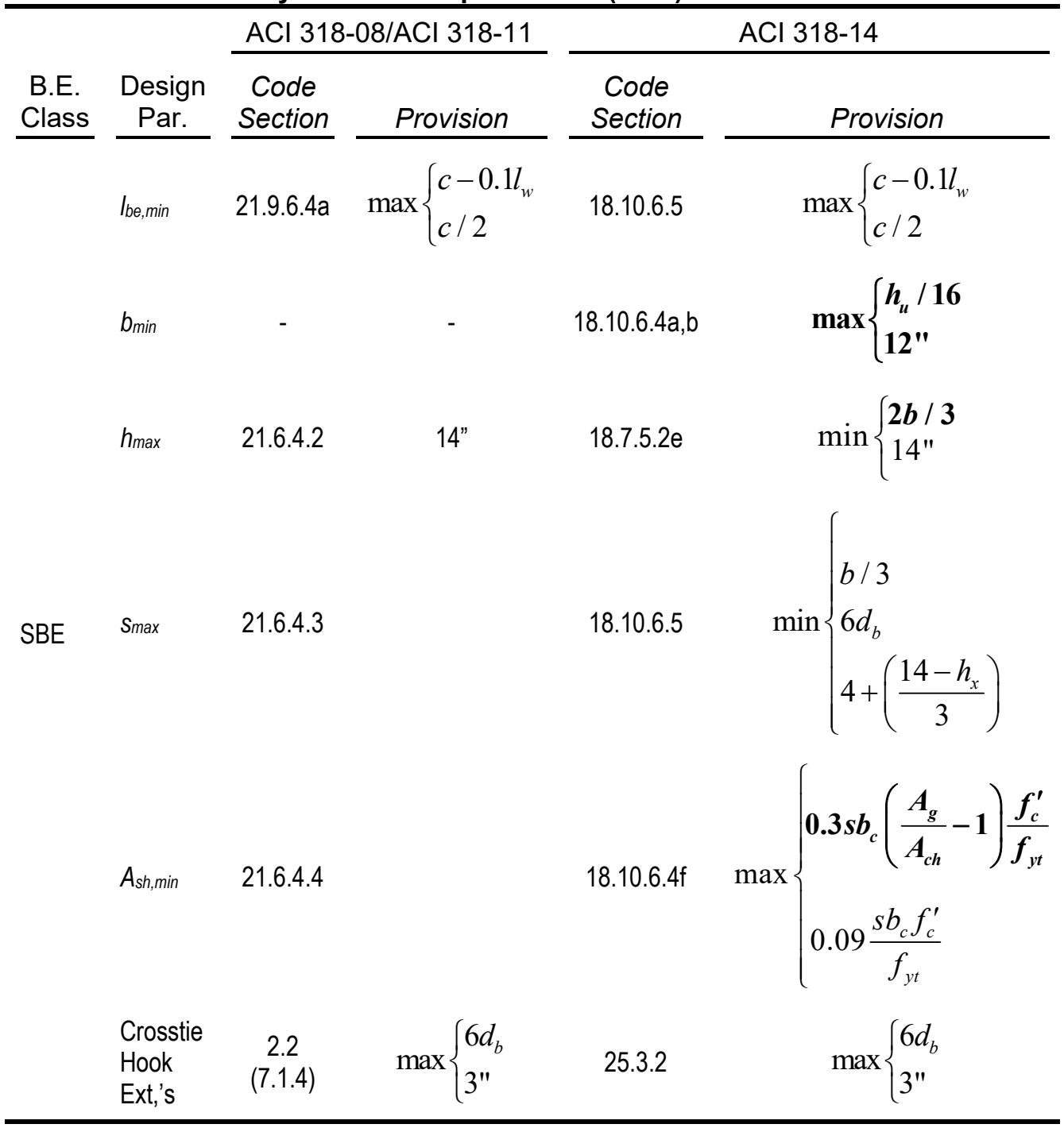

In Table 7-2, SBE refers to the confinement requirements for a special boundary element; OBE (or ordinary boundary element) confinement requirements are needed for walls not meeting the SBE limits. For additional information regarding confinement area and spacing requirements, the reader is referred to Welt et al. (2017).

\subsection{Performance and Damage Progression}

Figure 7-7 shows the progression of damage for walls subjected to flexure, as outlined in Figure 7-6. For flexure-controlled walls, response to increasing lateral displacement demand is typically characterized by the following: (1) development of horizontal cracks in the extreme tension region of the wall and diagonal cracks in the interior of the wall; (2) tensile yielding of longitudinal reinforcement in the extreme tension region of the wall; (3) spalling of cover concrete in the extreme compression region of the wall (Figure 7-7a); (4) core damage resulting in exposure of the 
longitudinal reinforcement (Figure 7-7b); (5) buckling of longitudinal reinforcement in the extreme compression region of the wall (Figure 7-7c); (6) crushing of the core concrete in the boundary element and/or web (Figure 7-7e); and (7) fracture of the longitudinal bar in some cases (Figure 7-7f). The final damage state (crushing/buckling of the compressive region and/or fracture of multiple longitudinal bars in the boundary element) leads to loss of lateral load carrying capacity due to concrete crushing, simultaneous concrete crushing and reinforcement buckling or tensile rupture of previously buckled longitudinal reinforcement.

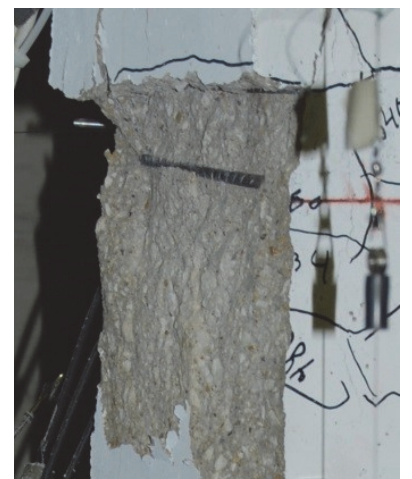

(a)

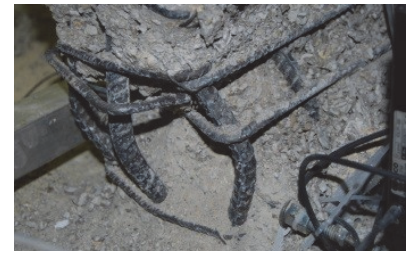

(d)

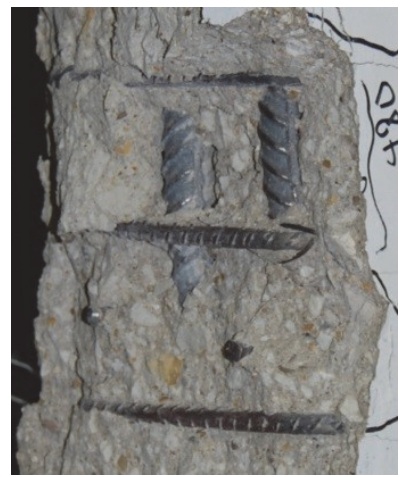

(b)

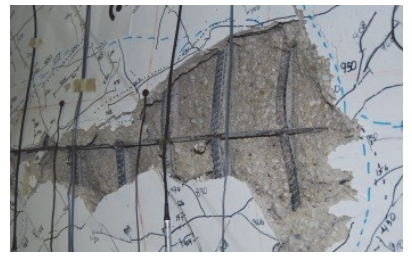

(e)

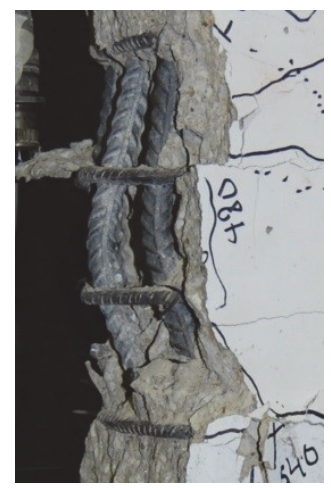

(c)

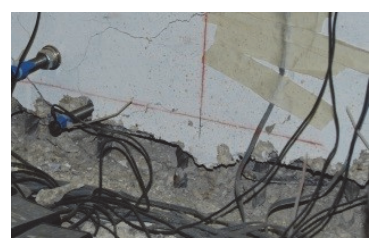

(f)

Figure 7-7 Selected damage states of reinforced concrete slender walls

(a) cover spalling; (b) exposed longitudinal reinforcement;

(c) bar buckling; (d) boundary element core crushing; (e) web core crushing; and (f) bar fracture (from Birely (2012)).

Figure 7-8 provides an overview of the damage progression in the web and flanges of a C-shaped wall subjected to cyclic unidirectional lateral loading in the symmetric direction and constant axial loading. The figures show distributed cracking corresponding to yielding of the flexural reinforcement. Initial cover spalling is most noticeable in the wall flanges and web. Failure results from extensive crushing and bar buckling of the concrete in both the web and flanges. In addition, the web of the wall exhibits long and open diagonal cracks, some of which sustain crushing along their length. 


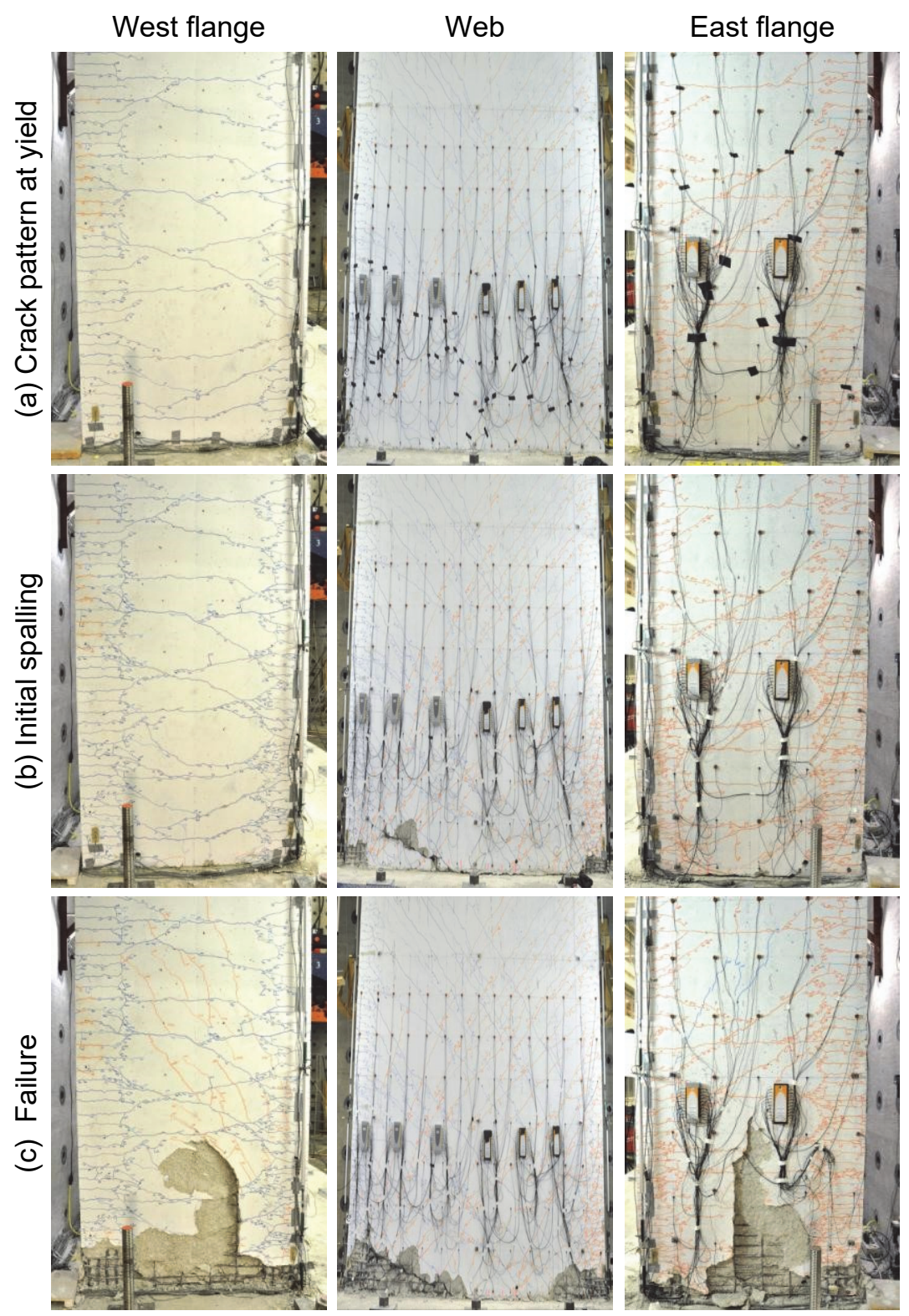

Figure 7-8 Damage progression for C-shaped flanged wall exhibiting buckling-rupture flexural failure (from Behrouzi et al. (2015)).

\subsection{Recommendations for Modeling}

The nonlinear response of flexure-controlled reinforced concrete walls can be simulated using a variety models. Figure 7-9 shows idealizations of the most commonly used wall response models, and Table 7-3 provides defining characteristics as well as advantages and disadvantages of these models with respect to response simulation, demand assessment, and performance evaluation for structural design and evaluation. Of the models presented in Figure 7-9 and Table $7-3$, models $a, b$, and $d$ are likely the most commonly employed models as they are readily available in commercial and research software and provide a desirable 
balance of computational efficiency, numerical robustness, accuracy in simulating cyclic response and/or geometric modeling capabilities. Recommendation for simulating wall response using these models are presented in Sections 7.4.2 through 7.4.4. Section 7.4.1 discusses the one-dimensional concrete and steel constitutive models that are employed in these models and provides recommendations for regularizing material response to achieve accurate, mesh-objective prediction of strength loss. Section 7.4.5 discusses response assessment for flexural walls with high shear demand and/or large cross-sectional aspect ratios that could be expected to exhibit a compression-shear failure mode.

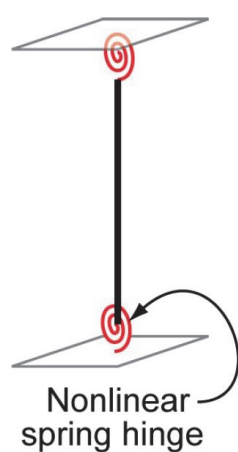

(a)

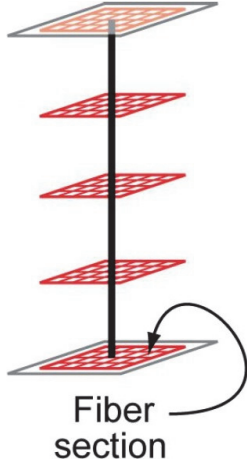

(b)

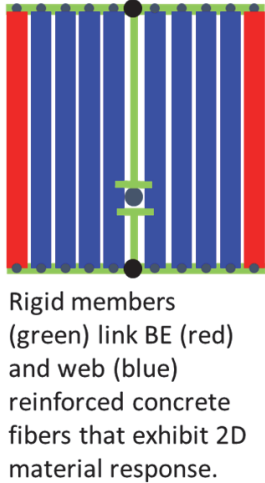

(c)

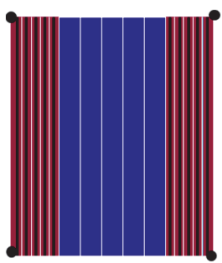

$B E(r e d)$ and web (blue) concrete and rebar (black) fibers

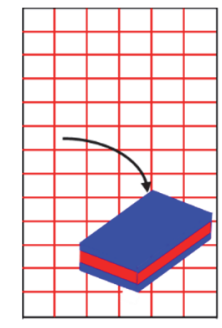

Unreinforced (blue) and reinforced concrete (red) layers

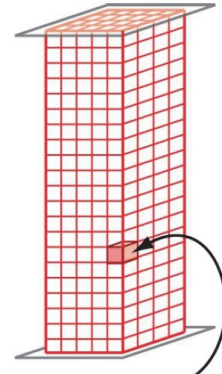

Finite element

(d)

Figure 7-9 Idealizations of models used for simulating the nonlinear response of concrete walls $(a, b, f$ from Figure 2-1 (NIST, 2010c)). (a) zero-length hinge model (e.g.; SAP2000 or PERFORM); (b) fiber-type beam-column element (e.g., OpenSees); (c) beam-column element with flexure-shear interaction; (d) fiber-shell element (e.g.; PERFORM); (e) layered-shell element (e.g., LS Dyna or Abaqus); and (f) 3D continuum elements (e.g., Atena or Abaqus).

\section{Table 7-3 Comparison of Wall Modeling Methods}

(a) Inelastic flexural response at the critical zero-length section is defined by a moment-rotation rotational response model; axial and shear response spring

(a) zero-length fiber-hinge are assumed to be linear elastic. Momentrotation response may be defined using an ASCE 41-type envelope combined with recommendations for characterizing unloading and reloading or using the results of a section-level moment-curvature response history and a plastic-hinge length.

Inelastic flexural response at the critical section is defined by a fiber-type section model and a user-defined plastic-hinge length.

\begin{tabular}{|c|c|}
\hline Advantages & Disadvantages \\
\hline \multirow[t]{4}{*}{$\begin{array}{l}\text { Highly computationally } \\
\text { efficient. }\end{array}$} & $\begin{array}{l}\text { Does not provide accurate simulation of } \\
\text { response. }\end{array}$ \\
\hline & $\begin{array}{l}\text { Does not simulate the impact on flexural } \\
\text { strength of variation in axial load. }\end{array}$ \\
\hline & $\begin{array}{l}\text { Does not simulate flexure-shear } \\
\text { interaction. }\end{array}$ \\
\hline & $\begin{array}{l}\text { May be difficult to model the geometry of } \\
\text { the structure and interaction of } \\
\text { components using this model. }\end{array}$ \\
\hline
\end{tabular}

Computationally efficient. Does not provide accurate simulation of response.

Does not simulate flexure-shear interaction.

May be difficult to model the geometry of the structure and the interaction of the wall with other components. 
Table 7-3 Comparison of Wall Modeling Methods (cont)

\begin{tabular}{|c|c|c|c|}
\hline \multirow{3}{*}{$\begin{array}{l}\text { (b) } \\
\text { fiber-type } \\
\text { beam-column } \\
\text { element }\end{array}$} & Model Characteristics ${ }^{1}$ & Advantages & Disadvantages \\
\hline & $\begin{array}{l}\text { Force- or displacement-based beam column } \\
\text { elements are used over the height of the }\end{array}$ & $\begin{array}{l}\text { Can provide accurate } \\
\text { simulation of response if }\end{array}$ & $\begin{array}{l}\text { Does not simulated flexure-shear } \\
\text { interaction. }\end{array}$ \\
\hline & $\begin{array}{l}\text { wall. Inelastic flexural response at any } \\
\text { section within the wall is simulated using a } \\
\text { fiber-type section model and nonlinear } \\
\text { concrete and steel material response }\end{array}$ & $\begin{array}{l}\text { shear does not significantly } \\
\text { affect response. }\end{array}$ & $\begin{array}{l}\text { May be difficult to model the geometry } \\
\text { of the structure and the interaction of } \\
\text { the wall with other components. }\end{array}$ \\
\hline
\end{tabular}

\begin{tabular}{|c|c|}
\hline $\begin{array}{l}\text { (b) } \\
\text { fiber-type } \\
\text { beam-column } \\
\text { element with } \\
\text { flexure-shear } \\
\text { interaction }\end{array}$ & $\begin{array}{l}\text { Force- or displacement-based beam column } \\
\text { elements are used over the height of the } \\
\text { wall. Inelastic flexure and shear response at } \\
\text { any section within the wall is simulated using } \\
\text { a fiber-type section model. Fiber strains } \\
\text { include normal and shear strains. Multi- } \\
\text { dimensional nonlinear concrete response is } \\
\text { employed. }\end{array}$ \\
\hline
\end{tabular}

(d) The model employs plane stress elements in Represents the 3D fiber-shell which the normal stress-strain response is configuration of the wall and element determined by the 1D stress-strain response facilitates system modeling of horizontal and vertical strips that lie within and interaction between the the plane of the element. Shear stress wall and other structural response is defined by a 1D shear stress- components. strain model.
(e) layered-shell element
The model employs 3D shell elements that
comprise multiple 2D plane-stress layers.
Each layer is defined by a 2D concrete
continuum model with or without embedded
reinforcing steel. Multiple shell elements are
employed along the height of the wall and
along the length of the wall cross-section.
Represents the 3D
configuration of the wall and facilitates system modeling and interaction between the wall and other structural components.
(f) The model employs 3D solid elements, a 3D Represents the 3D 3D continuum concrete constitutive model and a 1D elements reinforcing steel model. Multiple elements are required along the height, length and through the thickness of the wall. configuration of the wall.
Simulates the impact of design parameters on response.

May be difficult to model the geometry of the structure and the interaction of the wall with other components.

Not implemented in commonly employed commercial and research software.

Does not simulate flexure-shear interaction.

Moderately computationally demanding as relatively few elements are required along the length of the wall.

Computationally demanding and multiple elements are required along the height and length of the wall.

2D concrete constitutive model may reduce numerical robustness.

Too computationally demanding for system analysis.

\footnotetext{
1 With the exception of the moment-rotation hinge model, all response models require regularization of material constitutive models using a mesh-dependent characteristic length and a measure of energy dissipation to achieve accurate simulation of deformation capacity defined by the onset of strength loss.
}

\subsubsection{Constitutive Modeling}

Fiber-type plastic-hinge models, beam-column elements and shell element employ one-dimensional material response models to predict the flexural response of a wall cross section. Numerous models exist in the literature for simulating the onedimensional cyclic response of concrete and reinforcing steel. Figure 7-10 shows examples of recommended concrete and steel models (Pugh et al., 2015).

Fundamental characteristics of the concrete model are: (1) multi-linear unload-reload 
curve; and (2) damage-type response in tension. The fundamental characteristic of the steel model is a curvilinear unload-reload curve that simulates the Bauschinger effect. Other response models that predict similar cyclic response histories are appropriate for use.

For unconfined concrete, maximum compressive strength may be defined equal to the cylinder compressive strength, $f_{c}^{\prime}$. For confined concrete, compressive strength is increased using a confined concrete strength model. Models such as that proposed by Mander et al. (1988) and Saatcioglu and Razvi (1992) are used commonly; however, recent research by Welt (2015) provides a more accurate definition of confined concrete strength for rectangular (as opposed to square) boundary element regions of walls.

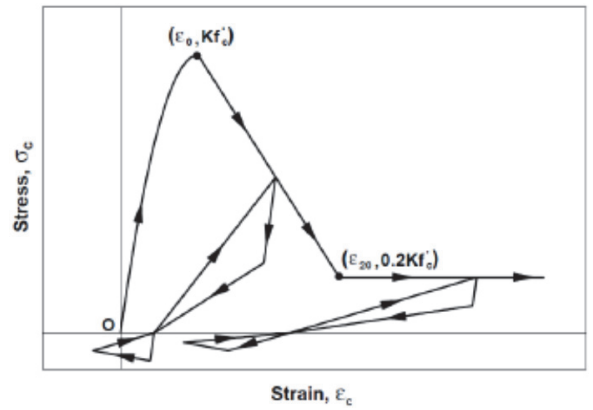

(a)

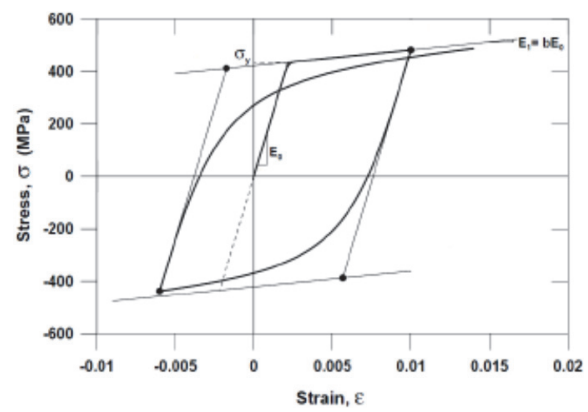

(b)

Figure 7-10 Nonlinear 1D cyclic material models. (a) Yassin cyclic concrete model, which employs the monotonic Modified Kent-Park model (Yassin, 1994) as presented by Orakcal et al. (2009). Note that compressive stress-strain is positive. (b) Menegotto-PintoFilippou reinforcing steel model (Filippou et al.,1983) as presented by Orakcal et al. (2009).

A critical aspect of modeling RC components that exhibit softening response, is material regularization. Slender concrete walls typically lose lateral strength due to concrete crushing and steel buckling in the flexural compression region of the wall. Research by Coleman and Spacone (2011), Pugh et al. (2015) and Almeida et al. (2016) shows that if wall response is simulated using common concrete material models (e.g. Mander et al. 1988, Saatcioglu and Razvi 1992) to define the softening portion of the compressive stress-strain envelope, simulated drift at onset of lateral strength loss is inaccurate and a function of the number of elements and/or fiber cross sections used per element.

Figure 7-11 shows simulated base shear versus drift for a concrete wall. Data in the figure were generated using a fiber-type beam-column element with concrete material response defined using the Saatcioglu and Razvi (1992) constitutive model, without material regularization. These data show that as the number of fiber sections per element increases, drift capacity is reduced. The mesh-sensitive results shown in Figure 7-11 results from localization of deformation demands at a single critical 
section. As the failing fiber section loses flexural strength, it experiences increasing curvature and strain demands; surrounding fiber sections "unload" and experience smaller curvature and strain demands. Total wall drift is determined by fiber section rotations, which are the product of the fiber section curvature and the vertical length of wall attributed to the fiber section. As more fiber sections are added to the model, the length associated with the critical fiber section is reduced, and the section rotation and total wall drift at a given section curvature and fiber strain demand are reduced. Thus, increased mesh refinement results in reduced drift capacity.

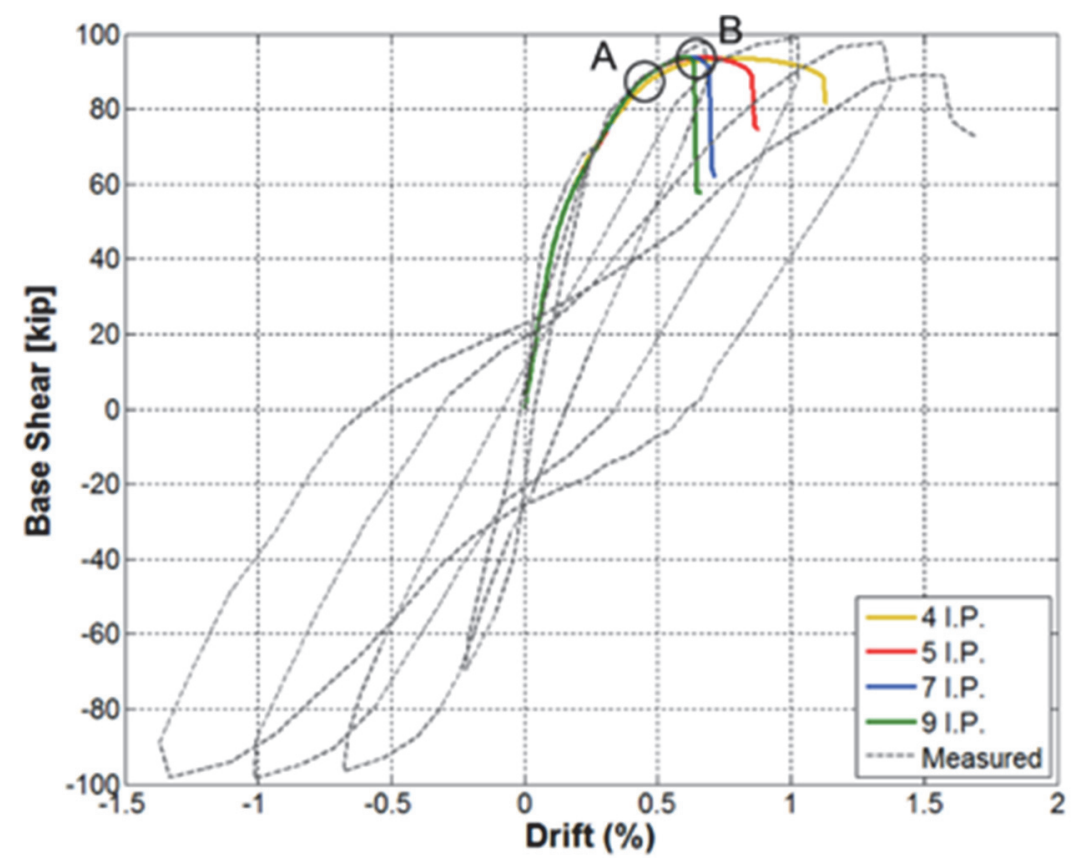

Figure 7-11 Impact of mesh size on models without material regularization. Note that results are provided for beam-column elements with different levels of mesh refinement, resulting from different numbers of integration points (IP) used within the element.

Mesh-sensitive simulation of softening response can be mitigated by "regularizing" the softening portion of material response models. Material response is regularized by adopting stress versus deformation, not stress versus strain, as the fundamental measure of material response. The need for material regularization is demonstrated by the reinforced concrete prism shown in Figure 7-12a. The deformation associated with axial strength loss for the pier localizes in a limited region. While the stress versus deformation response of the prism is unique, the stress-strain response of the prism is entirely dependent on the gage length used to compute the strain and over which strain is inherently assumed to be constant (Figure 7-12b). This localization of deformation associated with damage and strength loss is observed also in the field (Figure 7-12c). 


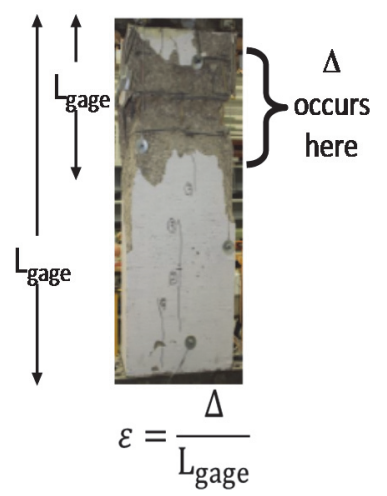

(a) RC prism subjected to axial loading in the laboratory (Welt, 2015)

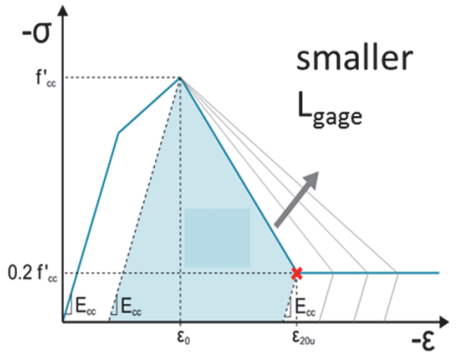

(b) impact of gage length on "measured" stress-strain response

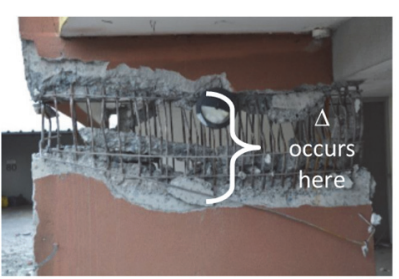

(c) localization of damage in a RC wall pier (image from Dragovich)

Figure 7-12 Localization of damage and non-uniqueness of stress-strain response.

Regularization of concrete material response for nonlinear response simulation is most easily accomplished by defining concrete strain capacity to be a function of a mesh-dependent characteristic length and the concrete crushing energy, where crushing energy describes the energy dissipated as the concrete goes from the point of maximum strength to the point of residual compressive strength. Research by Jansen and Shah (1997) and Nakamura and Higai (2001) demonstrates that crushing energy, and not strain corresponding to the residual compressive strength, is a fundamental concrete material property. Although reinforcing steel may harden to the point of failure, regularization of steel response is required when the reinforcedconcrete section softens and deformations localize to a single fiber section. For reinforcing steel, post-yield response and strain capacity are defined as a function of a mesh-dependent characteristic length and a steel yielding energy. Figure 7-13 shows regularized response envelopes for unconfined concrete, confined concrete, and reinforcing steel and graphically define unconfined concrete crushing energy, $G f_{c}$, confined concrete crushing, $G_{f c c}$, and the reinforcing steel energy, $G_{f s}$, associated with softening section response.

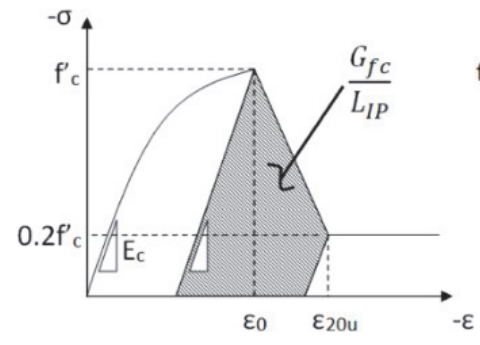

(a) unconfined concrete compression envelope

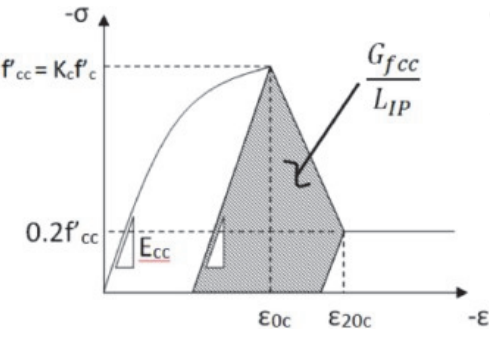

(b) confined concrete compression envelope

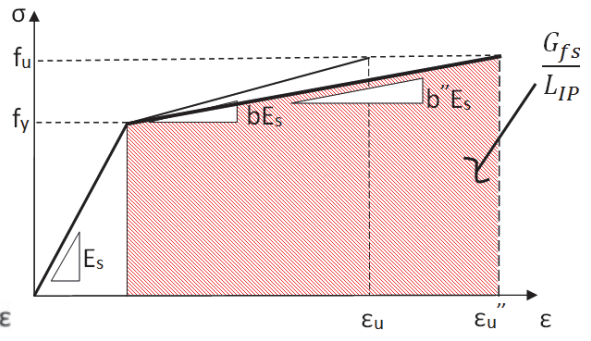

(c) reinforcing steel response envelope for tension and compression

Figure 7-13 Regularization of concrete and steel material response using mesh dependent length. 
Using experimental data for planar wall specimens tested in the laboratory, Pugh et al. (2015) determined that for the force-based beam-column element:

$$
\begin{gathered}
G_{f c}=2 f_{c}^{\prime} \mathrm{N} / \mathrm{mm} \text { for } f_{c}^{\prime} \text { in } \mathrm{MPa} \\
G_{f c}=0.079 f_{c}^{\prime} \mathrm{lb} / \text { in for } f_{c}^{\prime} \text { in psi } \\
G_{f c c}=1.70 G_{f c} \\
G_{s}=\frac{1}{2}\left(\varepsilon_{u_{-} \exp }-\varepsilon_{y}\right)\left(f_{u}+f_{y}\right) L_{g a g e}
\end{gathered}
$$

where $f_{c}^{\prime}$ is the concrete compressive strength in MPa for Equation 7-1a and psi for Eq. $7-1 \mathrm{~b}, \varepsilon_{u_{-} \text {exp }}$ is the measured tensile strain at steel ultimate strength, $\varepsilon_{y}$ is the steel yield strain, $f_{u}$ and $f_{y}$ are, respectively, the measured ultimate and yield strengths, and $L_{\text {gage }}$ is the gage length used in laboratory testing of the reinforcing steel, which may be assumed equal to the $200 \mathrm{~mm} / 8$ in. length required by the ASTM A370 specification steel testing. It should be noted that $G_{f c}$ determined by Equation 7-1 is larger than that computed using the recommendations of Jansen and Shah $\left(G_{f_{c}}=\right.$ $0.71 f_{c}^{\prime} \mathrm{N} / \mathrm{mm}$ for $f_{c}^{\prime}$ in $\mathrm{MPa}, G_{f_{c}}=0.030 f_{c}^{\prime} \mathrm{lb} /$ in for $f_{c}^{\prime}$ in psi) and Nakamura and Higai $\left(G_{f_{c}}=8.8 \sqrt{f_{c}^{\prime}}=1.5 f_{c}^{\prime} \mathrm{N} / \mathrm{mm}\right.$ for $35 \mathrm{MPa}$ concrete, $G_{f_{c}}=4.2 \sqrt{f_{c}^{\prime}}=0.059 f_{c}^{\prime}$ $\mathrm{lb} / \mathrm{in}$ for $5076 \mathrm{psi}$ concrete). This is attributed to the fact that these previous studies determined $G_{f c}$ from compression tests of plain concrete cylinders while Equation 7-1 was calibrated using data from tests of wall with longitudinal and vertical reinforcement, which could be expected to enhance concrete crushing energy.

Research by Welt (2015) provides a definition of $G_{f c c}$ in which $G_{f c c}$ is defined by the geometry of the boundary element section and the boundary element confining reinforcement configuration. Different concrete crushing energy values are required for use with the displacement-based beam-column element, which exhibits large variations in section axial load over the length of an element. Pugh (2015) presents crushing energies for use with displacement-based elements. Using the energies defined in Equation 7-1 through Equation 7-3, critical strain values identified in Figure 7-13 are defined as follows:

$$
\begin{aligned}
& \varepsilon_{20 u}=\frac{G_{f c}}{0.6 f_{c} L_{I P}}-\frac{(1-\alpha) f_{c}^{\prime}}{E_{c}} \\
& \varepsilon_{20 c}=\frac{G_{f c c}}{0.6 f_{c} L_{I P}}-\frac{(1-\alpha) K f_{c}^{\prime}}{E_{c}} \\
& \varepsilon_{u}^{\prime \prime}=\varepsilon_{y}+\left(\frac{L_{g a g e}}{L_{I P}}\right)\left(\varepsilon_{u}-\varepsilon_{y}\right)
\end{aligned}
$$


where $\alpha$ is the multiplier on $f_{c}^{\prime}$ to determine residual concrete compressive strength (in Figures 7-13a and 7-13b this value is approximated as 0.2 ), $K$ is the confined concrete strength factor, $L_{I P}$ is the mesh-dependent length taken equal to the integration/quadrature-rule length associated with the wall section for which material response is defined, $E_{0}$ is the initial elastic modulus for the concrete and all other variables are defined previously. It should be noted that concrete and steel strain capacities are a function of $L_{I P}$; thus, each fiber section with a unique $L_{I P}$ has a set of unique unconfined concrete, confined concrete, and reinforcing steel stress-strain response models.

The data in Figure 7-11 show that without regularization of material softening, drift capacity is determined by mesh size. This is explained by considering the dependence of the maximum deformation on the strain and the element length (for the beam-column element, the integration length is the parameter that determines the deformation of the wall). The critical response value of interest is the displacement capacity of the wall. If a single value of strain is used for any discretization, the axial deformation of the story will depend on $L_{I P}$ where an increase in $L_{I P}$ results in a larger axial deformation for a given strain capacity, thus increasing the drift. Using the regularization, the axial deformation of the story is given, and therefore the strain capacity is determined from $L_{I P}$ and all mesh sizes have the same capacity.

Equation 7-1 through Equation 7-6 define concrete and steel strain capacities using a regularization approach. However, for reinforced concrete sections subjected to reversed cyclic loading, reinforcing steel prematurely loses compressive strength due to severe buckling and prematurely loses tensile strength due to low-cycle fatigue and fracture. Research by Pugh et al. (2015) shows that accurate simulation of onset of strength loss can be achieved by assuming that reinforcing steel compressive strength is lost due to severe buckling when confined concrete is completely crushed; thus, it is recommended that the reinforcement compressive strength capacity to be equal to $\varepsilon_{20 c}$ (Equation 7-2). Further, because buckling and straightening of reinforcing steel under cyclic loading results in large, localized tensile strain demand, reinforcing steel loses tensile strain capacity at an average strain that is substantially smaller than the measured fracture strain, $\mathcal{E}_{u}$. Thus, Whitman (2015) recommends that onset of steel fracture be defined to occur at $0.5 \varepsilon_{u}^{\prime \prime}$, where $\varepsilon_{u}^{\prime \prime}$ is defined per Equation 7-6.

\subsubsection{Fiber-Type Beam-Column Elements}

Accurate, computationally efficient and numerically robust simulation of the cyclic response, including failure, of flexure-controlled concrete walls with low to moderate shear demands is most easily achieved using fiber-type beam-column elements. Using these models, nonlinear flexural response (i.e., moment and axial load) is simulated at the section level using a fiber-type discretization of the wall crosssection in which one-dimensional, cyclic stress-strain models are used to define 
concrete and steel fiber response. Use of a fiber-type section model enables simulation of impact of axial load on flexural response (i.e., P-M interaction). Accurate assessment of local demands (i.e., strains and rotation) and accurate simulation of strength loss require regularization of concrete and steel material response using a mesh-dependent characteristic length and a measure of energy dissipated in the post-yield or post-peak regime. Section response is integrated, numerically, along the length of the element to define element response using either a force- or displacement-based beam-column element formulation.

Accurately capturing the $\mathrm{P}-\mathrm{M}$ interaction response is critical to accurate simulation; as such, a force-based element formulation is preferred because it exactly represents the moment and axial load distribution over the height of the wall using a single element. In contrast, multiple displacement-based elements are required to provide acceptably accurate simulation of the moment distribution in yielding regions. Even if many displacement-based elements are employed, errors in section-level axial loads are large and may affect simulation of flexural strength and deformation capacity (Pugh et al., 2015).

Fiber-sections are discretized to provide accurate simulation of cyclic, momentcurvature response of the wall section; multiple elements are introduced to provide accurate simulation of the load and deformation field up the height of wall. Wall performance may be assessed on the basis of simulated local, section or story-level deformation demand at critical locations within the wall. Story drift or rotation are preferred as these quantities may be extracted directly from simulation output; because strain is not a fundamental response quantity, use of strain to assess performance requires calculation of relative strain demand using the section-specific stress-strain history calculated using Equations 7-1 through 7-4.

Figures 7-14 and 7-15 show measured and simulated response histories for two planar walls. Simulated histories were generated using fiber-type force-based beamcolumn elements with three elements used to model the wall specimen and with 3,5 or 7 integration points (IP) used along the length of each element. An integration point is a fiber-based discretization of the cross section, such as shown in Figure 7-12. The data in these models show that the fiber-type force-based beam-column element model provides accurate simulation of the measured cyclic response, including onset of loss of lateral load carrying capacity. Pugh et al. (2015) compared simulated and measured response data, such as shown in Figures 7-14 and 7-15, for wall test specimens and found the following for the case of five fiber sections per force-based element and one force-based element per story:

- Ratio of simulated to measured displacement at predicted yield strength is 1.03 with a coefficient of variation (COV) of $9 \%$

- Ratio of simulated to measured maximum strength is 0.96 with a COV of $6 \%$ 
- Ratio of simulated to measured drift at $20 \%$ strength loss is 1.04 with a COV of $19 \%$

Similar results were found for meshes employing three and seven fiber sections per element, demonstrating mesh independence.

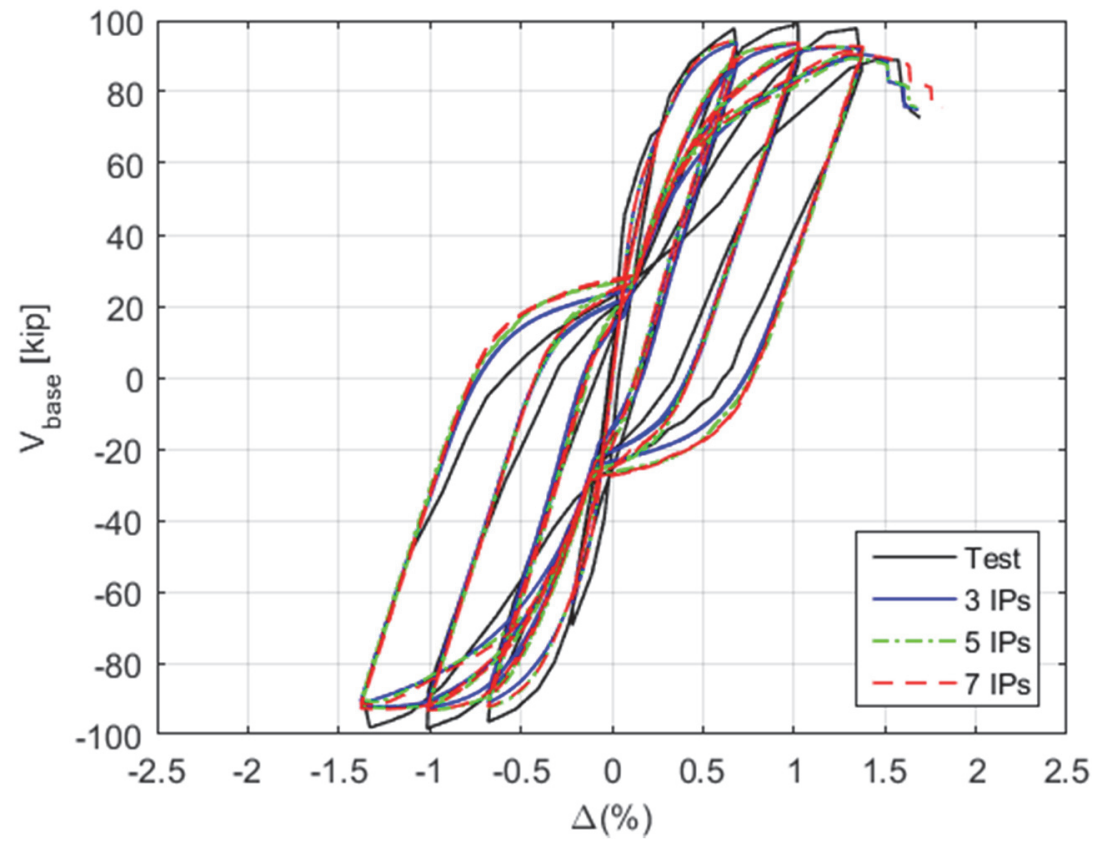

Figure 7-14 Measured (black) and simulated (red, blue and green) response histories for planar wall WSH4 tested by Dazio et al. (2009).

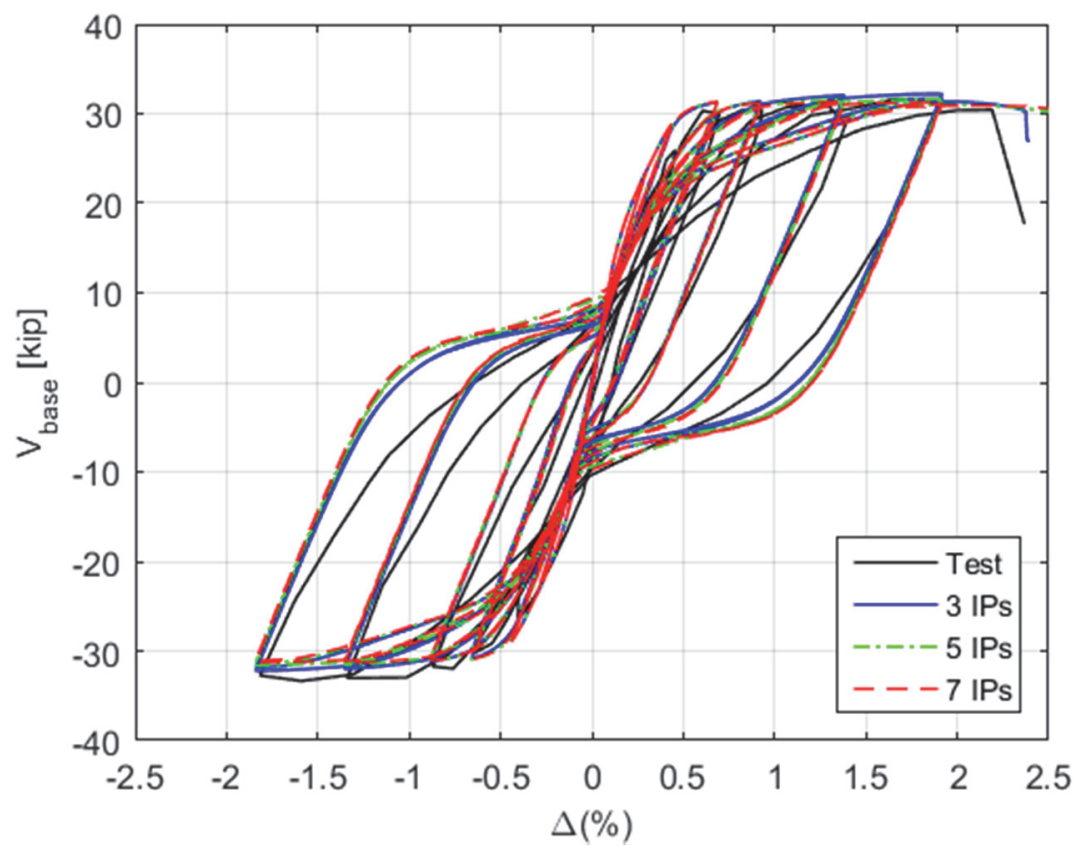

Figure 7-15 Measured (black) and simulated (red, blue and green) base shear versus drift response histories for planar wall RW1 tested by Thomsen and Wallace (1995). 
Within the typical fiber-type beam-column element formulation, nonlinear flexural response at the section level is simulated using a fiber-type discretization of the wall section and nonlinear one-dimensional cyclic material response models. Figure 7-16 shows a fiber-type discretization of a planar wall section; the section comprises unconfined and confined concrete fibers as well as steel fibers. Pugh et al. (2015) recommend that there are approximately 30 fibers within the boundary element region of the wall. Shear deformations on the section are assumed to be negligible such that plane sections remain plane and perpendicular to the neutral axis; thus, the strain demand in an individual fiber is defined by the curvature and axial strain demand. Nonlinear one-dimensional material response models define fiber stressstrain relations. Section moment and axial load are computed by integrating fiber stresses along the length of the cross section.

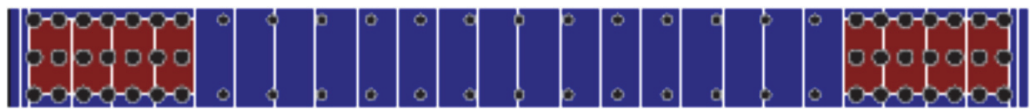

Figure 7-16

Fiber discretization of planar reinforced concrete wall. Red and blue indicate confined and unconfined concrete, respectively; black indicates reinforcing steel.

\subsubsection{Definition of the Force- or Displacement-Based Fiber-Type Beam- Column Element}

The force- and displacement-based fiber-type beam-column element formulations provide a procedure for integrating, numerically, fiber-section response to determine element-level response. The displacement-based element formulation employs the assumption of a linear curvature field and constant axial strain field along the length of the element. Section strains and curvatures are defined, explicitly, by nodal displacements and rotations; nodal forces and moments may be computed directly from section moments and axial load using numerical integration. Research by Pugh (2012) suggests that more than five displacement-based elements are required per story and five sections are required per element to achieve acceptably accurate simulation of the deformation and load distributions that develop in a walled building. Although this level of mesh refinement provides acceptable accuracy, perceptible errors will exist in the axial load, flexural strength deformation capacity predicted at individual sections.

The force-based element formulation employs the assumption of a linear moment distribution and constant axial load distribution along the length of the element. Multiple nonlinear solutions are required within the element to determine the nodal moments and forces that produce section deformations that, when integrated along the length of the element, result in nodal displacements and rotations that are equal to those imposed on the element as part of the displacement-based analysis. Because walls in a building typically have a linear moment and constant axial load 
distribution, only one force-based element with five or more fiber sections is required to accurately simulation response (Pugh, 2012). Using the force-based element formulation, once a converged solution state is achieved, section-level moments, axial loads, and associated deformations are exact.

\subsubsection{Shear Response Models for Use with Fiber-Type Beam-Column Elements}

The force- and displacement-based beam-column element models described above employ the assumptions of zero shear strain and no flexure-shear interaction for computation of sectional response. For walls with low to moderate axial load, this modeling approach alone can provide accurate simulation of flexural response; however, model accuracy can be improved by introducing a shear-response model. For the displacement-based element, a shear spring is introduced at each floor in series with the beam-column element; for force-based elements, a shear spring is introduced at each fiber section. In recent years a number of linear (Pugh, 2012; ATC/PEER, 2010) and nonlinear (Pugh, 2012; Moehle et al., 2011) shear response models have been proposed for use in simulating the response of flexure-controlled walls. Figure 7-17 shows linear and nonlinear models, which are considered appropriate for use with the fiber-type beam-column elements. (Note that, in Figure $7-17, A_{v}$ is the area of the wall activated in carrying the applied shear load and $G_{c}$ is the concrete shear modulus, defined equal to $40 \%$ of the concrete elastic modulus.) Based on research by Pugh (2012), it is recommended that a linear elastic shear model be employed with an effective shear stiffness of $0.1 G_{c} A_{v}$ in regions of yielding and $1.0 G_{c} A_{v}$ elsewhere.

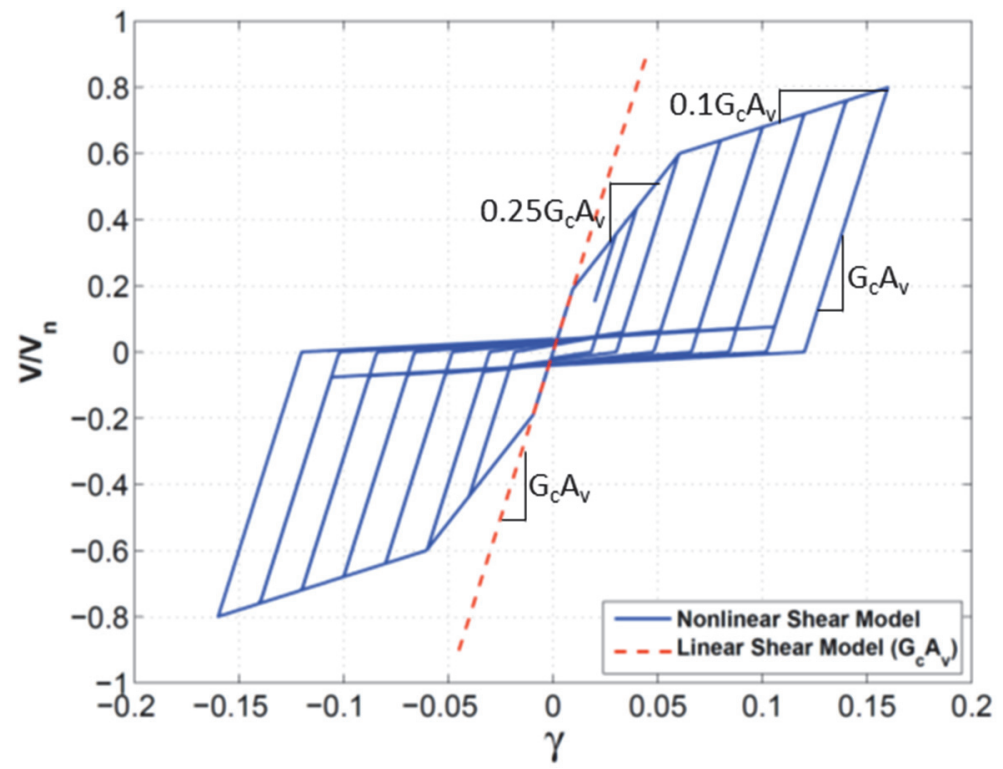

Figure 7-17 Linear and nonlinear shear response models for use with fiber-type beam-column elements. 


\subsubsection{Limitations of the Fiber-Type Beam-Column Element Model}

Research using nonlinear continuum-type finite element analysis shows that the modeling procedure described above can provide accurate simulation of the forcedeformation response for flexure-controlled concrete walls exhibiting failure due to simultaneous concrete crushing and reinforcement buckling (compression-buckling failure) or rupture of previously buckled reinforcement (buckling-rupture failure) (Pugh et al., 2015). However, this model cannot provide accurate simulation of drift capacity for walls that fail due to combined shear and flexural loading, nor can the model provide accurate prediction of cyclic response for walls for eight walls in which the normal strain distributions on the wall cross section are highly nonlinear. A method for predicting onset of strength loss for walls that fail due to flexure-shear interaction is addressed in Section 7.5; the fiber-type shell model presented in Section 7.4.4 may be used to predict the response of nonplanar walls with highly nonlinear cross-sectional strain distributions.

Whitman (2015) employed nonlinear continuum analysis using solid elements to investigate the behavior of planar concrete walls subjected to lateral and axial loading. The results of this study included identification of a compression-shear failure mode characterized by crushing of unconfined concrete in the vicinity of the interface between the boundary element and the web region of the wall; high principal compressive stresses develop at this interface due to superposition of shear stress and normal stress resulting from flexure. Figure 7-18 developed by Whitman (2015) shows simulated shear stress demand at failure, normalized by $\sqrt{f_{c}^{\prime}} A_{g}$ with $f_{c}^{\prime}$ in psi, plotted versus cross-sectional aspect ratio (ratio of wall length to thickness) for planar walls with detailing that meets ACI 318-14 requirements, i.e., all walls have shear capacities that exceed shear demand; shear capacity is computed per ACI

Code requirements with the exception that the shear strength limit of $10 \sqrt{f_{c}^{\prime}} A_{c v}$, with $f_{c}^{\prime}$ in psi, is ignored. The data in Figure 7-18 show that this compression-shear failure mode develops in planar walls with high shear stress demands and/or large cross-sectional aspect ratios. Because the fiber-type beam-column element does not simulate the impact of flexure-shear interaction, it cannot provide accurate simulation of strength loss due to compression-shear failure. Section 7.5 provides guidance on assessing deformation capacity for planar walls exhibiting compression-shear failure.

Research by Pugh (2012) shows that the fiber-type beam-column element cannot provide accurate simulation of stiffness or the drift capacity of nonplanar walls that have highly nonlinear strain distributions. This includes T-shaped and H-shaped walls with large flange-to-web length ratios. Comparison of simulated and measured response histories suggests that this does not include C-shaped walls (Pugh, 2012). The fiber-type shell element model presented in Section 7.4.4 may be used to predict the response of nonplanar walls with highly nonlinear cross-sectional strain distributions. 


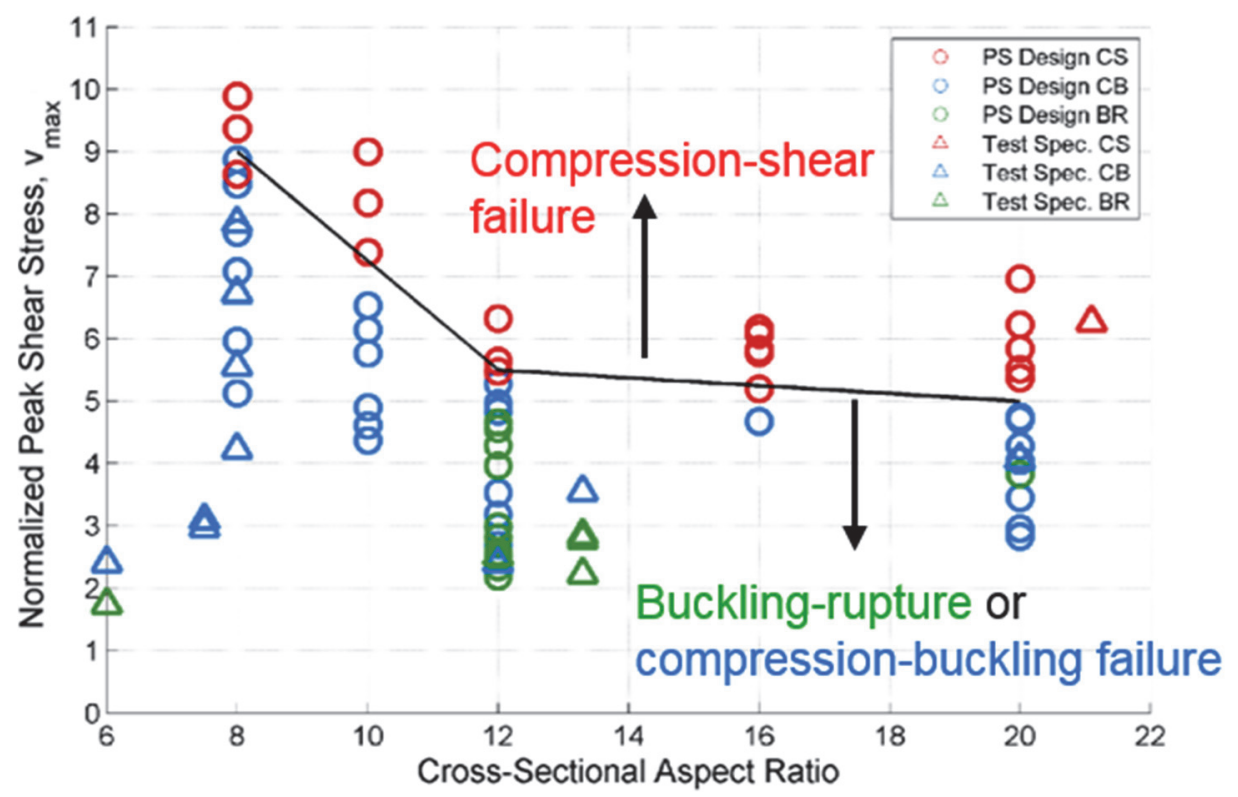

Figure 7-18 Planar wall design criteria triggering compression-shear versus flexural only failure modes (Whitman, 2015).

\subsubsection{Zero-Length Hinge (or Plastic-Hinge) Models}

Nonlinear zero-length hinge models are used to simulate nonlinear response mechanisms as part of an earthquake performance assessment. Recommendations for modeling wall response using fiber-type beam-column elements presented in Section 7.4.2 may be extended for use of zero-length hinge models as follows:

- Specify an appropriate plastic hinge length. Kazas (2013) and Bohl and Adebar (2011) review previously proposed plastic-hinge length definitions and proposed new definitions. A length equal to half the horizontal length of the wall (Birely, 2013) is employed in ASCE/SEI 41, Seismic Rehabilitation of Existing Buildings.

- Employ the recommendations presented in Section 7.4.1 for modeling the flexural response of a concrete wall using a fiber-type section model. The length associated with the fiber section, $L_{I P}$, should be taken equal to the plastic hinge length, $L_{p}$.

- Introduce a zero-length shear hinge and the location of the zero-length rotational hinge to simulate flexibility associated with shear. Recommendations presented in Section 7.4.2.3 may be used to calibrate the shear hinge.

- Limitations presented in Section 7.4.2 on the applicability and accuracy of the fiber-type beam-column element model apply also to the rotational hinge model. 


\subsubsection{Fiber-Type Shell Elements}

Fiber-type shell elements are used commonly in practice to simulate nonlinear wall response. These models are desirable as they facilitate representation of wall geometry and connectivity between walls and other structural components, and provide a mechanism for representing a nonlinear normal strain distribution across a wall cross section. Recommendations for modeling wall response using fiber-type beam-column elements presented in Section 7.4.2 may be extended for use of fibertype shell elements as follows:

- Determine an appropriate level of mesh refinement that enables accurate simulation of the normal strain distribution up the height of the wall and across the wall cross section.

- For definition of concrete and reinforcing steel material response, Equation 7-1 and Equation 7-2 defining concrete crushing energy for unconfined and confined concrete, respectively, should be replaced by the following equations:

$$
\begin{gathered}
G_{f c}=90 \mathrm{~N} / \mathrm{mm}=514 \mathrm{lb} / \mathrm{in} \\
G_{f c c}=8\left(\frac{f_{c c}}{f_{c}}-1\right) \text { with } 90 \leq G_{f c c} \leq 220 \mathrm{~N} / \mathrm{mm} \\
G_{f c c}=8\left(\frac{f_{c c}}{f_{c}}-1\right) \text { with } 514 \leq G_{f c c} \leq 1,256 \mathrm{lb} / \mathrm{in}
\end{gathered}
$$

per (Lowes et al., 2017). In Equation 7-4 through Equation 7-6, which define concrete compressive strain capacity and reinforcing steel fracture strain, the length associated with the fiber section, $L_{I P}$, should be taken equal to the vertical length of the fiber-shell element.

- Calibrate shear response model incorporated in the fiber-type shell element using the recommendations presented in Section 7.4.2.

- Similar to the fiber-type beam-column element, the fiber-type shell element does not simulated flexure-shear interaction and thus cannot provide accurate simulation of drift capacity for walls exhibiting compression-shear failure as defined by the criteria in Figure 7-18.

\subsection{Deformation Capacity}

The models presented in Section 7.4.1 through Section 7.4.3 have been shown, through comparison with experimental data (Pugh et al., 2016; Lowes et al., 2017), to provide accurate simulation of the cyclic response, including onset of loss of lateral load carrying capacity, for walls exhibiting failure due to simultaneous crushing of concrete and buckling of reinforcement (compression-buckling failure) or rupture of previously buckled longitudinal reinforcement (buckling-rupture failure). Data in 
Figure 7-18, which were generated using nonlinear continuum-type finite element analysis Whitman (2015), define the range of shear demands and cross-sectional aspect ratios for which these failure modes can be expected in planar walls.

Circumstances, however, may dictate the use of models other than those in Sections 7.4.1 through Section 7.4.3. In this event, data are provided in Table 7-4 indicating the deformation demand at onset of loss of lateral strength in planar walls.

Table 7-4 Deformation Demands at Onset of Strength Loss for Walls Exhibiting Different Failure Modes

\begin{tabular}{|c|c|c|c|c|}
\hline \multirow{4}{*}{$\begin{array}{l}\text { Planar walls exhibiting } \\
\text { compression-buckling } \\
\text { or buckling-rupture } \\
\text { failure per Figure } 7-18\end{array}$} & \multirow{3}{*}{$\begin{array}{l}\text { Story Drift (\%) } \\
\text { Total Hinge } \\
\text { Rotation (rad) }\end{array}$} & $\begin{array}{l}\text { Rotational } \\
\text { Hinge }\end{array}$ & $\begin{array}{l}\text { Fiber-Type Hinge, } \\
\text { Beam-Column, and } \\
\text { Shell Elements } \\
\end{array}$ & $\begin{array}{l}\text { Layered Shell and } \\
\text { 3D Continuum } \\
\text { Models }\end{array}$ \\
\hline & & $\begin{array}{l}\text { mean }=2.0 \\
\mathrm{COV}=0.3\end{array}$ & \multirow{2}{*}{ Use drift and rotation } & \\
\hline & & $\begin{array}{c}\text { mean }=0.016 \\
\operatorname{COV}=0.3\end{array}$ & & Ils to verily model \\
\hline & Concrete Strain & NA & \multicolumn{2}{|c|}{ Eq. 7-5 with $L_{I P}$ and $G_{f c c}$ depending on model } \\
\hline \multirow{3}{*}{$\begin{array}{l}\text { Planar walls exhibiting } \\
\text { compression-shear } \\
\text { failure per Figure 7-18 }\end{array}$} & Story Drift (\%) & $\begin{array}{l}\text { mean }=1.2 \\
\mathrm{COV}=0.16\end{array}$ & \multirow{2}{*}{$\begin{array}{l}\text { Model defines stiffness and } \\
\text { strength; drift and rotation } \\
\text { limits define onset of } \\
\text { strength loss }\end{array}$} & \multirow{2}{*}{$\begin{array}{l}\text { Use drift and rotation } \\
\text { limits to verify model }\end{array}$} \\
\hline & $\begin{array}{l}\text { Total Hinge } \\
\text { Rotation (rad) }\end{array}$ & $\begin{aligned} \text { mean } & =0.009 \\
\operatorname{COV} & =0.15\end{aligned}$ & & \\
\hline & Concrete Strain & NA & NA & $\begin{array}{l}\text { Eq. 7-5 with } L_{I P} \text { and } G_{f c c} \\
\text { depending on model }\end{array}$ \\
\hline
\end{tabular}

As discussed previously, research using nonlinear continuum-type finite element analysis shows that the models presented in Section 7.4.2 through Section 7.4.4 cannot predict loss of lateral-load carrying capacity due to compression-shear interaction in the boundary element and web regions. For walls expected to exhibit compression-shear failure per Figure 7-14, the models presented in Section 7.4.2 through Section 7.4.4 may be used to simulate cyclic response with the expressions in Table 7-4 used to determine the onset of strength loss. Figure 7-19 shows simulated response for a wall expected to exhibit compression-shear failure. For simulation using a fiber-type hinge model (black line), strength loss due to compression-shear failure is not simulated and post-processing of data to identify the drift at strength loss (vertical red line) is required. For simulation using a rotational hinge model, for which a multi-linear moment rotation envelope is defined, the rotation at strength loss due to compression-shear failure may be explicitly included in the model definition (blue line). Limited experimental data exist to support development of recommendations for defining post-peak response of walls exhibiting compressionshear failure. The dashed blue line defines a plausible post-peak curve. 

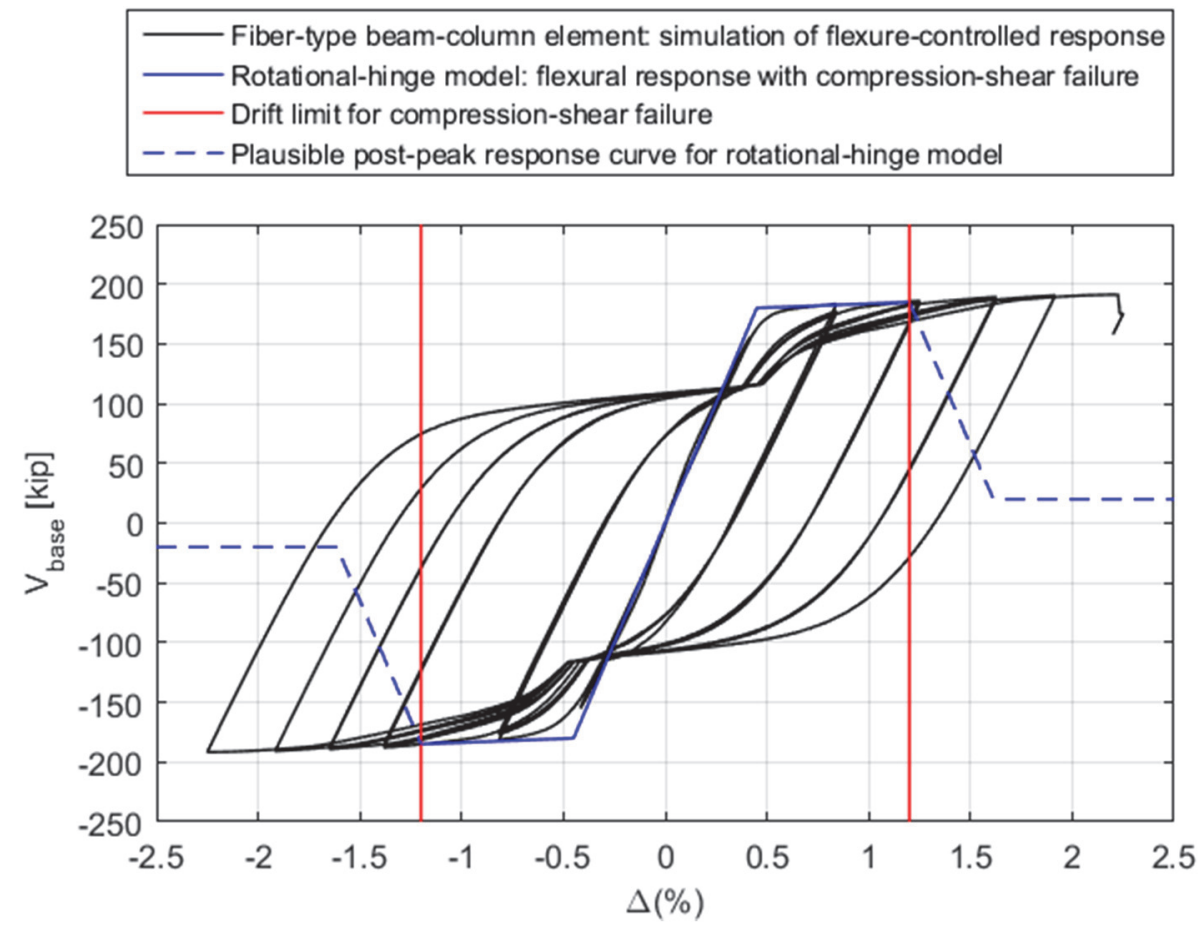

Figure 7-19 Simulated response for a slender wall exhibiting compressionshear failure per Figure 7-14. 


\section{Chapter 8}

\section{Shear-Controlled Reinforced Concrete Walls}

\subsection{Overview of Geometry and Behavior}

Shear-resisting mechanisms generally dictate the seismic behavior of squat (low aspect ratio) reinforced concrete shear walls. Such walls are widely used in low-rise commercial buildings and energy-related facilities.

\subsubsection{Classification of Shear Walls by Geometry in Plan and Elevation}

In this report, low aspect ratio shear walls are described using plan geometry as one of the following terms:

- Rectangular or planar walls: These walls may have zones of substantially increased vertical reinforcement, with or without corresponding increases in transverse reinforcement, at each end, and those zones of increased reinforcement, with respect to the web, are termed boundary zones herein.

- Barbell walls: These are rectangular walls constructed between, but cast with, boundary columns, forming the shape of a barbell.

- Flanged walls: These are composed of at least three components, a rectangular wall forming a web and two flanges, with four parallel faces, typically of the same thickness as the web.

In elevation, there are an infinite number of geometries of low aspect ratio shear walls. Herein, walls are described as either a cantilever pier or as a pier in a punched wall. It is noted that nearly all the physical tests completed to date have been performed on cantilever piers. A few tests have been performed on specimens that mimic a pier in a wall with multiple openings. The major differences between the two are boundary conditions and axial load effects. Wall piers may experience boundary conditions that approach fixed-fixed at the top and bottom, whereas cantilever piers have significant rotational restraint at the bottom but are free to rotate at the top. A wall pier close to one end of a shear wall may experience significant axial force, compressive or tensile, associated with flexure in the shear wall, whereas the net axial force in low aspect ratio shear walls is generally small. 


\subsubsection{Hysteretic Response of Low Aspect Ratio Shear Walls}

Unlike high aspect ratio walls, where behavior is principally affected by flexure and axial force, the response due to shearing force generally dominates the cyclic behavior of low aspect ratio shear walls. The peak strength of a low aspect ratio wall is limited by the following: (1) maximum stress in web reinforcement in the wall, which is loosely described as diagonal tension in the literature; (2) limiting axial stress in the diagonal concrete struts that form in the wall, which is loosely described as diagonal compression in the literature; or (3) some combination of the two, because the behaviors are closely related. Base sliding does not control the peak shear strength of a low aspect ratio wall, but may dominate the hysteretic response of a wall in displacement cycles following the attainment of peak shear strength.

Figure 8-1 presents the lateral force versus lateral displacement relationship for a wall (SW9) tested by Luna et al. (2015). This wall had an aspect ratio of 0.54 , a web horizontal reinforcement ratio of $0.67 \%$, and a web vertical reinforcement ratio of $1.5 \%$. The reinforcement was ASTM Grade 706, with a minimum yield strength of $60 \mathrm{ksi}$, and the uniaxial compressive strength of the concrete on the day of testing was 4300 psi. The hysteretic response of SW9 is not ductile: the initial stiffness is much greater than the secant stiffness to peak strength, strength and stiffness degrade rapidly with repeated cycling, and base sliding dominates the cyclic response at displacements associated with peak strength. Although the peak strengths in the first and third quadrants of loading are similar for SW9, this was not the case for a number of the walls tested by Luna: damage in the first quadrant of loading affected peak strength in the subsequent reversed cycle (third quadrant) of loading.

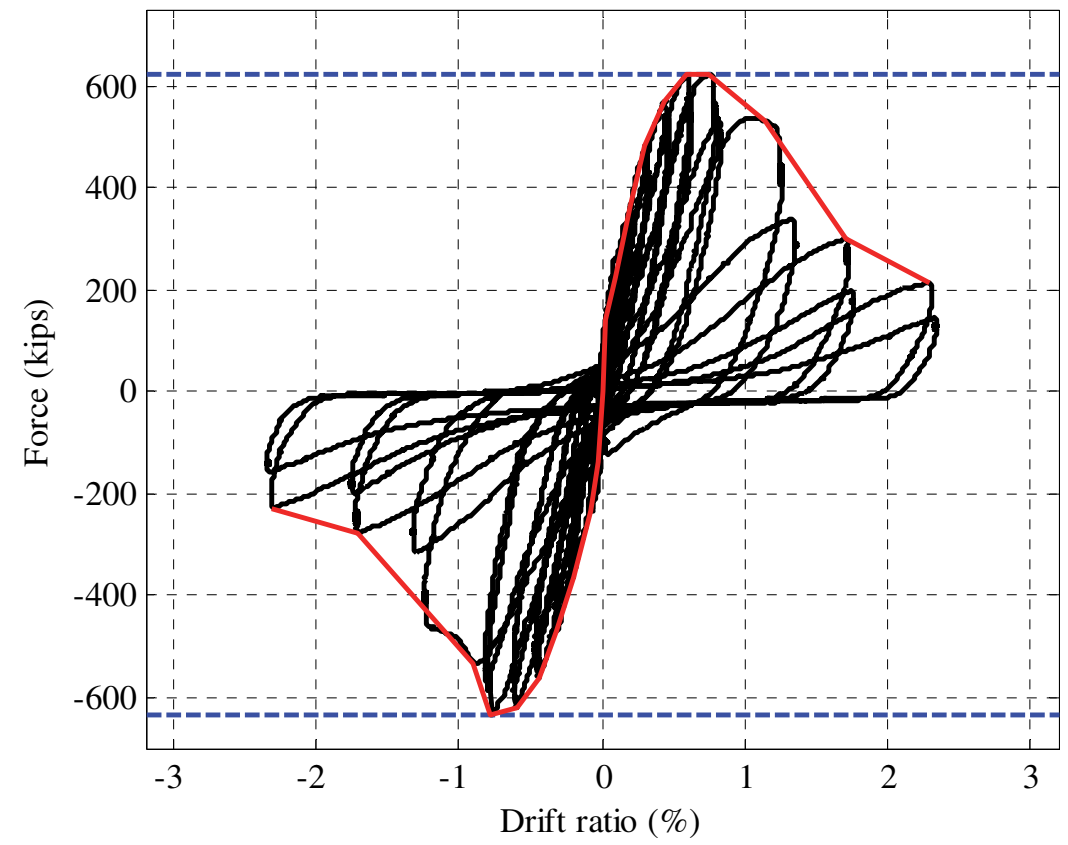

Figure 8-1 Hysteretic response of a low aspect ratio shear wall (Luna et al. 2015). 


\subsection{Summary of Design Code Provisions}

The shear-strength design provisions for low aspect ratio walls in ACI 318, Building Code Requirements for Structural Concrete and Commentary, have not changed materially in nearly 30 years. The ACI 318 equations for nominal shear strength for resistance to non-seismic and seismic loadings, and the upper limits on shear stress, presented in ACI 318-14 (ACI, 2014) are identical to those presented in ACI 318-89 (1989).

Barda et al. (1977) proposed an equation for the nominal shear strength of low-rise reinforced concrete walls based on analysis of data from tests of 8 walls constructed with flanges. This predictive equation accounts for concrete compressive strength, wall aspect ratio, axial force, and vertical web reinforcement ratio. This equation has been widely used in the nuclear industry, noting that nearly all walls inside containment vessels are flanged.

Other predictive equations have been proposed for the shear strength of low aspect ratio walls, including Wood (1990), Gulec and Whittaker (2011), Gulec et al. (2008; 2009), Moehle (2015), Kassem (2015), and Luna et al. (2015). None of these equations have been widely used in design practice and so are not discussed here.

\subsubsection{Modeling Parameters in ASCE/SEI 41-13}

A cyclic backbone curve is constructed from the measured hysteretic response of a wall by joining the (force, displacement) coordinates from the first loading excursion to a given lateral displacement. The red line in Figure 8-1 is the cyclic backbone curve derived from the measured cyclic response of SW9.

ASCE/SEI 41-13, Seismic Evaluation and Retrofit of Existing Buildings, (ASCE, 2014) presents a tri-linear lateral force-lateral deformation relationship to peak strength (see Figure 8-2) for shear-controlled reinforced walls. This relationship adopts Wallace's modification (2007) to the corresponding relationship in ASCE/SEI 41-06 (2007): the addition of a point on the curve (F in Figure 8-2) associated with the onset of cracking. The force-deformation relationship of Figure 8-2, also known as a cyclic backbone curve, is a capacity envelope that reflects the loss of strength due to reversed cyclic loading. This curve is generally different from that obtained under unidirectional monotonic loading (NIST, 2010c).

In Figure 8-2, the shear resistance is normalized by peak shear strength, and lateral deformation is normalized by story height, $h$. Here, lateral deformation normalized by story height is termed drift ratio. Parameters $\mathrm{g}$, $\mathrm{d}$, and e in Figure 8-2 represent yield drift ratio, drift ratio corresponding to the onset of loss of shear strength, and ultimate drift ratio, respectively. Parameters $f$ and $\mathrm{c}$ are associated with the onset of cracking in the wall panel, and residual strength of the wall panel, respectively. 


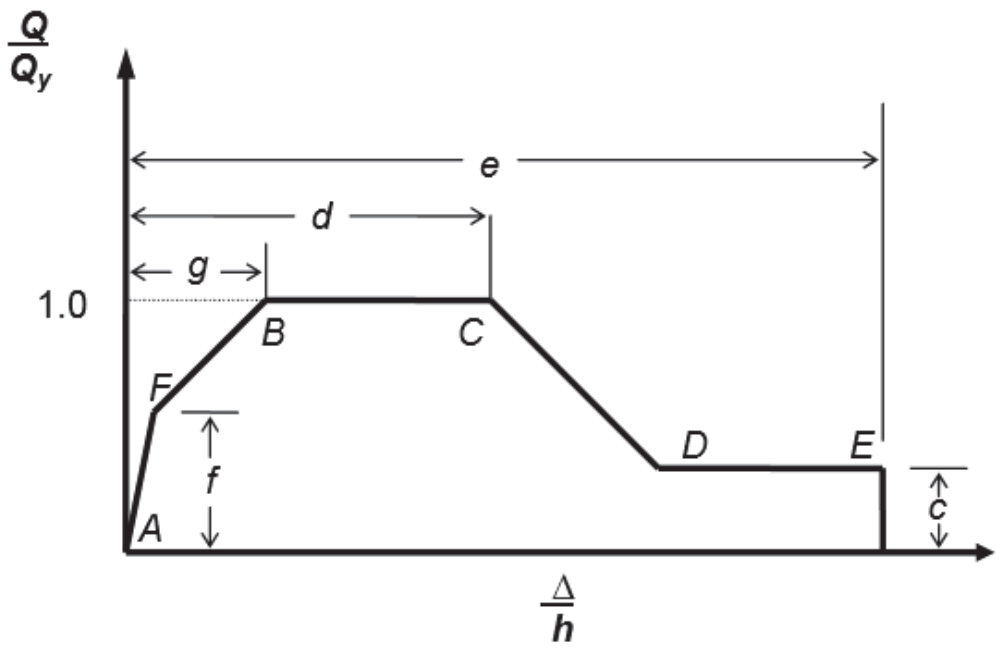

Figure 8-2 Load-deformation relationship for members controlled by shear (ASCE, 2014; with permission from ASCE).

Table 8-1 reproduces Table 10.20 of ASCE/SEI 41-13, which provides recommended values for modeling parameters and for acceptance criteria for nonlinear analysis of shear-critical RC walls. (Acceptance criteria are provided for the performance levels of Immediate Occupancy, Life Safety, and Collapse Prevention.) Per Table 8-1, the normalized force at cracking is 0.6 and the yield drift ratio is 0.4 : values that are assumed independent of axial load. Axial load is assumed to affect the drift at peak strength, the ultimate drift ratio, and residual force ratio.

Table 8-1 Modeling Parameters and Acceptance Criteria for Nonlinear Procedures-R/C Shear Walls and Wall Segments Controlled by Shear (ASCE, 2014)

\begin{tabular}{|c|c|c|c|c|c|c|c|c|}
\hline \multirow[b]{3}{*}{ Conditions } & & & & \multirow{2}{*}{\multicolumn{2}{|c|}{$\begin{array}{c}\text { Strength } \\
\text { Ratio } \\
\end{array}$}} & \multicolumn{3}{|c|}{$\begin{array}{l}\text { Acceptable Total } \\
\text { Drift Ratio (\%) }\end{array}$} \\
\hline & \multicolumn{3}{|c|}{ Total Drift Ratio (\%) } & & & \multicolumn{3}{|c|}{ Performance Level } \\
\hline & $d$ & $e$ & $g$ & $c$ & $f$ & 10 & $L S$ & $\mathrm{CP}$ \\
\hline$\frac{\left(A_{s}-A_{s}^{\prime}\right) f_{y}+P}{t_{w} l_{w} f_{c}^{\prime}} \leq 0.05$ & 1.0 & 2.0 & 0.4 & 0.2 & 0.6 & 0.4 & 1.5 & 2.0 \\
\hline$\frac{\left(A_{s}-A_{s}^{\prime}\right) f_{y}+P}{t_{w} l_{w} f_{c}^{\prime}}>0.05$ & 0.75 & 1.0 & 0.4 & 0.0 & 0.6 & 0.4 & 0.75 & 1.0 \\
\hline
\end{tabular}

\subsection{Database Development}

A significant number of experimental studies have been performed over many decades to characterize the behavior of shear-controlled reinforced concrete walls. Gulec and Whittaker (2011a) compiled a comprehensive database of 434 low-aspect ratio $\mathrm{RC}$ walls tested by other researchers prior to 2009. Luna et al. (2015) summarized experimental data collected between 2010 and 2015. Analytical and 
numerical studies have also been performed but there are no validated analytical models for the analysis of shear-critical walls that are capable of reproducing cyclic backbone response to levels of lateral drift corresponding to failure (measured here in terms of a significant loss of strength and stiffness), and validated cyclic macro models suitable for nonlinear dynamic analysis.

Data from the cyclic testing of 240 low-aspect ratio reinforced concrete walls were assembled to evaluate the utility of the ASCE/SEI 41-13 backbone curve of Figure 8-2. Specific information on the experimental setup of each wall in the database is presented in Appendix B.

The force-displacement relationship reported in the literature for each specimen was digitized, and reported properties including dimensions, details of reinforcement, and material properties, were logged. (The accuracy of the reported properties was not investigated, which introduces uncertainty into the calculations presented below.) The procedures used to establish the coordinates of the key points on the backbone curve are explained in the following sections.

\subsection{Peak Shear Strength}

Experimentally measured peak shear strengths of rectangular, barbell, and flanged walls in the database were compared with predictions obtained using three equations: equations from Chapters 11 and 18 of ACI 318-14 (2014) and Barda et al. (1977). The ACI equations do not recognize the presence of boundary columns (i.e., barbell wall) or boundary flanges (i.e., flanged wall). The Barda et al. (1977) equations were derived from tests of flanged walls.

Other equations have been proposed for predicting the shear strength of low aspect ratio walls, including Wood (1990), Gulec and Whittaker (2011), Gulec et al. (2008; 2009), Moehle (2015), Kassem (2015), and Luna et al. (2015). The Wood equation is empirical and calculates peak strength, within limits on average shear stress, using a shear-friction-type equation. The Gulec and Whittaker equations have a physical basis but are calibrated to a dataset developed in the mid-2000s, and so are considered empirical. The Kassem equation is similarly calibrated to a dataset. The Moehle equation applies best to very low aspect ratio walls, and predictions for walls with aspect ratios of 1.0 and greater are not supported by data. The Luna equation is physics-based but relies on insight gained from stress and strain measurements from his experiments; similar data from prior experiments are not available and the Luna studies are yet to be published in the archival literature. Because these other equations are not widely used in design practice in the United States, they are not investigated further in this section. 


\subsubsection{Chapter 11 of ACI 318-14}

The ACI equations in Chapter 11 of ACI 318-14 involve a diagonal tension check with an upper limit on shear stress that represents an indirect check on diagonal compression failure. Section 11.5 of ACI 318-14 provides equations for the peak shear strength of low aspect ratio shear walls. The nominal shear strength is given by:

$$
V_{n}=V_{c}+V_{s} \leq 10 \sqrt{f_{c}^{\prime}} d t_{w}
$$

where $V_{c}(\mathrm{lb})$ is the nominal shear force carried by concrete, $V_{s}(\mathrm{lb})$ is the nominal shear strength provided by shear reinforcement, $f_{c}^{\prime}$ (psi) is the compressive strength of concrete, $d$ (in) is the distance of the extreme compression fiber to the centroid of the tensile reinforcement (taken as $0.8 l_{w}$, where $l_{w}$ is the length of the wall), and $t_{w}$ (in) is the thickness of the wall. The concrete contribution, $V_{c}$, is the smaller of:

$$
\begin{gathered}
V_{c}=3.3 \lambda \sqrt{f_{c}^{\prime}} d t_{w}+\frac{N_{u} d}{4 l_{w}} \\
V_{c}=\left[0.6 \lambda \sqrt{f_{c}^{\prime}}+\frac{l_{w}\left(1.25 \lambda \sqrt{f_{c}^{\prime}}+\frac{0.2 N_{u}}{l_{w} t_{w}}\right)}{\frac{M_{u}}{V_{u}}-\frac{l_{w}}{2}}\right] d t_{w}
\end{gathered}
$$

where $N_{u}(\mathrm{lb})$ is the factored axial compressive force, $M_{u}(\mathrm{lb}-\mathrm{in})$ is the factored moment, $V_{u}(\mathrm{lb})$ is the factored shear force and $\lambda=1.0$ for normal weight concrete. If $\left(M_{u} / V_{u}-l_{w} / 2\right)$ is negative, Equation 8-3 is not applicable. The rebar contribution, $V_{s}$, is given by:

$$
V_{s}=\frac{A_{v} f_{y} d}{s}
$$

where $s$ (in) is the spacing of horizontal reinforcement, and $A_{v}\left(\mathrm{in}^{2}\right)$ is the area of horizontal reinforcement within distance $s$. The minimum horizontal reinforcement ratio, $\rho_{t}$, shall not be less than 0.0025 . The spacing $s$ must not exceed the smallest of $l_{w} / 5,3 h$, and 18 inches. The minimum vertical reinforcement ratio, $\rho_{l}$, must not be less than the greater of 0.0025 and that given by:

$$
\rho_{l}=0.0025+0.5\left(2.5-\frac{h_{w}}{l_{w}}\right)\left(\rho_{t}-0.0025\right)
$$

The spacing of the vertical reinforcement should not exceed the smallest of $l_{w} / 3,3 h$, and 18 inches. 


\subsubsection{Chapter 18 of ACI 318-14}

The nominal peak shear strength in section 18.10 of ACI 318-14 is given by:

$$
V_{n}=\left(\alpha_{c} \lambda \sqrt{f_{c}^{\prime}}+\rho_{t} f_{y h}\right) A_{w} \leq 10 \sqrt{f_{c}^{\prime}} A_{w}
$$

where $\alpha_{c}$ is the coefficient defining the relative contribution of concrete strength and is equal to 3.0 for $h_{w} / l_{w} \leq 1.5,2.0$ for $h_{w} / l_{w} \geq 2.0$ and varies linearly between 3.0 and 2.0 for $h_{w} / l_{w}$ between 1.5 and $2.0 ; \lambda=1.0$ for normal weight concrete; $f_{c}^{\prime}$ (psi) is the compressive strength of concrete; $\rho_{t}$ is the horizontal reinforcement ratio; $f_{y h}$ (psi) is the yield stress of the horizontal web reinforcement; and $A_{w}\left(\mathrm{in}^{2}\right)$ is the area of the wall.

\subsubsection{Barda et al. (1977)}

The equation of Barda et al. (1977) to predict the peak shear strength was derived based on tests of eight low aspect ratio walls with heavily reinforced flanges, and better predictions of peak strength are expected for flanged walls than for rectangular walls (and walls with barbells). The equation is applied to the flanged walls only. The nominal peak strength, $V_{n}$, is given by:

$$
V_{n}=\left(8 \sqrt{f_{c}^{\prime}}-2.5 \sqrt{f_{c}^{\prime}} \frac{h_{w}}{l_{w}}+\frac{N_{u}}{4 l_{w} t_{w}}+\rho_{l} f_{y v}\right) d t_{w}
$$

where $f_{c}^{\prime}$ (psi) is the compressive strength of concrete, $h_{w}$ (in) is the height of the wall, $l_{w}$ (in) is the length of the wall, $N_{u}(\mathrm{lb})$ is the factored axial compressive force, $t_{w}$ (in) is the thickness of the web, $\rho_{l}$ is the vertical web reinforcement ratio, $f_{y v}$ (psi) is the yield stress of vertical web reinforcement, and $d$ (in) is the distance of the extreme compression fiber to the centroid of the tensile reinforcement.

\subsubsection{Evaluation of Results and Recommendations}

Figure 8-3 presents predicted normalized peak strength, $V_{p p} / d_{w} t_{w}$, versus measured normalized peak strength, $V_{p t} / d_{w} t_{w}$, where $V_{p p}$ and $V_{p t}$ are predicted and measured peak lateral strengths. The ACI equations are applied to all walls and the Barda et al. equation is applied to flanged walls only. The statistically estimated values of the average, standard deviation, and coefficient of variation for each set of empirical predictive equation are presented in the graphs.

Two sets of results are presented in Figures 8-3a and 8-3b to investigate the effect of the upper shear stress limits on the accuracy of the ACI-based predictions of peak shear strength. The solid black circles are predictions that implement the upper limit on average shear stress, and the solid red squares are predictions that set aside the upper limit on shear stress. 

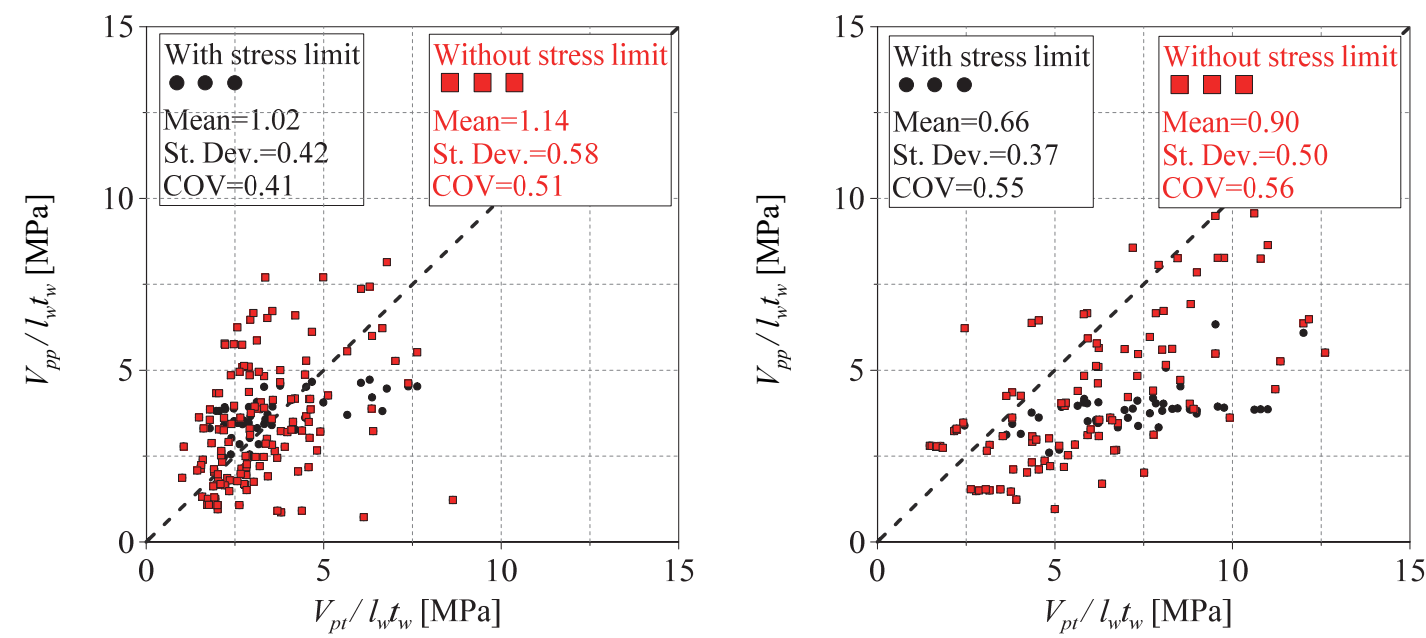

(a) ACI 318-14 Chapter 11; rectangular walls (left panel), and barbell and flanged walls (right panel)
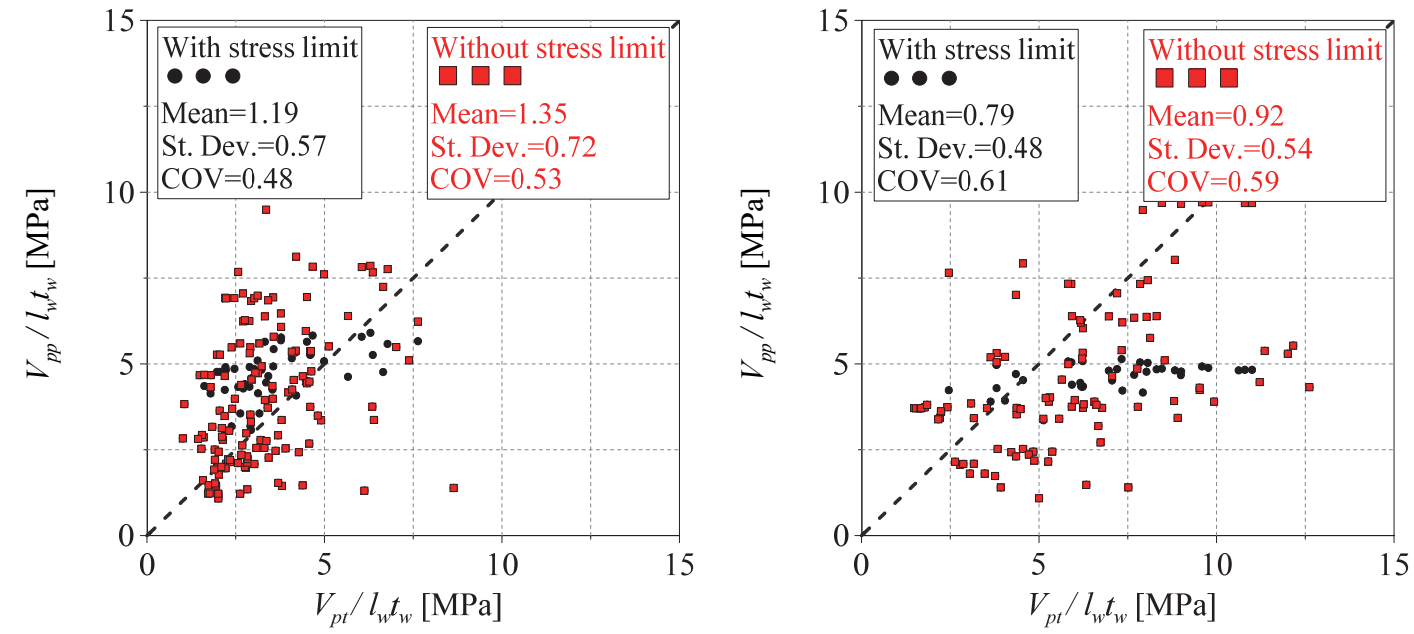

(b) ACI 318-14 Chapter 18; rectangular walls (left panel), and barbell and flanged walls (right panel)

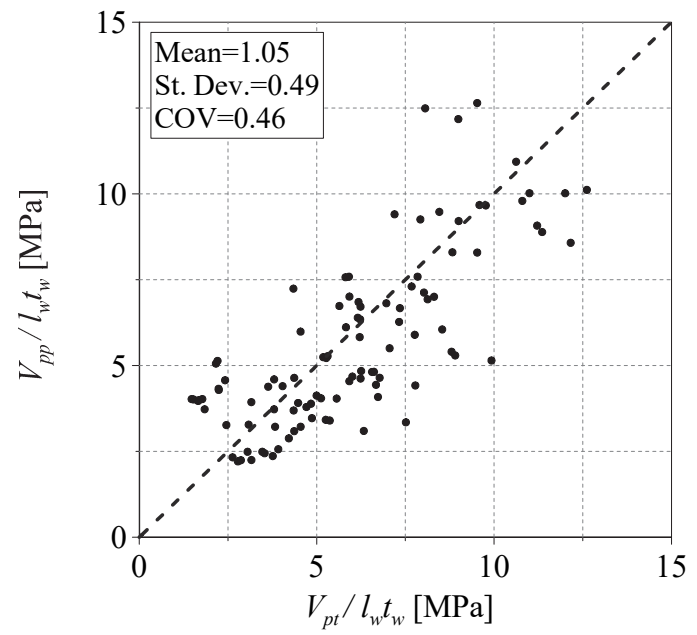

(c) Barda et al. (1977); flanged walls

Figure 8-3 Predicted and measured normalized lateral strength of reinforced concrete shear walls. 
The upper limit in Section 11.5.4.3 of ACI 318-14 of $10 \sqrt{f_{c}^{\prime}} h d$, where $h$ is the wall thickness and $d$ can be taken to be $80 \%$ of the length of the wall, is "imposed to guard against diagonal compression failure in shear walls" (Gulec and Whittaker, 2011a).

The upper limit in Section 18.10.4.4 of ACI 318-14 of $8 \sqrt{f_{c}^{\prime}} A_{c v}$, where $A_{c v}$ is the gross area of the cross section, is effectively identical to the that of Section 11.5.4.3 if the effective depth is assumed equal to $80 \%$ of the length of the wall.

Recommendations for predicting the peak shear strength of a low aspect ratio shear wall are as follows:

- For rectangular walls, the equation for nominal shear strength provided in Section 11.5.4.3 of ACI 318-14 should be used including the upper limit on nominal shear stress, and using expected rebar and concrete strengths for the calculation.

- For barbell or flanged walls, the Barda et al. equation should be used with expected rebar and concrete strengths in the calculation.

It is noted that the predictive equations are reasonable in a mean sense only: the scatter, measured here in terms of coefficient of variation, is great.

\subsection{Cyclic Backbone Curves}

The database of test results was used to investigate the effects of the following design variables on the cyclic backbone curve:

- Wall aspect (moment-to-shear) ratio, $M / V l_{w}$

- Vertical web reinforcement ratio $^{1}, \rho_{v} f_{y v}$

- Horizontal web reinforcement ratio, $\rho_{h} f_{y h}$

- Vertical reinforcement ratio in the boundary element ${ }^{2}, \rho_{v b} f_{y v b}$

- $\quad$ Normalized axial compressive load, $P / A_{g} f_{c}^{\prime}$

- Concrete uniaxial compressive strength, $f_{c}^{\prime}$

\subsubsection{Constructing a Cyclic Backbone Curve}

Following ASCE/SEI 41-13, the pre-peak-strength response of a low aspect ratio reinforced concrete wall was idealized as a tri-linear force-displacement relationship, corresponding to regions of elastic (origin-A), post-cracking (A-B), and post-yielding (B-C) response, as presented in Figure 8-4. The displacements and forces at the point of cracking ( $\delta_{A}$ and $F_{A}$ in Figure 8-4), and at the point of peak strength (termed the

${ }^{1}$ Ratios are multiplied by the yield stress of the reinforcement.

${ }^{2} \mathrm{~A}$ boundary element in a rectangular wall is that portion at the end of the wall that is locally strengthened by vertical reinforcement. A boundary element in barbell wall or a flanged wall is the lateral extension of the wall beyond the web. 
peak point hereafter) ( $\delta_{C}$ and $F_{C}$ in Figure 8-4) were obtained directly from the measured cyclic backbone curve. Because the drift ratio and lateral force at the onset of visible cracking were generally not reported, the point of cracking was associated herein to the onset of nonlinearity in the force-displacement relationship. The displacements and forces corresponding to point B were calculated using an equalenergy method (i.e., the area under the calculated backbone curve up to the peak point set equal to the corresponding area under the idealized backbone curve) as presented in Figure 8-4. Per Figure 8-5, the slope of the post-peak branch (C-D) of the idealized backbone curve was calculated as the average of slopes of the lines in the post-peak region; see the dashed red lines presented in Figure 8-5. (For example, line with slope $m_{2}$ joins the peak point and the local maximum corresponding to the second displacement increment after that to the peak point.) Three levels of postpeak force were considered for point D: $\mathrm{D}^{20}, \mathrm{D}^{40}$, and $\mathrm{D}^{80}$; corresponding to $20 \%$, $40 \%$, and $80 \%$ of peak strength, respectively.

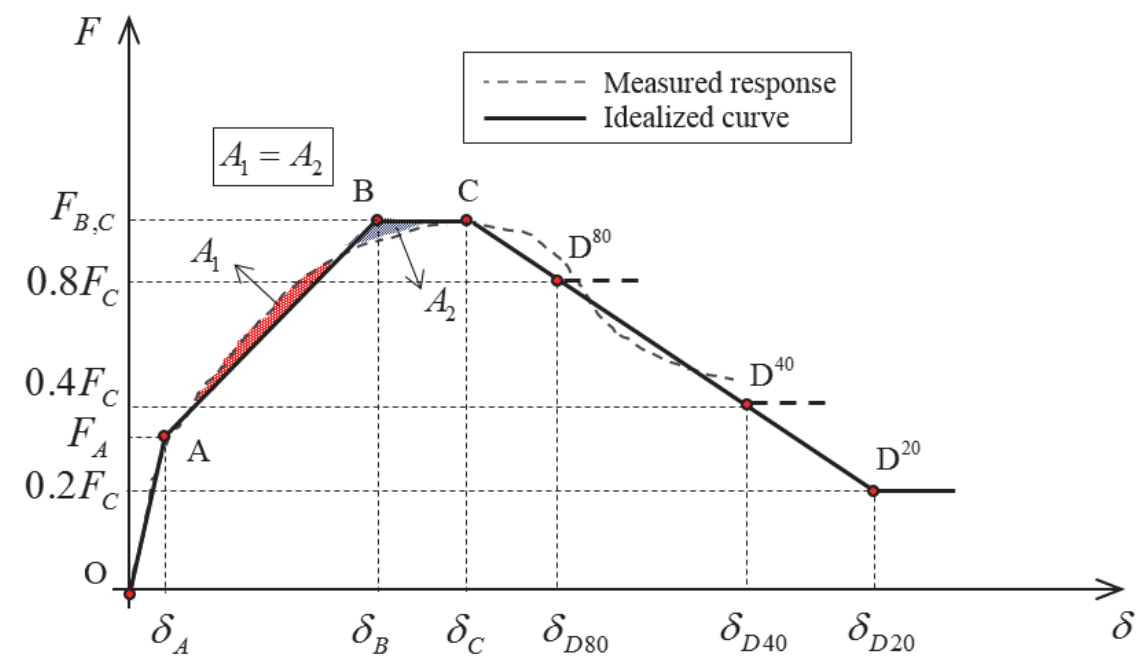

Figure 8-4 Measured and idealized cyclic backbone curves.

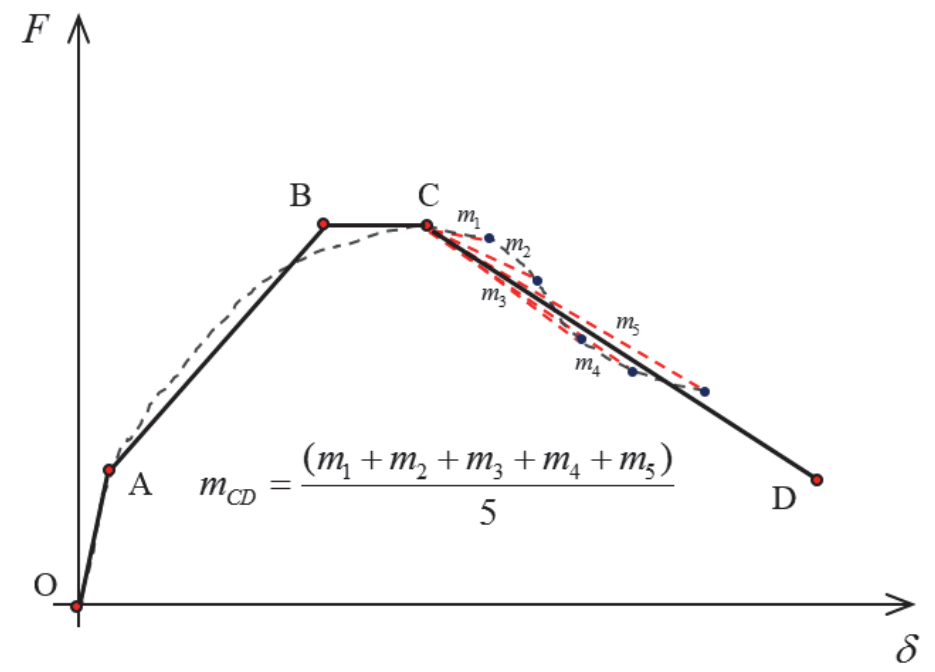

Figure 8-5 Describing post-peak response. 


\subsubsection{Control Points for Cyclic Backbone Curves}

The effects of the design variables on the values of drift ratio and lateral force at the key points on the idealized backbone curve are presented in Figure 8-6 through 8-17. The figures include the test data, a trend line (solid red) based on a least squares fit, and lower and upper bounds (solid black lines) for a confidence level of $95 \%{ }^{3}$. The properties of the trend line and the parameters of the statistical evaluation are presented in Appendix B.

\subsubsection{Drift at Cracking}

The effects of design variables on the drift ratio at cracking are presented in Figure 8-6. Of the six design variables considered here, $f_{c}^{\prime}$ has the greatest effect on rectangular (planar) walls: the greater the value of $f_{c}^{\prime}$, the greater the drift ratio at cracking. The effect of $f_{c}^{\prime}$ on the response of walls with barbells and flanges is small. Per the data, the drift ratio at cracking is also affected by aspect (moment-to-shear) ratio, axial compressive force, and boundary element reinforcement ratio. As expected, the drift ratio at cracking, $\delta_{A}$, increases with aspect ratio. The trend lines in both panels of Figure 8-6f indicates that an increase in axial compressive force leads to a decrease in the drift at the onset of cracking, which is counter intuitive, calling into question the following: (1) the use of trend lines for widely scattered data; (2) the accuracy of the reported data; and (3) the association here of cracking with the first deviation from initial stiffness (or onset of nonlinear response). The vertical reinforcement ratio in the boundary elements should have no effect on the drift ratio at the onset of cracking because the corresponding rebar strains will be tiny.

\subsubsection{Normalized Force at Cracking}

Figure 8-7 shows the effects of design variables on the normalized force at cracking defined here as the ratio of the force at cracking (as defined above) to the peak lateral force: $V_{A} / V_{C}$. The dashed blue line in Figure 8-7 corresponds to a ratio of 0.6: the value identified in ASCE/SEI 41-13. Figure 8-7b suggests that $f_{c}^{\prime}$ affects only the normalized force at cracking for flanged and barbell walls. The measure of vertical web reinforcement, $\rho_{v} f_{v v}$, has a significant effect on $V_{A} / V_{C}$. An increase in $\rho_{v} f_{v v}$ leads to a decrease in $V_{A} / V_{C}$ in both rectangular and flanged walls because of the following: (1) the lateral strength of a low aspect ratio wall is dependent on the vertical reinforcement ratio; and (2) the force at cracking should be independent of $\rho_{v} f_{y v}$. Increases in $\rho_{h} f_{v h}$ and $\rho_{v b} f_{v v b}$ reduce $V_{A} / V_{C}$ for rectangular walls, with a smaller effect observed for flanged walls. Axial compression affects the normalized force at cracking: as the axial force increases, $V_{A} / V_{C}$ decreases.

${ }^{3}$ Confidence intervals are used to indicate the reliability of an estimate. A $95 \%$ confidence level means there is $95 \%$ probability that the mean of the statistical population falls within the range of the mean plus/minus the standard deviation. 

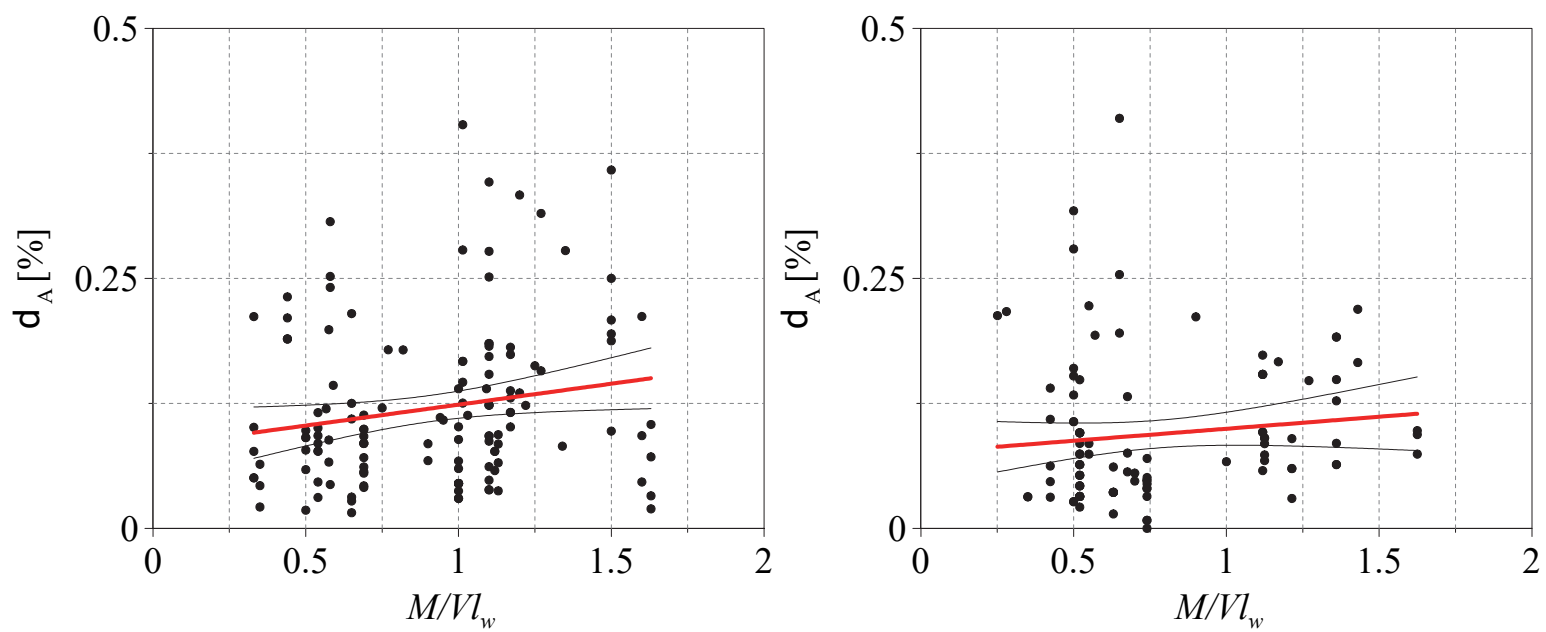

(a) $M / V I_{w}$ : rectangular walls (left panel), and barbell and flanged walls (right panel)
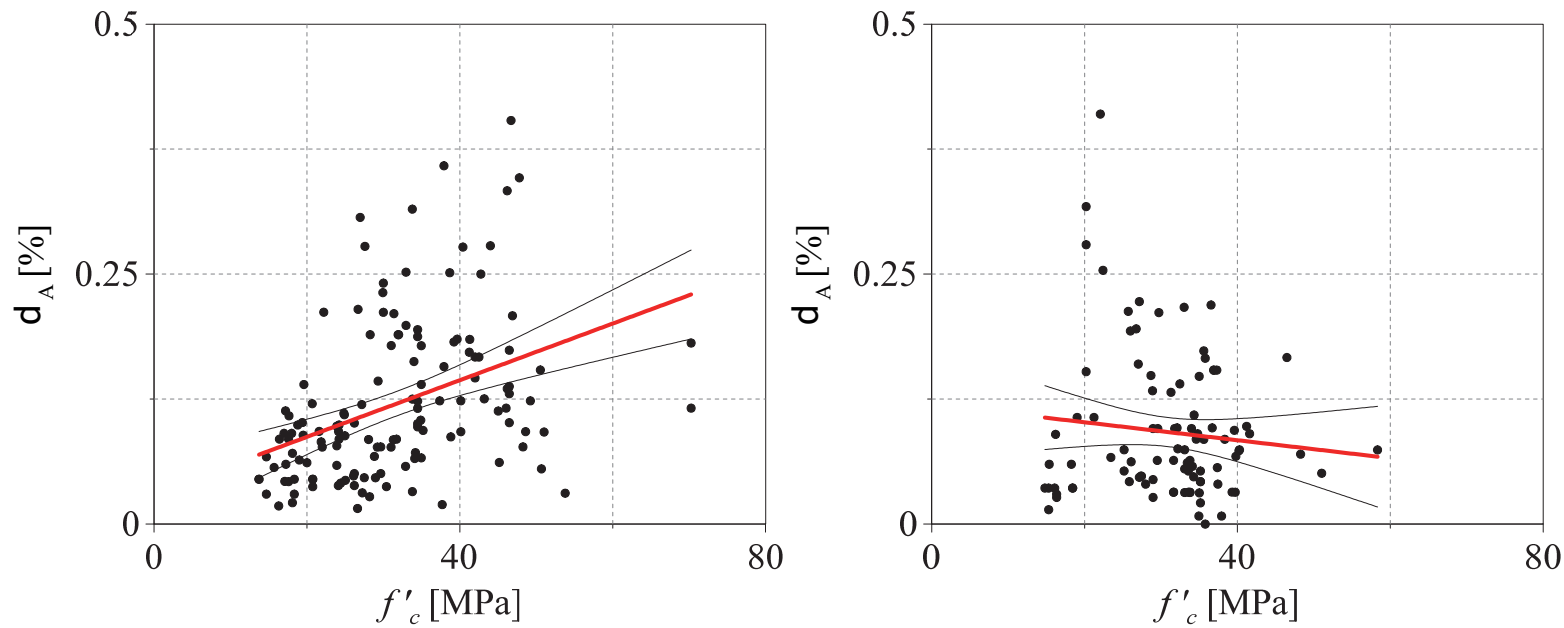

(b) $f_{c}^{\prime}$ : rectangular walls (left panel), and barbell and flanged walls (right panel)
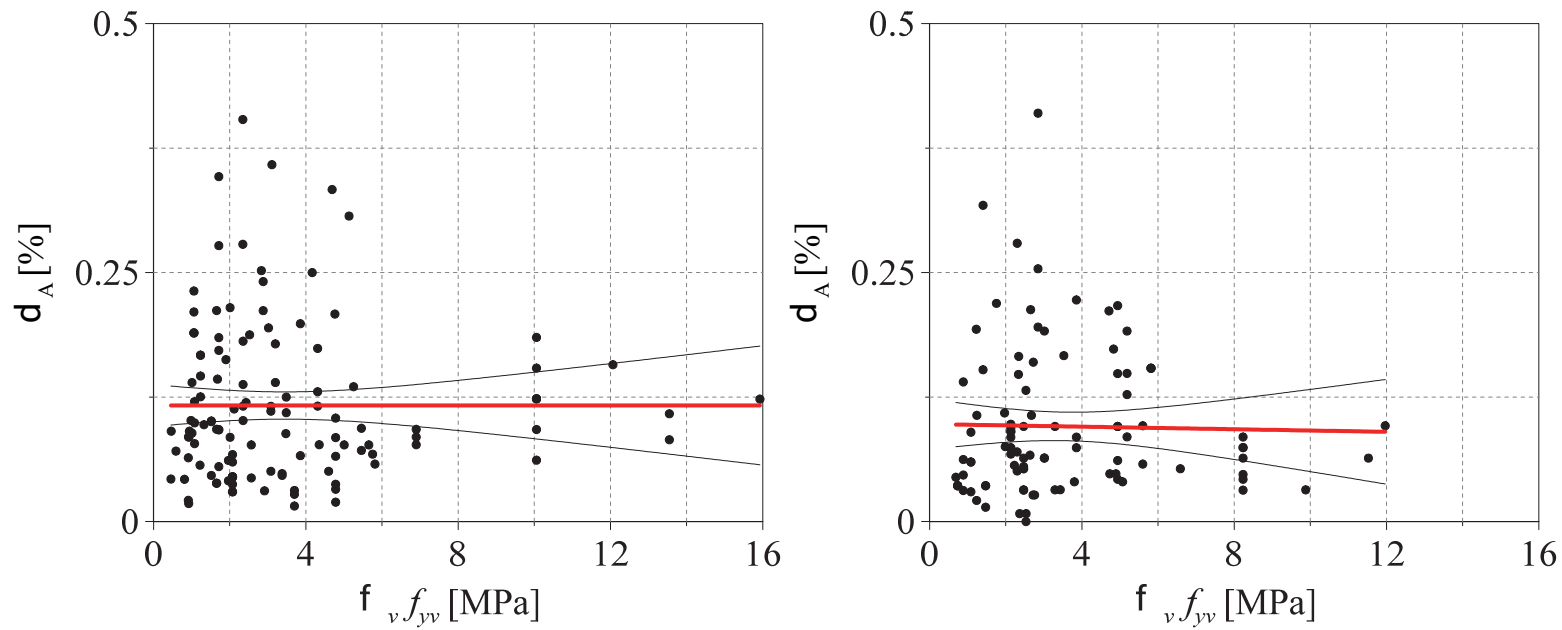

(c) $\rho_{v} f_{y v}$ : rectangular walls (left panel), and barbell and flanged walls (right panel)

Figure 8-6 Effect of design variables on drift ratio at cracking, $\delta_{A}$. 

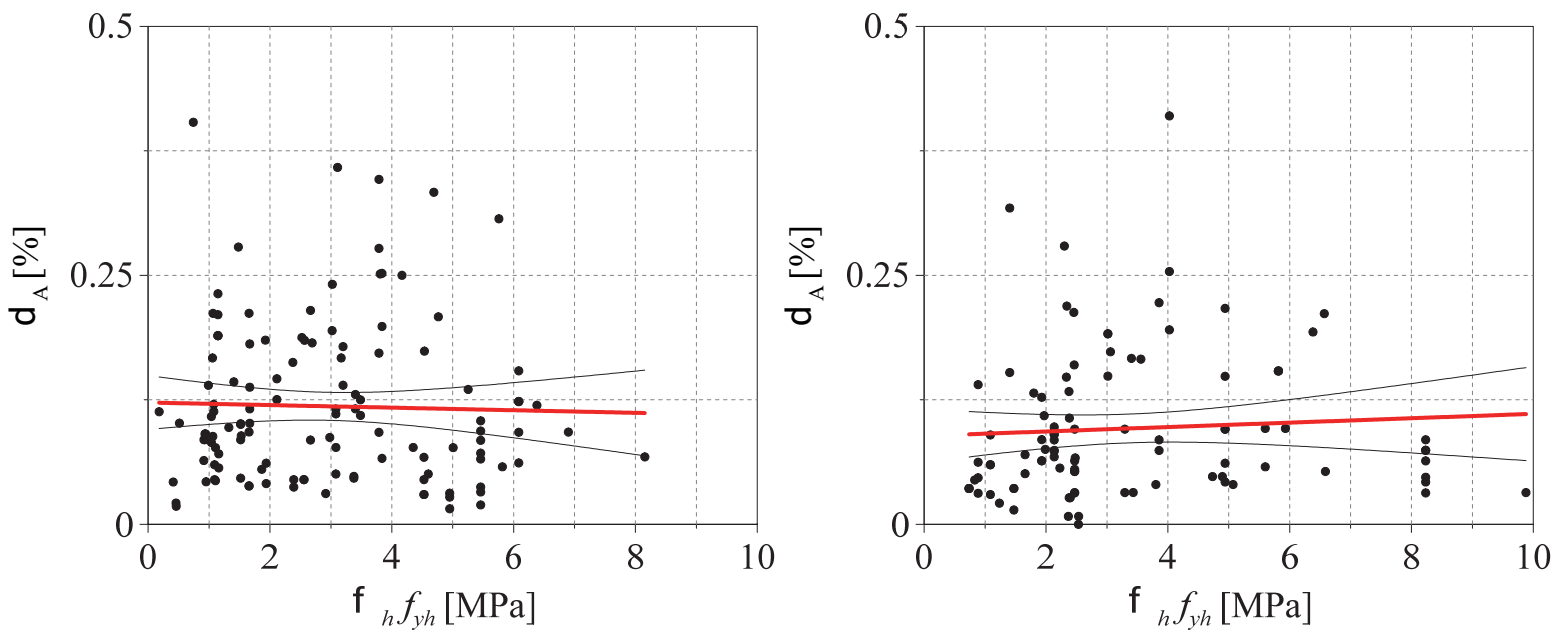

(d) $\rho_{h} f_{v h}$ : rectangular walls (left panel), and barbell and flanged walls (right panel)
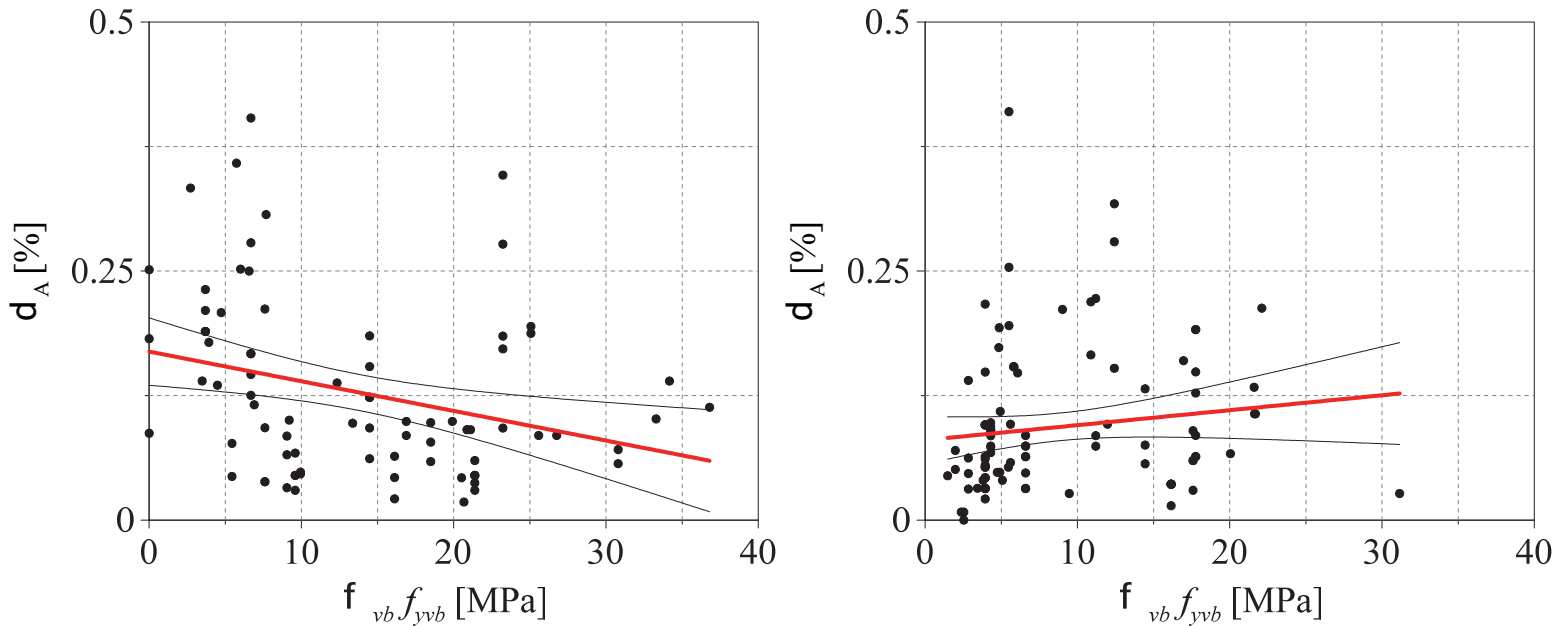

(e) $\rho_{v b} f_{v v b}$ : rectangular walls (left panel), and barbell and flanged walls (right panel)
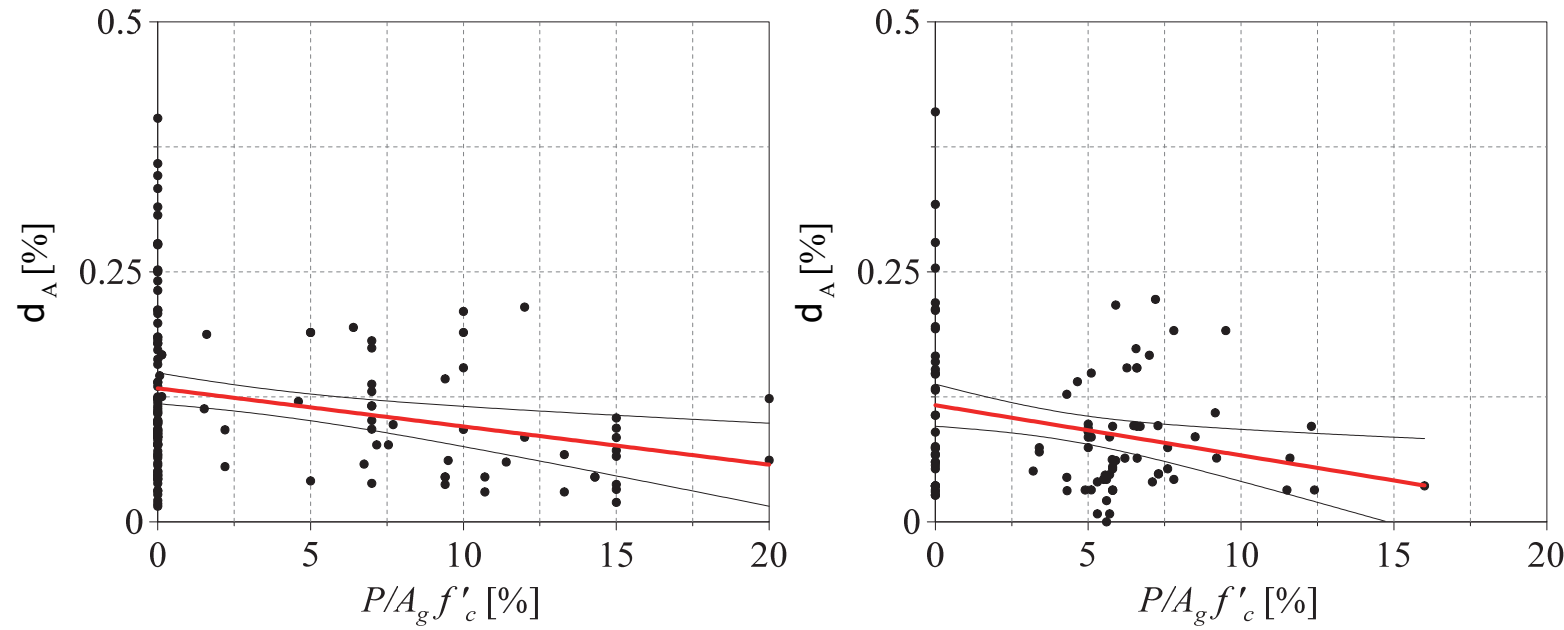

(f): $P / A_{g} f_{c}^{\prime}$ : rectangular walls (left panel), and barbell and flanged walls (right panel)

Figure 8-6 Effect of design variables on drift ratio at cracking, $\delta_{A}$ (continued). 

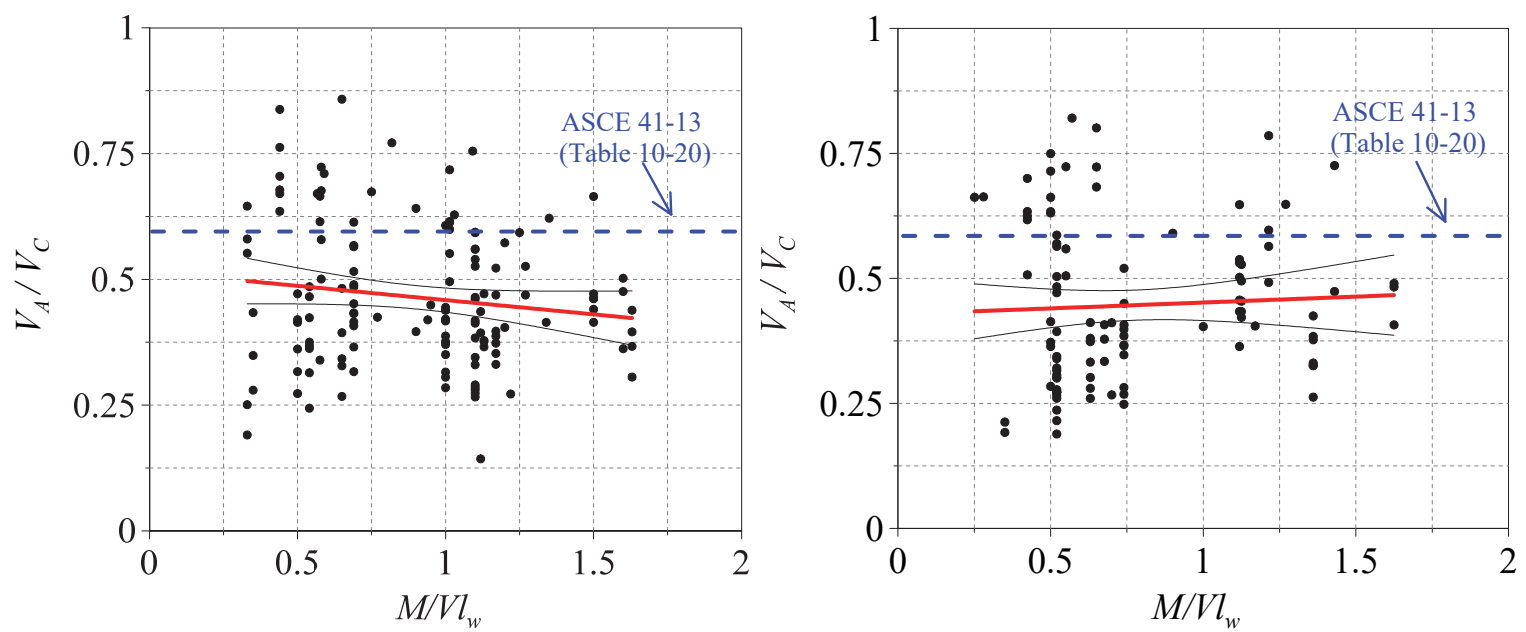

(a) $M / V I_{w}$ : rectangular walls (left panel), and barbell and flanged walls (right panel)
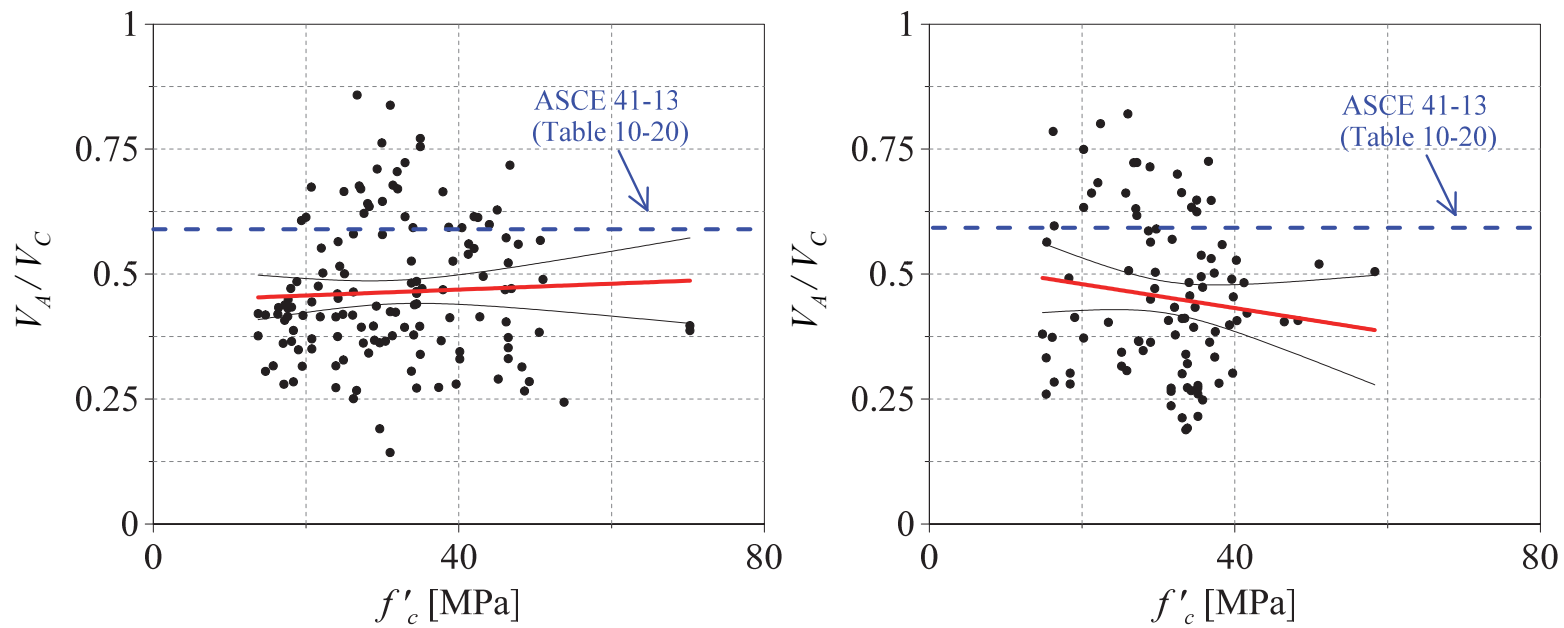

(b) $f_{c}^{\prime}$ : rectangular walls (left panel), and barbell and flanged walls (right panel)
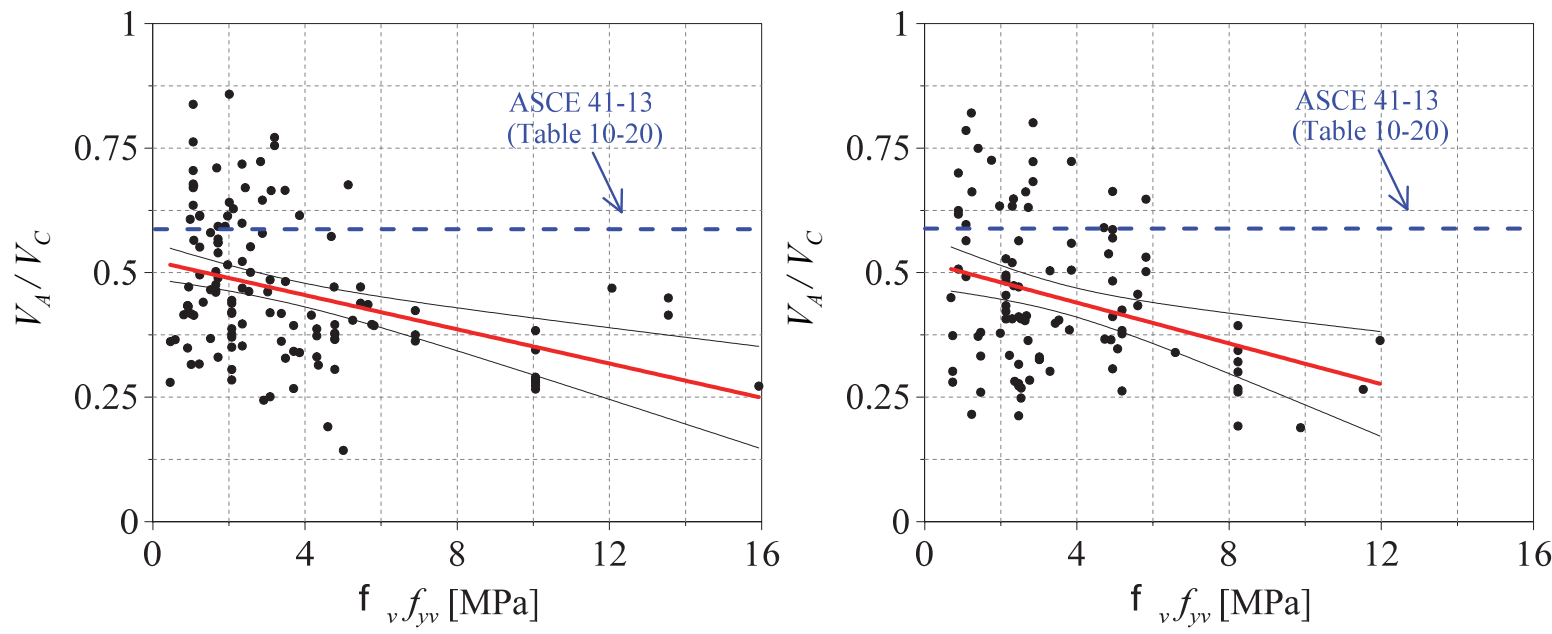

(c) $\rho_{v} f_{y v}$ : rectangular walls (left panel), and barbell and flanged walls (right panel)

Figure 8-7 Effect of design variables on normalized force at cracking, $V_{A} / V_{C}$. 

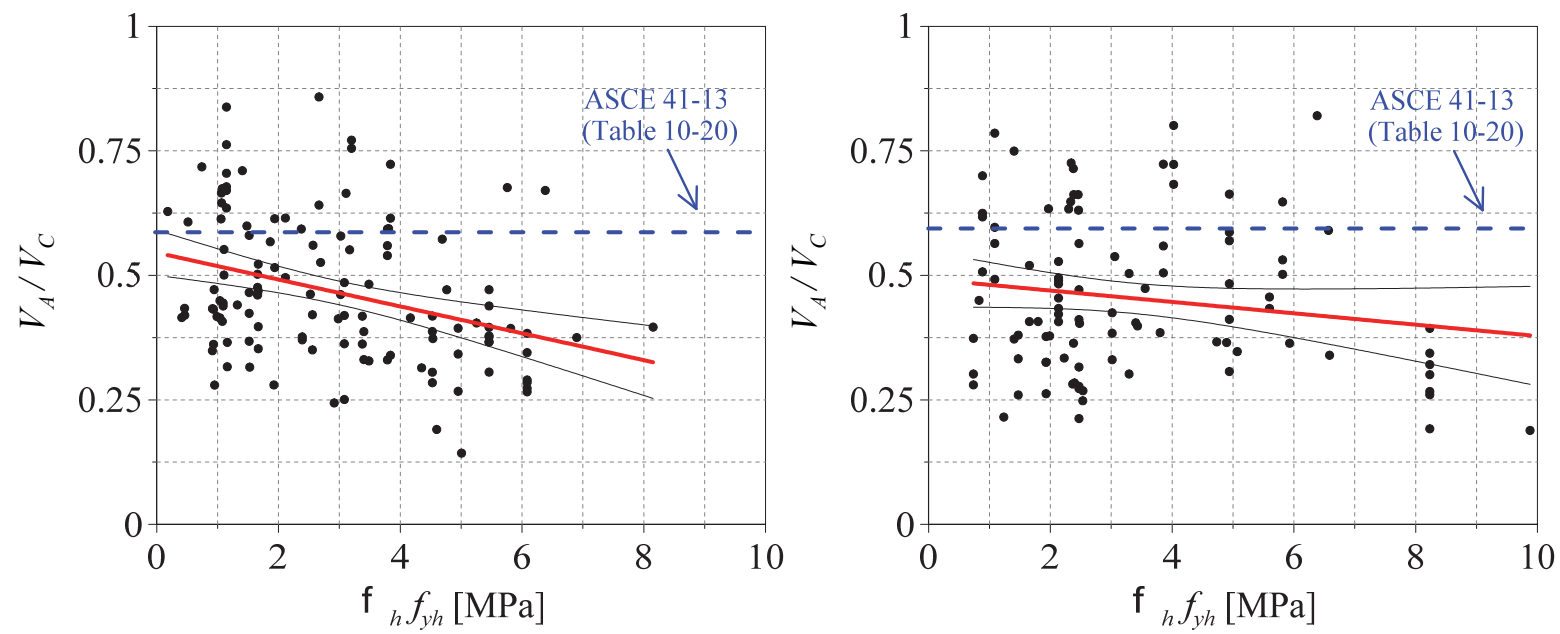

(d) $\rho_{h} f_{v h:}$ rectangular walls (left panel), and barbell and flanged walls (right panel)
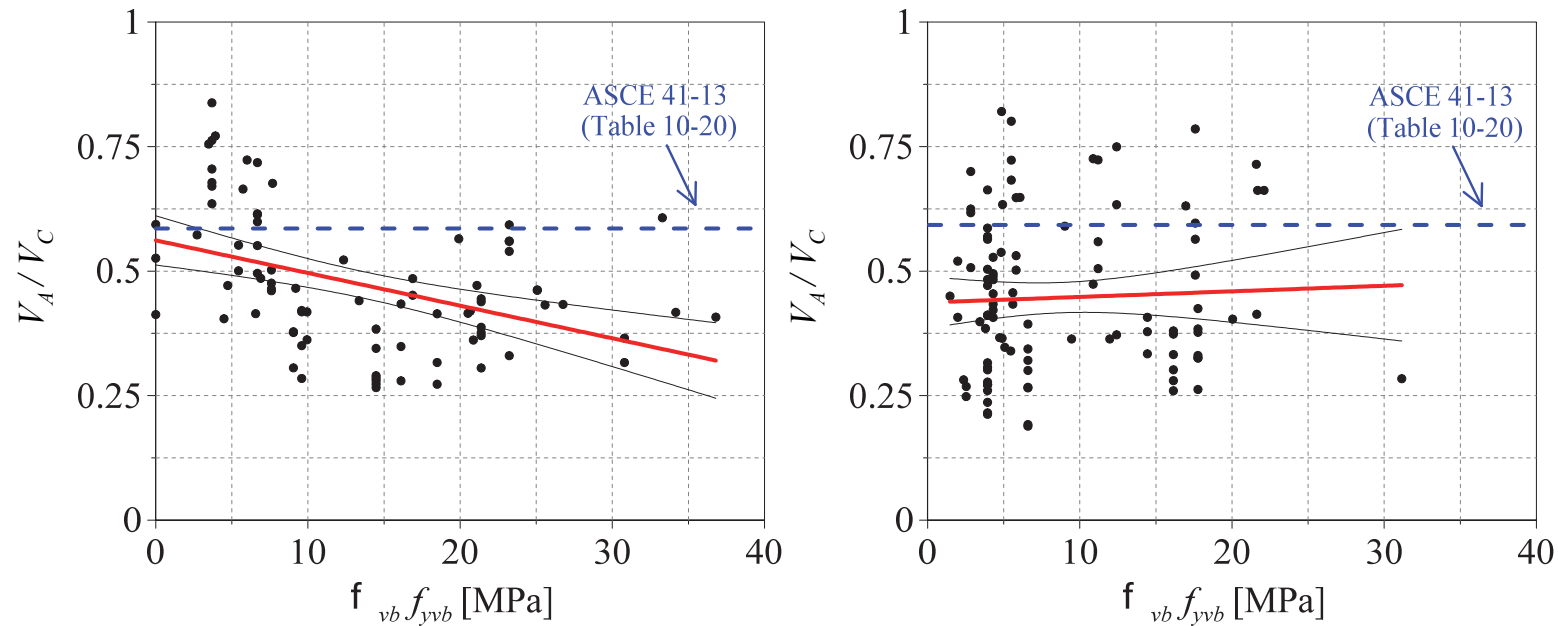

(e) $\rho_{v b} f_{v v b}$ : rectangular walls (left panel), and barbell and flanged walls (right panel)
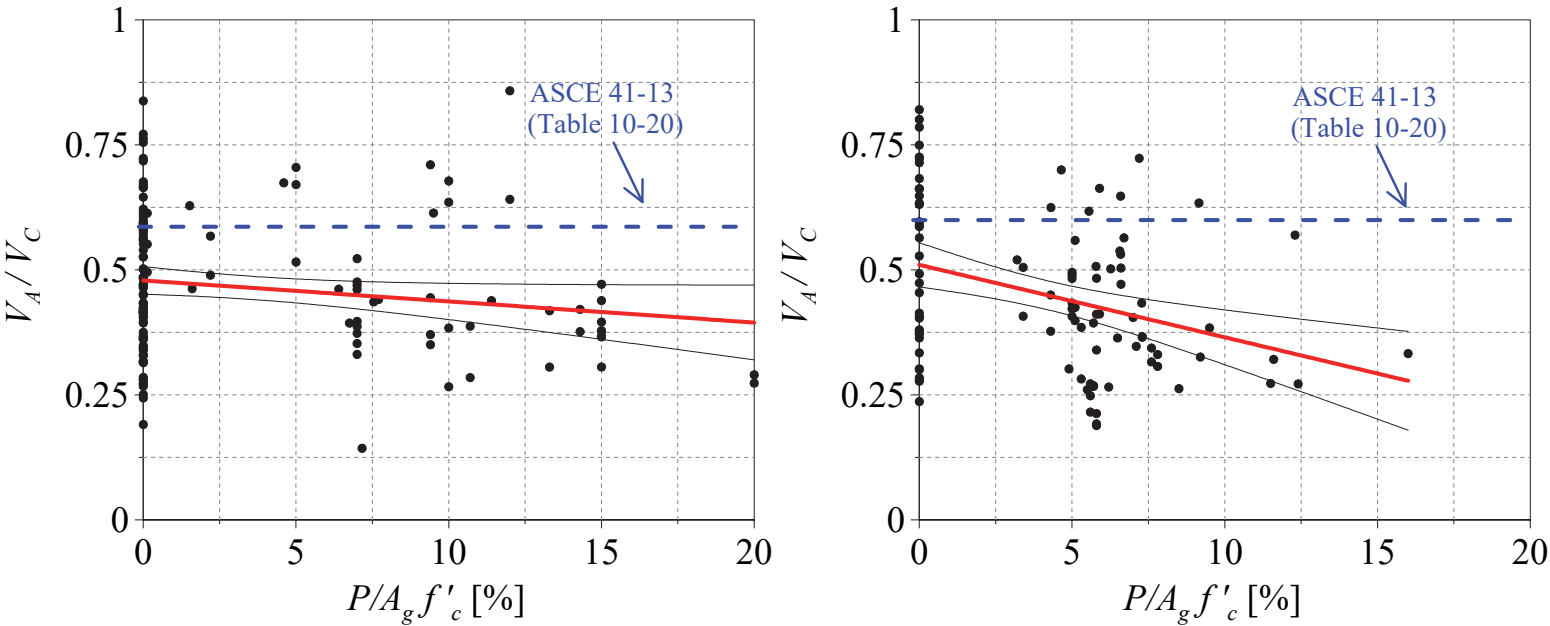

(f) $P / A_{g} f_{c}^{\prime}$ : rectangular walls (left panel), and barbell and flanged walls (right panel)

Figure 8-7 Effect of design variables on normalized force at cracking, $V_{A} / V_{C}$ (continued). 


\subsubsection{Drift Ratio at Yield}

Figure 8-8 presents data for drift ratio at yield, $\delta_{B}$. The dashed blue line in Figure 8-8 corresponds to the ASCE/SEI 41-13 value of 0.4\% (see Table 8-1), which underestimates most of the recorded data. Wall aspect ratio affects the yield drift ratio, although the effect on rectangular walls is small. Concrete uniaxial compressive strength, $f_{c}^{\prime}$, has a significant effect on the yield drift ratio for both types of wall.

\subsubsection{Drift Ratio at Peak Strength}

Figure 8-9 presents data for drift ratio at peak strength, $\delta_{C}$. Two dashed lines are shown in the panels. The green dashed line corresponds to a drift ratio of $1 \%$ (ASCE/SEI 41-13 value if the axial force ratio is 0.05 or smaller). The blue dashed line corresponds to $0.75 \%$ (ASCE/SEI $41-13$ value if the axial force ratio is greater than 0.05). Of the six design variables considered in this study, $f_{c}^{\prime}$ and $M / V l_{w}$ have the greatest effect on flanged walls: as $f_{c}^{\prime}$ or $M / V l_{w}$ increase, the drift ratio at peak strength increases. The drift ratio at peak strength decreases with increasing axial force ratio: the values proposed in ASCE/SEI 41-13 are not unreasonable.

\subsubsection{Normalized Peak Lateral Strength}

Figure 8-10 presents the effects of design variables on normalized peak lateral strength, $V_{d} / d_{w} t_{w}$, where the normalized peak strength was calculated as the peak lateral strength divided by the area of the web, $d_{w} t_{w}$. (The flanges contribute to shear resistance, as is clear from the y-axis range on the reported data, in the left and right columns of Figure 8-10. Substantially greater values of normalized peak strength are achieved in the flanged walls because only the web area is used to normalize the peak lateral strength.) The normalized peak lateral strength of the rectangular and flanged walls vary with $f_{c}^{\prime}, \rho_{v} f_{y v}$, and $\rho_{h} f_{y h}$ : as these values increase, $V_{c} / d_{w} t_{w}$ increases. Aspect ratio also influences normalized peak lateral strength of flanges walls, with an increase in aspect ratio leading to a decrease in normalized strength. Interestingly, this trend is not seen in the rectangular walls. Axial compressive force increases normalized peak lateral strength.

\subsubsection{Force at Peak Lateral Strength}

Section 8.4 presents recommendations for predicting the peak shear strength of rectangular, barbell, and flanged, low aspect ratio shear walls. 

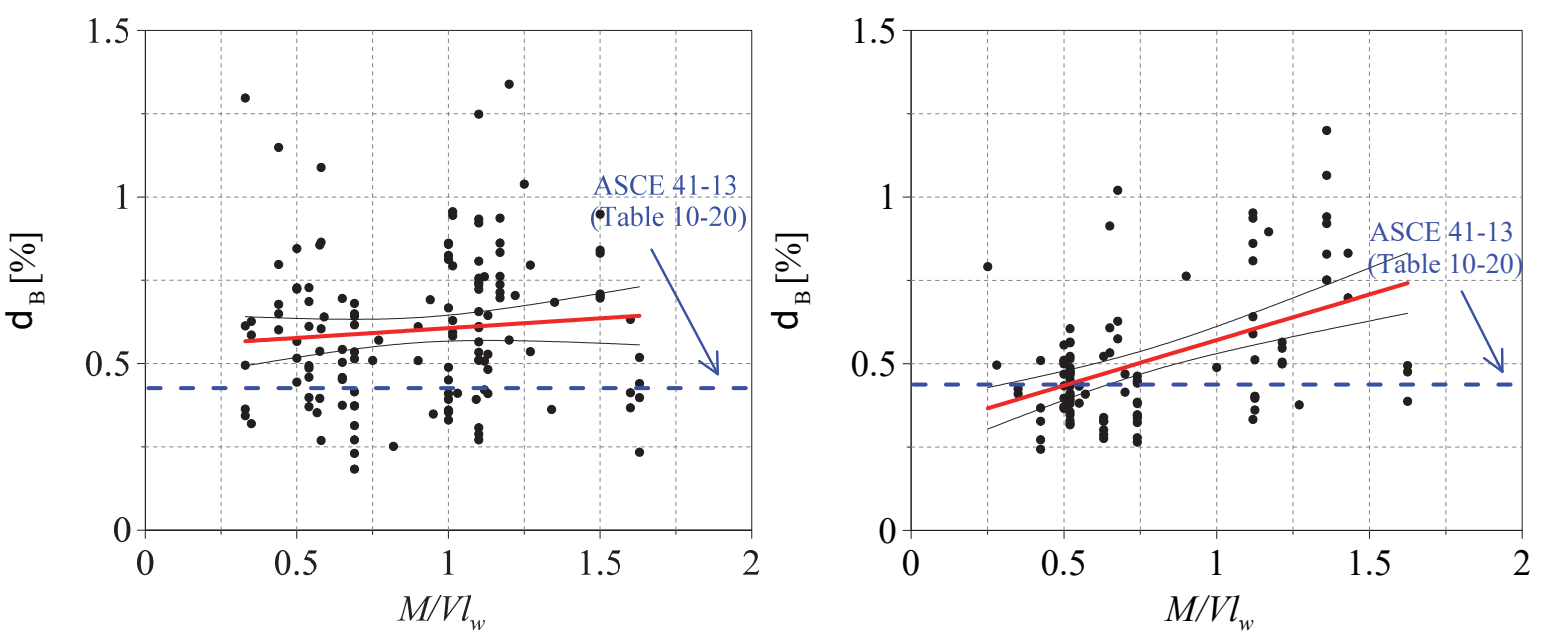

(a) $M / V I$ w: rectangular walls (left panel), and barbell and flanged walls (right panel)
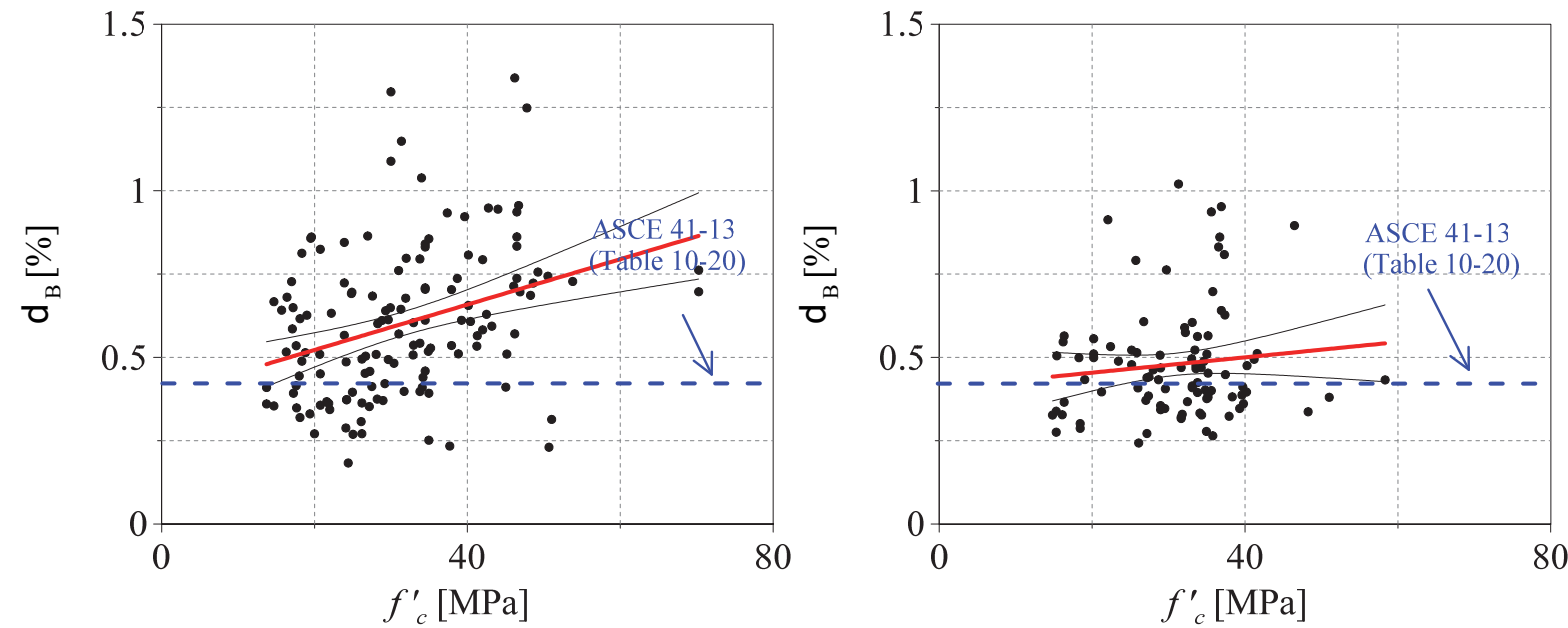

(b) $f_{c}^{\prime}$ : rectangular walls (left panel), and barbell and flanged walls (right panel)
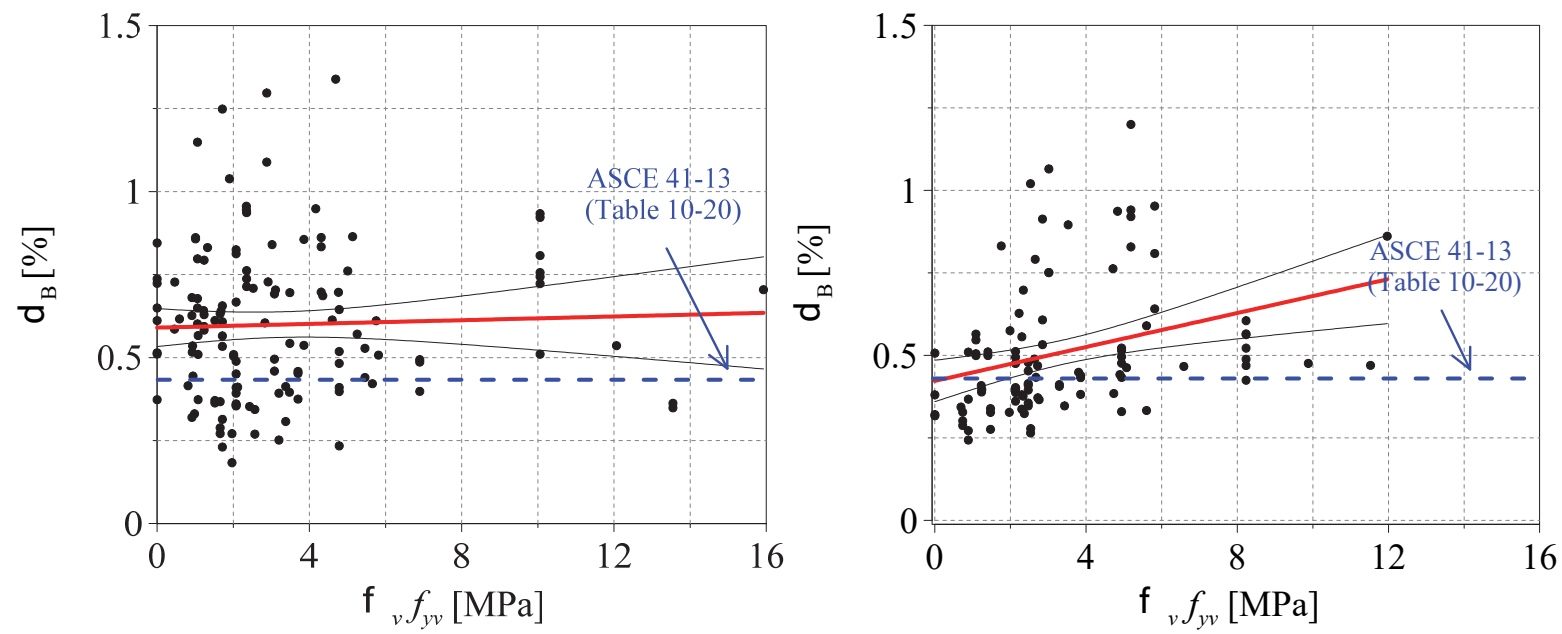

(c) $\rho_{v} f_{y v}$ : rectangular walls (left panel), and barbell and flanged walls (right panel)

Figure 8-8 Effect of design variables on drift ratio at yield, $\delta_{B}$. 

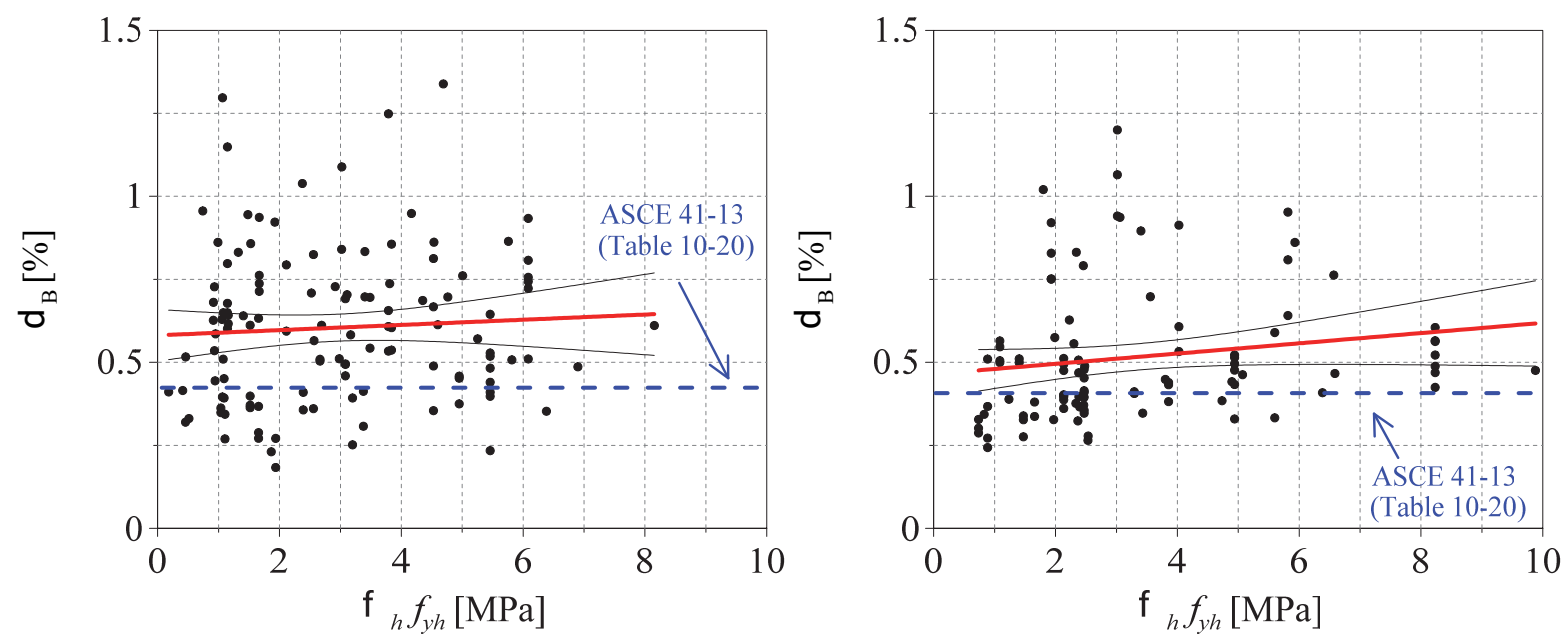

(d) $\rho_{h} f_{v h}$ rectangular walls (left panel), and barbell and flanged walls (right panel)
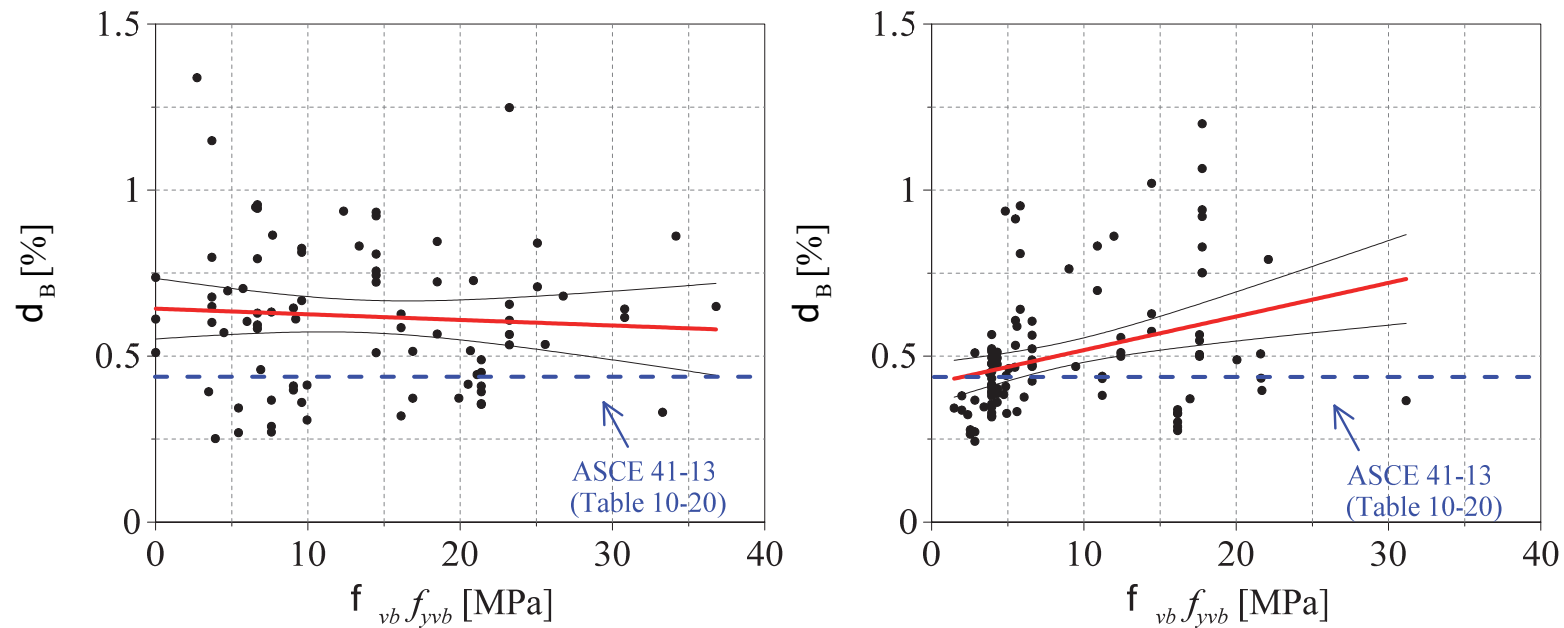

(e) $\rho_{v b} f_{v v b}$ : rectangular walls (left panel), and barbell and flanged walls (right panel)
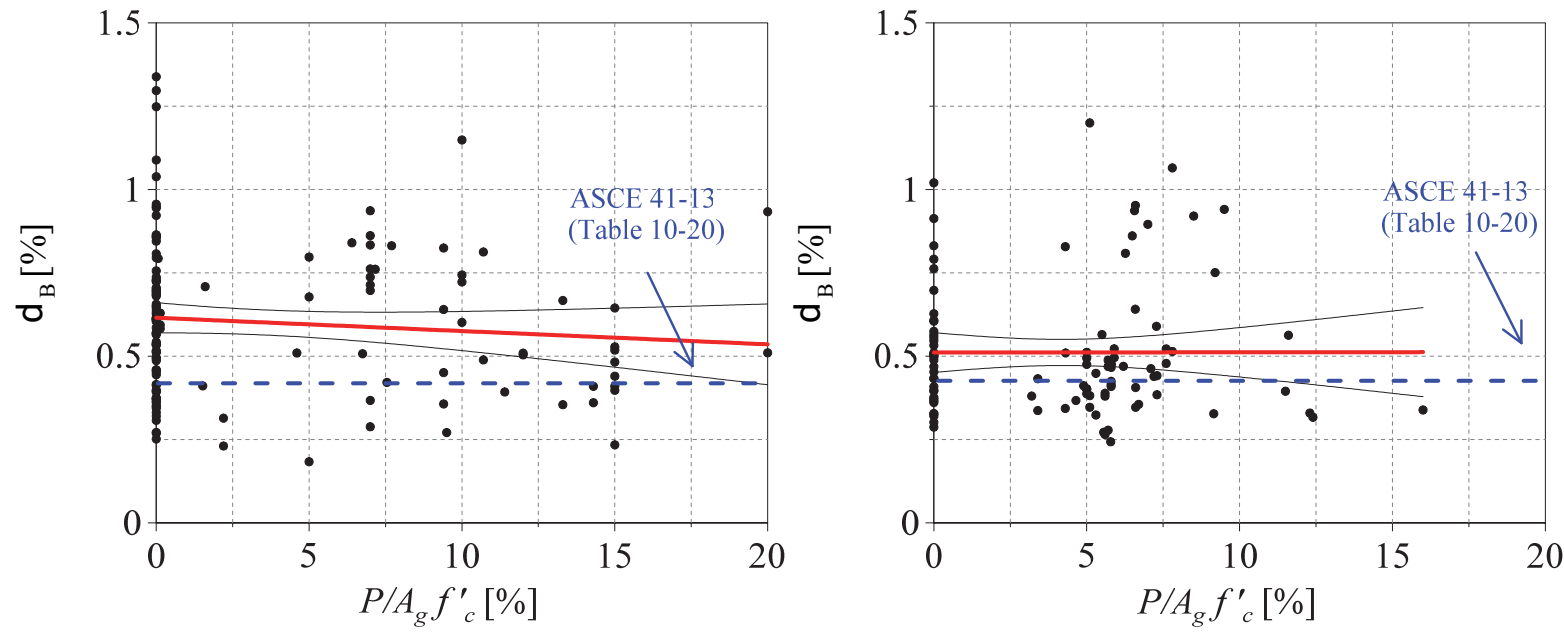

(f) $P / A_{g} f_{c}^{\prime}$ : rectangular walls (left panel), and barbell and flanged walls (right panel)

Figure 8-8 Effect of design variables on drift ratio at yield, $\delta_{B}$ (continued). 

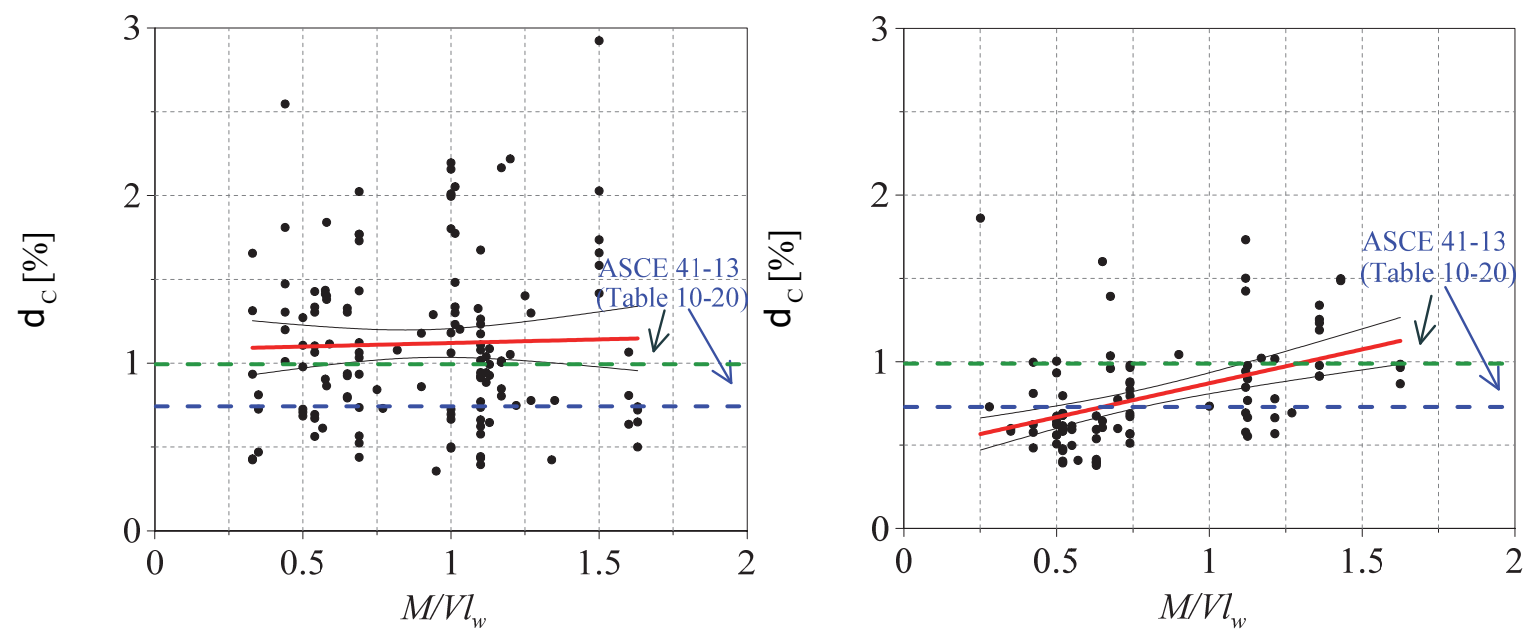

(a) $M / V I_{w}$ : rectangular walls (left panel), and barbell and flanged walls (right panel)
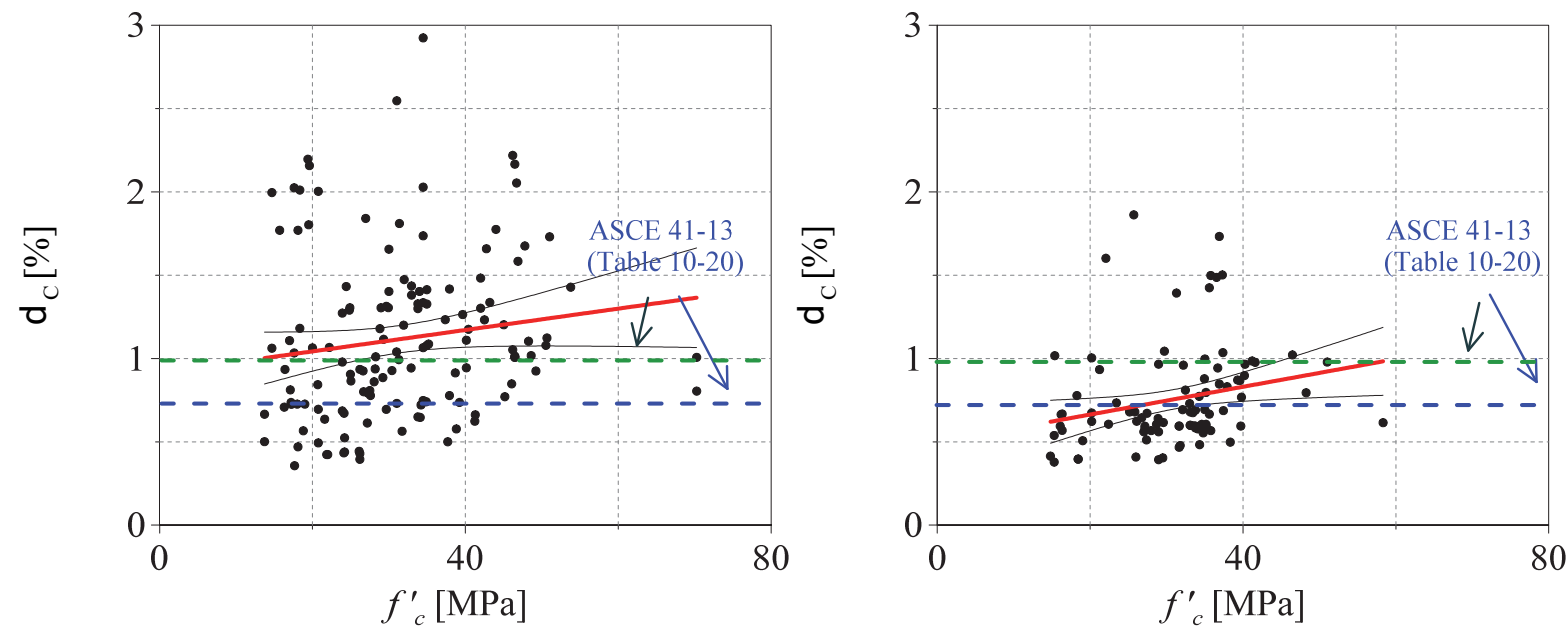

(b) $f_{c}^{\prime}$ : rectangular walls (left panel), and barbell and flanged walls (right panel)
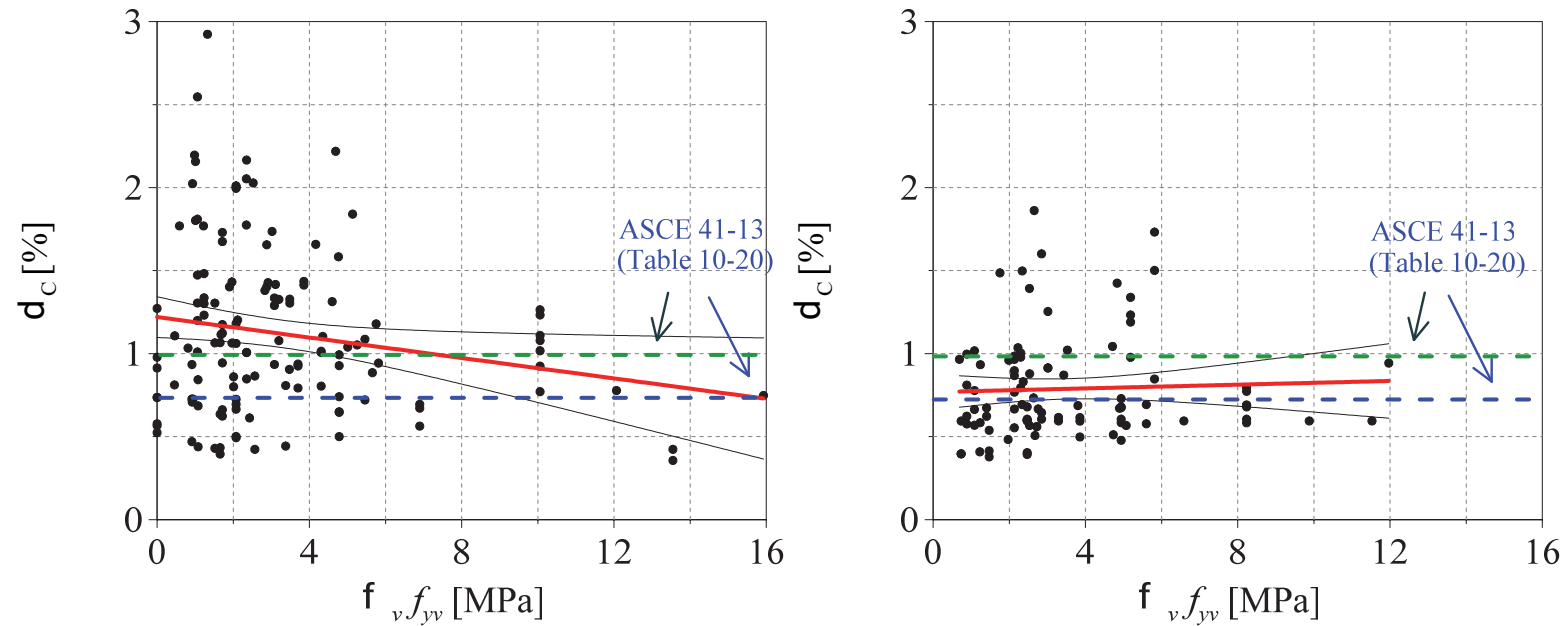

(c) $\rho_{v} f_{y v}$ : rectangular walls (left panel), and barbell and flanged walls (right panel)

Figure 8-9 Effect of design variables on drift ratio at peak strength, $\delta c$. 

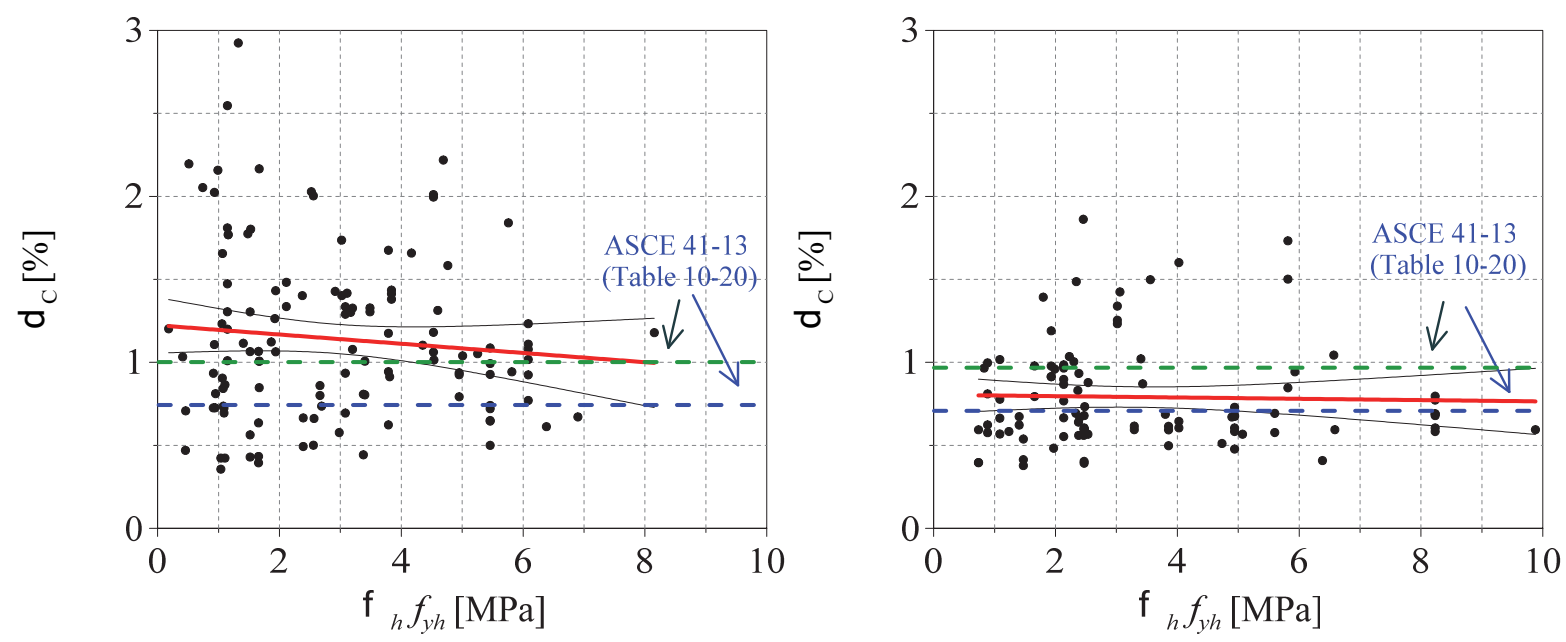

(d) $\rho_{h} f_{v h:}$ rectangular walls (left panel), and barbell and flanged walls (right panel)
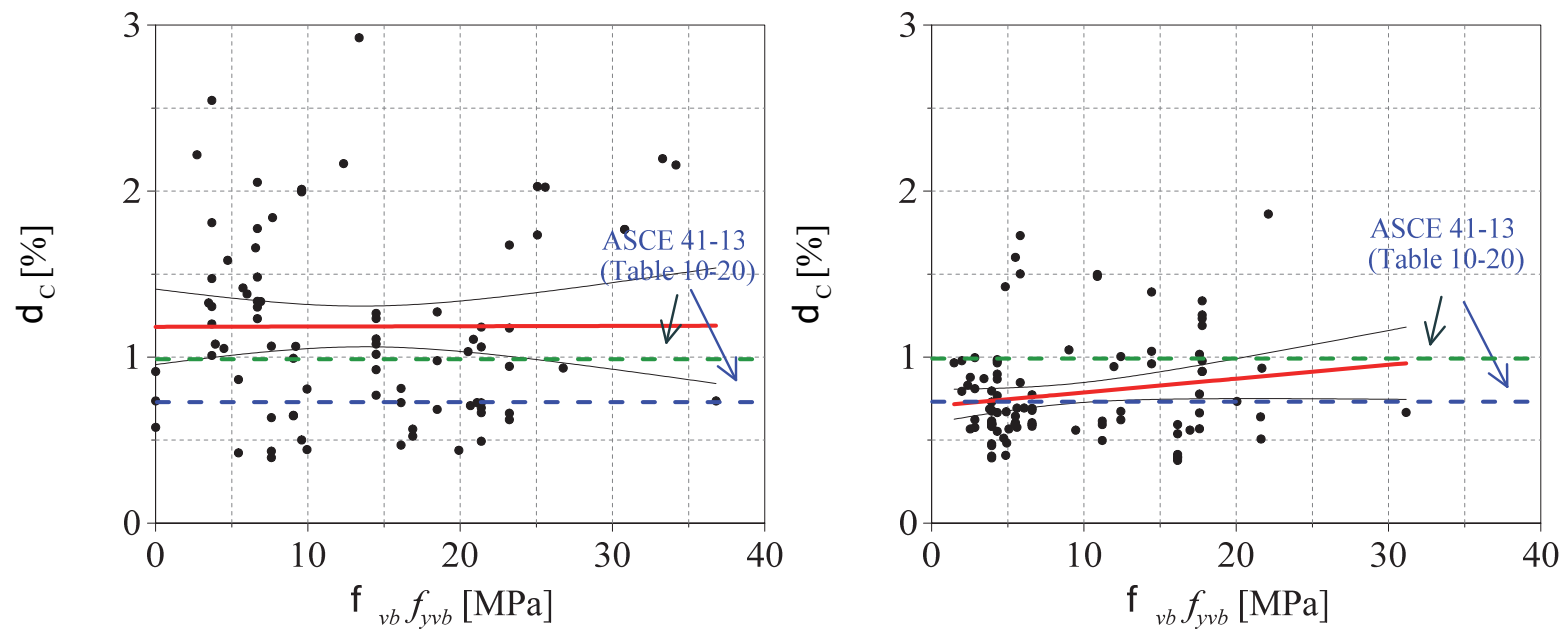

(e) $\rho_{v b} f_{v v b}$ : rectangular walls (left panel), and barbell and flanged walls (right panel)
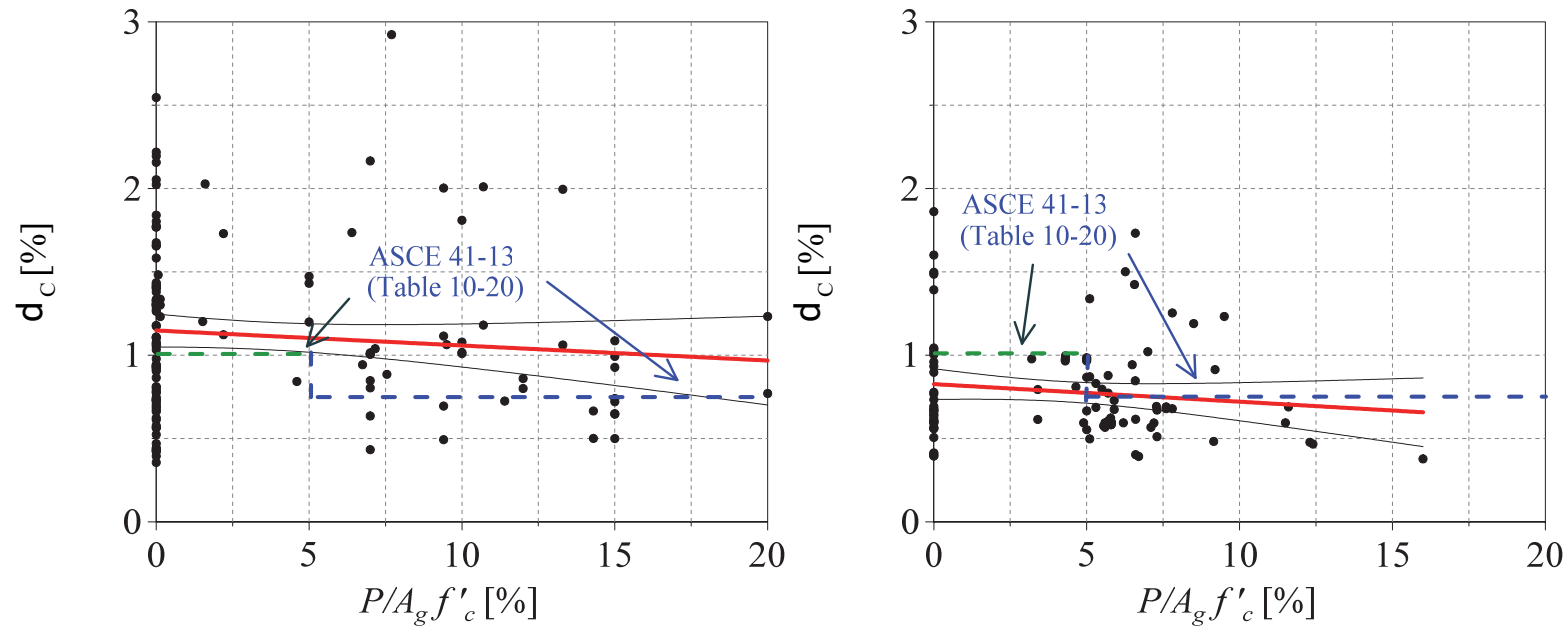

(f) $P / A_{g} f_{c}^{\prime}$ : rectangular walls (left panel), and barbell and flanged walls (right panel)

Figure 8-9 Effect of design variables on drift ratio at peak strength, $\delta_{c}$ (continued). 

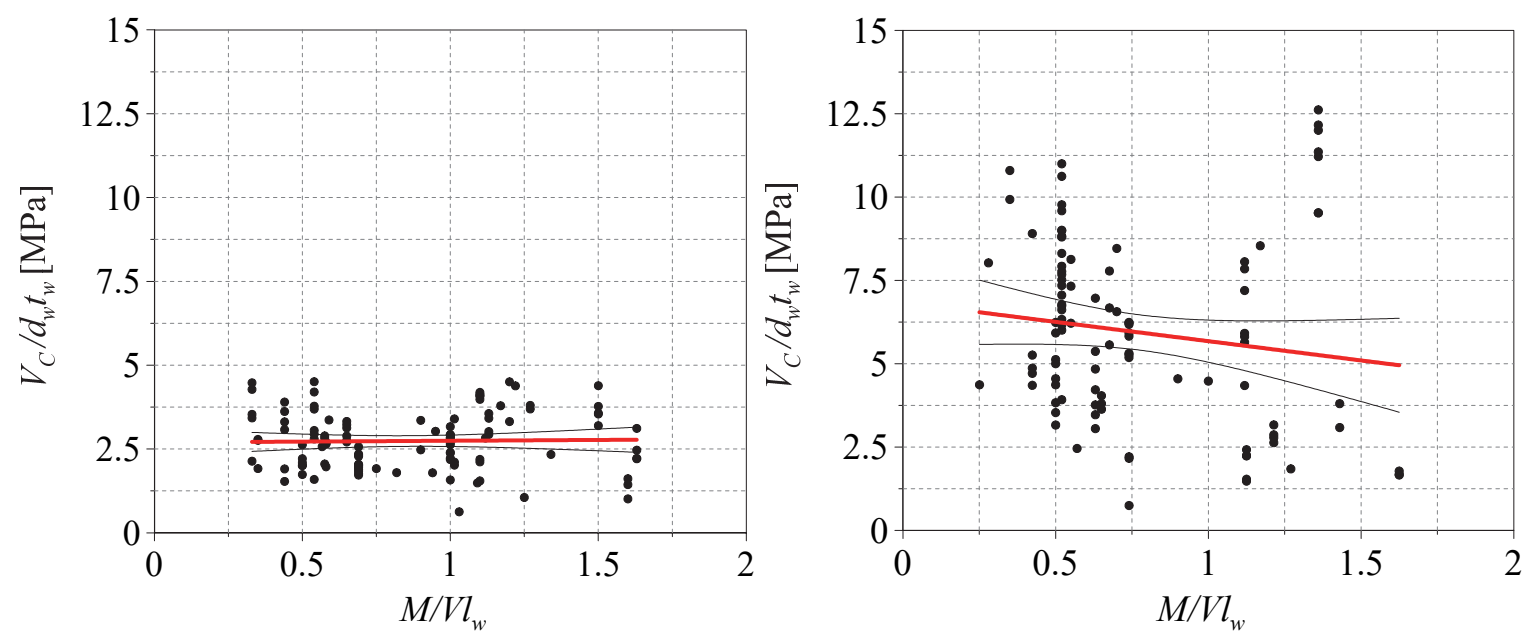

(a) $M / V I_{w}$ : rectangular walls (left panel), and barbell and flanged walls (right panel)
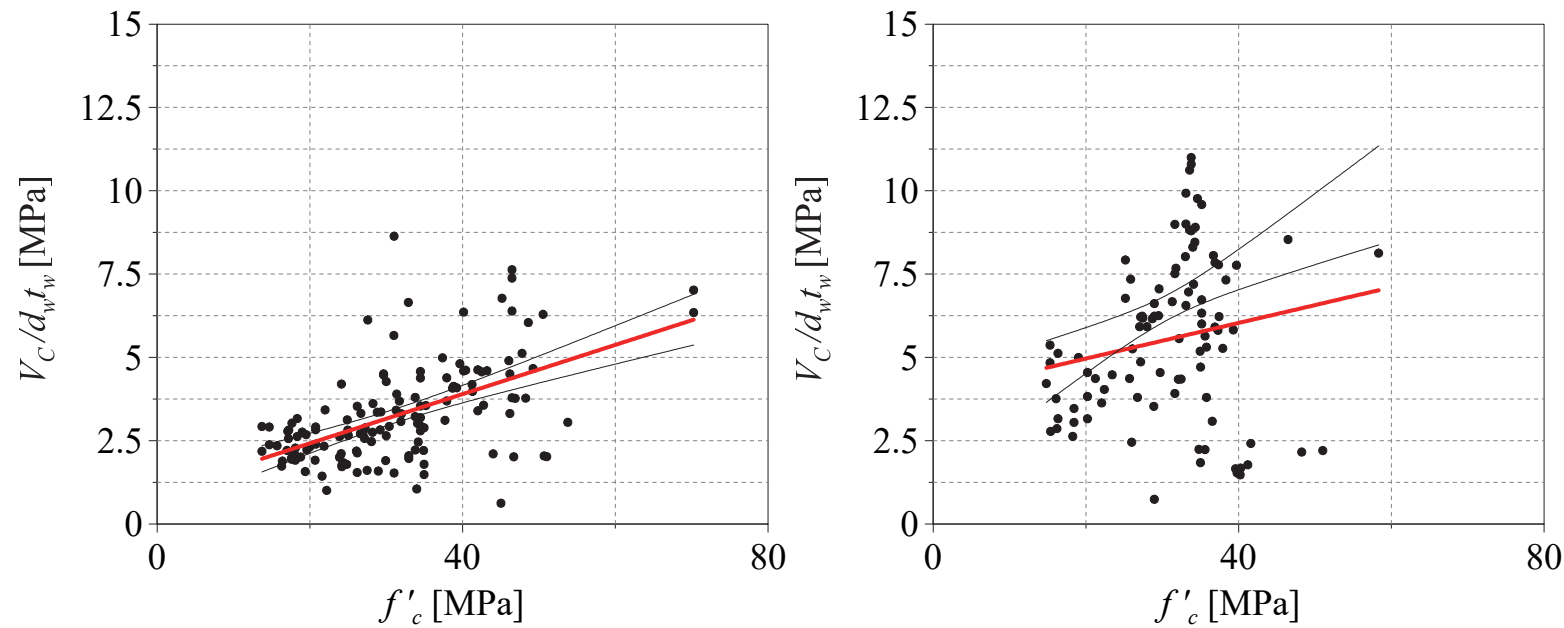

(b) $f_{c}^{\prime}$ : rectangular walls (left panel), and barbell and flanged walls (right panel)
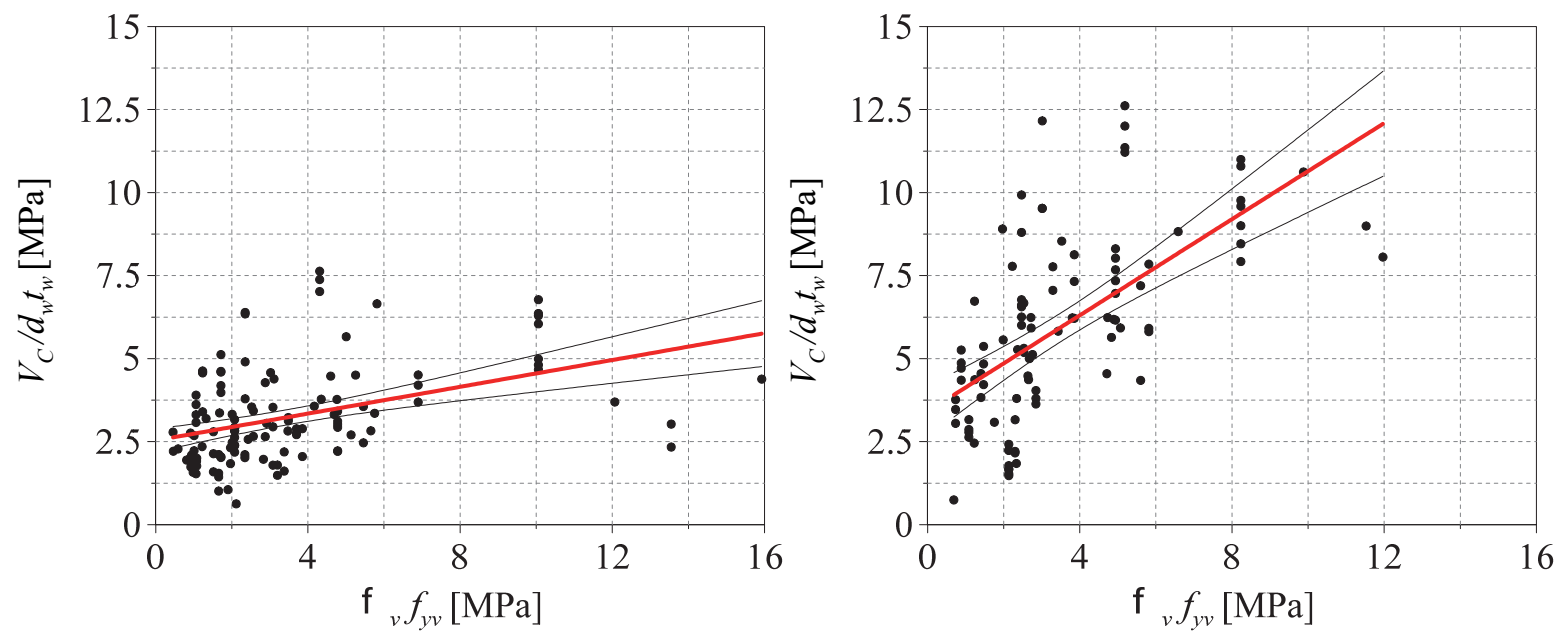

(c) $\rho_{v} f_{y v}$ : rectangular walls (left panel), and barbell and flanged walls (right panel)

Figure 8-10 Effect of design variables on normalized peak lateral strength, $V_{c} / d_{w} t_{w}$. 

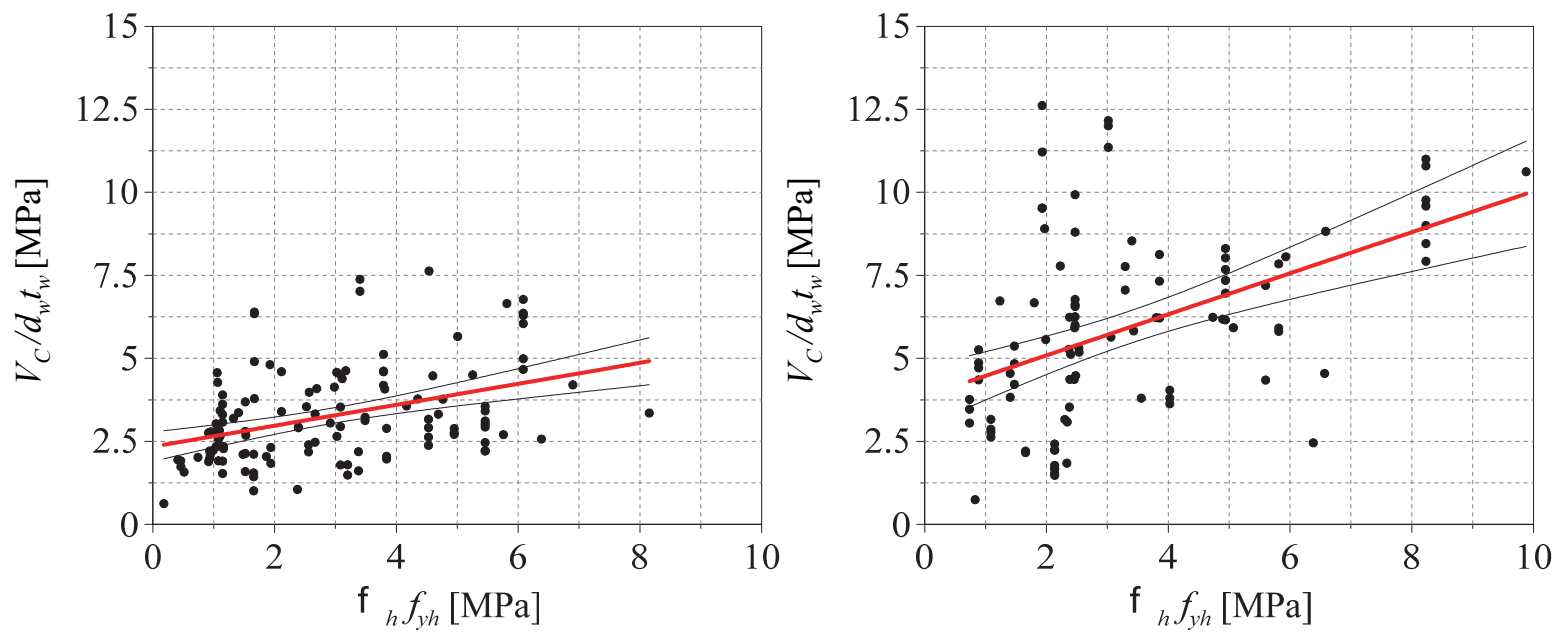

(d) $\rho_{h} f_{v h}$ rectangular walls (left panel), and barbell and flanged walls (right panel)
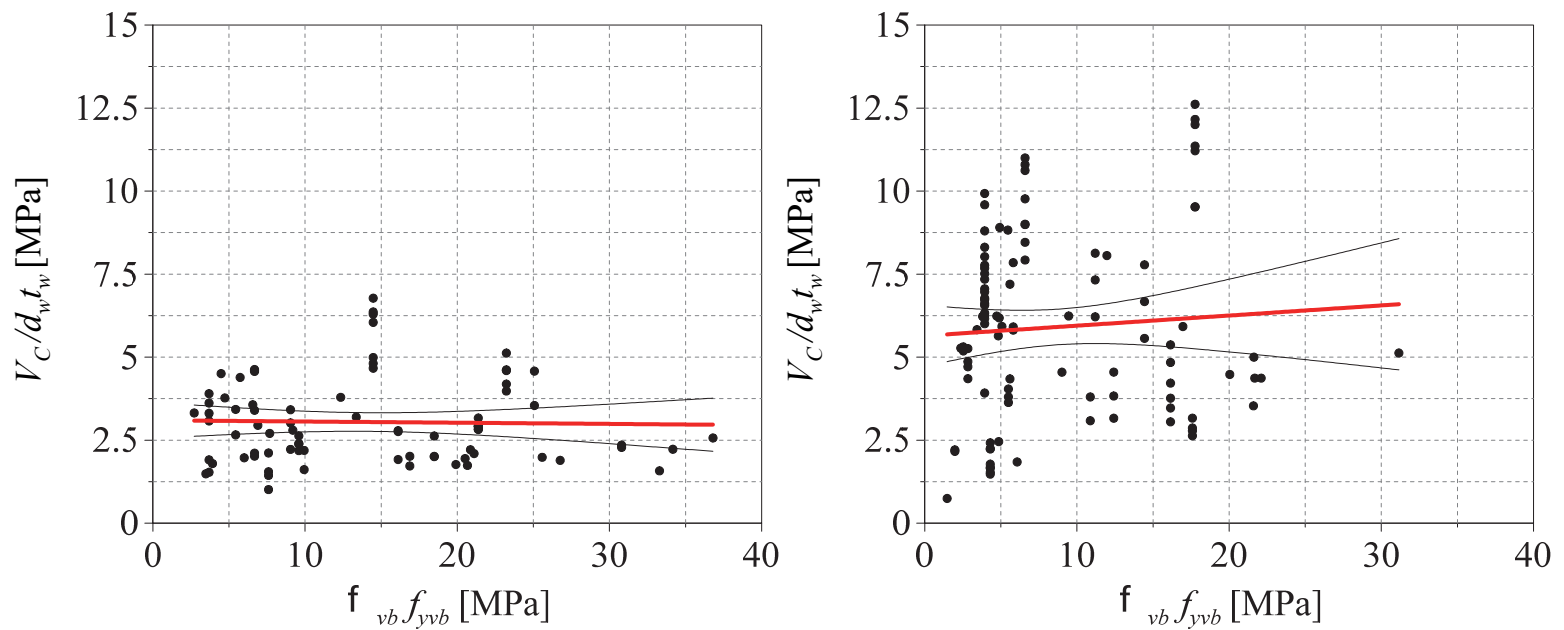

(e) $\rho_{v b} f_{v v b}$ : rectangular walls (left panel), and barbell and flanged walls (right panel)
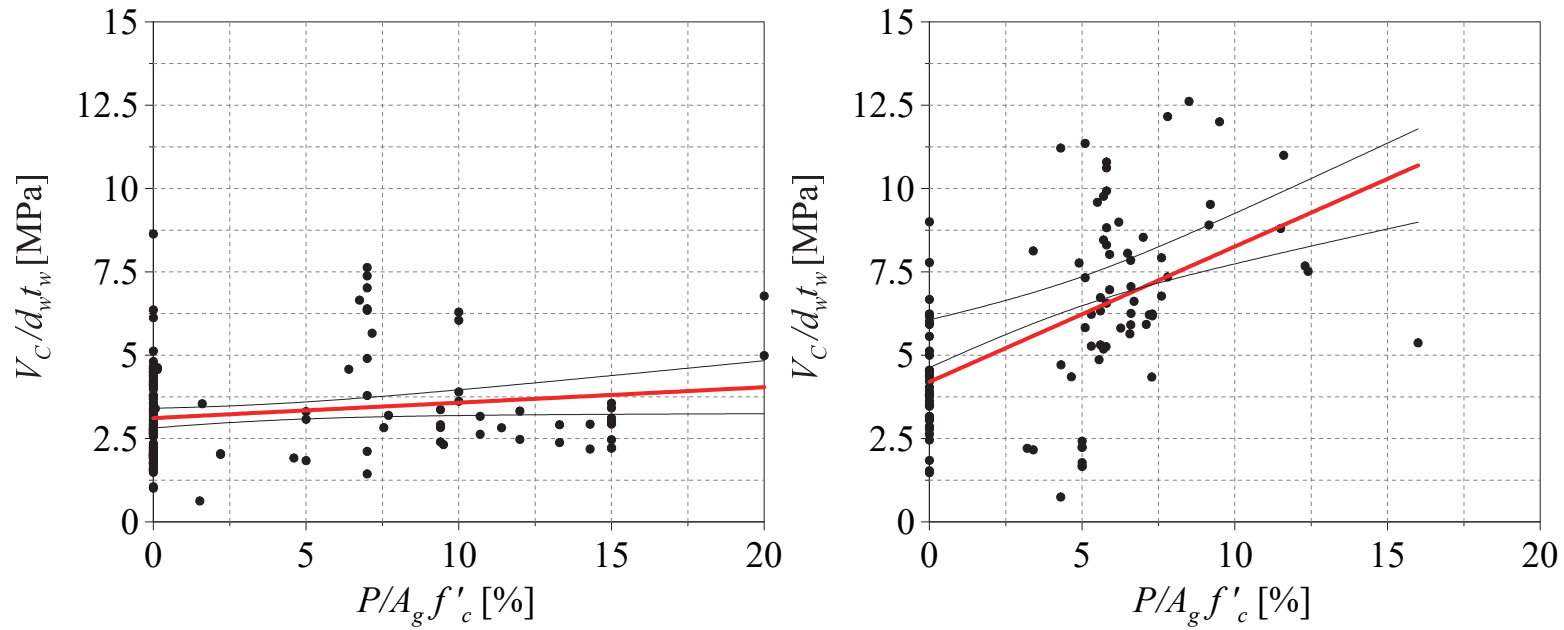

(f) $P / A_{g} f_{c}^{\prime}$ : rectangular walls (left panel), and barbell and flanged walls (right panel)

Figure 8-10 Effect of design variables on normalized peak lateral strength, $V_{c} / d_{w} t_{w}$ (continued). 


\subsubsection{Drift Ratio at Specified Residual Strength}

Table 10.20 of ASCE/SEI 41-13 (Table 8-1) sets the residual strength ratio and the ultimate drift ratio (i.e., point $\mathrm{E}$ on the backbone curve of Figure 8-2) equal to 0 and $1.0 \%$, respectively, if the axial force ratio, $\left[\left(A_{s}-A_{s}^{\prime}\right) f_{y}+P\right] /\left(t_{w} l_{w} f_{c}^{\prime}\right)$, is greater than 0.05 , and 0.2 and $2.0 \%$, respectively, otherwise. The effects of design variables on the drift ratio at zero residual strength, $\delta_{D}^{0}$, and at residual strength equal to $20 \%$, $40 \%$, and $80 \%$ of peak strength, $\delta_{D}^{20}, \delta_{D}^{40}$, and $\delta_{D}^{80}$, respectively, are presented in Figure 8-11 through Figure 8-14, respectively.

The panels of Figure 8-11 present the experimentally measured or projected ${ }^{4}$ drift ratio at point $\mathrm{D}$ for zero residual strength. The dashed blue line corresponds to the ASCE/SEI 41-13 ultimate drift ratio of 1.0\% for values of the axial force ratio greater than 0.05 , for which the residual strength ratio is set equal to 0 . For values of the axial force ratio in the range from 0.05 to $0.10,1.0 \%$ is a low estimate of $\delta_{D}^{0}$. The value of $\delta_{D}^{0}$ decreases as the axial force ratio increases.

Figure 8-12 presents information for drift ratio at a residual strength ratio of 0.2: the value assigned in ASCE/SEI 41-13 for values of the axial force ratio of less than or equal to 0.05 . The dashed blue line represents the corresponding ASCE/SEI 41-13 ultimate drift ratio of $2.0 \%$. For values of the axial force ratio in the range from 0 to $0.05,2.0 \%$ is a high estimate of $\delta_{D}^{20}$.

Values of the drift ratio at residual strength of $40 \%$ and $80 \%$ of peak strength are presented in Figures 8-13 and 8-14, respectively. ASCE/SEI 41-13 data are not included in these figures because residual strength is capped at $20 \%$ of the peak strength.

4 The projected drift ratio at zero residual strength was calculated using the displacement corresponding to the point at the intersection of the post-peak branch (C-D) of the idealized backbone curve, per Figure 8-2, with displacement axis. 

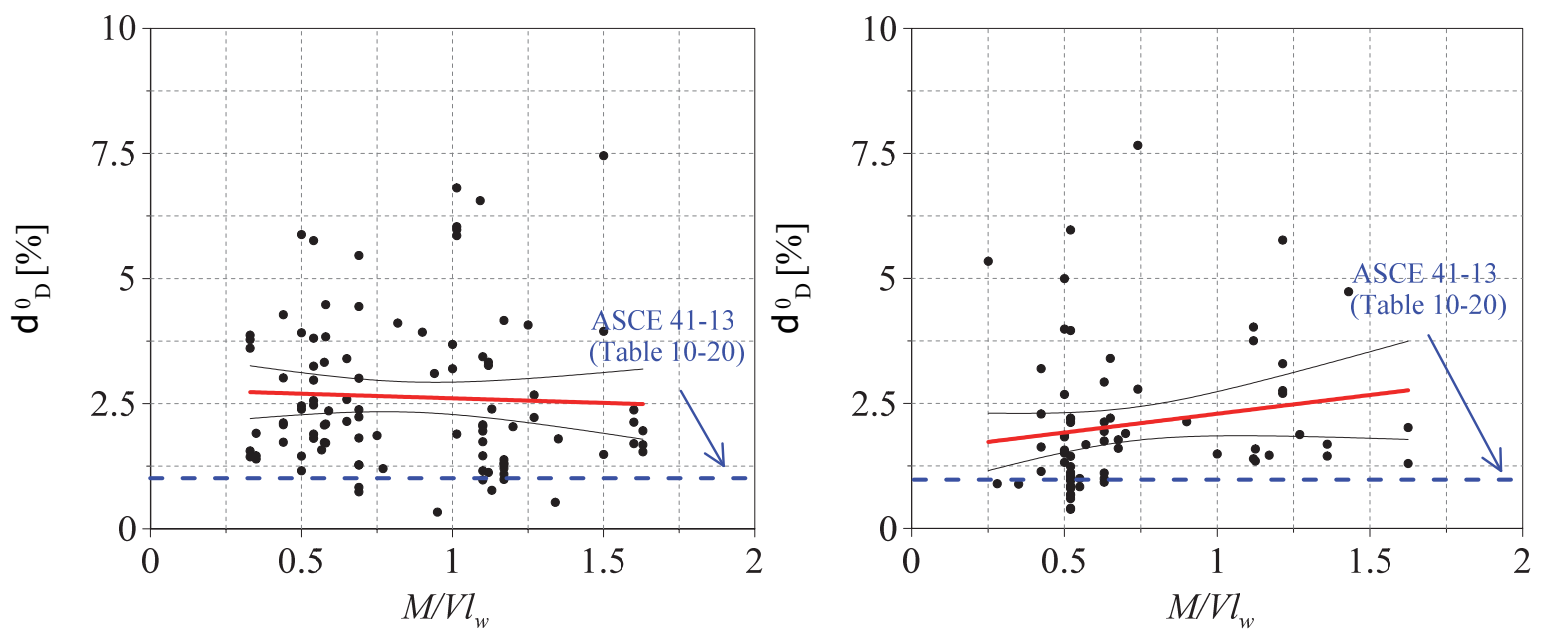

(a) $M / V l_{w}$ : rectangular walls (left panel), and barbell and flanged walls (right panel)
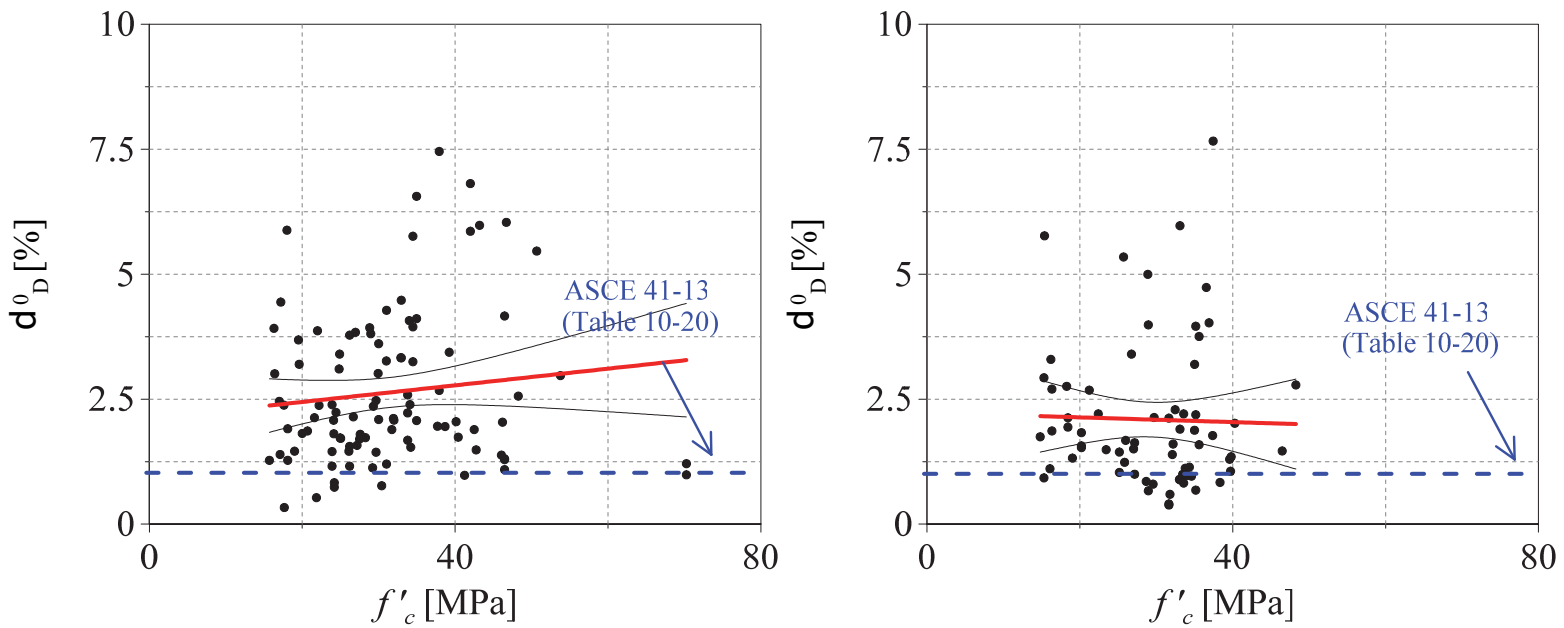

(b) $f_{c}^{\prime}$ : rectangular walls (left panel), and barbell and flanged walls (right panel)
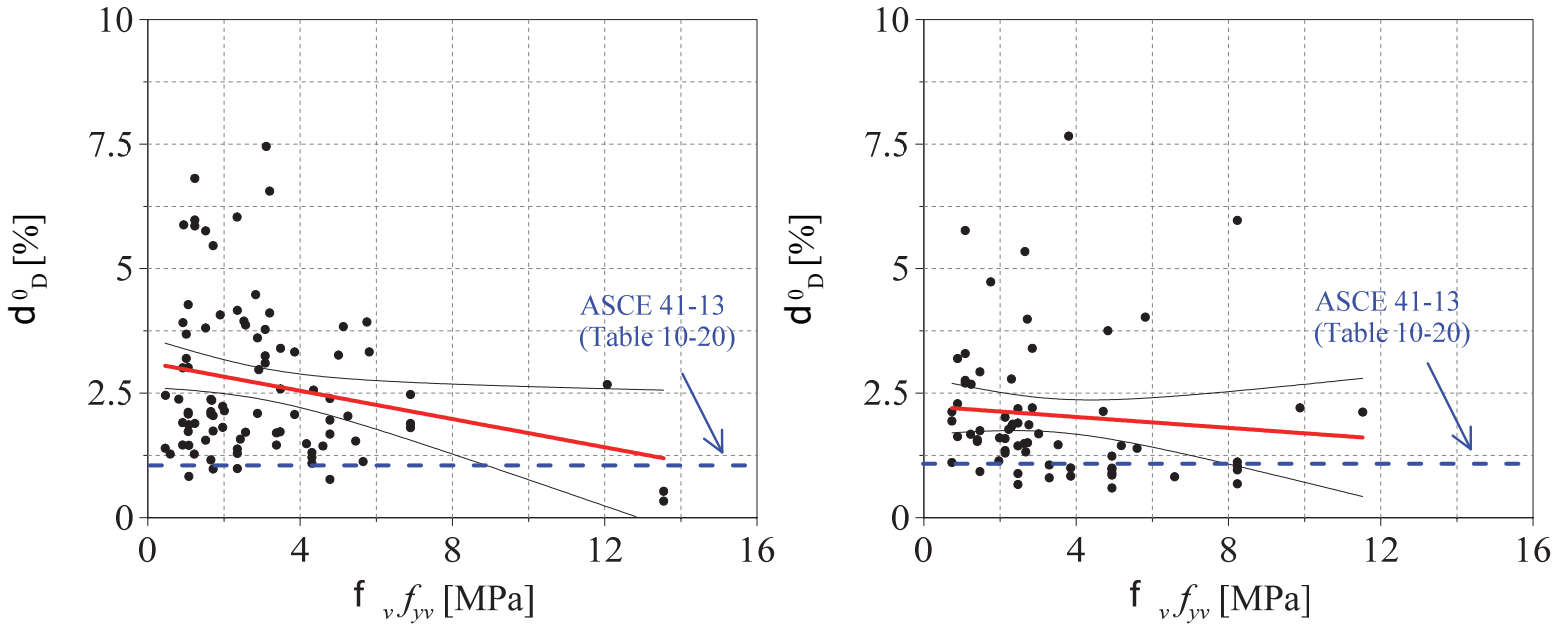

(c) $\rho_{v} f_{y v}$ : rectangular walls (left panel), and barbell and flanged walls (right panel)

Figure 8-11 Effect of design variables on drift ratio at zero residual strength, $\delta_{D}^{0}$. 

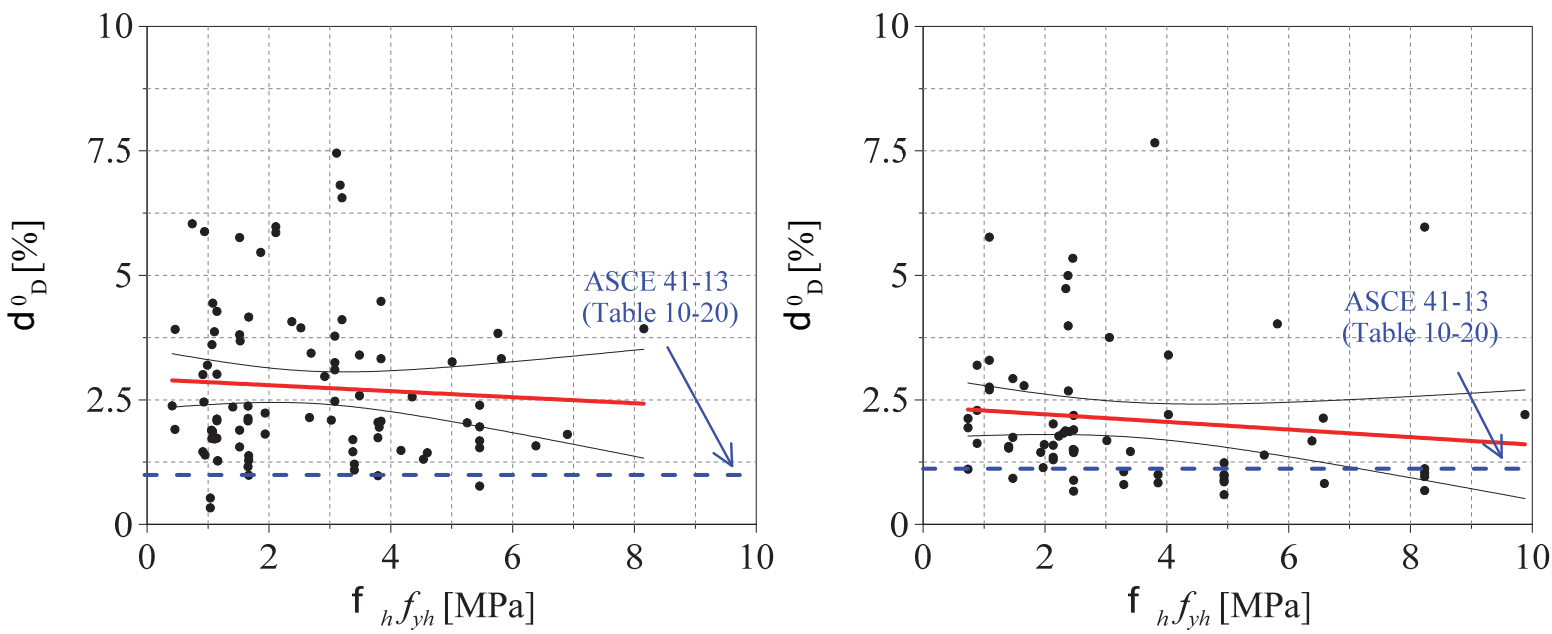

(d) $\rho_{h} f_{v h}$ : rectangular walls (left panel), and barbell and flanged walls (right panel)
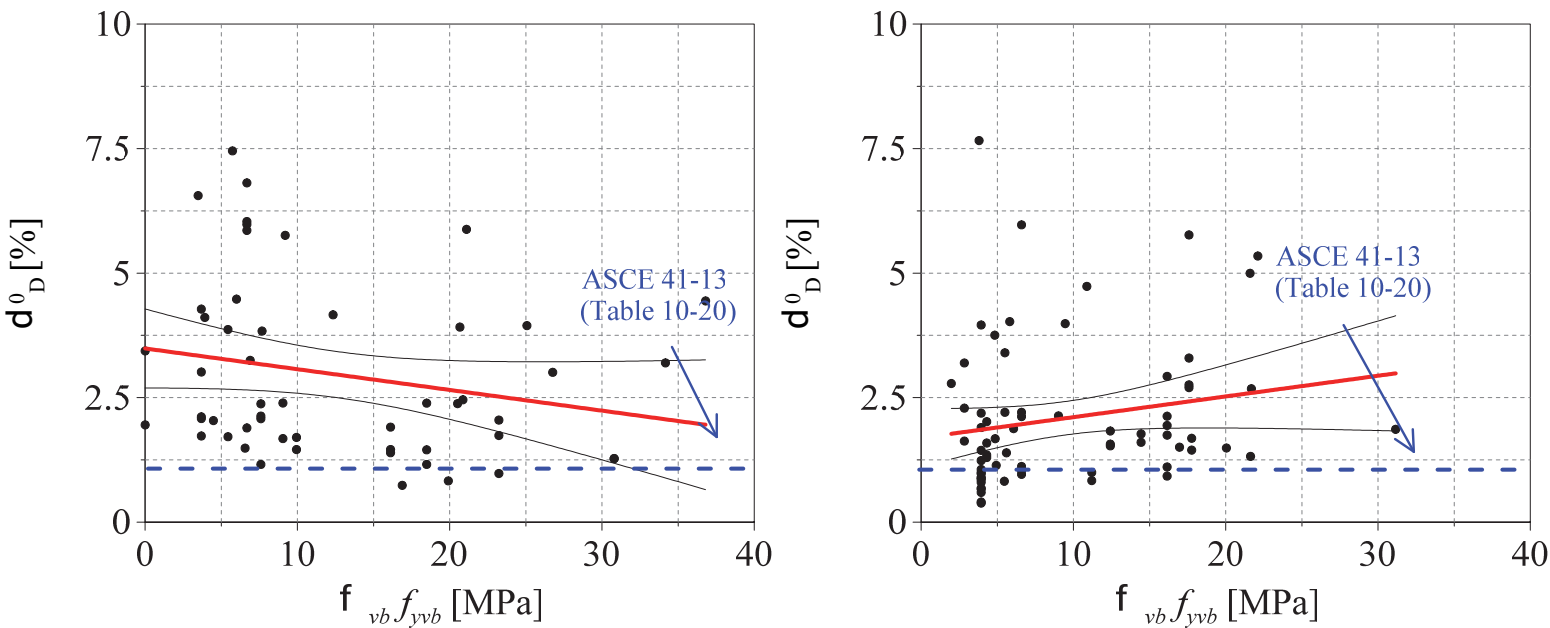

(e) $\rho_{v b} f_{v v b}$ : rectangular walls (left panel), and barbell and flanged walls (right panel)
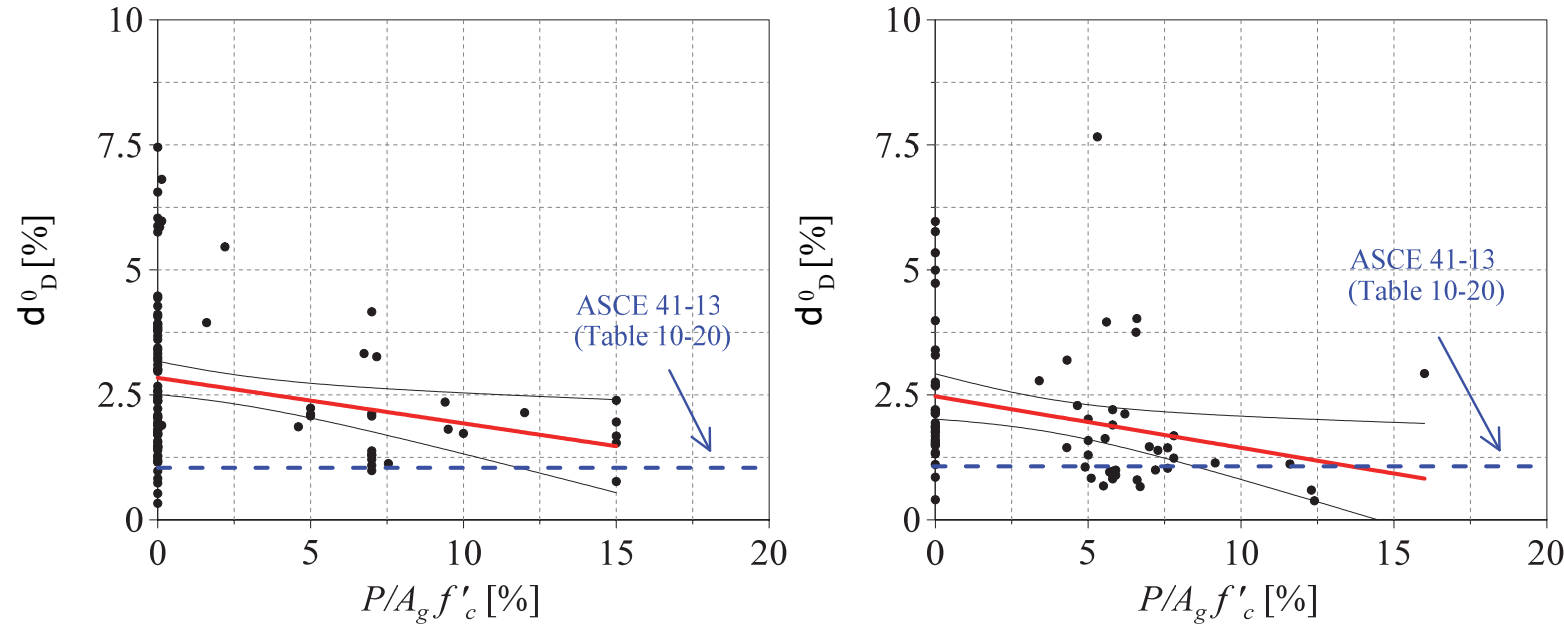

(f) $P / A_{g} f_{c}^{\prime}$ : rectangular walls (left panel), and barbell and flanged walls (right panel)

Figure 8-11 Effect of design variables on drift ratio at zero residual strength, $\delta_{D}^{0}$ (continued). 

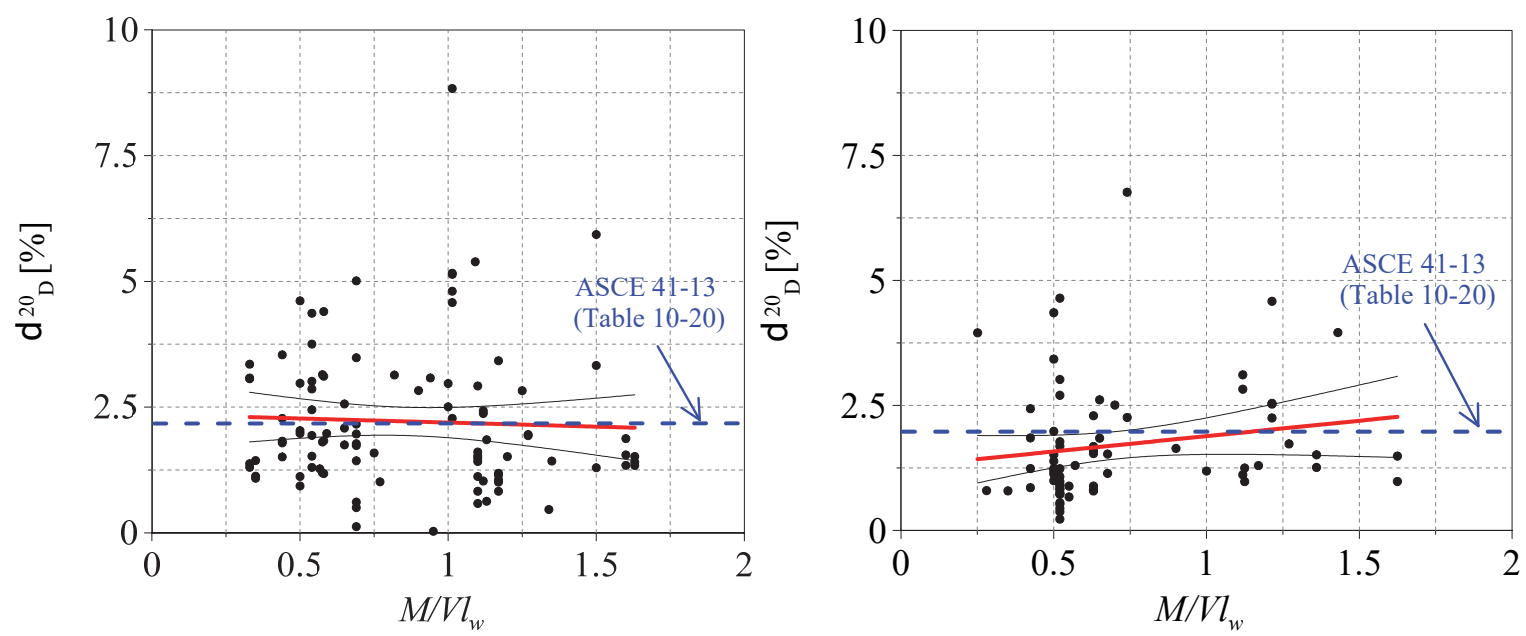

(a) $M / V I_{w}$ : rectangular walls (left panel), and barbell and flanged walls (right panel)
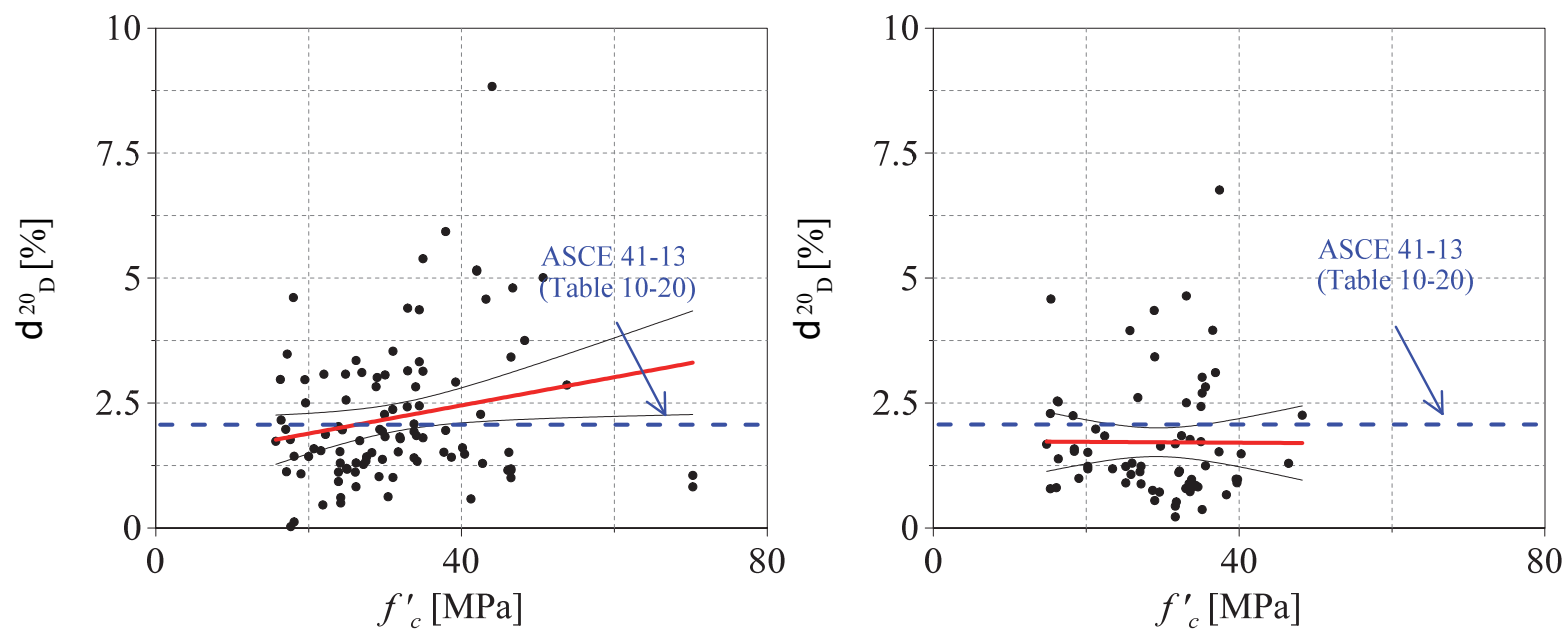

(b) $f_{c}^{\prime}$ : rectangular walls (left panel), and barbell and flanged walls (right panel)
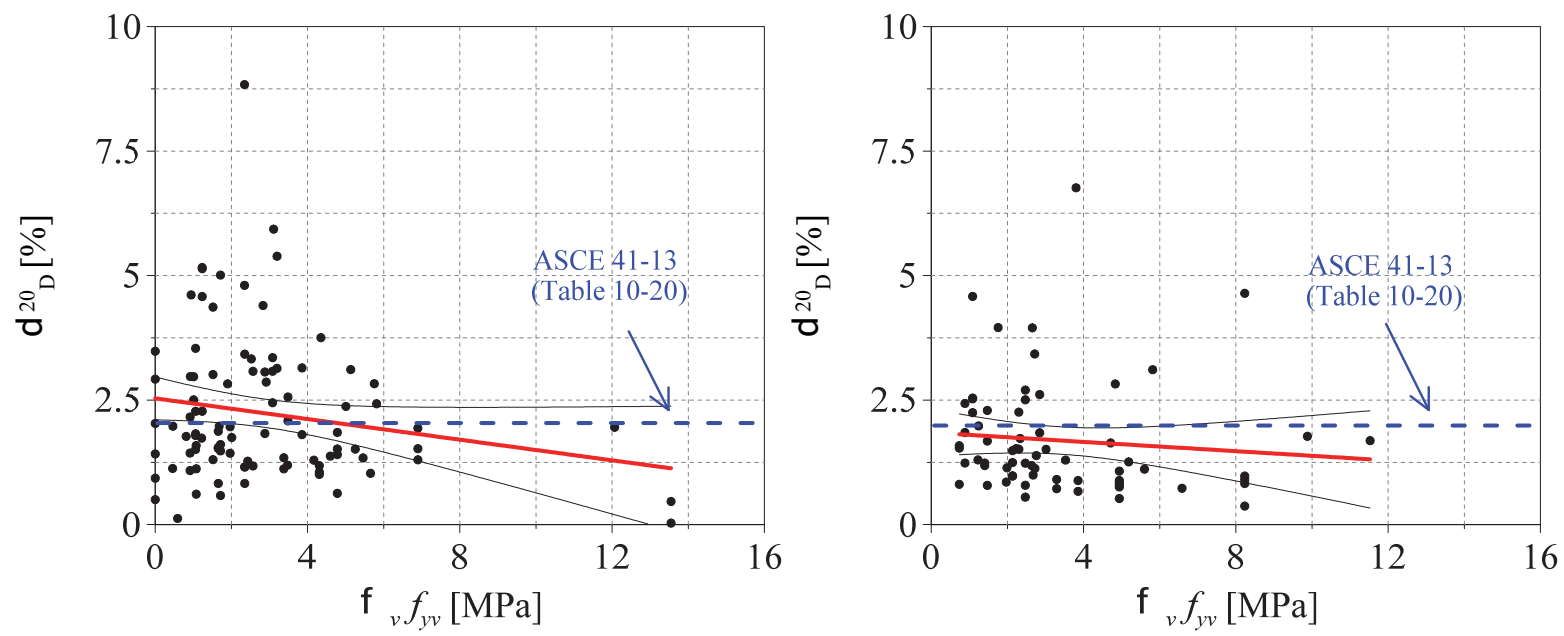

(c) $\rho_{v} f_{y v}$ : rectangular walls (left panel), and barbell and flanged walls (right panel)

Figure 8-12 Effect of design variables on drift ratio at $20 \%$ residual strength, $\delta_{D}^{20}$. 

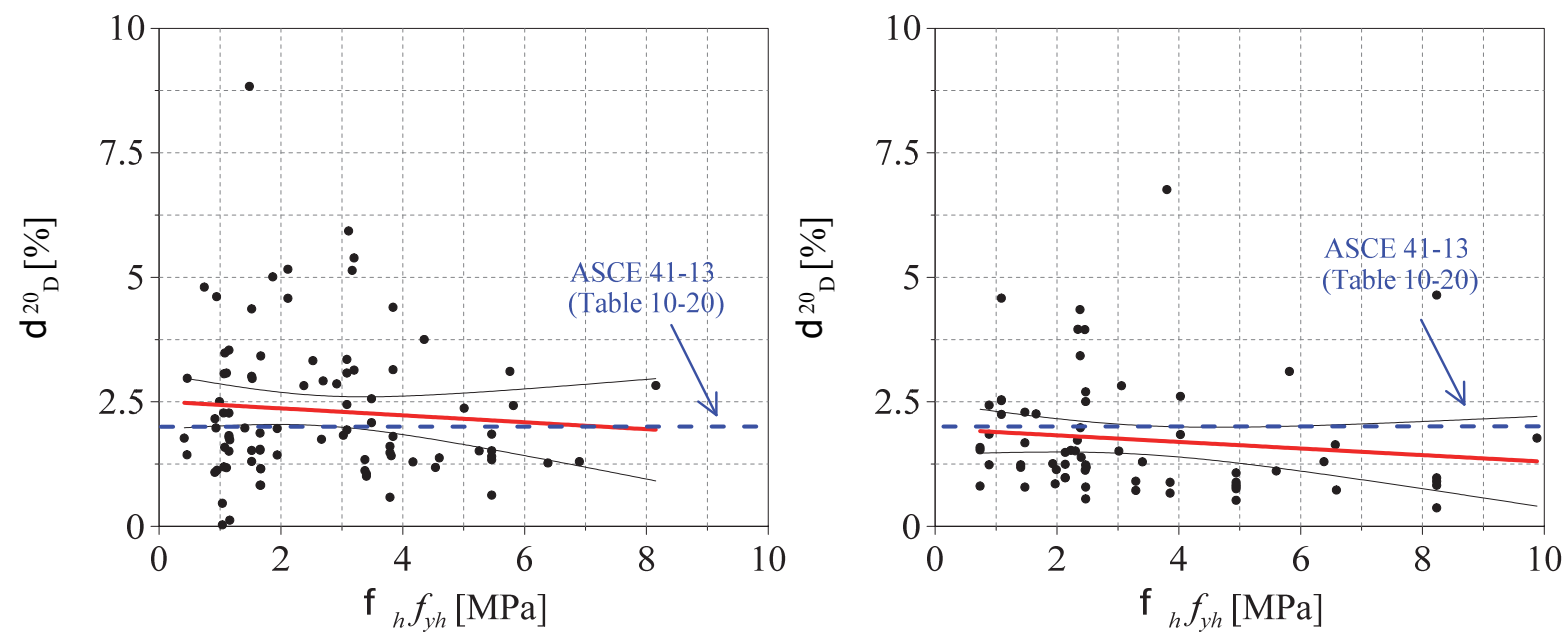

(d) $\rho_{h} f_{v h}$ : rectangular walls (left panel), and barbell and flanged walls (right panel)
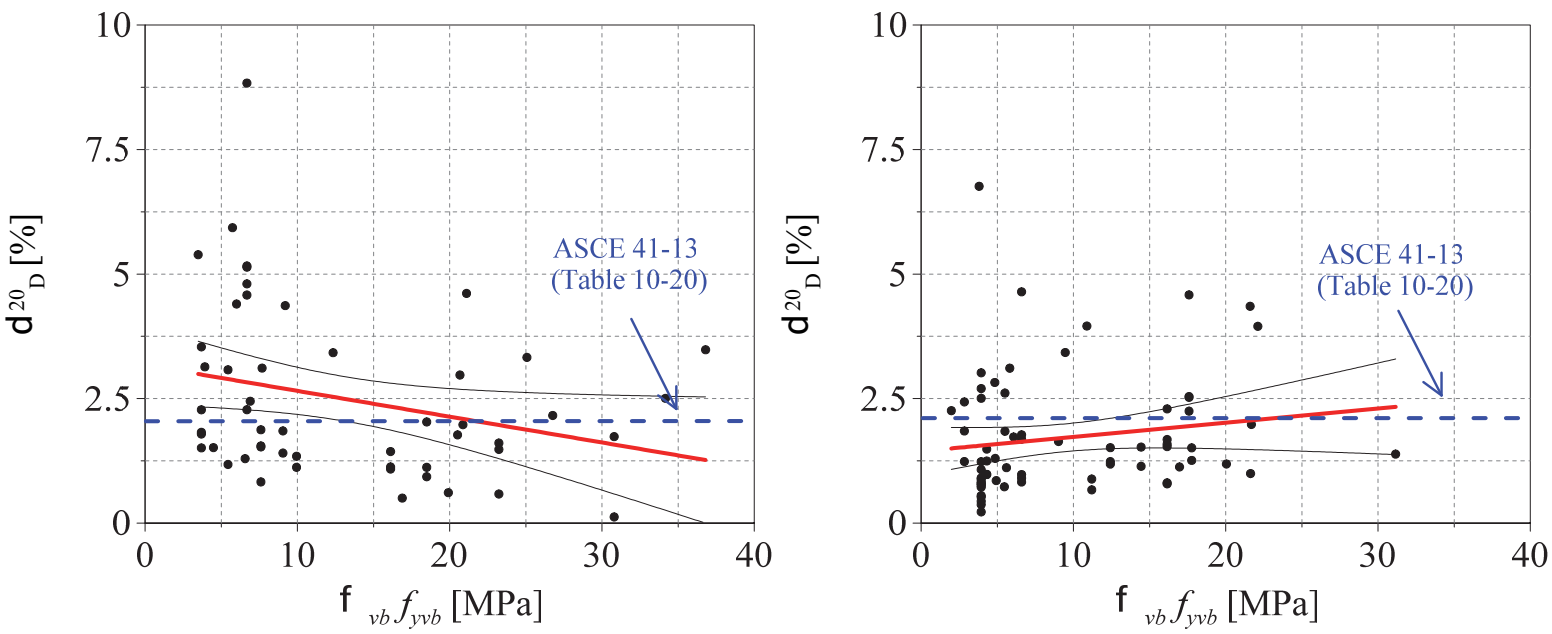

(e) $\rho_{v b} f_{v v b}$ : rectangular walls (left panel), and barbell and flanged walls (right panel)
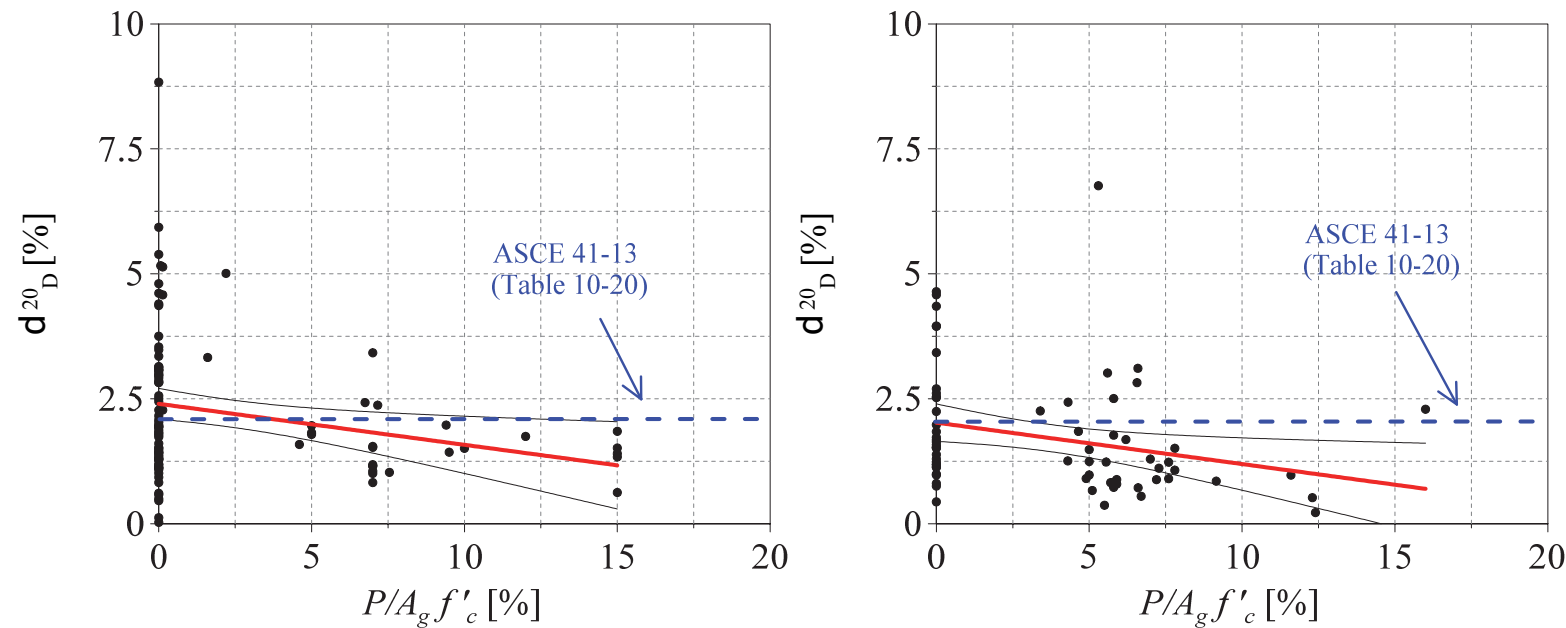

(f) $P / A_{g} f_{c}^{\prime}$ : rectangular walls (left panel), and barbell and flanged walls (right panel)

Figure 8-12 Effect of design variables on drift ratio at $20 \%$ residual strength, $\delta_{D}^{20}$ (continued). 

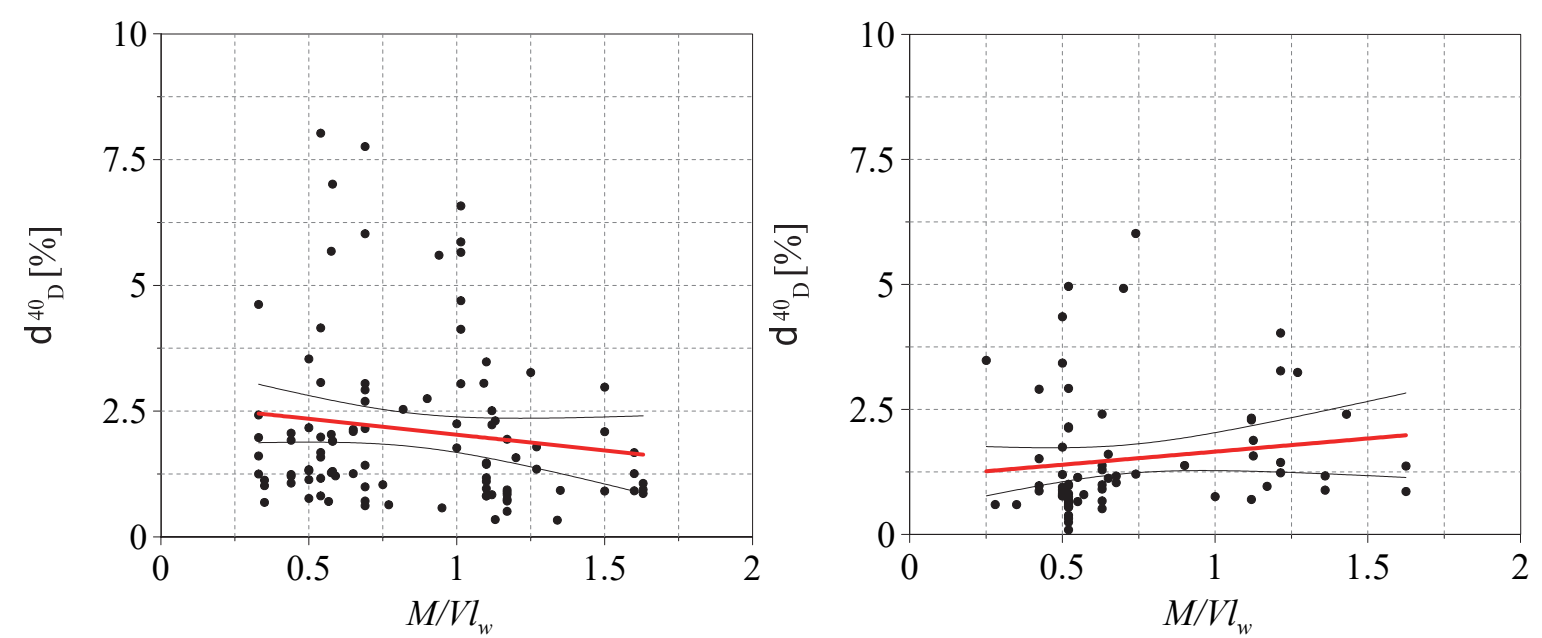

(a) $M / V I_{w}$ : rectangular walls (left panel), and barbell and flanged walls (right panel)
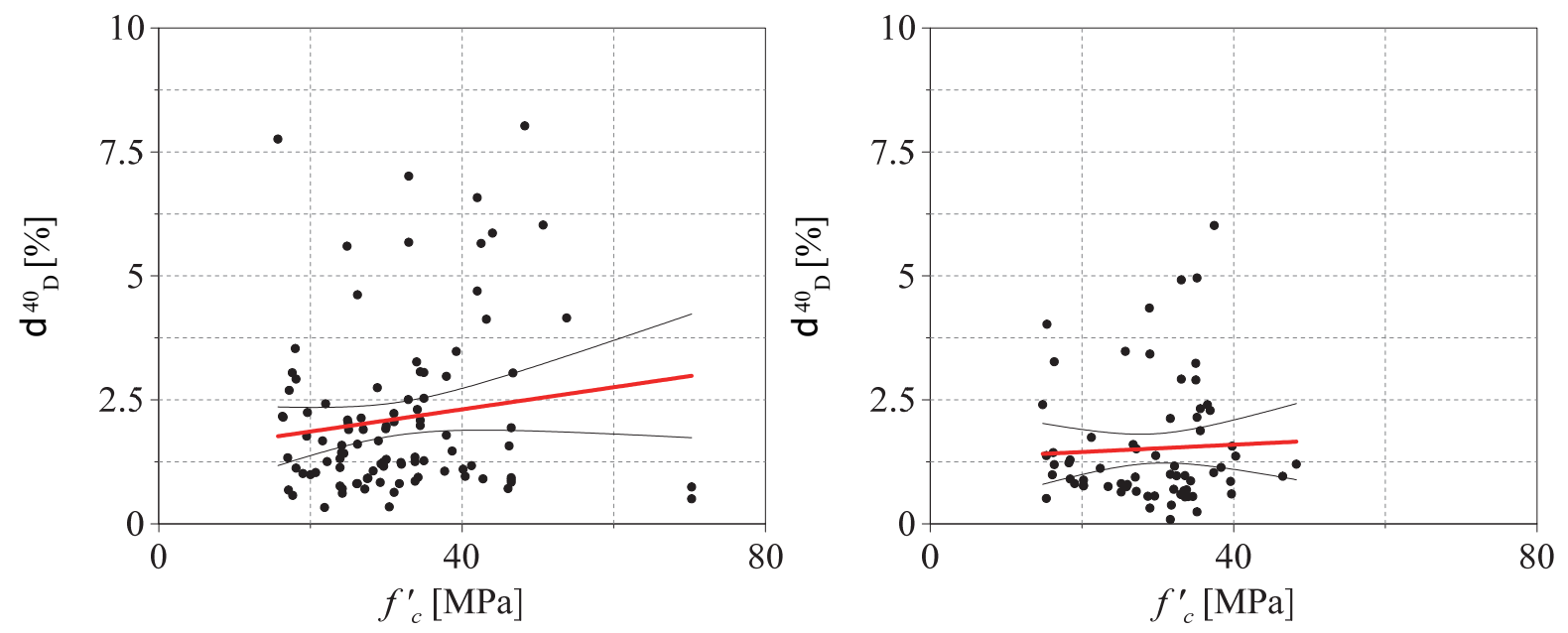

(b) $f_{c}^{\prime}$ : rectangular walls (left panel), and barbell and flanged walls (right panel)
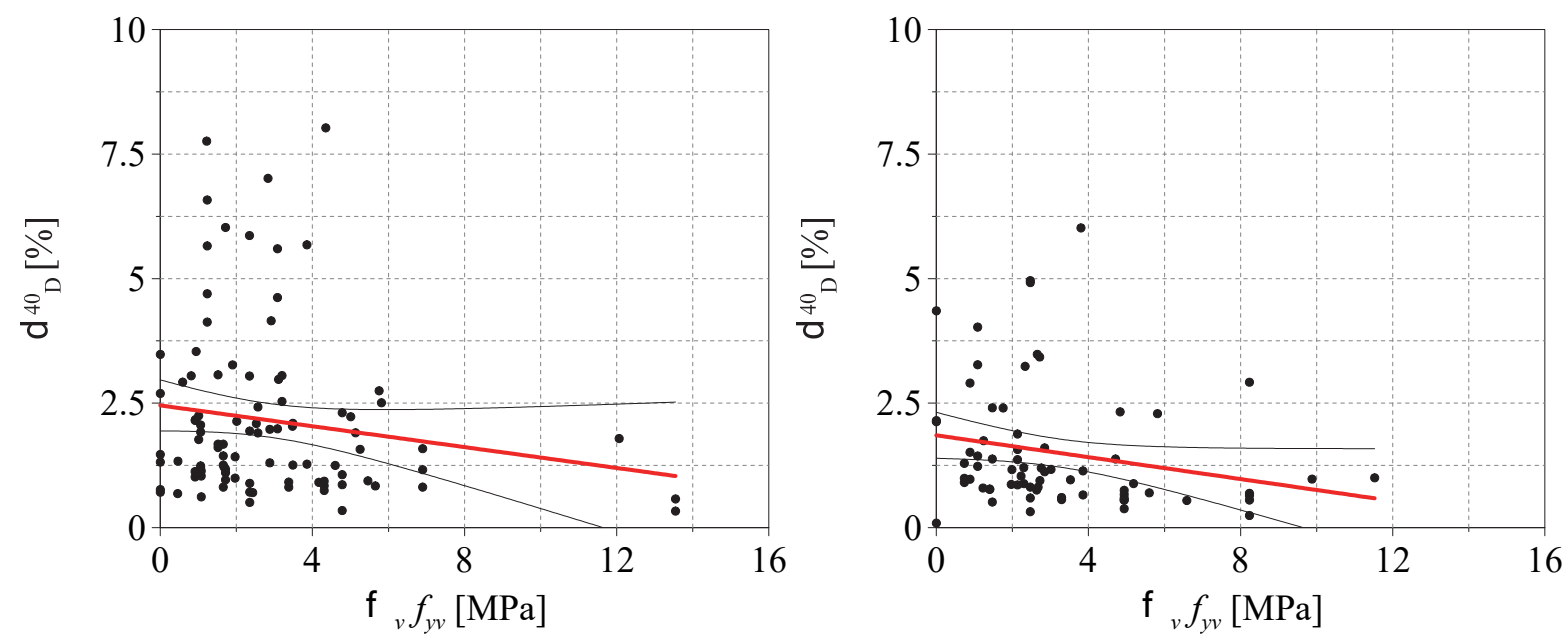

(c) $\rho_{v} f_{y v}$ : rectangular walls (left panel), and barbell and flanged walls (right panel)

Figure 8-13 Effect of design variables on drift ratio at $40 \%$ residual strength, $\delta_{D}^{40}$. 

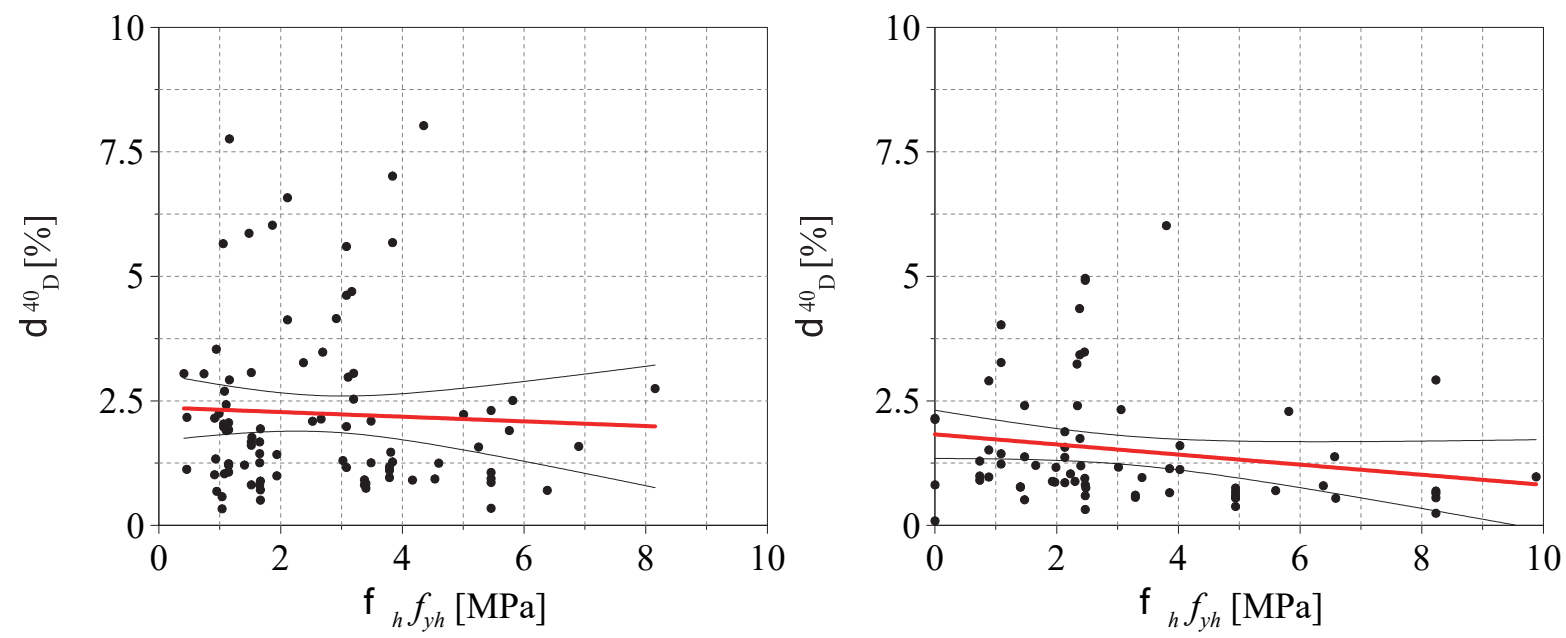

(d) $\rho_{h} f_{v h}$ : rectangular walls (left panel), and barbell and flanged walls (right panel)
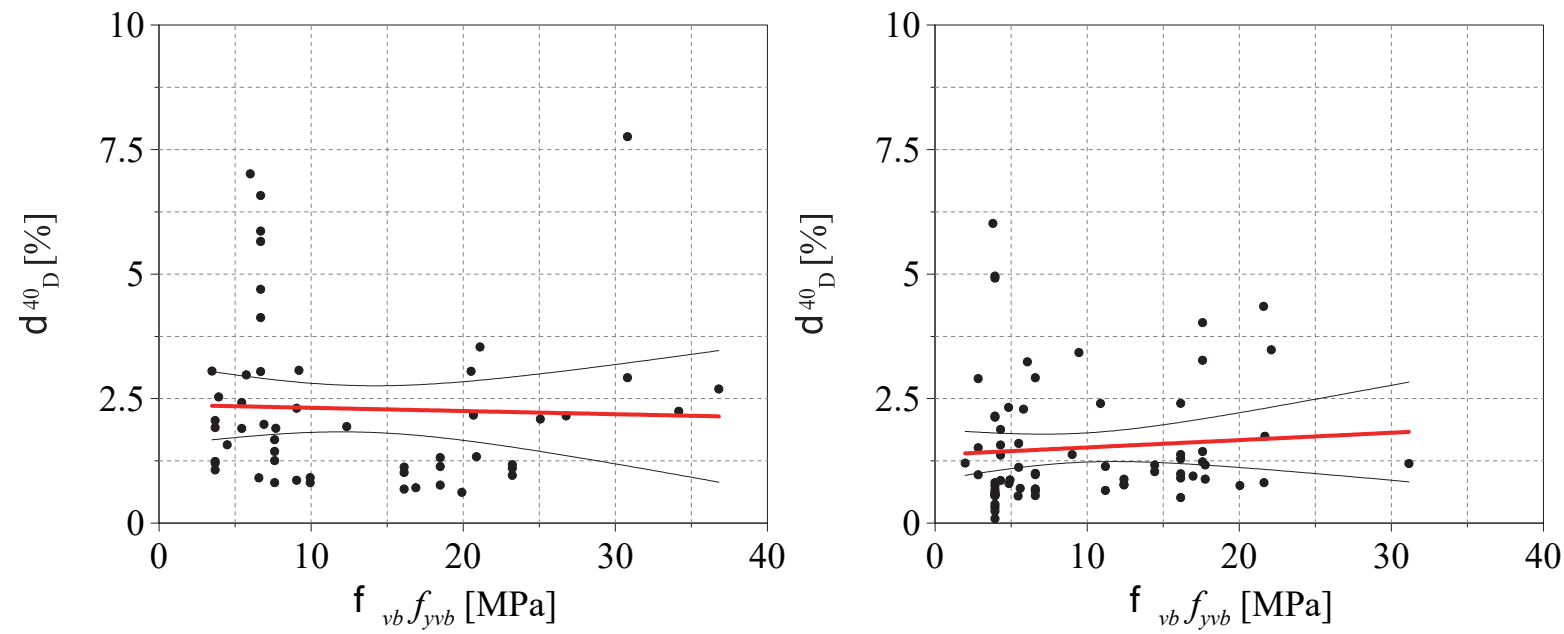

(e) $\rho_{v b} f_{v v b}$ : rectangular walls (left panel), and barbell and flanged walls (right panel)
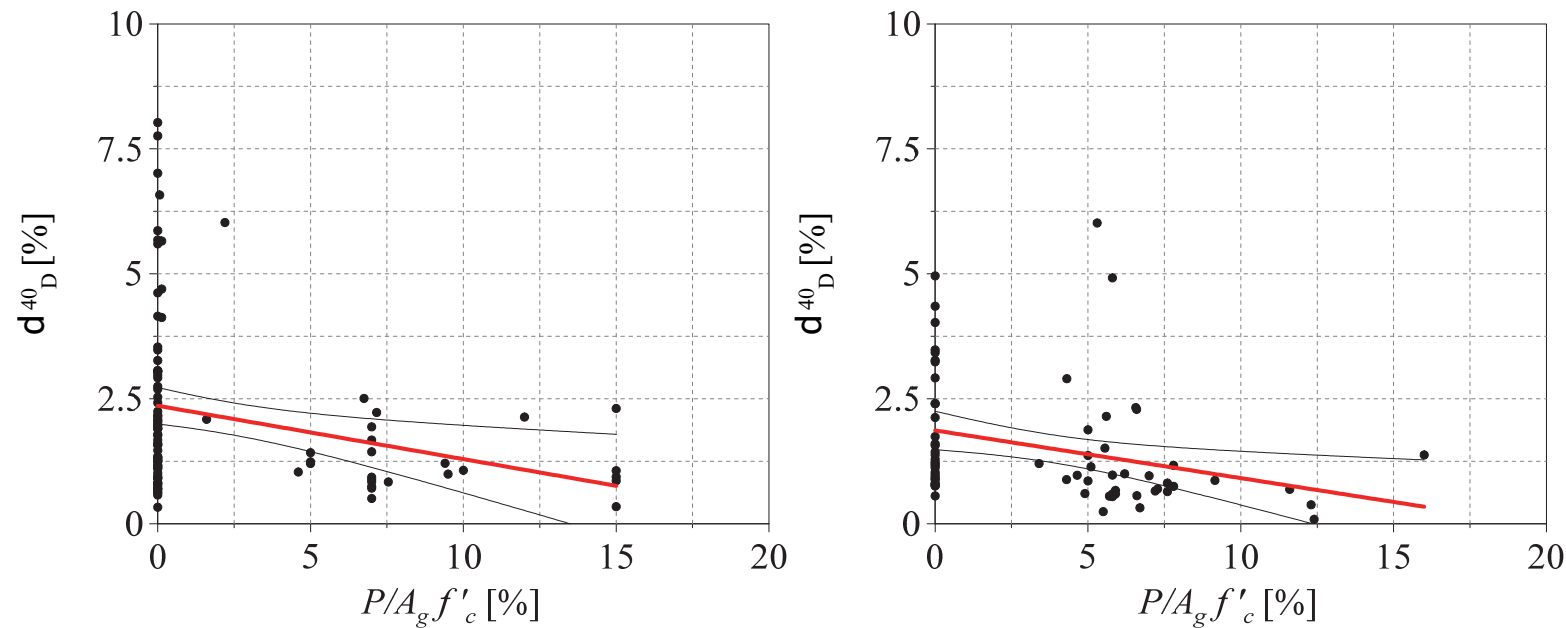

(f) $P / A_{g} f_{c}^{\prime}$ : rectangular walls (left panel), and barbell and flanged walls (right panel)

Figure 8-13 Effect of design variables on drift ratio at $40 \%$ residual strength, $\delta_{D}^{40}$ (continued). 

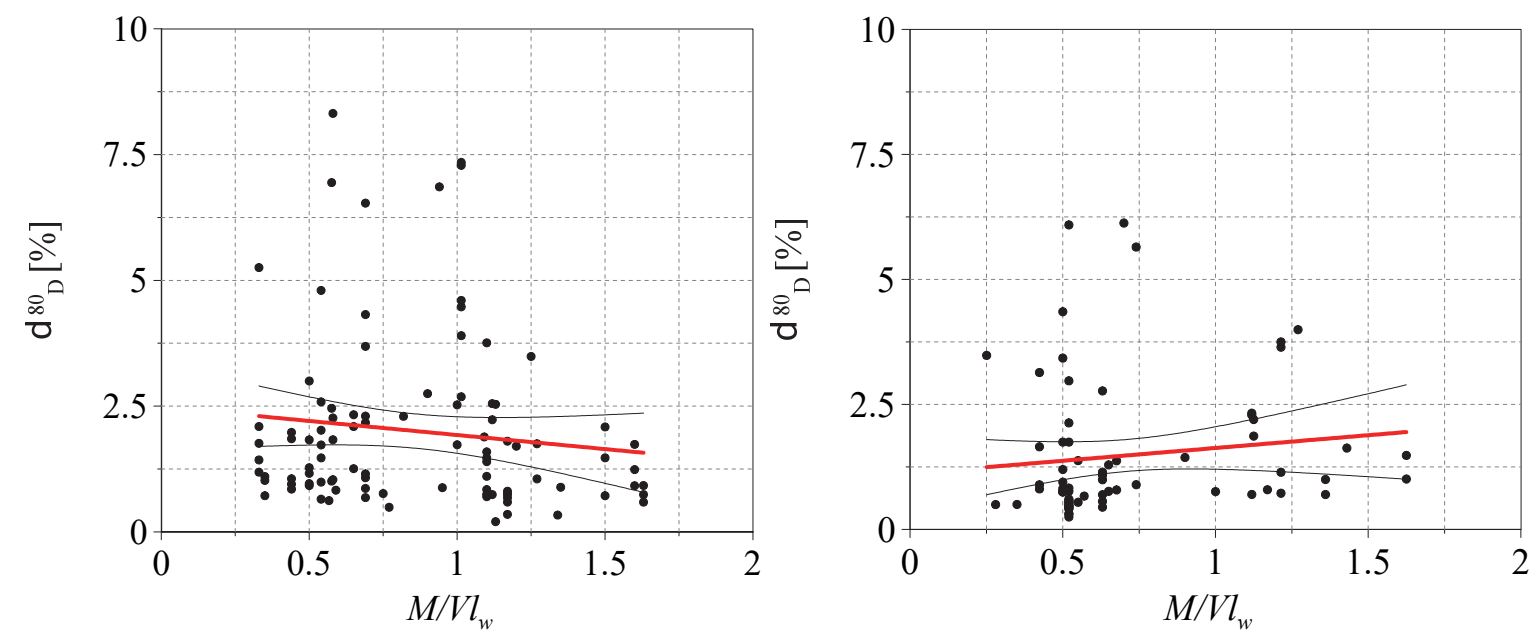

(a) $M / V I_{w}$ : rectangular walls (left panel), and barbell and flanged walls (right panel)
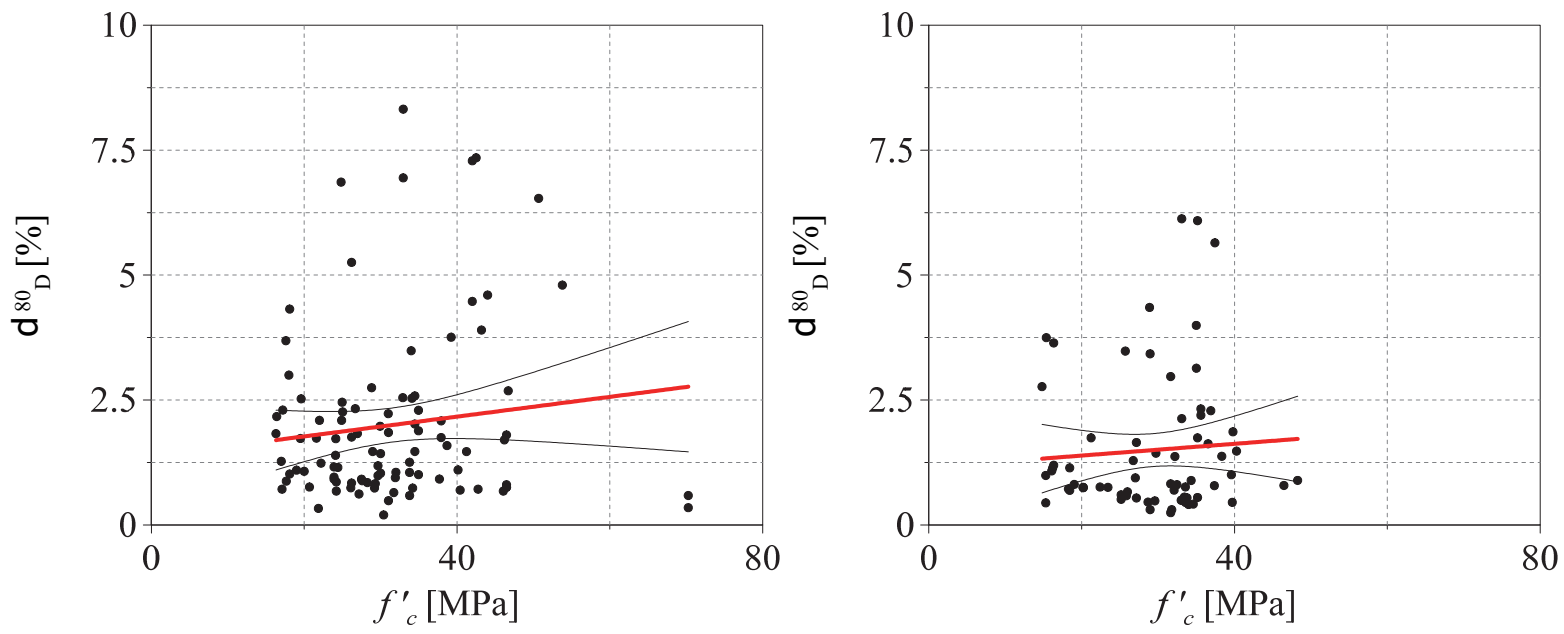

(b) $f_{c}^{\prime}$ : rectangular walls (left panel), and barbell and flanged walls (right panel)
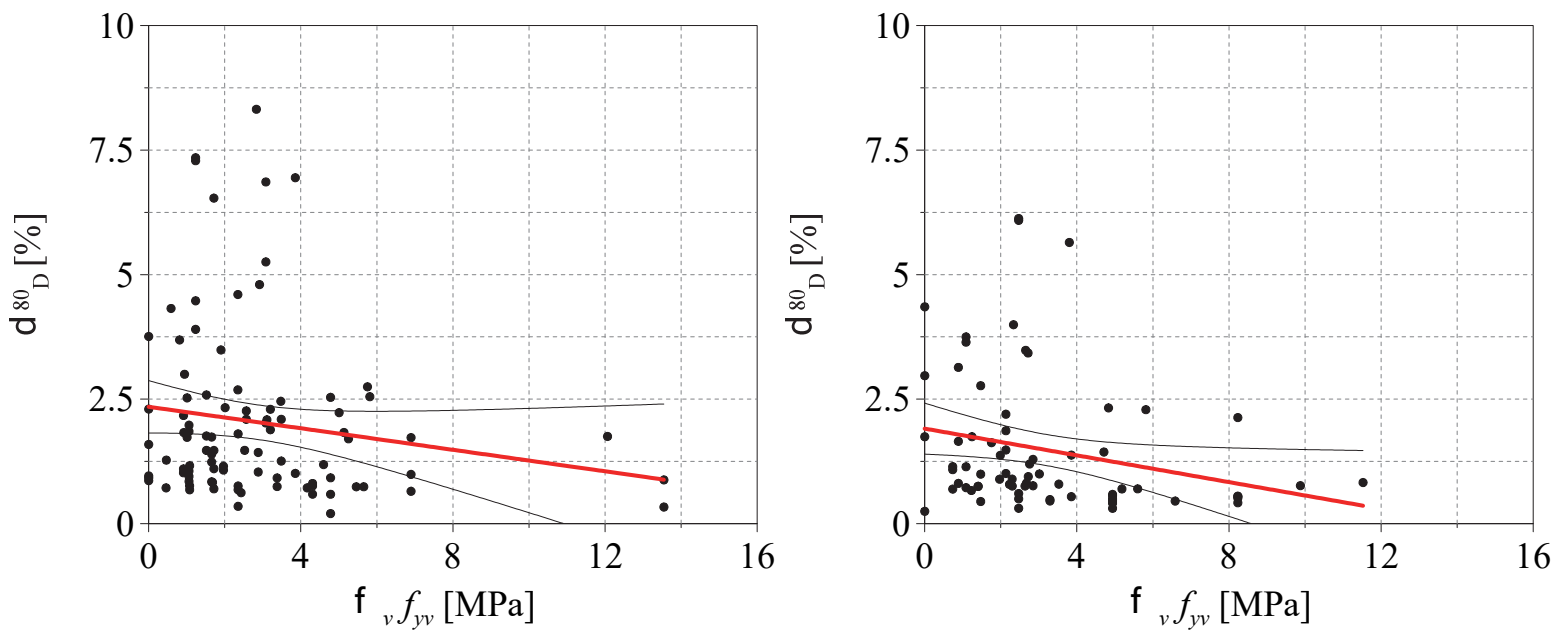

(c) $\rho_{v} f_{y v}$ : rectangular walls (left panel), and barbell and flanged walls (right panel)

Figure 8-14 Effect of design variables on drift ratio at $80 \%$ residual strength, $\delta_{D}^{80}$. 

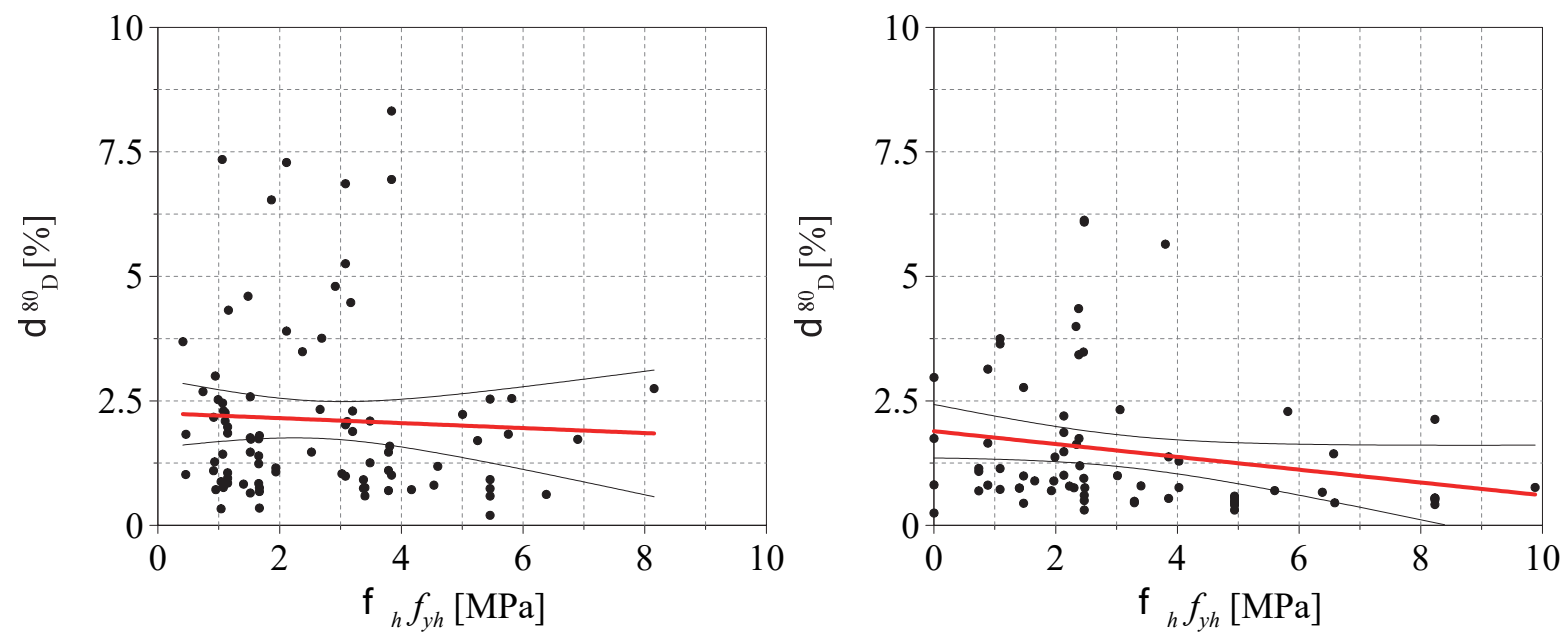

(d) $\rho_{h} f_{v h}$ : rectangular walls (left panel), and barbell and flanged walls (right panel)
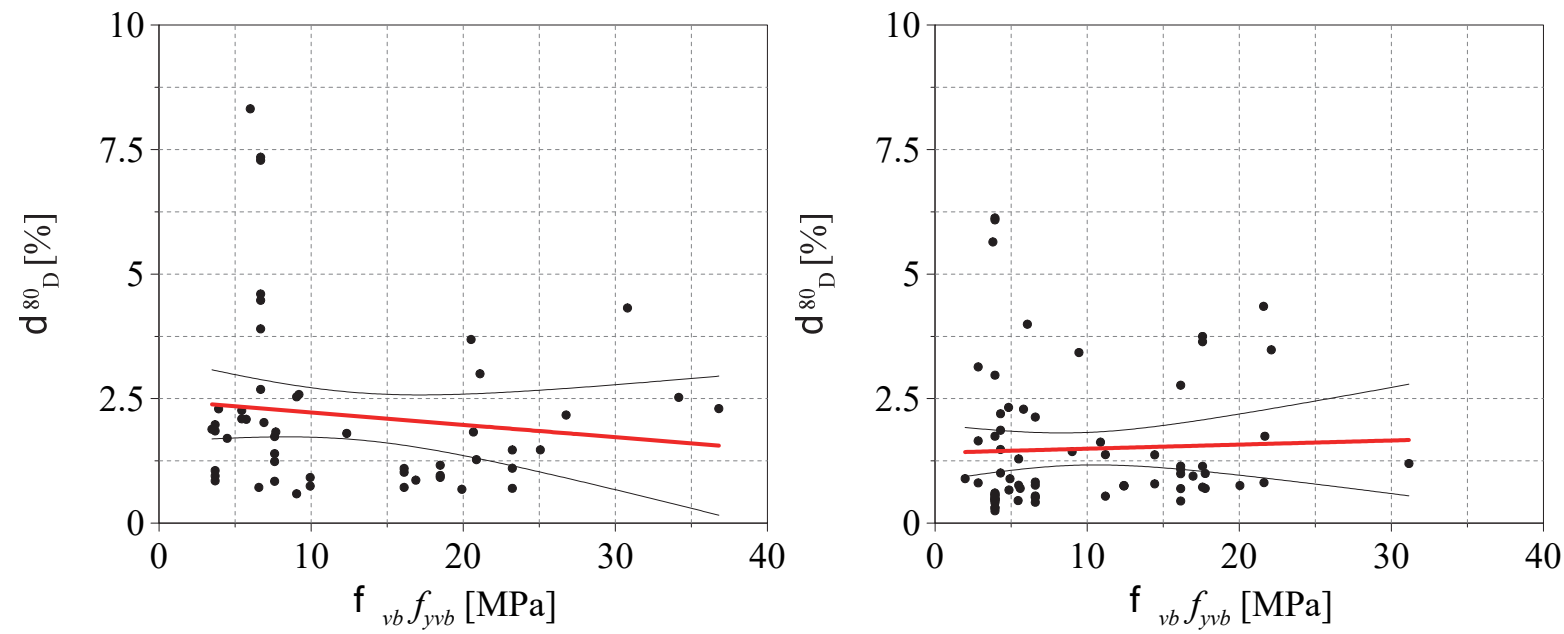

(e) $\rho_{v b} f_{y v b}$ : rectangular walls (left panel), and barbell and flanged walls (right panel)
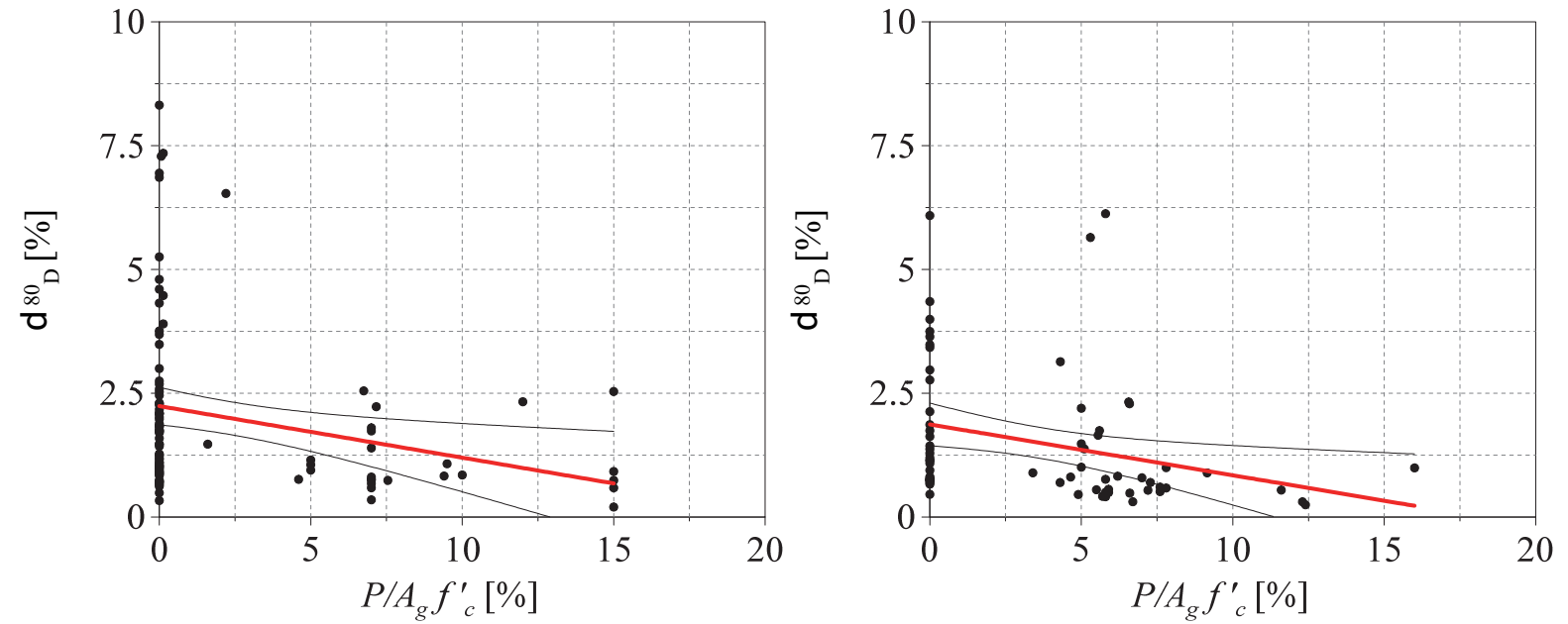

(f) $P / A_{g} f_{c}^{\prime}$ : rectangular walls (left panel), and barbell and flanged walls (right panel)

Figure 8-14 Effect of design variables on drift ratio at $80 \%$ residual strength, $\delta_{D}^{80}$ (continued). 


\subsubsection{Normalized initial, yield and post-peak stiffness}

Figure 8-15 presents the effects of some of the design variables on normalized initial stiffness, $K_{e} / K_{t}$, calculated as the secant stiffness to the point of cracking $\left(K_{e}\right)$ divided by the theoretical uncracked stiffness $\left(K_{t}\right)$, calculated as:

$$
K_{t}=\left(k_{f}^{-1}+k_{s}^{-1}\right)^{-1}
$$

where the flexural stiffness, $k_{f}$, is equal to $3 E_{c} I_{g} / h_{w}{ }^{3}$ and the shear stiffness, $k_{s}$, is calculated as $G_{c} A_{w} / h_{w}$, where $E_{c}$ is the modulus of elasticity of concrete (calculated per ACI 318-14); $I_{g}$ is the moment of inertia (second moment of area); $h_{w}$ is the height of the wall measured from the top of the foundation to the centerline of loading; $G_{c}$ is the shear modulus of concrete; and $A_{w}$ is the web area of the wall. Here, the onset of nonlinearity corresponds to point $\mathrm{F}$ in Figure 8-2. The data of Figure 8-15 make it clear the initial (assumed uncracked) stiffness of low aspect ratio walls is generally a small fraction of the theoretical uncracked stiffness, with typical values of the order of 0.25 . The dependence of $K_{e} / K_{t}$ on reinforcement ratios make clear the challenges associated with data interpretation: the secant stiffness to the onset of cracking should be independent of reinforcement ratio. Axial force ratio, as expected, influences $K_{e} / K_{t}$ : an increase in axial compressive stress increases the measured initial stiffness.

Figures 8-16 and 8-17 present information on the second and fourth branches of the cyclic backbone curve of Figure 8-2: normalized yield and post-peak stiffness, $K_{y} / K_{t}$ and $K_{p} / K_{t}$, respectively. The yield stiffness, $K_{y}$, is the slope of the line connecting points A and B in Figure 8-2. The post-peak stiffness, $K_{p}$, is the slope of the line connecting points $\mathrm{C}$ and $\mathrm{D}$ in Figure 8-2. As expected, the range on $K_{y} / K_{t}$ is smaller than the range on $K_{e} / K_{t}$. Here point D is defined using a residual strength equal to $20 \%$ of the peak lateral strength. 

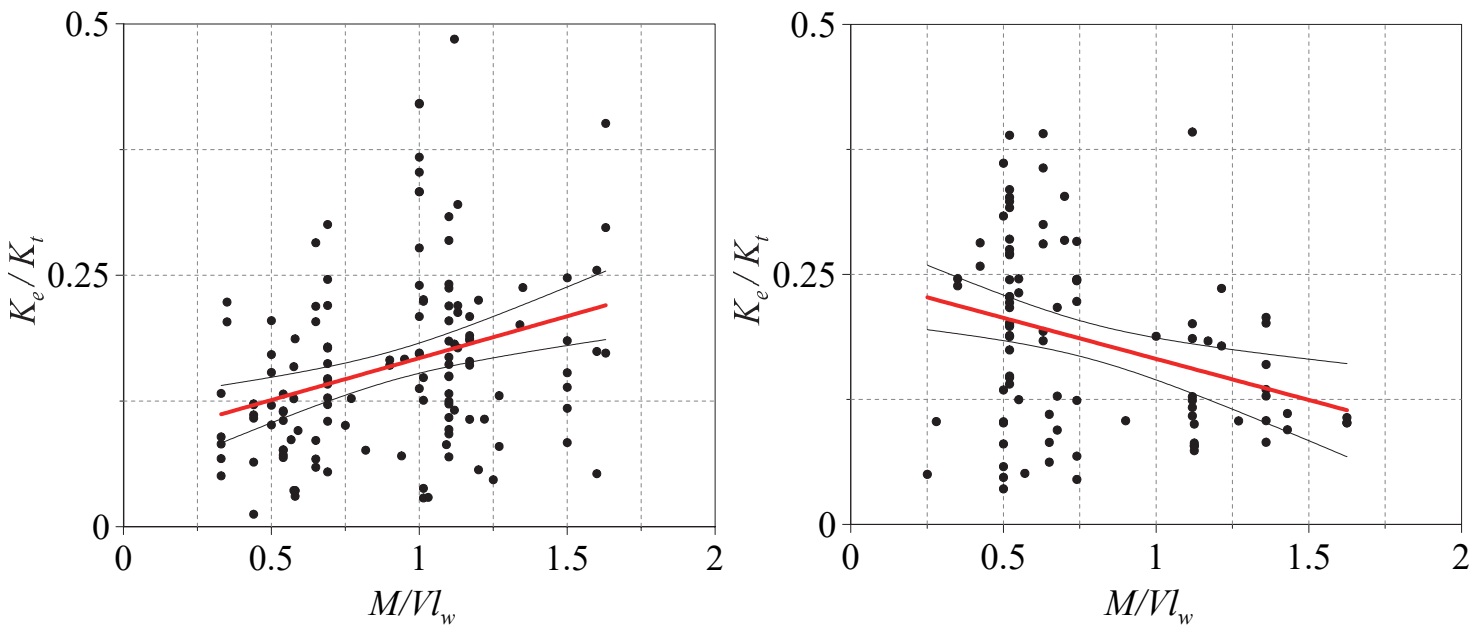

(a) $M / V I_{w}$ : rectangular walls (left panel), and barbell and flanged walls (right panel)
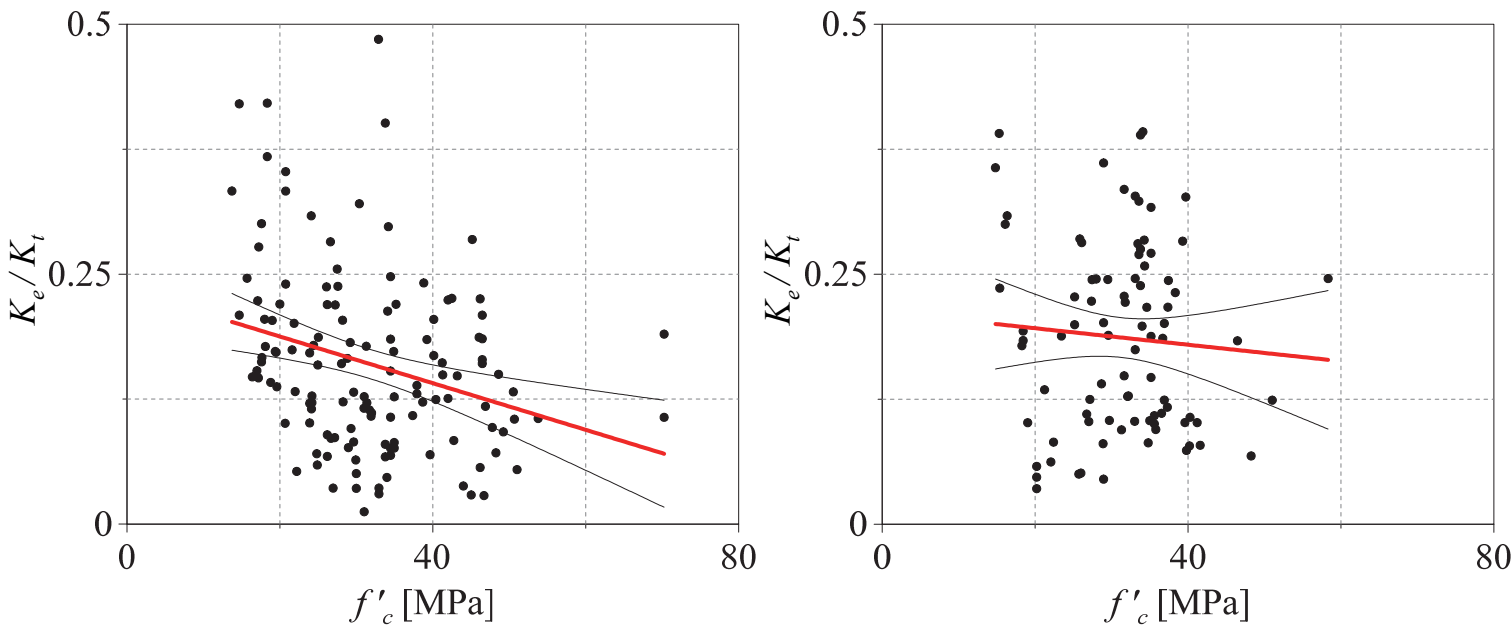

(b) $f_{c}^{\prime}$ : rectangular walls (left panel), and barbell and flanged walls (right panel)
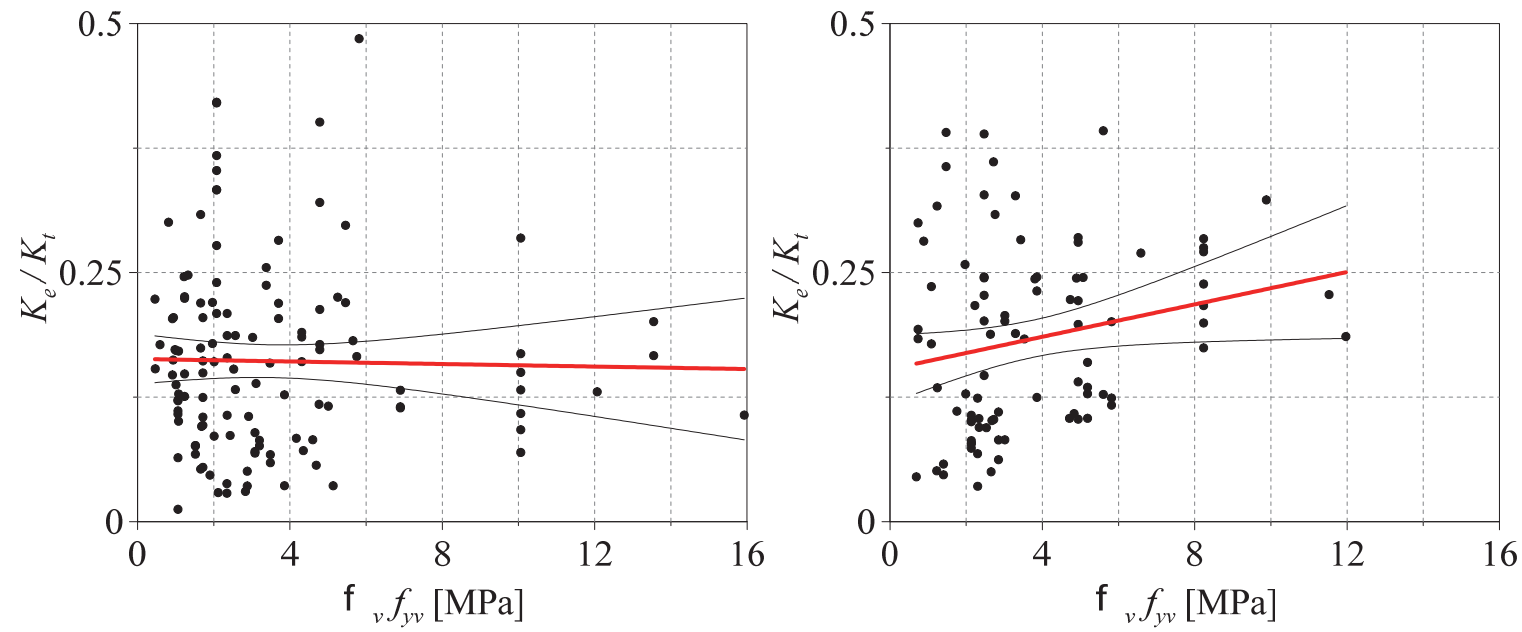

(c) $\rho_{v} f_{y v}$ : rectangular walls (left panel), and barbell and flanged walls (right panel)

Figure 8-15 Effect of design variables on normalized initial stiffness, $K_{e} / K_{t}$. 

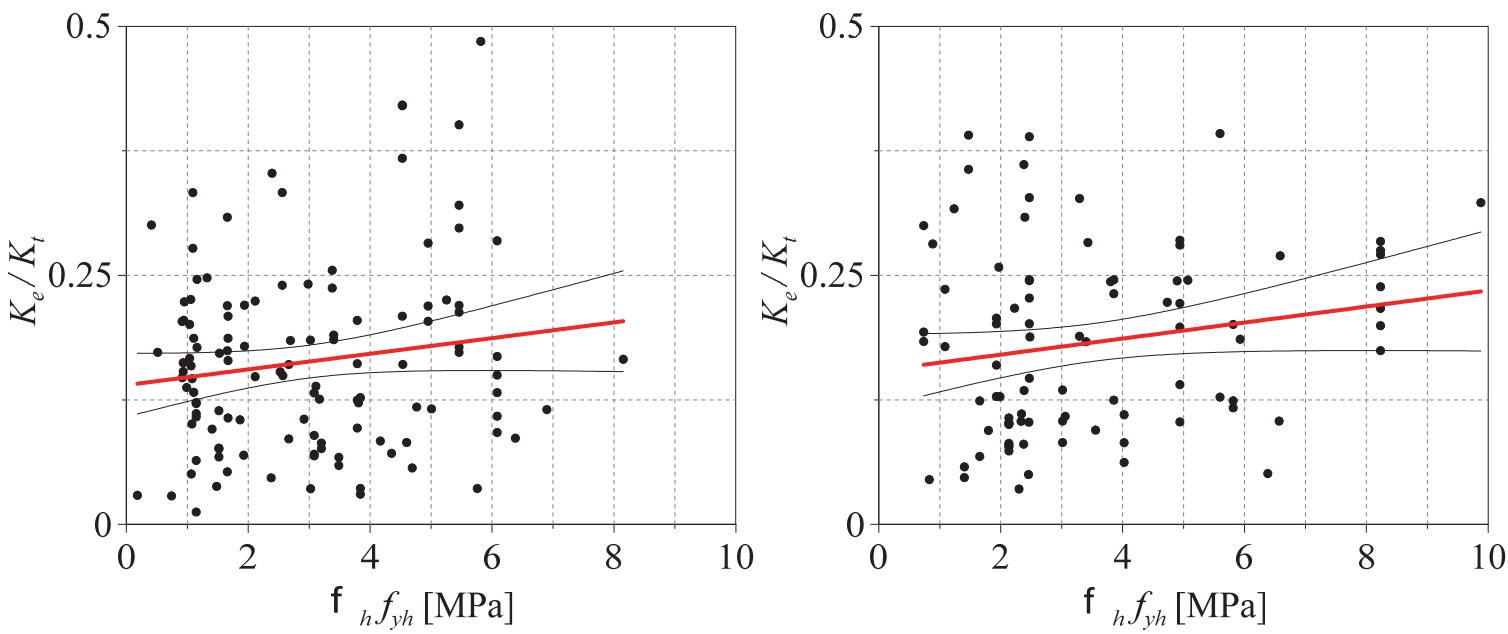

(d) $\rho_{h} f_{v h}$ rectangular walls (left panel), and barbell and flanged walls (right panel)
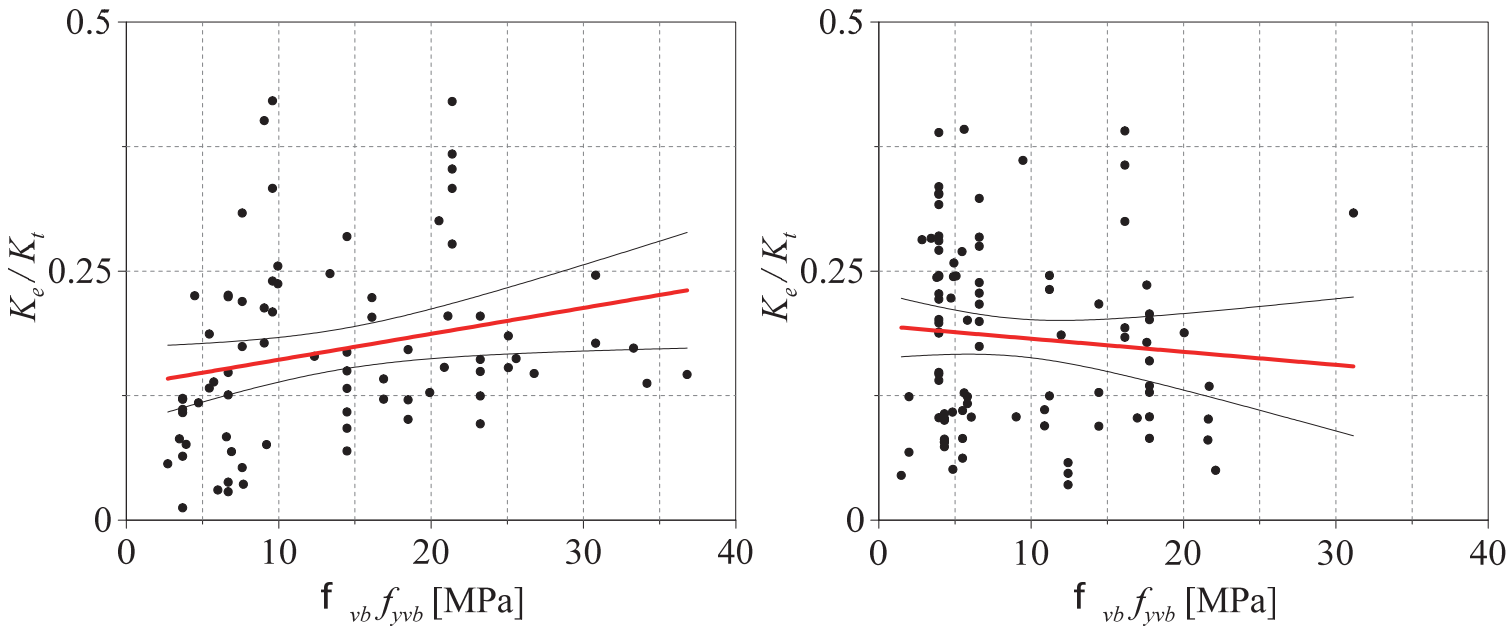

(e) $\rho_{v b} f_{v v b}$ : rectangular walls (left panel), and barbell and flanged walls (right panel)
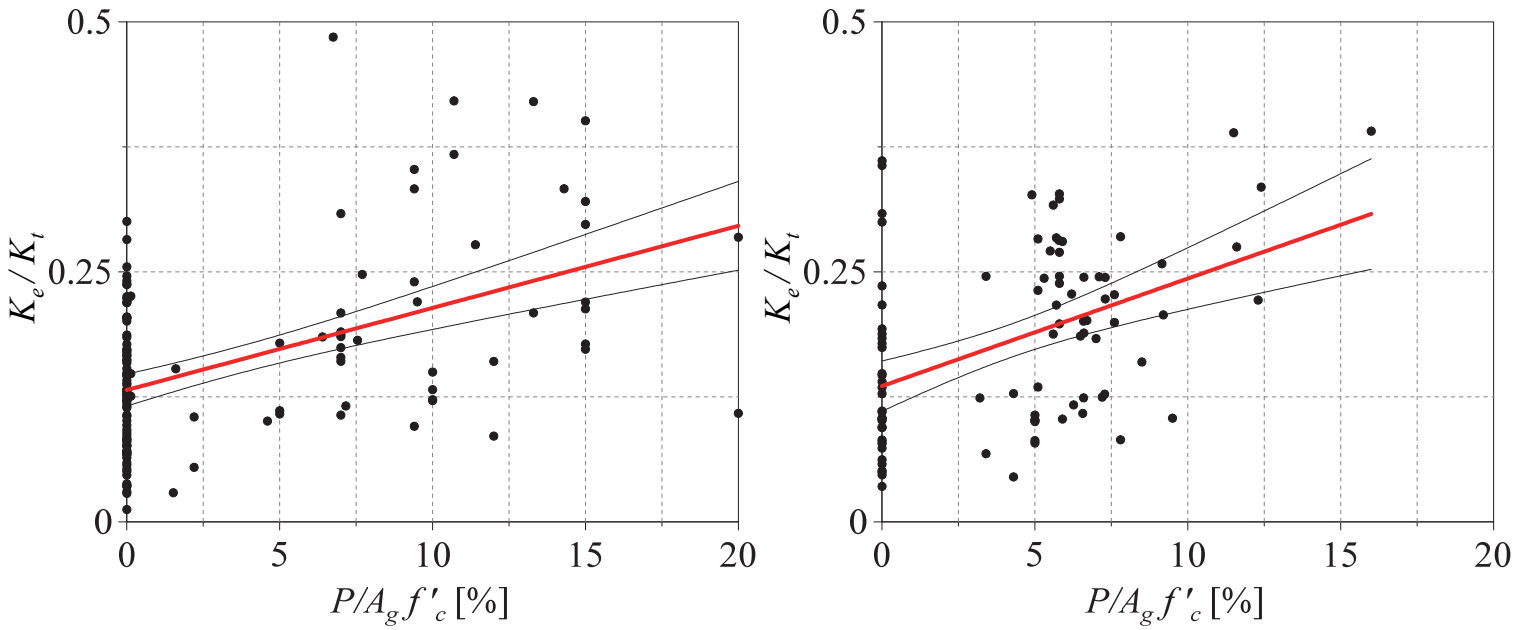

(f) $P / A_{g} f_{c}^{\prime}$ : rectangular walls (left panel), and barbell and flanged walls (right panel)

Figure 8-15 Effect of design variables on normalized initial stiffness, $K_{e} / K_{t}$ (continued). 

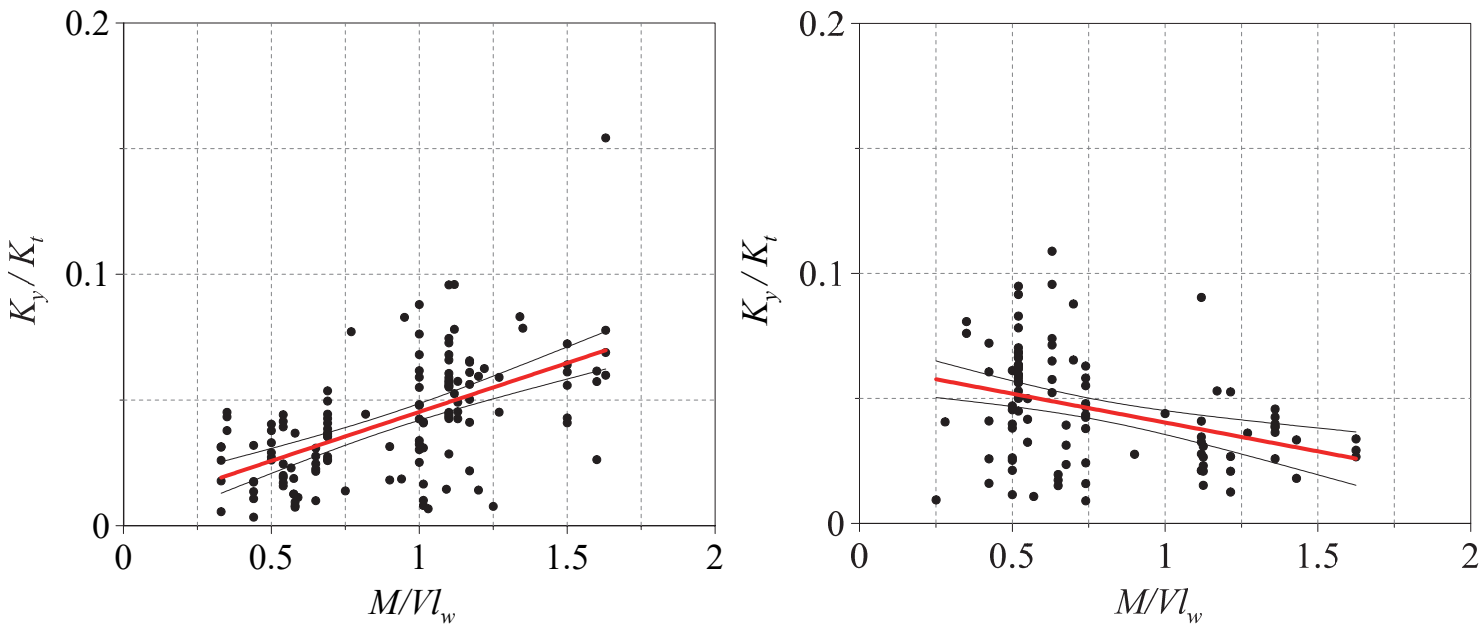

(a) $M / V l_{w}$ : rectangular walls (left panel), and barbell and flanged walls (right panel)
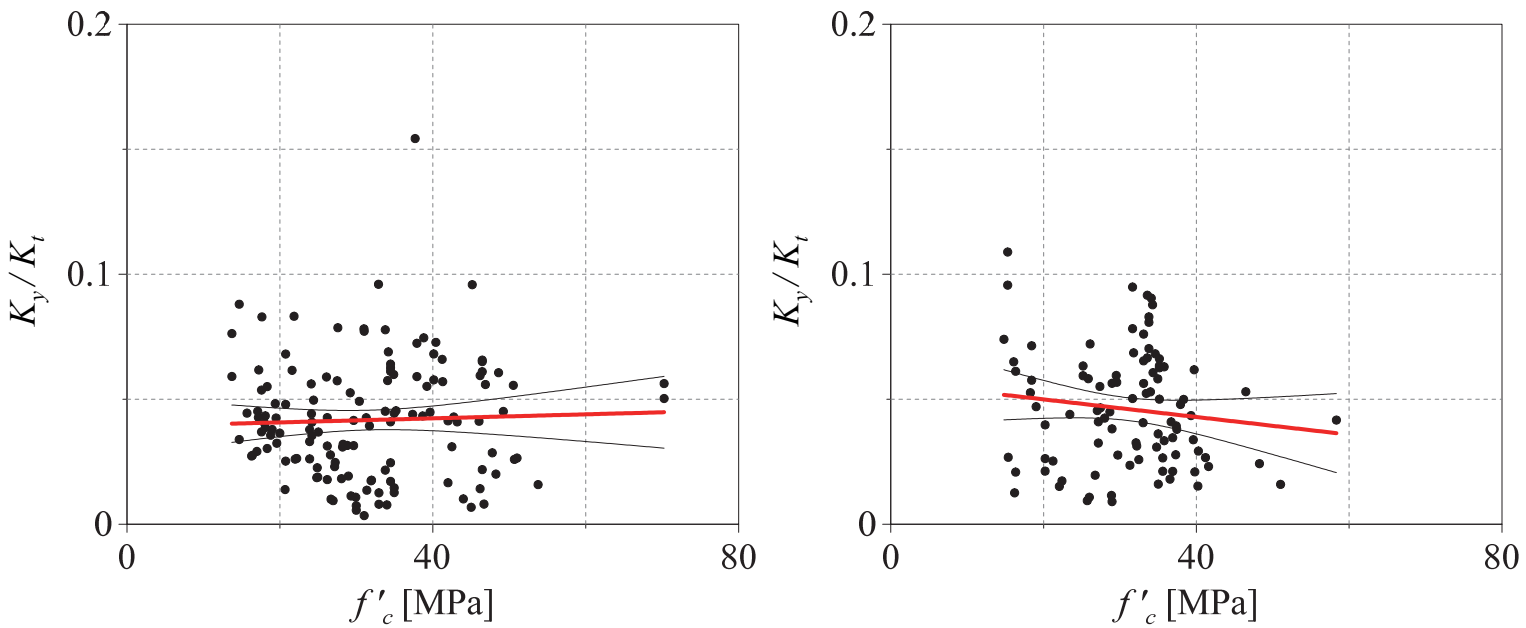

(b) $f_{c}^{\prime}$ : rectangular walls (left panel), and barbell and flanged walls (right panel)
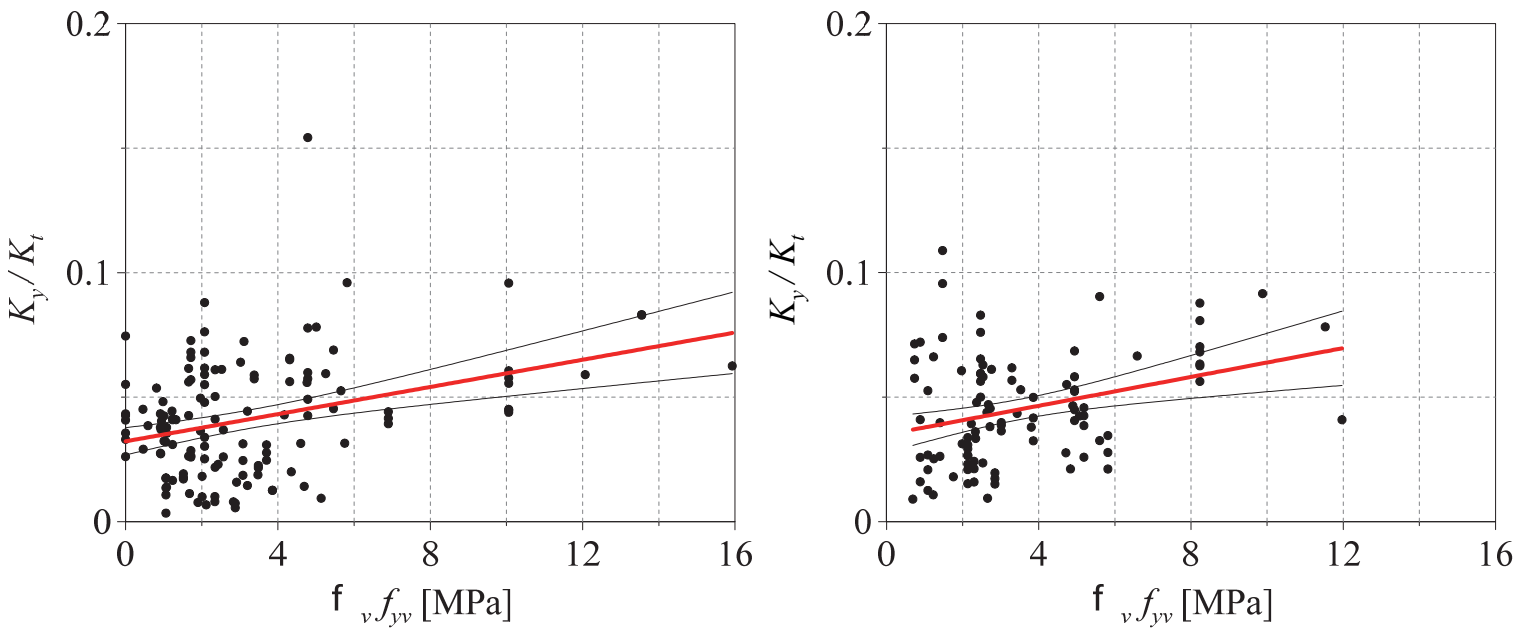

(c) $\rho_{v} f_{y v}$ : rectangular walls (left panel), and barbell and flanged walls (right panel)

Figure 8-16 Effect of design variables on normalized yield stiffness, $K_{y} / K_{t}$. 

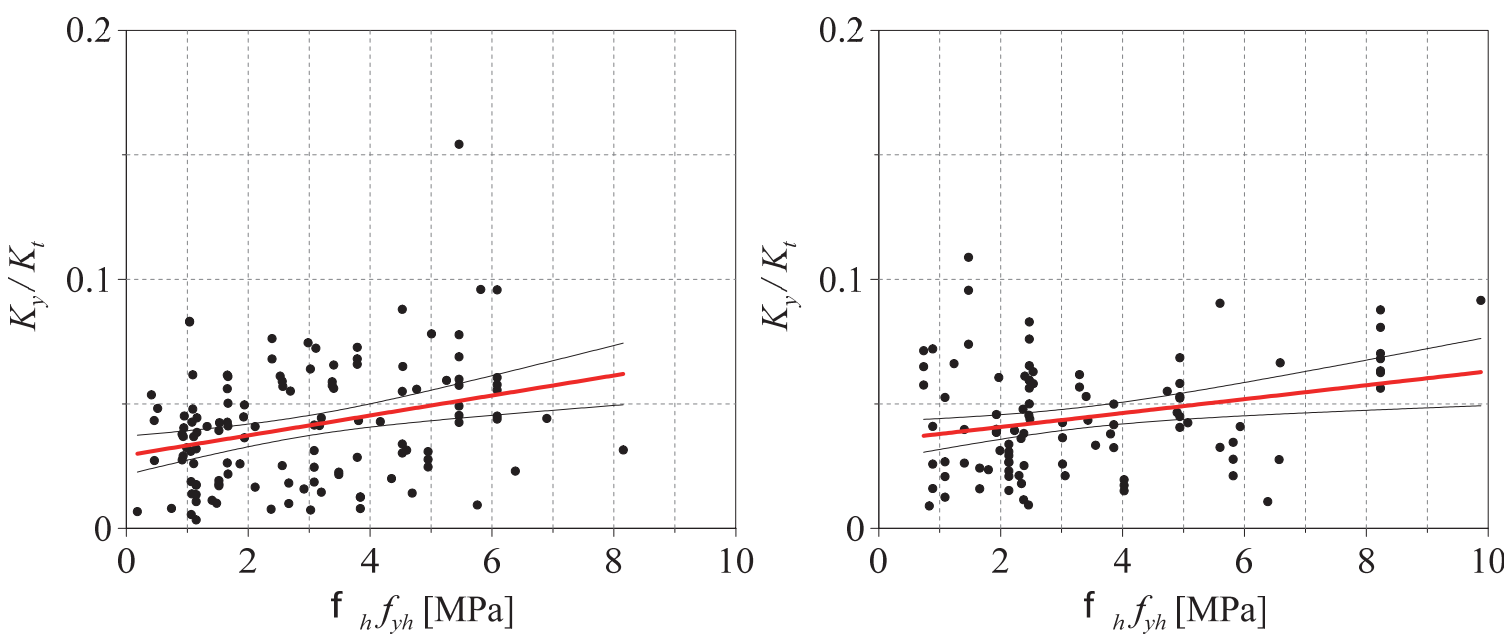

(d) $\rho_{h} f_{v h}$ rectangular walls (left panel), and barbell and flanged walls (right panel)
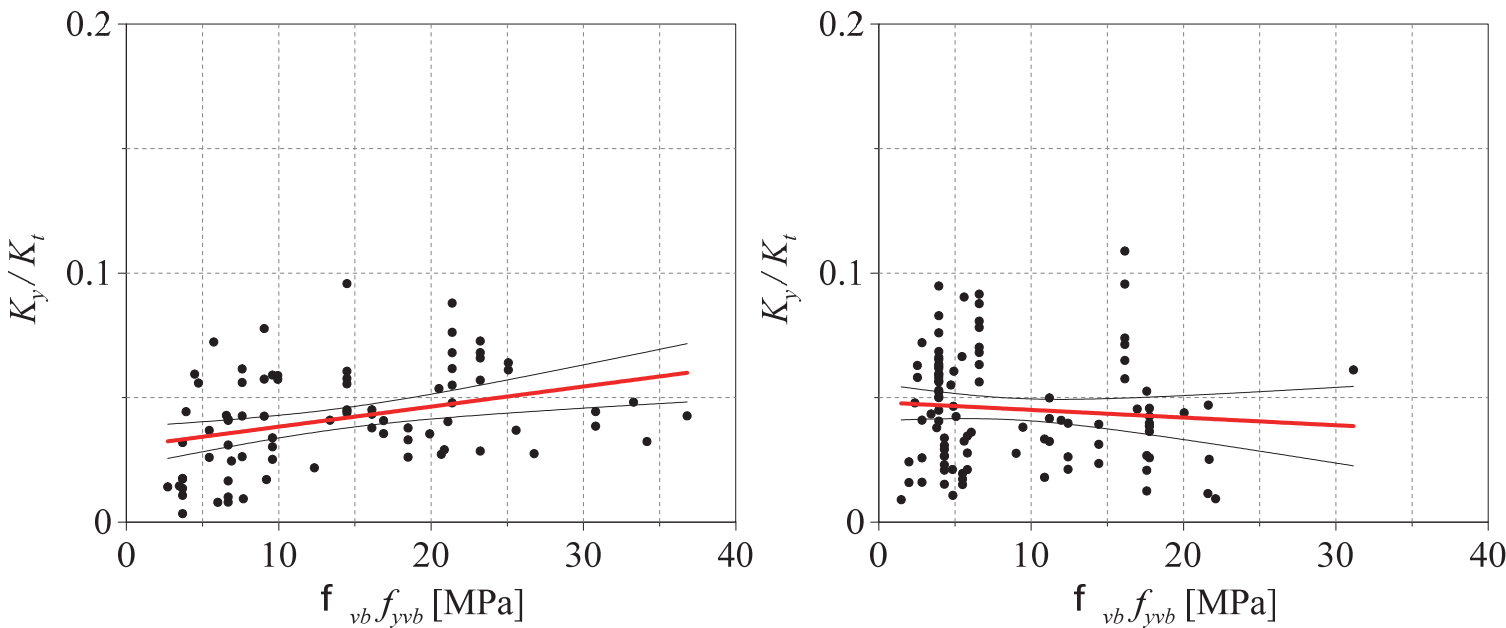

(e) $\rho_{v b} f_{v v b}$ : rectangular walls (left panel), and barbell and flanged walls (right panel)
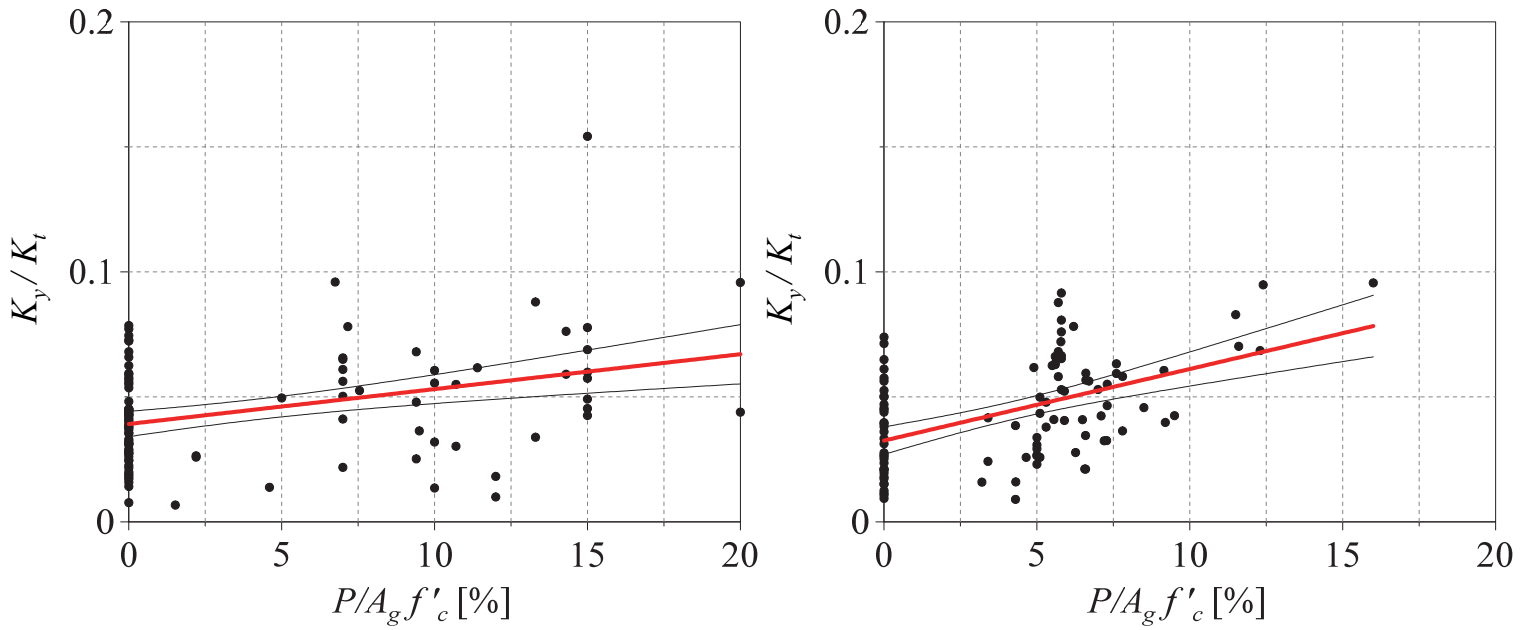

(f) $P / A_{g} f_{c}^{\prime}$ : rectangular walls (left panel), and barbell and flanged walls (right panel)

Figure 8-16 Effect of design variables on normalized yield stiffness, $K_{y} / K_{t}$ (continued). 

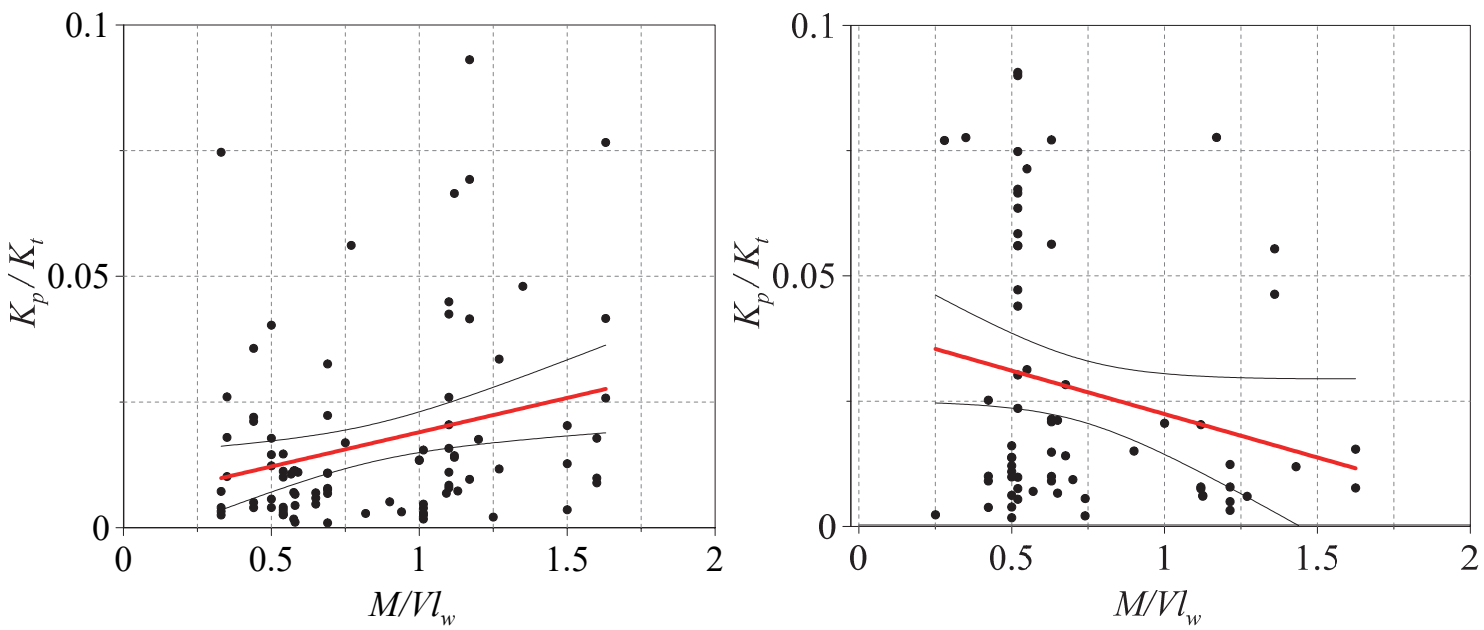

(a) $M / V I_{w}$ : rectangular walls (left panel), and barbell and flanged walls (right panel)
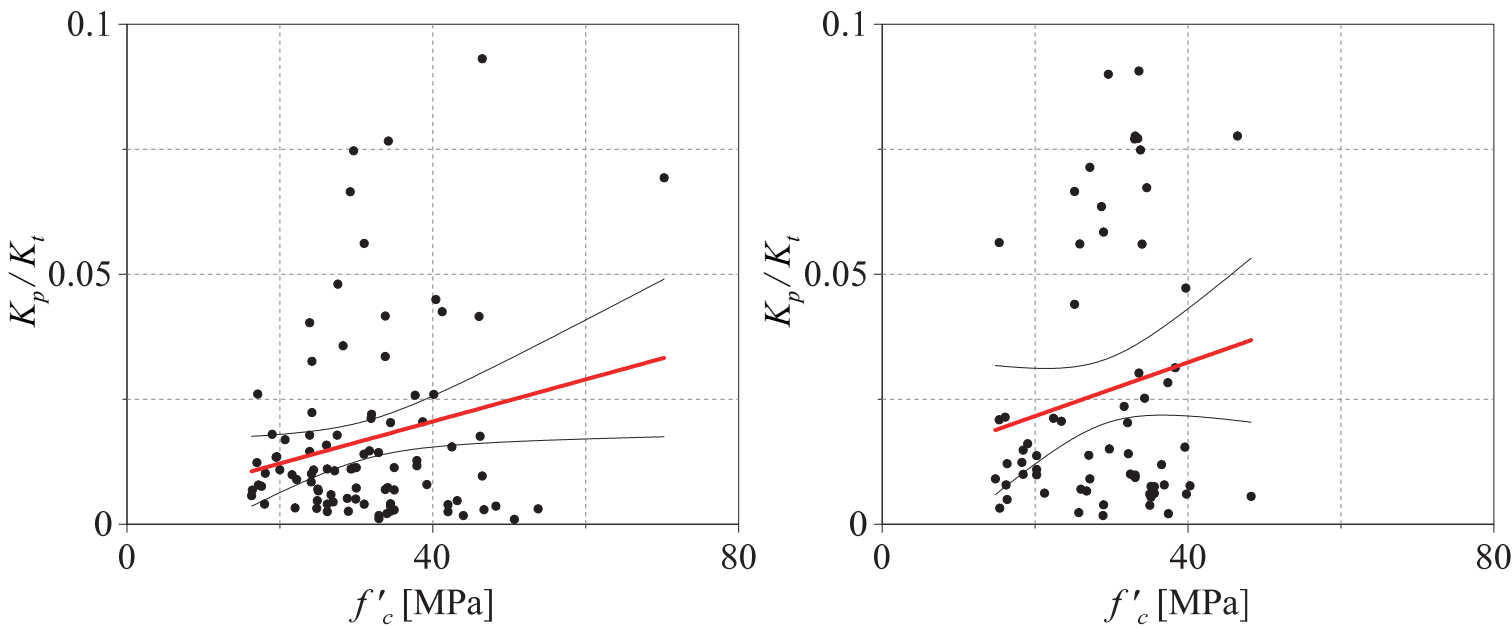

(b) $f_{c}^{\prime}$ : rectangular walls (left panel), and barbell and flanged walls (right panel)
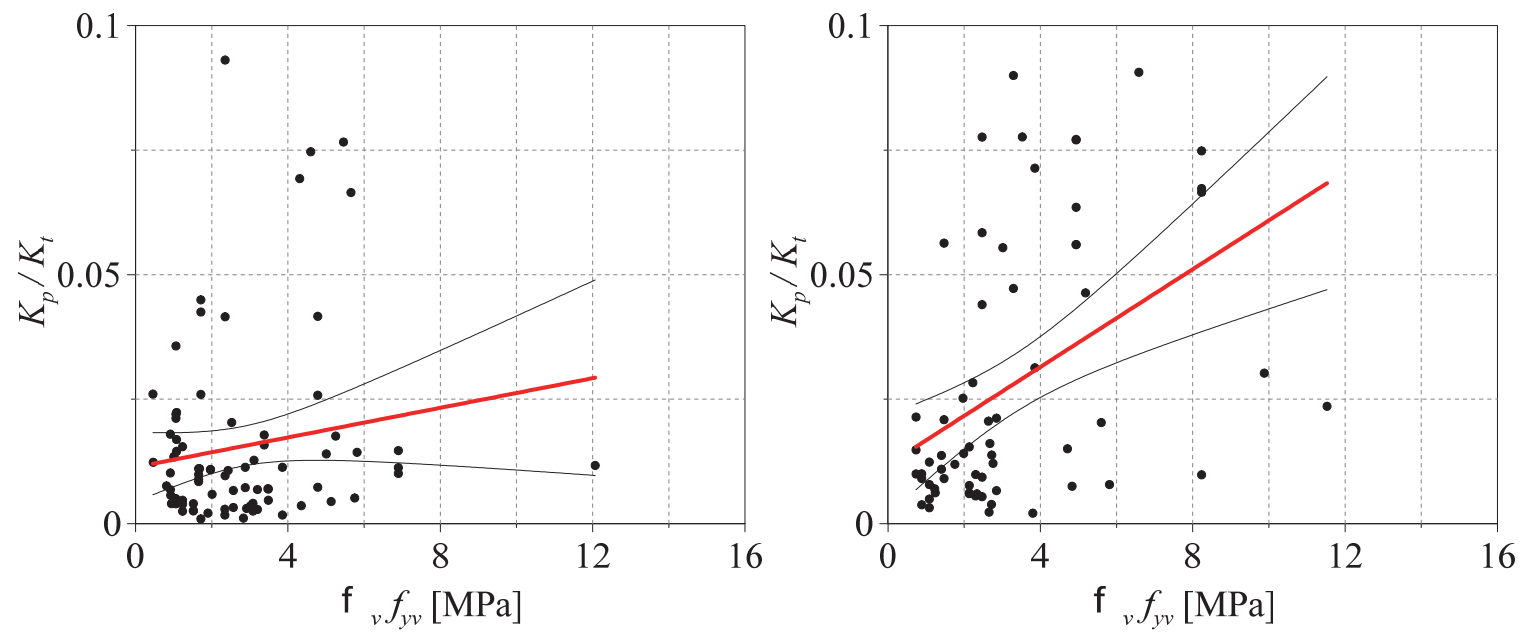

(c) $\rho_{v} f_{y v}$ : rectangular walls (left panel), and barbell and flanged walls (right panel)

Figure 8-17 Effect of design variables on normalized post-peak stiffness, $K_{p} / K_{t}$. 

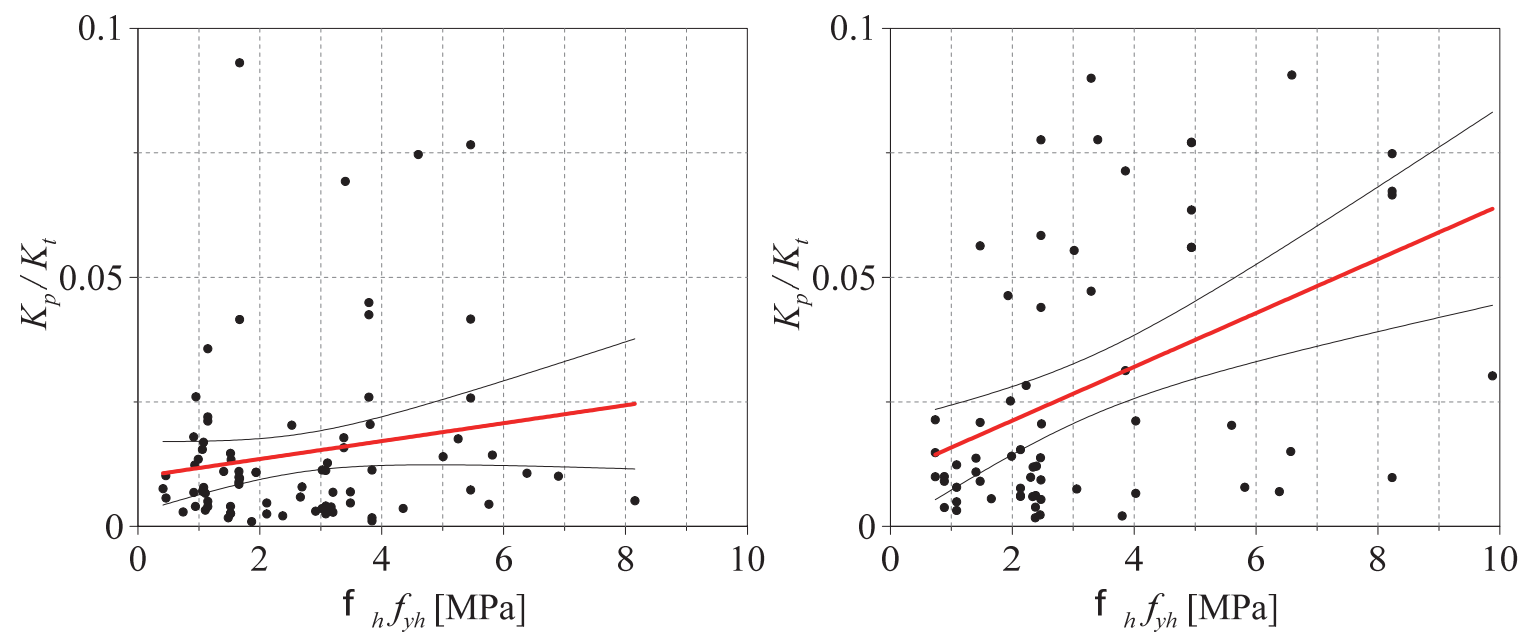

(d) $\rho_{h} f_{v h}:$ rectangular walls (left panel), and barbell and flanged walls (right panel)
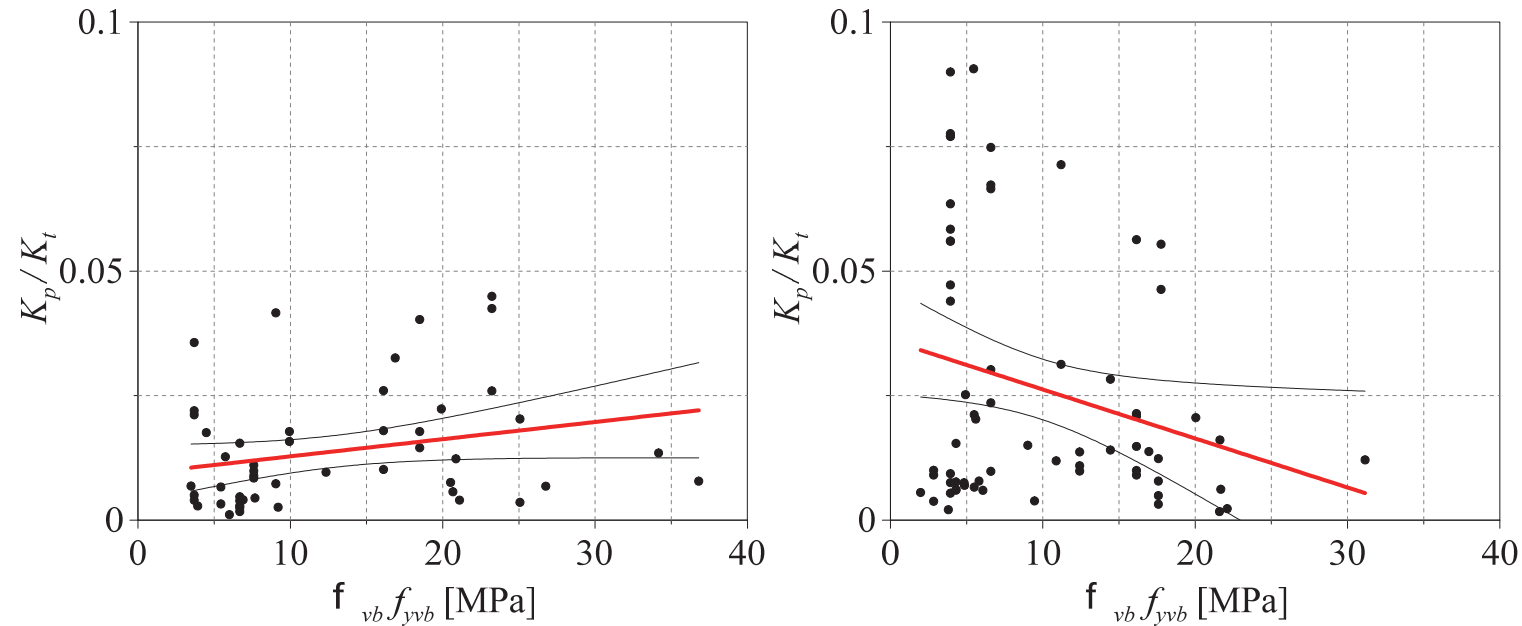

(e) $\rho_{v b} f_{v v b}$ : rectangular walls (left panel), and barbell and flanged walls (right panel)
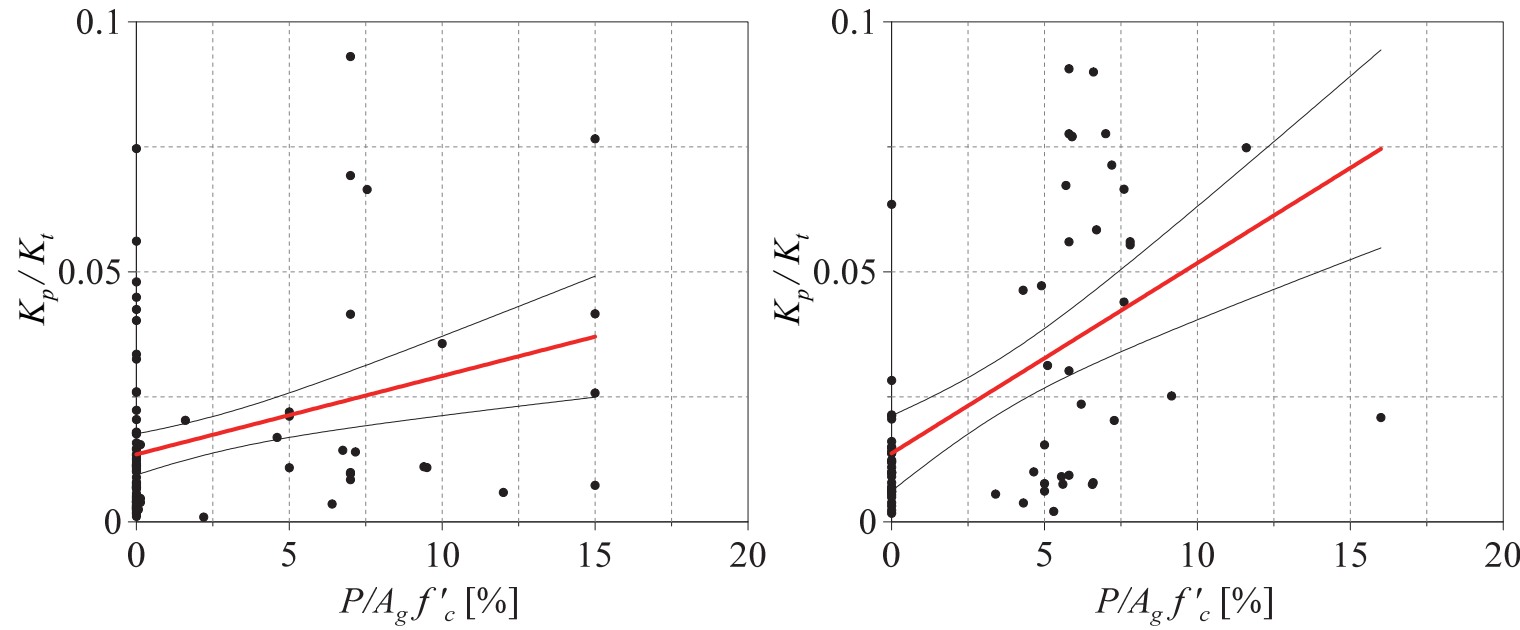

(f) $P / A_{g} f_{c}^{\prime}$ : rectangular walls (left panel), and barbell and flanged walls (right panel)

Figure 8-17 Effect of design variables on normalized post-peak stiffness, $K_{p} / K_{t}$ (continued). 


\subsubsection{Recommendations for Modeling and Control Points}

The data presented above show that variability is immense and that key design parameters should be expressed in a functional form, which accommodates those important design variables that impact calculations. However, in keeping with the format of ASCE/SEI 41-13 for shear walls and other framing systems, recommended values for key data points (see Figure 8-2) do not have a functional form, with the exception of peak lateral strength.

The data presented previously are re-binned to calculate the following: (1) drift ratios at cracking, yielding, peak load, $0 \%, 20 \%, 40 \%$, and $80 \%$ residual strength; (2) forces at cracking and peak load; and (3) initial stiffness, yield stiffness, and post-peak stiffness. Peak load was defined as the average of the maximum strengths in the first and third quadrants of loading.

Data are binned per Table 8-2, by aspect ratio, axial compressive force ratio, and wall geometry (rectangular and barbell/flanged). Data from tests with specimens that fall outside the domains below were not included in the analysis. Appendix $\mathrm{C}$ presents the binned data and a summary of the results by wall geometry and data bin $(1,1 \mathrm{a}, 2$, 2a), including mean (average) values, standard deviation of the natural logarithm, and percentiles, as well as the mean ACI-predicted to mean measured peak strength for the data bin $(1,1 \mathrm{a}, 2,2 \mathrm{a})$.

Table 8-2 Data Bins for Developing Updated Guidance

\begin{tabular}{lccc}
\hline & Bin 1 and $1 \mathrm{a}$ & & Bin 2 and 2a \\
\cline { 2 - 2 } Aspect ratio & $0.25 \leq h_{w} / l_{w} \leq 1$ & & $1<h_{w} / l_{w} \leq 2$ \\
Concrete compressive strength (MPa) & $10 \leq f_{c}^{\prime} \leq 80$ & & $10 \leq f_{c}^{\prime} \leq 80$ \\
Vertical web reinforcement (MPa) & $0 \leq \rho_{v} f_{y v} \leq 16$ & & $0 \leq \rho_{v} f_{y v} \leq 16$ \\
Horizontal web reinforcement (MPa) & $0 \leq \rho_{h} f_{y h} \leq 10$ & & $0 \leq \rho_{h} f_{y h} \leq 10$ \\
Boundary element reinforcement (MPa) & $0 \leq \rho_{v b} f_{y v b} \leq 40$ & & $0 \leq \rho_{v b} f_{y v b} \leq 40$ \\
Axial force ratio (Bins 1 and 2) & $P / A_{g} f_{c}^{\prime}=0$ & & $P / A_{g} f_{c}^{\prime}=0$ \\
Axial force ratio (Bins 1a and 2a) & $0.05 \leq P / A_{g} f_{c}^{\prime} \leq 0.20$ & & $0.05 \leq P / A_{g} f_{c}^{\prime} \leq 0.20$ \\
\hline
\end{tabular}

Based on the data and recognizing the significant variability and uncertainty (in the data and introduced by assigning discrete values and setting aside functional forms), the cyclic backbone curve of Figure 8-2 can be established as follows, noting that point E collapses to point D:

It is noted that the ratio of mean peak strength predicted by ACI equations to those measured varies from 0.87 to 1.27 and it varies from 0.98 to 1.13 , per equations from Chapters 11 and 18 of ACI 318-14, respectively. This indicates that the ACI equations are underpredicting the peak strengths of the flanged and barbell walls significantly. Table 8-3 summarizes the cyclic backbone control points. 


\begin{tabular}{|c|c|c|}
\hline \multicolumn{3}{|c|}{$\begin{array}{l}\text { Cyclic Backbone Control Points for Reinforced } \\
\text { Concrete Walls Controlled by Shear }\end{array}$} \\
\hline Control Point & $V$ & $\delta$ \\
\hline A initiation of cracking & $0.5 V_{n}$ & $0.1 \%$ \\
\hline B- Peak strength & $V_{n}$ & $0.5 \%$ \\
\hline C- Initiation of Degradation & $V_{n}$ & \\
\hline Rectangular walls & & $1.0 \%$ \\
\hline Flanged walls, $h_{w} / l_{w}<1$ & & $0.7 \%$ \\
\hline Flanged walls $1<h_{w} / /_{W}<2$ & & $0.9 \%$ \\
\hline$D^{80}$ & $0.8 V_{n}$ & Linear interpolation \\
\hline$D^{40}$ & $0.4 V_{n}$ & Linear interpolation \\
\hline$D^{20}-20 \% V_{n}$ & $0.2 V_{n}$ & \\
\hline Rectangular walls $h_{w} / /_{w}<1$ & & $2.1 \%$ \\
\hline Rectangular walls $1<h_{w} / l_{w}<2$ & & $2.5 \%$ \\
\hline $\begin{array}{l}\text { Flanged walls } h_{w} / \|_{W}<1 \text { and } \\
P / P_{n}<0.05\end{array}$ & & $1.8 \%$ \\
\hline $\begin{array}{l}\text { Flanged walls } h_{w} / l_{w}<1 \text { and } \\
0.05<P I P_{n}<0.2\end{array}$ & & $1.4 \%$ \\
\hline $\begin{array}{l}\text { Flanged walls } 1<h_{w} / \|_{w}<2 \text { and } \\
P / P_{n}<0.05\end{array}$ & & $2.6 \%$ \\
\hline $\begin{array}{l}\text { Flanged walls } h_{w} / l_{w}<1 \text { and } \\
0.05<P / P_{n}<0.2\end{array}$ & & $1.6 \%$ \\
\hline
\end{tabular}

\subsection{Cyclic Hysteretic Models}

The Ibarra-Krawinkler Pinching (IKP) model (Ibarra et al., 2005) is used to simulate the cyclic hysteretic response of shear-critical reinforced concrete walls. This model incorporates multiple, user-selected models for cyclic deterioration. Ibarra et al. simulated the inelastic behavior of two tested RC columns subjected to axial and cyclic lateral loadings using this model. Gulec and Whittaker (2011b) also used this model to simulate the cyclic behavior of seven shear-critical RC walls.

The IKP model is constructed using the piece-wise linear backbone curve of Figure $8-18 \mathrm{a}$ and the hysteretic rules presented in Figure 8-18b. The backbone curve is bilinear in the pre-peak-strength region and linear in the post-peak-strength softening regime. The unloading branch of the cyclic response is assumed to be linear. The reloading branch consists of two segments: the first reloading path (points 3-4 and $7-8$ in Figure 8-18b) is directed towards a break point (points 4 and 8), and the second path (4-5 and 8-9) connects the break point to the point corresponding to the maximum displacement of earlier cycles in the same quadrant (points 2 and 6 in Figure 8-18b). The break point is a function of the maximum force, $F_{\max }$, and 
residual displacement, $\delta_{\text {perl }}$, achieved in the previous cycle in the same quadrant, and is established using displacement and force coordinates equal to $(1-\mathrm{k} d) \delta_{\text {perl }}$ and $k_{f} F_{\max }$, respectively.

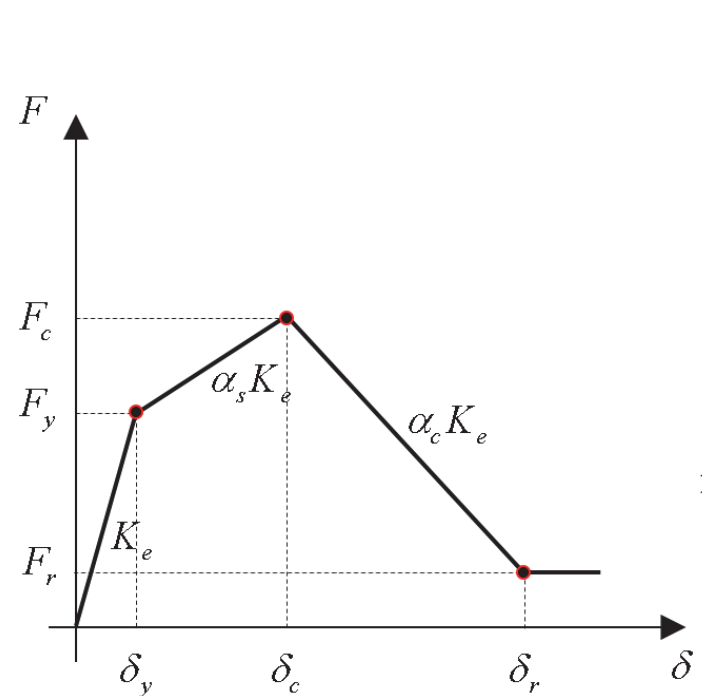

(a) Backbone curve

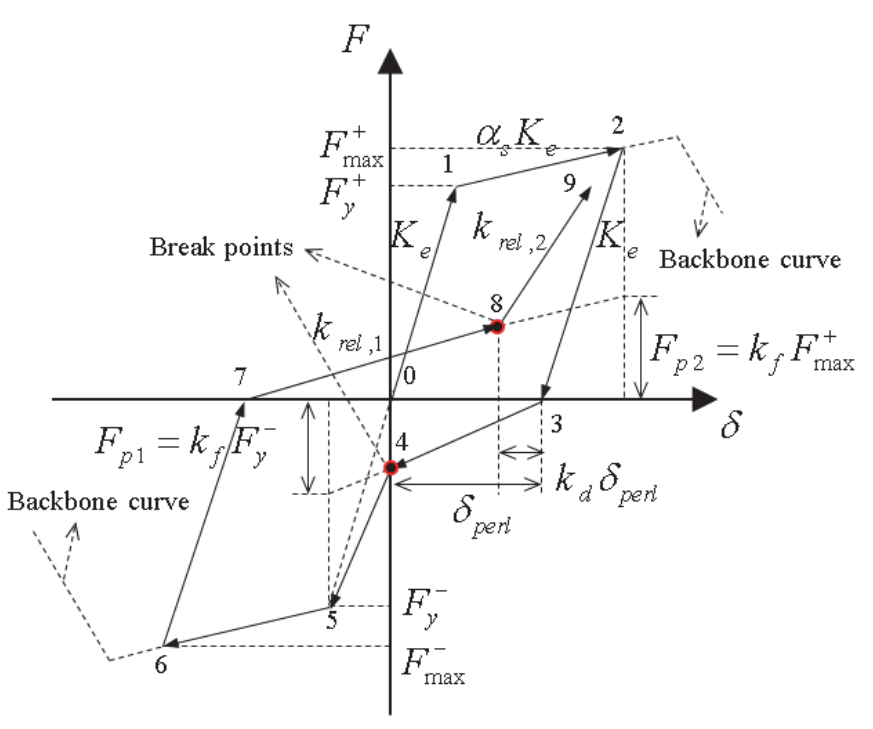

(b) Hysteretic model

Figure 8-18 IKP model (Ibarra et al., 2005).

The IKP model includes four models for deterioration, namely, basic-strength, postcapping strength, unloading stiffness, and accelerated stiffness, as drawn in Figure 8-19. Details are available in Ibarra et al. (2005).

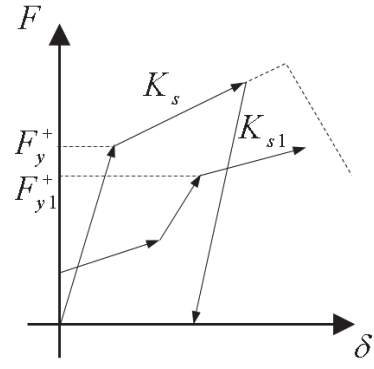

(a) Basic strength

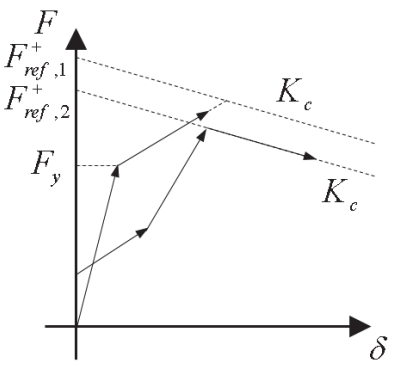

(b) Post-capping strength

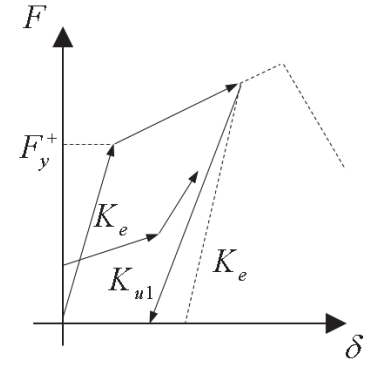

(c) Unloading-stiffness

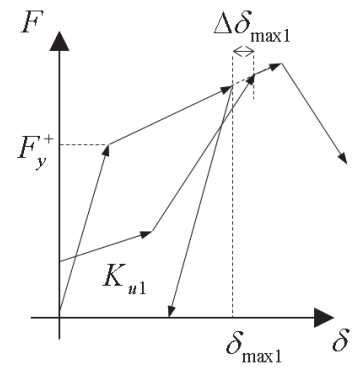

(d) Accelerated stiffness

Figure 8-19 IKP deterioration models.

Ibarra et al. use an energy-based parameter to determine the rate deterioration. This parameter is based on the hysteretic energy dissipated in cyclic loading, and is calculated as:

$$
\beta=\left(\frac{E_{i}}{E_{t}-\sum_{i=1}^{n} E_{j}}\right)^{d}
$$

where $E_{i}$ is the hysteretic energy dissipated in excursion $I ; E_{t}$ is the inherent hysteretic energy dissipation capacity equal to $\gamma F_{y} \delta_{y}$, where $F_{y}$ and $\delta_{y}$ are the yield force and the coresponding displacement, calculated by an equal-energy method; $\sum E_{j}$ is the sum 
of the hysteretic energy dissipated in all previous cycles, and $d$ is the rate of deterioration. Each time the sign of the force changes, the value of $\beta_{i}$ is re-calculated and the parameters of the chosen deterioration model are updated. The value of $\beta_{i}$ is calculated twice in each displacement cycle, once for loading in the positive direction and again for loading in the negative direction. The parameter $\beta_{i}$ takes values within the interval $0<\beta_{i} \leq 1$.

A reasonable range for $d$ in Equation 8-9 is between 1.0 and 2.0. For cyclic loading at a constant displacement amplitude, $d=1$ identifies a constant rate of deterioration and $d=2$ identifies an increasing rate of deterioration under cyclic loading.

Analysis of force-displacement relationships developed from data from tests of RC walls showed that IKP model did not capture the measured pre-peak strength and unloading branches of response. Further, the definition of $\beta_{i}$ per Equation 8-9 did not necessarily lead to increased deterioration with an increasing number of cycles for shear-critical RC walls. Modifications to the IKP model are made here for simulating the response of shear-critical RC walls: (1) a tri-linear relationship for the pre-peak-strength response that is characterized by concrete cracking, yielding of reinforcement, and crushing of concrete; (2) a bilinear curve for the unloading branch; (3) a tri-linear curve for the re-loading branch; and (4) an updated equation for the deterioration parameter:

$$
\beta_{i}=\left(\frac{\sum_{i=1}^{n} E_{j}}{E_{t}}\right)^{d}
$$

These modifications to the backbone curve and hysteretic force-displacement relationship of the IKP model are presented in Figure 8-20. Figure 8-20a presents the proposed cyclic backbone curve. Figure $8-20 \mathrm{~b}$ presents the backbone curve of the IKP model (dotted line), the hysteretic force-displacement relationship proposed by Ibarra et al. (solid line), and the modified IKP (MIKP) cyclic force-displacement relationship (dashed line).

The unloading and reloading branches of the MIKP force-displacement relationship consists of two segments with stiffness $k_{u n l, 1}^{\prime}$ and $k_{u n l, 2}^{\prime}$, and three segments with stiffness $k_{r e l, 1}^{\prime}, k_{r e l, 2}^{\prime}$, and $k_{r e l, 3}^{\prime}$, respectively. The first unloading path targets a force equal to a fraction, $\zeta$, of the maximum force, $F_{\max }$, achieved in the same quadrant in the previous cycle. The first control point of the reloading branch (points $a$ and $d$ in Figure 8-20b) on the force axis is established using the coordinate of the residual displacement in the previous cycle and stiffness $k_{\text {unl }, l}^{\prime}$. The calculated force at this point should not exceed $k_{f} F_{\max }$ (see Figure 8-20b). The second part of the reloading branch targets the second control point of the reloading branch (points $b$ and $e$ in Figure 8-20b) with the displacement and force coordinates of $\left(1-k_{d}\right) \delta_{p e r l}$ and $k_{f} F_{\max }$, 
respectively. The third part of the reloading branch targets the maximum displacement achieved in the previous cycle.

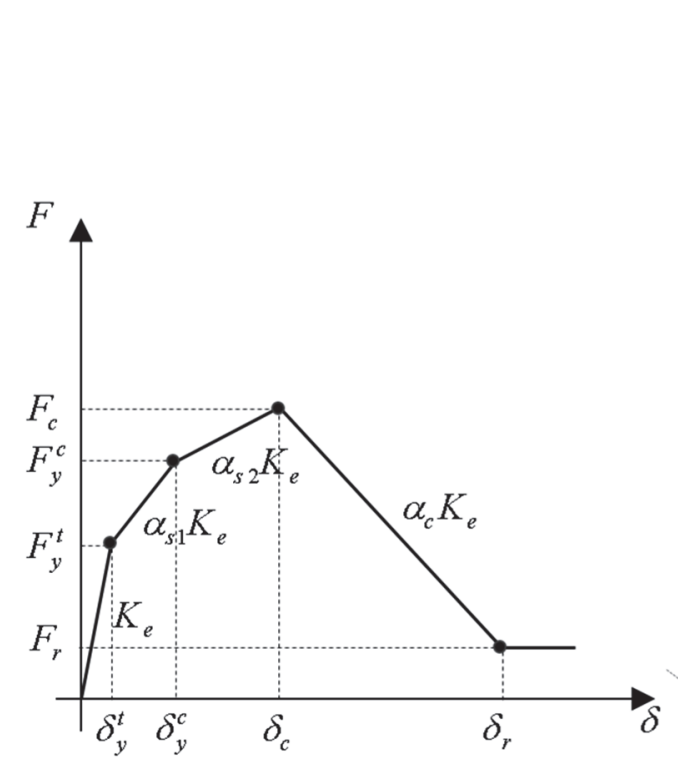

(a) Backbone curve

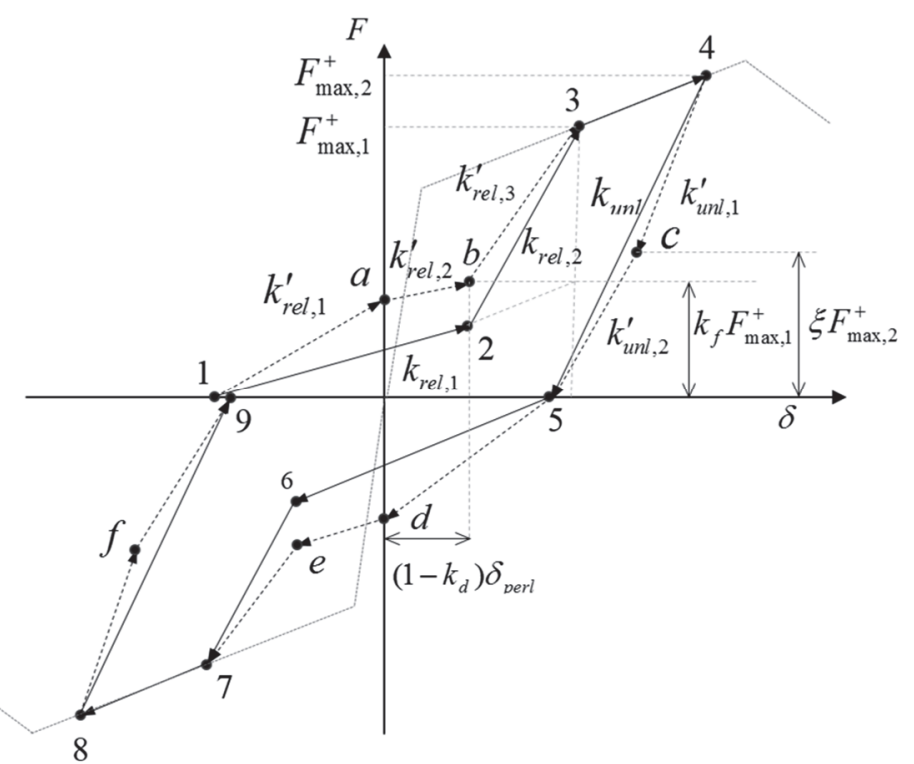

(b) Cyclic response

Figure 8-20 Modified backbone curve and hysteretic force-displacement relationship of the IKP model.

In this study, the cyclic backbone curve derived by digitizing experimental results of $63 \mathrm{RC}$ walls is used as input to the MIKP model in MATLAB. The pinching, deterioration, and rate parameters calculated for each wall are also used as input for the MIKP predictions of cyclic response. Basic strength and post-capping strength deterioration are not considered because the starting point for these simulations is a cyclic backbone curve and not a monotonic envelope curve.

Appendix B.3 presents the values of the pinching $\left(k_{f}, k_{d}, \zeta\right)$ and deterioration $\left(\gamma_{u n l, 1}\right.$, $\left.\gamma_{u n l, 2}, \gamma_{a}\right)$ parameters calibrated from the digitized force-displacement relationships. Appendix B.3 shows that the design variables affect the values of pinching parameters $k_{f}$ and $k_{d}$, and deterioration parameters, $\gamma_{u n l, 1}, \gamma_{u n l, 2}, \gamma_{a}$, but have no impact on $\zeta$.

The data from $63 \mathrm{RC}$ wall tests presented in Appendix B.3 for rectangular, flanged, and barbell walls, are re-binned to calculate the pinching and deterioration parameters, according to Table 8-2. Data from tests with specimens that fall outside the domains below were not included in the analysis. Table 8-4 summarizes results by wall geometry and data bin $(1,1 \mathrm{a}, 2,2 \mathrm{a})$, including mean (average) values, standard deviation, and coefficient of variation. 


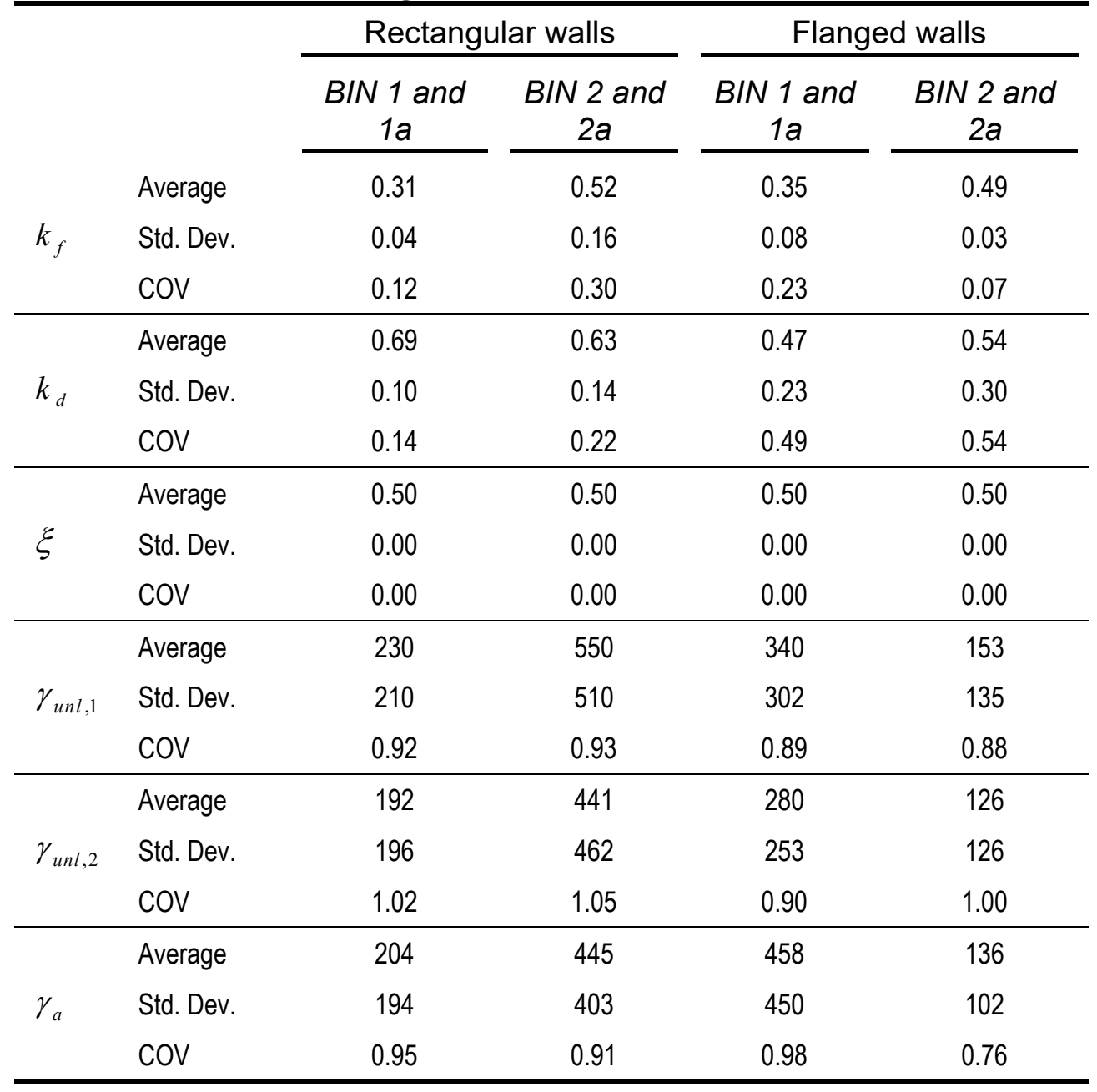

On the basis of the data of Table 8-4, and recognizing the significant variability and uncertainty in the data, and introduced here by assigning discrete values and setting aside functional forms, the cyclic response of Figure 8-20b can be established using the following test-independent values for the pinching and deterioration parameters:

- $\quad$ Pinching parameter, $k_{f}$

$\circ \quad k_{f}=0.3$ for rectangular and flanged walls, aspect ratio less than 1.0, and all axial compressive force ratios

$\circ k_{f}=0.5$ for rectangular and flanged walls, aspect ratio between 1.0 and 2.0, and all axial compressive force ratios

- Pinching parameter, $k_{d}$

○ $k_{d}=0.65$ for rectangular walls, aspect ratio between 0 and 2.0, and all axial compressive force ratios

$\circ k_{d}=0.50$ for flanged walls, aspect ratio between 0 and 2.0, and all axial compressive force ratios 
- Pinching parameter, $\xi$

o $\xi=0.50$ for rectangular and flanged walls, aspect ratio between 0 and 2.0, and all axial compressive force ratios

- Deterioration parameter, $\gamma_{u n l, l}$

o $\gamma_{u n l, l}=230$ for rectangular walls, aspect ratio less than 1.0 , and all axial compressive force ratios

- $\gamma_{u n l, l}=550$ for rectangular walls, aspect ratio between 1.0 and 2.0, and all axial compressive force ratios

- $\gamma_{u n l, l}=340$ for flanged walls, aspect ratio less than 1.0, and all axial compressive force ratios

- $\gamma_{u n l, l}=150$ for flanged walls, aspect ratio between 1.0 and 2.0, and all axial compressive force ratios

- Deterioration parameter, $\gamma_{u n l, 2}$

- $\gamma_{u n l, 2}=190$ for rectangular walls, aspect ratio less than 1.0, and all axial compressive force ratios

- $\gamma_{u n l, 2}=450$ for rectangular walls, aspect ratio between 1.0 and 2.0, and all axial compressive force ratios

- $\gamma_{u n l, 2}=280$ for flanged walls, aspect ratio less than 1.0, and all axial compressive force ratios

o $\quad \gamma_{u n l, 2}=130$ for flanged walls, aspect ratio between 1.0 and 2.0, and all axial compressive force ratios

- Deterioration parameter, $\gamma_{a}$

- $\gamma_{a}=200$ for rectangular walls, aspect ratio less than 1.0, and all axial compressive force ratios

- $\gamma_{a}=450$ for rectangular walls, aspect ratio between 1.0 and 2.0, and all axial compressive force ratios

- $\gamma_{a}=460$ for flanged walls, aspect ratio less than 1.0 , and all axial compressive force ratios

- $\gamma_{a}=140$ for flanged walls, aspect ratio between 1.0 and 2.0, and all axial compressive force ratios

The recomended values of the pinching and deterioration parameters are used here to simulate the hysteretic response of the $63 \mathrm{RC}$ walls, with aspect ratio between 0.5 and 2 , reinforcement ratio between $0.2 \%$ and $1 \%$, and low axial loads. Figure $8-21$ presents information on panel 4 of Alexander et al. (1973): the force-displacement relationship digitized from test data is shown in Figure 8-21a, the MIKP-predicted 
relationship using test-specific pinching and deterioration parameters in Figure $8-21$ b, and the cyclic relationship predicted using the best estimate cyclic backbone curves from Section 8.5 and the recommended test-independent values of the pinching and deterioration parameters, in Figure 8-21c. The predictions of Figure 8-21 use the recorded loading history as input. Comparisons for all $63 \mathrm{RC}$ walls are presented in Appendix D. In summary, the MIKP model, with a test-specific backbone curve and calibrated factors for deterioration, reasonably simulates the cyclic response of the RC walls: the post-peak pinched behavior, the continuity of the response in the pre- and post-peak-strength regions, and the unloading branch of the cyclic force-displacement relationship are captured well.

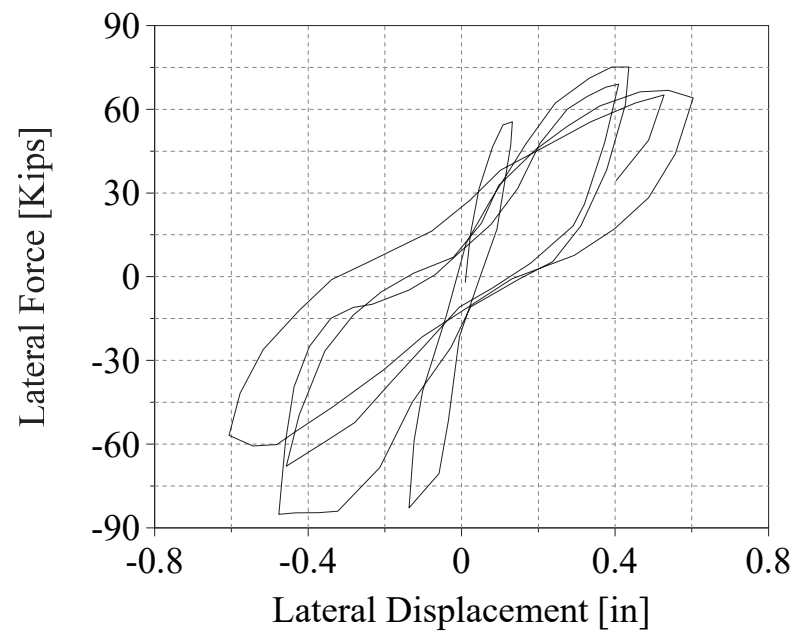

(a)

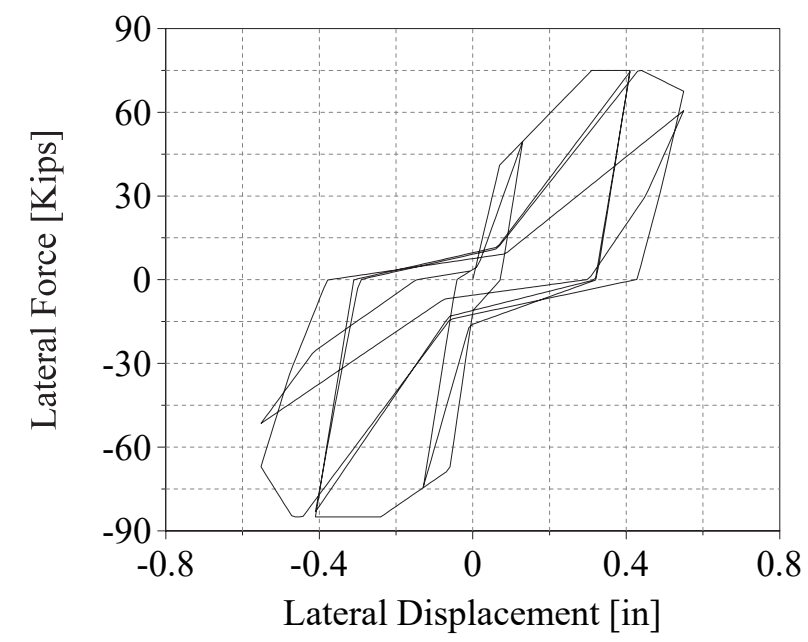

(b)

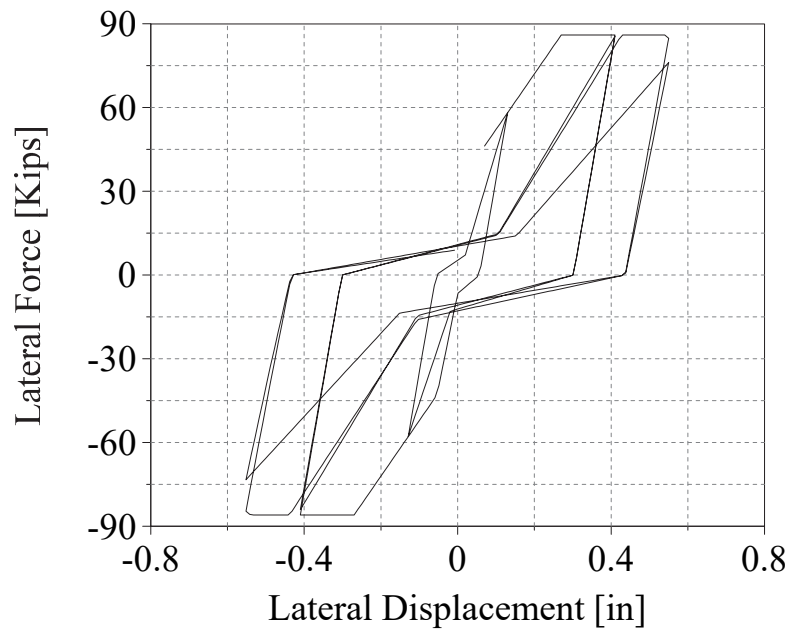

(c)

Figure 8-21 Comparison of (a) test data, (b) MIKP prediction, and (c) proposed-model prediction for Alexander et al. (1973) panel 4. 


\section{Chapter 9}

\section{Reinforced Masonry Walls}

\subsection{Overview of Geometry and Behavior}

\subsubsection{Wall Configurations in Buildings}

Reinforced masonry shear walls resist lateral forces from wind and seismic actions and carry gravity loads under normal service conditions. They may also serve architectural functions as building envelopes, internal partitions, or core walls enclosing stairwells and elevator shafts in reinforced concrete or steel frame structures.

Reinforced masonry shear walls are most commonly constructed of hollow concrete or clay units, which can be fully or partially filled with grout. Vertical reinforcing bars are placed in the hollow cells of these units, and horizontal bars are placed in bond-beam units that can be U-blocks (for lintels) or regular blocks with part or all of their end webs removed to allow bar placement. This type of wall is very similar to reinforced concrete shear walls, except that masonry walls normally do not have special confined boundary elements to enhance their flexural ductility because of the practicality in construction. Partially grouted walls have only the reinforced cells filled with grout.

Reinforced masonry walls designed according to current codes, ASCE/SEI 7-10, Minimum Designs Loads for Buildings and Other Structures, (ASCE, 2010) and TMS 402/ACI 530/ASCE 5, Building Code Requirements for Masonry Structures, (TMS, 2015) can be categorized into three types: ordinary, intermediate, and special. In addition to other detailing requirements, special walls, which are mandatory for Seismic Design Category (SDC) D or above, must have the vertical and horizontal reinforcing bars spaced at no more than 48 inches on center. Because of the close bar spacing, special walls are often fully grouted, which has been the common practice in California. However, partial grouting is the prevalent construction practice in most areas of the United States, where ordinary walls are often permitted. In partially grouted masonry walls, horizontal reinforcement usually only consists of wire mesh embedded in masonry bed joints. Another type of masonry construction that can be found in older buildings is fully grouted cavity walls, which consist of two wythes of solid or perforated brick units sandwiching a grouted, reinforced collar joint. This type of structures generally did not perform well in earthquakes, as shown in the 1994 earthquake in Northridge, California. Laboratory test data on the behavior of partially grouted walls is scarce, and that for cavity walls is even more difficult to 
find. Hence, this chapter focuses predominantly on the behavior and modeling of fully grouted masonry walls constructed of hollow units, but the differences in behavior and modeling for partially grouted walls are also discussed. Cavity walls are not covered.

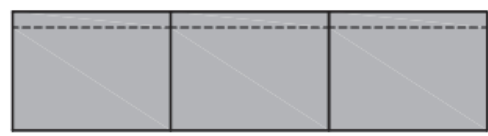

(a) Squat, shear-dominated wall, showing control joints

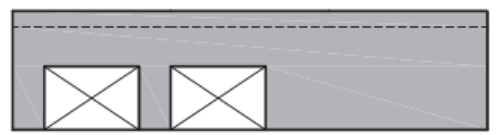

(b) Single line of resistance with disparate wall element stiffnesses

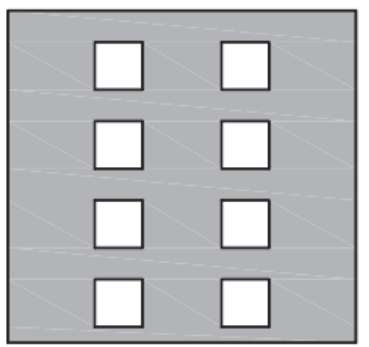

(c) Perforated wall - beam governed

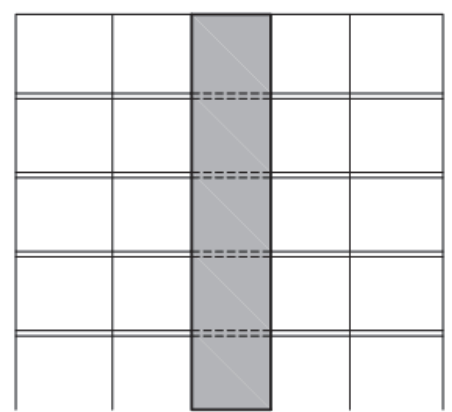

(e) Cantilever Wall

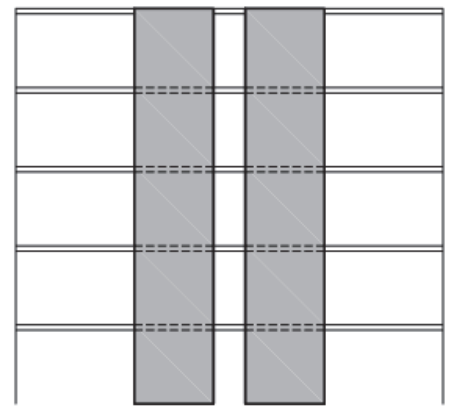

(f) Slab-coupled Wall
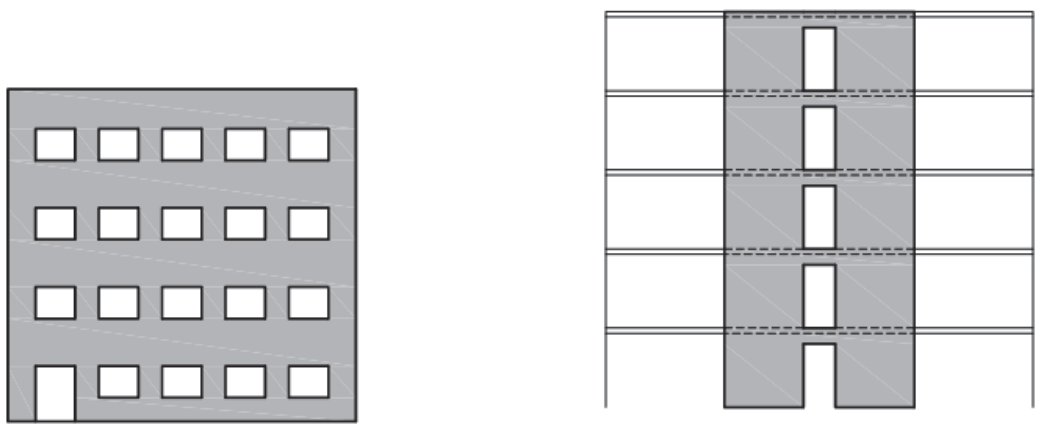

(d) Perforated wall - pier governed

(g) Beam-coupled Wall

Figure 9-1 Configurations of reinforced masonry wall systems (NIST, 2014).

Depending on the intended functions and architectural needs, masonry wall systems may have a number of possible configurations, as shown in Figure 9-1. Figures 9-1a through 9-1d show typical wall systems that also function as building envelopes in single- and multi-story masonry buildings. Single-story walls can have squat or slender wall components as shown in Figure 9-1b. Walls with configurations shown in Figure 9-1c and Figure 9-1d are called perforated walls, which are characterized by multiple small openings. These walls consist of piers, the components spanning 
vertically between openings, and beams. Reinforced masonry cantilever walls, as shown in Figure 9-1e, can be found in frame structures as exterior walls or core walls enclosing elevator shafts or stairways. It is not uncommon that a reinforced masonry building has multiple shear walls coupled by horizontal diaphragms, as shown in Figure 9-1f, or by masonry beams in addition to horizontal diaphragms, as shown in Figure 9-1g. These are coupled walls, whose behavior depends significantly on the strength and ductility of the coupling elements.

Masonry walls can be subjected to in-plane as well as out-of-plane loads from wind and seismic actions. However, this chapter focuses only on the strength and ductility of walls subjected to in-plane seismic forces. The performance of a wall system subjected to in-plane seismic forces depends on its geometric configuration, which defines the boundary conditions and aspect ratios of the wall components that constitute the system, the amount of reinforcement and design details, and the interaction of the wall components with each other and with horizontal diaphragms. Wall components can be piers and beams in a perforated wall, wall segments separated by control joints in a single-story building, or wall segments spanning the height of each story in a multi-story building. Behavior of different types of wall systems, and possible load-resistance and failure mechanisms of wall components are discussed in the following section.

\subsubsection{Behavior of Wall Systems}

Based on their anticipated structural behavior, wall systems shown in Figure 9-1 can be broadly classified into three main categories, namely, cantilever walls, coupled walls, and perforated walls. Cantilever walls can exhibit a relatively ductile flexuredominated behavior. The failure mechanism and associated ductility of a wall depend on the level of axial load the wall is subjected to, the shear-span ratio of the wall, and the amount of vertical and horizontal reinforcement (NIST, 2014). However, in a coupled wall, wall components may have their shear-span ratios significantly reduced by the coupling actions, and are thus vulnerable to brittle sheardominated behavior. The same is true for the piers and beams in a perforated wall. These components generally have low shear-span ratios.

The distinction between cantilever walls and coupled walls is often as not clear-cut as one may expect. Squat wall components separated by control joints in a single-story building, such as that shown in Figure 9-1a, can be considered cantilever walls provided the roof diaphragm is weak and not able to develop significant shear to restrain the rotation of the top end of the walls. Horizontal diaphragms in a multistory reinforced masonry building can be constructed of cast-in-place reinforced concrete slabs, precast hollow-core planks with cast-in-place topping, or metal decks with or without concrete topping. They may or may not develop strong coupling actions depending on the design and detailing of the slabs. A study by Seible et al. 
(1994) has shown that diaphragms constructed of hollow-core planks, with reinforced concrete topping, spanning parallel to the walls, could experience brittle shear failure if the concrete element between two planks above a door opening does not have transverse reinforcement. In that case, the coupling actions of the slabs cannot be relied upon and the walls shown in Figure 9-1f can be treated as cantilever walls. However, if the concrete elements above door openings have adequate transverse reinforcement, the slabs can behave as ductile coupling elements. Masonry beams in a wall system, like that shown in Figure 9-1g, can introduce strong coupling actions. This was observed in a full-scale, three-story, special reinforced masonry wall system tested on a shake table (Stavridis et al., 2016). The walls were designed according to ASCE/SEI 7-10 and TMS 402/ACI 530/ASCE 5 (TMS, 2011), and were securely connected to horizontal diaphragms, which were constructed of 6 inch thick hollowcore planks running parallel to the walls with 3 -inch concrete topping. The system had masonry beams above door openings for architectural purposes. The walls were designed as cantilever walls based on the detail that the horizontal reinforcement in the beams was debonded in the vicinity of the control joint at each end of the beam above each door opening. In spite of this, strong coupling actions were developed, which eventually resulted in the shear failure of the two exterior walls in the first story, as shown in Figure 9-2.

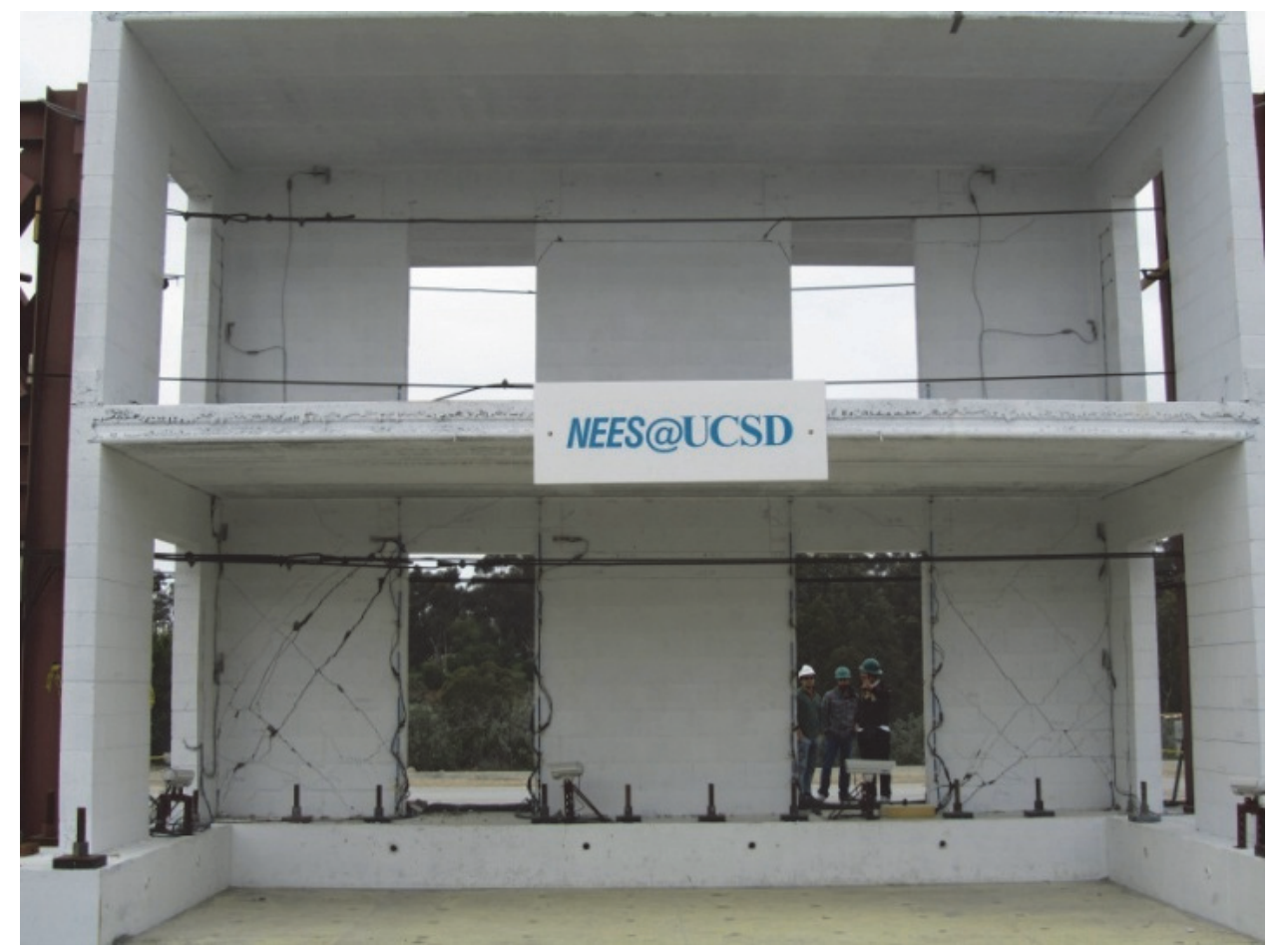

Figure 9-2 Shear failure of reinforced masonry walls in a three-story building tested on a shake table (Stavridis et al., 2016).

In a perforated wall, the piers and beams between openings normally have low shearspan ratios, and therefore will very likely exhibit brittle shear behavior. Figure 9-3 
shows brittle shear failure of piers adjacent to a window opening in a two-story reinforced masonry structure tested on a shake table (Mavros et al., 2016). However, failures may occur either in the beams or in the piers, depending on whether it has strong piers with weak beams, as in Figure 9-1c, or weak piers with strong beams, as in Figure 9-1d.

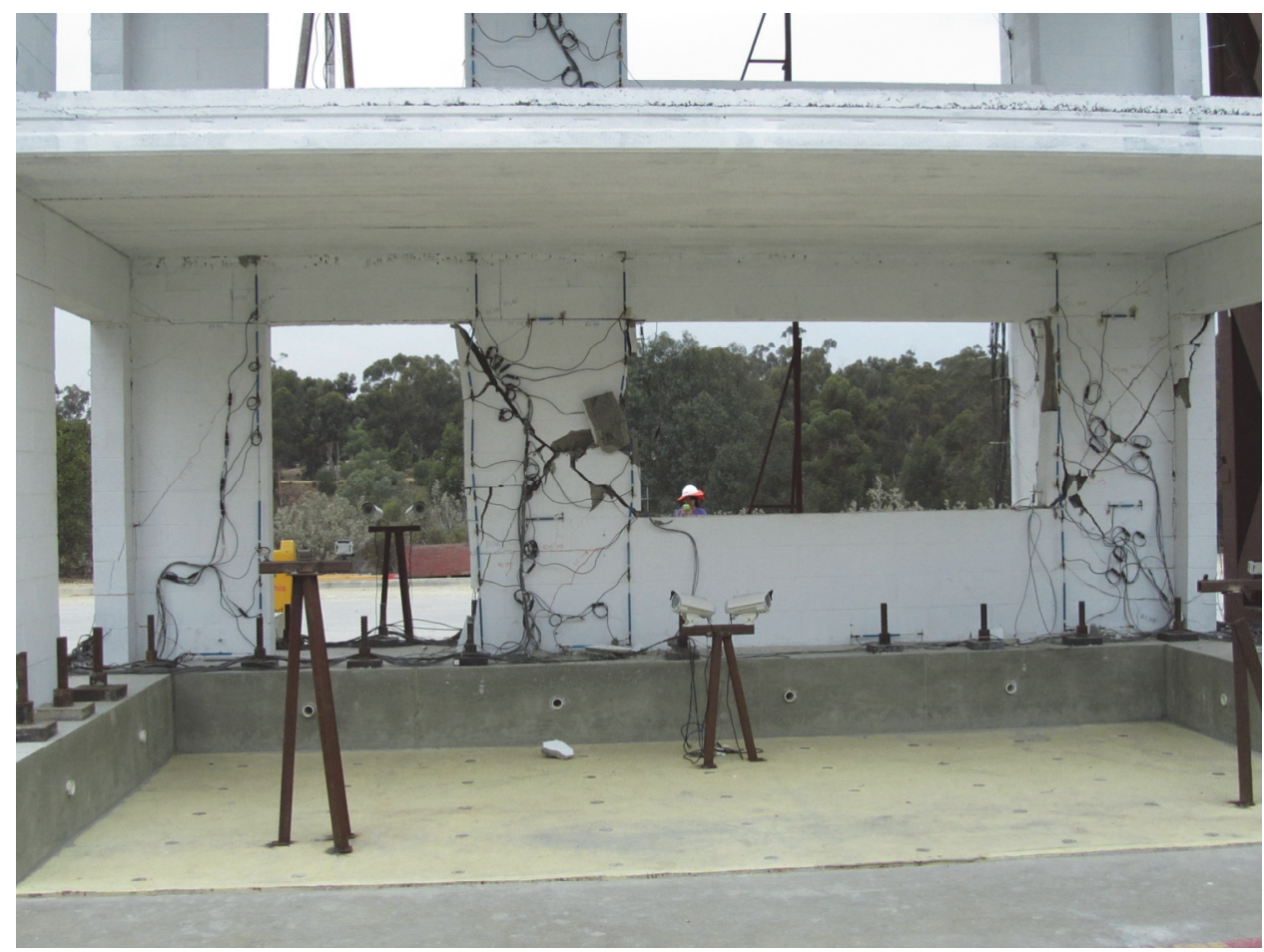

Figure 9-3 Shear failure of reinforced masonry piers in a two-story building tested on a shake table (Mavros et al., 2016).

To assess the seismic performance of a reinforced masonry wall system, it is important that the analytical model has the capabilities to capture the system behavior as well as the nonlinear behavior and failure mechanisms of the wall components.

\subsubsection{Behavior of Wall Components}

The ultimate strength and post-peak behavior of a reinforced masonry wall component can be governed by flexure, diagonal shear, and/or shear sliding.

A flexure-dominated mechanism may include flexural cracking, the yielding, buckling, and fracture of the vertical reinforcement, masonry crushing at wall toes, the failure of lap splices, and the out-of-plane buckling of a wall as discussed in FEMA 306 (FEMA, 1998). Walls dominated by flexure can exhibit a relatively ductile behavior. Figure 9-4 shows the in-plane lateral load-vs.-displacement hysteresis curves obtained from a reinforced masonry cantilever wall tested by Sherman (2011). As can be seen from the hysteresis curves, the wall had a relatively ductile behavior. In general, a wall with a higher axial compressive load and/or a 
higher amount of vertical reinforcement will have less ductile flexural behavior (NIST, 2014). Unlike reinforced concrete walls, masonry walls normally do not have confined boundary elements. Therefore, the extreme vertical reinforcing bars in a masonry wall are vulnerable to buckling because severe spalling may occur in the surrounding masonry, as shown in Figure 9-5. After a buckled bar has been subjected to a few cycles of straightening and bending as the wall has been pushed back and forth with cyclic load reversals, bar fracture may soon follow. This will lead to severe strength degradation in the wall.

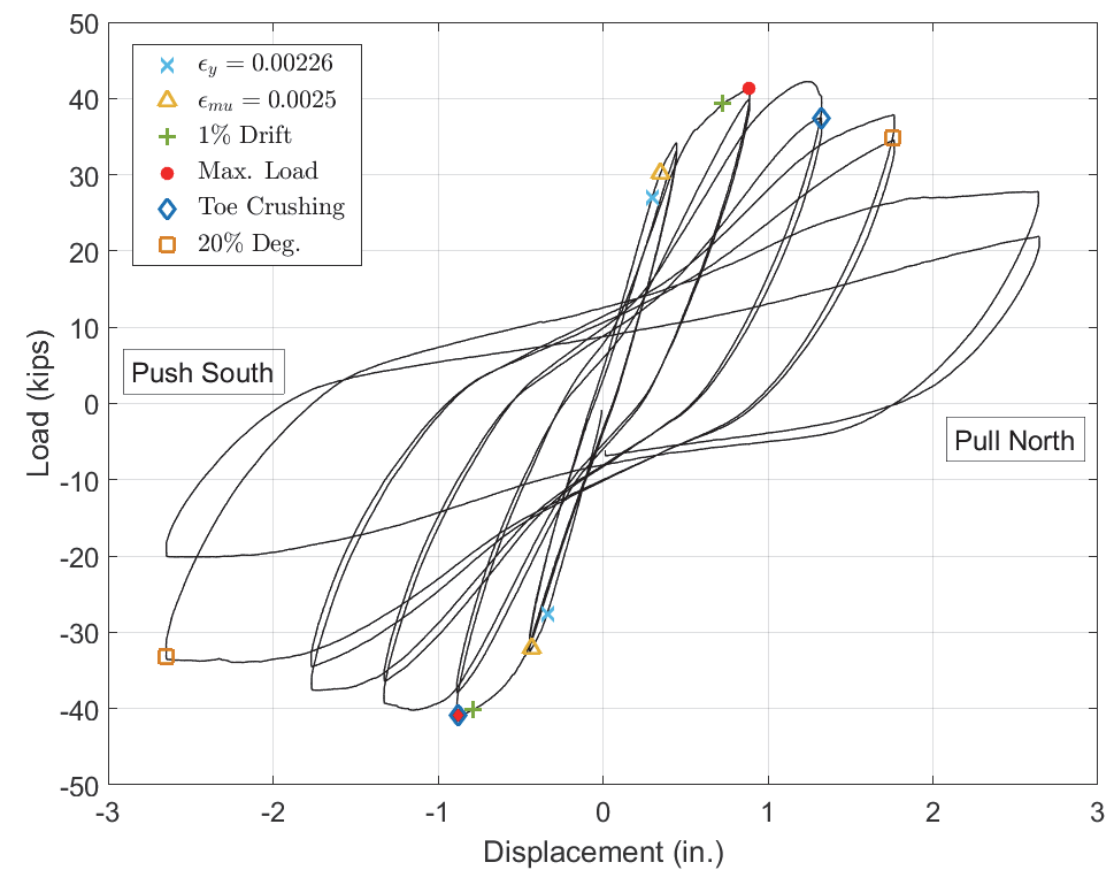

Figure 9-4 In-plane lateral load-vs.-displacement curves for a flexuredominated wall (WSU-W-1A tested by Sherman, 2011).
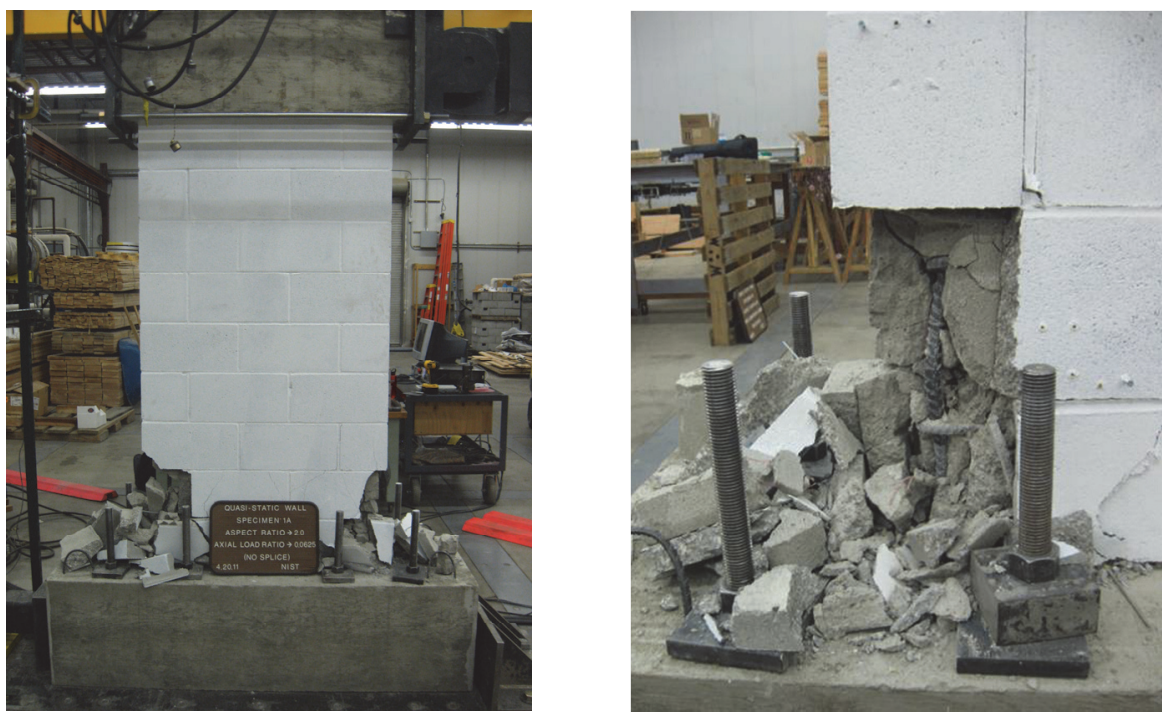

Figure 9-5 Crushing and bar buckling in a flexure-dominated wall (WSU-W-1A tested by Sherman, 2011). 
The behavior of walls dominated by diagonal shear cracks is often very brittle as shown by the lateral load vs. displacement hysteresis curves in Figure 9-6 for two such walls (Ahmadi, 2012).

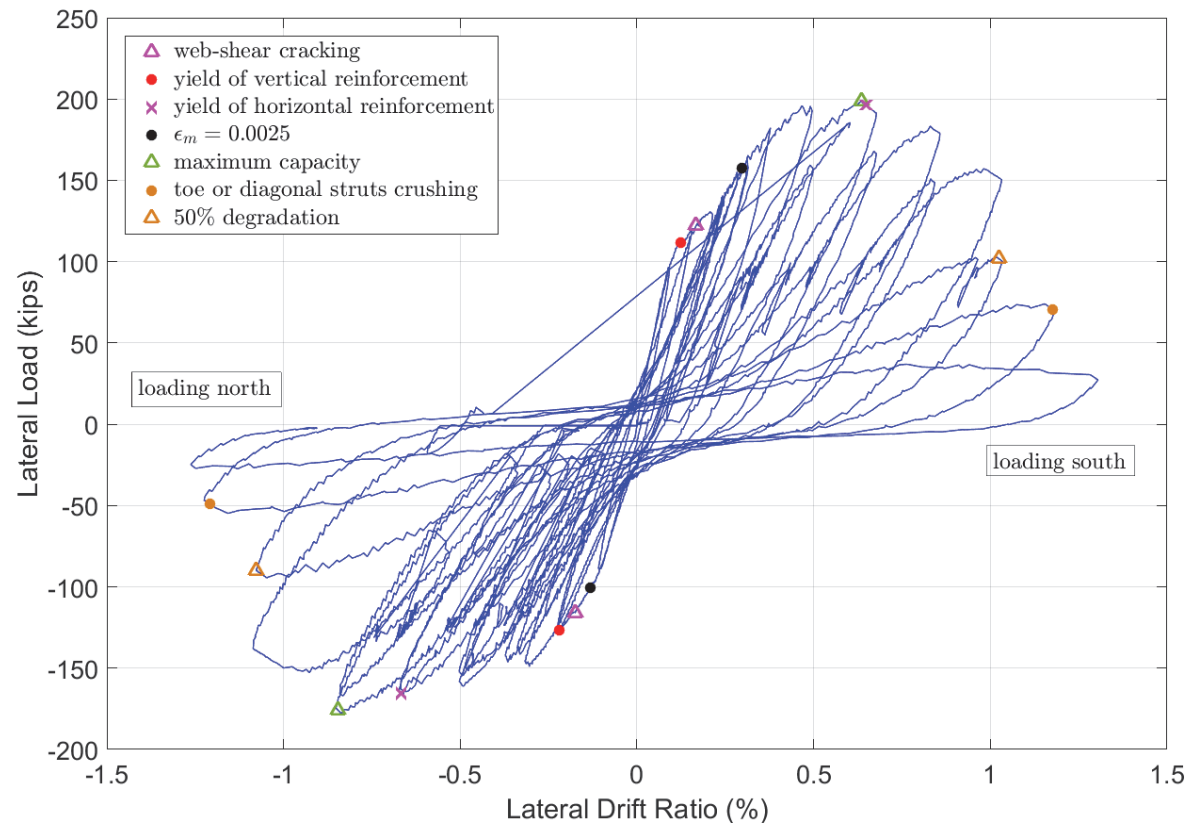

UT-PBS-01 (vert. reinf. \#6@8 in.; horiz. reinf. \#4@8 in.)

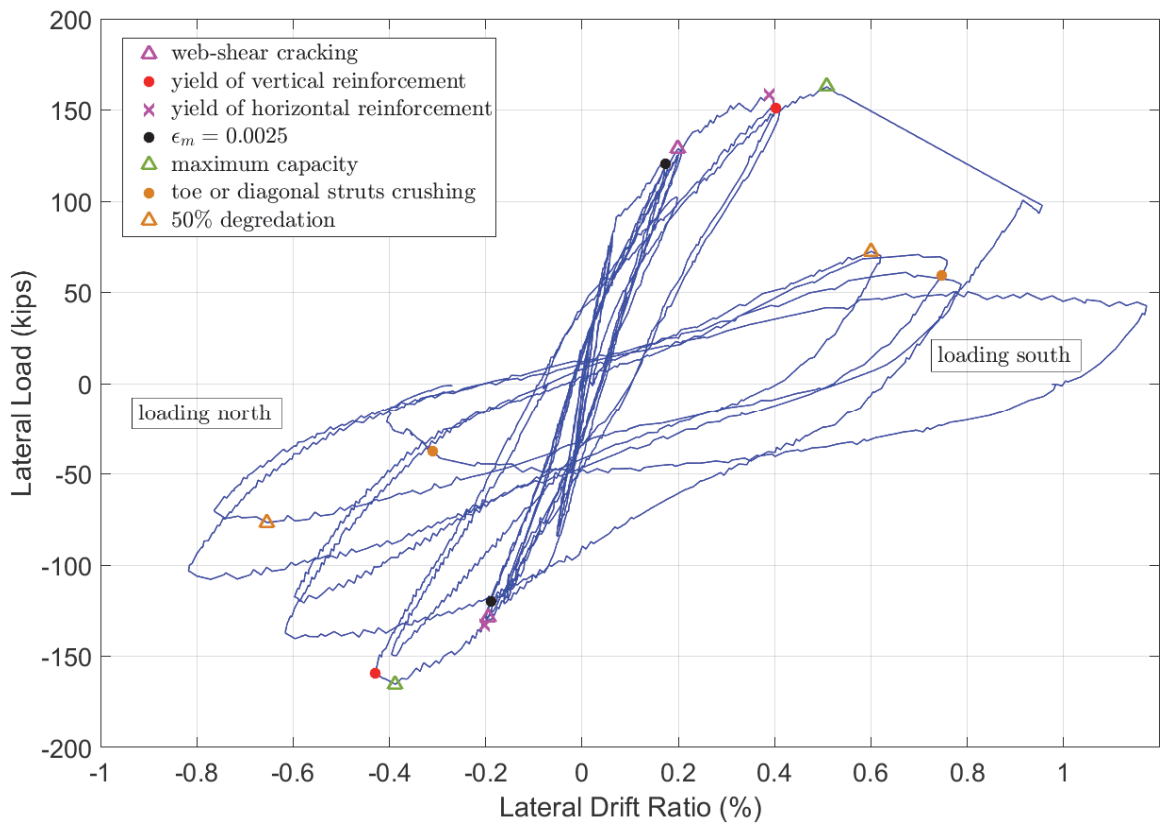

UT-PBS-02 (vert. reinf. \#6@16 in.; horiz. reinf. \#4@16 in.)

Figure 9-6 In-plane lateral load vs. displacement curves for diagonal sheardominated walls (Ahmadi, 2012).

The failure of these walls is normally preceded by the development of diagonal shear cracks, which at the beginning may be restrained from opening by the horizontal shear reinforcement. However, once the diagonal shear capacity has been reached, 
dominant shear cracks will open, as shown in Figure 9-7, and the lateral resistance of the wall may drop rapidly in a sudden manner. This may be accompanied by the tensile fracture or anchorage failure of the horizontal shear reinforcement. Limited ductility can be developed by a shear-dominated wall if sufficient horizontal reinforcement is present as shown by the first plot in Figure 9-6. The vertical reinforcement may also contribute to the shear resistance through the bending resistance of the bars crossing the diagonal cracks. This is referred to as the dowel action, and is normally small as compared to that provided by the horizontal reinforcement. Furthermore, if the top of the wall is restrained from vertical displacement, diagonal tension can develop in the vertical reinforcement, crossing the cracks as the diagonal cracks open. The shear strength of a wall depends on the wall aspect ratio, the strength of the masonry, the quantity of the shear reinforcement, and the applied axial compressive load. Walls with low shear-span ratios and insufficient amount of shear reinforcement will most likely exhibit shear-dominated behavior. A significant portion of the shear resistance of a wall can be provided by the shear resistance of masonry in the compression toe of the wall. Because of this, diagonal shear failure may occur after the flexural strength of a wall has been reached and the masonry in the compressed toe has been crushed. This is called flexure/shear failure (Shing et al., 1991; FEMA, 1998), and can significantly impair the ductility of a flexure-dominated wall.

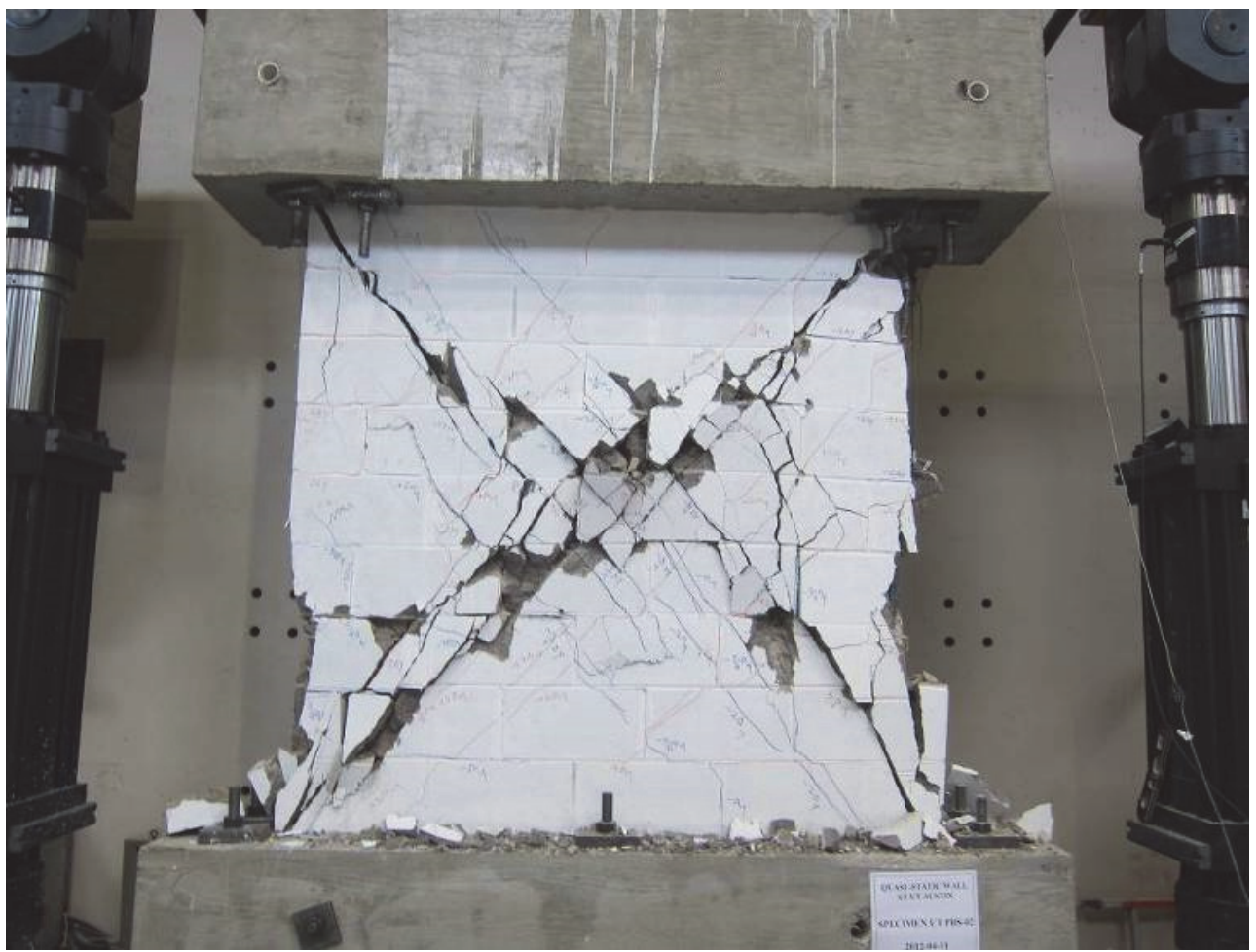

Figure 9-7 Failure of a shear-dominated wall (UT-PBS-02 tested by Ahmadi, 2012). 
Shear sliding may occur along the base of a wall or in a bed joint a few courses away from the base. The sliding shear resistance of a wall depends on the surface roughness, the clamping force developed by the vertical reinforcement crossing the shear plane, the applied axial compressive load, and, to a lesser extent, the dowel action of the vertical reinforcement. It can be calculated as a shear friction resistance. The 2016 Edition of TMS 402 (TMS, 2016) provides formulas to calculate the shear friction resistance in a wall. Walls with a low aspect ratio and low axial compressive load have a high tendency to develop shear sliding. It may occur early, or after the peak flexural resistance of a wall has been reached and the sliding shear resistance has been weakened by the flexural damage at the wall base. The latter is called flexure/sliding shear failure (FEMA, 1998; Shing et al., 1991). Walls dominated by shear sliding can exhibit a gradual degradation of the lateral load resistance, as shown in Figure 9-8. Shear sliding can exacerbate masonry crushing in the vicinity of the sliding plane, cause the damage of masonry due to the dowel action, and lead to the shear fracture of the vertical bars crossing the shear plane. Significant base sliding may also induce severe damage to other structural and nonstructural components, such as walls perpendicular to the direction of sliding, and may jeopardize the stability of the gravity frame that is present in the structure.

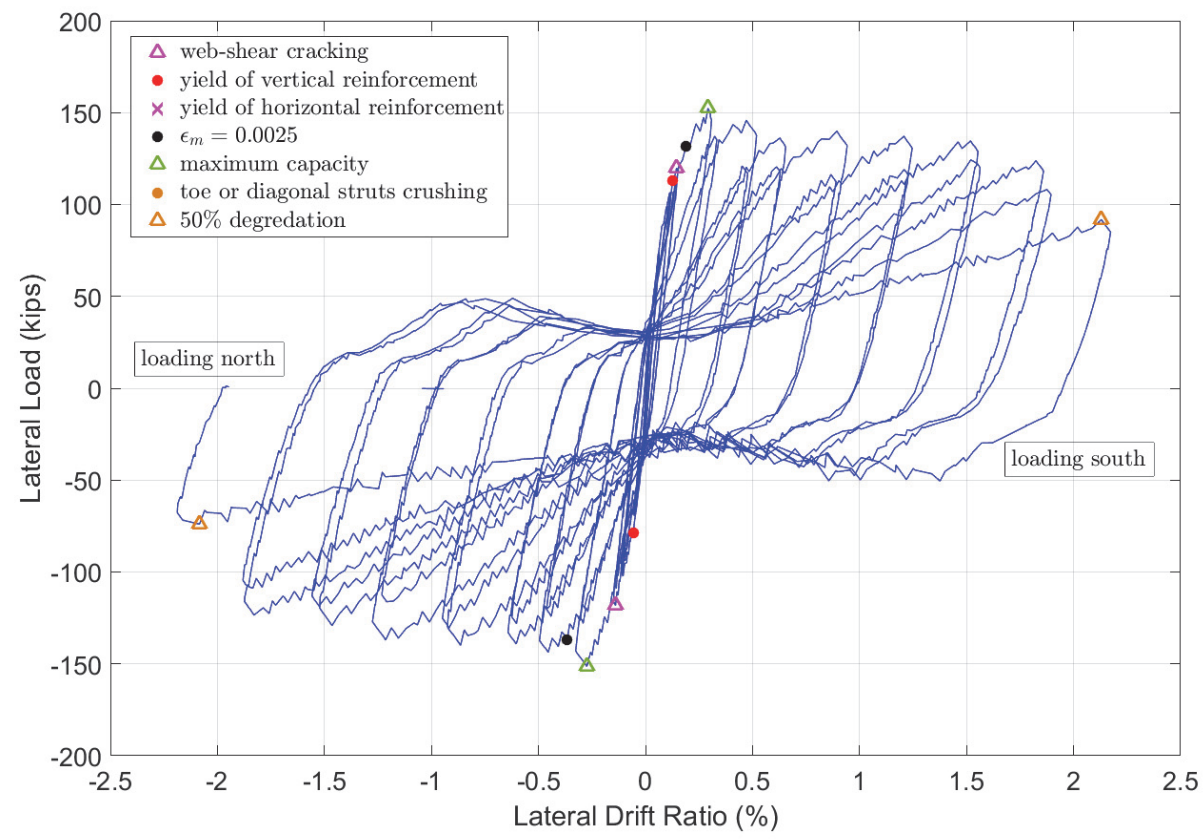

Figure 9-8 In-plane lateral load vs. displacement curves for a wall dominated by shear sliding (UT-PBS-05 tested by Ahmadi, 2012).

\subsection{Summary of Design Code Provisions}

Since 2003, after the introduction of the International Building Code (IBC), the design of masonry structures gradually started to follow Building Code Requirements 
for Masonry Structures developed by the Masonry Standards Joint Committee (MSJC), which is currently published as TMS 402. Currently, the IBC (ICC, 2012) and ASCE/SEI 7-10 reference TMS 402 for masonry design. Prior to this transition, the Uniform Building Code (UBC) was used predominantly for masonry design in the western United States. The last edition of the UBC was published in 1997, which had both allowable stress design (ASD) and strength design provisions for masonry. In the MSJC code (TMS 402), strength design was first introduced in 2002.

However, the move towards strength design has been slow, and ASD is still being widely used for masonry design. In recent years, the MJSC has made significant efforts to harmonize the two design approaches. To conform to the seismic design specifications in the IBC and ASCE/SEI 7, the 2002 MSJC code classifies reinforced masonry shear walls into three categories: special, intermediate, and ordinary, each with different prescriptive reinforcing requirements, and requires special walls to be used for SDC D or above. Reinforced masonry walls can be either fully or partially grouted; however, special walls are mostly fully grouted. The close spacing of reinforcing bars in these walls justifies full grouting from the construction efficiency standpoint. Older reinforced masonry shear wall structures not conforming to the special wall requirements, and ordinary and intermediate shear walls built in areas of low seismic hazards are mostly partially grouted. Studies (Minaie et al., 2010; Bolhassani, 2015) have shown that both the shear strength and the displacement capacity of partially grouted walls are in general inferior to those of fully grouted walls. Partially grouted walls with wider spacing of grouted cells tend to have worse performance. The 2013 Edition of TMS 402 (TMS, 2013) has introduced a reduction factor of 0.75 to the shear strength of partially grouted masonry walls. Prior to this, the shear strength of partially grouted walls was calculated with exactly the same formula as for fully grouted walls.

\subsection{Recommendations for Modeling}

The nonlinear behavior of a reinforced masonry wall structure can be modeled in a detailed manner using shell elements with a smeared-crack constitutive model for masonry, cohesive crack interface elements, and elastic-plastic bar elements for steel (Mavros, 2015; Mavros et al., 2015). Shell elements can intrinsically account for both the shear and flexural mechanisms of wall components and the behavior of panel zones, and can also model the response of a wall component to simultaneous in-plane and out-of-plane loads. Nevertheless, analysis using nonlinear shell elements normally requires significant model building and computational efforts. For this reason, frame models, in which wall components are represented by beamcolumn elements, are commonly used for the performance assessment of reinforced masonry wall structures. 
In a frame model, beam-column elements are used to represent the vertical and horizontal wall segments, while the dimensions of the segments and the panel zones connecting these segments can be simulated with rigid end zones specified for the beam-column elements. Examples of frame models representing different reinforced masonry wall configurations are shown in Figures 9-9 and 9-10.
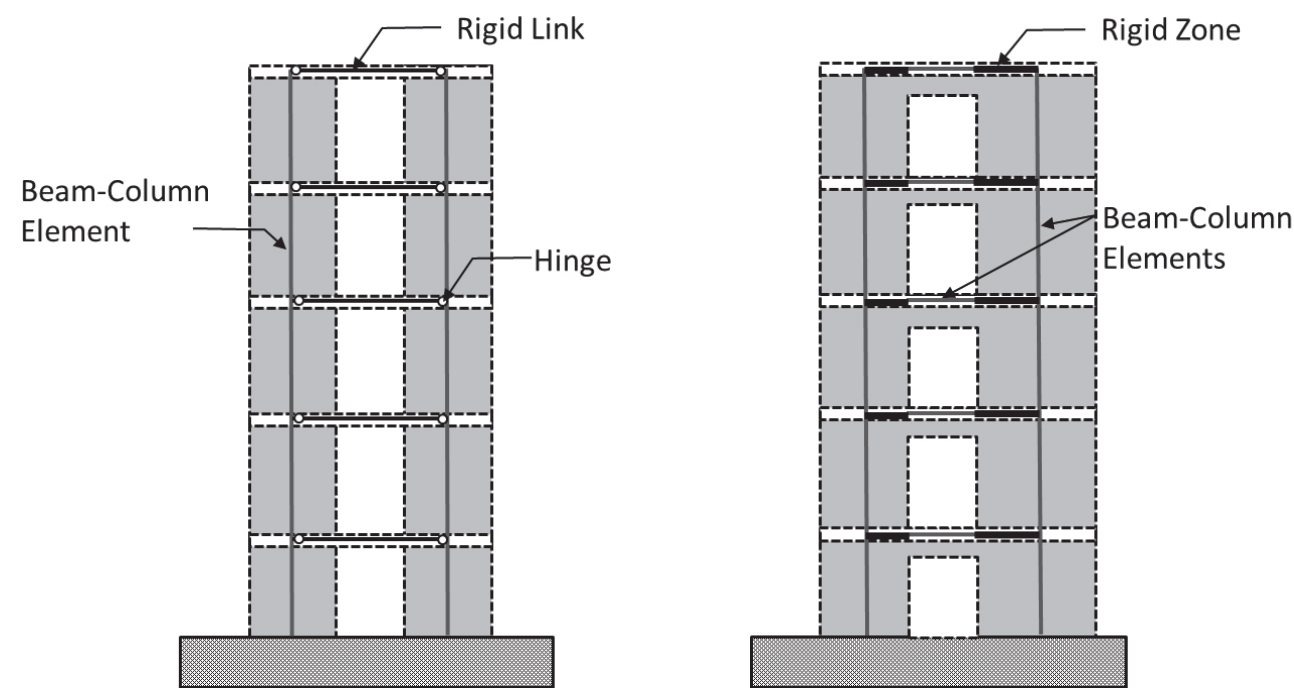

Figure 9-9 Frame models of cantilever and coupled walls (NIST, 2014).
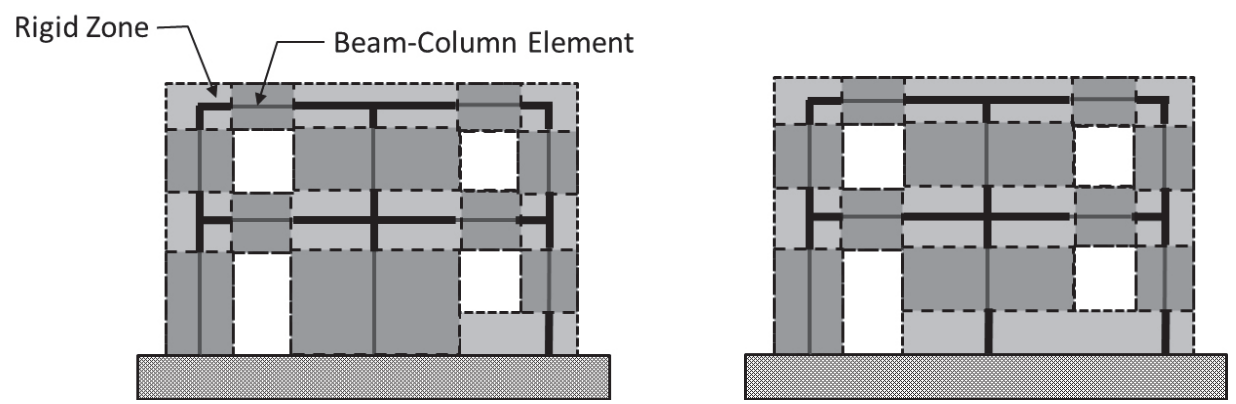

(a) Regular arrangement of openings (two possible models)

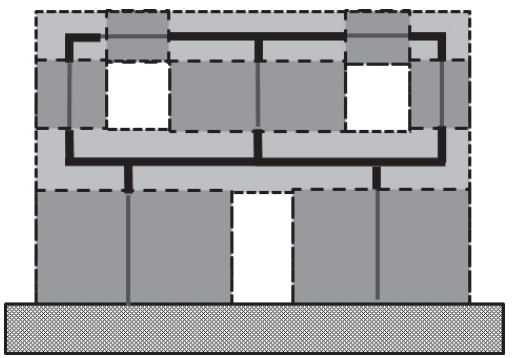

(b) Irregular arrangement of openings

Figure 9-10 Frame models of perforated walls (NIST, 2014).

Sometimes, frame idealizations have to take into account different possible damage patterns in the wall system so that the most critical condition can be identified. Figure 9-10a shows such a case. The model on the left corresponds to a situation that the entire middle wall in the bottom story is separated from the adjacent wall by a 
crack propagating from the lower left corner of the lower window into the middle wall. The one on the right represents a situation that the panel underneath the lower window remains intact. The first situation may result in the middle wall being dominated by flexure, while the second situation introduces a shear-critical condition because of the low shear span ratio of the middle wall. However, it is likely that the actual behavior of the wall system is somewhere in-between because the horizontal reinforcement beneath the window opening may prevent a complete separation of the middle wall when a crack develops.

\subsection{Modeling of Wall Components with Beam-Column Elements}

\subsubsection{Types of Beam-Column Elements}

For modeling the nonlinear behavior of reinforced masonry wall structures subjected to seismic forces, a basic requirement for beam-column elements is that they are able to accurately simulate the flexural behavior of wall components, including the moment-curvature relation for a wall section and the effective plastic-hinge length, in which plastic deformation tends to localize. The effective plastic-hinge length should account for the propagation of plastic deformation in the longitudinal bars into the foundation slab or the panel zones between wall elements, which is often referred to as the plastic strain penetration. These elements also have to be able to simulate the shear mechanism in an approximate but adequate manner. This is especially important for walls with low shear-span ratios. However, typical beam-column element formulations do not account for inelastic shear deformation, and, in particular, the behavior dominated by diagonal shear cracks. To overcome this limitation, the inelastic shear behavior of a wall can be simulated with a shear spring connected in series with the lateral degree of freedom of a beam-column element. The shear spring assumes a phenomenological hysteretic law to describe the shear force-shear deformation relation in an approximate manner. Such an approach has been used by Marini and Spacone (2006).

Beam-column element formulations can be either displacement- or force-based depending on how the displacement compatibility and equilibrium conditions are enforced. While displacement-based elements are computationally more efficient and robust, force-based elements enforce the equilibrium between the element nodal forces and the internal stress resultants at any beam section in an exact manner, and can accurately represent the localization of plastic deformation in a beam or wall with a single element.

The inelastic flexural behavior of a wall can be modeled with beam-column elements in two distinct ways. One is to have the plastic deformation concentrated in a zerolength hinge at one or both ends of an element. The other is to allow the plastic deformation spread along the element according to the deformation demands at the 
element sections. The former is called the plastic-hinge model and the latter is the distributed plasticity model. For the plastic-hinge model, the moment-rotation relation for the hinge has to be defined. This can be deduced from the momentcurvature relation for the beam or wall section and the effective plastic-hinge length represented by the zero-length hinge. Very often, phenomenological hysteretic moment-rotation laws are used for such elements, with or without the consideration of the effect of the axial force. If the axial force-moment interaction effect is to be considered, the element must provide the capability to define a failure surface to represent this interaction.

For elements with distributed plasticity, the nonlinear material properties have to be defined in terms of uniaxial stress-strain relations. For the curvature and axial strain given at an element section, the internal stresses can be determined from the constitutive relations and the stress resultants, i.e., the moment and the axial force, can be subsequently computed with numerical integration. To compute the stresses and stress resultants, the cross-sectional area of the element can be divided into small areas or fibers. This approach naturally accounts for the axial force-moment interaction effect. However, as will be discussed in the next section, in calibrating the constitutive models for this type of elements, one needs to have a good estimate of the effective plastic-hinge length of the wall when plastic deformation occurs.

Beam-column elements can also have a predefined plastic-hinge zone at one or both ends. They differ from plastic-hinge elements in that plastic deformation is spread over the predefined zones. The moment - curvature and axial force-axial deformation responses and their interaction in the plastic zone can be calculated with the fiber-section approach. The main advantage of this type of elements over the plastic-hinge or distributed-plasticity elements is that it allows the effective plastichinge length to be precisely specified while retaining the salient features of distributed plasticity. A force-based element of this type has been developed and implemented in OpenSEES (Scott and Fenves, 2006).

Regardless of the type of beam-column elements used for modeling, to capture the nonlinear flexural behavior of a wall component in an accurate manner, it is essential to have good estimates of the locations of potential plastic-hinge zones and the effective plastic-hinge lengths. Furthermore, the constitutive models used to simulate the behavior of masonry and reinforcing steel should be able to represent different possible local damage phenomena, such as the crushing of masonry and the buckling and fracture of reinforcing bars, exhibited by a wall. They have to be appropriately calibrated taking into consideration the element size, the element formulation, and the effective plastic-hinge length expected for the wall. The following section will present constitutive models appropriate for reinforced masonry wall analysis, and recommendations for model calibration. 


\subsubsection{Material Models for Masonry and Steel}

\subsubsection{Masonry}

To model the flexural behavior of a reinforced masonry wall, the tensile strength of masonry can be ignored. The compressive stress-strain behavior of masonry is very similar to that of concrete, and can, therefore, be represented by the Kent-Park model (Kent and Park, 1971), which is shown in Figure 9-11. For both concrete and masonry, the calibration of the post-peak regime of the model requires special considerations because it can have a significant influence on the flexural ductility exhibited in the analysis result. Whether it is in a laboratory test or computational analysis, the softening behavior of a material often leads to the localization of plastic deformation in the structural member. Nevertheless, the extent of the plastic strain localization zone in the analysis may differ significantly from that observed in a test. This is because plastic strains tend to localize in a single element, an integration, or a row of elements in a computational model, which is more of a numerical artifact than the real physical behavior. Because of this, the post-peak behavior of the modeled structure is highly sensitive to the size of the elements in which plastic strains are localized. One method to circumvent this problem is to adjust the post-peak stressstrain relation of the material in accordance with the element size to maintain the objectivity of the total inelastic energy dissipated in the model.

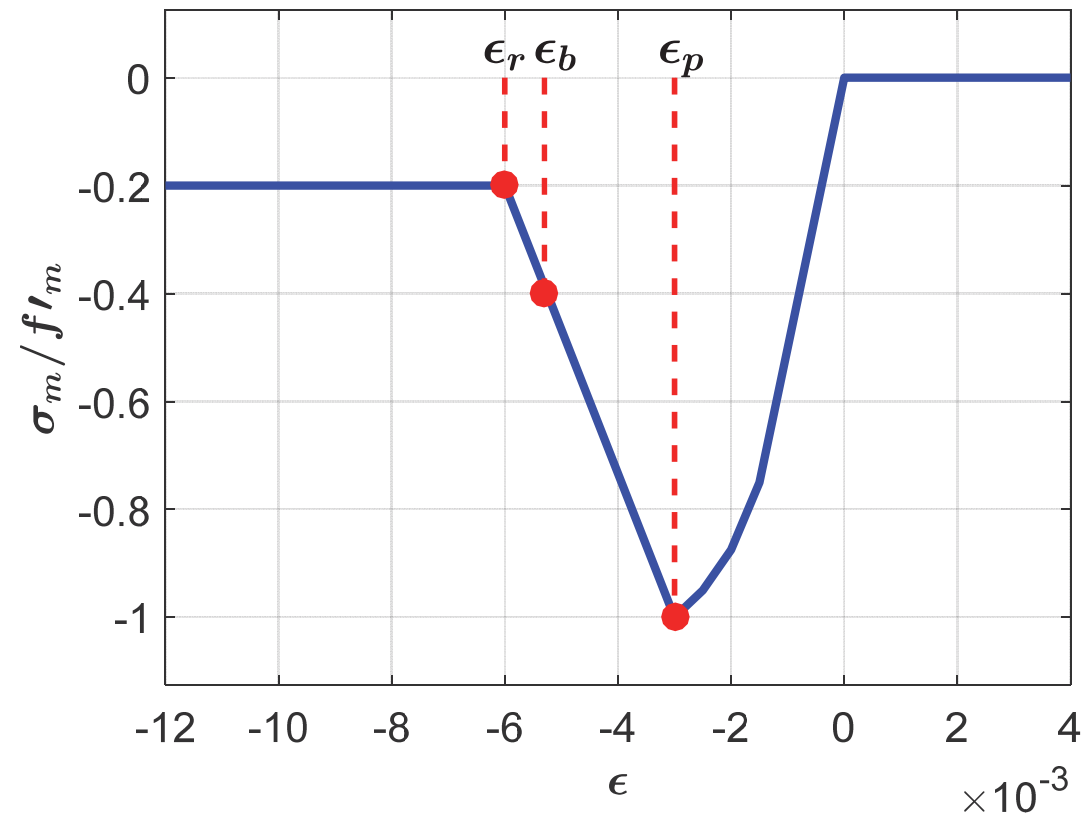

Figure 9-11 Baseline stress-strain curve for the compression behavior of fully grouted masonry.

It was observed in laboratory tests that when a concrete cylinder was subjected to compression, damage tended to localize in the mid-height region of the specimen. Jansen and Shah (1997) further observed in their tests that the post-peak slopes of the 
compressive stress vs. average axial strain curves were steeper for taller specimens. This is attributed to the elastic unloading occurring in the undamaged region of the specimen when the damage zone experienced post-peak strength degradation. The length of the damage zone was observed to be more or less independent of the specimen length. Hence, a taller specimen would experience more elastic strain recovery, resulting in more severe plastic strains in the damage zone. To characterize the post-peak compressive stress-strain behavior of concrete in an objective manner, Jansen and Shah have defined a post-peak compression fracture energy term, $G_{f}$, which is the energy dissipated per unit loaded area in the damage zone after the peak stress has been reached. Dividing this fracture energy term by the length of the damage zone, $h$, provides a basic material parameter that characterizes the post-peak stress-strain behavior of the damage zone (Coleman and Spacone, 2001). For masonry, such data can be obtained from masonry prism tests. Nevertheless, the damage zone observed in masonry prism tests is less distinct than that in concrete cylinder tests. One should also be cautioned that the value of $h$ identified in a masonry prism or concrete cylinder test may not necessarily reflect that developed in a column or wall subjected to flexure, because of the difference in the stress gradients induced by different loading conditions.

Plastic strain (or damage) localization also occurs in wall analysis using beamcolumn elements with distributed plasticity after the material has reached the peak stress. While this is consistent with test observations, the phenomenon that plastic strains tend to localize in a single element or integration point is a numerical artifact that does not necessarily reflect that observed in a test. For displacement-based elements, plastic deformation tends to localize in a single element regardless of the number of elements used to represent the wall. As a result, if the post-peak stressstrain relation for the material model remains the same, the post-peak response obtained in the wall analysis will change if the length of the element in which the plastic strain is localized changes. For force-based elements, plastic deformation tends to localize at an integration point at one or both ends of the element (Coleman and Spacone, 2001). Hence, the numerical results will be sensitive to the tributary length of the integration point, which is a function of the element length and the number of integration points in the element.

Because of the strain localization issues discussed above, data from masonry prism tests or concrete cylinder tests alone are not sufficient to calibrate the post-peak stress-stress relation of the material. Additional data from structural component tests are needed to determine the stress-strain relation that will best represent the actual post-peak behavior of the structural component to be analyzed for the given element length or the tributary length of an integration point. If the element length or the tributary length of an integration point chosen closely represents the actual effective plastic-hinge length of the structural component, the stress-strain relation that 
provides a good match of the component test results can be taken as the baseline relation, based on which appropriate post-peak stress-strain curves can be determined for different element sizes or numbers of integration points. This discussion for masonry wall analysis will follow.

To obtain the baseline stress-strain relation for masonry with the Kent-Park model, data from select fully grouted reinforced masonry cantilever walls tested by Ahmadi (2012), Sherman (2011), Kapoi (2012), Shedid et al. (2008), and Shing et al. (1991) are considered. Each wall was modeled with a beam-column element that had a predetermined plastic-hinge length (Scott and Fenves, 2006) corresponding to the effective plastic-hinge length of the wall, which is assumed to be $20 \%$ of the wall height as recommended for reinforced masonry wall analysis in NIST (2010a). The baseline model obtained is shown in Figure 9-11. While the value of $\varepsilon_{p}$ is based on masonry prism tests (Atkinson and Kingsley, 1985), the value of $\varepsilon_{r}$, which governs the post-peak slope of the curve, is so determined that the numerical results provide a good match of the test data.

When length of the beam-column element or the tributary length of an integration point closely represent the effective plastic-hinge length of the wall, the baseline stress-strain relation shown in Figure 9-11 can be used for the wall analysis.

Otherwise, the post-peak compressive stress-strain relation (i.e., the value of $\varepsilon_{r}$ ) has to be modified so that the total fracture energy dissipated in the model will remain unchanged (Bažant and Oh, 1983). Based on the fracture energy argument of Bažant and Oh (1983) and Jansen and Shah (1997), Coleman and Spacone (2001) have derived the following equation to calculate the value of $\varepsilon_{r}$ for any element size with the Kent-Park model:

$$
\varepsilon_{r}=-\left(\frac{G_{f}}{0.6 f_{m}^{\prime} L_{e}}-\frac{0.8 f_{m}^{\prime}}{E_{m}}-\varepsilon_{p}\right)
$$

where $f_{m}^{\prime}$ and $E_{m}$ are the compressive strength and the modulus of elasticity of masonry, respectively, $\varepsilon_{p}$ is the strain at the peak compressive stress, $L_{e}$ is the length of the plastic zone represented by the beam-column element, and $G_{f}$ is the total fracture energy per unit area, which should remain constant regardless of the value of $L_{e}$ if the results are to be objective. The value of $\varepsilon_{r}$ calculated with Equation 9-1 is the baseline value when $L_{e}$ is equal to the effective plastic-hinge length, $L_{p}$, of the wall. Hence, if the baseline stress-strain relation for the masonry (as shown in Figure 9-11) and the effective plastic-hinge length are known, Equation 9-1 can be rearranged to calculate the value of $G_{f}$ as follows:

$$
G_{f}=-0.6\left(\varepsilon_{r}-\varepsilon_{p}\right) f_{m}^{\prime} L_{p}+\frac{0.48 f_{m}^{\prime 2} L_{p}}{E_{m}}
$$


Even though the baseline model presented in Figure 9-11 is determined with test data on fully grouted masonry, it may be used for partially grouted masonry. This is certainly true for the grouted portion of a partially grouted wall. For the ungrouted portion, only the face shells of the masonry units should be considered and, strictly speaking, the stress-strain relation pertaining to the units should be used. However, there are not enough test data on partially grouted masonry to make such a distinction.

\subsubsection{Steel Reinforcement}

Load degradation occurring in a flexure-dominated reinforced masonry wall is often associated with one or more of the following phenomenon: the crushing and spalling of masonry in the compression toes, and the buckling and fracture of the vertical reinforcing bars after the loss of the confining masonry. It is important that the nonlinear model used for wall analysis be able to account for bar buckling and fracture. With beam-column elements, these phenomena can be incorporated in an approximate manner in the axial stress-strain relation for the reinforcing steel (NIST, 2010c). To this end, a baseline phenomenological stress-strain law for steel, as shown in Figure 9-12, is proposed. This law is obtained in the same calibration study using wall test data to obtain the baseline material law for masonry, as discussed in Section 9.4.2.1. In this law, the tensile strength of steel is assumed to be 1.5 times the yield strength, based on the expected properties of Grade 60 steel. The buckling of a reinforcing bar is represented by a sudden drop of the compressive stress as shown in the figure. The criterion for buckling is strain based with the assumption that the strain in a vertical bar is the same as the axial strain in the adjacent masonry and that a bar will buckle when the adjacent masonry reaches the compressive strain, $\varepsilon_{b}$, at which the masonry strength drops to $40 \%$ of its peak strength, signifying the occurrence of severe spalling. In the baseline stress-strain relation for masonry shown in Figure 9-11, $\varepsilon_{b}=-0.0053$. This value will change with the value of $\varepsilon_{r}$ calculated with Equation 9-1 when the element length (or the value of $L_{e}$ ) is changed. After a bar buckles, the compressive strength of the bar drops linearly with respect to strain increments to $10 \%$ of the yield strength when the compressive strain reaches a limiting value $\varepsilon_{10}$, which is set to be $\left(\varepsilon_{r}-0.01\right)$ to represent a sufficiently rapid stress drop and at the same time avoid numerical problems.

The strain at which a bar fractures depends on the loading history. Cyclic buckling and straightening of a bar as a wall is subjected to cyclic loading will lead to early bar fracture because of the low-cyclic fatigue induced by the severe stress cycles. In the proposed law, two types of loading histories are considered. One is monotonically increasing tension, which will occur in reinforcing bars when a wall is loaded in one direction only. For this case, based on tensile stress-strain curves obtained from tension tests, it is assumed that a bar reaches the peak tensile stress at the strain of 
$\varepsilon_{p s}=0.10$, after which the stress drops rapidly to zero at the strain of $\varepsilon_{0}=0.15$, simulating tensile fracture, as shown in Figure 9-12.

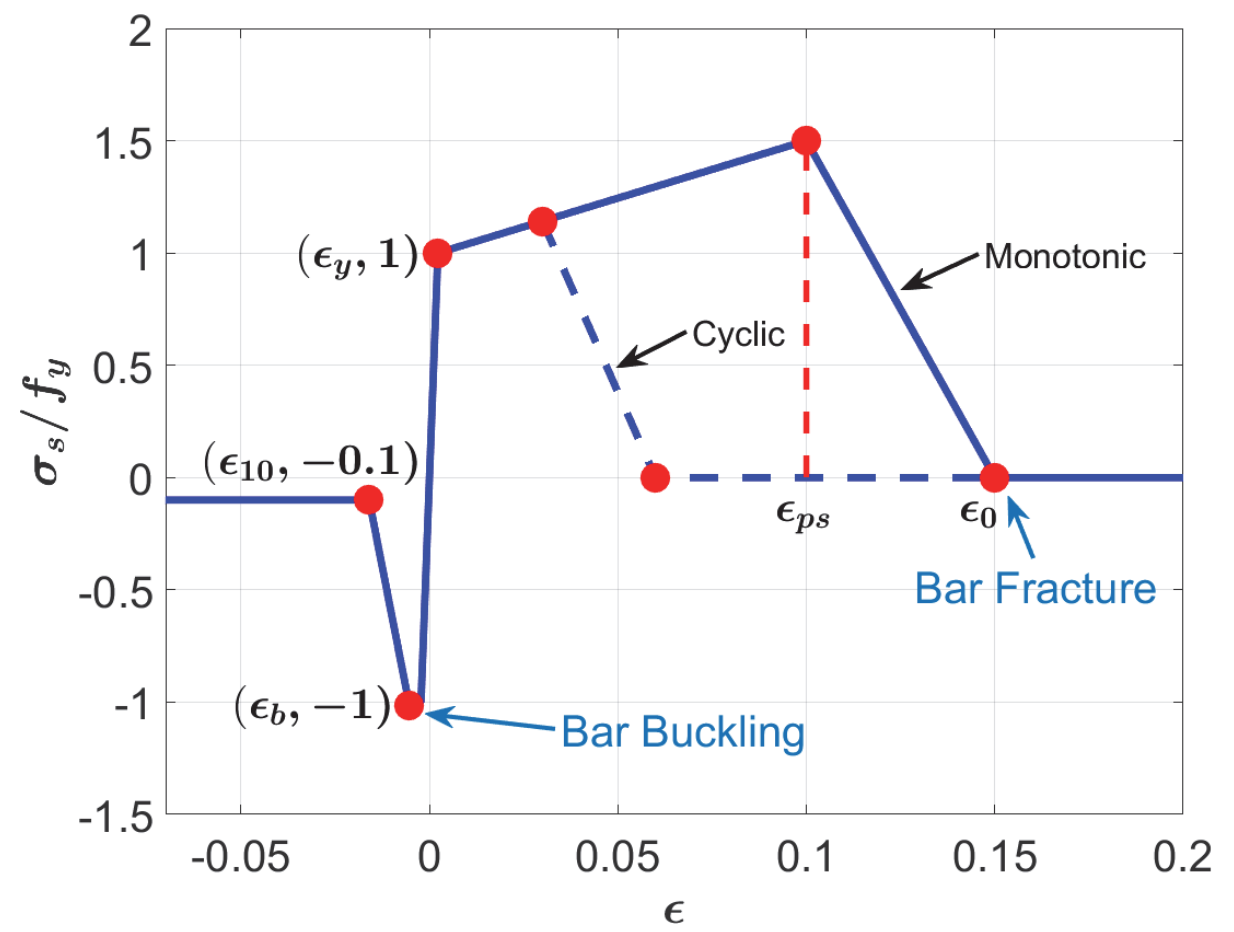

Figure 9-12 Stress-strain relation for steel considering bar buckling and fracture.

The second case is cyclic loading. It is assumed that bar fracture starts at a reduced strain $\varepsilon_{p s}$ between 0.03 and 0.072 , accounting for low-cycle fatigue, also shown in Figure 9-12. It is assumed that $\varepsilon_{0}=\varepsilon_{p s}+0.03$ to avoid too sharp a decline of the tensile stress, which may cause numerical problems. Walls having a larger amount of vertical reinforcement or subjected to a larger axial compressive stress are expected to have earlier masonry spalling, and thus earlier buckling of the vertical bars. Hence, it is assumed that $\varepsilon_{p s}$ is a function of the following parameters $\alpha$, reinforcing index, and $\beta$, axial compression load ratio, as shown in Figure 9-13.

$$
\begin{gathered}
\alpha=\frac{f_{y}}{f_{m}^{\prime}} \rho_{v} \\
\beta=\frac{P}{f_{m}^{\prime} A_{n}}
\end{gathered}
$$

where $A_{n}$ is the net horizontal cross-sectional area of the wall, $f_{y}$ is the yield strength of the vertical steel, $\rho_{v}$ is ratio of the total cross-sectional area of the vertical steel to $A_{n}$, and $P$ is the axial compressive load. The above relations provide numerical results that match the wall test data well. 


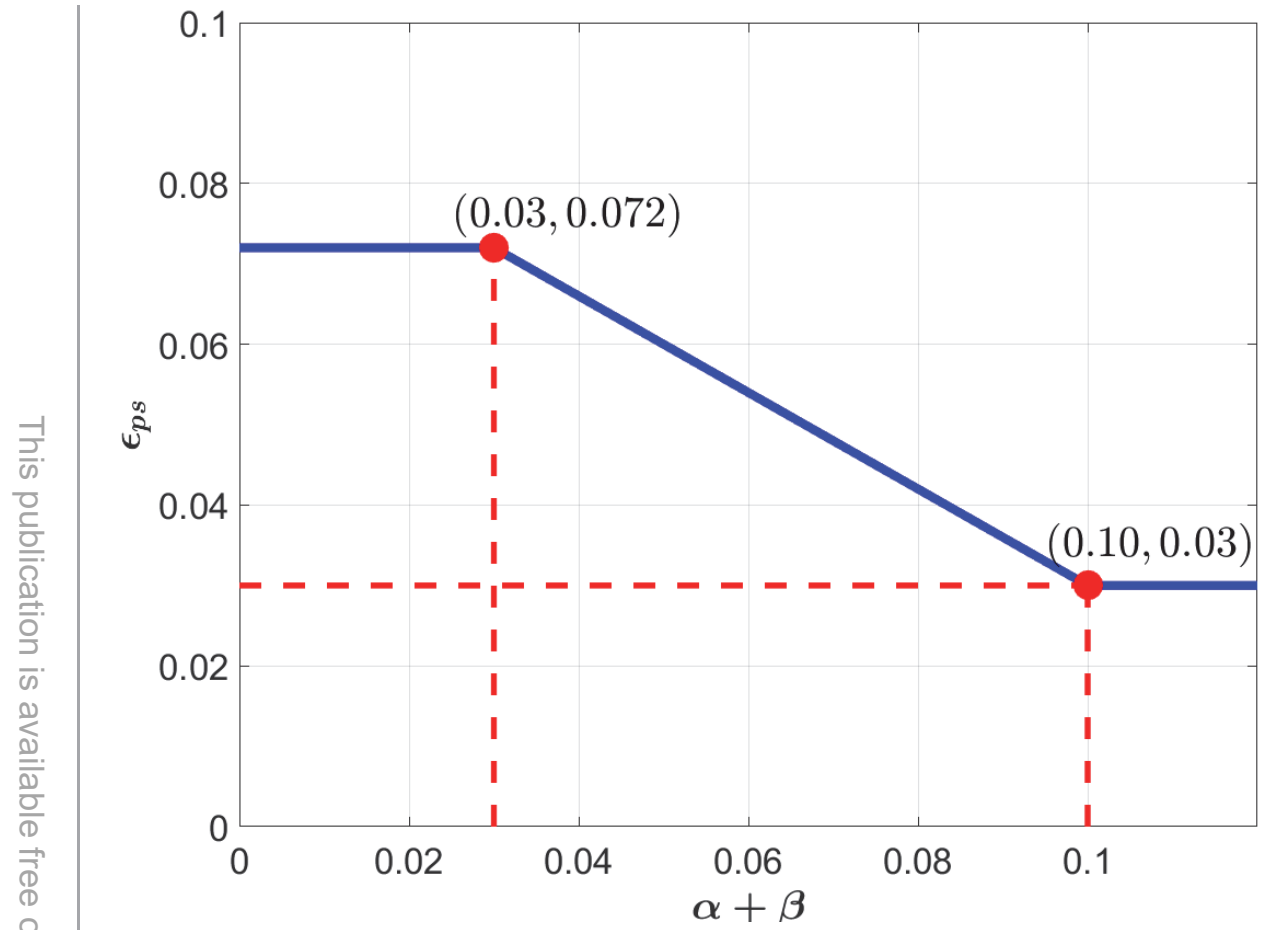

Figure 9-13 Tensile strain at bar fracture as a function of steel quantity and axial compressive load for cyclic analysis.

Similar to that for masonry, the proposed baseline material law for steel has to be modified when the element size selected does not represent the actual plastic-hinge length expected for the wall. This is because the length of the element in which plastic strains localize affects the displacement of the wall at which bar fracture occurs. As the element size is smaller, more plastic strains will develop for the same wall displacement, which will trigger premature bar fracture. Hence, to maintain the objectivity of the numerical results, the following formulas are used to update the values of $\varepsilon_{p s}$ and $\varepsilon_{0}$, as suggested in NIST (2010a).

$$
\begin{gathered}
\varepsilon_{p s, m}=\varepsilon_{y}+\left(\varepsilon_{p s}-\varepsilon_{y}\right) \frac{L_{p}}{L_{e}} \\
\varepsilon_{0, m}=\varepsilon_{p s, m}+\left(\varepsilon_{0}-\varepsilon_{p s}\right) \frac{L_{p}}{L_{e}}
\end{gathered}
$$

where $\varepsilon_{p s, m}$ and $\varepsilon_{0, m}$ are the modified values, while $\varepsilon_{p s}$ and $\varepsilon_{0}$ represent the respective baseline values given in Figures 9-12 and 9-13; $L_{e}$ is the length of the plastic zone represented by the beam-column element, and $L_{p}$ is the effective plastichinge length expected for the actual wall.

\subsubsection{Effective Plastic-Hinge Length}

As the flexural deformation of a wall increases, the region in which plastic deformation or damage localizes may grow. However, for modeling purposes, the 
length of this region can be assumed to be a constant and is often referred to as the effective plastic-hinge length. Based on laboratory observations, empirical formulas have been proposed to estimate the effective plastic-hinge lengths for reinforced concrete and masonry walls (e.g., Paulay and Priestley, 1992; Priestley et al., 2007). They are originally intended for use in simplified analytical methods to calculate the flexural displacement of a wall. Even though not exactly intended for this purpose, such formulas may be used to determine the stress-strain relations and lengths of beam-column elements that are suitable for wall analysis with the approach discussed in the previous section. For reinforced masonry walls, Priestley et al. (2007) have suggested the following equation for the effective plastic-hinge length.

$$
L_{p}=0.04 h_{e}+0.1 l_{w}+L_{s p} \geq 3 L_{s p}
$$

where:

$$
L_{s p}=0.15 f_{y e} d_{b}
$$

In the above equation, $h_{e}$ is the effective wall height, $l_{w}$ is the length of the wall, $L_{s p}$ accounts for plastic strain penetration, $d_{b}$ is the diameter of the vertical bars, and $f_{y e}$ is the expected yield strain of the bars. All units are in kips and inches. The effective wall height is defined as the distance between the section at which the maximum moment develops and the section with zero moment. For a cantilever wall, this is simply the wall height.

In a prior study (NIST, 2010a), it has been found that the above equation underestimates the effective plastic-hinge length observed in wall tests and may result in more brittle wall behavior than what was observed in those tests. Instead, the aforementioned study has suggested an effective plastic-hinge length that is $20 \%$ of the effective wall height for wall analysis. This has been adopted in this study.

\subsubsection{Shear Deformation}

For a flexure-dominated wall, it is appropriate to use an elastic shear spring connected in series with the lateral degree of freedom of a beam-column element to account for the shear deformation. The elastic shear stiffness of an uncracked wall segment is given by the following theoretical formula.

$$
k_{v}=\frac{A_{v} G_{m}}{h}
$$

where $h$ is the height of the wall segment, $A_{v}$ is the effective shear area, and $G_{m}$ is the shear modulus of masonry, which can be assumed to be $40 \%$ of the Young's modulus, $E_{m}$. However, flexural and shear cracks may develop in a reinforced masonry wall before the applied lateral force reaches the peak value. When cracks develop, the shear stiffness of the wall decreases significantly. Experimental data of 
Shing et al. (1991) show that the effective shear stiffness of a reinforced masonry cantilever wall, with a height-to-length ratio of one, can be reduced to $50 \%$ of the theoretical value given by Equation 9-8 when the applied lateral force reaches 50\% its shear capacity, and can be as low as $20 \%$ of the theoretical stiffness when major diagonal shear cracks develop. Based on this observation and data from other tests (Ahmadi, 2012), it is suggested that the effective elastic shear stiffness of a flexuredominated reinforced masonry wall be equal to $35 \%$ of the theoretical value given by Equation 9-8. Furthermore, for a wall loaded beyond the elastic regime, the shear stiffness can be assumed to be $20 \%$ of the theoretical value.

\subsubsection{Evaluation of Beam-Column Model with Test Data}

The experimental data of Ahmadi (2012), Sherman (2011), Kapoi (2012), Shedid et al (2008), and Shing et al. (1991) are used to demonstrate the capability of beamcolumn models to capture the flexure-dominated behavior of reinforced masonry walls with the material laws presented in Section 9.4.2. A total of 21 cantilever concrete masonry walls are considered. Eleven of these walls had vertical reinforcement lap-sliced at the base. The characteristics of these walls and the material properties are shown in Table E-1 in Appendix E. These wall specimens have aspect ratios $\left(h / l_{w}\right)$ ranging from 0.78 to 4.5 , the ratio of the area of the vertical reinforcement to the wall cross-sectional area, $\rho_{v}$, between 0.16 and $1.31 \%$, and the axial compression ratio, $\beta=P /\left(f_{m}^{\prime} A_{n}\right)$, varying from 0 to 0.125 .

The analyses were conducted with OpenSEES using a force-based element that has a pre-defined plastic-hinge length (Scott and Fenves, 2006). Each wall specimen is modeled with a single force-based element with a plastic-hinge length equal to $20 \%$ of the wall height at the base of the wall. Since the plastic-hinge lengths assumed in the beam-column elements are equal to those expected for the actual walls, the baseline material properties shown in Figures 9-11 and 9-12 are used. It should be mentioned that these baseline material properties are calibrated with these wall test data. Masonry is represented by the Kent-Scott-Park model (identified as Concrete01 in OpenSEES), and the vertical reinforcement is modeled with the Hysteretic Material model in OpenSEES that incorporates the pinching of the hysteresis loops as well as load degradation. The compressive strength of masonry prisms and the yield strength of steel reported for the wall specimens are used. For the hysteretic material model representing the behavior of steel, the parameters pinchX and pinchY, which control the pinching of the hysteresis loops, are both set to 1.0, and the damage parameters, dmg 1 and dmg2, which govern the load degradation, are set to 0.0001 and 0.02 , respectively. No stiffness degradation is assumed for unloading. These parameters are calibrated with the load-displacement hysteresis curves obtained from the wall tests. 
The shear behavior of the wall specimens is assumed to be elastic with the shear stiffness equal to $20 \%$ of the theoretical shear stiffness of an uncracked wall (given by Equation 9-8). To calculate the elastic shear stiffness, the Young's modulus of masonry, $E_{m}$, is assumed to be 900 times the compressive strength $f_{m}^{\prime}$ as recommended for concrete masonry in TMS 402 (TMS, 2013), and the shear modulus is equal to $0.4 E_{m}$.

The lateral load vs. lateral displacement curves obtained from the analyses and the tests are compared in Appendix E. Figures 9-14 through 9-16 show the comparison for select wall specimens, which had different amounts of vertical steel, axial compression rations, and aspect ratios, respectively. As shown, the beam-column models can capture the peak strengths, strength degradation, and hysteretic behavior of the wall specimens well, with the numerical results slightly underestimating the peak strengths and overestimating the strength degradation for most of the cases.

Figure 9-14 shows the results for walls UT-PBS-03 and UT-PBS-04, which had an aspect ratio of one and were not subjected to any axial load. Both walls had exactly the same geometry; but UT-PBS-03 had \#4 vertical bars spaced at 8 in. on center, while UT-PBS-04 had \#4 bars spaced at 16 in. on center. Because of the lower amount of vertical reinforcement, UT-PBS-04 exhibited a more ductile behavior in that its peak resistance was reached at a drift ratio of $1.75 \%$, while the peak load for UT-PBS-03 was reached at about $0.80 \%$. Nevertheless, UT-PBS-04 exhibited a more rapid strength drop after passing its peak strength. This can be attributed to the fact that UT-PBS-04 had a smaller number of vertical bars and a wider bar spacing. Therefore, the loss of the vertical bars due to fracture at the extreme ends in that wall had a more significant consequence than that in UT-PBS-03. As shown in Figure 9-14, this phenomenon is well captured by the beam-column model. However, for UT-PBS-04, the numerical result shows slightly less ductile behavior than the experimental results. This could be attributed to the fact that base sliding, which was more significant for that wall, is not accounted for in the model. Base sliding is, in general, more severe for walls with a lower amount of vertical steel or axial load.

Figure 9-15 shows the results for more slender walls, which had an aspect ratio of two. Both walls had the same amount of vertical reinforcement but one of them was not subjected to an axial load. For both walls, the analyses underestimate the peak strengths reached in the tests. For these walls, the models indicate bar fracture, while the tests did not. However, necking was observed in one of the extreme vertical bars in wall WSU-W-08 during the test.

Figure 9-16 shows the results for two very slender walls, which had aspect ratios of 3 and 4.5 , respectively. Both walls had an axial compressive load ratio of $5 \%$ and $\rho_{v}=0.72 \%$. While the load-displacement hysteresis curves for wall UT-W-17 are well captured, the analysis significantly underestimates the strength degradation 
shown by UT-W-13. The severe strength degradation exhibited by that wall specimen is believed to be caused by the failure of the lap splices in the vertical reinforcement, which is not modeled in the analysis. The failure of the lap splices was caused by severe masonry spalling.

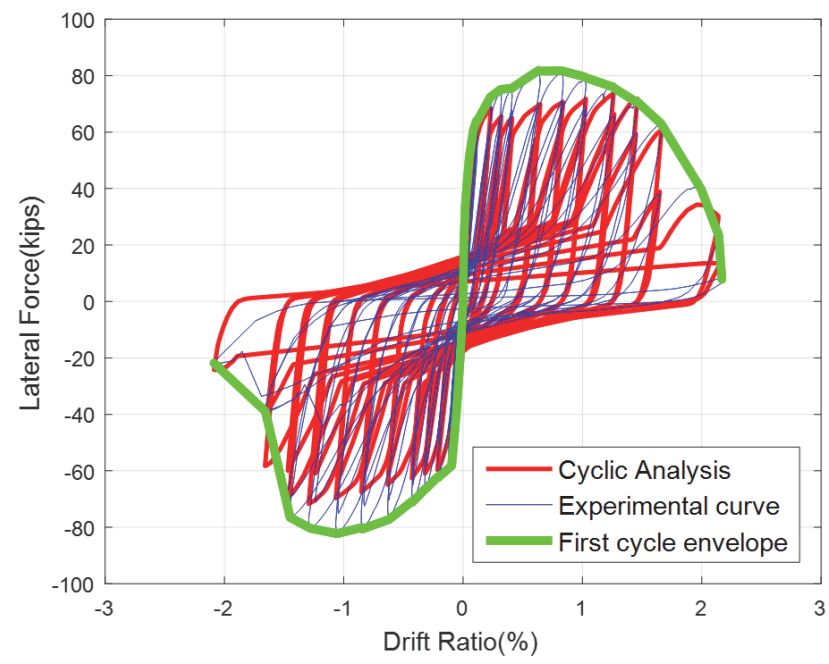

(a) UT-PBS-03, $\rho_{v}=0.33 \%$

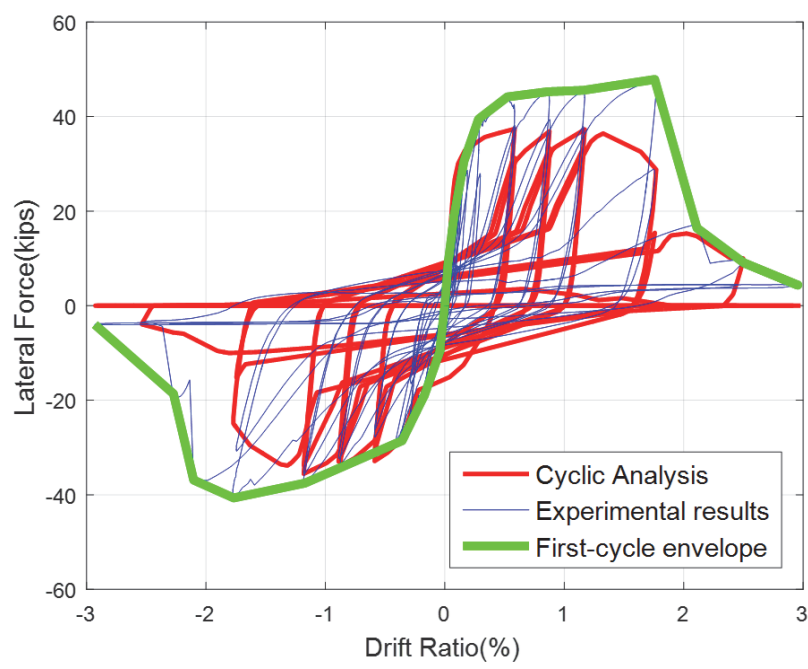

(b) UT-PBS-04, $\rho_{v}=0.16 \%$

Figure 9-14 Comparison of numerical results with experimental data for flexure-dominated walls with varying amounts of vertical steel [with an aspect ratio of 1 and no axial load].

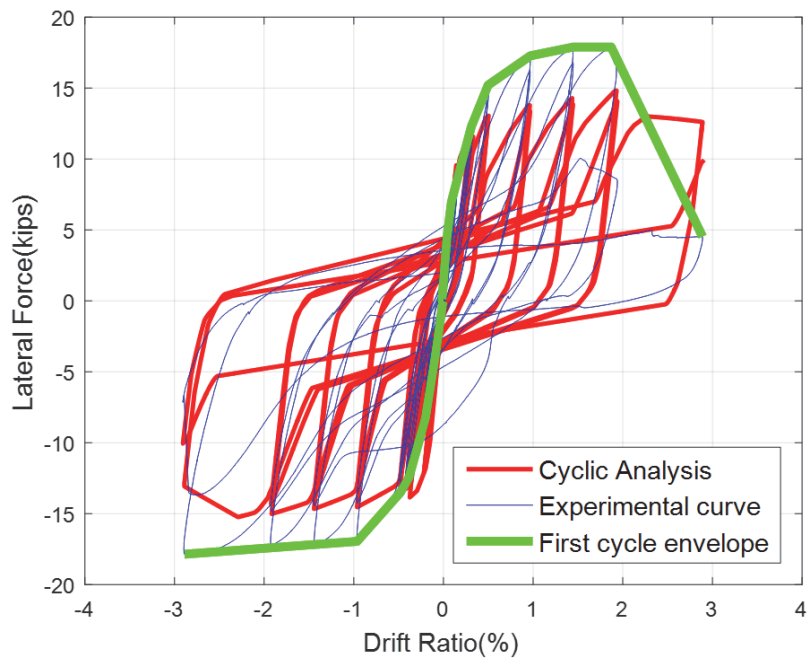

(a) WSU-W-07(C1), zero axial load

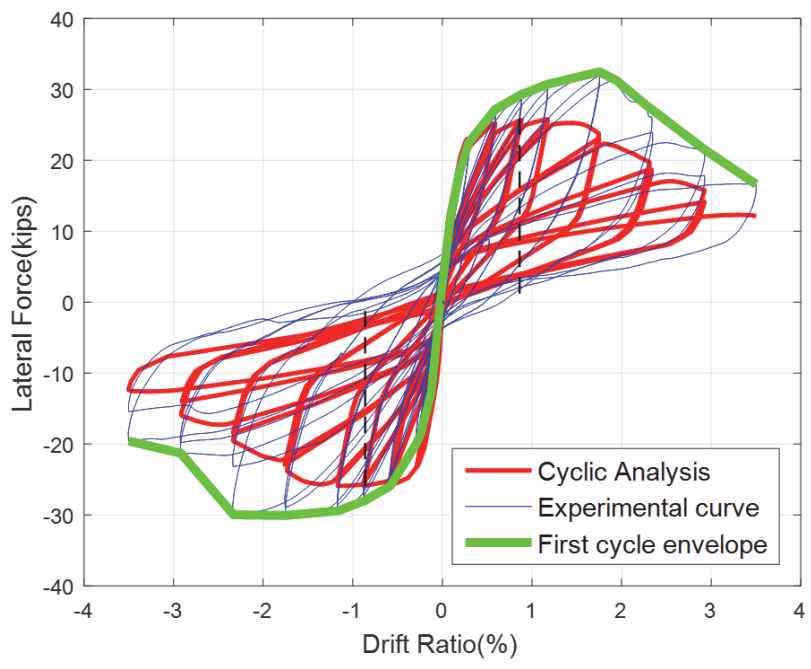

(b) WSU-W-08(C2), axial load ratio of $6.25 \%$

Figure 9-15 Comparison of numerical results with experimental data for flexure-dominated walls with varying amounts of axial load [with an aspect ratio of 2 and $\rho_{v}=0.33 \%$ ]. 


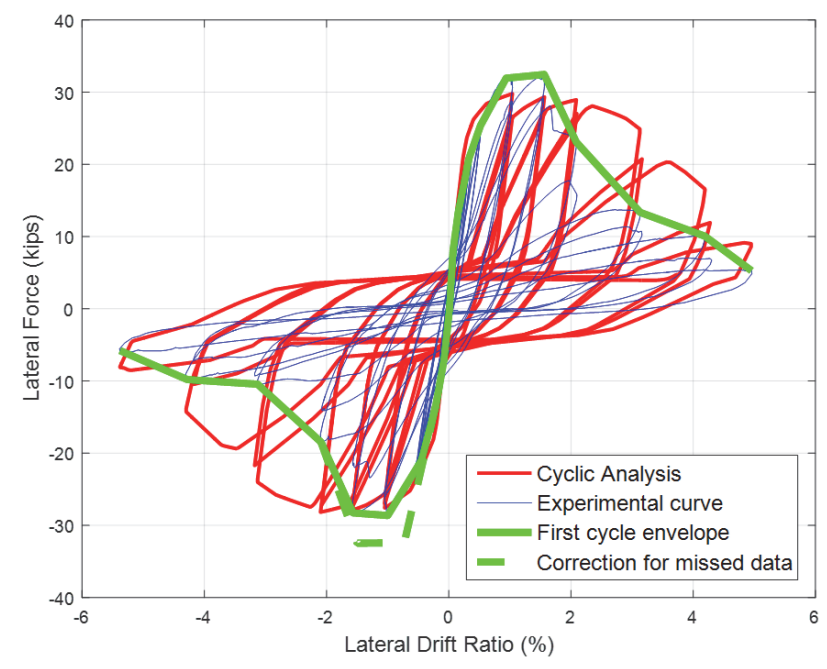

(a) UT-W-13, aspect ratio of 3

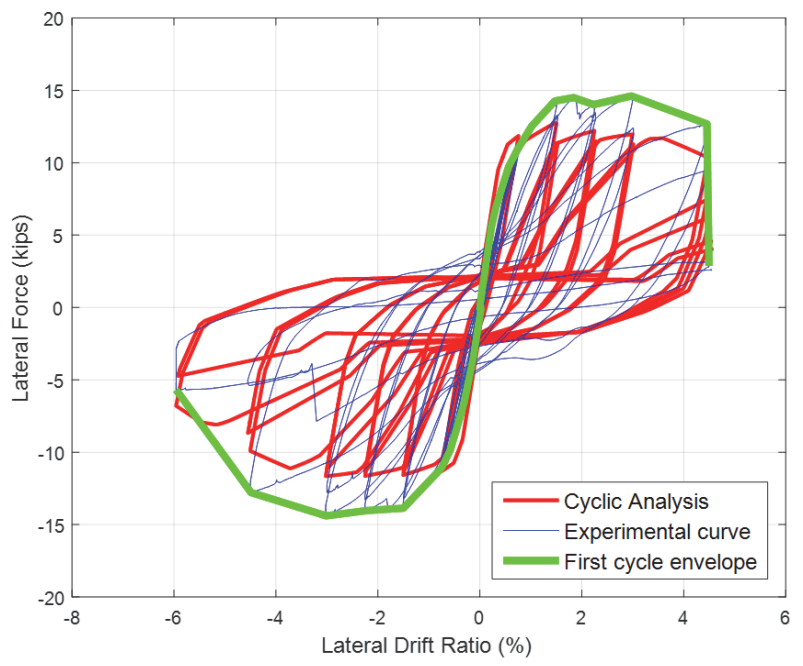

(b) UT-W-17, aspect ratio of 4.5

Figure 9-16 Comparison of numerical results with experimental data for flexure-dominated walls with varying aspect ratio [with axial load ratio of $2.8 \%$ and $\rho_{v}=0.72 \%$.

\subsubsection{Influence of Lap Splices}

Sherman (2011) tested two pairs of walls to study the influence of lap splices on the lateral load vs. lateral displacement hysteresis curves exhibited by the walls. Each pair had identical designs, except that one wall had lap splices at the base, while the other did not. The lengths of the lap splices complied with TMS 402 (TMS, 2011). Results for one pair are shown in Figure 9-17 and those for the other pair are shown in Appendix E.1. It can be seen that the wall with lap splices had more rapid strength degradation and more pinched hysteresis curves than those without. The numerical results provide a good match of the pinched hysteresis curves exhibited by the walls with lap splices but underestimate the hysteretic energy dissipated by the walls without lap splices. The shape of the hysteretic load-displacement curves obtained with the beam-column models is heavily influenced by the hysteretic material model for steel. Hence, the calibration of the hysteretic model for steel seems to be biased towards walls that had lap splices. However, the load degradation exhibited by the beam-column models, which is strongly influenced by bar fracture, matches the test results well regardless the walls had lap splices or not, as shown in Appendix E.1. This could be attributed to the fact that the tensile fracture behavior calibrated for the steel model also includes the influence of lap splices as the calibration is based on the global load-displacement response exhibited by the wall specimens. 


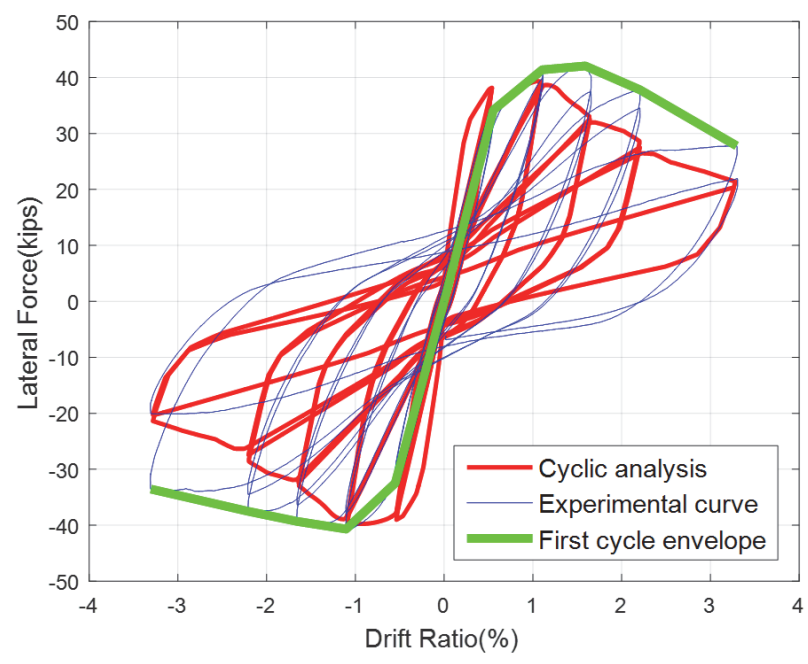

(a) Wall WSU-W-1A - without lap splices

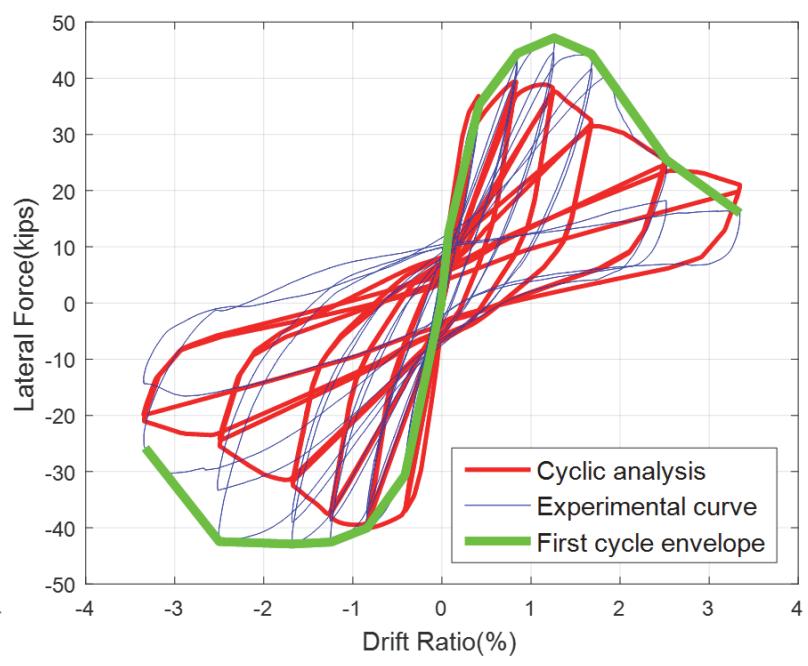

(b) Wall WSU-W-1B - with lap splices

Figure 9-17 Comparison of numerical results with experimental data for flexure-dominated walls for influence of lap splices.

\subsubsection{Influence of Loading History}

Experimental data on reinforced masonry walls subjected to monotonically increasing displacements are not available to identify the influence of the loading or displacement history on wall behavior. Hence, beam-column models are used to examine the difference in the load-displacement response of a wall subjected to monotonic and cyclic loading histories, respectively. For these analyses, the tensile stress-strain curves corresponding to the respective loading histories, as shown in Figure 9-13, are used for the vertical reinforcing steel. Figure 9-18 shows the comparisons for walls with three different values of $(\alpha+\beta)$, where $\alpha$ and $\beta$ represent the reinforcing index and axial load ratio, respectively, as defined in Equations 9-3 and 9-4. However, there are no test data to confirm these observations. The numerical results show that the behavior of walls that have lower amounts of vertical steel and lower axial compressive loads is more sensitive to the loading history, being significantly more ductile under monotonically increasing loads than under cyclic loads. This can be attributed to the fact that the behavior of these walls is significantly influenced by that of the steel reinforcement, while the behavior of walls having higher axial loads and reinforcement ratios is dominated by the crushing of masonry. 


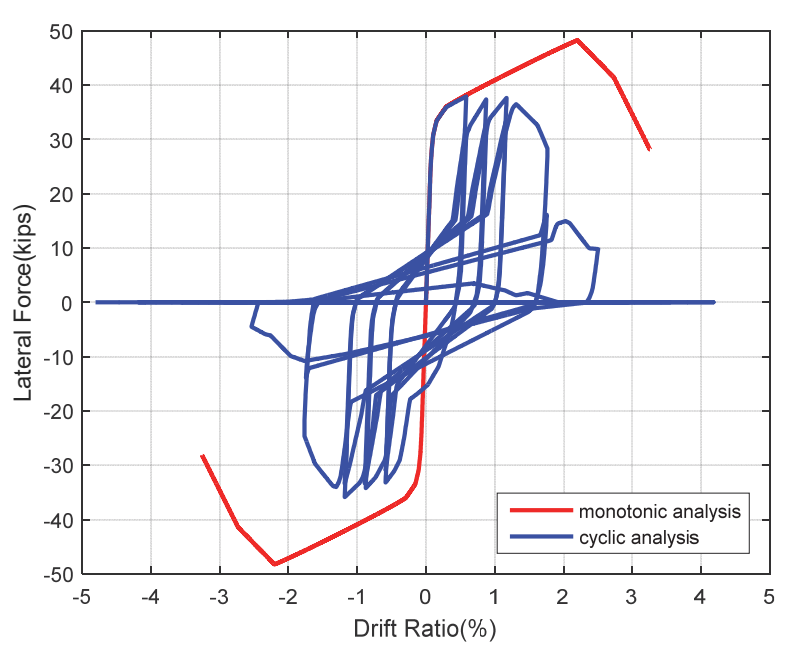

(a) UT-PBS-04, $\alpha+\beta=0.022$

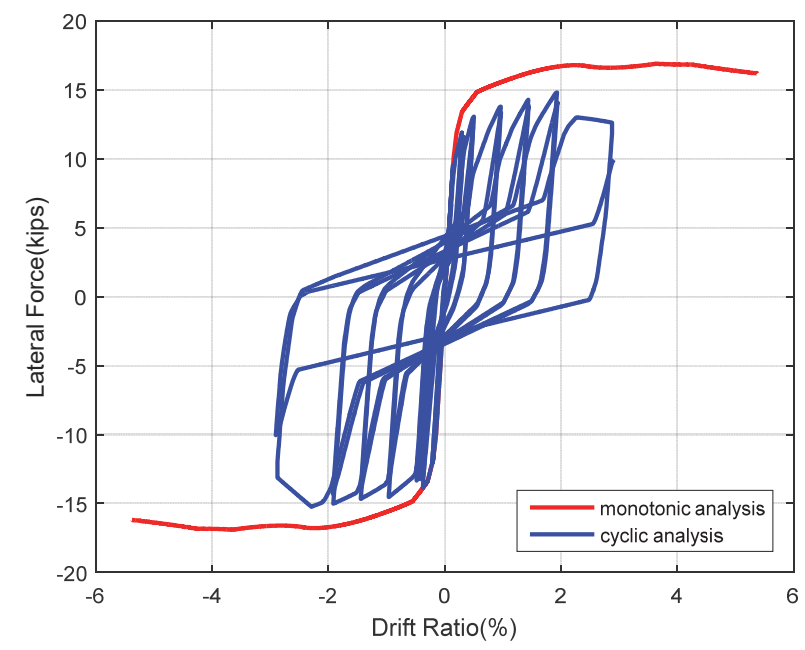

(b) WSU-W-07(C1), $\alpha+\beta=0.071$

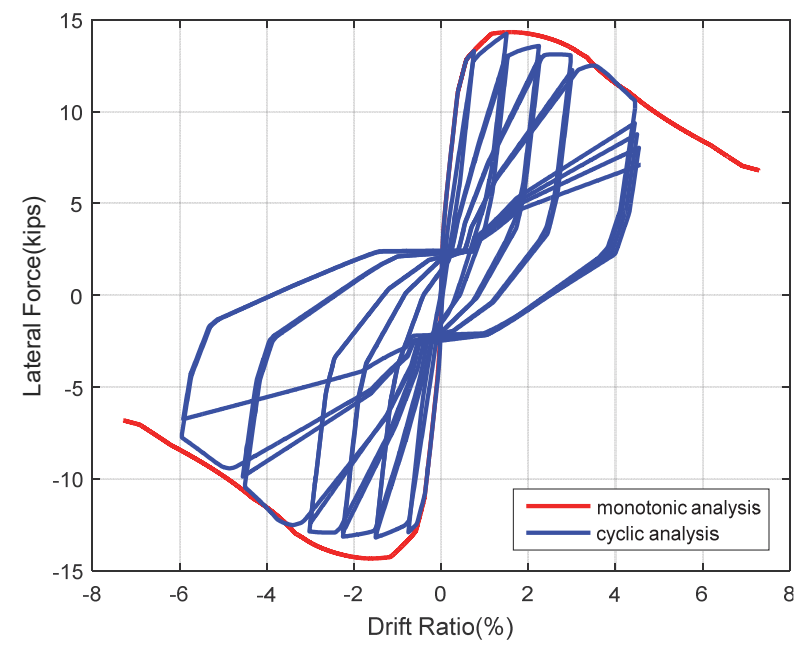

(c) UT-W-17, $\alpha+\beta=0.155$

Figure 9-18 Influence of loading history on flexure-dominated wall behavior.

\subsection{Backbone Force-Displacement Curves for Flexure-Dominated Walls}

\subsubsection{Construction of Idealized Backbone Curves}

Figure 9-19 shows an idealized backbone curve proposed here to represent the loaddisplacement relation for flexure-dominated wall components. This is based on the wall test data presented in Appendix E.1. It can be calibrated to represent the firstcycle envelope curves for walls subjected to cyclic loading.

The proposed backbone curve is defined in terms of five parameters, namely, the effective elastic stiffness of the wall, $k$, the expected maximum lateral load resistance of the wall, $Q_{\max }$, the displacement at which the maximum resistance develops, $\Delta_{m}$, the displacement at which the post-peak resistance drops to $75 \%$ of $Q_{\max }, \Delta_{75}$, and the capping displacement, $\Delta_{c}$, after which the lateral load resistance of the wall can be 
ignored. Here, the capping displacement, $\Delta_{c}$, is taken to be the point at which the post-peak resistance drops to $50 \%$ of $Q_{\max }$ because data from wall tests beyond this point are scarce. Furthermore, as will be explained in Section 9.5.3, $\Delta_{c}$ should not exceed $4 \%$ of the wall height.

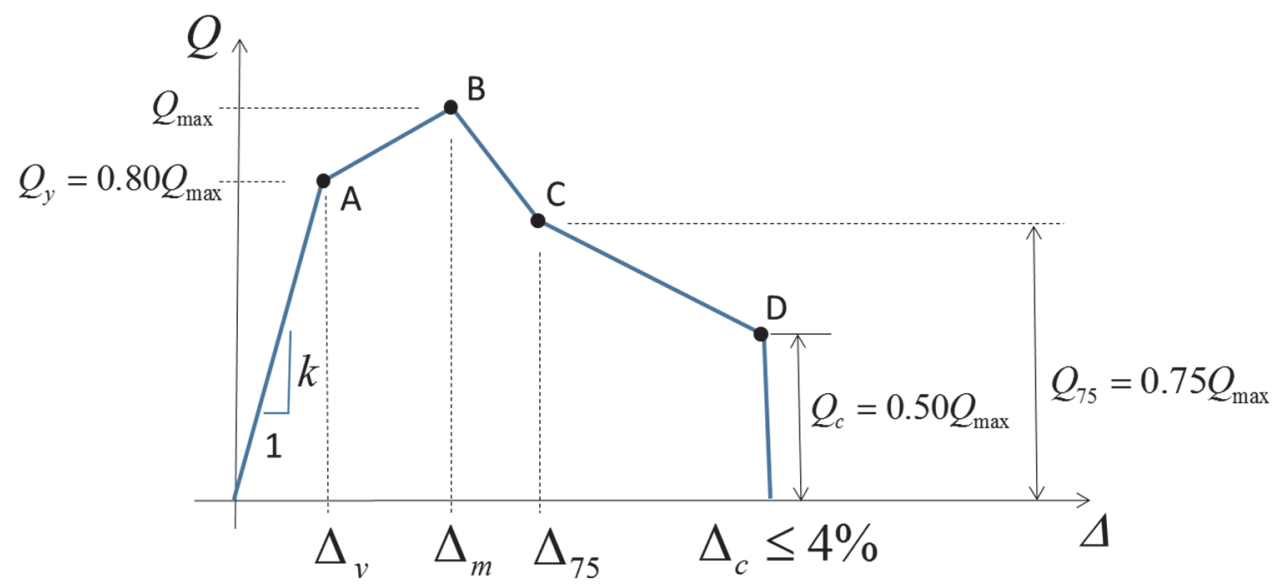

Figure 9-19 Backbone load-displacement curve for flexure-dominated reinforced masonry walls.

The values of the five parameters defining the backbone curve depend on the properties of the wall and the loading conditions, including the aspect ratio, boundary conditions, and reinforcement contents of the wall, the material properties, and the axial force exerted on the wall. The post-peak branch of the curve is also influenced by the loading history. Wall test data currently available cannot comprehensively cover this many variables and the range of possible variation for each variable. Hence, in this study, an analytical procedure is proposed to construct the backbone curve for a wall based on the given properties and loading conditions.

In the proposed analytical procedure, the values of $Q_{\max }, \Delta_{m}, \Delta_{75}$, and $\Delta_{c}$ are determined from the moment-curvature relation for the wall section, as that shown in Figure 9-20. The moment-curvature curve can be calculated with the material models presented in Section 9.4.2 and the assumption that a plane section remains plane during bending. Using simple beam theory, for a given axial load and curvature, the bending moment can be calculated from the stresses developed at the section with the force and moment equilibrium conditions. For the purpose of constructing the backbone load-displacement curve, only the values of the peak moment $M_{\max }$, and the respective curvatures, $\phi_{m}, \phi_{75}$, and $\phi_{c}$, as shown in Figure 9-20, are needed. Cyclic analysis is not needed to construct a backbone curve that represents the first-cycle envelope because the main factor that distinguishes the behavior of a cyclically loaded reinforced masonry wall from that of a monotonically loaded wall is the low-cycle fatigue behavior of the vertical reinforcing bars, as shown in Section 9.4.7. Hence, the cyclic load effect can be included in a monotonic 
load analysis if an appropriate steel model that accounts for the low-cycle fatigue induced by bar buckling, as presented in Figure 9-12, is used.

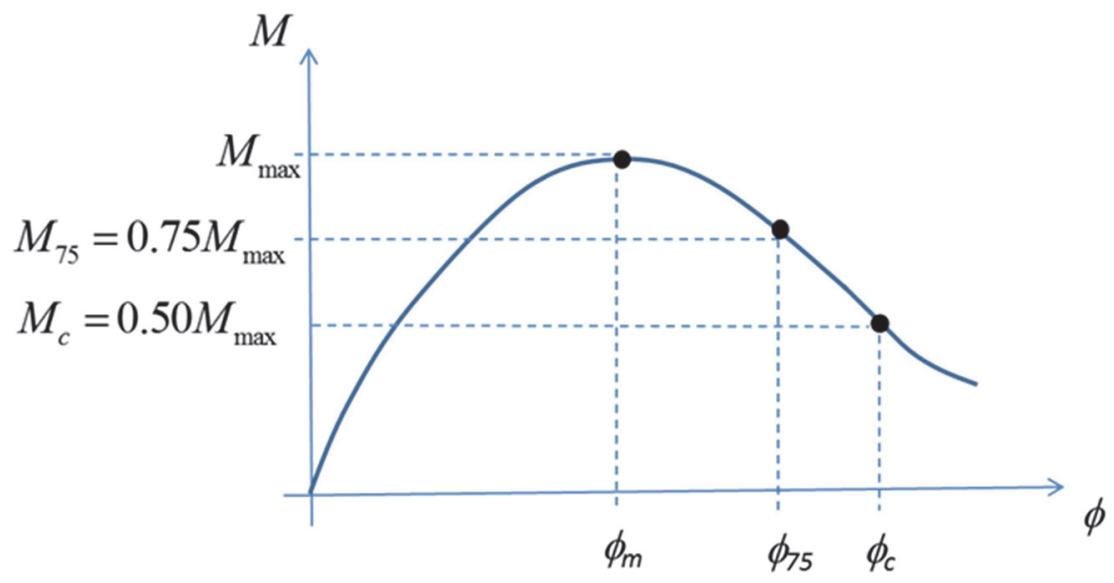

Figure 9-20 Moment-curvature relation for a wall section.

The procedure for the construction of a backbone load-displacement curve using a moment-curvature relation is presented below for a cantilever wall, with the dimensions shown in Figure 9-21, as an example.

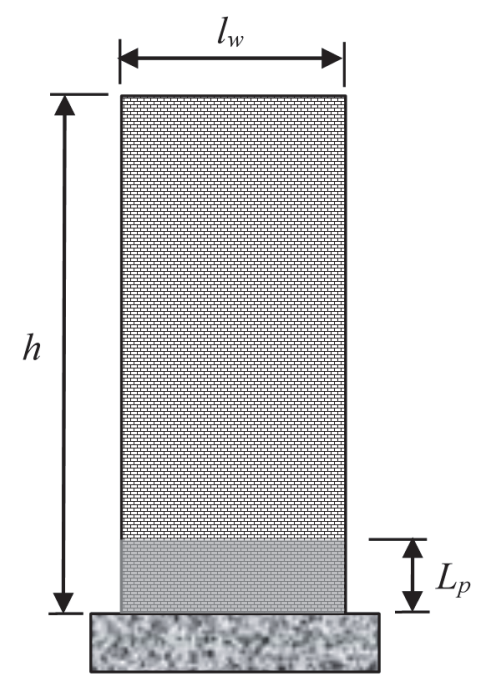

Figure 9-21 Cantilever wall.

As suggested in Section 9.4.3, the plastic-hinge length, $L_{p}$, for the wall is assumed to be $0.2 \mathrm{~h}$. The initial stiffness of the wall is calculated with the following equation, which is based on the elastic beam theory but with the consideration of stiffness reduction caused by cracking:

$$
k=\frac{1}{\frac{h^{3}}{3 E_{m} I_{e}}+\frac{h}{0.35 A_{v} G_{m}}}
$$


where the Young's modulus $E_{m}$ is equal to $900 f_{m}^{\prime}$ for concrete masonry and $700 f_{m}^{\prime}$ for clay masonry, and the shear modulus $G_{m}=0.4 E_{m}$, following the recommendations in TMS 402 (TMS, 2013). The effective moment of inertia, $I_{e}$, is assumed to be $15 \%$ of that of an uncracked wall section, and a reduction factor of 0.35 is applied to the shear stiffness to account for cracking. These stiffness reduction factors are deduced from experimental data as explained in NIST (2014). For a rectangular wall section, the effective shear area, $A_{v}$, equal to $5 A_{n} / 6$, where $A_{n}$ is the net cross-sectional area of the wall.

For a cantilever wall with a lateral load applied at the top, the maximum load capacity $Q_{\max }=M_{\max } / h$, and the displacement $\Delta_{m}$ at which this load is reached consists of a flexural component $\Delta_{f i}$ and a shear component $\Delta_{v m}$ :

$$
\Delta_{m}=\Delta_{f m}+\Delta_{v m}
$$

where:

$$
\begin{gathered}
\Delta_{f m}=\frac{M_{\max }}{E I_{e}} \frac{h^{3}}{3}+\left(\phi_{m}-\frac{M_{\max }}{E I_{e}}\right) L_{p}\left(h-\frac{L_{p}}{2}\right) \\
\Delta_{v m}=Q_{\max } \frac{h}{0.20 A_{v} G_{m}}
\end{gathered}
$$

Equation 9-10c has the shear stiffness at the peak load reduced by a factor of 0.20 to account for increased cracking.

The displacements $\Delta_{75}$ and $\Delta_{c}$ can be calculated in a similar way as follows:

$$
\Delta_{75 / c}=\Delta_{f 75 / c}+\Delta_{v 75 / c}
$$

where:

$$
\begin{gathered}
\Delta_{f 75 / c}=\frac{M_{75 / c}}{E I_{e}} \frac{h^{3}}{3}+\left(\phi_{75 / c}-\frac{M_{75 / c}}{E I_{e}}\right) L_{p}\left(h-\frac{L_{p}}{2}\right) \\
\Delta_{v 75 / c}=Q_{75 / c} \frac{h}{0.20 A_{v} G_{m}}
\end{gathered}
$$

\subsubsection{Nondimensionalized Moment-Curvature Relations for Fully Grouted Rectangular Wall Sections}

To reduce the number of independent variables that need to be considered for a general backbone curve, one can express the moment-curvature relation for a wall section in a dimensionless form. This is especially attractive for a fully grouted symmetric rectangular wall section, whose moment-curvature relation can be represented by a few variables. Figure 9-22 shows such a section, which is subjected to a compressive force $P$ and a bending moment $M$. It should be noted that the 
derivation of the following nondimensionalized equations is merely intended to identify the basic dimensionless variables required to construct a nondimensionalized moment-curvature curve, while actual calculations can be performed numerically using a computer software, such as OpenSEES.

(a)

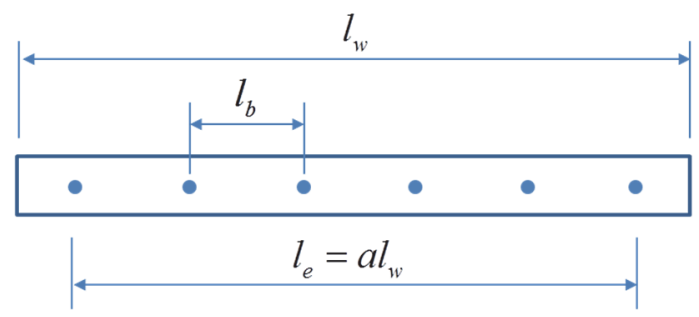

(b)

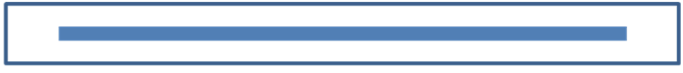

(c)

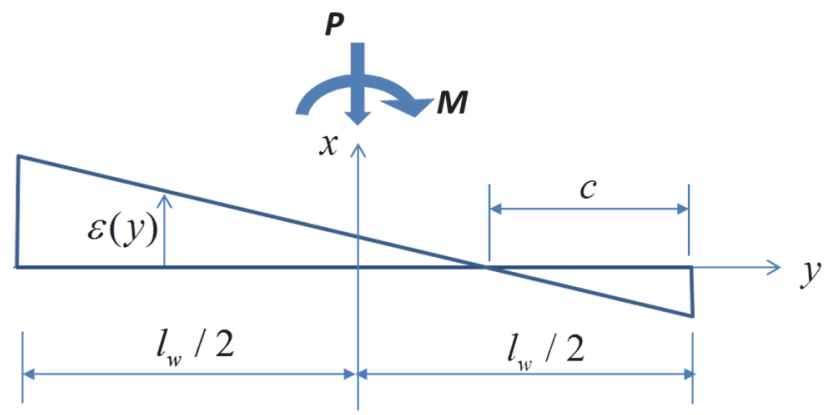

Figure 9-22 Fully grouted symmetric rectangular wall section subjected to compression and bending.

With the assumption that the vertical reinforcement is uniformly distributed over a distance of $l_{e}=a l_{w}$ (see Figure 9-21b), where $l_{w}$ is the length of the wall section and $l_{e}$ is the distance between the extreme vertical bars, the axial load and the bending moment about the centroidal axis of the wall section can be expressed as follows.

$$
\begin{gathered}
P=-t \int_{-\frac{l_{w}}{2}}^{\frac{l_{w}}{2}} \sigma_{m}(\varepsilon(y)) d y-\frac{t}{a} \rho_{v} \int_{-\frac{l_{e}}{2}}^{\frac{l_{e}}{2}} \sigma_{s}(\varepsilon(y)) d y \\
M=-t \int_{-\frac{l_{w}}{2}}^{\frac{l_{w}}{2}} \sigma_{m}(\varepsilon(y))\left(y-\frac{l_{w}}{2}\right) d y-\frac{t}{a} \rho_{v} \int_{-\frac{l_{e}}{2}}^{\frac{l_{e}}{2}} \sigma_{s}(\varepsilon(y))\left(y-\frac{l_{w}}{2}\right) d y
\end{gathered}
$$

where $y$ is the distance from the centroidal axis, $t$ is the thickness of the wall, $\rho_{v}$ is the vertical steel ratio with respect to the net cross-sectional area of the wall $\left(A_{n}=t l_{w}\right.$ ), and $\sigma_{m}$ and $\sigma_{s}$ are the normal stresses in the masonry and the steel, respectively, which are functions of the strain $\varepsilon$ and the strain history induced by the axial load and bending. Assuming that the plane section remains plane during bending:

$$
\varepsilon(y)=\phi\left(\frac{l_{w}}{2}-c-y\right)=\phi l_{w}\left(\frac{1}{2}-\frac{c}{l_{w}}-\frac{y}{l_{w}}\right)
$$


where $c$ is the distance of the neutral axis of bending from the extreme compression fiber of the section, and $\phi$ is the wall curvature.

Dividing Equation 9-12 by $f_{m}^{\prime} t l_{w}$ and Equation 9-13 by $f_{m}^{\prime} t l_{w}^{2}$ and with simple mathematical manipulations, the following nondimensionalized equations are obtained.

$$
\begin{gathered}
\beta=-\int_{-\frac{1}{2}}^{\frac{1}{2}} \sigma_{m}^{\prime}\left(\varepsilon\left(y^{\prime}\right)\right) d y^{\prime}-\frac{1}{a} \alpha \int_{-\frac{a}{2}}^{\frac{a}{2}} \sigma_{s}^{\prime}\left(\varepsilon\left(y^{\prime}\right)\right) d y^{\prime} \\
M^{\prime}=-\int_{-\frac{1}{2}}^{\frac{1}{2}} \sigma_{m}^{\prime}\left(\varepsilon\left(y^{\prime}\right)\right)\left(y^{\prime}-\frac{1}{2}\right) d y^{\prime}-\frac{1}{a} \alpha \int_{-\frac{a}{2}}^{\frac{a}{2}} \sigma_{s}^{\prime}\left(\varepsilon\left(y^{\prime}\right)\right)\left(y^{\prime}-\frac{1}{2}\right) d y^{\prime}
\end{gathered}
$$

in which $\alpha$ is the reinforcing index and $\beta$ is the axial load ratio, as defined in Equations 9-3 and 9-4, respectively, and the other dimensionless variables are defined below:

$$
\begin{gathered}
M^{\prime}=\frac{M}{f_{m}^{\prime} t l_{w}^{2}}=\frac{M}{f_{m}^{\prime} A_{n} l_{w}} \\
\sigma_{m}^{\prime}=\frac{\sigma_{m}}{f_{m}^{\prime}} \\
\sigma_{s}^{\prime}=\frac{\sigma_{s}}{f_{y}} \\
\varepsilon\left(y^{\prime}\right)=\phi l_{w}\left(\frac{1}{2}-c^{\prime}-y^{\prime}\right) \\
c^{\prime}=\frac{c}{l_{w}} \\
y^{\prime}=\frac{y}{l_{w}}
\end{gathered}
$$

For the case of monotonic loading, when the values of $\alpha, \beta$, and $a$ (which is equal to $l_{e} / l_{w}$ ) are given, Equation 9-15 can be used to compute the value of $c^{\prime}$ for any given value of $\phi l_{w}$, using the normalized stress-strain relations for masonry and steel shown in Figures 9-11 and 9-12. Once the value of $c^{\prime}$ has been determined, Equation 9-16 can be used to compute $M^{\prime}$. By repeating the calculations for different values of $\phi l_{w}$, the $M^{\prime}$ vs. $\phi l_{w}$ curve can be obtained.

In hollow block concrete masonry walls, the extreme vertical reinforcing bars are normally 4 inches away from the edges of the wall section. Hence, the value of $a=l_{e} / l_{w}$ varies with the wall length $l_{w}$. The influence of this value on the $M^{\prime}$ vs. $\phi l_{w}$ relation is shown in Figure 9-23. These results are obtained with OpenSEES using the material models presented in Section 9.4.2. It can be seen that the influence 
of $a$ is not significant for a wide range of wall lengths varying from 40 in. ( $a=0.80)$ to $240 \mathrm{in}$. $(a=0.97)$. The influence diminishes with the increase of the axial compressive load.

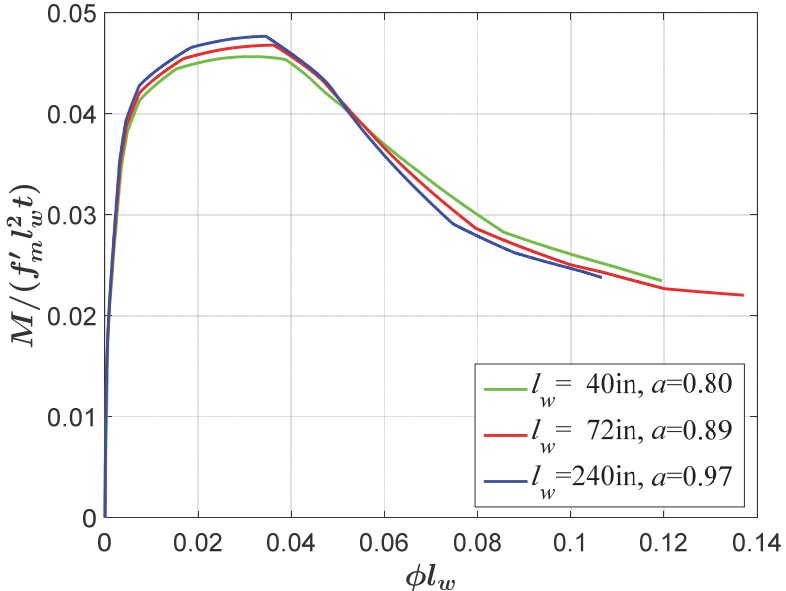

(a) Zero axial load

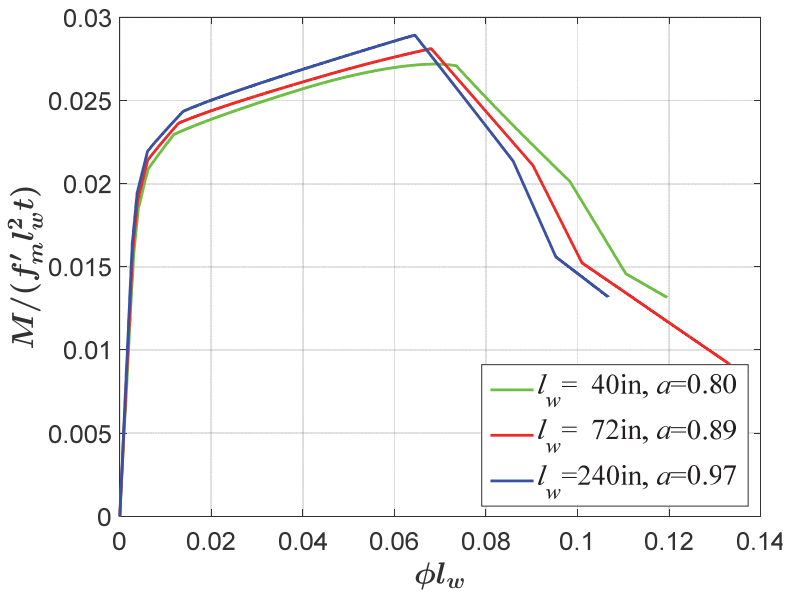

(b) Axial load ratio $\beta=0.05$

Figure 9-23 Influence of $a=l_{e} / I_{w}$ for walls with $\alpha=0.05$ and $I_{b} / I_{w}=0.25$.

Furthermore, Figure 9-24 shows that the bar spacing, i.e., the $l_{b} / l_{e}$ ratio, can have some influence on the $M^{\prime}$ vs. $\phi l_{w}$ relation. The influence is most significant when the axial compressive load is zero. It becomes negligible as the axial load ratio approaches $5 \%$.

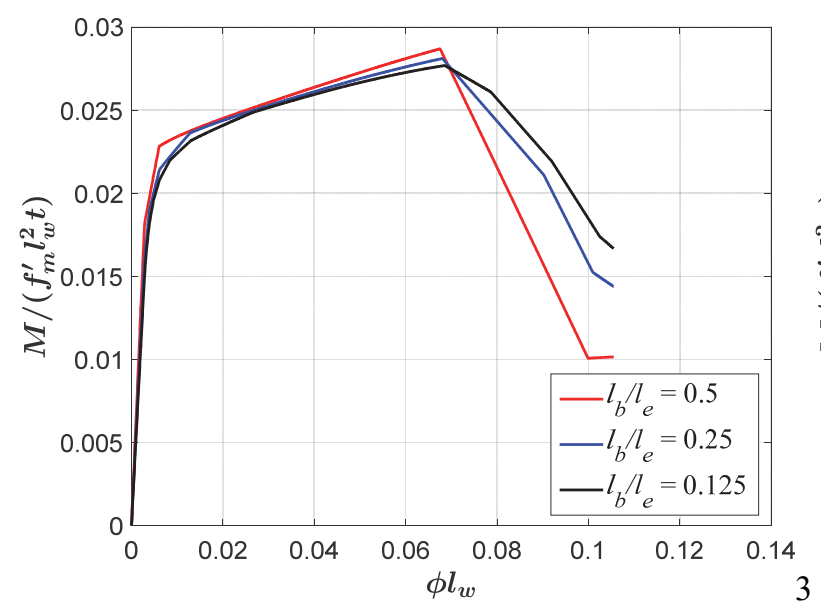

(a) Zero axial load

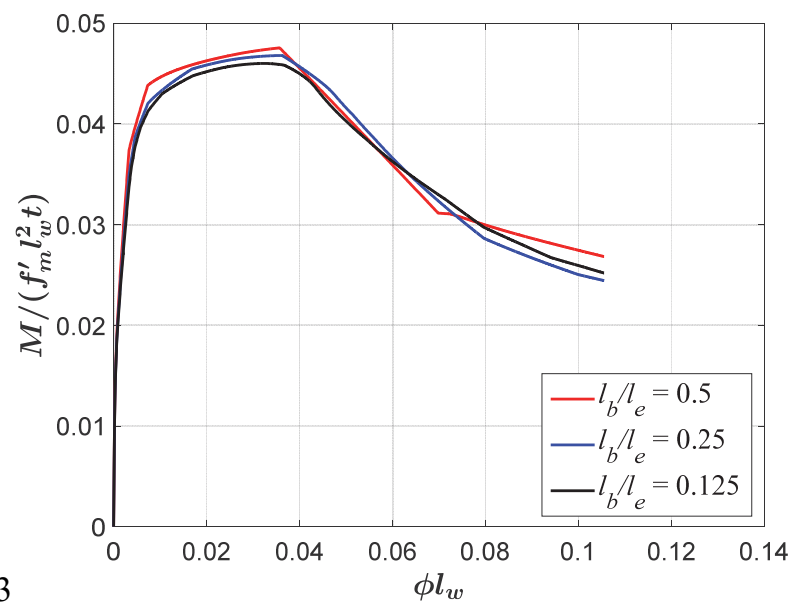

(b) Axial load ratio $\beta=0.05$

Figure 9-24 Influence of bar spacing for walls with $\alpha=0.05$ and $I_{w}=72 \mathrm{in}$.

Hence, one can assume that the nondimensionalized moment-curvature relation for a fully grouted rectangular wall section is governed by two independent variables, $\alpha$ and $\beta$. For the convenience of constructing backbone load-displacement curves, the values of the dimensionless parameters $M_{\max }^{\prime}, \phi_{m} l_{w}, \phi_{75} l_{w}$, and $\phi_{c} l_{w}$ are calculated for fully grouted rectangular wall sections using a fiber-section model with OpenSEES. A wide range of $\alpha$ and $\beta$ values are considered to represent typical wall sections. For 
these analyses, the material models presented in Section 9.4.2 are used. The tensile properties of steel considered accounts for the cyclic load effect. The results are presented in Table 9-1. For the calculations, the wall length $l_{w}$ is assumed to be 72 in., which results in $a=0.89$, and $l_{b} / l_{e}=0.25$. Because of the small influence of the values of $a$ and $l_{b} / l_{e}$ on the results, the values in Table 9-1 are representative rectangular wall sections with different lengths. For values of $\alpha$ and $\beta$ not shown in the table but within the range considered, the values of $M_{\max }^{\prime}, \phi_{m} l_{w}, \phi_{75} l_{w}$, and $\phi_{c} l_{w}$ can be computed by interpolation.

Table 9-1 Nondimensionalized Moment-Curvature Backbone Curve Values for Fully Grouted Rectangular Wall Sections Subjected to Cyclic Loading

\begin{tabular}{|c|c|c|c|c|c|}
\hline $\begin{array}{c}\begin{array}{c}\text { Reinforcement } \\
\text { Index }\end{array} \\
\alpha=\left(f_{y} / f_{m}^{\prime}\right) \rho_{v} \\
\end{array}$ & $\begin{array}{c}\text { Axial Compression } \\
\text { Load Ratio } \\
\beta=P /\left(f_{m}^{\prime} A_{n}\right) \\
\end{array}$ & $\phi_{m} I_{w}$ & $\phi_{75} / w$ & $\phi_{c} l_{w}$ & $M_{\max } /\left(f_{m}^{\prime} A_{n} l_{w}\right)$ \\
\hline \multirow{4}{*}{0.01} & 0 & 0.0782 & 0.1002 & 0.1210 & 0.0060 \\
\hline & 0.05 & 0.0481 & 0.1290 & 0.2099 & 0.0285 \\
\hline & 0.1 & 0.0224 & 0.0505 & 0.0785 & 0.0487 \\
\hline & 0.15 & 0.0151 & 0.0289 & 0.0435 & 0.0662 \\
\hline \multirow{4}{*}{0.05} & 0 & 0.0681 & 0.0904 & 0.1073 & 0.0285 \\
\hline & 0.05 & 0.0362 & 0.0631 & 0.1132 & 0.0472 \\
\hline & 0.1 & 0.0201 & 0.0415 & 0.0947 & 0.0653 \\
\hline & 0.15 & 0.0139 & 0.0271 & 0.0408 & 0.0810 \\
\hline \multirow{4}{*}{0.1} & 0 & 0.0355 & 0.0540 & 0.0673 & 0.0490 \\
\hline & 0.05 & 0.0244 & 0.0535 & 0.0849 & 0.0677 \\
\hline & 0.1 & 0.0171 & 0.0376 & 0.0767 & 0.0833 \\
\hline & 0.15 & 0.0125 & 0.0260 & 0.0383 & 0.0978 \\
\hline \multirow{4}{*}{0.15} & 0 & 0.0328 & 0.0567 & 0.0752 & 0.0712 \\
\hline & 0.05 & 0.0206 & 0.0498 & 0.0841 & 0.0870 \\
\hline & 0.1 & 0.0151 & 0.0350 & 0.0685 & 0.1012 \\
\hline & 0.15 & 0.0115 & 0.0248 & 0.0363 & 0.1147 \\
\hline \multirow{4}{*}{0.2} & 0 & 0.0232 & 0.0529 & 0.0763 & 0.0904 \\
\hline & 0.05 & 0.0179 & 0.0478 & 0.0872 & 0.1042 \\
\hline & 0.1 & 0.0136 & 0.0324 & 0.0616 & 0.1178 \\
\hline & 0.15 & 0.0121 & 0.0238 & 0.0350 & 0.1302 \\
\hline
\end{tabular}

Even though the values in Table 9-1 are obtained with monotonic moment-curvature analyses, they represent the first-cycle envelope curves for walls subjected to cyclic loading because of the steel material law used. Furthermore, as discussed in Section 9.4.6, since the experimental database used to calibrate the steel model includes a 
large number of wall specimens that had vertical reinforcement lap-spliced near the base, the steel model, to a certain extent, indirectly accounts for the influence of the lap splices but not the splice failure.

To produce a similar table for wall sections subjected to monotonically increasing moments, analyses have been repeated with steel properties that reflect behavior under monotonic loading, which essentially consist of a higher tensile strength and strain at the peak stress as shown in Figure 9-12. The results are shown in Table 9-2. Similar to the observations in Section 9.4.7, wall sections with a low reinforcing index and axial load ratio tend to show significantly more ductile behavior under monotonically increasing moments than under cyclic moment reversals. The influence of the loading history becomes negligible when the values of the reinforcing index and the axial load ratio increase. However, because of the lack of test data to confirm these results, the values in Table 9-2 should be used with caution.

\section{Table 9-2 Nondimensionalized Moment-Curvature Envelope Values for Fully Grouted Rectangular Wall Sections Subjected to Monotonically Increasing Loading}

\begin{tabular}{|c|c|c|c|c|c|}
\hline $\begin{array}{l}\text { Reinf. Index } \\
\alpha=\left(f_{y} / f^{\prime} m\right) \rho_{v}\end{array}$ & $\begin{array}{c}\text { Axial Compression Ratio } \\
\quad \beta=P /\left(f^{\prime} m A_{n}\right)\end{array}$ & $\phi_{m} l_{w}$ & $\phi_{75} / w$ & $\phi c l_{w}$ & $M_{\max } /\left(f_{m}^{\prime} A_{n} l_{w}\right)$ \\
\hline \multirow{4}{*}{0.01} & 0 & 0.1083 & 0.1446 & 0.1731 & 0.0066 \\
\hline & 0.05 & 0.0488 & 0.2028 & very large & 0.0285 \\
\hline & 0.1 & 0.0225 & 0.0509 & very large & 0.0487 \\
\hline & 0.15 & 0.0151 & 0.0289 & 0.0435 & 0.0663 \\
\hline \multirow{4}{*}{0.05} & 0 & 0.1172 & 0.1582 & 0.1901 & 0.0304 \\
\hline & 0.05 & 0.0361 & 0.1728 & 0.3226 & 0.0476 \\
\hline & 0.1 & 0.0203 & 0.0416 & 0.2789 & 0.0655 \\
\hline & 0.15 & 0.0139 & 0.0271 & 0.0409 & 0.0811 \\
\hline \multirow{4}{*}{0.1} & 0 & 0.0511 & 0.1736 & 0.2249 & 0.0516 \\
\hline & 0.05 & 0.0250 & 0.0638 & 0.2933 & 0.0682 \\
\hline & 0.1 & 0.0173 & 0.0378 & very large & 0.0836 \\
\hline & 0.15 & 0.0127 & 0.0261 & 0.0384 & 0.0979 \\
\hline \multirow{4}{*}{0.15} & 0 & 0.0331 & very large & very large & 0.0723 \\
\hline & 0.05 & 0.0209 & 0.0545 & 0.2859 & 0.0875 \\
\hline & 0.1 & 0.0152 & 0.0350 & very large & 0.1015 \\
\hline & 0.15 & 0.0116 & 0.0248 & 0.0364 & 0.1148 \\
\hline \multirow{4}{*}{0.2} & 0 & 0.0236 & 0.1072 & very large & 0.0913 \\
\hline & 0.05 & 0.0182 & 0.0476 & 0.2840 & 0.1048 \\
\hline & 0.1 & 0.0137 & 0.0325 & 0.0619 & 0.1181 \\
\hline & 0.15 & 0.0121 & 0.0239 & 0.0352 & 0.1304 \\
\hline
\end{tabular}




\subsubsection{Comparison of Backbone Curves with Test Data for Fully Grouted Walls}

Backbone load-displacement curves are constructed for the 21 flexure-dominated wall specimens summarized in Appendix E.1 using the procedure presented in Section 9.5.1 and the dimensionless moment and curvature values presented in Table 9-1. Select results are shown in Figures 9-25 through 9-27, and the rest are shown in Appendix E.1. For comparison, backbone curves determined with the current recommendations in ASCE/SEI 41-13, Seismic Evaluation and Retrofit of Existing Buildings, (ASCE, 2014) are also shown in the figures. The proposed backbone curves show good correlation with the test data, while the curves based on ASCE/SEI 41-13 show overly brittle behavior. The mismatch between the proposed curve and the test result for wall UT-W-13, as shown in Figure 9-27(a), can be attributed to the failure of the lap splices, which is not captured by the model, as explained in Section 9.4.5. Results for wall UT-W-17, as shown in Figure 9-27(b), suggest that numerical results obtained with the proposed method can significantly overestimate the wall resistance when the story drift exceeds $4 \%$. Hence, it is recommended that that a $4 \%$ cap be imposed on the maximum allowable drift. Otherwise, except for a few cases as shown in Appendix E.1, the proposed curves seem appropriate for drift levels less than $4 \%$.

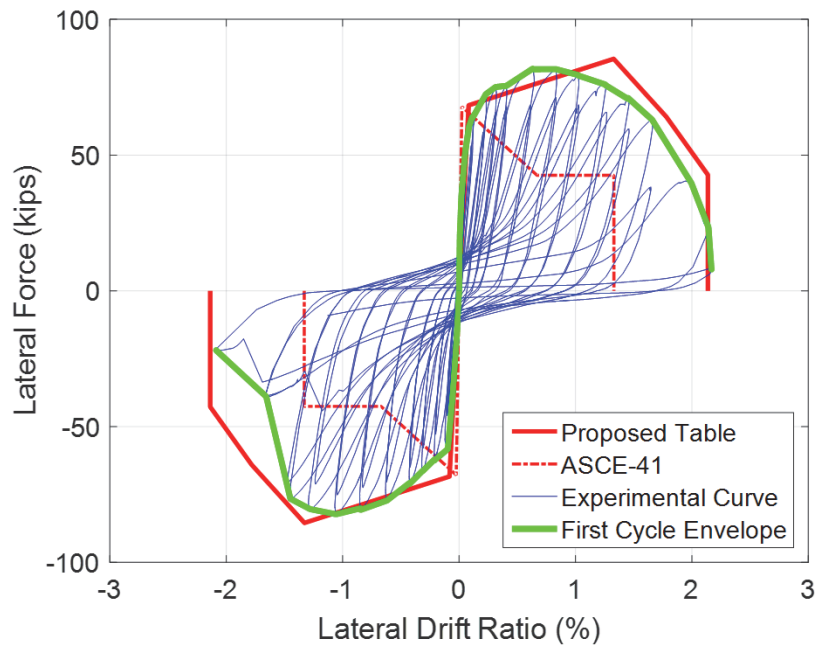

(a) Wall UT-PBS-03, $\rho_{v}=0.33 \%$

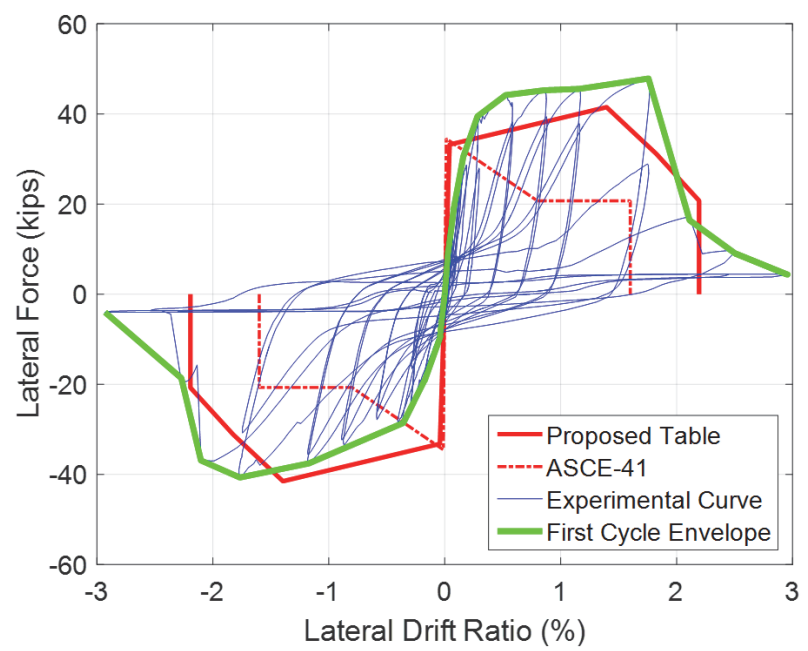

(b) Wall UT-PBS-04, $\rho_{v}=0.16 \%$

Figure 9-25 Comparison of proposed backbone curves with experimental data for flexure-dominated walls with an aspect ratio of one and zero axial load.

The discrepancies between the backbone curves calculated with the proposed method and the test results are quantified in terms of the coordinates of four control points, A, B, C, and D, on the idealized backbone curve, as shown in Figure 9-19. For this purpose, experimental backbone curves representing the first-cycle envelopes of the hysteresis curves from wall tests are obtained, and the control points are identified on the backbone curves. An idealized experimental backbone curve is then constructed 
by connecting the control points with straight lines. Figure 9-28 shows one example comparing an idealized backbone curve with that calculated by the analytical method.

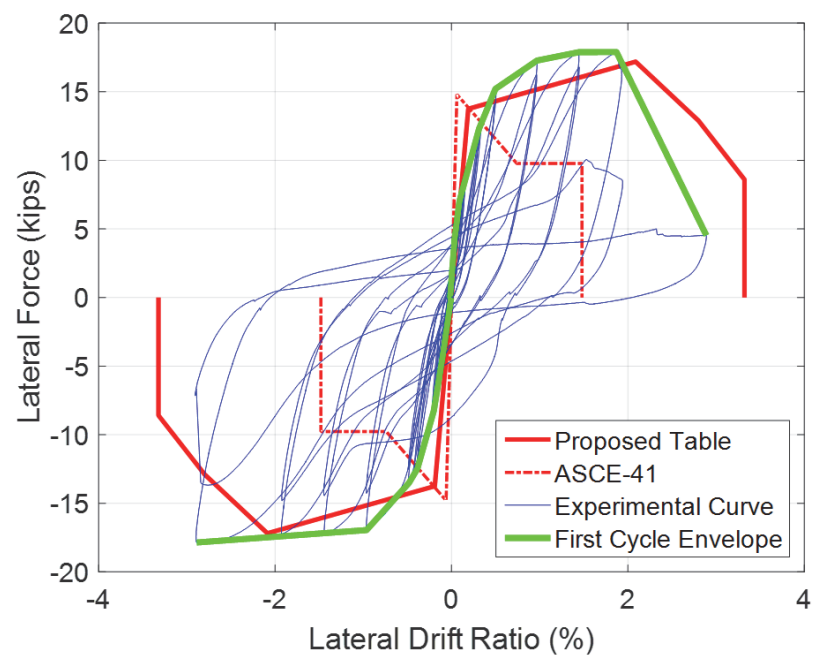

(a) WSU-W-07(C1), zero axial load

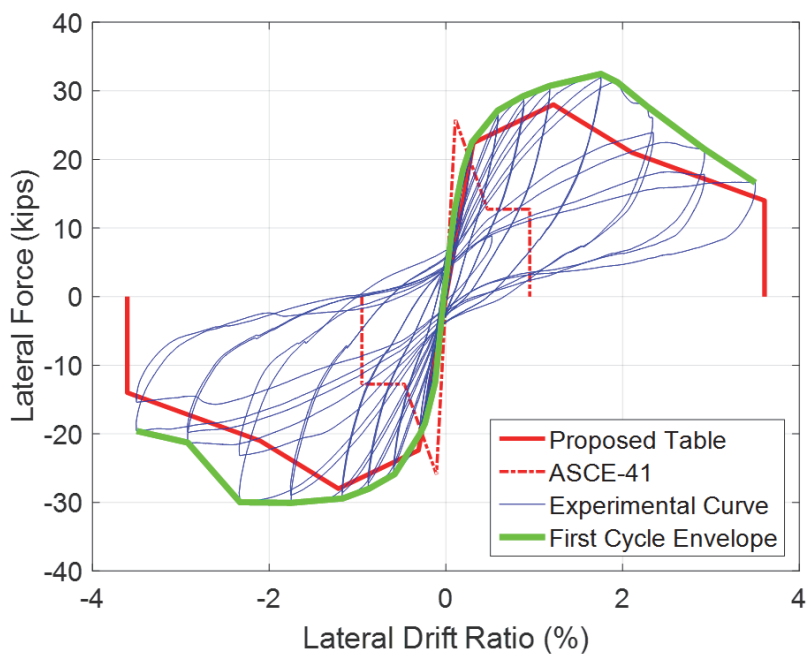

(b) WSU-W-08(C2), axial load ratio of $6.25 \%$

Figure 9-26 Comparison of proposed backbone curves with experimental data for flexure-dominated walls with an aspect ratio of two and $\rho_{v}=0.33 \%$.

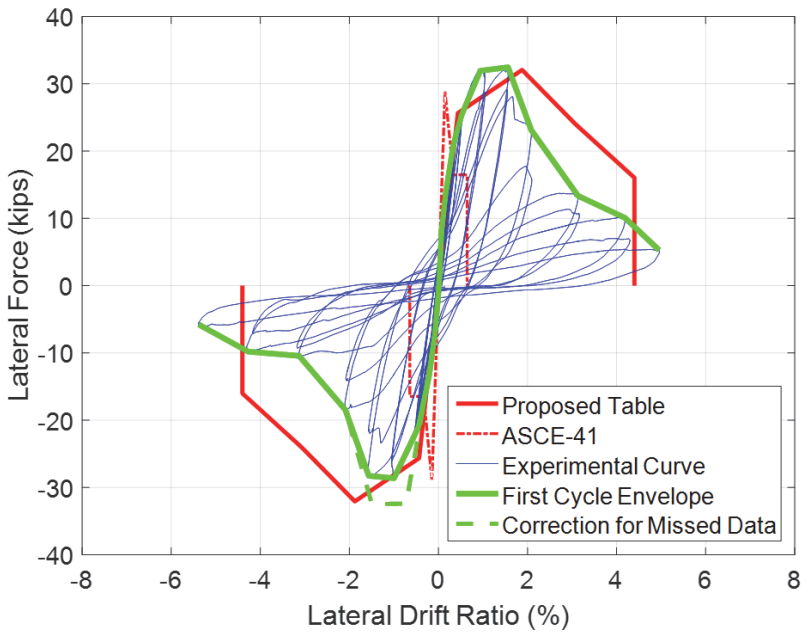

(a) UT-W-13, aspect ratio of 3

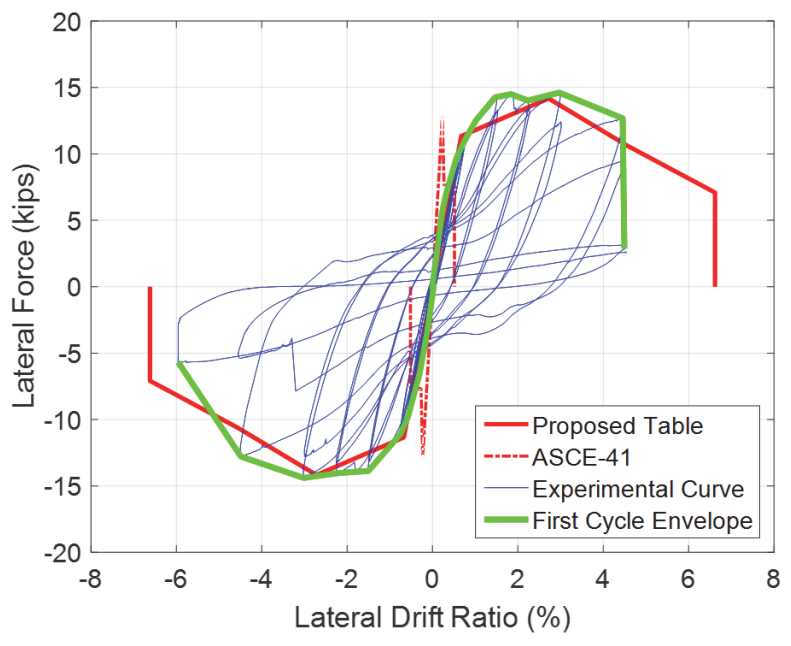

(b) UT-W-17, aspect ratio of 4.5

Figure 9-27 Comparison of proposed backbone curves with experimental data for flexure-dominated walls with an axial load ratio of $5 \%$ and $\rho_{v}=0.72 \%$.

The control points are given by the values of $Q_{\max }, \Delta_{m}, \Delta_{75}$, and $\Delta_{c}$, as shown in Figure 9-19. The discrepancies between the experimental and calculated backbone curves are expressed in terms of the ratios of the above values calculated for the respective control points to the corresponding values identified from the experimental backbone curves. Data from 32 wall tests are considered, including the 21 wall specimens shown in Table E-1, which have been used for the calibration of the material models and analysis method used in the calculations. Eleven wall specimens from the same sources are added to provide a more objective assessment of the method. The mean, 
median, standard deviation, and coefficient of variation for each of these ratios are presented in Table 9-3.

It can be seen that the values of $Q_{\max }$ are well predicted with a mean ratio of 1.02 and a coefficient of variation of 0.11 . However, the displacement ratios have a much larger dispersion. Since no experimental data are available for walls subjected to monotonically increasing loads, such evaluation cannot be performed on backbone curves constructed with the values in Table 9-2.

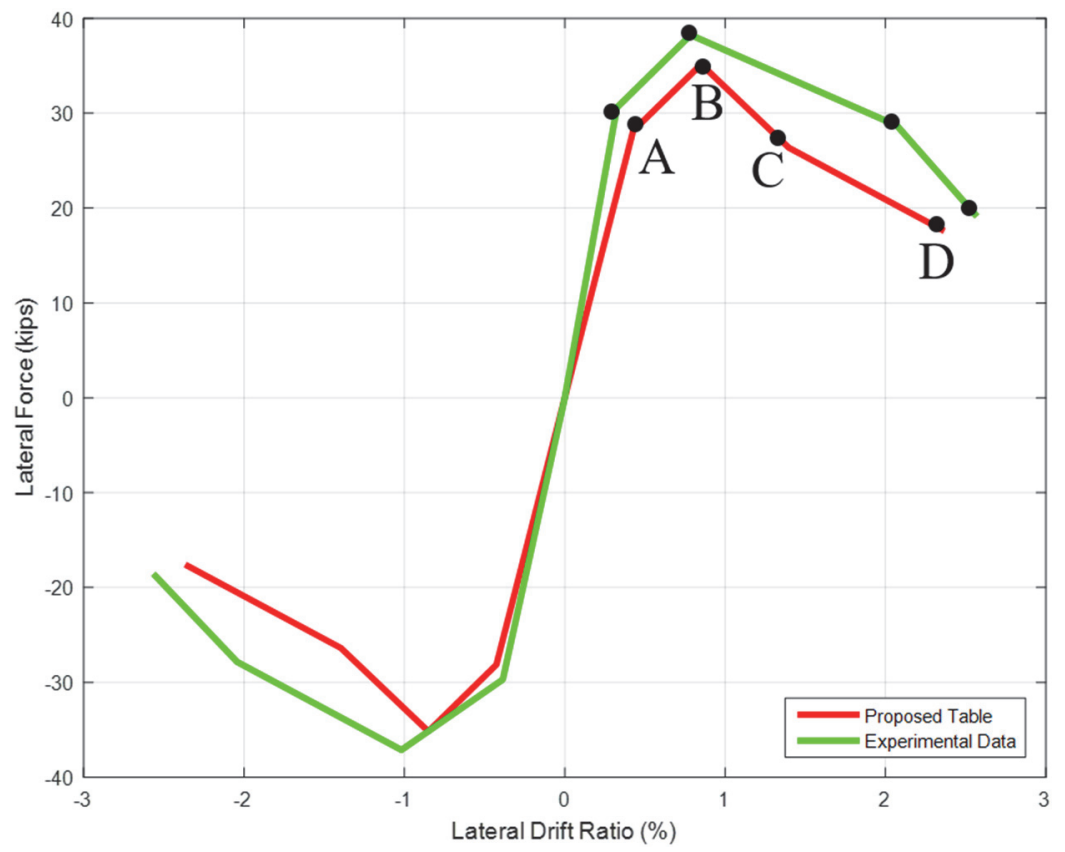

Figure 9-28 Backbone curves for wall WSU-W-2B.

Table 9-3 Statistical Data on Ratios of Calculated Values to Corresponding Experimental Values for Control Points on Backbone Curves

\begin{tabular}{|c|c|c|c|c|c|}
\hline & \multicolumn{5}{|c|}{ Calculated/Experimental } \\
\hline & $Q_{\max }$ & $\Delta y$ & $\Delta_{m}$ & $\Delta_{75}$ & $\Delta_{c}$ \\
\hline Mean & 1.02 & 0.84 & 1.05 & 0.94 & 1.2 \\
\hline Median & 1.02 & 0.78 & 0.93 & 0.92 & 1.11 \\
\hline Standard Dev. & 0.11 & 0.36 & 0.48 & 0.31 & 0.36 \\
\hline Coef. of Variation & 0.11 & 0.43 & 0.46 & 0.33 & 0.30 \\
\hline
\end{tabular}

\subsubsection{Additional Remarks}

Flanged walls have additional variables to consider, such as the configuration of the cross-section, the lengths of the flanges, and the amount of vertical reinforcement in a flange as compared to that in the web. These walls have a lot more variables to 
consider as compared to rectangular wall sections. The same applies to partially grouted walls, which can have a wide range of spacing of reinforcement and grouted cells. To construct backbone curves for those walls, moment-curvature analyses have to be conducted for the specific wall section under consideration.

\subsection{Backbone Force-Displacement Curves for Shear-Dominated Walls}

\subsubsection{Idealized Backbone Curves for Fully Grouted Walls}

For fully grouted shear-dominated walls, the strength and ductility depend on the wall aspect ratio, the amount of horizontal and vertical reinforcement, and the axial compressive load. The influence of the vertical reinforcement is through the dowel action. The shear strength can be calculated with the formulas given in TMS 402 (TMS, 2013), which ignores the dowel action of the vertical steel. As to the ductility of a wall, experimental results have shown that walls with higher amounts of horizontal reinforcement and lower axial loads tend to be more ductile. However, data on the influence of the axial compressive load on the ductility of sheardominated walls are not entirely conclusive. For this reason, it is ignored here.

Based on the test data of Voon and Ingham (2006), Ahmadi (2012), and Shing et al. (1991), the backbone lateral load-vs.-story drift ratio curve shown in Figure 9-29 is proposed for fully grouted shear-dominated walls.

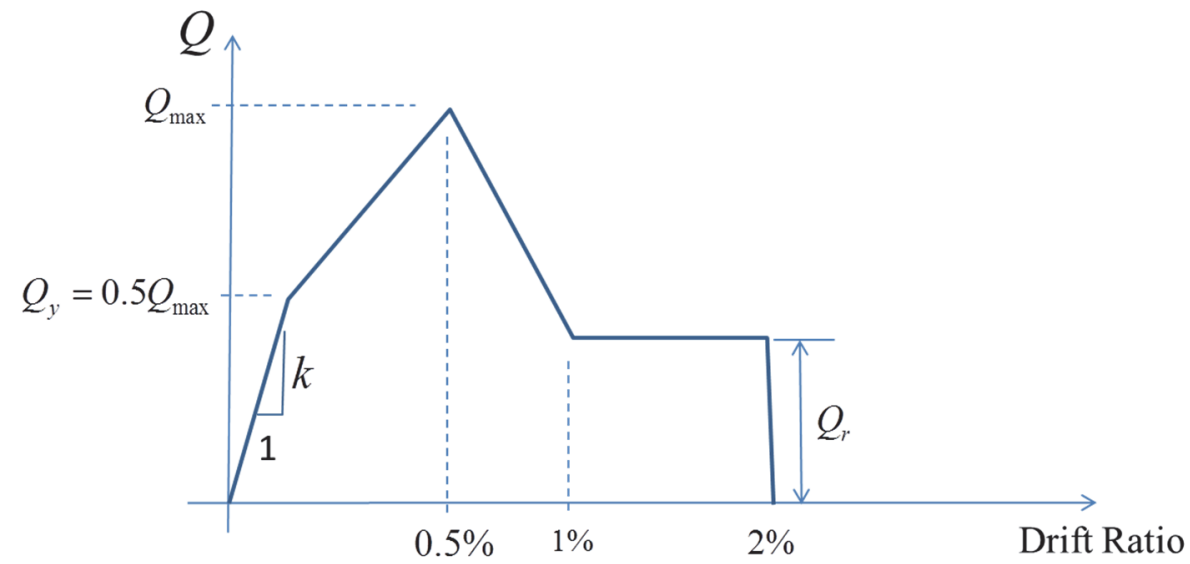

Figure 9-29 Backbone lateral load-vs.-story drift ratio curve for sheardominated reinforced masonry walls.

For a cantilever wall, the initial stiffness $k$ can be calculated with Equation 9-9. For a wall with fixed-fixed end conditions, the flexural flexibility term in that equation should be modified accordingly. According to the shear strength formula in TMS 402, $Q_{\max }$ can be calculated as follows:

$$
Q_{\max }=V_{n}=V_{n m}+V_{n s}
$$


where:

$$
\begin{gathered}
V_{n m}=\left[4.0-1.75 \frac{M}{V l_{w}}\right] A_{n} \sqrt{f_{m}{ }^{\prime}}+0.25 P \\
V_{n s}=0.5 \frac{A_{v}}{s} f_{y} d_{v}
\end{gathered}
$$

where $V_{n m}$ is the shear strength provided by the masonry, $V_{n s}$ is the resistance of the shear reinforcement, $M / V l_{w}$ is the shear-span ratio of the wall, $A_{n v}$ is the net shear area of the wall (only the net cross-sectional area of the web should be considered for a flanged wall), $P$ is the axial load (positive for compression), $A_{v}$ is the crosssectional area of shear reinforcement in one masonry course, $s$ is the vertical spacing of the shear reinforcement, and $d_{v}$ is the actual depth of the wall in the direction of shear and is equal to the wall length $l_{w}$. It is recommended that $V_{n m}$ be zero when $P$ is a tension force in lieu of the specification in TMS 402 that $P$ be negative for tension. The residual strength $Q_{r}$ is assumed to be entirely provided by the shear reinforcement. Therefore, the following formula can be used.

$$
Q_{r}=V_{n s}=0.5 \frac{A_{v}}{s} f_{y} d_{v}
$$

The maximum allowable drift ratio of $2 \%$ used here is based on the recommendation in ASCE/SEI 41-13, which appears to be overly unconservative as compared to quasi-static wall test data but is deemed adequate if the behavior of an entire wall system is considered. Shake-table tests of multi-story fully grouted reinforced masonry wall structures (Stavridis et al., 2016; Mavros et al., 2016) have shown that shear-dominated walls could sustain story drift ratios beyond $1.5 \%$ while retaining a good portion of their lateral resistance. This could be partly attributed to the wall flanges and partly to their interaction with other gravity load carrying elements.

\subsubsection{Comparison of Backbone Curves with Test Data for Fully Grouted Walls}

The backbone curve shown is Figure 9-29 is compared to the test data of Voon and Ingham (2006), Ahmadi (2012), and Shing et al. (1991). It can be seen that the backbone curves proposed here match the test results much better than those based on ASCE/SEI 41-13, which tend to result in more brittle behavior. The properties of the 16 wall specimens selected are shown in Table E-2 in Appendix E.2. The shear-span ratios, $M / V l_{w}$, of these walls range from 0.5 to 2 , with most of the walls having $M / V l_{w}=1$. They are fully grouted concrete masonry walls. The backbone curves constructed with the proposed method are compared to the test data in Figure 9-30 for select wall specimens. The backbone curves constructed with the ASCE 41 recommendation are also shown for comparison. The complete set of results is presented in Appendix E.2. 


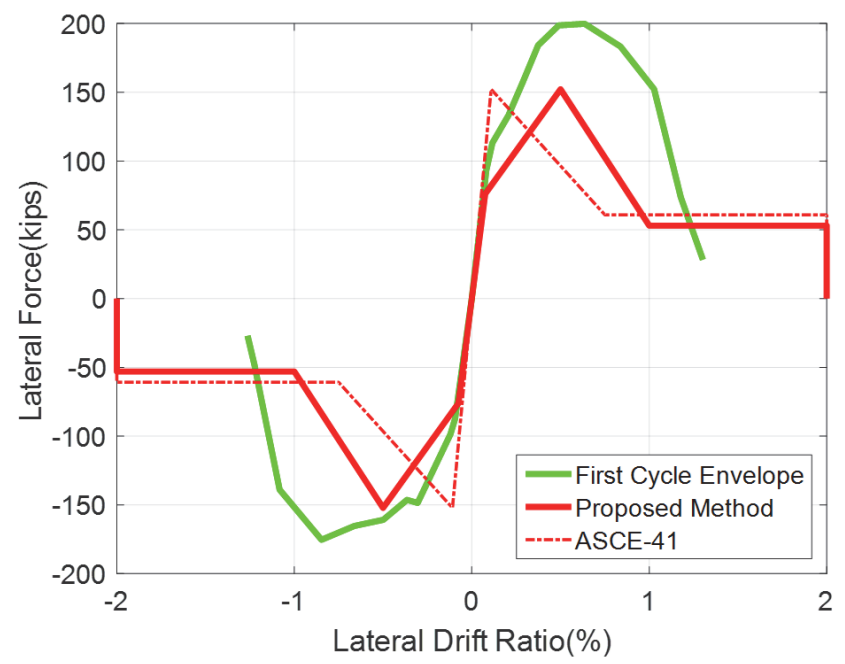

(a) UT-PBS-02, $M / V l_{w}=0.5$

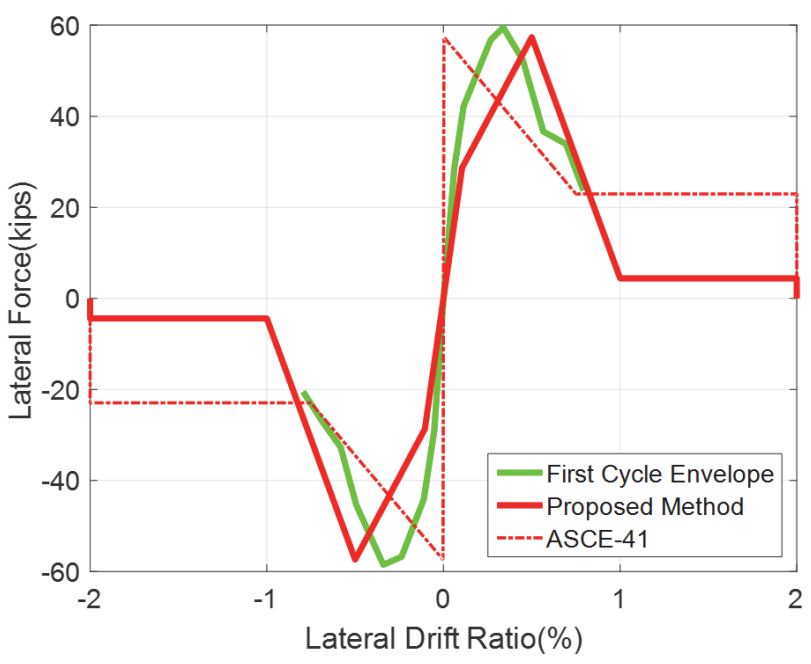

(b) Auckland-7, $M / V l_{w}=1.0$

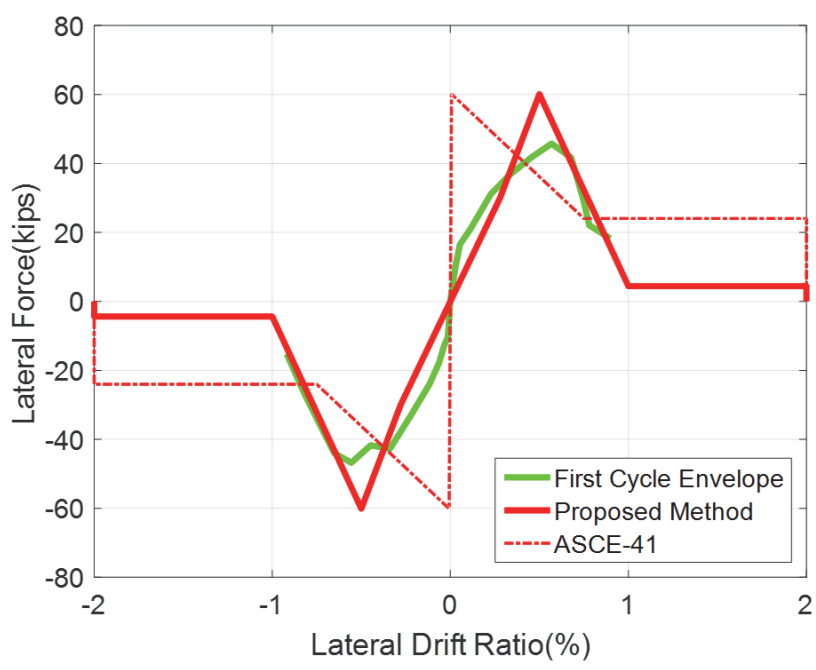

(c) Auckland-9, $M / V l_{w}=2.0$

Figure 9-30 Comparison of proposed backbone curves with experimental data for shear-dominated reinforced masonry walls.

\subsubsection{Idealized Backbone Curves for Partially Grouted Walls}

In addition to the parameters mentioned for fully grouted walls, the strength and ductility of a shear-dominated partially grouted wall are heavily influenced by the spacing of the grouted cells. Studies (Minaie et al., 2010; Bolhassani, 2015) have shown that Equation 9-18 can significantly overestimate the shear strength of partially grouted walls. While the spacing of the grouted cells has an important influence, the 2013 Edition of TMS 402 has introduced a reduction factor of 0.75 to the shear strength calculated with Equation 9-18 for partially grouted walls, regardless of the grouted-cell spacing.

Furthermore, partially grouted walls can have significantly lower displacement capacity than fully grouted walls. Test data from Minaie et al. (2010) and Bolhassani 
(2015) have shown that the story drift ratios at which the peak strengths of partially grouted walls were reached were between 0.2 and $0.5 \%$, depending on the spacing of the grouted cells, the aspect ratios of the walls, and whether there were side-by-side double grouted cells. Walls with side-by-side double grouted cells were more ductile. Walls with an aspect ratio of one and a wide spacing of grouted cells (of 72 inches or more) had the peak strength occurring at a drift ratio of $0.2 \%$.

Based on the limited experimental data discussed above, it is recommended that the backbone curve shown in Figure 9-29 for shear-dominated fully grouted walls be modified as follows for partially grouted walls. The shear strengths, $Q_{\max }$ and $Q_{r}$, of partially grouted walls can be calculated with Equation (9-18) and reduced by a factor of 0.75 as suggested by TMS 402 (TMS, 2013). The drift at the peak strength $Q_{\max }$ should be reduced from $0.5 \%$ to $0.2 \%$, and that corresponding to $Q_{r}$ be reduced from $1 \%$ to $0.4 \%$. The maximum allowable drift is to be capped at $0.8 \%$ rather than $2 \%$. 



\section{Chapter 10}

\section{Wood Shear Wall Systems}

\subsection{Overview of Geometry and Behavior}

Wood shear walls are the primary elements of the seismic force-resisting system in virtually all light-frame wood buildings. Wood shear walls are an assembly of a number of components including dimension lumber (e.g., $2 \times 4,2 \times 6$ ), sheathing (straight or diagonal boards, plaster, gypsum wallboard, plywood or oriented strand board (OSB)), fasteners (typically nails), and often, hold down devices. Larger nails are used to assemble the dimension lumber framing by nailing through the top plate and sill plate into the end grain of the vertical wood members. The strength of these nails in the end grain is very low and pull out occurs regularly. The framing is often assembled on the ground and then erected and placed into the building as a unit prior to attaching sheathing to one or both sides, unless the shear walls were preassembled offsite, a method becoming more common. Figure 10-1 shows a schematic of a typical wood shear wall with finish materials.

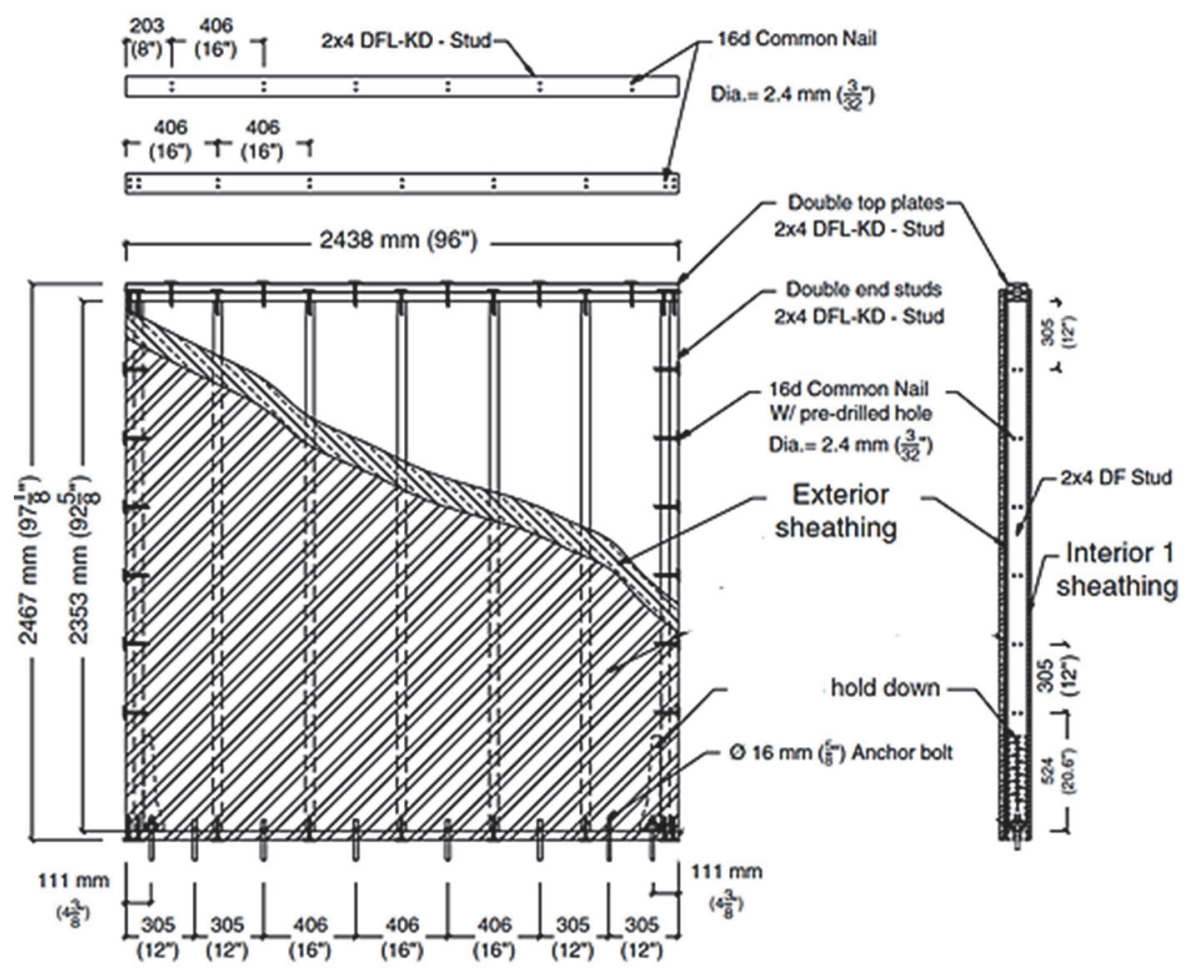

Figure 10-1 Typical components of a wood shear wall with conventional hold downs (Bahmani and van de Lindt, 2014). 
The sill plate is fastened to the foundation or floor system using anchor bolts which are usually 0.5 inches to 0.625 inches in diameter. A key component of modern engineered wood shear wall is the addition of overturning restraint at the shear wall end posts. Overturning/uplift restraint can take several different forms. Hold downs, illustrated in Figure 10-1, are steel bracket-like devices that connect to the inside of the vertical end posts with bolts or wood screws and then receive a bolt vertically from the foundation or floor system below. Figure 10-2 presents a schematic of a wood shear walls assembly with a continuous rod type hold down device.

Continuous rod systems are typically installed in taller wood-frame buildings (sometimes three stories and higher) and consist of steel rods with bearing plates at the end posts of the shear walls stacks. Bearing plates provide the transfer of uplift forces from the wall to the steel rod and into compression on the sill plate. The rods are located between the studs making up the stud pack at the ends of the designated shear walls.

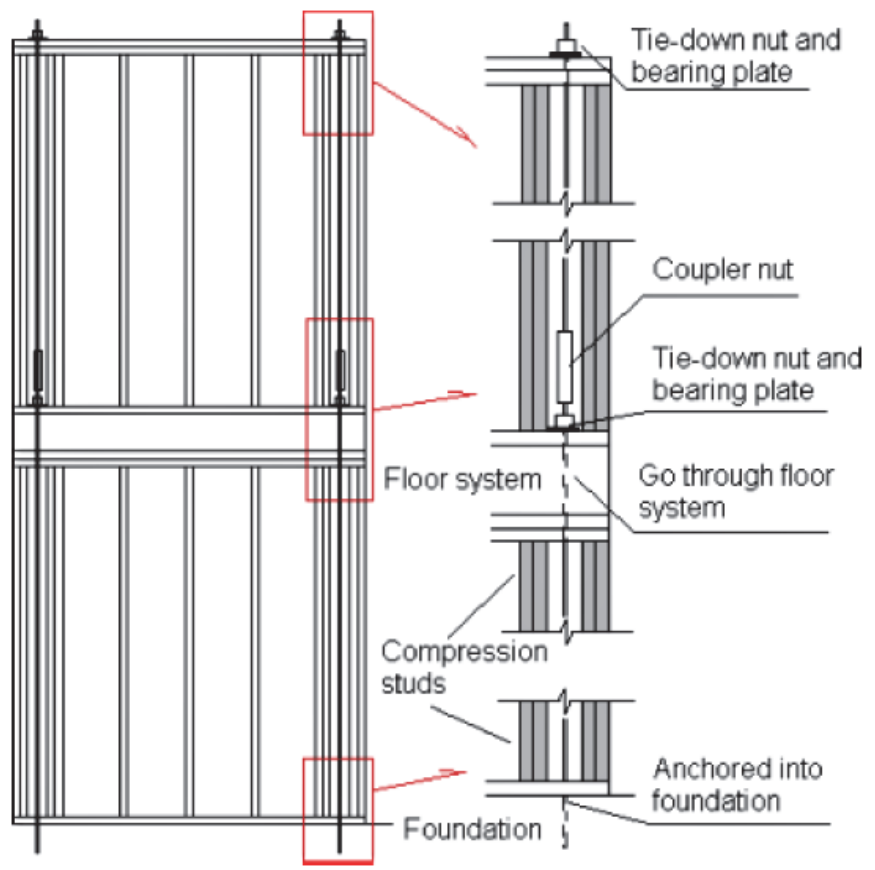

Figure 10-2 Illustration of a stacked wood shear wall system (Pei and van de Lindt, 2009).

Wood shear wall resistance varies depending on the sheathing type, nail schedule, and nail type used. In high seismic regions of the United States, modern building codes require structural panel sheathing (plywood or OSB) with $8 \mathrm{~d}$ or $10 \mathrm{~d}$ common nails used for sheathing-to-framing connections, and nail spacing selected depending on required shear capacity. Structural panel sheathing is available in $4 \times 8$ sheets ranging from $7 / 16$ inches to $1-1 / 8$ inches in thickness, with the most common thicknesses 7/16 inches, 15/32 inches, and 5/8 inches. Nail spacing around the perimeter of each sheathing panel is either 6 inches, 4 inches, 3 inches, or 2 inches on-center, with specific rules governing edge spacing and when to stagger to avoid 
splitting of the vertical studs. Field nailing, which connects the sheathing to the vertical studs in the interior of the panel, is typically spaced at 12 inches and is used mainly to prevent buckling of the panel, with little direct contribution to the shear capacity.

\subsection{Summary of Design Code Provisions}

The design of wood diaphragms was first introduced in design codes in the United States in the 1949 Uniform Building Code (UBC) (ICBO, 1949), while the first horizontal fully blocked plywood diaphragm properties (i.e., plywood thickness, nail size and framing member width) were tabulated in the 1952 UBC edition (ICBO, 1952). In the 1955 UBC revision (ICBO, 1955), a maximum diaphragm dimension ratios table was introduced for horizontal and vertical diaphragms, which was the first time that the code starts permitting unblocked wood diaphragms. No significant changes were made in the 1958 and 1964 UBC (ICBO, 1958; 1964) editions, while information on the design of unblocked wood diaphragms was first tabulated in the 1961 UBC (ICBO, 1961). The 1967 UBC edition (ICBO, 1967) permitted the use of both blocked and unblocked diaphragms, and two different tables were included in this edition, one for the design of horizontal plywood diaphragms and one for the design of wood shear walls. Although seismic design requirements in general have undergone substantial changes since 1971, particularly with regard to required strength levels, design criteria specific to wood shear wall design have evolved slowly over the last five decades. The 1976 UBC (ICBO, 1976) set specific requirements to limit earthquake induced story drift, though this was commonly neglected by many designers of light wood-frame buildings. The 1982 UBC (ICBO, 1982) permitted reduced design forces for light frame structures, three stories or less in height, where plywood sheathing was used on shear walls and diaphragms. The 1988 UBC (ICBO, 1988) permitted this reduction in design forces regardless of diaphragm sheathing used. This code also required that plywood sheets be at least 4 foot by 8 foot, and required shear wall sheathing in high seismic areas, i.e., Seismic Zones 3 and 4, to be blocked. The 1994 UBC (ICBO, 1994) included aspect ratio limits for high seismic regions. The 2000 International Building Code (ICC, 2000) prohibited the use of sheathing materials other than wood structural panels in Seismic Design Categories E and F, or in buildings exceeding three stories in height in Seismic Design Category D. This code also transcribed a formula for computation of shear wall deflections and specifically required its use, formalizing the requirement to compute story drift that had been introduced many years earlier. The 2006 IBC (ICC, 2006) introduced the concept of perforated shear walls, where reductions in allowable shear capacity are provided for perforated walls when local chords are not provided around the openings. The 2006 IBC also prescribed the use of plate washers on the anchor bolts for shear walls in Seismic Design Categories D, E, and F to minimize the potential for sill plate splitting under seismic response. Unblocked 
wood structural panels for seismic design appeared first in the 2008 Special Design Provisions for Wind and Seismic (AWC, 2008).

Although the building codes have changed relatively little with regard to design criteria for wood shear walls, design practice has evolved substantially over the years. Prior to the mid-1970s, many designers of light wood-frame structures neglected overturning effects and sized wood walls only for shear. Within a line of force, shear was commonly apportioned to different walls based on their length, neglecting flexural effects on wall stiffness. Although the building code introduced requirements to limit story drift in the 1970s, many designers of woodframe structures neglected this requirement until the 1994 Northridge Earthquake resulted in collapse of several structures, due to excessive deflection. Following the Northridge Earthquake, designers began to more rigorously consider deflection in design and also to apportion loads between walls based on relative stiffness, rather than just length.

\subsection{Performance and Damage Progression}

Light-frame wood buildings have historically performed well during ground shaking with regards to life safety. This is primarily due to their high relative strength to weight ratio as their name implies, and their high deformation capacity that has been shown to be many times the design-level deformations (van de Lindt, 2008). However, financial losses have been significant in recent earthquakes as a result of damage to other sheathing layers, such as gypsum wall board (GWB) and exterior stucco, particularly during the 1994 Northridge Earthquake. Life threatening damage has typically been limited to collapse of weak stories, common in residential buildings constructed over parking areas.

The relatively good performance of wood-frame buildings in earthquakes but more pointedly the large discrepancy in their strength and stiffness at the system level compared to many numerical models used for design and analysis, is at least partially the result of not including these traditionally noncontributing walls and frame members. This inherent conservatism has become embedded in design approaches but should be avoided in nonlinear modeling as the behavior of these elements can significantly affect building response. Section 10.4 of this chapter addresses this issue further.

\subsubsection{Seismic Force-Resisting Systems}

Figure 10-3a shows the lines of force for a simple wood-frame structure. The roof or building mass (stories above) accelerate in one direction requiring walls parallel to the line of action to resist the shear forces. In design, certain walls are designated as wood shear walls and together with the diaphragms, they make up the seismic forceresisting system. In Figure 10-3b, a wall with a door and window opening is shown. 
The wall segment to the left of the door is too narrow to be designated as part of the seismic force-resisting system in typical force-based design methods.

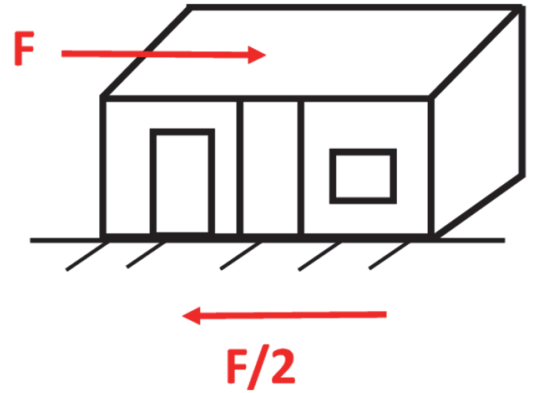

(a)

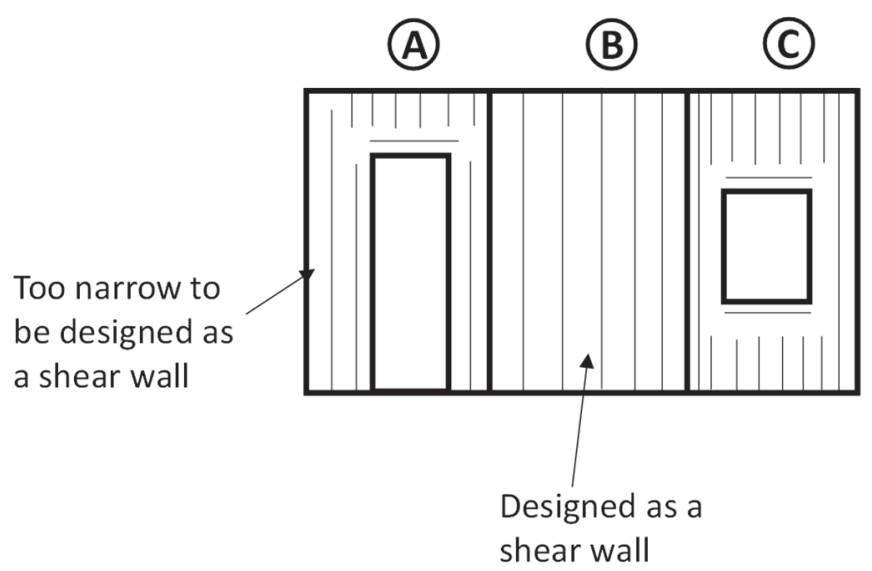

(b)

Figure 10-3 Typical wood shear wall in a wall line.

The segment labeled B in Figure 10-3b is the designated shear wall. Similar to the left side of the wall line, segment $\mathrm{C}$ would be neglected in force-based design (e.g., equivalent lateral force procedure). If the shear force in the wall was $F / 2$, this would need to be resisted solely by the length of segment $\mathrm{B}$. In reality, the wall segments around $\mathrm{A}$ and $\mathrm{C}$ and even their framing contribute to the seismic response of the wall line and entire building.

Earthquake damage to wood shear walls generally consists of the following failure mechanisms:

1. Nail withdrawal, pull through, or tear out are the most common failure mechanisms for wood shear walls. These are pictured in Figure 10-4. At large drifts, they can result in sheathing rotating off of the framing, causing instability of the wall or system as shown in Figure 10-5.

2. Splitting of the sill plate around the anchor bolts has been routinely observed in earthquakes and testing. Figure 10-6 shows a 1/2 inch wide split in the sill plate following a full-scale system level shake table test (Filiatrault et al., 2010) of a building designed to the 1988 Uniform Building Code. The result is due to the inability of shear to be transferred from the wall down into the shear wall sill plate and concrete foundation, thus resulting in a loss of wall shear capacity.

3. Excessive uplift or rocking has been observed when anchorage fails or overturning restraint is inadequate. If the hold downs fail, the result is excessive drift, which in turn can result in the failures previously described. 


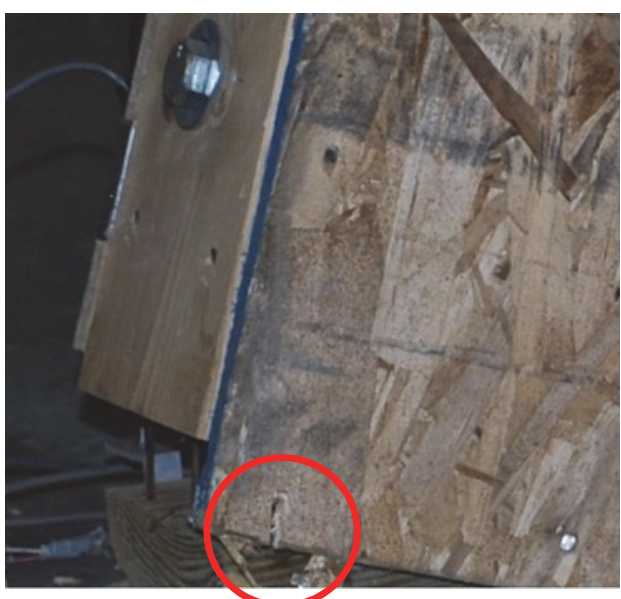

Figure 10-4 Corner nail pulling through and tearing out of the bottom edge of a 15/32" OSB sheathing panel due to excessive drift during a shear wall test.

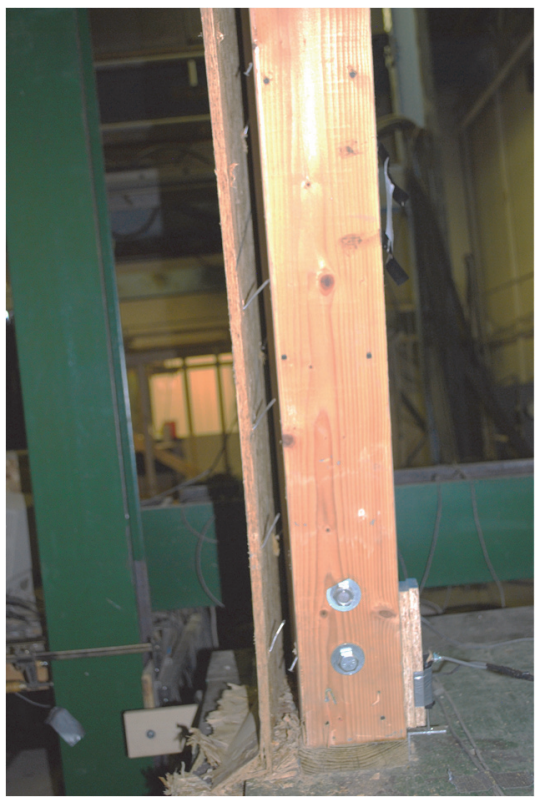

Figure 10-5 Sheathing rotating off resulting in complete loss of shear strength for the wood shear wall.

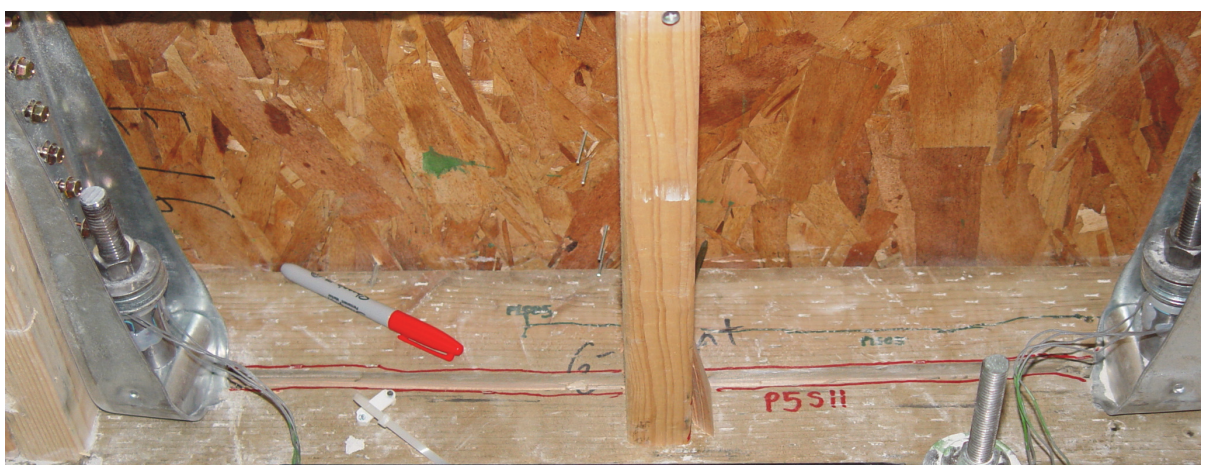

Figure 10-6 A $1 / 2$ " wide sill plate split along a garage wall during a full scale building shake table test performed by Filiatrault et al. (2010). 


\subsubsection{Finishes}

The three failure mechanisms described in 10.3.1 represent structural damage and initiate failure at the assembly or even system level, but the majority of the losses in wood-frame buildings during earthquakes are the result of damage to gypsum wallboard (GWB), stucco, and other finishes which are historically not part of the seismic force-resisting system.

Table 10-1, reproduced from Christovasilis et al. (2009), is based on full-scale testing of a wood-frame two-story townhouse with an integrated two-car garage and indicates the progression of damage at different story drifts. At small story drifts, i.e., below $1 \%$, some sheathing nail withdraw starts and there may be the start of splitting of the sill plate around the anchor bolts, but it is minor. However, at this level of story drift, the shear wall still has full capacity. At this small level of story drift, the start of diagonal cracks in the GWB extending from the corners of the window and door openings will be noticeable, particularly in lower floors where the wall drift is shear dominated. At slightly larger levels of story drift between $1 \%$ and $2 \%$, differential movement between panels is noticeable and may not return to flush after racking, the splitting of the sill plates has been observed, and the corner sheathing nails will be noticeably pulled out but not completely dislodged. Significant shear wall capacity remains but when unloading occurs, there will be stiffness and strength degradation on the returning cycle. The GWB will suffer damage at the taped joints and the corners may crush depending on the gap and location of the adjacent panel. At larger story drifts between $2 \%$ and $4 \%$, significant sill plate splitting is expected and vertical wall studs may begin to split. At larger levels of story drift, the GWB will have significant damage with screws pulling or pulled through and crushed edges. Extensive repair and replacement of most panels would be needed at this story drift level. At much larger levels of story drift from $4 \%$ to $7 \%$, corner nails will be fully or partially out and the shear wall will have little capacity remaining. The shear wall will be in its flatter hysteresis loops. Story drifts in excess of 7\% have been used in recent studies (e.g., FEMA, 2009; van de Lindt et al., 2014b) to represent collapse, but it is noted here that system responses have been observed well in excess of $7 \%$ story drift.

Additionally, the full-scale testing of a two-story wood-frame townhouse demonstrated that the garage wall line was capable of deforming to $3.5 \%$ story drift without nearing collapse. Van de Lindt et al. (2012) showed that an isolated shake table test on a one bay garage wall frame was capable of achieving almost $7 \%$ story drift with high levels of tributary weight without collapse. Testing of a four-story soft-story wood-frame building in 2013 (van de Lindt et al., 2014b) demonstrated that even these types of wood-frame buildings have deformation capacities well in excess of $10 \%$ story drift. 
If the model used for wood performance-based seismic design is as described in Section 10.4 below, then a collapse prevention drift of the order of $6-7 \%$ is recommended.

Table 10-1 Damage Progression for a Typical Modern Wood-Frame Building Based on Full-Scale Experiment (Christovasilis et al., 2009)

\begin{tabular}{|c|c|c|}
\hline $\begin{array}{l}\text { Corresponding } \\
\text { Peak Story } \\
\text { Drift (\%) } \\
\end{array}$ & $\begin{array}{l}\text { Wood Framing and } \\
\text { OSB/Plywood Sheathing }\end{array}$ & Gypsum Wall Board (GWB) \\
\hline $0.1-1.0 \%$ & $\begin{array}{l}\text { - Minor splitting and cracking of sill plates } \\
\text { (some propagation) } \\
\text { - Slight sheathing nail withdraw }\end{array}$ & $\begin{array}{l}\text { - Slight cracking of GWB } \\
\text { - Diagonal propagation from } \\
\text { door/window openings } \\
\text { - Partial screw withdraw } \\
\text { - Cracking at ceiling-to-wall } \\
\text { interface }\end{array}$ \\
\hline $1.0-2.0 \%$ & $\begin{array}{l}\text { - Permanent differential movement of } \\
\text { adjacent panels } \\
\text { - Corner sheathing nail pullout } \\
\text { - Cracking/splitting of sill/top plates }\end{array}$ & $\begin{array}{l}\text { - Crushing at corners of GWB } \\
\text { - Cracking of GWB taped/mud } \\
\text { joints }\end{array}$ \\
\hline $2.0-4.0 \%$ & $\begin{array}{l}\text { - Splitting of sill plates equal to anchor bolt } \\
\text { diameter } \\
\text { - Cracking of studs above anchor bolts } \\
\text { - Possible failure of anchor bolts }\end{array}$ & $\begin{array}{l}\text { - Separation of GWB corners in } \\
\text { ceiling } \\
\text { - Buckling of GWB at openings }\end{array}$ \\
\hline $4.0-7.0 \%$ & $\begin{array}{l}\text { - Severe damage across edge nail lines, } \\
\text { separation of sheathing } \\
\text { - Vertical posts uplifted } \\
\text { - Failure of anchor bolts }\end{array}$ & $\begin{array}{l}\text { - Large pieces separated from } \\
\text { framing } \\
\text { - Entire joints separated and } \\
\text { dislodged }\end{array}$ \\
\hline
\end{tabular}

\subsection{Recommendations for Modeling}

\subsubsection{Introduction and Challenges}

Numerical modeling of wood shear walls has been conducted since the early 1970 s with efforts in the 1980s focusing on classical finite element models, such as those developed by Cheung and Itani (1983), and more simplified models such as a five degree-of-freedom model developed by Gupta and Kuo (1987). Efforts into the 1990s included both complex models with significant degrees of freedom (e.g., Falk and Itani, 1989) to one of the first simplified single-degree-of-freedom models that used a piecewise linear segment formulation for the hysteresis (Dolan and Filiatrault, 1990). In wood shear wall modeling, there is a necessary balance between model complexity and model accuracy, and it is essential to capture key characteristics of shear wall behavior while neglecting other behaviors. Folz and Filiatrault (2001) developed a numerical model for wood shear walls under quasi-static loading. They used three structural components: rigid framing members, linear elastic sheathing 
panels, and nonlinear hysteretic sheathing to framing connectors. They then presented a procedure for calibrating a SDOF model to predict the nonlinear dynamic response of shear walls under seismic loading. Figure 10-7 presents the resulting hysteric model that has since become known as the CUREE model since it was developed as part of the CUREE-Caltech Woodframe Project. Folz and Filiartrault went on to develop and demonstrate the use of the 10-parameter model at the system level (Folz and Filiatrault, 2004a; 2004b). The 10 physically identifiable parameters are defined in Table 10-2. Other hysteretic formulations that are simpler or more complex have been developed since, but the CUREE model is widely used for wood shear walls, particularly in systems behaving as deep shear beams.

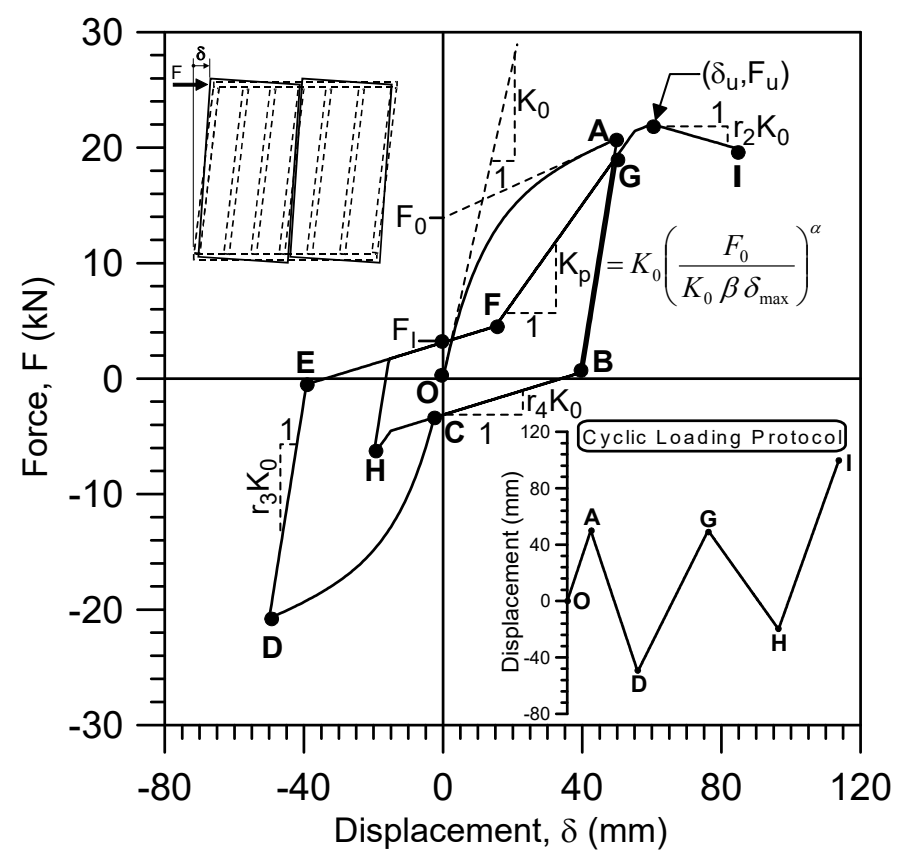

Figure 10-7 CUREE hysteretic model force-displacement response of wood shear walls under cyclic loading (Folz and Filiatrault, 2001).

Recent experimental studies of wood structural panel shear walls with additional layers of GWB, stucco, or horizontal wood siding (HWS) have identified the following as key challenges:

- The generalized force-deformation models that have been used in the past for wood structural panels (i.e., plywood or OSB) do not provide as good a fit for other sheathing materials.

- A refined combination rule is needed for walls sheathed with layers of different materials. Recent analytical studies (FEMA, 2012b, and the ongoing FEMAfunded ATC-116 project) have created backbone curves combining $100 \%$ of the capacity of the strongest wall bracing/finish material with $50 \%$ of the capacity of weaker materials. Walls with combinations of layers are thought to be stronger 
than implied by this combination rule, however, deformation capacity is reduced and strength degradation seems to occur at a lower drift. There is a lack of test data available for these combinations.

- Commercial software does not yet have a good hysteretic model that accurately pinches and degrades in a manner similar to that observed for wood walls.

Research oriented software such as OpenSees (McKenna and Fenves, 2001) provides this capability and has incorporated the 10-parameter CUREE model described earlier in this chapter.

\section{Table 10-2 Definition of Hysteretic Parameters of CUREE Hysteretic Model}

\begin{tabular}{|c|c|}
\hline $\begin{array}{c}\text { Parameter } \\
\text { in Figure 10-7 }\end{array}$ & Definition \\
\hline$K_{0}$ & Initial stiffness \\
\hline$F_{0}$ & Force intercept of the asymptotic stiffness at ultimate strength \\
\hline$F_{l}$ & Zero-displacement load intercept \\
\hline$\delta u$ & Displacement at ultimate load \\
\hline$r_{1}$ & Asymptotic stiffness ratio under monotonic load \\
\hline$r_{2}$ & Post-capping strength stiffness ratio under monotonic load \\
\hline$r_{3}$ & Unloading stiffness ratio \\
\hline$r_{4}$ & Reloading pinched stiffness ratio \\
\hline$\alpha$ & Hysteretic parameter for stiffness degradation \\
\hline$\beta$ & Hysteretic parameter for stiffness degradation \\
\hline
\end{tabular}

\subsubsection{Development of Backbone Curve Data Synthesis}

In order to address these challenges, an extensive synthesis of backbone curve data for a large number of wood shear walls incorporating different wood sheathing materials and material combinations was developed. This set of backbone curves included wood planks, drywall, stucco, plywood, gypsum, and wood siding materials. The test data considered for the backbone synthesis are mainly experimental data synthesized in FEMA P-807, Seismic Evaluation and Retrofit of Multi-Unit Woodframe Buildings with Weak First Stories (FEMA, 2012), as well as wood structural panel tests conducted by Line et al. (2008) and AWC (2016). Detailed discussion on the testing conditions of the datasets considered in the synthesis is out of the scope of this document, however, such a discussion is included in FEMA P-807 and Line et al. (2008). The shear capacity-displacement values of the monotonic backbone curves and the statistically computed values (mean/average, maximum, minimum, standard deviation and COV) of shear resistance at different drift ratios for the different wall material combinations considered are presented in Appendix F. 
Figure 10-8 presents the average, average plus, and minus one standard deviation backbone curves for each wall material combination. As shown in Figure 10-8, there is a large variation in the data considered for the various material combinations and this is mainly attributed to differences in system level effects, testing methods, including differences in loading protocols, boundary conditions, gravity load application, cyclic vs. monotonic testing as well as wall design variability (e.g., nailing size and pattern).
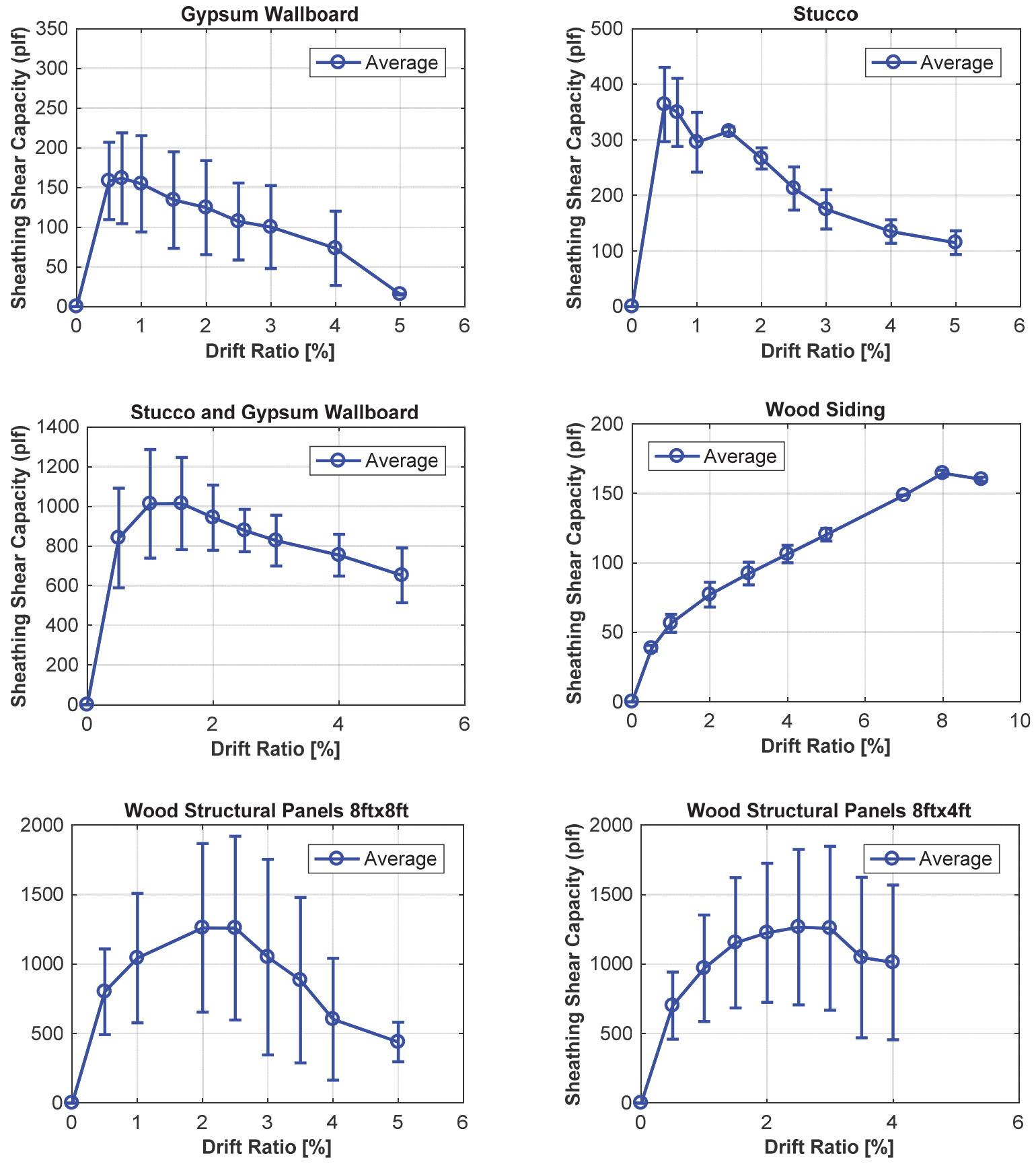

Figure 10-8 Force-displacement response of wood shear walls for varying material combinations. 
For backbone curve data of wood structural panels (WSP), it should be noted that the average backbone curve is developed from averaging the positive and negative excursions of multiple tests without simultaneously applied axial load.

It is noted that the average backbone curve may be conservative for determination of strength and drift capacity of wood shear walls in real building structures. Reasons may include lack of gravity load in testing, use of eccentric hold down details with greater eccentricity in stronger walls due to greater number of end studs, lack of tension continuity at the top of end studs, and plate material that terminated precisely at ends of walls, therefore increasing the compression deformations.

For example, per Salenikovich and Payeur (2010), the vertical loads did not significantly influence the lateral resistance of fully anchored light-frame WSP. However, with regards to drift capacity, improved drift capacity was observed with the addition of the vertical gravity load. Another study by Johnston et al. (2006), showed that the application of vertical load during testing as well as the use of hold down anchors influenced the cyclic response of WSP by limiting the displacement to \pm 3 inches as well as increasing both stiffness and energy dissipation. It was also found in this study that the current design procedures are conservative for walls that carry vertical compressive load.

Furthermore, for WSP, the allowable stress design (ASD) design strength is defined for each wall test considered (see Appendix F) and the shear strength ratio vs. drift ratio plot is developed (see Figure 10-9) for 1:1 WSP aspect ratio. This illustration avoids the appearance of "plf" variability in WSP walls due to difference nail schedules.

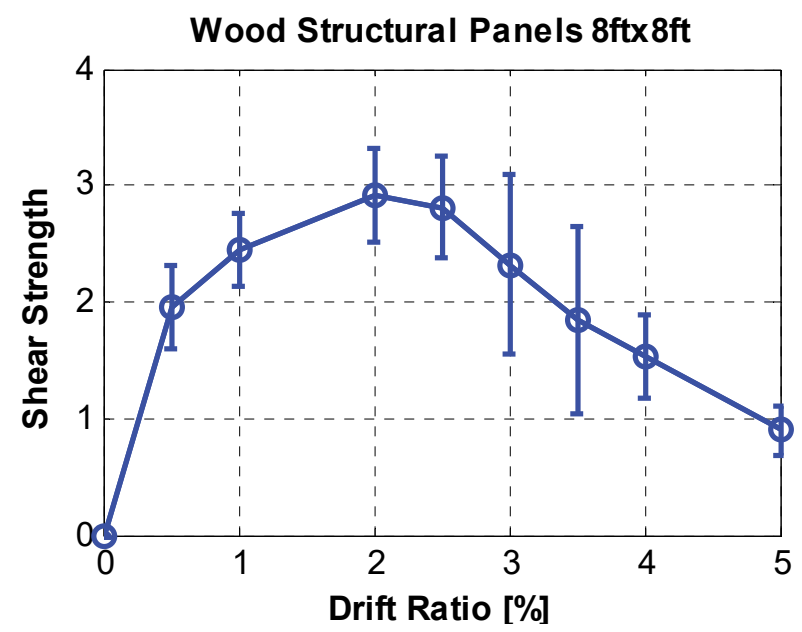

Figure 10-9 Shear strength ratio-displacement response of wood structural panels with aspect ratio 1:1.

The average and average plus and minus one standard deviation values were fit in to the 10-parameter CUREE hysteretic model. A set of MATLAB (2013) codes was 
created to optimize the hysteretic parameters associated with the CUREE hysteretic model. The optimal hysteretic parameters were computed through an identification process to match numerical and experimental (statistical) data of each wall material combination by minimizing the differences in force and deformation. The identification process was set as a constrained nonlinear least squares problem. A starting point vector, $x_{0}$, for all the hysteretic parameters associated with the CUREE model as well as lower, $l b$, and upper bound vectors, $u b$, of these parameters were defined to be used for the estimation of the force and deformation of each wall combination at each time step. The initial, upper, and lower bound values/vectors of the hysteretic properties of and CUREE model were defined based on trial and error. The Trust Region Reflective (TRR) algorithm was used to solve the least square minimization problem formed. The TRR is one of the available algorithm options in the MATLAB optimization toolbox for solving the least squares optimization problem along with the Lavenberg-Marquardt and Gauss-Newton algorithms. However, the TRR algorithm was considered in this study since it is robust and reliable (Yuan, 2000). The fitted hysteretic parameters for each wall material combination are summarized in Table 10-3, while the fitted and backbone synthesis statistical backbone curve data are plotted Figure 10-10 for illustrative purposes.

Table 10-3 CUREE Hysteretic Parameters for the Wood Wall Material Combinations Considered in this Chapter (Average Fitted Data)

\begin{tabular}{|c|c|c|c|c|c|c|c|c|c|c|}
\hline $\begin{array}{c}\text { Wood Material } \\
\text { Combination }\end{array}$ & $\begin{array}{c}K_{0} \\
{[\mathrm{lb} / \mathrm{in} / \mathrm{ft}]}\end{array}$ & $\begin{array}{c}F_{0} \\
{[\mathrm{lb} / \mathrm{ft}]}\end{array}$ & $\begin{array}{c}F_{1} \\
{[\mathrm{lb} / \mathrm{ft}]}\end{array}$ & $r_{1}$ & $r_{2}$ & $r_{3}$ & $r_{4}$ & $\begin{array}{c}\delta u \\
{[\mathrm{in} / \mathrm{in}]}\end{array}$ & $\alpha$ & $\beta$ \\
\hline Gypsum wallboard & 430 & 126 & 10 & 0.10 & -0.060 & 1.07 & 0.007 & 0.71 & 0.75 & 1.05 \\
\hline Stucco & 800 & 280 & 22 & 0.10 & -0.082 & 1.00 & 0.005 & 1.13 & 0.85 & 1.05 \\
\hline $\begin{array}{l}\text { Stucco \& gypsum } \\
\text { wallboard }\end{array}$ & 1550 & 890 & 40 & 0.10 & -0.075 & 1.00 & 0.008 & 1.13 & 0.85 & 1.05 \\
\hline $\begin{array}{l}\text { Horizontal wood } \\
\text { siding }\end{array}$ & 110 & 50 & 20 & 0.13 & -0.050 & 1.00 & 0.070 & 8.00 & 0.40 & 1.10 \\
\hline $\begin{array}{l}\text { Wood struct. panels } \\
\left(8^{\prime} \times 8^{\prime}\right)\end{array}$ & 1580 & 780 & 93 & 0.15 & -0.17 & 1.00 & 0.015 & 2.00 & 0.85 & 1.10 \\
\hline $\begin{array}{l}\text { Wood struct. panels } \\
\left(8^{\prime} \times 4^{\prime}\right)\end{array}$ & 1300 & 650 & 16 & 0.04 & -0.20 & 1.00 & 0.015 & 3.00 & 0.85 & 1.05 \\
\hline
\end{tabular}

\subsubsection{Numerical Modeling Recommendations}

\subsubsection{General Modeling Guidelines}

Wood shear walls could be modeled explicitly by modeling each individual sheathing and fastener in a variety of commercial available software. However, the variability in the parameters used for wood shear wall modeling vary from analyst to analyst and has been notable for several reasons. First, in some cases, the fitting of hysteretic parameters is conducted manually, therefore judgment is included. Furthermore, 
other sources of variability, such as wood species, moisture content, and nail brand, are often neglected, and slight differences in data introduce epistemic uncertainty; and finally, load protocols followed during experimental tests can result in data sets that are challenging to fit. In order to provide an example of typical variability, consider the single fastener tests presented in Christovasilis et al. (2007) for 8d common and $10 \mathrm{~d}$ common nails connected to $7 / 16$ inch OSB with either $2 \times 4$ or $2 \times 6$ framing as summarized in Koliou (2014) and presented in Table 10-4.
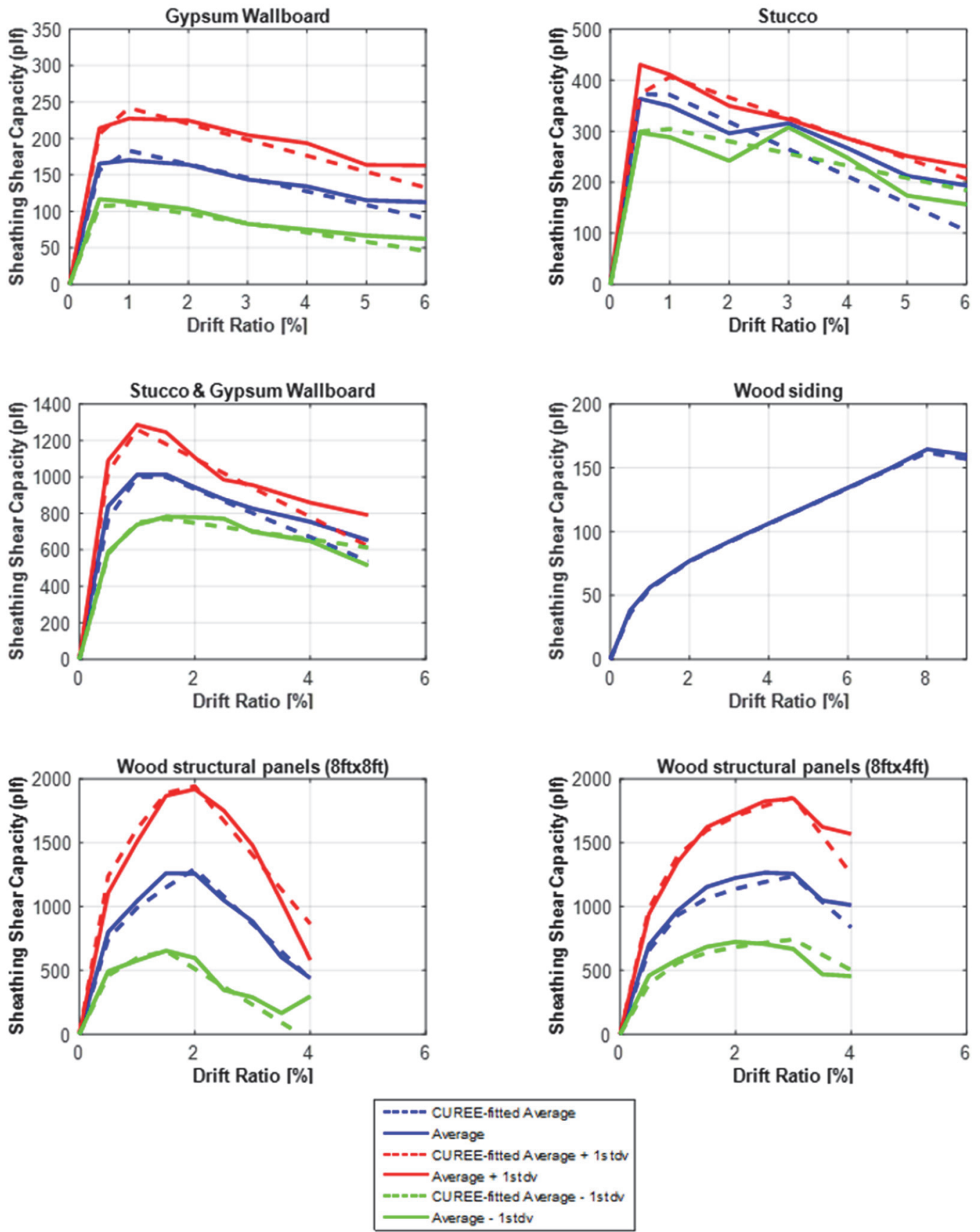

Figure 10-10 10-Parameter CUREE hysteresis model fit to data varying material wall combinations. 
The specimens used to develop the statistics were nominally identical yet the coefficients of variation (COV) can be seen to be significant for some parameters and less significant for others. Lumping the statistics for each of the combinations of nail type, dimension lumber, and panel thickness the table presents the COV's for each parameter which would serve as a more realistic level of variation for a modeler developing a wood shear wall model from fastener data.

Table 10-4 Common Wood Shear Wall Fastener Statistics for the CUREE Model

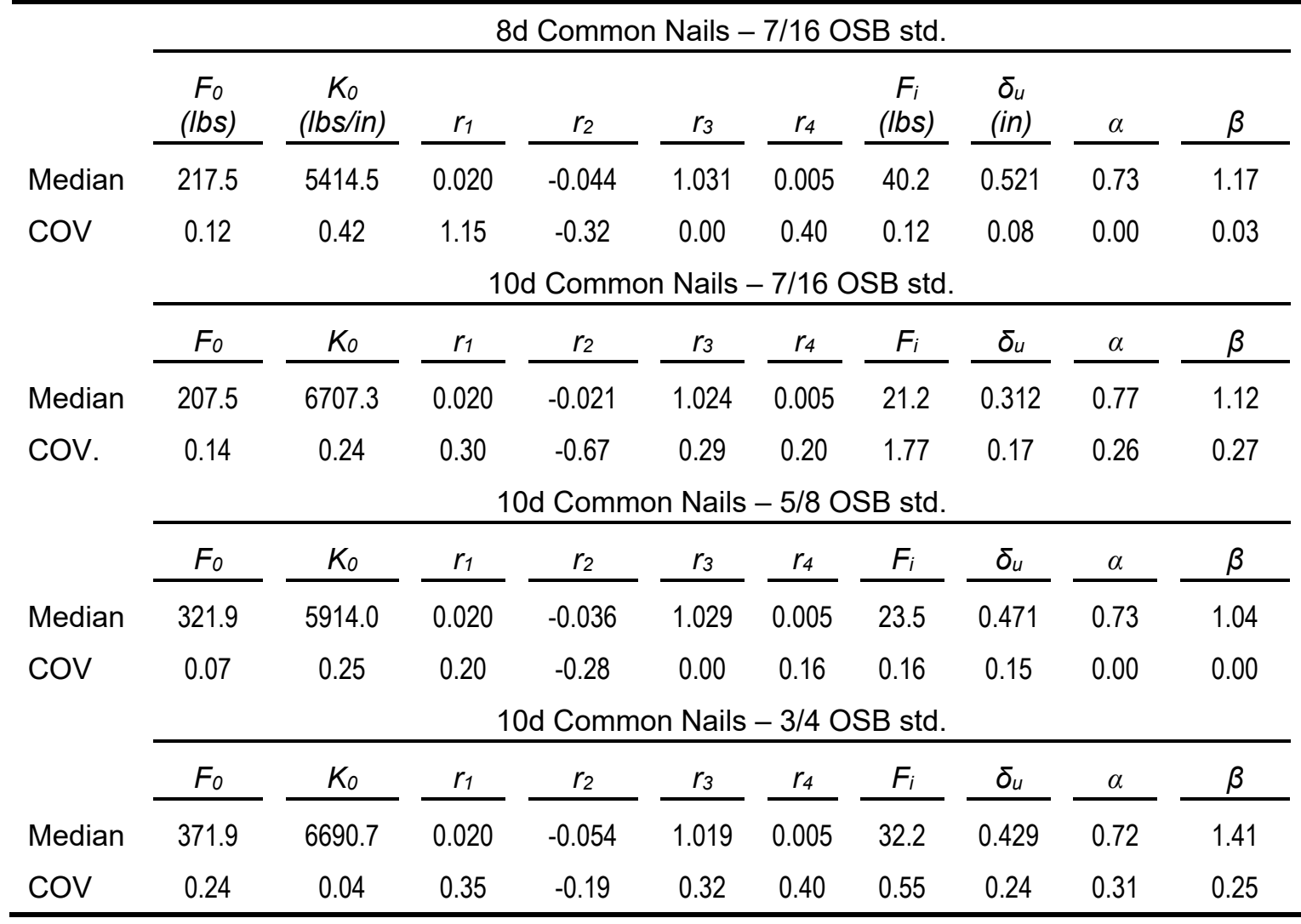

Using the wide synthesis of wood wall material combinations, the following recommendations are proposed for modeling wood shear walls and their systems:

- A hysteretic model similar to the CUREE model shown in Figure 10-7 that has a reasonable level of pinching such that it does not over predict energy dissipation should be used.

- The hysteretic model should be capable of both strength and stiffness degradation. These are important factors for post-peak drifts because this is where life safety and collapse prevention limit states currently lie.

- One of the critical parameters in wood-frame response estimation is the initial stiffness, $K_{0}$. There is some subjectivity in this estimation process, thus a more standardized approach, e.g., utilizing a formula or database developed using experimental data, would be essential for accurate modeling. 
- Because there is only a finite number of wall combinations and types that can be used with typical wood-frame retrofit and construction, a synthesis of wood shear wall combinations is recommended to aid in modeling decisions.

- The recommended hysteric properties from the 10-parameter CUREE model can be considered when using commercial software. Most of the commercially available structural analysis programs have the option of user-defined hysteretic models. In this case, the modeler can define a backbone curve based on the 10parameter CUREE model parameters for the wall system (see values summarized in Table 10-3).

- If the modeler would like to consider a less detailed backbone curve, it could be generated at a user-defined hysteresis (in commercial software) using the proposed monotonic backbone curve envelope introduced in Section 10.4.3.2.

\subsubsection{Force-Displacement Envelopes for Modeling under Monotonic Loading}

A number of force-displacement envelopes have been developed over the years as discussed in Section 10.4.1 with most of those now serving as the backbone of the hysteretic model with strength and stiffness degradation rules in place for nonlinear time history analysis.

A detailed backbone curve which is based on the exponential envelope of the 10-parameter CUREE model can be considered as shown in Figure 10-11. Equation 10-1 describes the rules of the recommended exponential backbone curve based on the hysteretic parameters of the CUREE model.

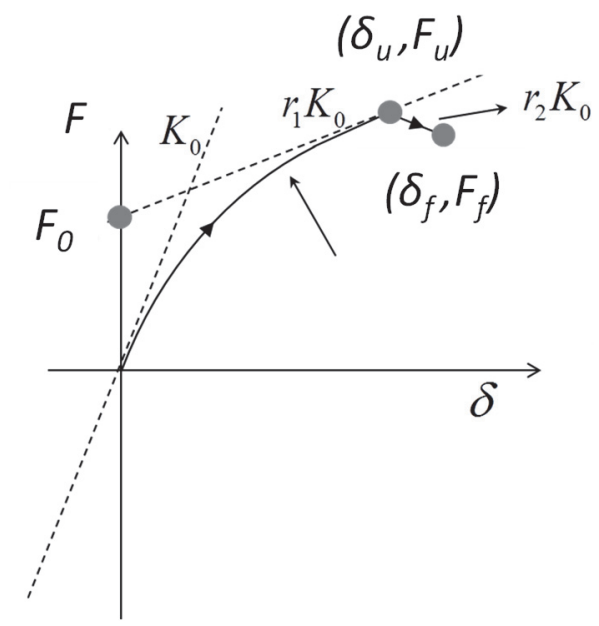

Figure 10-11 Monotonic envelope curve described in Equation 10-1.

$$
F=\left\{\begin{array}{cll}
\operatorname{sign}(\delta)\left(F_{0}+r_{1} K_{0}|\delta|\right)\left[1-\exp \left(-K_{0}|\delta| / F_{0}\right)\right], & \text { for } & |\delta| \leq\left|\delta_{u}\right| \\
\operatorname{sign}(\delta) F_{u}+r_{2} K_{0}\left[\delta-\operatorname{sign}(\delta) \delta_{u}\right], & \text { for } & \left|\delta_{u}\right|<|\delta| \leq\left|\delta_{F}\right| \\
0, & \text { for } & |\delta|>\left|\delta_{F}\right|
\end{array}\right.
$$


It can be seen that there is no distinct yield point for the ascending branch of the backbone curve. This is true for components, as well as system level behavior, making modeling of wood systems a non-conventional prospect since many generic models rely on a yield deformation/force. The $K_{0}$ parameter provides the initial stiffness of the model and is used in the calculation of the fundamental elastic period. As mentioned, Equation 10-1 then provides the equation of the curve up to $\left(X_{0}, F_{0}\right)$ where the descending branch begins and is defined as a line whose slope is the product of a dimensionless parameter, $r_{2}$, and the initial stiffness, $K_{0}$.

Despite the accuracy of the exponential envelope curve proposed by Folz and Filiatrault (2001), in an attempt to simplify the modeling steps and to be easily incorporated in commercially used software, a multi-linear envelope curve accounting for residual strength is introduced herein. The shape of the proposed envelope curve is schematically shown in Figure 10-12. Note that the parameters considered to define the shape of this curve are well aligned with some hysteretic parameters of the CUREE model and their values have been defined for the different wall combinations earlier in this chapter. Using the average fitted values of each wall system included in the synthesis presented in Section 10.4.2, the proposed envelope curves are plotted for each case and are compared with the average $F$ - $\Delta$ curves in Figure 10-13 for wall panels other than WSP. For wood shear panels, the simplified proposed envelope curve is recommended to be generated based on the nail scheduling and type of each set of wall tests. The proposed envelope curves are plotted for each case and are compared with the average $F$ - $\Delta$ curves in Figures 10-14 and $10-15$ for $8 \times 8 \mathrm{ft}$. WSP and $8 \times 4 \mathrm{ft}$. WSP, respectively. The results of Figures $10-14$ and $10-15$ are presented in normalized form in the y-axis (load/ASD).

The values recommended to be used for generating the simplified envelope curve for the different wall material configurations are summarized in Table 10-5.

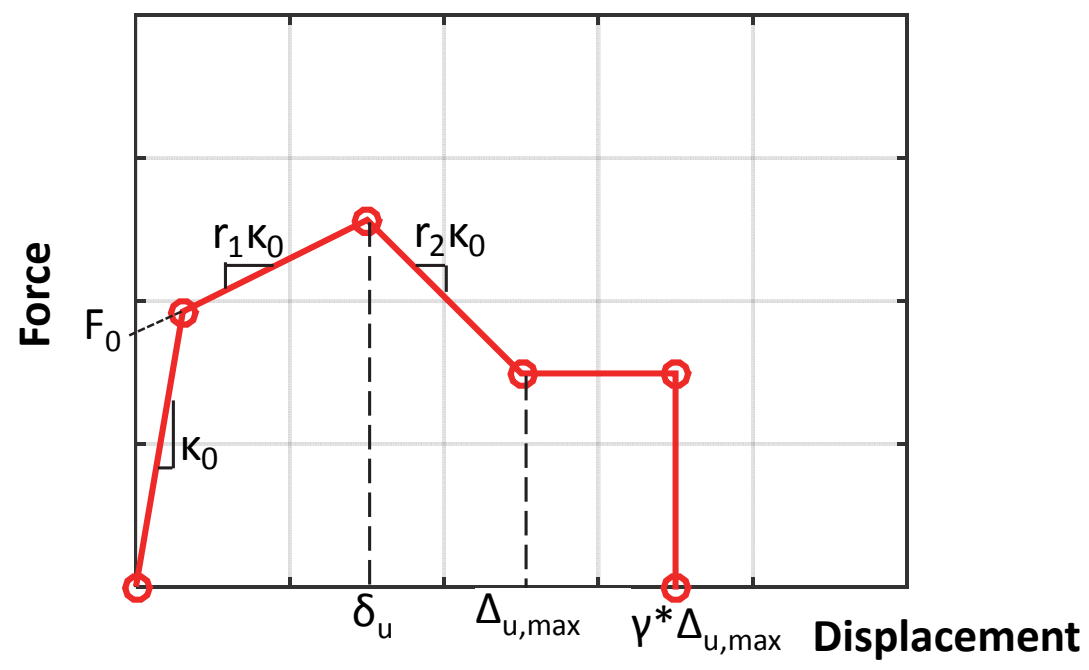

Figure 10-12 Proposed monotonic envelope curve. 

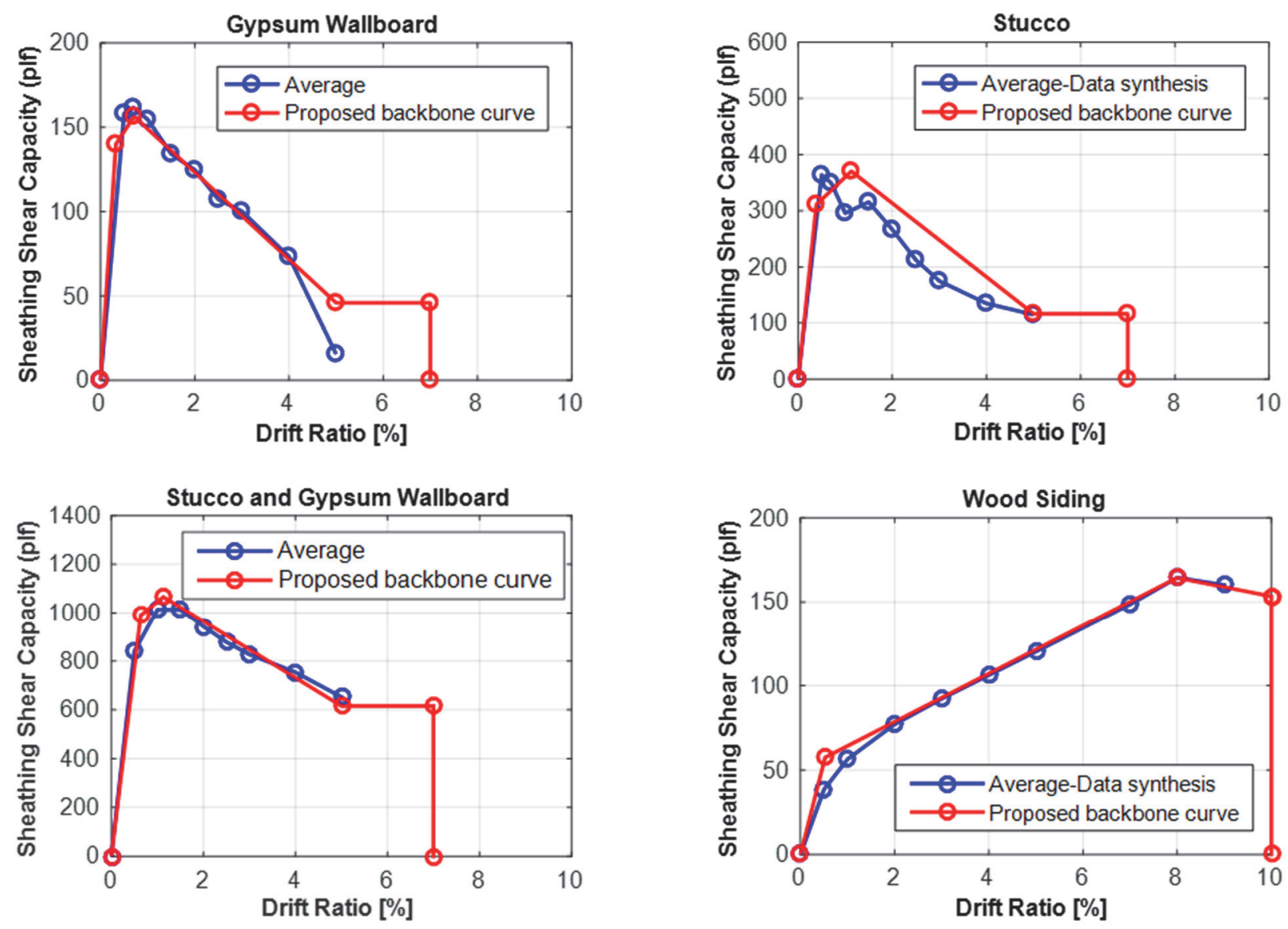

Figure 10-13 Proposed monotonic envelope compared to the average synthesis data for each wall combination for wall panels other than WSP.

The rationale for the inclusion of a residual strength and displacements in the range of $6 \%$ to $7 \%\left(\gamma \Delta_{u, \max }\right)$ drift for the WSP recommended model is based on the results of a number of reversed-cyclic tests and shake table tests. There is a key discrepancy that occurs when comparing light wood-frame models for walls to whole buildings. Isolated wood shear walls are well-modeled with the hysteretic and backbone models described earlier. Whereas whole building models present a significant challenge in that during larger deformations, components within the building begin to bear on other components. There is no model currently available that can predict or model this phenomena. However, it is clear from isolated shear walls tests (van de Lindt, 2008; Pei et al., 2013) that $6 \%$ is achievable without consideration of bearing and system-level effects. If these other types of whole building effects are considered, drifts can easily exceed 10\% (see van de Lindt et al., 2015). Therefore, for WSP, a $\gamma$ value of 1.4-1.5 is recommended. For wood wall panels other than WSP, similar rationale may be considered for a value of residual displacement in the range of $1.2-1.5 \Delta_{u, \max }(\gamma=1.2-1.5)$. 

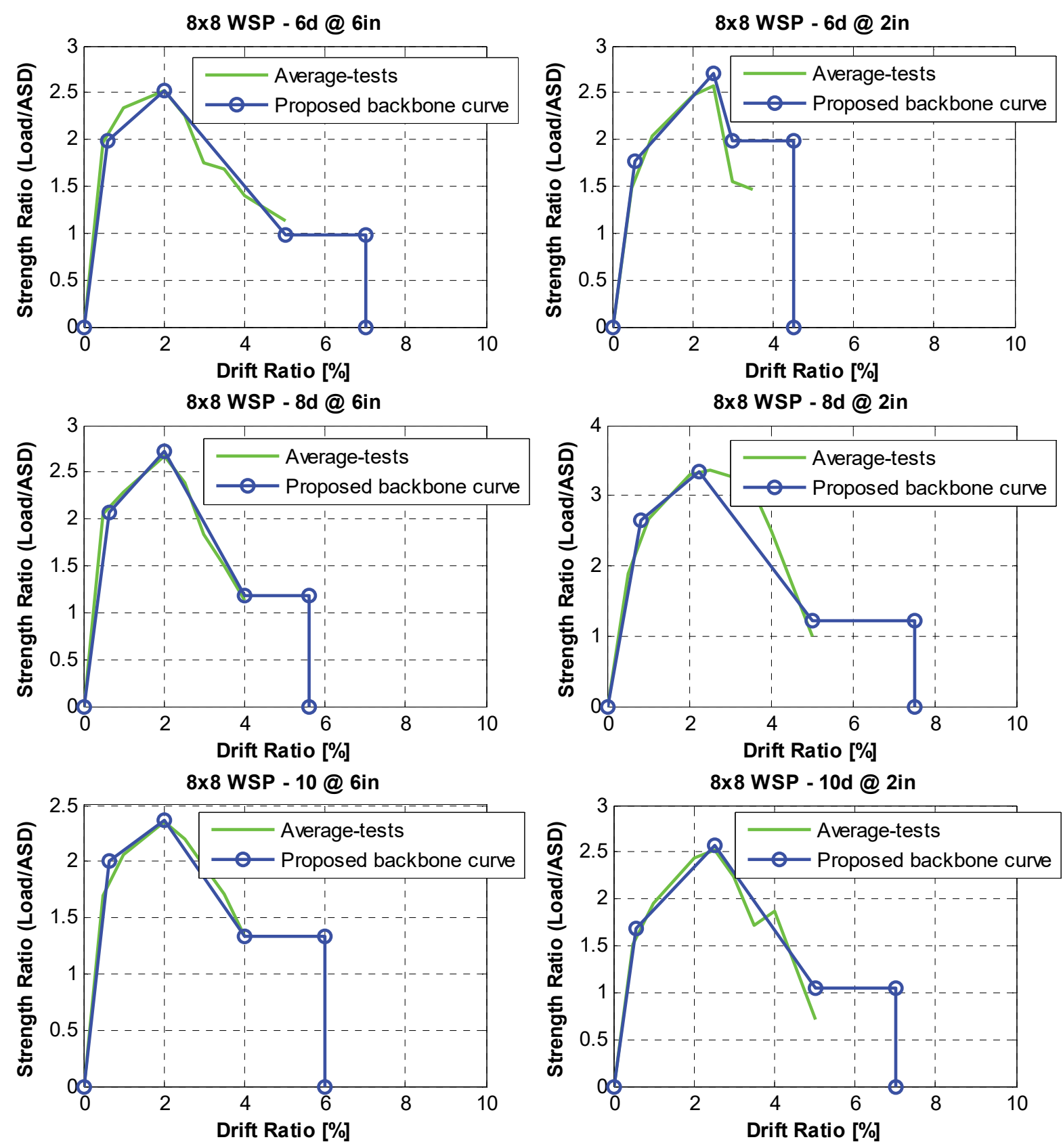

Figure 10-14 Proposed monotonic envelope compared to the average synthesis data for each wall combination for $8 \times 8 \mathrm{WSP}$. 

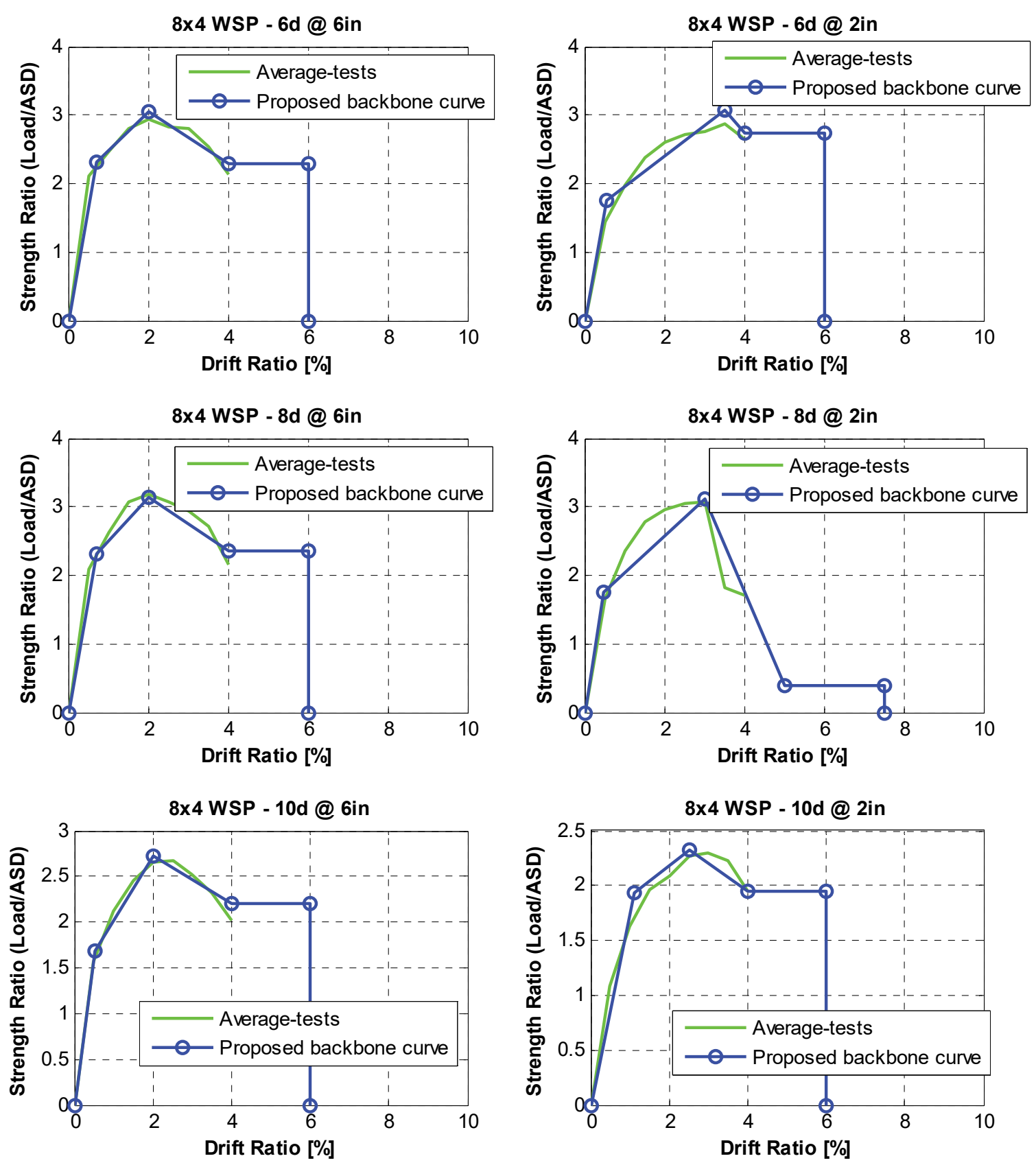

Figure 10-15 Proposed monotonic envelope compared to the average synthesis data for each wall combination for $8 \times 4$ WSP. 
Table 10-5 Parameters for Proposed Envelope Curve for Wood Wall Material Combinations Considered

\begin{tabular}{|c|c|c|c|c|c|c|c|}
\hline Combination & $K_{0}[\mathrm{lb} / \mathrm{in} / \mathrm{ft}]$ & $\begin{array}{c}F_{0} \\
{[\mathrm{lb} / \mathrm{ft}]}\end{array}$ & $r_{1}$ & $r_{2}$ & $\begin{array}{c}\delta u \\
{[\mathrm{in} / \mathrm{in}]}\end{array}$ & $\begin{array}{l}\Delta, \text { umax } \\
{[\mathrm{in} / \mathrm{in}]}\end{array}$ & $V$ \\
\hline Gypsum wallboard & 430 & 126 & 0.10 & -0.060 & 0.71 & 5.0 & 1.4 \\
\hline Stucco & 800 & 280 & 0.10 & -0.082 & 1.13 & 5.0 & 1.4 \\
\hline $\begin{array}{l}\text { Stucco \& gypsum } \\
\text { wallboard }\end{array}$ & 1550 & 890 & 0.10 & -0.075 & 1.13 & 5.0 & 1.4 \\
\hline $\begin{array}{l}\text { Horizontal wood } \\
\text { siding }\end{array}$ & 110 & 50 & 0.13 & -0.050 & 8.00 & 9.0 & 1.2 \\
\hline 8x8 WSP - 6d@6" & 712 & 389 & 0.12 & -0.16 & 2.00 & 5.0 & 1.5 \\
\hline 8x8 WSP - 6d@2" & 1812 & 849 & 0.15 & -0.45 & 2.50 & 3.0 & 1.5 \\
\hline 8x8 WSP - 8d@6" & 769 & 422 & 0.15 & -0.24 & 1.50 & 4.0 & 1.5 \\
\hline 8x8 WSP - 8d@2" & 1911 & 1345 & 0.15 & -0.24 & 2.25 & 5.0 & 1.5 \\
\hline 8x8 WSP - 10d@6" & 1088 & 649 & 0.08 & -0.16 & 2.00 & 4.0 & 1.5 \\
\hline 8x8 WSP - 10d@2" & 2645 & 1240 & 0.15 & -0.20 & 2.50 & 5.0 & 1.5 \\
\hline 8x4 WSP - 6d@6" & 712 & 423 & 0.18 & -0.12 & 2.00 & 4.0 & 1.5 \\
\hline 8x4 WSP - 6d@2" & 1812 & 580 & 0.14 & -0.20 & 3.50 & 4.0 & 1.5 \\
\hline 8x4 WSP - 8d@6" & 768 & 1776 & 0.20 & -0.12 & 2.00 & 4.0 & 1.5 \\
\hline 8x4 WSP - 8d@2" & 2195 & 915 & 0.15 & -0.38 & 3.00 & 5.0 & 1.5 \\
\hline 8x4 WSP - 10d@6" & 1095 & 629 & 0.22 & -0.08 & 2.00 & 4.0 & 1.5 \\
\hline 8x4 WSP - 10d@2" & 1497 & 1393 & 0.17 & -0.15 & 2.50 & 4.0 & 1.5 \\
\hline
\end{tabular}

\subsection{Illustrative Example Summary}

An illustrative example was conducted to evaluate the response of a wood lightframe system with structural panel sheathing considering the modeling recommendations introduced earlier in this document. The simplified proposed backbone curve (see Figure 10-12) as well as the backbone curve published in ASCE/SEI 41-13, Seismic Evaluation and Retrofit of Existing Buildings (ASCE, 2014), were both considered in this study for comparison.

A typical 5-story multi-family dwelling located in San Francisco, California was considered to conduct nonlinear time history analyses accounting for the two different designs for backbone curves (ASCE/SEI 41-13 and the simplified backbone curve presented in Figure 10-12). The building archetype was designed per ASCE/SEI 7-10, Minimum Design Loads for Buildings and Other Structures (ASCE, 2010), for Seismic Design Category D, Risk Category II and a response modification factor (R-factor) of 6.5 for building frame systems with light-frame walls sheathed with wood structural panels. The shear walls used $15 / 32$ Structural I sheathing with 
$10 \mathrm{~d}$ common nails with a maximum penetration of 1.5 inches into the framing members. Details of the building archetype are included in the Appendix G.

This illustrative example consisted of two stages: (1) component (wood shear walls); and (2) system level analyses. In the first stage, a typical wood shear wall panel from the building archetype was modeled in the SAPWood software (Pei and van de Lindt, 2007). Both the proposed simplified backbone curve as well as the ASCE/SEI 41-13 recommendation were considered in the modeling. Nonlinear response analyses at increasing seismic intensities (IDAs) (Vamvatsikos and Cornell, 2002) were conducted using the FEMA P-695 (FEMA, 2009) ground motion set to evaluate the collapse capacity of the wall frame system considering the two modeling approaches.

In the second stage of this example, the 5-story building archetype was modeled in the SAPWood software considering the modeling assumptions for the wall frame systems considered in the first stage of this study. Similar to the first stage, IDAs were conducted for the building structure under the FEMA P-695 far-field ground motion ensemble to evaluate its collapse performance for both modeling considerations, namely ASCE/SEI 41-13 and simplified backbone curve of Figure $10-12$.

Based on the results of this analytical effort both at the component (wood shear walls) and system level, the proposed backbone curve (Figure 10-12) was found to be associated with higher collapse capacities compared to the ASCE/SEI-41 recommendation. Thus, the non-trivial residual strength level and increased component displacement capacity accounted for the wall assemblies in the proposed backbone curve result in a more realistic representation of the response of woodframe wall systems aligning well with observed earthquake performance.

Details for this illustrative example are presented in Appendix G. 


\section{Appendix A}

\section{Steel Moment-Frame Systems - Application Case Study}

This appendix summarizes comparisons between the nonlinear modeling recommendations proposed in Chapter 4 and the recommendations provided in ASCE/SEI 41-13, Seismic Evaluation and Retrofit of Existing Buildings (ASCE, 2014), for selected steel components in steel moment-frame systems and for three archetype buildings.

\section{A.1 Steel Beams in Fully-Restrained Beam-to-Column Moment Connections}

Figure A-1 illustrates a comparison of the monotonic and first-cycle envelope curves based on the proposed modeling recommendations for nonlinear modeling of nonreduced beam section (RBS) steel beams and beams with RBS with experimental data. The beam depths range from W18 to W36. Superimposed in the same figure are the first-cycle envelope curves based on the modeling recommendations in ASCE/SEI 41-13. The following are observed in Figure A-1:

- The post-yield component behavior based on the ASCE/SEI 41-13 component model is based on a constant $3 \%$ strain hardeing ratio. However, the experimental data show that this may not always be the case. In that sense, the proposed $M_{\max }^{*} / M_{y}^{*}$ ratio is a more stable parameter than what has been historically used in ASCE/SEI 41-13 to represent the post-yield component behavior for steel beams. This agrees with prior work by Lignos and Krawinkler (2011).

- The pre-peak plastic deformation $\theta_{p}^{*}$ (i.e., " $a$ " value based on ASCE/SEI 41-13, typically expressed either as a constant value or a function of beam depth, $d$ ) of a steel beam is strongly dependent on its cross-section web and flange local slenderness ratios. The proposed nonlinear modeling recommendations explicitly capture this effect.

- The pre-peak plastic deformation $\theta_{p}^{*}$ is typically smaller than what is currently used in ASCE/SEI 41-13. This is to be expected because the proposed nonlinear modeling recommendations are based on the idealized curve that caps at the maximum moment, $M_{\max }$ as measured from each test and not at $80 \% M_{\max }$. The latter was done in ASCE/SEI 41-13 (ASCE, 2014). For steel beams with depths, $d>21$ inches the current " $a$ " value based on the ASCE/SEI 41-13 
recommendations is much larger than what it should be in reality (see Figures A-1c to A-1f). For beam depths smaller than 21 inches the " $a$ " value based on ASCE/SEI 41-13 seems to be more reasonable as shown in Figures A-1a and $\mathrm{A}-1 \mathrm{~b}$. This indicates that there may be some bias in the current " $a$ " and " $b$ " values of the ASCE/SEI 41-13 (see Figure A-1a and refer to Figure 4-35) component model from experimental data on small depth steel beams.

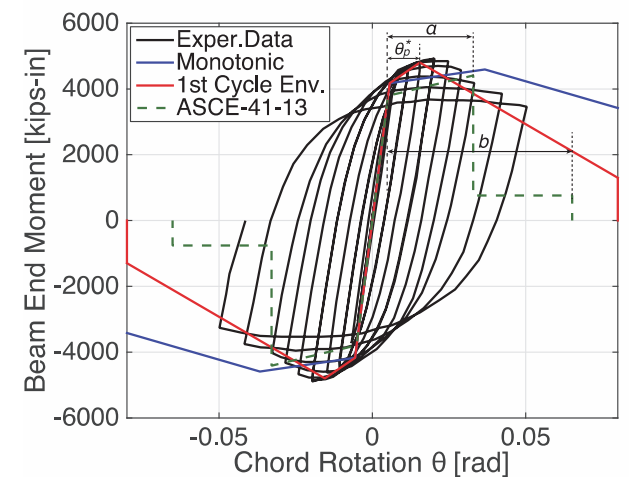

(a) W18x40 beam with WUF-B

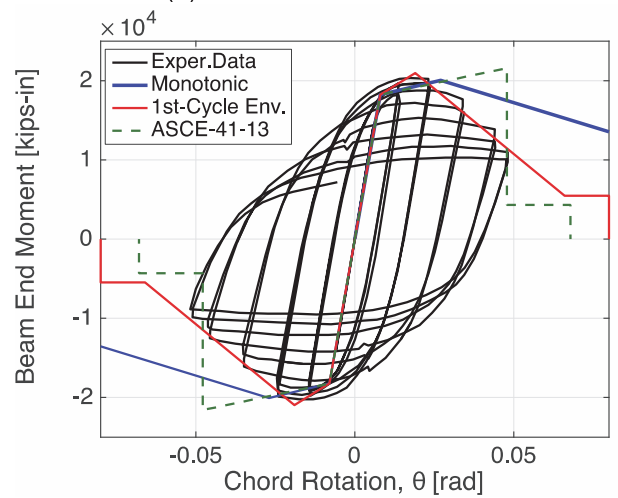

(c) W33 130 beam with RBS

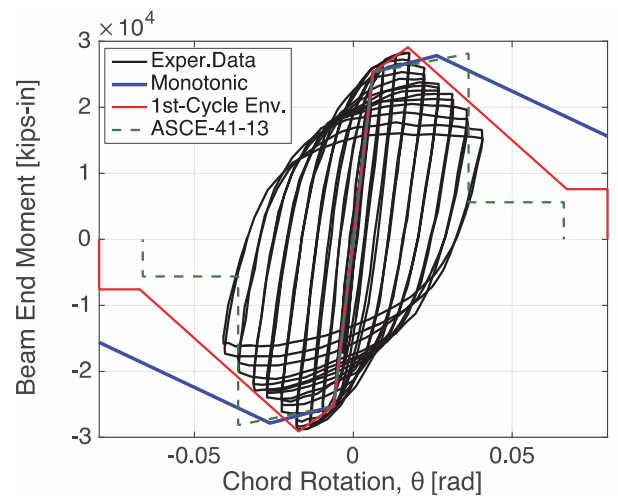

(e) W36x150 beam with welded flange plate

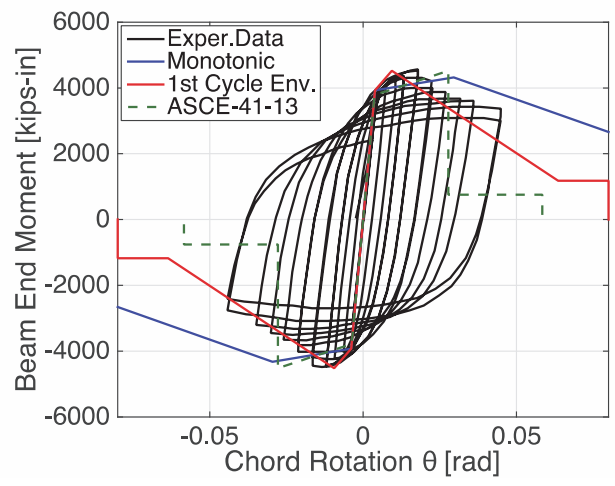

(b) W21x44 beam with WUF-B

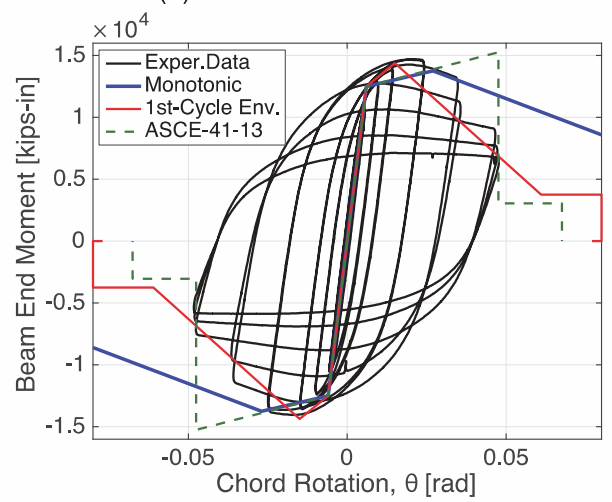

(d) W30x99 beam with RBS

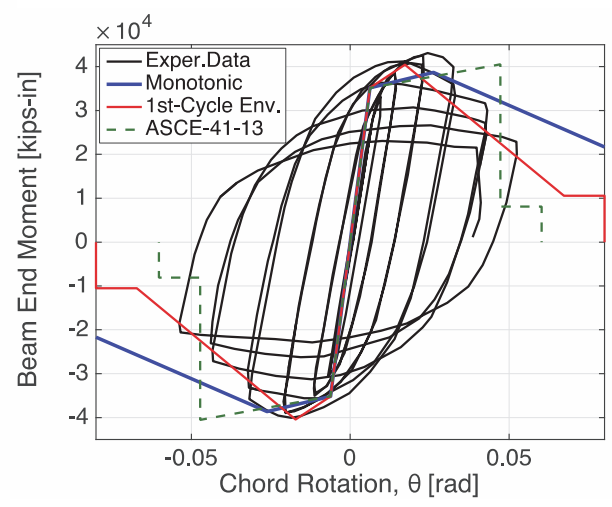

(f) W36x150 beam with WUF-W

Figure A-1 Comparisons of proposed modeling recommendations with ASCE/SEI 41-13 for bare steel beams [(a) and (b) data from Tsai and Popov, 1988; (c) data from Popov et al., 1997; (d) Chi and Uang, 2002; (e) Engelhardt et al., 1997; (f) Ricles et al., 2002]. 
- The flexural strength of a steel beam deteriorates gradually with the increasing lateral loading amplitude regardless of the beam size and connection type. This is captured fairly well based on the post-peak plastic deformation, $\theta_{p c}^{*}$, as computed from the proposed modeling recommendations.

- The large discontinuity in flexural strength of steel beams based on the ASCE/SEI 41-13 component model is not reflected in the experimental data for steel beams as part of fully restrained beam-to-column connections regardless of the respective beam depth. Historically, this discontinuity caused numerical instabilities in nonlinear structural analysis software that is being used by the engineering profession.

- The pre-peak plastic deformation, $\theta_{p}^{*}$ of the first cycle envelope curve is on average 0.65 times the initial backbone curve value based on monotonic loading, $\theta_{p}$. This is fairly consistent with PEER/ATC-72-1 (ATC, 2010) modeling recommendations for Option 3 and Option 1 (i.e., recommended values was $0.70)$.

- The ratio between the mean value of the total inelastic deformation, $\theta_{u l t}$ to $\theta_{u l t}^{*}$ at which loss of gravity load carrying capacity is expected to occur in steel beams as part of post-Northridge beam-to-column connections is in the range of 3.5. This is fairly consistent with Chapter 16 of ASCE/SEI 7-16, Minimum Design Loads and Associated Criteria for Buildings and Other Structures (ASCE, 2017).

\section{A.1.1 Composite Steel Beams in Fully Restrained Beam-to-Column Moment Connections}

Figure A-2 illustrates a comparison of the monotonic and first-cycle envelope curves based on the proposed nonlinear modeling recommendations for composite steel beams. The ASCE/SEI 41-13 component model (ASCE, 2014) is also superimposed in the same figure. From Figure A-2, it is evident that the current ASCE/SEI 41-13 component model considerably overestimates the pre-peak plastic deformation $\theta_{p}^{*}$ of composite beams when the slab is in tension (i.e., negative loading direction). The same component model seems to underestimate by $20 \%$, on average, the flexural strength of a composite beam when the slab is in compression (i.e., positive loading direction). This can be fairly important for the nonlinear evaluation of steel momentframe systems because the flexural strength of steel beams is directly related with the employed strong-column/weak-beam ratio. The computation of the effective yield flexural strength of a composite beam when the slab is in compression based on Section I3 of ANSI/AISC 360-10, Specification for Structural Steel Buildings (AISC, 2010c), seems to be effective in terms of adjusting the component backbone curve for the composite beam effects. This is consistent with what was found in recent studies by Elkady and Lignos (2014). 


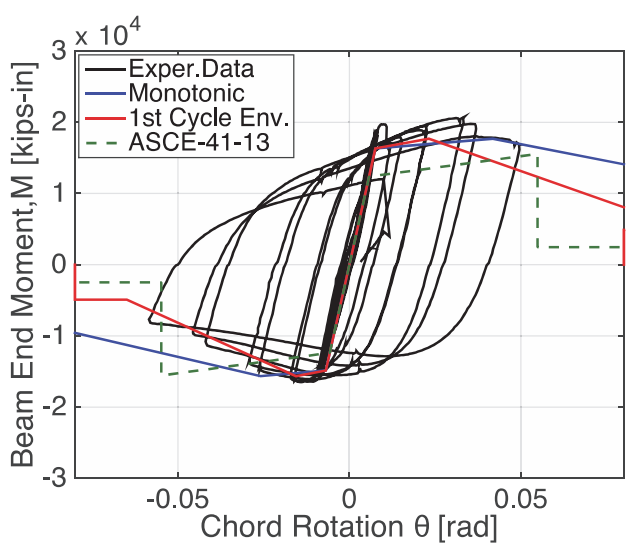

(a) W30×108 steel beam with RBS

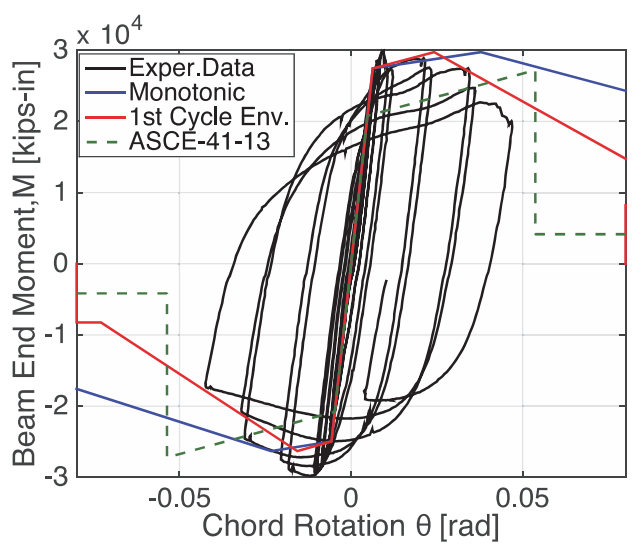

(b) W36×150 steel beam with RBS

Figure A-2 Comparisons of proposed modeling recommendations with ASCE/SEI 41-13 for composite steel beams (data from Zhang and Ricles, 2006).

\section{A.2 Beams with Pre-Northridge WUF-B Connections}

Figure A-3 illustrates a number of examples for steel beams as part of pre-Northridge beam-to-column connections. The connection type in most cases was welded flange unreinforced web with a bolted shear tab (WUF-B). In few of the examples shown in the same figure, a weld reinforcement was used in the shear tab (i.e., WUF-W). Superimposed in the same figure are the nonlinear modeling recommendations discussed in this chapter as well as the current ASCE/SEI 41-13 recommendations. The following observations hold true,

- For steel beams that experience fracture at chord rotations larger than 0.015 radians their effective yield strength is in general underpredicted by both nonlinear modeling approaches (see Figures A-3a and A-3c). This is attributed to cyclic hardening prior to the occurrence of fracture, which is not captured by Equation 4-17. However, based on the test data, Equation 4-17 is, on average, a reasonable estimate of the effective yield strength of a steel beam.

- For steel beams with $d<24$ " (see Figure A-3c) the first-cycle envelope curve based on both modeling recommendations is approximately the same for all practical purposes. The use of the proposed nonlinear model discussed in this chapter is likely to eliminate problems associated with numerical instabilities witihin a nonlinear analysis software because it does not include the instantaneous vertical drop in flexural strength that the ASCE/SEI 41-13 component model does. Same observations hold true for beams that utilize a W30 cross-section (see Figure A-3e).

- The ASCE/SEI 41-13 component model seems to underestimate the pre-peak plastic rotation $\theta_{p}^{*}$ (i.e., parameter " $a$ ") of steel beams with $d>30$ " (see Figures A-3a, A-3b, A-3d, A-3f) by $50 \%$, on average. 


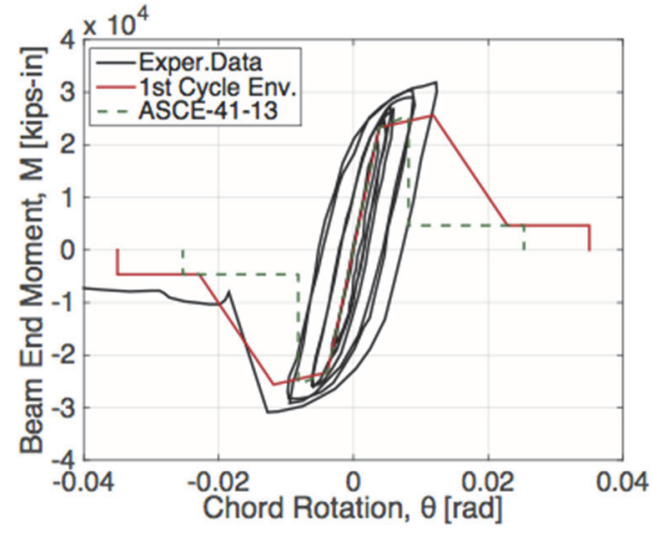

(a) W36x150 Beam (WUF-B)

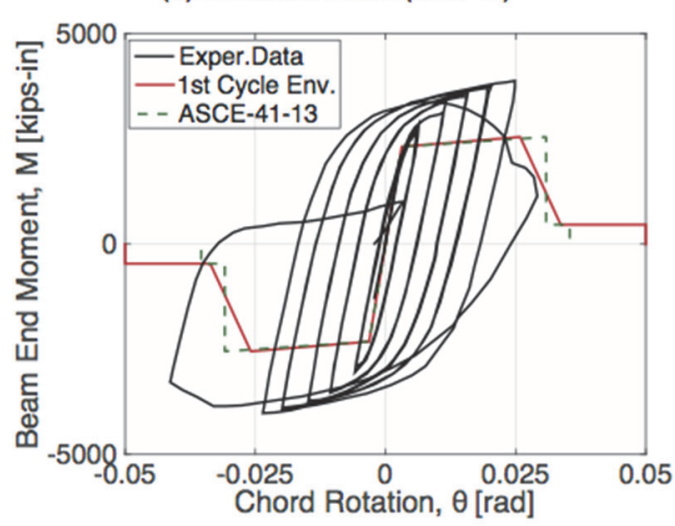

(c) W18x40 Beam (WUF-B)

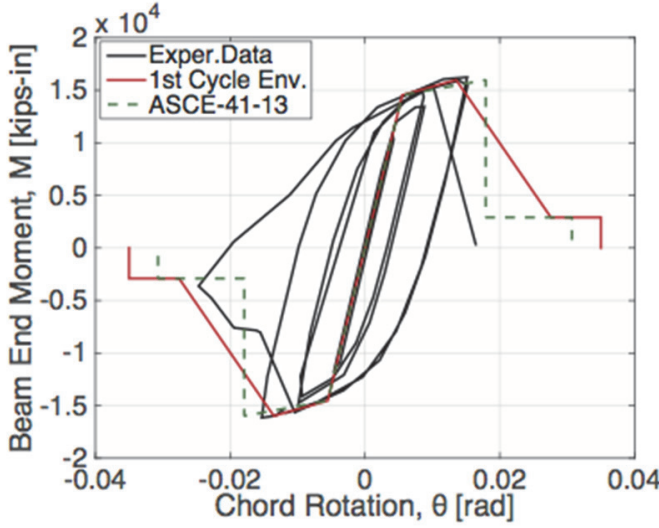

(e) W30x99 Beam (WUF-W)

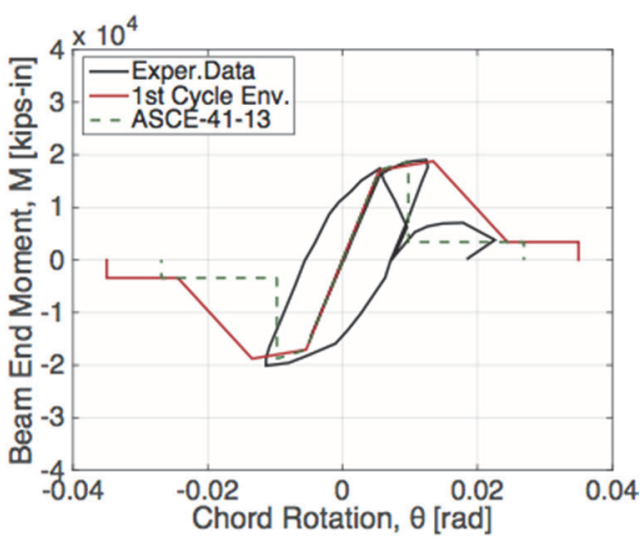

(b) W36x150 Beam (WUF-B)

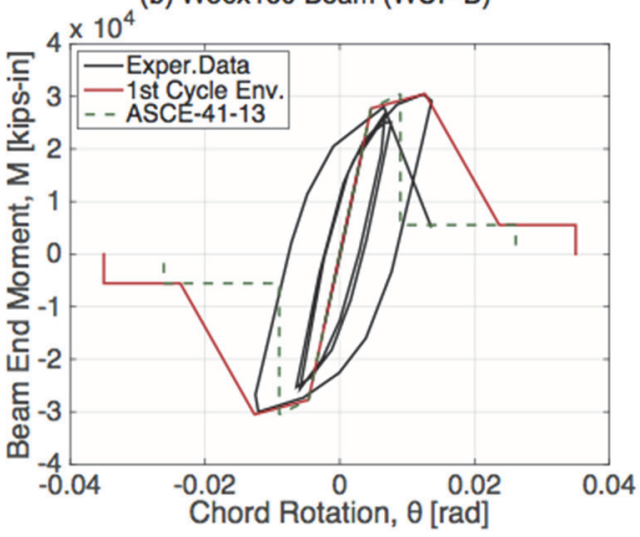

(d) W36x150 Beam (WUF-W)

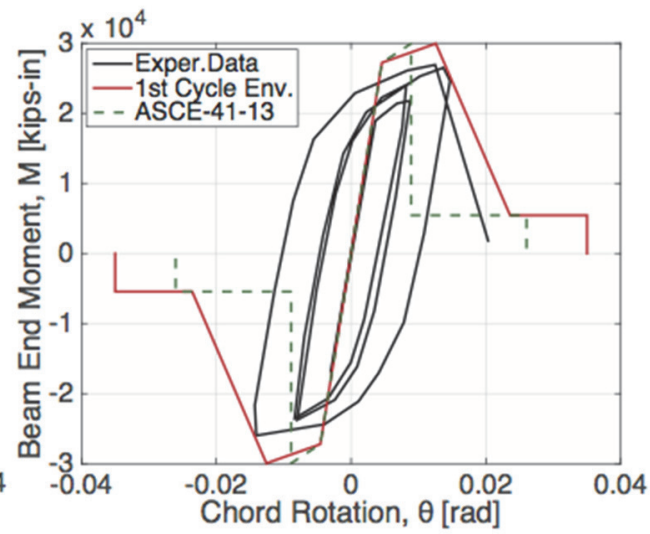

(f) W36x150 Beam (WUF-W)

Figure A-3 Comparisons of proposed modeling recommendations with ASCE 41-13 for steel beams as part of pre-Northridge beam-to-column connections [FEMA, 1997c; Tsai and Popov, 1988; Engelhardt and Sabol, 1994].

\section{A.3 Steel Wide-Flange Beam-Columns}

This section provides comparisons between the proposed nonlinear modeling recommendations discussed in Section 4.5.4.1 and the ones summarized in ASCE/SEI 41-13 for steel wide-flange beam-columns. The comparison is done with available experimental data. Figure A-4 includes the deduced column end moment- 
chord rotation relation for a wide range of wide-flange steel columns subjected to monotonic (see Figures A-4a and A-4b) and reversed cyclic lateral loading (see Figures A-4c to A-4f). In all cases the compressive axial load was kept constant throughout the loading history. The main observations are summarized as follows:

- In Figures A-4a and A-4b, the proposed monotonic curve for nonlinear modeling of wide-flange beam-columns represent the experimental data fairly well including the post-peak plastic deformation range regardless of the applied axial load ratio. The observed differences in the predicted effective yield strength compared to the experimental data are due to the material variability (expected versus measured yield stress) and due to the fact that the proposed component model captures only approximately the effect of kinematic hardening on the monotonic response of steel columns.

- In Figures A-4c to A-4f, the proposed first-cycle envelope curve represent the measured response of the steel columns relatively well, regardless of the crosssectional local slenderness ratio and the applied compressive axial load ratio. Based on the same figures, the ASCE/SEI 41-13 component model overestimates by a considerable amount the pre-peak plastic deformation of steel columns subjected to $P_{g} / P_{y e}=0.20$ and 0.30 (see Figures A-4c to A-4e). This is attributed to the fact that the ASCE/SEI 41-13 component model does not capture the cross-section local slenderness effects on the parameter " $a$." In addition, the ASCE/SEI 41-13 component model does not directly capture the effect of global slenderness ratio on the pre-peak plastic deformation parameter " $a$." This is only done through the calculation of $P_{c r}$ but this load is not relevant for triggering global-out-of-plane instabilities associated with lateral torsional buckling of a steel column under lateral loading.

- In Figures A-4b and A-4f, steel columns that utilize cross sections within the limits of highly ductile members as per AISC 341-10 (AISC, 2010a) and subjected to $P_{g} / P_{y e}=0.50$ (i.e., $P / P_{c r}>0.50$ ) show an appreciable plastic deformation capacity that is significantly underestimated by the ASCE/SEI 41-13 component model. This model treats such members as force-controlled elements. However, experimental evidence (Suzuki and Lignos, 2015; Lignos et al., 2016) confirmed with detailed finite element studies (Elkady and Lignos, 2015b) indicates that they should be treated as deformation-controlled elements.

Figures A-5, A-6 and Tables A-1 to A-3 provide estimates of the plastic deformation parameters that define the monotonic backbone and the first-cycle envelope curves shown in Figure 4-35 for 13ft long steel columns based on the proposed nonlinear modeling recommendations for wide-flange steel columns. These columns utilize a range of cross-sections subjected to $P_{g} / P_{y e}=0.05$ (see Table A-1), 0.20 (see Table A-2) and 0.50 (see Table A-3). From these figures and tables the main observations are summarized as follows: 

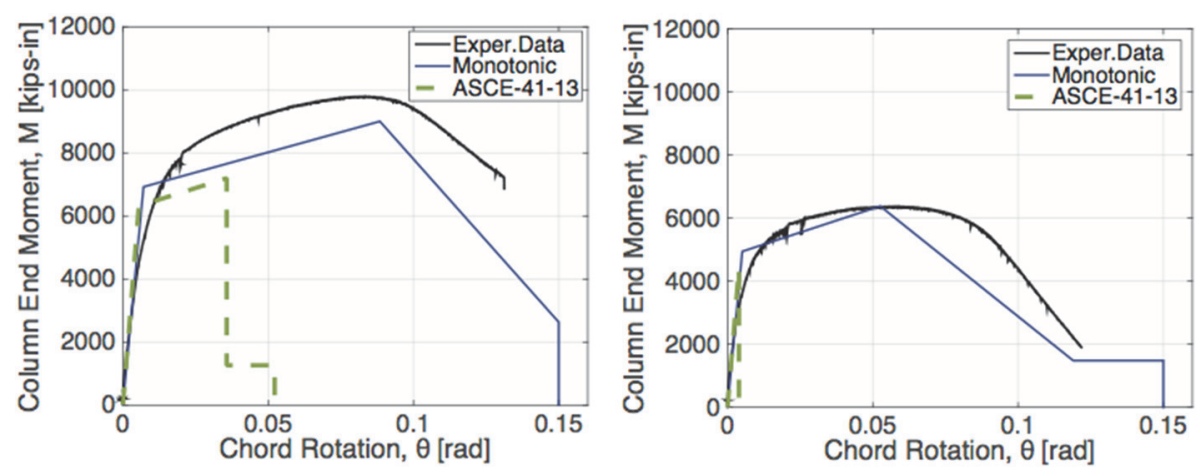

(a) $\mathrm{W} 14 \times 82, \mathrm{P}_{\mathrm{g}} / \mathrm{P}_{\mathrm{ye}}=0.30$, Monotonic

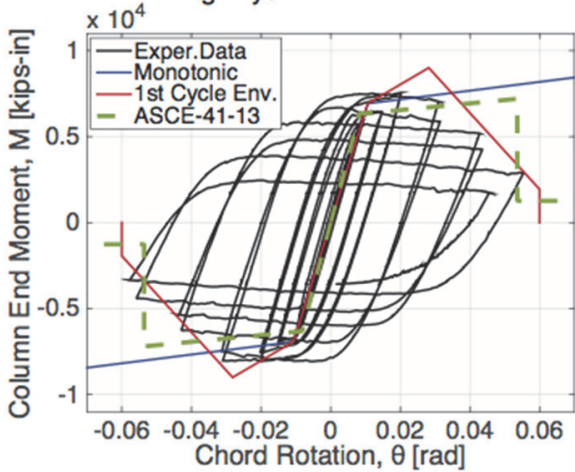

(b) $\mathrm{W} 14 \times 82, \mathrm{P}_{\mathrm{g}} / \mathrm{P}_{\mathrm{ye}}=0.50$, Monotonic

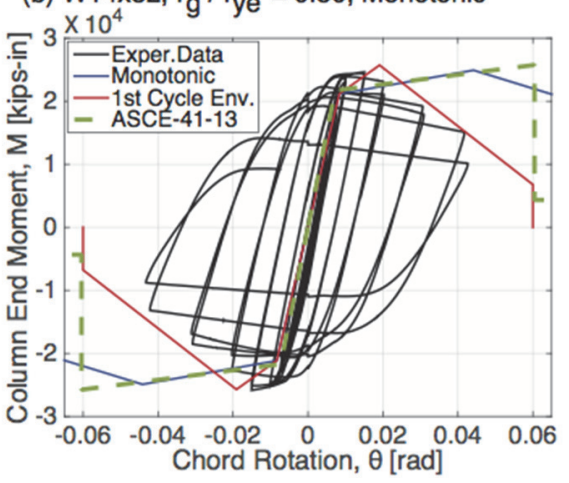

(c) $\mathrm{W} 14 \times 82, \mathrm{P}_{\mathrm{g}} / \mathrm{P}_{\mathrm{ye}}=0.30$, Reversed Cyclic

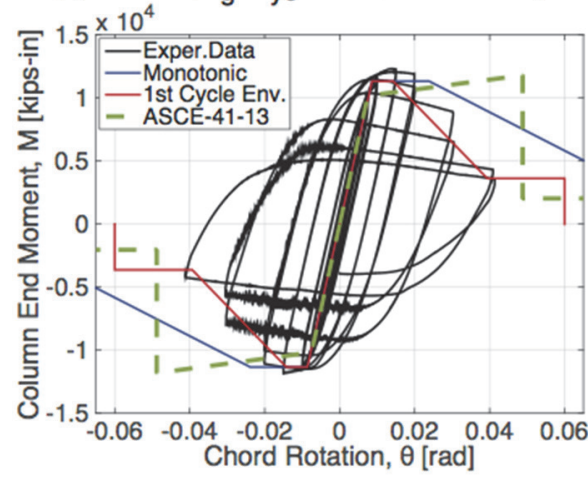

(e) W24x84, $P_{g} / P_{y e}=0.20$, Reversed Cyclic

(d) W24x146, $\mathrm{P}_{\mathrm{g}} / \mathrm{P}_{\mathrm{ye}}=0.20$, Reversed Cyclic

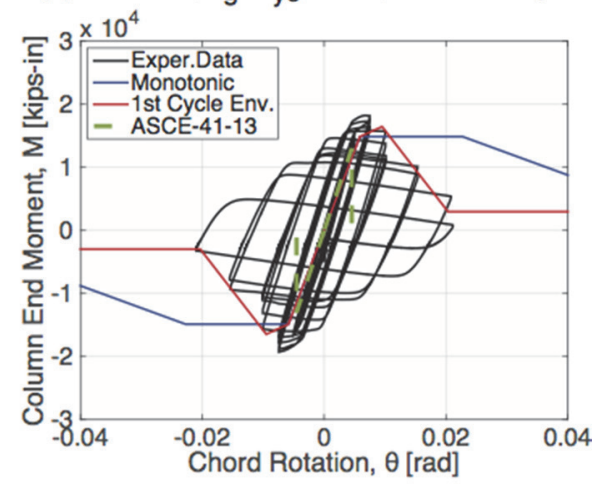

(f) W24x146, $P_{g} / P_{y e}=0.50$, Reversed Cyclic

Figure A-4 Comparisons of proposed modeling recommendations with ASCE 41-13 for steel wide-flange beam-columns [data from Suzuki and Lignos, 2015; Elkady and Lignos, 2016; Lignos et al., 2016].

- The pre-peak plastic deformation, $\theta_{p}^{*}$, of the first-cycle envelope curve of wideflange beam-columns is, on average, two to three times smaller than the corresponding value of the idealized monotonic curve. This difference decreases while the applied compressive axial load increases.

- The ratio between the mean value of the total plastic deformation of a monotonic curve with respect to that of a first-cycle envelope curve for the same crosssection is, on average, 2.8. This value is consistent with what is currently reported in Chapter 16 of ASCE/SEI 7-16 for deformation-controlled elements (i.e., recommended value is 3.0). 
- In Table A-3, steel columns with $L_{b} / r_{y}=80$ that utilize cross-sections near the current compactness limits for highly ductile member as per AISC 341-10 have a finite plastic deformation capacity even when they are subjected to $P_{g} / P_{y e}=0.50$ (i.e., $\left.P / P_{c r}>0.50\right)$. This indicates that columns subjected to axial load ratios in that range should not be treated as force-controlled elements as currently suggested by ASCE/SEI 41-13. This agrees with recent findings of the ASCE/SEI 41 steel subcommittee (Bech et al., 2015).

- In Figures A-5 and A-6, stocky columns (i.e., $\left.h / t_{w}<15\right)$ show a relative large plastic deformation capacity regardless of the applied axial compressive load ratio. This is consistent with prior experimental data by Newell and Uang (2006).

- In Figures A-5 and A-6, the scatter in the computed pre- and post-peak plastic deformation capacity of steel columns is smaller for higher levels of axial compressive load. This indicates that the higher the applied axial load ratio the smaller the influence of the cross-sectional slenderness on the column behavior.

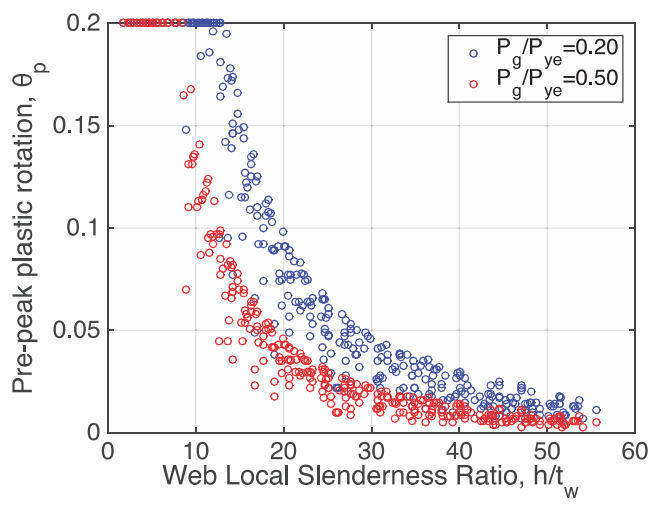

(a) Pre-peak plastic rotation, $\theta p$

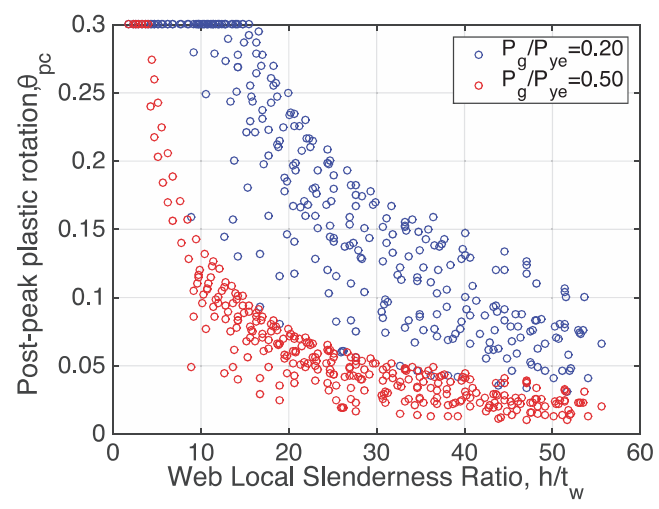

(b) Post-peak plastic rotation, $\theta p c$

Figure A-5 Trends of pre- and post-peak plastic deformations with respect to the cross-section web local slenderness ratio for modeling the monotonic backbone curve of steel wide-flange columns.

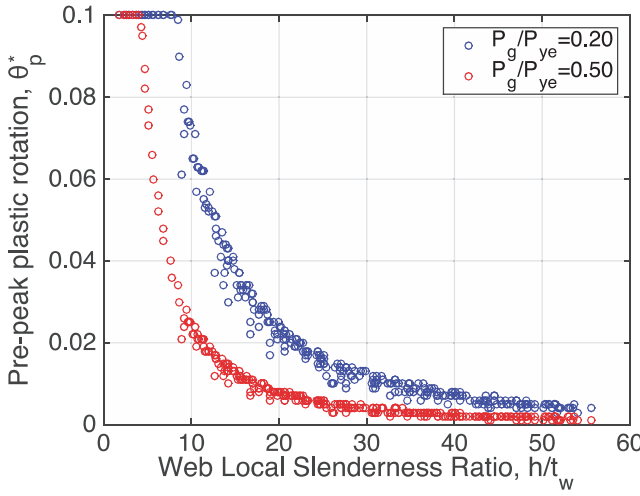

(a) Pre-peak plastic rotation, $\theta_{p}^{*}$

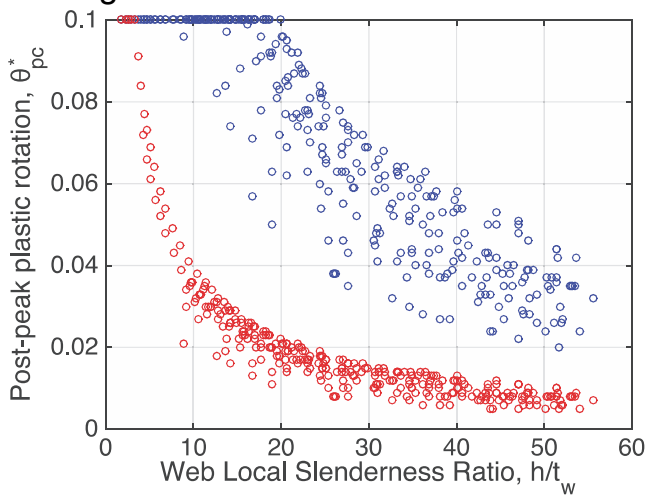

(b) Post-peak plastic rotation, $\theta_{\mathrm{pc}}^{*}$

Figure A-6 Trends of pre- and post-peak plastic deformations with respect to the cross-section web local slenderness ratio for modeling the first-cycle envelope curve of steel wide-flange columns. 
Table A-1 Modeling Parameters for Various Steel Column Sizes Based on Regression Equations (Assumed Column Height 13ft and A992 Gr. 50 steel, $\left.P_{g} / P_{y e}=0.05\right)$

\begin{tabular}{|c|c|c|c|c|c|c|c|}
\hline Section & $h / t_{w}$ & $P_{g} / P_{y e}$ & $L / r_{y}$ & $\begin{array}{c}\theta_{p} \\
{[\mathrm{rad}]}\end{array}$ & $\begin{array}{c}\theta_{p c} \\
{[\mathrm{rad}]}\end{array}$ & $\begin{array}{c}\theta_{p}^{*} \\
{[\mathrm{rad}]}\end{array}$ & $\begin{array}{c}\theta_{p c}^{*} \\
{[\mathrm{rad}]}\end{array}$ \\
\hline W27×307 & 20.6 & 0.05 & 46 & 0.103 & 0.337 & 0.033 & 0.156 \\
\hline W27×235 & 26.2 & 0.05 & 47 & 0.067 & 0.273 & 0.023 & 0.127 \\
\hline$W 27 \times 146$ & 39.4 & 0.05 & 49 & 0.033 & 0.191 & 0.012 & 0.090 \\
\hline$W 24 \times 146$ & 33.2 & 0.05 & 60 & 0.038 & 0.186 & 0.014 & 0.093 \\
\hline$W 24 \times 103$ & 39.2 & 0.05 & 78 & 0.024 & 0.131 & 0.010 & 0.071 \\
\hline W24×84 & 45.9 & 0.05 & 80 & 0.018 & 0.114 & 0.008 & 0.062 \\
\hline$W 14 \times 370$ & $6.89^{*}$ & 0.05 & $37^{*}$ & 0.200 & 0.300 & 0.100 & 0.100 \\
\hline$W 14 \times 233$ & $10.7^{*}$ & 0.05 & $38^{*}$ & 0.200 & 0.300 & 0.100 & 0.100 \\
\hline
\end{tabular}

* values outside the range of applicability of predictive equations

Table A-2 Modeling Parameters for Various Steel Column Sizes Based on Regression Equations (Assumed Column Height 13ft and A992 Gr. 50 steel, $\left.P_{g} / P_{\text {ye }}=0.20\right)$

\begin{tabular}{|c|c|c|c|c|c|c|c|}
\hline Section & $h / t_{w}$ & $P_{g} / P_{y e}$ & $L / r_{y}$ & $\begin{array}{c}\theta_{p} \\
\text { [rad] }\end{array}$ & $\begin{array}{c}\theta_{p c} \\
\text { [rad] }\end{array}$ & $\begin{array}{c}\theta_{p}^{*} \\
{[\mathrm{rad}]}\end{array}$ & $\begin{array}{c}\theta_{p c}^{*} \\
{[\mathrm{rad}]}\end{array}$ \\
\hline W27×307 & 20.6 & 0.2 & 46 & 0.065 & 0.235 & 0.023 & 0.090 \\
\hline W27×235 & 26.2 & 0.2 & 47 & 0.042 & 0.190 & 0.015 & 0.073 \\
\hline W27×146 & 39.4 & 0.2 & 49 & 0.021 & 0.133 & 0.008 & 0.052 \\
\hline W24×146 & 33.2 & 0.2 & 60 & 0.024 & 0.130 & 0.010 & 0.054 \\
\hline$W 24 \times 103$ & 39.2 & 0.2 & 78 & 0.015 & 0.091 & 0.007 & 0.041 \\
\hline$W 24 \times 84$ & 45.9 & 0.2 & 80 & 0.011 & 0.079 & 0.005 & 0.036 \\
\hline W14×370 & $6.89^{*}$ & 0.2 & $37^{*}$ & 0.200 & 0.300 & 0.100 & 0.100 \\
\hline W14×233 & $10.7^{*}$ & 0.2 & $38^{*}$ & 0.200 & 0.300 & 0.068 & 0.100 \\
\hline
\end{tabular}

* values outside the range of applicability of predictive equations 
Table A-3 Modeling Parameters for Various Steel Column Sizes Based on Regression Equations (Assumed Column Height 13ft and A992 Gr.50 steel, $P_{g} / P_{y e}=0.50$ )

\begin{tabular}{|c|c|c|c|c|c|c|c|}
\hline Section & $h / t_{w}$ & $P_{g} / P_{y e}$ & $L / r_{y}$ & $\begin{array}{c}\theta_{p} \\
{[\mathrm{rad}]}\end{array}$ & $\begin{array}{c}\theta_{p c} \\
{[\mathrm{rad}]}\end{array}$ & $\begin{array}{c}\theta_{p}^{*} \\
{[\mathrm{rad}]}\end{array}$ & $\begin{array}{c}\theta_{p c}^{*} \\
{[\mathrm{rad}]}\end{array}$ \\
\hline$W 27 \times 307$ & 20.6 & 0.5 & 46 & 0.018 & 0.088 & 0.008 & 0.020 \\
\hline W27×235 & 26.2 & 0.5 & 47 & 0.012 & 0.071 & 0.005 & 0.016 \\
\hline$W 27 \times 146$ & 39.4 & 0.5 & 49 & 0.006 & 0.050 & 0.003 & 0.012 \\
\hline$W 24 \times 146$ & 33.2 & 0.5 & 60 & 0.007 & 0.048 & 0.003 & 0.012 \\
\hline$W 24 \times 103$ & 39.2 & 0.5 & 78 & 0.004 & 0.034 & 0.002 & 0.009 \\
\hline$W 24 \times 84$ & 45.9 & 0.5 & 80 & 0.003 & 0.030 & 0.002 & 0.008 \\
\hline W14×370 & $6.89^{*}$ & 0.5 & $37^{*}$ & 0.137 & 0.252 & 0.047 & 0.054 \\
\hline W14×233 & $10.7^{*}$ & 0.5 & $38^{*}$ & 0.063 & 0.172 & 0.023 & 0.037 \\
\hline
\end{tabular}

\section{A.4 Hollow Structural Steel Beam-Columns}

This section provides illustrative comparisons of the proposed nonlinear modeling recommendations for steel HSS beam-columns. The comparison is done with available experimental data. Figure A-7 includes the deduced column end momentchord rotation relation for a range of HSS steel columns subjected to reversed cyclic lateral loading. In all cases the compressive axial load was kept constant throughout the loading history. In addition, Figure A-8 shows the trends of pre- and post-peak plastic deformations with respect to the cross-section local slenderness ratio, $D / t$ for steel beam-columns utilizing HSS cross-sections. Tables A-4 to A-6 provide estimates of the plastic deformation parameters that define the monotonic backbone and the first-cycle envelope curves for selected HSS beam-columns. These columns are subjected to $P_{g} / P_{y e}=0.05$ (see Table A-4), 0.20 (see Table A-5), and 0.50 (see Table A-6). The following observations can be made:

- In Figure A-7, the proposed modeling recommendations capture relatively well the pre- and post-peak plastic deformation capacities, $\theta_{p}^{*}$ and $\theta_{p c}^{*}$, respectively, for both slender (i.e., see Figure A-7a) and highly compact (see Figure A-7b) HSS columns.

- In Figure A-7a and Tables A-5 and A-6, slender HSS columns ( $D / t>27$ or so) do not seem to harden cyclically for axial load ratios $P_{g} / P_{y e}>0.20$. This is attributed to the early occurrence of local buckling. For highly ductile HSS members (see Figure A-7b), the amount of hardening is appreciable.

- The ratio between the mean value of the total plastic deformation, $\theta_{p}$, with respect to $\theta_{p}^{*}$ is, on average, 2.0. This value is smaller than what is currently 
reported in Chapter 16 of ASCE/SEI 7-16 for deformation-controlled elements (i.e., recommended value is 3.0).

- In Figure A-8b and Tables A-4 to A-6, the applied $P_{g} / P_{y e}$ dominates the postbuckling behavior of HSS columns. On the other hand, in Figure A-8a, the influence of $D / t$ ratio on the pre-buckling behavior of HSS columns is significant even in cases that the applied compressive axial load is fairly large (i.e., $P_{g} / P_{y e}>$ $0.20)$.

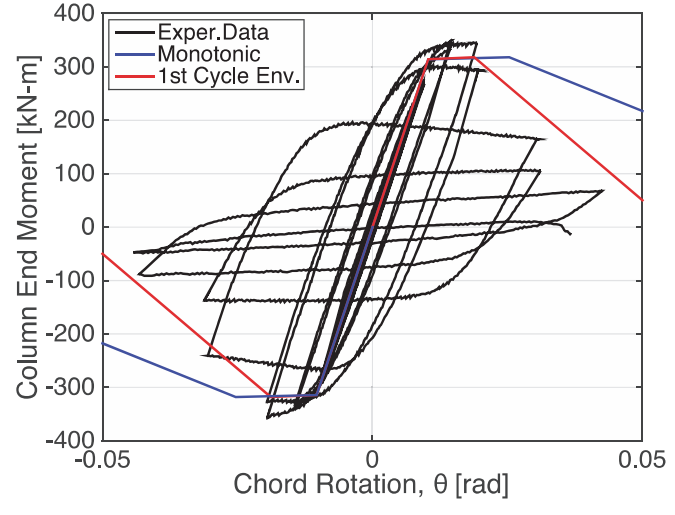

(a) HSS $10 \times 10 \times 3 / 8, P_{g} / P_{y e}=0.30$, Reversed Cyclic

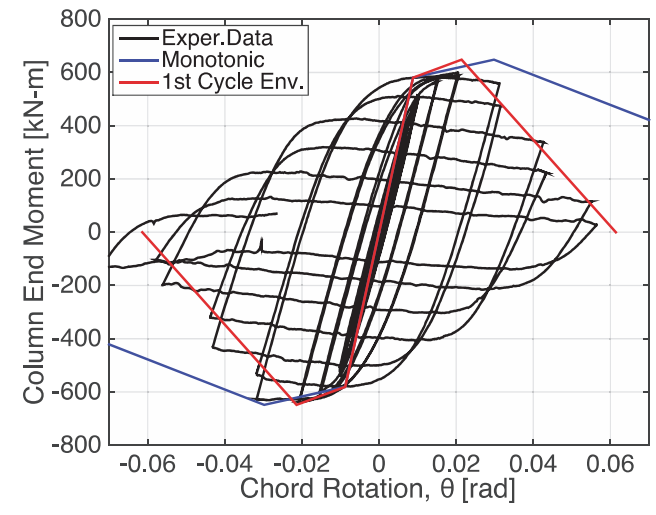

(b) HSS $12 \times 12 \times 5 / 8, P_{g} / P_{y e}=0.30$, Reversed Cyclic

Figure A-7 Illustration of proposed modeling recommendations for HSS beamcolumns [data from Suzuki and Lignos, 2015].

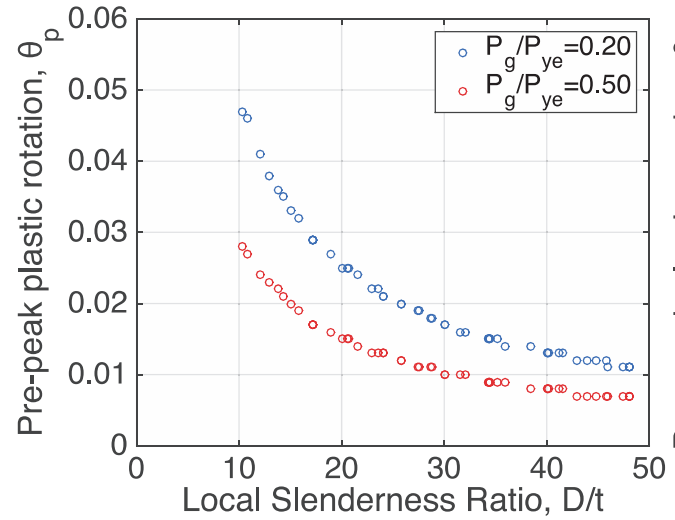

(a)

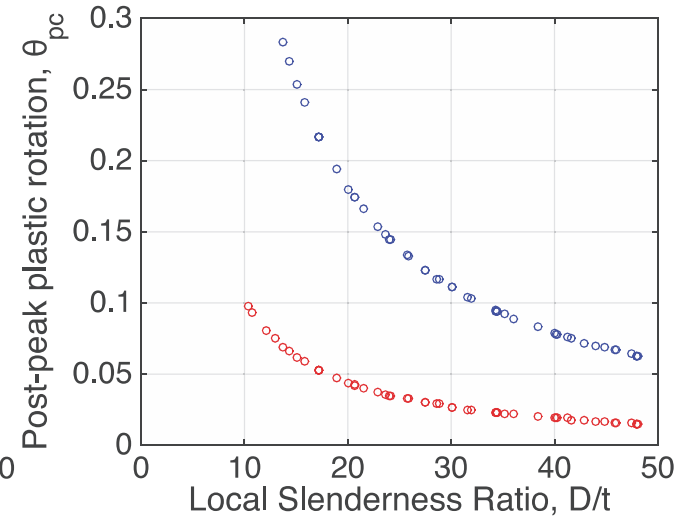

(b)

Figure A-8 Trends of pre- and post-peak plastic deformations with respect to the cross-section local slenderness ratio for modeling the monotonic backbone curve of HSS beam-columns. 
Table A-4 Modeling Parameters for Various HSS Column Sizes Based on Regression Equations [Assumed Steel Material ASTM A500Gr.B (nominal $f_{y}=42 \mathrm{ksi}$ ), $\left.P_{g} / P_{y e}=0.05\right]$

\begin{tabular}{|c|c|c|c|c|c|c|}
\hline Section & $D / t$ & $P_{g} / P_{y e}$ & $\begin{array}{c}\theta_{p} \\
\text { [rad] }\end{array}$ & $\begin{array}{c}\theta_{p c} \\
{[\mathrm{rad}]}\end{array}$ & $\begin{array}{c}\theta_{p}^{*} \\
{[\mathrm{rad}]}\end{array}$ & $\begin{array}{c}\theta^{*} p c \\
{[\mathrm{rad}]}\end{array}$ \\
\hline HSS20×20×5/8 & 32.0 & 0.05 & 0.020 & 0.172 & 0.013 & 0.078 \\
\hline HSS20×20×3/8 & $53.3^{*}$ & 0.05 & 0.012 & 0.093 & 0.008 & 0.040 \\
\hline HSS16×16×5/8 & 27.5 & 0.05 & 0.023 & 0.206 & 0.016 & 0.094 \\
\hline HSS16×16×3/8 & $45.8^{*}$ & 0.05 & 0.014 & 0.112 & 0.009 & 0.049 \\
\hline HSS12×12×5/8 & 20.7 & 0.05 & 0.030 & 0.291 & 0.021 & 0.137 \\
\hline HSS12×12×3/8 & 34.4 & 0.05 & 0.018 & 0.158 & 0.012 & 0.071 \\
\hline HSS $10 \times 10 \times 5 / 8$ & 17.2 & 0.05 & 0.035 & 0.363 & 0.026 & 0.174 \\
\hline HSS $10 \times 10 \times 3 / 8$ & 28.7 & 0.05 & 0.022 & 0.197 & 0.015 & 0.090 \\
\hline
\end{tabular}

* values outside the range of applicability of predictive equations

Table A-5 Modeling Parameters for Various HSS Column Sizes Based on Regression Equations [Assumed Steel Material ASTM A500Gr.B (nominal $f_{y}=42 \mathrm{ksi}$ ), $\left.P_{g} / P_{y e}=0.20\right]$

\begin{tabular}{|c|c|c|c|c|c|c|}
\hline Section & $D / t$ & $P_{g} / P_{y e}$ & $\begin{array}{c}\theta_{p} \\
{[\mathrm{rad}]}\end{array}$ & $\begin{array}{c}\theta_{p c} \\
\text { [rad] }\end{array}$ & $\begin{array}{c}\theta_{p}^{*} \\
\text { [rad] }\end{array}$ & $\begin{array}{c}\theta_{p c}^{*} \\
\text { [rad] }\end{array}$ \\
\hline HSS $20 \times 20 \times 5 / 8$ & 32.0 & 0.20 & 0.016 & 0.103 & 0.010 & 0.050 \\
\hline HSS $20 \times 20 \times 3 / 8$ & $53.3^{*}$ & 0.20 & 0.010 & 0.055 & 0.006 & 0.026 \\
\hline HSS $16 \times 16 \times 5 / 8$ & 27.5 & 0.20 & 0.019 & 0.123 & 0.012 & 0.060 \\
\hline HSS $16 \times 16 \times 3 / 8$ & $45.8^{*}$ & 0.20 & 0.012 & 0.067 & 0.007 & 0.031 \\
\hline HSS $12 \times 12 \times 5 / 8$ & 20.7 & 0.20 & 0.025 & 0.174 & 0.017 & 0.088 \\
\hline HSS12×12×3/8 & 34.4 & 0.20 & 0.015 & 0.094 & 0.010 & 0.045 \\
\hline HSS $10 \times 10 \times 5 / 8$ & 17.2 & 0.20 & 0.029 & 0.217 & 0.021 & 0.111 \\
\hline HSS10 $\times 10 \times 3 / 8$ & 28.7 & 0.20 & 0.018 & 0.117 & 0.012 & 0.057 \\
\hline
\end{tabular}


Table A-6 Modeling Parameters for Various HSS Column Sizes Based on Regression Equations [Assumed steel material ASTM A500Gr.B (nominal $\left.\left.f_{y}=42 \mathrm{ksi}\right), P_{g} / P_{y e}=0.50\right]$

\begin{tabular}{|c|c|c|c|c|c|c|}
\hline Section & $D / t$ & $P_{g} / P_{y e}$ & $\begin{array}{c}\theta_{p} \\
{[\mathrm{rad}]}\end{array}$ & $\begin{array}{c}\theta_{p c} \\
{[\mathrm{rad}]}\end{array}$ & $\begin{array}{c}\theta_{p}^{*} \\
{[\mathrm{rad}]}\end{array}$ & $\begin{array}{c}\theta_{p c}^{*} \\
{[\mathrm{rad}]}\end{array}$ \\
\hline HSS20×20×5/8 & 32.0 & 0.50 & 0.010 & 0.025 & 0.005 & 0.015 \\
\hline HSS $20 \times 20 \times 3 / 8$ & $53.3^{*}$ & 0.50 & 0.006 & 0.014 & 0.003 & 0.008 \\
\hline HSS16×16×5/8 & 27.5 & 0.50 & 0.011 & 0.030 & 0.006 & 0.018 \\
\hline HSS $16 \times 16 \times 3 / 8$ & $45.8^{*}$ & 0.50 & 0.007 & 0.016 & 0.004 & 0.009 \\
\hline HSS12×12×5/8 & 20.7 & 0.50 & 0.015 & 0.042 & 0.009 & 0.026 \\
\hline HSS12×12×3/8 & 34.4 & 0.50 & 0.009 & 0.023 & 0.005 & 0.013 \\
\hline HSS10×10×5/8 & 17.2 & 0.50 & 0.017 & 0.053 & 0.011 & 0.033 \\
\hline HSS $10 \times 10 \times 3 / 8$ & 28.7 & 0.50 & 0.011 & 0.029 & 0.006 & 0.017 \\
\hline
\end{tabular}

* values outside the range of applicability of predictive equations

\section{A.5 Case Studies}

In order to evaluate the performance of the proposed modeling recommendations provided in Chapter 4 for steel moment-resisting frame systems, three archetype buildings with perimeter steel special moment frames as their primary lateral load resisting system were evaluated. The archetype buildings were chosen as 4-, 12-, and 20 -story office buildings located in urban California. The evaluation of the component models was done through a series of single-mode nonlinear static analysis procedures (NSP) and nonlinear response history analysis procedures (NRHA). The objectives of the case studies were to compare the implementation of the proposed modeling recommendations of Chapter 4 to the recommendations of ASCE/SEI 41-13; assess the effects of modeling the composite beam action; and to investigate the effects of explicitly modeling the panel zone on the overall seismic performance of the archetype buildings.

\section{A.5.1 Description of Archetype Buildings}

The selected archetype buildings reflect typical office buildings designed for downtown Los Angeles, California, with 3-bay perimeter SMFs as the seismic force resisting system (NIST, 2010a; Lignos et al., 2015). These SMFs were designed based on response spectrum analysis for a seismic design category $D_{\max }$ in California. The following provisions were used in the design of the archetype buildings: AISC 341-05 (AISC, 2005a), AISC 358-05 (AISC, 2005b), and ASCE/SEI 7-05 (ASCE, 2006). The first story height of each of the structures is specified as 15 feet, the typical story height is 13 feet, and the column center-to-center bay width is 20 feet. The typical plan view of the buildings is shown in Figure A-9. The steel of all structural components is ASTM A992 Gr. 50. All fully restrained beam-to-column 
connections are specified as reduced beam section (RBS) moment connections as per AISC 358-05. In order to account for the effects of the composite slab, a composite floor system was designed with a depth of the rib of the steel deck to be $d_{r i b}=3.5$ inches. The total depth of the concrete slab, $t_{s}$, is 5.0 inches. Full composite action is assumed between the steel beams and the slab, and the specified compressive strength of the concrete was assumed to be $f_{c}^{\prime}=5000$ psi. Column splices are provided every two stories at mid-story height, with the first splice starting between stories two and three. A detailed description of the design assumptions for the archetype buildings can be found in NIST (2010a) and Lignos et al. (2015).

\section{A.5.2 Ground Motion Selection and Scaling}

In order to conduct nonlinear response history analysis (NRHA), the set of 44 farfield ground motions from FEMA P-695 (FEMA, 2009b) were selected to evaluate the building performance. The ground motions were scaled such that the mean of the absolute acceleration spectrum from the unscaled intensities of the 44 ground motions represents the absolute acceleration design spectrum in urban California for soil type D. The building performance and comparisons between various component models were based on three different levels of ground motion intensity of interest to the engineering profession, including a service level earthquake (SLE), with a 50\% probability of exceedence over 50 years; a design basis earthquake (DBE), with a $10 \%$ probability of exceedence over 50 years; and a maximum considered earthquake (MCE), with a $2 \%$ probability of exceedence over 50 years.

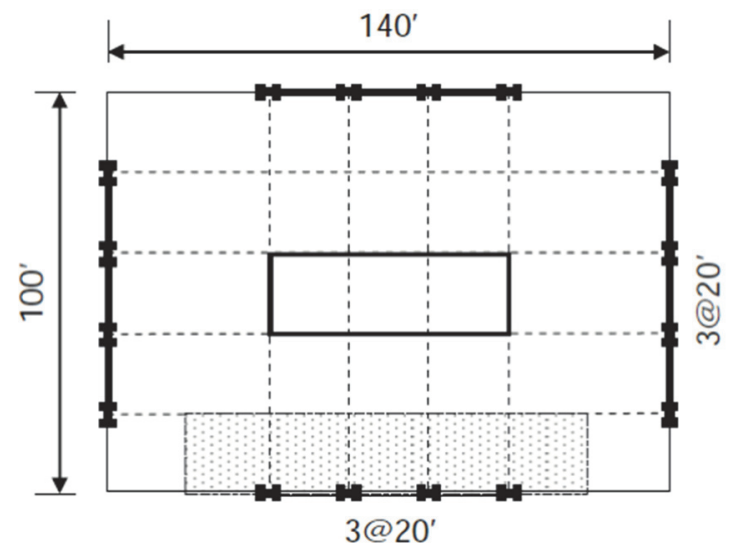

Figure A-9 Plan view of archetype buildings from NIST (2010a).

\section{A.5.3 Nonlinear Building Models}

Nonlinear building models were developed based on the East-West 3-bay perimeter SMF (see Figure A-9) from each of the archetype buildings using the Open System for Earthquake Engineering Simulation (OpenSees) (McKenna, 1997) version 2.5.0. The nonlinear behavior of the structural components was modeled using a concentrated plasticity approach. The structural components were modeled with 
elastic beam-columns with multi-linear spring elements at their ends as shown in Figure A-10a. In this case, the modified Ibarra-Medina-Krawinkler (IMK) model was employed (Ibarra et al., 2005). In the case that a first-mode NSP was carried out, the parameters of the multi-linear spring elements followed either the ASCE/SEI 41-13 nonlinear component model, or the first-cycle envelope proposed in Chapter 4. In the case that a NRHA was carried out, the parameters of the backbone curve of the respective structural component were based off of the proposed monotonic backbone curve modeling recommendations of Chapter 4. The cyclic and in-cycle deterioration of the beams and columns were modeled explicitly using the modified IMK deterioration model through the use of the reference cumulative plastic rotation, $\Lambda$, as discussed in Lignos and Krawinkler (2011) that was adopted in PEER/ATC-72 (PEER/ATC, 2010) nonlinear modeling guidelines. Beam-to-column joint panel zones were modeled using the Krawinkler model as discussed in Gupta and Krawinkler (1999). When the panel zones were not modeled explicitly (i.e., centerline model), the beam and column elements were extended to the mid-point of the panel zone using the respective flexural properties of the elements (i.e., not considering a rigid offset) as shown in Figure A-10b. P-delta effects were considered with a leaning column. For NRHA, a damping ratio of $2.5 \%$ was applied to the first natural period, $T_{l}$ and to $20 \% T_{l}$ as suggested in NIST (2010a).

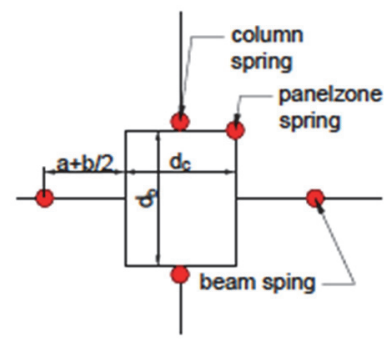

(a)

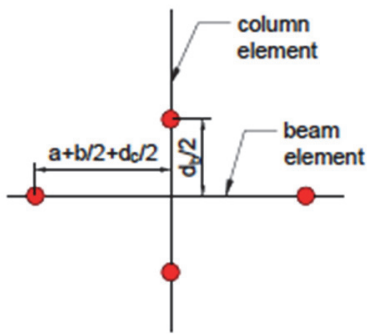

(b)

Figure A-10 Schematic representation of the analytical model representations of the beam-to-column joint panel zones (Hartloper and Lignos, 2016).

\section{A.5.4 Results of the Nonlinear Static Analysis}

Nonlinear static analysis was conducted for all three archetype buildings discussed in Section A.5.3 by using a first mode lateral load pattern. This section presents the results for the 12-story building for illustration but the findings are applicable to the other two archetypes that were analyzed. Figure A-11 shows the global pushover curve based on the bare and composite model representations of the 12-story archetype building. Predicted response from three types of models (5 total) are superimposed: (1) Beams and columns modeled with current ASCE/SEI 41-13 nonlinear modeling provisions (i.e., noted "PZ-ASCE-41-13"); (2) beams and columns modeled with the nonlinear modeling recommendations of Chapter 4 (i.e., noted "PZ-114-1st-Cycle Env." and "Composite- PZ-114-1st-Cycle Env."); and (3) a 
bare and composite centerline model in which beams and columns are modeled with the proposed 114 nonlinear modeling recommendations (i.e., "CL-114-1st-Cycle Env.", "Composite-CL-114-1st-Cycle Env."). In this figure, the base shear force, $V$, is normalized with respect to the seismic weight, $W$, of the archetype in the EW loading direction. The roof displacement, $\Delta_{r}$ is normalized with respect to the total height, $H$, of the archetype building. The following observations hold true:

- When the predicted nonlinear static response of steel SMFs designed according to the AISC seismic provisions is based on the bare properties of steel beams (i.e., no consideration of composite beam effects), there is practically no need to explicitly consider the parallelogram panel zone model (Gupta and Krawinkler, 1999) in the numerical model representation of the steel SMF.

- The post-yield global stiffness of the archetype buildings based on the ASCE/SEI 41-13 model is significantly underestimated compared to the proposed model of Chapter 4 . This is primarily attributed to the assumed fixed 3\% strain-hardening ratio of the ASCE/SEI 41-13 component model regardless of the compactness of the employed cross-section of the respective beam and/or column.

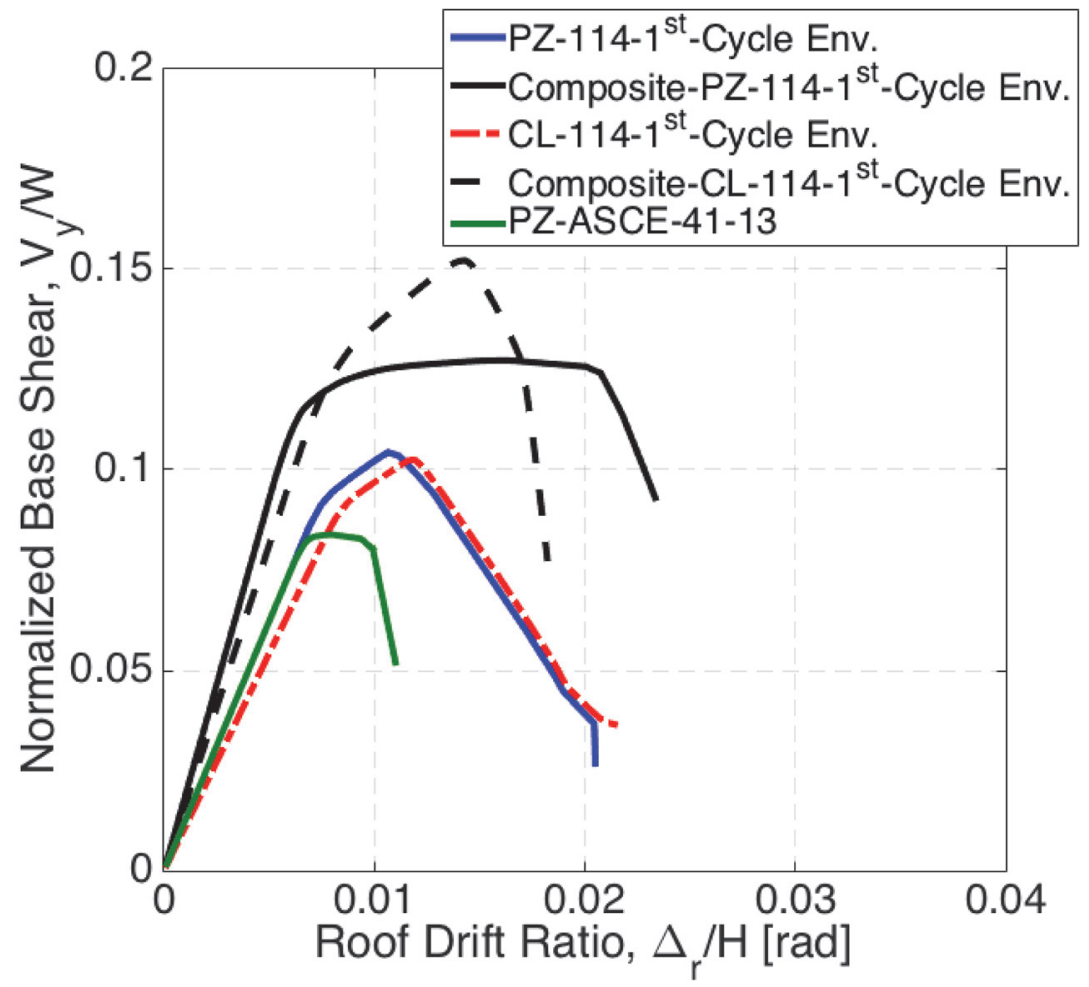

Figure A-11 Global pushover curves based on single-mode nonlinear static analysis procedure and various component-modeling options. 
- The post-capping global stiffness of the archetype buildings based on the ASCE/SEI 41-13 model is very steep due to the characteristic vertical strength drop in the ASCE/SEI 41-13 component model. This issue is likely to cause convergence problems as discussed in prior studies (Lignos et al., 2015).

- Table A-7 summarizes the overstrength factors for the three archetype buildings that were analyzed based on various modeling approaches. Based on Table A-7 and Figure A-11, the predicted overstrength, $\Omega_{s}$, obtained from single-mode NSPs based on the composite nonlinear building model is on the order of 1.3 times larger than the one predicted based on a bare nonlinear building model. $\Omega_{s}=V_{\max } / V$, where $V_{\max }$ is the maximum base shear from a pushover analysis and $V$ is the design base shear as per ASCE/SEI 7-05. It is also observed that the composite beam effects also increase the global elastic lateral stiffness of the respective numerical model by 1.4 times, on average. These findings are consistent with recent studies by Elkady and Lignos (2014; 2015b). In reality, the static overstrength should be expected to be more than the tabulated values if the contribution of the gravity framing system to the lateral strength of the archetype is considered. Such contributions have been quantified in recent studies (Elkady and Lignos, 2015b).

Table A-7 Model Periods and Static Overstrength for Nonlinear Static Analysis

\begin{tabular}{|c|c|c|c|c|c|c|c|c|c|}
\hline \multirow[b]{2}{*}{ Stories } & \multirow{2}{*}{$\begin{array}{c}\begin{array}{c}\text { Period, } T \\
(\mathrm{sec})\end{array} \\
T=C_{u} T_{a} \\
\end{array}$} & \multicolumn{4}{|c|}{ Computed Period, $T_{a}(\mathrm{sec})$} & \multicolumn{4}{|c|}{ Static Overstrength $\Omega_{s}, V_{\max } / V$} \\
\hline & & $\begin{array}{c}\text { Bare- } \\
\text { PZ } \\
\end{array}$ & $\begin{array}{c}\text { Comp- } \\
P Z \\
\end{array}$ & $\begin{array}{c}\text { Bare- } \\
\text { CL } \\
\end{array}$ & $\begin{array}{c}\text { Comp- } \\
\text { CL } \\
\end{array}$ & $\begin{array}{c}\text { Bare- } \\
\text { PZ } \\
\end{array}$ & $\begin{array}{c}\text { Comp- } \\
P Z \\
\end{array}$ & $\begin{array}{c}\text { Bare- } \\
\text { CL } \\
\end{array}$ & $\begin{array}{c}\text { Comp- } \\
\text { CL }\end{array}$ \\
\hline 4 & 0.95 & 1.46 & 1.19 & 1.55 & 1.24 & 2.09 & 2.43 & 2.19 & 2.85 \\
\hline 12 & 2.25 & 2.76 & 2.26 & 2.97 & 2.41 & 2.81 & 3.43 & 2.76 & 4.11 \\
\hline 20 & 3.37 & 3.95 & 3.16 & 4.26 & 3.42 & 2.27 & 2.95 & 2.24 & 3.43 \\
\hline
\end{tabular}

- In Figure A-11, when the composite effects are considered in the nonlinear building model, the predicted nonlinear static response of the archetypes is inherently more ductile than that predicted based on bare nonlinear building models. This is in part attributed to the delay of the local buckling formation at the top flange of the respective steel beams due to the slab restraint and the shift of the neutral axis towards the top flange prior to concrete slab cracking. It should be noted that the effective beam depth is larger than that of the bare beam when the slab is considered in the nonlinear building model; therefore, the panel zone shear demands are larger than those predicted by bare models. This results in panel zone shear yielding. This is shown in Figure A-12 that illustrates the predicted panel zone behavior in terms of panel zone moment demands versus panel zone shear distortion, based on various nonlinear component-modeling options. When the composite beam action is neglected, the predicted panel zone 
response is elastic, which could be misleading especially if the predicted response of a steel moment-resisting frame system is based on centerline models. This illustrates the significance of the composite action in the predicted response of steel SMFs.

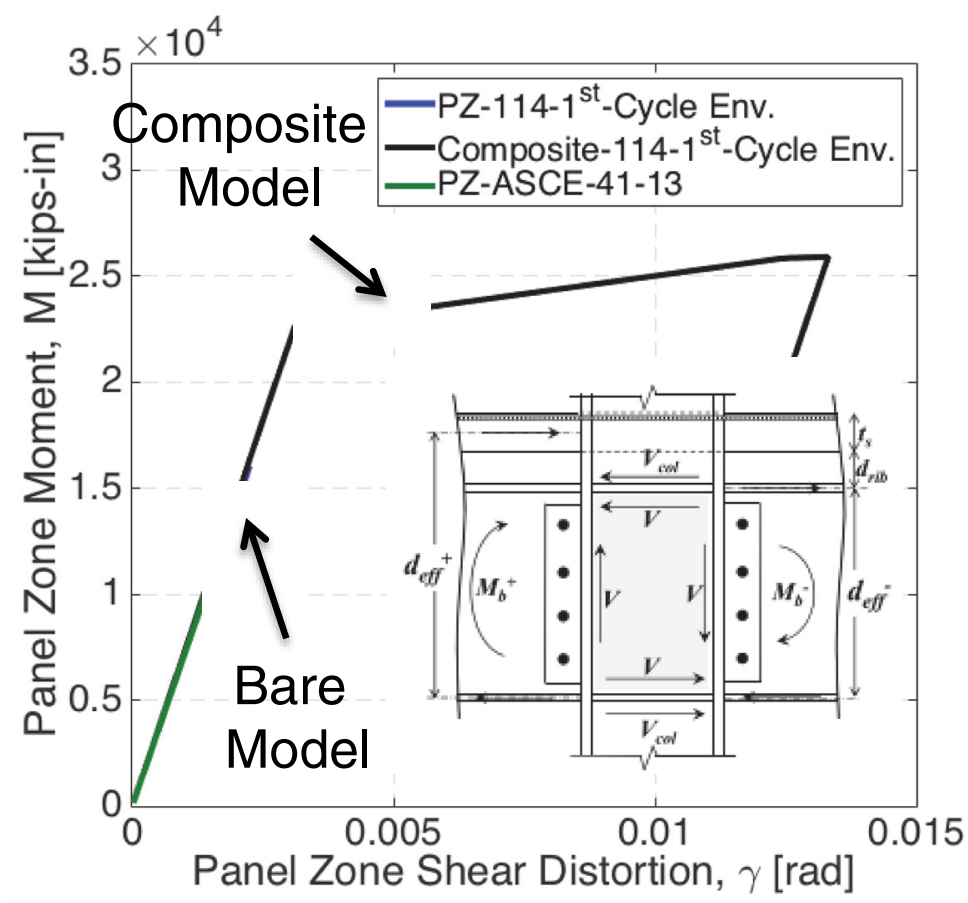

Figure A-12 Prediction of the nonlinear static response of the first story interior beam-to-column panel zone joint of the 12-story steel SMF based on first-mode NSP.

The implication of various nonlinear modeling options is also illustrated based on the assessment of story-based demand parameters of the respective archetypes based on nonlinear static analysis. Figure A-13 shows the predicted peak story drift ratios along the height of the 12-story archetype building for two target roof drift ratios and based on various nonlinear modeling options. From Figure A-13a, at 1\% target roof drift ratio, the predicted peak story drift ratios of the 12-story archetype are practically not influenced from the employed component model. The reason is that most of the structural components are not far into their inelastic range. However, from Figure A-13b (far into the inelastic range), the predicted peak story drift ratios of the 12-story archetype are very sensitive to the choice of the employed nonlinear component model. In particular, in Figure A-13b, the consideration of composite beam effects in the nonlinear building model can shift the global collapse mechanism of the respective archetype. This is consistent with recent findings by Elkady and Lignos (2014) regarding the composite beam effects. It should also be noted that a modeler should tie the selected numerical model complexity with the performance objective of interest as discussed in Lignos et al. (2015). 


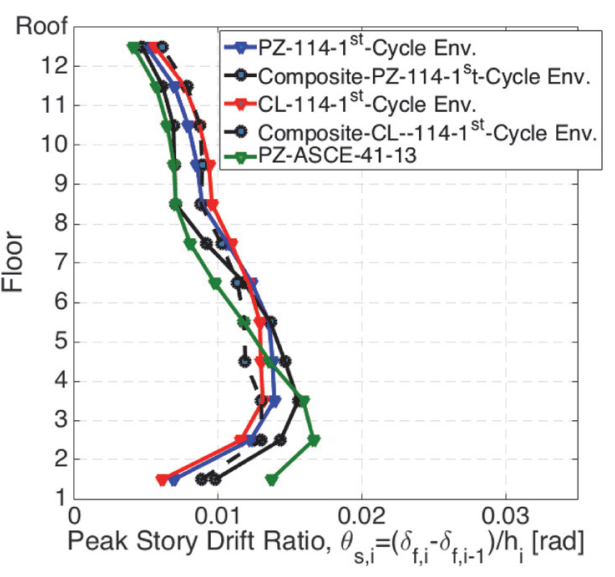

(a) Target roof drift ratio, $\Delta_{r} / H=1 \%$

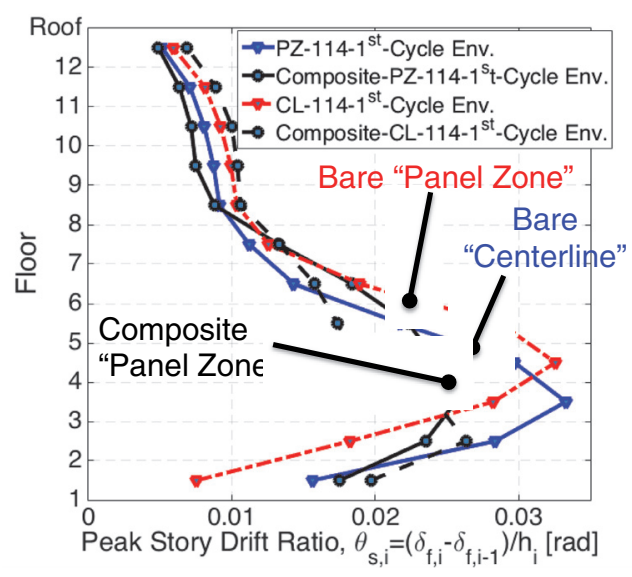

(b) Target roof drift ratio, $\Delta_{r} / H=1.5 \%$

Figure A-13 Predicted peak story drift ratios along the height of the 12-story archetype based on NSP, first-mode lateral load pattern.

\section{A.5.5 Results of the Nonlinear Response History Analysis}

This section discusses the main findings of the present study based on story-based peak engineering demand parameter comparisons between the predicted nonlinear static response of the building archetypes based on a single-mode NSP that were considered and that obtained based on rigorous NRHA. The building archetypes were subjected to the set of 44 far-field ground motions from FEMA P-695 as discussed in Section A.5.2. The counted median response of 44 nonlinear building simulations corresponding to each one of the employed ground motions is compared with the single-mode NSP predictions for three story-based demand parameters of interest, including peak story drift ratios; peak story shear force $\left(V_{I+P-4}\right)$; and peak overturning moment $\left(O T M_{I+P-4}\right)$ demands including the effects of P-delta. The discussion presented here is based on the comparisons shown for the 12-story archetype building. However, the findings are applicable in all cases that were analyzed.

The NSP to NRHA comparisons for all three story-based demand parameters of interest are shown in Figure A-14 for two seismic intensities: a DBE that corresponds to a $10 \%$ probability of exceedance over 50 years and a MCE that corresponds to a $2 \%$ probability of exceedance over 50 years. It should be noted that the NRHA predictions are based on the composite model representations of the archetype buildings that explicitly consider the possibility for panel zone yielding due to shear distortion (noted as "NRHA-Median"). The NSP predictions summarized in Figure A-14 are based on three selected nonlinear building models that their structural components were modeled based on the proposed nonlinear modeling recommendations proposed in Chapter 4: (1) the bare model with explicit consideration of the panel zone (noted as "NSP-Bare-PZ"); (2) a bare centerline 
model (noted as "NSP-Bare-CL"); and (3) a composite model that explicitly captures the panel zone (noted as "NSP-Composite-PZ").

Figures A-14a and A-14b show the peak story drift ratios along the height of the 12story archetype as predicted by NSP and NRHA for the DBE and MCE seismic intensities, respectively. For both seismic intensities, peak story drift ratios are underestimated in the upper stories. This is attributed to the higher-mode effects that are not explicitly captured by single-mode NSPs (Chopra and Goel, 2004;

Krawinkler and Seneviratna, 1998). In Figure A-14b (far into the inelastic range), peak story drift ratios are overpredicted by all single-mode NSP options in the lower stories. This is attributed to the fact that the first-mode lateral load pattern that is employed to conduct the NSPs remains constant throughout the nonlinear static procedure (Krawinkler and Seneviratna, 1998). However, the composite model tends to predict the story drift distribution of the 12-story archetype much closer to NRHA than the other models. Figures A-14c and A-14d show the normalized peak story shear predictions based on various NSP options for the 12-story archetype for DBE and MCE seismic intensities, respectively. From this figure, all NSP predictions underestimate the peak story shear demands in mid and upper stories of the 12-story archetype. For seismic intensities associated with low probability of occurrence earthquakes (i.e., MCE) the maximum base shear force from the NSP is approximately $20 \%$ less than that predicted based on the median response from the NRHA. When the composite beam effects are considered in the nonlinear building model the corresponding base shear force is under predicted by approximately $10 \%$ compared to the NRHA median base shear. These effects, which increase with the number of stories, are attributed to the dynamic redistribution of story shear forces during the nonlinear building response. This tends to amplify story shear forces compared to those obtained from a pre-determined in single-mode NSPs (Krawinkler et al., 2011).

Figures A-14e and A-14f show the NSP peak floor OTM predictions for the 12-story archetype building for DBE and MCE seismic intensities, respectively. From both figures, the distribution of the OTM is almost linear. This is to be expected from the peak story shear force distribution shown in Figures A-14c and A-14d. From Figures A-14e and A-14f, at the base, the difference between single-mode NSP predictions and those from NRHA are smaller compared to those from peak story shear forces shown in Figure A-14c and A-14f for the DBE and MCE seismic intensities, respectively. This is attributed to the fact that maximum shear forces in individual stories along the height of the archetypes occur at different times. 


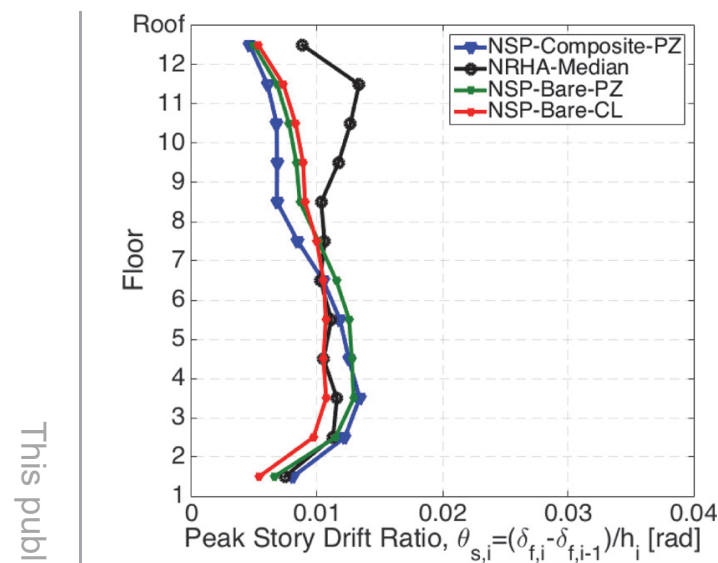

(a)

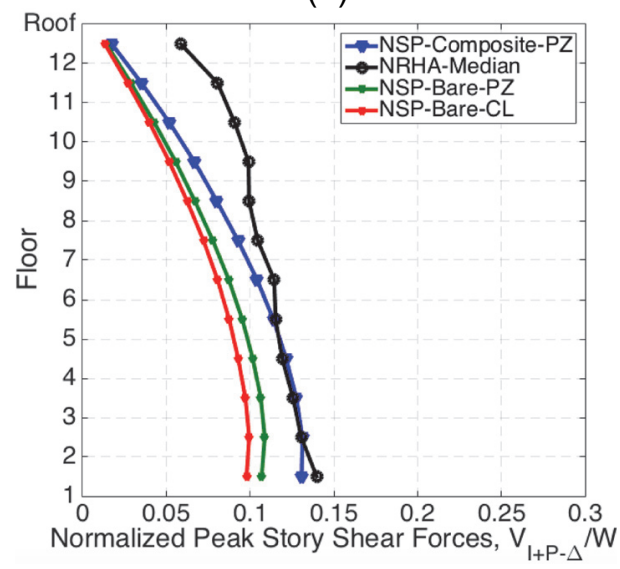

(c)

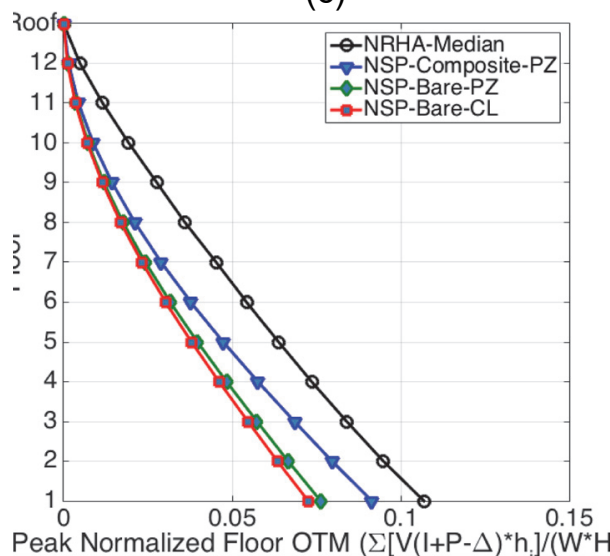

(e)

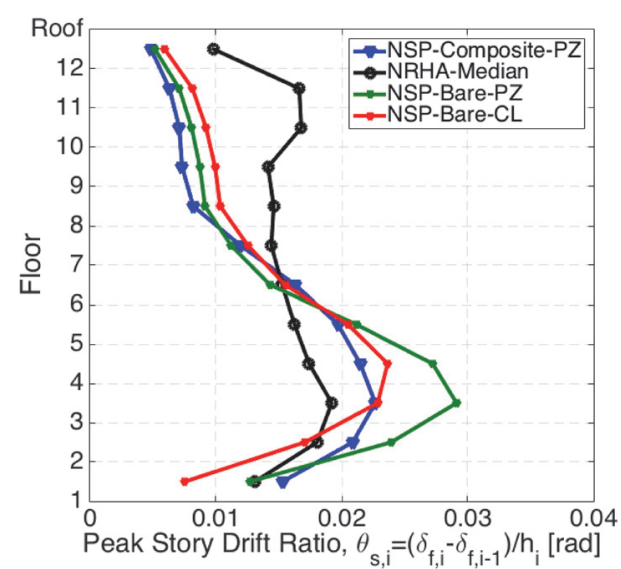

(b)

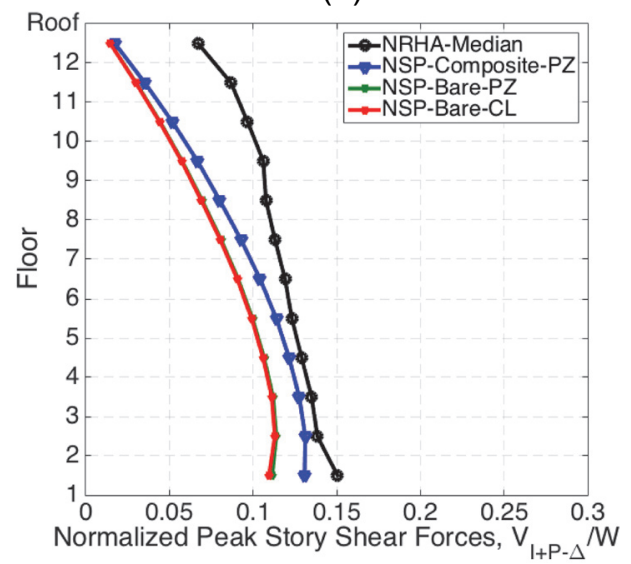

(d)

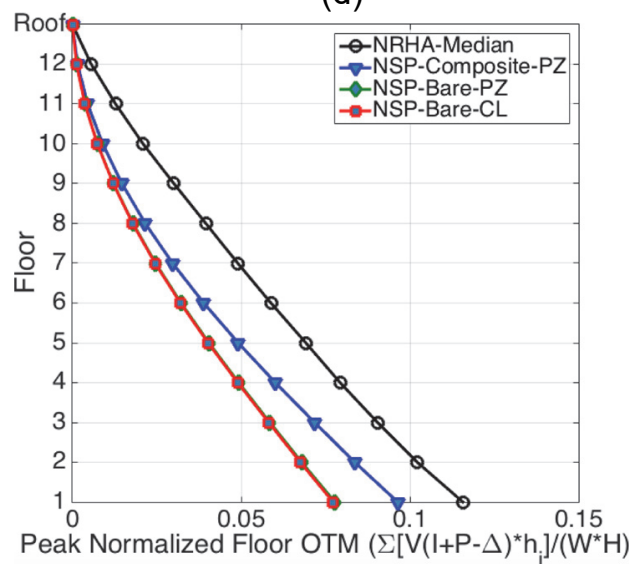

(f)

Figure A-14 NSP to NRHA comparison of predicted story-based engineering demand parameters, 12-story archetype; left DBE seismic intensity; right MCE seismic intensity. 



\section{Appendix B}

\section{Shear-Controlled Reinforced Concrete Walls - Database}

\section{B.1 Loading Properties of Reinforced Concrete Walls}

Table B-1 through Table B-6 present information on rectangular (planar) walls and the Table B-7 through Table B-12 present information on walls pwith barbells and flanges. In Table B-1 and Table B-7, $P$ is the axial compressive load; $A_{g}$ is the total cross-sectional area; and $f_{c}^{\prime}$ is the concrete compressive strength. In Table B-2 and Table B-8, $t_{w}$ is the wall thickness; $h_{w}$ is the clear height of the wall; $l_{w}$ is the length of the wall; $l_{f}$ is the width of the flanges; $t_{f}$ is the flange thickness; $l_{c}$ is the width of the boundary column; $t_{c}$ is the depth of the boundary column; and $l_{b e}$ is the depth of the boundary element. In Table B-3 and Table B-9, $\rho_{v}^{w}$ and $\rho_{h}^{w}$ are the vertical and horizontal web reinforcement ratio, respectively; $S_{h}^{w}$ and $S_{v}^{w}$ are the spacing of the horizontal and vertical web reinforcement, respectively; $\rho_{v}^{f}$ and $\rho_{h}^{f}$ are the vertical and horizontal reinforcement ratio in the flanges, respectively; $S_{h}^{f}$ and $S_{v}^{f}$ are the spacing of the horizontal and vertical reinforcement in the flanges; $\rho_{v}^{b e}$ is the vertical reinforcement ratio of the boundary columns; $S_{h}^{b e}$ is the tie spacing in the boundary elements. In Table B-4 and Table B-10, $f_{v v}^{w}$ and $f_{u v}^{w}$ are the yield and ultimate strengths of the vertical web reinforcement; $f_{v h}^{w}$ and $f_{u h}^{w}$ are the yield and ultimate strengths of the horizontal web reinforcement; $f_{v v}^{f}$ and $f_{u v}^{f}$ are the yield and ultimate strengths of the vertical reinforcement in the flanges; and $f_{v h}^{f}$ and $f_{u h}^{f}$ are the yield and ultimate strengths of the horizontal reinforcement in the flanges. In Table B-5, Table B-6, Table B-11, and Table B-12, $\Delta_{A}, \Delta_{B}, \Delta_{C}$, and $\Delta_{D}$ are the displacements of points $A, B, C$, and $D$ on the idealized backbone curve, presented in Figure 8-1, and $F_{A}, F_{B}$, $F_{C}$, and $F_{D}$ are the corresponding forces.

Table B-1 Loading Properties of the RC Walls with Rectangular Cross-Section

\begin{tabular}{|c|c|c|c|c|c|}
\hline Test No. & Researcher & Spec ID & Wall type & Loading type & $\begin{array}{c}P / A_{g} f_{c}^{\prime} \\
(\%)\end{array}$ \\
\hline 1 & Alexander et al. (1973) & 4 & Rectangular & Cyclic & 4.6 \\
\hline 2 & & 72 & Rectangular & Cyclic & 11.4 \\
\hline 3 & & 73 & Rectangular & Cyclic & 9.4 \\
\hline 4 & Hirosawa (1975) & 74 & Rectangular & Cyclic & 9.4 \\
\hline 5 & & 75 & Rectangular & Cyclic & 14.3 \\
\hline 6 & & 76 & Rectangular & Cyclic & 13.3 \\
\hline
\end{tabular}


Table B-1 Loading Properties of the RC Walls with Rectangular Cross-Section (continued)

\begin{tabular}{|c|c|c|c|c|c|}
\hline Test No. & Researcher & Spec ID & Wall type & Loading type & $\begin{array}{c}P / A_{g} f_{c}^{\prime} \\
(\%)\end{array}$ \\
\hline 7 & \multirow{5}{*}{ Hirosawa (1975) } & 77 & Rectangular & Cyclic & 10.7 \\
\hline 8 & & 78 & Rectangular & Cyclic & 9.4 \\
\hline 9 & & 79 & Rectangular & Cyclic & 14.3 \\
\hline 10 & & 80 & Rectangular & Cyclic & 13.3 \\
\hline 11 & & 81 & Rectangular & Cyclic & 10.7 \\
\hline 12 & Synge (1980) & WALL 1 & Rectangular & Cyclic & 0.0 \\
\hline 13 & \multirow{2}{*}{ Wiradinata (1985) } & WALL1 & Rectangular & Cyclic & 0.0 \\
\hline 14 & & WALL2 & Rectangular & Cyclic & 0.0 \\
\hline 15 & \multirow{3}{*}{ Maier (1985) } & S4 & Rectangular & Monotonic & 6.7 \\
\hline 16 & & S9 & Rectangular & Monotonic & 7.5 \\
\hline 17 & & S10 & Rectangular & Monotonic & 7.2 \\
\hline 18 & \multirow{2}{*}{ Pilette (1987) } & WALL 4 & Rectangular & Cyclic & 0.0 \\
\hline 19 & & WALL 5 & Rectangular & Cyclic & 0.0 \\
\hline 20 & \multirow{2}{*}{ Huang and Sheu (1988) } & SWN-1D & Rectangular & Cyclic & 12.0 \\
\hline 21 & & SWN-5D & Rectangular & Cyclic & 12.0 \\
\hline 22 & \multirow{2}{*}{ Wasiewicz (1988) } & WALL 3 & Rectangular & Cyclic & 0.0 \\
\hline 23 & & WALL 6 & Rectangular & Cyclic & 0.0 \\
\hline 24 & \multirow{7}{*}{ Lefas et al. (1990) } & SW11 & Rectangular & Monotonic & 0.0 \\
\hline 25 & & SW12 & Rectangular & Monotonic & 10.0 \\
\hline 26 & & SW13 & Rectangular & Monotonic & 20.0 \\
\hline 27 & & SW14 & Rectangular & Monotonic & 0.0 \\
\hline 28 & & SW15 & Rectangular & Monotonic & 10.0 \\
\hline 29 & & SW16 & Rectangular & Monotonic & 20.0 \\
\hline 30 & & SW17 & Rectangular & Monotonic & 0.0 \\
\hline 31 & \multirow{3}{*}{ Lopes and Elnashai (1991) } & SW11 & Rectangular & Monotonic & 0.0 \\
\hline 32 & & SW12 & Rectangular & Cyclic & 0.0 \\
\hline 33 & & SW13 & Rectangular & Cyclic & 0.0 \\
\hline 34 & \multirow{5}{*}{ Lopes and Elnashai (1991) } & SW14 & Rectangular & Cyclic & 0.0 \\
\hline 35 & & SW15 & Rectangular & Cyclic & 0.0 \\
\hline 36 & & SW16 & Rectangular & Cyclic & 0.0 \\
\hline 37 & & SW17 & Rectangular & Cyclic & 0.0 \\
\hline 38 & & SW18 & Rectangular & Cyclic & 0.0 \\
\hline
\end{tabular}


Table B-1 Loading Properties of the RC Walls with Rectangular Cross-Section (continued)

\begin{tabular}{|c|c|c|c|c|c|}
\hline Test No. & Researcher & Spec ID & Wall type & Loading type & $\begin{array}{c}P / A_{g} f_{c}^{\prime} \\
(\%)\end{array}$ \\
\hline 39 & \multirow{3}{*}{ Saatcioglu (1991) } & Wall-1 & Rectangular & Cyclic & 0.0 \\
\hline 40 & & Wall-4 & Rectangular & Cyclic & 0.0 \\
\hline 41 & & Wall-6 & Rectangular & Cyclic & 0.0 \\
\hline 42 & \multirow{3}{*}{ Cheng (1992) } & SW4A & Rectangular & Cyclic & 0.0 \\
\hline 43 & & SW5 & Rectangular & Cyclic & 0.0 \\
\hline 44 & & SW6 & Rectangular & Cyclic & 0.0 \\
\hline 45 & \multirow{2}{*}{ Mohammadi (1994) } & Wall 7 & Rectangular & Cyclic & 0.0 \\
\hline 46 & & Wall 8 & Rectangular & Cyclic & 0.0 \\
\hline 47 & \multirow{3}{*}{ Cheng (1992) } & SW-OE & Rectangular & Cyclic & 0.0 \\
\hline 48 & & SW-1E & Rectangular & Cyclic & 0.0 \\
\hline 49 & & SW-9E & Rectangular & Cyclic & 0.0 \\
\hline 50 & \multirow{6}{*}{ Salonikios et al. (1999) } & LSW1 & Rectangular & Cyclic & 0.0 \\
\hline 51 & & LSW2 & Rectangular & Cyclic & 0.0 \\
\hline 52 & & LSW3 & Rectangular & Cyclic & 7.0 \\
\hline 53 & & MSW1 & Rectangular & Cyclic & 0.0 \\
\hline 54 & & MSW2 & Rectangular & Cyclic & 0.0 \\
\hline 55 & & MSW3 & Rectangular & Cyclic & 7.0 \\
\hline 56 & Xia and Xiao (2000) & W1-A & Rectangular & Cyclic & 9.4 \\
\hline 57 & \multirow{11}{*}{ Hidalgo et al. (2002) } & 1 & Rectangular & Cyclic & 0.0 \\
\hline 58 & & 2 & Rectangular & Cyclic & 0.0 \\
\hline 59 & & 4 & Rectangular & Cyclic & 0.0 \\
\hline 60 & & 6 & Rectangular & Cyclic & 0.0 \\
\hline 61 & & 7 & Rectangular & Cyclic & 0.0 \\
\hline 62 & & 8 & Rectangular & Cyclic & 0.0 \\
\hline 63 & & 9 & Rectangular & Cyclic & 0.0 \\
\hline 64 & & 10 & Rectangular & Cyclic & 0.0 \\
\hline 65 & & 11 & Rectangular & Cyclic & 0.0 \\
\hline 66 & & 12 & Rectangular & Cyclic & 0.0 \\
\hline 67 & & 13 & Rectangular & Cyclic & 0.0 \\
\hline 68 & \multirow{3}{*}{ Hidalgo et al. (2002) } & 14 & Rectangular & Cyclic & 0.0 \\
\hline 69 & & 15 & Rectangular & Cyclic & 0.0 \\
\hline 70 & & 16 & Rectangular & Cyclic & 0.0 \\
\hline
\end{tabular}


Table B-1 Loading Properties of the RC Walls with Rectangular Cross-Section (continued)

\begin{tabular}{|c|c|c|c|c|c|}
\hline Test No. & Researcher & Spec ID & Wall type & Loading type & $\begin{array}{c}P / A_{g} f_{c}^{\prime} \\
(\%)\end{array}$ \\
\hline 71 & \multirow{7}{*}{ Hidalgo et al. (2002) } & 21 & Rectangular & Cyclic & 0.0 \\
\hline 72 & & 22 & Rectangular & Cyclic & 0.0 \\
\hline 73 & & 23 & Rectangular & Cyclic & 0.0 \\
\hline 74 & & 24 & Rectangular & Cyclic & 0.0 \\
\hline 75 & & 25 & Rectangular & Cyclic & 0.0 \\
\hline 76 & & 26 & Rectangular & Cyclic & 0.0 \\
\hline 77 & & 28 & Rectangular & Cyclic & 0.0 \\
\hline 78 & \multirow{6}{*}{ Massone (2006) } & WP111-9 & Rectangular & Cyclic & 10.0 \\
\hline 79 & & WP111-10 & Rectangular & Cyclic & 10.0 \\
\hline 80 & & WP1105-8 & Rectangular & Cyclic & 5.0 \\
\hline 81 & & WP1105-7 & Rectangular & Cyclic & 5.0 \\
\hline 82 & & WP110-5 & Rectangular & Cyclic & 0.0 \\
\hline 83 & & WP110-6 & Rectangular & Monotonic & 0.0 \\
\hline 84 & \multirow{4}{*}{ Greifenhagen (2005) } & M1 & Rectangular & Cyclic & 2.2 \\
\hline 85 & & M2 & Rectangular & Cyclic & 2.2 \\
\hline 86 & & M3 & Rectangular & Cyclic & 9.5 \\
\hline 87 & & M4 & Rectangular & Cyclic & 5.0 \\
\hline 88 & \multirow{8}{*}{ Kuang and $\mathrm{Ho}$ (2008) } & U 1.0 & Rectangular & Cyclic & 15.0 \\
\hline 89 & & U 1.5 & Rectangular & Cyclic & 15.0 \\
\hline 90 & & C 1.0 & Rectangular & Cyclic & 15.0 \\
\hline 91 & & C 1.5 & Rectangular & Cyclic & 15.0 \\
\hline 92 & & U $1.0 \mathrm{BC}$ & Rectangular & Cyclic & 15.0 \\
\hline 93 & & U1.5 BC & Rectangular & Cyclic & 15.0 \\
\hline 94 & & U $1.0 \mathrm{BC} 2$ & Rectangular & Cyclic & 15.0 \\
\hline 95 & & $\mathrm{U} 1.0 \mathrm{CT}$ & Rectangular & Cyclic & 15.0 \\
\hline 96 & \multirow{5}{*}{ Athanasopoulou (2010) } & S1 & Rectangular & Cyclic & 0.0 \\
\hline 97 & & S4 & Rectangular & Cyclic & 0.0 \\
\hline 98 & & S6 & Rectangular & Cyclic & 0.0 \\
\hline 99 & & S9 & Rectangular & Cyclic & 0.0 \\
\hline 100 & & S10 & Rectangular & Cyclic & 0.0 \\
\hline
\end{tabular}


Table B-1 Loading Properties of the RC Walls with Rectangular Cross-Section (continued)

\begin{tabular}{|c|c|c|c|c|c|}
\hline Test No. & Researcher & Spec ID & Wall type & Loading type & $\begin{array}{c}P / A_{g} f_{c}^{\prime} \\
(\%) \\
\end{array}$ \\
\hline 101 & Tran and Wallace (2012) & RW-A15-P10-S51 & Rectangular & Cyclic & 7.7 \\
\hline 102 & \multirow{2}{*}{ Tran and Wallace (2012) } & RW-A15-P10-S78 & Rectangular & Cyclic & 6.4 \\
\hline 103 & & RW-A15-P2.5-S64 & Rectangular & Cyclic & 1.6 \\
\hline 104 & El-Sokkary and Galal (2013) & $\mathrm{CW}$ & Rectangular & Cyclic & 1.5 \\
\hline 105 & \multirow{7}{*}{ Park et al. (2015) } & S1 & Rectangular & Cyclic & 7.0 \\
\hline 106 & & S2 & Rectangular & Cyclic & 7.0 \\
\hline 107 & & S3 & Rectangular & Cyclic & 7.0 \\
\hline 108 & & S5 & Rectangular & Cyclic & 7.0 \\
\hline 109 & & S6 & Rectangular & Cyclic & 7.0 \\
\hline 110 & & S7 & Rectangular & Cyclic & 7.0 \\
\hline 111 & & S8 & Rectangular & Cyclic & 7.0 \\
\hline 112 & \multirow{5}{*}{ Hajyalikhani (2015) } & SW1 & Rectangular & Cyclic & 0.0 \\
\hline 113 & & SW2 & Rectangular & Cyclic & 0.0 \\
\hline 114 & & SW3 & Rectangular & Cyclic & 0.0 \\
\hline 115 & & SW5 & Rectangular & Cyclic & 0.0 \\
\hline 116 & & SW6 & Rectangular & Cyclic & 0.0 \\
\hline 117 & \multirow{6}{*}{ Peng et al. (2015) } & RCWS1 & Rectangular & Cyclic & 0.1 \\
\hline 118 & & RCWS2 & Rectangular & Cyclic & 0.1 \\
\hline 119 & & RCWS3 & Rectangular & Cyclic & 0.1 \\
\hline 120 & & RCWS4 & Rectangular & Cyclic & 0.1 \\
\hline 121 & & RCWS5 & Rectangular & Cyclic & 0.0 \\
\hline 122 & & RCWS6 & Rectangular & Cyclic & 0.0 \\
\hline 123 & \multirow{10}{*}{ Luna et al. (2015) } & SW1 & Rectangular & Cyclic & 0.0 \\
\hline 124 & & SW2 & Rectangular & Cyclic & 0.0 \\
\hline 125 & & SW3 & Rectangular & Cyclic & 0.0 \\
\hline 126 & & SW4 & Rectangular & Cyclic & 0.0 \\
\hline 127 & & SW5 & Rectangular & Cyclic & 0.0 \\
\hline 128 & & SW6 & Rectangular & Cyclic & 0.0 \\
\hline 129 & & SW7 & Rectangular & Cyclic & 0.0 \\
\hline 130 & & SW8 & Rectangular & Cyclic & 0.0 \\
\hline 131 & & SW9 & Rectangular & Cyclic & 0.0 \\
\hline 132 & & SW10 & Rectangular & Cyclic & 0.0 \\
\hline
\end{tabular}


Table B-1 Loading Properties of the RC Walls with Rectangular Cross-Section (continued)

\begin{tabular}{|c|c|c|c|c|c|}
\hline Test No. & Researcher & Spec ID & Wall type & Loading type & $\begin{array}{c}P / A_{g} f_{c}^{\prime} \\
(\%)\end{array}$ \\
\hline 133 & \multirow{2}{*}{ Luna et al. (2015) } & SW11 & Rectangular & Cyclic & 0.0 \\
\hline 134 & & SW12 & Rectangular & Cyclic & 0.0 \\
\hline 135 & Li et al. (2015) & S1 & Rectangular & Cyclic & 0.0 \\
\hline 136 & \multirow{2}{*}{ Woods et al. (2016) } & CW1 & Rectangular & Cyclic & 0.0 \\
\hline 137 & & cW2 & Rectangular & Cyclic & 0.0 \\
\hline
\end{tabular}

Table B-2 Geometric Properties of the RC Walls with Rectangular Cross-Section

\begin{tabular}{|c|c|c|c|c|c|c|c|c|c|c|c|c|c|}
\hline $\begin{array}{l}\text { Test } \\
\text { No. }\end{array}$ & Researcher & Spec ID & $\begin{array}{l}t_{w} \\
\text { (in) }\end{array}$ & $\begin{array}{l}h_{w} \\
\text { (in) }\end{array}$ & $\begin{array}{l}l_{w} \\
\text { (in) }\end{array}$ & $\frac{h_{w}}{l_{w}}$ & $\frac{M}{V l_{w}}$ & $\frac{l_{w}}{t_{w}}$ & $\begin{array}{l}l_{f} \\
\text { (in) }\end{array}$ & $\begin{array}{l}t_{f} \\
\text { (in) }\end{array}$ & $\begin{array}{l}l_{c} \\
\text { (in) }\end{array}$ & $\begin{array}{c}t_{c} \\
\text { (in) }\end{array}$ & $\begin{array}{l}l_{b e} \\
\text { (in) }\end{array}$ \\
\hline 1 & $\begin{array}{c}\text { Alexander et al. } \\
\text { (1973) }\end{array}$ & 4 & 4.0 & 54.0 & 72.0 & 0.8 & 0.8 & 18.0 & - & - & - & - & - \\
\hline 2 & \multirow{10}{*}{ Hirosawa (1975) } & 72 & 6.3 & 63.0 & 66.9 & 0.9 & 1.0 & 10.6 & - & - & - & - & 6.7 \\
\hline 3 & & 73 & 6.3 & 63.0 & 66.9 & 0.9 & 1.0 & 10.6 & - & - & - & - & 6.7 \\
\hline 4 & & 74 & 6.3 & 63.0 & 66.9 & 0.9 & 1.0 & 10.6 & - & - & - & - & 6.7 \\
\hline 5 & & 75 & 6.3 & 63.0 & 66.9 & 0.9 & 1.0 & 10.6 & - & - & - & - & 6.7 \\
\hline 6 & & 76 & 6.3 & 63.0 & 66.9 & 0.9 & 1.0 & 10.6 & - & - & - & - & 6.7 \\
\hline 7 & & 77 & 6.3 & 63.0 & 66.9 & 0.9 & 1.0 & 10.6 & - & - & - & - & 6.7 \\
\hline 8 & & 78 & 6.3 & 63.0 & 66.9 & 0.9 & 1.0 & 10.6 & - & - & - & - & 6.7 \\
\hline 9 & & 79 & 6.3 & 63.0 & 66.9 & 0.9 & 1.0 & 10.6 & - & - & - & - & 6.7 \\
\hline 10 & & 80 & 6.3 & 63.0 & 66.9 & 0.9 & 1.0 & 10.6 & - & - & - & - & 6.7 \\
\hline 11 & & 81 & 6.3 & 63.0 & 66.9 & 0.9 & 1.0 & 10.6 & - & - & - & - & 6.7 \\
\hline 12 & Synge (1980) & WALL 1 & 3.9 & 59.1 & 118.1 & 0.5 & 0.6 & 30.1 & - & - & - & - & - \\
\hline 13 & \multirow{2}{*}{ Wiradinata (1985) } & WALL1 & 3.9 & 39.4 & 78.7 & 0.5 & 0.6 & 20.0 & - & - & - & - & 12.6 \\
\hline 14 & & WALL2 & 3.9 & 19.7 & 78.7 & 0.3 & 0.3 & 20.0 & - & - & - & - & 12.6 \\
\hline 15 & \multirow{3}{*}{ Maier (1985) } & S4 & 3.9 & 47.2 & 46.5 & 1.0 & 1.1 & 11.8 & - & - & - & - & - \\
\hline 16 & & S9 & 3.9 & 47.2 & 46.5 & 1.0 & 1.1 & 11.8 & - & - & - & - & - \\
\hline 17 & & S10 & 3.9 & 47.2 & 46.5 & 1.0 & 1.1 & 11.8 & - & - & - & - & - \\
\hline 18 & \multirow{2}{*}{ Pilette (1987) } & WALL 4 & 3.9 & 39.4 & 78.7 & 0.5 & 0.6 & 20.0 & - & - & - & - & 12.6 \\
\hline 19 & & WALL 5 & 3.9 & 39.4 & 78.7 & 0.5 & 0.6 & 20.0 & - & - & - & - & 9.8 \\
\hline 20 & \multirow{2}{*}{$\begin{array}{l}\text { Huang and Sheu } \\
\text { (1988) }\end{array}$} & SWN-1D & 3.9 & 19.7 & 39.4 & 0.5 & 0.7 & 10.0 & - & - & - & - & - \\
\hline 21 & & SWN-5D & 3.9 & 29.5 & 39.4 & 0.7 & 0.9 & 10.0 & - & - & - & - & - \\
\hline 22 & \multirow{2}{*}{ Wasiewicz (1988) } & WALL 3 & 3.9 & 19.7 & 78.7 & 0.3 & 0.3 & 20.0 & - & - & - & - & - \\
\hline 23 & & WALL 6 & 3.9 & 39.4 & 78.7 & 0.5 & 0.6 & 20.0 & - & - & - & - & - \\
\hline
\end{tabular}


Table B-2 Geometric Properties of the RC Walls with Rectangular Cross-Section (continued)

\begin{tabular}{|c|c|c|c|c|c|c|c|c|c|c|c|c|c|}
\hline $\begin{array}{l}\text { Test } \\
\text { No. }\end{array}$ & Researcher & Spec ID & $\begin{array}{l}t_{w} \\
\text { (in) } \\
\end{array}$ & $\begin{array}{l}h_{w} \\
\text { (in) } \\
\end{array}$ & $\begin{array}{r}l_{w} \\
\text { (in) } \\
\end{array}$ & $\frac{h_{w}}{l_{w}}$ & $\frac{M}{V l_{w}}$ & $\frac{l_{w}}{t_{w}}$ & $\begin{array}{l}l_{f} \\
\text { (in) }\end{array}$ & $\begin{array}{l}t_{f} \\
\text { (in) } \\
\end{array}$ & $\begin{array}{r}l_{c} \\
\text { (in) } \\
\end{array}$ & $\begin{array}{r}t_{c} \\
\text { (in) } \\
\end{array}$ & $\begin{array}{l}l_{b e} \\
\text { (in) }\end{array}$ \\
\hline 24 & \multirow{7}{*}{ Lefas et al. (1990) } & SW11 & 2.8 & 29.5 & 29.5 & 1.0 & 1.1 & 10.7 & - & - & - & - & 5.5 \\
\hline 25 & & SW12 & 2.8 & 29.5 & 29.5 & 1.0 & 1.1 & 10.7 & - & - & - & - & 5.5 \\
\hline 26 & & SW13 & 2.8 & 29.5 & 29.5 & 1.0 & 1.1 & 10.7 & - & - & - & - & 5.5 \\
\hline 27 & & SW14 & 2.8 & 29.5 & 29.5 & 1.0 & 1.1 & 10.7 & - & - & - & - & 5.5 \\
\hline 28 & & SW15 & 2.8 & 29.5 & 29.5 & 1.0 & 1.1 & 10.7 & - & - & - & - & 5.5 \\
\hline 29 & & SW16 & 2.8 & 29.5 & 29.5 & 1.0 & 1.1 & 10.7 & - & - & - & - & 5.5 \\
\hline 30 & & SW17 & 2.8 & 29.5 & 29.5 & 1.0 & 1.1 & 10.7 & - & - & - & - & 5.5 \\
\hline 31 & \multirow{6}{*}{$\begin{array}{l}\text { Lopes and Elnashai } \\
\qquad(1991)\end{array}$} & SW11 & 1.8 & 33.7 & 17.7 & 1.9 & 1.1 & 10.0 & - & - & - & - & 3.5 \\
\hline 32 & & SW12 & 1.8 & 33.7 & 17.7 & 1.9 & 1.1 & 10.0 & - & - & - & - & 3.5 \\
\hline 33 & & SW13 & 1.8 & 33.7 & 17.7 & 1.9 & 1.1 & 10.0 & - & - & - & - & 3.5 \\
\hline 34 & & SW14 & 1.8 & 33.7 & 17.7 & 1.9 & 1.1 & 10.0 & - & - & - & - & 3.5 \\
\hline 35 & & SW15 & 1.8 & 33.7 & 17.7 & 1.9 & 1.1 & 10.0 & - & - & - & - & 3.5 \\
\hline 36 & & SW16 & 1.8 & 33.7 & 17.7 & 1.9 & 1.1 & 10.0 & - & - & - & - & 3.5 \\
\hline 37 & \multirow{2}{*}{$\begin{array}{l}\text { Lopes and Elnashai } \\
\text { (1991) }\end{array}$} & SW17 & 1.8 & 33.7 & 17.7 & 1.9 & 1.1 & 10.0 & - & - & - & - & 3.5 \\
\hline 38 & & SW18 & 1.8 & 33.7 & 17.7 & 1.9 & 1.1 & 10.0 & - & - & - & - & 3.5 \\
\hline 39 & \multirow{3}{*}{ Saatcioglu (1991) } & Wall-1 & 3.9 & 39.3 & 78.7 & 0.5 & 0.6 & 20.0 & - & - & - & - & - \\
\hline 40 & & Wall-4 & 3.9 & 39.3 & 78.7 & 0.5 & 0.6 & 20.0 & - & - & - & - & - \\
\hline 41 & & Wall-6 & 3.9 & 39.3 & 78.7 & 0.5 & 0.6 & 20.0 & - & - & - & - & - \\
\hline 42 & \multirow{3}{*}{ Cheng (1992) } & SW4A & 3.9 & 19.7 & 39.4 & 0.5 & 0.7 & 10.0 & - & - & - & - & - \\
\hline 43 & & SW5 & 3.9 & 19.7 & 39.4 & 0.5 & 0.7 & 10.0 & - & - & - & - & - \\
\hline 44 & & SW6 & 3.9 & 19.7 & 39.4 & 0.5 & 0.7 & 10.0 & - & - & - & - & - \\
\hline 45 & \multirow{2}{*}{ Mohammadi (1994) } & Wall 7 & 3.9 & 59.1 & 78.7 & 0.7 & 0.8 & 20.0 & - & - & - & - & 12.6 \\
\hline 46 & & Wall 8 & 3.9 & 59.1 & 59.1 & 1.0 & 1.1 & 15.0 & - & - & - & - & 14.1 \\
\hline 47 & \multirow{3}{*}{ Cheng (1992) } & SW-OE & 3.9 & 19.7 & 39.4 & 0.5 & 0.7 & 10.0 & - & - & - & - & - \\
\hline 48 & & SW-1E & 3.9 & 19.7 & 39.4 & 0.5 & 0.7 & 10.0 & - & - & - & - & - \\
\hline 49 & & SW-9E & 3.9 & 29.5 & 39.4 & 0.7 & 0.9 & 10.0 & - & - & - & - & - \\
\hline 50 & \multirow{6}{*}{$\begin{array}{l}\text { Salonikios et al. } \\
\qquad(1999)\end{array}$} & LSW1 & 3.9 & 47.2 & 47.2 & 1.0 & 1.1 & 12.0 & - & - & - & - & 9.4 \\
\hline 51 & & LSW2 & 3.9 & 47.2 & 47.2 & 1.0 & 1.1 & 12.0 & - & - & - & - & 9.4 \\
\hline 52 & & LSW3 & 3.9 & 47.2 & 47.2 & 1.0 & 1.1 & 12.0 & - & - & - & - & 9.4 \\
\hline 53 & & MSW1 & 3.9 & 70.8 & 47.2 & 1.5 & 1.6 & 12.0 & - & - & - & - & 9.4 \\
\hline 54 & & MSW2 & 3.9 & 70.8 & 47.2 & 1.5 & 1.6 & 12.0 & - & - & - & - & 9.4 \\
\hline 55 & & MSW3 & 3.9 & 70.8 & 47.2 & 1.5 & 1.6 & 12.0 & - & - & - & - & 9.4 \\
\hline
\end{tabular}


Table B-2 Geometric Properties of the RC Walls with Rectangular Cross-Section (continued)

\begin{tabular}{|c|c|c|c|c|c|c|c|c|c|c|c|c|c|}
\hline $\begin{array}{l}\text { Test } \\
\text { No. }\end{array}$ & Researcher & Spec ID & $\begin{array}{r}t_{w} \\
\text { (in) } \\
\end{array}$ & $\begin{array}{c}h_{w} \\
\text { (in) }\end{array}$ & $\begin{array}{r}l_{w} \\
\text { (in) } \\
\end{array}$ & $\frac{h_{w}}{l_{w}}$ & $\frac{M}{V l_{w}}$ & $\frac{l_{w}}{t_{w}}$ & $\begin{array}{l}l_{f} \\
\text { (in) }\end{array}$ & $\begin{array}{l}t_{f} \\
\text { (in) }\end{array}$ & $\begin{array}{c}l_{c} \\
\text { (in) }\end{array}$ & $\begin{array}{r}t_{c} \\
\text { (in) } \\
\end{array}$ & $\begin{array}{l}l_{b e} \\
\text { (in) }\end{array}$ \\
\hline 56 & Xia and Xiao (2000) & W1-A & 6.0 & 48.0 & 96.0 & 0.5 & 0.6 & 16.0 & - & - & - & - & - \\
\hline 57 & \multirow{14}{*}{ Hidalgo et al. (2002) } & 1 & 4.7 & 78.7 & 39.4 & 2.0 & 1.0 & 8.3 & - & - & - & - & 3.9 \\
\hline 58 & & 2 & 4.7 & 78.7 & 39.4 & 2.0 & 1.0 & 8.3 & - & - & - & - & 3.9 \\
\hline 59 & & 4 & 4.7 & 78.7 & 39.4 & 2.0 & 1.0 & 8.3 & - & - & - & - & 3.9 \\
\hline 60 & & 6 & 4.7 & 70.9 & 51.2 & 1.4 & 0.7 & 10.8 & - & - & - & - & 5.1 \\
\hline 61 & & 7 & 4.7 & 70.9 & 51.2 & 1.4 & 0.7 & 10.8 & - & - & - & - & 5.1 \\
\hline 62 & & 8 & 4.7 & 70.9 & 51.2 & 1.4 & 0.7 & 10.8 & - & - & - & - & 5.1 \\
\hline 63 & & 9 & 3.9 & 70.9 & 51.2 & 1.4 & 0.7 & 13.0 & - & - & - & - & 5.1 \\
\hline 64 & & 10 & 3.2 & 70.9 & 51.2 & 1.4 & 0.7 & 16.3 & - & - & - & - & 5.1 \\
\hline 65 & & 11 & 3.9 & 55.1 & 55.1 & 1.0 & 0.5 & 14.0 & - & - & - & - & 5.5 \\
\hline 66 & & 12 & 3.9 & 55.1 & 55.1 & 1.0 & 0.5 & 14.0 & - & - & - & - & 5.5 \\
\hline 67 & & 13 & 3.9 & 55.1 & 55.1 & 1.0 & 0.5 & 14.0 & - & - & - & - & 5.5 \\
\hline 68 & & 14 & 3.2 & 47.2 & 66.9 & 0.7 & 0.4 & 21.2 & - & - & - & - & 6.7 \\
\hline 69 & & 15 & 3.2 & 47.2 & 66.9 & 0.7 & 0.4 & 21.2 & - & - & - & - & 6.7 \\
\hline 70 & & 16 & 3.2 & 47.2 & 66.9 & 0.7 & 0.4 & 21.2 & - & - & - & - & 6.7 \\
\hline 71 & \multirow{7}{*}{ Hidalgo et al. (2002) } & 21 & 3.9 & 70.9 & 51.2 & 1.4 & 0.7 & 13.0 & - & - & - & - & 5.1 \\
\hline 72 & & 22 & 3.9 & 70.9 & 51.2 & 1.4 & 0.7 & 13.0 & - & - & - & - & 5.1 \\
\hline 73 & & 23 & 3.9 & 70.9 & 51.2 & 1.4 & 0.7 & 13.0 & - & - & - & - & 5.1 \\
\hline 74 & & 24 & 3.9 & 70.9 & 51.2 & 1.4 & 0.7 & 13.0 & - & - & - & - & 5.1 \\
\hline 75 & & 25 & 3.9 & 55.1 & 51.1 & 1.1 & 0.5 & 13.0 & - & - & - & - & 5.5 \\
\hline 76 & & 26 & 3.9 & 55.1 & 51.1 & 1.1 & 0.5 & 13.0 & - & - & - & - & 5.5 \\
\hline 77 & & 28 & 3.9 & 55.1 & 51.1 & 1.1 & 0.5 & 13.0 & - & - & - & - & 5.5 \\
\hline 78 & \multirow{6}{*}{ Massone (2006) } & WP111-9 & 6.0 & 48.0 & 54.0 & 0.9 & 0.4 & 9.0 & - & - & - & - & 7.5 \\
\hline 79 & & WP111-10 & 6.0 & 48.0 & 54.0 & 0.9 & 0.4 & 9.0 & - & - & - & - & 7.5 \\
\hline 80 & & WP1105-8 & 6.0 & 48.0 & 54.0 & 0.9 & 0.4 & 9.0 & - & - & - & - & 7.5 \\
\hline 81 & & WP1105-7 & 6.0 & 48.0 & 54.0 & 0.9 & 0.4 & 9.0 & - & - & - & - & 7.5 \\
\hline 82 & & WP110-5 & 6.0 & 48.0 & 54.0 & 0.9 & 0.4 & 9.0 & - & - & - & - & 7.5 \\
\hline 83 & & WP110-6 & 6.0 & 48.0 & 54.0 & 0.9 & 0.4 & 9.0 & - & - & - & - & 7.5 \\
\hline 84 & \multirow{3}{*}{ Greifenhagen (2005) } & M1 & 3.9 & 24.0 & 39.4 & 0.6 & 0.7 & 10.0 & - & - & - & - & - \\
\hline 85 & & M2 & 3.9 & 24.0 & 39.4 & 0.6 & 0.7 & 10.0 & - & - & - & - & - \\
\hline 86 & & M3 & 3.2 & 24.0 & 35.4 & 0.7 & 0.7 & 11.2 & - & - & - & - & - \\
\hline
\end{tabular}


Table B-2 Geometric Properties of the RC Walls with Rectangular Cross-Section (continued)

\begin{tabular}{|c|c|c|c|c|c|c|c|c|c|c|c|c|c|}
\hline $\begin{array}{l}\text { Test } \\
\text { No. }\end{array}$ & Researcher & Spec ID & $\begin{array}{l}t_{w} \\
\text { (in) } \\
\end{array}$ & $\begin{array}{c}h_{w} \\
\text { (in) }\end{array}$ & $\begin{array}{r}l_{w} \\
\text { (in) } \\
\end{array}$ & $\frac{h_{w}}{l_{w}}$ & $\frac{M}{V l_{w}}$ & $\frac{l_{w}}{t_{w}}$ & $\begin{array}{l}l_{f} \\
\text { (in) }\end{array}$ & $\begin{array}{l}t_{f} \\
\text { (in) }\end{array}$ & $\begin{array}{c}l_{c} \\
\text { (in) }\end{array}$ & $\begin{array}{r}t_{c} \\
\text { (in) } \\
\end{array}$ & $\begin{array}{l}l_{b e} \\
\text { (in) }\end{array}$ \\
\hline 87 & Greifenhagen (2005) & M4 & 3.2 & 24.0 & 35.4 & 0.7 & 0.7 & 11.2 & - & - & - & - & - \\
\hline 88 & \multirow{8}{*}{ Kuang and $\mathrm{Ho}(2008)$} & U 1.0 & 3.9 & 47.2 & 47.2 & 1.0 & 1.1 & 12.0 & - & - & - & - & - \\
\hline 89 & & U 1.5 & 3.9 & 70.9 & 47.2 & 1.5 & 1.6 & 12.0 & - & - & - & - & - \\
\hline 90 & & C 1.0 & 3.9 & 47.2 & 47.2 & 1.0 & 1.1 & 12.0 & - & - & - & - & - \\
\hline 91 & & C 1.5 & 3.9 & 70.9 & 47.2 & 1.5 & 1.6 & 12.0 & - & - & - & - & - \\
\hline 92 & & $\mathrm{U} 1.0 \mathrm{BC}$ & 3.9 & 47.2 & 47.2 & 1.0 & 1.1 & 12.0 & - & - & - & - & 9.4 \\
\hline 93 & & $\mathrm{U} 1.5 \mathrm{BC}$ & 3.9 & 70.9 & 47.2 & 1.5 & 1.6 & 12.0 & - & - & - & - & 9.4 \\
\hline 94 & & U $1.0 \mathrm{BC} 2$ & 3.9 & 47.2 & 47.2 & 1.0 & 1.1 & 12.0 & - & - & - & - & 9.4 \\
\hline 95 & & U $1.0 \mathrm{CT}$ & 3.9 & 47.2 & 47.2 & 1.0 & 1.6 & 12.0 & - & - & - & - & - \\
\hline 96 & \multirow{5}{*}{$\begin{array}{l}\text { Athanasopoulou } \\
\text { (2010) }\end{array}$} & S1 & 4.0 & 40.0 & 40.0 & 1.0 & 1.2 & 10.0 & - & - & - & - & 3.5 \\
\hline 97 & & S4 & 4.0 & 40.0 & 40.0 & 1.0 & 1.2 & 10.0 & - & - & - & - & 5.0 \\
\hline 98 & & S6 & 4.0 & 52.0 & 40.0 & 1.3 & 1.5 & 10.0 & - & - & - & - & 3.5 \\
\hline 99 & & S9 & 4.0 & 52.0 & 40.0 & 1.3 & 1.5 & 10.0 & - & - & - & - & 5.0 \\
\hline 100 & & S10 & 4.0 & 52.0 & 40.0 & 1.3 & 1.5 & 10.0 & - & - & - & - & 5.0 \\
\hline 101 & \multirow{3}{*}{$\begin{array}{l}\text { Tran and Wallace } \\
\text { (2012) }\end{array}$} & $\begin{array}{l}\text { RW-A15- } \\
\text { P10-S51 }\end{array}$ & 6.0 & 81.0 & 48.0 & 1.7 & 1.5 & 8.0 & - & - & - & - & 9.0 \\
\hline 102 & & $\begin{array}{l}\text { RW-A15- } \\
\text { P10-S78 }\end{array}$ & 6.0 & 81.0 & 48.0 & 1.7 & 1.5 & 8.0 & - & - & - & - & 9.0 \\
\hline 103 & & $\begin{array}{l}\text { RW-A15- } \\
\text { P2.5-S64 }\end{array}$ & 6.0 & 81.0 & 48.0 & 1.7 & 1.5 & 8.0 & - & - & - & - & 9.0 \\
\hline 104 & $\begin{array}{l}\text { El-Sokkary and Galal } \\
\qquad(2013)\end{array}$ & CW & 3.2 & 41.1 & 47.2 & 0.9 & 1.0 & 15.0 & - & - & - & - & 3.2 \\
\hline 105 & \multirow{7}{*}{ Park et al. (2015) } & S1 & 7.8 & 59.0 & 59.0 & 1.0 & 1.2 & 7.6 & - & - & - & - & 11.8 \\
\hline 106 & & S2 & 7.8 & 59.0 & 59.0 & 1.0 & 1.2 & 7.6 & - & - & - & - & 11.8 \\
\hline 107 & & S3 & 7.8 & 59.0 & 59.0 & 1.0 & 1.2 & 7.6 & - & - & - & - & 11.8 \\
\hline 108 & & S5 & 7.8 & 59.0 & 59.0 & 1.0 & 1.2 & 7.6 & - & - & - & - & 7.9 \\
\hline 109 & & S6 & 7.8 & 59.0 & 59.0 & 1.0 & 1.2 & 7.6 & - & - & - & - & 7.9 \\
\hline 110 & & S7 & 7.8 & 59.0 & 59.0 & 1.0 & 1.2 & 7.6 & - & - & - & - & 7.9 \\
\hline 111 & & S8 & 7.8 & 59.0 & 59.0 & 1.0 & 1.2 & 7.6 & - & - & - & - & 7.9 \\
\hline 112 & \multirow{5}{*}{ Hajyalikhani (2015) } & SW1 & 4.0 & 40.0 & 40.0 & 1.0 & 1.2 & 10.0 & - & - & - & - & - \\
\hline 113 & & SW2 & 4.0 & 40.0 & 40.0 & 1.0 & 1.3 & 10.0 & - & - & - & - & - \\
\hline 114 & & SW3 & 4.0 & 40.0 & 40.0 & 1.0 & 1.3 & 10.0 & - & - & - & - & - \\
\hline 115 & & SW5 & 4.0 & 40.0 & 40.0 & 1.0 & 1.4 & 10.0 & - & - & - & - & - \\
\hline 116 & & SW6 & 4.0 & 20.0 & 40.0 & 0.5 & 0.8 & 10.0 & - & - & - & - & - \\
\hline
\end{tabular}


Table B-2 Geometric Properties of the RC Walls with Rectangular Cross-Section (continued)

\begin{tabular}{|c|c|c|c|c|c|c|c|c|c|c|c|c|c|}
\hline $\begin{array}{l}\text { Test } \\
\text { No. }\end{array}$ & Researcher & Spec ID & $\begin{array}{l}t_{w} \\
\text { (in) } \\
\end{array}$ & $\begin{array}{c}h_{w} \\
\text { (in) }\end{array}$ & $\begin{array}{r}l_{w} \\
\text { (in) } \\
\end{array}$ & $\frac{h_{w}}{l_{w}}$ & $\frac{M}{V l_{w}}$ & $\frac{l_{w}}{t_{w}}$ & $\begin{array}{l}l_{f} \\
\text { (in) }\end{array}$ & $\begin{array}{l}t_{f} \\
\text { (in) } \\
\end{array}$ & $\begin{array}{c}l_{c} \\
\text { (in) } \\
\end{array}$ & $\begin{array}{c}t_{c} \\
\text { (in) } \\
\end{array}$ & $\begin{array}{l}l_{b e} \\
\text { (in) }\end{array}$ \\
\hline 117 & \multirow{6}{*}{ Peng et al. (2015) } & RCWS1 & 7.1 & 63.0 & 70.9 & 0.9 & 1.0 & 10.0 & - & - & - & - & 14.2 \\
\hline 118 & & RCWS2 & 7.1 & 63.0 & 70.9 & 0.9 & 1.0 & 10.0 & - & - & - & - & 14.2 \\
\hline 119 & & RCWS3 & 7.1 & 63.0 & 70.9 & 0.9 & 1.0 & 10.0 & - & - & - & - & 14.2 \\
\hline 120 & & RCWS4 & 7.1 & 63.0 & 70.9 & 0.9 & 1.0 & 10.0 & - & - & - & - & 14.2 \\
\hline 121 & & RCWS5 & 7.1 & 63.0 & 70.9 & 0.9 & 1.0 & 10.0 & - & - & - & - & 14.2 \\
\hline 122 & & RCWS6 & 7.1 & 63.0 & 70.9 & 0.9 & 1.0 & 10.0 & - & - & - & - & 14.2 \\
\hline 123 & \multirow{12}{*}{ Luna et al. (2015) } & SW1 & 8.0 & 112.8 & 120.0 & 0.9 & 0.9 & 15.0 & - & - & - & - & - \\
\hline 124 & & SW2 & 8.0 & 64.8 & 120.0 & 0.5 & 0.5 & 15.0 & - & - & - & - & - \\
\hline 125 & & SW3 & 8.0 & 64.8 & 120.0 & 0.5 & 0.5 & 15.0 & - & - & - & - & - \\
\hline 126 & & SW4 & 8.0 & 64.8 & 120.0 & 0.5 & 0.5 & 15.0 & - & - & - & - & - \\
\hline 127 & & SW5 & 8.0 & 39.6 & 120.0 & 0.3 & 0.3 & 15.0 & - & - & - & - & - \\
\hline 128 & & SW6 & 8.0 & 39.6 & 120.0 & 0.3 & 0.3 & 15.0 & - & - & - & - & - \\
\hline 129 & & SW7 & 8.0 & 39.6 & 120.0 & 0.3 & 0.3 & 15.0 & - & - & - & - & - \\
\hline 130 & & SW8 & 8.0 & 64.8 & 120.0 & 0.5 & 0.5 & 15.0 & - & - & - & - & - \\
\hline 131 & & SW9 & 8.0 & 64.8 & 120.0 & 0.5 & 0.5 & 15.0 & - & - & - & - & - \\
\hline 132 & & SW10 & 8.0 & 64.8 & 120.0 & 0.5 & 0.5 & 15.0 & - & - & - & - & - \\
\hline 133 & & SW11 & 8.0 & 64.8 & 120.0 & 0.5 & 0.5 & 15.0 & - & - & - & - & 15.5 \\
\hline 134 & & SW12 & 8.0 & 64.8 & 120.0 & 0.5 & 0.5 & 15.0 & - & - & - & - & 15.5 \\
\hline 135 & Li et al. (2015) & S1 & 4.7 & 90.6 & 78.7 & 1.1 & 1.3 & 16.7 & - & - & - & - & - \\
\hline 136 & \multirow{2}{*}{ Woods et al. (2016) } & CW1 & 3.9 & 70.9 & 59.1 & 1.2 & 1.3 & 15.0 & - & - & - & - & - \\
\hline 137 & & CW2 & 5.5 & 70.9 & 82.7 & 0.9 & 1.0 & 15.0 & - & - & - & - & - \\
\hline
\end{tabular}

Table B-3 Reinforcement Properties of the RC Walls with Rectangular Cross-Section

\begin{tabular}{|c|c|c|c|c|c|c|c|c|c|c|c|c|}
\hline $\begin{array}{l}\text { Test } \\
\text { No. }\end{array}$ & Researcher & Spec ID & $\begin{array}{l}\rho_{v}^{w} \\
(\%) \\
\end{array}$ & $\begin{array}{l}\rho_{h}^{w} \\
(\%) \\
\end{array}$ & $\begin{array}{c}s_{h}^{w} \\
\text { (in) } \\
\end{array}$ & $\begin{array}{l}S_{v}^{w} \\
\text { (in) }\end{array}$ & $\begin{array}{l}\rho_{v}^{f} \\
(\%)\end{array}$ & $\begin{array}{c}\rho_{h}^{f} \\
(\%) \\
\end{array}$ & $\begin{array}{l}s_{h}^{f} \\
\text { (in) }\end{array}$ & $\begin{array}{c}s_{v}^{f} \\
\text { (in) }\end{array}$ & $\begin{array}{l}\rho_{v}^{b e} \\
(\%)\end{array}$ & $\begin{array}{l}s_{h}^{b e} \\
\text { (in) }\end{array}$ \\
\hline 1 & Alexander et al. (1973) & 4 & 0.30 & 0.30 & 4.50 & 4.50 & - & - & - & - & - & - \\
\hline 2 & & 72 & 0.51 & 0.26 & 5.20 & 5.20 & - & - & - & - & 5.68 & - \\
\hline 3 & & 73 & 0.51 & 0.26 & 5.20 & 5.20 & - & - & - & - & 5.68 & - \\
\hline 4 & & 74 & 0.51 & 0.57 & 2.60 & 5.20 & - & - & - & - & 5.68 & - \\
\hline 5 & Hirosawa (1975) & 75 & 0.51 & 0.57 & 2.60 & 5.20 & - & - & - & - & 5.68 & - \\
\hline 6 & & 76 & 0.51 & 1.08 & 1.30 & 5.20 & - & - & - & - & 5.68 & - \\
\hline 7 & & 77 & 0.51 & 1.08 & 1.30 & 5.20 & - & - & - & - & 5.68 & - \\
\hline 8 & & 78 & 0.51 & 0.61 & 2.60 & 5.20 & - & - & - & - & 2.51 & - \\
\hline
\end{tabular}


Table B-3 Reinforcement Properties of the RC Walls with Rectangular Cross-Section (continued)

\begin{tabular}{|c|c|c|c|c|c|c|c|c|c|c|c|c|}
\hline $\begin{array}{l}\text { Test } \\
\text { No. }\end{array}$ & Researcher & Spec ID & $\begin{array}{l}\rho_{v}^{w} \\
(\%) \\
\end{array}$ & $\begin{array}{l}\rho_{h}^{w} \\
(\%)\end{array}$ & $\begin{array}{l}s_{h}^{w} \\
\text { (in) }\end{array}$ & $\begin{array}{l}s_{v}^{w} \\
\text { (in) }\end{array}$ & $\begin{array}{l}\rho_{v}^{f} \\
(\%)\end{array}$ & $\begin{array}{l}\rho_{h}^{f} \\
(\%) \\
\end{array}$ & $\begin{array}{c}s_{h}^{f} \\
\text { (in) } \\
\end{array}$ & $\begin{array}{l}s_{v}^{f} \\
\text { (in) } \\
\end{array}$ & $\begin{array}{l}\rho_{v}^{b e} \\
(\%) \\
\end{array}$ & $\begin{array}{l}s_{h}^{b e} \\
\text { (in) }\end{array}$ \\
\hline 9 & \multirow{3}{*}{ Hirosawa (1975) } & 79 & 0.51 & 0.61 & 2.60 & 5.20 & - & - & - & - & 2.51 & - \\
\hline 10 & & 80 & 0.51 & 1.08 & 1.30 & 5.20 & - & - & - & - & 2.51 & - \\
\hline 11 & & 81 & 0.51 & 1.08 & 1.30 & 5.20 & - & - & - & - & 2.51 & - \\
\hline 12 & Synge (1980) & WALL 1 & 0.81 & 1.68 & 4.72 & 5.51 & - & - & - & - & 7.50 & 1.96 \\
\hline 13 & \multirow{2}{*}{ Wiradinata (1985) } & WALL1 & 0.59 & 0.26 & 11.81 & 11.20 & - & - & - & - & 1.25 & 3.15 \\
\hline 14 & & WALL2 & 0.59 & 0.26 & 11.81 & 11.20 & - & - & - & - & 1.25 & 3.15 \\
\hline 15 & \multirow{3}{*}{ Maier (1985) } & S4 & 1.01 & 1.01 & 3.94 & 3.94 & - & - & - & - & - & - \\
\hline 16 & & S9 & 1.01 & 0.00 & 3.94 & 0.00 & - & - & - & - & - & - \\
\hline 17 & & S10 & 1.01 & 1.01 & 3.94 & 3.94 & - & - & - & - & - & - \\
\hline 18 & \multirow{2}{*}{ Pilette (1987) } & WALL 4 & 0.59 & 0.80 & 9.80 & 11.20 & - & - & - & - & 1.25 & - \\
\hline 19 & & WALL 5 & 1.07 & 1.20 & 6.90 & 6.90 & - & - & - & - & 1.60 & - \\
\hline 20 & \multirow{2}{*}{ Huang and Sheu (1988) } & SWN-1D & 0.43 & 0.57 & 5.90 & 7.10 & - & - & - & - & - & - \\
\hline 21 & & SWN-5D & 0.43 & 0.57 & 5.10 & 7.10 & - & - & - & - & - & - \\
\hline 22 & \multirow{2}{*}{ Wasiewicz (1988) } & WALL 3 & 0.60 & 0.43 & 5.90 & 10.20 & - & - & - & - & - & - \\
\hline 23 & & WALL 6 & 0.60 & 0.63 & 9.84 & 10.20 & - & - & - & - & - & - \\
\hline 24 & \multirow{7}{*}{ Lefas et al. (1990) } & SW11 & 2.14 & 1.17 & 3.10 & 2.40 & - & - & - & - & 3.08 & 2.36 \\
\hline 25 & & SW12 & 2.14 & 1.17 & 3.10 & 2.40 & - & - & - & - & 3.08 & 2.36 \\
\hline 26 & & SW13 & 2.14 & 1.17 & 3.10 & 2.40 & - & - & - & - & 3.08 & 2.36 \\
\hline 27 & & SW14 & 2.14 & 1.17 & 3.10 & 2.40 & - & - & - & - & 3.08 & 2.36 \\
\hline 28 & & SW15 & 2.14 & 1.17 & 3.10 & 2.40 & - & - & - & - & 3.08 & 2.36 \\
\hline 29 & & SW16 & 2.14 & 1.17 & 3.10 & 2.40 & - & - & - & - & 3.08 & 2.36 \\
\hline 30 & & SW17 & 2.14 & 0.37 & 3.10 & 2.40 & - & - & - & - & 3.08 & 2.36 \\
\hline 31 & \multirow{4}{*}{ Lopes and Elnashai (1991) } & SW11 & 0.41 & 0.92 & - & - & - & - & - & - & 5.61 & - \\
\hline 32 & & SW12 & 0.41 & 0.92 & - & - & - & - & - & - & 5.61 & - \\
\hline 33 & & SW13 & 0.41 & 0.92 & - & - & - & - & - & - & 5.61 & - \\
\hline 34 & & SW14 & 0.41 & 0.92 & - & - & - & - & - & - & 5.61 & - \\
\hline 35 & \multirow{4}{*}{ Lopes and Elnashai (1991) } & SW15 & 0.41 & 0.62 & - & - & - & - & - & - & 5.61 & - \\
\hline 36 & & SW16 & 0.00 & 0.92 & - & - & - & - & - & - & 5.61 & - \\
\hline 37 & & SW17 & 0.00 & 0.65 & - & - & - & - & - & - & 5.61 & - \\
\hline 38 & & SW18 & 0.00 & 0.72 & - & - & - & - & - & - & 5.61 & - \\
\hline 39 & \multirow{2}{*}{ Saatcioglu (1991) } & Wall-1 & 0.80 & 0.25 & - & - & - & - & - & - & - & - \\
\hline 40 & & Wall-4 & 0.80 & 0.80 & - & - & - & - & - & - & - & - \\
\hline
\end{tabular}


Table B-3 Reinforcement Properties of the RC Walls with Rectangular Cross-Section (continued)

\begin{tabular}{|c|c|c|c|c|c|c|c|c|c|c|c|c|}
\hline $\begin{array}{l}\text { Test } \\
\text { No. }\end{array}$ & Researcher & Spec ID & $\begin{array}{l}\rho_{v}^{w} \\
(\%) \\
\end{array}$ & $\begin{array}{l}\rho_{h}^{w} \\
(\%) \\
\end{array}$ & $\begin{array}{c}s_{h}^{w} \\
\text { (in) } \\
\end{array}$ & $\begin{array}{l}S_{v}^{w} \\
\text { (in) } \\
\end{array}$ & $\begin{array}{l}\rho_{v}^{f} \\
(\%) \\
\end{array}$ & $\begin{array}{l}\rho_{h}^{f} \\
(\%) \\
\end{array}$ & $\begin{array}{c}S_{h}^{f} \\
\text { (in) } \\
\end{array}$ & $\begin{array}{c}S_{v}^{f} \\
\text { (in) } \\
\end{array}$ & $\begin{array}{l}\rho_{v}^{b e} \\
(\%) \\
\end{array}$ & $\begin{array}{l}s_{h}^{b e} \\
\text { (in) }\end{array}$ \\
\hline 41 & Saatcioglu (1991) & Wall-6 & 0.80 & 0.80 & - & - & - & - & - & - & - & - \\
\hline 42 & \multirow{3}{*}{ Cheng (1992) } & SW4A & 0.77 & 1.03 & 5.90 & 7.10 & - & - & - & - & - & - \\
\hline 43 & & SW5 & 0.77 & 1.03 & 5.90 & 7.10 & - & - & - & - & - & - \\
\hline 44 & & SW6 & 0.77 & 1.03 & 5.90 & 7.10 & - & - & - & - & - & - \\
\hline 45 & \multirow{2}{*}{ Mohammadi (1994) } & Wall 7 & 0.80 & 0.80 & 11.10 & 11.10 & - & - & - & - & 0.98 & 3.14 \\
\hline 46 & & Wall 8 & 0.80 & 0.80 & 9.84 & 9.84 & - & - & - & - & 0.87 & 3.14 \\
\hline 47 & \multirow{3}{*}{ Cheng (1992) } & SW-OE & 0.71 & 0.71 & 3.90 & 4.10 & - & - & - & - & - & - \\
\hline 48 & & SW-1E & 0.71 & 0.71 & 3.90 & 4.10 & - & - & - & - & - & - \\
\hline 49 & & SW-9E & 1.27 & 1.80 & 4.30 & 4.10 & - & - & - & - & - & - \\
\hline 50 & \multirow{6}{*}{ Salonikios et al. (1999) } & LSW1 & 0.57 & 0.57 & 3.90 & 3.90 & - & - & - & - & 1.70 & 1.06 \\
\hline 51 & & LSW2 & 0.28 & 0.28 & 3.90 & 3.90 & - & - & - & - & 1.30 & 1.06 \\
\hline 52 & & LSW3 & 0.28 & 0.28 & 3.90 & 3.90 & - & - & - & - & 1.30 & 1.06 \\
\hline 53 & & MSW1 & 0.57 & 0.57 & 3.90 & 3.90 & - & - & - & - & 1.70 & 1.65 \\
\hline 54 & & MSW2 & 0.28 & 0.28 & 3.90 & 3.90 & - & - & - & - & 1.30 & 1.65 \\
\hline 55 & & MSW3 & 0.28 & 0.28 & 3.90 & 3.90 & - & - & - & - & 1.30 & 1.65 \\
\hline 56 & Xia and Xiao (2000) & W1-A & 0.37 & 0.31 & 13.50 & 13.50 & - & - & - & - & 0.00 & - \\
\hline 57 & \multirow{12}{*}{ Hidalgo et al. (2002) } & 1 & 0.25 & 0.13 & - & - & - & - & - & - & 8.50 & - \\
\hline 58 & & 2 & 0.25 & 0.25 & - & - & - & - & - & - & 8.50 & - \\
\hline 59 & & 4 & 0.25 & 0.38 & - & - & - & - & - & - & 10.58 & - \\
\hline 60 & & 6 & 0.26 & 0.13 & - & - & - & - & - & - & 6.54 & - \\
\hline 61 & & 7 & 0.13 & 0.25 & - & - & - & - & - & - & 6.54 & - \\
\hline 62 & & 8 & 0.26 & 0.25 & - & - & - & - & - & - & 6.54 & - \\
\hline 63 & & 9 & 0.26 & 0.26 & - & - & - & - & - & - & 7.00 & - \\
\hline 64 & & 10 & 0.25 & 0.25 & - & - & - & - & - & - & 7.31 & - \\
\hline 65 & & 11 & 0.26 & 0.13 & - & - & - & - & - & - & 5.71 & - \\
\hline 66 & & 12 & 0.13 & 0.26 & - & - & - & - & - & - & 5.71 & - \\
\hline 67 & & 13 & 0.26 & 0.26 & - & - & - & - & - & - & 5.71 & - \\
\hline 68 & & 14 & 0.25 & 0.13 & - & - & - & - & - & - & 4.41 & - \\
\hline 69 & \multirow{4}{*}{ Hidalgo et al. (2002) } & 15 & 0.13 & 0.26 & - & - & - & - & - & - & 4.41 & - \\
\hline 70 & & 16 & 0.25 & 0.25 & - & - & - & - & - & - & 4.41 & - \\
\hline 71 & & 21 & 0.00 & 0.00 & - & - & - & - & - & - & 4.62 & - \\
\hline 72 & & 22 & 0.00 & 0.00 & - & - & - & - & - & - & 4.62 & - \\
\hline
\end{tabular}


Table B-3 Reinforcement Properties of the RC Walls with Rectangular Cross-Section (continued)

\begin{tabular}{|c|c|c|c|c|c|c|c|c|c|c|c|c|}
\hline $\begin{array}{l}\text { Test } \\
\text { No. }\end{array}$ & Researcher & Spec ID & $\begin{array}{l}\rho_{v}^{w} \\
(\%)\end{array}$ & $\begin{array}{l}\rho_{h}^{w} \\
(\%)\end{array}$ & $\begin{array}{l}s_{h}^{w} \\
\text { (in) }\end{array}$ & $\begin{array}{l}s_{v}^{w} \\
\text { (in) }\end{array}$ & $\begin{array}{l}\rho_{v}^{f} \\
(\%)\end{array}$ & $\begin{array}{l}\rho_{h}^{f} \\
(\%)\end{array}$ & $\begin{array}{l}s_{h}^{f} \\
\text { (in) }\end{array}$ & $\begin{array}{l}s_{v}^{f} \\
\text { (in) }\end{array}$ & $\begin{array}{l}\rho_{v}^{b e} \\
(\%)\end{array}$ & $\begin{array}{l}s_{h}^{b e} \\
\text { (in) }\end{array}$ \\
\hline 73 & \multirow{5}{*}{ Hidalgo et al. (2002) } & 23 & 0.00 & 0.25 & - & - & - & - & - & - & 8.54 & - \\
\hline 74 & & 24 & 0.25 & 0.00 & - & - & - & - & - & - & 4.62 & - \\
\hline 75 & & 25 & 0.00 & 0.00 & - & - & - & - & - & - & 4.29 & - \\
\hline 76 & & 26 & 0.00 & 0.00 & - & - & - & - & - & - & 4.29 & - \\
\hline 77 & & 28 & 0.25 & 0.00 & - & - & - & - & - & - & 4.29 & - \\
\hline 78 & \multirow{6}{*}{ Massone (2006) } & WP111-9 & 0.25 & 0.27 & 12.00 & 13.00 & - & - & - & - & 0.87 & - \\
\hline 79 & & WP111-10 & 0.25 & 0.27 & 12.00 & 13.00 & - & - & - & - & 0.87 & - \\
\hline 80 & & WP1105-8 & 0.25 & 0.27 & 12.00 & 13.00 & - & - & - & - & 0.87 & - \\
\hline 81 & & WP1105-7 & 0.25 & 0.27 & 12.00 & 13.00 & - & - & - & - & 0.87 & - \\
\hline 82 & & WP110-5 & 0.25 & 0.27 & 12.00 & 13.00 & - & - & - & - & 0.87 & - \\
\hline 83 & & WP110-6 & 0.25 & 0.27 & 12.00 & 13.00 & - & - & - & - & 0.87 & - \\
\hline 84 & \multirow{4}{*}{ Greifenhagen (2005) } & M1 & 0.34 & 0.37 & 7.90 & 7.50 & - & - & - & - & - & - \\
\hline 85 & & M2 & 0.34 & 0.00 & 0.00 & 7.50 & - & - & - & - & - & - \\
\hline 86 & & M3 & 0.39 & 0.26 & 4.80 & 8.60 & - & - & - & - & - & - \\
\hline 87 & & M4 & 0.39 & 0.26 & 4.80 & 8.60 & - & - & - & - & - & - \\
\hline 88 & \multirow{8}{*}{ Kuang and $\mathrm{Ho}$ (2008) } & U 1.0 & 0.92 & 1.05 & 5.90 & 7.08 & - & - & - & - & - & - \\
\hline 89 & & U 1.5 & 0.92 & 1.05 & 5.90 & 7.08 & - & - & - & - & - & - \\
\hline 90 & & C 1.0 & 1.05 & 1.05 & 5.90 & 11.81 & - & - & - & - & - & - \\
\hline 91 & & C 1.5 & 1.05 & 1.05 & 5.90 & 11.81 & - & - & - & - & - & - \\
\hline 92 & & $\mathrm{U} 1.0 \mathrm{BC}$ & 0.92 & 1.05 & 5.90 & 7.08 & - & - & - & - & 1.74 & 5.90 \\
\hline 93 & & U1.5 BC & 0.92 & 1.05 & 5.90 & 7.08 & - & - & - & - & 1.74 & 5.90 \\
\hline 94 & & U $1.0 \mathrm{BC} 2$ & 0.92 & 1.05 & 5.90 & 7.08 & - & - & - & - & 1.74 & 2.95 \\
\hline 95 & & $\mathrm{U} 1.0 \mathrm{CT}$ & 0.92 & 1.05 & 5.90 & 7.08 & - & - & - & - & - & - \\
\hline 96 & \multirow{5}{*}{ Athanasopoulou (2010) } & S1 & 0.71 & 0.71 & 3.50 & 3.50 & - & - & - & - & 0.53 & 1.00 \\
\hline 97 & & S4 & 0.83 & 0.83 & 3.00 & 3.00 & - & - & - & - & 1.00 & 1.00 \\
\hline 98 & & S6 & 0.71 & 0.71 & 3.50 & 3.50 & - & - & - & - & 0.98 & 4.00 \\
\hline 99 & & S9 & 0.67 & 0.67 & 3.00 & 3.00 & - & - & - & - & 1.40 & 1.00 \\
\hline 100 & & S10 & 0.50 & 0.50 & 4.00 & 4.00 & - & - & - & - & 1.20 & 4.00 \\
\hline 101 & \multirow{3}{*}{ Tran and Wallace (2012) } & RW-A15-P10-S51 & 0.32 & 0.32 & 4.50 & 4.50 & - & - & - & - & 3.23 & 2.00 \\
\hline 102 & & RW-A15-P10-S78 & 0.73 & 0.73 & 5.00 & 5.00 & - & - & - & - & 6.06 & 2.00 \\
\hline 103 & & RW-A15-P2.5-S64 & 0.61 & 0.61 & 6.00 & 6.00 & - & - & - & - & 6.06 & 2.00 \\
\hline 104 & El-Sokkary and Galal (2013) & CW & 0.47 & 0.04 & 8.46 & 8.13 & - & - & - & - & - & 3.14 \\
\hline
\end{tabular}


Table B-3 Reinforcement Properties of the RC Walls with Rectangular Cross-Section (continued)

\begin{tabular}{|c|c|c|c|c|c|c|c|c|c|c|c|c|}
\hline $\begin{array}{l}\text { Test } \\
\text { No. }\end{array}$ & Researcher & Spec ID & $\begin{array}{l}\rho_{v}^{w} \\
(\%) \\
\end{array}$ & $\begin{array}{l}\rho_{h}^{w} \\
(\%) \\
\end{array}$ & $\begin{array}{c}s_{h}^{w} \\
\text { (in) } \\
\end{array}$ & $\begin{array}{c}S_{v}^{w} \\
\text { (in) } \\
\end{array}$ & $\begin{array}{c}\rho_{v}^{f} \\
(\%) \\
\end{array}$ & $\begin{array}{l}\rho_{h}^{f} \\
(\%) \\
\end{array}$ & $\begin{array}{c}S_{h}^{f} \\
\text { (in) } \\
\end{array}$ & $\begin{array}{l}s_{v}^{f} \\
\text { (in) } \\
\end{array}$ & $\begin{array}{l}\rho_{v}^{b e} \\
(\%) \\
\end{array}$ & $\begin{array}{l}s_{h}^{b e} \\
\text { (in) }\end{array}$ \\
\hline 105 & \multirow{7}{*}{ Park et al. (2015) } & S1 & 0.66 & 0.51 & 9.80 & 9.80 & - & - & - & - & 9.70 & 9.80 \\
\hline 106 & & S2 & 0.66 & 0.68 & 7.09 & 9.80 & - & - & - & - & 9.70 & 9.80 \\
\hline 107 & & S3 & 0.66 & 0.51 & 9.80 & 9.80 & - & - & - & - & 9.70 & 9.80 \\
\hline 108 & & S5 & 0.36 & 0.25 & 19.60 & 9.80 & - & - & - & - & 9.70 & 2.17 \\
\hline 109 & & S6 & 0.36 & 0.25 & 19.60 & 9.80 & - & - & - & - & 9.70 & 2.17 \\
\hline 110 & & S7 & 0.36 & 0.25 & 19.60 & 9.80 & - & - & - & - & 9.70 & 2.17 \\
\hline 111 & & S8 & 0.36 & 0.25 & 19.60 & 9.80 & - & - & - & - & 2.00 & 2.17 \\
\hline 112 & \multirow{5}{*}{ Hajyalikhani (2015) } & SW1 & 3.30 & - & 4.00 & 9.50 & - & - & - & - & - & - \\
\hline 113 & & SW2 & 4.42 & - & 4.50 & 9.00 & - & - & - & - & - & - \\
\hline 114 & & SW3 & 2.50 & - & 2.25 & 4.00 & - & - & - & - & - & - \\
\hline 115 & & SW5 & 6.07 & - & 2.00 & 4.00 & - & - & - & - & - & - \\
\hline 116 & & SW6 & 6.07 & - & 2.00 & 4.50 & - & - & - & - & - & - \\
\hline 117 & \multirow{6}{*}{ Peng et al. (2015) } & RCWS1 & 0.31 & 0.87 & 3.93 & 7.09 & - & - & - & - & 1.40 & 2.95 \\
\hline 118 & & RCWS2 & 0.31 & 0.58 & 5.91 & 7.09 & - & - & - & - & 1.40 & 2.95 \\
\hline 119 & & RCWS3 & 0.31 & 0.58 & 5.91 & 7.09 & - & - & - & - & 1.40 & 2.95 \\
\hline 120 & & RCWS4 & 0.31 & 0.29 & 11.81 & 7.09 & - & - & - & - & 1.40 & 2.95 \\
\hline 121 & & RCWS5 & 0.65 & 0.37 & 5.91 & 5.31 & - & - & - & - & 1.40 & 2.95 \\
\hline 122 & & RCWS6 & 0.65 & 0.19 & 11.81 & 5.31 & - & - & - & - & 1.40 & 2.95 \\
\hline 123 & \multirow{12}{*}{ Luna et al. (2015) } & SW1 & 0.67 & 0.67 & 8.00 & 8.00 & - & - & - & - & - & - \\
\hline 124 & & SW2 & 1.00 & 1.00 & 5.00 & 5.00 & - & - & - & - & - & - \\
\hline 125 & & SW3 & 0.67 & 0.67 & 7.00 & 7.00 & - & - & - & - & - & - \\
\hline 126 & & SW4 & 0.33 & 0.33 & 16.00 & 16.00 & - & - & - & - & - & - \\
\hline 127 & & SW5 & 1.00 & 1.00 & 5.00 & 5.00 & - & - & - & - & - & - \\
\hline 128 & & SW6 & 0.67 & 0.67 & 7.00 & 7.00 & - & - & - & - & - & - \\
\hline 129 & & SW7 & 0.33 & 0.33 & 14.00 & 14.00 & - & - & - & - & - & - \\
\hline 130 & & SW8 & 1.50 & 1.50 & 3.00 & 3.00 & - & - & - & - & - & - \\
\hline 131 & & SW9 & 1.50 & 0.67 & 7.00 & 3.50 & - & - & - & - & - & - \\
\hline 132 & & SW10 & 1.50 & 0.33 & 15.00 & 3.50 & - & - & - & - & - & - \\
\hline 133 & & SW11 & 0.67 & 0.67 & 7.00 & 7.00 & - & - & - & - & 1.50 & 3.25 \\
\hline 134 & & SW12 & 0.33 & 0.33 & 15.00 & 15.00 & - & - & - & - & 2.00 & 2.50 \\
\hline 135 & Li et al. (2015) & S1 & 0.40 & 0.50 & 7.87 & 7.08 & - & - & - & - & - & - \\
\hline 136 & \multirow{2}{*}{ Woods et al. (2016) } & CW1 & 3.00 & 0.25 & 10.62 & 5.51 & - & - & - & - & - & - \\
\hline 137 & & CW2 & 3.00 & 0.25 & 7.08 & 5.51 & - & - & - & - & - & - \\
\hline
\end{tabular}


Table B-4 Material Properties of the RC Walls with Rectangular Cross-Section

\begin{tabular}{|c|c|c|c|c|c|c|c|c|c|c|c|}
\hline $\begin{array}{l}\text { Test } \\
\text { No. }\end{array}$ & Researcher & Spec ID & $\begin{array}{c}f_{c}^{\prime} \\
(\mathrm{ksi})\end{array}$ & $\begin{array}{c}f_{y v}^{w} \\
(\mathrm{ksi})\end{array}$ & $\begin{array}{c}f_{u v}^{w} \\
(\mathrm{ksi})\end{array}$ & $\begin{array}{c}f_{y h}^{w} \\
(\mathrm{ksi})\end{array}$ & $\begin{array}{c}f_{u h}^{w} \\
(\mathrm{ksi})\end{array}$ & $\begin{array}{c}f_{y v}^{f} \\
(\mathrm{ksi})\end{array}$ & $\begin{array}{c}f_{u v}^{f} \\
(\mathrm{ksi})\end{array}$ & $\begin{array}{c}f_{y h}^{f} \\
(\mathrm{ksi})\end{array}$ & $\begin{array}{c}f_{u h}^{f} \\
\text { (ksi) }\end{array}$ \\
\hline 1 & $\begin{array}{c}\text { Alexander et al. } \\
\quad(1973)\end{array}$ & 4 & 3.0 & 52.0 & - & 52.0 & - & - & - & - & - \\
\hline 2 & \multirow{10}{*}{ Hirosawa (1975) } & 72 & 2.5 & 59.0 & - & 60.8 & - & 54.6 & - & 54.6 & - \\
\hline 3 & & 73 & 3.0 & 59.0 & - & 60.8 & - & 54.6 & - & 54.6 & - \\
\hline 4 & & 74 & 3.0 & 59.0 & - & 60.8 & - & 54.6 & - & 54.6 & - \\
\hline 5 & & 75 & 2.0 & 59.0 & - & 60.8 & - & 54.6 & - & 54.6 & - \\
\hline 6 & & 76 & 2.1 & 59.0 & - & 60.8 & - & 54.6 & - & 54.6 & - \\
\hline 7 & & 77 & 2.7 & 59.0 & - & 60.8 & - & 54.6 & - & 54.6 & - \\
\hline 8 & & 78 & 3.0 & 59.0 & - & 60.8 & - & 55.4 & - & 55.4 & - \\
\hline 9 & & 79 & 2.0 & 59.0 & - & 60.8 & - & 55.4 & - & 55.4 & - \\
\hline 10 & & 80 & 2.1 & 59.0 & - & 60.8 & - & 55.4 & - & 55.4 & - \\
\hline 11 & & 81 & 2.7 & 59.0 & - & 60.8 & - & 55.4 & - & 55.4 & - \\
\hline 12 & Synge (1980) & WALL 1 & 3.9 & 43.5 & 66.7 & 55.1 & 83.5 & 43.5 & 66.7 & - & - \\
\hline 13 & \multirow{2}{*}{ Wiradinata (1985) } & WALL1 & 3.6 & 63.1 & 94.3 & 61.6 & 79.8 & 63.1 & 94.3 & 63.1 & 94.3 \\
\hline 14 & & WALL2 & 3.2 & 63.1 & 94.3 & 61.6 & 79.8 & 63.1 & 94.3 & 63.1 & 94.3 \\
\hline 15 & \multirow{3}{*}{ Maier (1985) } & S4 & 4.8 & 83.5 & 110.0 & 83.5 & 110.0 & 83.5 & 110.0 & 83.5 & 110.0 \\
\hline 16 & & S9 & 4.2 & 81.2 & 81.2 & - & - & 81.2 & 81.2 & - & - \\
\hline 17 & & $\mathrm{~S} 10$ & 4.5 & 71.9 & 103.8 & 71.9 & 103.8 & 71.9 & 103.8 & 71.9 & 103.8 \\
\hline 18 & \multirow{2}{*}{ Pilette (1987) } & WALL 4 & 4.8 & 69.6 & 111.7 & 69.6 & 111.7 & 69.6 & 111.7 & 69.6 & 111.7 \\
\hline 19 & & WALL 5 & 3.9 & 69.6 & 111.7 & 69.6 & 111.7 & 69.6 & 111.7 & 69.6 & 111.7 \\
\hline 20 & \multirow{2}{*}{$\begin{array}{l}\text { Huang and Sheu } \\
\text { (1988) }\end{array}$} & SWN-1D & 3.9 & 67.8 & 104.7 & 67.8 & 104.7 & - & - & - & - \\
\hline 21 & & SWN-5D & 4.1 & 67.8 & 104.7 & 67.8 & 104.7 & - & - & - & - \\
\hline 22 & \multirow{2}{*}{ Wasiewicz (1988) } & WALL 3 & 4.4 & 69.6 & 112.1 & 35.9 & 43.5 & - & - & - & - \\
\hline 23 & & WALL 6 & 4.4 & 69.6 & 112.1 & 69.6 & 112.1 & - & - & - & - \\
\hline 24 & \multirow{7}{*}{ Lefas et al. (1990) } & SW11 & 7.1 & 68.2 & 82.0 & 75.4 & 88.5 & - & - & 60.9 & 71.1 \\
\hline 25 & & SW12 & 7.3 & 68.2 & 82.0 & 75.4 & 88.5 & - & - & 60.9 & 71.1 \\
\hline 26 & & SW13 & 5.4 & 68.2 & 82.0 & 75.4 & 88.5 & - & - & 60.9 & 71.1 \\
\hline 27 & & SW14 & 5.8 & 68.2 & 82.0 & 75.4 & 88.5 & - & - & 60.9 & 71.1 \\
\hline 28 & & SW15 & 7.1 & 68.2 & 82.0 & 75.4 & 88.5 & - & - & 60.9 & 71.1 \\
\hline 29 & & SW16 & 6.6 & 68.2 & 82.0 & 75.4 & 88.5 & - & - & 60.9 & 71.1 \\
\hline 30 & & SW17 & 5.8 & 68.2 & 82.0 & 75.4 & 88.5 & - & - & 60.9 & 71.1 \\
\hline 31 & \multirow{2}{*}{$\begin{array}{l}\text { Lopes and Elnashai } \\
\qquad(1991)\end{array}$} & SW11 & 5.8 & 60.1 & - & 60.1 & - & - & - & - & - \\
\hline 32 & & SW12 & 6.0 & 60.1 & - & 60.1 & - & - & - & - & - \\
\hline
\end{tabular}


Table B-4 Material Properties of the RC Walls with Rectangular Cross-Section (continued)

\begin{tabular}{|c|c|c|c|c|c|c|c|c|c|c|c|}
\hline $\begin{array}{l}\text { Test } \\
\text { No. }\end{array}$ & Researcher & $\begin{array}{l}\text { Spec } \\
\text { ID }\end{array}$ & $\begin{array}{c}f_{c}^{\prime} \\
(\mathrm{ksi}) \\
\end{array}$ & $\begin{array}{r}f_{y v}^{w} \\
(\mathrm{ksi}) \\
\end{array}$ & $\begin{array}{l}f_{u v}^{w} \\
(\mathrm{ksi})\end{array}$ & $\begin{array}{c}f_{y h}^{w} \\
(\mathrm{ksi})\end{array}$ & $\begin{array}{c}f_{u h}^{w} \\
(\mathrm{ksi})\end{array}$ & $\begin{array}{r}f_{y v}^{f} \\
(\mathrm{ksi})\end{array}$ & $\begin{array}{c}f_{u v}^{f} \\
(\mathrm{ksi})\end{array}$ & $\begin{array}{r}f_{y h}^{f} \\
(\mathrm{ksi})\end{array}$ & $\begin{array}{c}f_{u h}^{f} \\
\text { (ksi) } \\
\end{array}$ \\
\hline 33 & \multirow{2}{*}{$\begin{array}{l}\text { Lopes and Elnashai } \\
\text { (1991) }\end{array}$} & SW13 & 6.9 & 60.1 & - & 60.1 & - & - & - & - & - \\
\hline 34 & & SW14 & 5.9 & 60.1 & - & 60.1 & - & - & - & - & - \\
\hline 35 & \multirow{4}{*}{$\begin{array}{l}\text { Lopes and Elnashai } \\
\qquad(1991)\end{array}$} & SW15 & 6.0 & 60.1 & - & 60.1 & - & - & - & - & - \\
\hline 36 & & SW16 & 5.6 & 0.0 & - & 60.1 & - & - & - & - & - \\
\hline 37 & & SW17 & 5.7 & 0.0 & - & 60.1 & - & - & - & - & - \\
\hline 38 & & SW18 & 5.6 & 0.0 & - & 60.1 & - & - & - & - & - \\
\hline 39 & \multirow{3}{*}{ Saatcioglu (1991) } & Wall-1 & 3.6 & 63.0 & - & 61.6 & - & - & - & - & - \\
\hline 40 & & Wall-4 & 4.8 & 69.9 & - & 69.6 & - & - & - & - & - \\
\hline 41 & & Wall-6 & 5.1 & 69.9 & - & 69.6 & - & - & - & - & - \\
\hline 42 & \multirow{3}{*}{ Cheng (1992) } & SW4A & 3.9 & 69.7 & 113.4 & 69.7 & 113.4 & - & - & - & - \\
\hline 43 & & SW5 & 4.0 & 69.7 & 113.4 & 69.7 & 113.4 & - & - & - & - \\
\hline 44 & & SW6 & 4.1 & 69.7 & 113.4 & 69.7 & 113.4 & - & - & - & - \\
\hline 45 & \multirow{2}{*}{ Mohammadi (1994) } & Wall 7 & 5.1 & 58.0 & - & 58.0 & - & - & - & - & - \\
\hline 46 & & Wall 8 & 5.1 & 58.0 & - & 58.0 & - & - & - & - & - \\
\hline 47 & \multirow{3}{*}{ Cheng (1992) } & SW-OE & 3.6 & 71.2 & 109.9 & 71.2 & 109.9 & - & - & - & - \\
\hline 48 & & SW-1E & 4.9 & 71.2 & 109.9 & 71.2 & 109.9 & - & - & - & - \\
\hline 49 & & SW-9E & 4.2 & 65.7 & 102.4 & 65.7 & 102.4 & - & - & - & - \\
\hline 50 & \multirow{6}{*}{$\begin{array}{l}\text { Salonikios et al. } \\
\qquad(1999)\end{array}$} & LSW1 & 3.8 & 86.7 & - & 86.7 & - & 84.8 & - & 84.8 & - \\
\hline 51 & & LSW2 & 3.8 & 86.7 & - & 86.7 & - & 84.8 & - & 84.8 & - \\
\hline 52 & & LSW3 & 3.5 & 86.7 & - & 86.7 & - & 84.8 & - & 84.8 & - \\
\hline 53 & & MSW1 & 4.0 & 86.7 & - & 86.7 & - & 84.8 & - & 84.8 & - \\
\hline 54 & & MSW2 & 3.2 & 86.7 & - & 86.7 & - & 84.8 & - & 84.8 & - \\
\hline 55 & & MSW3 & 3.1 & 86.7 & - & 86.7 & - & 84.8 & - & 84.8 & - \\
\hline 56 & Xia and Xiao (2000) & W1-A & 4.3 & 65.8 & 106.2 & 65.8 & 106.2 & - & - & - & - \\
\hline 57 & \multirow{9}{*}{ Hidalgo et al. (2002) } & 1 & 2.8 & 56.8 & - & 56.8 & - & - & - & - & - \\
\hline 58 & & 2 & 2.8 & 58.3 & - & 58.3 & - & - & - & - & - \\
\hline 59 & & 4 & 2.8 & 58.3 & - & 58.3 & - & - & - & - & - \\
\hline 60 & & 6 & 2.6 & 45.5 & - & 45.5 & - & - & - & - & - \\
\hline 61 & & 7 & 2.6 & 68.3 & - & 68.3 & - & - & - & - & - \\
\hline 62 & & 8 & 2.3 & 68.3 & - & 68.3 & - & - & - & - & - \\
\hline 63 & & 9 & 2.6 & 53.0 & - & 53.0 & - & - & - & - & - \\
\hline 64 & & 10 & 2.4 & 53.1 & - & 53.1 & - & - & - & - & - \\
\hline 65 & & 11 & 2.4 & 52.5 & - & 52.5 & - & - & - & - & - \\
\hline
\end{tabular}


Table B-4 Material Properties of the RC Walls with Rectangular Cross-Section (continued)

\begin{tabular}{|c|c|c|c|c|c|c|c|c|c|c|c|}
\hline $\begin{array}{l}\text { Test } \\
\text { No. }\end{array}$ & Researcher & Spec ID & $\begin{array}{c}f_{c}^{\prime} \\
(\mathrm{ksi}) \\
\end{array}$ & $\begin{array}{r}f_{y v}^{w} \\
(\mathrm{ksi})\end{array}$ & $\begin{array}{c}f_{u v}^{w} \\
(\mathrm{ksi})\end{array}$ & $\begin{array}{c}f_{y h}^{w} \\
(\mathrm{ksi})\end{array}$ & $\begin{array}{c}f_{u h}^{w} \\
(\mathrm{ksi})\end{array}$ & $\begin{array}{c}f_{y v}^{f} \\
(\mathrm{ksi})\end{array}$ & $\begin{array}{c}f_{u v}^{f} \\
(\mathrm{ksi})\end{array}$ & $\begin{array}{c}f_{y h}^{f} \\
(\mathrm{ksi})\end{array}$ & $\begin{array}{c}f_{u h}^{f} \\
\text { (ksi) }\end{array}$ \\
\hline 66 & \multirow{3}{*}{ Hidalgo et al. (2002) } & 12 & 2.5 & 53.0 & - & 53.0 & - & - & - & - & - \\
\hline 67 & & 13 & 2.6 & 53.6 & - & 53.6 & - & - & - & - & - \\
\hline 68 & & 14 & 2.6 & 53.0 & - & 53.0 & - & - & - & - & - \\
\hline 69 & \multirow{9}{*}{ Hidalgo et al. (2002) } & 15 & 2.5 & 53.0 & - & 53.0 & - & - & - & - & - \\
\hline 70 & & 16 & 2.8 & 53.0 & - & 53.0 & - & - & - & - & - \\
\hline 71 & & 21 & 2.7 & 53.0 & - & 53.0 & - & - & - & - & - \\
\hline 72 & & 22 & 3.5 & 53.0 & - & 53.0 & - & - & - & - & - \\
\hline 73 & & 23 & 2.5 & 62.5 & - & 62.5 & - & - & - & - & - \\
\hline 74 & & 24 & 3.5 & 62.5 & - & 62.5 & - & - & - & - & - \\
\hline 75 & & 25 & 3.5 & 62.5 & - & 62.5 & - & - & - & - & - \\
\hline 76 & & 26 & 3.5 & 62.5 & - & 62.5 & - & - & - & - & - \\
\hline 77 & & 28 & 3.5 & 62.5 & - & 62.5 & - & - & - & - & - \\
\hline 78 & \multirow{6}{*}{ Massone (2006) } & WP111-9 & 4.1 & 61.5 & 91.5 & 61.5 & 91.5 & 61.5 & 91.5 & - & - \\
\hline 79 & & WP111-10 & 4.6 & 61.5 & 91.5 & 61.5 & 91.5 & 61.5 & 91.5 & - & - \\
\hline 80 & & WP1105-8 & 4.6 & 61.5 & 91.5 & 61.5 & 91.5 & 61.5 & 91.5 & - & - \\
\hline 81 & & WP1105-7 & 4.6 & 61.5 & 91.5 & 61.5 & 91.5 & 61.5 & 91.5 & - & - \\
\hline 82 & & WP110-5 & 4.3 & 61.5 & 91.5 & 61.5 & 91.5 & 61.5 & 91.5 & - & - \\
\hline 83 & & WP110-6 & 4.5 & 61.5 & 91.5 & 61.5 & 91.5 & 61.5 & 91.5 & - & - \\
\hline 84 & \multirow{4}{*}{ Greifenhagen (2005) } & M1 & 7.4 & 73.1 & 92.0 & 73.1 & 92.0 & - & - & - & - \\
\hline 85 & & M2 & 7.4 & 73.1 & 92.0 & 73.1 & 92.0 & - & - & - & - \\
\hline 86 & & M3 & 2.9 & 73.1 & 92.0 & 108.1 & 116.0 & - & - & - & - \\
\hline 87 & & M4 & 3.5 & 73.1 & 92.0 & 108.1 & 116.0 & - & - & - & - \\
\hline 88 & \multirow{8}{*}{ Kuang and Ho (2008) } & U 1.0 & 4.4 & 75.4 & - & 75.4 & - & 75.4 & - & 75.4 & - \\
\hline 89 & & U 1.5 & 5.1 & 75.4 & - & 75.4 & - & 75.4 & - & 75.4 & - \\
\hline 90 & & C 1.0 & 5.1 & 75.4 & - & 75.4 & - & 75.4 & - & 75.4 & - \\
\hline 91 & & C 1.5 & 5.0 & 75.4 & - & 75.4 & - & 75.4 & - & 75.4 & - \\
\hline 92 & & U $1.0 \mathrm{BC}$ & 4.5 & 75.4 & - & 75.4 & - & 75.4 & - & 75.4 & - \\
\hline 93 & & U1.5 BC & 4.9 & 75.4 & - & 75.4 & - & 75.4 & - & 75.4 & - \\
\hline 94 & & U $1.0 \mathrm{BC} 2$ & 4.9 & 75.4 & - & 75.4 & - & 75.4 & - & 75.4 & - \\
\hline 95 & & U $1.0 \mathrm{CT}$ & 5.5 & 75.4 & - & 75.4 & - & 75.4 & - & 75.4 & - \\
\hline 96 & \multirow{3}{*}{$\begin{array}{l}\text { Athanasopoulou } \\
\text { (2010) }\end{array}$} & S1 & 6.7 & 95.8 & 99.7 & 95.8 & 99.7 & 74.3 & 108.0 & 74.3 & 108.0 \\
\hline 97 & & S4 & 6.7 & 91.8 & 94.6 & 91.8 & 94.6 & 65.0 & 101.0 & 65.0 & 101.0 \\
\hline 98 & & S6 & 6.8 & 97.3 & 98.4 & 97.3 & 98.4 & 70.0 & 105.0 & 70.0 & 105.0 \\
\hline
\end{tabular}


Table B-4 Material Properties of the RC Walls with Rectangular Cross-Section (continued)

\begin{tabular}{|c|c|c|c|c|c|c|c|c|c|c|c|}
\hline $\begin{array}{l}\text { Test } \\
\text { No. }\end{array}$ & Researcher & Spec ID & $\begin{array}{c}f_{c}^{\prime} \\
(\mathrm{ksi})\end{array}$ & $\begin{array}{l}f_{y v}^{w} \\
(\mathrm{ksi})\end{array}$ & $\begin{array}{c}f_{u v}^{w} \\
(\mathrm{ksi})\end{array}$ & $\begin{array}{c}f_{y h}^{w} \\
(\mathrm{ksi})\end{array}$ & $\begin{array}{c}f_{u h}^{w} \\
(\mathrm{ksi})\end{array}$ & $\begin{array}{c}f_{y v}^{f} \\
(\mathrm{ksi})\end{array}$ & $\begin{array}{c}f_{u v}^{f} \\
(\mathrm{ksi})\end{array}$ & $\begin{array}{c}f_{y h}^{f} \\
(\mathrm{ksi})\end{array}$ & $\begin{array}{c}f_{u h}^{f} \\
(\mathrm{ksi})\end{array}$ \\
\hline 99 & \multirow{2}{*}{$\begin{array}{c}\text { Athanasopoulou } \\
(2010)\end{array}$} & S9 & 6.2 & 90.2 & 95.2 & 90.2 & 95.2 & 68.0 & 103.0 & 68.0 & 103.0 \\
\hline 100 & & S10 & 5.5 & 90.2 & 95.2 & 90.2 & 95.2 & 69.3 & 106.0 & 69.3 & 106.0 \\
\hline 101 & \multirow{3}{*}{$\begin{array}{l}\text { Tran and Wallace } \\
(2012)\end{array}$} & $\begin{array}{l}\text { RW-A15- } \\
\text { P10-S51 }\end{array}$ & 5.0 & 60.0 & - & 60.0 & - & 60.0 & - & 40.0 & - \\
\hline 102 & & $\begin{array}{l}\text { RW-A15- } \\
\text { P10-S78 }\end{array}$ & 5.0 & 60.0 & - & 60.0 & - & 60.0 & - & 40.0 & - \\
\hline 103 & & $\begin{array}{l}\text { RW-A15- } \\
\text { P2.5-S64 }\end{array}$ & 5.0 & 60.0 & - & 60.0 & - & 60.0 & - & 40.0 & - \\
\hline 104 & $\begin{array}{l}\text { El-Sokkary and } \\
\text { Galal (2013) }\end{array}$ & CW & 6.5 & 65.3 & 73.8 & 65.3 & 79.8 & 89.9 & 104.4 & 89.9 & 104.4 \\
\hline 105 & \multirow{7}{*}{ Park et al. (2015) } & S1 & 6.7 & 94.7 & - & 96.7 & - & 89.5 & - & 96.7 & - \\
\hline 106 & & S2 & 6.7 & 94.7 & - & 96.7 & - & 89.5 & - & 96.7 & - \\
\hline 107 & & S3 & 10.2 & 94.7 & - & 96.7 & - & 89.5 & - & 96.7 & - \\
\hline 108 & & S5 & 6.7 & 94.7 & - & 96.7 & - & 89.5 & - & 96.7 & - \\
\hline 109 & & S6 & 10.2 & 94.7 & - & 96.7 & - & 89.5 & - & 96.7 & - \\
\hline 110 & & S7 & 6.7 & 94.7 & - & 96.7 & - & 89.5 & - & 96.7 & - \\
\hline 111 & & S8 & 6.7 & 94.7 & - & 96.7 & - & 89.5 & - & 96.7 & - \\
\hline 112 & \multirow{5}{*}{ Hajyalikhani (2015) } & SW1 & 5.0 & 70.0 & - & 70.0 & - & - & - & - & - \\
\hline 113 & & SW2 & 4.9 & 70.0 & - & 70.0 & - & - & - & - & - \\
\hline 114 & & SW3 & 5.5 & 70.0 & - & 70.0 & - & - & - & - & - \\
\hline 115 & & SW5 & 4.0 & 70.0 & - & 70.0 & - & - & - & - & - \\
\hline 116 & & SW6 & 4.5 & 70.0 & - & 70.0 & - & - & - & - & - \\
\hline 117 & \multirow{6}{*}{ Peng et al. (2015) } & RCWS1 & 6.1 & 57.7 & 75.1 & 52.6 & 69.9 & 69.2 & 91.1 & 52.6 & 69.9 \\
\hline 118 & & RCWS2 & 6.1 & 57.7 & 75.1 & 52.6 & 69.9 & 69.2 & 91.1 & 52.6 & 69.9 \\
\hline 119 & & RCWS3 & 6.3 & 57.7 & 75.1 & 52.6 & 75.1 & 69.2 & 91.1 & 52.6 & 69.9 \\
\hline 120 & & RCWS4 & 6.2 & 57.7 & 75.1 & 52.6 & 75.1 & 69.2 & 91.1 & 52.6 & 69.9 \\
\hline 121 & & RCWS5 & 6.4 & 52.6 & 69.9 & 57.7 & 75.1 & 69.2 & 91.1 & 52.6 & 69.9 \\
\hline 122 & & RCWS6 & 6.8 & 52.6 & 69.9 & 57.7 & 75.1 & 69.2 & 91.1 & 52.6 & 69.9 \\
\hline 123 & \multirow{7}{*}{ Luna et al. (2015) } & SW1 & 3.6 & 66.7 & 101.0 & 66.7 & 101.0 & - & - & - & - \\
\hline 124 & & SW2 & 7.0 & 63.1 & 87.0 & 63.1 & 87.0 & - & - & - & - \\
\hline 125 & & SW3 & 7.8 & 63.1 & 87.0 & 63.1 & 87.0 & & & & \\
\hline 126 & & SW4 & 4.2 & 66.7 & 101.0 & 66.7 & 101.0 & - & - & - & - \\
\hline 127 & & SW5 & 4.3 & 66.7 & 101.0 & 66.7 & 101.0 & - & - & - & - \\
\hline 128 & & SW6 & 3.8 & 66.7 & 101.0 & 66.7 & 101.0 & - & - & - & - \\
\hline 129 & & SW7 & 3.8 & 66.7 & 101.0 & 66.7 & 101.0 & - & - & - & - \\
\hline
\end{tabular}


Table B-4 Material Properties of the RC Walls with Rectangular Cross-Section (continued)

\begin{tabular}{|c|c|c|c|c|c|c|c|c|c|c|c|}
\hline $\begin{array}{l}\text { Test } \\
\text { No. }\end{array}$ & Researcher & Spec ID & $\begin{array}{c}f_{c}^{\prime} \\
(\mathrm{ksi})\end{array}$ & $\begin{array}{c}f_{y v}^{w} \\
(\mathrm{ksi})\end{array}$ & $\begin{array}{c}f_{u v}^{w} \\
\text { (ksi) }\end{array}$ & $\begin{array}{c}f_{y h}^{w} \\
(\mathrm{ksi})\end{array}$ & $\begin{array}{c}f_{u h}^{w} \\
(\mathrm{ksi})\end{array}$ & $\begin{array}{c}f_{y v}^{f} \\
(\mathrm{ksi})\end{array}$ & $\begin{array}{c}f_{u v}^{f} \\
(\mathrm{ksi})\end{array}$ & $\begin{array}{c}f_{y h}^{f} \\
(\mathrm{ksi})\end{array}$ & $\begin{array}{c}f_{u h}^{f} \\
\text { (ksi) }\end{array}$ \\
\hline 130 & \multirow{5}{*}{ Luna et al. (2015) } & SW8 & 3.5 & 66.7 & 101.0 & 66.7 & 101.0 & - & - & - & - \\
\hline 131 & & SW9 & 4.3 & 66.7 & 101.0 & 66.7 & 101.0 & - & - & - & - \\
\hline 132 & & SW10 & 4.6 & 66.7 & 101.0 & 66.7 & 101.0 & - & - & - & - \\
\hline 133 & & SW11 & 5.0 & 66.7 & 101.0 & 66.7 & 101.0 & 66.7 & 101.0 & 66.7 & 101.0 \\
\hline 134 & & SW12 & 5.0 & 66.7 & 101.0 & 66.7 & 101.0 & 66.7 & 101.0 & 66.7 & 101.0 \\
\hline 135 & Li et al. (2015) & S1 & 4.9 & 68.9 & 100.0 & 68.9 & 100.0 & - & - & - & - \\
\hline 136 & \multirow{2}{*}{$\begin{array}{l}\text { Woods et al. } \\
\quad(2016)\end{array}$} & CW1 & 3.2 & 65.5 & - & 60.2 & - & - & - & - & - \\
\hline 137 & & CW2 & 2.6 & 65.5 & - & 60.2 & - & - & - & - & - \\
\hline
\end{tabular}

Table B-5 Test Results of the RC Walls with Rectangular Cross-Section in the First Quadrant

\begin{tabular}{|c|c|c|c|c|c|c|c|c|c|c|}
\hline $\begin{array}{l}\text { Test } \\
\text { No. }\end{array}$ & Researcher & Spec ID & $\begin{array}{c}\Delta_{A} \\
\text { (in) } \\
\end{array}$ & $\begin{array}{l}\Delta_{B} \\
\text { (in) }\end{array}$ & $\begin{array}{c}\Delta_{C} \\
\text { (in) } \\
\end{array}$ & $\begin{array}{l}\Delta_{D} \\
\text { (in) } \\
\end{array}$ & $\begin{array}{c}F_{A} \\
\text { (kips) }\end{array}$ & $\begin{array}{c}F_{B} \\
\text { (kips) }\end{array}$ & $\begin{array}{c}F_{C} \\
\text { (kips) } \\
\end{array}$ & $\begin{array}{c}F_{D} \\
\text { (kips) }\end{array}$ \\
\hline 1 & $\begin{array}{l}\text { Alexander et } \\
\text { al. (1973) }\end{array}$ & 4 & 0.070 & 0.314 & 0.440 & 1.321 & 41 & 75 & 75 & 15 \\
\hline 2 & \multirow{10}{*}{$\begin{array}{c}\text { Hirosawa } \\
(1975)\end{array}$} & 72 & 0.050 & 0.267 & 0.630 & - & 88 & 180 & 180 & - \\
\hline 3 & & 73 & 0.030 & 0.241 & 0.330 & 1.879 & 70 & 163 & 163 & 33 \\
\hline 4 & & 74 & 0.030 & 0.243 & 0.330 & 1.400 & 70 & 185 & 185 & 37 \\
\hline 5 & & 75 & 0.010 & 0.225 & 0.340 & - & 46 & 182 & 182 & - \\
\hline 6 & & 76 & 0.020 & 0.220 & 0.760 & 1.078 & 63 & 181 & 181 & 36 \\
\hline 7 & & 77 & 0.020 & 0.336 & 0.930 & - & 64 & 200 & 200 & - \\
\hline 8 & & 78 & 0.020 & 0.700 & 1.340 & - & 46 & 153 & 153 & - \\
\hline 9 & & 79 & 0.020 & 0.219 & 0.330 & - & 55 & 140 & 140 & - \\
\hline 10 & & 80 & 0.050 & 0.546 & 1.340 & - & 68 & 158 & 158 & - \\
\hline 11 & & 81 & 0.010 & 0.562 & 1.340 & - & 46 & 171 & 171 & - \\
\hline 12 & Synge (1980) & WALL 1 & 0.060 & 0.295 & 0.400 & 1.431 & 97 & 179 & 179 & 36 \\
\hline 13 & \multirow{2}{*}{$\begin{array}{l}\text { Wiradinata } \\
(1985)\end{array}$} & WALL1 & 0.020 & 0.116 & 0.380 & 1.000 & 64 & 130 & 130 & 26 \\
\hline 14 & & WALL2 & 0.030 & 0.100 & 0.100 & 1.532 & 102 & 153 & 153 & 31 \\
\hline 15 & \multirow{3}{*}{ Maier (1985) } & S4 & 0.030 & 0.265 & 0.490 & 2.472 & 70 & 177 & 177 & 35 \\
\hline 16 & & S9 & 0.040 & 0.219 & 0.460 & 0.660 & 33 & 75 & 75 & 15 \\
\hline 17 & & S10 & 0.040 & 0.395 & 0.540 & 2.389 & 22 & 150 & 150 & 30 \\
\hline 18 & \multirow{2}{*}{ Pilette (1987) } & WALL 4 & 0.100 & 0.279 & 0.640 & 2.892 & 53 & 85 & 85 & 17 \\
\hline 19 & & WALL 5 & 0.160 & 0.423 & 0.900 & 2.356 & 79 & 131 & 131 & 26 \\
\hline 20 & \multirow{2}{*}{$\begin{array}{l}\text { Huang and } \\
\text { Sheu (1988) }\end{array}$} & SWN-1D & 0.040 & 0.110 & 0.260 & 0.810 & 60 & 72 & 72 & 14 \\
\hline 21 & & SWN-5D & 0.030 & 0.196 & 0.330 & - & 36 & 55 & 55 & - \\
\hline
\end{tabular}


Table B-5 Test Results of the RC Walls with Rectangular Cross-Section in the First Quadrant (continued)

\begin{tabular}{|c|c|c|c|c|c|c|c|c|c|c|}
\hline $\begin{array}{l}\text { Test } \\
\text { No. }\end{array}$ & Researcher & Spec ID & $\begin{array}{c}\Delta_{A} \\
\text { (in) } \\
\end{array}$ & $\begin{array}{l}\triangle_{B} \\
\text { (in) } \\
\end{array}$ & $\begin{array}{c}\Delta_{C} \\
\text { (in) } \\
\end{array}$ & $\begin{array}{l}\Delta_{D} \\
\text { (in) } \\
\end{array}$ & $\begin{array}{c}F_{A} \\
\text { (kips) } \\
\end{array}$ & $\begin{array}{c}F_{B} \\
\text { (kips) } \\
\end{array}$ & $\begin{array}{c}F_{C} \\
\text { (kips) } \\
\end{array}$ & $\begin{array}{c}F_{D} \\
\text { (kips) }\end{array}$ \\
\hline 22 & \multirow{2}{*}{$\begin{array}{c}\text { Wasiewicz } \\
\text { (1988) }\end{array}$} & WALL 3 & 0.050 & 0.295 & 0.400 & 1.211 & 127 & 204 & 204 & 41 \\
\hline 23 & & WALL 6 & 0.090 & 0.480 & 0.650 & 1.154 & 55 & 118 & 118 & 24 \\
\hline 24 & \multirow{7}{*}{$\begin{array}{l}\text { Lefas et al. } \\
\text { (1990) }\end{array}$} & SW11 & 0.040 & 0.245 & 0.300 & - & 16 & 55 & 55 & - \\
\hline 25 & & SW12 & 0.050 & 0.241 & 0.350 & - & 29 & 74 & 74 & - \\
\hline 26 & & SW13 & 0.040 & 0.303 & 0.400 & - & 16 & 59 & 59 & - \\
\hline 27 & & SW14 & 0.040 & 0.262 & 0.360 & - & 26 & 75 & 75 & - \\
\hline 28 & & SW15 & 0.030 & 0.235 & 0.330 & - & 19 & 71 & 71 & - \\
\hline 29 & & SW16 & 0.020 & 0.166 & 0.250 & - & 23 & 80 & 80 & - \\
\hline 30 & & SW17 & 0.060 & 0.299 & 0.410 & - & 16 & 57 & 57 & - \\
\hline 31 & \multirow{6}{*}{$\begin{array}{c}\text { Lopes and } \\
\text { Elnashai (1991) }\end{array}$} & SW11 & 0.018 & 0.128 & 0.184 & 0.528 & 7 & 21 & 21 & 4 \\
\hline 32 & & SW12 & 0.033 & 0.093 & 0.110 & 0.219 & 11 & 18 & 18 & 4 \\
\hline 33 & & SW13 & 0.080 & 0.243 & 0.333 & 0.626 & 14 & 24 & 24 & 5 \\
\hline 34 & & SW14 & 0.046 & 0.124 & 0.228 & 0.403 & 12 & 21 & 21 & 4 \\
\hline 35 & & SW15 & 0.040 & 0.116 & 0.130 & 0.694 & 10 & 17 & 17 & 3 \\
\hline 36 & & SW16 & 0.048 & 0.154 & 0.193 & 0.516 & 10 & 19 & 19 & 4 \\
\hline 37 & \multirow{2}{*}{$\begin{array}{c}\text { Lopes and } \\
\text { Elnashai (1991) }\end{array}$} & SW17 & 0.057 & 0.132 & 0.133 & 0.974 & 13 & 19 & 19 & 4 \\
\hline 38 & & SW18 & 0.017 & 0.098 & 0.132 & - & 7 & 21 & 21 & - \\
\hline 39 & \multirow{3}{*}{$\begin{array}{l}\text { Saatcioglu } \\
\text { (1991) }\end{array}$} & Wall-1 & 0.060 & 0.204 & 0.390 & 0.983 & 101 & 134 & 134 & 27 \\
\hline 40 & & Wall-4 & 0.110 & 0.261 & 0.650 & 2.016 & 54 & 87 & 87 & 17 \\
\hline 41 & & Wall-6 & 0.010 & 0.398 & 0.630 & 1.107 & 39 & 127 & 127 & 25 \\
\hline 42 & \multirow{3}{*}{ Cheng (1992) } & SW4A & 0.004 & 0.116 & 0.237 & - & 16 & 61 & 61 & - \\
\hline 43 & & SW5 & 0.008 & 0.117 & 0.203 & - & 26 & 65 & 65 & - \\
\hline 44 & & SW6 & 0.007 & 0.096 & 0.240 & - & 21 & 62 & 62 & - \\
\hline 45 & \multirow{2}{*}{$\begin{array}{l}\text { Mohammadi } \\
\text { (1994) }\end{array}$} & Wall 7 & 0.050 & 0.090 & 0.840 & 3.962 & 54 & 80 & 80 & 16 \\
\hline 46 & & Wall 8 & 0.080 & 0.241 & 0.690 & 6.083 & 36 & 50 & 50 & 10 \\
\hline 47 & \multirow{3}{*}{ Cheng (1992) } & SW-OE & 0.028 & 0.178 & 0.334 & 1.192 & 23 & 70 & 70 & 14 \\
\hline 48 & & SW-1E & 0.032 & 0.139 & 0.340 & 0.855 & 35 & 73 & 73 & 15 \\
\hline 49 & & SW-9E & 0.024 & 0.217 & 0.418 & 1.977 & 30 & 76 & 76 & 15 \\
\hline 50 & \multirow{5}{*}{$\begin{array}{c}\text { Salonikios et al. } \\
\text { (1999) }\end{array}$} & LSW1 & 0.020 & 0.132 & 0.230 & 1.073 & 27 & 60 & 60 & 12 \\
\hline 51 & & LSW2 & 0.020 & 0.119 & 0.190 & 0.824 & 22 & 43 & 43 & 9 \\
\hline 52 & & LSW3 & 0.020 & 0.146 & 0.190 & 1.555 & 27 & 60 & 60 & 12 \\
\hline 53 & & MSW1 & 0.040 & 0.364 & 0.620 & 1.699 & 16 & 44 & 44 & 9 \\
\hline 54 & & MSW2 & 0.150 & 0.489 & 0.820 & 2.392 & 12 & 27 & 27 & 5 \\
\hline
\end{tabular}


Table B-5 Test Results of the RC Walls with Rectangular Cross-Section in the First Quadrant (continued)

\begin{tabular}{|c|c|c|c|c|c|c|c|c|c|c|}
\hline $\begin{array}{l}\text { Test } \\
\text { No. }\end{array}$ & Researcher & Spec ID & $\begin{array}{c}\Delta_{A} \\
\text { (in) } \\
\end{array}$ & $\begin{array}{c}\Delta_{B} \\
\text { (in) } \\
\end{array}$ & $\begin{array}{c}\Delta_{C} \\
\text { (in) } \\
\end{array}$ & $\begin{array}{l}\Delta_{D} \\
\text { (in) } \\
\end{array}$ & $\begin{array}{c}F_{A} \\
\text { (kips) } \\
\end{array}$ & $\begin{array}{c}F_{B} \\
\text { (kips) } \\
\end{array}$ & $\begin{array}{c}F_{C} \\
\text { (kips) } \\
\end{array}$ & $\begin{array}{c}F_{D} \\
\text { (kips) }\end{array}$ \\
\hline 55 & $\begin{array}{c}\text { Salonikios et al. } \\
\text { (1999) }\end{array}$ & MSW3 & 0.050 & 0.259 & 0.480 & 2.279 & 17 & 39 & 39 & 8 \\
\hline 56 & $\begin{array}{l}\text { Xia and Xiao } \\
(2000)\end{array}$ & W1-A & 0.087 & 0.335 & 0.455 & 1.580 & 202 & 280 & 280 & 56 \\
\hline 57 & \multirow{16}{*}{$\begin{array}{l}\text { Hidalgo et al. } \\
\text { (2002) }\end{array}$} & 1 & 0.050 & 0.115 & 1.000 & - & 27 & 37 & 37 & - \\
\hline 58 & & 2 & 0.030 & 0.287 & 1.000 & 1.654 & 21 & 59 & 59 & 12 \\
\hline 59 & & 4 & 0.030 & 0.325 & 0.710 & 1.896 & 22 & 71 & 71 & 14 \\
\hline 60 & & 6 & 0.020 & 0.218 & 0.290 & 1.050 & 31 & 80 & 80 & 16 \\
\hline 61 & & 7 & 0.020 & 0.214 & 0.800 & - & 29 & 79 & 79 & - \\
\hline 62 & & 8 & 0.030 & 0.184 & 0.800 & - & 31 & 80 & 80 & - \\
\hline 63 & & 9 & 0.040 & 0.201 & 0.860 & - & 27 & 58 & 58 & - \\
\hline 64 & & 10 & 0.030 & 0.230 & 0.340 & 1.511 & 20 & 45 & 45 & 9 \\
\hline 65 & & 11 & 0.000 & 0.139 & 0.190 & 1.604 & 19 & 55 & 55 & 11 \\
\hline 66 & & 12 & 0.040 & 0.198 & 0.350 & 0.945 & 32 & 71 & 71 & 14 \\
\hline 67 & & 13 & 0.010 & 0.121 & 0.200 & 2.471 & 28 & 67 & 67 & 13 \\
\hline 68 & & 14 & 0.010 & 0.083 & 0.110 & 0.649 & 25 & 59 & 59 & 12 \\
\hline 69 & & 15 & 0.010 & 0.131 & 0.190 & 0.408 & 31 & 85 & 85 & 17 \\
\hline 70 & & 16 & 0.010 & 0.155 & 0.180 & 0.454 & 33 & 84 & 84 & 17 \\
\hline 71 & & 21 & 0.040 & 0.185 & 0.210 & 0.465 & 31 & 60 & 60 & 12 \\
\hline 72 & & 22 & 0.040 & 0.143 & 0.200 & 0.321 & 28 & 55 & 55 & 11 \\
\hline 73 & \multirow{5}{*}{$\begin{array}{l}\text { Hidalgo et al. } \\
\text { (2002) }\end{array}$} & 23 & 0.050 & 0.273 & 0.320 & 2.414 & 30 & 82 & 82 & 16 \\
\hline 74 & & 24 & 0.040 & 0.144 & 0.170 & 0.390 & 31 & 53 & 53 & 11 \\
\hline 75 & & 25 & 0.030 & 0.305 & 0.370 & 0.826 & 30 & 89 & 89 & 18 \\
\hline 76 & & 26 & 0.010 & 0.191 & 0.280 & 0.354 & 15 & 61 & 61 & 12 \\
\hline 77 & & 28 & 0.010 & 0.204 & 0.250 & 0.563 & 25 & 66 & 66 & 13 \\
\hline 78 & \multirow{6}{*}{ Massone (2006) } & WP111-9 & 0.050 & 0.152 & 0.280 & 0.553 & 109 & 178 & 178 & 36 \\
\hline 79 & & WP111-10 & 0.040 & 0.210 & 0.440 & 0.747 & 109 & 172 & 172 & 34 \\
\hline 80 & & WP1105-8 & 0.040 & 0.161 & 0.310 & 0.658 & 94 & 145 & 145 & 29 \\
\hline 81 & & WP1105-7 & 0.050 & 0.230 & 0.410 & 0.640 & 104 & 159 & 159 & 32 \\
\hline 82 & & WP110-5 & 0.040 & 0.175 & 0.320 & 0.969 & 62 & 90 & 90 & 18 \\
\hline 83 & & WP110-6 & 0.260 & 0.410 & 0.590 & 1.245 & 67 & 78 & 78 & 16 \\
\hline 84 & \multirow{2}{*}{$\begin{array}{l}\text { Greifenhagen } \\
(2005)\end{array}$} & M1 & 0.020 & 0.070 & 0.350 & 2.235 & 35 & 46 & 46 & 9 \\
\hline 85 & & M2 & 0.030 & 0.108 & 0.470 & 1.763 & 26 & 46 & 46 & 9 \\
\hline
\end{tabular}


Table B-5 Test Results of the RC Walls with Rectangular Cross-Section in the First Quadrant (continued)

\begin{tabular}{|c|c|c|c|c|c|c|c|c|c|c|}
\hline $\begin{array}{l}\text { Test } \\
\text { No. }\end{array}$ & Researcher & Spec ID & $\begin{array}{c}\Delta_{A} \\
\text { (in) }\end{array}$ & $\begin{array}{c}\triangle_{B} \\
\text { (in) }\end{array}$ & $\begin{array}{c}\Delta_{C} \\
\text { (in) } \\
\end{array}$ & $\begin{array}{l}\Delta_{D} \\
\text { (in) }\end{array}$ & $\begin{array}{c}F_{A} \\
\text { (kips) } \\
\end{array}$ & $\begin{array}{c}F_{B} \\
\text { (kips) } \\
\end{array}$ & $\begin{array}{c}F_{C} \\
\text { (kips) } \\
\end{array}$ & $\begin{array}{c}F_{D} \\
\text { (kips) } \\
\end{array}$ \\
\hline 86 & & M3 & 0.010 & 0.065 & 0.260 & 0.553 & 22 & 38 & 38 & 8 \\
\hline 87 & $\begin{array}{l}\text { Greifenhagen } \\
\quad(2005)\end{array}$ & M4 & 0.010 & 0.038 & 0.350 & 0.664 & 17 & 30 & 30 & 6 \\
\hline 88 & \multirow{8}{*}{$\begin{array}{l}\text { Kuang and Ho } \\
\text { (2008) }\end{array}$} & U 1.0 & 0.020 & 0.311 & 0.580 & - & 27 & 77 & 77 & - \\
\hline 89 & & U 1.5 & 0.110 & 0.375 & 0.580 & - & 25 & 61 & 61 & - \\
\hline 90 & & C 1.0 & 0.070 & 0.317 & 0.580 & - & 46 & 94 & 94 & - \\
\hline 91 & & C 1.5 & 0.070 & 0.329 & 0.440 & 1.446 & 30 & 66 & 66 & 13 \\
\hline 92 & & U $1.0 \mathrm{BC}$ & 0.060 & 0.380 & 0.530 & 0.981 & 40 & 90 & 90 & 18 \\
\hline 93 & & $\mathrm{U} 1.5 \mathrm{BC}$ & 0.030 & 0.317 & 0.550 & 1.817 & 18 & 57 & 57 & 11 \\
\hline 94 & & U $1.0 \mathrm{BC} 2$ & 0.050 & 0.225 & 0.280 & 1.770 & 35 & 81 & 81 & 16 \\
\hline 95 & & U $1.0 \mathrm{CT}$ & 0.010 & 0.201 & 0.410 & 2.206 & 32 & 83 & 83 & 17 \\
\hline 96 & \multirow{5}{*}{$\begin{array}{l}\text { Athanasopoulou } \\
(2010)\end{array}$} & S1 & 0.150 & 0.472 & 0.960 & - & 55 & 81 & 81 & - \\
\hline 97 & & S4 & 0.110 & 0.304 & 0.600 & 1.358 & 48 & 102 & 102 & 20 \\
\hline 98 & & S6 & 0.110 & 0.359 & 0.860 & - & 44 & 87 & 87 & - \\
\hline 99 & & S9 & 0.150 & 0.524 & 1.100 & - & 34 & 83 & 83 & - \\
\hline 100 & & S10 & 0.330 & 0.579 & 0.620 & 6.414 & 95 & 108 & 108 & 22 \\
\hline 101 & \multirow{2}{*}{$\begin{array}{c}\text { Tran and } \\
\text { Wallace (2012) }\end{array}$} & $\begin{array}{l}\text { RW-A15- } \\
\text { P10-S51 }\end{array}$ & 0.060 & 0.624 & 2.060 & 3.483 & 58 & 136 & 136 & 27 \\
\hline 102 & & $\begin{array}{l}\text { RW-A15- } \\
\text { P10-S78 }\end{array}$ & 0.160 & 0.625 & 1.460 & 16.209 & 92 & 195 & 195 & 39 \\
\hline 103 & $\begin{array}{c}\text { Tran and } \\
\text { Wallace }(2010)\end{array}$ & $\begin{array}{l}\text { RW-A15- } \\
\text { P2.5-S64 }\end{array}$ & 0.180 & 0.494 & 1.470 & 3.679 & 84 & 150 & 150 & 30 \\
\hline 104 & $\begin{array}{l}\text { El-Sokkary and } \\
\text { Galal (2013) }\end{array}$ & CW & 0.040 & 0.159 & 0.550 & 0.691 & 8 & 14 & 14 & 3 \\
\hline 105 & \multirow{7}{*}{ Park et al. (2015) } & S1 & 0.060 & 0.568 & 0.700 & 0.792 & 132 & 484 & 484 & 97 \\
\hline 106 & & S2 & 0.080 & 0.585 & 0.690 & 1.014 & 153 & 526 & 526 & 105 \\
\hline 107 & & S3 & 0.050 & 0.500 & 0.580 & 1.027 & 141 & 477 & 477 & 95 \\
\hline 108 & & S5 & 0.080 & 0.473 & 0.660 & 1.247 & 141 & 323 & 323 & 65 \\
\hline 109 & & S6 & 0.120 & 0.497 & 0.710 & - & 185 & 434 & 434 & - \\
\hline 110 & & S7 & 0.070 & 0.548 & 0.700 & 1.012 & 170 & 463 & 463 & 93 \\
\hline 111 & & S8 & 0.060 & 0.478 & 1.520 & 3.724 & 151 & 257 & 257 & 51 \\
\hline 112 & \multirow{3}{*}{$\begin{array}{l}\text { Hajyalikhani } \\
\text { (2015) }\end{array}$} & SW1 & 0.050 & 0.365 & 0.390 & - & 21 & 110 & 110 & - \\
\hline 113 & & SW2 & 0.120 & 0.376 & 0.640 & 1.391 & 42 & 89 & 89 & 18 \\
\hline 114 & & SW3 & 0.060 & 0.271 & 0.400 & 1.939 & 37 & 91 & 91 & 18 \\
\hline
\end{tabular}


Table B-6 Test Results of the RC Walls with Rectangular Cross-Section in the Third Quadrant

\begin{tabular}{|c|c|c|c|c|c|c|c|c|c|c|}
\hline $\begin{array}{l}\text { Test } \\
\text { No. }\end{array}$ & Researcher & Spec ID & $\begin{array}{c}\Delta_{A} \\
\text { (in) } \\
\end{array}$ & $\begin{array}{c}A_{B} \\
\text { (in) } \\
\end{array}$ & $\begin{array}{c}\Delta_{C} \\
\text { (in) } \\
\end{array}$ & $\begin{array}{l}\Delta_{D} \\
\text { (in) } \\
\end{array}$ & $\begin{array}{c}F_{A} \\
\text { (kips) } \\
\end{array}$ & $\begin{array}{c}F_{B} \\
\text { (kips) } \\
\end{array}$ & $\begin{array}{c}F_{C} \\
\text { (kips) } \\
\end{array}$ & $\begin{array}{c}F_{D} \\
\text { (kips) }\end{array}$ \\
\hline 1 & $\begin{array}{l}\text { Alexander et } \\
\text { al. (1973) }\end{array}$ & 4 & -0.060 & -0.236 & -0.470 & -0.779 & -68 & -85 & -85 & -17 \\
\hline 2 & \multirow{10}{*}{$\begin{array}{c}\text { Hirosawa } \\
\text { (1975) }\end{array}$} & 72 & -0.030 & -0.258 & -0.340 & - & -64 & -165 & -165 & - \\
\hline 3 & & 73 & -0.030 & -0.362 & -0.600 & - & -84 & -183 & -183 & - \\
\hline 4 & & 74 & -0.020 & -0.234 & -0.330 & - & -62 & -171 & -171 & - \\
\hline 5 & & 75 & -0.050 & -0.323 & -0.550 & - & -88 & -176 & -176 & - \\
\hline 6 & & 76 & -0.020 & -0.255 & -0.660 & - & -46 & -175 & -175 & - \\
\hline 7 & & 77 & -0.040 & -0.318 & -0.650 & - & -85 & -187 & -187 & - \\
\hline 8 & & 78 & -0.040 & -0.403 & -1.340 & - & -56 & -140 & -140 & - \\
\hline 9 & & 79 & -0.040 & -0.263 & -0.340 & - & -57 & -127 & -127 & - \\
\hline 10 & & 80 & -0.040 & -0.346 & -1.330 & - & -54 & -133 & -133 & - \\
\hline 11 & & 81 & -0.030 & -0.526 & -1.350 & - & -45 & -150 & -150 & - \\
\hline 12 & Synge (1980) & WALL 1 & -0.100 & -0.177 & -0.420 & -1.014 & -134 & -167 & -167 & -33 \\
\hline 13 & \multirow{2}{*}{$\begin{array}{l}\text { Wiradinata } \\
(1985)\end{array}$} & WALL1 & -0.020 & -0.130 & -0.410 & -2.353 & -56 & -109 & -109 & -22 \\
\hline 14 & & WALL2 & -0.010 & -0.078 & -0.120 & -0.870 & -68 & -155 & -155 & -31 \\
\hline 15 & \multirow{3}{*}{ Maier (1985) } & S4 & -0.030 & -0.262 & -0.490 & -2.643 & -69 & -176 & -176 & -35 \\
\hline 16 & & s9 & -0.040 & -0.219 & -0.460 & -0.661 & -33 & -75 & -75 & -15 \\
\hline 17 & & S10 & -0.040 & -0.395 & -0.540 & -2.394 & -21 & -150 & -150 & -30 \\
\hline 18 & \multirow{2}{*}{ Pilette (1987) } & WALL 4 & -0.130 & -0.273 & -0.620 & -7.644 & -76 & -92 & -92 & -18 \\
\hline 19 & & WALL 5 & -0.120 & -0.366 & -0.780 & -1.968 & -84 & -113 & -113 & -23 \\
\hline 20 & \multirow{2}{*}{$\begin{array}{l}\text { Huang and } \\
\text { Sheu (1988) }\end{array}$} & SWN-1D & -0.070 & -0.148 & -0.150 & -1.096 & -68 & -78 & -78 & -16 \\
\hline 21 & & SWN-5D & -0.030 & -0.165 & -0.280 & - & -35 & -56 & -56 & - \\
\hline 22 & \multirow{2}{*}{$\begin{array}{c}\text { Wasiewicz } \\
(1988)\end{array}$} & WALL 3 & -0.060 & -0.378 & -0.460 & -0.781 & -120 & -180 & -180 & -36 \\
\hline 23 & & WALL 6 & -0.130 & -0.514 & -0.630 & -1.091 & -83 & -120 & -120 & -24 \\
\hline 24 & \multirow{7}{*}{$\begin{array}{l}\text { Lefas et al. } \\
(1990)\end{array}$} & SW11 & -0.040 & -0.245 & -0.300 & - & -16 & -55 & -55 & - \\
\hline 25 & & SW12 & -0.050 & -0.241 & -0.350 & - & -29 & -74 & -74 & - \\
\hline 26 & & SW13 & -0.040 & -0.303 & -0.400 & - & -16 & -59 & -59 & - \\
\hline 27 & & SW14 & -0.040 & -0.262 & -0.360 & - & -26 & -75 & -75 & - \\
\hline 28 & & SW15 & -0.030 & -0.235 & -0.330 & - & -19 & -71 & -71 & - \\
\hline 29 & & SW16 & -0.020 & -0.166 & -0.250 & - & -23 & -80 & -80 & - \\
\hline 30 & & SW17 & -0.060 & -0.299 & -0.410 & - & -16 & -57 & -57 & - \\
\hline 31 & \multirow{3}{*}{$\begin{array}{c}\text { Lopes and } \\
\text { Elnashai } \\
\text { (1991) }\end{array}$} & SW11 & -0.018 & -0.128 & -0.184 & -0.528 & -7 & -21 & -21 & -4 \\
\hline 32 & & SW12 & -0.034 & -0.115 & -0.133 & -0.703 & -10 & -20 & -20 & -4 \\
\hline 33 & & SW13 & -0.055 & -0.244 & -0.320 & - & -12 & -23 & -23 & - \\
\hline
\end{tabular}


Table B-6 Test Results of the RC Walls with Rectangular Cross-Section in the Third Quadrant (continued)

\begin{tabular}{|c|c|c|c|c|c|c|c|c|c|c|}
\hline $\begin{array}{l}\text { Test } \\
\text { No. }\end{array}$ & Researcher & Spec ID & $\begin{array}{c}\Delta_{A} \\
\text { (in) } \\
\end{array}$ & $\begin{array}{l}\Delta_{B} \\
\text { (in) }\end{array}$ & $\begin{array}{l}\Delta_{C} \\
\text { (in) } \\
\end{array}$ & $\begin{array}{l}\Delta_{D} \\
\text { (in) } \\
\end{array}$ & $\begin{array}{c}F_{A} \\
\text { (kips) } \\
\end{array}$ & $\begin{array}{c}F_{B} \\
\text { (kips) } \\
\end{array}$ & $\begin{array}{c}F_{C} \\
\text { (kips) } \\
\end{array}$ & $\begin{array}{c}F_{D} \\
\text { (kips) }\end{array}$ \\
\hline 34 & \multirow{3}{*}{$\begin{array}{c}\text { Lopes and } \\
\text { Elnashai } \\
\text { (1991) }\end{array}$} & SW14 & -0.062 & -0.113 & -0.230 & -0.460 & -13 & -21 & -21 & -4 \\
\hline 35 & & SW15 & -0.032 & -0.105 & -0.128 & - & -10 & -19 & -19 & - \\
\hline 36 & & SW16 & -0.050 & -0.134 & -0.163 & -0.674 & -12 & -18 & -18 & -4 \\
\hline 37 & \multirow{2}{*}{$\begin{array}{c}\text { Lopes and } \\
\text { Elnashai } \\
\text { (1991) }\end{array}$} & SW17 & -0.014 & -0.106 & -0.154 & -1.431 & -6 & -19 & -19 & -4 \\
\hline 38 & & SW18 & -0.017 & -0.102 & -0.093 & - & -8 & -16 & -16 & - \\
\hline 39 & \multirow{3}{*}{$\begin{array}{l}\text { Saatcioglu } \\
\text { (1991) }\end{array}$} & Wall-1 & -0.020 & -0.154 & -0.430 & -2.548 & -69 & -119 & -119 & -24 \\
\hline 40 & & Wall-4 & -0.070 & -0.225 & -0.650 & -6.605 & -59 & -97 & -97 & -19 \\
\hline 41 & & Wall-6 & -0.050 & -0.378 & -0.650 & -1.136 & -50 & -132 & -132 & -26 \\
\hline 42 & \multirow{3}{*}{ Cheng (1992) } & SW4A & -0.004 & -0.116 & -0.237 & - & -16 & -61 & -61 & - \\
\hline 43 & & SW5 & -0.008 & -0.117 & -0.203 & - & -26 & -65 & -65 & - \\
\hline 44 & & SW6 & -0.007 & -0.096 & -0.240 & - & -21 & -62 & -62 & - \\
\hline 45 & \multirow{2}{*}{$\begin{array}{l}\text { Mohammadi } \\
\text { (1994) }\end{array}$} & Wall 7 & -0.180 & -0.235 & -0.550 & -2.444 & -71 & -81 & -81 & -16 \\
\hline 46 & & Wall 8 & -0.100 & -0.266 & -1.020 & -1.649 & -39 & -50 & -50 & -10 \\
\hline 47 & \multirow{3}{*}{ Cheng (1992) } & SW-OE & -0.028 & -0.178 & -0.334 & -1.192 & -23 & -70 & -70 & -14 \\
\hline 48 & & SW-1E & -0.032 & -0.139 & -0.340 & -0.855 & -35 & -73 & -73 & -15 \\
\hline 49 & & SW-9E & -0.024 & -0.217 & -0.418 & -1.977 & -30 & -76 & -76 & -15 \\
\hline 50 & \multirow{6}{*}{$\begin{array}{l}\text { Salonikios et } \\
\text { al. (1999) }\end{array}$} & LSW1 & -0.030 & -0.188 & -0.230 & -0.793 & -22 & -59 & -59 & -12 \\
\hline 51 & & LSW2 & -0.020 & -0.162 & -0.220 & -0.964 & -17 & -40 & -40 & -8 \\
\hline 52 & & LSW3 & -0.020 & -0.154 & -0.260 & -1.438 & -26 & -54 & -54 & -11 \\
\hline 53 & & MSW1 & -0.030 & -0.259 & -0.600 & -1.696 & -16 & -43 & -43 & -9 \\
\hline 54 & & MSW2 & -0.170 & -0.467 & -0.790 & -2.247 & -16 & -28 & -28 & -6 \\
\hline 55 & & MSW3 & -0.090 & -0.296 & -0.480 & -2.662 & -20 & -38 & -38 & -8 \\
\hline 56 & $\begin{array}{c}\text { Xia and Xiao } \\
(2000)\end{array}$ & W1-A & -0.075 & -0.390 & -0.808 & -1.414 & -198 & -282 & -282 & -56 \\
\hline 57 & \multirow{9}{*}{$\begin{array}{l}\text { Hidalgo et al. } \\
\quad(2002)\end{array}$} & 1 & -0.030 & -0.146 & -0.730 & - & -24 & -48 & -48 & - \\
\hline 58 & & 2 & -0.080 & -0.392 & -0.700 & -2.228 & -29 & -61 & -61 & -12 \\
\hline 59 & & 4 & -0.040 & -0.351 & -0.710 & -1.778 & -24 & -74 & -74 & -15 \\
\hline 60 & & 6 & -0.010 & -0.076 & -0.440 & -3.006 & -25 & -56 & -56 & -11 \\
\hline 61 & & 7 & -0.030 & -0.221 & -0.450 & -2.427 & -30 & -81 & -81 & -16 \\
\hline 62 & & 8 & -0.010 & -0.269 & -0.450 & -4.706 & -21 & -85 & -85 & -17 \\
\hline 63 & & 9 & -0.020 & -0.177 & -0.570 & - & -23 & -58 & -58 & - \\
\hline 64 & & 10 & -0.030 & -0.251 & -0.320 & -1.542 & -18 & -44 & -44 & -9 \\
\hline 65 & & 11 & -0.010 & -0.146 & -0.200 & -0.864 & -27 & -55 & -55 & -11 \\
\hline
\end{tabular}


Table B-6 Test Results of the RC Walls with Rectangular Cross-Section in the Third Quadrant (continued)

\begin{tabular}{|c|c|c|c|c|c|c|c|c|c|c|}
\hline $\begin{array}{l}\text { Test } \\
\text { No. }\end{array}$ & Researcher & Spec ID & $\begin{array}{l}\Delta_{A} \\
\text { (in) }\end{array}$ & $\begin{array}{l}\Delta_{B} \\
\text { (in) }\end{array}$ & $\begin{array}{c}\Delta_{C} \\
\text { (in) }\end{array}$ & $\begin{array}{l}\Delta_{D} \\
\text { (in) }\end{array}$ & $\begin{array}{c}F_{A} \\
\text { (kips) }\end{array}$ & $\begin{array}{c}F_{B} \\
\text { (kips) }\end{array}$ & $\begin{array}{c}F_{C} \\
\text { (kips) }\end{array}$ & $\begin{array}{c}F_{D} \\
\text { (kips) }\end{array}$ \\
\hline 66 & $\begin{array}{l}\text { Hidalgo et al. } \\
\qquad(2002)\end{array}$ & 12 & -0.010 & -0.203 & -0.260 & -0.724 & -19 & -69 & -69 & -14 \\
\hline 67 & \multirow{6}{*}{$\begin{array}{l}\text { Hidalgo et al. } \\
\qquad(2002)\end{array}$} & 13 & -0.040 & -0.124 & -0.200 & -1.285 & -34 & -65 & -65 & -13 \\
\hline 68 & & 14 & 0.000 & -0.067 & -0.110 & -0.453 & -26 & -58 & -58 & -12 \\
\hline 69 & & 15 & -0.010 & -0.143 & -0.190 & -0.472 & -17 & -85 & -85 & -17 \\
\hline 70 & & 16 & -0.020 & -0.138 & -0.160 & -0.586 & -26 & -85 & -85 & -17 \\
\hline 71 & & 21 & -0.030 & -0.178 & -0.190 & - & -26 & -58 & -58 & - \\
\hline 72 & & 22 & -0.020 & -0.121 & -0.170 & -0.720 & -18 & -46 & -46 & -9 \\
\hline 73 & \multirow{5}{*}{$\begin{array}{l}\text { Hidalgo et al. } \\
\qquad(2002)\end{array}$} & 23 & -0.030 & -0.186 & -0.200 & -1.182 & -31 & -69 & -69 & -14 \\
\hline 74 & & 24 & -0.030 & -0.120 & -0.140 & -0.535 & -27 & -51 & -51 & -10 \\
\hline 75 & & 25 & -0.020 & -0.127 & -0.280 & -0.555 & -19 & -64 & -64 & -13 \\
\hline 76 & & 26 & -0.020 & -0.178 & -0.220 & -0.612 & -17 & -57 & -57 & -11 \\
\hline 77 & & 28 & -0.030 & -0.085 & -0.100 & -0.466 & -23 & -52 & -52 & -10 \\
\hline 78 & \multirow{6}{*}{ Massone (2006) } & WP111-9 & -0.040 & -0.134 & -0.200 & -0.345 & -107 & -162 & -162 & -32 \\
\hline 79 & & WP111-10 & -0.060 & -0.336 & -0.420 & - & -140 & -194 & -194 & - \\
\hline 80 & & WP1105-8 & -0.050 & -0.161 & -0.260 & -0.467 & -110 & -144 & -144 & -29 \\
\hline 81 & & WP1105-7 & -0.040 & -0.149 & -0.290 & -0.614 & -105 & -152 & -152 & -30 \\
\hline 82 & & WP110-5 & -0.070 & -0.134 & -0.300 & -1.058 & -74 & -89 & -89 & -18 \\
\hline 83 & & WP110-6 & -0.210 & -0.387 & -0.620 & -1.369 & -54 & -66 & -66 & -13 \\
\hline 84 & \multirow{4}{*}{$\begin{array}{l}\text { Greifenhagen } \\
\quad(2005)\end{array}$} & M1 & -0.010 & -0.056 & -0.260 & -3.250 & -17 & -46 & -46 & -9 \\
\hline 85 & & M2 & -0.020 & -0.063 & -0.470 & - & -19 & -45 & -45 & - \\
\hline 86 & & M3 & -0.020 & -0.067 & -0.260 & -0.713 & -24 & -37 & -37 & -7 \\
\hline 87 & & M4 & -0.010 & -0.052 & -0.350 & -0.565 & -14 & -30 & -30 & -6 \\
\hline 88 & \multirow{8}{*}{$\begin{array}{l}\text { Kuang and Ho } \\
\text { (2008) }\end{array}$} & U 1.0 & -0.020 & -0.204 & -0.410 & -0.712 & -32 & -82 & -82 & -16 \\
\hline 89 & & U 1.5 & -0.050 & -0.423 & -0.560 & - & -22 & -58 & -58 & - \\
\hline 90 & & C 1.0 & -0.030 & -0.246 & -0.580 & - & -45 & -98 & -98 & - \\
\hline 91 & & C 1.5 & -0.040 & -0.348 & -0.670 & -0.893 & -29 & -67 & -67 & -13 \\
\hline 92 & & U $1.0 \mathrm{BC}$ & -0.030 & -0.308 & -0.530 & - & -29 & -94 & -94 & - \\
\hline 93 & & U1.5 BC & -0.020 & -0.296 & -0.450 & -0.860 & -19 & -63 & -63 & -13 \\
\hline 94 & & U $1.0 \mathrm{BC} 2$ & -0.020 & -0.213 & -0.410 & -2.874 & -27 & -82 & -82 & -16 \\
\hline 95 & & U $1.0 \mathrm{CT}$ & -0.020 & -0.159 & -0.360 & -1.278 & -30 & -86 & -86 & -17 \\
\hline 96 & \multirow{2}{*}{$\begin{array}{l}\text { Athanasopoulou } \\
\text { (2010) }\end{array}$} & S1 & -0.170 & -0.813 & -1.170 & - & -34 & -73 & -73 & - \\
\hline 97 & & S4 & -0.020 & -0.244 & -0.410 & -1.665 & -36 & -107 & -107 & -21 \\
\hline
\end{tabular}


Table B-6 Test Results of the RC Walls with Rectangular Cross-Section in the Third Quadrant (continued)

\begin{tabular}{|c|c|c|c|c|c|c|c|c|c|c|}
\hline $\begin{array}{l}\text { Test } \\
\text { No. }\end{array}$ & Researcher & Spec ID & $\begin{array}{l}\Delta_{A} \\
\text { (in) } \\
\end{array}$ & $\begin{array}{l}\Delta_{B} \\
\text { (in) }\end{array}$ & $\begin{array}{c}\Delta_{C} \\
\text { (in) } \\
\end{array}$ & $\begin{array}{l}\Delta_{D} \\
\text { (in) } \\
\end{array}$ & $\begin{array}{c}F_{A} \\
\text { (kips) } \\
\end{array}$ & $\begin{array}{c}F_{B} \\
\text { (kips) } \\
\end{array}$ & $\begin{array}{c}F_{C} \\
\text { (kips) } \\
\end{array}$ & $\begin{array}{c}F_{D} \\
\text { (kips) }\end{array}$ \\
\hline 98 & \multirow{3}{*}{$\begin{array}{l}\text { Athanasopoulou } \\
\text { (2010) }\end{array}$} & S6 & -0.140 & -0.477 & -1.040 & - & -38 & -88 & -88 & - \\
\hline 99 & & S9 & -0.150 & -0.614 & -0.890 & -1.350 & -35 & -82 & -82 & -16 \\
\hline 100 & & S10 & -0.100 & -0.265 & -1.080 & -2.594 & -43 & -96 & -96 & -19 \\
\hline 101 & \multirow{2}{*}{$\begin{array}{l}\text { Tran and Wallace } \\
\text { (2012) }\end{array}$} & $\begin{array}{l}\text { RW-A15- } \\
\text { P10-S51 }\end{array}$ & -0.080 & -0.573 & -2.150 & - & -59 & -131 & -131 & - \\
\hline 102 & & $\begin{array}{l}\text { RW-A15- } \\
\text { P10-S78 }\end{array}$ & -0.120 & -0.585 & -1.040 & -11.64 & -85 & -188 & -188 & -38 \\
\hline 103 & $\begin{array}{c}\text { Tran and Wallace } \\
(2010)\end{array}$ & $\begin{array}{l}\text { RW-A15- } \\
\text { P2.5-S64 }\end{array}$ & -0.090 & -0.527 & -1.450 & -2.805 & -53 & -146 & -146 & -29 \\
\hline 104 & $\begin{array}{l}\text { El-Sokkary and } \\
\text { Galal (2013) }\end{array}$ & $\mathrm{CW}$ & -0.070 & -0.241 & -0.620 & - & -9 & -13 & -13 & - \\
\hline 105 & \multirow{7}{*}{ Park et al. (2015) } & S1 & -0.120 & -0.583 & -0.690 & -1.058 & -195 & -501 & -501 & -100 \\
\hline 106 & & S2 & -0.160 & -0.605 & -0.710 & -1.077 & -224 & -493 & -493 & -99 \\
\hline 107 & & S3 & -0.110 & -0.463 & -0.530 & -0.937 & -220 & -460 & -460 & -92 \\
\hline 108 & & S5 & -0.080 & -0.512 & -0.510 & -1.151 & -166 & -332 & -332 & -66 \\
\hline 109 & & S6 & -0.130 & -0.555 & -0.680 & -1.121 & -152 & -413 & -413 & -83 \\
\hline 110 & & S7 & -0.070 & -0.469 & -0.690 & -1.115 & -132 & -390 & -390 & -78 \\
\hline 111 & & S8 & -0.130 & -0.815 & -1.470 & -3.358 & -114 & -249 & -249 & -50 \\
\hline 112 & \multirow{5}{*}{ Hajyalikhani (2015) } & SW1 & -0.070 & -0.323 & -0.340 & - & -33 & -93 & -93 & - \\
\hline 113 & & SW2 & -0.200 & -0.432 & -0.680 & -1.120 & -51 & -87 & -87 & -17 \\
\hline 114 & & SW3 & -0.100 & -0.274 & -0.390 & -1.776 & -43 & -80 & -80 & -16 \\
\hline 115 & & SW5 & -0.230 & -0.394 & -0.410 & -1.130 & -107 & -134 & -134 & -27 \\
\hline 116 & & SW6 & -0.040 & -0.164 & -0.220 & -0.452 & -64 & -195 & -195 & -39 \\
\hline 117 & \multirow{6}{*}{ Peng et al. (2015) } & RCWS1 & -0.130 & -0.385 & -1.040 & -6.097 & -208 & -342 & -342 & -68 \\
\hline 118 & & RCWS2 & -0.180 & -0.608 & -1.000 & -10.093 & -166 & -243 & -243 & -49 \\
\hline 119 & & RCWS3 & -0.090 & -0.369 & -0.840 & -4.872 & -188 & -345 & -345 & -69 \\
\hline 120 & & RCWS4 & -0.200 & -0.490 & -0.930 & -11.396 & -230 & -330 & -330 & -66 \\
\hline 121 & & RCWS5 & -0.260 & -0.723 & -1.270 & -5.105 & -109 & -157 & -157 & -31 \\
\hline 122 & & RCWS6 & -0.350 & -0.745 & -1.370 & -3.881 & -120 & -165 & -165 & -33 \\
\hline 123 & \multirow{5}{*}{ Luna et al. (2015) } & SW1 & -0.120 & -0.717 & -1.440 & -16.077 & -109 & -246 & -246 & -49 \\
\hline 124 & & SW2 & -0.050 & -0.443 & -0.610 & -13.194 & -175 & -486 & -486 & -97 \\
\hline 125 & & SW3 & -0.020 & -0.320 & -0.490 & -5.440 & -105 & -381 & -381 & -76 \\
\hline 126 & & SW4 & -0.030 & -0.207 & -0.980 & -2.506 & -77 & -217 & -217 & -43 \\
\hline 127 & & SW5 & -0.020 & -0.224 & -0.520 & -0.641 & -108 & -542 & -542 & -108 \\
\hline
\end{tabular}


Table B-6 Test Results of the RC Walls with Rectangular Cross-Section in the Third Quadrant (continued)

\begin{tabular}{|c|c|c|c|c|c|c|c|c|c|c|}
\hline $\begin{array}{l}\text { Test } \\
\text { No. }\end{array}$ & Researcher & Spec ID & $\begin{array}{c}\Delta_{A} \\
\text { (in) }\end{array}$ & $\begin{array}{c}\triangle_{B} \\
\text { (in) }\end{array}$ & $\begin{array}{c}\Delta_{C} \\
\text { (in) } \\
\end{array}$ & $\begin{array}{c}\Delta_{D} \\
\text { (in) }\end{array}$ & $\begin{array}{c}F_{A} \\
\text { (kips) } \\
\end{array}$ & $\begin{array}{c}F_{B} \\
\text { (kips) } \\
\end{array}$ & $\begin{array}{c}F_{C} \\
\text { (kips) } \\
\end{array}$ & $\begin{array}{c}F_{D} \\
\text { (kips) }\end{array}$ \\
\hline 127 & \multirow{8}{*}{$\begin{array}{l}\text { Luna et al. } \\
(2015)\end{array}$} & SW5 & -0.020 & -0.224 & -0.520 & -0.641 & -108 & -542 & -542 & -108 \\
\hline 128 & & SW6 & -0.020 & -0.160 & -0.420 & -4.231 & -118 & -412 & -412 & -82 \\
\hline 129 & & SW7 & -0.030 & -0.124 & -0.160 & -1.356 & -169 & -277 & -277 & -55 \\
\hline 130 & & SW8 & -0.050 & -0.294 & -0.420 & -2.334 & -215 & -543 & -543 & -109 \\
\hline 131 & & SW9 & -0.040 & -0.332 & -0.510 & -1.450 & -228 & -629 & -629 & -126 \\
\hline 132 & & SW10 & -0.040 & -0.258 & -0.380 & -0.907 & -212 & -527 & -527 & -105 \\
\hline 133 & & SW11 & -0.070 & -0.273 & -0.890 & -3.063 & -199 & -400 & -400 & -80 \\
\hline 134 & & SW12 & -0.050 & -0.477 & -0.800 & -3.152 & -182 & -417 & -417 & -83 \\
\hline 135 & Li et al. (2015) & S1 & -0.150 & -1.318 & -1.420 & -7.340 & -33 & -50 & -50 & -10 \\
\hline 136 & \multirow{2}{*}{$\begin{array}{l}\text { Woods et al. } \\
\quad(2016)\end{array}$} & CW1 & 0.070 & 0.342 & 0.440 & 0.573 & 26 & 81 & 81 & 16 \\
\hline 137 & & CW2 & 0.060 & 0.280 & 0.300 & - & 97 & 226 & 226 & - \\
\hline
\end{tabular}

Table B-7 Loading Properties of the RC Walls with Barbell and Flanged Cross-Section

\begin{tabular}{|c|c|c|c|c|c|}
\hline $\begin{array}{l}\text { Test } \\
\text { No. }\end{array}$ & Researcher & Spec ID & $\begin{array}{l}\text { Wall } \\
\text { Type }\end{array}$ & $\begin{array}{l}\text { Loading } \\
\text { Type }\end{array}$ & $P / A_{g} f_{c}^{\prime}$ \\
\hline 1 & \multirow{6}{*}{ Shiga et al. (1973) } & WB1 & Barbell & Cyclic & 0.0 \\
\hline 2 & & WB2 & Barbell & Cyclic & 0.0 \\
\hline 3 & & WB3 & Barbell & Cyclic & 0.0 \\
\hline 4 & & WB6 & Barbell & Cyclic & 0.0 \\
\hline 5 & & WB7 & Barbell & Cyclic & 16.0 \\
\hline 6 & & WB8 & Barbell & Cyclic & 32.0 \\
\hline 7 & \multirow{8}{*}{ Barda et al. (1977) } & B1-1 & Flanged & Monotonic & 0.0 \\
\hline 8 & & B2-1 & Flanged & Monotonic & 0.0 \\
\hline 9 & & B3-2 & Flanged & Cyclic & 0.0 \\
\hline 10 & & B4-3 & Flanged & Cyclic & 0.0 \\
\hline 11 & & B5-4 & Flanged & Cyclic & 0.0 \\
\hline 12 & & B6-4 & Flanged & Cyclic & 0.0 \\
\hline 13 & & $B 7-5$ & Flanged & Cyclic & 0.0 \\
\hline 14 & & B8-5 & Flanged & Cyclic & 0.0 \\
\hline 15 & Synge (1980) & WALL 3 & Flanged & Cyclic & 0.0 \\
\hline 16 & \multirow{3}{*}{$\begin{array}{c}\text { Yagishita } \\
\text { (AlJ, 1986b) }\end{array}$} & CW-0.6-0.6-20a & Barbell & Cyclic & 6.7 \\
\hline 17 & & CW-0.6-0.8-20a & Barbell & Cyclic & 6.6 \\
\hline 18 & & CW-0.6-1.2-0 & Barbell & Cyclic & 0.0 \\
\hline
\end{tabular}


Table B-7 Loading Properties of the RC Walls with Barbell and Flanged Cross-Section (continued)

\begin{tabular}{|c|c|c|c|c|c|}
\hline $\begin{array}{l}\text { Test } \\
\text { No. }\end{array}$ & Researcher & Spec ID & $\begin{array}{l}\text { Wall } \\
\text { Type }\end{array}$ & $\begin{array}{l}\text { Loading } \\
\text { Type }\end{array}$ & $P / A_{g} f_{c}^{\prime}$ \\
\hline 19 & \multirow{8}{*}{$\begin{array}{c}\text { Chiba } \\
\text { (AlJ, 1985a) }\end{array}$} & CW-0.6-1.2-20 & Barbell & Cyclic & 5.8 \\
\hline 20 & & CW-0.6-0.6-20 & Barbell & Cyclic & 6.6 \\
\hline 21 & & CW-0.6-0.8-20 & Barbell & Cyclic & 4.9 \\
\hline 22 & & CW-0.6-1.6-20 & Barbell & Cyclic & 5.8 \\
\hline 23 & & CW-0.6-2.0-20 & Barbell & Cyclic & 5.7 \\
\hline 24 & & CW-0.6-1.2-40 & Barbell & Cyclic & 12.3 \\
\hline 25 & & CW-0.4-1.2-20 & Barbell & Cyclic & 5.9 \\
\hline 26 & & CW-0.8-1.2-20 & Barbell & Cyclic & 5.9 \\
\hline 27 & \multirow{8}{*}{$\begin{array}{l}\text { Fukuzawa } \\
\text { (AIJ, 1985b) }\end{array}$} & CW-0.6-0-20 & Barbell & Cyclic & 5.6 \\
\hline 28 & & CW-0.6-0.3-20 & Barbell & Cyclic & 5.6 \\
\hline 29 & & CW-0.6-2.4-20 & Barbell & Cyclic & 5.8 \\
\hline 30 & & CW-0.6-2.8-20 & Barbell & Cyclic & 6.2 \\
\hline 31 & & CW-0.6-0-0 & Barbell & Cyclic & 0.0 \\
\hline 32 & & CW-0.6-0-40 & Barbell & Cyclic & 12.4 \\
\hline 33 & & CW-0.6-0.6-0 & Barbell & Cyclic & 0.0 \\
\hline 34 & & CW-0.6-0.6-40 & Barbell & Cyclic & 11.5 \\
\hline 35 & Fukuzawa (1985) & CW-0.4-0.6-20 & Barbell & Cyclic & 5.8 \\
\hline 36 & \multirow{3}{*}{ Fukuzawa (1985) } & CW-0.8-0.6-20 & Barbell & Cyclic & 5.8 \\
\hline 37 & & CW-0.4-2.0-20 & Barbell & Cyclic & 5.8 \\
\hline 38 & & CW-0.8-2.0-20 & Barbell & Cyclic & 5.7 \\
\hline 39 & \multirow{6}{*}{$\begin{array}{c}\text { Hatori } \\
\text { (AlJ, 1986a) }\end{array}$} & CW-0.6-2-0 & Barbell & Cyclic & 0.0 \\
\hline 40 & & CW-0.6-2-40 & Barbell & Cyclic & 11.6 \\
\hline 41 & & CW-0.6-2-20B & Barbell & Cyclic & 5.5 \\
\hline 42 & & CW-0.6-0.6-20L & Barbell & Cyclic & 7.6 \\
\hline 43 & & CW-0.6-1.2-20L & Barbell & Cyclic & 7.8 \\
\hline 44 & & CW-0.6-2-20L & Barbell & Cyclic & 7.6 \\
\hline 45 & \multirow{3}{*}{$\begin{array}{c}\text { Taga } \\
\text { (AIJ, 1986c) }\end{array}$} & WALL1 & Barbell & Cyclic & 7.2 \\
\hline 46 & & WALL2 & Barbell & Cyclic & 5.1 \\
\hline 47 & & WALL3 & Barbell & Cyclic & 3.4 \\
\hline
\end{tabular}


Table B-7 Loading Properties of the RC Walls with Barbell and Flanged Cross-Section (continued)

\begin{tabular}{|c|c|c|c|c|c|}
\hline $\begin{array}{l}\text { Test } \\
\text { No. }\end{array}$ & Researcher & Spec ID & $\begin{array}{l}\text { Wall } \\
\text { Type }\end{array}$ & $\begin{array}{l}\text { Loading } \\
\text { Type }\end{array}$ & $P / A_{g} f_{c}^{\prime}$ \\
\hline 48 & \multirow{7}{*}{ Maier (1985) } & S1 & Flanged & Monotonic & 6.6 \\
\hline 49 & & S2 & Flanged & Monotonic & 6.6 \\
\hline 50 & & S3 & Flanged & Monotonic & 6.5 \\
\hline 51 & & S5 & Flanged & Cyclic & 6.3 \\
\hline 52 & & S6 & Flanged & Monotonic & 6.6 \\
\hline 53 & & S7 & Flanged & Cyclic & 27.3 \\
\hline 54 & & S8 & Flanged & Cyclic & 7.3 \\
\hline 55 & \multirow{5}{*}{ Tomii et al. (1988) } & T1-W87.5-N50 & Barbell & Cyclic & 4.3 \\
\hline 56 & & T2-W87.5-N50 & Barbell & Cyclic & 4.6 \\
\hline 57 & & H2-W87.5-N50 & Barbell & Cyclic & 5.6 \\
\hline 58 & & H3-W87.5-N50 & Barbell & Cyclic & 5.8 \\
\hline 59 & & H3-W40-N65 & Barbell & Cyclic & 9.2 \\
\hline 60 & \multirow{8}{*}{ Sato et al. (1989) } & $36 \mathrm{M} 8-30$ & Flanged & Cyclic & 5.1 \\
\hline 61 & & $36 \mathrm{M} 8-50$ & Flanged & Cyclic & 5.3 \\
\hline 62 & & $24 M 8-30$ & Flanged & Cyclic & 5.3 \\
\hline 63 & & $24 \mathrm{M} 8-40$ & Flanged & Cyclic & 5.6 \\
\hline 64 & & $24 \mathrm{M} 8-50$ & Flanged & Cyclic & 5.7 \\
\hline 65 & & $48 \mathrm{M} 8-30$ & Flanged & Cyclic & 7.3 \\
\hline 66 & & $48 \mathrm{M} 8-40$ & Flanged & Cyclic & 7.3 \\
\hline 67 & & 48M8-50 & Flanged & Cyclic & 7.1 \\
\hline 68 & Mansour et al. (2005) & W3 & Flanged & Cyclic & 0.0 \\
\hline 69 & \multirow{2}{*}{ Mansour et al. (2005) } & W4 & Flanged & Cyclic & 0.0 \\
\hline 70 & & W5 & Flanged & Cyclic & 0.0 \\
\hline 71 & \multirow{4}{*}{$\begin{array}{l}\text { Sheu and Huang } \\
\text { (1991) }\end{array}$} & SWB-4B & Barbell & Cyclic & 0.0 \\
\hline 72 & & SWB-6A & Barbell & Cyclic & 0.0 \\
\hline 73 & & SWB-8A & Barbell & Cyclic & 0.0 \\
\hline 74 & & SWB-17A & Barbell & Cyclic & 0.0 \\
\hline 75 & \multirow{3}{*}{ Mo and Kuo (1998) } & $\mathrm{H}-\mathrm{HZ} 4$ & Barbell & Shake & 0.0 \\
\hline 76 & & $\mathrm{H}-\mathrm{HZG}$ & Barbell & Shake & 0.0 \\
\hline 77 & & H-HZ8 & Barbell & Shake & 0.0 \\
\hline
\end{tabular}


Table B-7 Loading Properties of the RC Walls with Barbell and Flanged Cross-Section (continued)

\begin{tabular}{|c|c|c|c|c|c|}
\hline $\begin{array}{l}\text { Test } \\
\text { No. }\end{array}$ & Researcher & Spec ID & $\begin{array}{l}\text { Wall } \\
\text { Type }\end{array}$ & $\begin{array}{l}\text { Loading } \\
\text { Type }\end{array}$ & $P / A_{g} f_{c}^{\prime}$ \\
\hline 78 & Mo and Kuo (1998) & $\mathrm{H}-\mathrm{HZ} 10$ & Barbell & Shake & 0.0 \\
\hline 79 & \multirow{2}{*}{ Sittipunt (2001) } & WALL 1 & Barbell & Cyclic & 0.0 \\
\hline 80 & & WALL 2 & Barbell & Cyclic & 0.0 \\
\hline 81 & \multirow{3}{*}{ Effendy et al. (2006) } & SMAC & Barbell & Cyclic & 0.0 \\
\hline 82 & & SMAS & Barbell & Cyclic & 0.0 \\
\hline 83 & & SMAM & Barbell & Cyclic & 0.0 \\
\hline 84 & \multirow{7}{*}{$\begin{array}{l}\text { Farvashany et al. } \\
\qquad(2008)\end{array}$} & HSCW1 & Flanged & Monotonic & 4.3 \\
\hline 85 & & HSCW2 & Flanged & Monotonic & 8.5 \\
\hline 86 & & HSCW3 & Flanged & Monotonic & 9.2 \\
\hline 87 & & HSCW4 & Flanged & Monotonic & 21.6 \\
\hline 88 & & HSCW5 & Flanged & Monotonic & 9.5 \\
\hline 89 & & HSCW6 & Flanged & Monotonic & 5.1 \\
\hline 90 & & HSCW7 & Flanged & Monotonic & 7.8 \\
\hline 91 & Park et al. (2015) & S4 & Barbell & Cyclic & 7.0 \\
\hline 92 & \multirow{3}{*}{ Almeida et al. (2015) } & TW1 & Flanged & Cyclic & 4.3 \\
\hline 93 & & TW2 & Flanged & Cyclic & 3.2 \\
\hline 94 & & TW3 & Flanged & Cyclic & 3.4 \\
\hline 95 & \multirow{8}{*}{ Li et al. (2015) } & LW1 & Barbell & Cyclic & 0.0 \\
\hline 96 & & LW2 & Barbell & Cyclic & 5.0 \\
\hline 97 & & LW3 & Barbell & Cyclic & 5.0 \\
\hline 98 & & LW4 & Barbell & Cyclic & 0.0 \\
\hline 99 & & LW5 & Barbell & Cyclic & 5.0 \\
\hline 100 & & MW1 & Barbell & Cyclic & 5.0 \\
\hline 101 & & MW2 & Barbell & Cyclic & 5.0 \\
\hline 102 & & MW3 & Barbell & Cyclic & 5.0 \\
\hline 103 & Li et al. (2017) & SF1 & Flanged & Cyclic & 0.0 \\
\hline
\end{tabular}


Table B-8 Geometric Properties of the RC Walls with Barbell and Flanged Cross-Section

\begin{tabular}{|c|c|c|c|c|c|c|c|c|c|c|c|c|c|}
\hline $\begin{array}{l}\text { Test } \\
\text { No. }\end{array}$ & Researcher & Spec ID & $\begin{array}{l}t_{w} \\
\text { (in) } \\
\end{array}$ & $\begin{array}{l}h_{w} \\
\text { (in) } \\
\end{array}$ & $\begin{array}{r}l_{w} \\
\text { (in) } \\
\end{array}$ & $\frac{h_{w}}{l_{w}}$ & $\frac{M}{V l_{w}}$ & $\frac{l_{w}}{t_{w}}$ & $\begin{array}{l}l_{f} \\
\text { (in) } \\
\end{array}$ & $\begin{array}{l}t_{f} \\
\text { (in) } \\
\end{array}$ & $\begin{array}{c}l_{c} \\
\text { (in) }\end{array}$ & $\begin{array}{c}t_{c} \\
\text { (in) } \\
\end{array}$ & $\begin{array}{l}l_{b e} \\
\text { (in) }\end{array}$ \\
\hline 1 & \multirow{6}{*}{$\begin{array}{c}\text { Shiga et al. } \\
\text { (1973) }\end{array}$} & WB1 & 2.0 & 23.6 & 44.1 & 0.5 & 0.6 & 22.4 & - & - & - & - & 4.7 \\
\hline 2 & & WB2 & 2.0 & 23.6 & 44.1 & 0.5 & 0.6 & 22.4 & - & - & - & - & 4.7 \\
\hline 3 & & WB3 & 2.0 & 23.6 & 44.1 & 0.5 & 0.6 & 22.4 & - & - & - & - & 4.7 \\
\hline 4 & & WB6 & 2.0 & 23.6 & 44.1 & 0.5 & 0.6 & 22.4 & - & - & - & - & 4.7 \\
\hline 5 & & WB7 & 2.0 & 23.6 & 44.1 & 0.5 & 0.6 & 22.4 & - & - & - & - & 4.7 \\
\hline 6 & & WB8 & 2.0 & 23.6 & 44.1 & 0.5 & 0.6 & 22.4 & - & - & - & - & 4.7 \\
\hline 7 & \multirow{8}{*}{$\begin{array}{c}\text { Barda et al. } \\
\text { (1977) }\end{array}$} & B1-1 & 4.0 & 34.5 & 75.0 & 0.5 & 0.5 & 18.8 & 24.0 & 4.0 & - & - & - \\
\hline 8 & & B2-1 & 4.0 & 34.5 & 75.0 & 0.5 & 0.5 & 18.8 & 24.0 & 4.0 & - & - & - \\
\hline 9 & & B3-2 & 4.0 & 34.5 & 75.0 & 0.5 & 0.5 & 18.8 & 24.0 & 4.0 & - & - & - \\
\hline 10 & & B4-3 & 4.0 & 34.5 & 75.0 & 0.5 & 0.5 & 18.8 & 24.0 & 4.0 & - & - & - \\
\hline 11 & & B5-4 & 4.0 & 34.5 & 75.0 & 0.5 & 0.5 & 18.8 & 24.0 & 4.0 & - & - & - \\
\hline 12 & & B6-4 & 4.0 & 34.5 & 75.0 & 0.5 & 0.5 & 18.8 & 24.0 & 4.0 & - & - & - \\
\hline 13 & & B7-5 & 4.0 & 15.8 & 75.0 & 0.2 & 0.3 & 18.8 & 24.0 & 4.0 & - & - & - \\
\hline 14 & & B8-5 & 4.0 & 72.0 & 75.0 & 1.0 & 1.0 & 18.8 & 24.0 & 4.0 & - & - & - \\
\hline 15 & Synge (1980) & WALL 3 & 3.9 & 59.1 & 118.1 & 0.5 & 0.6 & 30.1 & 19.7 & 3.9 & - & - & - \\
\hline 16 & \multirow{3}{*}{ Yagishita (1986) } & CW-0.6-0.6-20a & 3.2 & 41.3 & 90.6 & 0.5 & 0.5 & 28.8 & - & - & - & - & 11.8 \\
\hline 17 & & CW-0.6-0.8-20a & 3.2 & 41.3 & 90.6 & 0.5 & 0.5 & 28.8 & - & - & - & - & 11.8 \\
\hline 18 & & CW-0.6-1.2-0 & 3.2 & 41.3 & 90.6 & 0.5 & 0.5 & 28.8 & - & - & - & - & 11.8 \\
\hline 19 & \multirow{8}{*}{ Chiba (1985) } & CW-0.6-1.2-20 & 3.2 & 41.3 & 90.6 & 0.5 & 0.5 & 28.8 & - & - & - & - & 11.8 \\
\hline 20 & & CW-0.6-0.6-20 & 3.2 & 41.3 & 90.6 & 0.5 & 0.5 & 28.8 & - & - & - & - & 11.8 \\
\hline 21 & & CW-0.6-0.8-20 & 3.2 & 41.3 & 90.6 & 0.5 & 0.5 & 28.8 & - & - & - & - & 11.8 \\
\hline 22 & & CW-0.6-1.6-20 & 3.2 & 41.3 & 90.6 & 0.5 & 0.5 & 28.8 & - & - & - & - & 11.8 \\
\hline 23 & & CW-0.6-2.0-20 & 3.2 & 41.3 & 90.6 & 0.5 & 0.5 & 28.8 & - & - & - & - & 11.8 \\
\hline 24 & & CW-0.6-1.2-40 & 3.2 & 41.3 & 90.6 & 0.5 & 0.5 & 28.8 & - & - & - & - & 11.8 \\
\hline 25 & & CW-0.4-1.2-20 & 3.2 & 25.6 & 90.6 & 0.3 & 0.3 & 28.8 & - & - & - & - & 11.8 \\
\hline 26 & & CW-0.8-1.2-20 & 3.2 & 57.1 & 90.6 & 0.6 & 0.6 & 28.8 & - & - & - & - & 11.8 \\
\hline 27 & \multirow{7}{*}{ Fukuzawa (1985) } & CW-0.6-0-20 & 3.2 & 41.3 & 90.6 & 0.5 & 0.5 & 28.8 & - & - & - & - & 11.8 \\
\hline 28 & & CW-0.6-0.3-20 & 3.2 & 41.3 & 90.6 & 0.5 & 0.5 & 28.8 & - & - & - & - & 11.8 \\
\hline 29 & & CW-0.6-2.4-20 & 3.2 & 41.3 & 90.6 & 0.5 & 0.5 & 28.8 & - & - & - & - & 11.8 \\
\hline 30 & & CW-0.6-2.8-20 & 3.2 & 41.3 & 90.6 & 0.5 & 0.5 & 28.8 & - & - & - & - & 11.8 \\
\hline 31 & & CW-0.6-0-0 & 3.2 & 41.3 & 90.6 & 0.5 & 0.5 & 28.8 & - & - & - & - & 11.8 \\
\hline 32 & & CW-0.6-0-40 & 3.2 & 41.3 & 90.6 & 0.5 & 0.5 & 28.8 & - & - & - & - & 11.8 \\
\hline 33 & & CW-0.6-0.6-0 & 3.2 & 41.3 & 90.6 & 0.5 & 0.5 & 28.8 & - & - & - & - & 11.8 \\
\hline
\end{tabular}


Table B-8 Geometric Properties of the RC Walls with Barbell and Flanged Cross-Section (continued)

\begin{tabular}{|c|c|c|c|c|c|c|c|c|c|c|c|c|c|}
\hline $\begin{array}{l}\text { Test } \\
\text { No. }\end{array}$ & Researcher & Spec ID & $\begin{array}{l}t_{w} \\
\text { (in) }\end{array}$ & $\begin{array}{l}h_{w} \\
\text { (in) }\end{array}$ & $\begin{array}{c}l_{w} \\
\text { (in) }\end{array}$ & $\frac{h_{w}}{l_{w}}$ & $\frac{M}{V l_{w}}$ & $\frac{l_{w}}{t_{w}}$ & $\begin{array}{l}l_{f} \\
\text { (in) }\end{array}$ & $\begin{array}{l}t_{f} \\
\text { (in) }\end{array}$ & $\begin{array}{c}l_{c} \\
\text { (in) }\end{array}$ & $\begin{array}{c}t_{c} \\
\text { (in) }\end{array}$ & $\begin{array}{l}l_{b e} \\
\text { (in) }\end{array}$ \\
\hline 34 & \multirow{2}{*}{ Fukuzawa (1985) } & CW-0.6-0.6-40 & 3.2 & 41.3 & 90.6 & 0.5 & 0.5 & 28.8 & - & - & - & - & 11.8 \\
\hline 35 & & CW-0.4-0.6-20 & 3.2 & 25.6 & 90.6 & 0.3 & 0.4 & 28.8 & - & - & - & - & 11.8 \\
\hline 36 & \multirow{3}{*}{ Fukuzawa (1985) } & CW-0.8-0.6-20 & 3.2 & 57.1 & 90.6 & 0.6 & 0.7 & 28.8 & - & - & - & - & 11.8 \\
\hline 37 & & CW-0.4-2.0-20 & 3.2 & 25.6 & 90.6 & 0.3 & 0.4 & 28.8 & - & - & - & - & 11.8 \\
\hline 38 & & CW-0.8-2.0-20 & 3.2 & 57.1 & 90.6 & 0.6 & 0.7 & 28.8 & - & - & - & - & 11.8 \\
\hline 39 & \multirow{6}{*}{ Hatori (1986) } & CW-0.6-2-0 & 3.2 & 41.3 & 90.6 & 0.5 & 0.5 & 28.8 & - & - & - & - & 11.8 \\
\hline 40 & & CW-0.6-2-40 & 3.2 & 41.3 & 90.6 & 0.5 & 0.5 & 28.8 & - & - & - & - & 11.8 \\
\hline 41 & & CW-0.6-2-20B & 3.2 & 41.3 & 90.6 & 0.5 & 0.5 & 28.8 & - & - & - & - & 11.8 \\
\hline 42 & & CW-0.6-0.6-20L & 3.2 & 41.3 & 90.6 & 0.5 & 0.5 & 28.8 & - & - & - & - & 11.8 \\
\hline 43 & & CW-0.6-1.2-20L & 3.2 & 41.3 & 90.6 & 0.5 & 0.5 & 28.8 & - & - & - & - & 11.8 \\
\hline 44 & & CW-0.6-2-20L & 3.2 & 41.3 & 90.6 & 0.5 & 0.5 & 28.8 & - & - & - & - & 11.8 \\
\hline 45 & \multirow{3}{*}{ Taga (1986) } & WALL1 & 3.2 & 41.3 & 85.8 & 0.5 & 0.6 & 27.2 & - & - & - & - & 7.1 \\
\hline 46 & & WALL2 & 3.2 & 41.3 & 85.8 & 0.5 & 0.6 & 27.2 & - & - & - & - & 7.1 \\
\hline 47 & & WALL3 & 3.2 & 41.3 & 85.8 & 0.5 & 0.6 & 27.2 & - & - & - & - & 7.1 \\
\hline 48 & \multirow{7}{*}{ Maier (1985) } & S1 & 3.9 & 47.2 & 46.5 & 1.0 & 1.1 & 11.8 & 15.8 & 3.9 & - & - & - \\
\hline 49 & & S2 & 3.9 & 47.2 & 46.5 & 1.0 & 1.1 & 11.8 & 15.8 & 3.9 & - & - & - \\
\hline 50 & & S3 & 3.9 & 47.2 & 46.5 & 1.0 & 1.1 & 11.8 & 15.8 & 3.9 & - & - & - \\
\hline 51 & & S5 & 3.9 & 47.2 & 46.5 & 1.0 & 1.1 & 11.8 & 15.8 & 3.9 & - & - & - \\
\hline 52 & & S6 & 3.9 & 47.2 & 46.5 & 1.0 & 1.1 & 11.8 & 15.8 & 3.9 & - & - & - \\
\hline 53 & & S7 & 3.9 & 47.2 & 46.5 & 1.0 & 1.1 & 11.8 & 15.8 & 3.9 & - & - & - \\
\hline 54 & & S8 & 3.9 & 47.2 & 46.5 & 1.0 & 1.1 & 11.8 & 15.8 & 3.9 & - & - & - \\
\hline 55 & \multirow{5}{*}{ Tomi et al. (1988) } & T1-W87.5-N50 & 3.4 & 32.1 & 75.8 & 0.4 & 0.4 & 22.0 & - & - & 6.9 & 6.9 & - \\
\hline 56 & & T2-W87.5-N50 & 3.4 & 32.1 & 75.8 & 0.4 & 0.4 & 22.0 & - & - & 6.9 & 6.9 & - \\
\hline 57 & & H2-W87.5-N50 & 3.4 & 32.1 & 75.8 & 0.4 & 0.4 & 22.0 & - & - & 6.9 & 6.9 & - \\
\hline 58 & & H3-W87.5-N50 & 3.4 & 32.1 & 75.8 & 0.4 & 0.4 & 22.0 & - & - & 6.9 & 6.9 & - \\
\hline 59 & & H3-W40-N65 & 1.6 & 32.1 & 75.8 & 0.4 & 0.4 & 48.3 & - & - & 6.9 & 6.9 & - \\
\hline 60 & \multirow{5}{*}{ Sato et al. (1989) } & $36 \mathrm{M} 8-30$ & 5.9 & 55.1 & 84.6 & 0.7 & 0.7 & 14.3 & 39.4 & 5.9 & - & - & - \\
\hline 61 & & $36 \mathrm{M} 8-50$ & 5.9 & 55.1 & 84.6 & 0.7 & 0.7 & 14.3 & 39.4 & 5.9 & - & - & - \\
\hline 62 & & $24 \mathrm{M} 8-30$ & 5.9 & 55.1 & 84.6 & 0.7 & 0.7 & 14.3 & 39.4 & 5.9 & - & - & - \\
\hline 63 & & $24 M 8-40$ & 5.9 & 55.1 & 84.6 & 0.7 & 0.7 & 14.3 & 39.4 & 5.9 & - & - & - \\
\hline 64 & & $24 M 8-50$ & 5.9 & 55.1 & 84.6 & 0.7 & 0.7 & 14.3 & 39.4 & 5.9 & - & - & - \\
\hline
\end{tabular}


Table B-8 Geometric Properties of the RC Walls with Barbell and Flanged Cross-Section (continued)

\begin{tabular}{|c|c|c|c|c|c|c|c|c|c|c|c|c|c|}
\hline $\begin{array}{l}\text { Test } \\
\text { No. }\end{array}$ & Researcher & Spec ID & $\begin{array}{l}t_{w} \\
\text { (in) }\end{array}$ & $\begin{array}{l}h_{w} \\
\text { (in) } \\
\end{array}$ & $\begin{array}{c}l_{w} \\
\text { (in) }\end{array}$ & $\frac{h_{w}}{l_{w}}$ & $\frac{M}{V l_{w}}$ & $\frac{l_{w}}{t_{w}}$ & $\begin{array}{l}l_{f} \\
\text { (in) }\end{array}$ & $\begin{array}{l}t_{f} \\
\text { (in) }\end{array}$ & $\begin{array}{c}l_{c} \\
\text { (in) }\end{array}$ & $\begin{array}{c}t_{c} \\
\text { (in) }\end{array}$ & $\begin{array}{l}l_{b e} \\
\text { (in) }\end{array}$ \\
\hline 65 & \multirow{3}{*}{ Sato et al. (1989) } & 48M8-30 & 5.9 & 55.1 & 84.6 & 0.7 & 0.7 & 14.3 & 39.4 & 5.9 & - & - & - \\
\hline 66 & & $48 \mathrm{M} 8-40$ & 5.9 & 55.1 & 84.6 & 0.7 & 0.7 & 14.3 & 39.4 & 5.9 & - & - & - \\
\hline 67 & & $48 M 8-50$ & 5.9 & 55.1 & 84.6 & 0.7 & 0.7 & 14.3 & 39.4 & 5.9 & - & - & - \\
\hline 68 & \multirow{3}{*}{$\begin{array}{l}\text { Mansour et al. } \\
\qquad(2005)\end{array}$} & W3 & 2.4 & 23.6 & 39.3 & 0.6 & 0.7 & 16.7 & - & - & 3.9 & 7.9 & - \\
\hline 69 & & W4 & 2.4 & 23.6 & 39.3 & 0.6 & 0.7 & 16.7 & - & - & 3.9 & 7.9 & - \\
\hline 70 & & W5 & 2.4 & 23.6 & 39.3 & 0.6 & 0.7 & 16.7 & - & - & 3.9 & 7.9 & - \\
\hline 71 & \multirow{4}{*}{$\begin{array}{l}\text { Sheu and Huang } \\
\text { (1991) }\end{array}$} & SWB-4B & 2.8 & 29.5 & 39.4 & 0.7 & 0.9 & 14.3 & - & - & - & - & 5.9 \\
\hline 72 & & SWB-6A & 2.8 & 19.7 & 39.4 & 0.5 & 0.7 & 14.3 & - & - & - & - & 5.9 \\
\hline 73 & & SWB-8A & 2.8 & 19.7 & 39.4 & 0.5 & 0.7 & 14.3 & - & - & - & - & 5.9 \\
\hline 74 & & SWB-17A & 2.8 & 19.7 & 39.4 & 0.5 & 0.7 & 14.3 & - & - & - & - & 5.9 \\
\hline 75 & \multirow{4}{*}{$\begin{array}{l}\text { Mo and Kuo } \\
(1998)\end{array}$} & $\mathrm{H}-\mathrm{HZ} 4$ & 1.2 & 15.7 & 13.8 & 1.1 & 1.2 & 11.7 & - & - & 2.0 & 5.1 & - \\
\hline 76 & & $\mathrm{H}-\mathrm{HZC}$ & 1.2 & 15.7 & 13.8 & 1.1 & 1.2 & 11.7 & - & - & 2.0 & 5.1 & - \\
\hline 77 & & $\mathrm{H}-\mathrm{HZZ}$ & 1.2 & 15.7 & 13.8 & 1.1 & 1.2 & 11.7 & - & - & 2.0 & 5.1 & - \\
\hline 78 & & $\mathrm{H}-\mathrm{HZ} 10$ & 1.2 & 15.7 & 13.8 & 1.1 & 1.2 & 11.7 & - & - & 2.0 & 5.1 & - \\
\hline 79 & \multirow{2}{*}{ Sittipunt (2001) } & WALL 1 & 3.9 & 78.7 & 59.1 & 1.3 & 1.4 & 15.0 & - & - & 9.8 & 9.8 & - \\
\hline 80 & & WALL 2 & 3.9 & 78.7 & 59.1 & 1.3 & 1.4 & 15.0 & - & - & 9.8 & 9.8 & - \\
\hline 81 & \multirow{3}{*}{$\begin{array}{l}\text { Effendy et al. } \\
(2006)\end{array}$} & SMAC & 4.7 & 31.0 & 78.7 & 0.4 & 0.5 & 16.7 & - & - & 7.1 & 9.5 & - \\
\hline 82 & & SMAS & 4.7 & 31.0 & 78.7 & 0.4 & 0.5 & 16.7 & - & - & 7.1 & 9.5 & - \\
\hline 83 & & SMAM & 4.7 & 31.0 & 78.7 & 0.4 & 0.5 & 16.7 & - & - & 7.1 & 9.5 & - \\
\hline 84 & \multirow{7}{*}{$\begin{array}{l}\text { Farvashany et al. } \\
\qquad(2008)\end{array}$} & HSCW1 & 3.0 & 43.3 & 34.6 & 1.3 & 1.4 & 11.7 & 14.8 & 3.5 & - & - & - \\
\hline 85 & & HSCW2 & 3.0 & 43.3 & 34.6 & 1.3 & 1.4 & 11.7 & 14.8 & 3.5 & - & - & - \\
\hline 86 & & HSCW3 & 3.0 & 43.3 & 34.6 & 1.3 & 1.4 & 11.7 & 14.8 & 3.5 & - & - & - \\
\hline 87 & & HSCW4 & 3.0 & 43.3 & 34.6 & 1.3 & 1.4 & 11.7 & 14.8 & 3.5 & - & - & - \\
\hline 88 & & HSCW5 & 3.0 & 43.3 & 34.6 & 1.3 & 1.4 & 11.7 & 14.8 & 3.5 & - & - & - \\
\hline 89 & & HSCW6 & 3.0 & 43.3 & 34.6 & 1.3 & 1.4 & 11.7 & 14.8 & 3.5 & - & - & - \\
\hline 90 & & HSCW7 & 3.0 & 43.3 & 34.6 & 1.3 & 1.4 & 11.7 & 14.8 & 3.5 & - & - & - \\
\hline 91 & Park et al. (2015) & S4 & 7.8 & 59.0 & 59.0 & 1.0 & 1.2 & 7.6 & - & - & 7.8 & 11.8 & - \\
\hline 92 & \multirow{3}{*}{$\begin{array}{l}\text { Almeida et al. } \\
\qquad(2015)\end{array}$} & TW1 & 3.2 & 78.7 & 106.3 & 0.7 & 0.7 & 33.7 & 17.3 & 3.1 & - & - & - \\
\hline 93 & & TW2 & 4.7 & 78.7 & 106.3 & 0.7 & 0.7 & 22.5 & 17.3 & 3.1 & - & - & - \\
\hline 94 & & TW3 & 4.7 & 78.7 & 106.3 & 0.7 & 0.7 & 22.5 & 17.3 & 3.1 & - & - & - \\
\hline
\end{tabular}


Table B-8 Geometric Properties of the RC Walls with Barbell and Flanged Cross-Section (continued)

\begin{tabular}{|c|c|c|c|c|c|c|c|c|c|c|c|c|c|}
\hline $\begin{array}{l}\text { Test } \\
\text { No. }\end{array}$ & Researcher & Spec ID & $\begin{array}{l}t_{w} \\
\text { (in) } \\
\end{array}$ & $\begin{array}{l}h_{w} \\
\text { (in) } \\
\end{array}$ & $\begin{array}{c}l_{w} \\
\text { (in) }\end{array}$ & $\frac{h_{w}}{l_{w}}$ & $\frac{M}{V l_{w}}$ & $\frac{l_{w}}{t_{w}}$ & $\begin{array}{l}l_{f} \\
\text { (in) } \\
\end{array}$ & $\begin{array}{l}t_{f} \\
\text { (in) } \\
\end{array}$ & $\begin{array}{c}l_{c} \\
\text { (in) }\end{array}$ & $\begin{array}{c}t_{c} \\
\text { (in) } \\
\end{array}$ & $\begin{array}{l}l_{b e} \\
\text { (in) }\end{array}$ \\
\hline 95 & \multirow{8}{*}{ Li et al. (2015) } & LW1 & 4.7 & 78.7 & 78.7 & 1.0 & 1.1 & 16.7 & - & - & 5.9 & 11.8 & - \\
\hline 96 & & LW2 & 4.7 & 78.7 & 78.7 & 1.0 & 1.1 & 16.7 & - & - & 5.9 & 11.8 & - \\
\hline 97 & & LW3 & 4.7 & 78.7 & 78.7 & 1.0 & 1.1 & 16.7 & - & - & 5.9 & 11.8 & - \\
\hline 98 & & LW4 & 4.7 & 78.7 & 78.7 & 1.0 & 1.1 & 16.7 & - & - & 5.9 & 11.8 & - \\
\hline 99 & & LW5 & 4.7 & 78.7 & 78.7 & 1.0 & 1.1 & 16.7 & - & - & 5.9 & 11.8 & - \\
\hline 100 & & MW1 & 4.7 & 118.1 & 78.7 & 1.5 & 1.6 & 16.7 & - & - & 5.9 & 11.8 & - \\
\hline 101 & & MW2 & 4.7 & 118.1 & 78.7 & 1.5 & 1.6 & 16.7 & - & - & 5.9 & 11.8 & - \\
\hline 102 & & MW3 & 4.7 & 118.1 & 78.7 & 1.5 & 1.6 & 16.7 & - & - & 5.9 & 11.8 & - \\
\hline 103 & Li et al. (2017) & SF1 & 4.7 & 90.6 & 69.3 & 1.3 & 1.3 & 14.7 & 4.7 & 15.8 & - & - & - \\
\hline
\end{tabular}

Table B-9 Reinforcement Properties of the RC Walls with Barbell and Flanged Cross-Section

\begin{tabular}{|c|c|c|c|c|c|c|c|c|c|c|c|c|}
\hline $\begin{array}{l}\text { Test } \\
\text { No. }\end{array}$ & Researcher & Spec ID & $\begin{array}{l}\rho_{v}^{w} \\
(\%) \\
\end{array}$ & $\begin{array}{l}\rho_{h}^{w} \\
(\%) \\
\end{array}$ & $\begin{array}{l}S_{h}^{w} \\
\text { (in) } \\
\end{array}$ & $\begin{array}{l}S_{v}^{w} \\
\text { (in) }\end{array}$ & $\begin{array}{l}\rho_{v}^{f} \\
(\%) \\
\end{array}$ & $\begin{array}{l}\rho_{h}^{f} \\
(\%) \\
\end{array}$ & $\begin{array}{l}S_{h}^{f} \\
\text { (in) } \\
\end{array}$ & $\begin{array}{l}S_{v}^{w} \\
\text { (in) } \\
\end{array}$ & $\begin{array}{l}\rho_{v}^{b e} \\
(\%)\end{array}$ & $\begin{array}{l}S_{h}^{b e} \\
\text { (in) }\end{array}$ \\
\hline 1 & \multirow{6}{*}{$\begin{array}{l}\text { Shiga et al. } \\
\text { (1973) }\end{array}$} & WB1 & 0.25 & 0.25 & 2.40 & 2.40 & - & - & - & - & 4.22 & - \\
\hline 2 & & WB2 & 0.25 & 0.25 & 2.40 & 2.40 & - & - & - & - & 4.22 & - \\
\hline 3 & & WB3 & 0.25 & 0.25 & 2.40 & 2.40 & - & - & - & - & 4.22 & - \\
\hline 4 & & WB6 & 0.50 & 0.50 & 1.20 & 1.20 & - & - & - & - & 4.22 & - \\
\hline 5 & & WB7 & 0.50 & 0.50 & 1.20 & 1.20 & - & - & - & - & 4.22 & - \\
\hline 6 & & WB8 & 0.50 & 0.50 & 1.20 & 1.20 & - & - & - & - & 4.22 & - \\
\hline 7 & \multirow{8}{*}{$\begin{array}{l}\text { Barda et al. } \\
\text { (1977) }\end{array}$} & B1-1 & 0.50 & 0.48 & 4.30 & 11.20 & 1.80 & 1.10 & 2.00 & 3.20 & - & - \\
\hline 8 & & B2-1 & 0.50 & 0.48 & 4.30 & 11.20 & 6.40 & 1.10 & 2.00 & 2.50 & - & - \\
\hline 9 & & B3-2 & 0.50 & 0.48 & 4.30 & 11.20 & 4.10 & 1.10 & 2.00 & 2.50 & - & - \\
\hline 10 & & B4-3 & 0.50 & 0.00 & - & 11.20 & 4.10 & 1.10 & 2.00 & 2.50 & - & - \\
\hline 11 & & B5-4 & 0.00 & 0.48 & 4.30 & - & 4.10 & 1.10 & 2.00 & 2.50 & - & - \\
\hline 12 & & B6-4 & 0.25 & 0.48 & 4.30 & 8.40 & 4.10 & 1.10 & 2.00 & 2.50 & - & - \\
\hline 13 & & B7-5 & 0.50 & 0.49 & 5.30 & 11.20 & 4.10 & 1.10 & 2.00 & 2.50 & - & - \\
\hline 14 & & B8-5 & 0.50 & 0.50 & 4.50 & 11.20 & 4.10 & 1.10 & 2.00 & 2.50 & - & - \\
\hline 15 & Synge (1980) & WALL 3 & 0.39 & 1.68 & 4.72 & 7.87 & 1.62 & 1.13 & 1.96 & 5.50 & - & - \\
\hline 16 & \multirow{3}{*}{$\begin{array}{c}\text { Yagishita } \\
\text { (1985) }\end{array}$} & CW-0.6-0.6-20a & 0.60 & 0.60 & 5.20 & 5.20 & - & - & - & - & 1.04 & - \\
\hline 17 & & CW-0.6-0.8-20a & 0.80 & 0.80 & 3.90 & 3.90 & - & - & - & - & 1.04 & - \\
\hline 18 & & CW-0.6-1.2-0 & 1.20 & 1.20 & 2.60 & 2.60 & - & - & - & - & 1.04 & - \\
\hline
\end{tabular}


Table B-9 Reinforcement Properties of the RC Walls with Barbell and Flanged Cross-Section (continued)

\begin{tabular}{|c|c|c|c|c|c|c|c|c|c|c|c|c|}
\hline $\begin{array}{l}\text { Test } \\
\text { No. }\end{array}$ & Researcher & Spec ID & $\begin{array}{l}\rho_{v}^{w} \\
(\%)\end{array}$ & $\begin{array}{l}\rho_{h}^{w} \\
(\%)\end{array}$ & $\begin{array}{l}S_{h}^{w} \\
\text { (in) }\end{array}$ & $\begin{array}{l}S_{v}^{w} \\
\text { (in) }\end{array}$ & $\begin{array}{l}\rho_{v}^{f} \\
(\%)\end{array}$ & $\begin{array}{l}\rho_{h}^{f} \\
(\%)\end{array}$ & $\begin{array}{l}S_{h}^{f} \\
\text { (in) }\end{array}$ & $\begin{array}{l}S_{v}^{w} \\
\text { (in) }\end{array}$ & $\begin{array}{l}\rho_{v}^{b e} \\
(\%)\end{array}$ & $\begin{array}{l}S_{h}^{b e} \\
\text { (in) }\end{array}$ \\
\hline 19 & \multirow{8}{*}{ Chiba (1985) } & CW-0.6-1.2-20 & 1.20 & 1.20 & 2.60 & 2.60 & - & - & - & - & 1.04 & - \\
\hline 20 & & CW-0.6-0.6-20 & 0.60 & 0.60 & 5.20 & 5.20 & - & - & - & - & 1.04 & - \\
\hline 21 & & CW-0.6-0.8-20 & 0.80 & 0.80 & 3.90 & 3.90 & - & - & - & - & 1.04 & - \\
\hline 22 & & CW-0.6-1.6-20 & 1.60 & 1.60 & 2.00 & 2.00 & - & - & - & - & 1.44 & - \\
\hline 23 & & CW-0.6-2.0-20 & 2.00 & 2.00 & 1.60 & 1.60 & - & - & - & - & 1.76 & - \\
\hline 24 & & CW-0.6-1.2-40 & 1.20 & 1.20 & 2.60 & 2.60 & - & - & - & - & 1.04 & - \\
\hline 25 & & CW-0.4-1.2-20 & 1.20 & 1.20 & 2.60 & 2.60 & - & - & - & - & 1.04 & - \\
\hline 26 & & CW-0.8-1.2-20 & 1.20 & 1.20 & 2.60 & 2.60 & - & - & - & - & 1.04 & - \\
\hline 27 & \multirow{12}{*}{$\begin{array}{c}\text { Fukuzawa } \\
(1985)\end{array}$} & CW-0.6-0-20 & 0.00 & 0.00 & - & - & - & - & - & - & 1.04 & - \\
\hline 28 & & CW-0.6-0.3-20 & 0.30 & 0.30 & 10.50 & 10.50 & - & - & - & - & 1.04 & - \\
\hline 29 & & CW-0.6-2.4-20 & 2.40 & 2.40 & 1.30 & 1.30 & - & - & - & - & 1.76 & - \\
\hline 30 & & CW-0.6-2.8-20 & 2.80 & 2.80 & 1.10 & 1.10 & - & - & - & - & 1.76 & - \\
\hline 31 & & CW-0.6-0-0 & 0.00 & 0.00 & - & - & - & - & - & - & 1.04 & - \\
\hline 32 & & CW-0.6-0-40 & 0.00 & 0.00 & - & - & - & - & - & - & 1.04 & - \\
\hline 33 & & CW-0.6-0.6-0 & 0.60 & 0.60 & 5.20 & 5.20 & - & - & - & - & 1.04 & - \\
\hline 34 & & CW-0.6-0.6-40 & 0.60 & 0.60 & 5.20 & 5.20 & - & - & - & - & 1.04 & - \\
\hline 35 & & CW-0.4-0.6-20 & 0.60 & 0.60 & 5.20 & 5.20 & - & - & - & - & 1.04 & - \\
\hline 36 & & CW-0.8-0.6-20 & 0.60 & 0.60 & 5.20 & 5.20 & - & - & - & - & 1.04 & - \\
\hline 37 & & CW-0.4-2.0-20 & 2.00 & 2.00 & 1.60 & 1.60 & - & - & - & - & 1.76 & - \\
\hline 38 & & CW-0.8-2.0-20 & 2.00 & 2.00 & 1.60 & 1.60 & - & - & - & - & 1.76 & - \\
\hline 39 & \multirow{6}{*}{ Hatori (1985) } & CW-0.6-2-0 & 2.00 & 2.00 & 1.60 & 1.60 & - & - & - & - & 1.76 & - \\
\hline 40 & & CW-0.6-2-40 & 2.00 & 2.00 & 1.60 & 1.60 & - & - & - & - & 1.76 & - \\
\hline 41 & & CW-0.6-2-20B & 2.00 & 2.00 & 1.60 & 1.60 & - & - & - & - & 1.04 & - \\
\hline 42 & & CW-0.6-0.6-20L & 0.60 & 0.60 & 5.20 & 5.20 & - & - & - & - & 1.04 & - \\
\hline 43 & & CW-0.6-1.2-20L & 1.20 & 1.20 & 2.60 & 2.60 & - & - & - & - & 1.04 & - \\
\hline 44 & & CW-0.6-2-20L & 2.00 & 2.00 & 1.60 & 1.60 & - & - & - & - & 1.76 & - \\
\hline 45 & \multirow{3}{*}{ Taga (1985) } & WALL1 & 1.20 & 1.20 & 2.60 & 2.60 & - & - & - & - & 2.89 & - \\
\hline 46 & & WALL2 & 1.20 & 1.20 & 2.60 & 2.60 & - & - & - & - & 2.89 & - \\
\hline 47 & & WALL3 & 1.20 & 1.20 & 2.60 & 2.60 & - & - & - & - & 2.89 & - \\
\hline 48 & \multirow{2}{*}{ Maier (1985) } & $\mathrm{S} 1$ & 1.01 & 1.01 & 3.94 & 3.94 & 1.01 & 1.01 & 3.94 & 3.94 & - & - \\
\hline 49 & & S2 & 1.01 & 1.01 & 3.94 & 3.94 & 1.01 & 1.01 & 3.94 & 3.94 & - & - \\
\hline
\end{tabular}


Table B-9 Reinforcement Properties of the RC Walls with Barbell and Flanged Cross-Section (continued)

\begin{tabular}{|c|c|c|c|c|c|c|c|c|c|c|c|c|}
\hline $\begin{array}{l}\text { Test } \\
\text { No. }\end{array}$ & Researcher & Spec ID & $\begin{array}{l}\rho_{v}^{w} \\
(\%)\end{array}$ & $\begin{array}{l}\rho_{h}^{w} \\
(\%) \\
\end{array}$ & $\begin{array}{l}S_{h}^{w} \\
\text { (in) }\end{array}$ & $\begin{array}{l}S_{v}^{w} \\
\text { (in) }\end{array}$ & $\begin{array}{l}\rho_{v}^{f} \\
(\%)\end{array}$ & $\begin{array}{l}\rho_{h}^{f} \\
(\%)\end{array}$ & $\begin{array}{l}S_{h}^{f} \\
\text { (in) }\end{array}$ & $\begin{array}{l}S_{v}^{w} \\
\text { (in) }\end{array}$ & $\begin{array}{l}\rho_{v}^{b e} \\
(\%)\end{array}$ & $\begin{array}{l}S_{h}^{b e} \\
\text { (in) }\end{array}$ \\
\hline 50 & \multirow{5}{*}{ Maier (1985) } & S3 & 2.26 & 1.03 & 3.94 & 3.94 & 2.26 & 1.03 & 3.94 & 3.94 & - & - \\
\hline 51 & & S5 & 1.01 & 1.01 & 3.94 & 3.94 & 1.01 & 1.01 & 3.94 & 3.94 & - & - \\
\hline 52 & & S6 & 1.01 & 0.57 & 3.94 & 3.94 & 1.01 & 0.57 & 3.94 & 3.94 & - & - \\
\hline 53 & & S7 & 1.01 & 1.01 & 3.94 & 3.94 & 1.01 & 1.01 & 3.94 & 3.94 & - & - \\
\hline 54 & & S8 & 1.01 & 1.01 & 3.94 & 3.94 & 1.01 & 1.01 & 3.94 & 3.94 & - & - \\
\hline 55 & \multirow{5}{*}{$\begin{array}{c}\text { Tomii et al. } \\
\text { (1988) }\end{array}$} & T1-W87.5-N50 & 0.31 & 0.31 & - & - & - & - & - & - & 0.84 & - \\
\hline 56 & & T2-W87.5-N50 & 0.31 & 0.31 & - & - & - & - & - & - & 0.84 & - \\
\hline 57 & & H2-W87.5-N50 & 0.31 & 0.31 & - & - & - & - & - & - & 0.84 & - \\
\hline 58 & & H3-W87.5-N50 & 0.31 & 0.31 & - & - & - & - & - & - & 0.84 & - \\
\hline 59 & & H3-W40-N65 & 0.69 & 0.69 & - & - & - & - & - & - & 1.46 & - \\
\hline 60 & \multirow{8}{*}{$\begin{array}{c}\text { Sato et al. } \\
\text { (1989) }\end{array}$} & $36 \mathrm{M} 8-30$ & 1.16 & 1.16 & 3.20 & 3.20 & 1.16 & 1.16 & 3.20 & 3.20 & - & - \\
\hline 61 & & $36 \mathrm{M} 8-50$ & 0.72 & 0.72 & 5.00 & 5.00 & 0.72 & 0.72 & 5.00 & 5.00 & - & - \\
\hline 62 & & $24 \mathrm{M} 8-30$ & 0.80 & 0.80 & 4.60 & 4.60 & 0.80 & 0.80 & 4.60 & 4.60 & - & - \\
\hline 63 & & 24M8-40 & 0.60 & 0.60 & 6.10 & 6.10 & 0.60 & 0.60 & 6.10 & 6.10 & - & - \\
\hline 64 & & $24 \mathrm{M} 8-50$ & 0.48 & 0.48 & 7.90 & 7.90 & 0.48 & 0.48 & 7.90 & 7.90 & - & - \\
\hline 65 & & 48M8-30 & 1.60 & 1.60 & 2.30 & 2.30 & 1.60 & 1.60 & 2.30 & 2.30 & - & - \\
\hline 66 & & $48 \mathrm{M} 8-40$ & 1.16 & 1.16 & 3.20 & 3.20 & 1.16 & 1.16 & 3.20 & 3.20 & - & - \\
\hline 67 & & 48M8-50 & 0.96 & 0.96 & 3.90 & 3.90 & 0.96 & 0.96 & 3.90 & 3.90 & - & - \\
\hline 68 & \multirow{3}{*}{$\begin{array}{l}\text { Mansour et al. } \\
\text { (1991) }\end{array}$} & W3 & 0.55 & 0.55 & - & - & - & - & - & - & 3.14 & 3.93 \\
\hline 69 & & W4 & 0.62 & 0.62 & - & - & - & - & - & - & 3.14 & 3.93 \\
\hline 70 & & W5 & 0.62 & 0.62 & - & - & - & - & - & - & 3.14 & 3.93 \\
\hline 71 & \multirow{4}{*}{$\begin{array}{l}\text { Sheu and } \\
\text { Huang (1991) }\end{array}$} & SWB-4B & 1.04 & 1.45 & 4.90 & 5.50 & - & - & - & - & 1.99 & - \\
\hline 72 & & SWB-6A & 0.58 & 0.82 & 4.90 & 5.50 & - & - & - & - & 1.12 & - \\
\hline 73 & & SWB-8A & 0.58 & 0.82 & 4.90 & 5.50 & - & - & - & - & 1.12 & - \\
\hline 74 & & SWB-17A & 0.58 & 0.82 & 4.90 & 5.50 & - & - & - & - & 1.12 & - \\
\hline 75 & \multirow{4}{*}{$\begin{array}{l}\text { Mo and Kuo } \\
(1998)\end{array}$} & $\mathrm{H}-\mathrm{HZ} 4$ & 0.42 & 0.42 & - & - & - & - & - & - & 7.80 & 3.93 \\
\hline 76 & & $\mathrm{H}-\mathrm{HZ6}$ & 0.42 & 0.42 & - & - & - & - & - & - & 7.80 & 3.93 \\
\hline 77 & & $\mathrm{H}-\mathrm{HZZ}$ & 0.42 & 0.42 & - & - & - & - & - & - & 7.80 & 3.93 \\
\hline 78 & & $\mathrm{H}-\mathrm{HZ} 10$ & 0.42 & 0.42 & - & - & - & - & - & - & 7.80 & 3.93 \\
\hline 79 & \multirow{2}{*}{ Sittipunt (2001) } & WALL 1 & 0.39 & 0.52 & 5.91 & 7.87 & - & - & - & - & 2.30 & 3.93 \\
\hline 80 & & WALL 2 & 0.52 & 0.79 & 3.94 & 5.91 & - & - & - & - & 2.30 & 3.93 \\
\hline
\end{tabular}


Table B-9 Reinforcement Properties of the RC Walls with Barbell and Flanged Cross-Section (continued)

\begin{tabular}{|c|c|c|c|c|c|c|c|c|c|c|c|c|}
\hline $\begin{array}{l}\text { Test } \\
\text { No. }\end{array}$ & Researcher & Spec ID & $\begin{array}{l}\rho_{v}^{w} \\
(\%)\end{array}$ & $\begin{array}{l}\rho_{h}^{w} \\
(\%)\end{array}$ & $\begin{array}{l}S_{h}^{w} \\
\text { (in) }\end{array}$ & $\begin{array}{l}S_{v}^{w} \\
\text { (in) }\end{array}$ & $\begin{array}{l}\rho_{v}^{f} \\
(\%)\end{array}$ & $\begin{array}{l}\rho_{h}^{f} \\
(\%)\end{array}$ & $\begin{array}{l}S_{h}^{f} \\
\text { (in) }\end{array}$ & $\begin{array}{l}S_{v}^{w} \\
\text { (in) }\end{array}$ & $\begin{array}{l}\rho_{v}^{b e} \\
(\%)\end{array}$ & $\begin{array}{l}S_{h}^{b e} \\
\text { (in) }\end{array}$ \\
\hline 81 & \multirow{3}{*}{$\begin{array}{l}\text { Effendy et al. } \\
\quad(2006)\end{array}$} & SMAC & 0.59 & 0.59 & 7.87 & 7.87 & - & - & - & - & 2.75 & 3.93 \\
\hline 82 & & SMAS & 0.36 & 0.36 & 13.11 & 13.11 & - & - & - & - & 2.75 & 3.93 \\
\hline 83 & & SMAM & 0.36 & 0.36 & 13.11 & 13.11 & - & - & - & - & 2.75 & 3.93 \\
\hline 84 & \multirow{7}{*}{$\begin{array}{c}\text { Faravashany et } \\
\text { al. (2008) }\end{array}$} & HSCW1 & 1.29 & 0.48 & 6.30 & 2.40 & 4.00 & 0.84 & 2.95 & 2.50 & - & - \\
\hline 85 & & HSCW2 & 1.29 & 0.48 & 6.30 & 2.40 & 4.00 & 0.84 & 2.95 & 2.50 & - & - \\
\hline 86 & & HSCW3 & 0.75 & 0.48 & 6.30 & 3.90 & 4.00 & 0.84 & 2.95 & 2.50 & - & - \\
\hline 87 & & HSCW4 & 0.75 & 0.48 & 6.30 & 3.90 & 4.00 & 0.84 & 2.95 & 2.50 & - & - \\
\hline 88 & & HSCW5 & 1.29 & 0.75 & 3.90 & 2.40 & 4.00 & 0.84 & 2.95 & 2.50 & - & - \\
\hline 89 & & HSCW6 & 1.29 & 0.75 & 3.90 & 2.40 & 4.00 & 0.84 & 2.95 & 2.50 & - & - \\
\hline 90 & & HSCW7 & 0.75 & 0.75 & 3.90 & 3.90 & 4.00 & 0.84 & 2.95 & 2.50 & - & - \\
\hline 91 & $\begin{array}{c}\text { Park et al. } \\
\text { (2013) }\end{array}$ & S4 & 0.54 & 0.51 & 9.80 & 11.81 & - & - & - & - & 9.70 & 2.17 \\
\hline 92 & \multirow{3}{*}{$\begin{array}{l}\text { Almeida et al. } \\
\quad(2015)\end{array}$} & TW1 & 0.15 & 0.18 & 7.80 & 9.40 & 0.32 & 0.18 & 7.80 & 9.40 & - & - \\
\hline 93 & & TW2 & 0.50 & 0.36 & 5.10 & 3.70 & 0.43 & 0.36 & 5.10 & 3.70 & - & - \\
\hline 94 & & TW3 & 0.50 & 0.36 & 5.10 & 3.70 & 0.43 & 0.36 & 5.10 & 3.70 & - & - \\
\hline 95 & \multirow{8}{*}{ Li et al. (2015) } & LW1 & 0.50 & 0.50 & 9.84 & 9.84 & - & - & - & - & 1.40 & 9.84 \\
\hline 96 & & LW2 & 0.50 & 0.50 & 9.84 & 9.84 & - & - & - & - & 1.40 & 9.84 \\
\hline 97 & & LW3 & 0.50 & 0.50 & 9.84 & 9.84 & - & - & - & - & 1.40 & 9.84 \\
\hline 98 & & LW4 & 0.50 & 0.50 & 9.84 & 9.84 & - & - & - & - & 1.40 & 9.84 \\
\hline 99 & & LW5 & 0.50 & 0.50 & 9.84 & 9.84 & - & - & - & - & 1.40 & 9.84 \\
\hline 100 & & MW1 & 0.50 & 0.50 & 9.84 & 9.84 & - & - & - & - & 1.40 & 9.84 \\
\hline 101 & & MW2 & 0.50 & 0.50 & 9.84 & 9.84 & - & - & - & - & 1.40 & 9.84 \\
\hline 102 & & MW3 & 0.50 & 0.50 & 9.84 & 9.84 & - & - & - & - & 1.40 & 9.84 \\
\hline 103 & Li et al. (2016) & SF1 & 0.50 & 0.50 & 10.62 & 9.84 & 1.30 & 0.20 & 7.87 & 4.33 & - & - \\
\hline
\end{tabular}


Table B-10 Material Properties of the RC Walls with Barbell and Flanged Cross-Section

\begin{tabular}{|c|c|c|c|c|c|c|c|c|c|c|c|}
\hline $\begin{array}{l}\text { Test } \\
\text { No. }\end{array}$ & Researcher & Spec ID & $\begin{array}{c}f_{c}^{\prime} \\
(\mathrm{ksi}) \\
\end{array}$ & $\begin{array}{c}f_{y v}^{w} \\
\text { (ksi) }\end{array}$ & $\begin{array}{c}f_{u v}^{w} \\
(\mathrm{ksi})\end{array}$ & $\begin{array}{c}f_{y h}^{w} \\
(\mathrm{ksi})\end{array}$ & $\begin{array}{c}f_{u h}^{w} \\
(\mathrm{ksi})\end{array}$ & $\begin{array}{c}f_{y v}^{f} \\
(\mathrm{ksi})\end{array}$ & $\begin{array}{c}f_{u v}^{f} \\
(\mathrm{ksi})\end{array}$ & $\begin{array}{c}f_{y h}^{f} \\
(\mathrm{ksi})\end{array}$ & $\begin{array}{c}f_{u h}^{f} \\
(\mathrm{ksi})\end{array}$ \\
\hline 1 & \multirow{6}{*}{$\begin{array}{l}\text { Shiga et al. } \\
\text { (1973) }\end{array}$} & WB1 & 2.7 & 42.7 & 58.3 & 42.7 & 58.3 & 55.5 & 79.6 & 55.5 & 79.6 \\
\hline 2 & & WB2 & 2.7 & 42.7 & 58.3 & 42.7 & 58.3 & 55.5 & 79.6 & 55.5 & 79.6 \\
\hline 3 & & WB3 & 2.3 & 42.7 & 58.3 & 42.7 & 58.3 & 55.5 & 79.6 & 55.5 & 79.6 \\
\hline 4 & & WB6 & 2.1 & 42.7 & 58.3 & 42.7 & 58.3 & 55.5 & 79.6 & 55.5 & 79.6 \\
\hline 5 & & WB7 & 2.2 & 42.7 & 58.3 & 42.7 & 58.3 & 55.5 & 79.6 & 55.5 & 79.6 \\
\hline 6 & & WB8 & 2.2 & 42.7 & 58.3 & 42.7 & 58.3 & 55.5 & 79.6 & 55.5 & 79.6 \\
\hline 7 & \multirow{8}{*}{$\begin{array}{l}\text { Barda et al. } \\
\text { (1977) }\end{array}$} & B1-1 & 4.2 & 78.8 & 123.1 & 71.9 & 98.6 & 76.2 & 118.7 & 72.5 & 97.4 \\
\hline 8 & & B2-1 & 2.4 & 80.0 & 126.5 & 72.4 & 97.0 & 70.6 & 119.0 & 70.5 & 96.8 \\
\hline 9 & & B3-2 & 3.9 & 79.0 & 123.3 & 74.4 & 97.5 & 60.0 & 96.7 & 70.0 & 96.3 \\
\hline 10 & & B4-3 & 2.8 & 77.6 & 119.6 & 77.6 & - & 76.5 & 117.2 & 71.2 & 99.0 \\
\hline 11 & & B5-4 & 4.2 & 71.8 & - & 71.8 & 98.3 & 76.4 & 117.0 & 68.7 & 96.7 \\
\hline 12 & & B6-4 & 3.1 & 72.0 & 94.8 & 72.0 & 94.8 & 76.7 & 116.3 & 72.0 & 94.8 \\
\hline 13 & & B7-5 & 3.7 & 77.0 & 120.4 & 72.7 & 96.0 & 78.2 & 115.1 & 72.7 & 96.0 \\
\hline 14 & & B8-5 & 3.4 & 76.5 & 109.5 & 71.9 & 97.9 & 70.9 & 112.8 & 69.3 & 96.8 \\
\hline 15 & Synge (1980) & WALL 3 & 3.8 & 45.6 & 63.8 & 55.1 & 83.5 & 43.5 & 66.7 & - & - \\
\hline 16 & \multirow{3}{*}{$\begin{array}{c}\text { Yagishita } \\
\text { (1985) }\end{array}$} & CW-0.6-0.6-20a & 4.2 & 59.7 & 77.8 & 59.7 & 77.8 & 54.9 & 83.4 & 54.9 & 83.4 \\
\hline 17 & & CW-0.6-0.8-20a & 4.3 & 59.7 & 77.8 & 59.7 & 77.8 & 54.9 & 83.4 & 54.9 & 83.4 \\
\hline 18 & & CW-0.6-1.2-0 & 4.2 & 59.7 & 77.8 & 59.7 & 77.8 & 54.9 & 83.4 & 54.9 & 83.4 \\
\hline 19 & \multirow{8}{*}{ Chiba (1985) } & CW-0.6-1.2-20 & 4.9 & 59.7 & 77.8 & 59.7 & 77.8 & 54.9 & 83.4 & 54.9 & 83.4 \\
\hline 20 & & CW-0.6-0.6-20 & 4.3 & 59.7 & 77.8 & 59.7 & 77.8 & 54.9 & 83.4 & 54.9 & 83.4 \\
\hline 21 & & CW-0.6-0.8-20 & 5.8 & 59.7 & 77.8 & 59.7 & 77.8 & 54.9 & 83.4 & 54.9 & 83.4 \\
\hline 22 & & CW-0.6-1.6-20 & 4.9 & 59.7 & 77.8 & 59.7 & 77.8 & 55.0 & 82.7 & 55.0 & 82.7 \\
\hline 23 & & CW-0.6-2.0-20 & 5.0 & 59.7 & 77.8 & 59.7 & 77.8 & 54.3 & 83.8 & 54.3 & 83.8 \\
\hline 24 & & CW-0.6-1.2-40 & 4.6 & 59.7 & 77.8 & 59.7 & 77.8 & 54.9 & 83.4 & 54.9 & 83.4 \\
\hline 25 & & CW-0.4-1.2-20 & 4.8 & 59.7 & 77.8 & 59.7 & 77.8 & 54.9 & 83.4 & 54.9 & 83.4 \\
\hline 26 & & CW-0.8-1.2-20 & 4.9 & 59.7 & 77.8 & 59.7 & 77.8 & 54.9 & 83.4 & 54.9 & 83.4 \\
\hline 27 & \multirow{6}{*}{$\begin{array}{c}\text { Fukuzawa } \\
\text { (1985) }\end{array}$} & CW-0.6-0-20 & 5.1 & 59.7 & - & 59.7 & - & 54.9 & 83.4 & 54.9 & 83.4 \\
\hline 28 & & CW-0.6-0.3-20 & 5.1 & 59.7 & 77.8 & 59.7 & 77.8 & 54.9 & 83.4 & 54.9 & 83.4 \\
\hline 29 & & CW-0.6-2.4-20 & 4.9 & 59.7 & 77.8 & 59.7 & 77.8 & 54.3 & 83.8 & 54.3 & 83.8 \\
\hline 30 & & CW-0.6-2.8-20 & 4.6 & 59.7 & 77.8 & 59.7 & 77.8 & 54.3 & 83.8 & 54.3 & 83.8 \\
\hline 31 & & CW-0.6-0-0 & 4.6 & 59.7 & - & 59.7 & - & 54.9 & 83.4 & 54.9 & 83.4 \\
\hline 32 & & CW-0.6-0-40 & 4.6 & 59.7 & - & 59.7 & - & 54.9 & 83.4 & 54.9 & 83.4 \\
\hline
\end{tabular}


Table B-10 Material Properties of the RC Walls with Barbell and Flanged Cross-Section (continued)

\begin{tabular}{|c|c|c|c|c|c|c|c|c|c|c|c|}
\hline $\begin{array}{l}\text { Test } \\
\text { No. }\end{array}$ & Researcher & Spec ID & $\begin{array}{c}f_{c}^{\prime} \\
(\mathrm{ksi}) \\
\end{array}$ & $\begin{array}{r}f_{y v}^{w} \\
(\mathrm{ksi}) \\
\end{array}$ & $\begin{array}{c}f_{u v}^{w} \\
(\mathrm{ksi}) \\
\end{array}$ & $\begin{array}{c}f_{y h}^{w} \\
(\mathrm{ksi})\end{array}$ & $\begin{array}{c}f_{u h}^{w} \\
(\mathrm{ksi})\end{array}$ & $\begin{array}{c}f_{y v}^{f} \\
(\mathrm{ksi})\end{array}$ & $\begin{array}{c}f_{u v}^{f} \\
(\mathrm{ksi})\end{array}$ & $\begin{array}{c}f_{y h}^{f} \\
(\mathrm{ksi}) \\
\end{array}$ & $\begin{array}{c}f_{u h}^{f} \\
(\mathrm{ksi})\end{array}$ \\
\hline 33 & \multirow{2}{*}{$\begin{array}{c}\text { Fukuzawa } \\
\text { (1985) }\end{array}$} & CW-0.6-0.6-0 & 5.1 & 59.7 & 77.8 & 59.7 & 77.8 & 54.9 & 83.4 & 54.9 & 83.4 \\
\hline 34 & & CW-0.6-0.6-40 & 4.9 & 59.7 & 77.8 & 59.7 & 77.8 & 54.9 & 83.4 & 54.9 & 83.4 \\
\hline 35 & \multirow{4}{*}{$\begin{array}{c}\text { Fukuzawa } \\
\text { (1985) }\end{array}$} & CW-0.4-0.6-20 & 4.8 & 59.7 & 77.8 & 59.7 & 77.8 & 54.9 & 83.4 & 54.9 & 83.4 \\
\hline 36 & & CW-0.8-0.6-20 & 4.8 & 59.7 & 77.8 & 59.7 & 77.8 & 54.9 & 83.4 & 54.9 & 83.4 \\
\hline 37 & & CW-0.4-2.0-20 & 4.9 & 59.7 & 77.8 & 59.7 & 77.8 & 54.3 & 83.8 & 54.3 & 83.8 \\
\hline 38 & & CW-0.8-2.0-20 & 5.0 & 59.7 & 77.8 & 59.7 & 77.8 & 54.3 & 83.8 & 54.3 & 83.8 \\
\hline 39 & \multirow{6}{*}{ Hatori (1985) } & CW-0.6-2-0 & 4.8 & 59.7 & 77.8 & 59.7 & 77.8 & 54.3 & 83.8 & 54.3 & 83.8 \\
\hline 40 & & CW-0.6-2-40 & 4.9 & 59.7 & 77.8 & 59.7 & 77.8 & 54.3 & 83.8 & 54.3 & 83.8 \\
\hline 41 & & CW-0.6-2-20B & 5.1 & 59.7 & 77.8 & 59.7 & 77.8 & 54.9 & 83.4 & 54.9 & 83.4 \\
\hline 42 & & CW-0.6-0.6-20L & 3.7 & 59.7 & 77.8 & 59.7 & 77.8 & 54.9 & 83.4 & 54.9 & 83.4 \\
\hline 43 & & CW-0.6-1.2-20L & 3.8 & 59.7 & 77.8 & 59.7 & 77.8 & 54.9 & 83.4 & 54.9 & 83.4 \\
\hline 44 & & CW-0.6-2-20L & 3.7 & 59.7 & 77.8 & 59.7 & 77.8 & 54.3 & 83.8 & 54.3 & 83.8 \\
\hline 45 & \multirow{3}{*}{ Taga (1985) } & WALL1 & 3.9 & 46.6 & 75.8 & 46.6 & 75.8 & 56.2 & 77.0 & 56.2 & 77.0 \\
\hline 46 & & WALL2 & 5.6 & 46.6 & 75.8 & 46.6 & 75.8 & 56.2 & 77.0 & 56.2 & 77.0 \\
\hline 47 & & WALL3 & 8.5 & 46.6 & 75.8 & 46.6 & 75.8 & 56.2 & 77.0 & 56.2 & 77.0 \\
\hline 48 & \multirow{7}{*}{ Maier (1985) } & S1 & 5.4 & 83.5 & 110.0 & 83.5 & 110.0 & 83.5 & 110.0 & 83.5 & 110.0 \\
\hline 49 & & S2 & 5.4 & 83.5 & 110.0 & 83.5 & 110.0 & 83.5 & 110.0 & 83.5 & 110.0 \\
\hline 50 & & S3 & 5.3 & 76.8 & 107.0 & 83.5 & 110.0 & 76.8 & 107.0 & 83.5 & 110.0 \\
\hline 51 & & S5 & 5.4 & 83.5 & 110.0 & 83.5 & 110.0 & 83.5 & 110.0 & 83.5 & 110.0 \\
\hline 52 & & S6 & 5.2 & 69.4 & 110.8 & 77.8 & 108.8 & 69.4 & 110.8 & 77.8 & 108.8 \\
\hline 53 & & S7 & 4.9 & 80.4 & 113.4 & 80.4 & 113.4 & 80.4 & 113.4 & 80.4 & 113.4 \\
\hline 54 & & S8 & 4.7 & 80.4 & 113.4 & 80.4 & 113.4 & 80.4 & 113.4 & 80.4 & 113.4 \\
\hline 55 & \multirow{5}{*}{$\begin{array}{c}\text { Tomi et al. } \\
\text { (1988) }\end{array}$} & T1-W87.5-N50 & 5.1 & 41.4 & - & 41.4 & - & 48.9 & - & 51.1 & - \\
\hline 56 & & T2-W87.5-N50 & 4.7 & 41.4 & - & 41.4 & - & 48.9 & - & 51.1 & - \\
\hline 57 & & H2-W87.5-N50 & 3.9 & 41.4 & - & 41.4 & - & 48.9 & - & 41.4 & - \\
\hline 58 & & H3-W87.5-N50 & 3.8 & 41.4 & - & 41.4 & - & 48.9 & - & 41.4 & - \\
\hline 59 & & H3-W40-N65 & 5.0 & 41.4 & - & 41.4 & - & 48.9 & - & 41.4 & - \\
\hline 60 & \multirow{4}{*}{$\begin{array}{c}\text { Sato et al. } \\
(1989)\end{array}$} & 36M8-30 & 5.7 & 42.9 & 69.9 & 42.9 & 69.6 & 42.9 & 69.6 & - & - \\
\hline 61 & & $36 M 8-50$ & 5.4 & 76.6 & 89.9 & 76.6 & 89.9 & 76.6 & 89.9 & - & - \\
\hline 62 & & 24M8-30 & 5.5 & 42.9 & 69.6 & 42.9 & 69.6 & 42.9 & 69.9 & - & - \\
\hline 63 & & $24 \mathrm{M} 8-40$ & 5.2 & 61.2 & 81.2 & 61.2 & 81.2 & 61.2 & 81.2 & - & - \\
\hline
\end{tabular}


Table B-10 Material Properties of the RC Walls with Barbell and Flanged Cross-Section (continued)

\begin{tabular}{|c|c|c|c|c|c|c|c|c|c|c|c|}
\hline $\begin{array}{l}\text { Test } \\
\text { No. }\end{array}$ & Researcher & Spec ID & $\begin{array}{c}f_{c}^{\prime} \\
(\mathrm{ksi})\end{array}$ & $\begin{array}{c}f_{y v}^{w} \\
(\mathrm{ksi})\end{array}$ & $\begin{array}{c}f_{u v}^{w} \\
(\mathrm{ksi})\end{array}$ & $\begin{array}{c}f_{y h}^{w} \\
(\mathrm{ksi})\end{array}$ & $\begin{array}{c}f_{u h}^{w} \\
(\mathrm{ksi})\end{array}$ & $\begin{array}{c}f_{y v}^{f} \\
(\mathrm{ksi})\end{array}$ & $\begin{array}{c}f_{u v}^{f} \\
(\mathrm{ksi})\end{array}$ & $\begin{array}{c}f_{y h}^{f} \\
(\mathrm{ksi})\end{array}$ & $\begin{array}{c}f_{u h}^{f} \\
(\mathrm{ksi})\end{array}$ \\
\hline 64 & \multirow{4}{*}{$\begin{array}{l}\text { Sato et al. } \\
\text { (1989) }\end{array}$} & $24 M 8-50$ & 5.1 & 76.6 & 89.9 & 76.6 & 89.9 & 76.6 & 89.9 & - & - \\
\hline 65 & & $48 \mathrm{M} 8-30$ & 4.0 & 42.9 & 69.9 & 42.9 & 69.9 & 42.9 & 69.6 & - & - \\
\hline 66 & & $48 \mathrm{M} 8-40$ & 4.0 & 61.2 & 81.2 & 61.2 & 81.2 & 61.2 & 81.2 & - & - \\
\hline 67 & & $48 \mathrm{M} 8-50$ & 4.1 & 76.6 & 89.9 & 76.6 & 89.9 & 76.6 & 89.9 & - & - \\
\hline 68 & \multirow{3}{*}{$\begin{array}{l}\text { Mansour et al. } \\
\quad(1991)\end{array}$} & W3 & 4.5 & 66.7 & 71.8 & 47.4 & 66.0 & 66.7 & 71.7 & 47.4 & 65.9 \\
\hline 69 & & W4 & 5.4 & 52.1 & 55.4 & 52.1 & 55.4 & 66.7 & 71.7 & 47.4 & 65.9 \\
\hline 70 & & W5 & 4.7 & 46.5 & 59.9 & 46.5 & 59.9 & 66.7 & 71.7 & 47.4 & 65.9 \\
\hline 71 & \multirow{4}{*}{$\begin{array}{l}\text { Sheu and } \\
\text { Huang (1991) }\end{array}$} & SWB-4B & 4.3 & 65.7 & 102.4 & 65.7 & 102.4 & 65.7 & 102.4 & 65.7 & 102.4 \\
\hline 72 & & SWB-6A & 3.9 & 71.2 & 103.6 & 71.2 & 103.6 & 71.2 & 103.6 & 71.2 & 103.6 \\
\hline 73 & & SWB-8A & 3.2 & 71.2 & 103.6 & 71.2 & 103.6 & 71.2 & 103.6 & 71.2 & 103.6 \\
\hline 74 & & SWB-17A & 3.3 & 71.2 & 103.6 & 71.2 & 103.6 & 71.2 & 103.6 & 71.2 & 103.6 \\
\hline 75 & \multirow{4}{*}{$\begin{array}{c}\text { Mo and Kuo } \\
\quad(1998)\end{array}$} & $\mathrm{H}-\mathrm{HZ} 4$ & 2.2 & 37.5 & - & 37.5 & - & 32.7 & - & 37.5 & - \\
\hline 76 & & $\mathrm{H}-\mathrm{HZ6}$ & 2.4 & 37.5 & - & 37.5 & - & 32.7 & - & 37.5 & - \\
\hline 77 & & $\mathrm{H}-\mathrm{HZZ}$ & 2.4 & 37.5 & - & 37.5 & - & 32.7 & - & 37.5 & - \\
\hline 78 & & $\mathrm{H}-\mathrm{HZ} 10$ & 2.7 & 37.5 & - & 37.5 & - & 32.7 & - & 37.5 & - \\
\hline 79 & \multirow{2}{*}{ Sittipunt (2001) } & WALL 1 & 5.3 & 65.3 & - & 65.3 & - & 68.6 & - & 64.3 & - \\
\hline 80 & & WALL 2 & 5.2 & 65.3 & - & 65.3 & - & 68.6 & - & 64.3 & - \\
\hline 81 & \multirow{3}{*}{$\begin{array}{l}\text { Effendy et al. } \\
\qquad(2006)\end{array}$} & SMAC & 2.9 & 56.6 & 82.0 & 56.6 & 82.0 & 65.5 & 100.5 & 56.6 & 82.0 \\
\hline 82 & & SMAS & 2.9 & 56.6 & 82.0 & 56.6 & 82.0 & 65.5 & 100.5 & 56.6 & 82.0 \\
\hline 83 & & SMAM & 2.9 & 56.6 & 82.0 & 56.6 & 82.0 & 65.5 & 100.5 & 56.6 & 82.0 \\
\hline 84 & \multirow{7}{*}{$\begin{array}{l}\text { Faravashany et } \\
\text { al. (2008) }\end{array}$} & HSCW1 & 15.1 & 58.3 & 79.8 & 58.3 & 79.8 & 64.4 & 77.6 & 64.4 & 77.6 \\
\hline 85 & & HSCW2 & 13.4 & 58.3 & 79.8 & 58.3 & 79.8 & 64.4 & 77.6 & 64.4 & 77.6 \\
\hline 86 & & HSCW3 & 12.5 & 58.3 & 79.8 & 58.3 & 79.8 & 64.4 & 77.6 & 64.4 & 77.6 \\
\hline 87 & & HSCW4 & 13.2 & 58.3 & 79.8 & 58.3 & 79.8 & 64.4 & 77.6 & 64.4 & 77.6 \\
\hline 88 & & HSCW5 & 12.2 & 58.3 & 79.8 & 58.3 & 79.8 & 64.4 & 77.6 & 64.4 & 77.6 \\
\hline 89 & & HSCW6 & 13.1 & 58.3 & 79.8 & 58.3 & 79.8 & 64.4 & 77.6 & 64.4 & 77.6 \\
\hline 90 & & HSCW7 & 14.8 & 58.3 & 79.8 & 58.3 & 79.8 & 64.4 & 77.6 & 64.4 & 77.6 \\
\hline 91 & $\begin{array}{l}\text { Park et al. } \\
\text { (2013) }\end{array}$ & S4 & 6.7 & 94.7 & - & 96.7 & - & 89.5 & - & 96.7 & - \\
\hline
\end{tabular}


Table B-10 Material Properties of the RC Walls with Barbell and Flanged Cross-Section (continued)

\begin{tabular}{|c|c|c|c|c|c|c|c|c|c|c|c|}
\hline $\begin{array}{l}\text { Test } \\
\text { No. }\end{array}$ & Researcher & Spec ID & $\begin{array}{c}f_{c}^{\prime} \\
(\mathrm{ksi})\end{array}$ & $\begin{array}{c}f_{y v}^{w} \\
(\mathrm{ksi})\end{array}$ & $\begin{array}{c}f_{u v}^{w} \\
(\mathrm{ksi})\end{array}$ & $\begin{array}{c}f_{y h}^{w} \\
(\mathrm{ksi})\end{array}$ & $\begin{array}{c}f_{u h}^{w} \\
(\mathrm{ksi})\end{array}$ & $\begin{array}{c}f_{y v}^{f} \\
(\mathrm{ksi})\end{array}$ & $\begin{array}{c}f_{u v}^{f} \\
\text { (ksi) }\end{array}$ & $\begin{array}{c}f_{y h}^{f} \\
(\mathrm{ksi})\end{array}$ & $\begin{array}{c}f_{u h}^{f} \\
(\mathrm{ksi})\end{array}$ \\
\hline 92 & \multirow{3}{*}{$\begin{array}{l}\text { Almeida et al. } \\
\quad(2015)\end{array}$} & TW1 & 4.2 & 66.7 & 90.6 & 66.7 & 90.6 & 66.7 & 90.6 & 66.7 & 90.6 \\
\hline 93 & & TW2 & 7.4 & 66.7 & 90.6 & 66.7 & 90.6 & 66.7 & 90.6 & 66.7 & 90.6 \\
\hline 94 & & TW3 & 7.0 & 66.7 & 90.6 & 66.7 & 90.6 & 66.7 & 90.6 & 66.7 & 90.6 \\
\hline 95 & \multirow{8}{*}{ Li et al. (2015) } & LW1 & 5.8 & 61.9 & 72.1 & 61.9 & 72.1 & 44.6 & 55.8 & 37.8 & 46.9 \\
\hline 96 & & LW2 & 6.0 & 61.9 & 72.1 & 61.9 & 72.1 & 44.6 & 55.8 & 37.8 & 46.9 \\
\hline 97 & & LW3 & 5.0 & 61.9 & 72.1 & 61.9 & 72.1 & 44.6 & 55.8 & 37.8 & 46.9 \\
\hline 98 & & LW4 & 5.8 & 61.9 & 72.1 & 61.9 & 72.1 & 44.6 & 55.8 & 37.8 & 46.9 \\
\hline 99 & & LW5 & 5.2 & 61.9 & 72.1 & 61.9 & 72.1 & 44.6 & 55.8 & 37.8 & 46.9 \\
\hline 100 & & MW1 & 6.0 & 61.9 & 72.1 & 61.9 & 72.1 & 44.6 & 55.8 & 37.8 & 46.9 \\
\hline 101 & & MW2 & 5.7 & 61.9 & 72.1 & 61.9 & 72.1 & 44.6 & 55.8 & 37.8 & 46.9 \\
\hline 102 & & MW3 & 5.8 & 61.9 & 72.1 & 61.9 & 72.1 & 44.6 & 55.8 & 37.8 & 46.9 \\
\hline 103 & Li et al. (2016) & SF1 & 5.1 & 67.7 & 78.4 & 67.7 & 78.4 & - & - & - & - \\
\hline
\end{tabular}

Table B-11 Test Results of the RC Walls with Barbell and Flanged Cross-Section in the First Quadrant

\begin{tabular}{|c|c|c|c|c|c|c|c|c|c|c|}
\hline $\begin{array}{l}\text { Test } \\
\text { No. }\end{array}$ & Researcher & Spec ID & $\begin{array}{c}\Delta_{A} \\
\text { (in) }\end{array}$ & $\begin{array}{c}\Delta_{B} \\
\text { (in) }\end{array}$ & $\begin{array}{c}\Delta_{C} \\
\text { (in) } \\
\end{array}$ & $\begin{array}{l}\Delta_{D} \\
\text { (in) }\end{array}$ & $\begin{array}{c}F_{A} \\
\text { (kips) } \\
\end{array}$ & $\begin{array}{c}F_{B} \\
\text { (kips) } \\
\end{array}$ & $\begin{array}{c}F_{C} \\
\text { (kips) } \\
\end{array}$ & $\begin{array}{c}F_{D} \\
\text { (kips) }\end{array}$ \\
\hline 1 & \multirow{6}{*}{$\begin{array}{l}\text { Shiga et al. } \\
\text { (1973) }\end{array}$} & WB1 & 0.010 & 0.084 & 0.110 & 0.797 & 12 & 39 & 39 & 8 \\
\hline 2 & & WB2 & 0.010 & 0.079 & 0.110 & 0.879 & 12 & 41 & 41 & 8 \\
\hline 3 & & WB3 & 0.010 & 0.108 & 0.220 & 0.448 & 18 & 47 & 47 & 9 \\
\hline 4 & & WB6 & 0.010 & 0.078 & 0.110 & 0.702 & 20 & 54 & 54 & 11 \\
\hline 5 & & WB7 & 0.010 & 0.082 & 0.100 & 1.233 & 23 & 69 & 69 & 14 \\
\hline 6 & & WB8 & 0.004 & 0.072 & 0.185 & 0.357 & 16 & 63 & 63 & 13 \\
\hline 7 & \multirow{8}{*}{$\begin{array}{l}\text { Barda et al. } \\
\quad(1977)\end{array}$} & B1-1 & 0.010 & 0.176 & 0.210 & 2.265 & 99 & 271 & 271 & 54 \\
\hline 8 & & B2-1 & 0.010 & 0.137 & 0.250 & 0.968 & 63 & 223 & 223 & 45 \\
\hline 9 & & B3-2 & 0.060 & 0.139 & 0.210 & 0.776 & 163 & 258 & 258 & 52 \\
\hline 10 & & B4-3 & 0.040 & 0.163 & 0.190 & 0.678 & 90 & 218 & 218 & 44 \\
\hline 11 & & B5-4 & 0.050 & 0.190 & 0.240 & 2.852 & 110 & 154 & 154 & 31 \\
\hline 12 & & B6-4 & 0.040 & 0.149 & 0.350 & 1.397 & 126 & 190 & 190 & 38 \\
\hline 13 & & B7-5 & 0.040 & 0.149 & 0.350 & 1.397 & 126 & 190 & 190 & 38 \\
\hline 14 & & B8-5 & 0.050 & 0.367 & 0.550 & 1.456 & 79 & 195 & 195 & 39 \\
\hline 15 & Synge (1980) & WALL 3 & 0.120 & 0.220 & 0.220 & 1.584 & 147 & 176 & 176 & 35 \\
\hline
\end{tabular}


Table B-11 Test Results of the RC Walls with Barbell and Flanged Cross-Section in the First Quadrant (continued)

\begin{tabular}{|c|c|c|c|c|c|c|c|c|c|c|}
\hline $\begin{array}{l}\text { Test } \\
\text { No. }\end{array}$ & Researcher & Spec ID & $\begin{array}{c}\Delta_{A} \\
\text { (in) }\end{array}$ & $\begin{array}{l}\triangle_{B} \\
\text { (in) }\end{array}$ & $\begin{array}{c}\Delta_{C} \\
\text { (in) }\end{array}$ & $\begin{array}{l}\Delta_{D} \\
\text { (in) }\end{array}$ & $\begin{array}{c}F_{A} \\
\text { (kips) }\end{array}$ & $\begin{array}{c}F_{B} \\
\text { (kips) }\end{array}$ & $\begin{array}{c}F_{C} \\
\text { (kips) }\end{array}$ & $\begin{array}{c}F_{D} \\
\text { (kips) }\end{array}$ \\
\hline 16 & \multirow{3}{*}{$\begin{array}{c}\text { Yagishita } \\
\text { (1985) }\end{array}$} & CW-0.6-0.6-20a & 0.040 & 0.168 & 0.190 & 0.396 & 160 & 287 & 287 & 57 \\
\hline 17 & & CW-0.6-0.8-20a & 0.040 & 0.183 & 0.290 & 0.429 & 145 & 294 & 294 & 59 \\
\hline 18 & & CW-0.6-1.2-0 & 0.040 & 0.193 & 0.290 & 0.478 & 123 & 255 & 255 & 51 \\
\hline 19 & \multirow{8}{*}{ Chiba (1985) } & CW-0.6-1.2-20 & 0.040 & 0.220 & 0.270 & 0.563 & 158 & 353 & 353 & 71 \\
\hline 20 & & CW-0.6-0.6-20 & 0.020 & 0.147 & 0.180 & 0.636 & 98 & 261 & 261 & 52 \\
\hline 21 & & CW-0.6-0.8-20 & 0.010 & 0.193 & 0.280 & 0.629 & 71 & 319 & 319 & 64 \\
\hline 22 & & CW-0.6-1.6-20 & 0.030 & 0.228 & 0.280 & 0.450 & 143 & 372 & 372 & 74 \\
\hline 23 & & CW-0.6-2.0-20 & 0.030 & 0.234 & 0.290 & 0.557 & 139 & 412 & 412 & 82 \\
\hline 24 & & CW-0.6-1.2-40 & 0.040 & 0.151 & 0.260 & 0.349 & 162 & 320 & 320 & 64 \\
\hline 25 & & CW-0.4-1.2-20 & 0.050 & 0.131 & 0.200 & 0.267 & 217 & 345 & 345 & 69 \\
\hline 26 & & CW-0.8-1.2-20 & 0.040 & 0.313 & 0.390 & 0.682 & 119 & 299 & 299 & 60 \\
\hline 27 & \multirow{10}{*}{$\begin{array}{c}\text { Fukuzawa } \\
\text { (1985) }\end{array}$} & CW-0.6-0-20 & 0.020 & 0.191 & 0.280 & 2.815 & 89 & 268 & 268 & 54 \\
\hline 28 & & CW-0.6-0.3-20 & 0.010 & 0.192 & 0.270 & 0.523 & 72 & 292 & 292 & 58 \\
\hline 29 & & CW-0.6-2.4-20 & 0.010 & 0.224 & 0.280 & 1.493 & 75 & 448 & 448 & 90 \\
\hline 30 & & CW-0.6-2.8-20 & 0.050 & 0.232 & 0.280 & 1.430 & 149 & 388 & 388 & 78 \\
\hline 31 & & CW-0.6-0-0 & 0.020 & 0.158 & 0.370 & 0.370 & 47 & 169 & 169 & 169 \\
\hline 32 & & CW-0.6-0-40 & 0.020 & 0.171 & 0.260 & - & 97 & 320 & 320 & - \\
\hline 33 & & CW-0.6-0.6-0 & 0.030 & 0.210 & 0.280 & 1.473 & 79 & 255 & 255 & 51 \\
\hline 34 & & CW-0.6-0.6-40 & 0.010 & 0.189 & 0.280 & - & 93 & 379 & 379 & - \\
\hline 35 & & CW-0.4-0.6-20 & 0.010 & 0.135 & 0.190 & 0.335 & 98 & 418 & 418 & 84 \\
\hline 36 & & CW-0.8-0.6-20 & 0.050 & 0.292 & 0.380 & 1.699 & 141 & 286 & 286 & 57 \\
\hline 37 & \multirow{2}{*}{$\begin{array}{c}\text { Fukuzawa } \\
(1985)\end{array}$} & CW-0.4-2.0-20 & 0.010 & 0.143 & 0.190 & - & 91 & 460 & 460 & - \\
\hline 38 & & CW-0.8-2.0-20 & 0.030 & 0.317 & 0.500 & - & 97 & 370 & 370 & - \\
\hline 39 & \multirow{6}{*}{ Hatori (1985) } & CW-0.6-2-0 & 0.040 & 0.293 & 0.320 & 4.307 & 124 & 385 & 385 & 77 \\
\hline 40 & & CW-0.6-2-40 & 0.040 & 0.276 & 0.330 & 0.652 & 175 & 464 & 464 & 93 \\
\hline 41 & & CW-0.6-2-20B & 0.020 & 0.261 & 0.430 & - & 111 & 398 & 398 & - \\
\hline 42 & & CW-0.6-0.6-20L & 0.020 & 0.229 & 0.320 & 0.894 & 86 & 289 & 289 & 58 \\
\hline 43 & & CW-0.6-1.2-20L & 0.020 & 0.233 & 0.320 & 0.739 & 94 & 312 & 312 & 62 \\
\hline 44 & & CW-0.6-2-20L & 0.030 & 0.257 & 0.330 & 0.589 & 100 & 342 & 342 & 68 \\
\hline
\end{tabular}


Table B-11 Test Results of the RC Walls with Barbell and Flanged Cross-Section in the First Quadrant (continued)

\begin{tabular}{|c|c|c|c|c|c|c|c|c|c|c|}
\hline $\begin{array}{l}\text { Test } \\
\text { No. }\end{array}$ & Researcher & Spec ID & $\begin{array}{c}\Delta_{A} \\
\text { (in) }\end{array}$ & $\begin{array}{l}A_{B} \\
\text { (in) }\end{array}$ & $\begin{array}{c}\Delta_{C} \\
\text { (in) }\end{array}$ & $\begin{array}{l}\Delta_{D} \\
\text { (in) }\end{array}$ & $\begin{array}{c}F_{A} \\
\text { (kips) }\end{array}$ & $\begin{array}{c}F_{B} \\
\text { (kips) }\end{array}$ & $\begin{array}{c}F_{C} \\
\text { (kips) }\end{array}$ & $\begin{array}{c}F_{D} \\
\text { (kips) }\end{array}$ \\
\hline 45 & \multirow{3}{*}{ Taga (1985) } & WALL1 & 0.050 & 0.202 & 0.270 & 0.575 & 118 & 247 & 247 & 49 \\
\hline 46 & & WALL2 & 0.050 & 0.181 & 0.280 & 0.534 & 173 & 299 & 299 & 60 \\
\hline 47 & & WALL3 & 0.020 & 0.196 & 0.290 & 0.914 & 153 & 329 & 329 & 66 \\
\hline 48 & \multirow{7}{*}{ Maier (1985) } & S1 & 0.080 & 0.497 & 0.900 & 2.806 & 83 & 157 & 157 & 31 \\
\hline 49 & & S2 & 0.080 & 0.332 & 0.440 & - & 135 & 208 & 208 & - \\
\hline 50 & & S3 & 0.050 & 0.448 & 0.490 & - & 78 & 214 & 214 & - \\
\hline 51 & & S5 & 0.070 & 0.480 & 0.830 & - & 80 & 159 & 159 & - \\
\hline 52 & & S6 & 0.090 & 0.486 & 0.740 & 2.675 & 81 & 150 & 150 & 30 \\
\hline 53 & & S7 & 0.040 & 0.186 & 0.320 & 0.424 & 95 & 188 & 188 & 38 \\
\hline 54 & & S8 & 0.050 & 0.306 & 0.360 & 0.941 & 50 & 115 & 115 & 23 \\
\hline 55 & \multirow{5}{*}{$\begin{array}{c}\text { Tomi et al. } \\
\text { (1988) }\end{array}$} & T1-W87.5-N50 & 0.000 & 0.147 & 0.320 & 1.449 & 99 & 182 & 182 & 36 \\
\hline 56 & & T2-W87.5-N50 & 0.000 & 0.069 & 0.240 & 0.999 & 100 & 162 & 162 & 32 \\
\hline 57 & & H2-W87.5-N50 & 0.030 & 0.096 & 0.200 & 0.740 & 110 & 191 & 191 & 38 \\
\hline 58 & & H3-W87.5-N50 & 0.030 & 0.080 & 0.210 & 2.015 & 101 & 199 & 199 & 40 \\
\hline 59 & & H3-W40-N65 & 0.050 & 0.117 & 0.200 & 0.537 & 94 & 157 & 157 & 31 \\
\hline 60 & \multirow{8}{*}{$\begin{array}{c}\text { Sato et al. } \\
(1989)\end{array}$} & 36M8-30 & 0.020 & 0.227 & 0.540 & 3.250 & 166 & 439 & 439 & 88 \\
\hline 61 & & $36 \mathrm{M} 8-50$ & 0.030 & 0.310 & 0.530 & 7.516 & 198 & 461 & 461 & 92 \\
\hline 62 & & $24 M 8-30$ & 0.000 & 0.209 & 0.500 & 1.751 & 128 & 391 & 391 & 78 \\
\hline 63 & & $24 M 8-40$ & 0.000 & 0.188 & 0.400 & 0.955 & 118 & 396 & 396 & 79 \\
\hline 64 & & $24 M 8-50$ & 0.000 & 0.171 & 0.550 & - & 116 & 382 & 382 & - \\
\hline 65 & & 48M8-30 & 0.030 & 0.254 & 0.330 & 1.320 & 168 & 460 & 460 & 92 \\
\hline 66 & & $48 \mathrm{M} 8-40$ & 0.040 & 0.289 & 0.520 & - & 170 & 458 & 458 & - \\
\hline 67 & & 48M8-50 & 0.020 & 0.307 & 0.390 & 1.178 & 142 & 441 & 441 & 88 \\
\hline 68 & \multirow{3}{*}{$\begin{array}{l}\text { Mansour et al. } \\
\quad(1991)\end{array}$} & W3 & 0.030 & 0.190 & 0.290 & 0.810 & 28 & 87 & 87 & 17 \\
\hline 69 & & W4 & 0.010 & 0.124 & 0.190 & 0.503 & 35 & 94 & 94 & 19 \\
\hline 70 & & W5 & 0.020 & 0.151 & 0.230 & 0.503 & 34 & 81 & 81 & 16 \\
\hline 71 & \multirow{4}{*}{$\begin{array}{l}\text { Sheu and } \\
\text { Huang (1991) }\end{array}$} & SWB-4B & 0.060 & 0.247 & 0.410 & 1.027 & 39 & 75 & 75 & 15 \\
\hline 72 & & SWB-6A & 0.020 & 0.091 & 0.110 & 1.239 & 34 & 60 & 60 & 12 \\
\hline 73 & & SWB-8A & 0.040 & 0.189 & 0.430 & 0.873 & 40 & 73 & 73 & 15 \\
\hline 74 & & SWB-17A & 0.050 & 0.173 & 0.210 & 0.865 & 61 & 96 & 96 & 19 \\
\hline
\end{tabular}


Table B-11 Test Results of the RC Walls with Barbell and Flanged Cross-Section in the First Quadrant (continued)

\begin{tabular}{|c|c|c|c|c|c|c|c|c|c|c|}
\hline $\begin{array}{l}\text { Test } \\
\text { No. }\end{array}$ & Researcher & Spec ID & $\begin{array}{l}\Delta_{A} \\
\text { (in) } \\
\end{array}$ & $\begin{array}{l}\Delta_{B} \\
\text { (in) } \\
\end{array}$ & $\begin{array}{c}\Delta_{C} \\
\text { (in) } \\
\end{array}$ & $\begin{array}{l}\Delta_{D} \\
\text { (in) } \\
\end{array}$ & $\begin{array}{c}F_{A} \\
\text { (kips) } \\
\end{array}$ & $\begin{array}{c}F_{B} \\
\text { (kips) } \\
\end{array}$ & $\begin{array}{c}F_{C} \\
\text { (kips) } \\
\end{array}$ & $\begin{array}{c}F_{D} \\
\text { (kips) }\end{array}$ \\
\hline 75 & \multirow{4}{*}{$\begin{array}{c}\text { Mo and Kuo } \\
(1998)\end{array}$} & $\mathrm{H}-\mathrm{HZ} 4$ & 0.010 & 0.063 & 0.190 & 1.459 & 3 & 6 & 6 & 1 \\
\hline 76 & & $\mathrm{H}-\mathrm{HZ} 6$ & 0.000 & 0.061 & 0.062 & 0.764 & 5 & 7 & 7 & 1 \\
\hline 77 & & $\mathrm{H}-\mathrm{HZZ}$ & 0.000 & 0.087 & 0.100 & 0.669 & 4 & 7 & 7 & 1 \\
\hline 78 & & $\mathrm{H}-\mathrm{HZ} 10$ & 0.010 & 0.028 & 0.080 & 0.609 & 4 & 6 & 6 & 1 \\
\hline 79 & \multirow{2}{*}{$\begin{array}{l}\text { Sittipunt } \\
(2001)\end{array}$} & WALL 1 & 0.080 & 0.411 & 1.260 & 5.644 & 58 & 110 & 110 & 22 \\
\hline 80 & & WALL 2 & 0.120 & 0.683 & 1.280 & - & 59 & 140 & 140 & - \\
\hline 81 & \multirow{3}{*}{$\begin{array}{l}\text { Effendy et al. } \\
\quad(2006)\end{array}$} & SMAC & 0.130 & 0.269 & 0.390 & 0.910 & 123 & 201 & 201 & 40 \\
\hline 82 & & SMAS & 0.050 & 0.179 & 0.250 & 0.812 & 101 & 254 & 254 & 51 \\
\hline 83 & & SMAM & 0.110 & 0.191 & 0.210 & 0.782 & 188 & 233 & 233 & 47 \\
\hline 84 & \multirow{7}{*}{$\begin{array}{l}\text { Faravashany } \\
\text { et al. (2008) }\end{array}$} & HSCW1 & 0.060 & 0.390 & 0.460 & 0.813 & 63 & 166 & 166 & 33 \\
\hline 85 & & HSCW2 & 0.040 & 0.433 & 0.560 & - & 49 & 187 & 187 & - \\
\hline 86 & & HSCW3 & 0.030 & 0.353 & 0.430 & - & 46 & 141 & 141 & - \\
\hline 87 & & HSCW4 & 0.030 & 0.353 & 0.430 & - & 46 & 141 & 141 & - \\
\hline 88 & & HSCW5 & 0.090 & 0.443 & 0.580 & - & 68 & 178 & 178 & - \\
\hline 89 & & HSCW6 & 0.070 & 0.565 & 0.630 & - & 71 & 168 & 168 & - \\
\hline 90 & & HSCW7 & 0.090 & 0.501 & 0.590 & 0.913 & 60 & 180 & 180 & 36 \\
\hline 91 & $\begin{array}{c}\text { Park et al. } \\
\text { (2013) }\end{array}$ & S4 & 0.060 & 0.573 & 0.700 & 1.187 & 182 & 578 & 578 & 116 \\
\hline 92 & \multirow{3}{*}{$\begin{array}{l}\text { Almeida et al. } \\
\quad(2015)\end{array}$} & TW1 & 0.040 & 0.184 & 0.880 & - & 15 & 33 & 33 & - \\
\hline 93 & & TW2 & 0.020 & 0.228 & 0.870 & - & 62 & 150 & 150 & - \\
\hline 94 & & TW3 & 0.030 & 0.196 & 0.610 & 3.115 & 48 & 146 & 146 & 29 \\
\hline 95 & \multirow{8}{*}{ Li et al. (2015) } & LW1 & 0.050 & 0.360 & 0.730 & 1.347 & 41 & 83 & 83 & 17 \\
\hline 96 & & LW2 & 0.070 & 0.435 & 0.880 & 2.860 & 52 & 133 & 133 & 27 \\
\hline 97 & & LW3 & 0.080 & 0.337 & 0.400 & 4.614 & 56 & 118 & 118 & 24 \\
\hline 98 & & LW4 & 0.050 & 0.271 & 0.770 & 1.590 & 34 & 83 & 83 & 17 \\
\hline 99 & & LW5 & 0.050 & 0.335 & 0.600 & 1.904 & 43 & 118 & 118 & 24 \\
\hline 100 & & MW1 & 0.110 & 0.592 & 1.250 & - & 45 & 96 & 96 & - \\
\hline 101 & & MW2 & 0.110 & 0.471 & 1.090 & 1.966 & 42 & 89 & 89 & 18 \\
\hline 102 & & MW3 & 0.070 & 0.554 & 1.210 & 3.359 & 28 & 93 & 93 & 19 \\
\hline 103 & Li et al. (2016) & SF1 & 0.110 & 0.274 & 0.440 & 2.095 & 58 & 86 & 86 & 17 \\
\hline
\end{tabular}


Table B-12 Test Results of the RC Walls with Barbell and Flanged Cross-Section in the Third Quadrant

\begin{tabular}{|c|c|c|c|c|c|c|c|c|c|c|}
\hline $\begin{array}{l}\text { Test } \\
\text { No. }\end{array}$ & Researcher & Spec ID & $\begin{array}{c}\Delta_{A} \\
\text { (in) }\end{array}$ & $\begin{array}{c}\Delta_{B} \\
\text { (in) }\end{array}$ & $\begin{array}{c}\Delta_{C} \\
\text { (in) } \\
\end{array}$ & $\begin{array}{l}\Delta_{D} \\
\text { (in) }\end{array}$ & $\begin{array}{c}F_{A} \\
\text { (kips) } \\
\end{array}$ & $\begin{array}{c}F_{B} \\
\text { (kips) } \\
\end{array}$ & $\begin{array}{c}F_{C} \\
\text { (kips) } \\
\end{array}$ & $\begin{array}{c}F_{D} \\
\text { (kips) }\end{array}$ \\
\hline 1 & \multirow{6}{*}{$\begin{array}{l}\text { Shiga et al. } \\
\text { (1973) }\end{array}$} & WB1 & -0.010 & -0.083 & -0.110 & -0.324 & -12 & -38 & -38 & -8 \\
\hline 2 & & WB2 & -0.010 & -0.080 & -0.110 & -0.553 & -12 & -46 & -46 & -9 \\
\hline 3 & & WB3 & -0.010 & -0.075 & -0.110 & -0.545 & -18 & -48 & -48 & -10 \\
\hline 4 & & WB6 & -0.010 & -0.104 & -0.120 & -1.521 & -20 & -52 & -52 & -10 \\
\hline 5 & & WB7 & -0.010 & -0.106 & -0.110 & -0.388 & -22 & -67 & -67 & -13 \\
\hline 6 & & WB8 & -0.004 & -0.081 & -0.114 & -0.247 & -16 & -59 & -59 & -12 \\
\hline 7 & \multirow{8}{*}{$\begin{array}{c}\text { Barda et al. } \\
\text { (1977) }\end{array}$} & B1-1 & -0.010 & -0.176 & -0.210 & -2.265 & -99 & -271 & -271 & -54 \\
\hline 8 & & B2-1 & -0.010 & -0.137 & -0.250 & -0.968 & -63 & -223 & -223 & -45 \\
\hline 9 & & B3-2 & -0.060 & -0.139 & -0.210 & -0.776 & -163 & -258 & -258 & -52 \\
\hline 10 & & B4-3 & -0.040 & -0.163 & -0.190 & -0.678 & -90 & -218 & -218 & -44 \\
\hline 11 & & B5-4 & -0.050 & -0.190 & -0.240 & -2.852 & -110 & -154 & -154 & -31 \\
\hline 12 & & B6-4 & -0.040 & -0.149 & -0.350 & -1.397 & -126 & -190 & -190 & -38 \\
\hline 13 & & B7-5 & -0.040 & -0.149 & -0.350 & -1.397 & -126 & -190 & -190 & -38 \\
\hline 14 & & B8-5 & -0.050 & -0.367 & -0.550 & -1.456 & -79 & -195 & -195 & -39 \\
\hline 15 & Synge (1980) & WALL 3 & -0.140 & -0.330 & -0.330 & -0.995 & -125 & -155 & -155 & -31 \\
\hline 16 & \multirow{3}{*}{$\begin{array}{c}\text { Yagishita } \\
\text { (1985) }\end{array}$} & CW-0.6-0.6-20a & -0.050 & -0.167 & -0.180 & -0.409 & -149 & -261 & -261 & -52 \\
\hline 17 & & CW-0.6-0.8-20a & -0.050 & -0.199 & -0.290 & -0.450 & -150 & -290 & -290 & -58 \\
\hline 18 & & CW-0.6-1.2-0 & -0.100 & -0.215 & -0.280 & -0.465 & -176 & -255 & -255 & -51 \\
\hline 19 & \multirow{8}{*}{ Chiba (1985) } & CW-0.6-1.2-20 & -0.050 & -0.229 & -0.280 & -0.516 & -174 & -335 & -335 & -67 \\
\hline 20 & & CW-0.6-0.6-20 & -0.040 & -0.180 & -0.200 & - & -145 & -257 & -257 & - \\
\hline 21 & & CW-0.6-0.8-20 & -0.020 & -0.195 & -0.280 & -0.499 & -124 & -324 & -324 & -65 \\
\hline 22 & & CW-0.6-1.6-20 & -0.020 & -0.211 & -0.280 & -0.456 & -105 & -359 & -359 & -72 \\
\hline 23 & & CW-0.6-2.0-20 & -0.050 & -0.226 & -0.280 & -0.522 & -178 & -396 & -396 & -79 \\
\hline 24 & & CW-0.6-1.2-40 & -0.050 & -0.160 & -0.190 & -0.372 & -200 & -316 & -316 & -63 \\
\hline 25 & & CW-0.4-1.2-20 & -0.060 & -0.121 & -0.170 & -0.305 & -223 & -319 & -319 & -64 \\
\hline 26 & & CW-0.8-1.2-20 & -0.030 & -0.283 & -0.380 & -0.585 & -119 & -278 & -278 & -56 \\
\hline 27 & \multirow{7}{*}{$\begin{array}{c}\text { Fukuzawa } \\
\text { (1985) }\end{array}$} & CW-0.6-0-20 & -0.020 & -0.167 & -0.280 & -1.290 & -54 & -256 & -256 & -51 \\
\hline 28 & & CW-0.6-0.3-20 & -0.010 & -0.175 & -0.280 & - & -49 & -265 & -265 & - \\
\hline 29 & & CW-0.6-2.4-20 & -0.020 & -0.224 & -0.280 & -0.695 & -90 & -431 & -431 & -86 \\
\hline 30 & & CW-0.6-2.8-20 & -0.010 & -0.211 & -0.280 & -0.770 & -53 & -356 & -356 & -71 \\
\hline 31 & & CW-0.6-0-0 & -0.010 & -0.144 & -0.190 & -1.779 & -30 & -156 & -156 & -31 \\
\hline 32 & & CW-0.6-0-40 & -0.010 & -0.128 & -0.180 & -0.477 & -73 & -302 & -302 & -60 \\
\hline 33 & & CW-0.6-0.6-0 & -0.020 & -0.216 & -0.290 & -5.740 & -59 & -242 & -242 & -48 \\
\hline
\end{tabular}


Table B-12 Test Results of the RC Walls with Barbell and Flanged Cross-Section in the Third Quadrant (continued)

\begin{tabular}{|c|c|c|c|c|c|c|c|c|c|c|}
\hline $\begin{array}{l}\text { Test } \\
\text { No. }\end{array}$ & Researcher & Spec ID & $\begin{array}{c}\Delta_{A} \\
\text { (in) }\end{array}$ & $\begin{array}{c}\triangle_{B} \\
\text { (in) }\end{array}$ & $\begin{array}{c}\Delta_{C} \\
\text { (in) }\end{array}$ & $\begin{array}{l}\Delta_{D} \\
\text { (in) }\end{array}$ & $\begin{array}{c}F_{A} \\
\text { (kips) }\end{array}$ & $\begin{array}{c}F_{B} \\
\text { (kips) }\end{array}$ & $\begin{array}{c}F_{C} \\
\text { (kips) }\end{array}$ & $\begin{array}{c}F_{D} \\
\text { (kips) }\end{array}$ \\
\hline 34 & \multirow{3}{*}{$\begin{array}{c}\text { Fukuzawa } \\
\text { (1985) }\end{array}$} & CW-0.6-0.6-40 & -0.020 & -0.183 & -0.280 & - & -105 & -350 & -350 & - \\
\hline 35 & & CW-0.4-0.6-20 & -0.010 & -0.124 & -0.190 & -0.290 & -77 & -404 & -404 & -81 \\
\hline 36 & & CW-0.8-0.6-20 & -0.020 & -0.234 & -0.380 & -7.822 & -85 & -258 & -258 & -52 \\
\hline 37 & \multirow{2}{*}{$\begin{array}{c}\text { Fukuzawa } \\
\text { (1985) }\end{array}$} & CW-0.4-2.0-20 & -0.010 & -0.127 & -0.180 & - & -81 & -434 & -434 & - \\
\hline 38 & & CW-0.8-2.0-20 & -0.030 & -0.278 & -0.480 & - & -90 & -330 & -330 & - \\
\hline 39 & \multirow{6}{*}{ Hatori (1985) } & CW-0.6-2-0 & -0.030 & -0.277 & -0.320 & -1.329 & -101 & -360 & -360 & -72 \\
\hline 40 & & CW-0.6-2-40 & -0.020 & -0.254 & -0.320 & -0.537 & -118 & -447 & -447 & -89 \\
\hline 41 & & CW-0.6-2-20B & -0.020 & -0.271 & -0.320 & -0.900 & -96 & -396 & -396 & -79 \\
\hline 42 & & CW-0.6-0.6-20L & -0.030 & -0.221 & -0.320 & -0.534 & -91 & -272 & -272 & -54 \\
\hline 43 & & CW-0.6-1.2-20L & -0.020 & -0.252 & -0.320 & -0.512 & -93 & -297 & -297 & -59 \\
\hline 44 & & CW-0.6-2-20L & -0.040 & -0.234 & -0.320 & -0.551 & -124 & -314 & -314 & -63 \\
\hline 45 & \multirow{3}{*}{ Taga (1985) } & WALL1 & -0.160 & -0.213 & -0.290 & -0.415 & -233 & -240 & -240 & -48 \\
\hline 46 & & WALL2 & -0.030 & -0.180 & -0.190 & -1.335 & -149 & -275 & -275 & -55 \\
\hline 47 & & WALL3 & -0.050 & -0.212 & -0.290 & - & -168 & -308 & -308 & - \\
\hline 48 & \multirow{7}{*}{ Maier (1985) } & S1 & -0.080 & -0.493 & -0.900 & -2.801 & -83 & -157 & -157 & -31 \\
\hline 49 & & S2 & -0.080 & -0.334 & -0.440 & - & -135 & -208 & -208 & - \\
\hline 50 & & S3 & -0.050 & -0.447 & -0.490 & - & -78 & -214 & -214 & - \\
\hline 51 & & S5 & -0.090 & -0.360 & -0.730 & - & -75 & -149 & -149 & - \\
\hline 52 & & S6 & -0.090 & -0.488 & -0.740 & -2.672 & -80 & -150 & -150 & -30 \\
\hline 53 & & S7 & -0.020 & -0.160 & -0.280 & - & -79 & -194 & -194 & - \\
\hline 54 & & S8 & -0.050 & -0.306 & -0.360 & -0.940 & -50 & -115 & -115 & -23 \\
\hline 55 & \multirow{5}{*}{$\begin{array}{c}\text { Tomi et al. } \\
\text { (1988) }\end{array}$} & T1-W87.5-N50 & -0.020 & -0.180 & -0.320 & -2.052 & -123 & -174 & -174 & -35 \\
\hline 56 & & T2-W87.5-N50 & -0.090 & -0.167 & -0.280 & -0.649 & -131 & -167 & -167 & -33 \\
\hline 57 & & H2-W87.5-N50 & 0.000 & -0.078 & -0.170 & -1.065 & -117 & -177 & -177 & -35 \\
\hline 58 & & H3-W87.5-N50 & -0.010 & -0.076 & -0.190 & - & -101 & -198 & -198 & - \\
\hline 59 & & H3-W40-N65 & -0.020 & -0.094 & -0.110 & -0.508 & -101 & -151 & -151 & -30 \\
\hline 60 & \multirow{5}{*}{$\begin{array}{l}\text { Sato et al. } \\
\text { (1989) }\end{array}$} & 36M8-30 & -0.020 & -0.208 & -0.550 & - & -170 & -406 & -406 & - \\
\hline 61 & & 36M8-50 & -0.020 & -0.252 & -0.330 & -5.452 & -150 & -442 & -442 & -88 \\
\hline 62 & & $24 M 8-30$ & -0.010 & -0.196 & -0.540 & - & -88 & -374 & -374 & - \\
\hline 63 & & $24 \mathrm{M} 8-40$ & 0.000 & -0.144 & -0.310 & - & -74 & -374 & -374 & - \\
\hline 64 & & 24M8-50 & -0.010 & -0.177 & -0.550 & - & -86 & -370 & -370 & - \\
\hline
\end{tabular}


Table B-12 Test Results of the RC Walls with Barbell and Flanged Cross-Section in the Third Quadrant (continued)

\begin{tabular}{|c|c|c|c|c|c|c|c|c|c|c|}
\hline $\begin{array}{l}\text { Test } \\
\text { No. }\end{array}$ & Researcher & Spec ID & $\begin{array}{c}\Delta_{A} \\
\text { (in) }\end{array}$ & $\begin{array}{c}\triangle_{B} \\
\text { (in) }\end{array}$ & $\begin{array}{c}\Delta_{C} \\
\text { (in) }\end{array}$ & $\begin{array}{l}\Delta_{D} \\
\text { (in) }\end{array}$ & $\begin{array}{c}F_{A} \\
\text { (kips) }\end{array}$ & $\begin{array}{c}F_{B} \\
\text { (kips) }\end{array}$ & $\begin{array}{c}F_{C} \\
\text { (kips) }\end{array}$ & $\begin{array}{c}F_{D} \\
\text { (kips) }\end{array}$ \\
\hline 65 & \multirow{3}{*}{$\begin{array}{l}\text { Sato et al. } \\
\text { (1989) }\end{array}$} & $48 \mathrm{M} 8-30$ & -0.030 & -0.227 & -0.310 & - & -164 & -444 & -444 & - \\
\hline 66 & & 48M8-40 & -0.020 & -0.264 & -0.320 & - & -158 & -438 & -438 & - \\
\hline 67 & & $48 \mathrm{M} 8-50$ & -0.030 & -0.272 & -0.320 & - & -156 & -419 & -419 & - \\
\hline 68 & \multirow{3}{*}{$\begin{array}{l}\text { Mansour et al. } \\
\quad(1991)\end{array}$} & W3 & -0.040 & -0.352 & -0.450 & - & -46 & -93 & -93 & - \\
\hline 69 & & W4 & -0.020 & -0.209 & -0.360 & -0.570 & -34 & -115 & -115 & -23 \\
\hline 70 & & W5 & -0.020 & -0.154 & -0.280 & -0.991 & -23 & -69 & -69 & -14 \\
\hline 71 & \multirow{4}{*}{$\begin{array}{l}\text { Sheu and } \\
\text { Huang (1991) }\end{array}$} & SWB-4B & -0.090 & -0.294 & -0.330 & -1.116 & -46 & -69 & -69 & -14 \\
\hline 72 & & SWB-6A & -0.080 & -0.220 & -0.220 & -0.709 & -53 & -60 & -60 & -12 \\
\hline 73 & & SWB-8A & -0.170 & -0.279 & -0.390 & - & -34 & -42 & -42 & - \\
\hline 74 & & SWB-17A & -0.080 & -0.100 & -0.100 & -0.185 & -30 & -31 & -31 & -6 \\
\hline 75 & \multirow{4}{*}{$\begin{array}{l}\text { Mo and Kuo } \\
(1998)\end{array}$} & $\mathrm{H}-\mathrm{HZ} 4$ & -0.010 & -0.106 & -0.150 & -1.048 & -4 & -7 & -7 & -1 \\
\hline 76 & & $\mathrm{H}-\mathrm{HZC}$ & -0.030 & -0.122 & -0.160 & -0.466 & -5 & -7 & -7 & -1 \\
\hline 77 & & $\mathrm{H}-\mathrm{HZZ}$ & -0.010 & -0.101 & -0.090 & -1.158 & -5 & -8 & -8 & -2 \\
\hline 78 & & $\mathrm{H}-\mathrm{HZ} 10$ & -0.010 & -0.139 & -0.180 & -0.331 & -3 & -6 & -6 & -1 \\
\hline 79 & \multirow{2}{*}{ Sittipunt (2001) } & WALL 1 & -0.290 & -0.993 & -1.250 & -2.113 & -91 & -98 & -98 & -20 \\
\hline 80 & & WALL 2 & -0.160 & -0.495 & -1.250 & - & -61 & -115 & -115 & - \\
\hline 81 & \multirow{3}{*}{$\begin{array}{l}\text { Effendy et al. } \\
\qquad(2006)\end{array}$} & SMAC & -0.090 & -0.169 & -0.400 & -0.874 & -91 & -140 & -140 & -28 \\
\hline 82 & & SMAS & -0.070 & -0.223 & -0.280 & -0.759 & -82 & -237 & -237 & -47 \\
\hline 83 & & SMAM & -0.140 & -0.202 & -0.280 & -0.798 & -124 & -180 & -180 & -36 \\
\hline 84 & \multirow{7}{*}{$\begin{array}{l}\text { Faravashany et } \\
\text { al. (2008) }\end{array}$} & HSCW1 & 0.060 & 0.390 & 0.460 & 0.813 & 63 & 166 & 166 & 33 \\
\hline 85 & & HSCW2 & 0.040 & 0.433 & 0.560 & - & 49 & 187 & 187 & - \\
\hline 86 & & HSCW3 & 0.030 & 0.353 & 0.430 & - & 46 & 141 & 141 & - \\
\hline 87 & & HSCW4 & 0.030 & 0.353 & 0.430 & - & 46 & 141 & 141 & - \\
\hline 88 & & HSCW5 & 0.090 & 0.443 & 0.580 & - & 68 & 178 & 178 & - \\
\hline 89 & & HSCW6 & 0.070 & 0.565 & 0.630 & - & 71 & 168 & 168 & - \\
\hline 90 & & HSCW7 & 0.090 & 0.501 & 0.590 & 0.913 & 60 & 180 & 180 & 36 \\
\hline 91 & $\begin{array}{l}\text { Park et al. } \\
\text { (2013) }\end{array}$ & S4 & -0.170 & -0.664 & -0.710 & -1.148 & -278 & -562 & -562 & -112 \\
\hline 92 & \multirow{3}{*}{$\begin{array}{l}\text { Almeida et al. } \\
\quad(2015)\end{array}$} & TW1 & -0.030 & -0.357 & -0.640 & - & -17 & -39 & -39 & - \\
\hline 93 & & TW2 & -0.060 & -0.370 & -0.670 & - & -107 & -171 & -171 & - \\
\hline 94 & & TW3 & -0.080 & -0.334 & -0.640 & -1.449 & -82 & -169 & -169 & -34 \\
\hline
\end{tabular}


Table B-12 Test Results of the RC Walls with Barbell and Flanged Cross-Section in the Third Quadrant (continued)

\begin{tabular}{|c|c|c|c|c|c|c|c|c|c|c|}
\hline $\begin{array}{l}\text { Test } \\
\text { No. }\end{array}$ & Researcher & Spec ID & $\begin{array}{c}\Delta_{A} \\
\text { (in) } \\
\end{array}$ & $\begin{array}{c}\Delta_{B} \\
\text { (in) }\end{array}$ & $\begin{array}{c}\Delta_{C} \\
\text { (in) } \\
\end{array}$ & $\begin{array}{l}\Delta_{D} \\
\text { (in) }\end{array}$ & $\begin{array}{c}F_{A} \\
\text { (kips) } \\
\end{array}$ & $\begin{array}{c}F_{B} \\
\text { (kips) } \\
\end{array}$ & $\begin{array}{c}F_{C} \\
\text { (kips) } \\
\end{array}$ & $\begin{array}{c}F_{D} \\
\text { (kips) }\end{array}$ \\
\hline 95 & \multirow{8}{*}{ Li et al. (2015) } & LW1 & -0.080 & -0.342 & -0.860 & - & -43 & -76 & -76 & - \\
\hline 96 & & LW2 & -0.090 & -0.471 & -0.850 & - & -58 & -128 & -128 & - \\
\hline 97 & & LW3 & -0.080 & -0.376 & -0.580 & - & -48 & -124 & -124 & - \\
\hline 98 & & LW4 & -0.070 & -0.368 & -0.590 & -3.513 & -41 & -82 & -82 & -16 \\
\hline 99 & & LW5 & -0.100 & -0.375 & -0.580 & -4.129 & -77 & -123 & -123 & -25 \\
\hline 100 & & MW1 & -0.140 & -0.673 & -1.270 & - & -48 & -96 & -96 & - \\
\hline 101 & & MW2 & -0.130 & -0.520 & -1.130 & -3.530 & -46 & -90 & -90 & -18 \\
\hline 102 & & MW3 & -0.120 & -0.662 & -1.260 & -4.561 & -45 & -88 & -88 & -18 \\
\hline 103 & Li et al. (2016) & SF1 & -0.150 & -0.389 & -0.780 & -7.749 & -55 & -89 & -89 & -18 \\
\hline
\end{tabular}

\section{B.2 Statistical Evaluation of the Measured Data}

A statistical evaluation of the measured data is summarized in Table B-13 through Table B-22. The design variables and the wall type are listed in the first and second columns, respectively. The equation of the linear trend line, presented in Table B-13 through Table $\mathrm{B}-10$, is $Y=M X+C$, where $Y$ is the predicted output variable and $X$ is the design variable. The values of the parameters $M$ and $C$ are listed in the third and fourth columns of the tables, respectively; $N$ is the number of data points used in the regression analysis; parameter $\mu$ is the average of the response variable; SSE is the residual sum of squares that is a measure of the difference between the test data and the trend line; SSR is the regression sum of squares that is the sum of the squares of the deviations of the predicted values from the mean value of the measured responses; and SST is the total sum of squares that is calculated as the sum of SSE and SSR. The coefficient of determination, $R^{2}$, is a measure of how close the data are to the fitted regression line. The standard deviation and the coefficient of variation are listed in the last two columns of the tables, respectively. 
Table B-13 Statistical Summary: $\delta_{A}$

\begin{tabular}{|c|c|c|c|c|c|c|c|c|c|c|c|}
\hline Var. & $\begin{array}{l}\text { Wall } \\
\text { Type } \\
\end{array}$ & $M$ & $C$ & $N$ & $\mu$ & SSE & SSR & SST & $R^{2}$ & $\sigma$ & $c_{v}$ \\
\hline \multirow{2}{*}{$M / V l_{w}$} & ReB. & 0.0419 & 0.082 & 136 & 0.12 & 0.82 & 0.03 & 0.85 & 0.034 & 0.079 & 0.66 \\
\hline & $\mathrm{Fl}$. & 0.0240 & 0.076 & 103 & 0.09 & 0.52 & 0.01 & 0.52 & 0.013 & 0.071 & 0.76 \\
\hline \multirow{2}{*}{$f_{c}^{\prime}$} & ReB. & 0.0028 & 0.031 & 136 & 0.12 & 0.71 & 0.13 & 0.85 & 0.159 & 0.079 & 0.66 \\
\hline & $\mathrm{Fl}$. & 0.0002 & 0.086 & 103 & 0.09 & 0.52 & 0.00 & 0.52 & 0.004 & 0.071 & 0.76 \\
\hline \multirow{2}{*}{$\rho_{v} f_{y v}$} & ReB. & 0.0000 & 0.117 & 125 & 0.12 & 0.75 & 0.00 & 0.75 & 0.000 & 0.077 & 0.66 \\
\hline & $\mathrm{Fl}$. & -0.0006 & 0.098 & 99 & 0.10 & 0.51 & 0.00 & 0.51 & 0.000 & 0.072 & 0.75 \\
\hline \multirow{2}{*}{$\rho_{h} f_{y h}$} & ReB. & -0.0013 & 0.122 & 123 & 0.12 & 0.77 & 0.00 & 0.77 & 0.001 & 0.079 & 0.67 \\
\hline & Fl. & 0.0022 & 0.089 & 98 & 0.10 & 0.51 & 0.00 & 0.51 & 0.004 & 0.072 & 0.75 \\
\hline \multirow{2}{*}{$\rho_{v b} f_{y v b}$} & ReB. & -0.0008 & 0.142 & 86 & 0.13 & 0.59 & 0.01 & 0.60 & 0.021 & 0.084 & 0.66 \\
\hline & $\mathrm{Fl}$. & 0.0015 & 0.081 & 103 & 0.09 & 0.51 & 0.01 & 0.52 & 0.027 & 0.071 & 0.76 \\
\hline \multirow{2}{*}{$P / A_{g} f_{c}^{\prime}$} & ReB. & -0.0038 & 0.134 & 136 & 0.12 & 0.79 & 0.06 & 0.85 & 0.068 & 0.079 & 0.66 \\
\hline & $\mathrm{Fl}$. & -0.0035 & 0.111 & 103 & 0.09 & 0.49 & 0.04 & 0.52 & 0.069 & 0.071 & 0.76 \\
\hline
\end{tabular}

Table B-14 Statistical Summary: $V_{A} / V_{C}$

\begin{tabular}{|c|c|c|c|c|c|c|c|c|c|c|c|}
\hline Var. & $\begin{array}{l}\text { Wall } \\
\text { Type }\end{array}$ & $M$ & $C$ & $N$ & $\mu$ & SSE & SSR & SST & $R^{2}$ & $\sigma$ & $c_{v}$ \\
\hline \multirow{2}{*}{$M / V l_{w}$} & ReB. & -0.0566 & 0.515 & 137 & 0.46 & 2.60 & 0.05 & 2.65 & 0.020 & 0.139 & 0.30 \\
\hline & $\mathrm{Fl}$. & 0.0235 & 0.428 & 103 & 0.45 & 2.46 & 0.01 & 2.47 & 0.003 & 0.155 & 0.35 \\
\hline \multirow{2}{*}{$f_{c}^{\prime}$} & ReB. & 0.0006 & 0.445 & 137 & 0.46 & 2.65 & 0.01 & 2.65 & 0.002 & 0.139 & 0.30 \\
\hline & $\mathrm{Fl}$. & -0.0018 & 0.511 & 103 & 0.45 & 2.36 & 0.11 & 2.47 & 0.044 & 0.155 & 0.35 \\
\hline \multirow{2}{*}{$\rho_{v} f_{y v}$} & ReB. & -0.0172 & 0.524 & 126 & 0.46 & 2.22 & 0.32 & 2.54 & 0.127 & 0.142 & 0.31 \\
\hline & $\mathrm{Fl}$. & -0.0204 & 0.521 & 99 & 0.45 & 2.05 & 0.24 & 2.29 & 0.104 & 0.152 & 0.34 \\
\hline \multirow{2}{*}{$\rho_{h} f_{y h}$} & ReB. & -0.0270 & 0.545 & 124 & 0.47 & 2.22 & 0.29 & 2.51 & 0.116 & 0.142 & 0.31 \\
\hline & $\mathrm{Fl}$. & -0.0114 & 0.492 & 98 & 0.45 & 2.26 & 0.06 & 2.32 & 0.025 & 0.154 & 0.34 \\
\hline \multirow{2}{*}{$\rho_{v b} f_{y v b}$} & ReB. & -0.0036 & 0.527 & 87 & 0.46 & 1.33 & 0.24 & 1.57 & 0.151 & 0.134 & 0.29 \\
\hline & $\mathrm{Fl}$. & 0.0004 & 0.443 & 103 & 0.45 & 2.47 & 0.00 & 2.47 & 0.000 & 0.155 & 0.35 \\
\hline \multirow{2}{*}{$P / A_{g} f_{c}^{\prime}$} & ReB. & -0.0042 & 0.479 & 137 & 0.46 & 2.58 & 0.07 & 2.65 & 0.026 & 0.139 & 0.30 \\
\hline & $\mathrm{Fl}$. & -0.0091 & 0.490 & 103 & 0.45 & 2.23 & 0.24 & 2.47 & 0.096 & 0.155 & 0.35 \\
\hline
\end{tabular}


Table B-15 Statistical Summary: $\delta_{B}$

\begin{tabular}{|c|c|c|c|c|c|c|c|c|c|c|c|}
\hline Var. & $\begin{array}{l}\text { Wall } \\
\text { Type }\end{array}$ & $M$ & $C$ & $N$ & $\mu$ & SSE & SSR & SST & $R^{2}$ & $\sigma$ & $c_{v}$ \\
\hline \multirow{2}{*}{$M / V l_{w}$} & ReB. & 0.0587 & 0.548 & 136 & 0.60 & 6.73 & 0.06 & 6.79 & 0.008 & 0.223 & 0.37 \\
\hline & $\mathrm{Fl}$. & 0.2733 & 0.298 & 103 & 0.51 & 3.15 & 0.91 & 4.06 & 0.224 & 0.199 & 0.39 \\
\hline \multirow{2}{*}{$f_{c}^{\prime}$} & ReB. & 0.0068 & 0.386 & 136 & 0.60 & 6.01 & 0.77 & 6.79 & 0.114 & 0.223 & 0.37 \\
\hline & $\mathrm{Fl}$. & 0.0061 & 0.295 & 103 & 0.51 & 2.88 & 1.19 & 4.06 & 0.292 & 0.199 & 0.39 \\
\hline \multirow{2}{*}{$\rho_{v} f_{y v}$} & ReB. & 0.0036 & 0.586 & 125 & 0.60 & 6.56 & 0.01 & 6.58 & 0.002 & 0.229 & 0.38 \\
\hline & $\mathrm{Fl}$. & 0.0247 & 0.428 & 99 & 0.51 & 3.62 & 0.35 & 3.97 & 0.087 & 0.200 & 0.39 \\
\hline \multirow{2}{*}{$\rho_{h} f_{y h}$} & ReB. & 0.0078 & 0.581 & 123 & 0.60 & 6.40 & 0.02 & 6.42 & 0.004 & 0.229 & 0.38 \\
\hline & $\mathrm{Fl}$. & 0.0154 & 0.465 & 98 & 0.52 & 3.86 & 0.11 & 3.96 & 0.027 & 0.201 & 0.39 \\
\hline \multirow{2}{*}{$\rho_{v b} f_{y v b}$} & ReB. & 0.0018 & 0.600 & 86 & 0.63 & 4.20 & 0.06 & 4.26 & 0.014 & 0.223 & 0.35 \\
\hline & $\mathrm{Fl}$. & 0.0092 & 0.425 & 103 & 0.51 & 3.51 & 0.56 & 4.06 & 0.137 & 0.199 & 0.39 \\
\hline \multirow{2}{*}{$P / A_{g} f_{c}^{\prime}$} & ReB. & -0.0040 & 0.615 & 136 & 0.60 & 6.72 & 0.06 & 6.79 & 0.009 & 0.223 & 0.37 \\
\hline & $\mathrm{Fl}$. & -0.0022 & 0.520 & 103 & 0.51 & 4.05 & 0.01 & 4.06 & 0.003 & 0.199 & 0.39 \\
\hline
\end{tabular}

Table B-16 Statistical Summary: $\delta_{C}$

\begin{tabular}{|c|c|c|c|c|c|c|c|c|c|c|c|}
\hline Var. & $\begin{array}{l}\text { Wall } \\
\text { Type } \\
\end{array}$ & $M$ & $C$ & $N$ & $\mu$ & SSE & SSR & SST & $R^{2}$ & $\sigma$ & $c_{v}$ \\
\hline \multirow{2}{*}{$M / V l_{w}$} & ReB. & 0.0427 & 1.078 & 137 & 1.12 & 33.06 & 0.03 & 33.09 & 0.001 & 0.491 & 0.44 \\
\hline & $\mathrm{Fl}$. & 0.4066 & 0.464 & 103 & 0.78 & 7.56 & 2.02 & 9.58 & 0.211 & 0.305 & 0.39 \\
\hline \multirow{2}{*}{$f_{c}^{\prime}$} & ReB. & 0.0064 & 0.914 & 137 & 1.12 & 32.40 & 0.69 & 33.09 & 0.021 & 0.491 & 0.44 \\
\hline & $\mathrm{Fl}$. & 0.0063 & 0.558 & 103 & 0.78 & 8.32 & 1.26 & 9.58 & 0.131 & 0.305 & 0.39 \\
\hline \multirow{2}{*}{$\rho_{v} f_{y v}$} & ReB. & -0.0417 & 1.285 & 126 & 1.14 & 29.50 & 1.89 & 31.39 & 0.060 & 0.499 & 0.44 \\
\hline & $\mathrm{Fl}$. & 0.0056 & 0.768 & 99 & 0.79 & 9.37 & 0.02 & 9.39 & 0.002 & 0.308 & 0.39 \\
\hline \multirow{2}{*}{$\rho_{h} f_{y h}$} & ReB. & -0.0278 & 1.223 & 124 & 1.14 & 30.38 & 0.31 & 30.69 & 0.010 & 0.498 & 0.44 \\
\hline & $\mathrm{Fl}$. & -0.0040 & 0.804 & 98 & 3.33 & 9.28 & 0.01 & 9.29 & 0.001 & 0.308 & 0.09 \\
\hline \multirow{2}{*}{$\rho_{v b} f_{y v b}$} & ReB. & -0.0037 & 1.256 & 87 & 1.19 & 26.16 & 0.25 & 26.41 & 0.009 & 0.551 & 0.46 \\
\hline & $\mathrm{Fl}$. & 0.0069 & 0.715 & 103 & 0.78 & 9.26 & 0.32 & 9.58 & 0.033 & 0.305 & 0.39 \\
\hline \multirow{2}{*}{$P / A_{g} f_{c}^{\prime}$} & ReB. & -0.0090 & 1.149 & 137 & 1.12 & 32.77 & 0.32 & 33.09 & 0.010 & 0.491 & 0.44 \\
\hline & $\mathrm{Fl}$. & -0.0080 & 0.818 & 103 & 0.78 & 9.40 & 0.18 & 9.58 & 0.019 & 0.305 & 0.39 \\
\hline
\end{tabular}


Table B-17 Statistical Summary: $\delta_{D^{0}}$

\begin{tabular}{|c|c|c|c|c|c|c|c|c|c|c|c|}
\hline Var. & $\begin{array}{l}\text { Wall } \\
\text { Type }\end{array}$ & $M$ & $C$ & $N$ & $\mu$ & SSE & SSR & SST & $R^{2}$ & $\sigma$ & $c_{v}$ \\
\hline \multirow{2}{*}{$M / V l_{w}$} & ReB. & -0.1841 & 2.791 & 98 & 2.63 & 222.71 & 0.45 & 223.16 & 0.002 & 1.509 & 0.57 \\
\hline & $\mathrm{Fl}$. & 0.7508 & 1.542 & 71 & 2.08 & 141.26 & 4.31 & 145.56 & 0.030 & 1.432 & 0.69 \\
\hline \multirow{2}{*}{$f_{c}^{\prime}$} & ReB. & 0.0167 & 2.110 & 98 & 2.63 & 220.09 & 3.07 & 223.16 & 0.014 & 1.509 & 0.57 \\
\hline & $\mathrm{Fl}$. & -0.0065 & 2.280 & 71 & 2.08 & 144.94 & 0.62 & 145.56 & 0.004 & 1.432 & 0.69 \\
\hline \multirow{2}{*}{$\rho_{v} f_{y v}$} & ReB. & -0.1418 & 3.114 & 89 & 2.68 & 198.83 & 11.00 & 209.83 & 0.052 & 1.535 & 0.57 \\
\hline & $\mathrm{Fl}$. & -0.0547 & 2.240 & 67 & 2.06 & 126.66 & 1.15 & 127.81 & 0.009 & 1.381 & 0.67 \\
\hline \multirow{2}{*}{$\rho_{h} f_{y h}$} & ReB. & -0.0603 & 2.916 & 88 & 2.76 & 205.26 & 0.92 & 206.18 & 0.004 & 1.531 & 0.56 \\
\hline & $\mathrm{Fl}$. & -0.0764 & 2.363 & 66 & 2.11 & 133.80 & 1.90 & 135.71 & 0.014 & 1.434 & 0.68 \\
\hline \multirow{2}{*}{$\rho_{v b} f_{y v b}$} & ReB. & -0.0373 & 3.490 & 58 & 2.80 & 151.87 & 22.74 & 174.61 & 0.130 & 1.735 & 0.62 \\
\hline & $\mathrm{Fl}$. & 0.0172 & 1.902 & 71 & 2.08 & 143.92 & 1.64 & 145.56 & 0.011 & 1.432 & 0.69 \\
\hline \multirow{2}{*}{$P / A_{g} f_{c}^{\prime}$} & ReB. & -0.0911 & 2.841 & 98 & 2.63 & 208.78 & 14.38 & 223.16 & 0.064 & 1.509 & 0.57 \\
\hline & $\mathrm{Fl}$. & -0.0761 & 2.386 & 71 & 2.08 & 135.02 & 10.55 & 145.56 & 0.072 & 1.432 & 0.69 \\
\hline
\end{tabular}

Table B-18 Statistical Summary: $\delta_{D^{20}}$

\begin{tabular}{|c|c|c|c|c|c|c|c|c|c|c|c|}
\hline Var. & $\begin{array}{l}\text { Wall } \\
\text { Type } \\
\end{array}$ & $M$ & $C$ & $N$ & $\mu$ & SSE & SSR & SST & $R^{2}$ & $\sigma$ & $c_{v}$ \\
\hline \multirow{2}{*}{$M / V l_{w}$} & ReB. & -0.1626 & 2.355 & 99 & 2.22 & 197.35 & 0.35 & 197.70 & 0.002 & 1.413 & 0.64 \\
\hline & $\mathrm{Fl}$. & 0.6151 & 1.269 & 71 & 1.71 & 96.19 & 2.89 & 99.08 & 0.029 & 1.181 & 0.69 \\
\hline \multirow{2}{*}{$f_{c}^{\prime}$} & ReB. & 0.0282 & 1.326 & 99 & 2.22 & 188.76 & 8.94 & 197.70 & 0.045 & 1.413 & 0.64 \\
\hline & $\mathrm{Fl}$. & -0.0034 & 1.816 & 71 & 1.71 & 98.91 & 0.18 & 99.08 & 0.002 & 1.181 & 0.69 \\
\hline \multirow{2}{*}{$\rho_{v} f_{y v}$} & ReB. & -0.1255 & 2.644 & 90 & 2.26 & 179.38 & 8.62 & 188.00 & 0.046 & 1.445 & 0.64 \\
\hline & $\mathrm{Fl}$. & -0.0466 & 1.847 & 67 & 1.69 & 85.71 & 0.83 & 86.55 & 0.010 & 1.137 & 0.67 \\
\hline \multirow{2}{*}{$\rho_{h} f_{y h}$} & ReB. & -0.0696 & 2.507 & 89 & 2.32 & 183.41 & 1.24 & 184.65 & 0.007 & 1.440 & 0.62 \\
\hline & $\mathrm{Fl}$. & -0.0661 & 1.959 & 66 & 1.74 & 91.56 & 1.43 & 92.98 & 0.015 & 1.187 & 0.68 \\
\hline \multirow{2}{*}{$\rho_{v b} f_{y v b}$} & ReB. & -0.0339 & 2.979 & 59 & 2.36 & 138.86 & 18.96 & 157.82 & 0.120 & 1.636 & 0.69 \\
\hline & $\mathrm{Fl}$. & 0.0120 & 1.586 & 71 & 1.71 & 98.29 & 0.79 & 99.08 & 0.008 & 1.181 & 0.69 \\
\hline \multirow{2}{*}{$P / A_{g} f_{c}^{\prime}$} & ReB. & -0.0820 & 2.401 & 99 & 2.22 & 186.00 & 11.70 & 197.70 & 0.059 & 1.413 & 0.64 \\
\hline & $\mathrm{Fl}$. & -0.0611 & 1.956 & 71 & 1.71 & 92.28 & 6.81 & 99.08 & 0.069 & 1.181 & 0.69 \\
\hline
\end{tabular}


Table B-19 Statistical Summary: $V_{c} / d_{w} t_{w}$

\begin{tabular}{|c|c|c|c|c|c|c|c|c|c|c|c|}
\hline Var. & $\begin{array}{l}\text { Wall } \\
\text { Type }\end{array}$ & $M$ & $C$ & $N$ & $\mu$ & SSE & SSR & SST & $R^{2}$ & $\sigma$ & $c_{v}$ \\
\hline \multirow{2}{*}{$M / V l_{w}$} & ReB. & 0.9672 & 2.399 & 137 & 3.28 & 285.05 & 15.35 & 300.39 & 0.051 & 1.481 & 0.45 \\
\hline & $\mathrm{Fl}$. & -1.1562 & 6.832 & 103 & 5.94 & 758.32 & 16.33 & 774.65 & 0.021 & 2.742 & 0.46 \\
\hline \multirow{2}{*}{$f_{c}^{\prime}$} & ReB. & 0.0738 & 0.947 & 137 & 3.28 & 209.34 & 91.06 & 300.39 & 0.303 & 1.481 & 0.45 \\
\hline & Fl. & 0.0831 & 3.013 & 103 & 5.94 & 555.22 & 219.43 & 774.65 & 0.283 & 2.742 & 0.46 \\
\hline \multirow{2}{*}{$\rho_{v} f_{y v}$} & ReB. & 0.2019 & 2.537 & 126 & 3.23 & 210.05 & 44.38 & 254.43 & 0.174 & 1.421 & 0.44 \\
\hline & $\mathrm{Fl}$. & 0.7238 & 3.409 & 99 & 5.96 & 464.52 & 297.58 & 762.09 & 0.390 & 2.775 & 0.47 \\
\hline \multirow{2}{*}{$\rho_{h} f_{y h}$} & ReB. & 0.3153 & 2.341 & 124 & 3.27 & 210.14 & 40.02 & 250.16 & 0.160 & 1.420 & 0.43 \\
\hline & $\mathrm{Fl}$. & 0.6177 & 3.856 & 98 & 5.91 & 588.25 & 169.41 & 757.67 & 0.224 & 2.781 & 0.47 \\
\hline \multirow{2}{*}{$\rho_{v b} f_{y v b}$} & ReB. & 0.0485 & 2.434 & 87 & 3.29 & 161.47 & 42.36 & 203.83 & 0.208 & 1.531 & 0.47 \\
\hline & $\mathrm{Fl}$. & 0.0387 & 5.577 & 103 & 5.94 & 764.73 & 9.92 & 774.65 & 0.013 & 2.742 & 0.46 \\
\hline \multirow{2}{*}{$P / A_{g} f_{c}^{\prime}$} & ReB. & 0.0464 & 3.112 & 137 & 3.28 & 291.92 & 8.47 & 300.39 & 0.028 & 1.481 & 0.45 \\
\hline & Fl. & 0.2065 & 4.934 & 103 & 5.94 & 651.78 & 122.87 & 774.65 & 0.159 & 2.742 & 0.46 \\
\hline
\end{tabular}

Table B-20 Statistical Summary: $K_{e} / K_{t}$

\begin{tabular}{|c|c|c|c|c|c|c|c|c|c|c|c|}
\hline Var. & $\begin{array}{l}\text { Wall } \\
\text { Type }\end{array}$ & $M$ & $C$ & $N$ & $\mu$ & SSE & SSR & SST & $R^{2}$ & $\sigma$ & $c_{v}$ \\
\hline \multirow{2}{*}{$M / V l_{w}$} & ReB. & 8.3324 & 8.432 & 133 & 16.00 & 9547.12 & 1069.08 & 10616.20 & 0.101 & 8.934 & 0.56 \\
\hline & $\mathrm{Fl}$. & -8.2047 & 24.754 & 94 & 18.35 & 7103.55 & 769.55 & 7873.10 & 0.098 & 9.152 & 0.50 \\
\hline \multirow{2}{*}{$f_{c}^{\prime}$} & ReB. & -0.2333 & 23.429 & 133 & 16.00 & 9749.21 & 867.00 & 10616.21 & 0.082 & 8.934 & 0.56 \\
\hline & $\mathrm{Fl}$. & -0.0722 & 20.945 & 94 & 18.35 & 7713.79 & 159.31 & 7873.10 & 0.020 & 9.152 & 0.50 \\
\hline \multirow{2}{*}{$\rho_{v} f_{y v}$} & ReB. & -0.0635 & 16.330 & 122 & 16.11 & 10318.50 & 4.32 & 10322.82 & 0.000 & 9.199 & 0.57 \\
\hline & $\mathrm{Fl}$. & 0.8131 & 15.307 & 90 & 18.34 & 7179.06 & 346.49 & 7525.55 & 0.046 & 9.144 & 0.50 \\
\hline \multirow{2}{*}{$\rho_{h} f_{y h}$} & ReB. & 0.7854 & 13.986 & 120 & 16.31 & 10000.00 & 236.51 & 10236.51 & 0.023 & 9.236 & 0.57 \\
\hline & $\mathrm{Fl}$. & 0.8040 & 15.436 & 89 & 18.26 & 7280.76 & 263.29 & 7544.05 & 0.035 & 9.207 & 0.50 \\
\hline \multirow{2}{*}{$\rho_{v b} f_{y v b}$} & ReB. & 0.0842 & 15.658 & 84 & 17.14 & 6541.62 & 127.55 & 6669.17 & 0.019 & 8.910 & 0.52 \\
\hline & $\mathrm{Fl}$. & -0.0770 & 19.083 & 94 & 18.35 & 7836.42 & 36.68 & 7873.10 & 0.005 & 9.152 & 0.50 \\
\hline \multirow{2}{*}{$P / A_{g} f_{c}^{\prime}$} & ReB. & 0.8214 & 13.182 & 133 & 16.00 & 8142.32 & 2473.88 & 10616.20 & 0.233 & 8.934 & 0.56 \\
\hline & $\mathrm{Fl}$. & 0.9001 & 14.178 & 94 & 18.35 & 6180.40 & 1692.70 & 7873.10 & 0.215 & 9.152 & 0.50 \\
\hline
\end{tabular}


Table B-21 Statistical Summary: $K_{y} / K_{t}$

\begin{tabular}{|c|c|c|c|c|c|c|c|c|c|c|c|}
\hline Var. & $\begin{array}{l}\text { Wall } \\
\text { Type }\end{array}$ & $M$ & C & $N$ & $\mu$ & SSE & SSR & SST & $R^{2}$ & $\sigma$ & $c_{v}$ \\
\hline \multirow{2}{*}{$M / V l_{w}$} & ReB. & 3.8951 & 0.632 & 137 & 4.16 & 498.42 & 248.91 & 747.33 & 0.333 & 2.336 & 0.56 \\
\hline & FI. & -2.3087 & 6.343 & 103 & 4.55 & 436.46 & 65.11 & 501.56 & 0.130 & 2.207 & 0.48 \\
\hline \multirow{2}{*}{$f_{c}^{\prime}$} & ReB. & 0.0081 & 3.910 & 137 & 4.16 & 746.24 & 1.09 & 747.33 & 0.001 & 2.336 & 0.56 \\
\hline & FI. & -0.0173 & 5.160 & 103 & 4.55 & 492.09 & 9.47 & 501.56 & 0.019 & 2.207 & 0.48 \\
\hline \multirow{2}{*}{$\rho_{v} f_{y v}$} & ReB. & 0.3025 & 3.050 & 126 & 4.09 & 604.36 & 99.67 & 704.04 & 0.142 & 2.364 & 0.58 \\
\hline & $\mathrm{Fl}$. & 0.2898 & 3.493 & 99 & 4.51 & 414.38 & 47.71 & 462.09 & 0.103 & 2.160 & 0.48 \\
\hline \multirow{2}{*}{$\rho_{h} f_{y h}$} & ReB. & 0.4012 & 2.931 & 124 & 4.11 & 640.85 & 64.77 & 705.62 & 0.092 & 2.385 & 0.58 \\
\hline & $\mathrm{Fl}$. & 0.2796 & 3.513 & 98 & 4.44 & 427.25 & 34.72 & 461.97 & 0.075 & 2.171 & 0.49 \\
\hline \multirow{2}{*}{$\rho_{v b} f_{y v b}$} & ReB. & 0.0467 & 3.448 & 87 & 4.27 & 287.61 & 39.36 & 326.97 & 0.120 & 1.939 & 0.45 \\
\hline & $\mathrm{Fl}$. & -0.0131 & 4.674 & 103 & 4.55 & 500.43 & 1.14 & 501.56 & 0.002 & 2.207 & 0.48 \\
\hline \multirow{2}{*}{$P / A_{g} f_{c}^{\prime}$} & ReB. & 0.1580 & 3.605 & 137 & 4.16 & 648.91 & 98.42 & 747.33 & 0.132 & 2.336 & 0.56 \\
\hline & $\mathrm{Fl}$. & 0.2237 & 3.468 & 103 & 4.55 & 357.40 & 144.17 & 501.56 & 0.287 & 2.207 & 0.48 \\
\hline
\end{tabular}

Table B-22 Statistical Summary: $K_{p} / K_{t}$

\begin{tabular}{|c|c|c|c|c|c|c|c|c|c|c|c|}
\hline Var. & $\begin{array}{l}\text { Wall } \\
\text { Type }\end{array}$ & $M$ & $C$ & $N$ & $\mu$ & SSE & SSR & SST & $R^{2}$ & $\sigma$ & $c_{v}$ \\
\hline \multirow{2}{*}{$M / V l_{w}$} & ReB. & 2.0283 & 0.195 & 93 & 1.92 & 503.00 & 53.24 & 556.24 & 0.096 & 2.446 & 1.27 \\
\hline & $\mathrm{Fl}$. & -1.9651 & 4.270 & 68 & 2.85 & 511.54 & 29.07 & 540.61 & 0.054 & 2.820 & 0.99 \\
\hline \multirow{2}{*}{$f_{c}^{\prime}$} & ReB. & 0.0442 & 0.537 & 93 & 1.92 & 539.07 & 17.17 & 556.24 & 0.031 & 2.446 & 1.27 \\
\hline & $\mathrm{Fl}$. & 0.0392 & 1.627 & 68 & 2.85 & 517.68 & 22.93 & 540.61 & 0.042 & 2.820 & 0.99 \\
\hline \multirow{2}{*}{$\rho_{v} f_{y v}$} & ReB. & 0.4524 & 0.491 & 84 & 1.81 & 436.50 & 85.97 & 522.46 & 0.165 & 2.494 & 1.37 \\
\hline & $\mathrm{Fl}$. & 0.5275 & 1.185 & 66 & 2.93 & 428.56 & 100.10 & 528.67 & 0.189 & 2.830 & 0.97 \\
\hline \multirow{2}{*}{$\rho_{h} f_{y h}$} & ReB. & 0.1696 & 1.290 & 83 & 1.74 & 493.30 & 6.79 & 500.10 & 0.014 & 2.455 & 1.41 \\
\hline & $\mathrm{Fl}$. & 0.5854 & 1.021 & 65 & 2.91 & 430.86 & 103.32 & 534.18 & 0.193 & 2.867 & 0.98 \\
\hline \multirow{2}{*}{$\rho_{v b} f_{y v b}$} & ReB. & 0.1009 & 0.167 & 55 & 1.86 & 131.87 & 127.20 & 259.07 & 0.491 & 2.170 & 1.17 \\
\hline & $\mathrm{Fl}$. & -0.0171 & 3.032 & 68 & 2.85 & 539.02 & 1.59 & 540.61 & 0.003 & 2.820 & 0.99 \\
\hline \multirow{2}{*}{$P / A_{g} f_{c}^{\prime}$} & ReB. & 0.1677 & 1.560 & 93 & 1.92 & 513.28 & 42.97 & 556.24 & 0.077 & 2.446 & 1.27 \\
\hline & $\mathrm{Fl}$. & 0.2798 & 1.740 & 68 & 2.85 & 404.88 & 135.73 & 540.61 & 0.251 & 2.820 & 0.99 \\
\hline
\end{tabular}

\section{B.3 Calculated Parameters for Cyclic Hysteresis Models}

The Modified IKP (MIKP) model is implemented in MATLAB and used to simulate the cyclic responses of $63 \mathrm{RC}$ walls. Table B-23 presents the values of the pinching $\left(k_{f}, k_{d}, \zeta\right)$ and deterioration $\left(\gamma_{u n l, 1}, \gamma_{u n l, 2}, \gamma_{a}\right)$ parameters calibrated from the digitized force-displacement relationships. The rate parameter was set equal to 1.0 in all cases. 


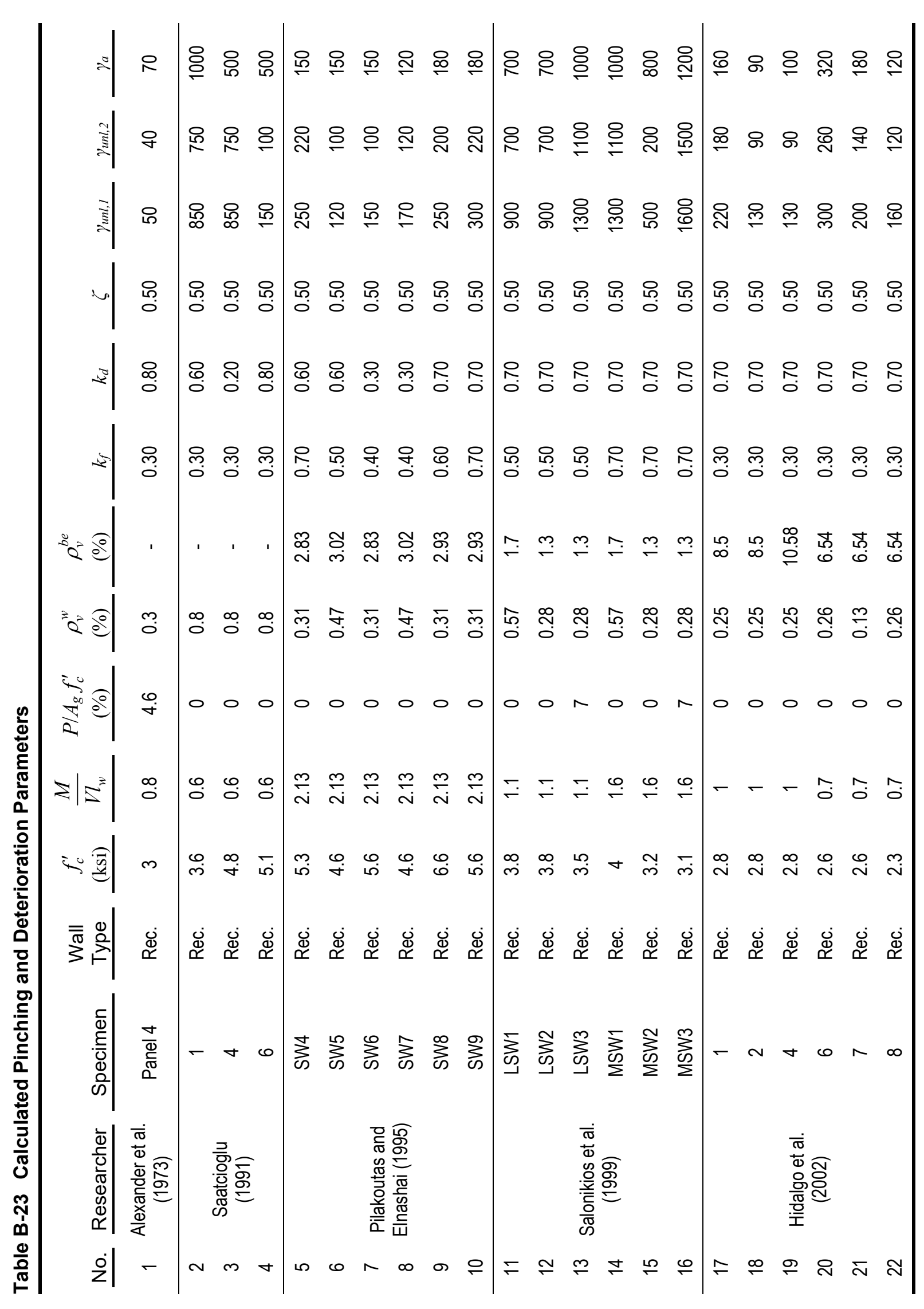




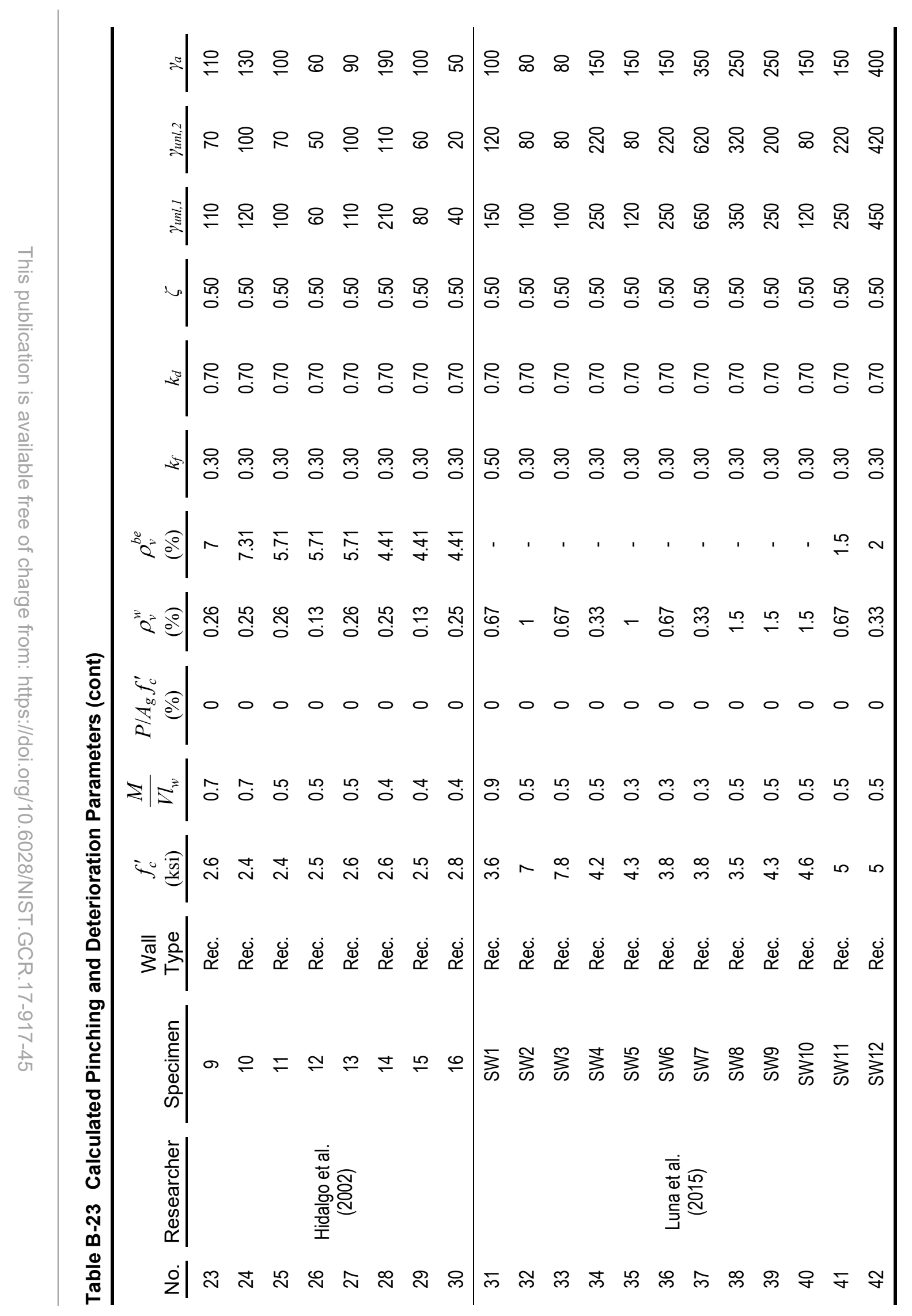




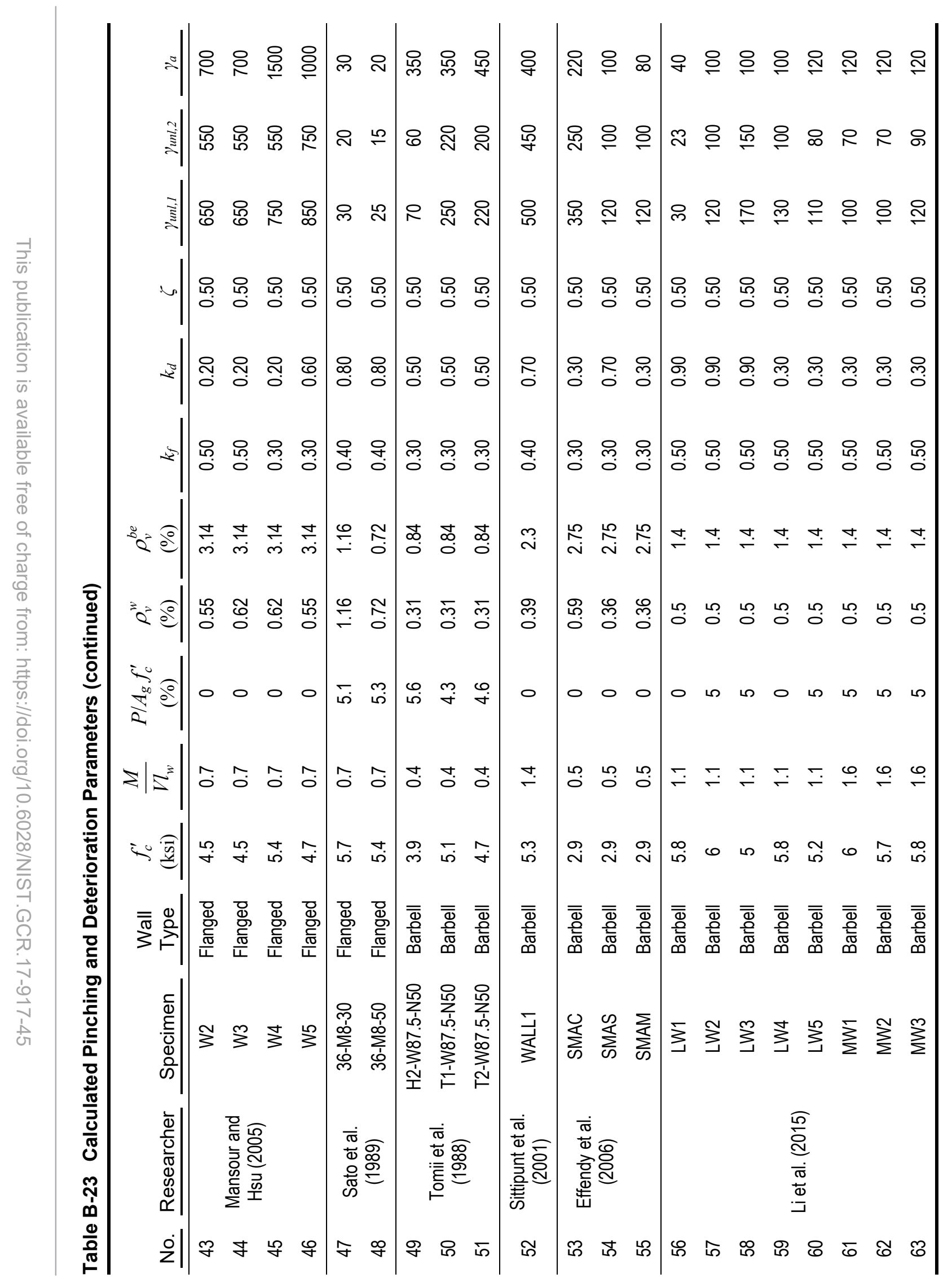




\section{Appendix C}

\section{Shear-Controlled Reinforced Concrete Walls - Data Analysis}

In order to develop recommendations for backbone curves and control points for modeling, the data presented in Appendix B were re-binned to calculate the following: (1) drift ratios at cracking, yielding, peak load, $0 \%, 20 \%, 40 \%$, and $80 \%$ residual strength; (2) forces at cracking and peak load; and (3) initial stiffness, yield stiffness, and post-peak stiffness. Peak load was defined as the average of the maximum strengths in the first and third quadrants of loading.

Figures $\mathrm{C}-1$ through $\mathrm{C}-12$ present data by bin and wall geometry. Table $\mathrm{C}-1$ summarizes the results by wall geometry and data bin (as described in Chapter 8), including mean (average) values, standard deviation of the natural logarithm, and percentiles, as well as the mean ACI-predicted to mean measured peak strength for the data bin. 

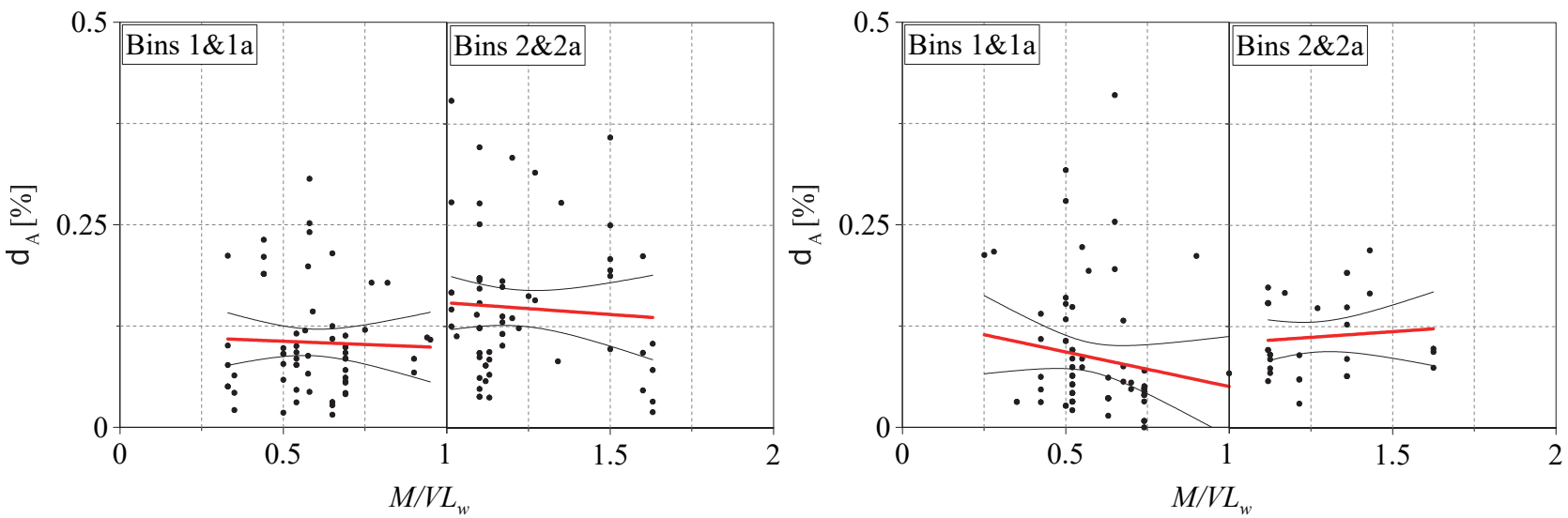

(a) $M / V I_{w}$ : rectangular walls (left panel), and barbell and flanged walls (right panel)
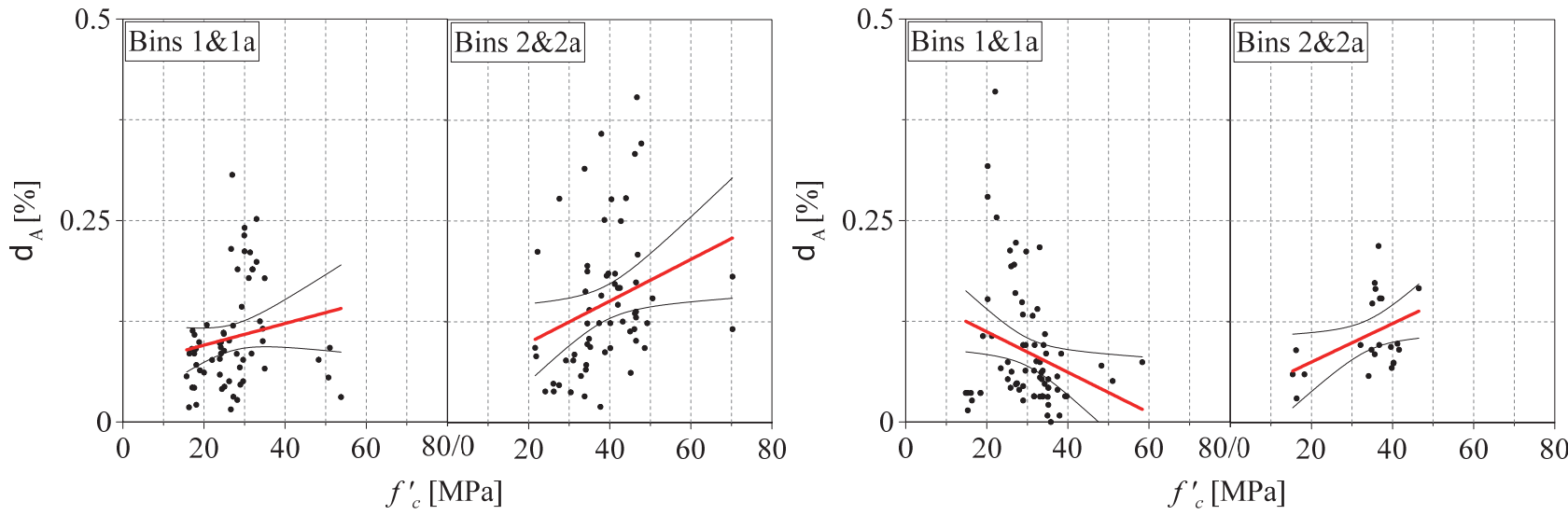

(b) $f_{c}^{\prime}$ : rectangular walls (left panel), and barbell and flanged walls (right panel)
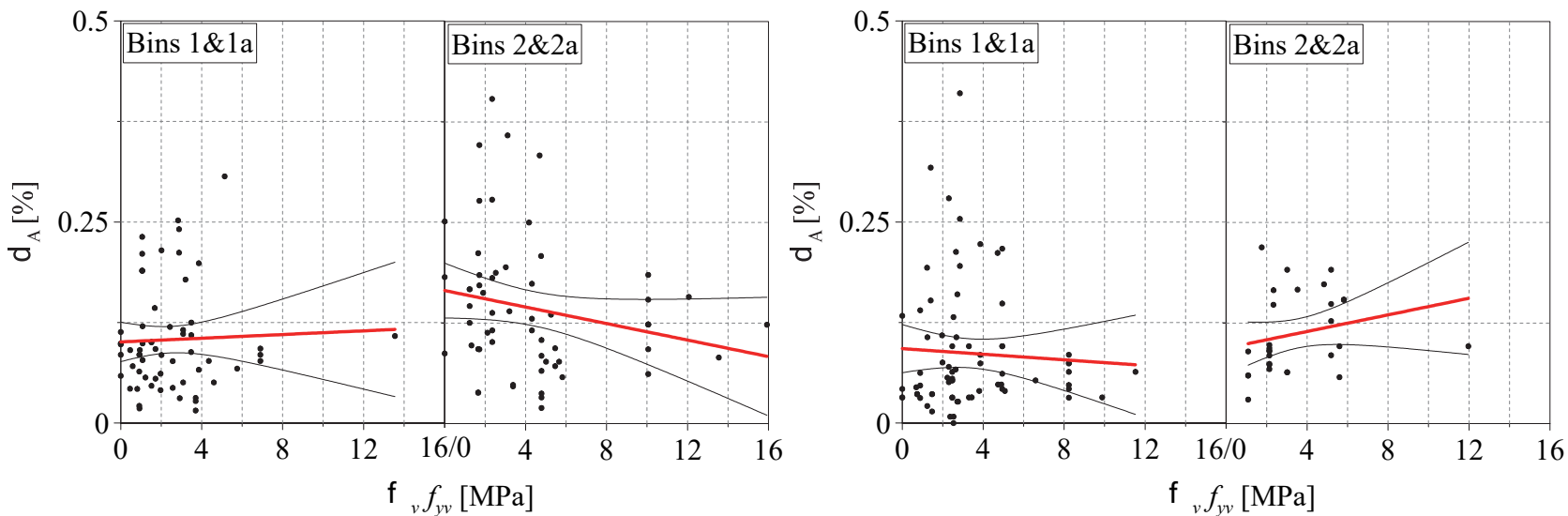

(c) $\rho_{v} f_{y v}$ : rectangular walls (left panel), and barbell and flanged walls (right panel)

Figure C-1 Effect of design variables on $\delta_{A}$. 

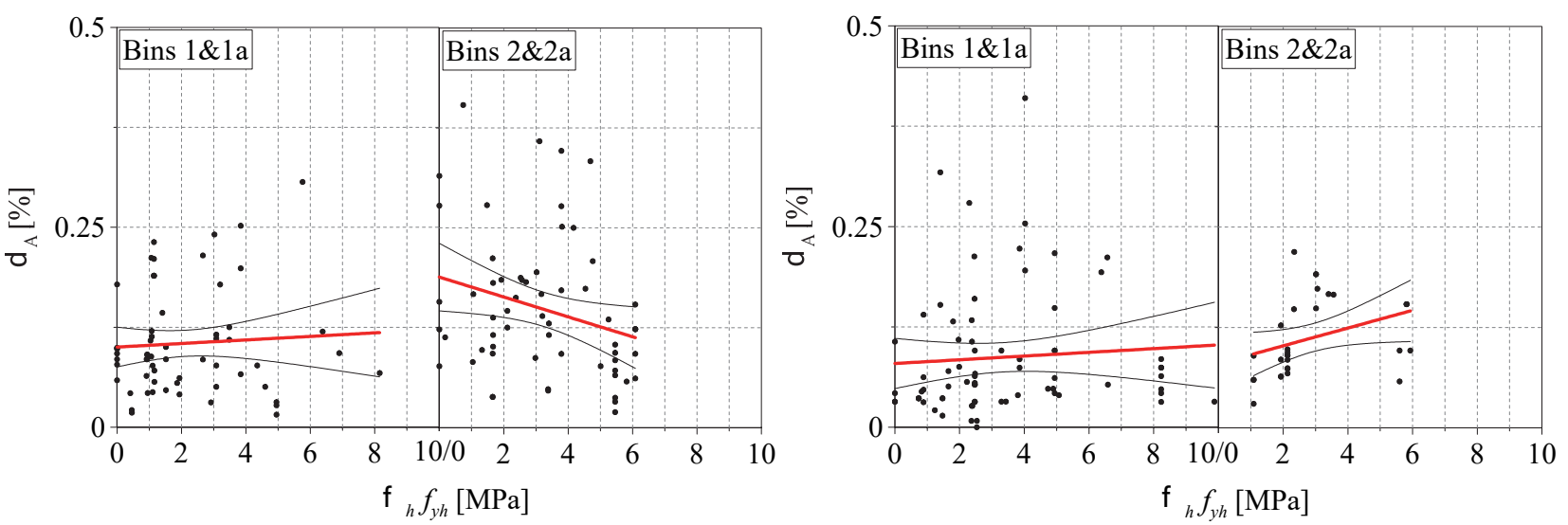

(d) $\rho_{h} f_{v h}$ : rectangular walls (left panel), and barbell and flanged walls (right panel)
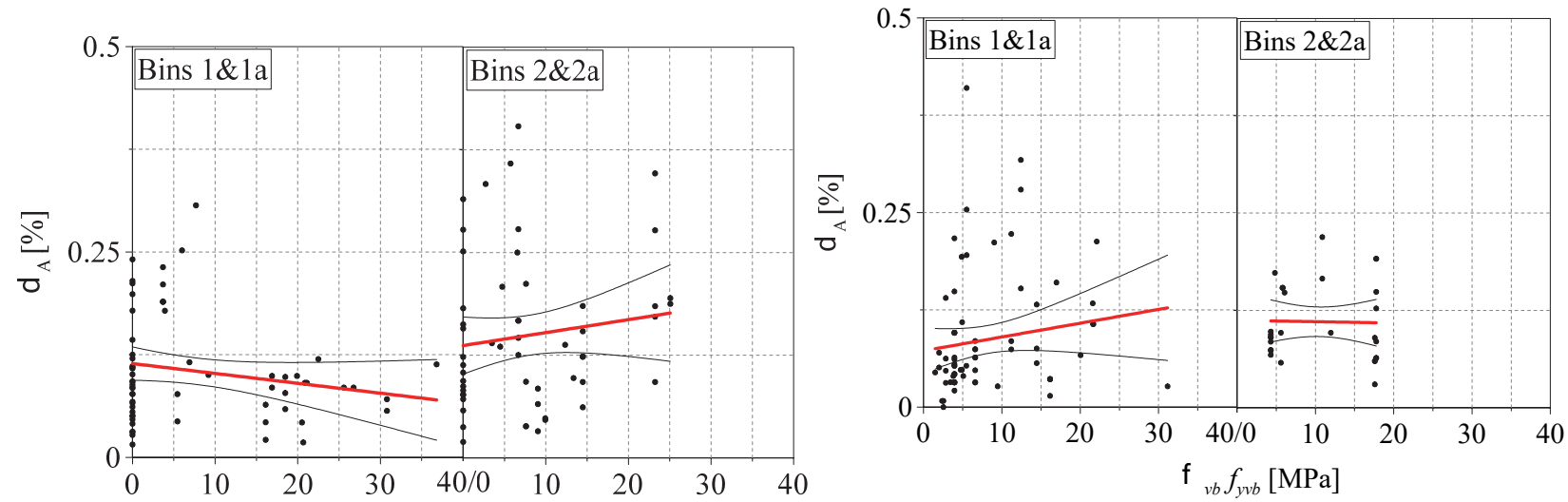

(e) $\rho_{v b} f_{v v b}$ : rectangular walls (left panel), and barbell and flanged walls (right panel)
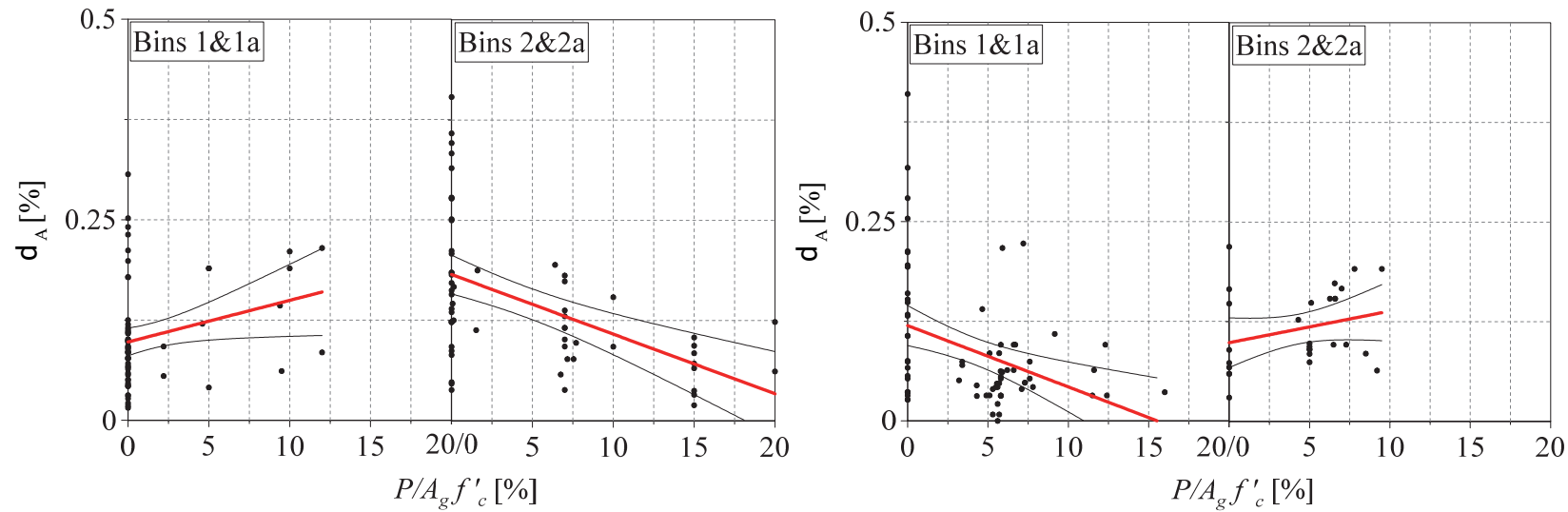

(f): $P / A_{g} f_{c}^{\prime}$ rectangular walls (left panel), and barbell and flanged walls (right panel)

Figure C-1 Effect of design variables on $\delta_{A}$ (cont). 

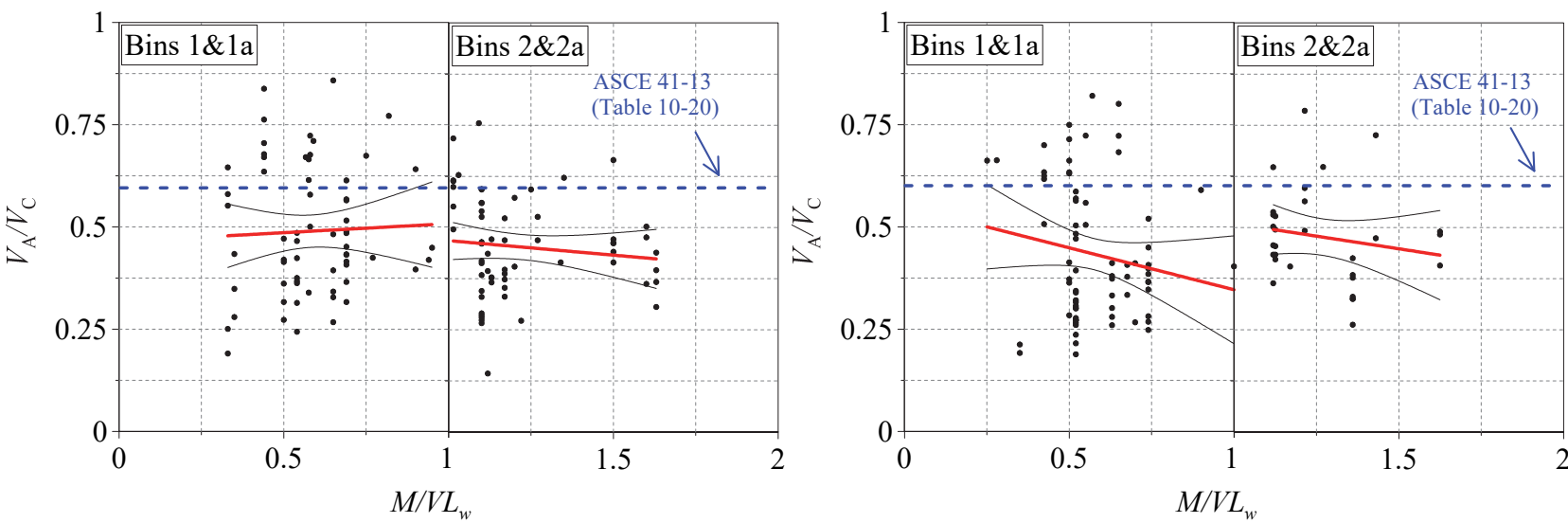

(a) $M / V I_{w}$ : rectangular walls (left panel), and barbell and flanged walls (right panel)
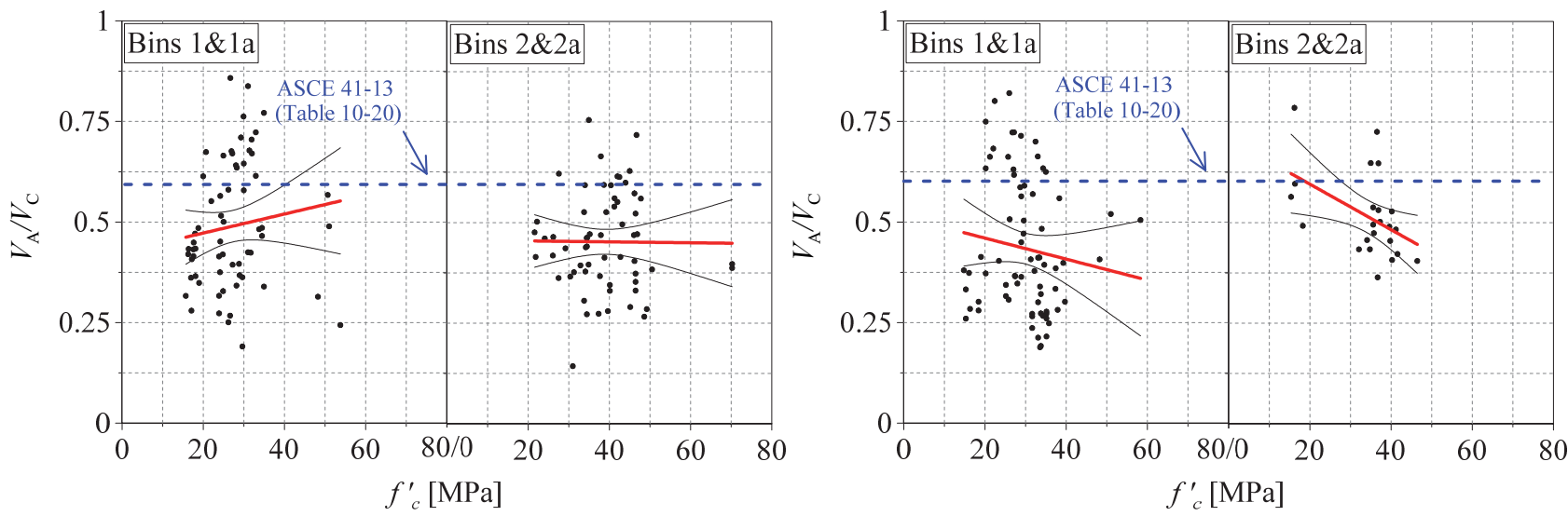

(b) $f_{c}^{\prime}$ : rectangular walls (left panel), and barbell and flanged walls (right panel)
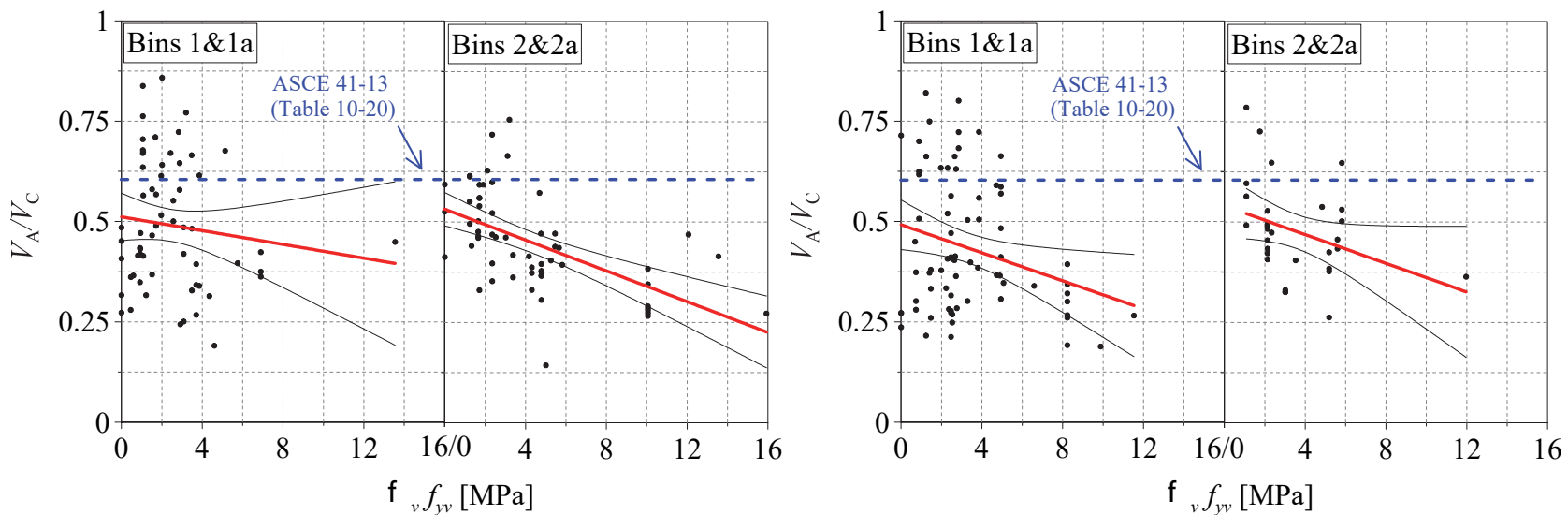

(c) $\rho_{v} f_{y v}$ : rectangular walls (left panel), and barbell and flanged walls (right panel)

Figure $\mathrm{C}-2 \quad$ Effect of design variables on $V_{A} / V_{C}$. 

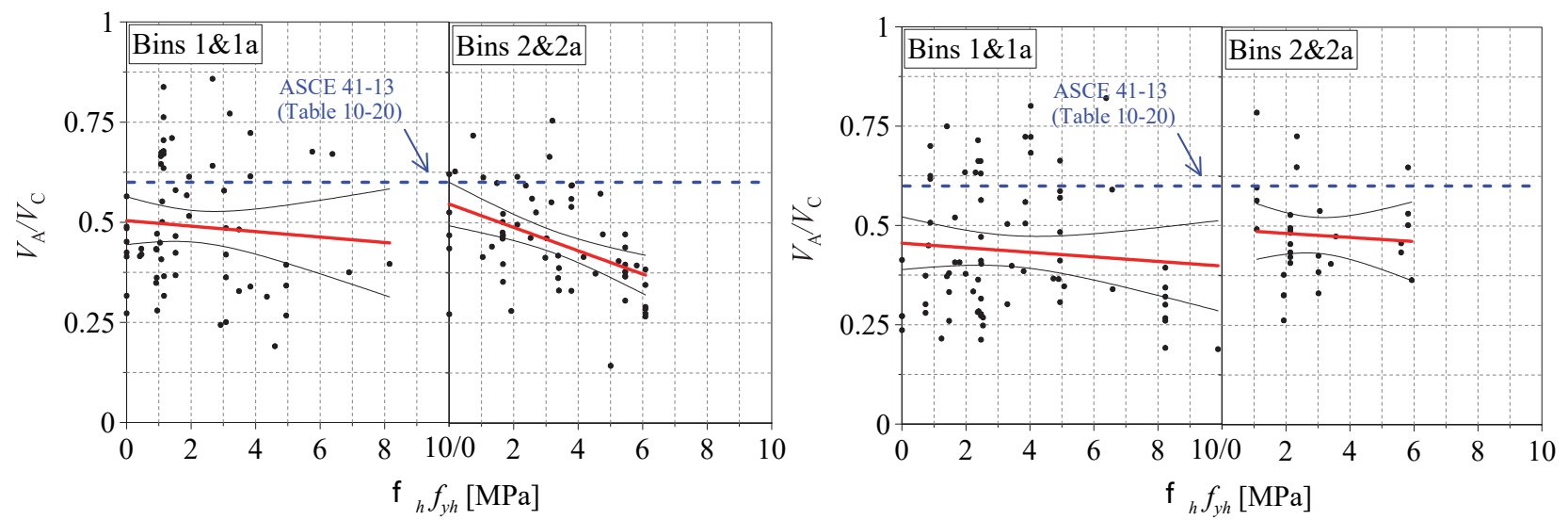

(d) $\rho_{h} f_{v h}$ : rectangular walls (left panel), and barbell and flanged walls (right panel)
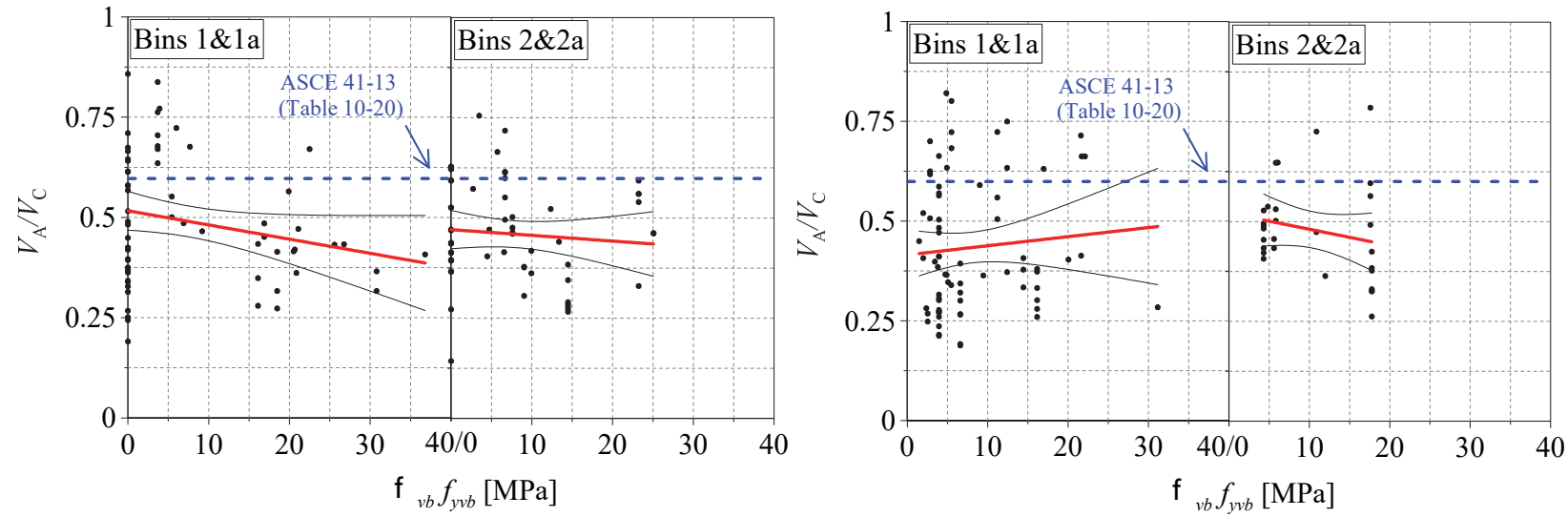

(e) $\rho_{v b} f_{v v b}$ : rectangular walls (left panel), and barbell and flanged walls (right panel)
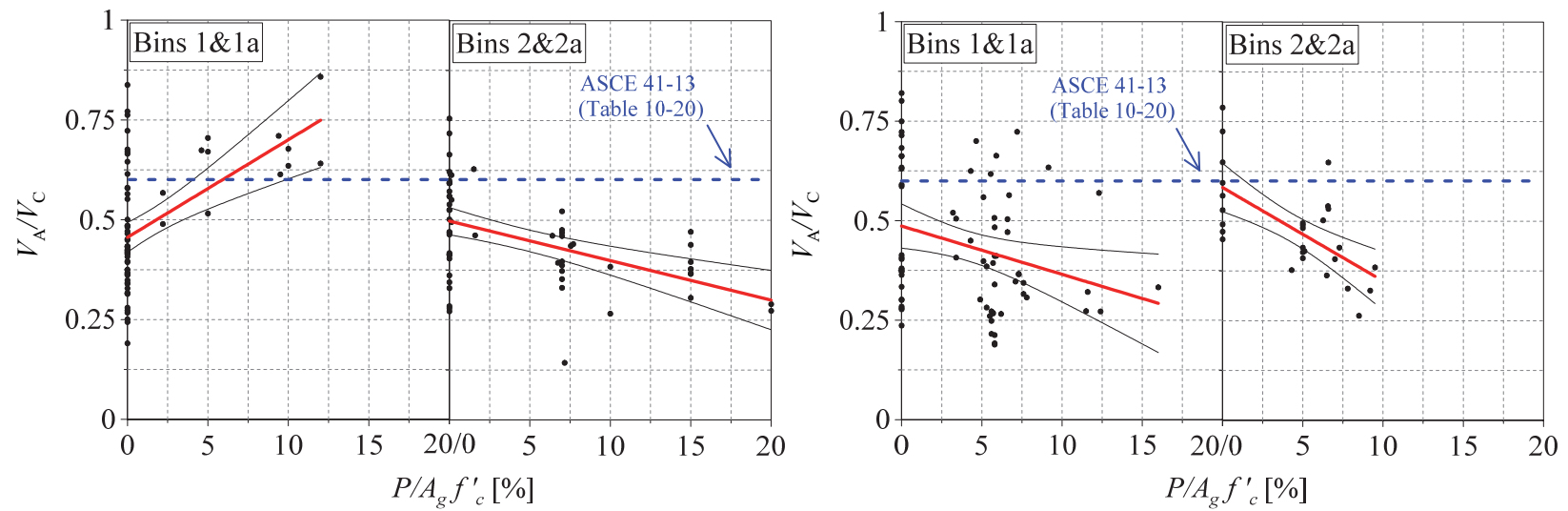

(f) $P / A_{g} f_{c}^{\prime}$ : rectangular walls (left panel), and barbell and flanged walls (right panel)

Figure C-2 Effect of design variables on $V_{A} / V_{C}$ (cont). 

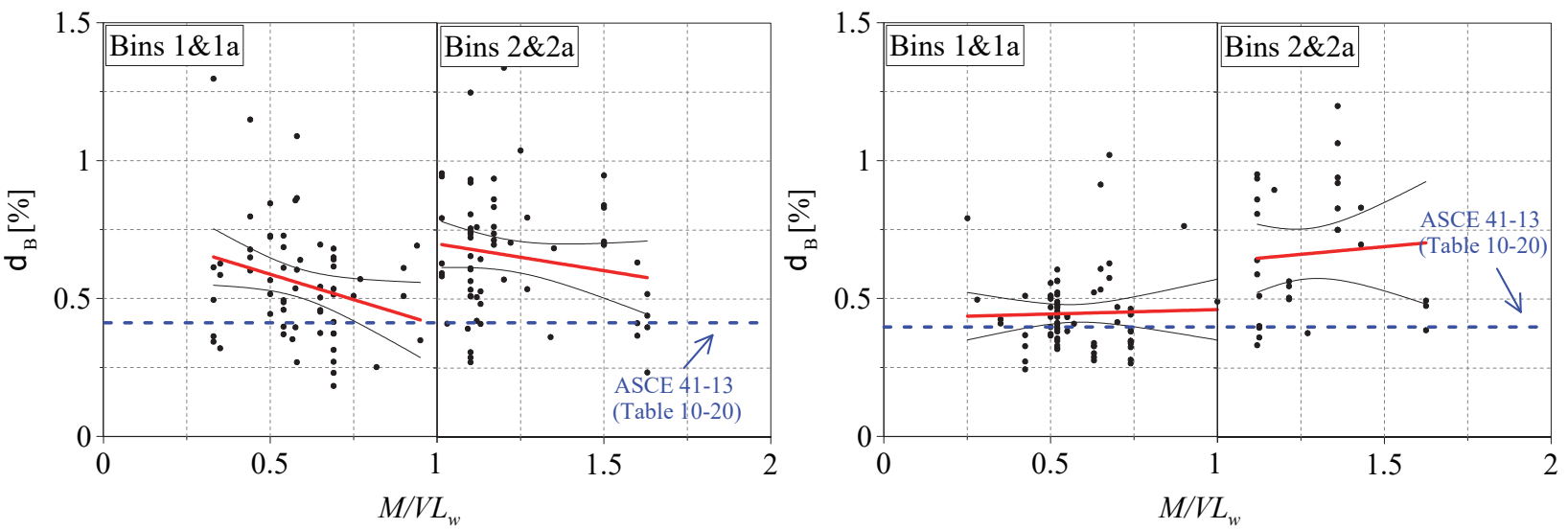

(a) $M / V I_{w}$ : rectangular walls (left panel), and barbell and flanged walls (right panel)
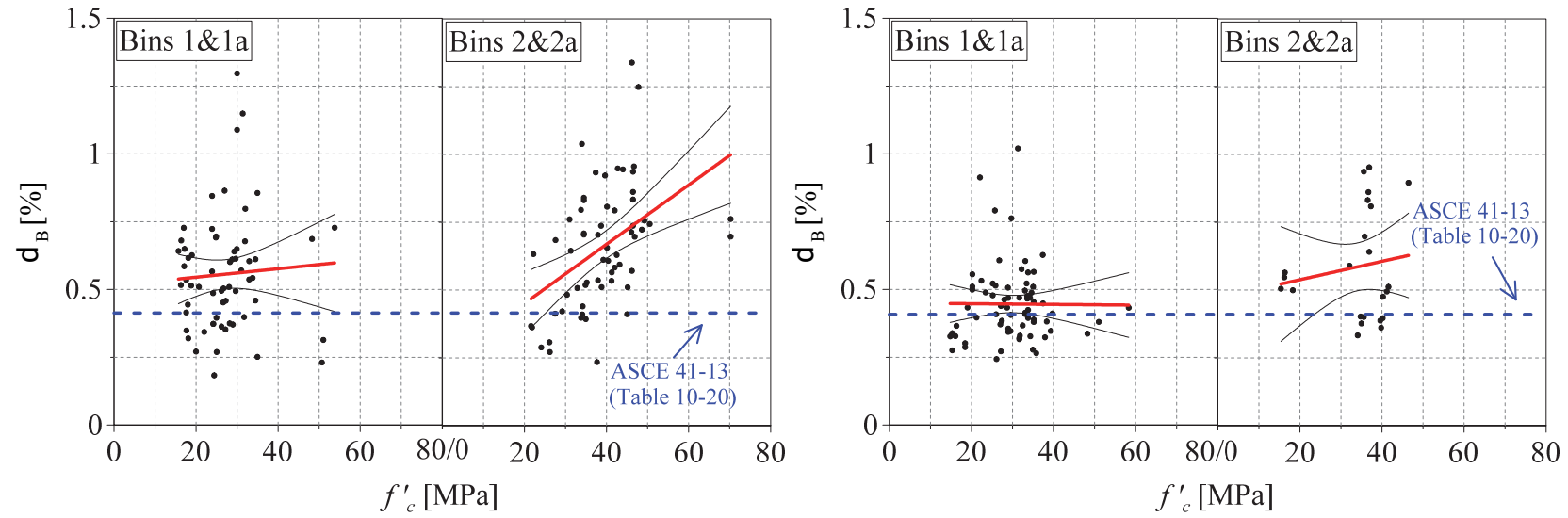

(b) $f_{c}^{\prime}$ : rectangular walls (left panel), and barbell and flanged walls (right panel)
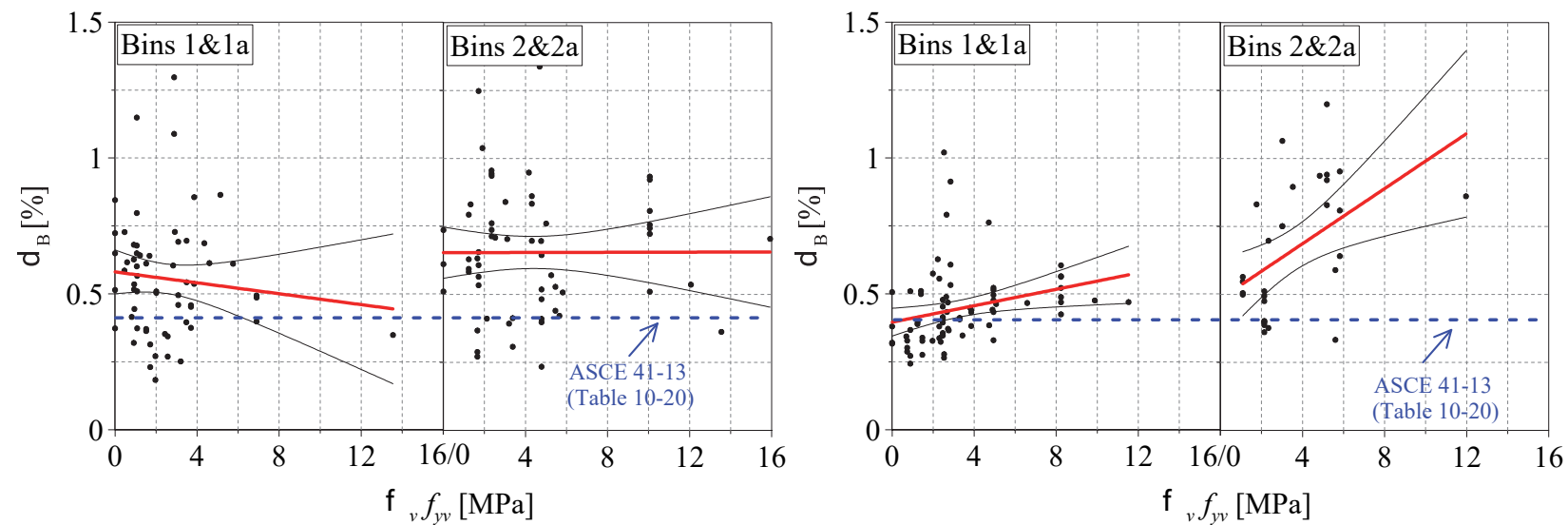

(c) $\rho_{v} f_{y v}$ : rectangular walls (left panel), and barbell and flanged walls (right panel)

Figure C-3 Effect of design variables on $\delta_{B}$. 

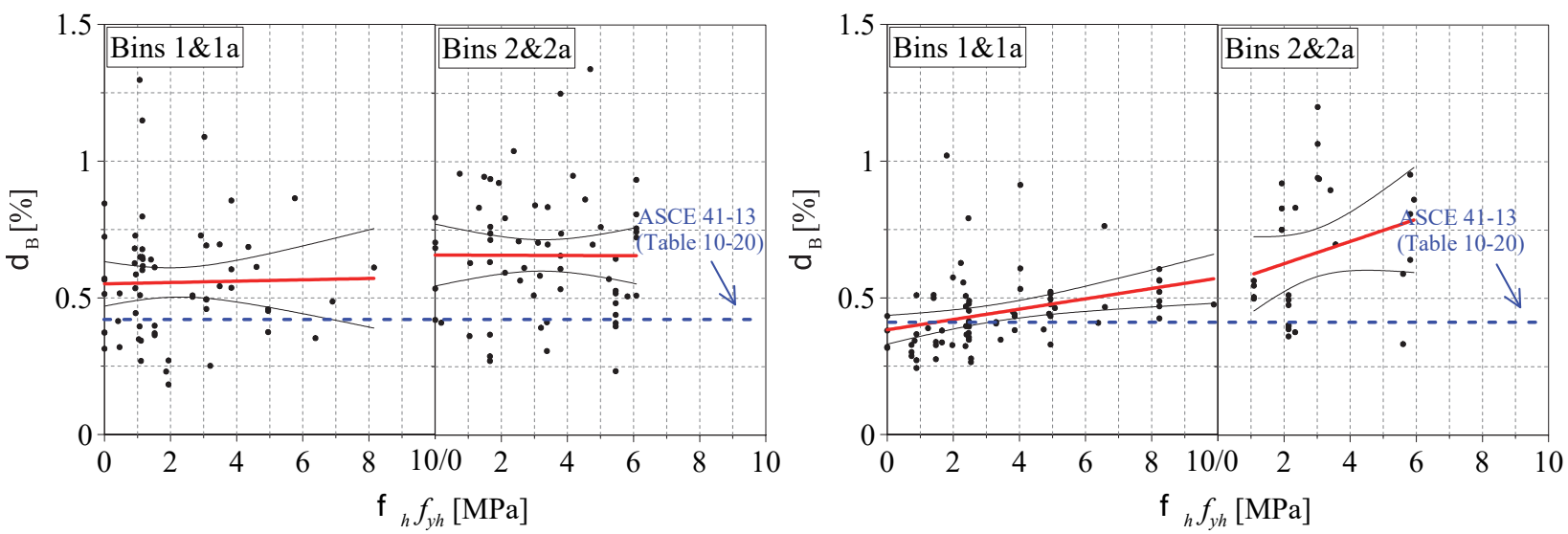

(d) $\rho_{h} f_{v h}$ : rectangular walls (left panel), and barbell and flanged walls (right panel)
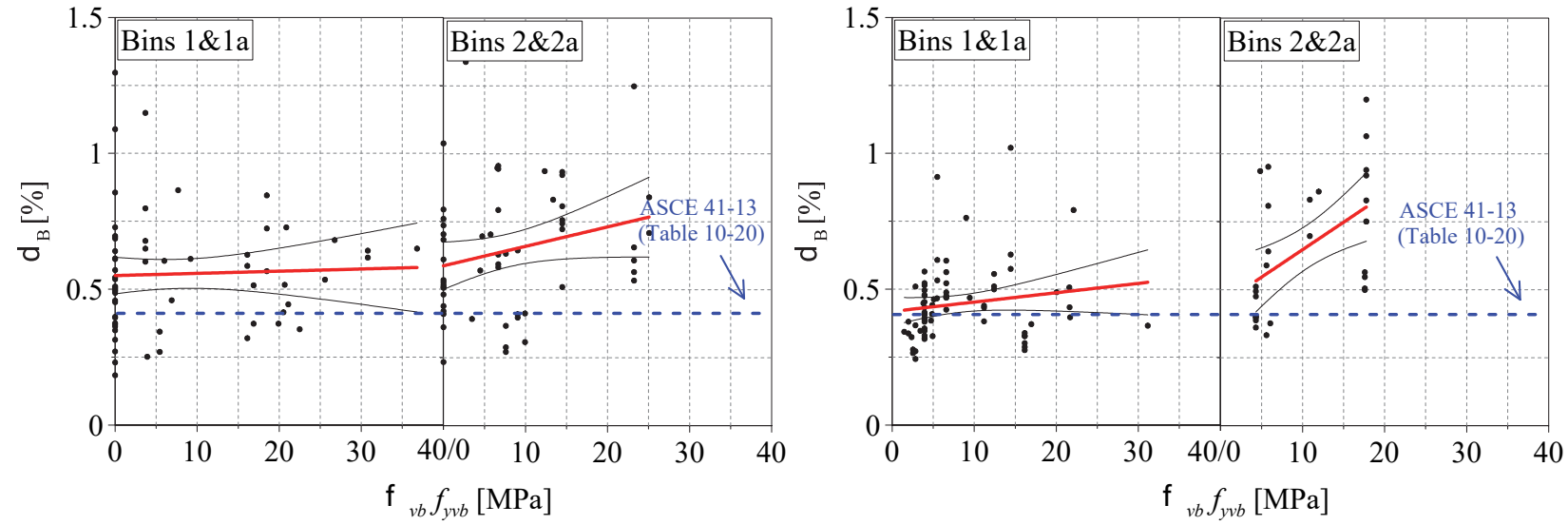

(e) $\rho_{v b} f_{v v b}$ : rectangular walls (left panel), and barbell and flanged walls (right panel)
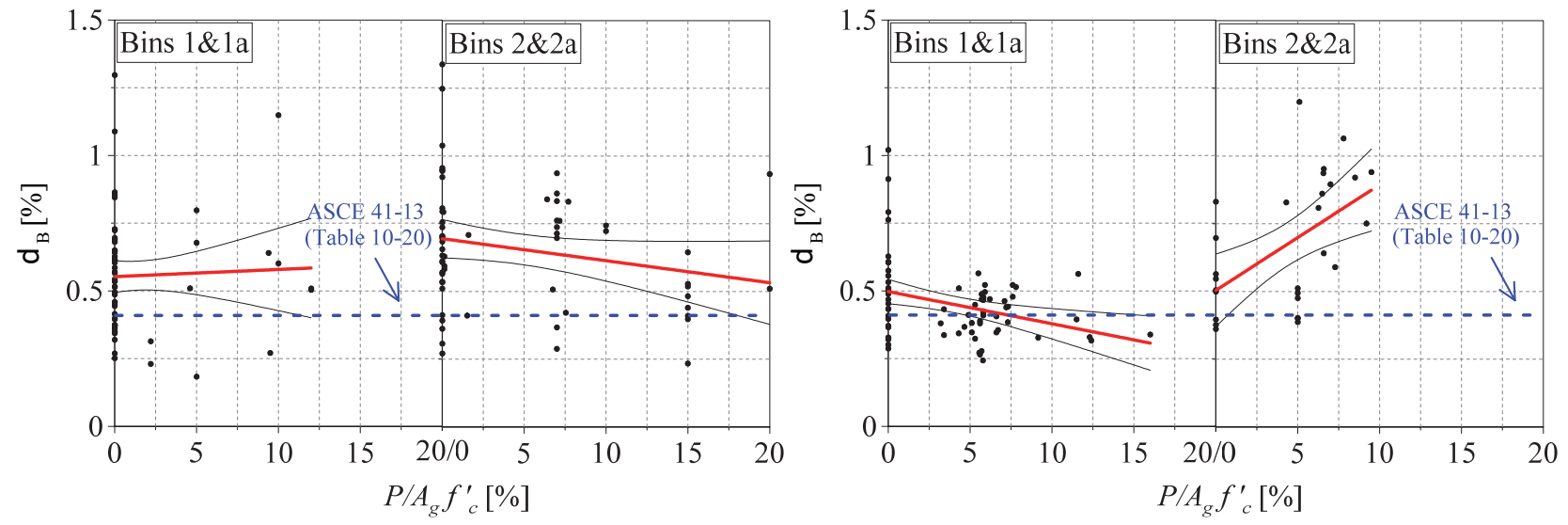

(f) $P / A_{g} f_{c}^{\prime}$ : rectangular walls (left panel), and barbell and flanged walls (right panel)

Figure C-3 Effect of design variables on $\delta_{B}$ (cont). 

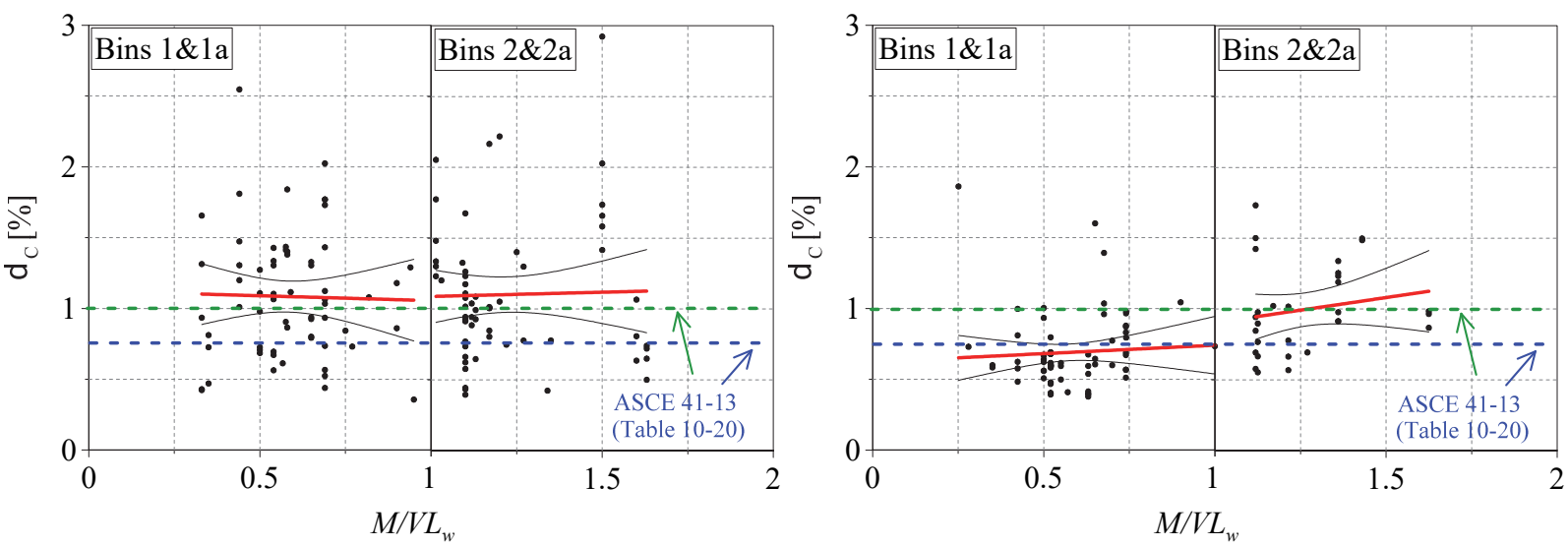

(a) $M / V l_{w}$ : rectangular walls (left panel), and barbell and flanged walls (right panel)
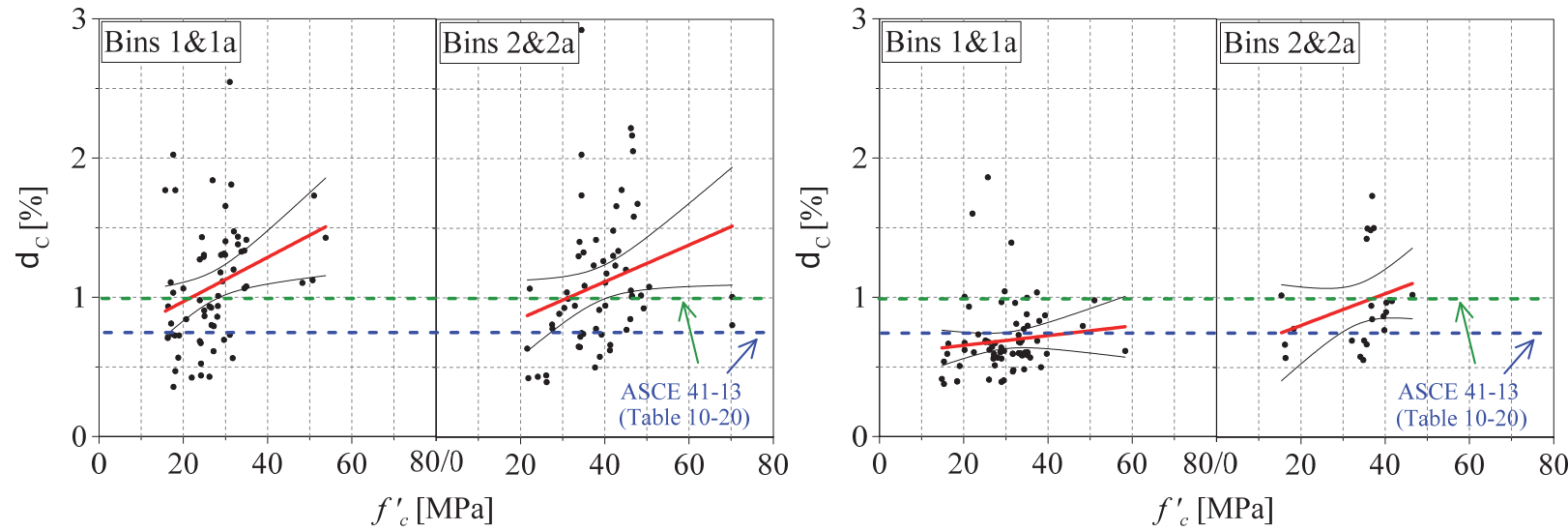

(b) $f_{c}^{\prime}$ : rectangular walls (left panel), and barbell and flanged walls (right panel)
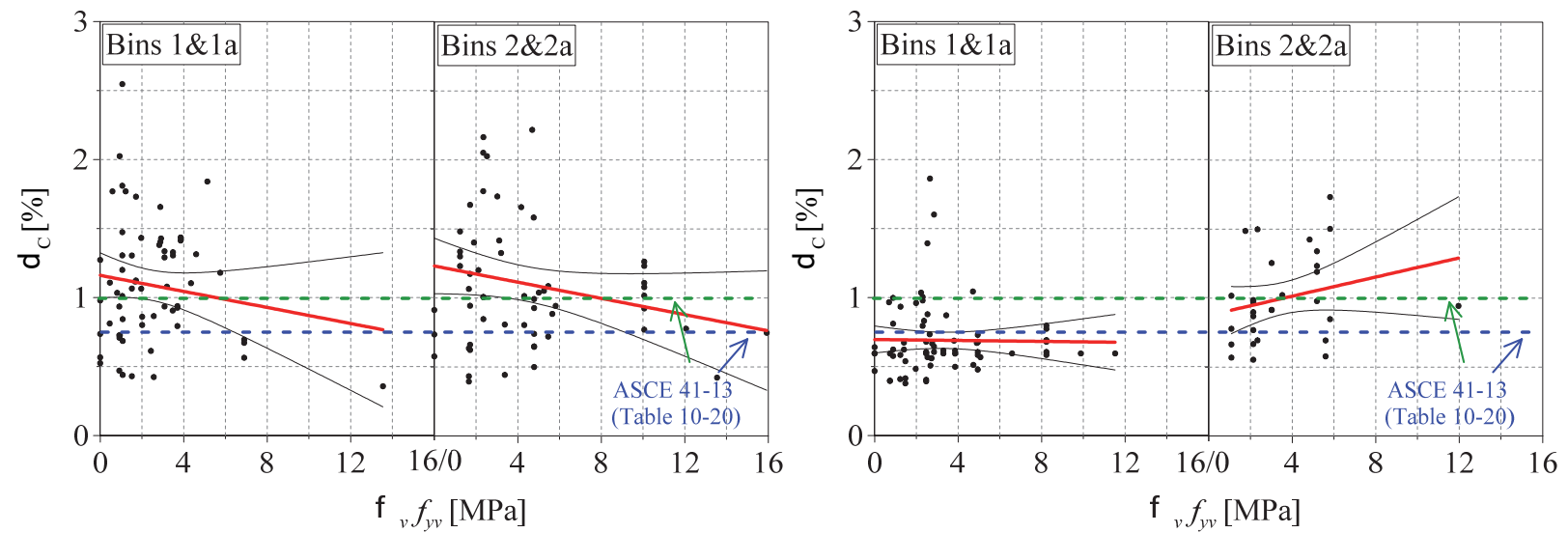

(c) $\rho_{v} f_{y v}$ : rectangular walls (left panel), and barbell and flanged walls (right panel)

Figure C-4 Effect of design variables on $\delta c$. 

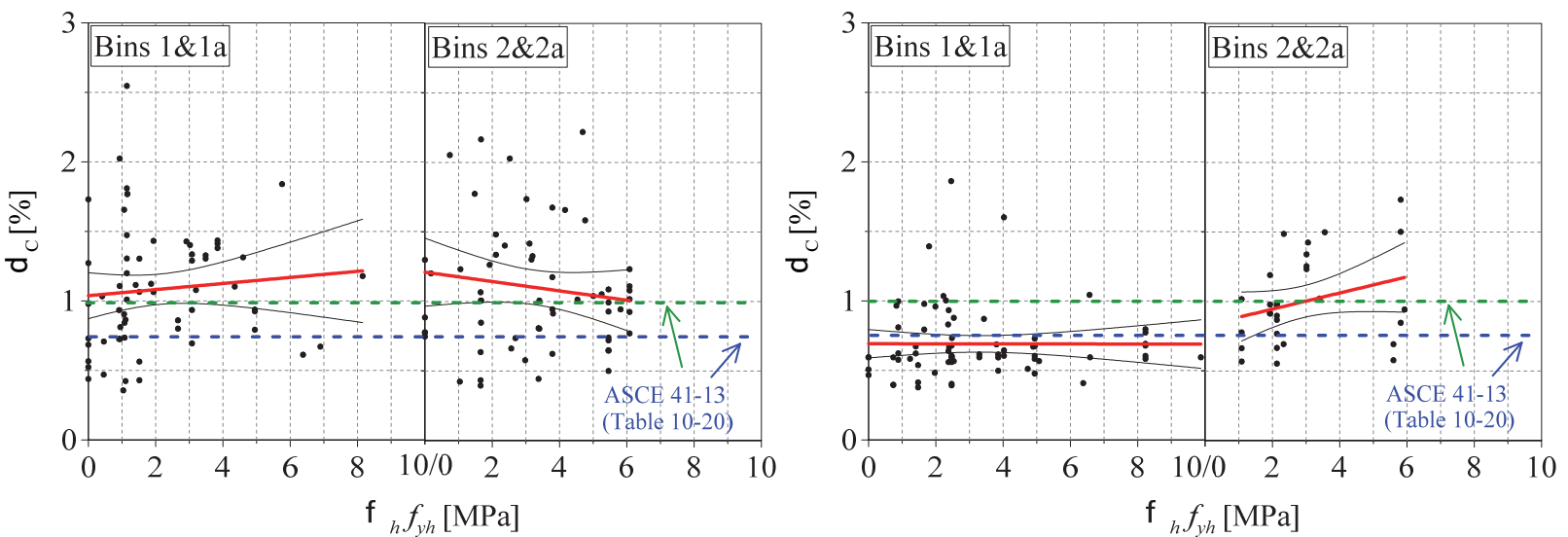

(d) $\rho_{h} f_{v h}$ : rectangular walls (left panel), and barbell and flanged walls (right panel)
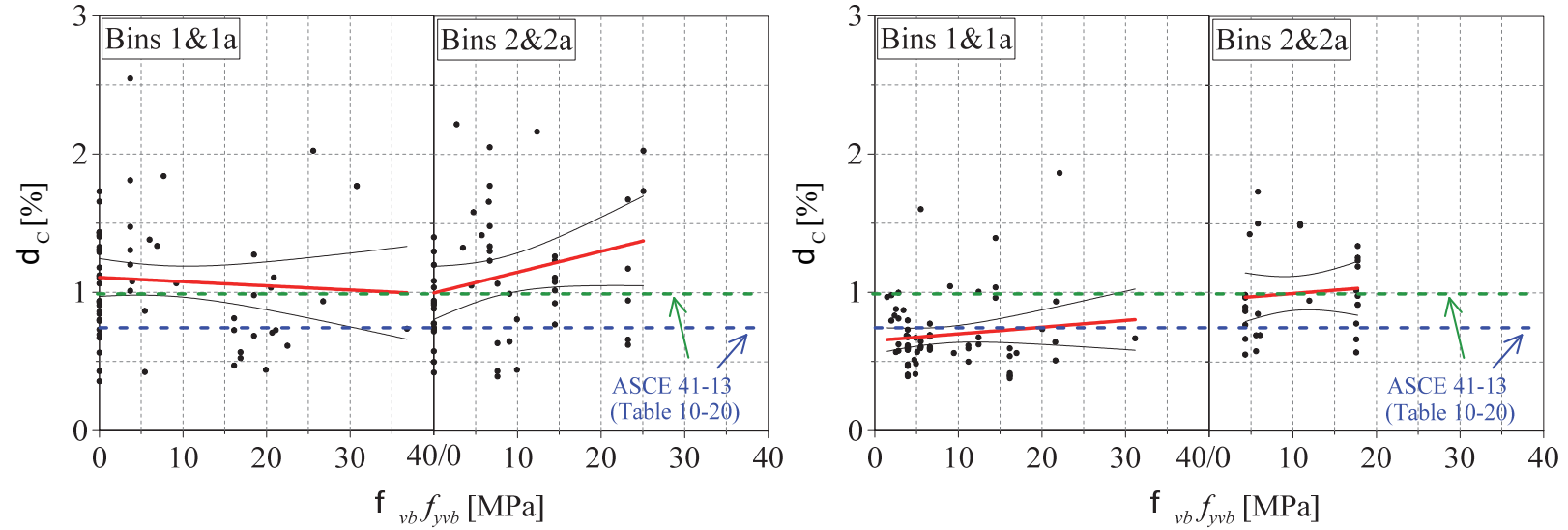

(e) $\rho_{v b} f_{v v b}$ : rectangular walls (left panel), and barbell and flanged walls (right panel)
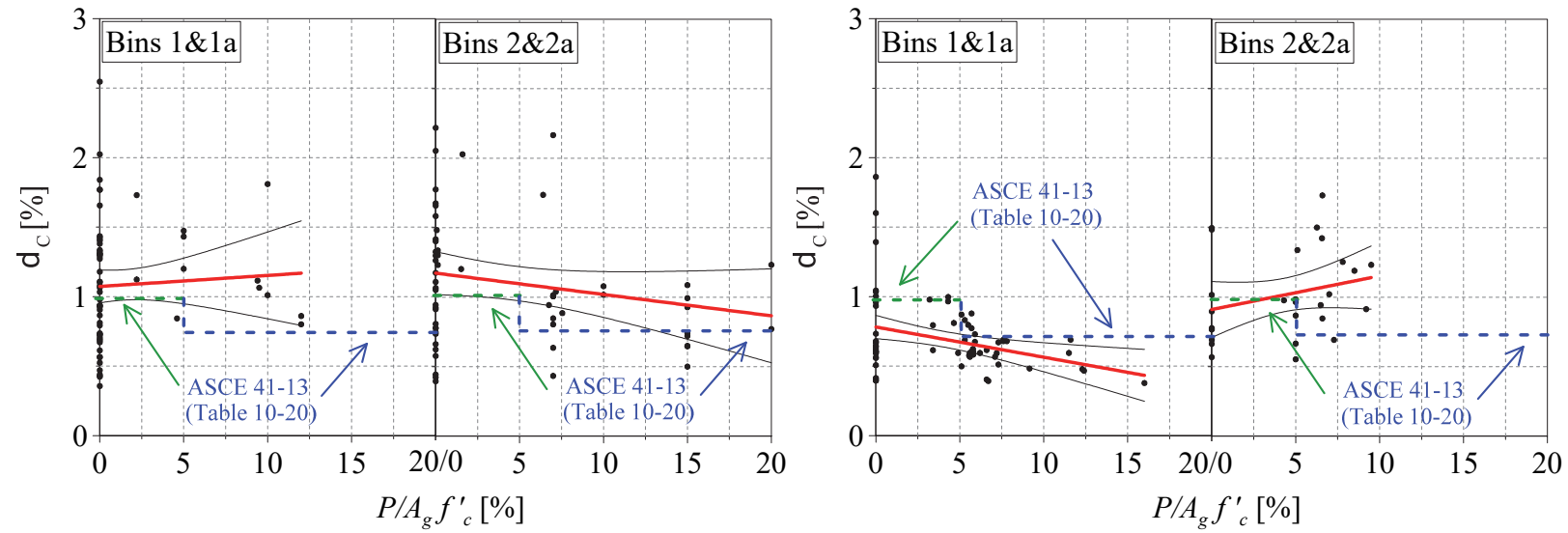

(f) $P / A_{g} f_{c}^{\prime}$ : rectangular walls (left panel), and barbell and flanged walls (right panel)

Figure C-4 Effect of design variables on $\delta_{c}$ (cont). 

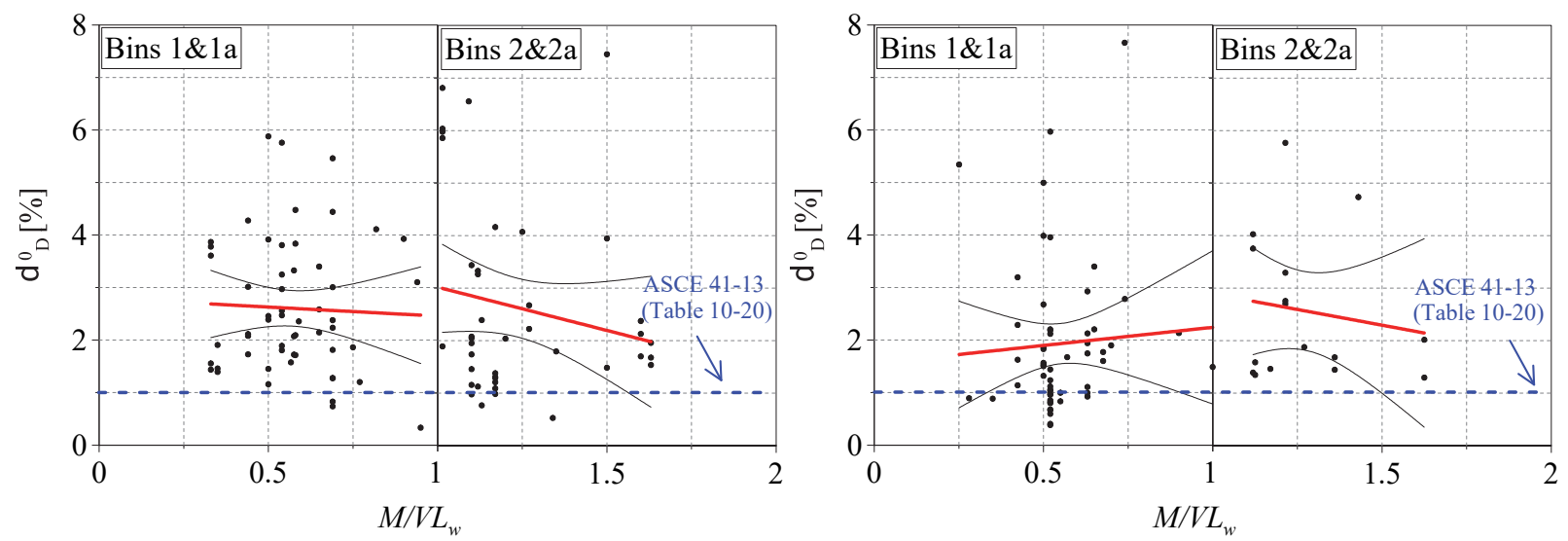

(a) $M / V I_{w}$ : rectangular walls (left panel), and barbell and flanged walls (right panel)
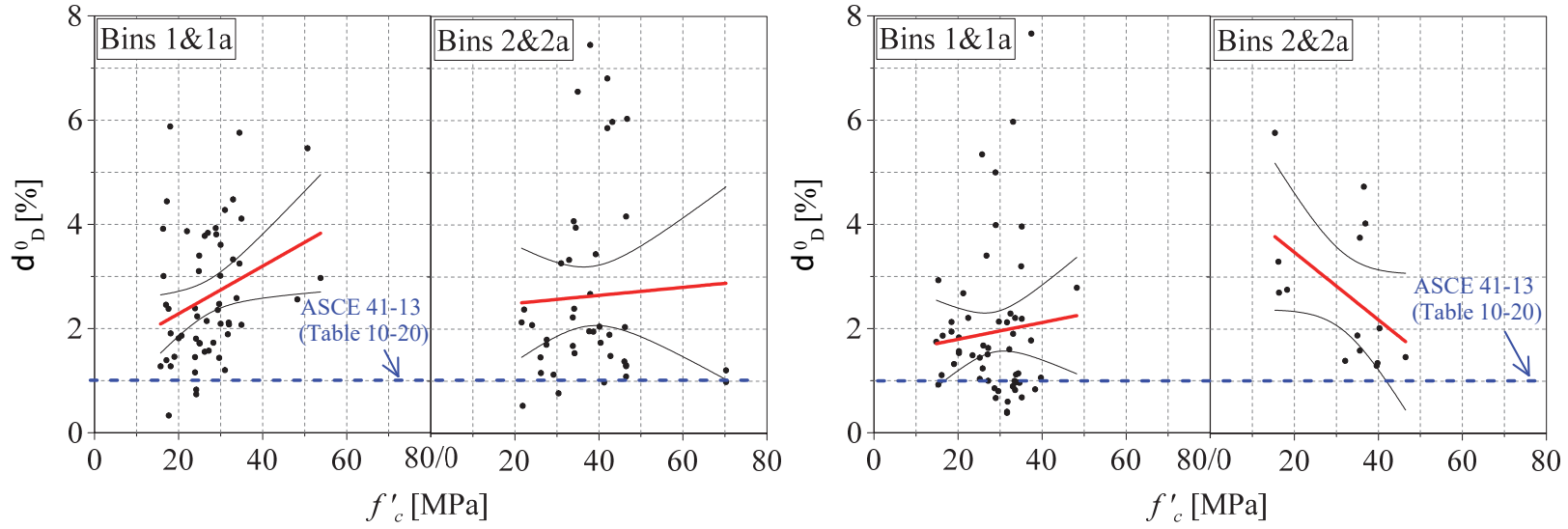

(b) $f_{c}^{\prime}$ : rectangular walls (left panel), and barbell and flanged walls (right panel)
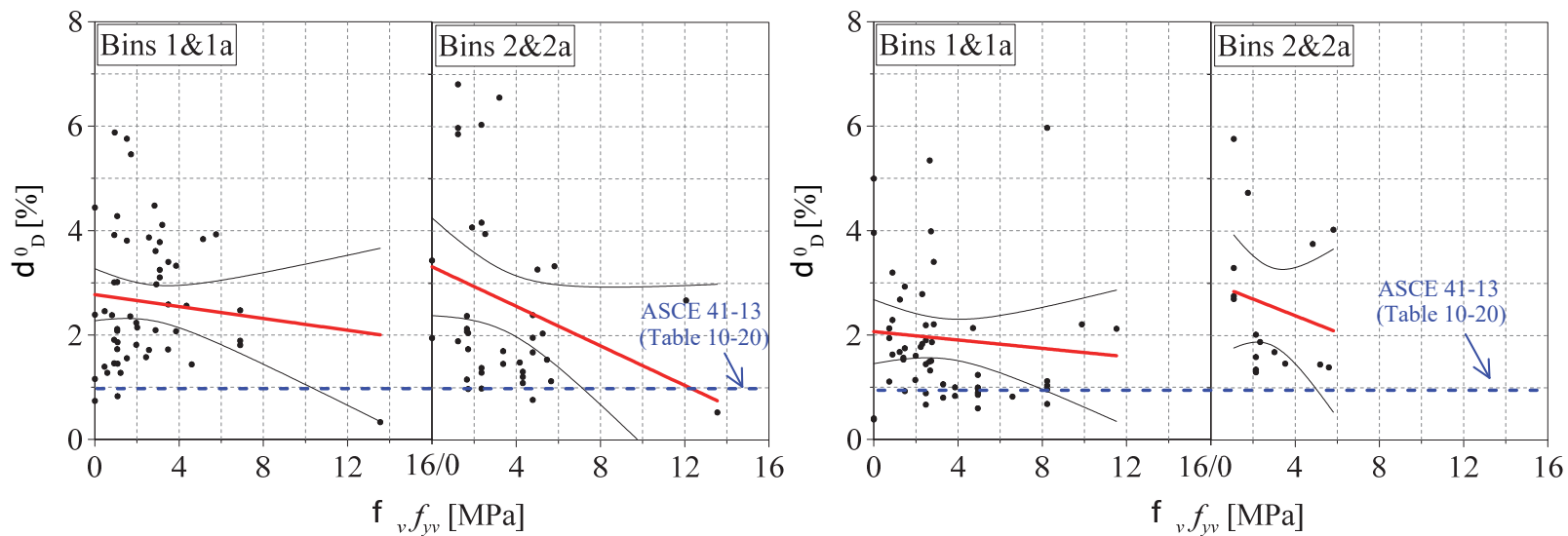

(c) $\rho_{v} f_{y v}$ : rectangular walls (left panel), and barbell and flanged walls (right panel)

Figure C-5 Effect of design variables on $\delta_{D}^{0}$. 

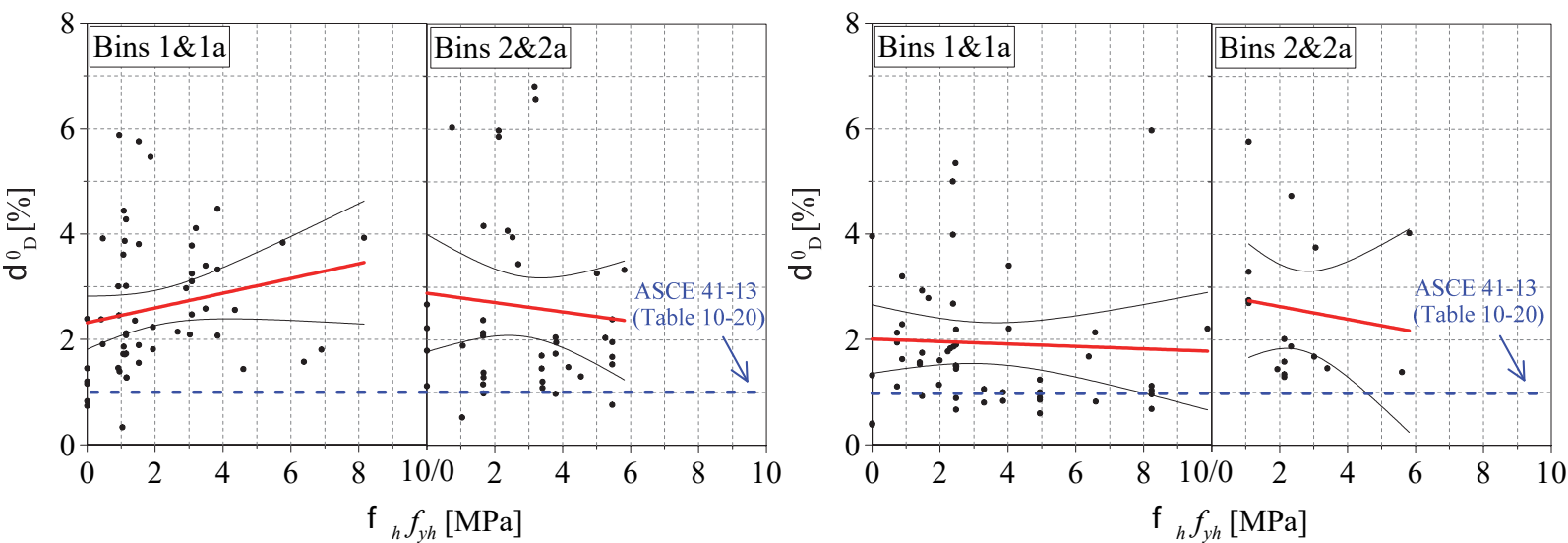

(d) $\rho_{h} f_{v h}$ : rectangular walls (left panel), and barbell and flanged walls (right panel)
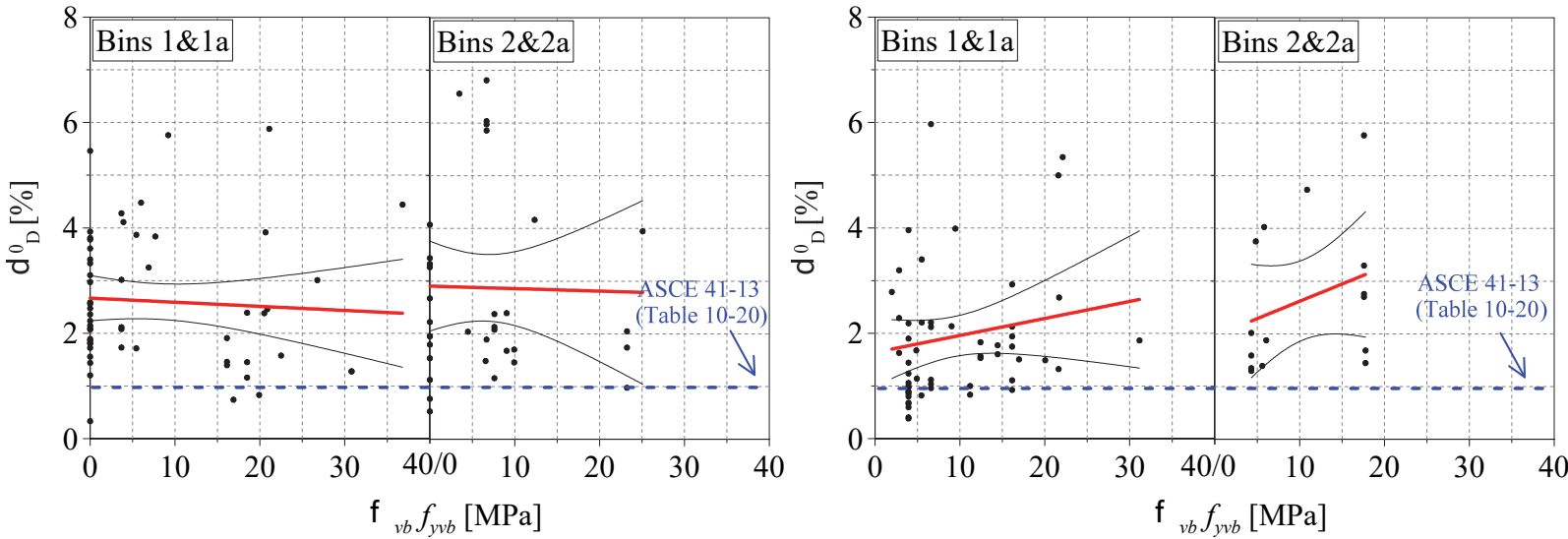

(e) $\rho_{v b} f_{v v b}$ : rectangular walls (left panel), and barbell and flanged walls (right panel)
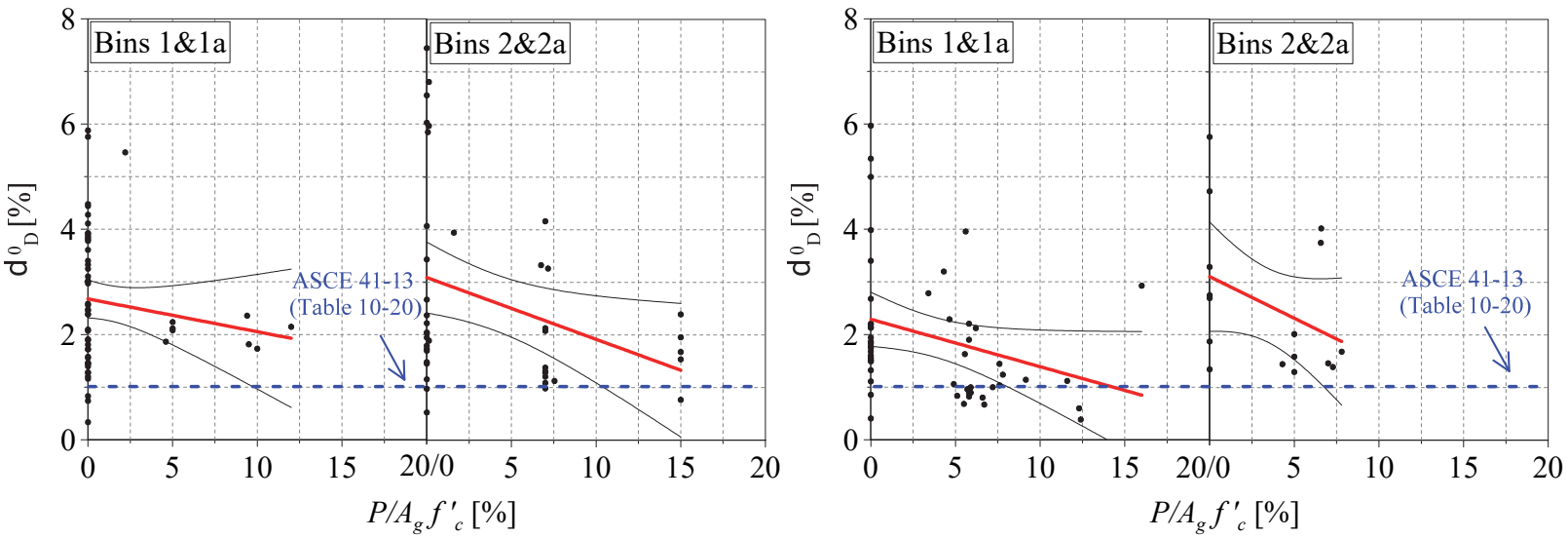

(f) $P / A_{g} f_{c}^{\prime}$ : rectangular walls (left panel), and barbell and flanged walls (right panel)

Figure $\mathrm{C}-5 \quad$ Effect of design variables on $\delta_{D}^{0}$ (cont). 

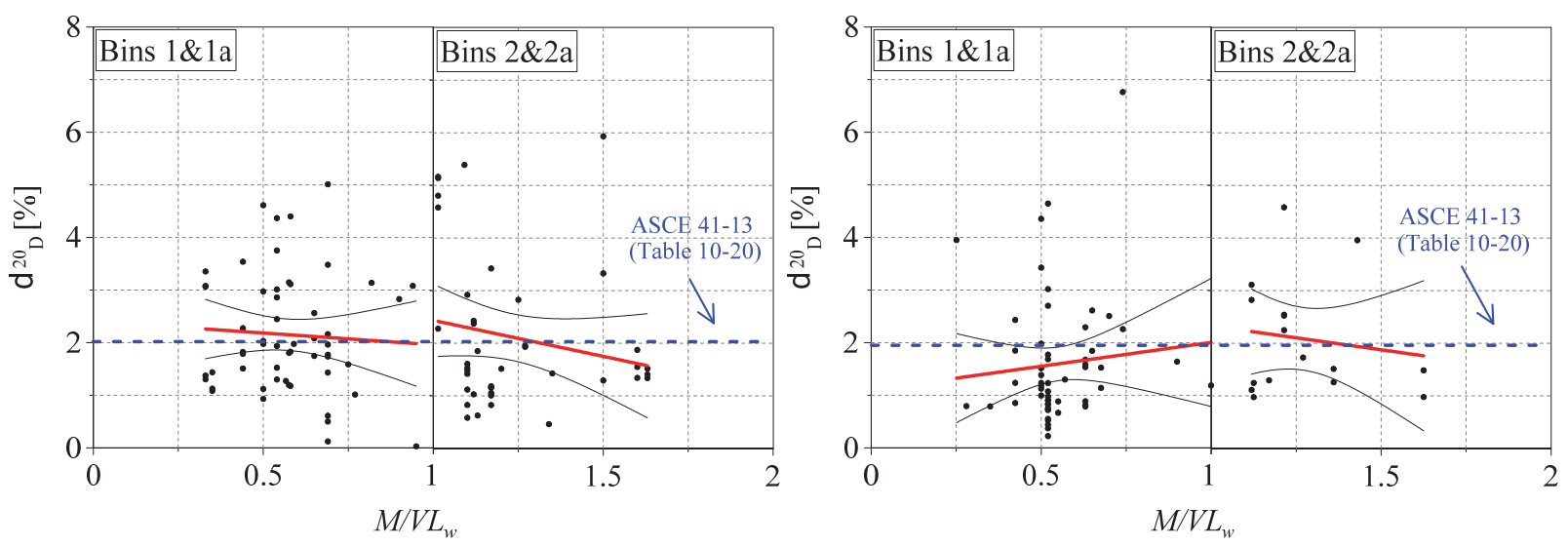

(a) $M / V I_{w}$ : rectangular walls (left panel), and barbell and flanged walls (right panel)
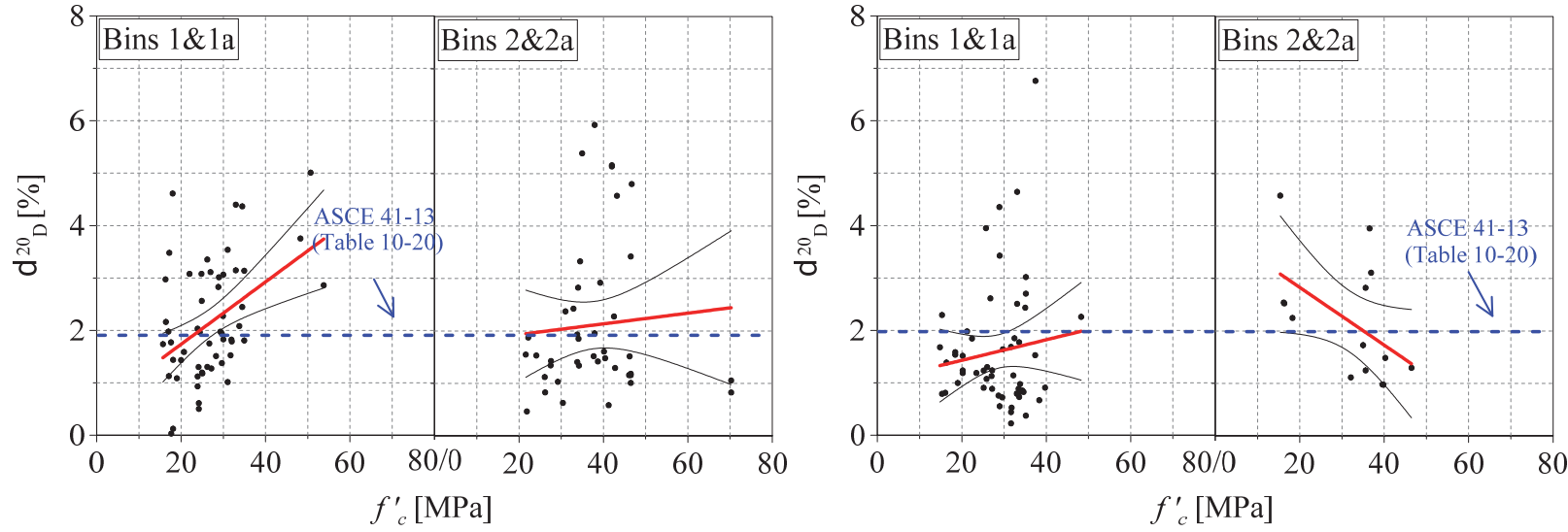

(b) $f_{c}^{\prime}$ : rectangular walls (left panel), and barbell and flanged walls (right panel)
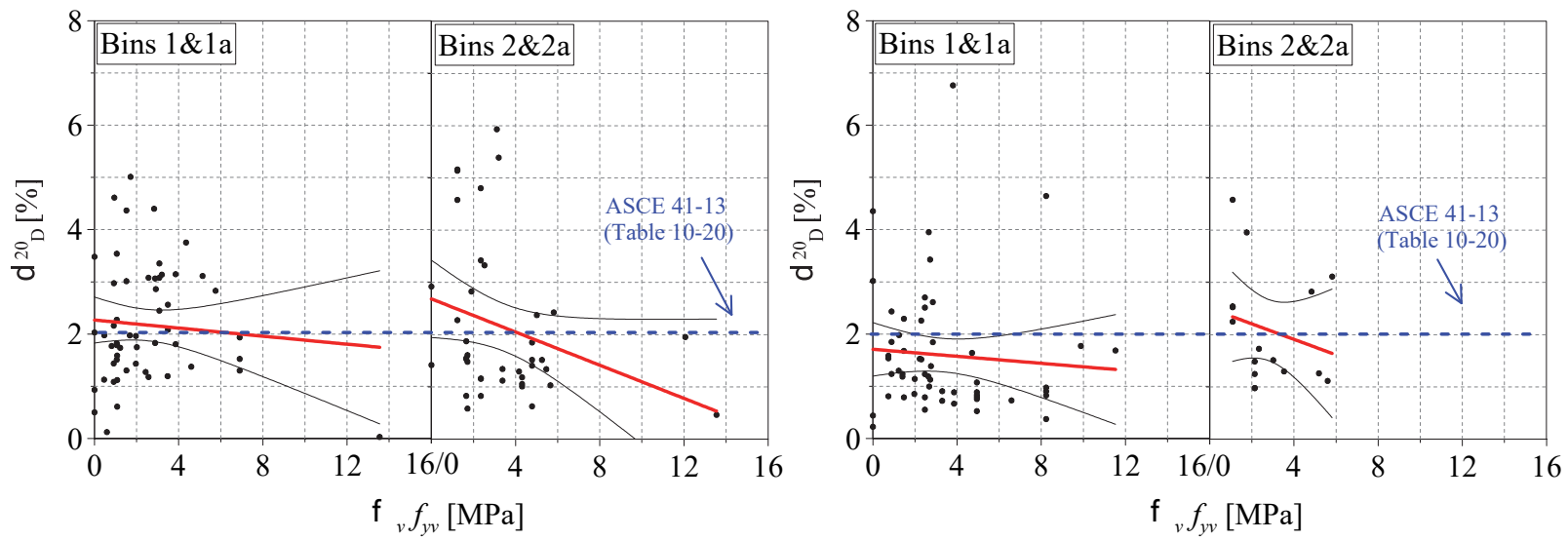

(c) $\rho_{v} f_{y v}$ : rectangular walls (left panel), and barbell and flanged walls (right panel)

Figure C-6 Effect of design variables on $\delta_{D}^{20}$. 

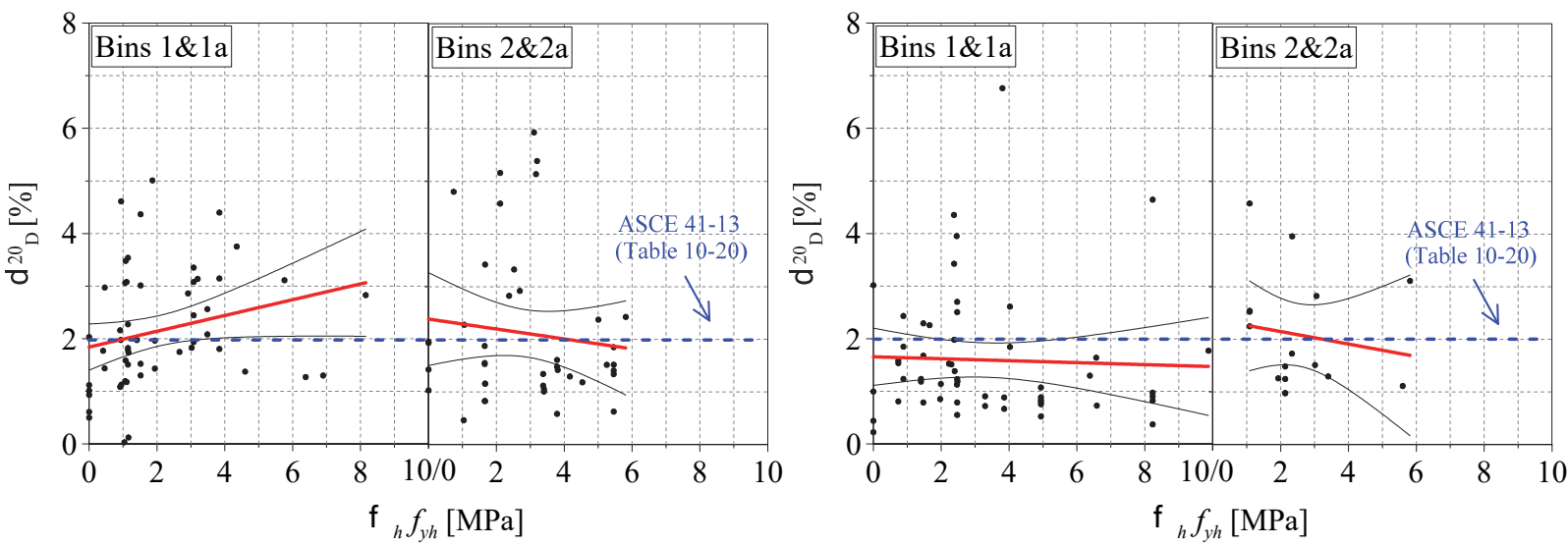

(d) $\rho_{h} f_{v h}$ : rectangular walls (left panel), and barbell and flanged walls (right panel)
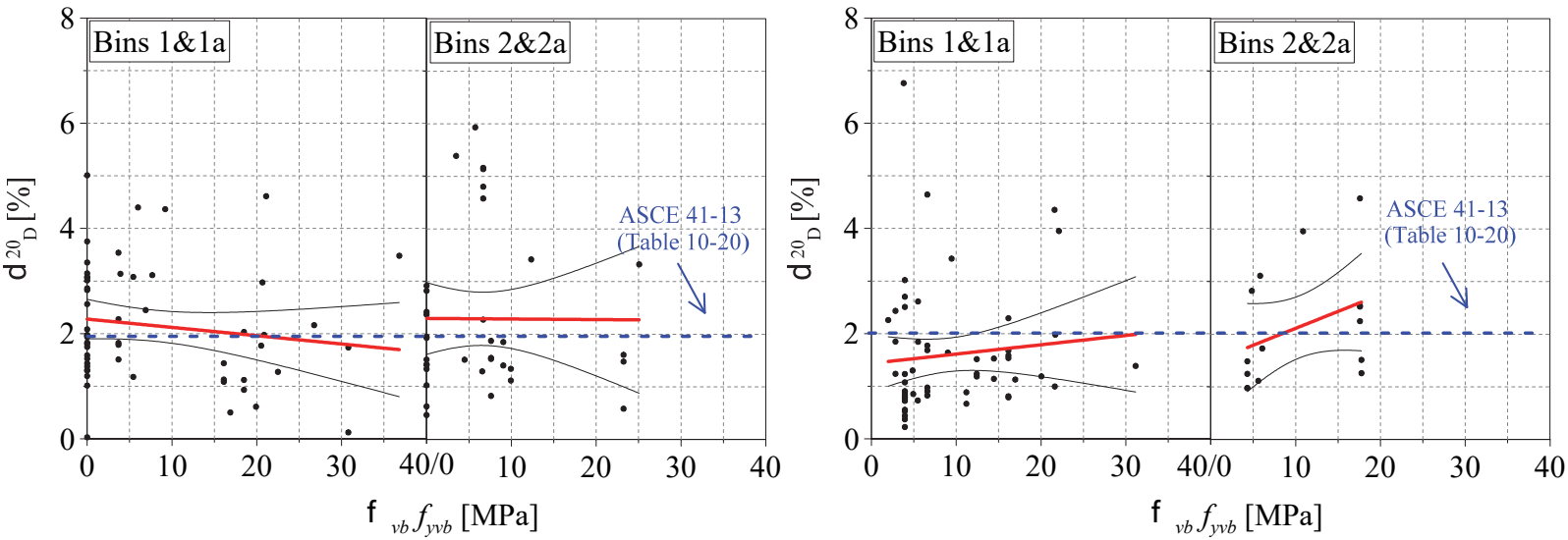

(e) $\rho_{v b} f_{v v b}$ : rectangular walls (left panel), and barbell and flanged walls (right panel)
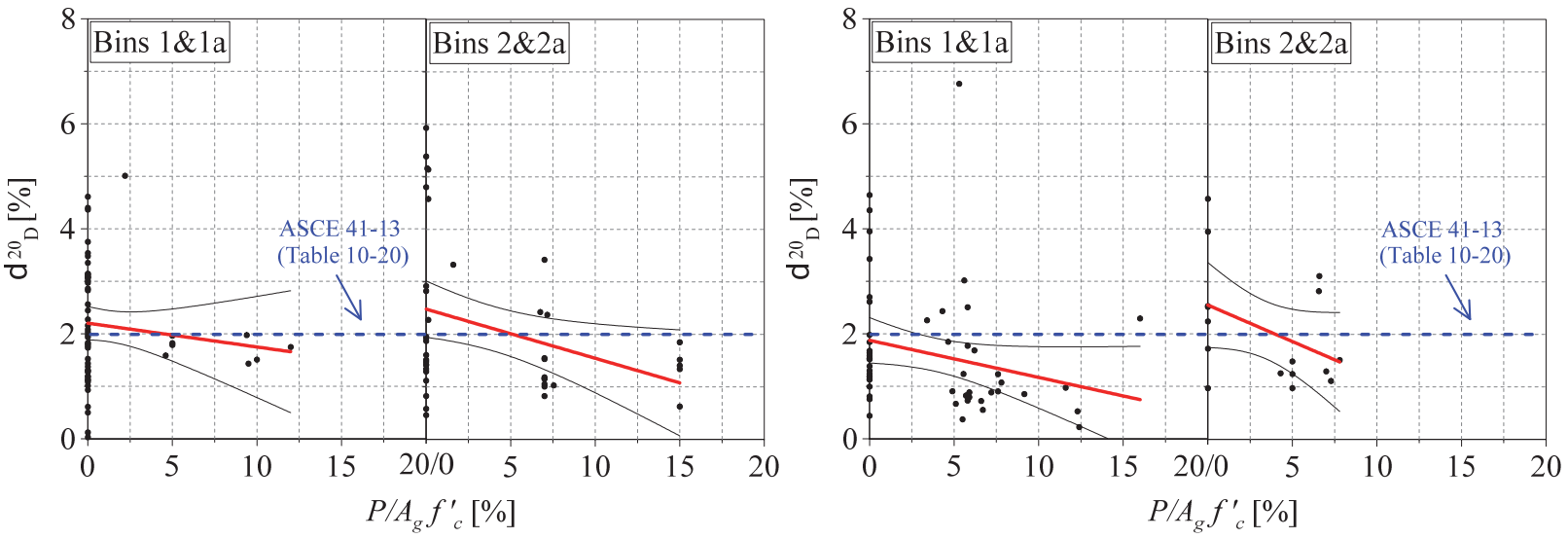

(f) $P / A_{g} f_{c}^{\prime}$ : rectangular walls (left panel), and barbell and flanged walls (right panel)

Figure C-6 Effect of design variables on $\delta_{D}^{20}$ (cont). 

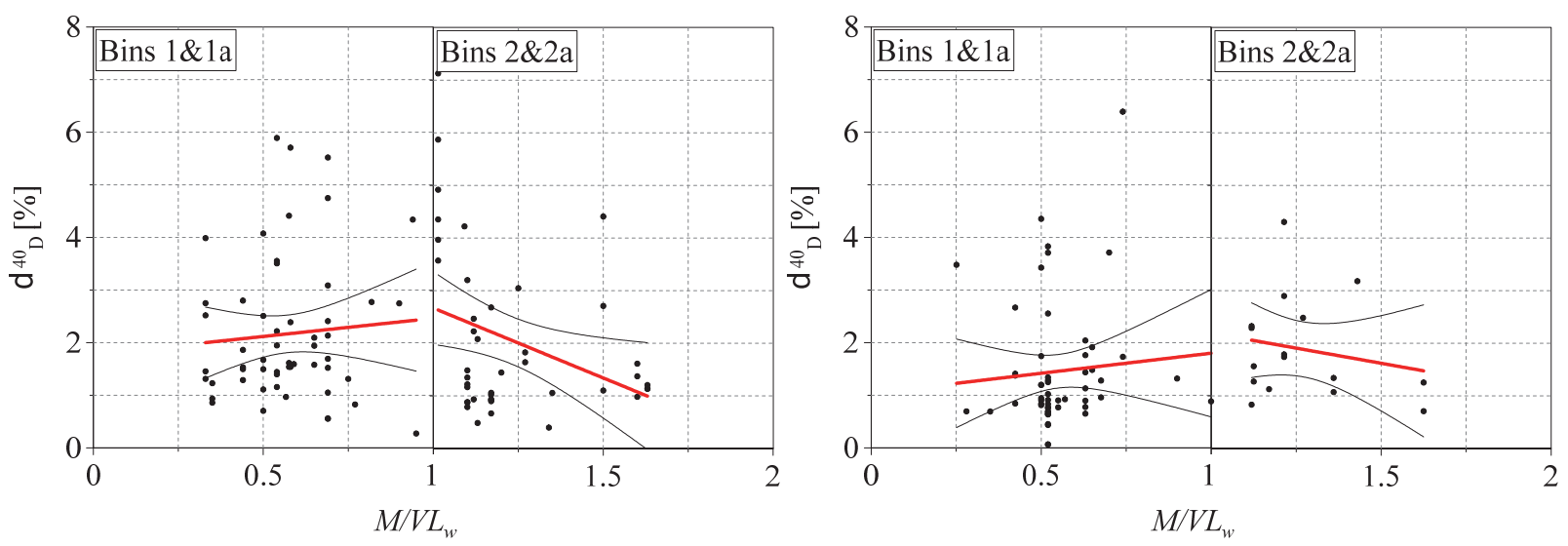

(a) $M / V I_{w}$ : rectangular walls (left panel), and barbell and flanged walls (right panel)
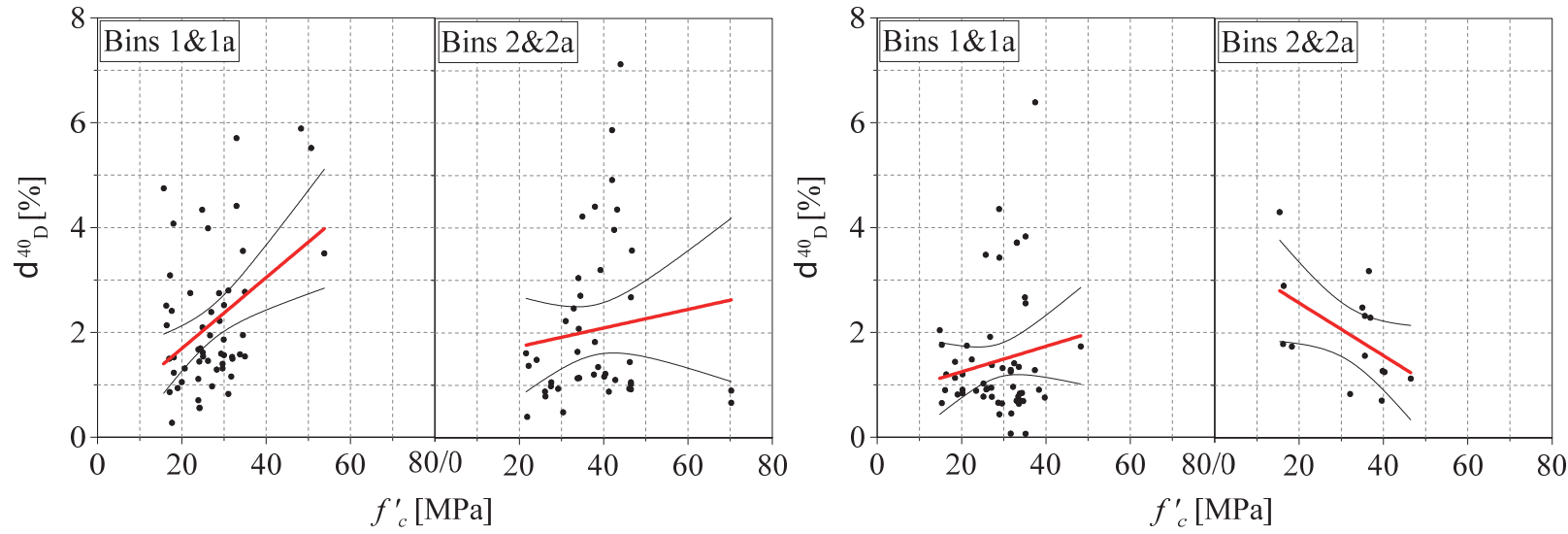

(b) $f_{c}^{\prime}$ : rectangular walls (left panel), and barbell and flanged walls (right panel)
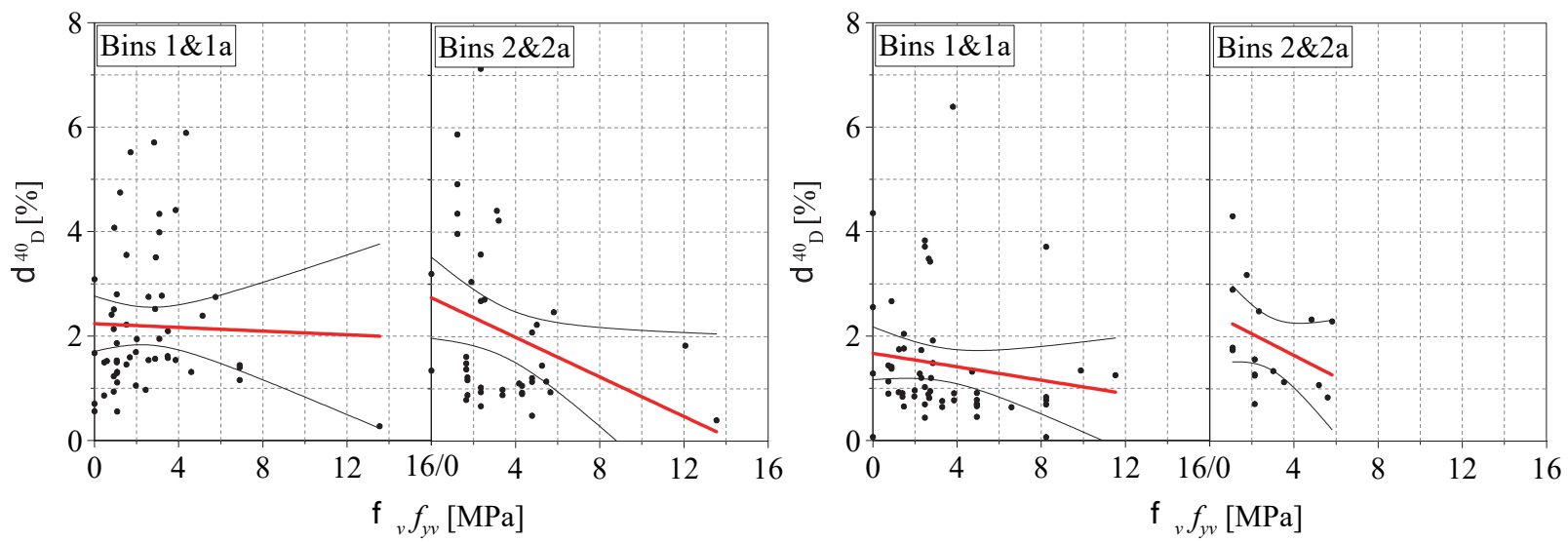

(c) $\rho_{v} f_{y v}$ : rectangular walls (left panel), and barbell and flanged walls (right panel)

Figure C-7 Effect of design variables on $\delta_{D}^{40}$. 

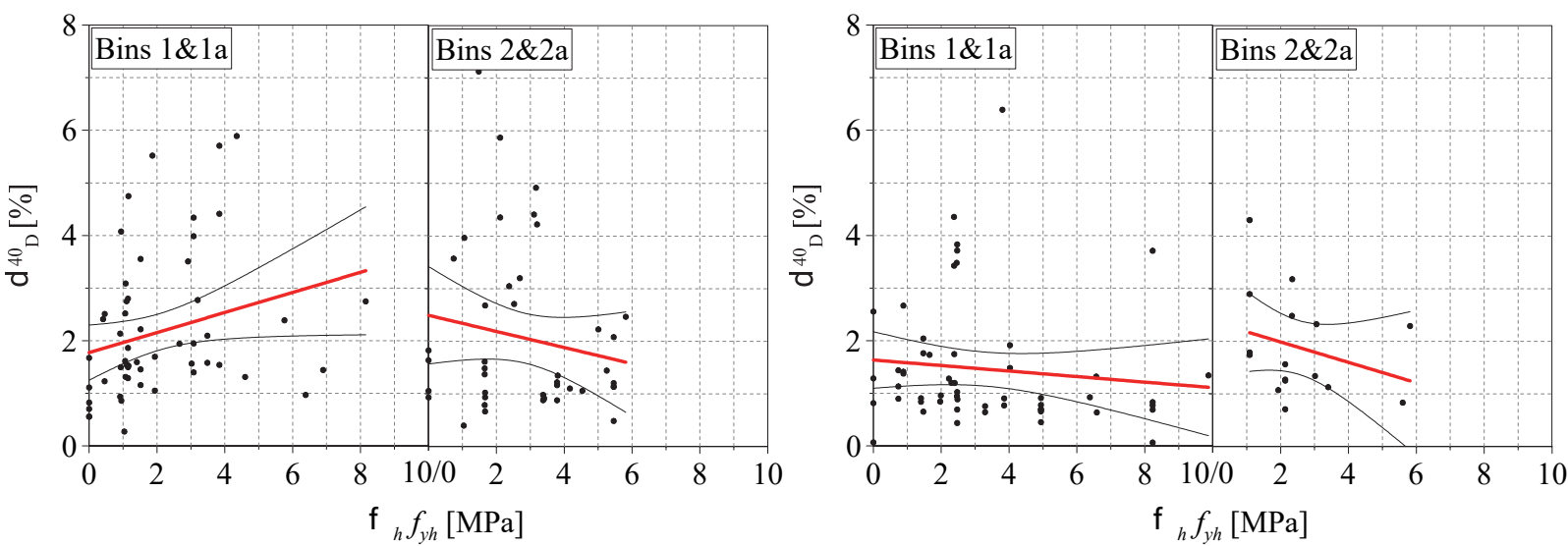

(d) $\rho_{h} f_{v h:}$ rectangular walls (left panel), and barbell and flanged walls (right panel)
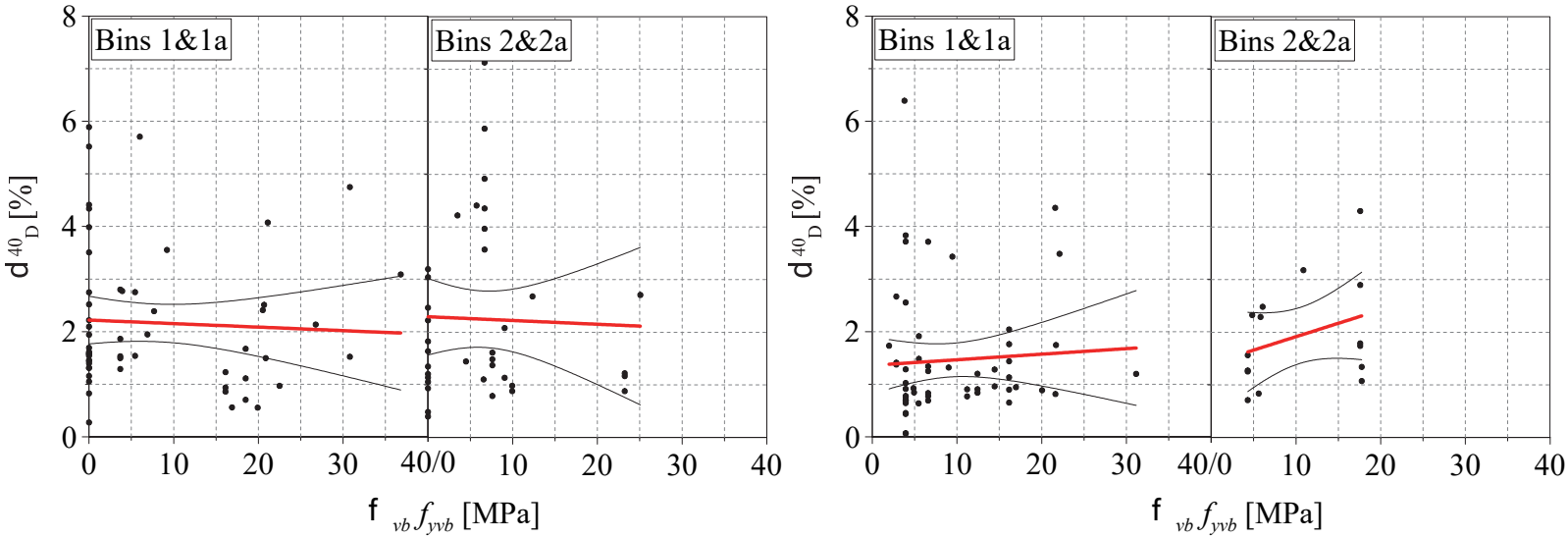

(e) $\rho_{v b} f_{v v b}$ : rectangular walls (left panel), and barbell and flanged walls (right panel)
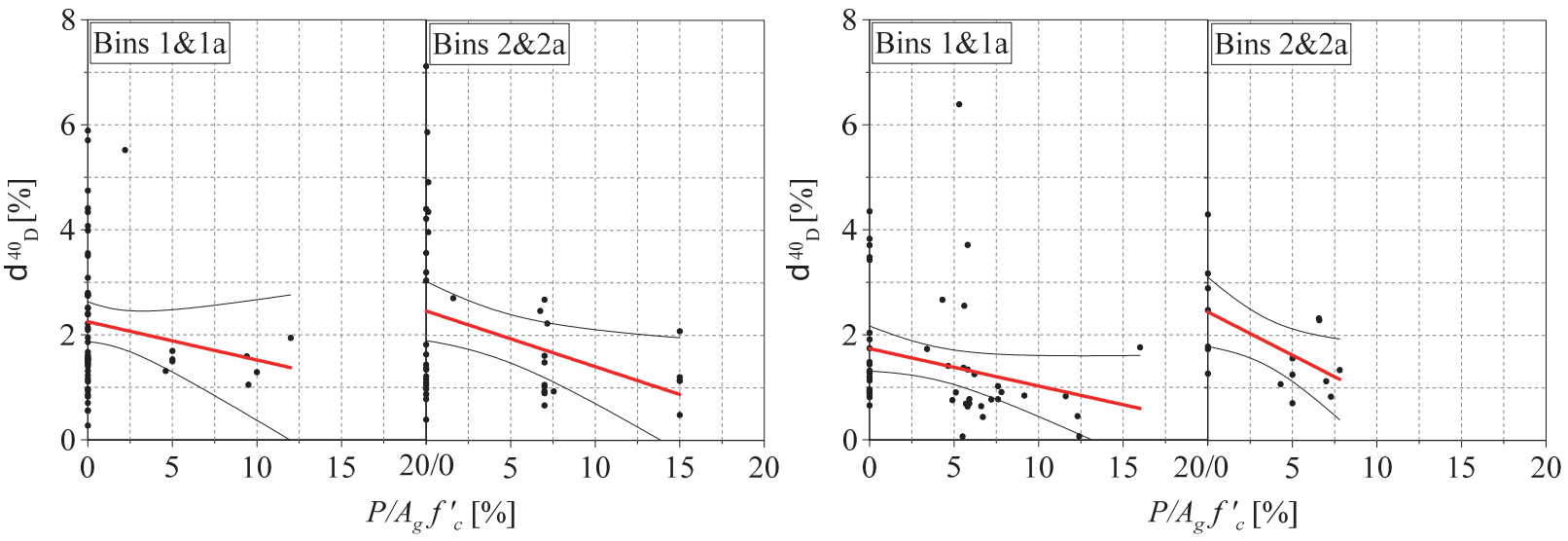

(f) $P / A_{g} f_{c}^{\prime}$ : rectangular walls (left panel), and barbell and flanged walls (right panel)

Figure C-7 Effect of design variables on $\delta_{D}^{40}$ (cont). 

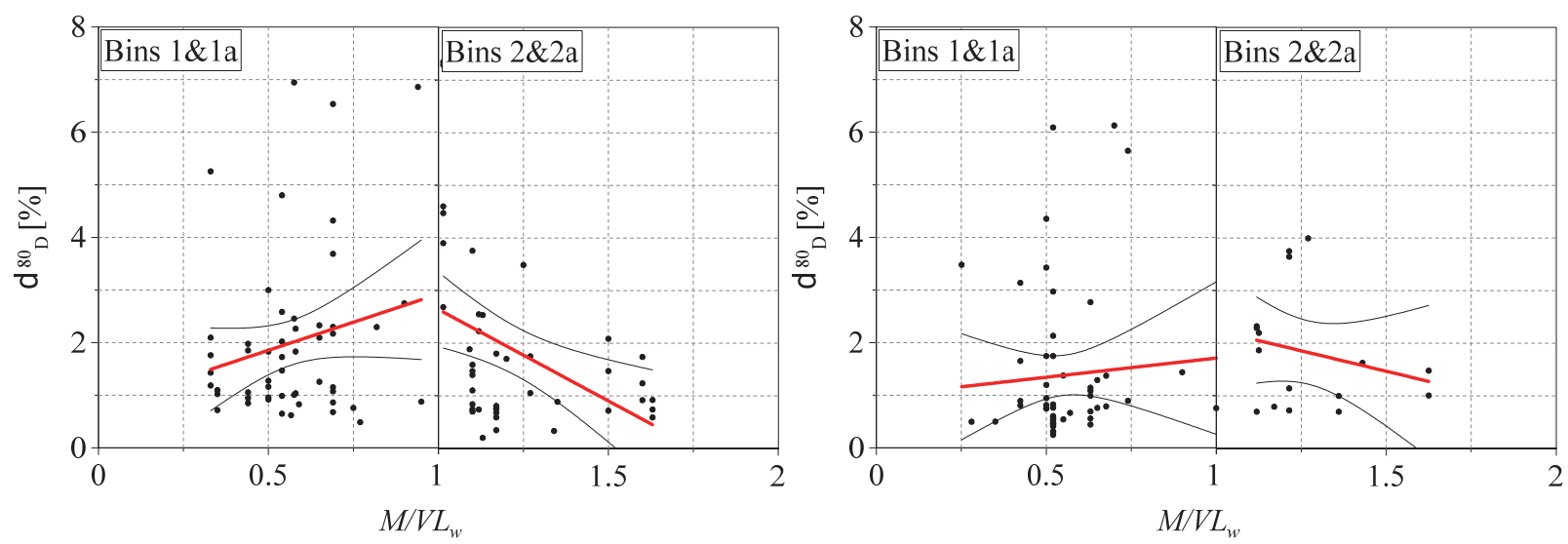

(a) $M / V I_{w}$ : rectangular walls (left panel), and barbell and flanged walls (right panel)
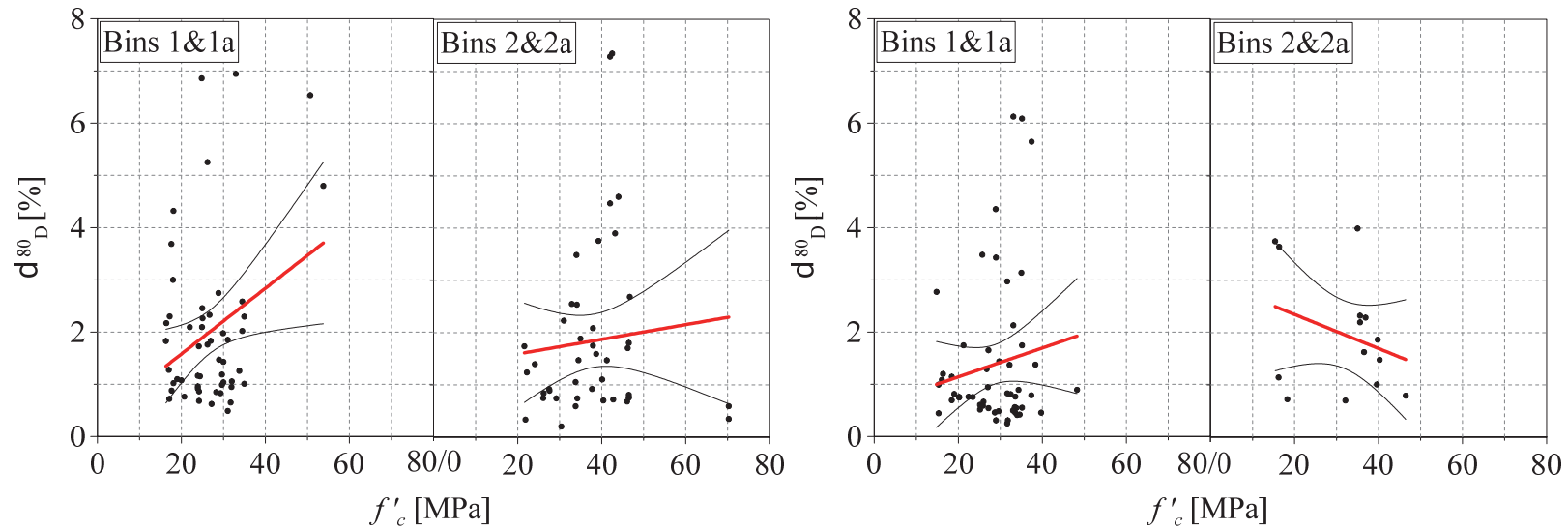

(b) $f_{c}^{\prime}$ : rectangular walls (left panel), and barbell and flanged walls (right panel)
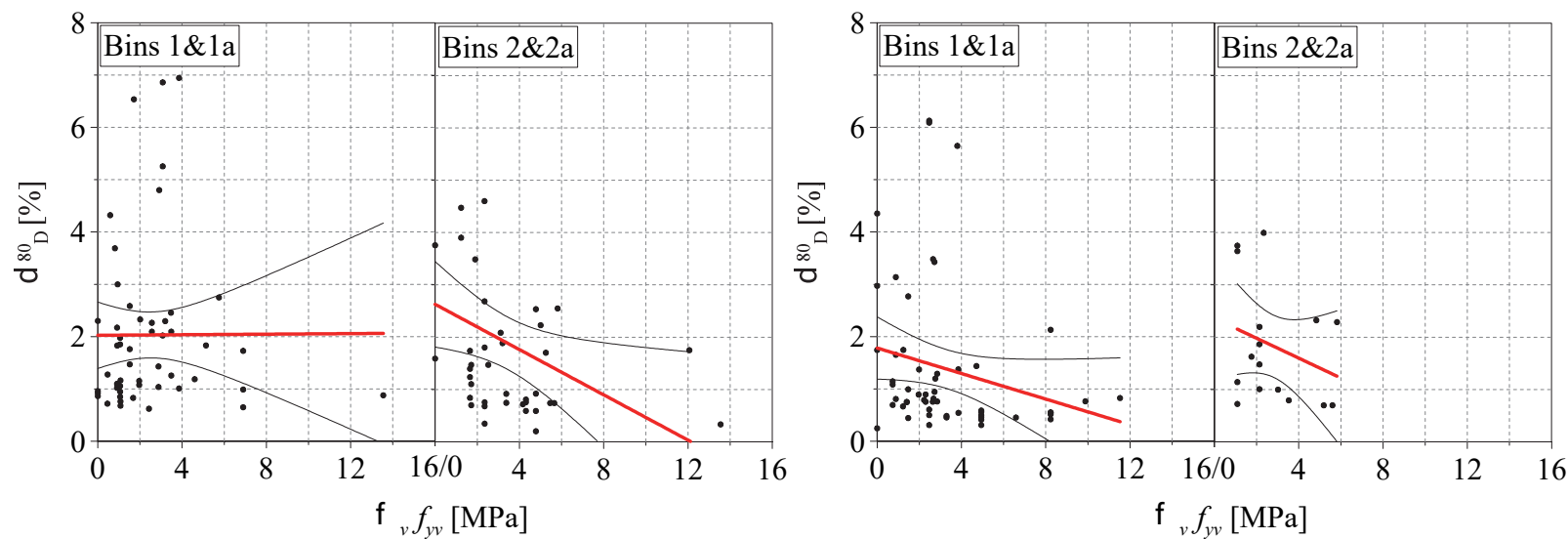

(c) $\rho_{v} f_{y v}$ : rectangular walls (left panel), and barbell and flanged walls (right panel)

Figure C-8 Effect of design variables on $\delta_{D}^{80}$. 

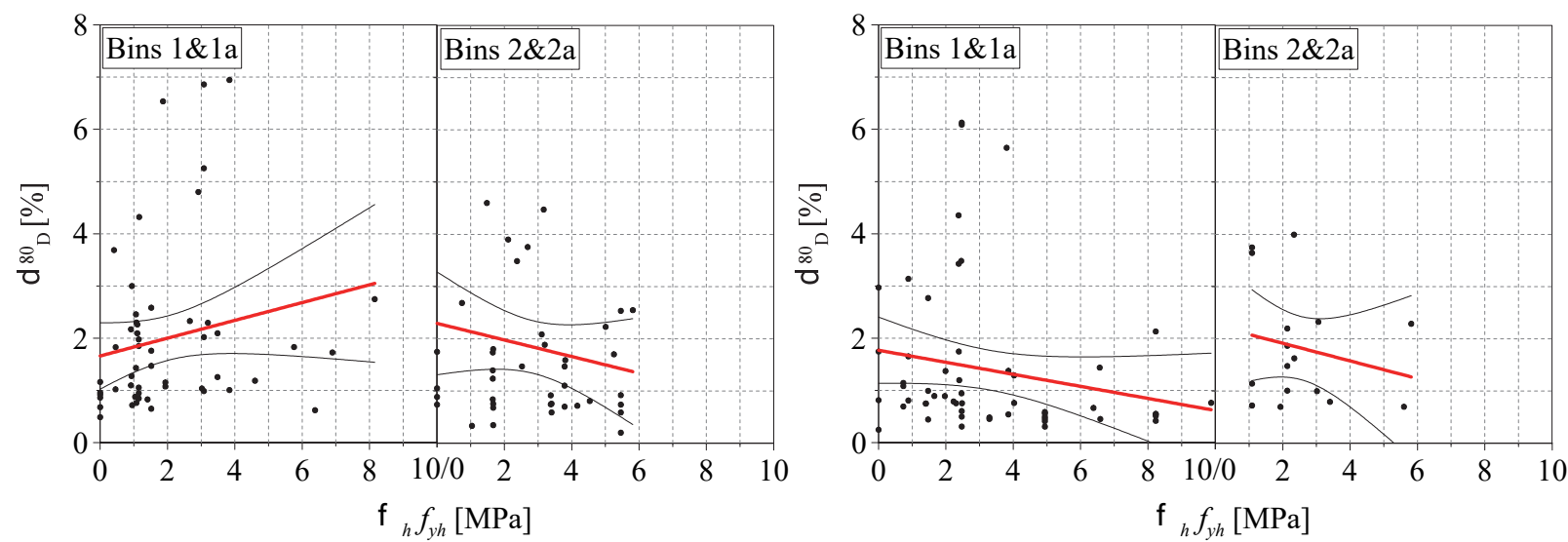

(d) $\rho_{h} f_{v h}$ : rectangular walls (left panel), and barbell and flanged walls (right panel)
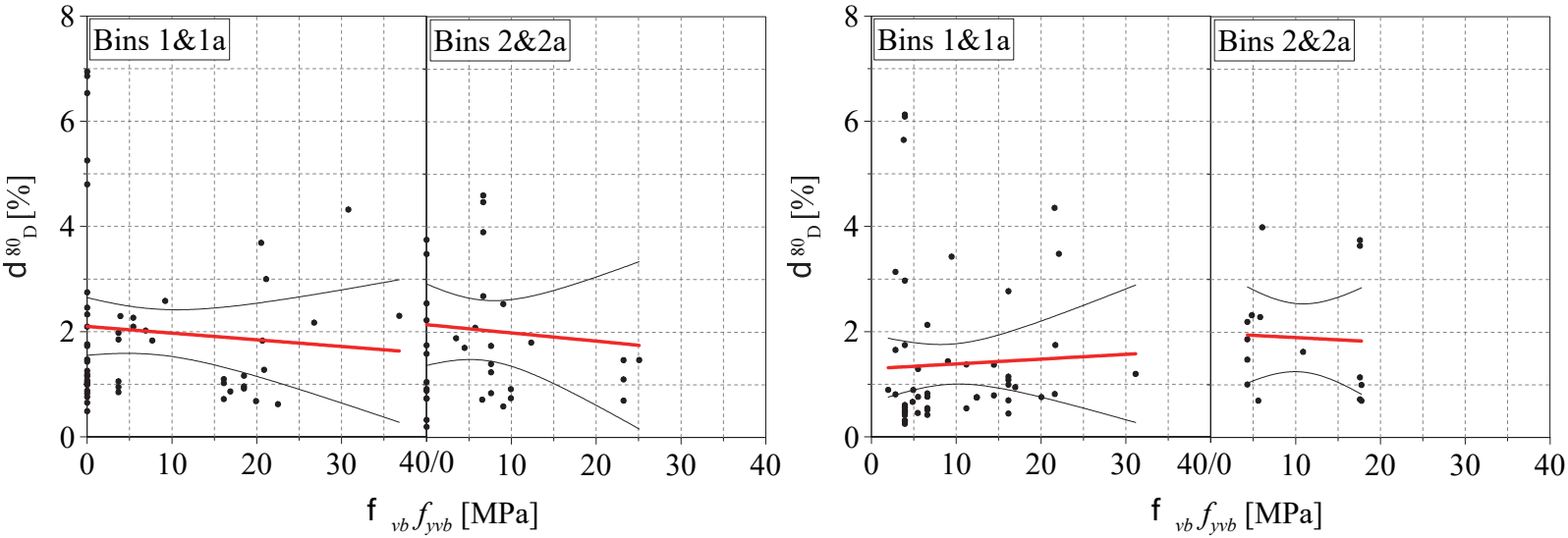

(e) $\rho_{v b} f_{v v b}$ : rectangular walls (left panel), and barbell and flanged walls (right panel)
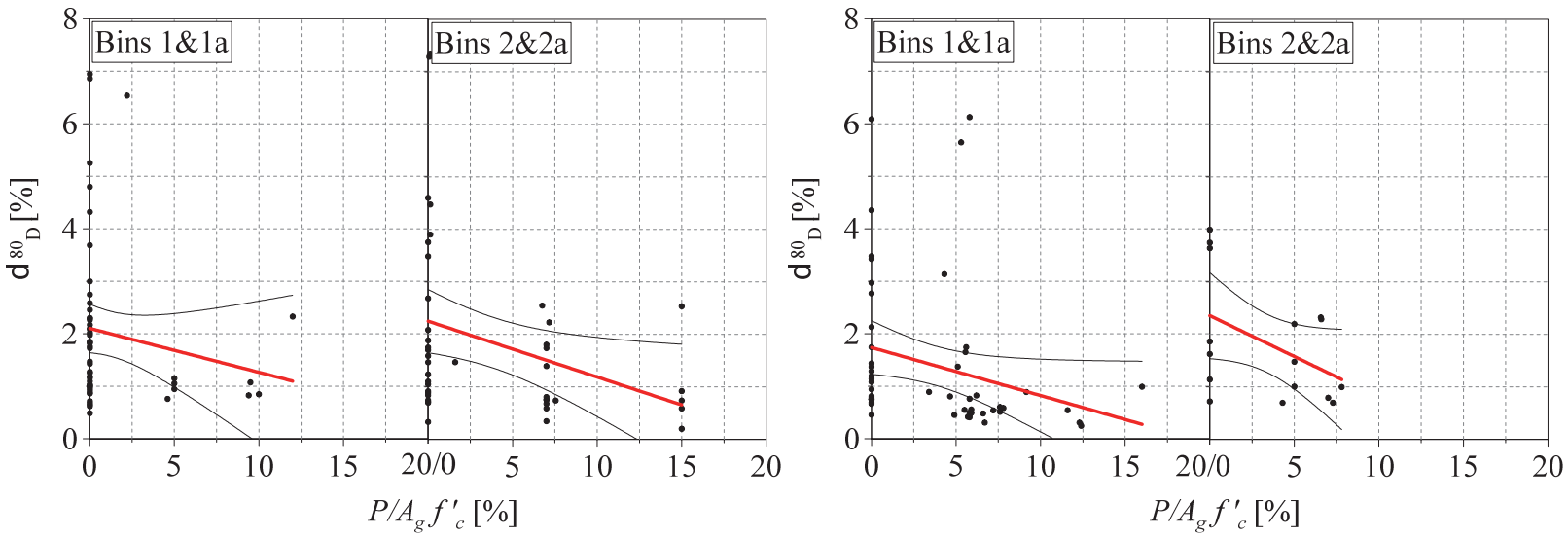

(f) $P / A_{g} f_{c}^{\prime}$ : rectangular walls (left panel), and barbell and flanged walls (right panel)

Figure C-8 Effect of design variables on $\delta_{D}^{80}$ (cont). 

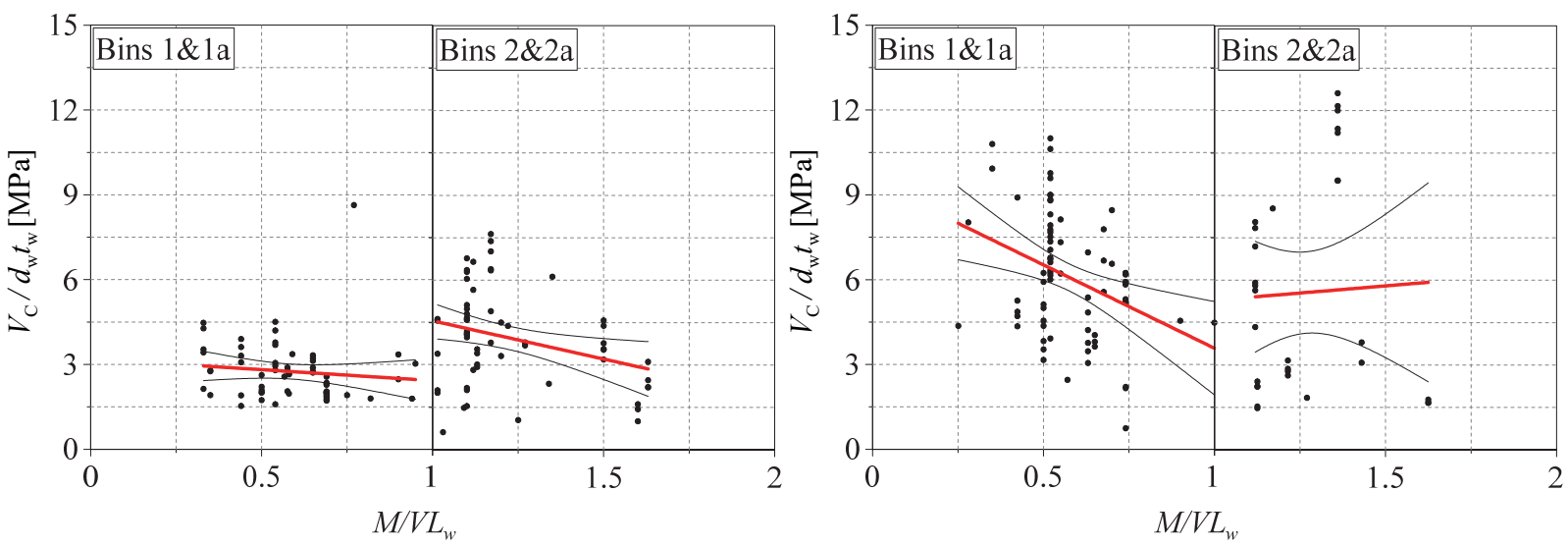

(a) $M / V l_{w}$ : rectangular walls (left panel), and barbell and flanged walls (right panel)
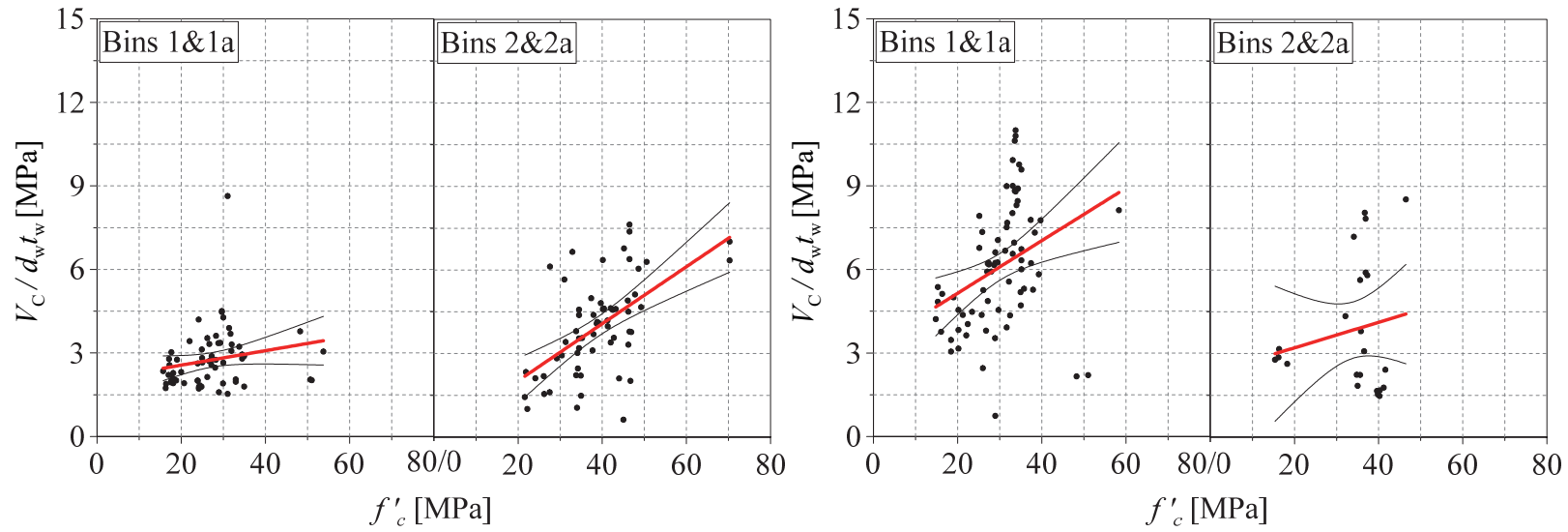

(b) $f_{c}^{\prime}$ : rectangular walls (left panel), and barbell and flanged walls (right panel)
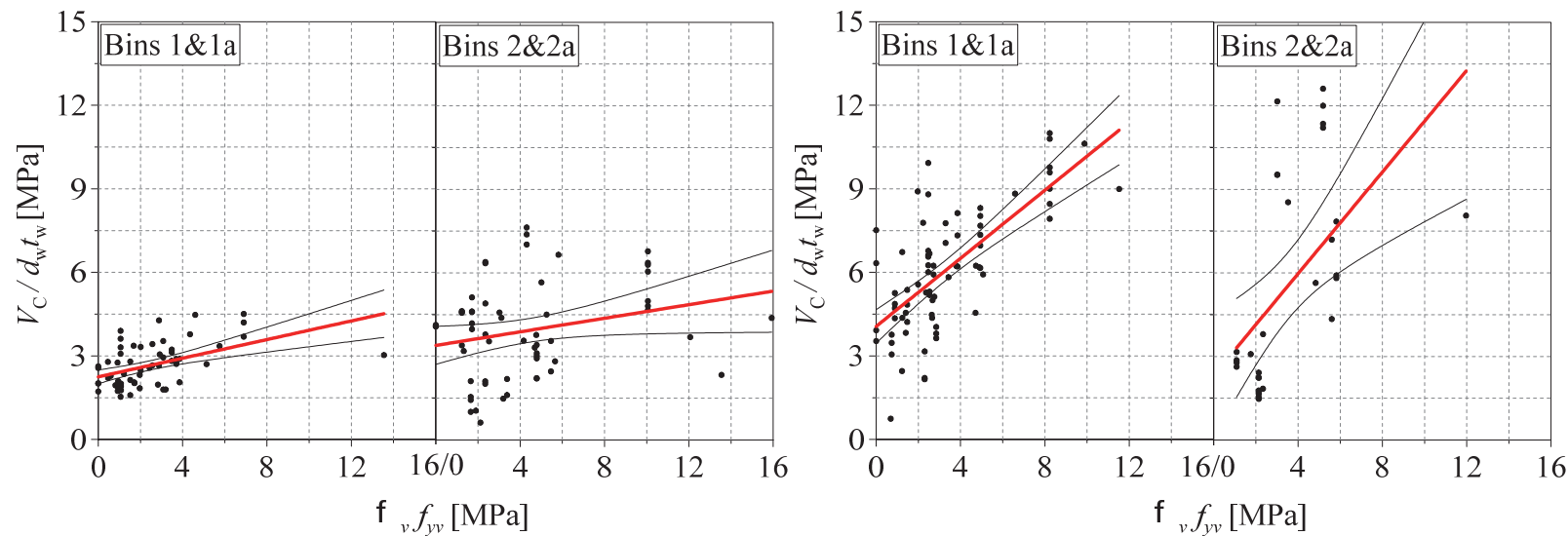

(c) $\rho_{v} f_{y v}$ : rectangular walls (left panel), and barbell and flanged walls (right panel)

Figure C-9 Effect of design variables on $V_{c} / d_{w} t_{w}$. 

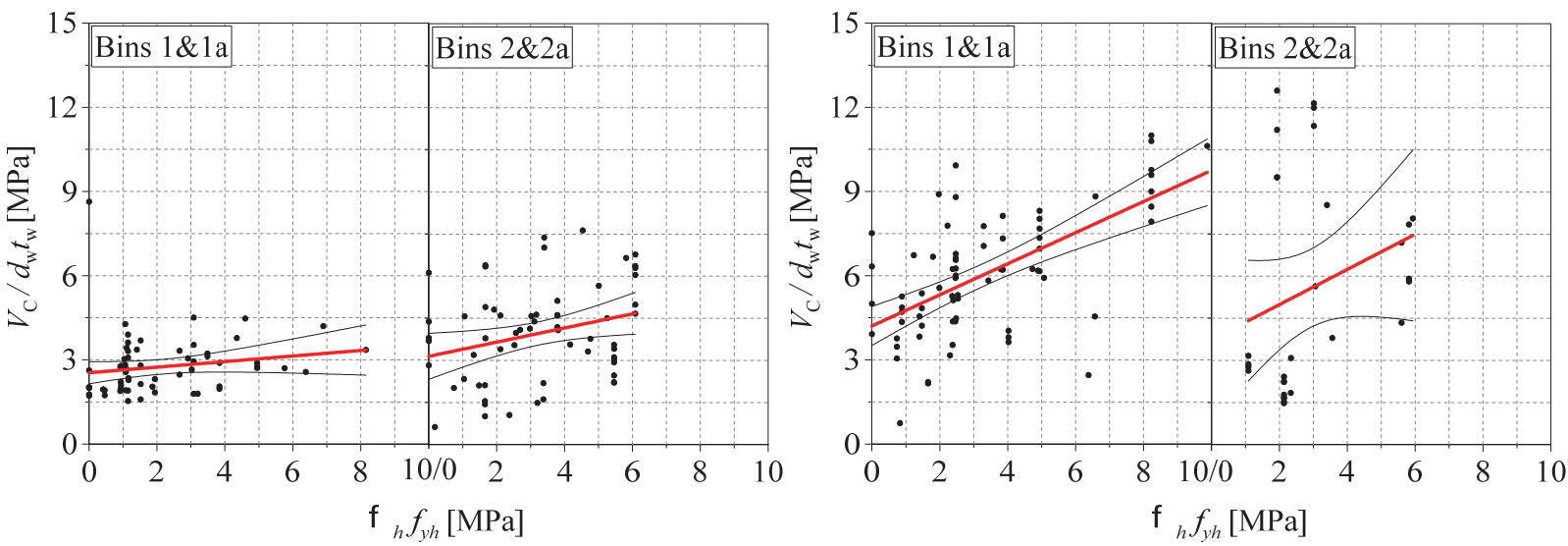

(d) $\rho_{h} f_{v h}$ rectangular walls (left panel), and barbell and flanged walls (right panel)
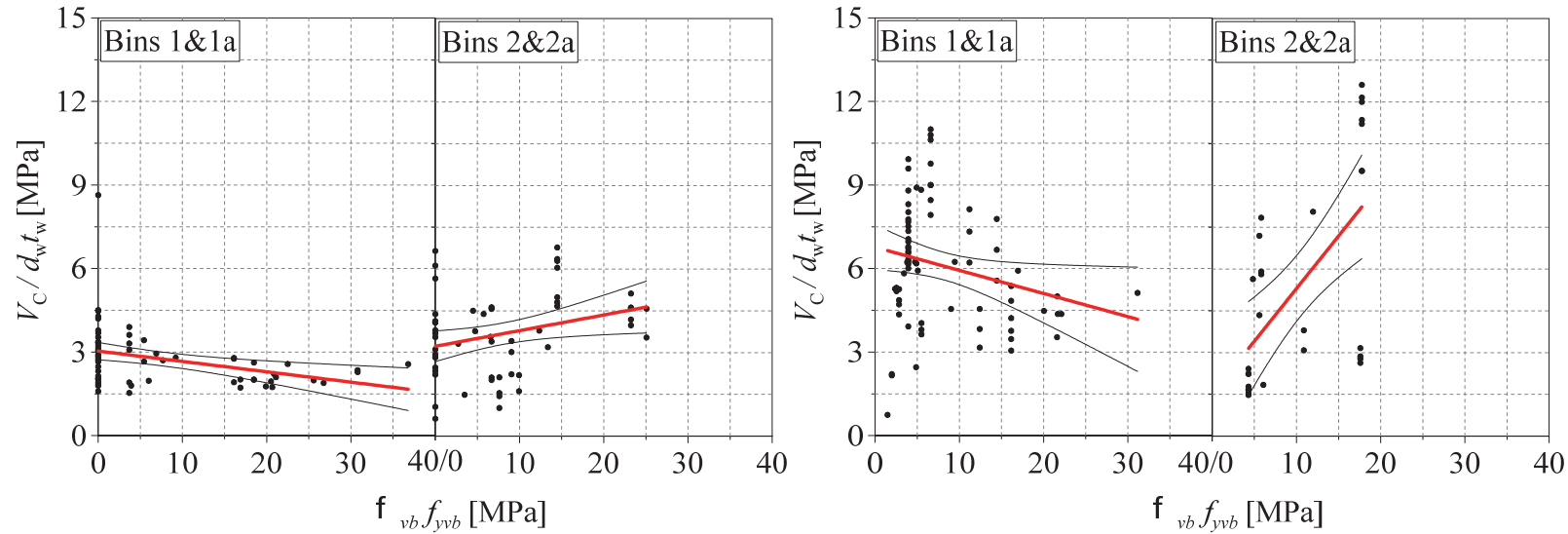

(e) $\rho_{v b} f_{v v b}$ : rectangular walls (left panel), and barbell and flanged walls (right panel)
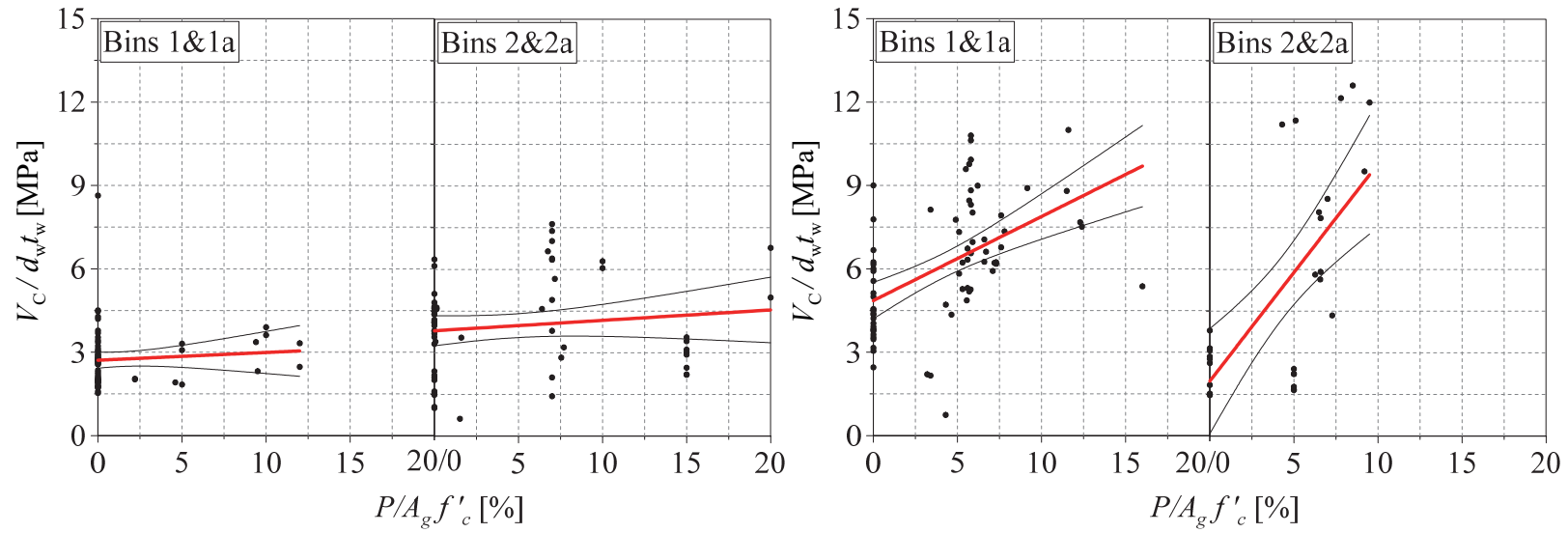

(f) $P / A_{g} f_{c}^{\prime}$ : rectangular walls (left panel), and barbell and flanged walls (right panel)

Figure C-9 Effect of design variables on $V_{c} / d_{w} t_{w}$ (cont). 

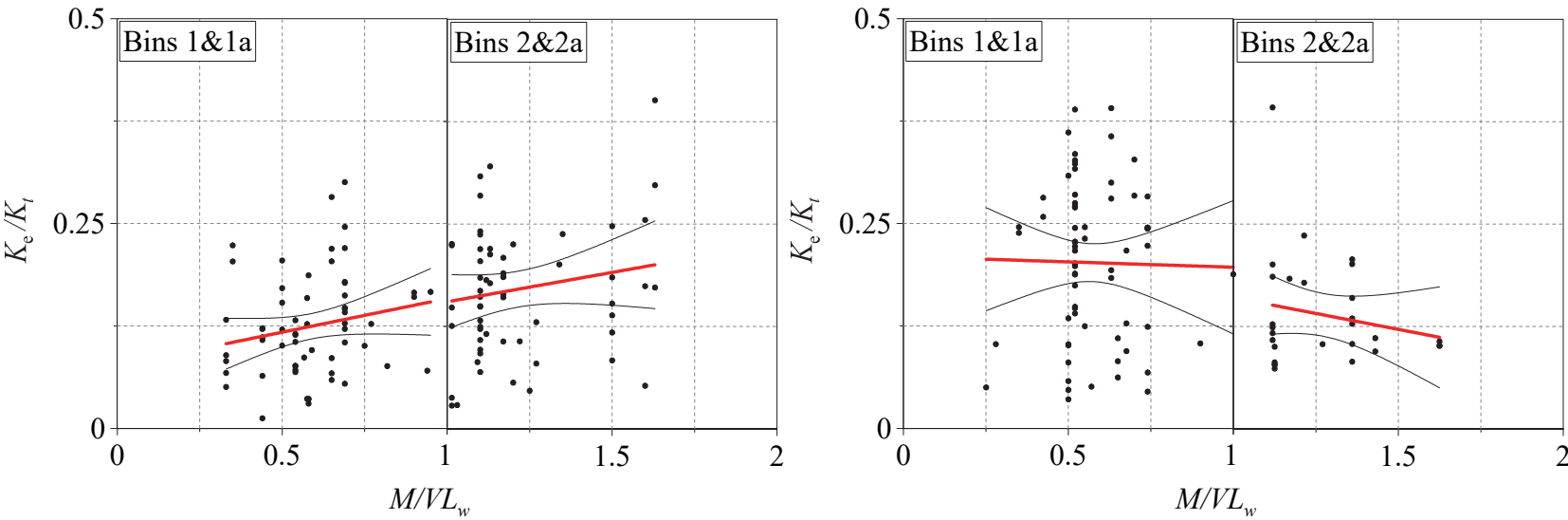

(a) $M / V I_{w}$ : rectangular walls (left panel), and barbell and flanged walls (right panel)
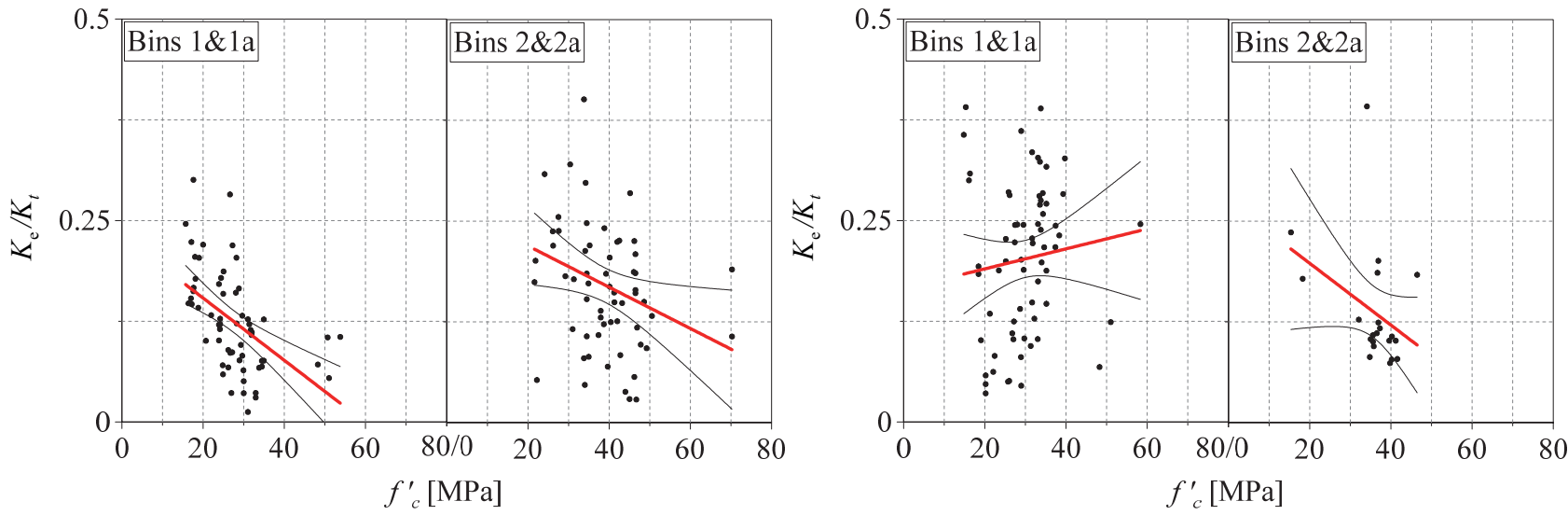

(b) $f_{c}^{\prime}$ : rectangular walls (left panel), and barbell and flanged walls (right panel)
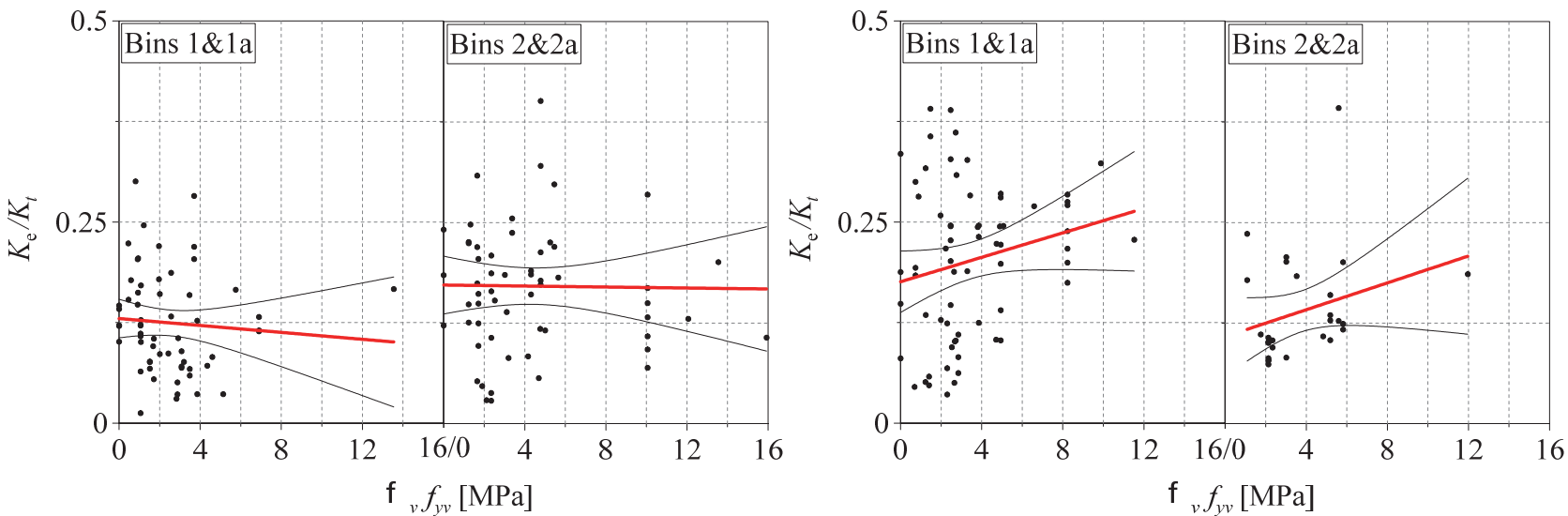

(c) $\rho_{v} f_{y v}$ : rectangular walls (left panel), and barbell and flanged walls (right panel)

Figure C-10 Effect of design variables on $K_{e} / K_{t}$. 

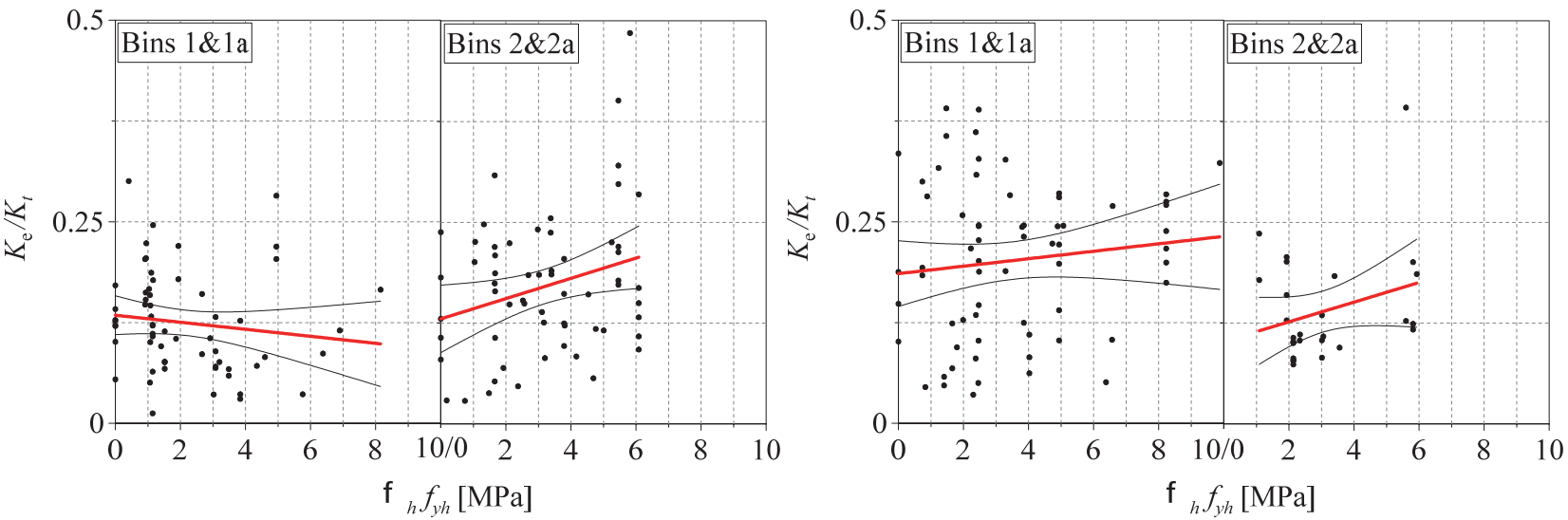

(d) $\rho_{h} f_{v h}$ : rectangular walls (left panel), and barbell and flanged walls (right panel)
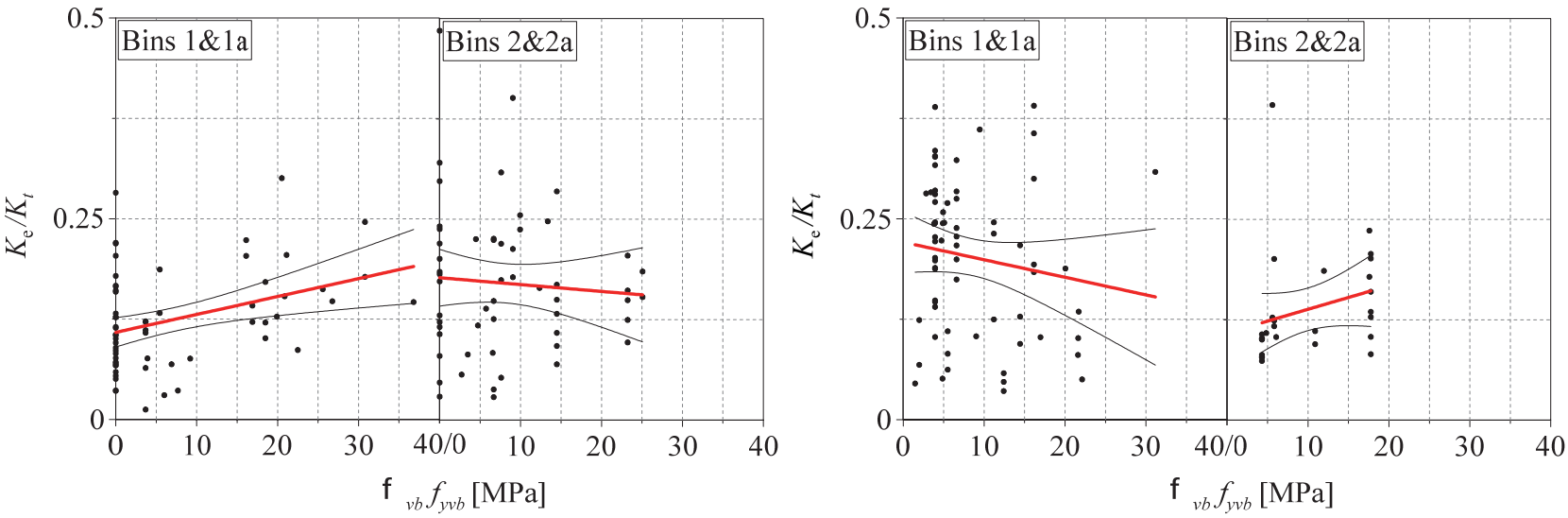

(e) $\rho_{v b} f_{v v b}$ : rectangular walls (left panel), and barbell and flanged walls (right panel)
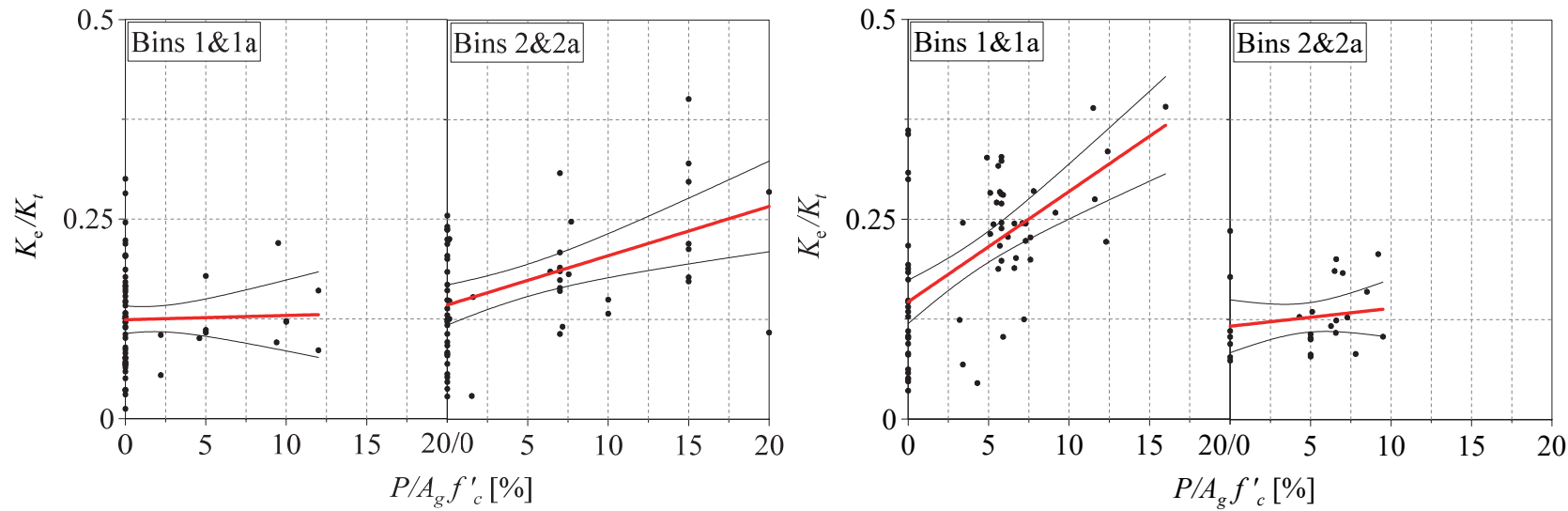

(f) $P / A_{g} f_{c}^{\prime}$ : rectangular walls (left panel), and barbell and flanged walls (right panel)

Figure C-10 Effect of design variables on $K_{e} / K_{t}$ (cont). 

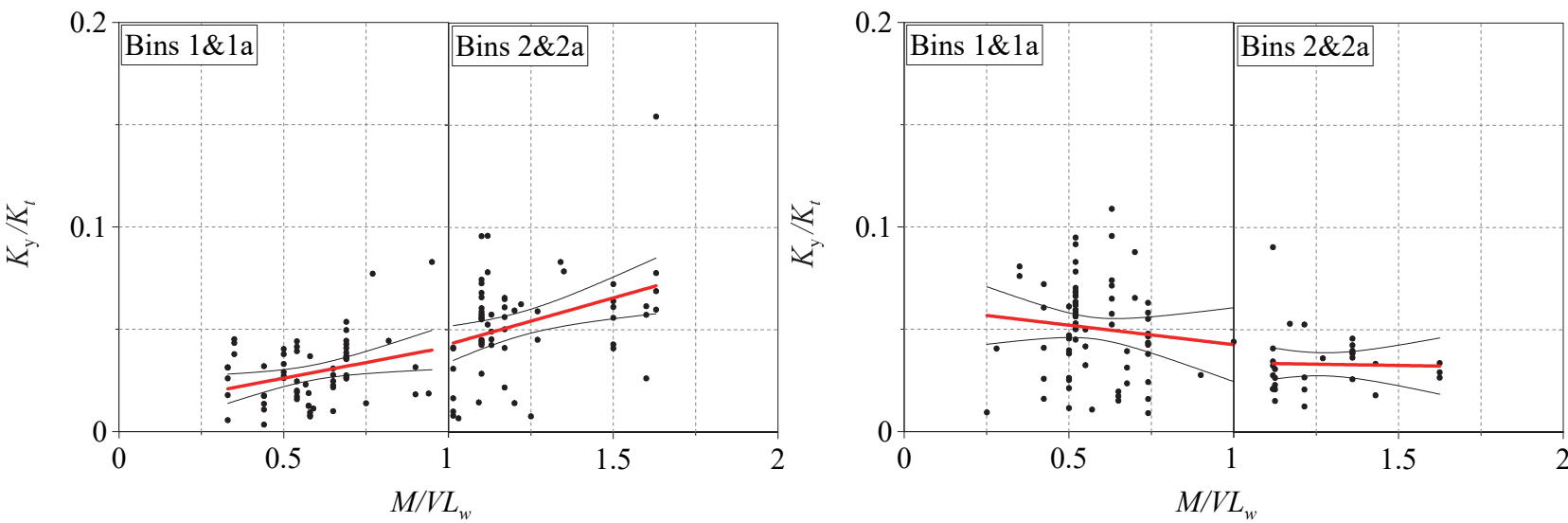

(a) $M / V I_{w}$ : rectangular walls (left panel), and barbell and flanged walls (right panel)
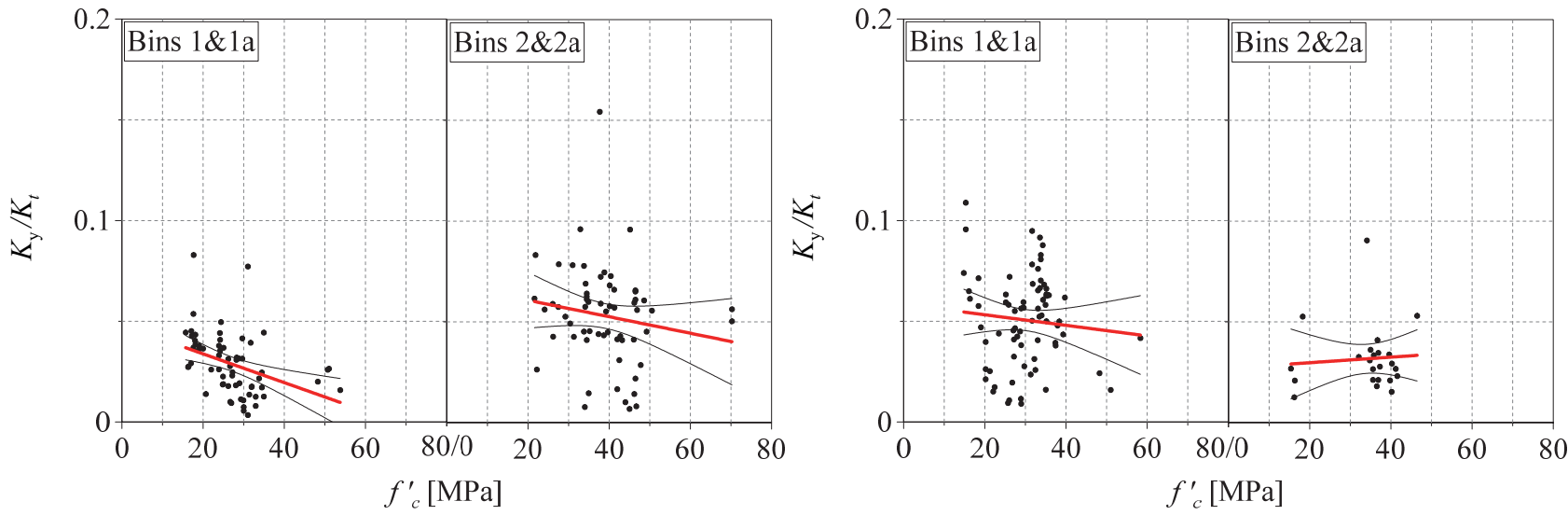

(b) $f_{c}^{\prime}$ : rectangular walls (left panel), and barbell and flanged walls (right panel)
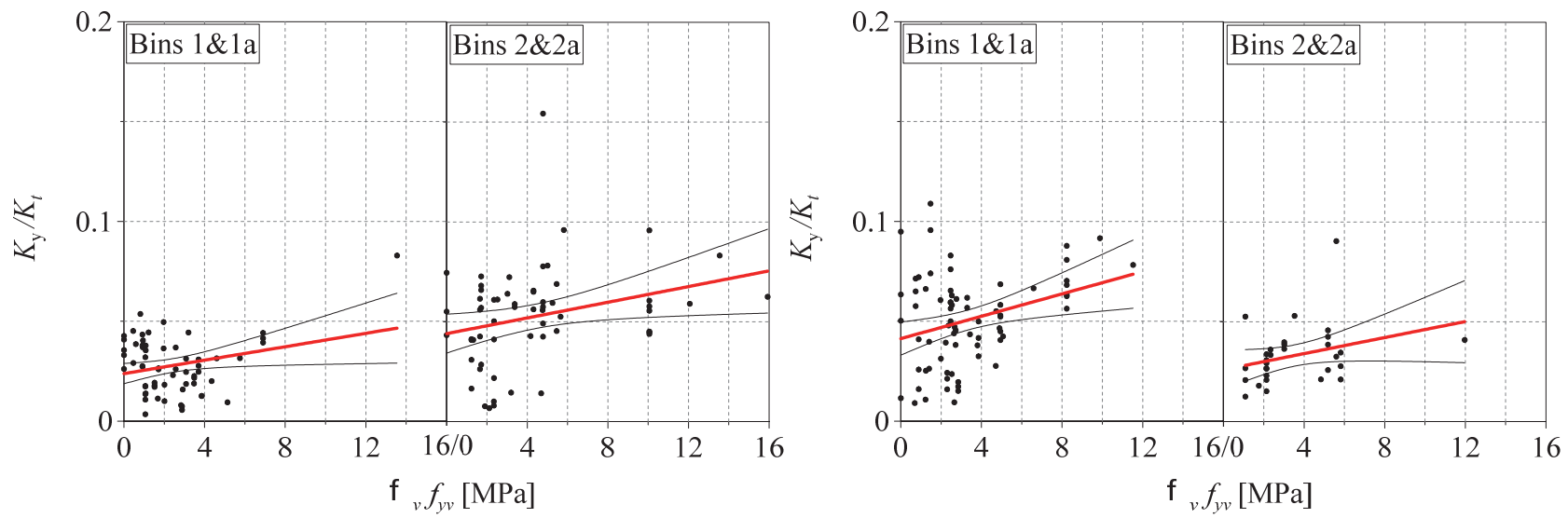

(c) $\rho_{v} f_{y v}$ : rectangular walls (left panel), and barbell and flanged walls (right panel)

Figure C-11 Effect of design variables on $K_{y} / K_{t}$. 

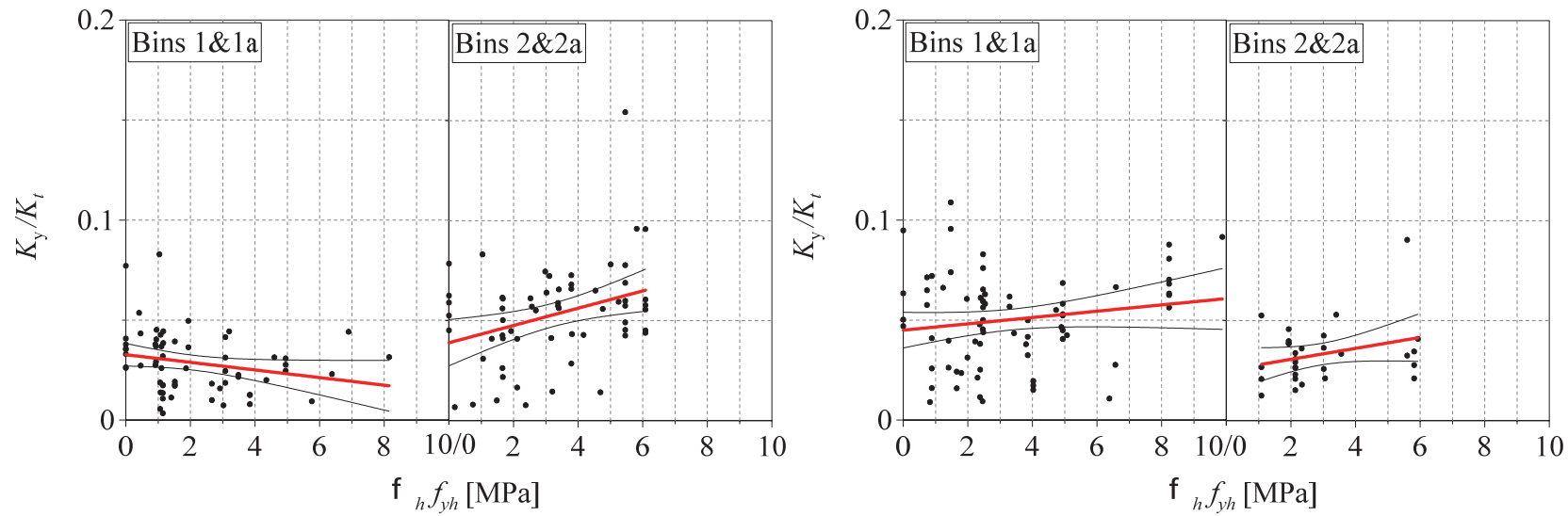

(d) $\rho_{h} f_{v h}$ : rectangular walls (left panel), and barbell and flanged walls (right panel)
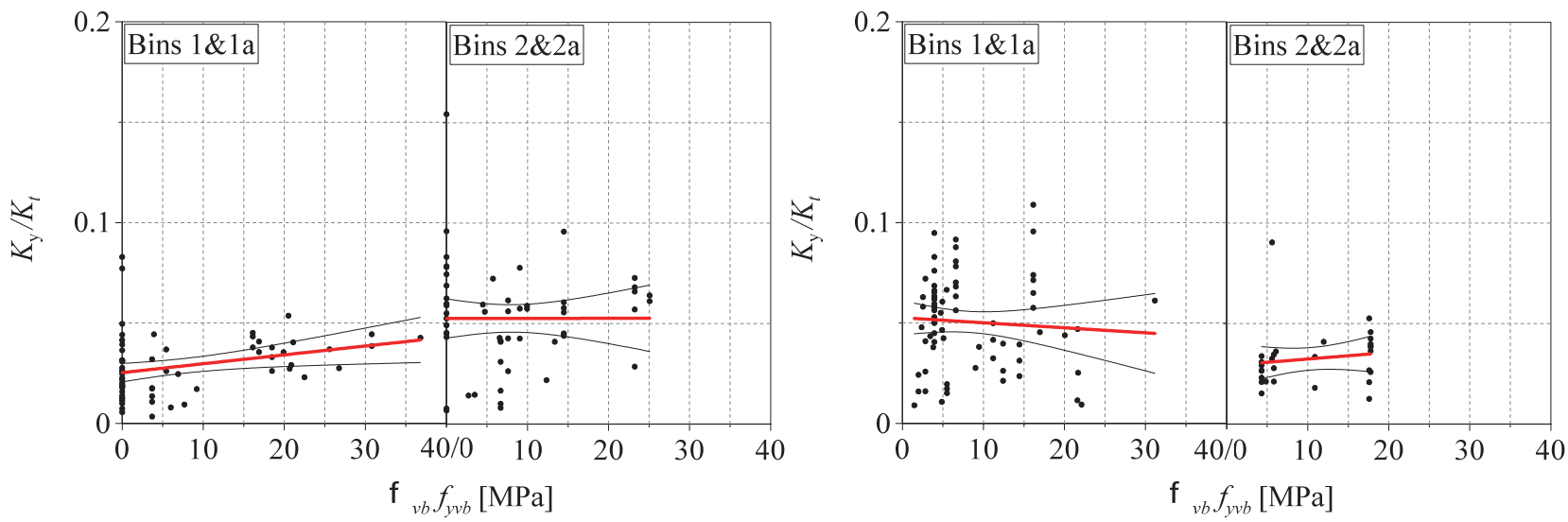

(e) $\rho_{v b} f_{v v b}$ : rectangular walls (left panel), and barbell and flanged walls (right panel)
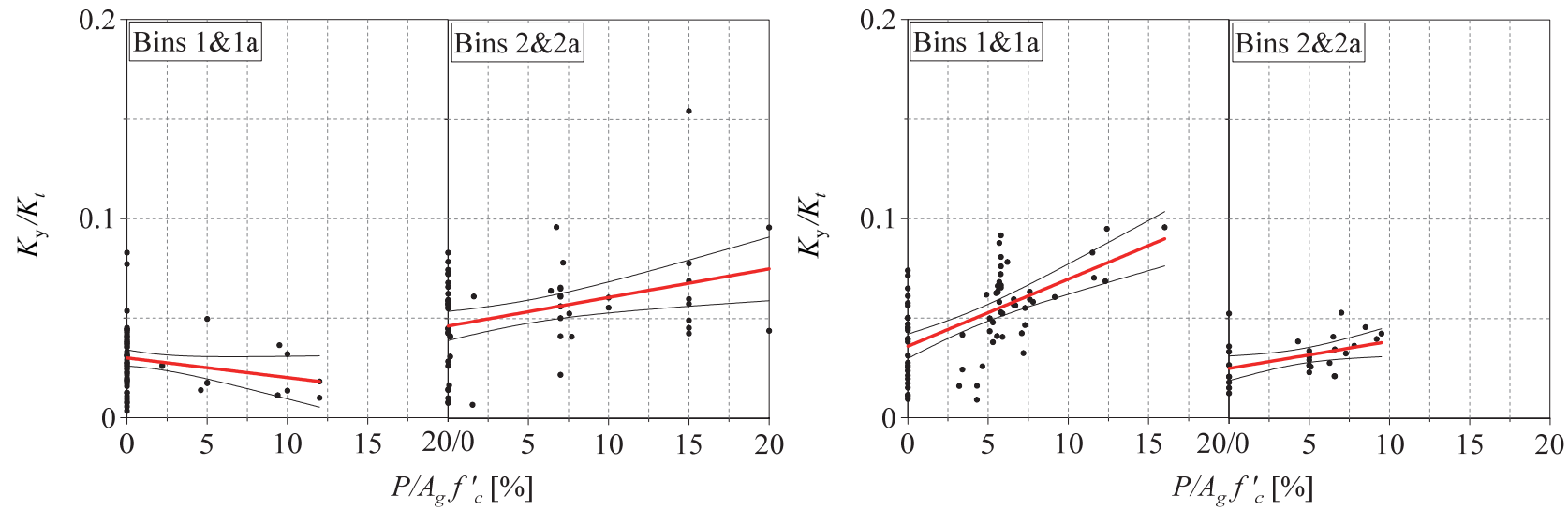

(f) $P / A_{g} f_{c}^{\prime}$ : rectangular walls (left panel), and barbell and flanged walls (right panel)

Figure C-11 Effect of design variables on $K_{y} / K_{t}$ (cont). 

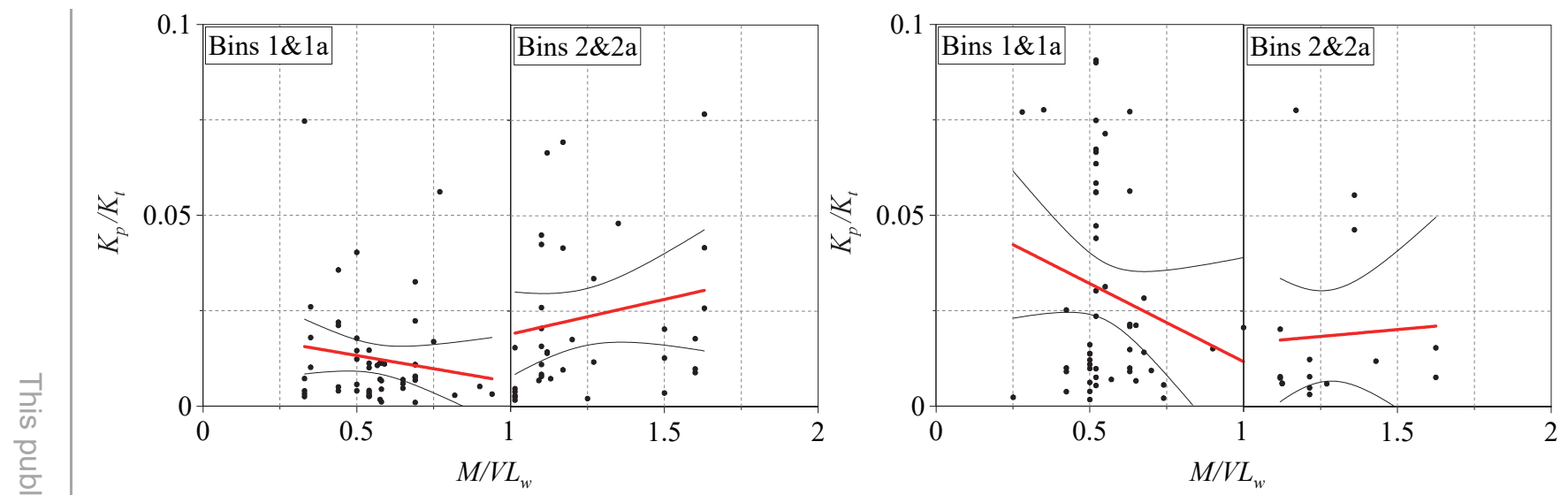

(a) $M / V I_{w}$ : rectangular walls (left panel), and barbell and flanged walls (right panel)
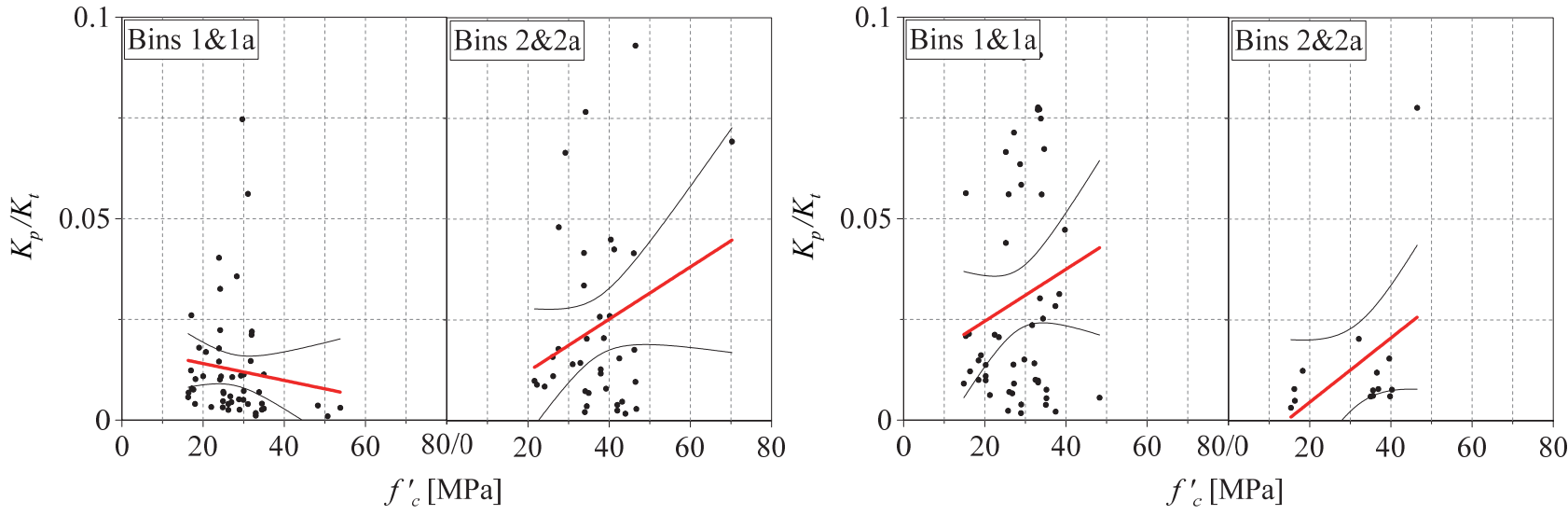

(b) $f_{c}^{\prime}$ : rectangular walls (left panel), and barbell and flanged walls (right panel)
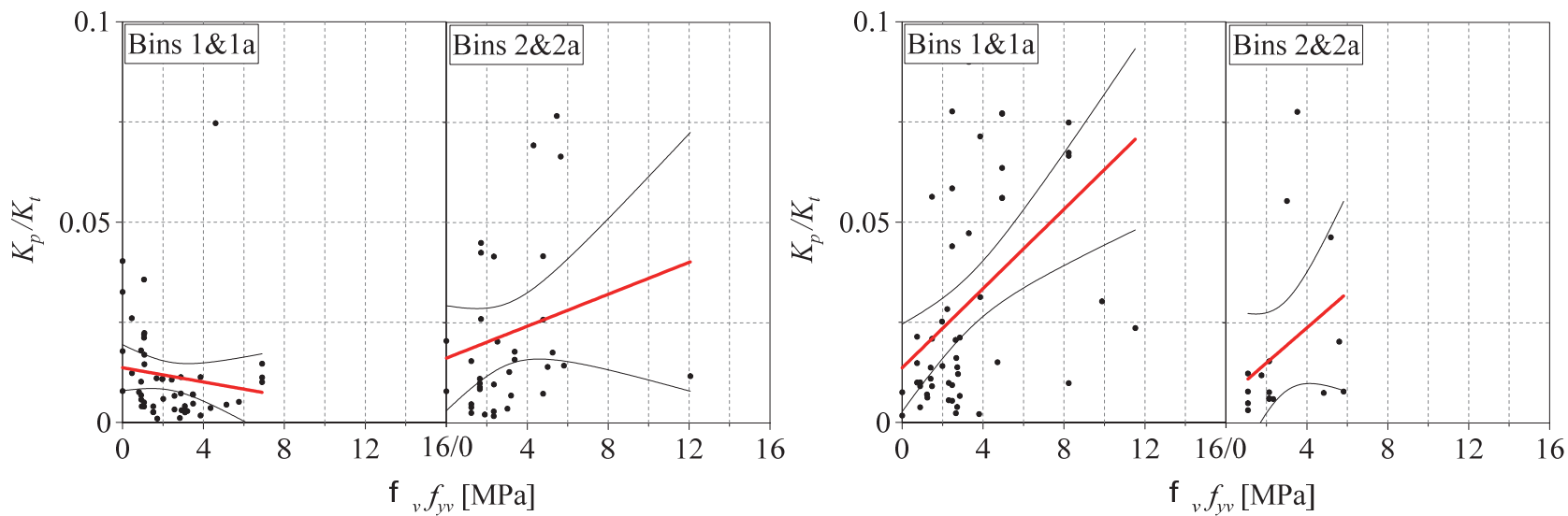

(c) $\rho_{v} f_{y v}$ rectangular walls (left panel), and barbell and flanged walls (right panel)

Figure C-12 Effect of design variables on $K_{p} / K_{t}$. 

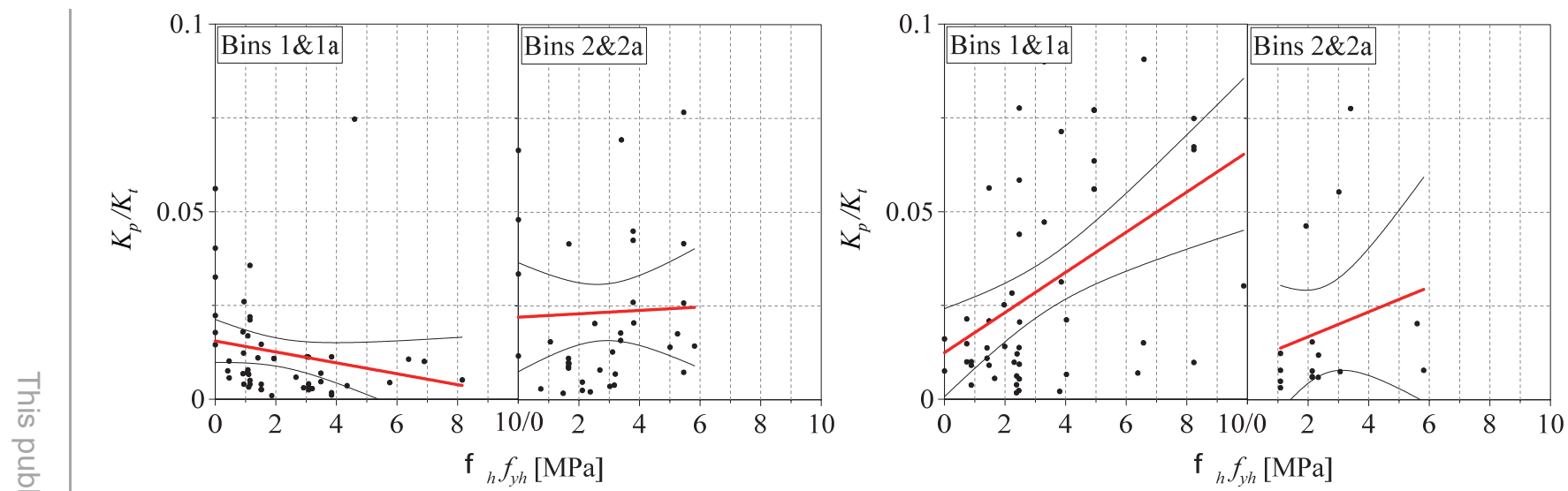

(d) $\rho_{h} f_{v h}$ : rectangular walls (left panel), and barbell and flanged walls (right panel)
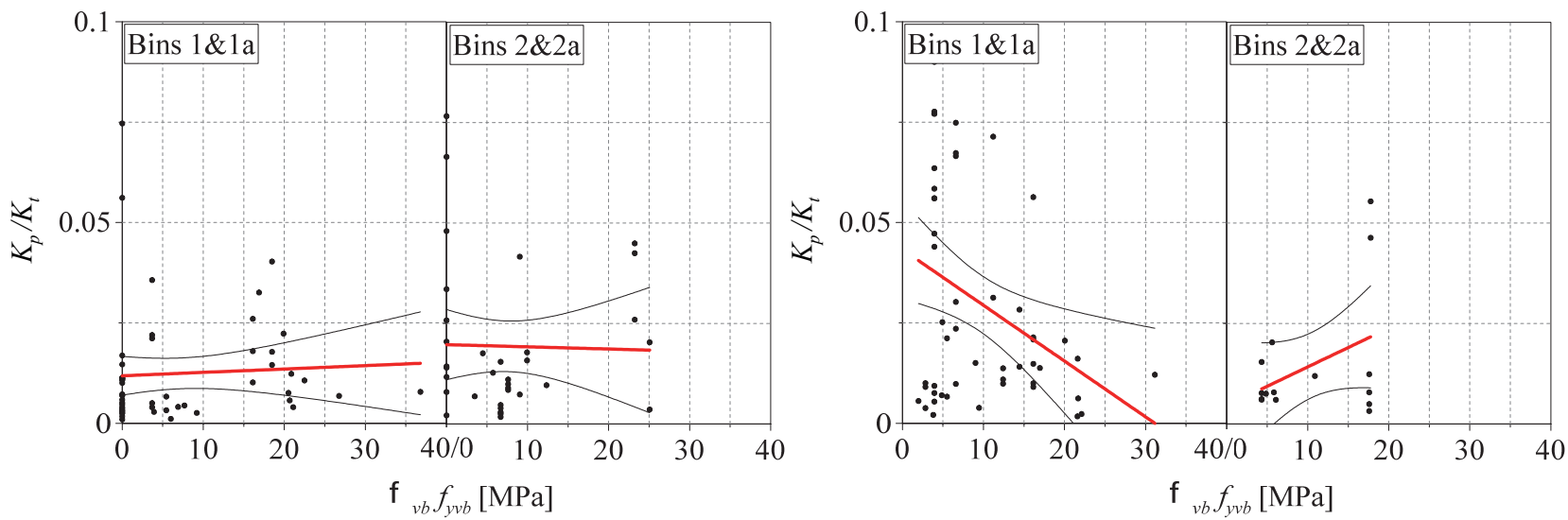

(e) $\rho_{v b} f_{v v b}$ : rectangular walls (left panel), and barbell and flanged walls (right panel)
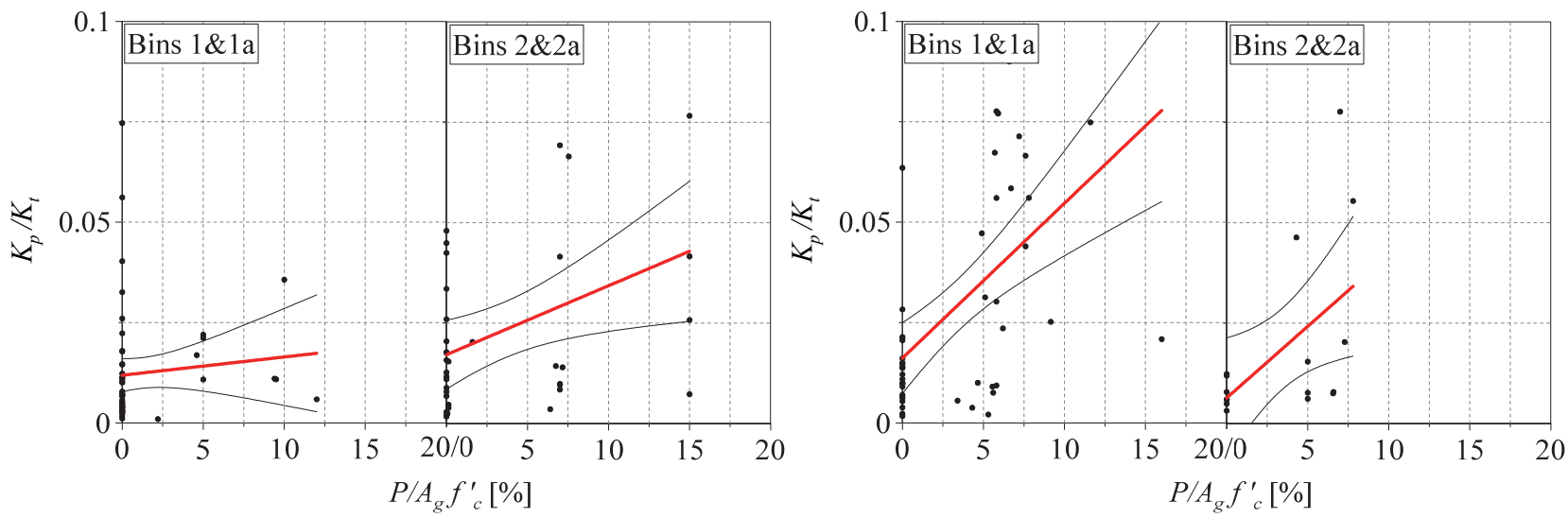

(f) $P / A_{g} f_{c}^{\prime}$ : rectangular walls (left panel), and barbell and flanged walls (right panel)

Figure C-12 Effect of design variables on $K_{p} / K_{t}$ (cont). 
Table C-1 Statistical Summary of Design Variables

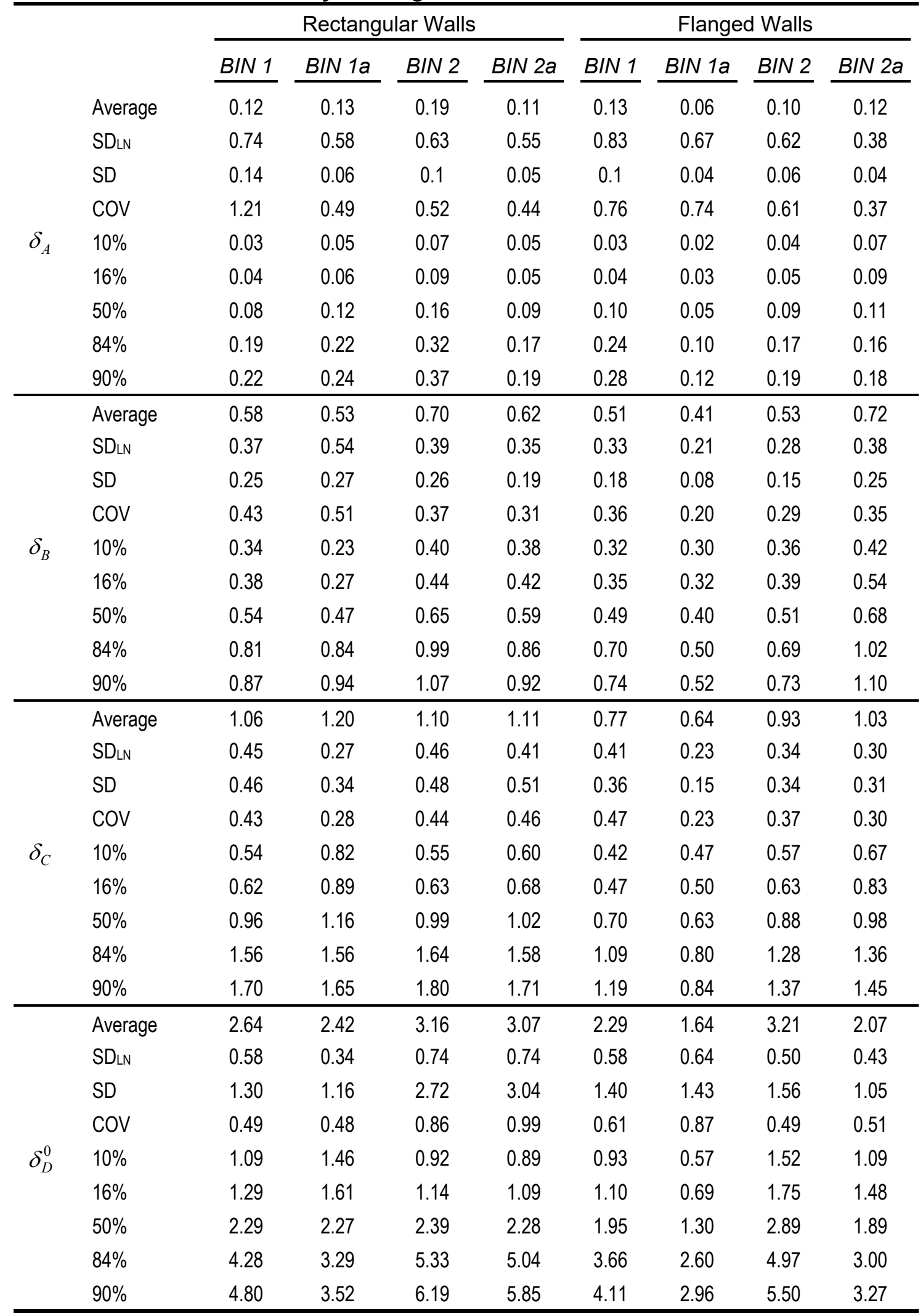


Table C-1 Statistical Summary of Design Variables (cont)

\begin{tabular}{|c|c|c|c|c|c|c|c|c|c|}
\hline & & \multicolumn{4}{|c|}{ Rectangular Walls } & \multicolumn{4}{|c|}{ Flanged Walls } \\
\hline & & BIN 1 & BIN $1 a$ & BIN 2 & BIN 2a & BIN 1 & BIN $1 a$ & BIN 2 & $B I N 2 a$ \\
\hline \multirow{9}{*}{$\delta_{D}^{20}$} & Average & 2.16 & 2.09 & 2.48 & 2.53 & 1.86 & 1.38 & 2.65 & 1.65 \\
\hline & SDLN & 0.90 & 0.37 & 0.76 & 0.71 & 0.56 & 0.68 & 0.52 & 0.40 \\
\hline & SD & 1.13 & 1.11 & 2.14 & 2.53 & 1.13 & 1.24 & 1.24 & 0.77 \\
\hline & COV & 0.52 & 0.53 & 0.87 & 1.00 & 0.61 & 0.9 & 0.47 & 0.47 \\
\hline & $10 \%$ & 0.54 & 1.20 & 0.71 & 0.76 & 0.78 & 0.45 & 1.23 & 0.91 \\
\hline & $16 \%$ & 0.70 & 1.33 & 0.88 & 0.93 & 0.91 & 0.55 & 1.43 & 1.20 \\
\hline & $50 \%$ & 1.71 & 1.93 & 1.86 & 1.90 & 1.59 & 1.08 & 2.38 & 1.52 \\
\hline & $84 \%$ & 4.52 & 2.90 & 4.21 & 4.11 & 2.92 & 2.24 & 4.16 & 2.35 \\
\hline & $90 \%$ & 5.41 & 3.12 & 4.90 & 4.75 & 3.27 & 2.57 & 4.62 & 2.54 \\
\hline \multirow{9}{*}{$\delta_{D}^{40}$} & Average & 2.21 & 1.94 & 2.08 & 2.45 & 1.71 & 1.25 & 2.52 & 1.39 \\
\hline & SDLN & 0.65 & 0.48 & 0.71 & 0.78 & 0.56 & 0.93 & 0.42 & 0.41 \\
\hline & SD & 1.35 & 1.37 & 1.68 & 2.48 & 1.11 & 1.24 & 1.04 & 0.58 \\
\hline & COV & 0.61 & 0.71 & 0.81 & 1.01 & 0.65 & 0.99 & 0.41 & 0.42 \\
\hline & $10 \%$ & 0.79 & 0.93 & 0.64 & 0.64 & 0.71 & 0.27 & 1.37 & 0.77 \\
\hline & $16 \%$ & 0.96 & 1.06 & 0.79 & 0.81 & 0.83 & 0.35 & 1.55 & 1.02 \\
\hline & $50 \%$ & 1.83 & 1.70 & 1.61 & 1.76 & 1.44 & 0.87 & 2.34 & 1.29 \\
\hline & $84 \%$ & 3.70 & 2.84 & 3.50 & 4.11 & 2.64 & 2.39 & 3.68 & 2.00 \\
\hline & $90 \%$ & 4.21 & 3.12 & 4.04 & 4.80 & 2.96 & 2.88 & 4.00 & 2.17 \\
\hline \multirow{9}{*}{$\delta_{D}^{80}$} & Average & 2.56 & 1.73 & 1.68 & 2.35 & 1.70 & 1.11 & 2.39 & 1.39 \\
\hline & SDLN & 0.76 & 0.69 & 0.66 & 1.01 & 0.69 & 0.78 & 0.66 & 0.51 \\
\hline & SD & 2.44 & 1.86 & 1.14 & 2.63 & 1.40 & 1.42 & 1.37 & 0.70 \\
\hline & COV & 0.95 & 1.08 & 0.68 & 1.12 & 0.82 & 1.28 & 0.57 & 0.51 \\
\hline & $10 \%$ & 0.70 & 0.53 & 0.59 & 0.39 & 0.55 & 0.27 & 0.86 & 0.64 \\
\hline & $16 \%$ & 0.87 & 0.65 & 0.71 & 0.52 & 0.67 & 0.34 & 1.04 & 0.92 \\
\hline & $50 \%$ & 1.86 & 1.29 & 1.37 & 1.42 & 1.32 & 0.74 & 2.02 & 1.24 \\
\hline & $84 \%$ & 4.25 & 2.73 & 2.79 & 4.25 & 2.78 & 1.73 & 4.12 & 2.14 \\
\hline & $90 \%$ & 4.96 & 3.13 & 3.19 & 5.21 & 3.20 & 2.03 & 4.71 & 2.37 \\
\hline \multirow{9}{*}{$V_{A} / V_{C}$} & Average & 0.45 & 0.65 & 0.49 & 0.42 & 0.49 & 0.40 & 0.59 & 0.43 \\
\hline & SDLN & 0.32 & 0.15 & 0.28 & 0.29 & 0.39 & 0.36 & 0.19 & 0.21 \\
\hline & SD & 0.15 & 1.16 & 0.13 & 0.11 & 0.19 & 0.14 & 0.12 & 0.09 \\
\hline & COV & 0.33 & 0.48 & 0.26 & 0.25 & 0.38 & 0.36 & 0.20 & 0.21 \\
\hline & $10 \%$ & 0.28 & 0.53 & 0.33 & 0.28 & 0.28 & 0.23 & 0.45 & 0.32 \\
\hline & $16 \%$ & 0.31 & 0.55 & 0.36 & 0.30 & 0.31 & 0.26 & 0.48 & 0.37 \\
\hline & $50 \%$ & 0.43 & 0.64 & 0.47 & 0.40 & 0.46 & 0.37 & 0.58 & 0.42 \\
\hline & $84 \%$ & 0.61 & 0.75 & 0.64 & 0.55 & 0.70 & 0.55 & 0.71 & 0.53 \\
\hline & $90 \%$ & 0.65 & 0.78 & 0.68 & 0.58 & 0.76 & 0.59 & 0.74 & 0.55 \\
\hline
\end{tabular}


Table C-1 Statistical Summary of Design Variables (cont)

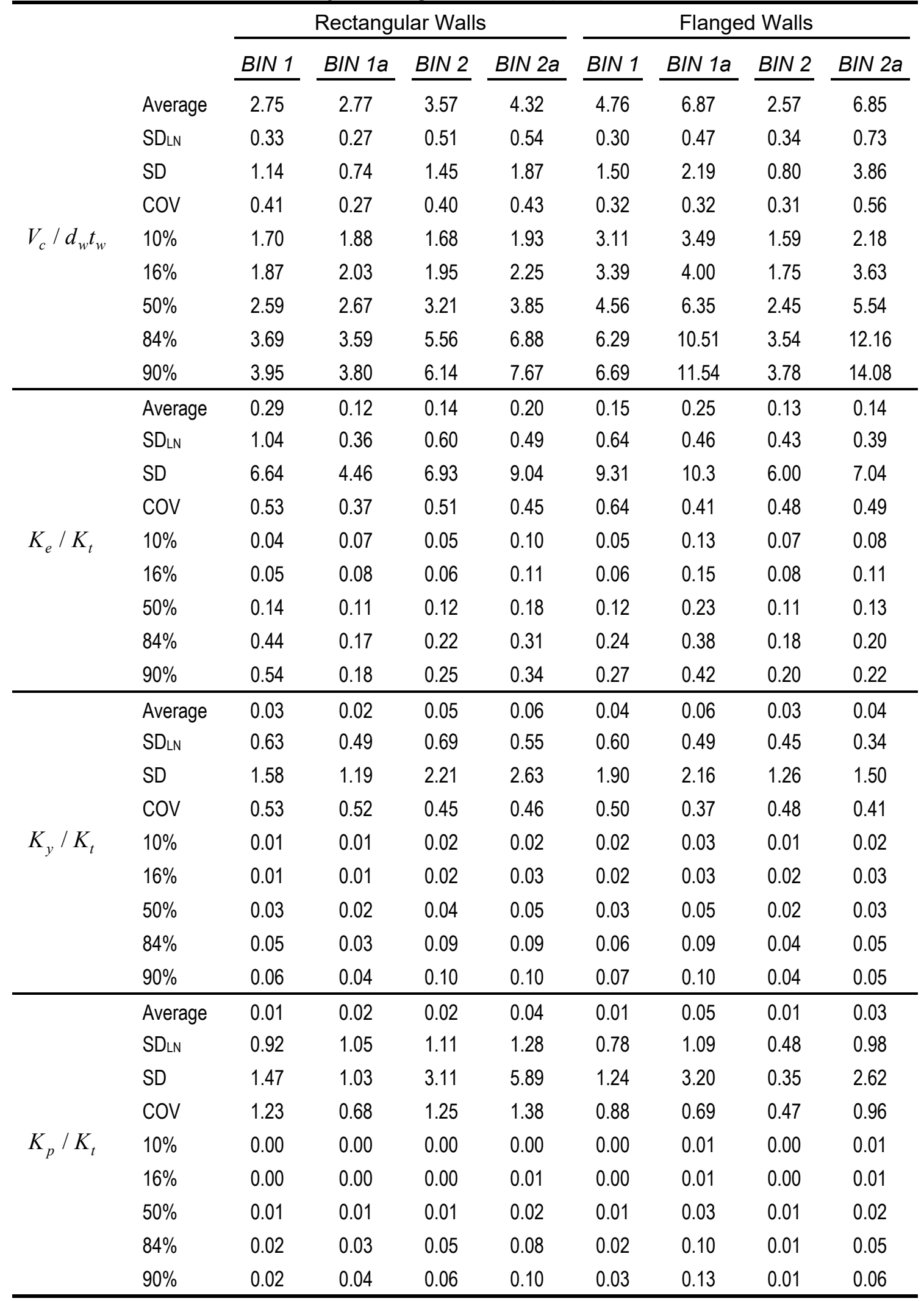


Table C-2 Ratio of the Mean ACI-Predicted to Mean Measured Peak Strength

\begin{tabular}{|c|c|c|c|c|c|c|c|c|c|}
\hline & \multicolumn{4}{|c|}{ Rectangular Walls } & \multicolumn{4}{|c|}{ Flanged Walls } \\
\hline & & BIN 1 & BIN 1a & BIN 2 & $B I N 2 a$ & BIN 1 & BIN 1a & $B I N 2$ & $B I N 2 a$ \\
\hline \multirow{2}{*}{$V_{c p} / V_{c m}$} & $\begin{array}{l}\text { ACI 318-14 } \\
\text { Chapter } 11\end{array}$ & 0.91 & 1.27 & 0.87 & 0.91 & 0.57 & 0.55 & 0.89 & 0.71 \\
\hline & $\begin{array}{l}\mathrm{ACl} 318-14 \\
\text { Chapter } 18\end{array}$ & 1.10 & 1.04 & 1.13 & 0.98 & 0.69 & 0.57 & 1.23 & 0.66 \\
\hline
\end{tabular}





\section{Appendix D}

\section{Shear-Controlled Reinforced Concrete Walls - Comparison Studies}

This appendix presents a comparison of test and MIKP-predicted response of 63 reinforced concrete walls. The calculated pinching and deteriorition parameters of the 63 walls are provided in Chapter 8. In the figures below, the Panel on the left displays test results and the right Panel presents the response predicted by using a modified Ibarra-Krawinkler cyclic force-displacement (MIKP) model, as described in Chapter 8.

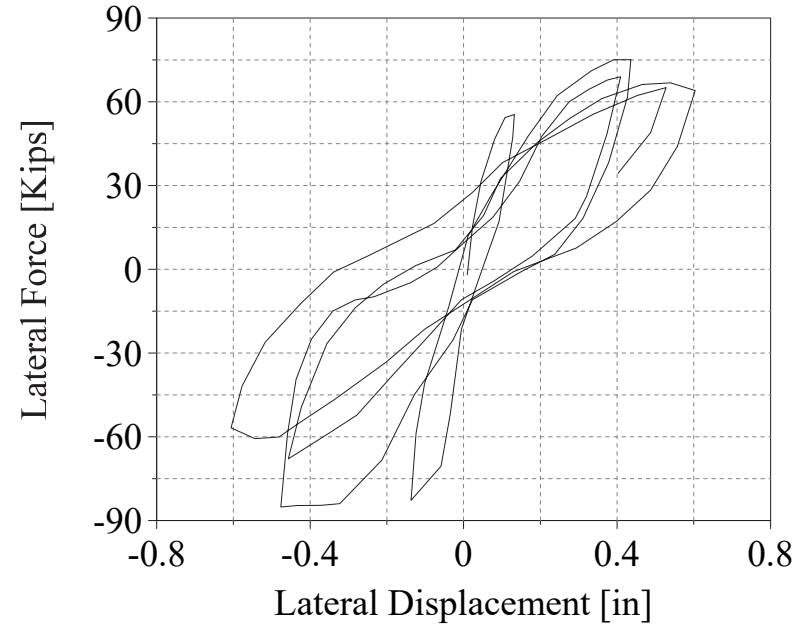

(a)

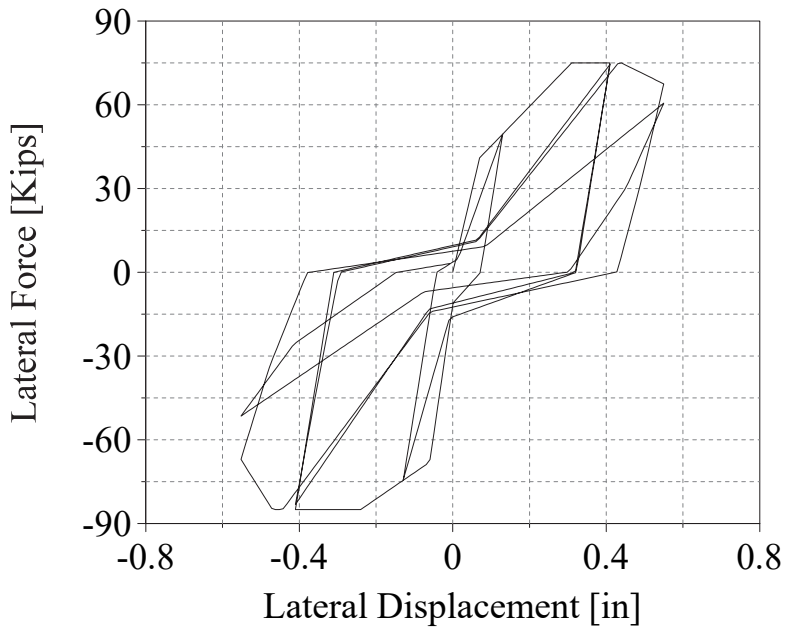

(b)

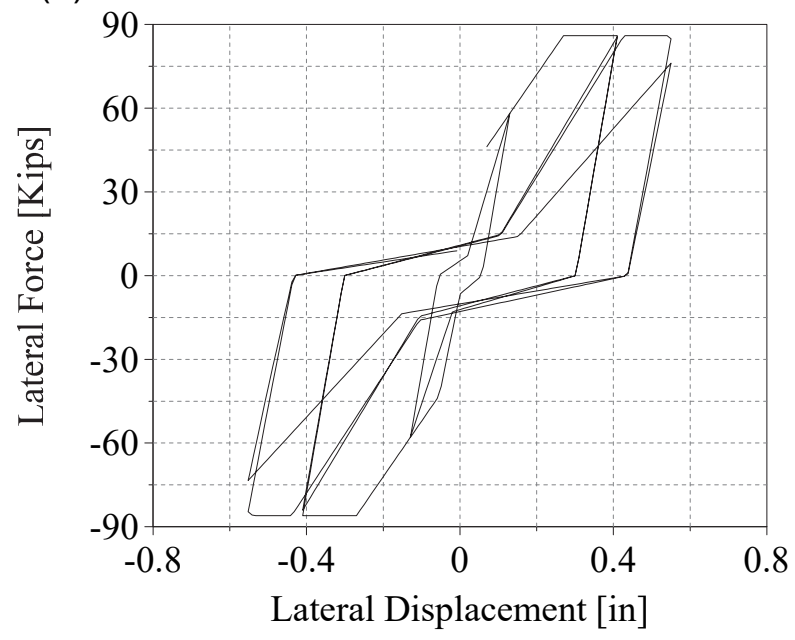

(c)

Figure D-1 Comparison of (a) test data, (b) MIKP prediction, and (c) proposed-model prediction for Alexander et al. (1973) panel 4. 


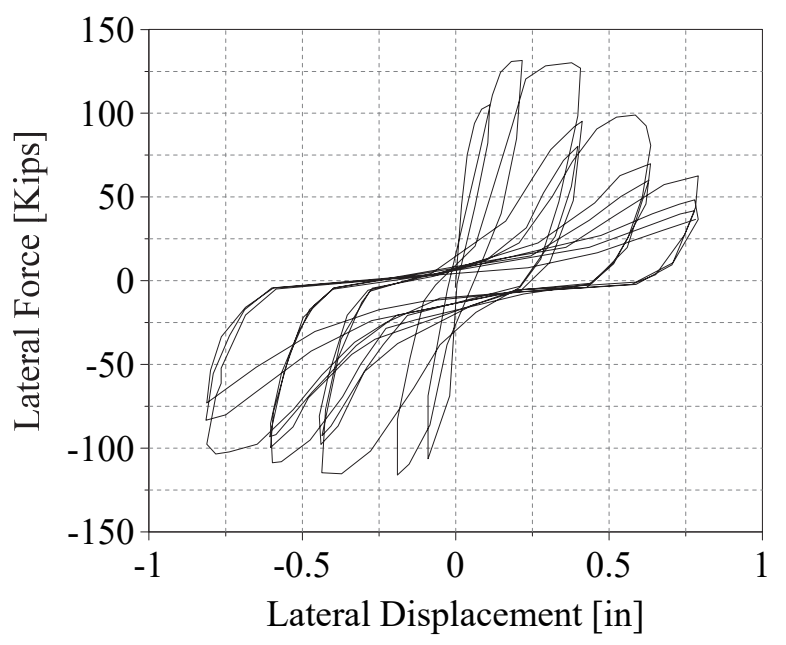

(a)

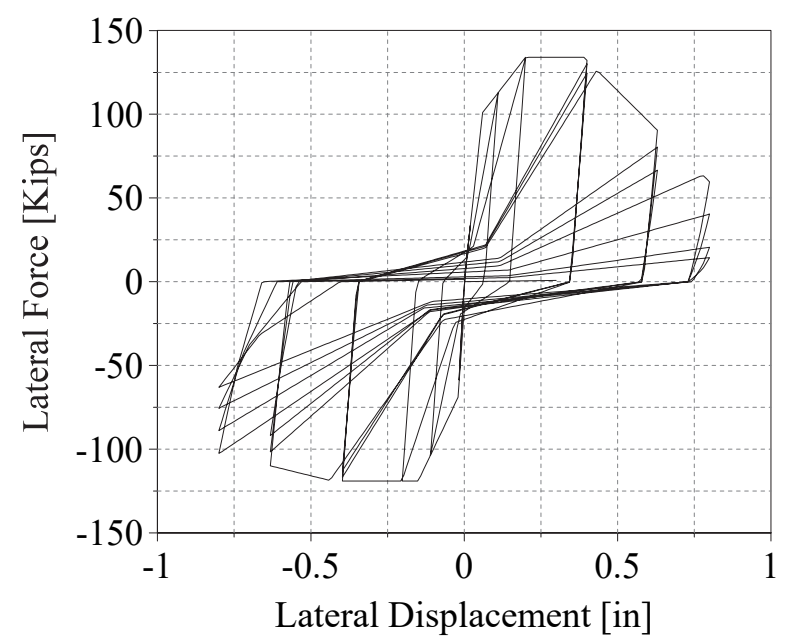

(b)

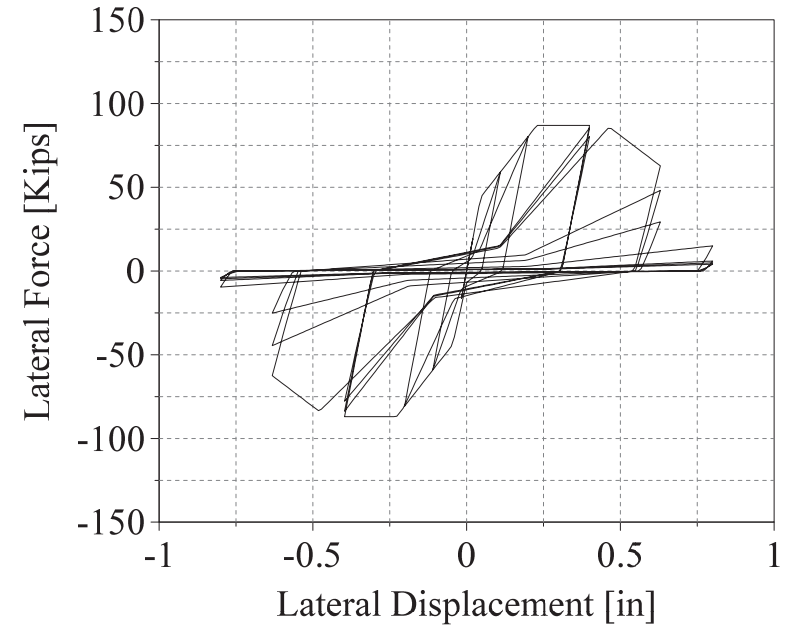

(c)

Figure D-2 Comparison of (a) test data, (b) MIKP prediction, and (c) proposed-model prediction for Saatcioglu (1991) Specimen 1. 


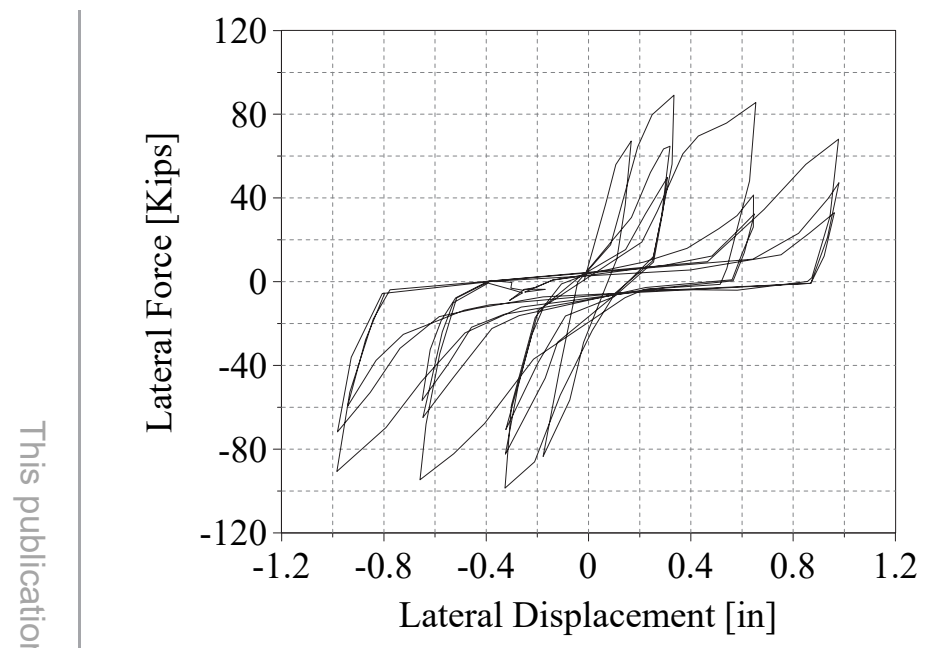

(a)

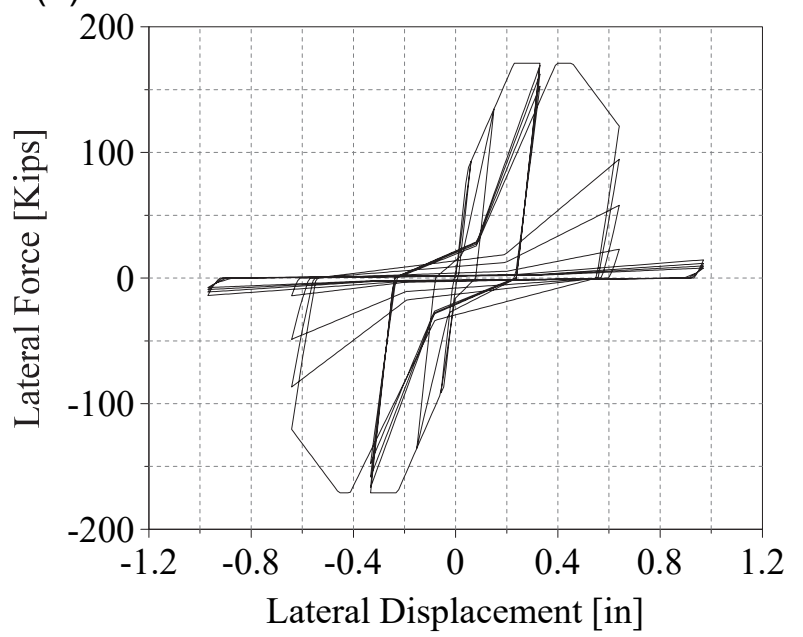

(c)

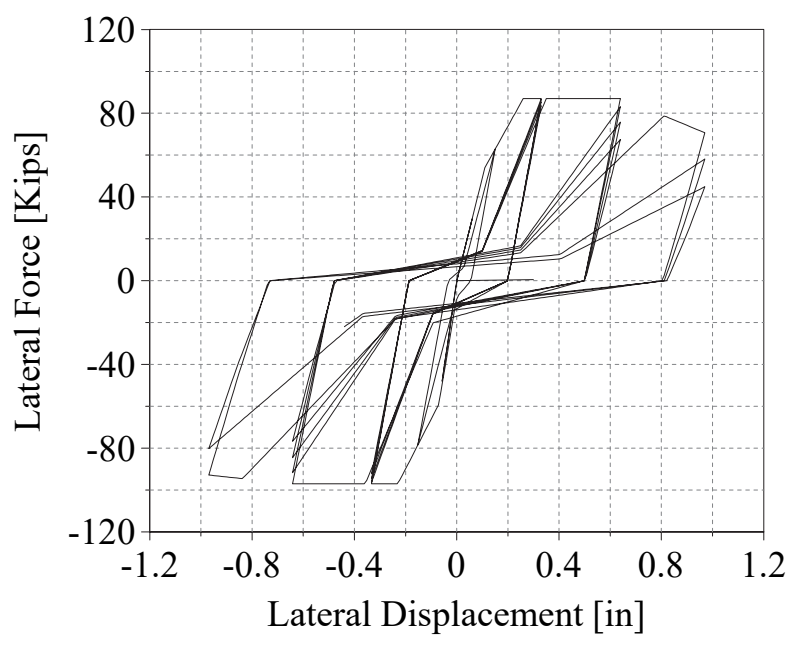

(b)

Figure D-3 Comparison of (a) test data, (b) MIKP prediction, and (c) proposed-model prediction for Saatcioglu (1991) Specimen 4. 


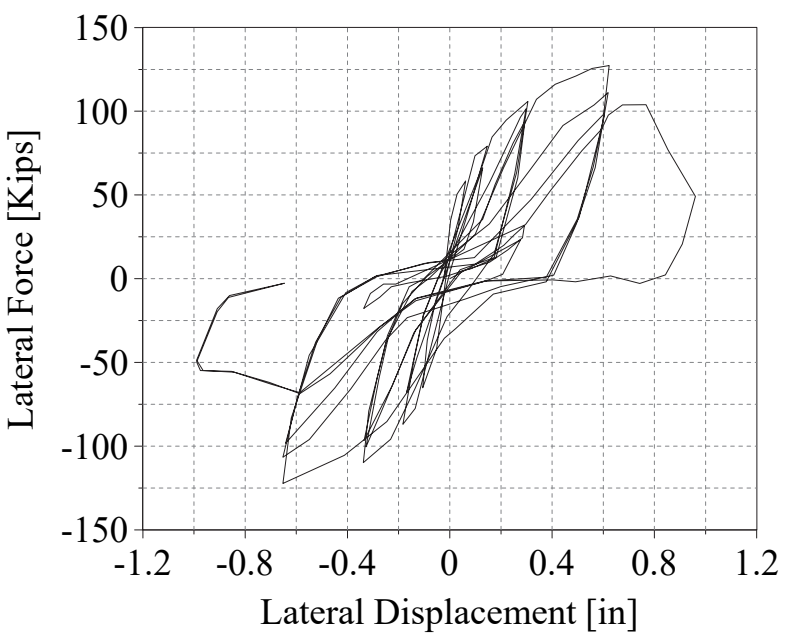

(a)

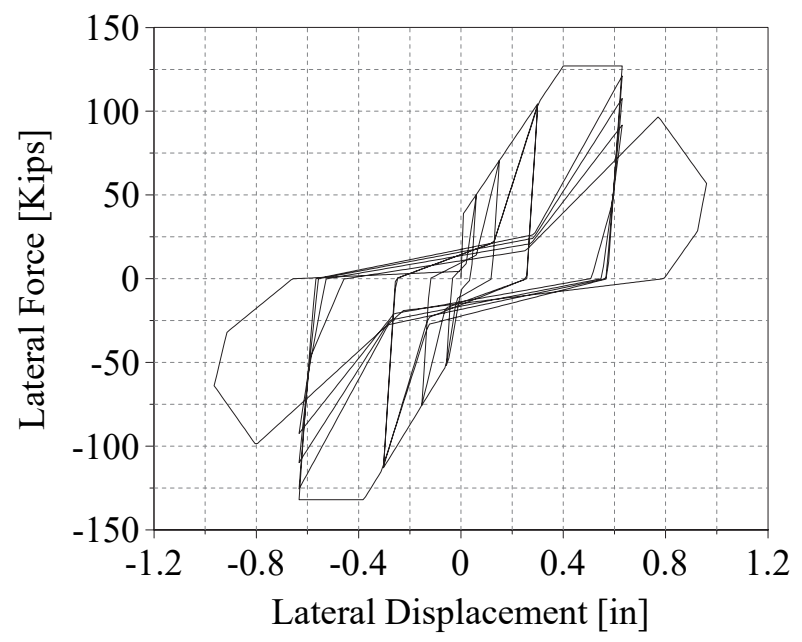

(b)

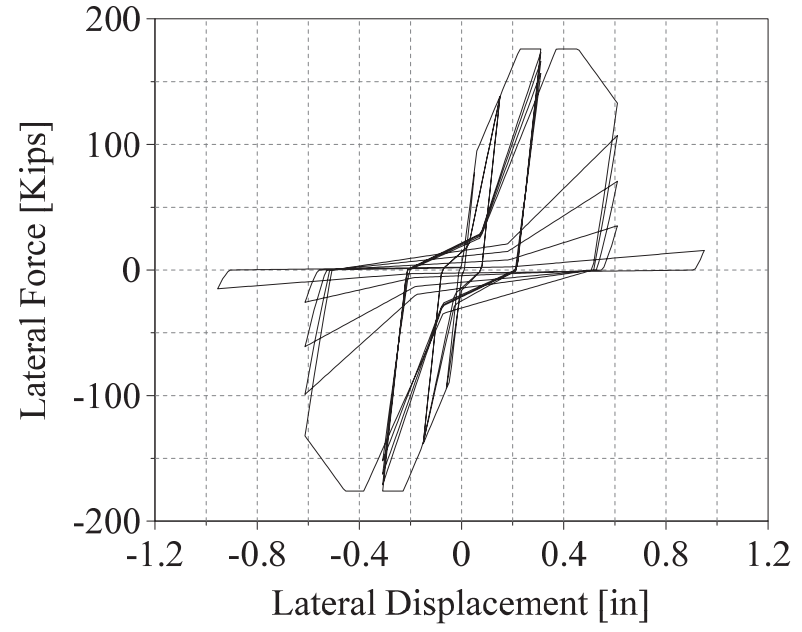

(c)

Figure D-4 Comparison of (a) test data, (b) MIKP prediction, and (c) proposed-model prediction for Saatcioglu (1991) Specimen 6. 


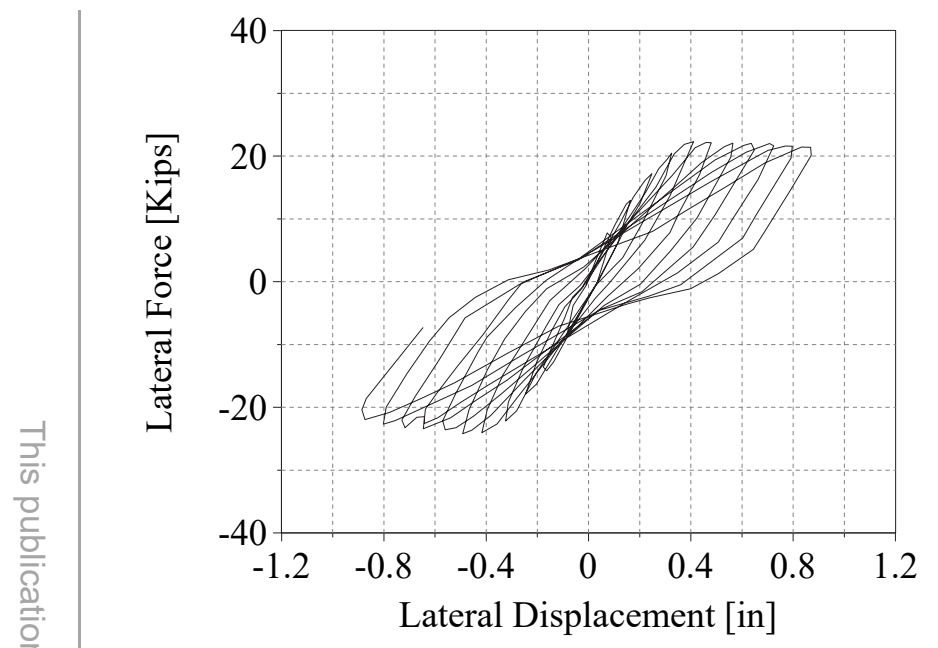

(a)

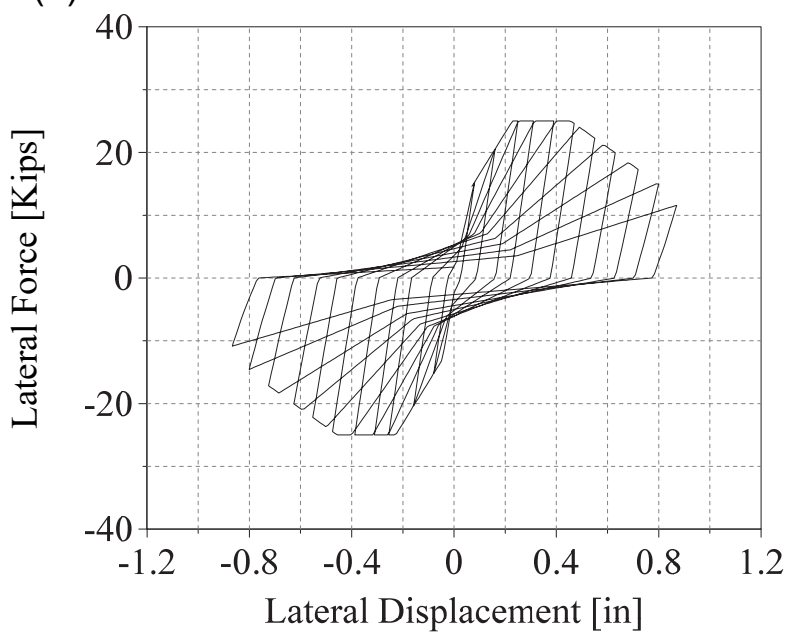

(c)

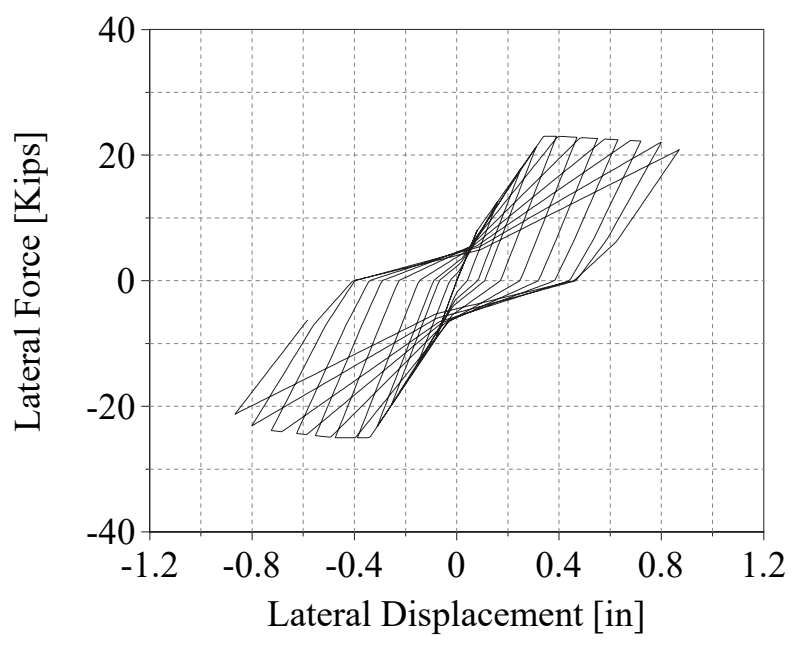

(b)

Figure D-5 Comparison of (a) test data, (b) MIKP prediction, and (c) proposed-model prediction for Pilakoutas and Elnashai (1995) Specimen SW4. 


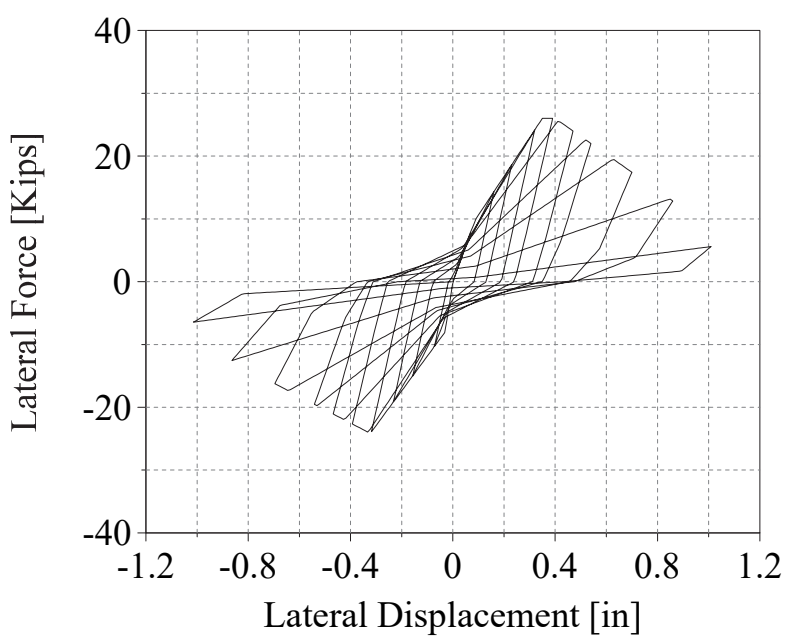

(a)

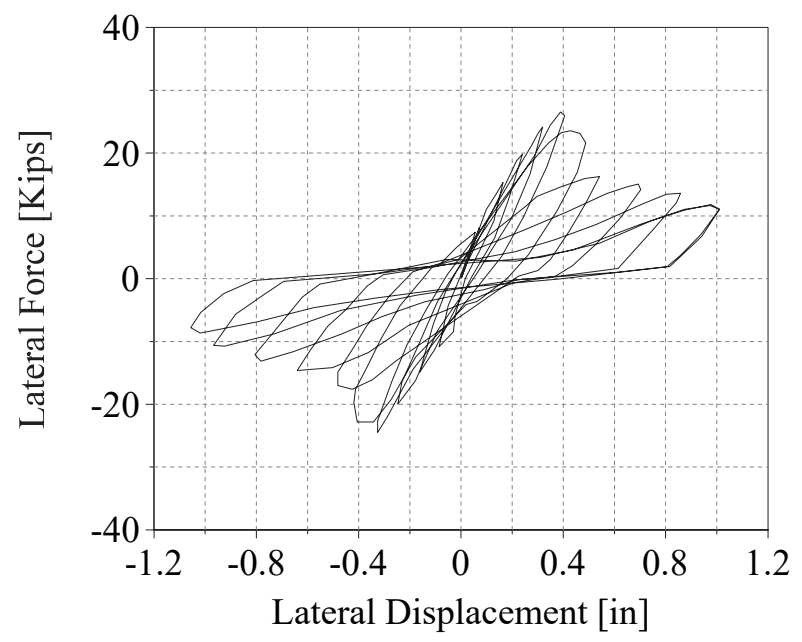

(b)

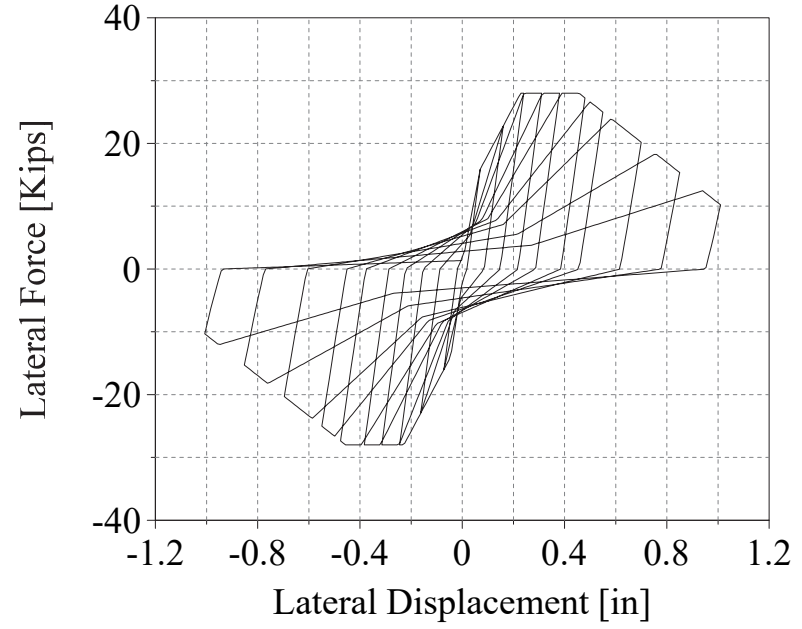

(c)

Figure D-6 Comparison of (a) test data, (b) MIKP prediction, and (c) proposed-model prediction for Pilakoutas and Elnashai (1995) Specimen SW5. 


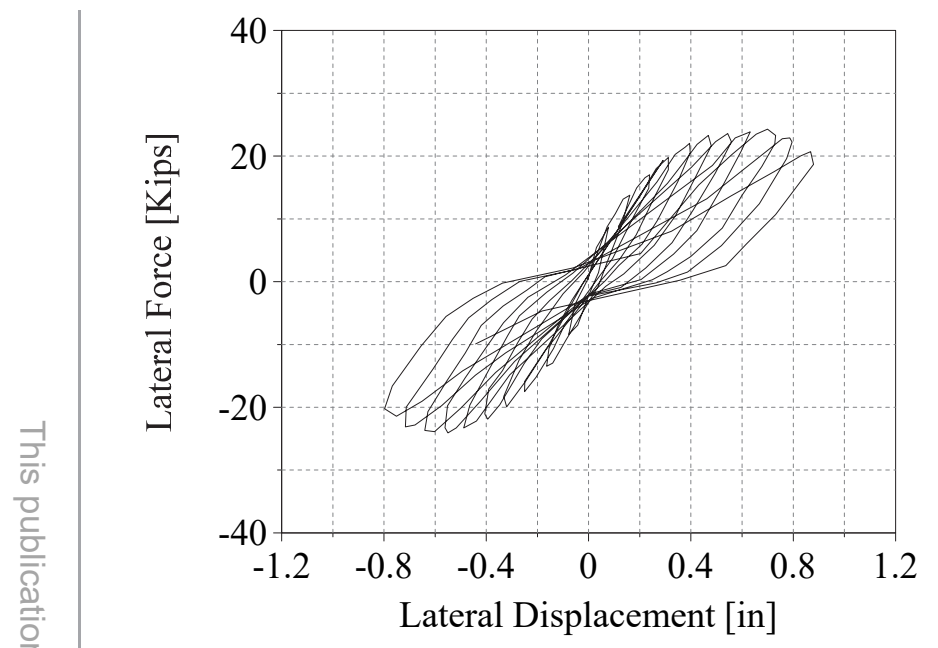

(a)

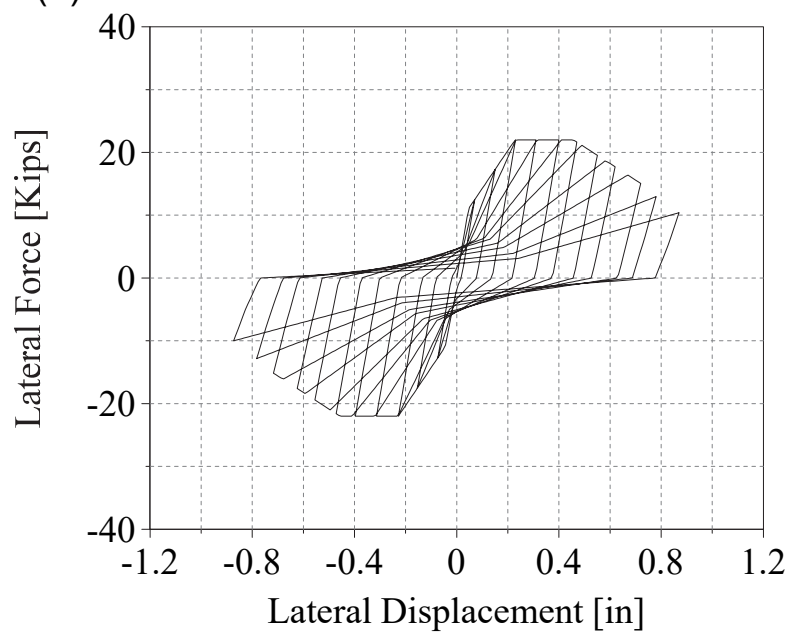

(c)

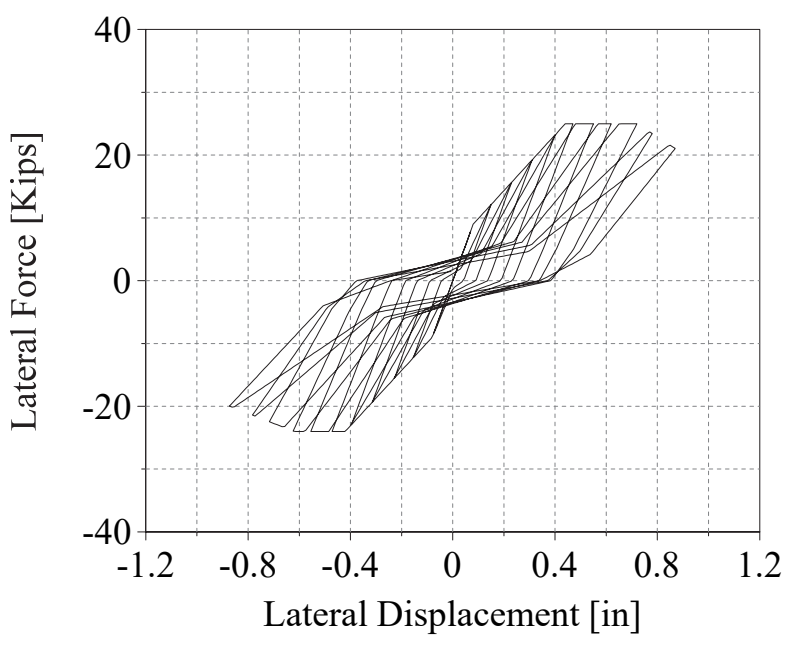

(b)

Figure D-7 Comparison of (a) test data, (b) MIKP prediction, and (c) proposed-model prediction for Pilakoutas and Elnashai (1995) Specimen SW6. 


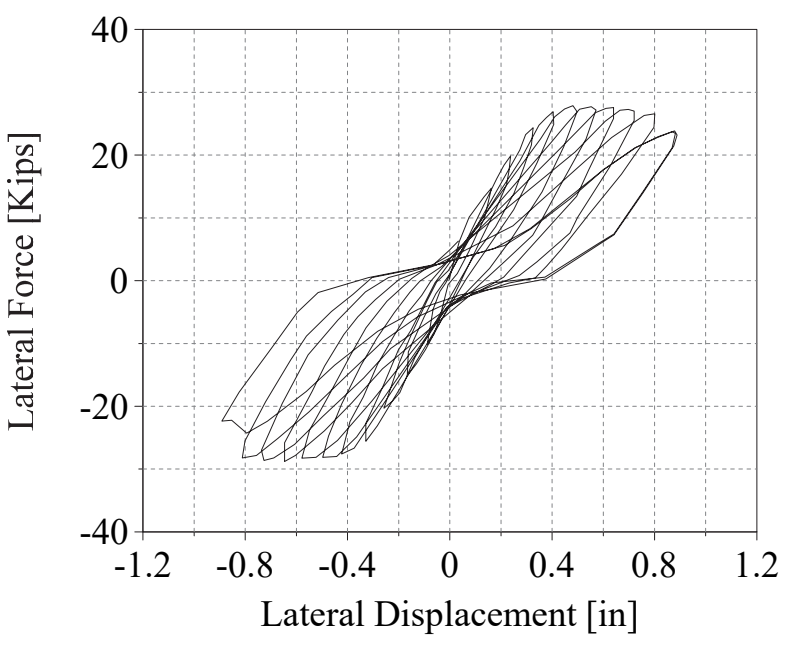

(a)

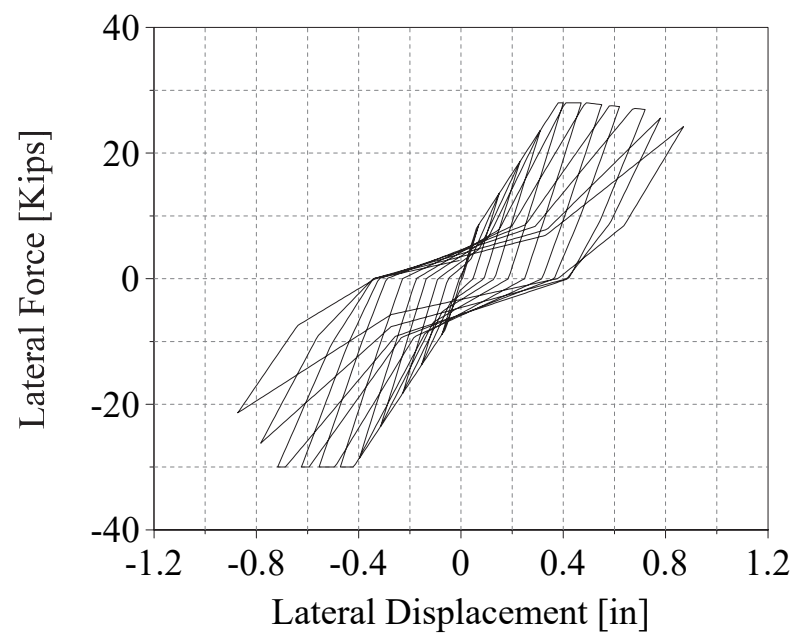

(b)

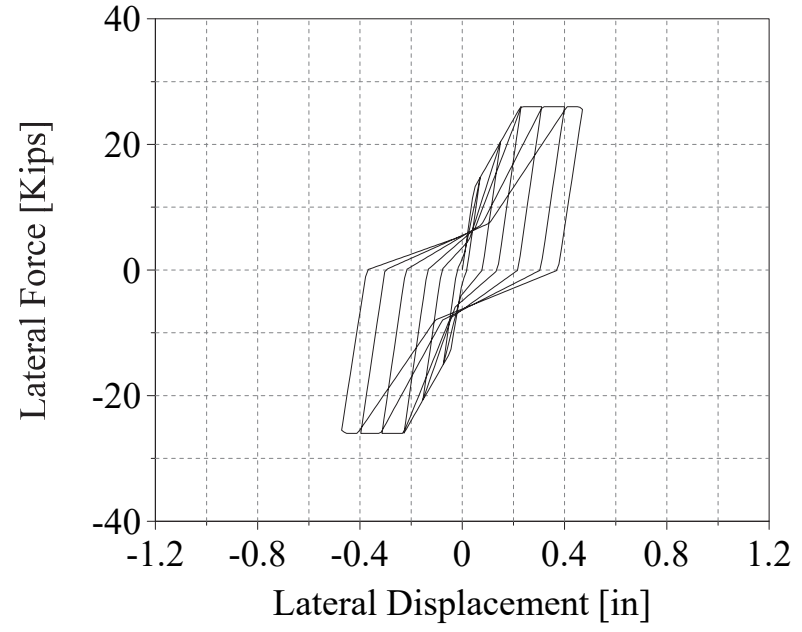

(c)

Figure D-8 Comparison of (a) test data, (b) MIKP prediction, and (c) proposed-model prediction for Pilakoutas and Elnashai (1995) Specimen SW7. 


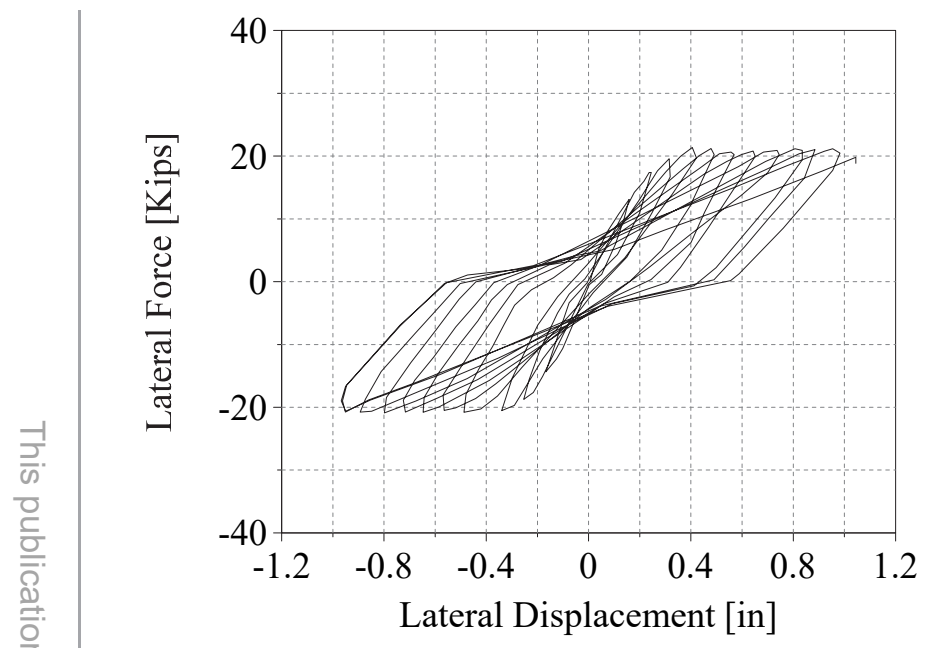

(a)

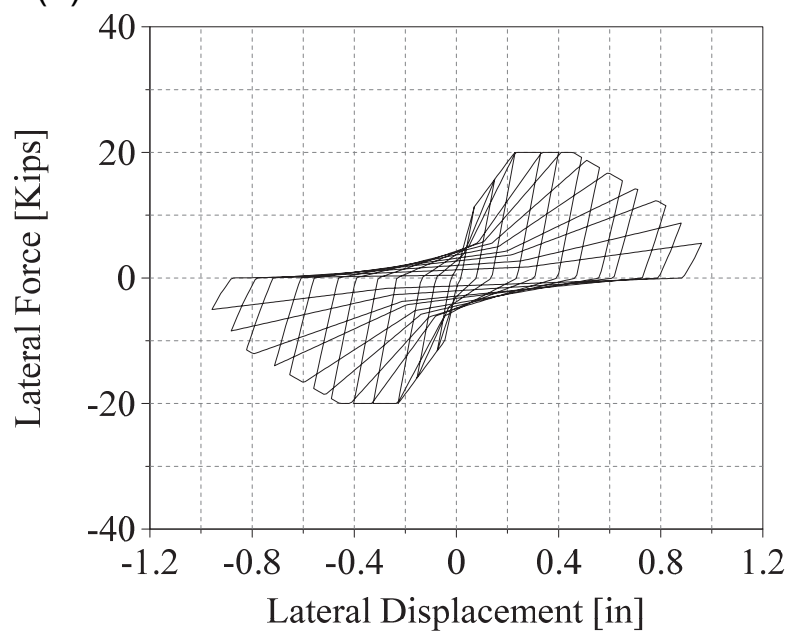

(c)

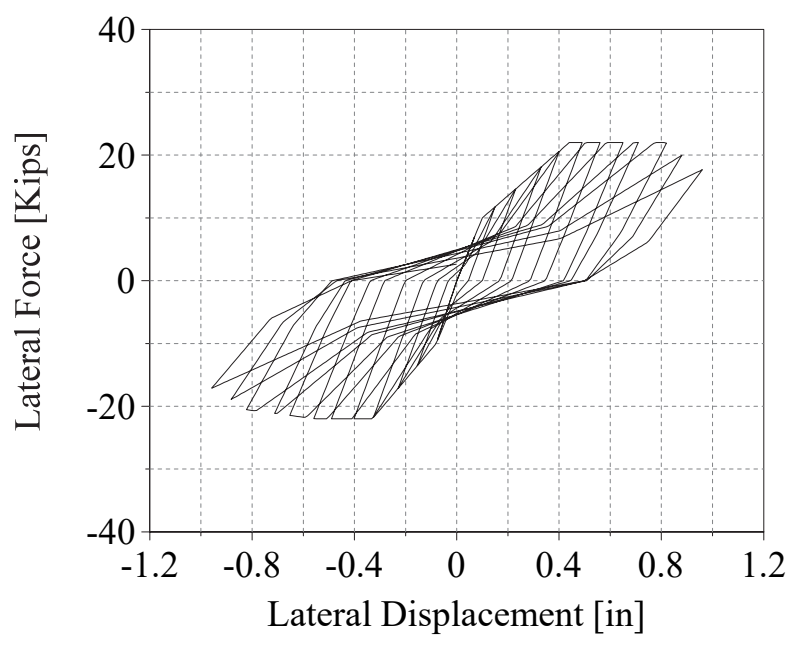

(b)

Figure D-9 Comparison of (a) test data, (b) MIKP prediction, and (c) proposed-model prediction for Pilakoutas and Elnashai (1995) Specimen SW8. 


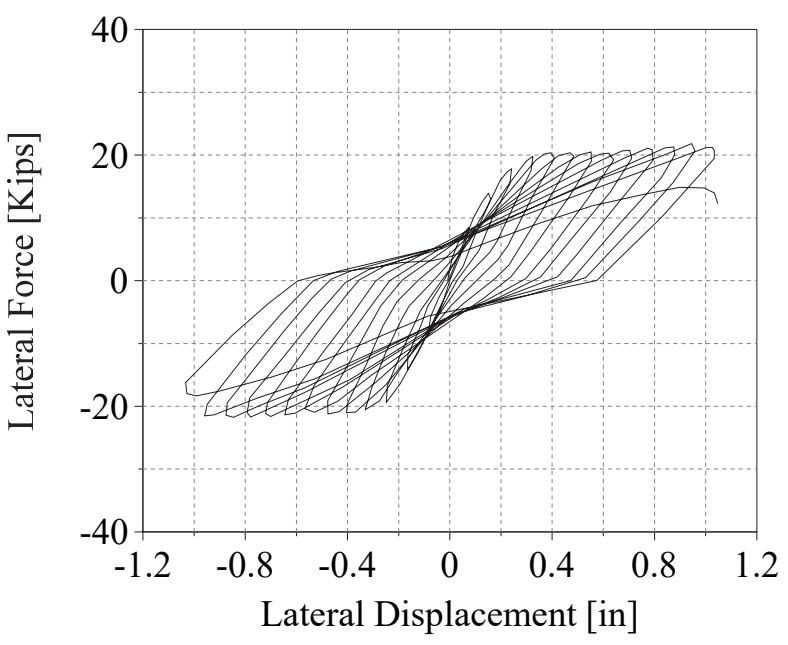

(a)

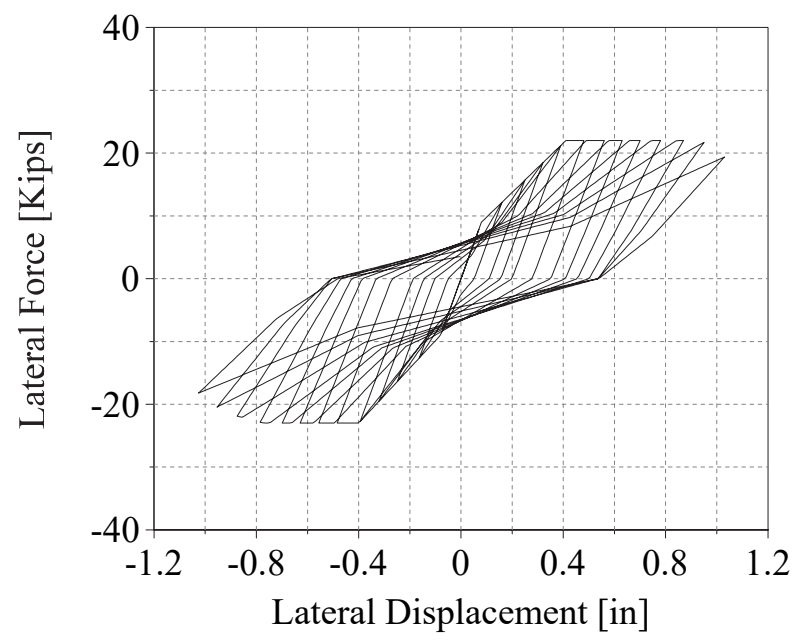

(b)

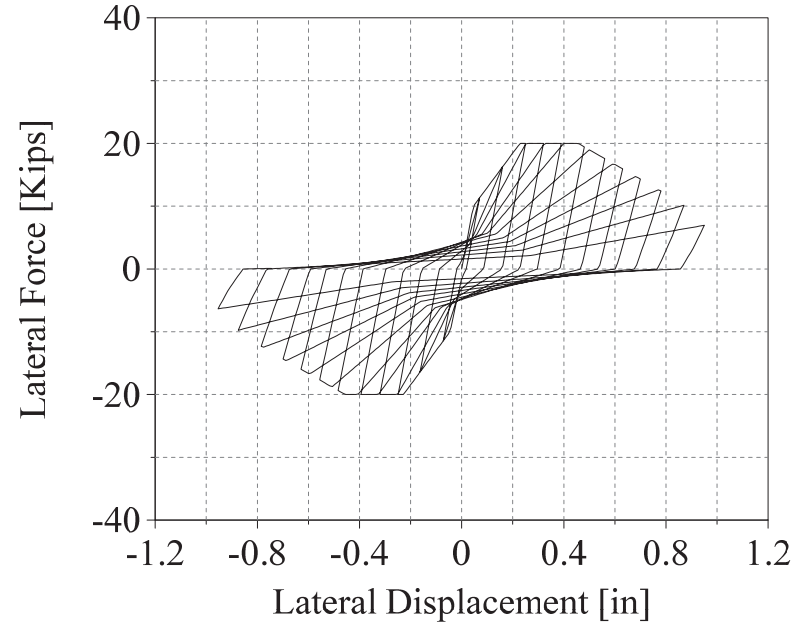

(c)

Figure D-10 Comparison of (a) test data, (b) MIKP prediction, and (c) proposed-model prediction for Pilakoutas and Elnashai (1995) Specimen SW9. 


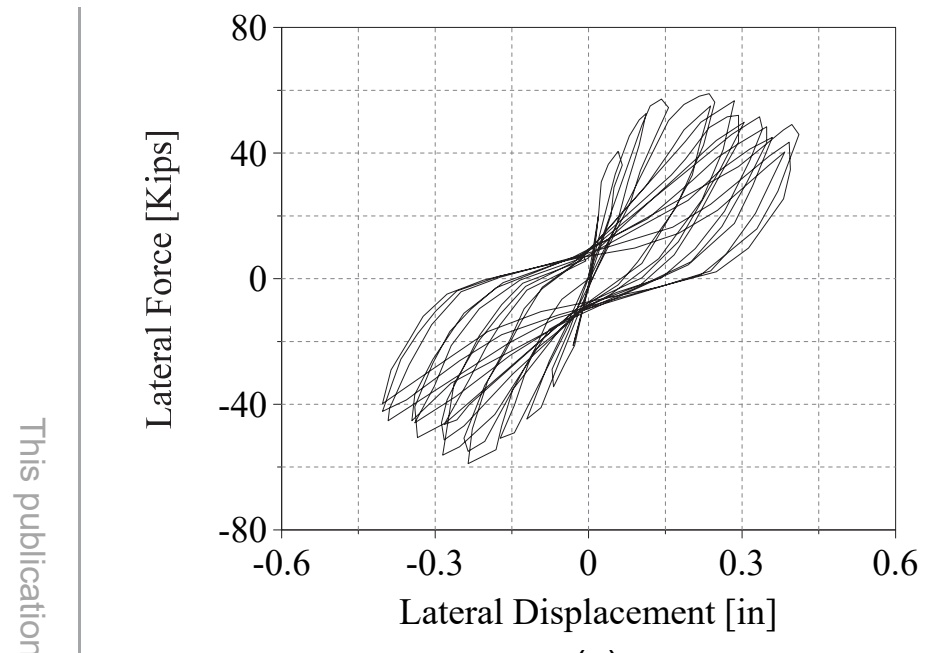

(a)

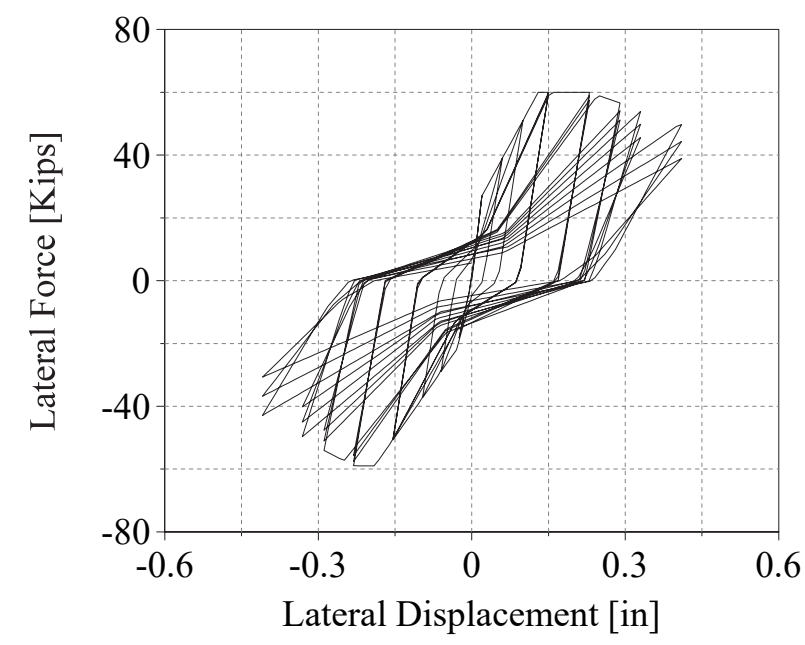

(b)

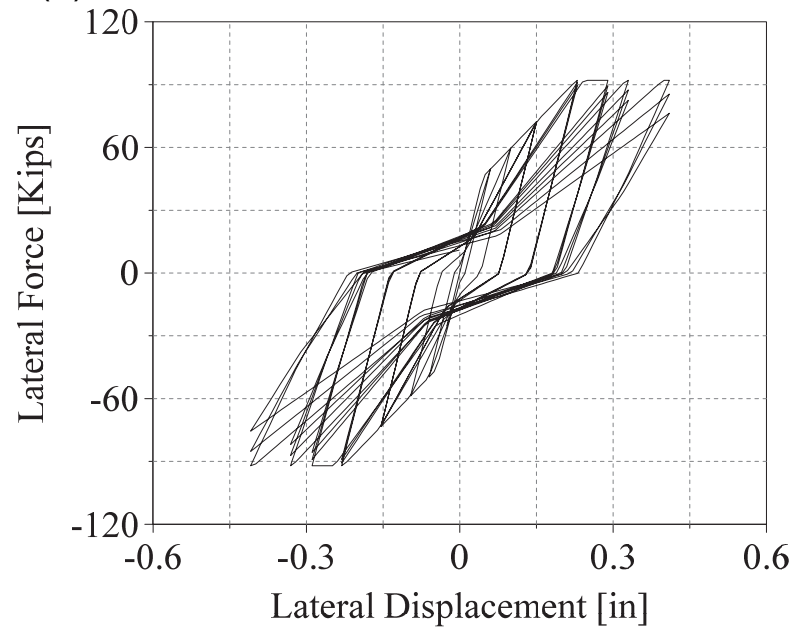

(c)

Figure D-11 Comparison of (a) test data, (b) MIKP prediction, and (c) proposed-model prediction for Salonikios et al. (1999) Specimen LSW1. 


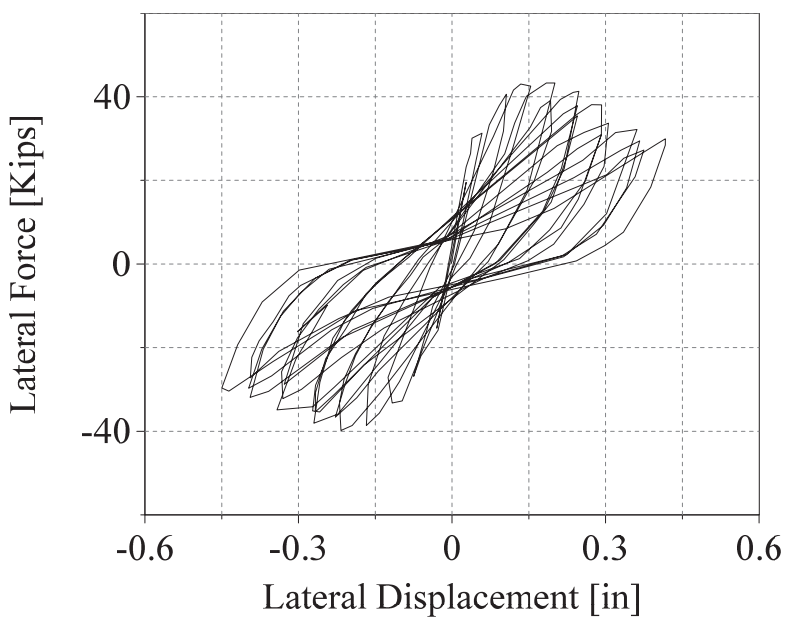

(a)

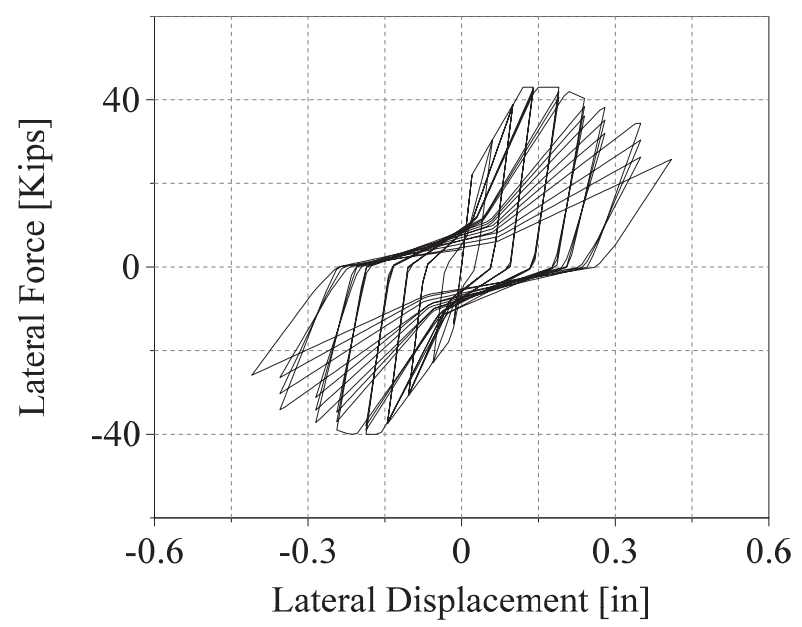

(b)

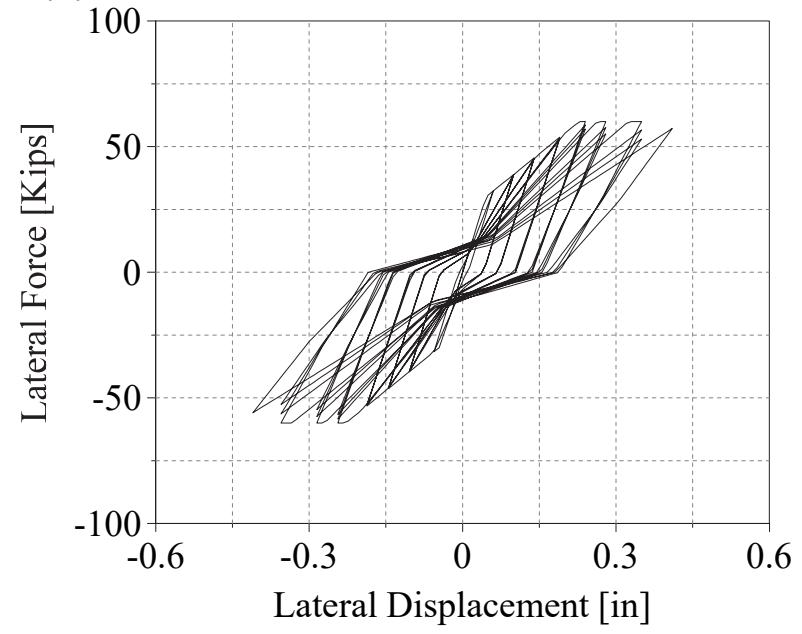

(c)

Figure D-12 Comparison of (a) test data, (b) MIKP prediction, and (c) proposed-model prediction for Salonikios et al. (1999) Specimen LSW2. 


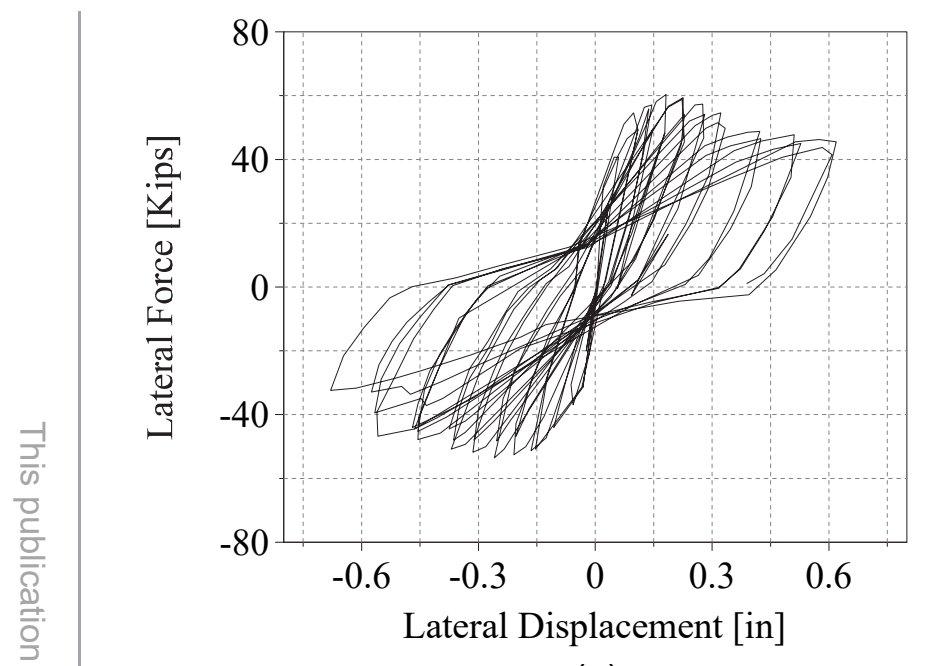

(a)

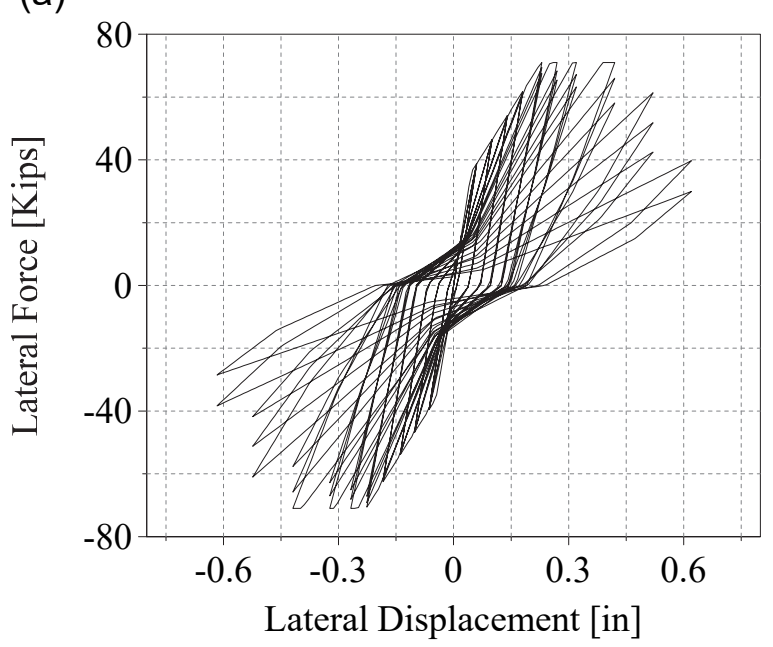

(c)

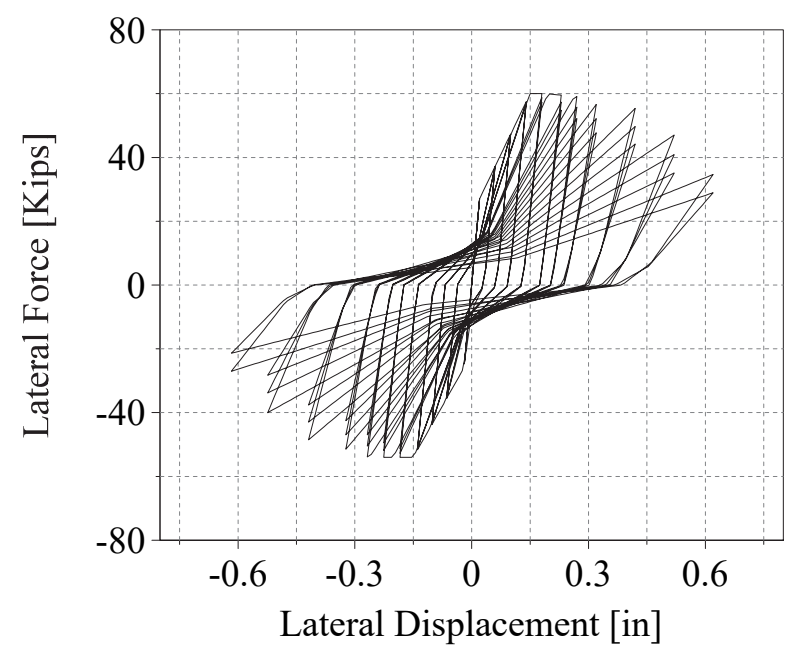

(b)

Figure D-13 Comparison of (a) test data, (b) MIKP prediction, and (c) proposed-model prediction for Salonikios et al. (1999) Specimen LSW3. 


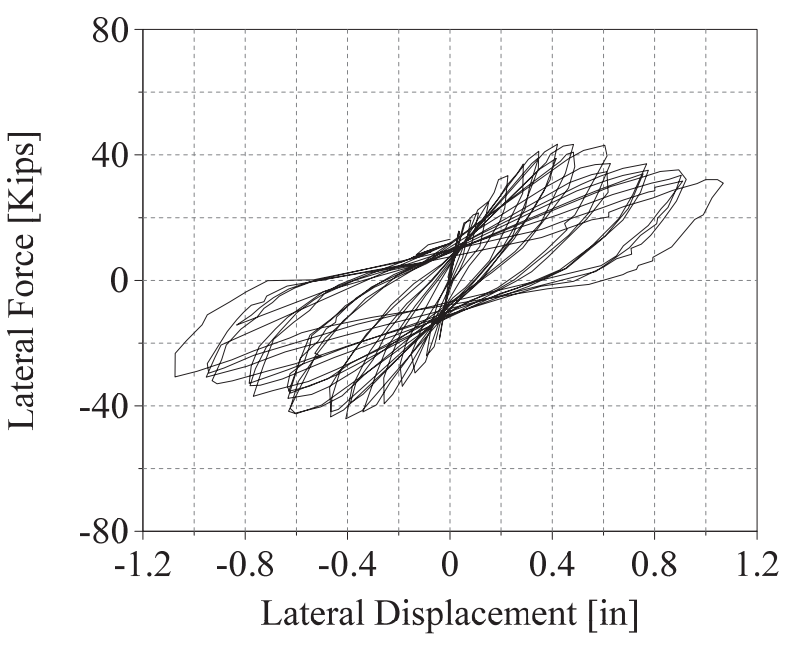

(a)

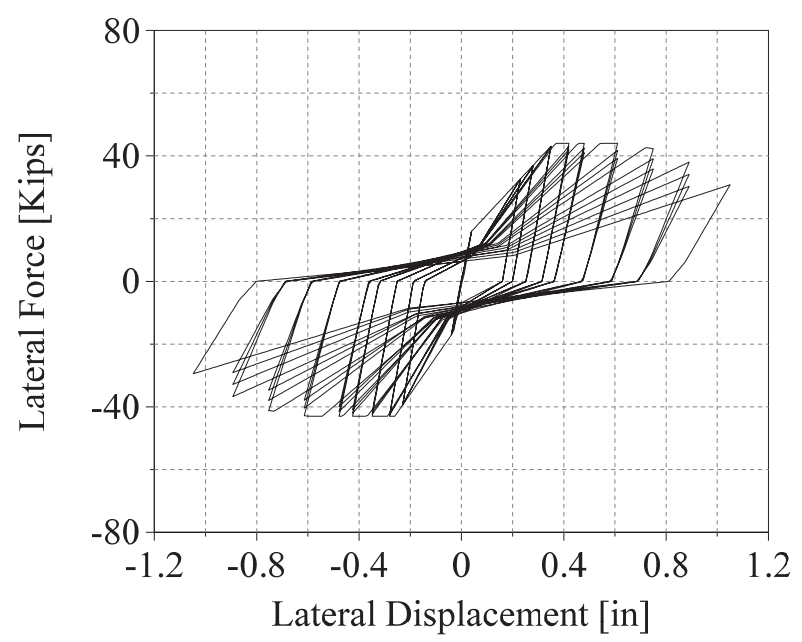

(b)

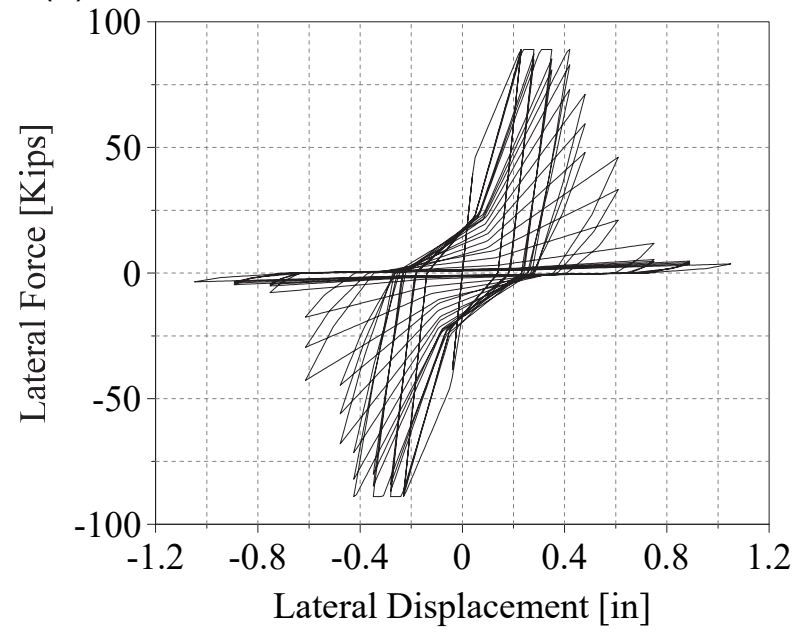

(c)

Figure D-14 Comparison of (a) test data, (b) MIKP prediction, and (c) proposed-model prediction for Salonikios et al. (1999) Specimen MSW1. 


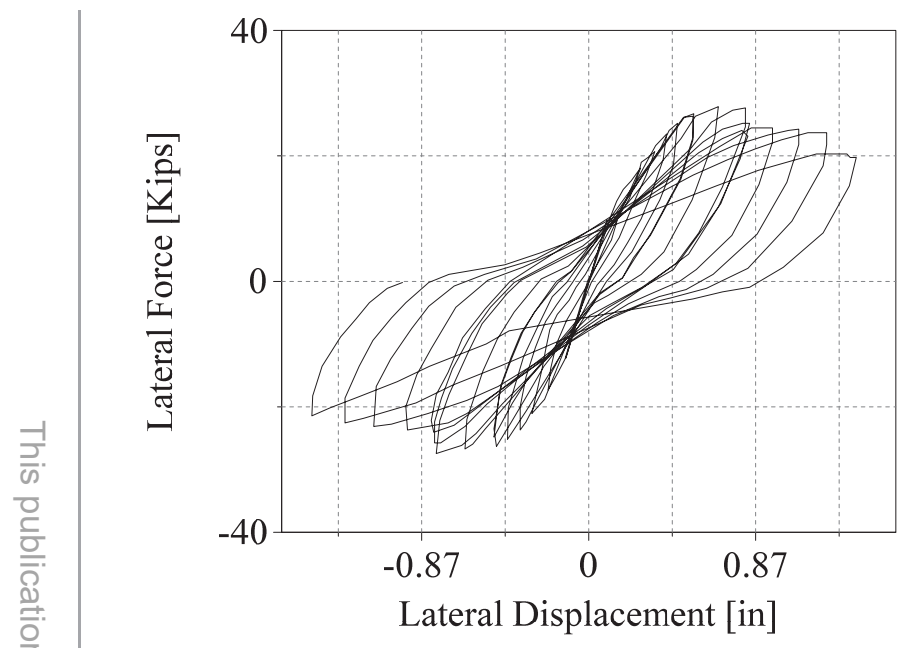

(a)

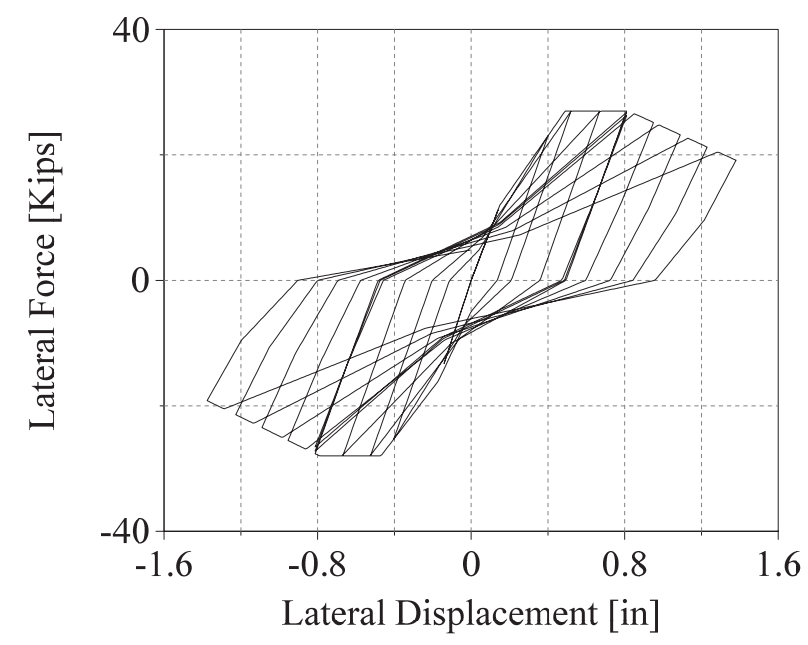

(b)

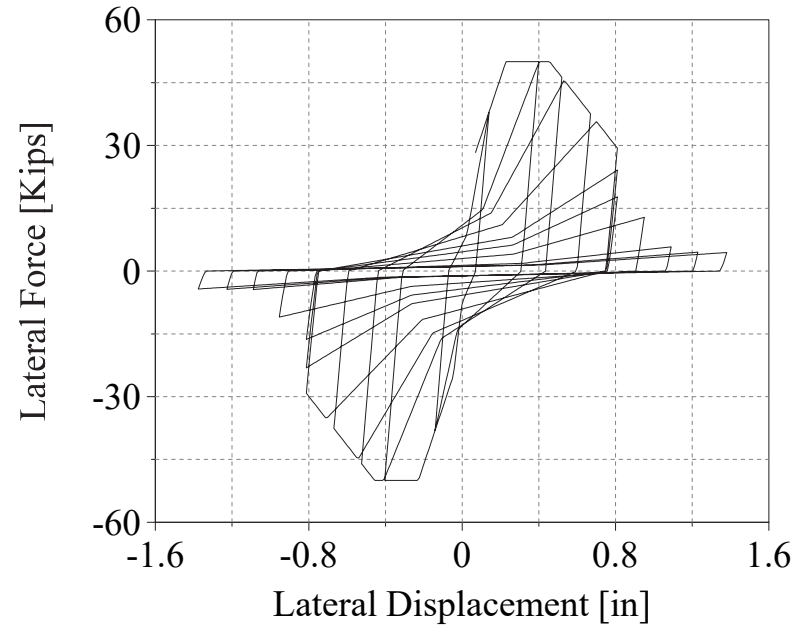

(c)

Figure D-15 Comparison of (a) test data, (b) MIKP prediction, and (c) proposed-model prediction for Salonikios et al. (1999) Specimen MSW2. 


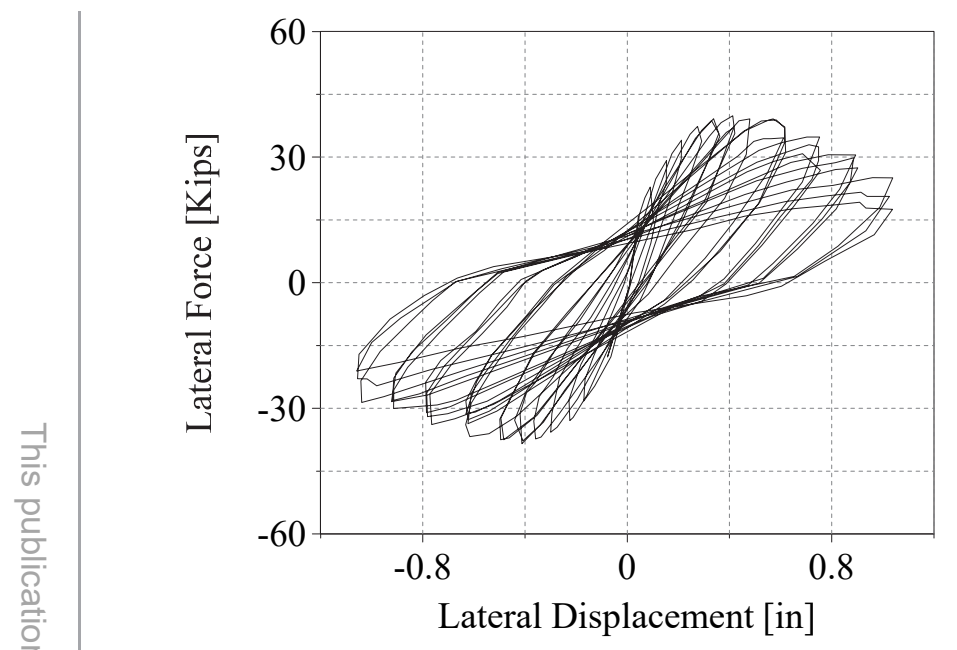

(a)

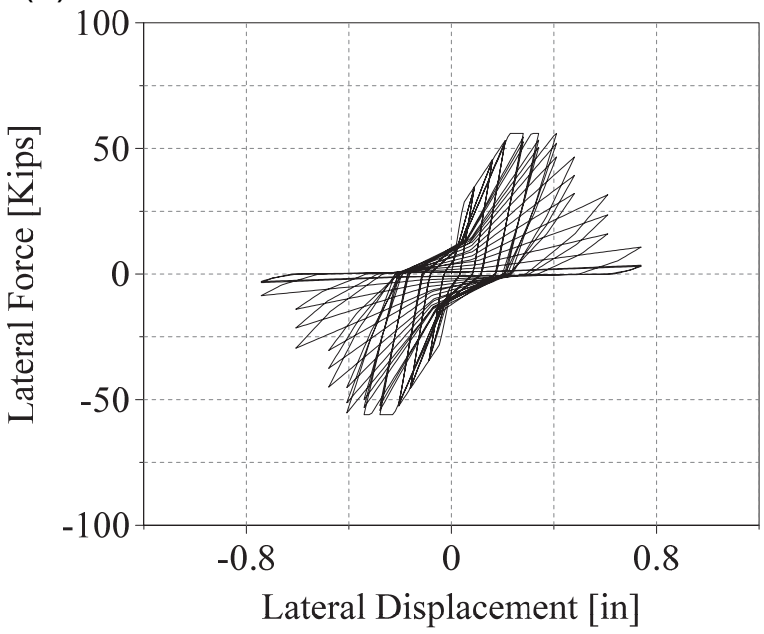

(c)

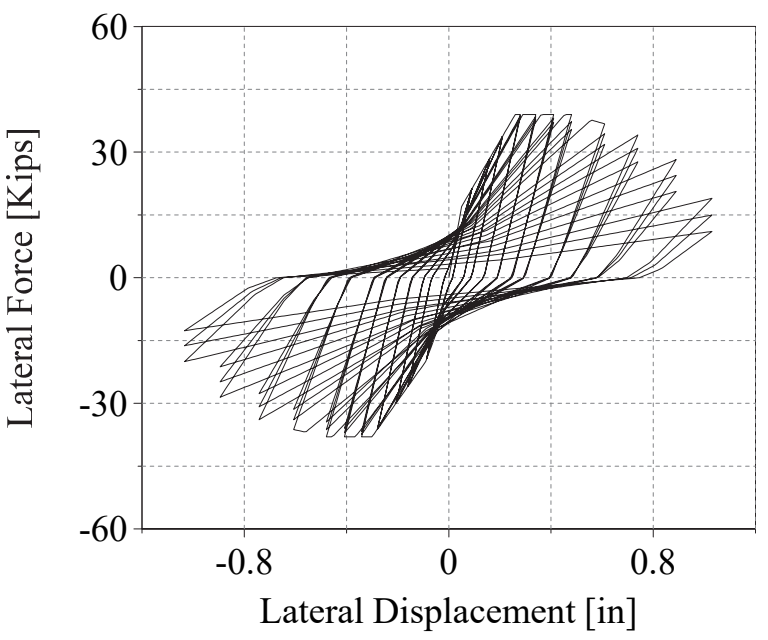

(b)

Figure D-16 Comparison of (a) test data, (b) MIKP prediction, and (c) proposed-model prediction for Salonikios et al. (1999) Specimen MSW3. 


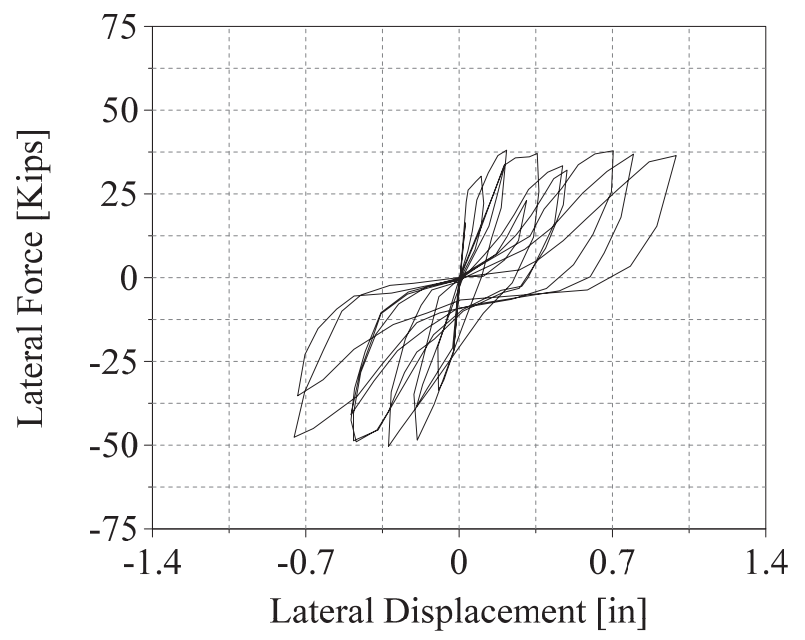

(a)

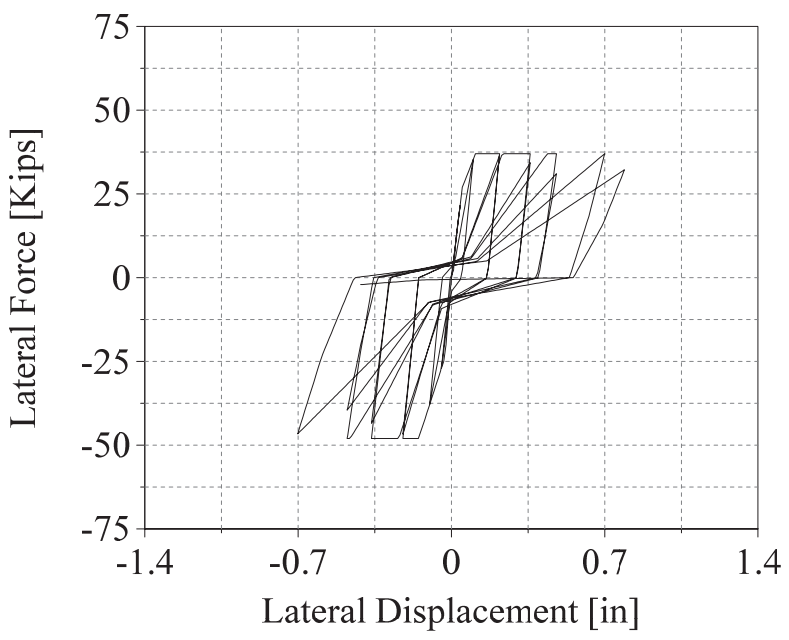

(b)

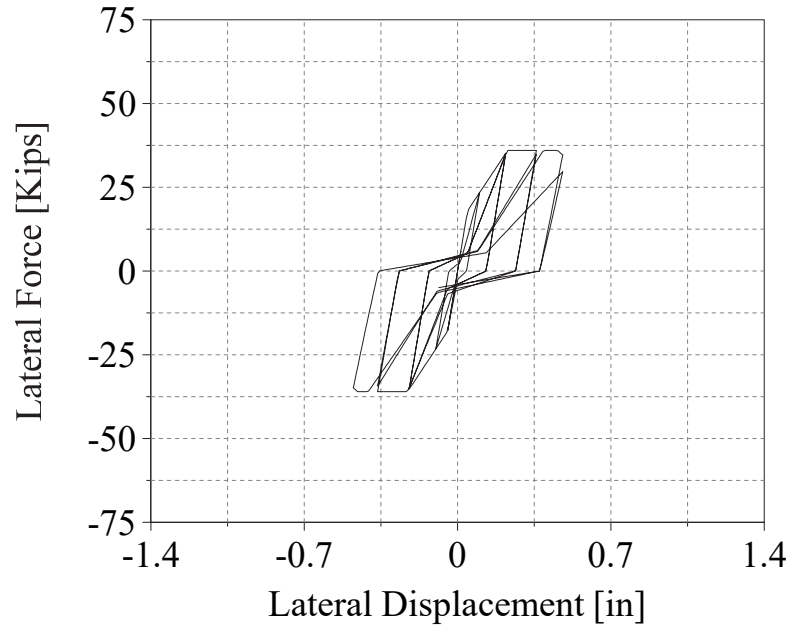

(c)

Figure D-17 Comparison of (a) test data, (b) MIKP prediction, and (c) proposed-model prediction for Hidalgo et al. (2002) Specimen 1. 


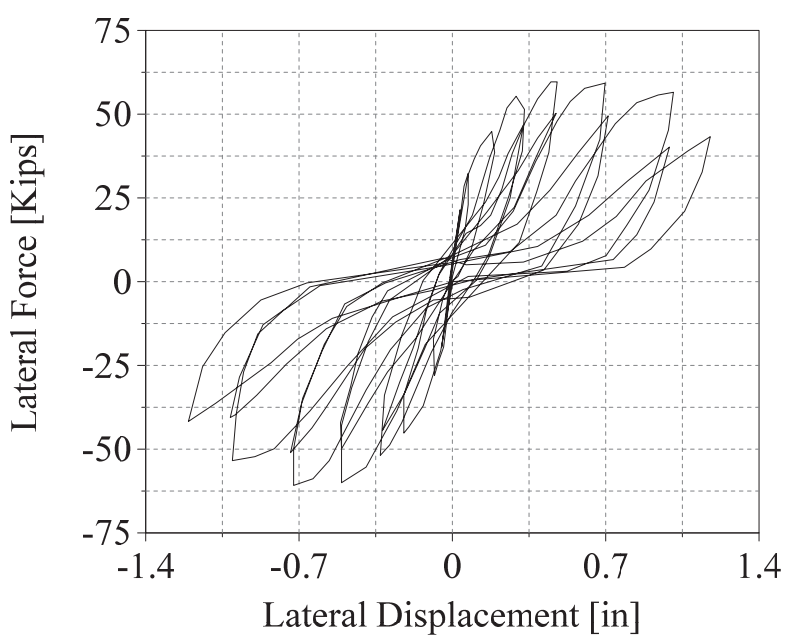

(a)

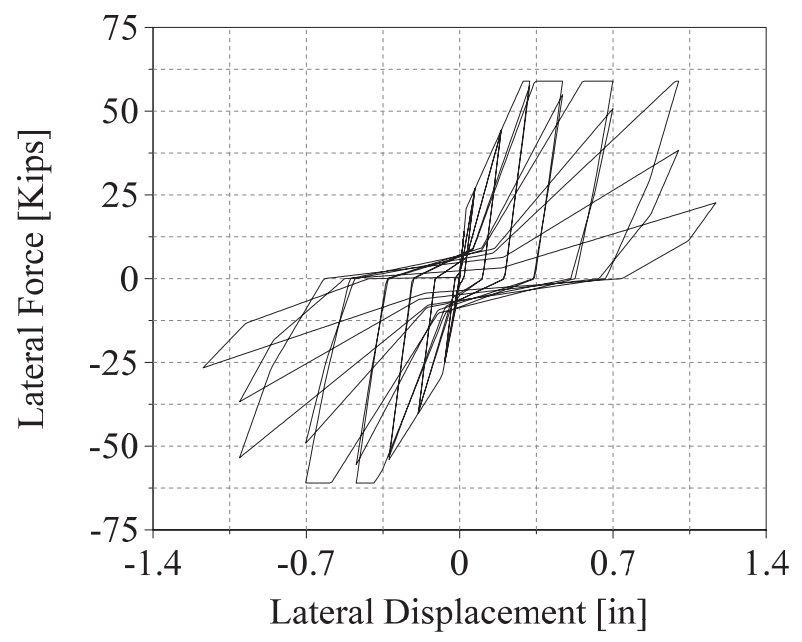

(b)

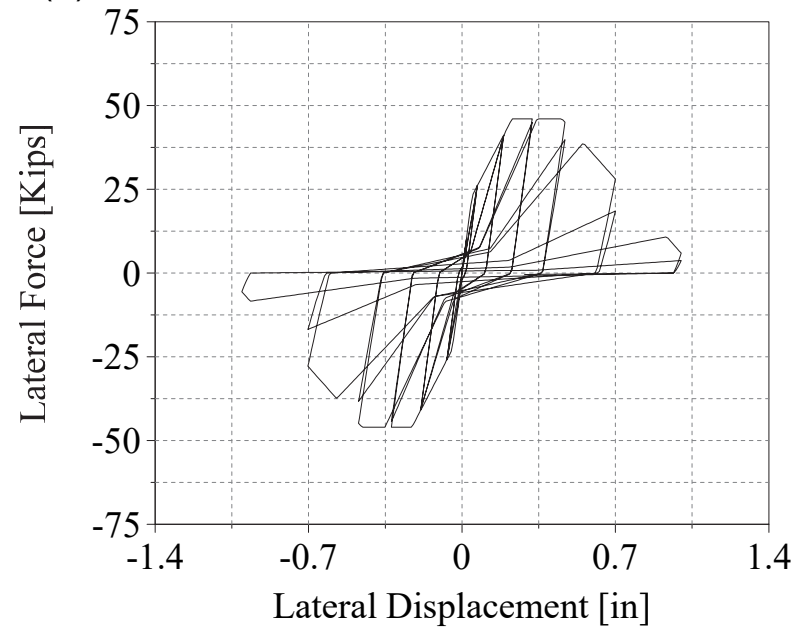

(c)

Figure D-18 Comparison of (a) test data, (b) MIKP prediction, and (c) proposed-model prediction for Hidalgo et al. (2002) Specimen 2. 


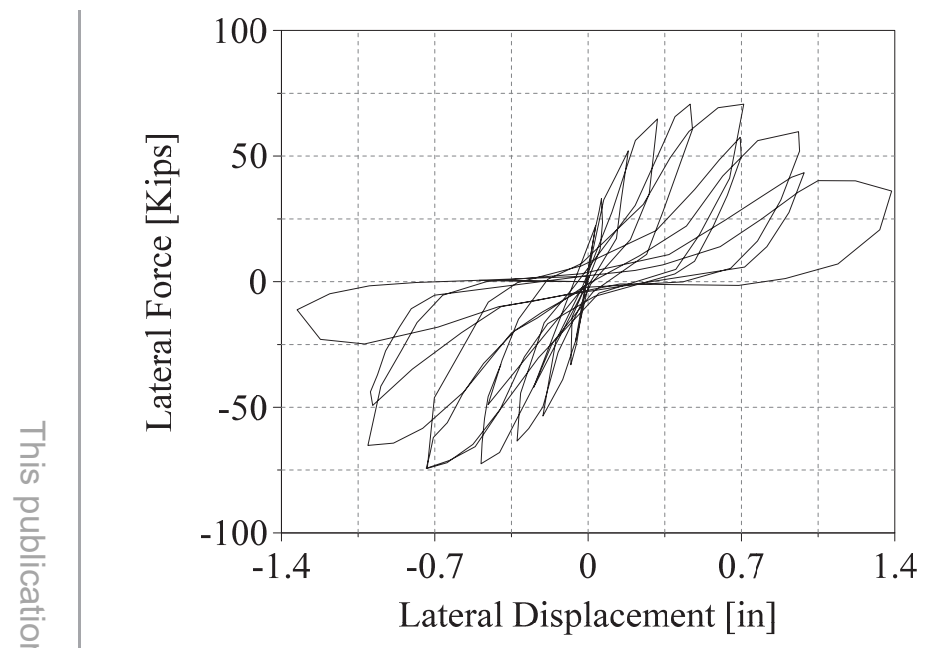

(a)

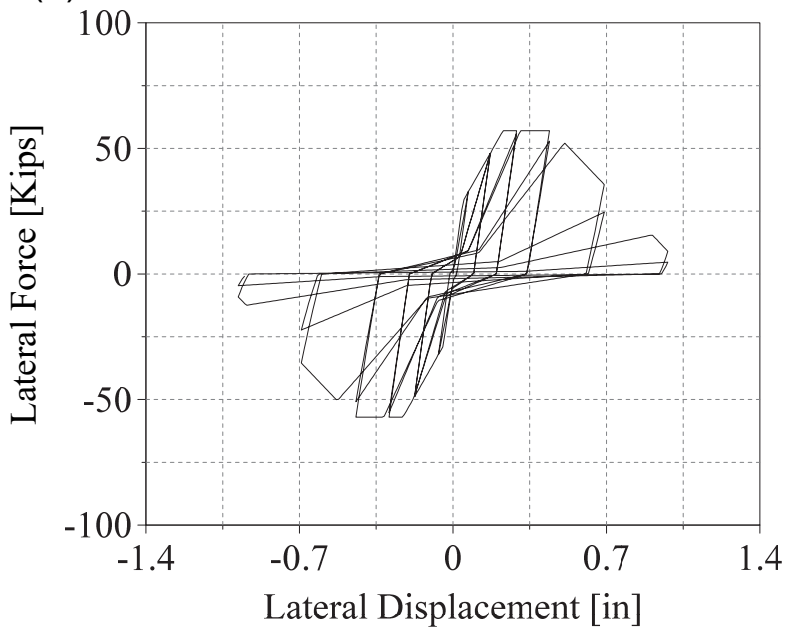

(c)

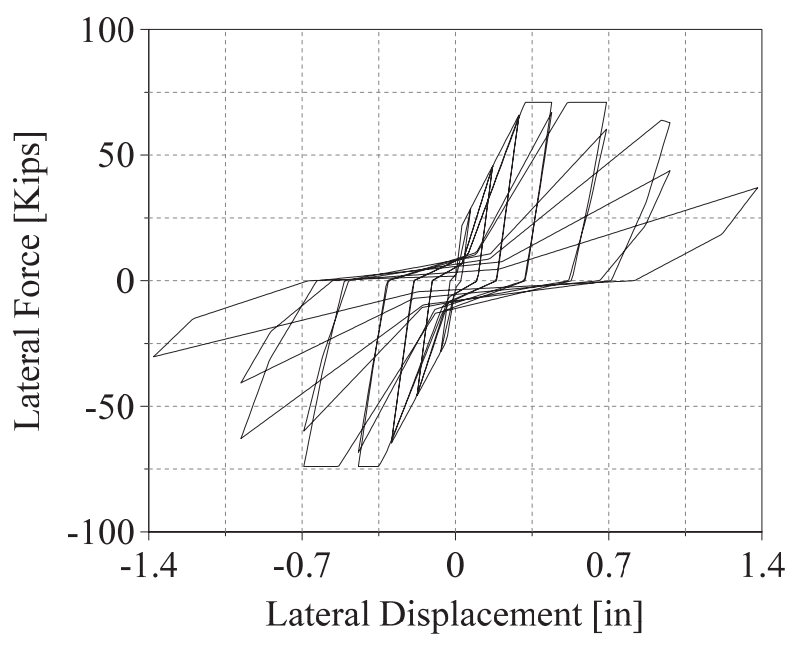

(b)

Figure D-19 Comparison of (a) test data, (b) MIKP prediction, and (c) proposed-model prediction for Hidalgo et al. (2002) Specimen 4. 


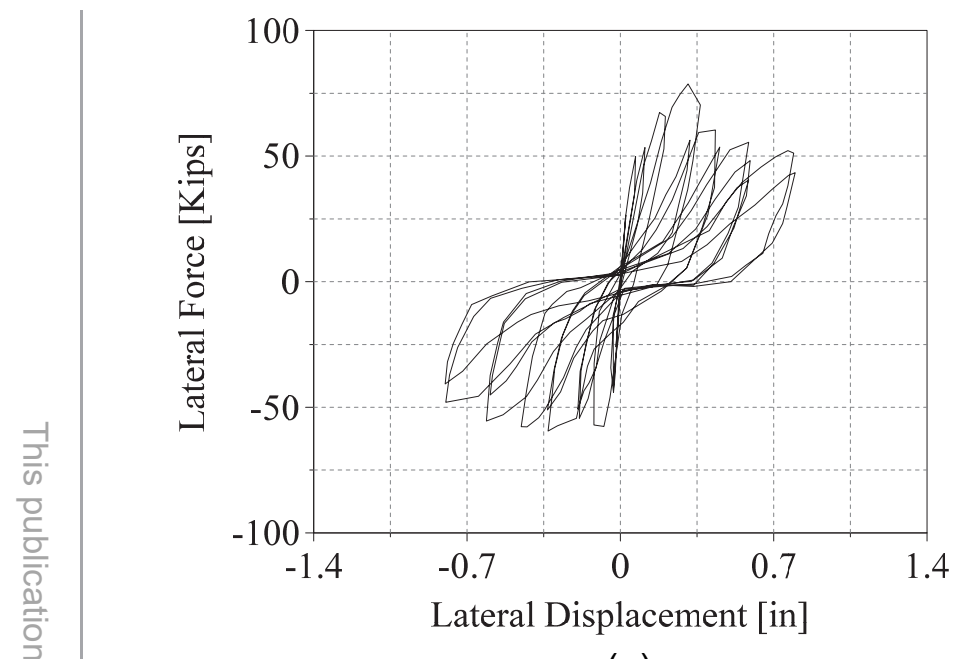

(a)

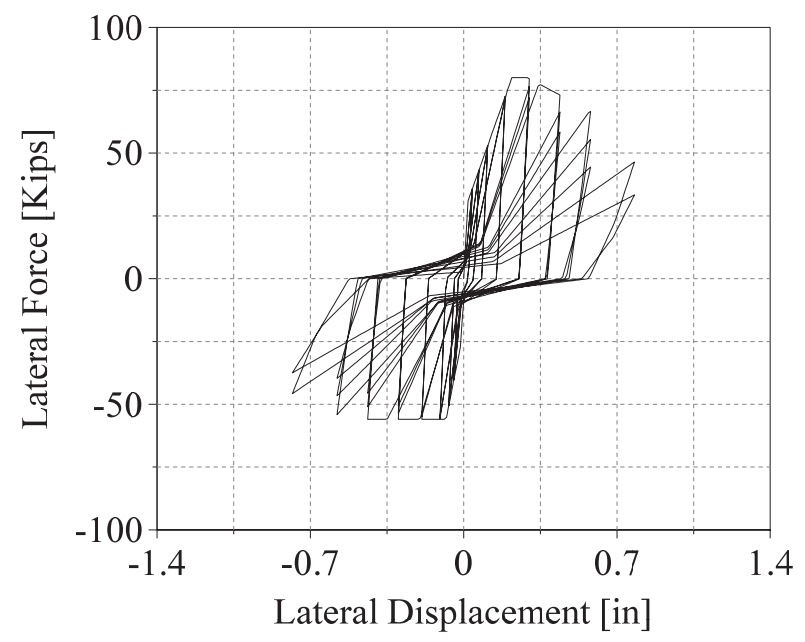

(b)

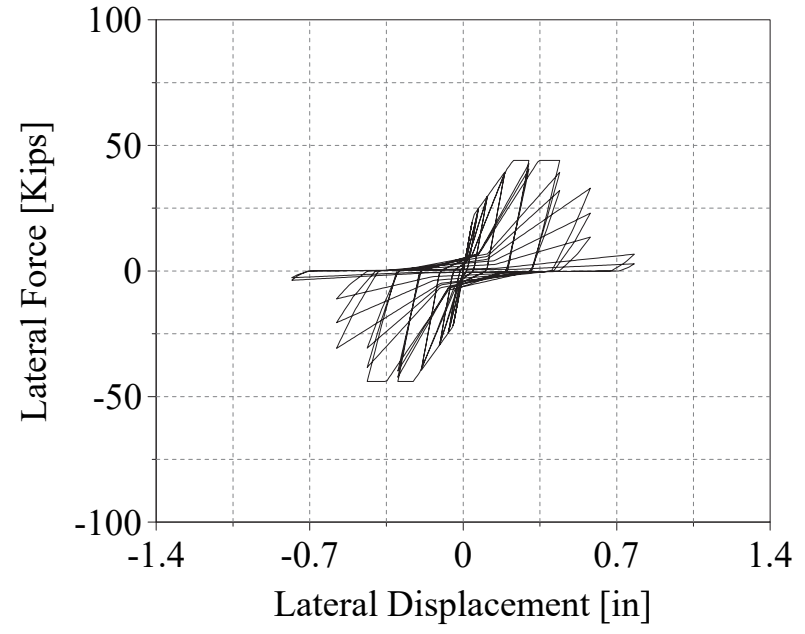

(c)

Figure D-20 Comparison of (a) test data, (b) MIKP prediction, and (c) proposed-model prediction for Hidalgo et al. (2002) Specimen 6. 


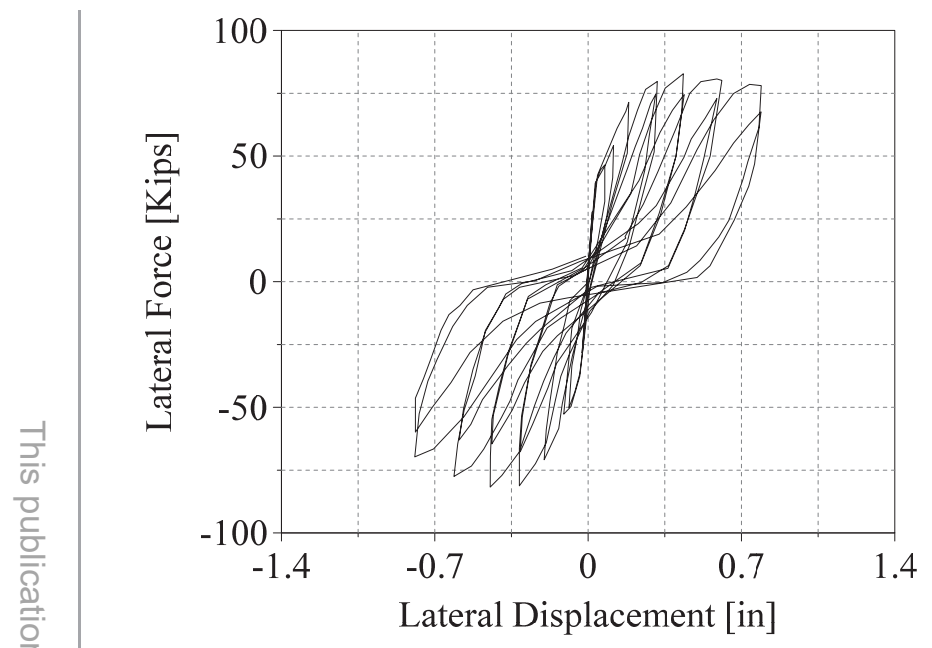

(a)

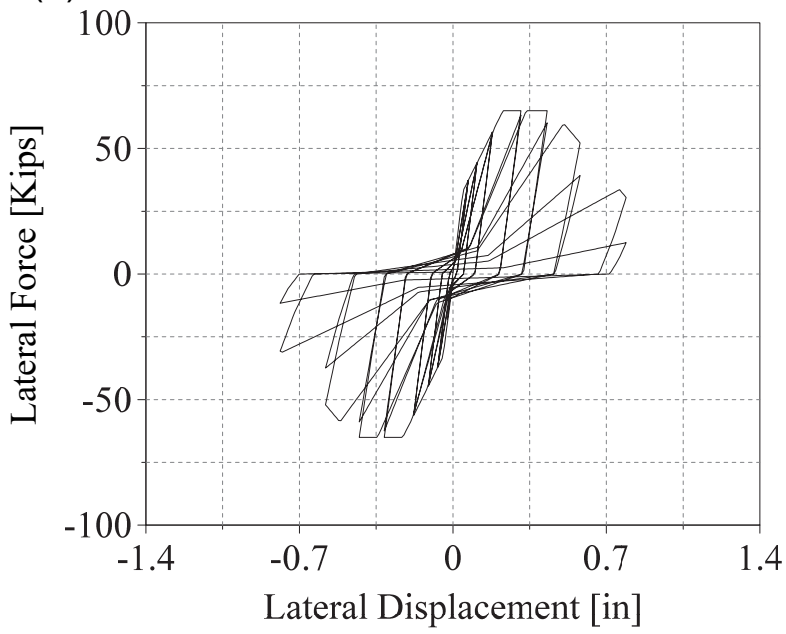

(c)

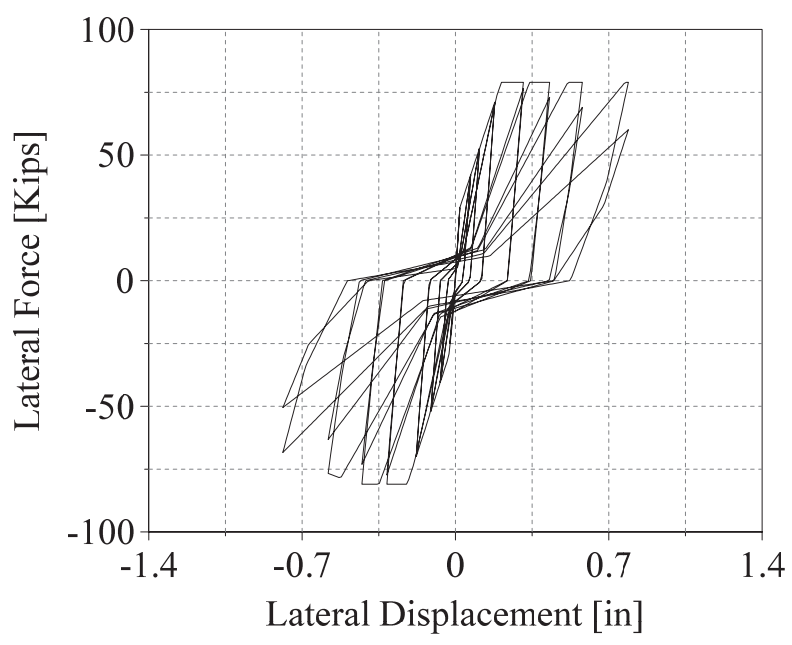

(b)

Figure D-21 Comparison of (a) test data, (b) MIKP prediction, and (c) proposed-model prediction for Hidalgo et al. (2002) Specimen 7. 


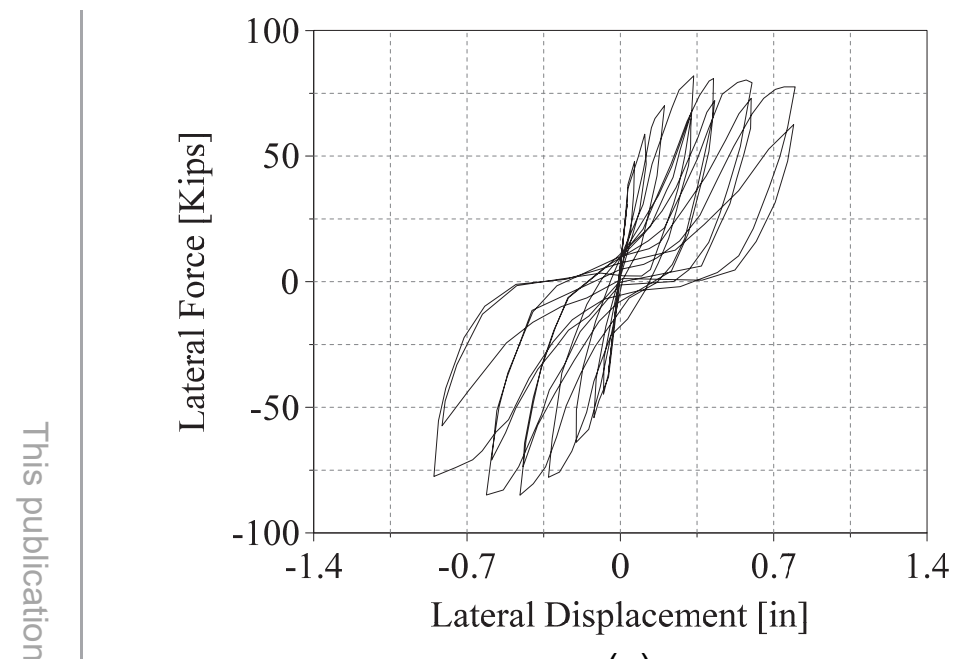

(a)

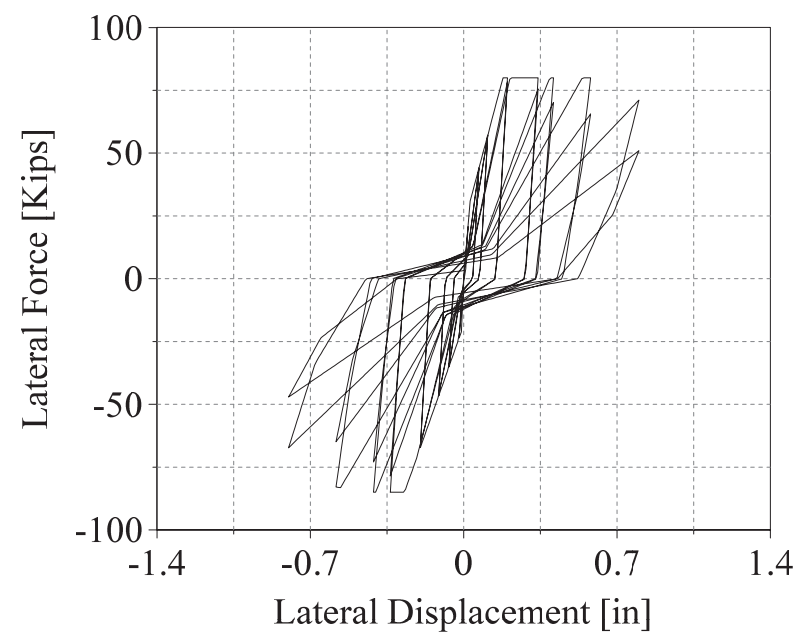

(b)

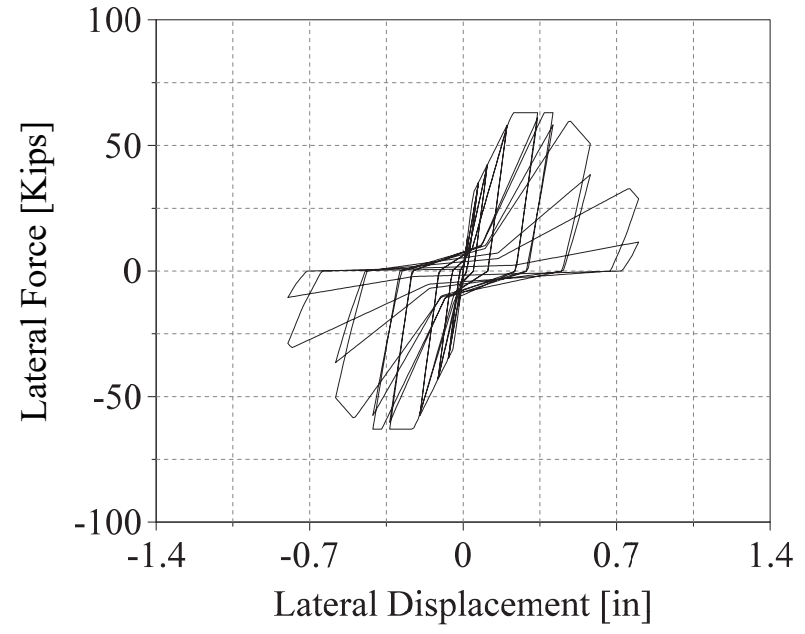

(c)

Figure D-22 Comparison of (a) test data, (b) MIKP prediction, and (c) proposed-model prediction for Hidalgo et al. (2002) Specimen 8. 


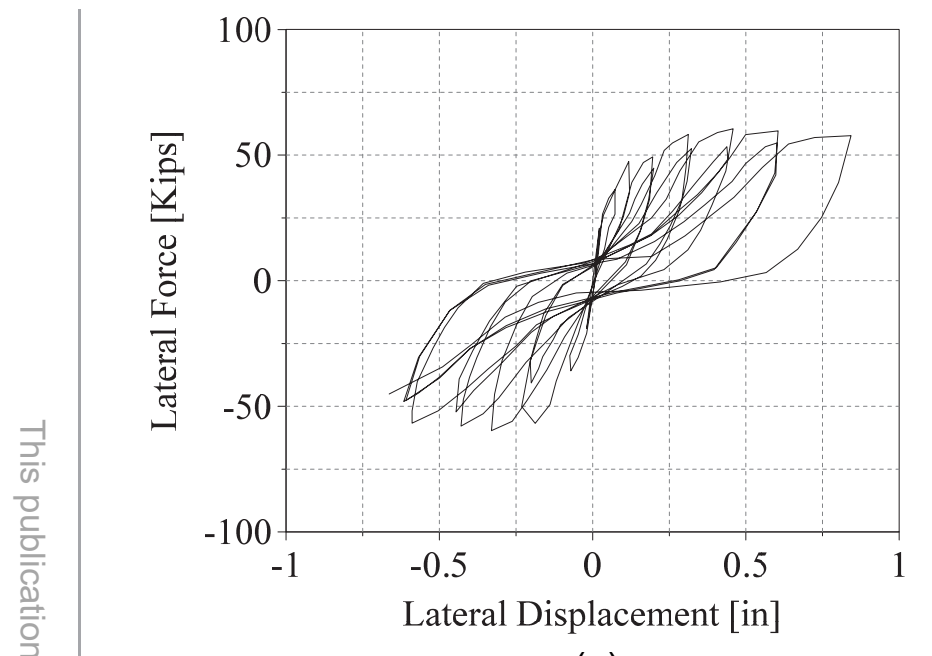

(a)

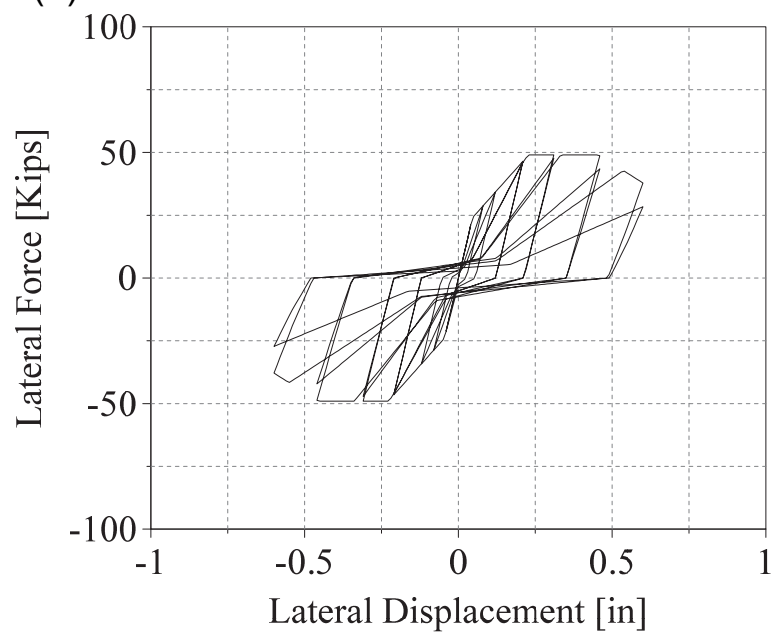

(c)

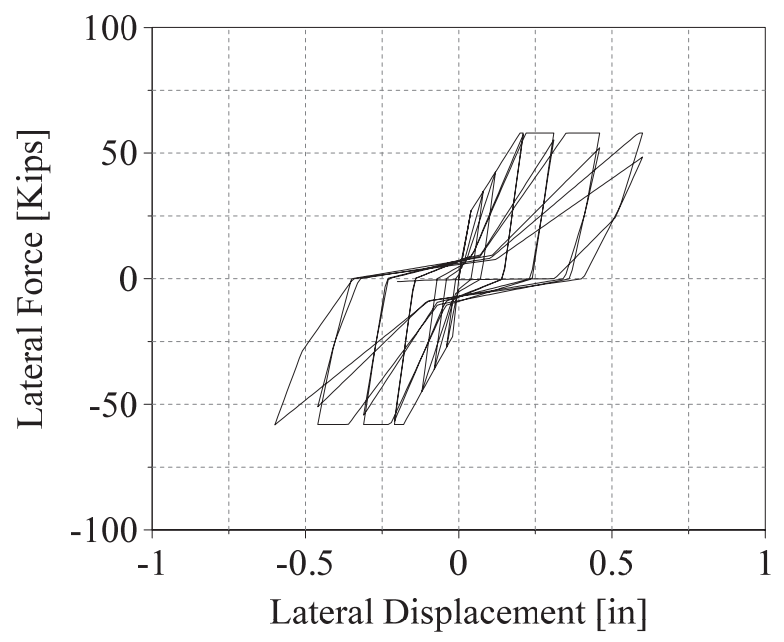

(b)

Figure D-23 Comparison of (a) test data, (b) MIKP prediction, and (c) proposed-model prediction for Hidalgo et al. (2002) Specimen 9. 


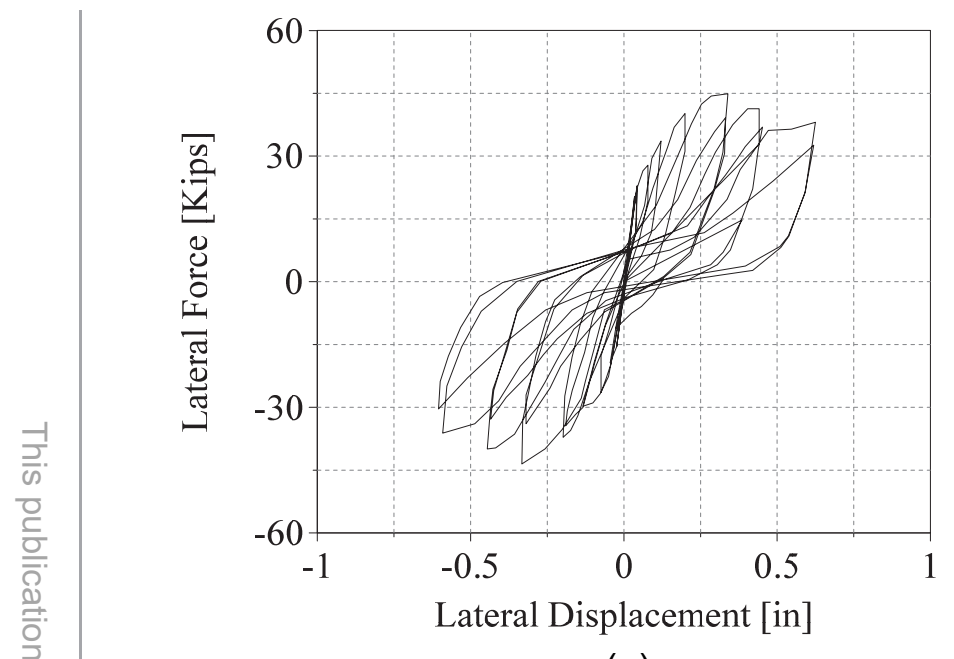

(a)

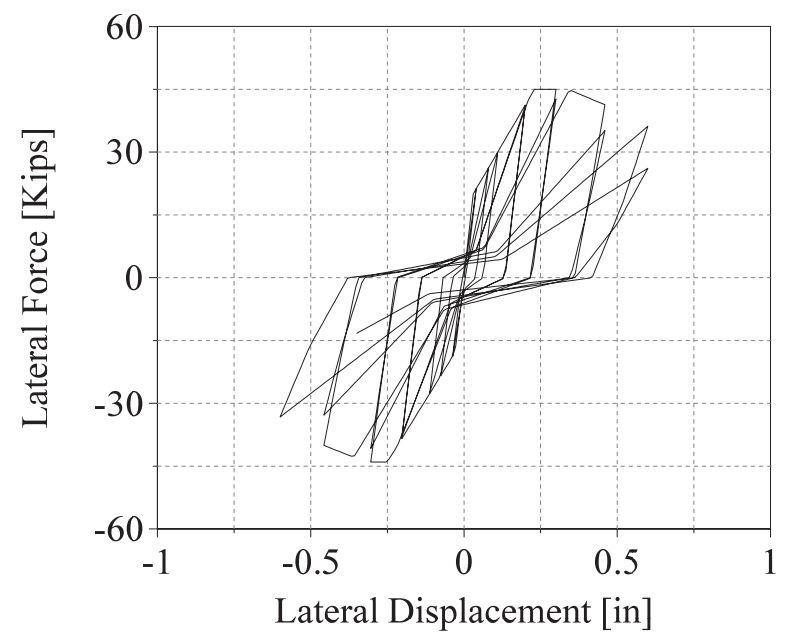

(b)

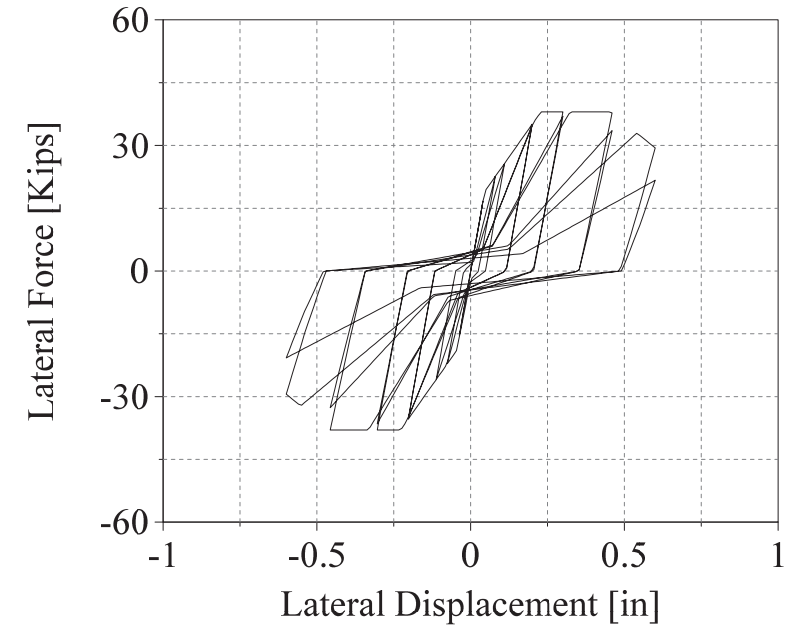

(c)

Figure D-24 Comparison of (a) test data, (b) MIKP prediction, and (c) proposed-model prediction for Hidalgo et al. (2002) Specimen 10. 


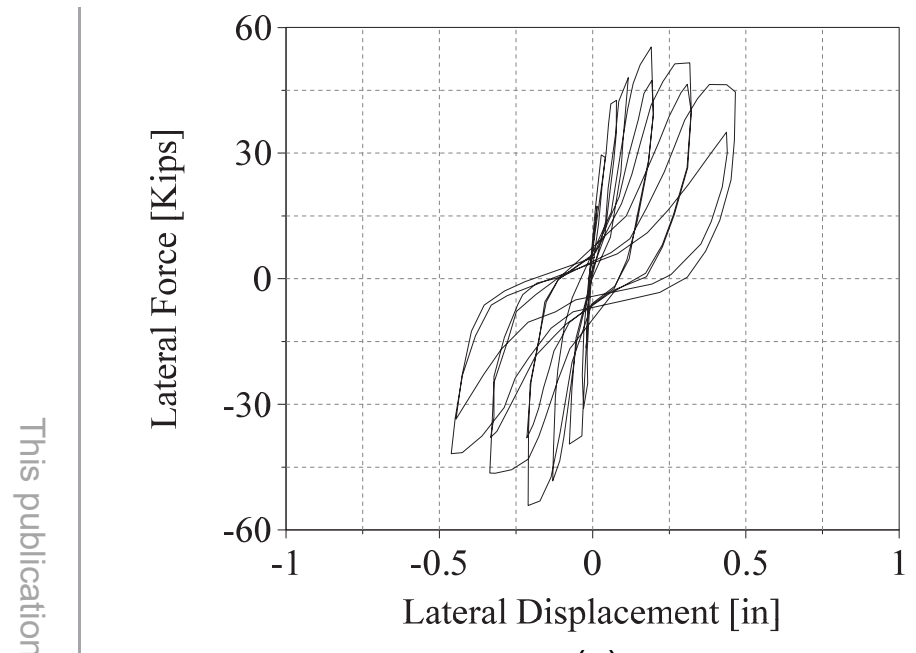

(a)

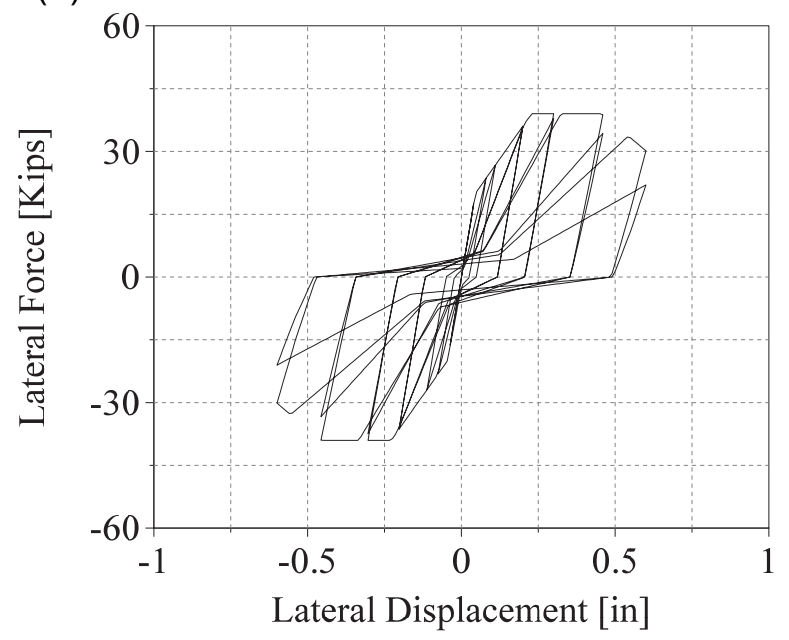

(c)

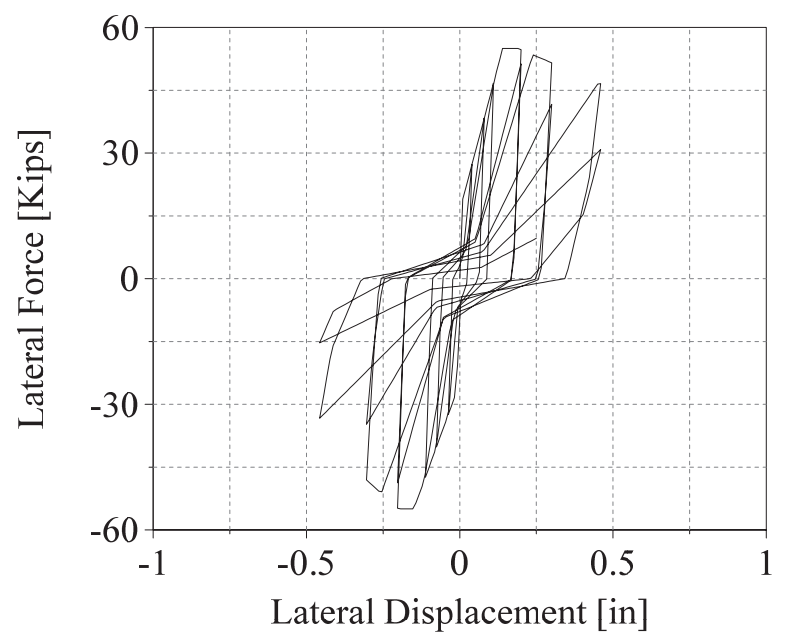

(b)

Figure D-25 Comparison of (a) test data, (b) MIKP prediction, and (c) proposed-model prediction for Hidalgo et al. (2002) Specimen 11. 


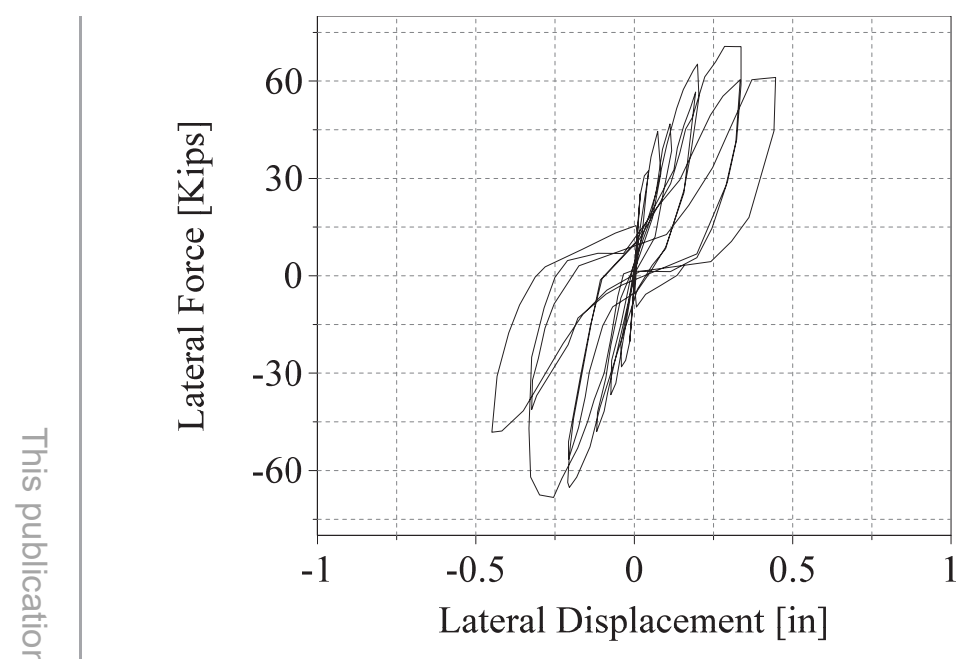

(a)

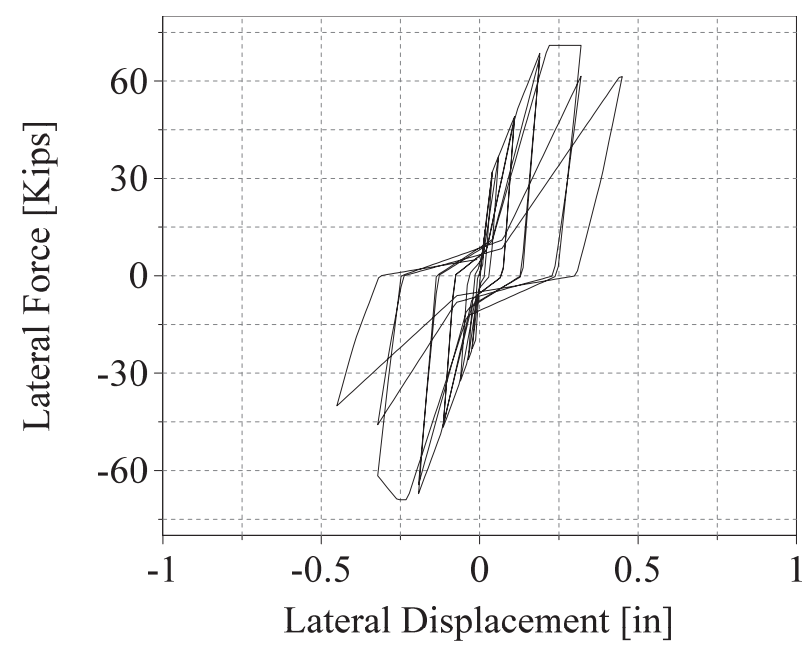

(b)

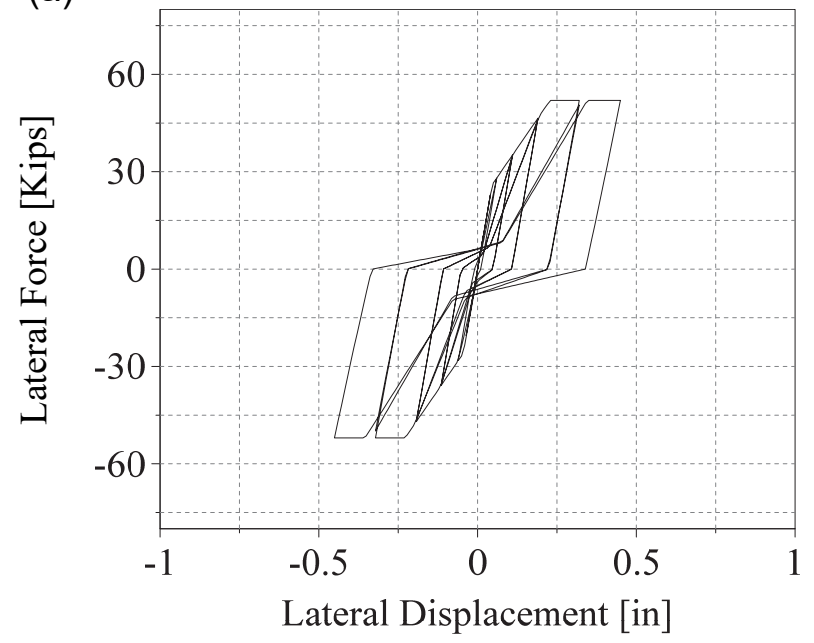

(c)

Figure D-26 Comparison of (a) test data, (b) MIKP prediction, and (c) proposed-model prediction for Hidalgo et al. (2002) Specimen 12. 


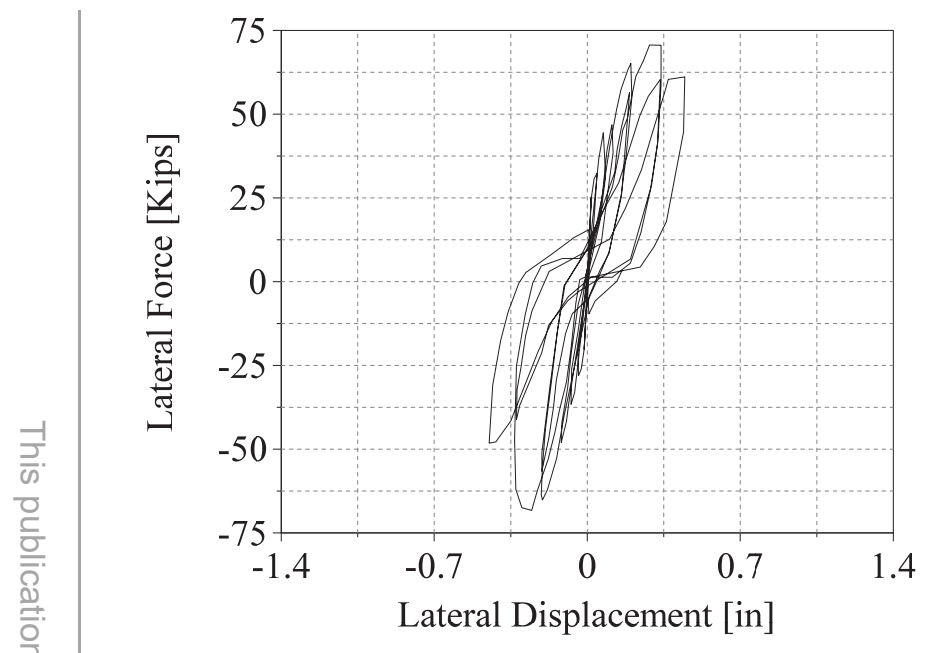

(a)

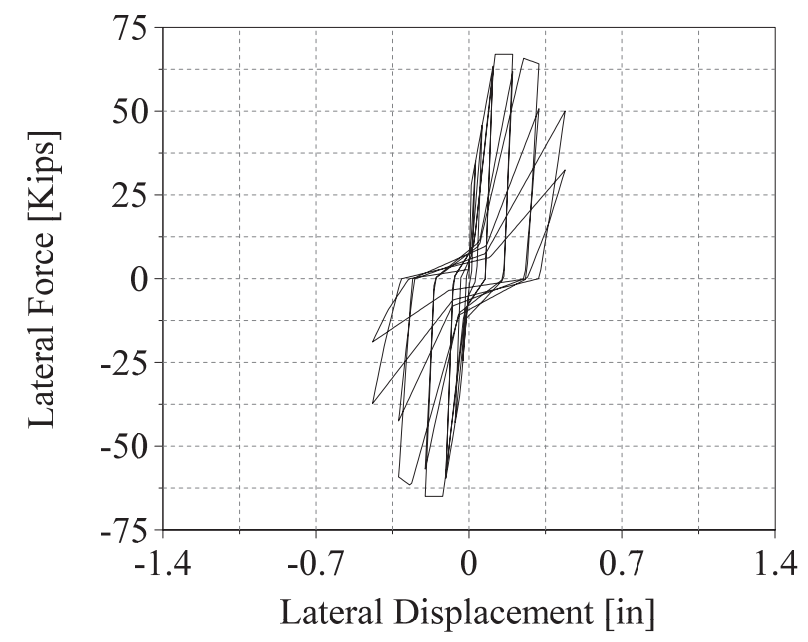

(b)

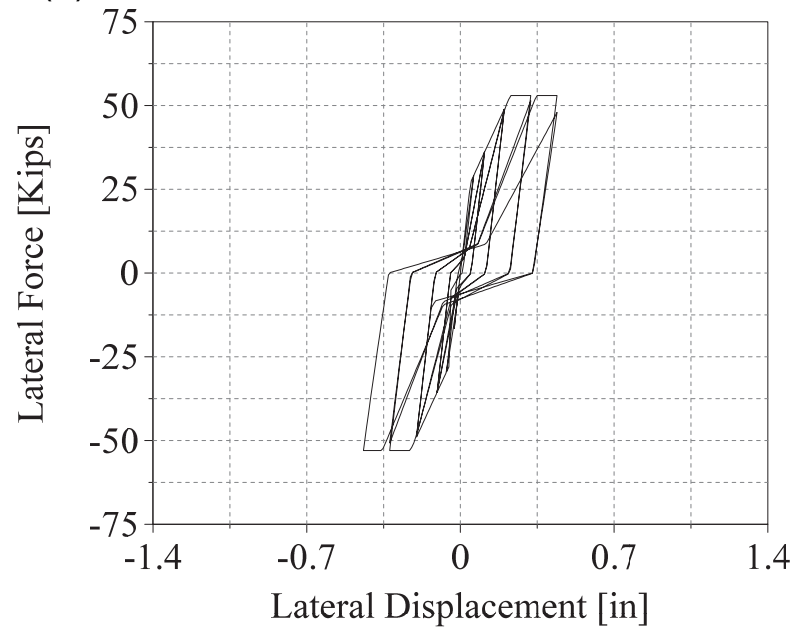

(c)

Figure D-27 Comparison of (a) test data, (b) MIKP prediction, and (c) proposed-model prediction for Hidalgo et al. (2002) Specimen 13. 


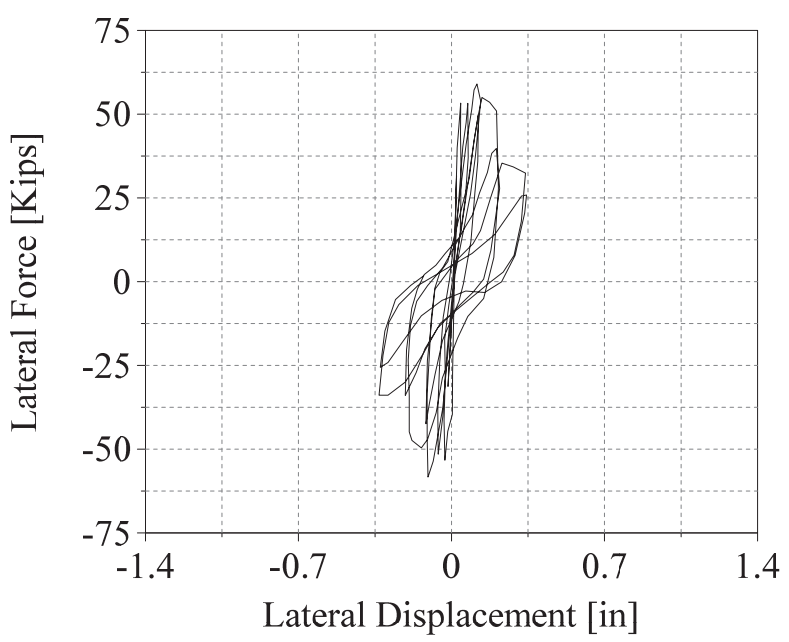

(a)

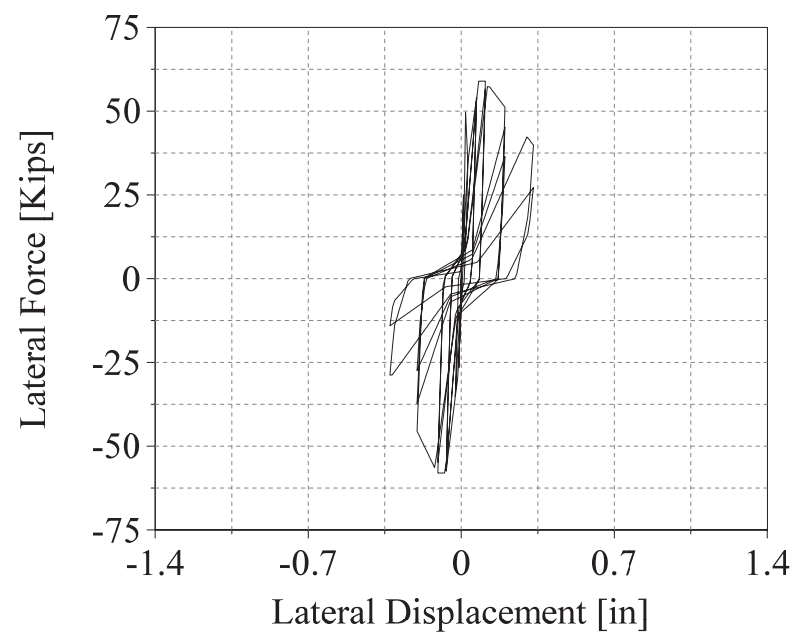

(b)

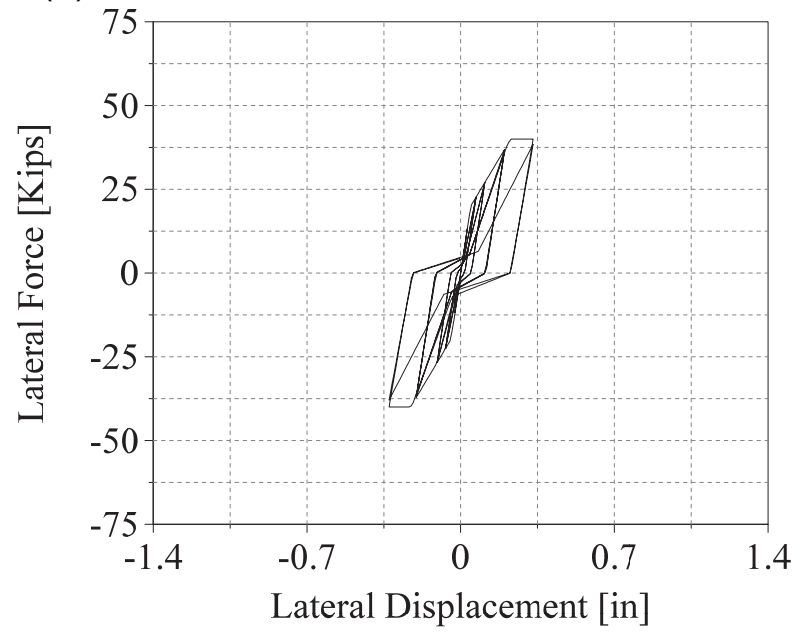

(c)

Figure D-28 Comparison of (a) test data, (b) MIKP prediction, and (c) proposed-model prediction for Hidalgo et al. (2002) Specimen 14. 


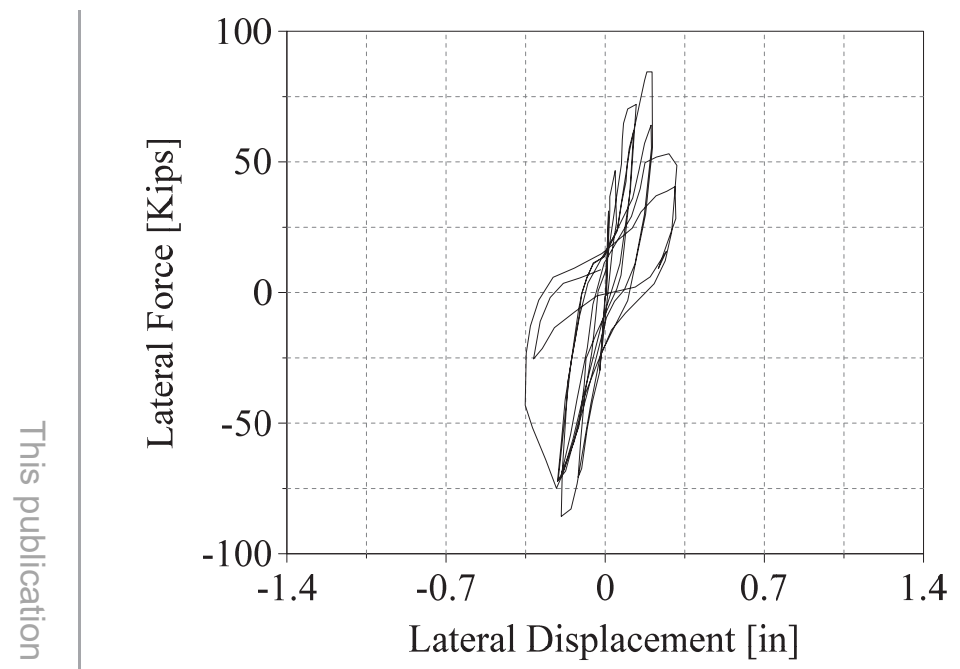

(a)

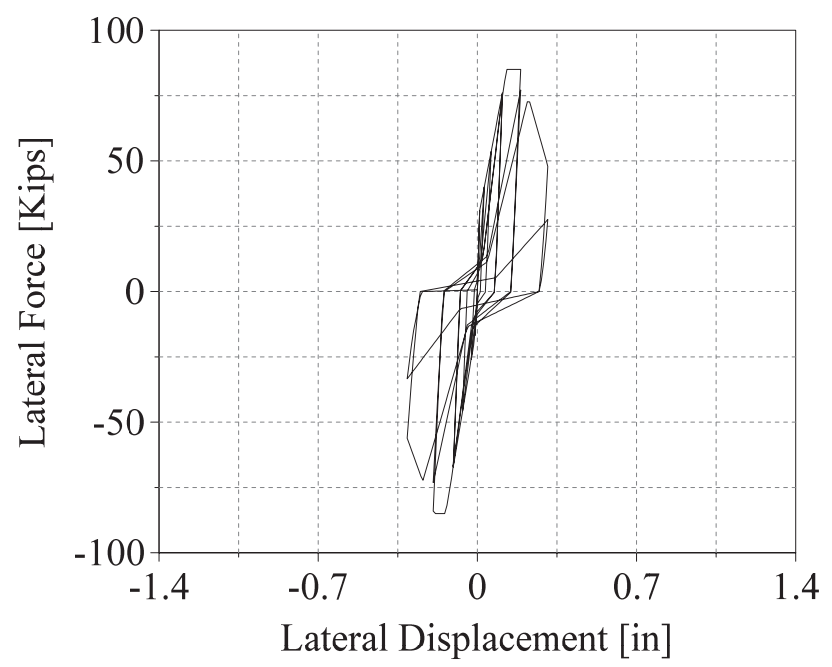

(b)

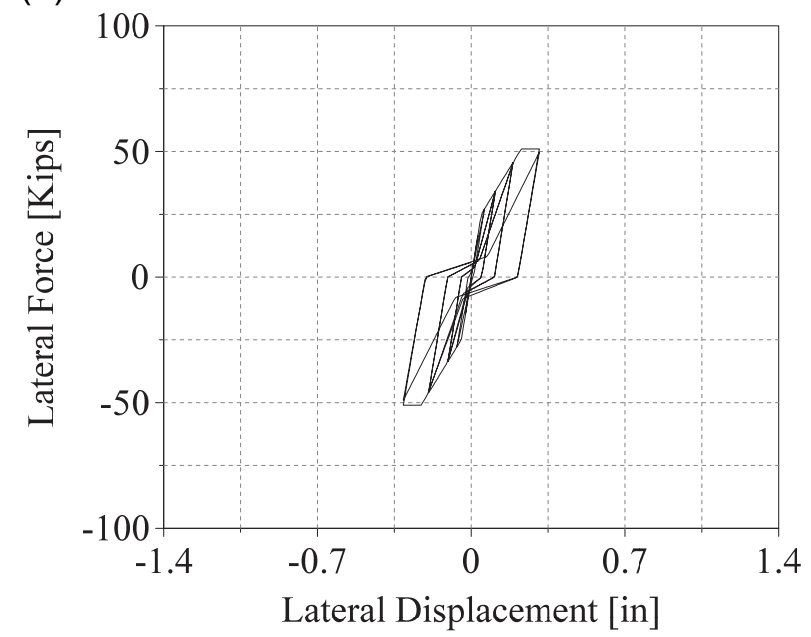

(c)

Figure D-29 Comparison of (a) test data, (b) MIKP prediction, and (c) proposed-model prediction for Hidalgo et al. (2002) Specimen 15. 


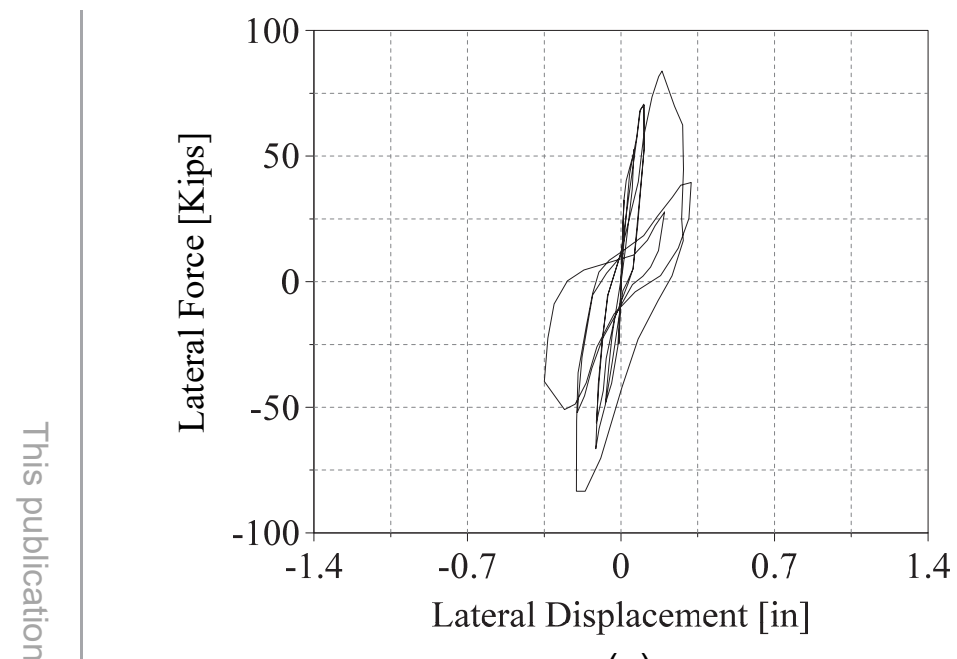

(a)

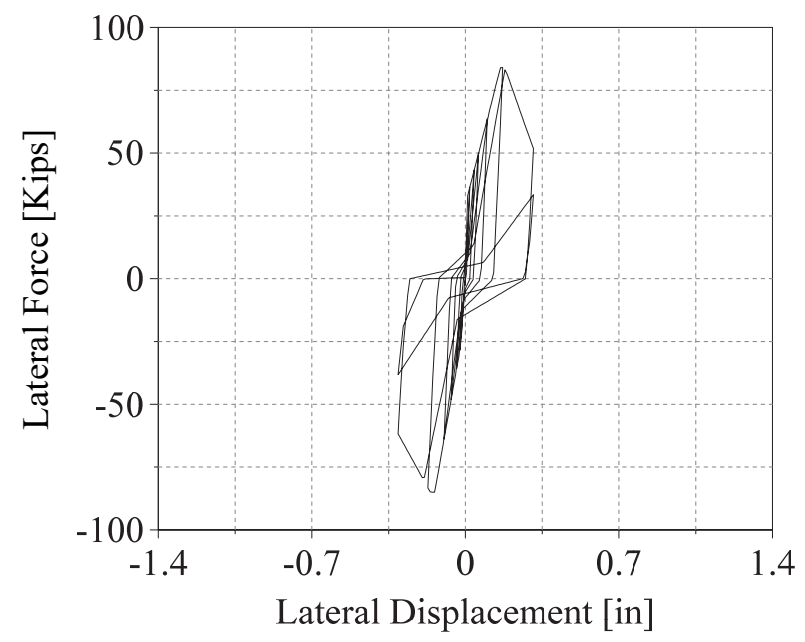

(b)

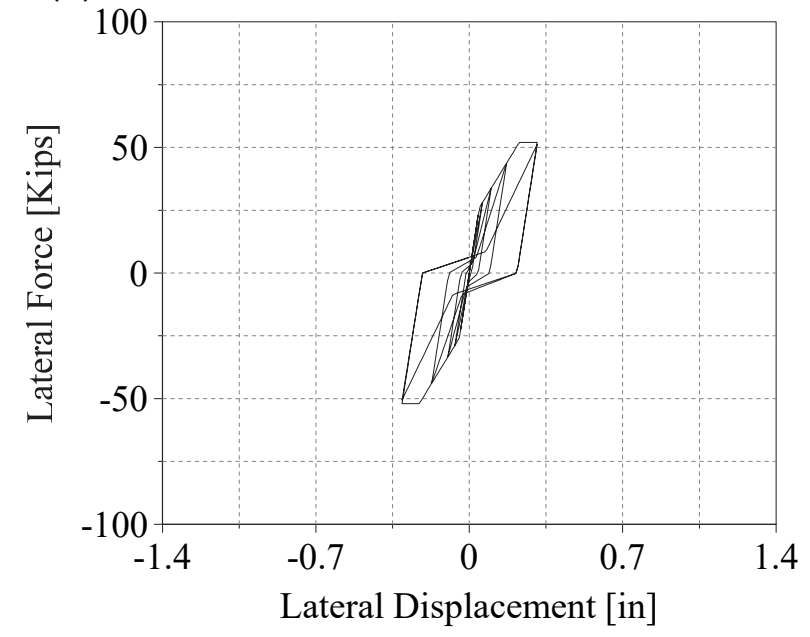

(c)

Figure D-30 Comparison of (a) test data, (b) MIKP prediction, and (c) proposed-model prediction for Hidalgo et al. (2002) Specimen 16. 


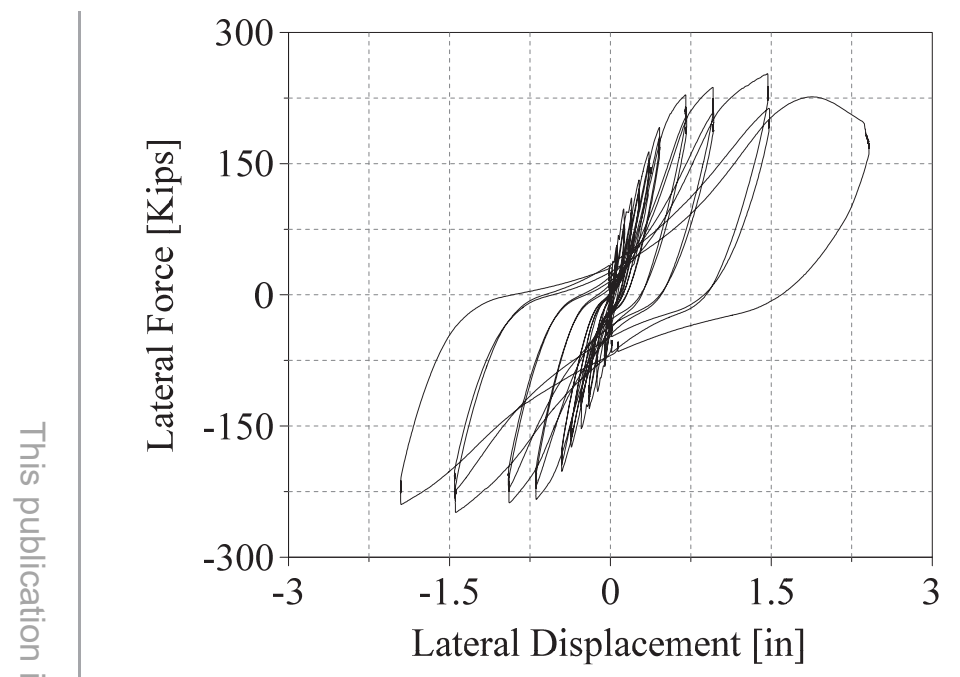

(a)

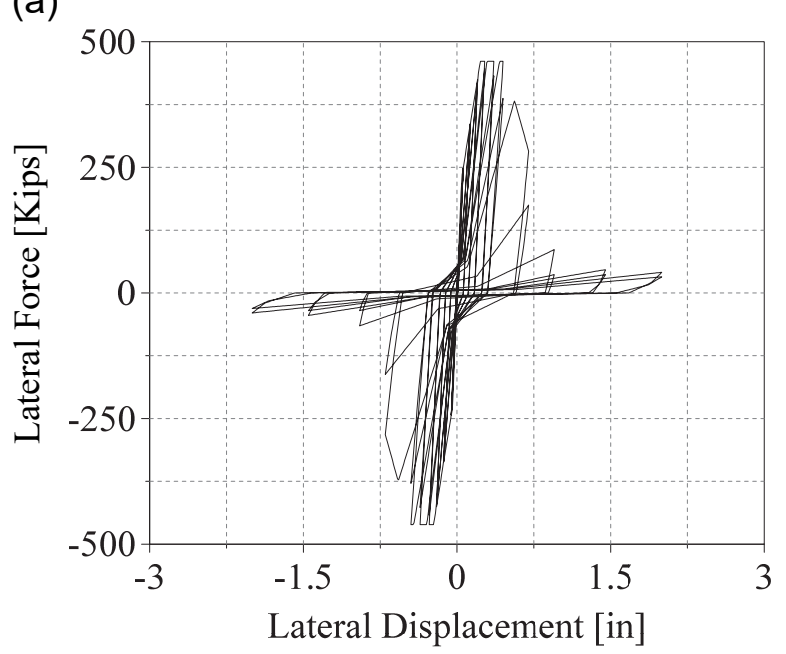

(c)

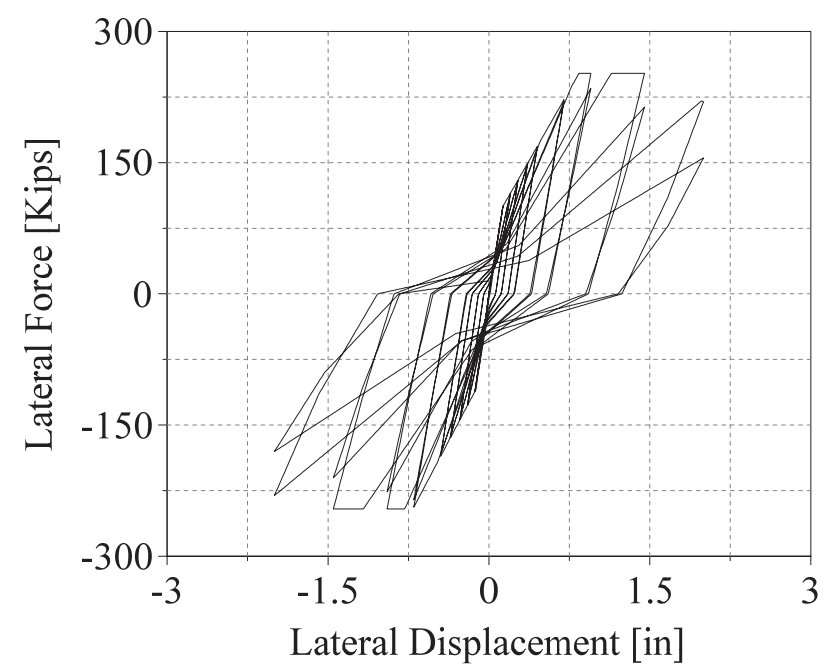

(b)

Figure D-31 Comparison of (a) test data, (b) MIKP prediction, and (c) proposed-model prediction for Luna et al. (2015) Specimen SW1. 


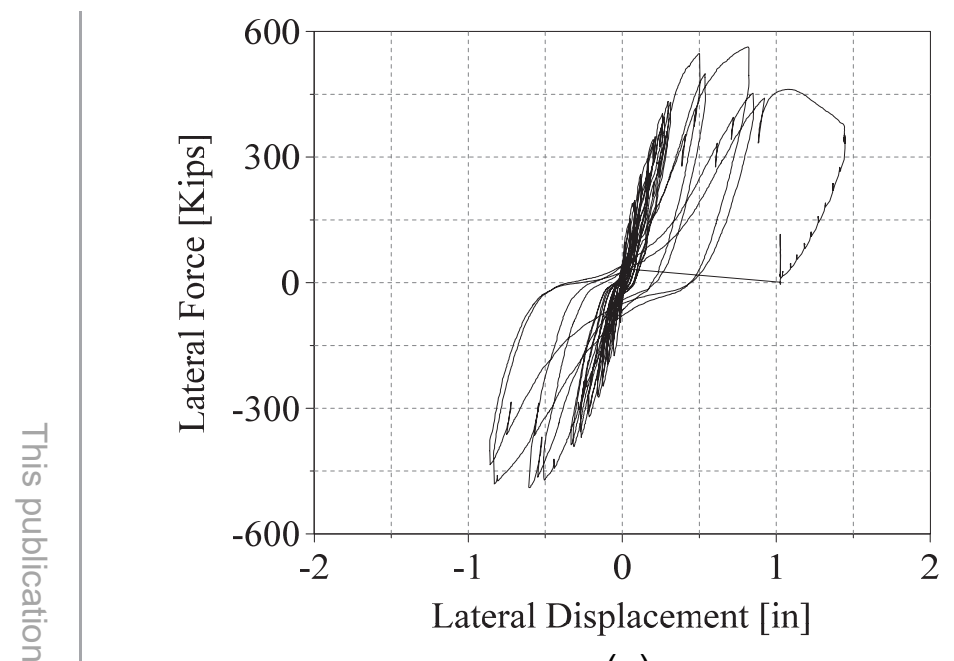

(a)

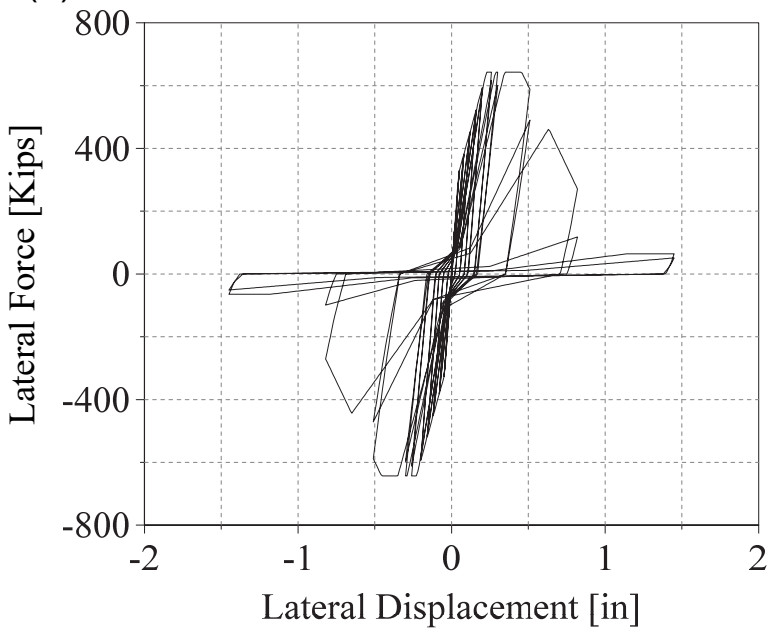

(c)

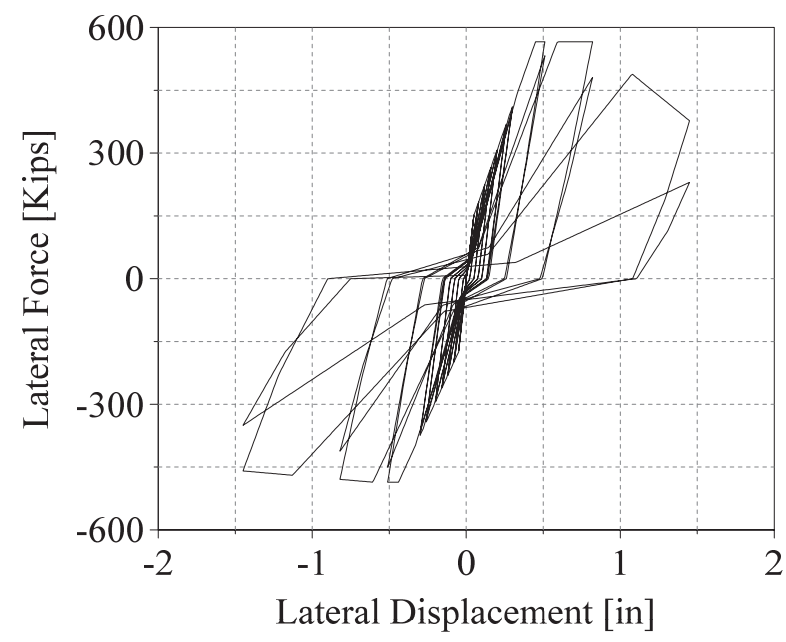

(b)

Figure D-32 Comparison of (a) test data, (b) MIKP prediction, and (c) proposed-model prediction for Luna et al. (2015) Specimen SW2. 


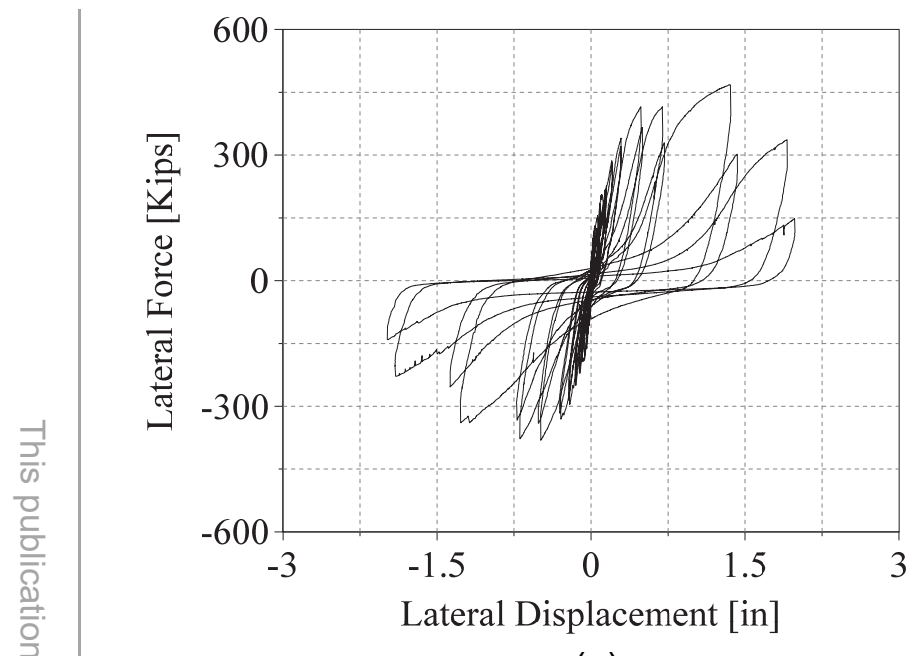

(a)

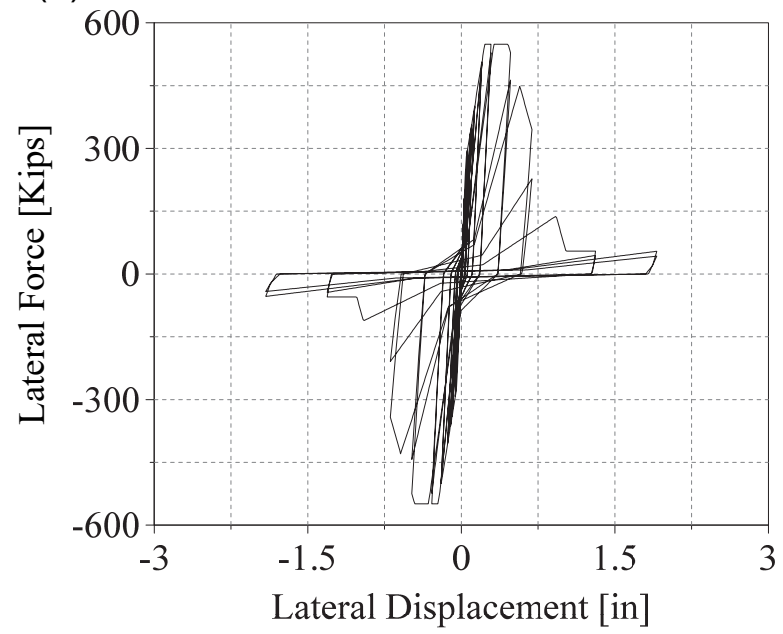

(c)

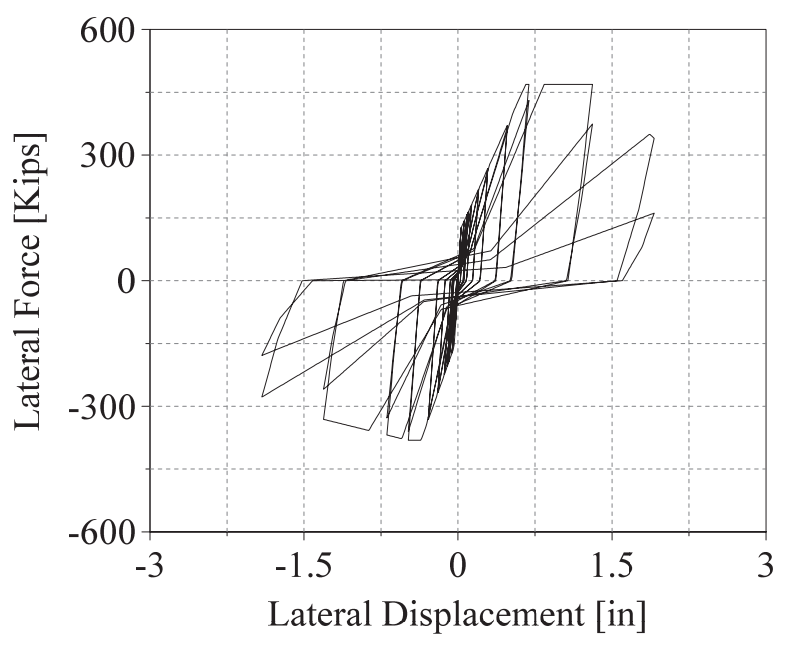

(b)

Figure D-33 Comparison of (a) test data, (b) MIKP prediction, and (c) proposed-model prediction for Luna et al. (2015) Specimen SW3. 


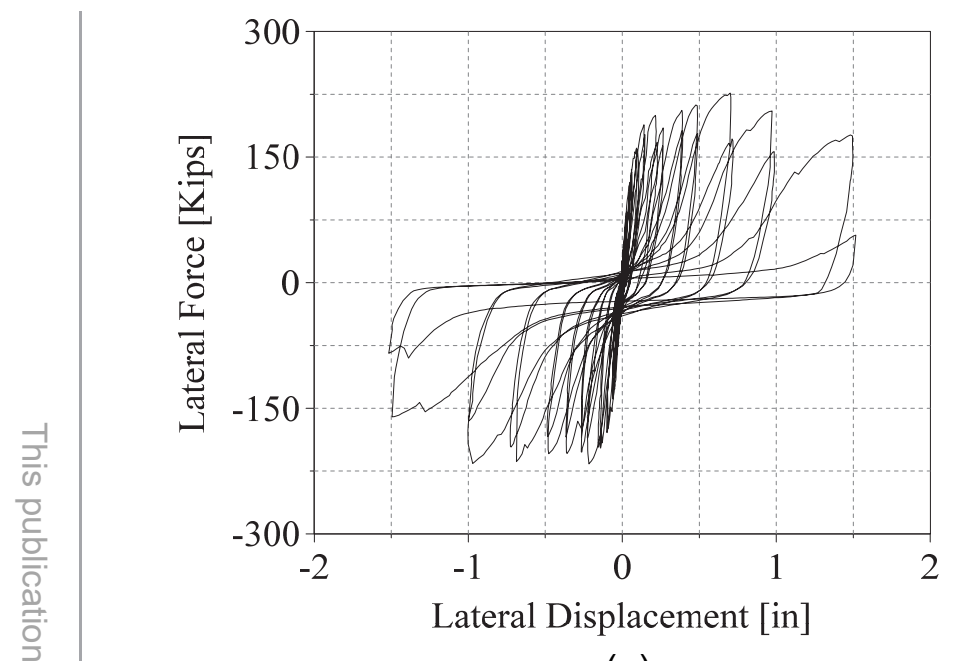

(a)

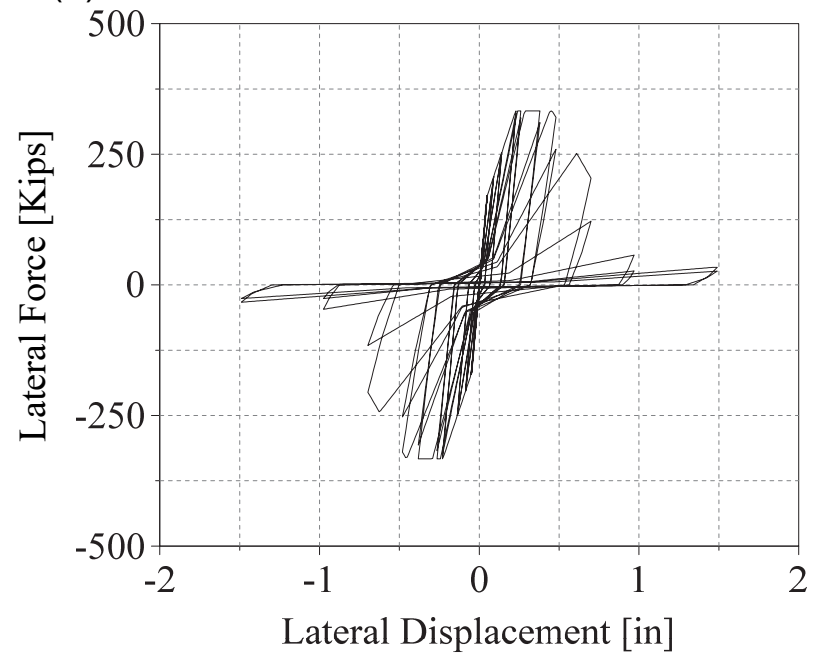

(c)

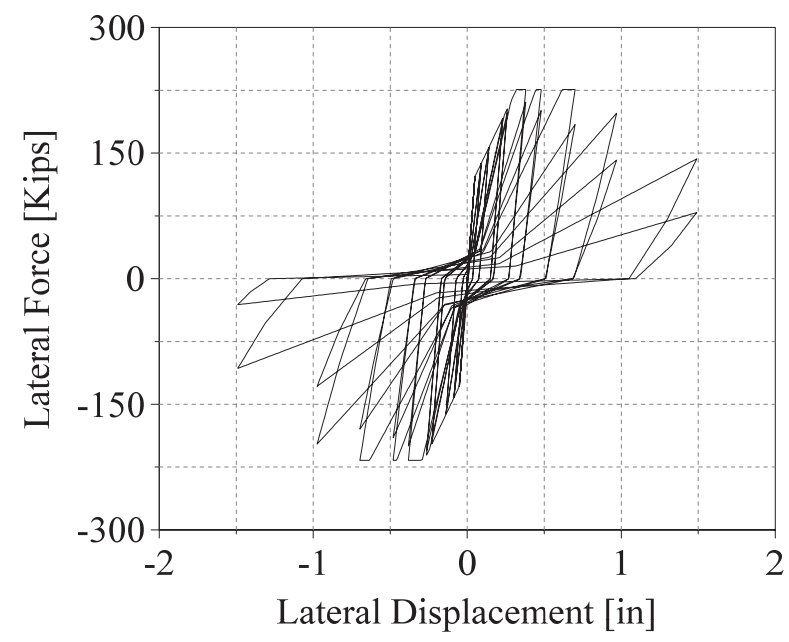

(b)

Figure D-34 Comparison of (a) test data, (b) MIKP prediction, and (c) proposed-model prediction for Luna et al. (2015) Specimen SW4. 


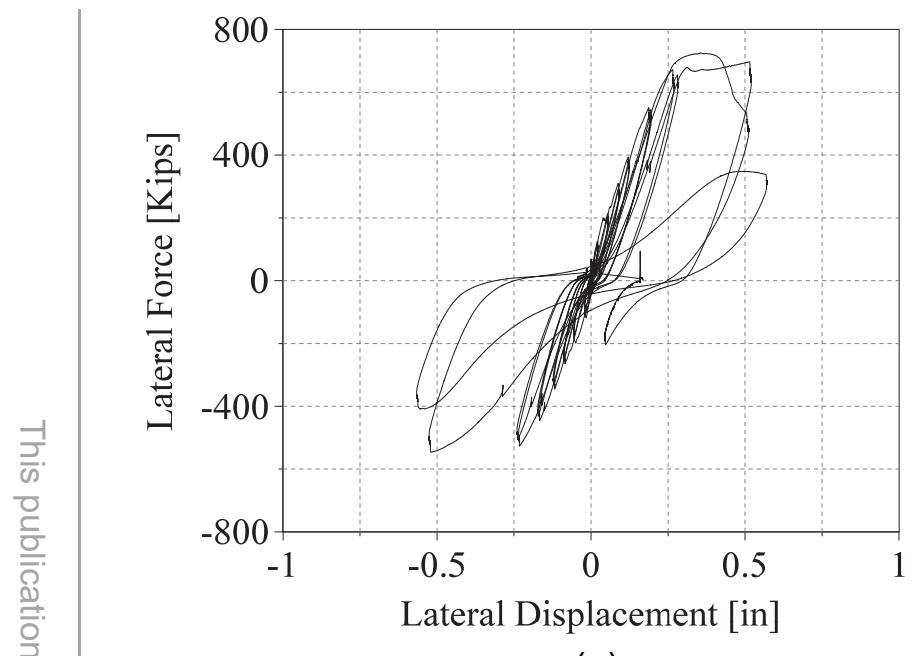

(a)

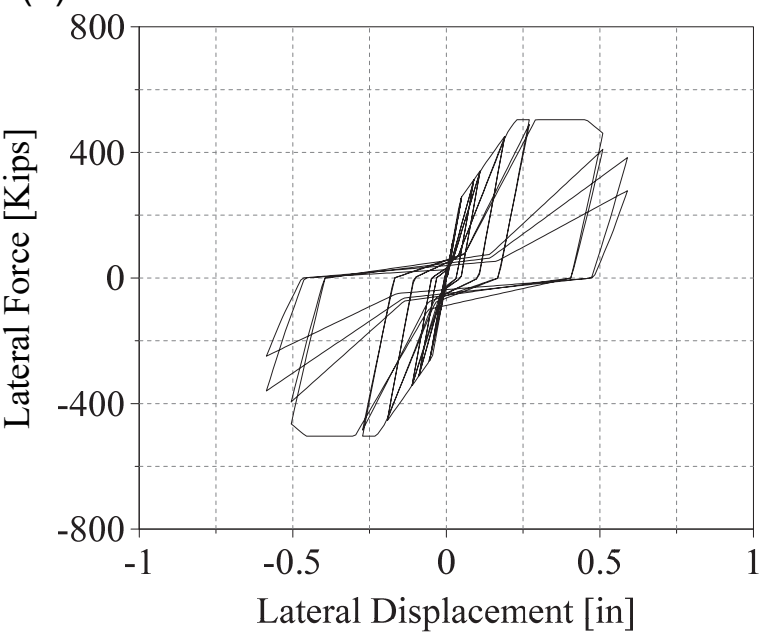

(c)

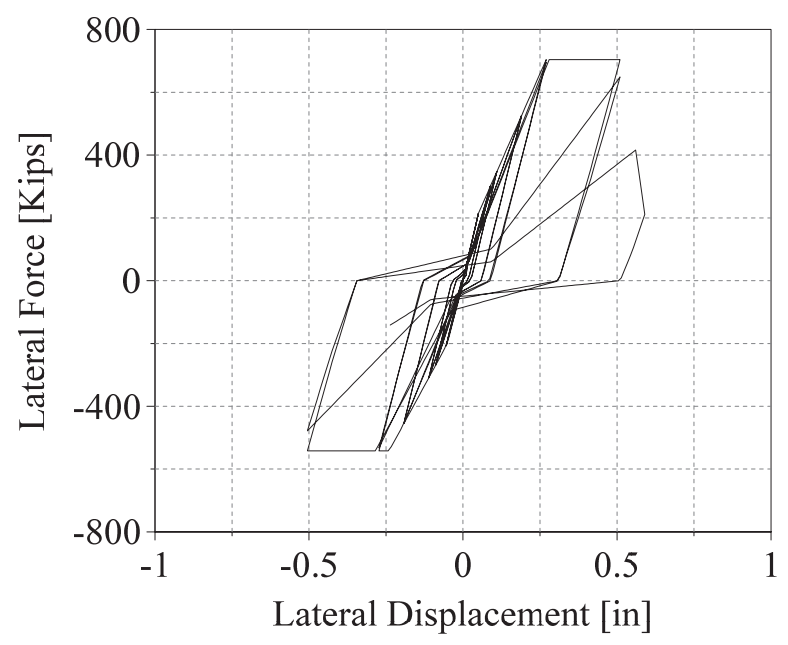

(b)

Figure D-35 Comparison of (a) test data, (b) MIKP prediction, and (c) proposed-model prediction for Luna et al. (2015) Specimen SW5. 


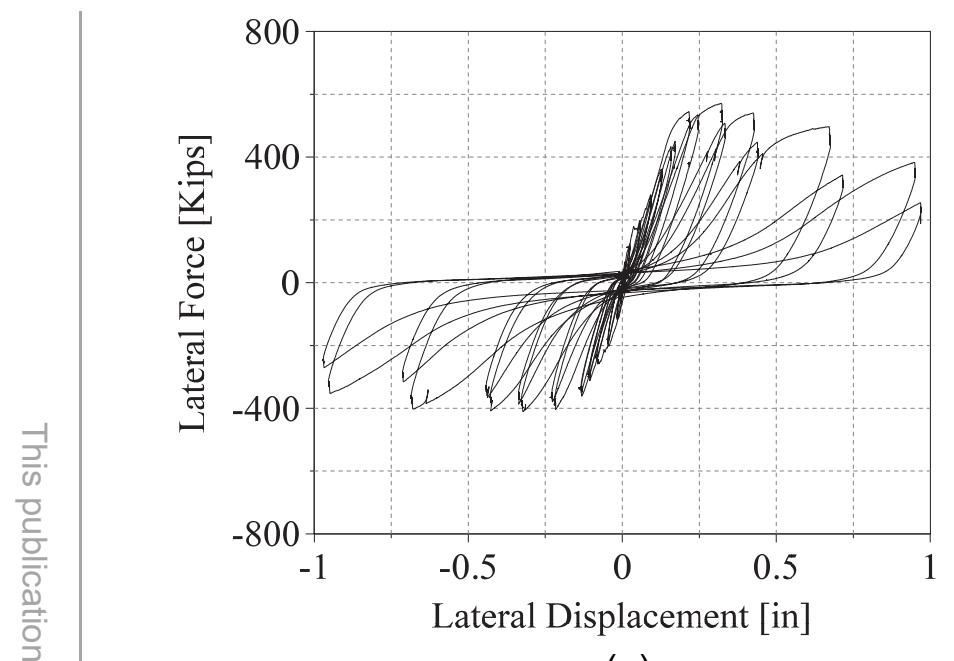

(a)

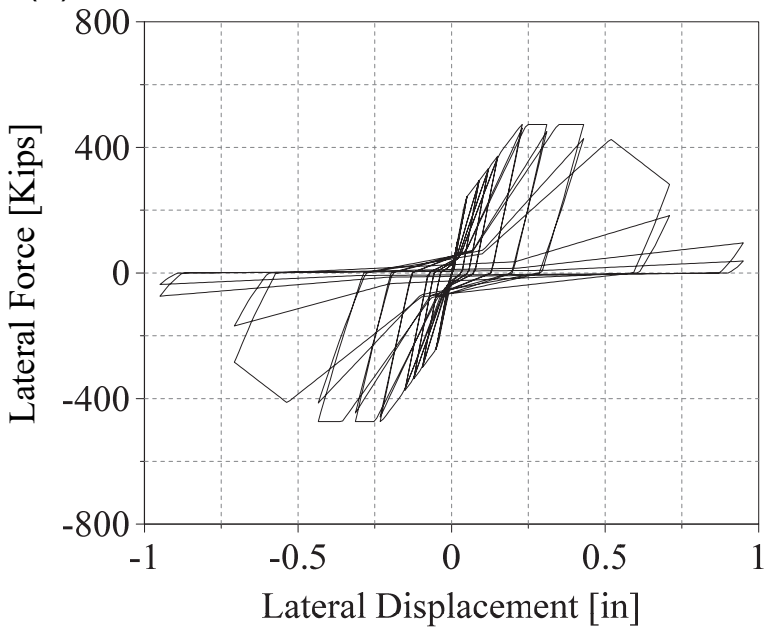

(c)

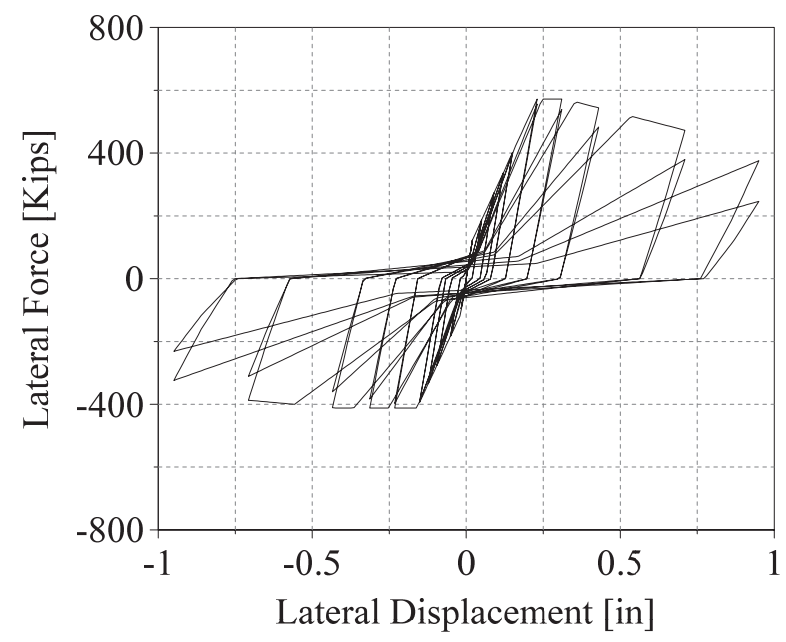

(b)

Figure D-36 Comparison of (a) test data, (b) MIKP prediction, and (c) proposed-model prediction for Luna et al. (2015) Specimen SW6. 


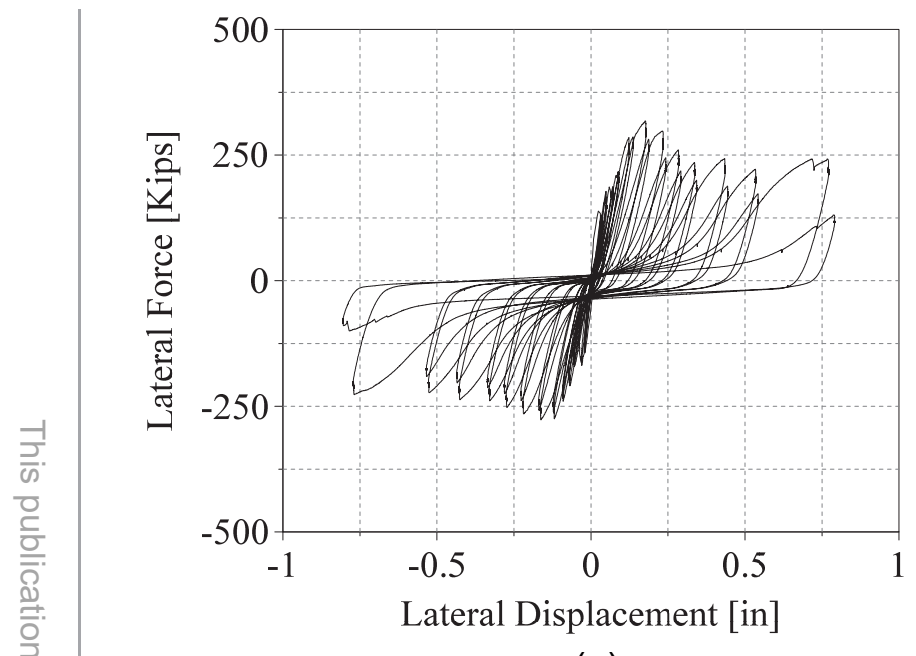

(a)

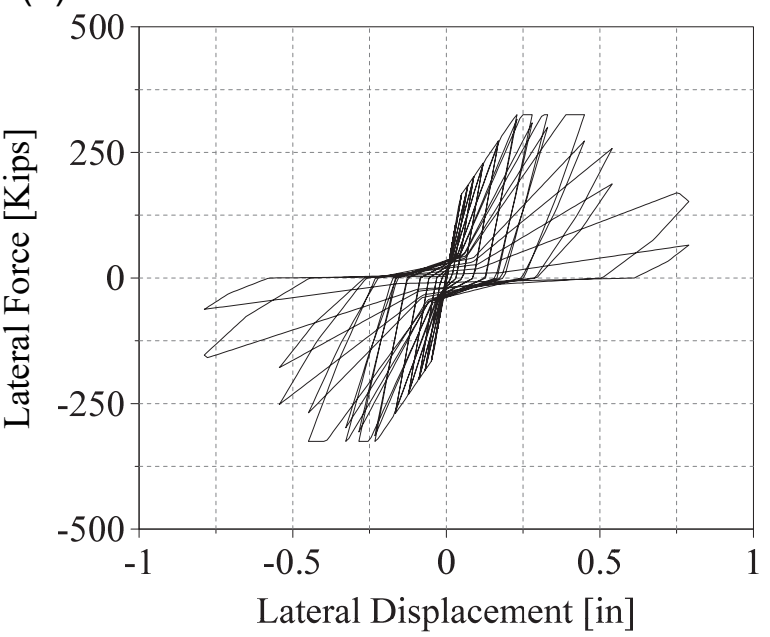

(c)

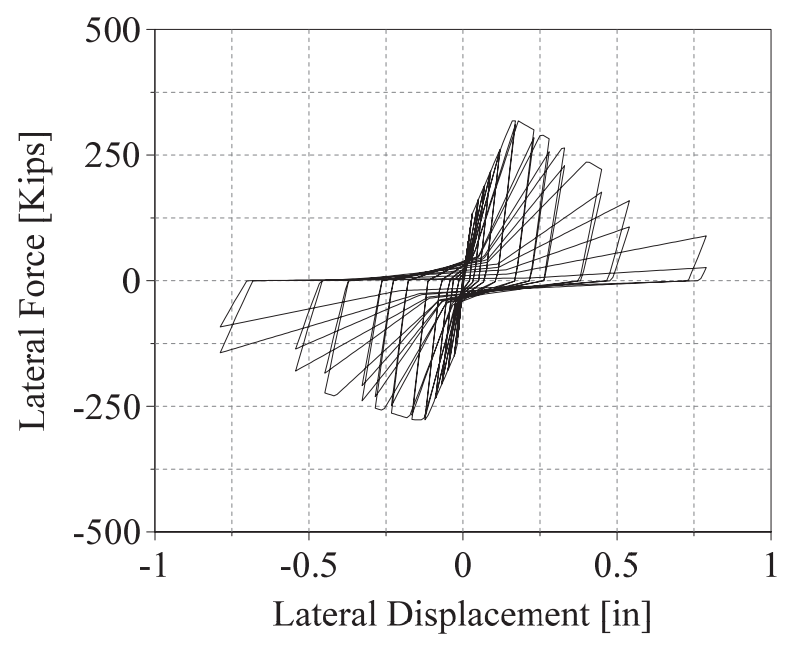

(b)

Figure D-37 Comparison of (a) test data, (b) MIKP prediction, and (c) proposed-model prediction for Luna et al. (2015) Specimen SW7. 


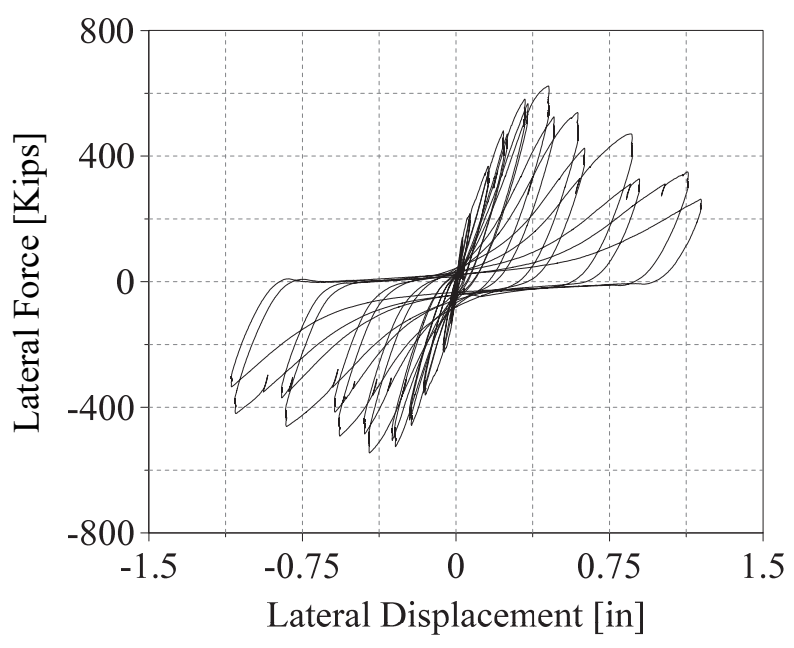

(a)

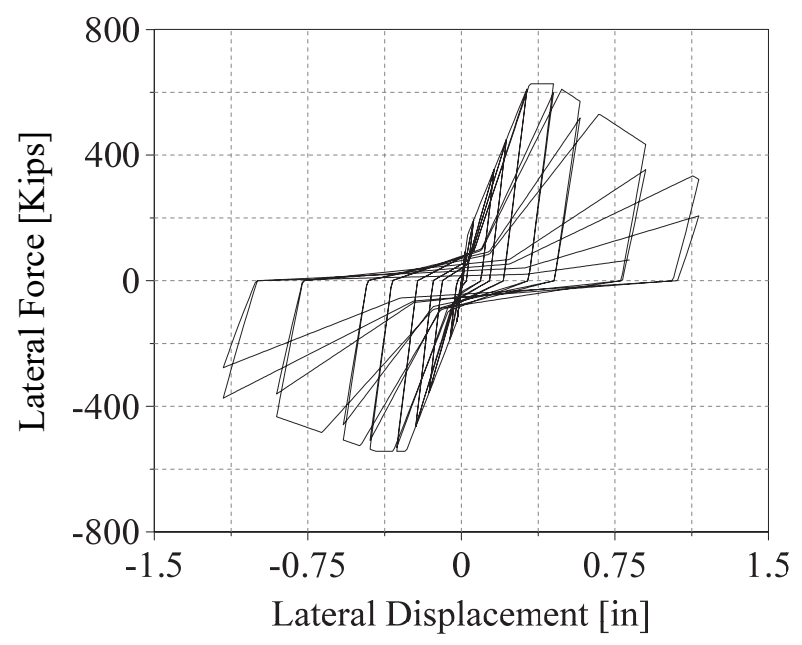

(b)

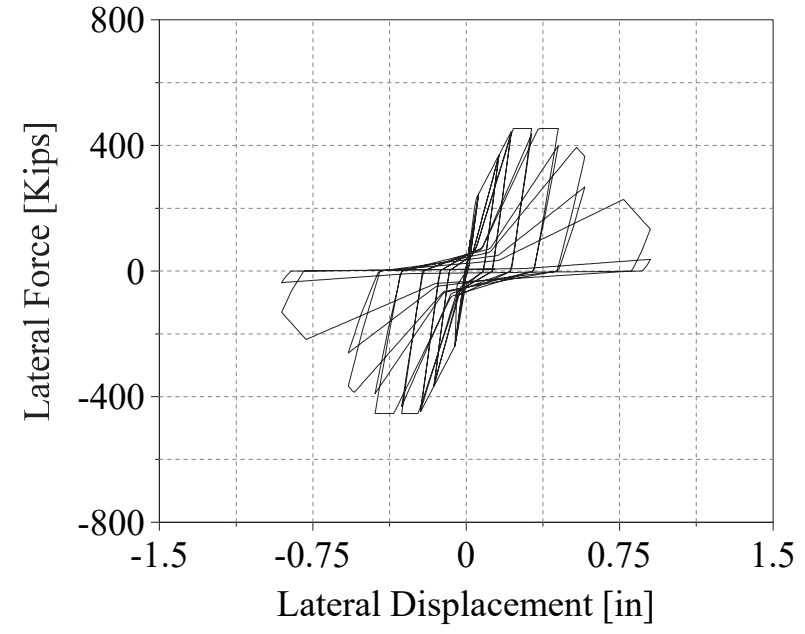

(c)

Figure D-38 Comparison of (a) test data, (b) MIKP prediction, and (c) proposed-model prediction for Luna et al. (2015) Specimen SW8. 


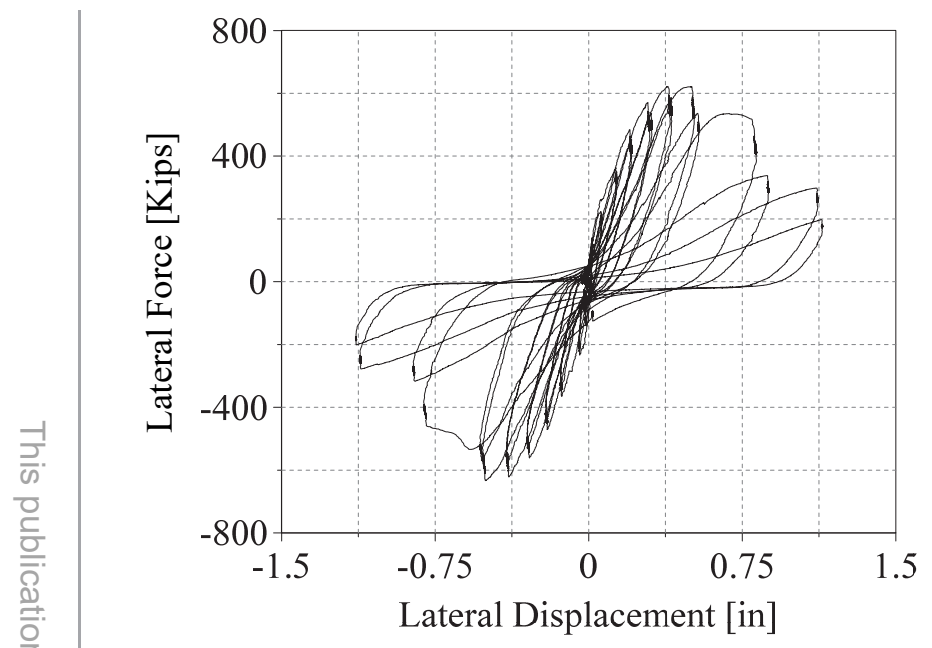

(a)

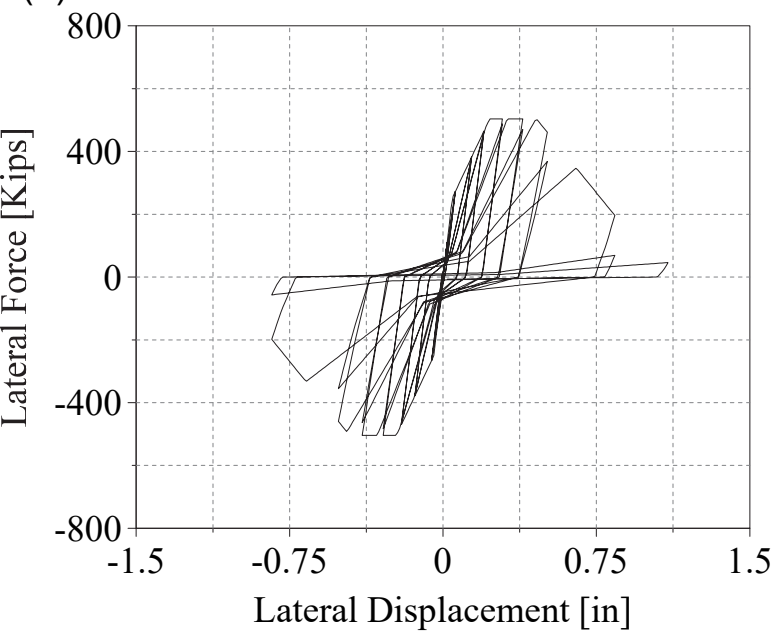

(c)

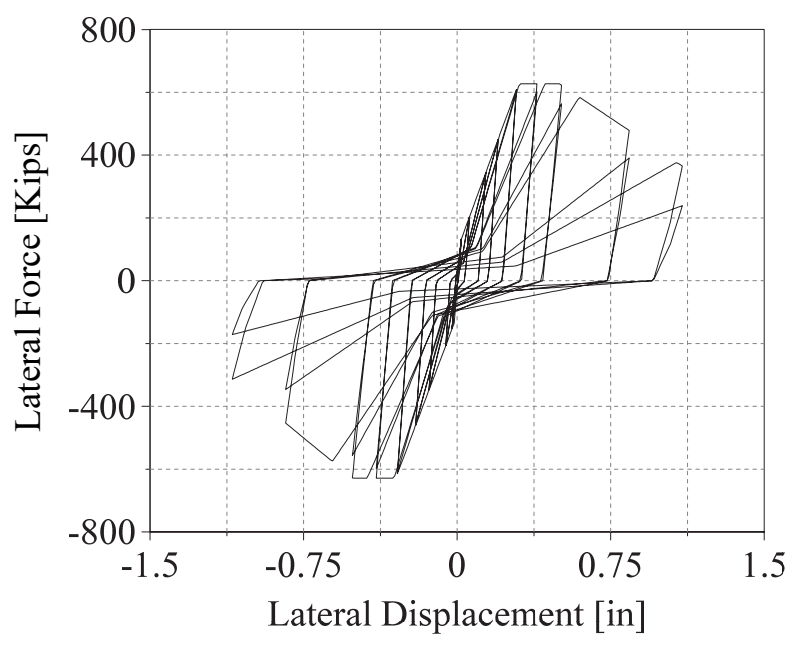

(b)

Figure D-39 Comparison of (a) test data, (b) MIKP prediction, and (c) proposed-model prediction for Luna et al. (2015) Specimen SW9. 


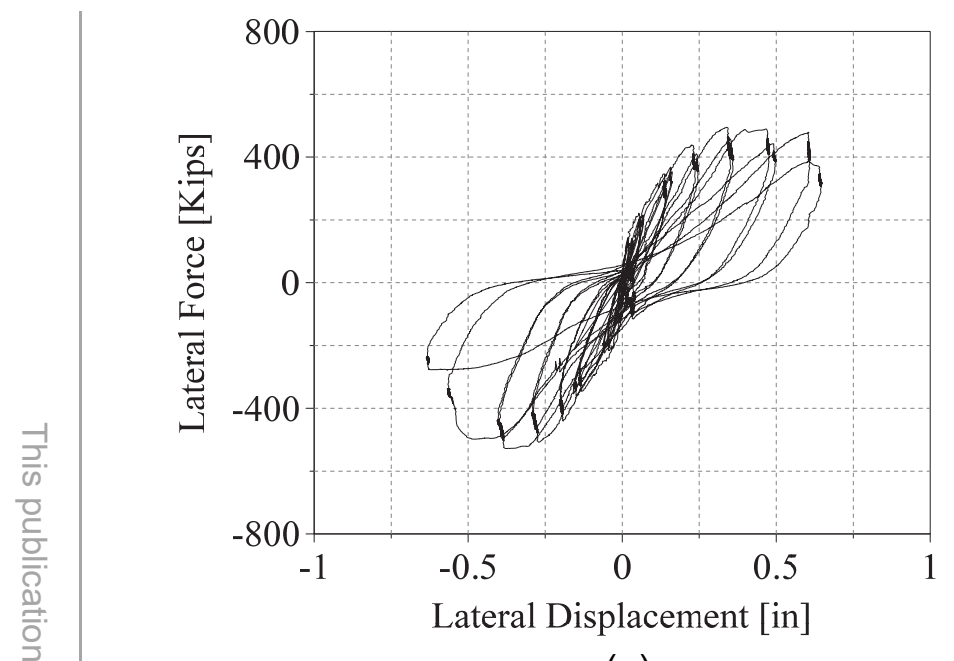

(a)

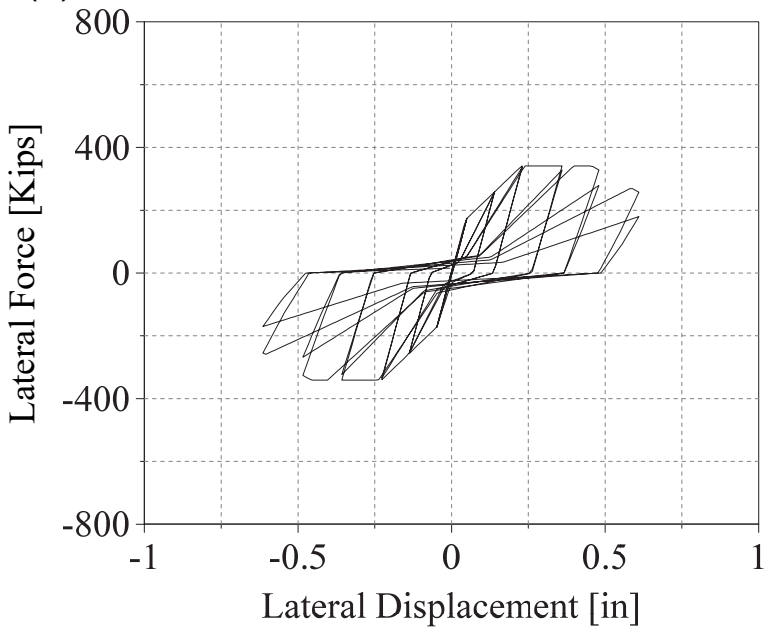

(c)

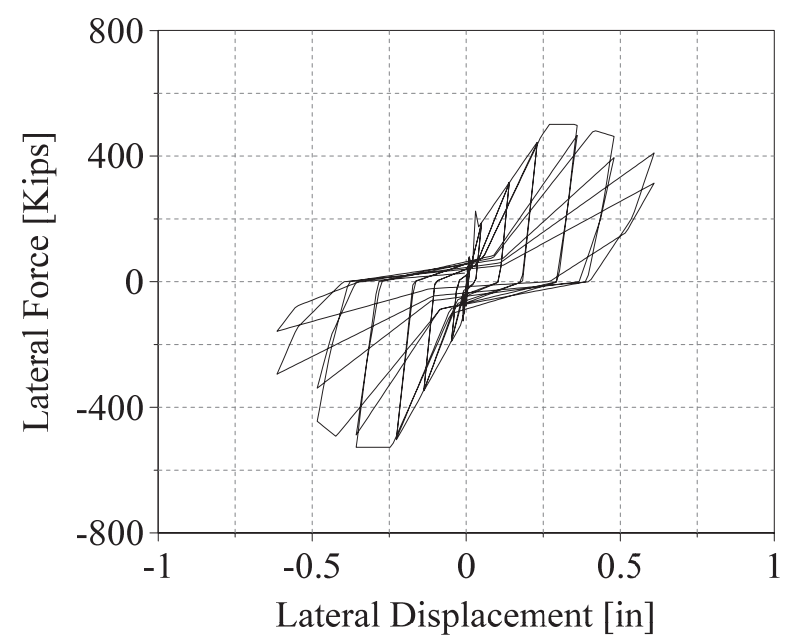

(b)

Figure D-40 Comparison of (a) test data, (b) MIKP prediction, and (c) proposed-model prediction
for Luna et al. (2015) Specimen SW10. 


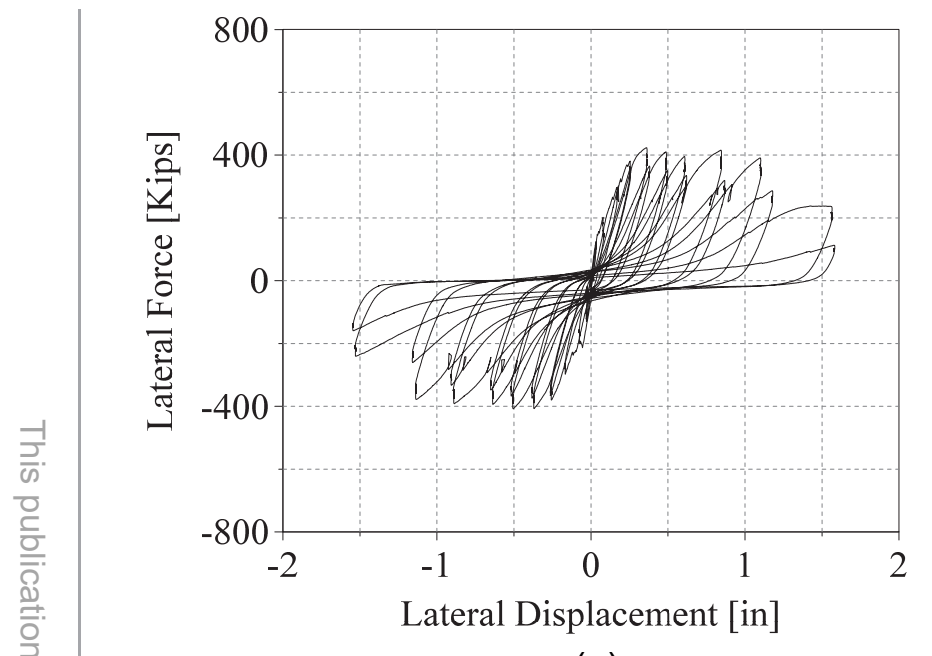

(a)

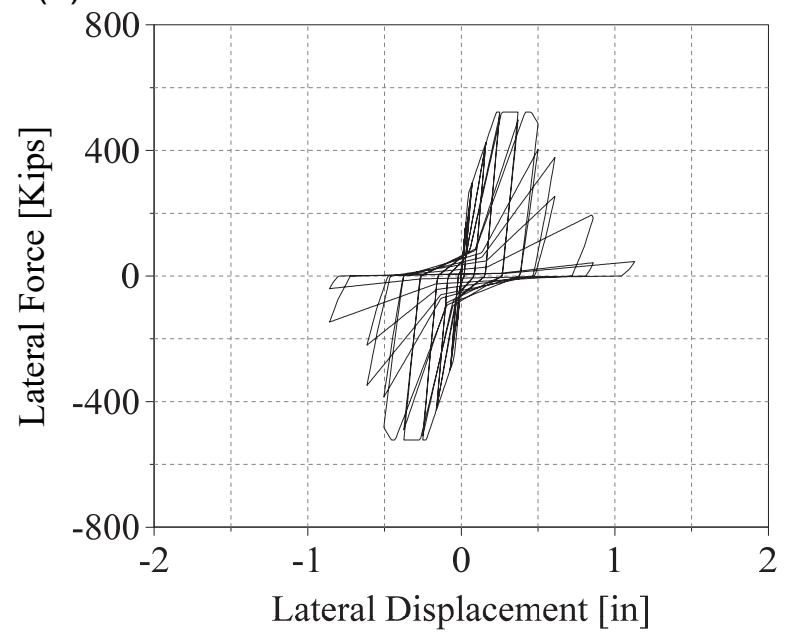

(c)

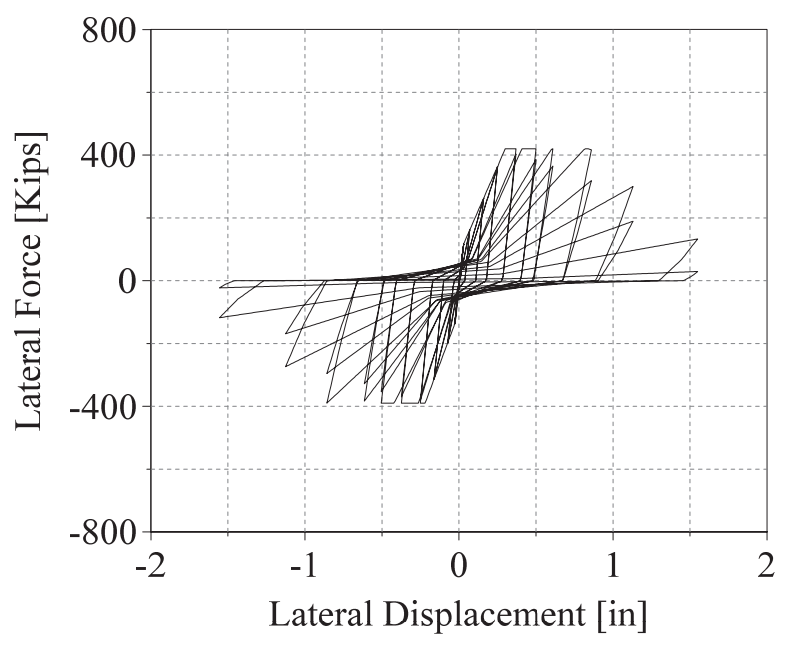

(b)

Figure D-41 Comparison of (a) test data, (b) MIKP prediction, and (c) proposed-model prediction for Luna et al. (2015) Specimen SW11. 


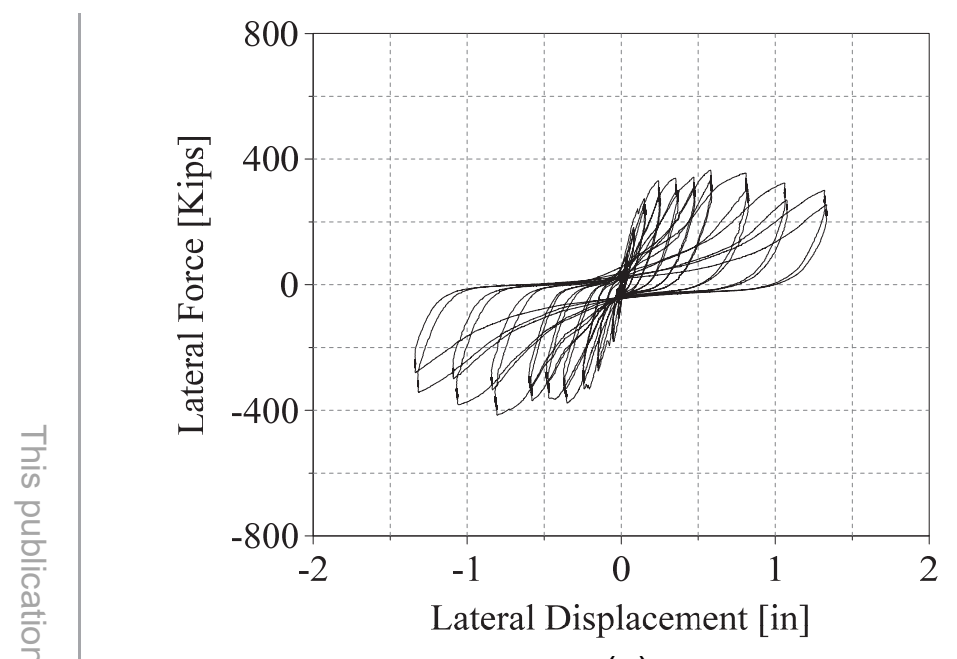

(a)

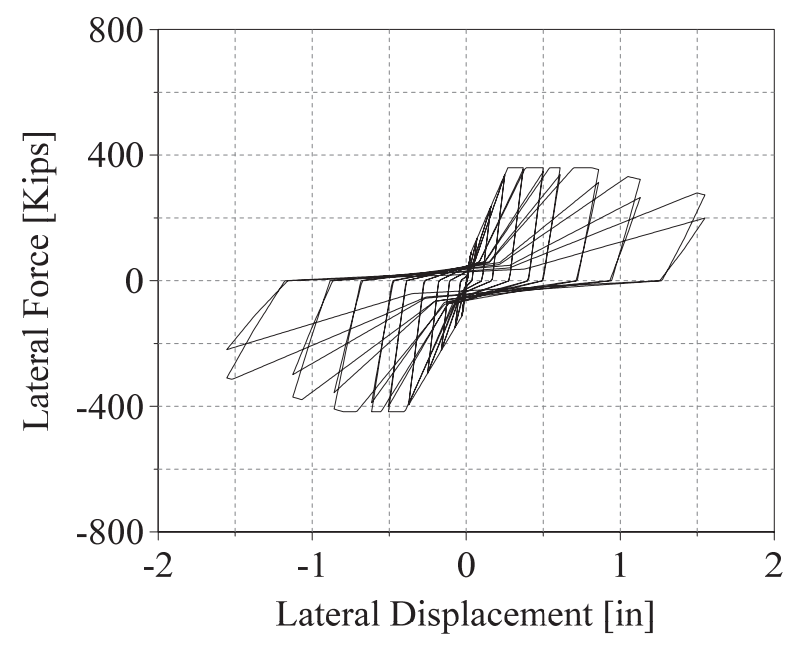

(b)

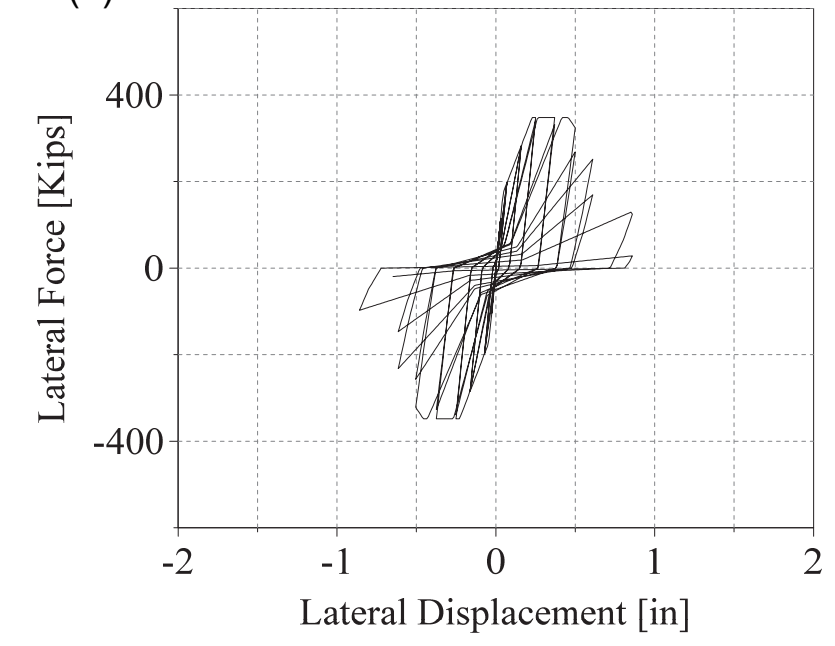

(c)

Figure D-42 Comparison of (a) test data, (b) MIKP prediction, and (c) proposed-model prediction for Luna et al. (2015) Specimen SW12. 


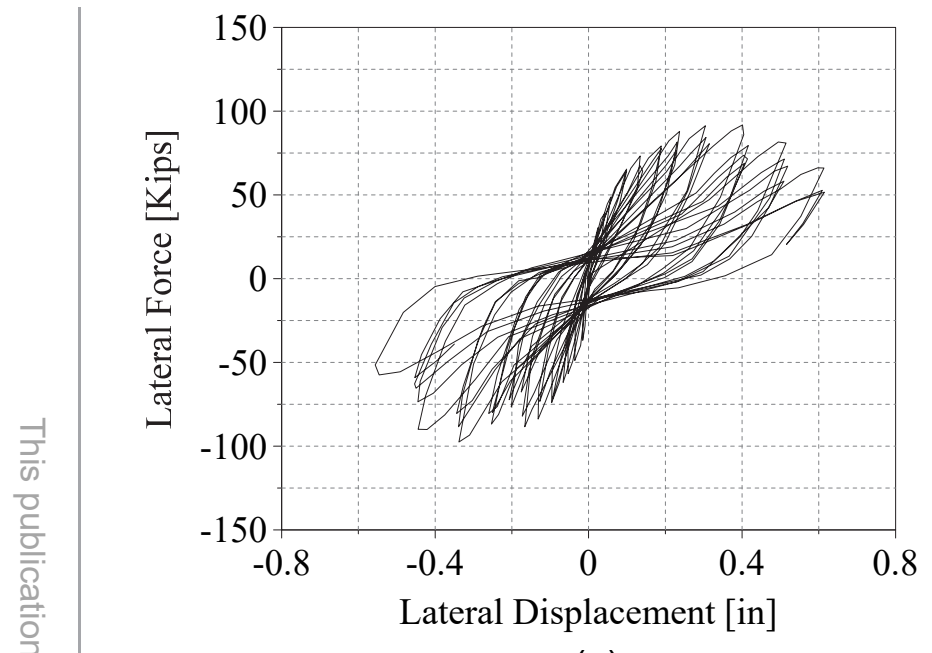

(a)

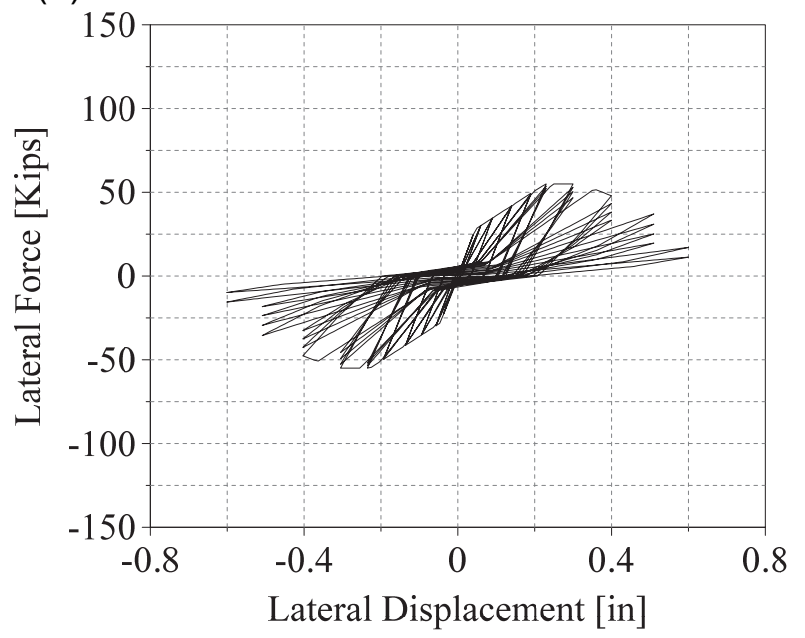

(c)

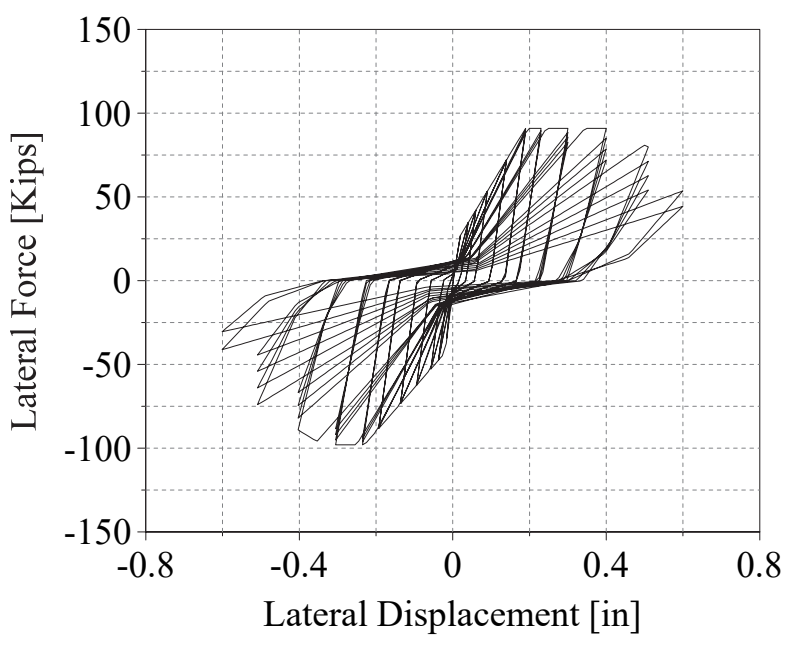

(b)

Figure D-43 Comparison of (a) test data, (b) MIKP prediction, and (c) proposed-model prediction for Mansour and Hsu (2005) Specimen W2. 


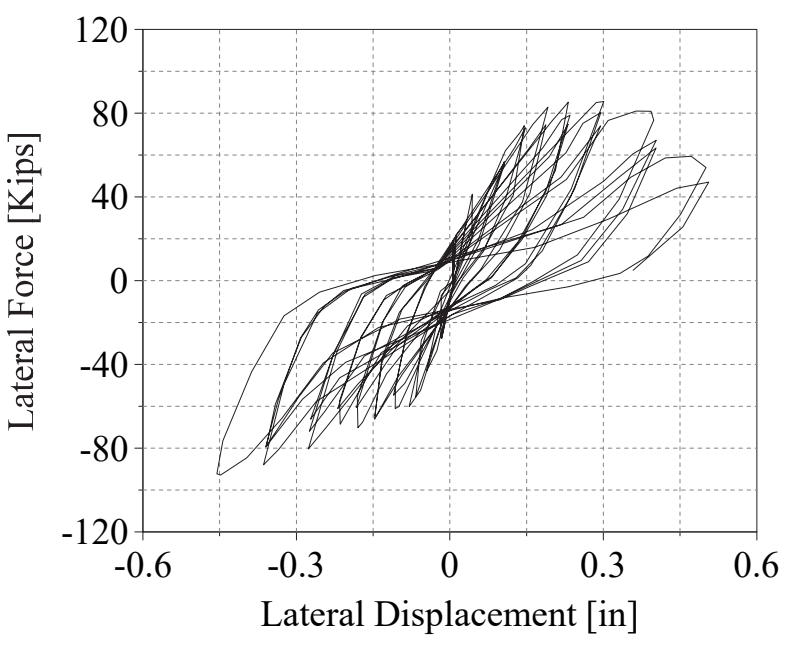

(a)

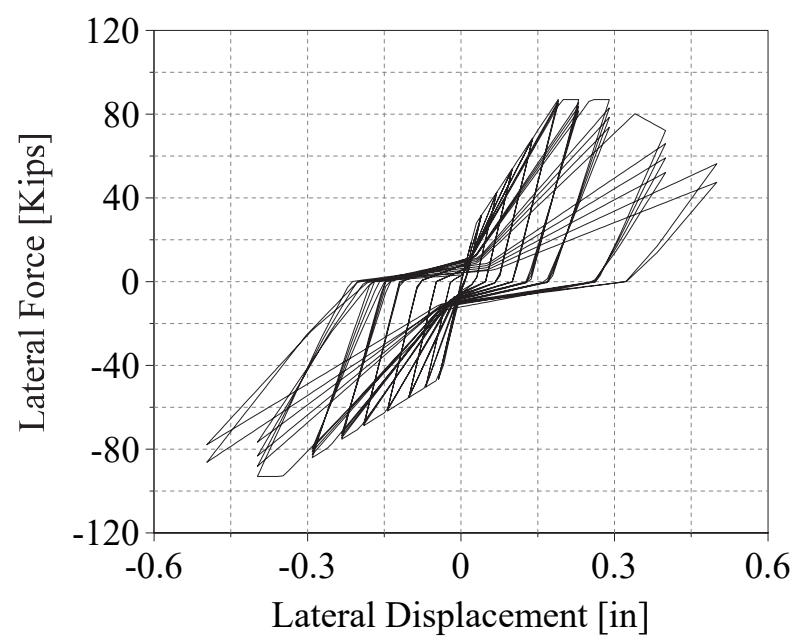

(b)

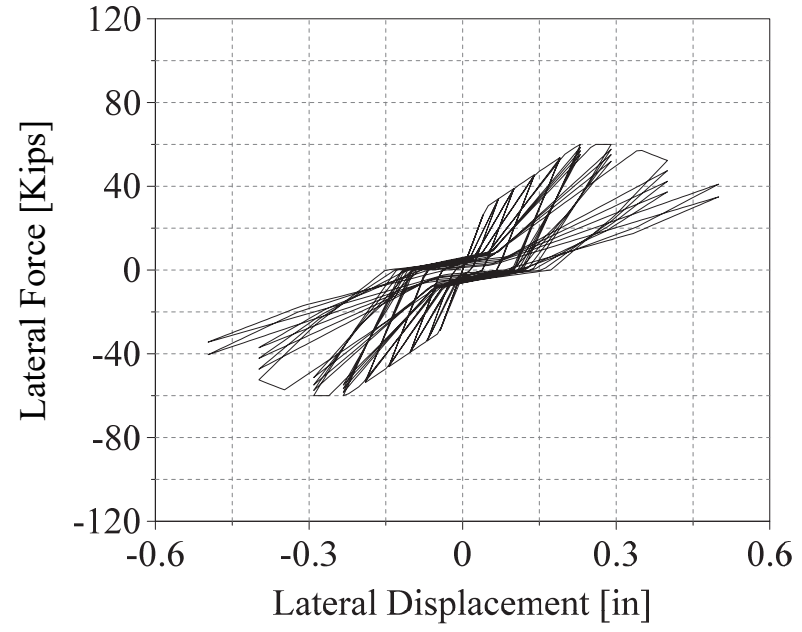

(c)

Figure D-44 Comparison of (a) test data, (b) MIKP prediction, and (c) proposed-model prediction for Mansour and Hsu (2005) Specimen W3. 


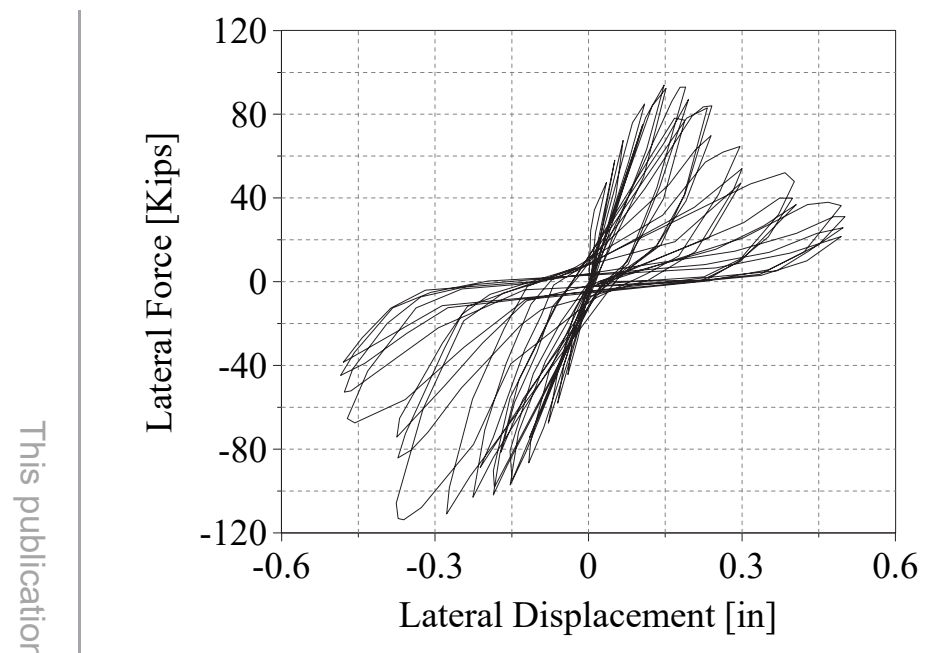

(a)

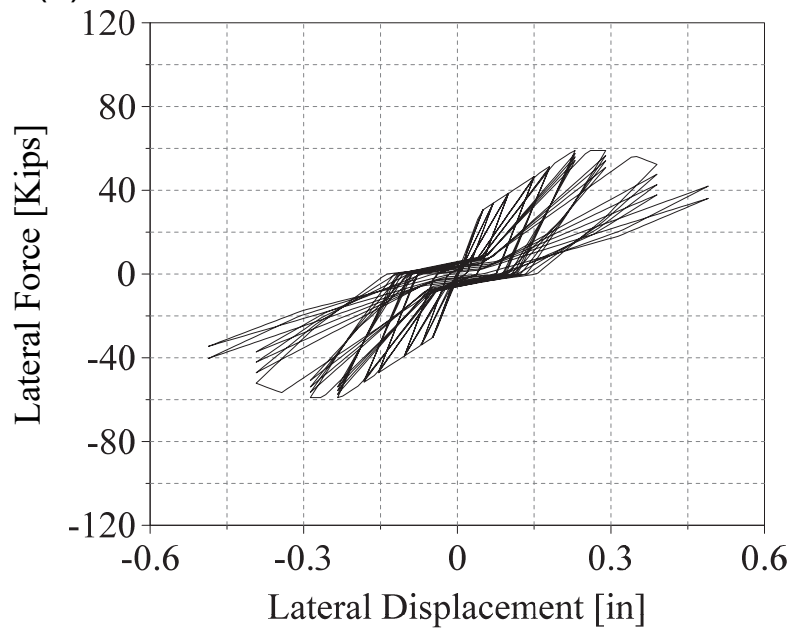

(c)

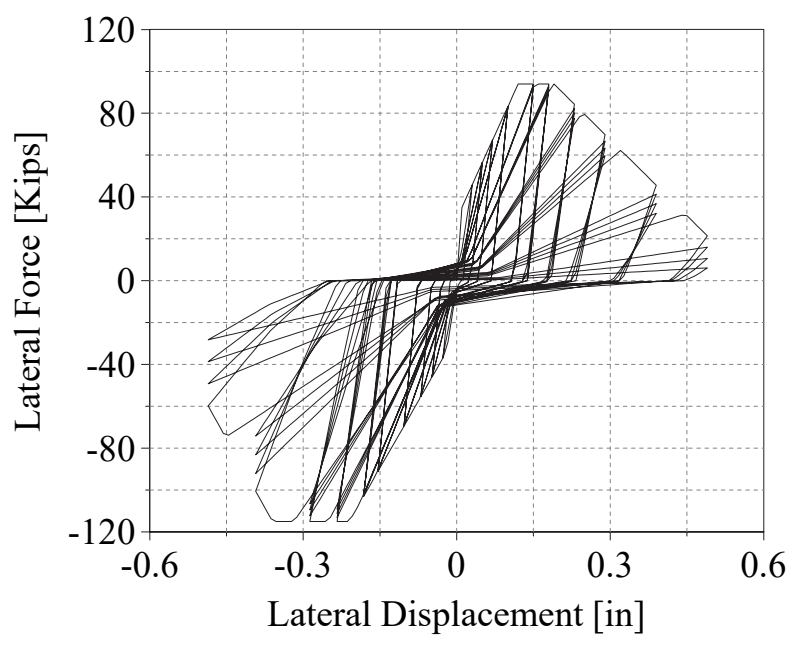

(b)

Figure D-45 Comparison of (a) test data, (b) MIKP prediction, and (c) proposed-model prediction for Mansour and Hsu (2005) Specimen W4. 


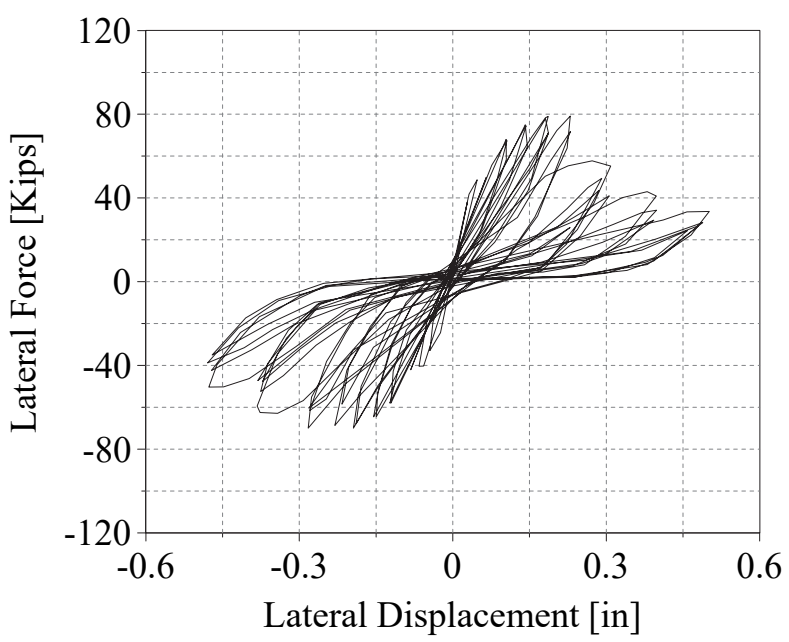

(a)

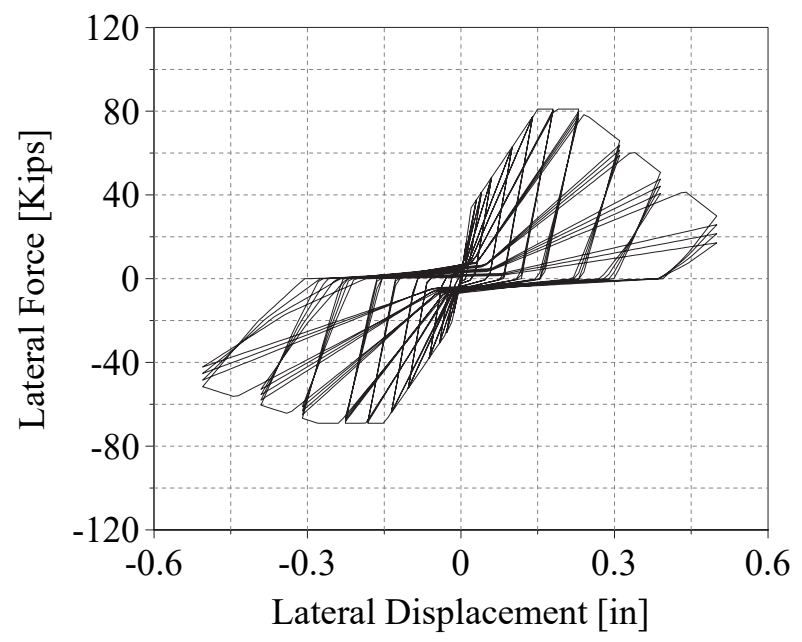

(b)

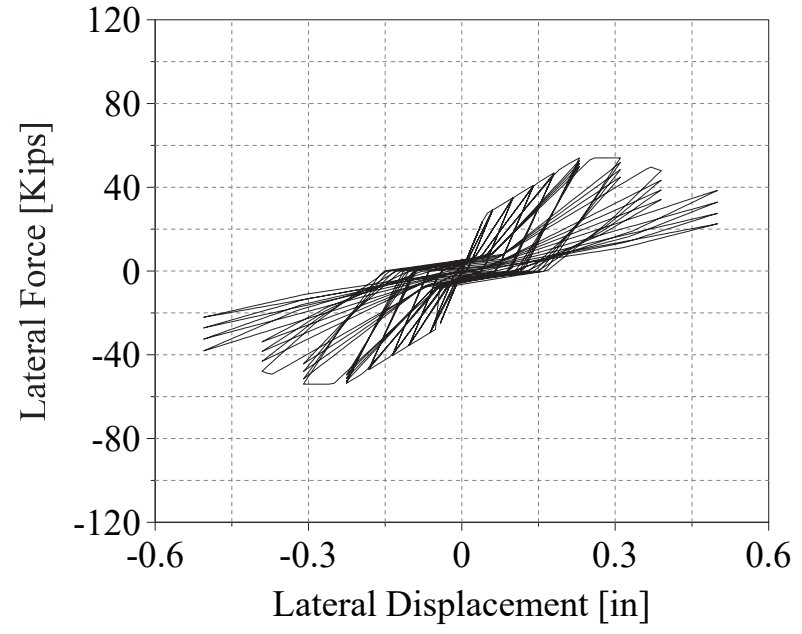

(c)

Figure D-46 Comparison of (a) test data, (b) MIKP prediction, and (c) proposed-model prediction for Mansour and Hsu (2005) Specimen W5. 


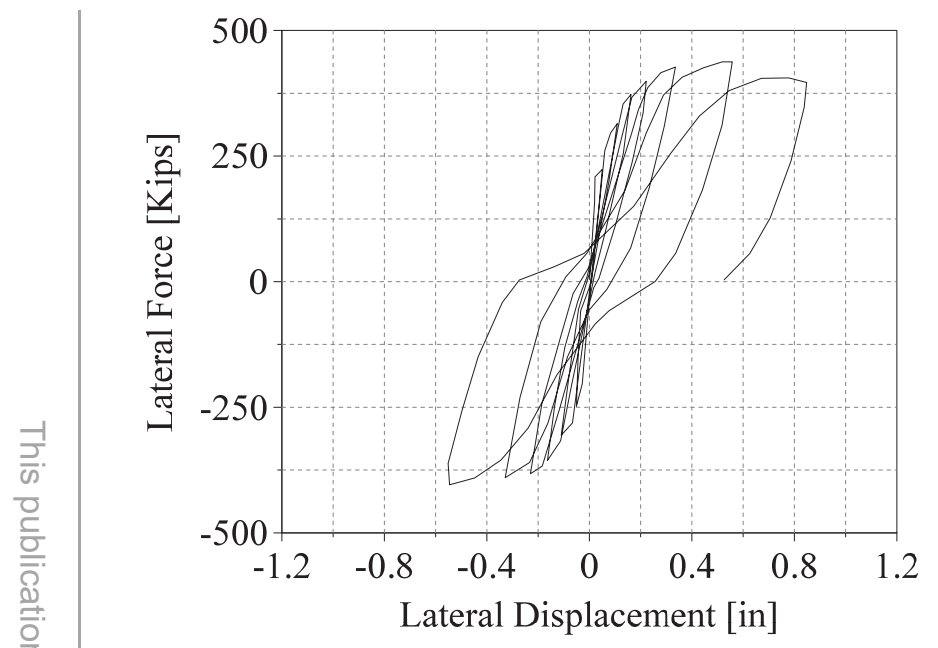

(a)

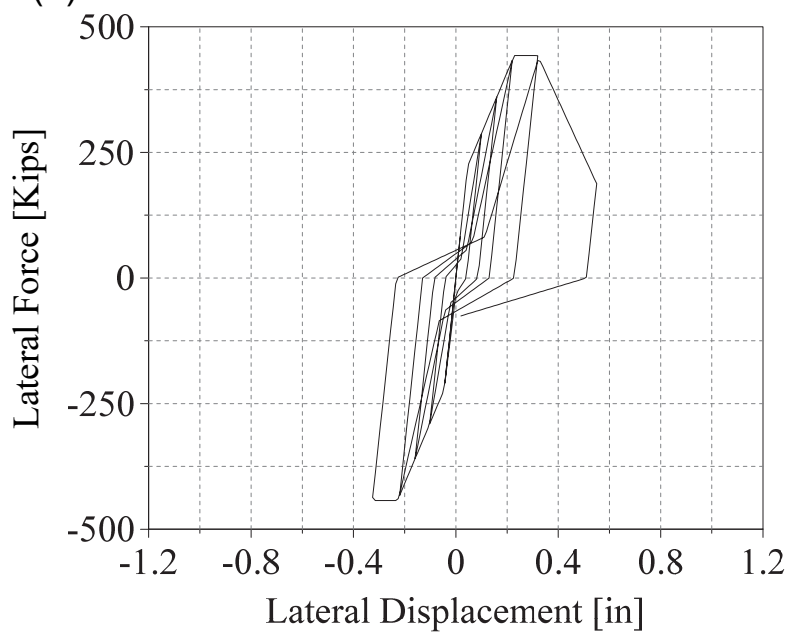

(c)

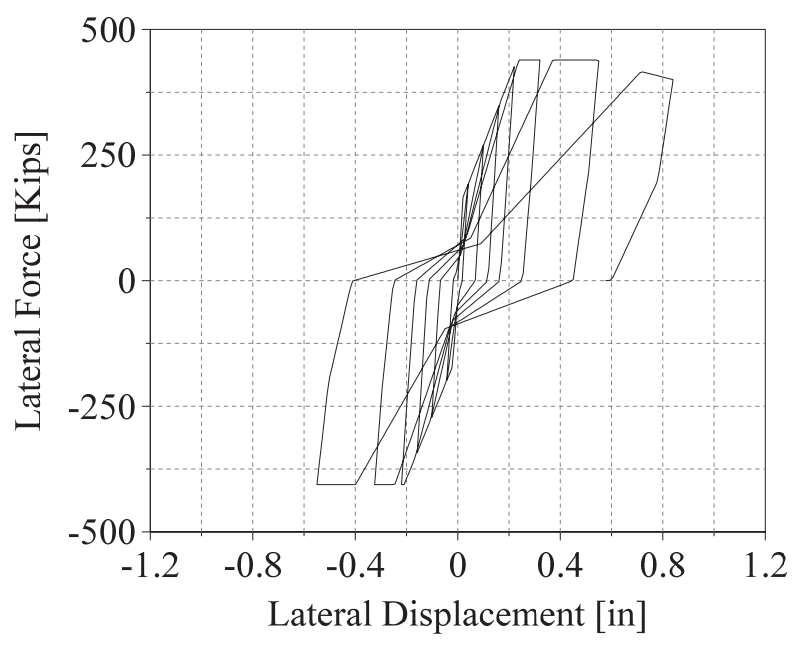

(b)

Figure D-47 Comparison of (a) test data, (b) MIKP prediction, and (c) proposed-model prediction for Sato et al. (1989) Specimen 36-M8-30. 


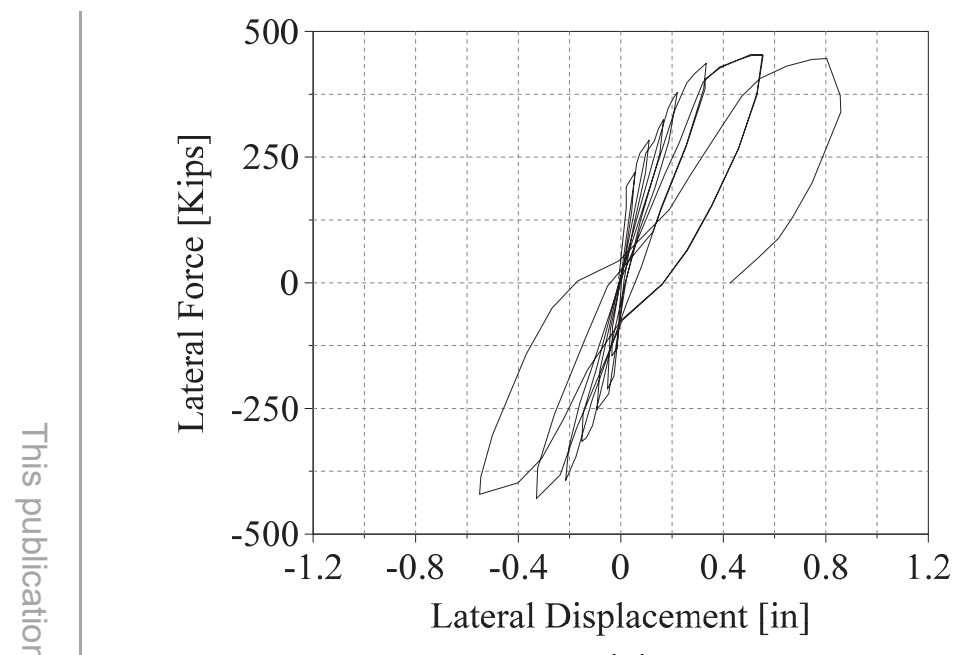

(a)

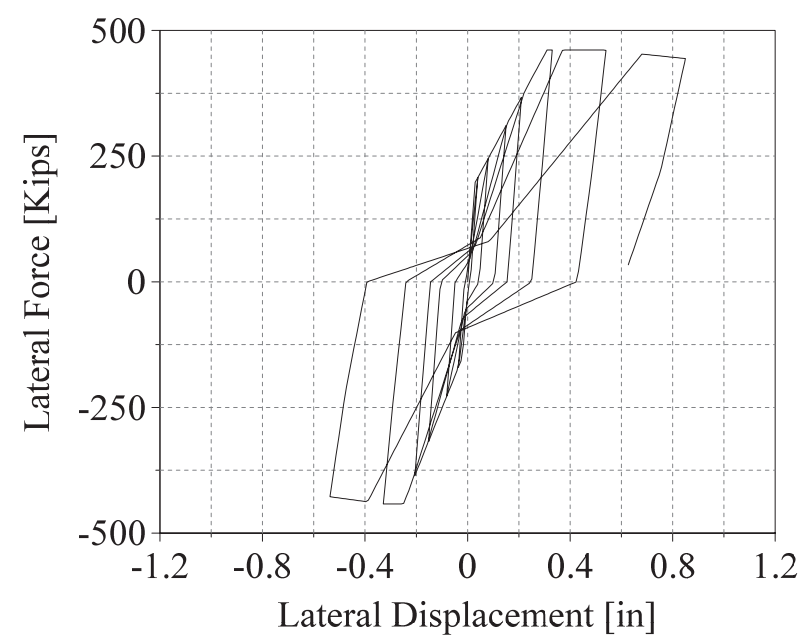

(b)

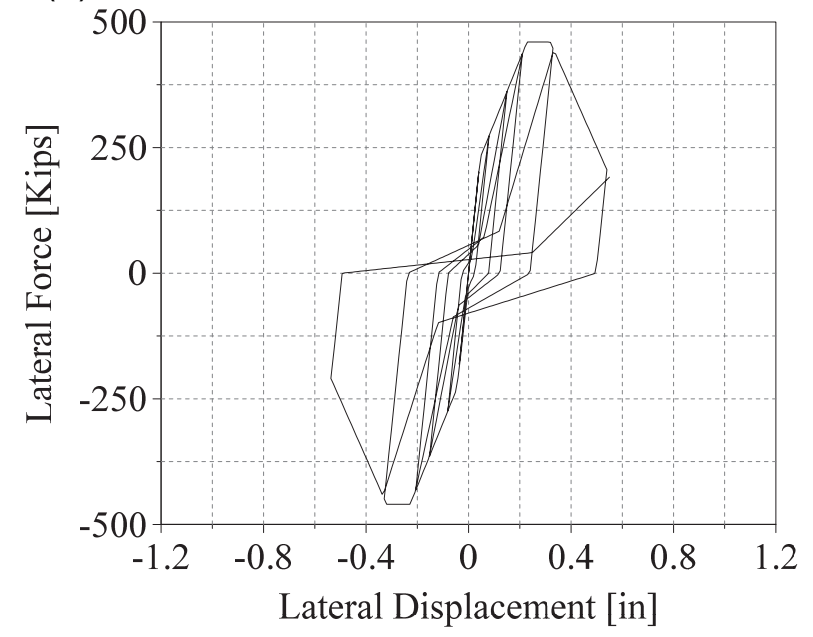

(c)

Figure D-48 Comparison of (a) test data, (b) MIKP prediction, and (c) proposed-model prediction for Sato et al. (1989) Specimen 36-M8-50. 


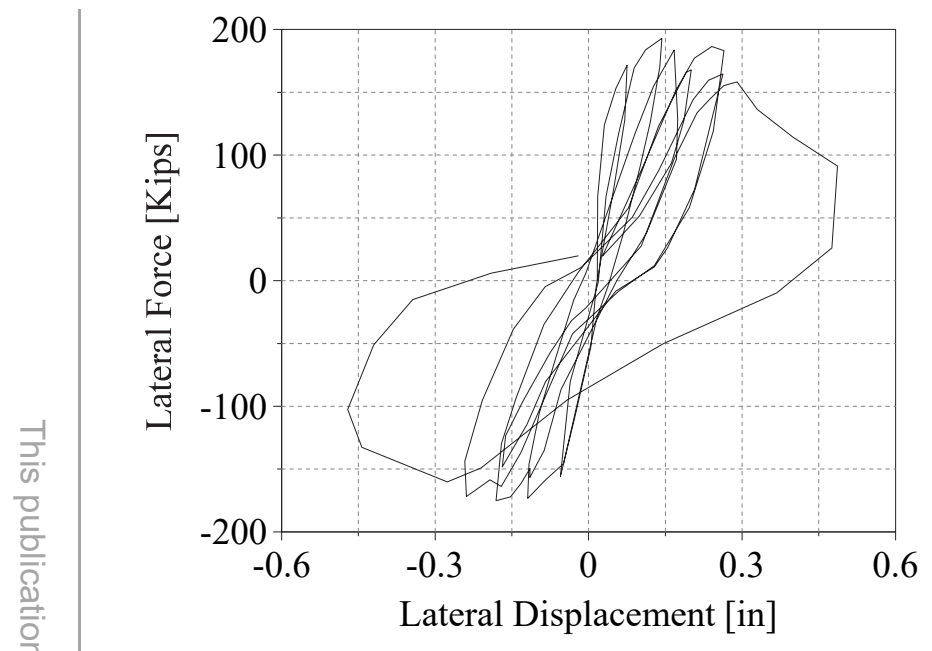

(a)

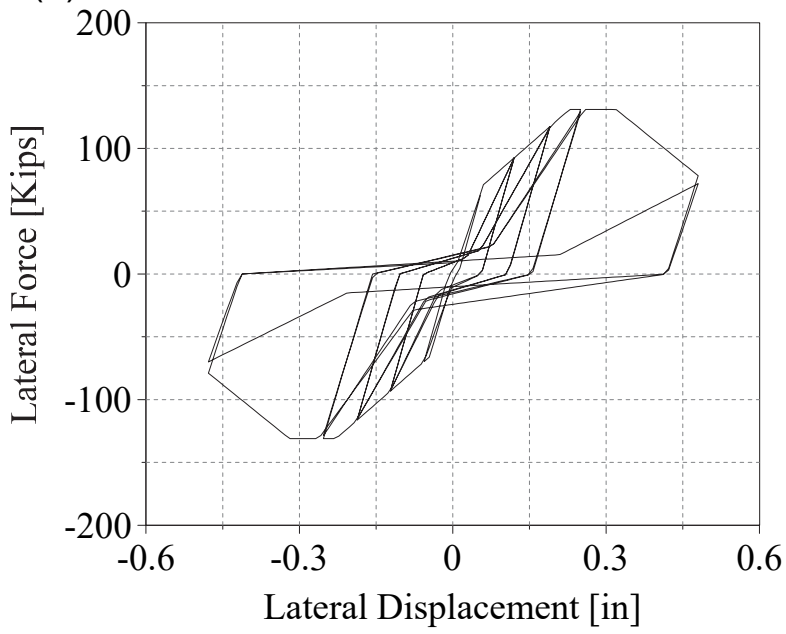

(c)

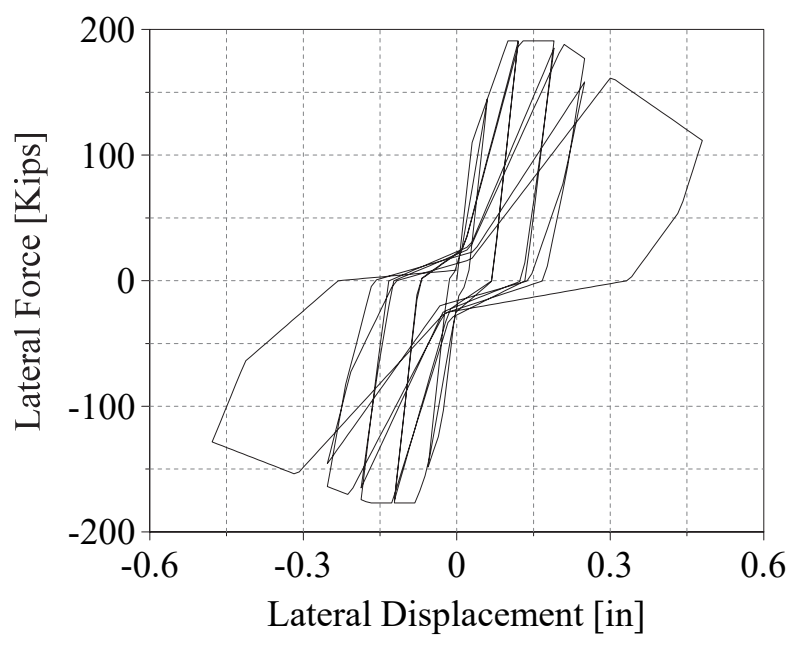

(b)

Figure D-49 Comparison of (a) test data, (b) MIKP prediction, and (c) proposed-model prediction for Tomii et al. (1988) Specimen H2-W87.5-N50. 


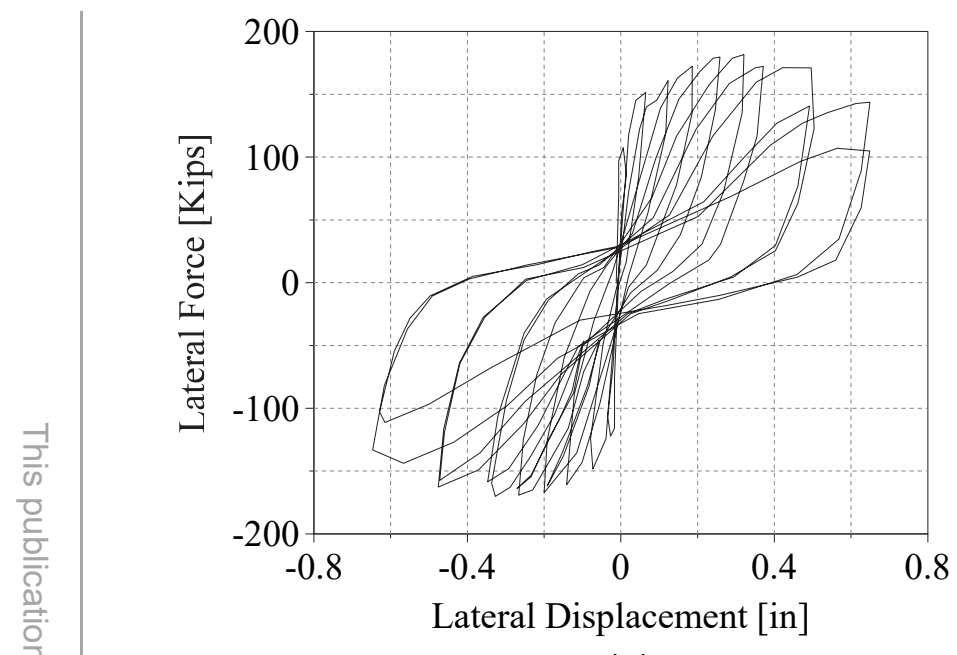

(a)

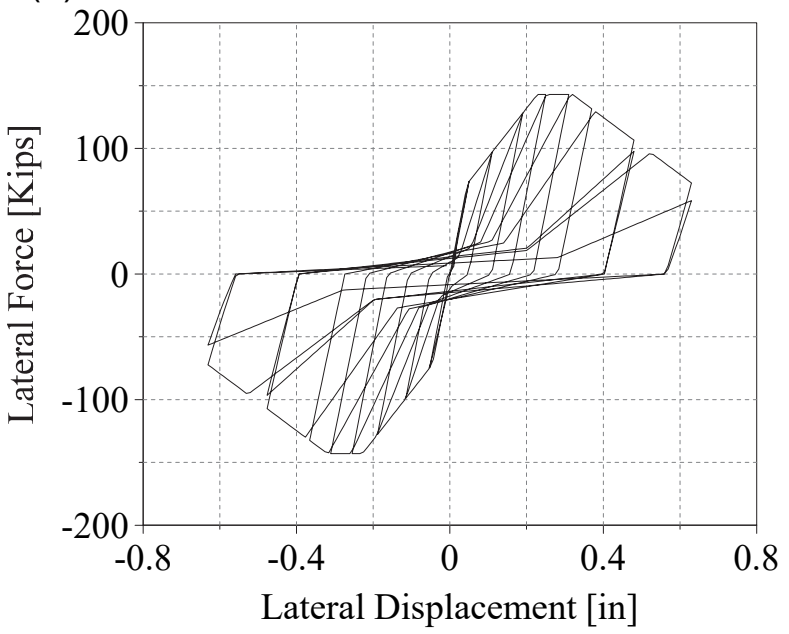

(c)

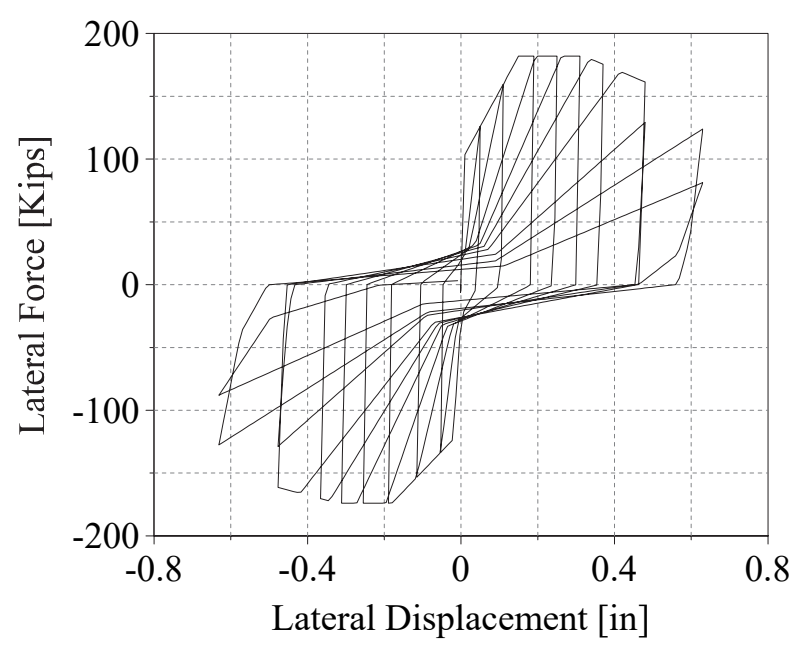

(b)

$\begin{array}{ll}\text { Figure D-50 } & \text { Comparison of (a) test data, (b) MIKP prediction, and (c) proposed-model prediction } \\ \text { for Tomii et al. (1988) Specimen T1-W87.5-N50. }\end{array}$ 


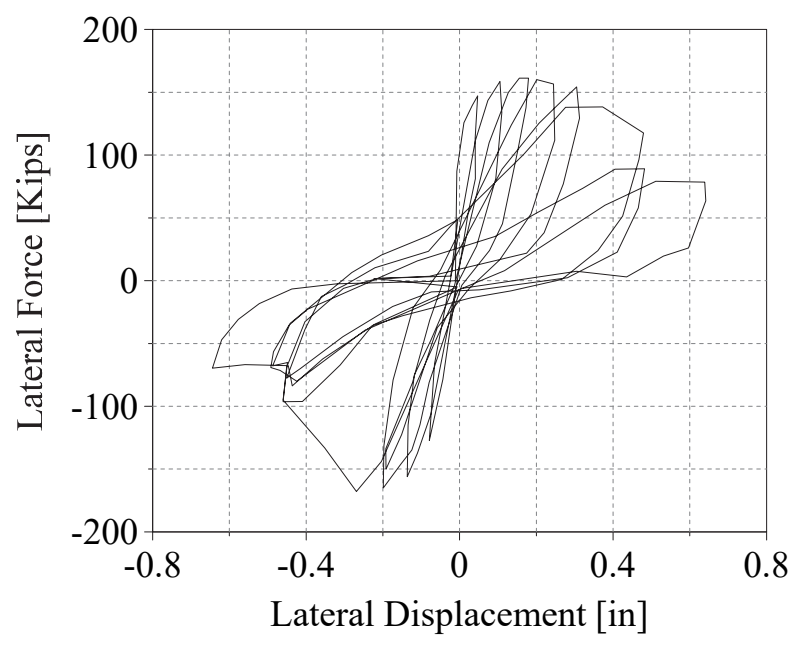

(a)

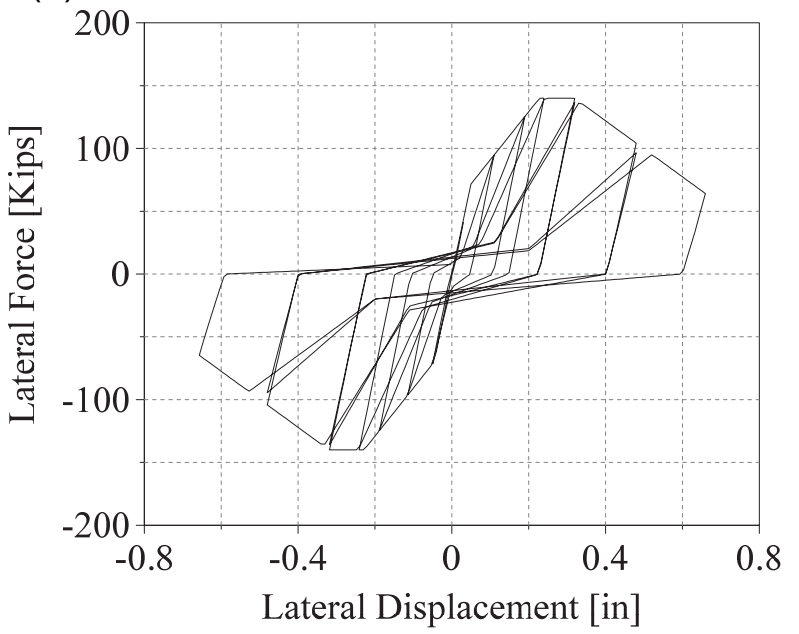

(c)

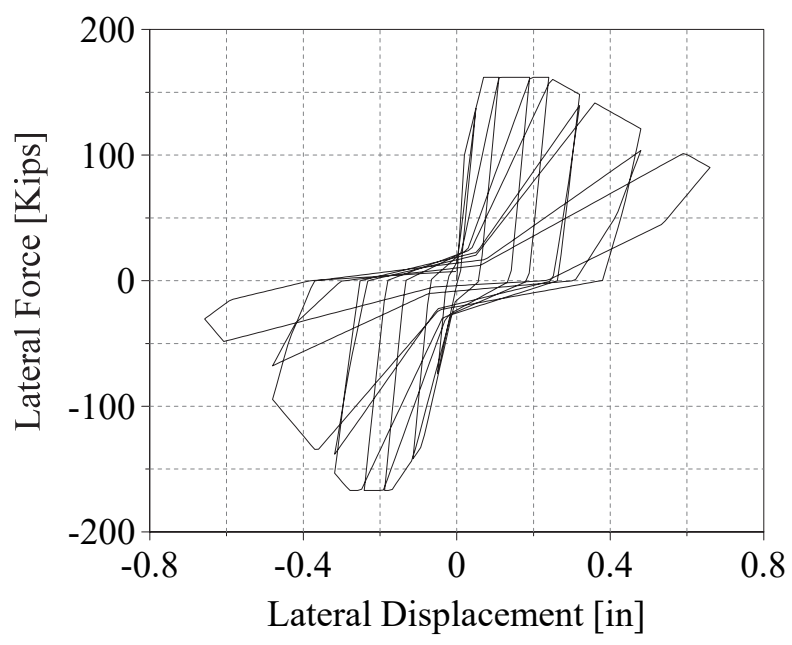

(b)

Figure D-51 Comparison of (a) test data, (b) MIKP prediction, and (c) proposed-model prediction for Tomii et al. (1988) Specimen T2-W87.5-N50. 


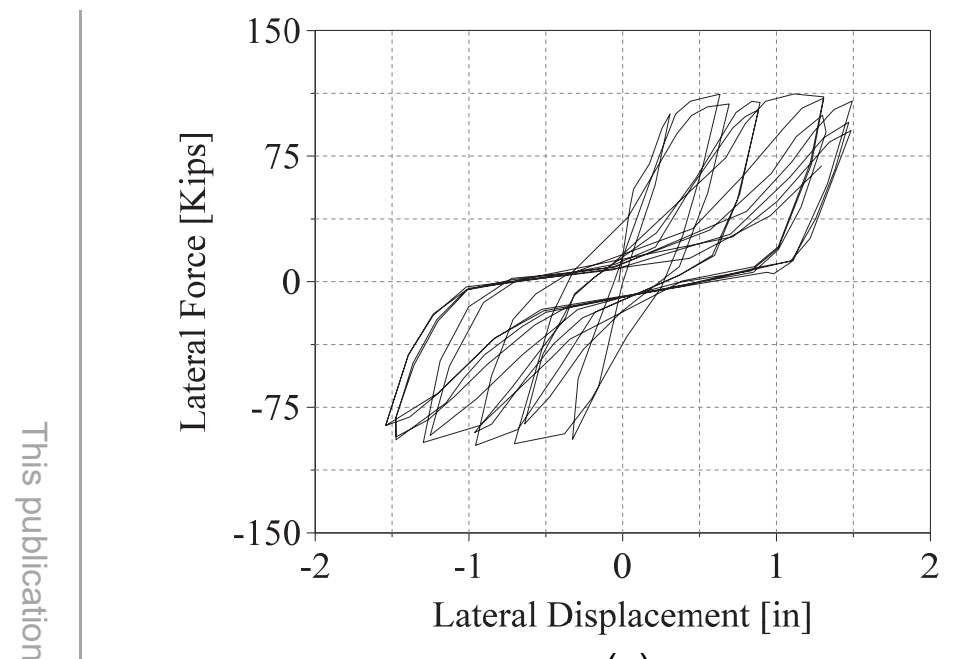

(a)

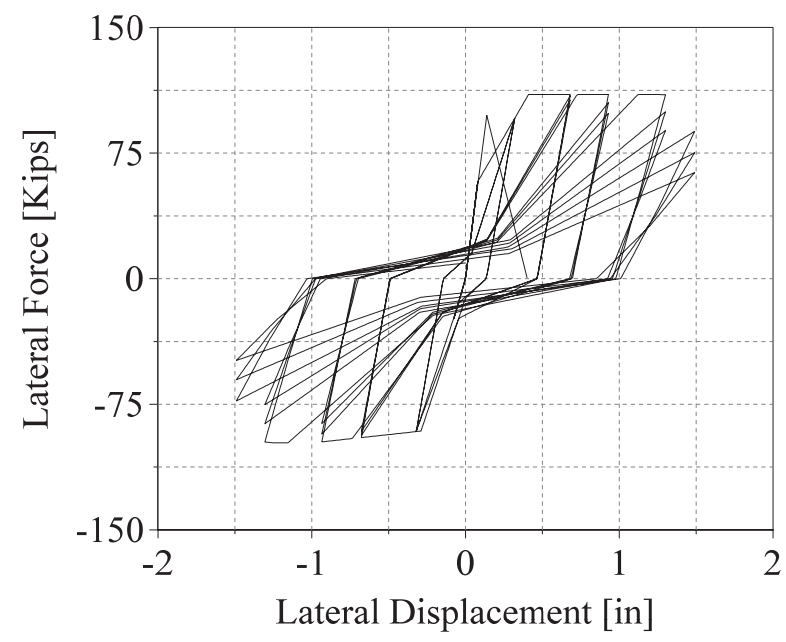

(b)

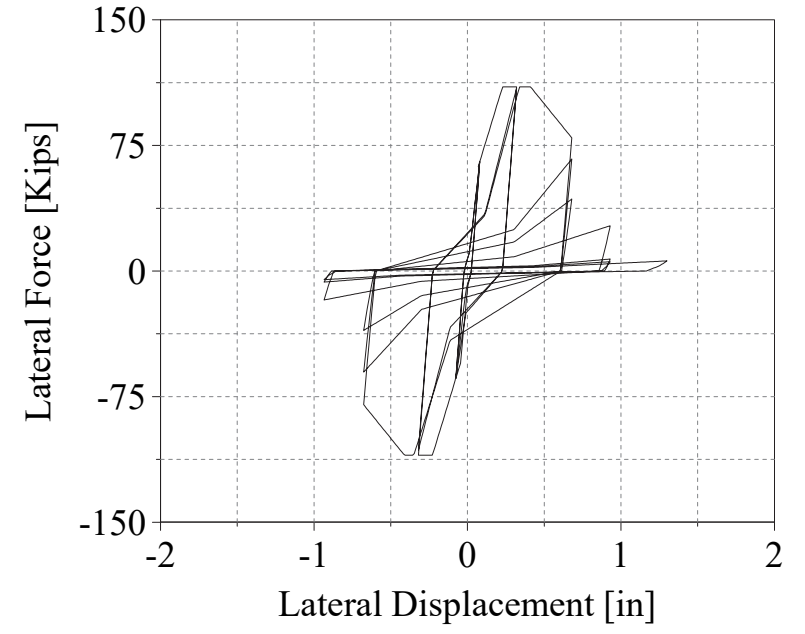

(c)

Figure D-52 Comparison of (a) test data, (b) MIKP prediction, and (c) proposed-model prediction for Sittupunt et al. (2001) Specimen WALL1. 


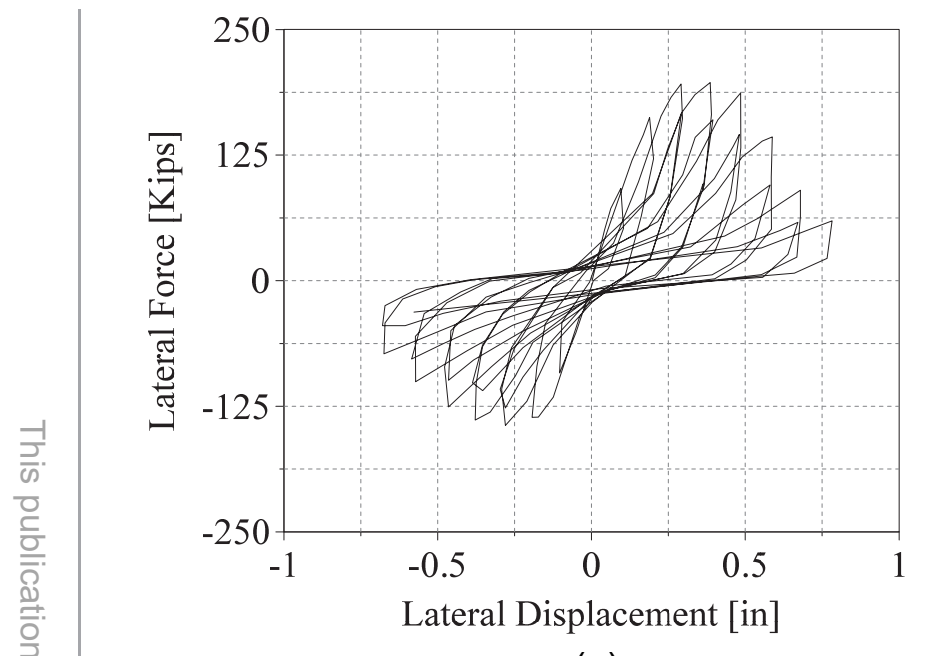

(a)

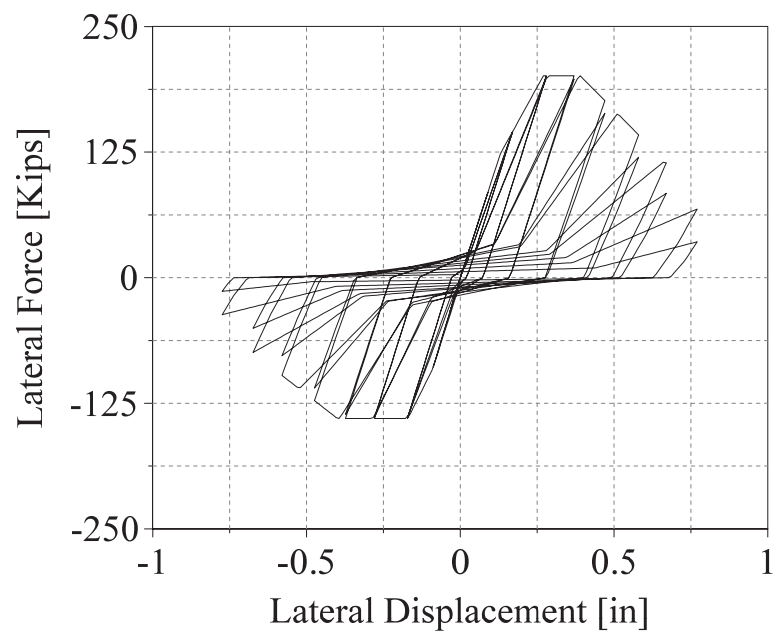

(b)

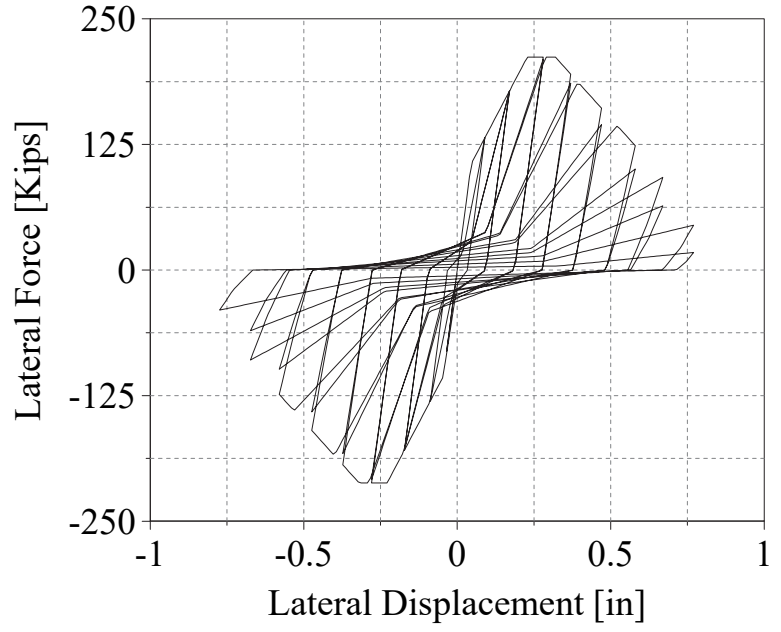

(c)

Figure D-53 Comparison of (a) test data, (b) MIKP prediction, and (c) proposed-model prediction for Effendy et al. (2006) Specimen SMAC. 


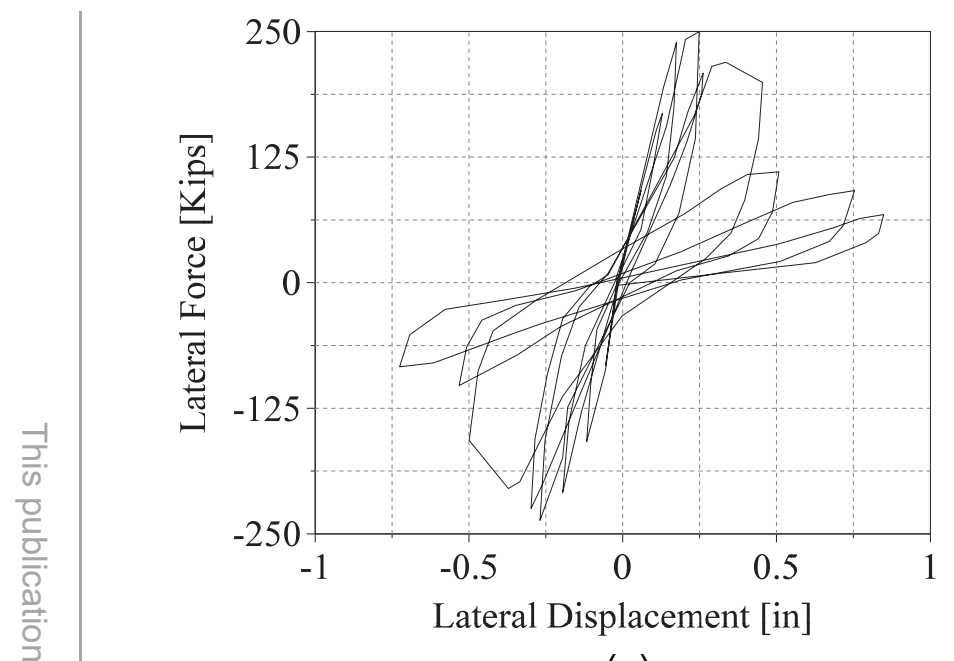

(a)

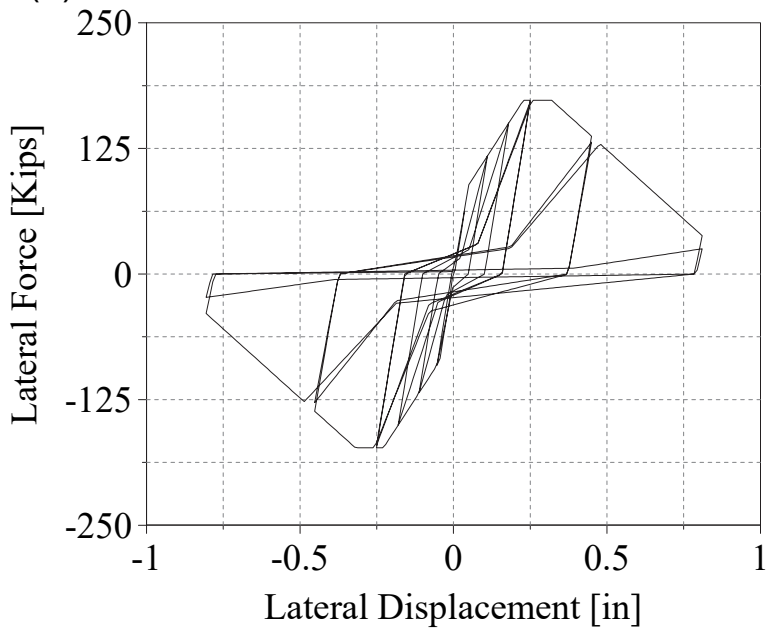

(c)

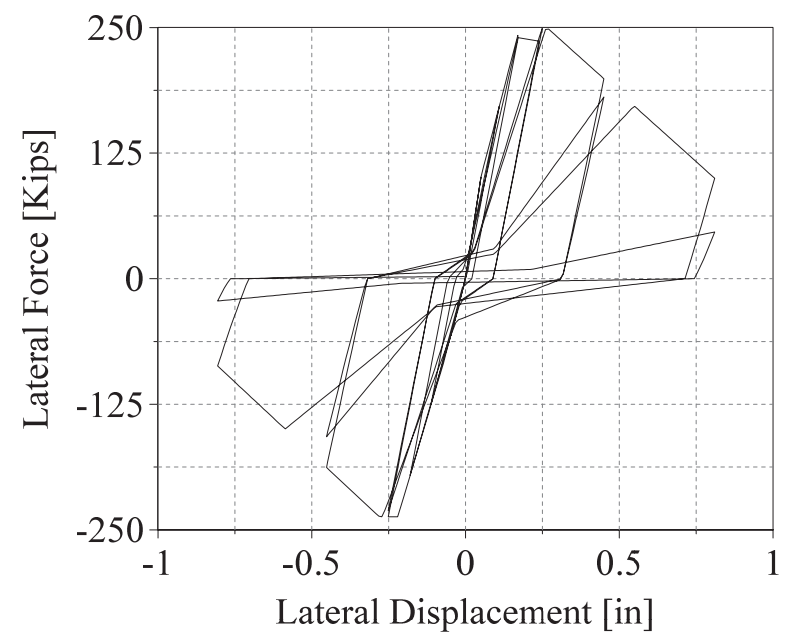

(b)

Figure D-54 Comparison of (a) test data, (b) MIKP prediction, and (c) proposed-model prediction for Effendy et al. (2006) Specimen SMAS. 


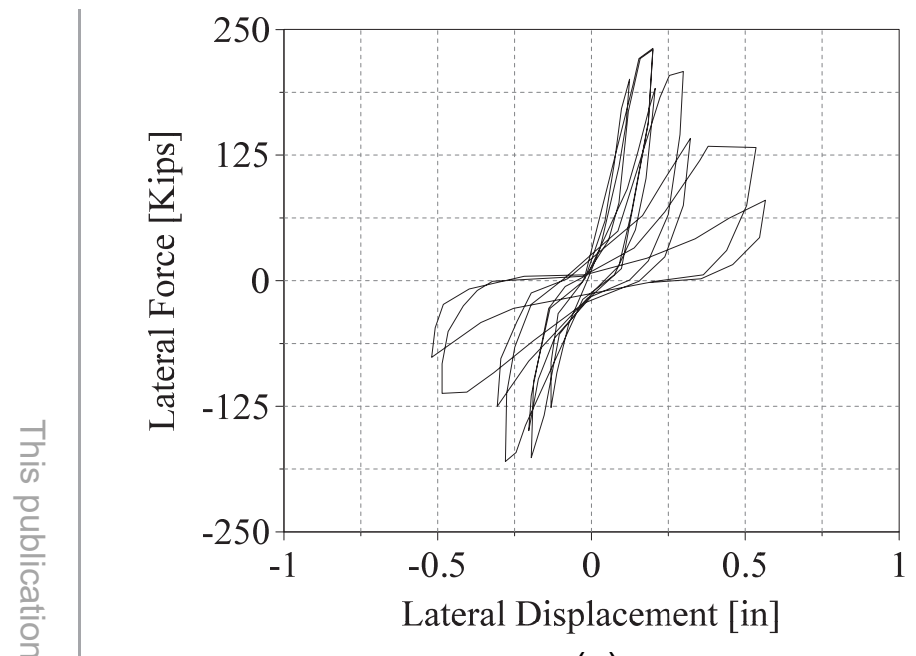

(a)

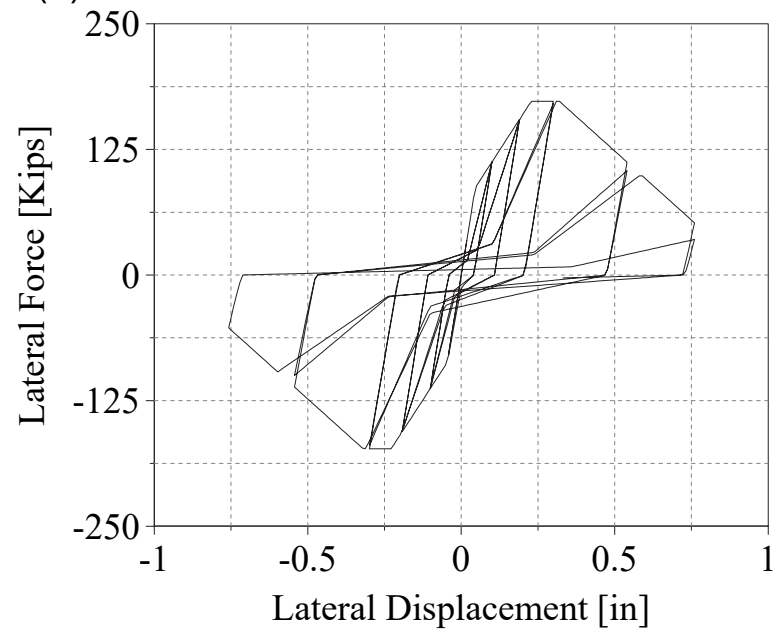

(c)

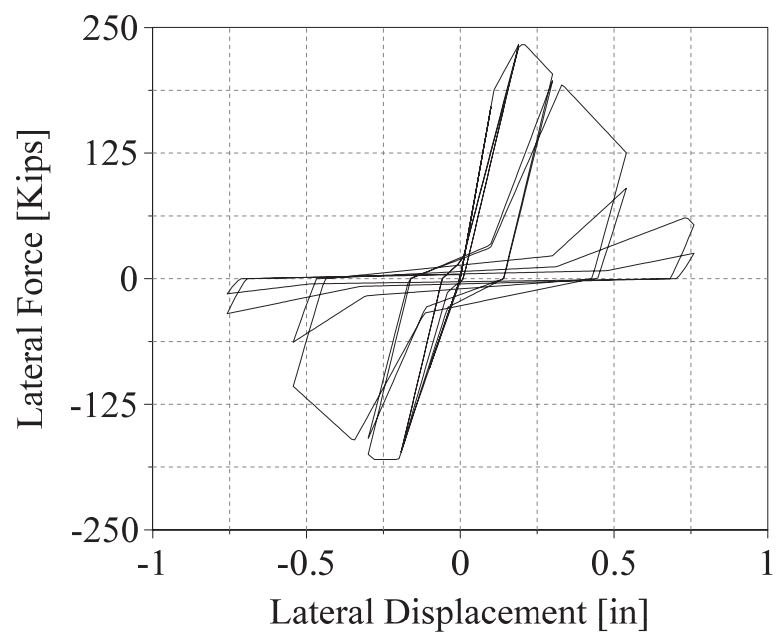

(b)

Figure D-55 Comparison of (a) test data, (b) MIKP prediction, and (c) proposed-model prediction for Effendy et al. (2006) Specimen SMAM. 


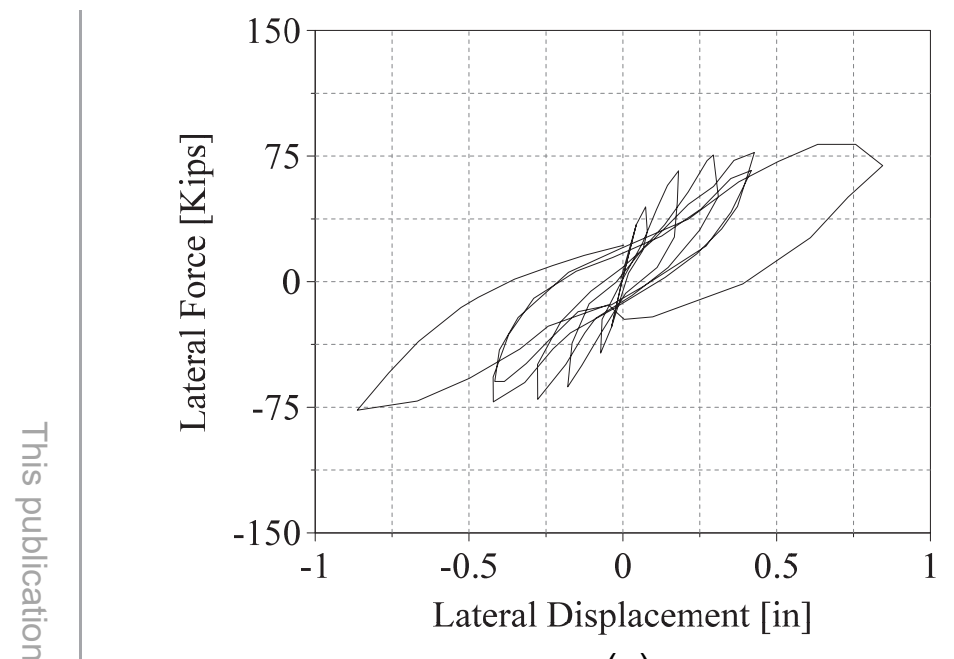

(a)

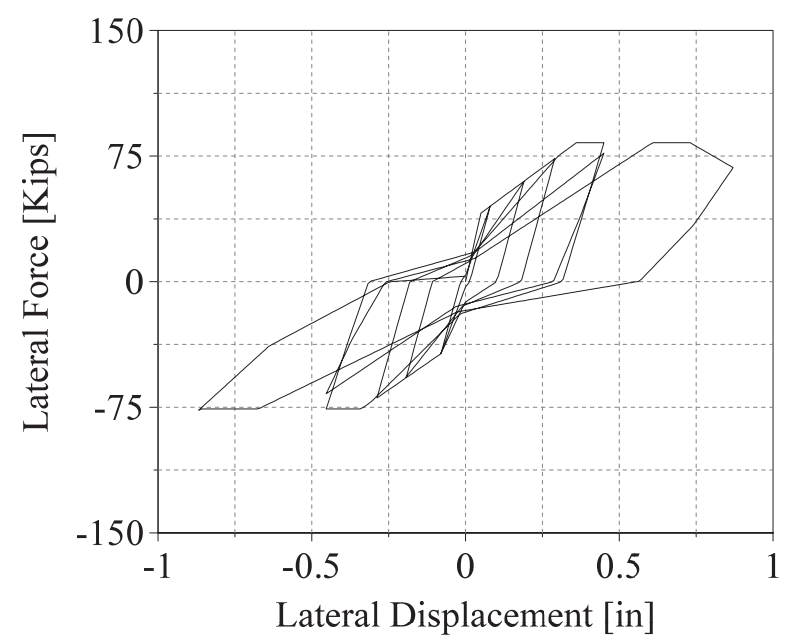

(b)

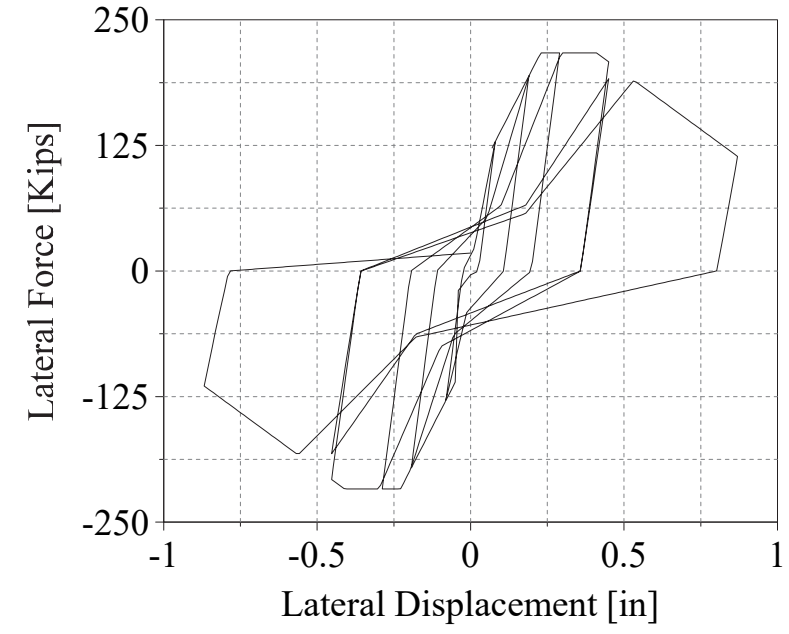

(c)

Figure D-56 Comparison of (a) test data, (b) MIKP prediction, and (c) proposed-model prediction for Li et al. (2006) Specimen LW1. 


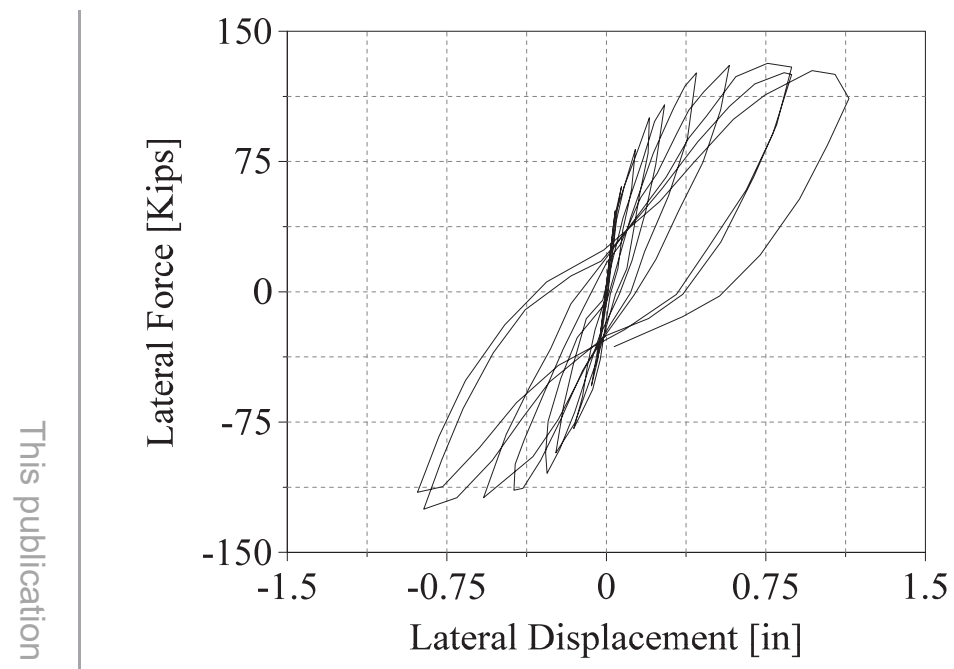

(a)

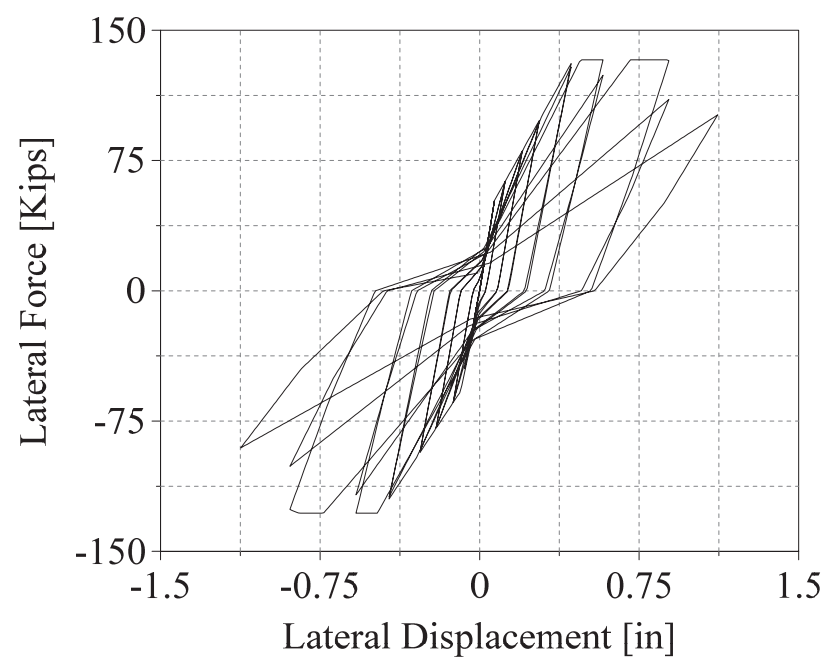

(b)

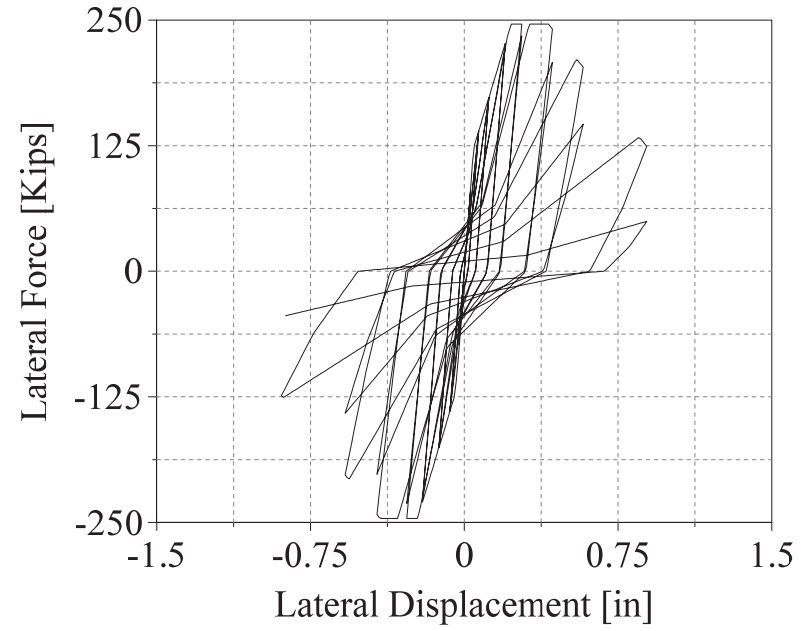

(c)

Figure D-57 Comparison of (a) test data, (b) MIKP prediction, and (c) proposed-model prediction for Li et al. (2006) Specimen LW2. 


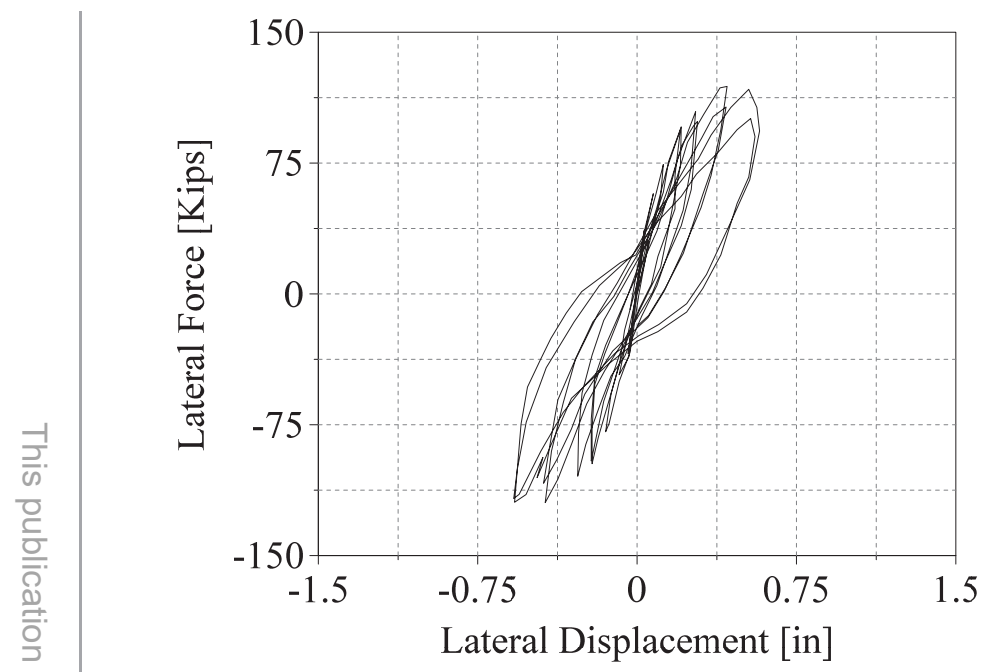

(a)

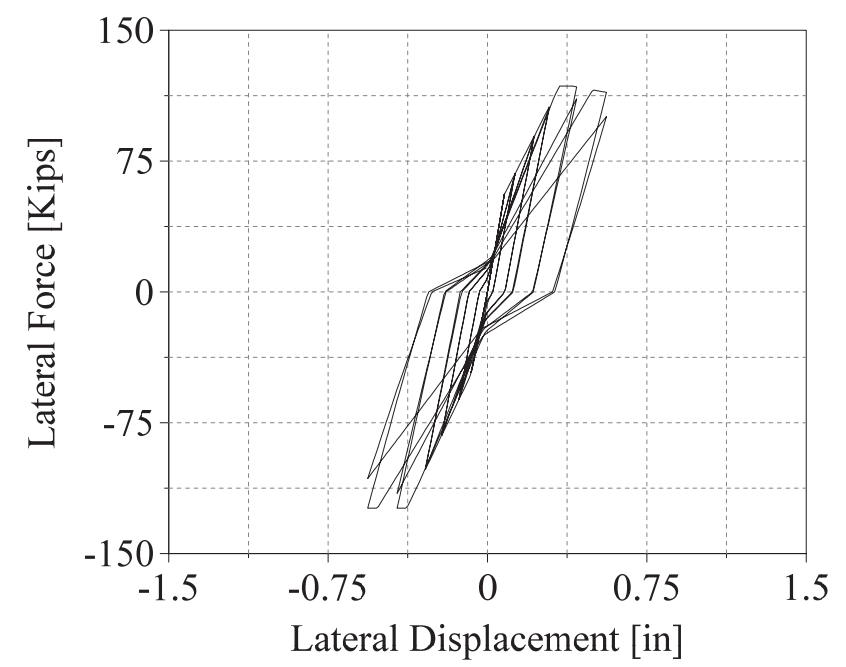

(b)

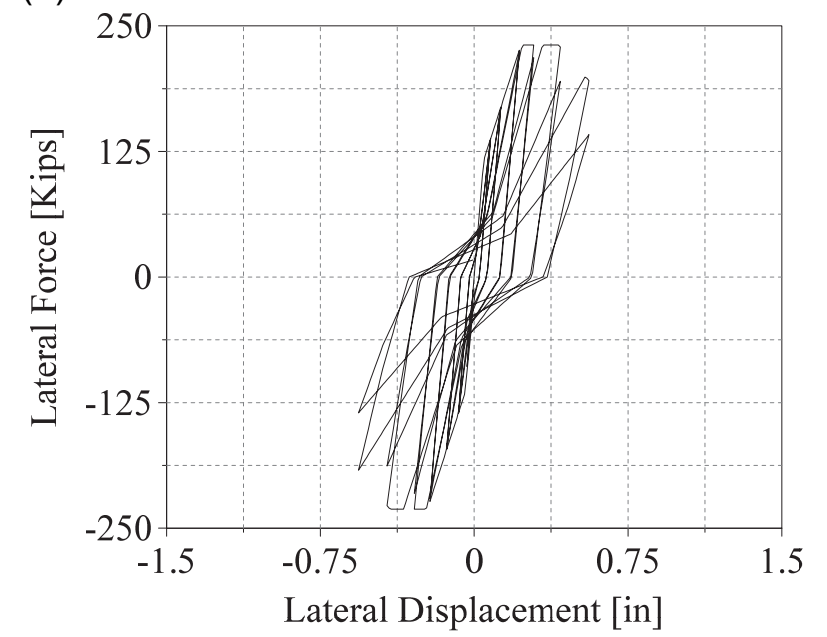

(c)

Figure D-58 Comparison of (a) test data, (b) MIKP prediction, and (c) proposed-model prediction for Li et al. (2006) Specimen LW3. 


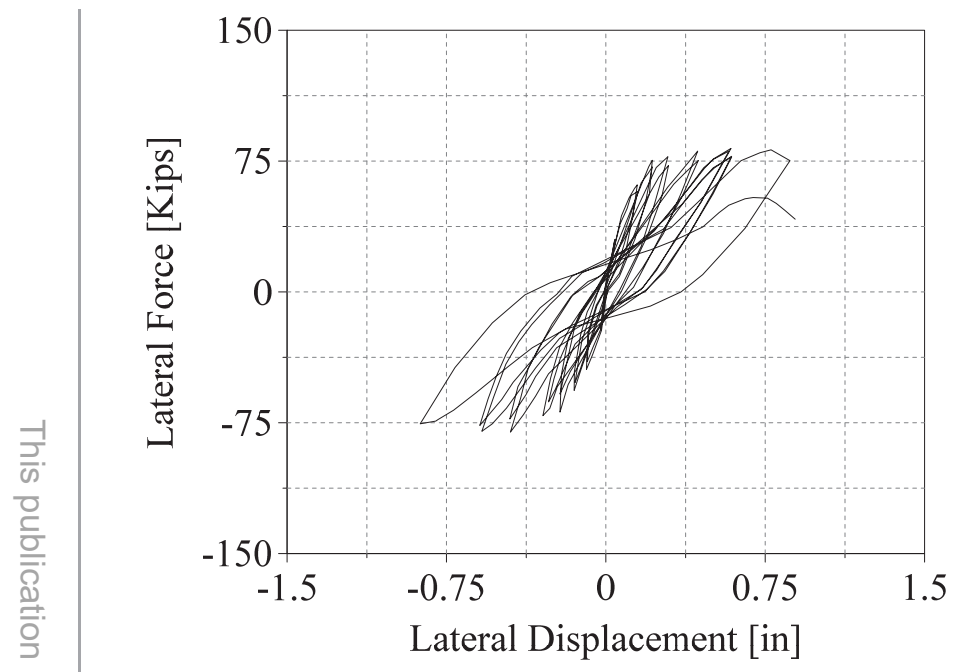

(a)

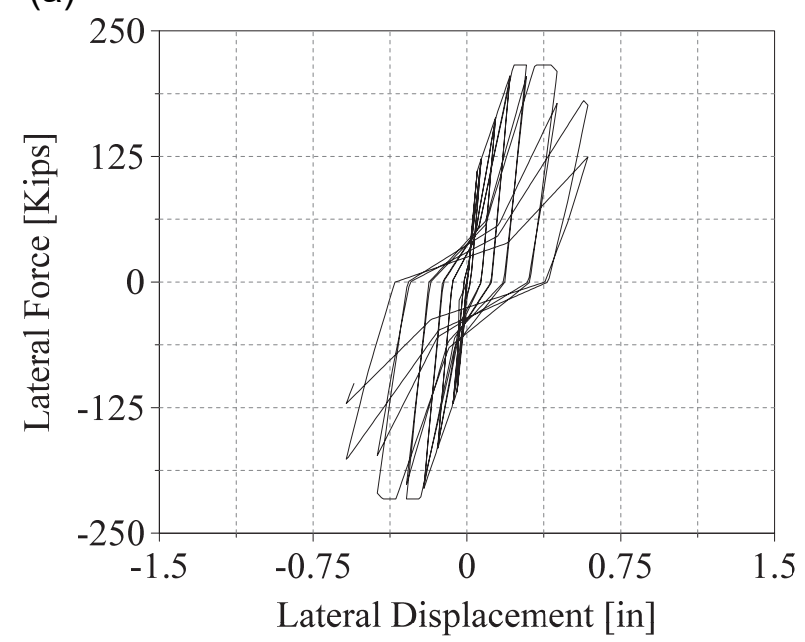

(c)

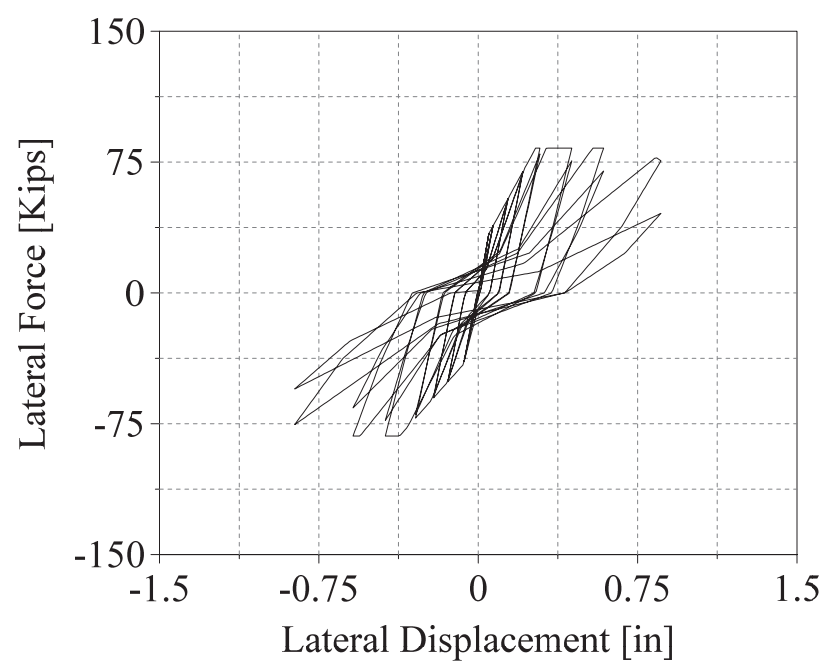

(b)

Figure D-59 Comparison of (a) test data, (b) MIKP prediction, and (c) proposed-model prediction for Li et al. (2006) Specimen LW4. 


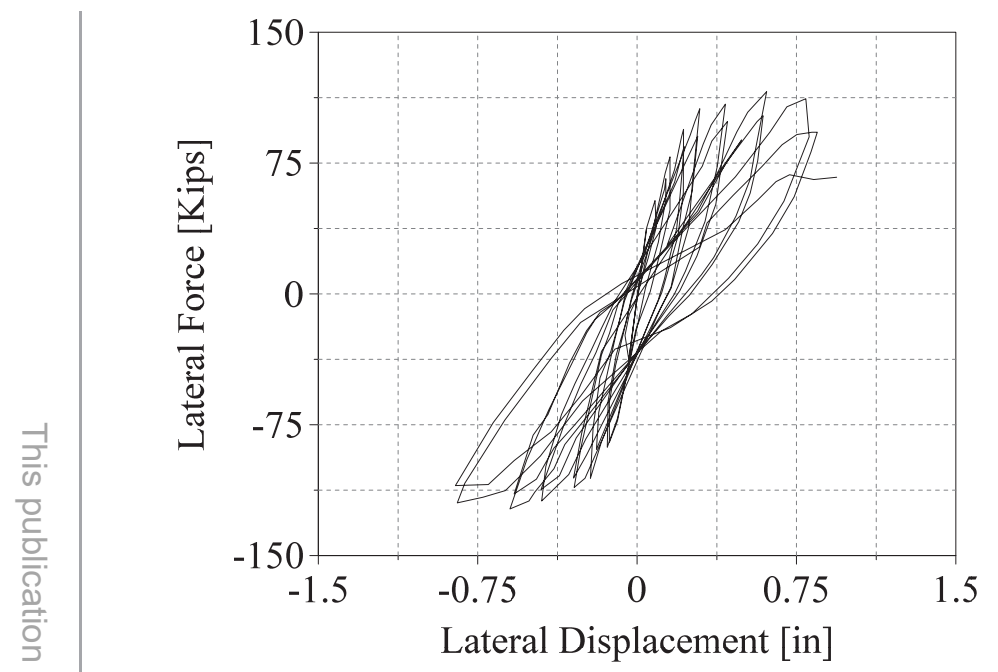

(a)

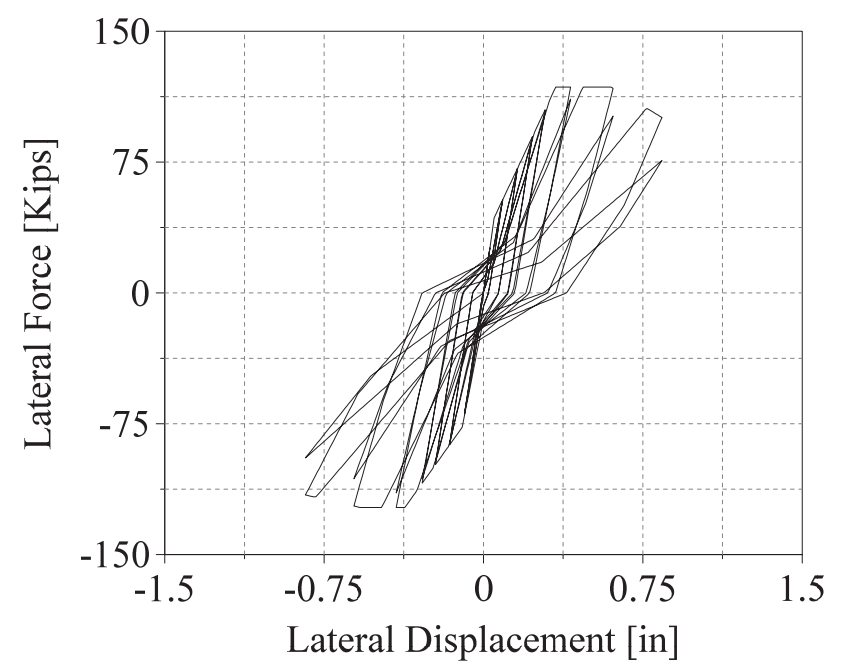

(b)

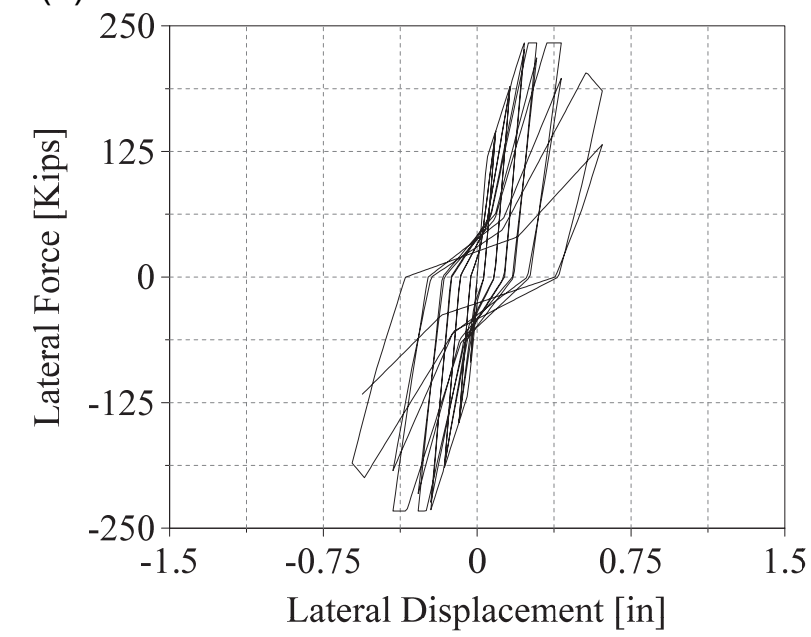

(c)

Figure D-60 Comparison of (a) test data, (b) MIKP prediction, and (c) proposed-model prediction for Li et al. (2006) Specimen LW5. 


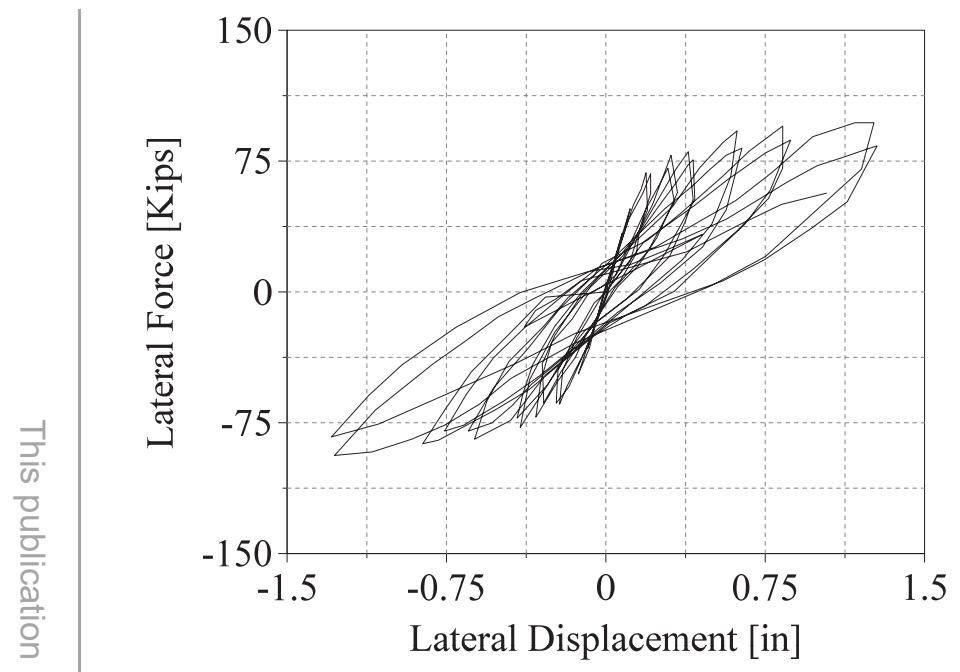

(a)

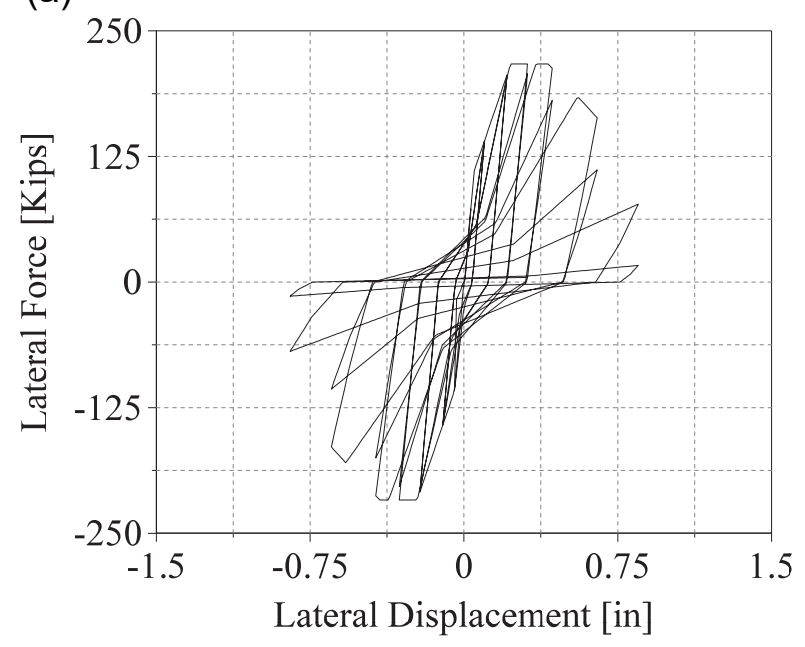

(c)

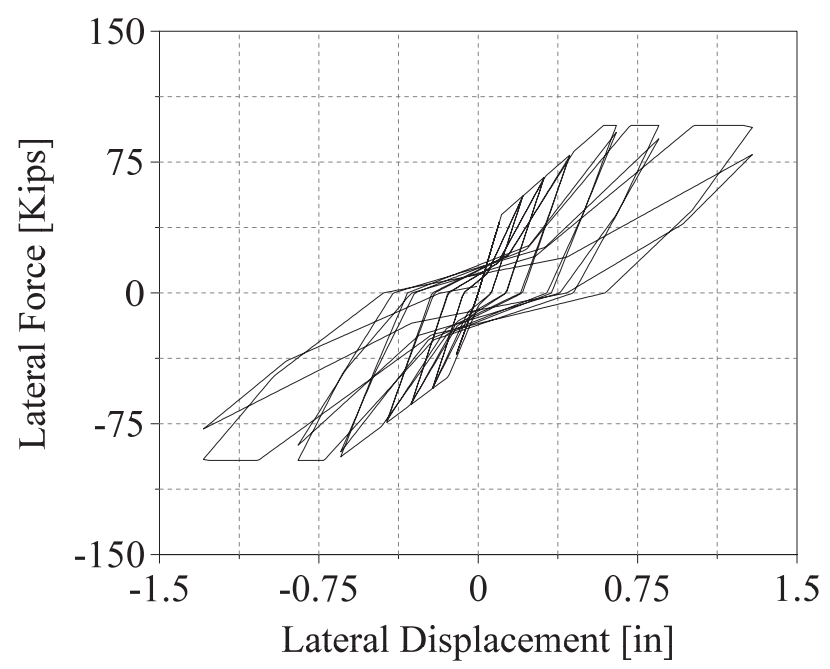

(b)

Figure D-61 Comparison of (a) test data, (b) MIKP prediction, and (c) proposed-model prediction for Li et al. (2006) Specimen MW1. 


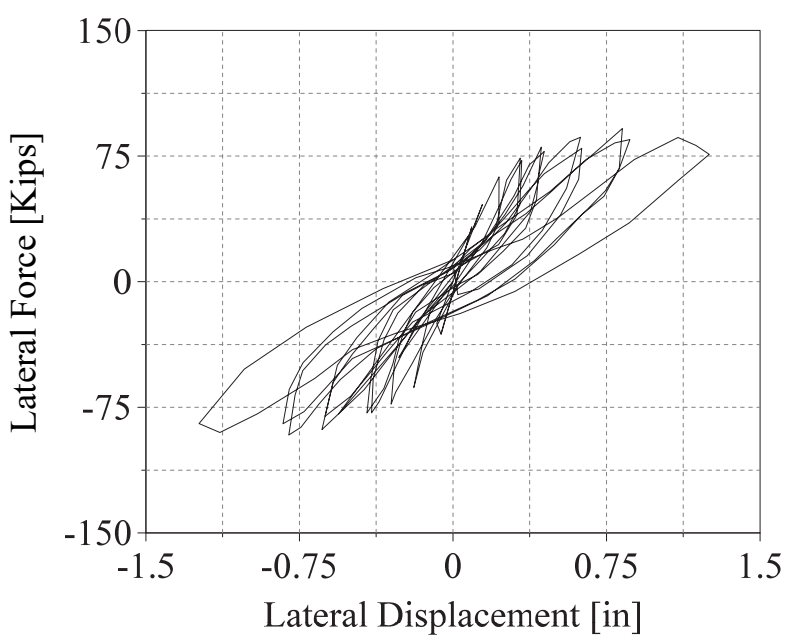

(a)

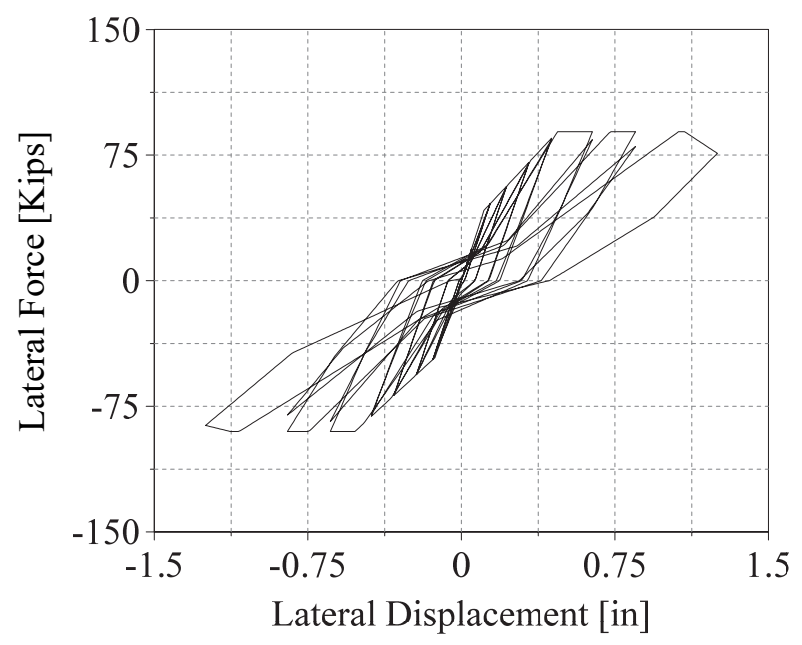

(b)

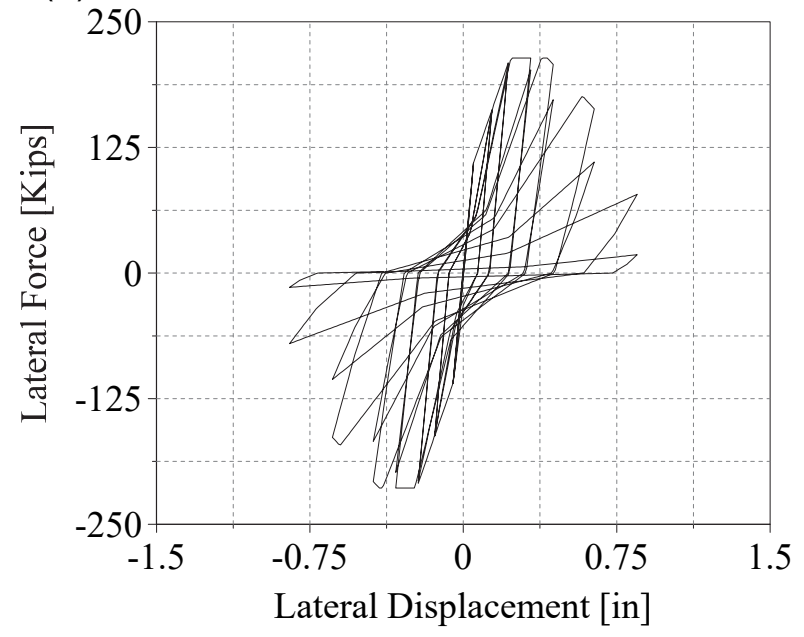

(c)

Figure D-62 Comparison of (a) test data, (b) MIKP prediction, and (c) proposed-model prediction for Li et al. (2006) Specimen MW2. 


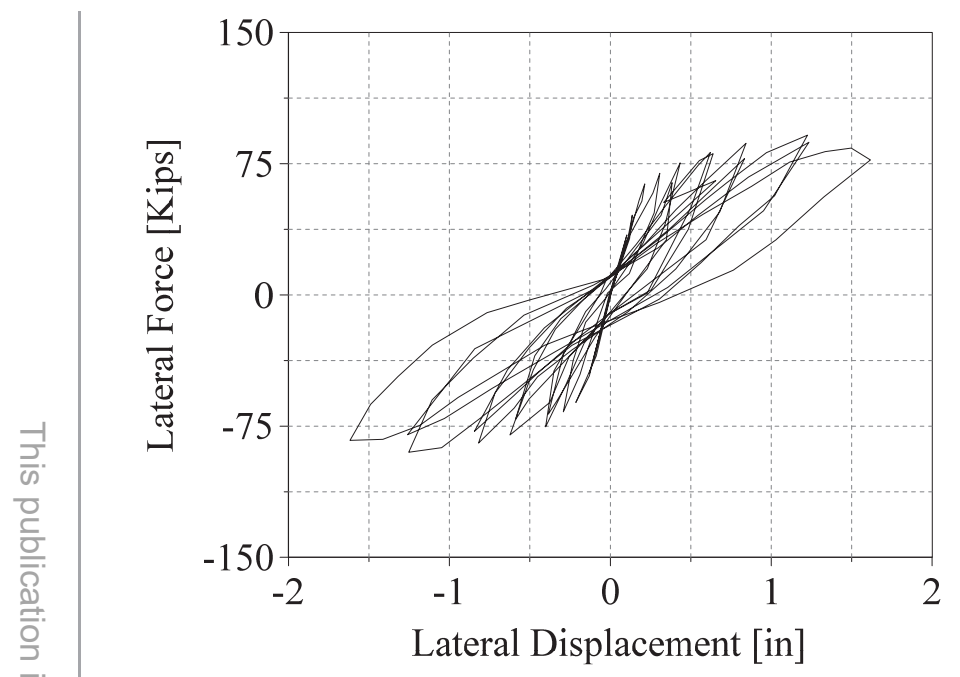

(a)

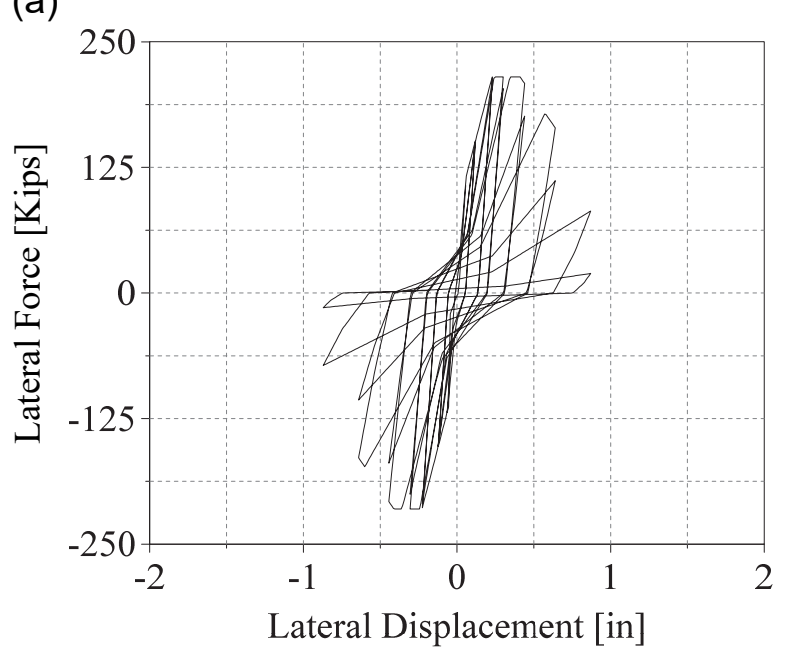

(c)

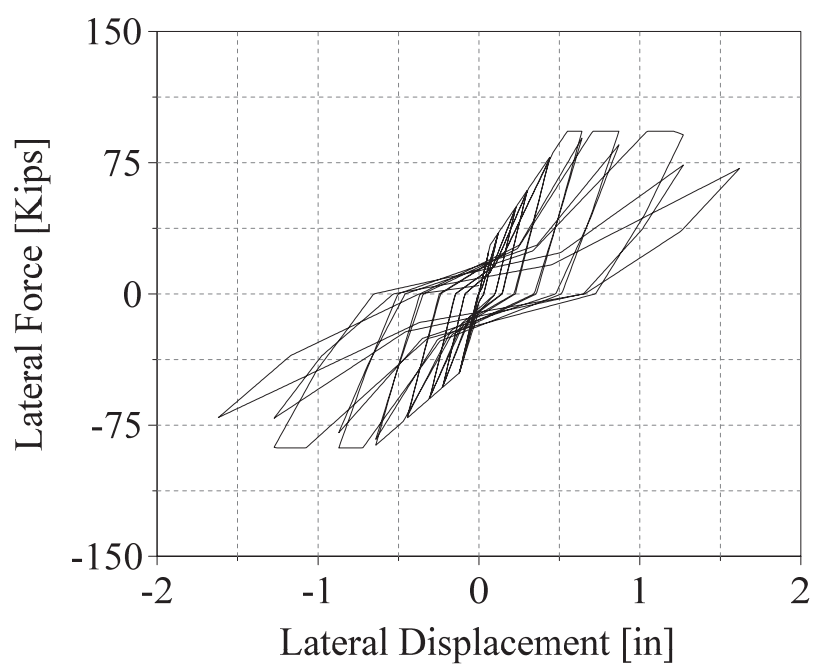

(b)

Figure D-63 Comparison of (a) test data, (b) MIKP prediction, and (c) proposed-model prediction for Li et al. (2006) Specimen MW3. 



\section{Appendix E}

\section{Reinforced Masonry Walls - \\ Database}

\section{E.1 Flexure-Dominated Walls}

Table E-1 Flexure-Dominated Wall Specimens Analyzed

\begin{tabular}{|c|c|c|c|c|c|c|c|c|c|}
\hline Specimen No. & $\begin{array}{l}\text { Height } \\
\text { (in) } \\
\end{array}$ & $\begin{array}{l}\text { Length } \\
\text { (in) } \\
\end{array}$ & $\begin{array}{l}\text { Width } \\
\text { (in) } \\
\end{array}$ & $\begin{array}{c}\text { Aspect } \\
\text { Ratio } \\
\end{array}$ & $\begin{array}{c}\text { Axial } \\
\text { Compression } \\
\text { Ratio }(\beta) \\
\end{array}$ & $\begin{array}{c}f_{y} \\
\text { (ksi) } \\
\end{array}$ & $\begin{array}{c}f_{m}^{\prime} \\
(\mathrm{ksi})\end{array}$ & $\begin{array}{c}\rho_{v} \\
(\%) \\
\end{array}$ & $\begin{array}{c}\rho_{v} f_{y} / f_{m}^{\prime} m \\
(\alpha)\end{array}$ \\
\hline UT-PBS-03* & 96 & 96 & 7.625 & 1 & 0.0000 & 61.4 & 4.447 & 0.33 & 0.046 \\
\hline UT-PBS-04* & 96 & 96 & 7.625 & 1 & 0.0000 & 61.4 & 4.447 & 0.16 & 0.022 \\
\hline WSU-W-1A & 80 & 40 & 7.625 & 2 & 0.0625 & 65.4 & 2.770 & 0.72 & 0.170 \\
\hline WSU-W-1B* & 80 & 40 & 7.625 & 2 & 0.0625 & 64.7 & 3.040 & 0.72 & 0.170 \\
\hline WSU-W-2A & 80 & 40 & 7.625 & 2 & 0.1250 & 66.2 & 2.770 & 0.33 & 0.079 \\
\hline WSU-W-2B* & 80 & 40 & 7.625 & 2 & 0.1250 & 65.3 & 3.040 & 0.33 & 0.079 \\
\hline WSU-W- $04^{*}$ & 72 & 72 & 7.625 & 1 & 0.0625 & 65.3 & 3.040 & 0.33 & 0.071 \\
\hline WSU-W-05* & 56 & 72 & 7.625 & 0.78 & 0.0000 & 65.3 & 3.040 & 0.33 & 0.071 \\
\hline WSU-W- $06^{*}$ & 56 & 72 & 7.625 & 0.78 & 0.0625 & 65.3 & 3.040 & 0.33 & 0.071 \\
\hline WSU-W-07 (C1)* & 80 & 40 & 7.625 & 2 & 0.0000 & 65.3 & 3.038 & 0.33 & 0.071 \\
\hline WSU-W-08 (C2)* & 80 & 40 & 7.625 & 2 & 0.0625 & 66.0 & 3.038 & 0.33 & 0.072 \\
\hline UT-W-13* & 144 & 48 & 7.625 & 3 & 0.0281 & 61.1 & 4.447 & 0.72 & 0.099 \\
\hline UT-W-17* & 144 & 32 & 7.625 & 4.5 & 0.0297 & 61.1 & 4.206 & 0.72 & 0.105 \\
\hline CU-Boulder-1 & 72 & 72 & 5.625 & 1 & 0.0833 & 64.0 & 2.916 & 0.38 & 0.101 \\
\hline CU-Boulder-2 & 72 & 72 & 5.625 & 1 & 0.0833 & 64.0 & 2.916 & 0.38 & 0.101 \\
\hline CU-Boulder-10 & 72 & 72 & 5.625 & 1 & 0.0394 & 64.0 & 3.237 & 0.38 & 0.096 \\
\hline CU-Boulder-12 & 72 & 72 & 5.625 & 1 & 0.0394 & 64.0 & 3.237 & 0.38 & 0.096 \\
\hline CU-Boulder-15 & 72 & 72 & 5.625 & 1 & 0.0347 & 65.0 & 3.342 & 0.54 & 0.122 \\
\hline McMaster-1 & 144 & 72 & 7.5 & 2 & 0.0000 & 72.8 & 2.466 & 0.29 & 0.071 \\
\hline McMaster-2 & 144 & 72 & 7.5 & 2 & 0.0000 & 72.8 & 2.466 & 0.78 & 0.191 \\
\hline McMaster-3 & 144 & 72 & 7.5 & 2 & 0.0000 & 72.8 & 2.466 & 0.73 & 0.179 \\
\hline
\end{tabular}

* Vertical bars lap-spliced at the base 


\section{E.1.1 Cyclic Analysis for Flexure-Dominated Walls}

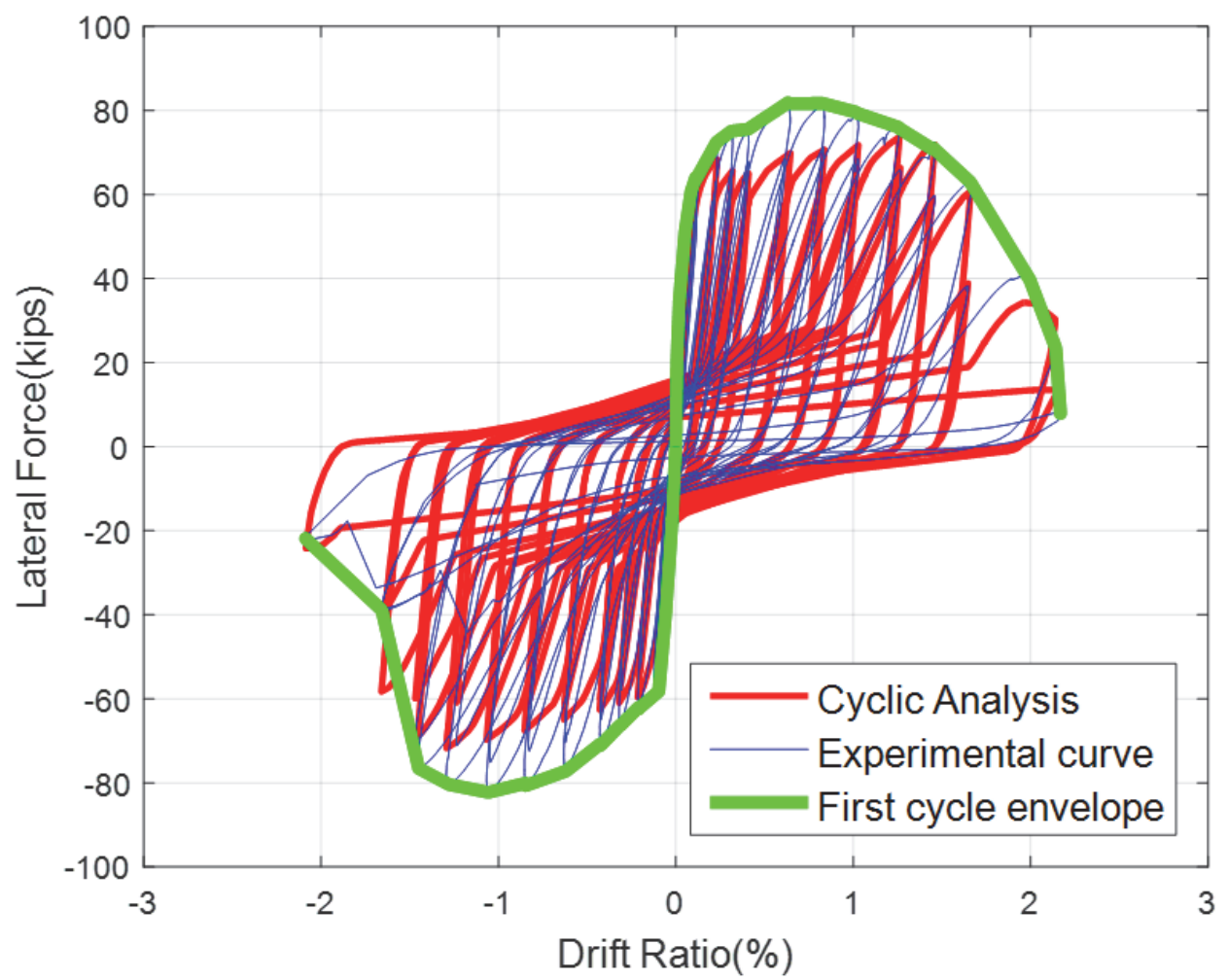

(a) Wall UT-PBS-03

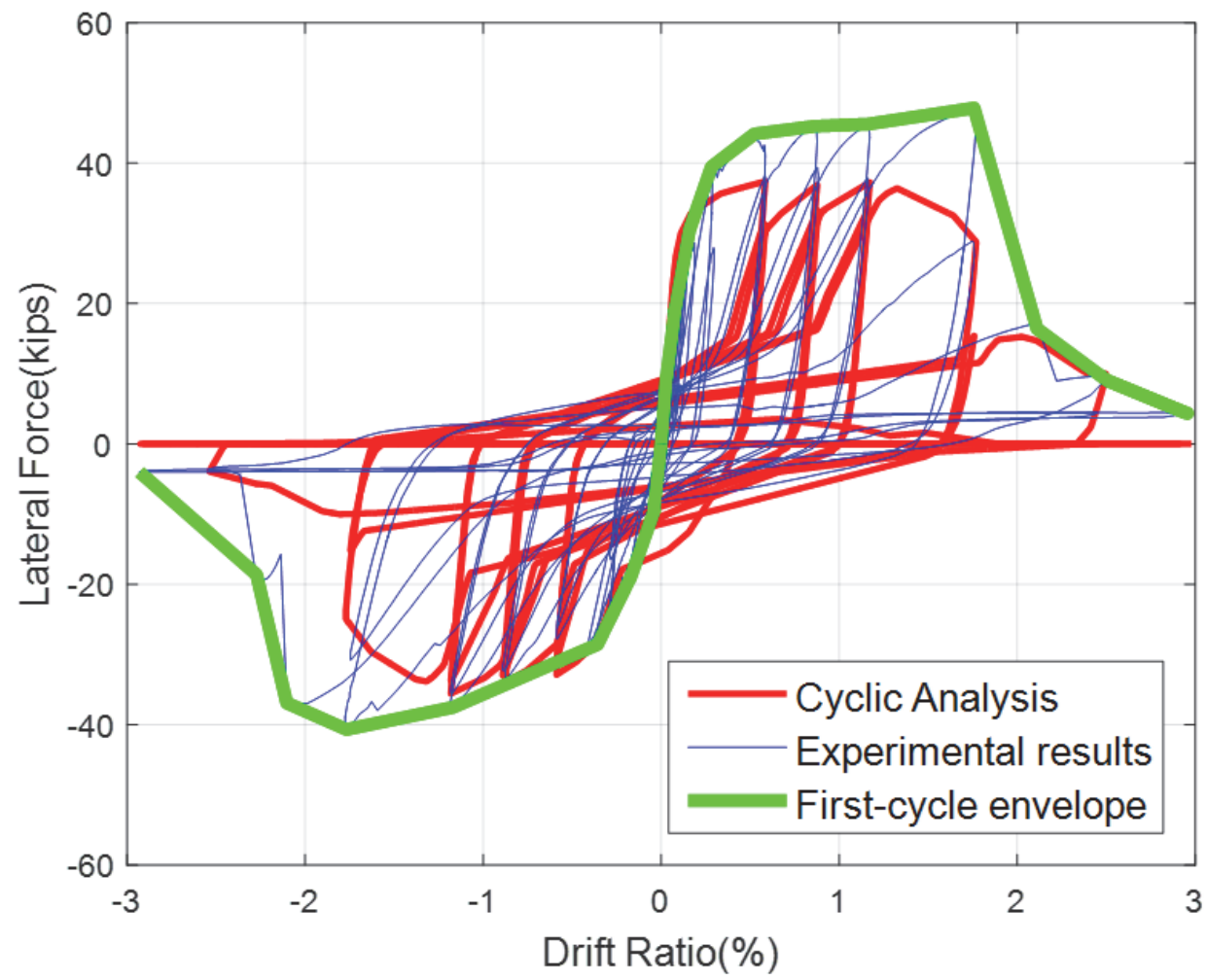

(b) Wall UT-PBS-04

Figure E-1 Cyclic analysis of flexure-dominated walls. 


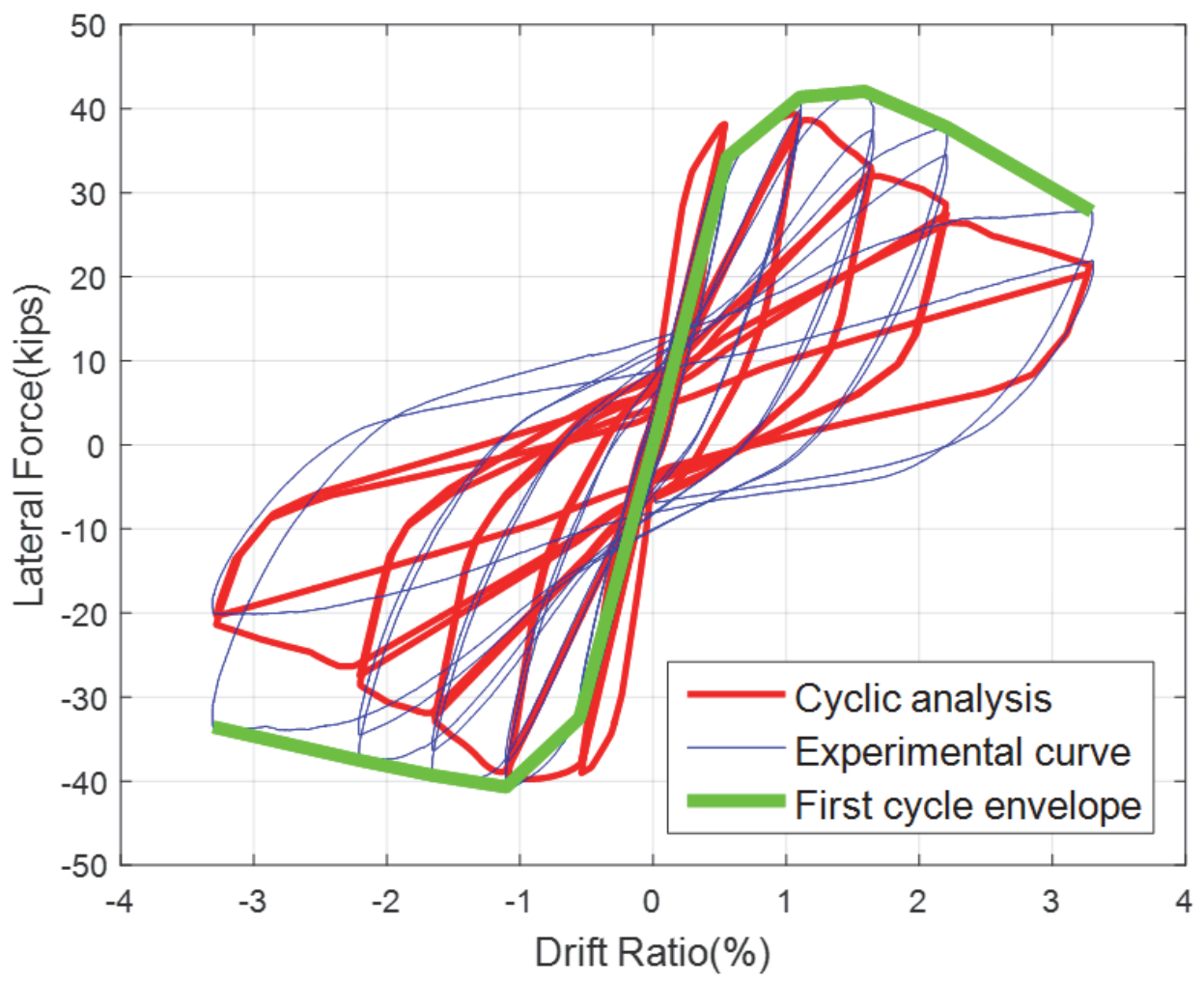

(c) Wall WSU-W-1A

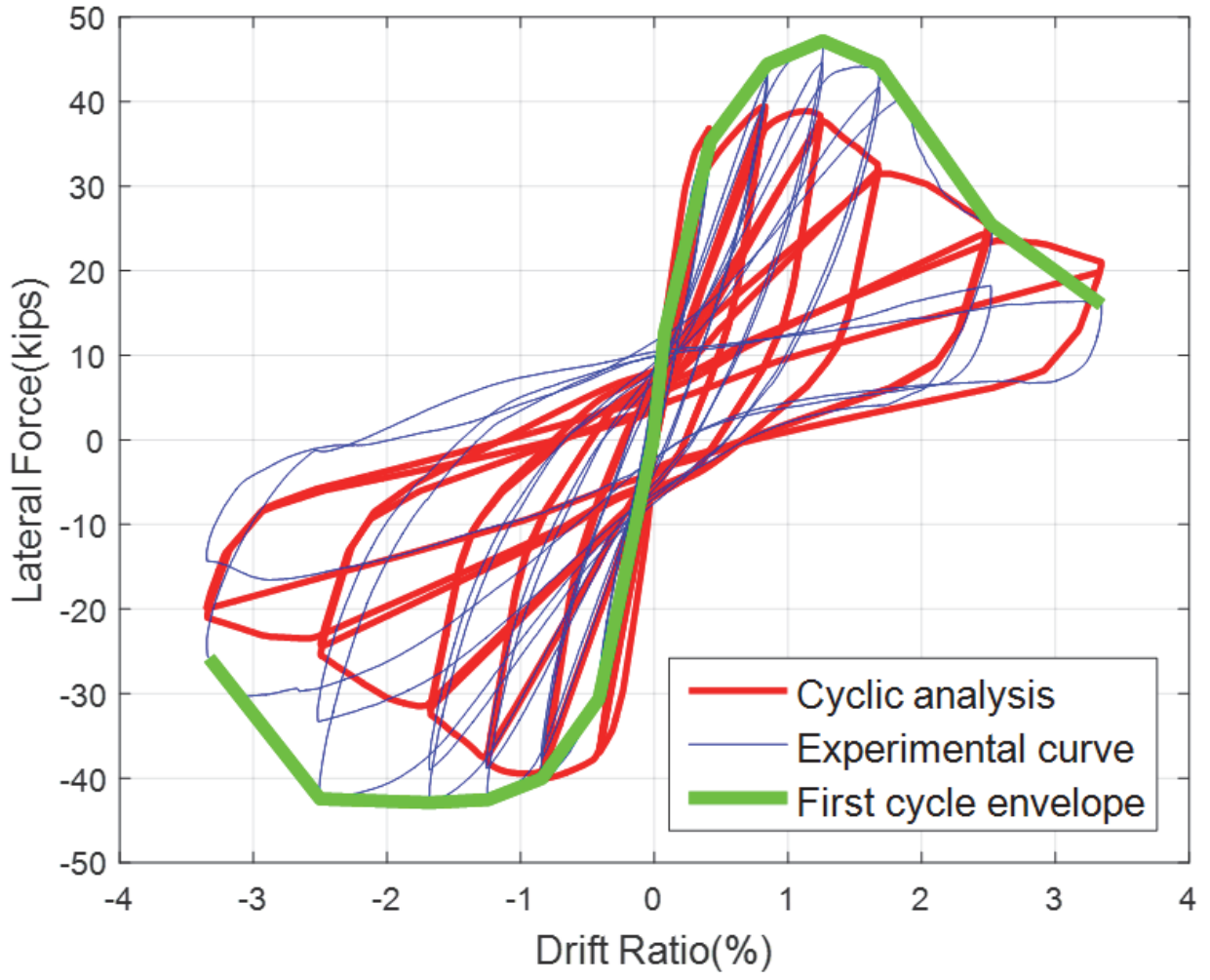

(d) Wall WSU-W-1B

Figure E-1 Cyclic analysis of flexure-dominated walls (continued). 


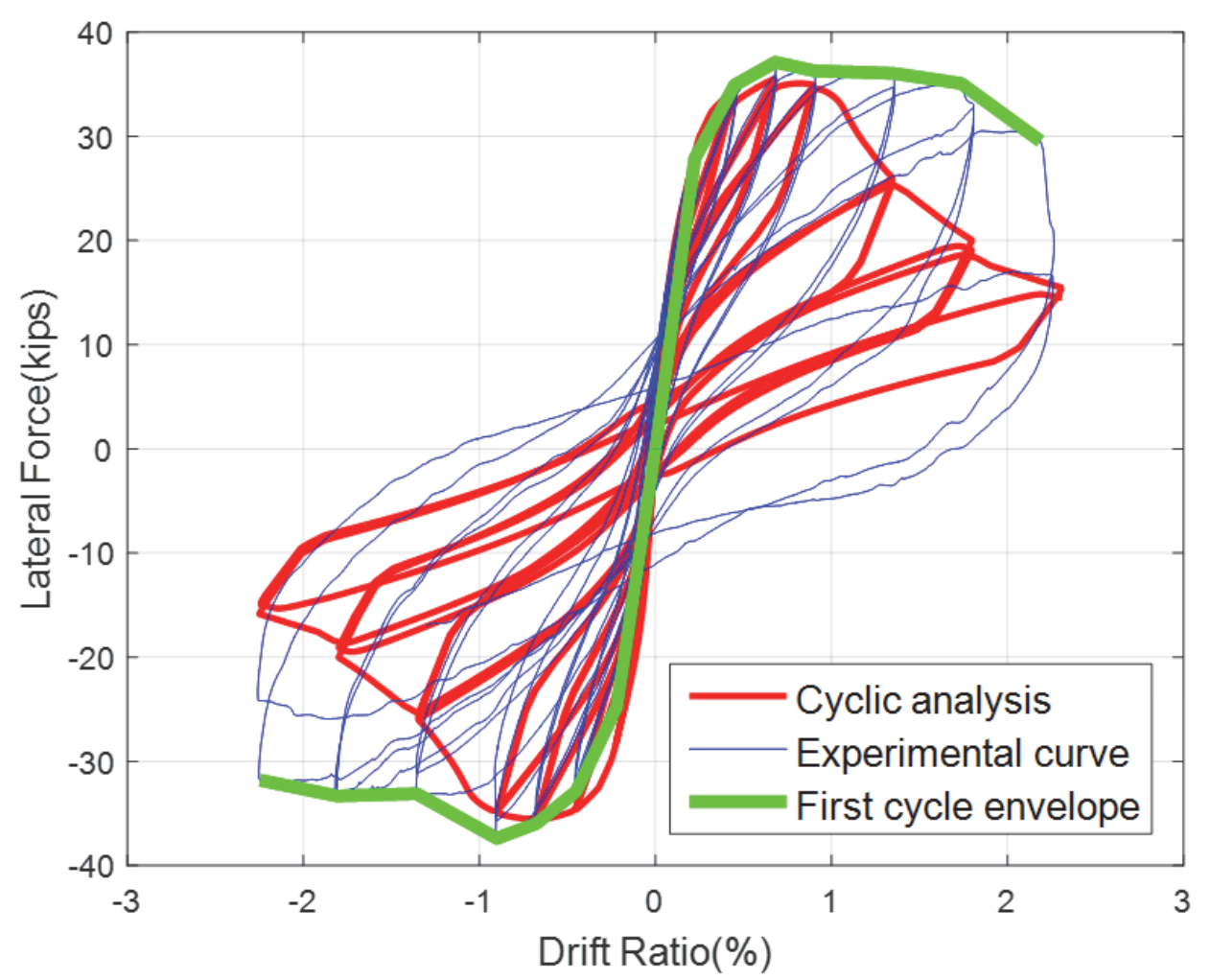

(e) Wall WSU-W-2A

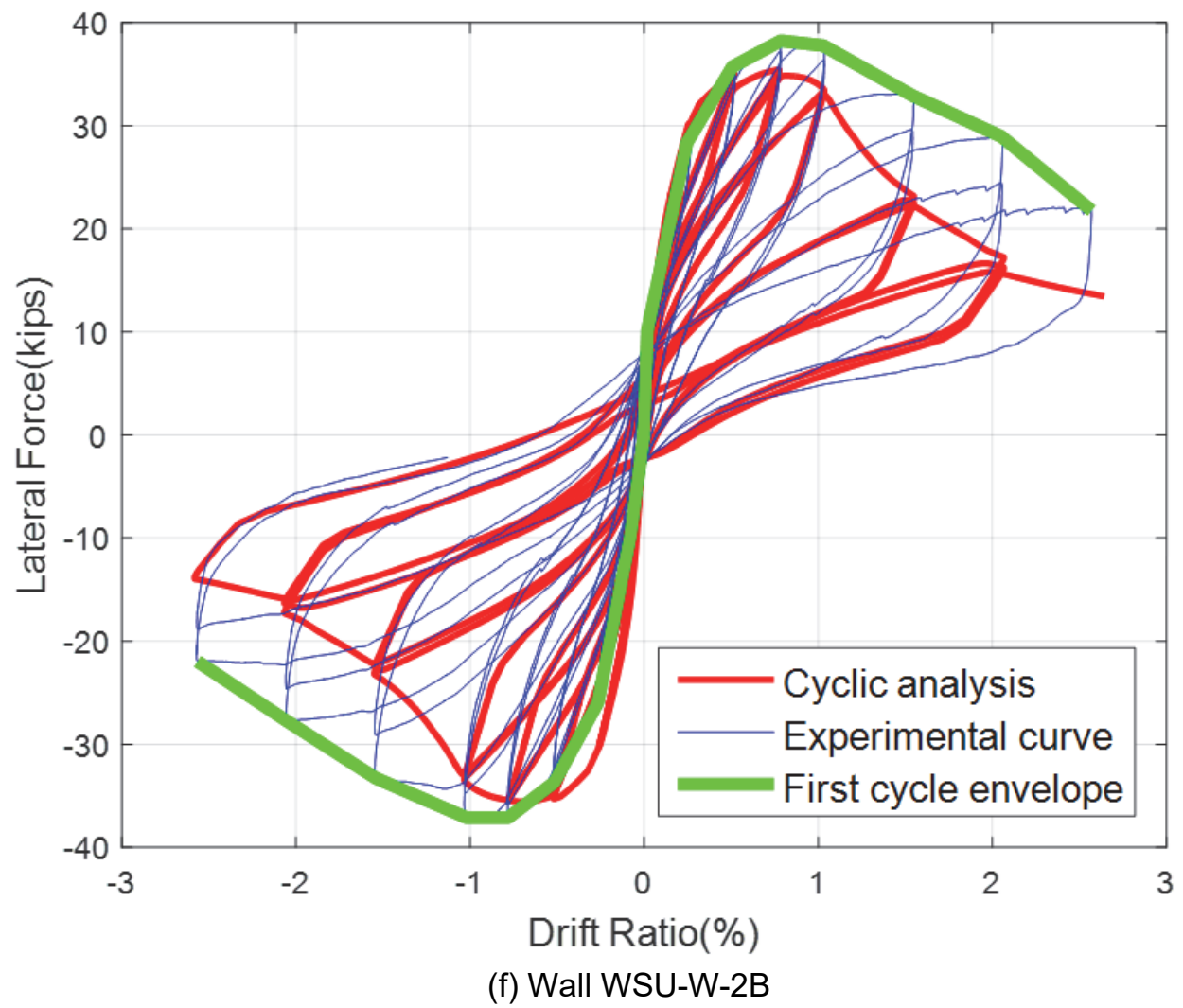

Figure E-1 Cyclic analysis of flexure-dominated walls (continued). 


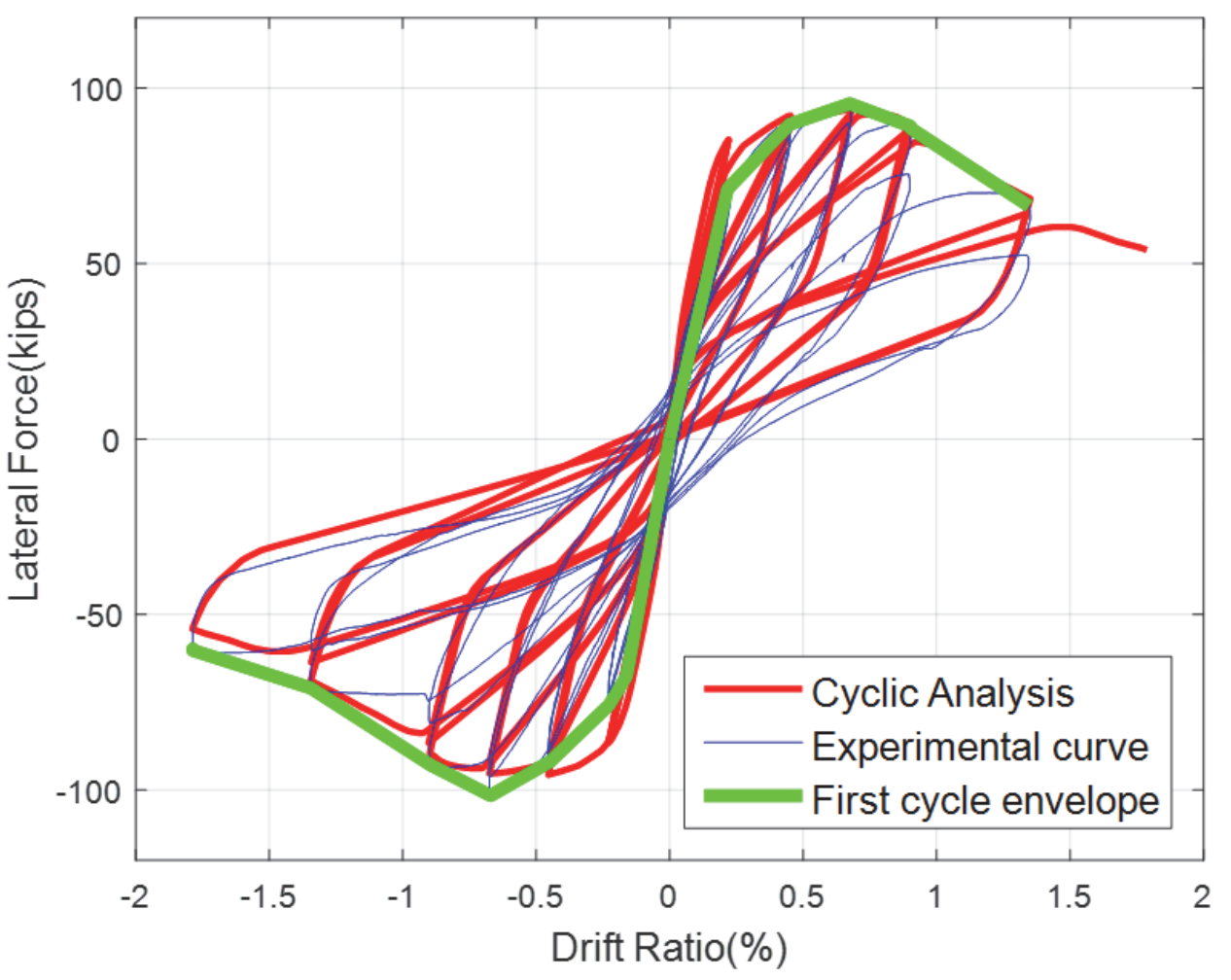

(g) Wall WSU-W-04

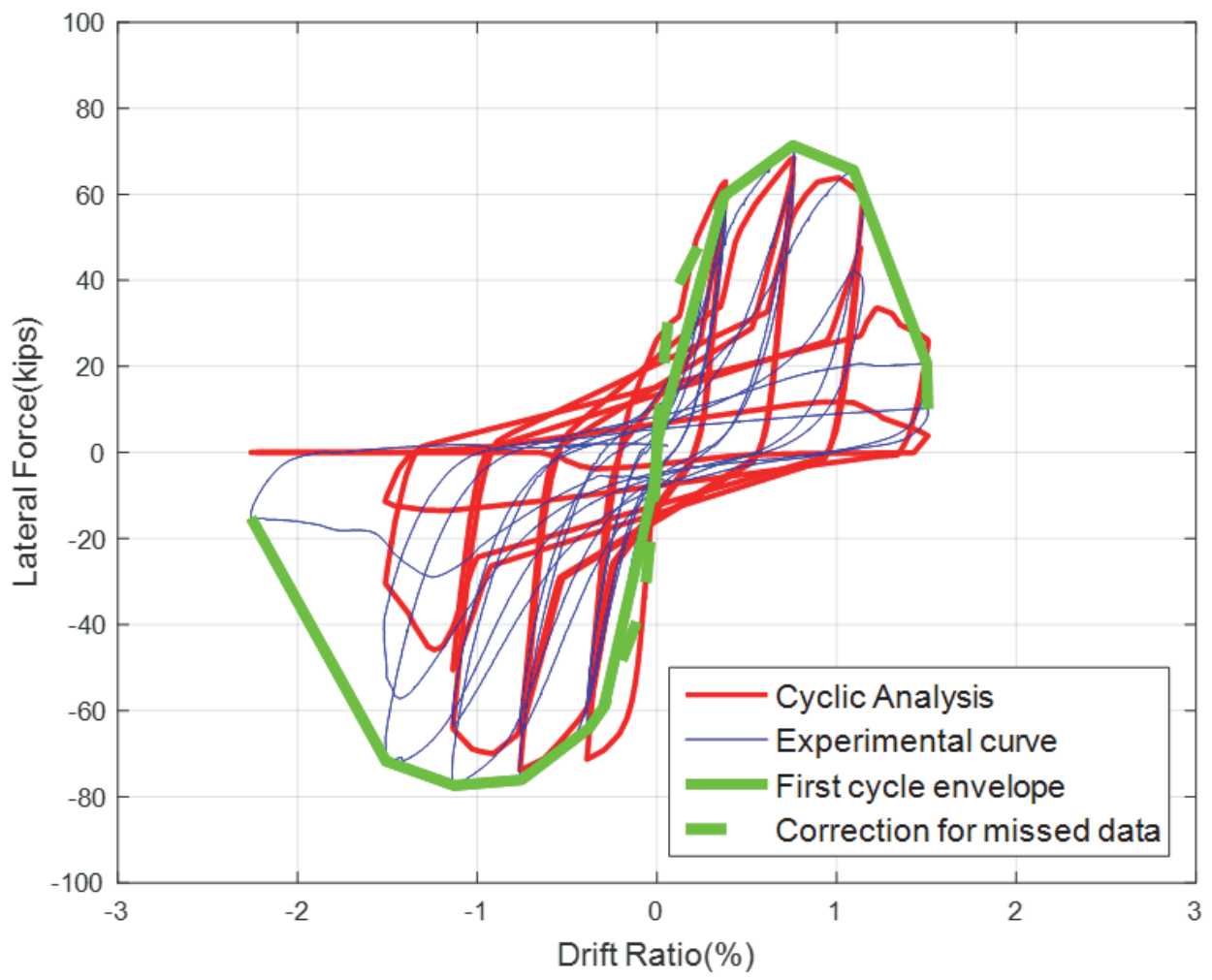

(h) Wall WSU-W-05

Figure E-1 Cyclic analysis of flexure-dominated walls (continued). 

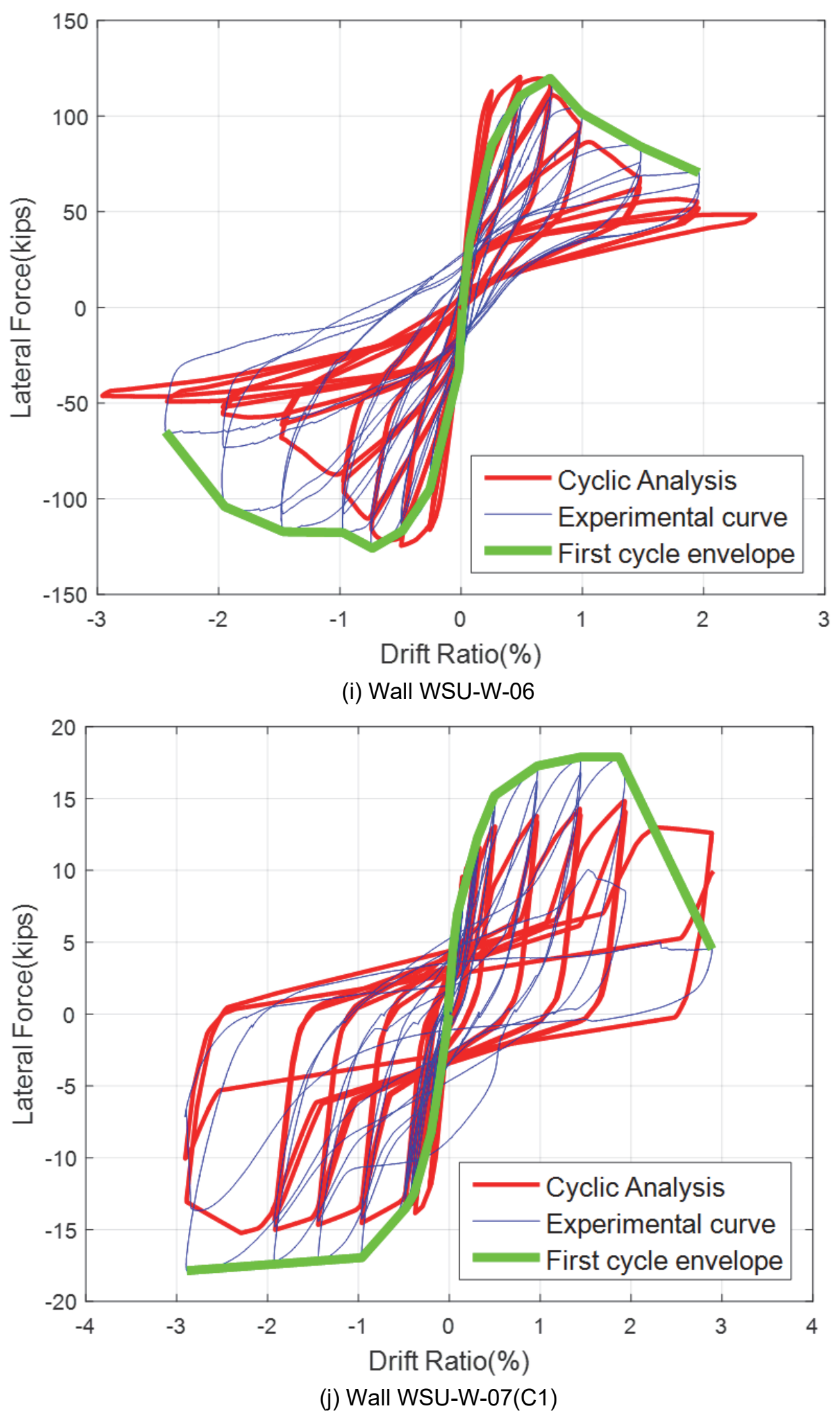

Figure E-1 Cyclic analysis of flexure-dominated walls (continued). 


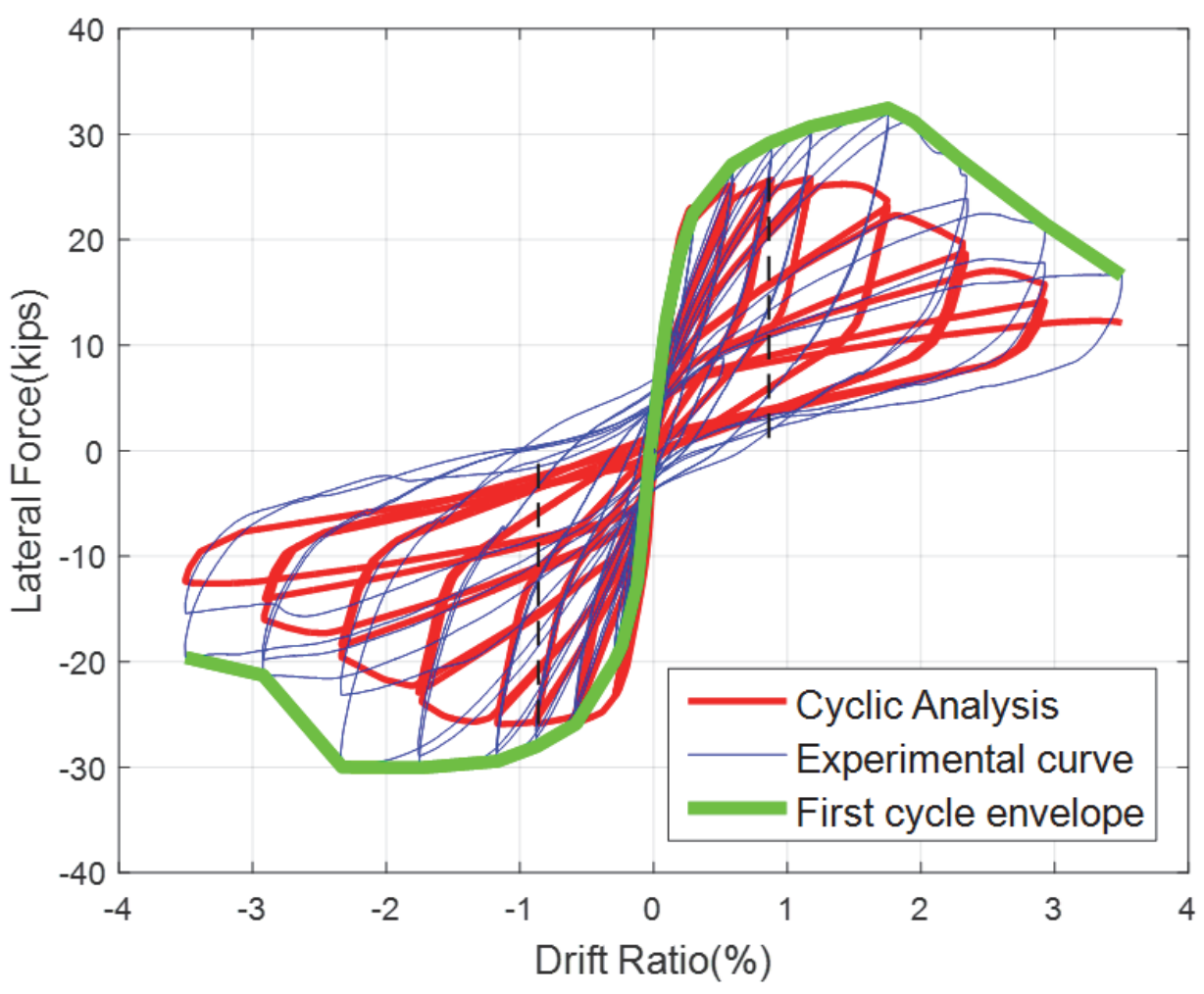

(k) Wall WSU-W-08(C2)

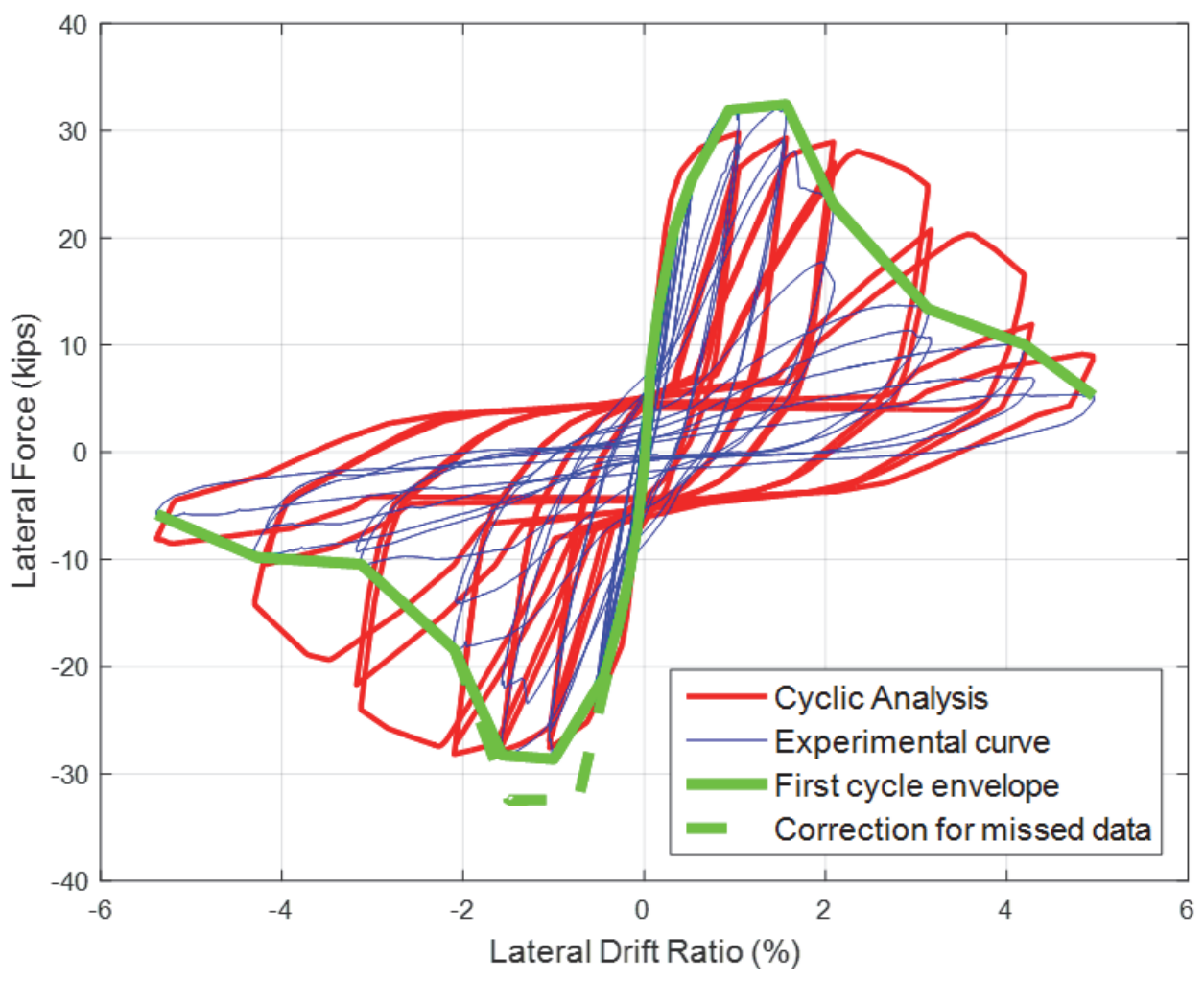

(I) Wall UT-W-13

Figure E-1 Cyclic analysis of flexure-dominated walls (continued). 


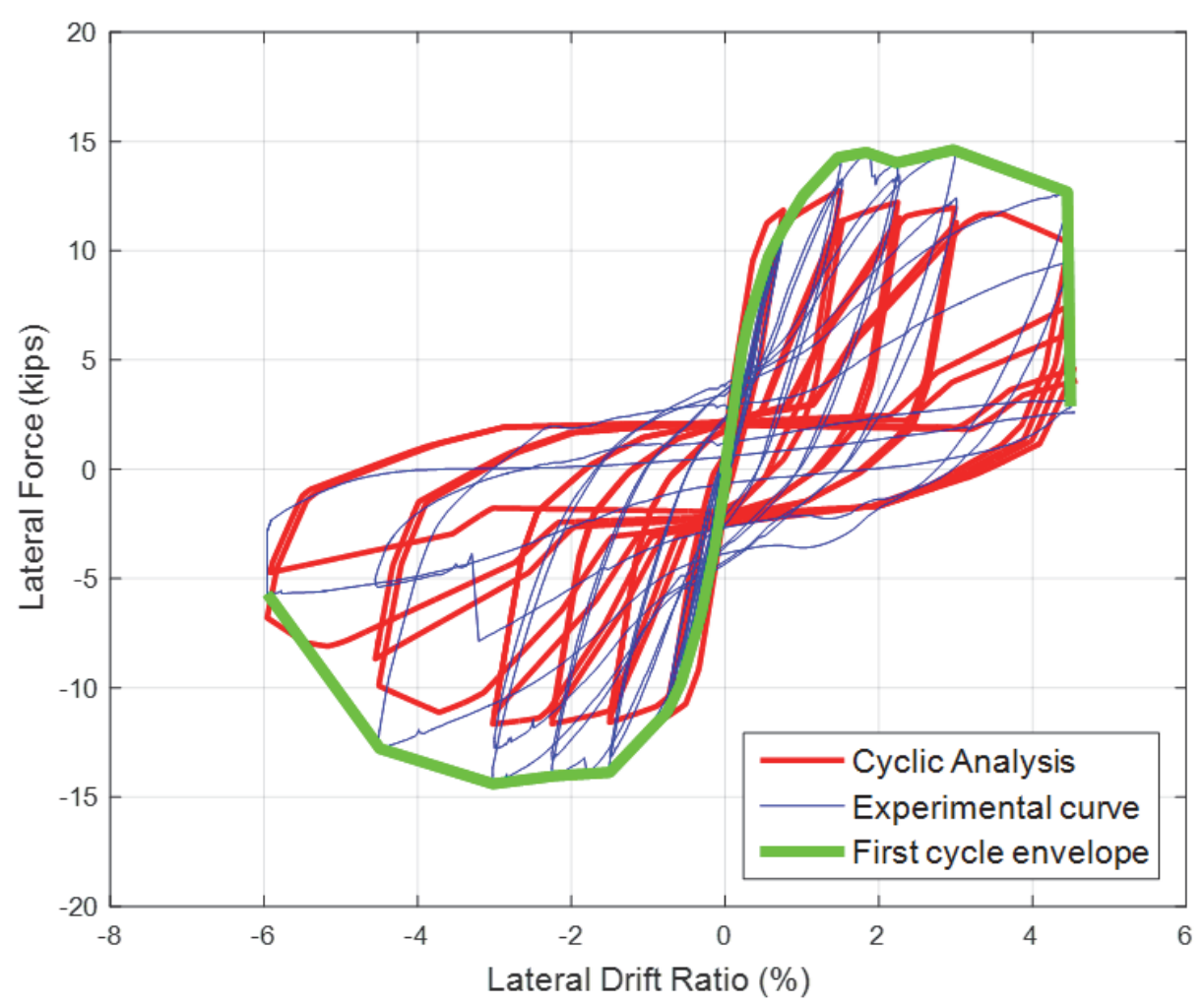

(m) Wall UT-W-17

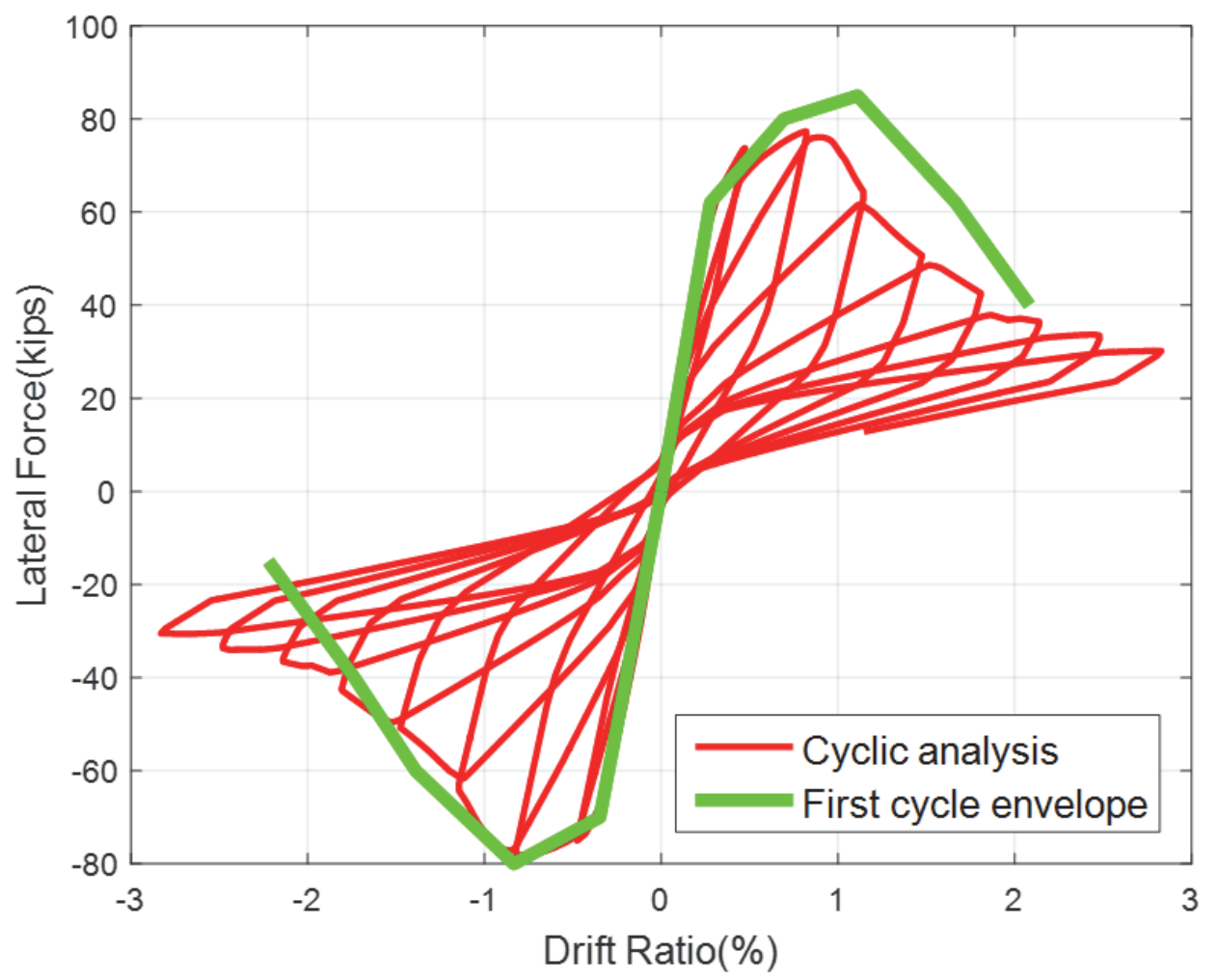

(n) Wall CU-Boulder-1

Figure E-1 Cyclic analysis of flexure-dominated walls (continued). 


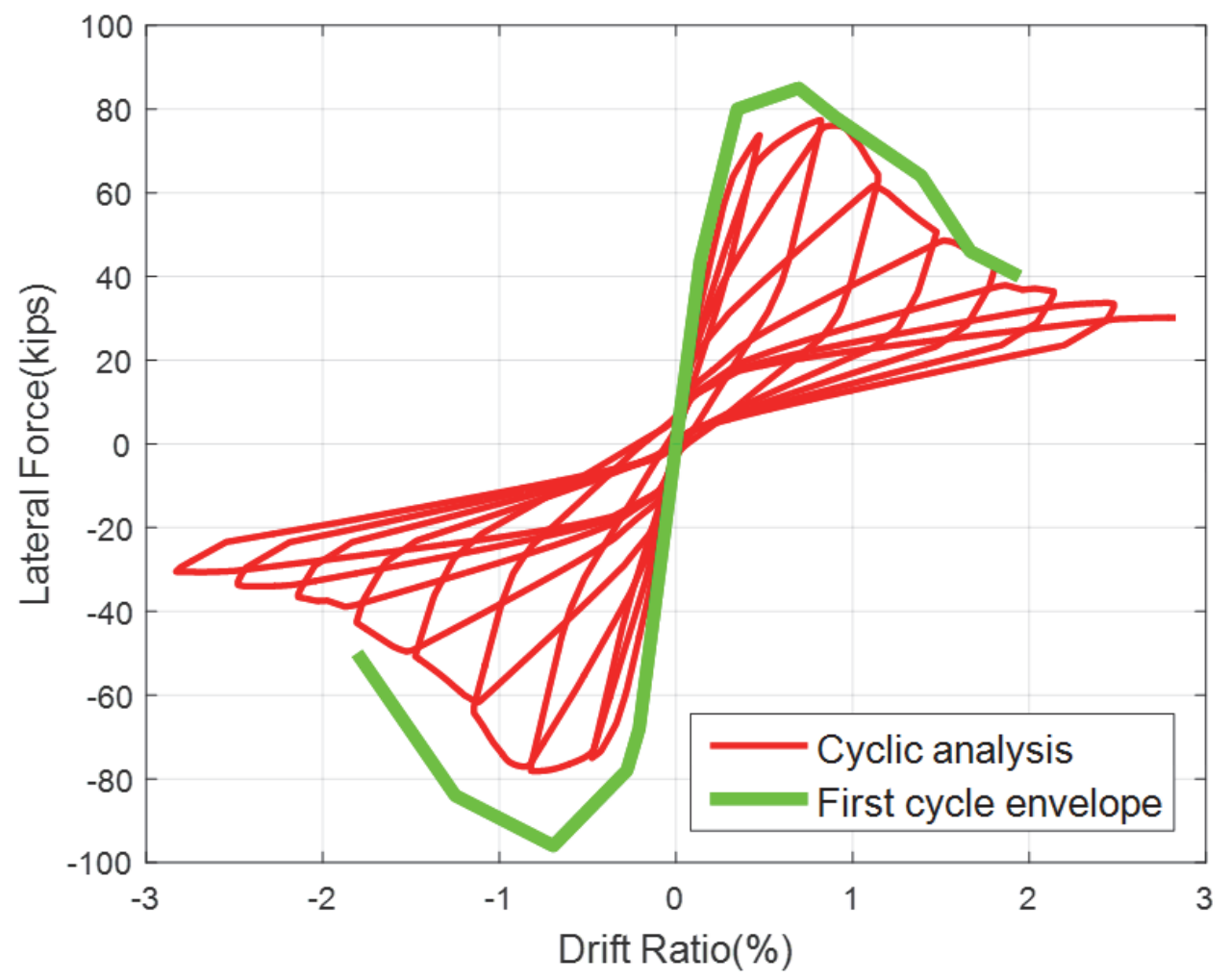

(o) Wall CU-Boulder-2

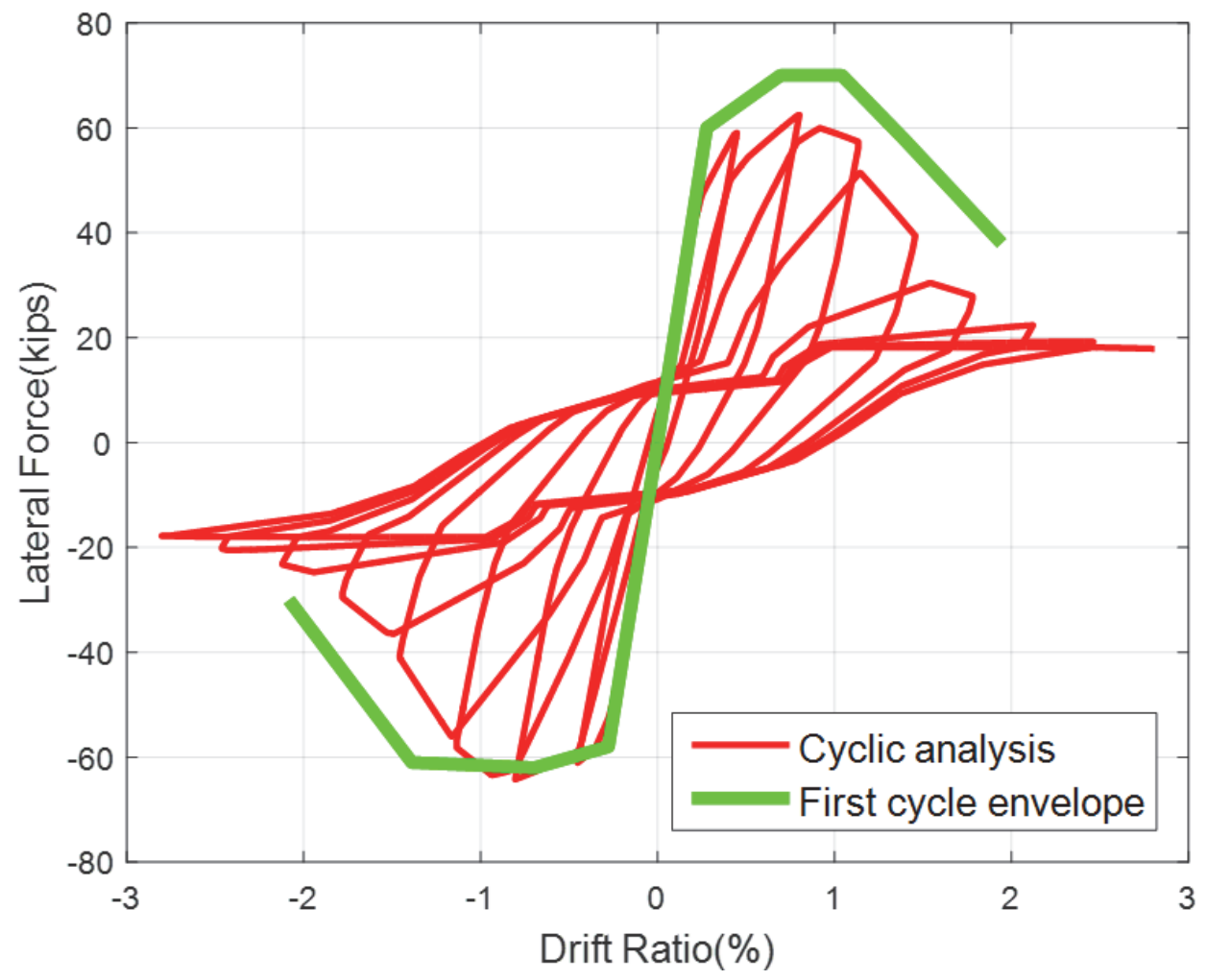

(p) Wall CU-Boulder-10

Figure E-1 Cyclic analysis of flexure-dominated walls (continued). 


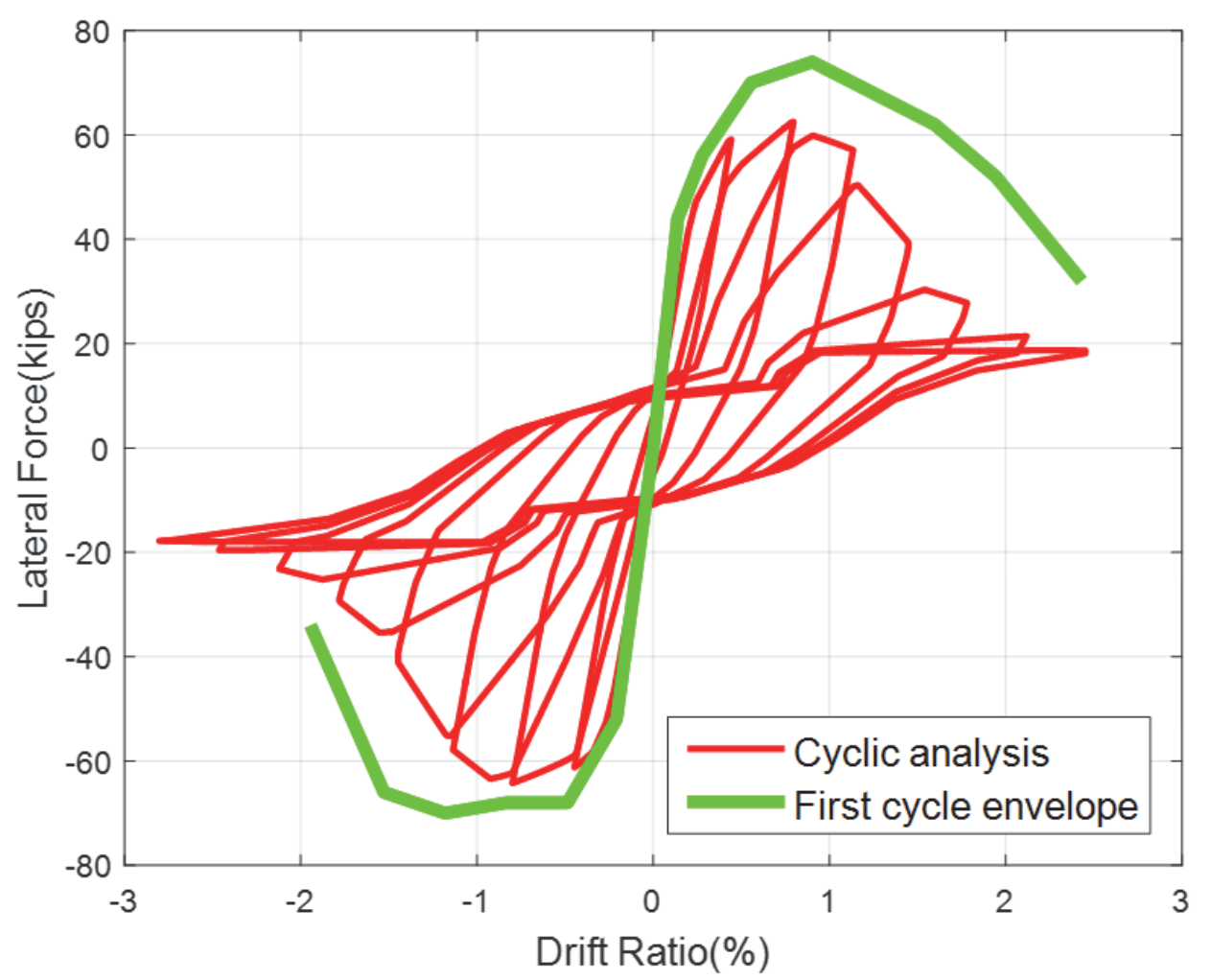

(q) Wall CU-Boulder-12

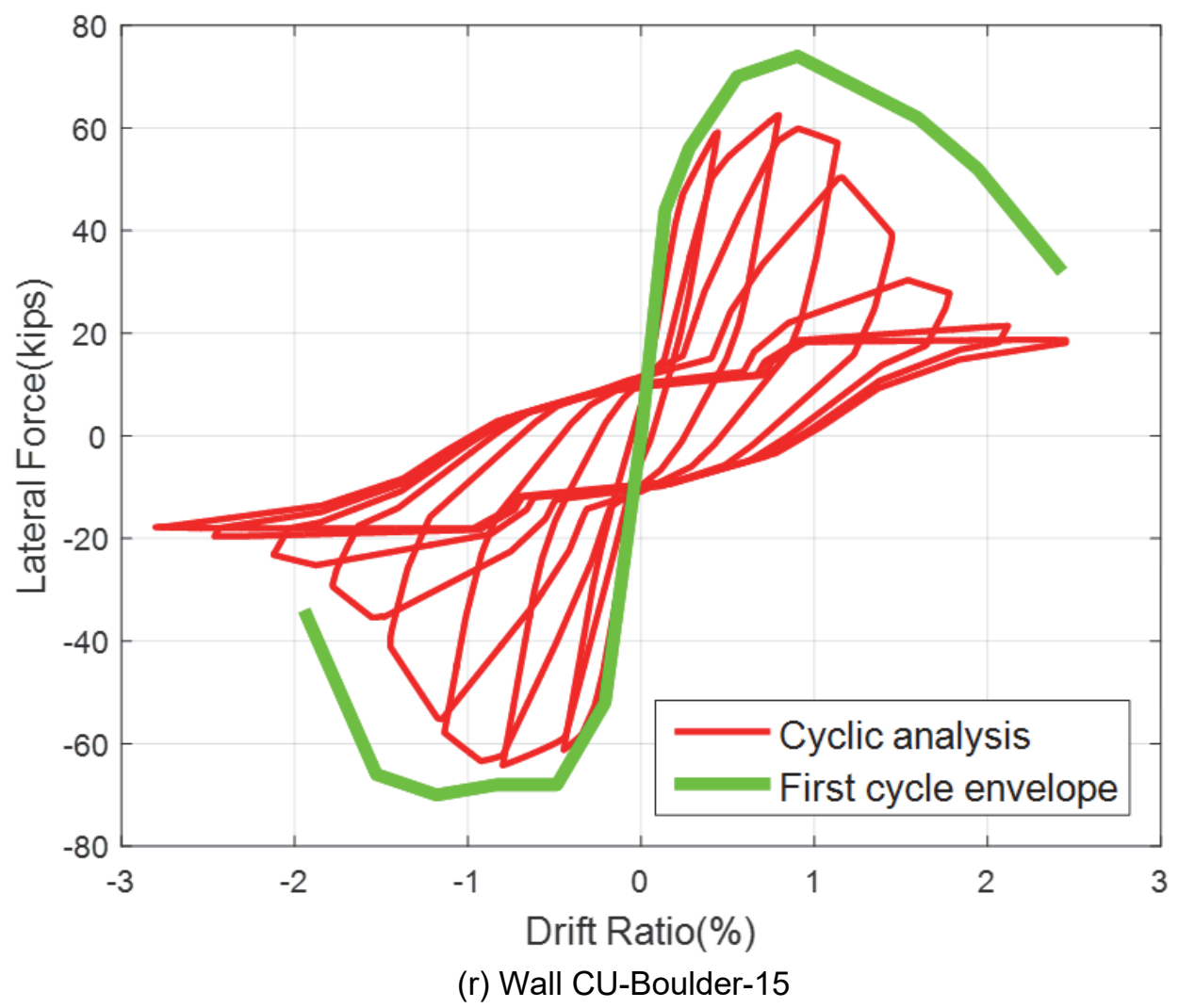

Figure E-1 Cyclic analysis of flexure-dominated walls (continued). 


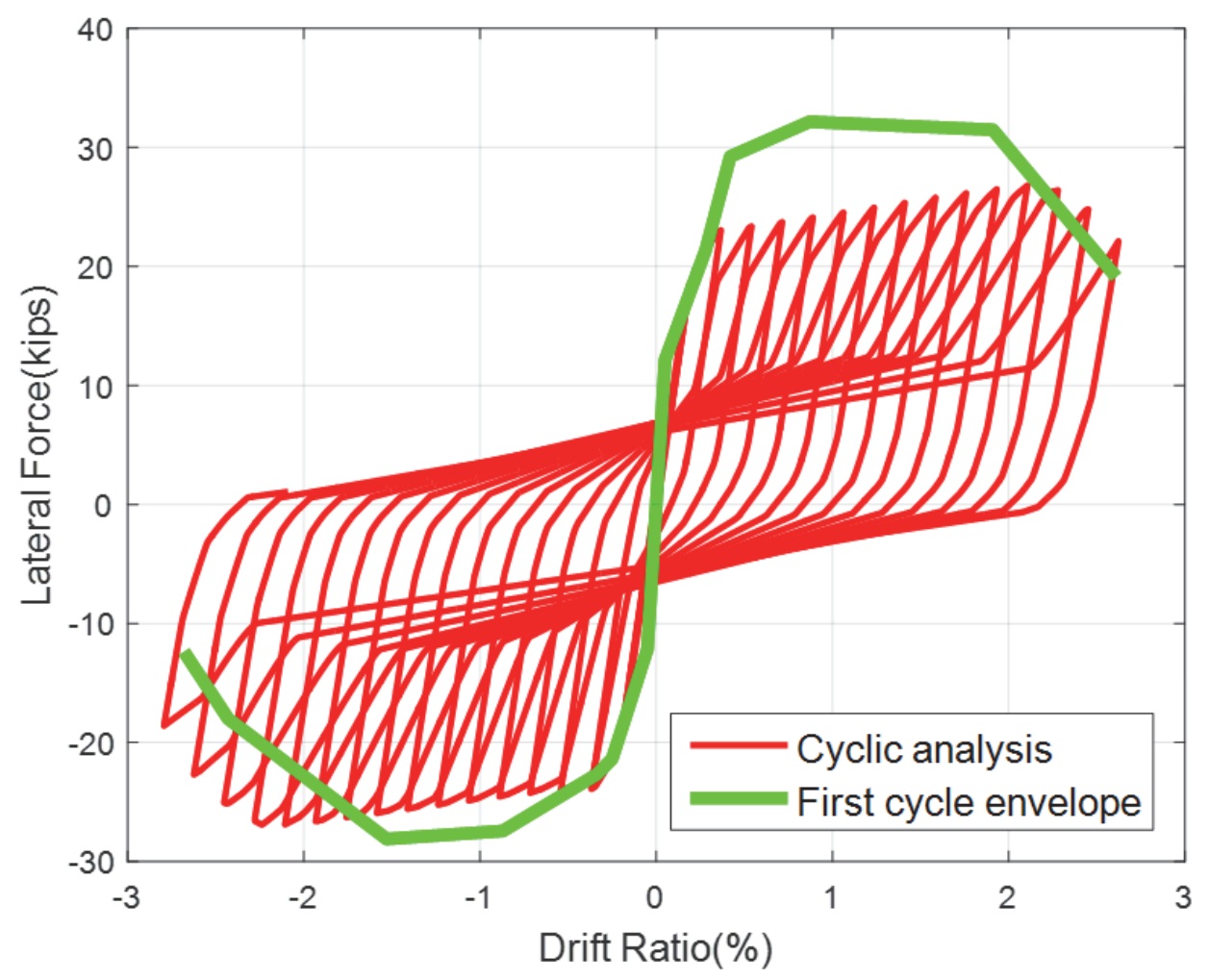

(s) Wall McMaster-1

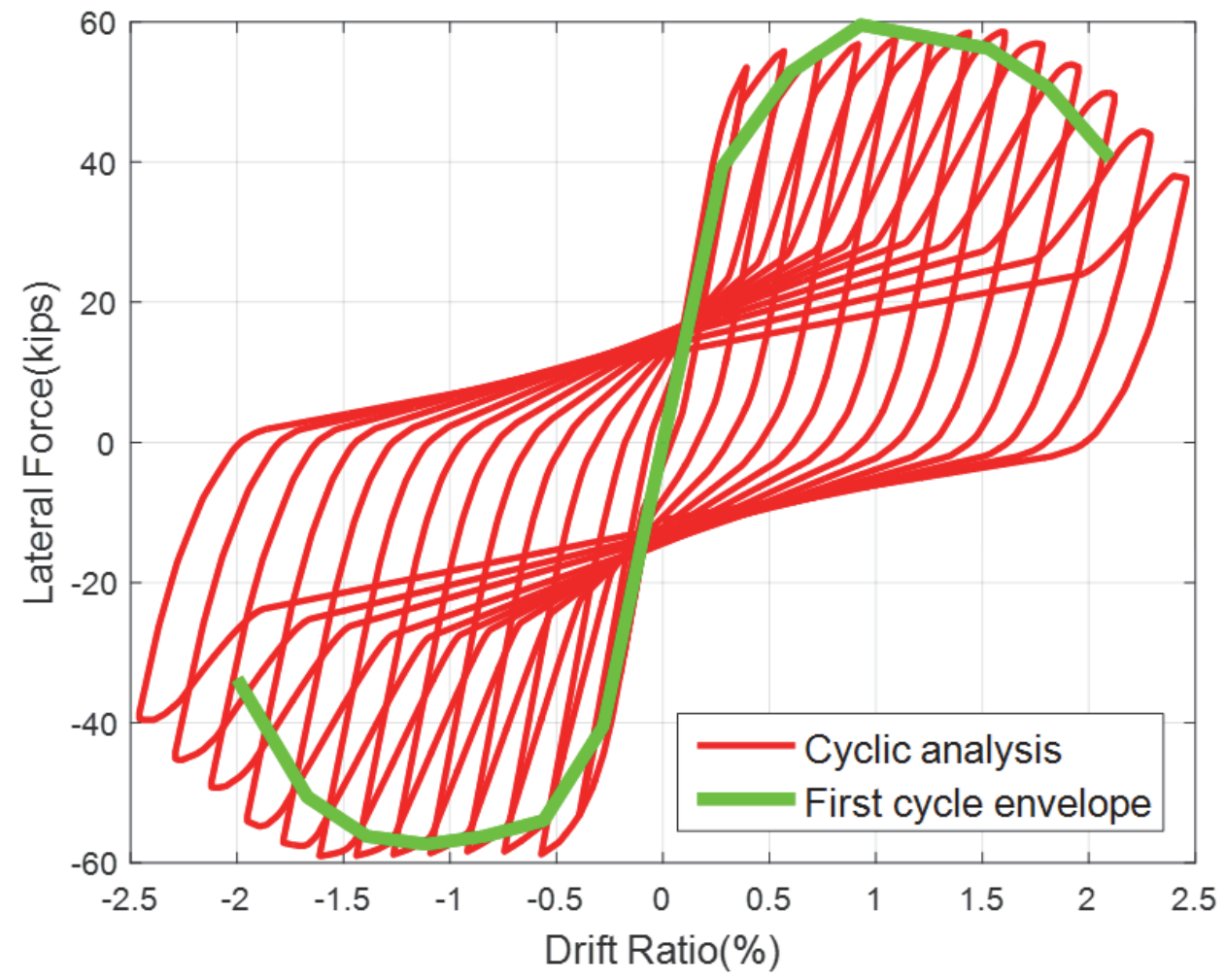

(t) Wall McMaster-2

Figure E-1 Cyclic analysis of flexure-dominated walls (continued). 


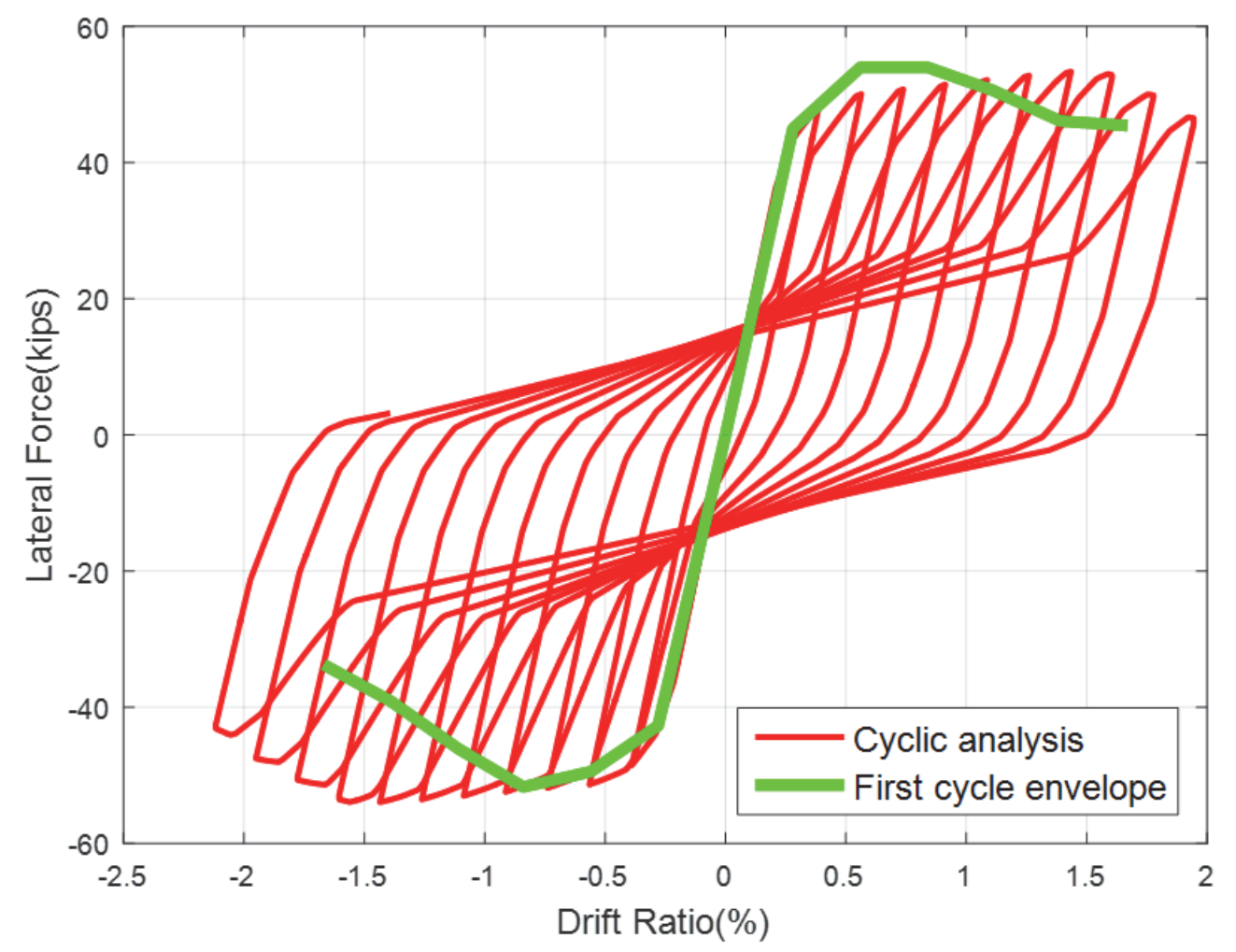

(u) Wall McMaster-3

Figure E-1 Cyclic analysis of flexure-dominated walls (continued).

\section{E.1.2 Backbone Curves for Flexure-Dominated Walls}

It should be noted that backbone curves for some of the walls cannot be computed with the current ASCE/SEI 41-13, Seismic Evaluation and Retrofit of Existing Buildings, (ASCE, 2014) recommendations because their properties are beyond the range of the tabulated values. 


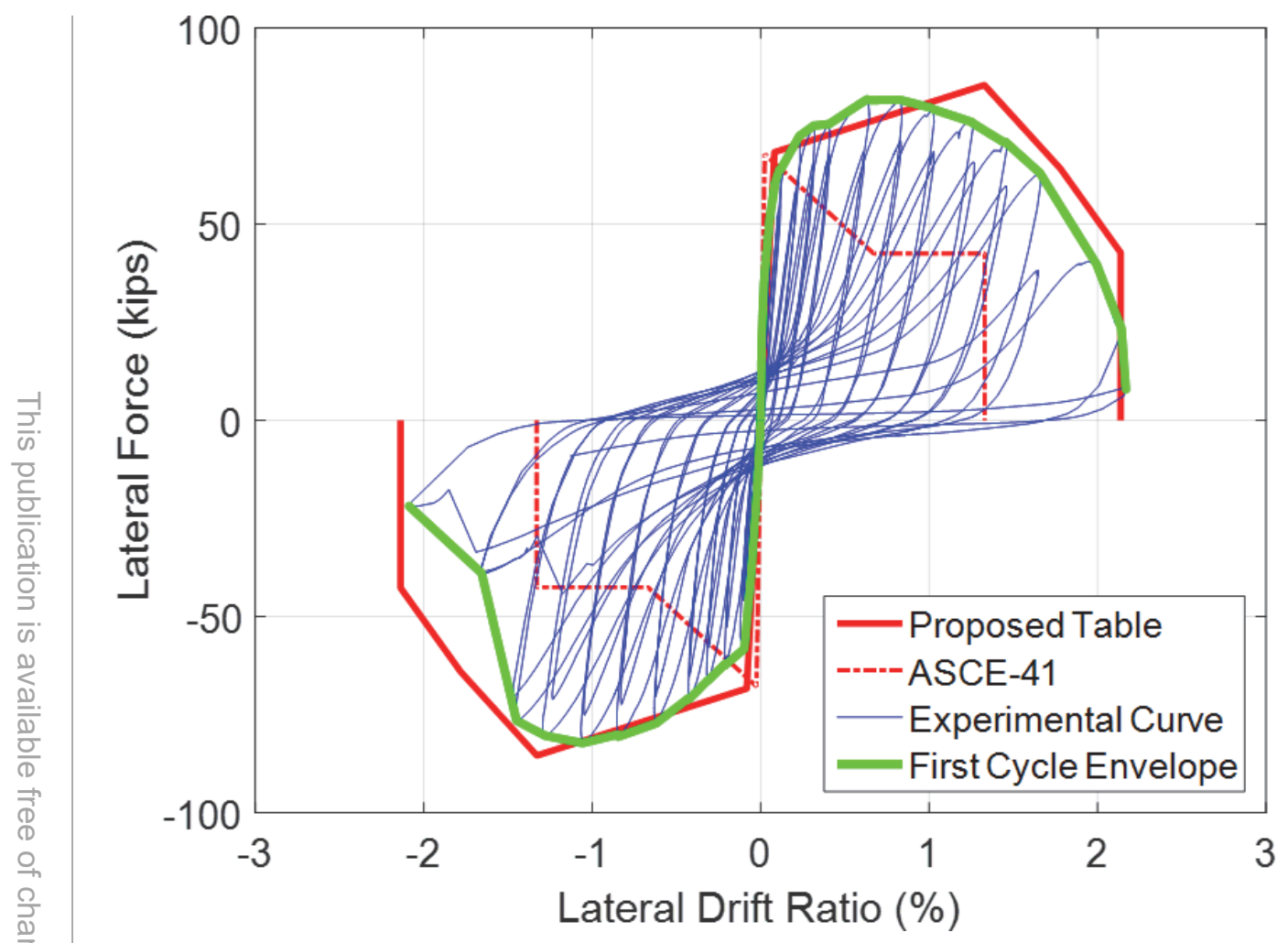

(a) Wall UT-PBS-03

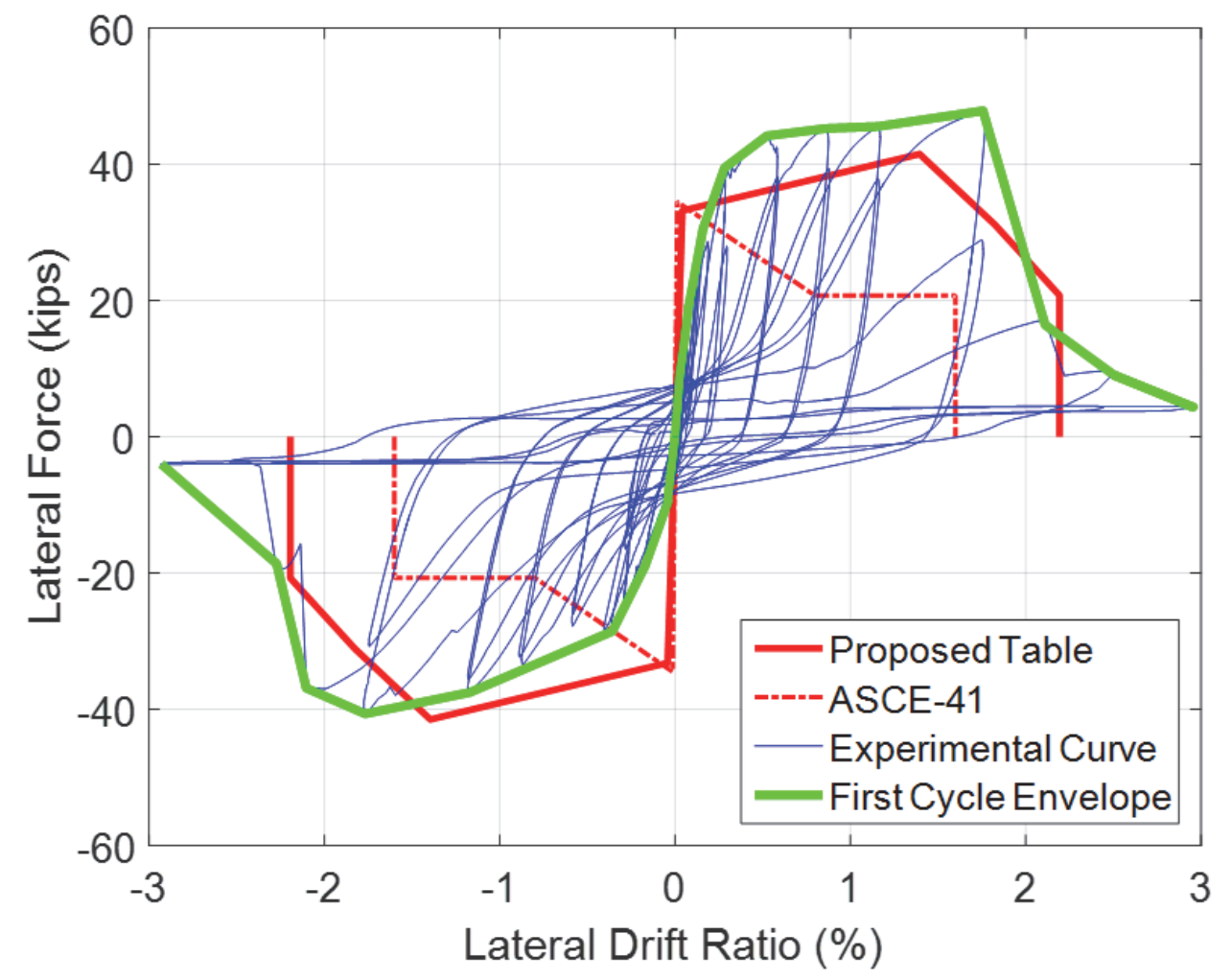

(b) Wall UT-PBS-04

Figure E-2 Backbone load-displacement curves for flexure-dominated walls. 


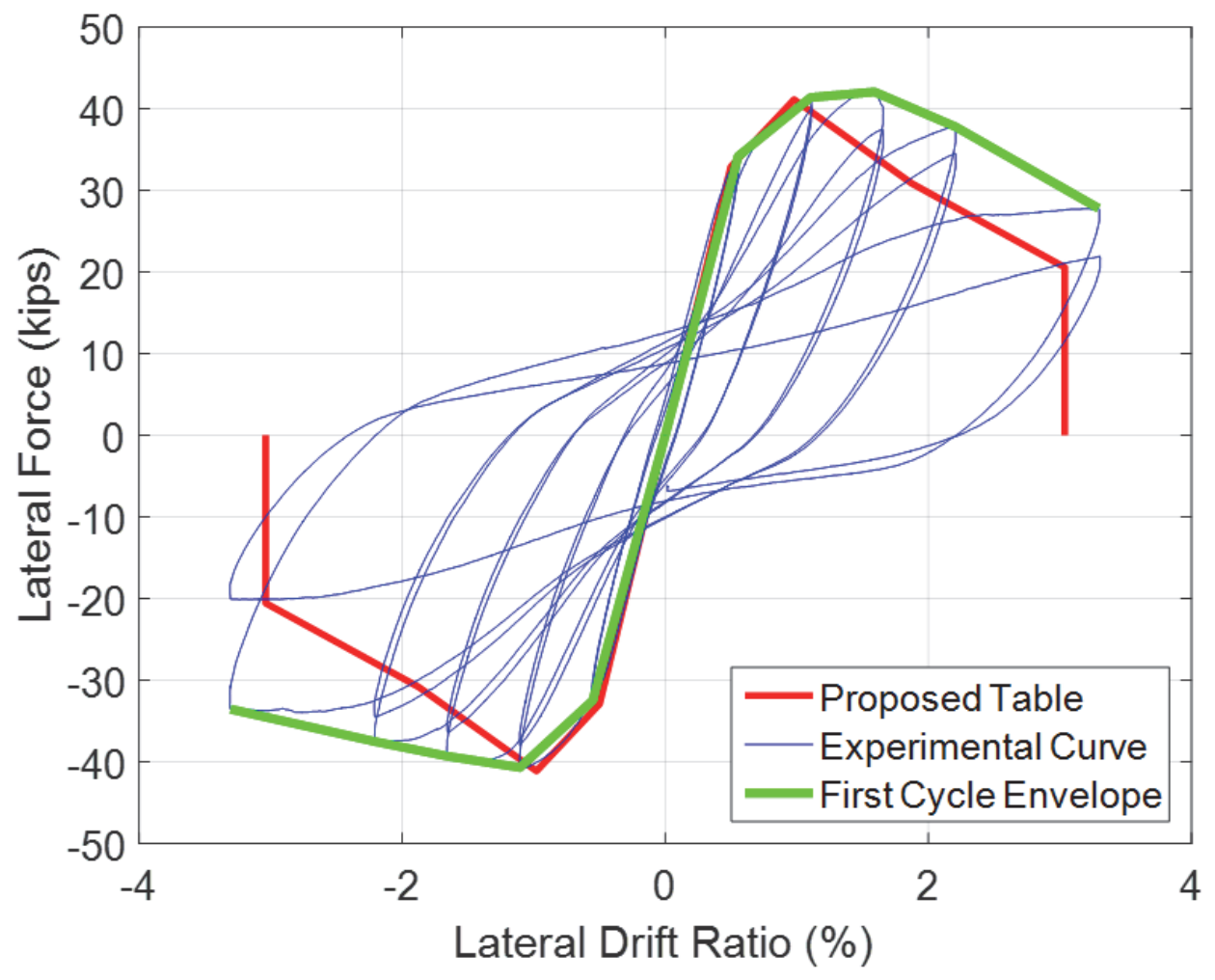

(c) Wall WSU-W-1A

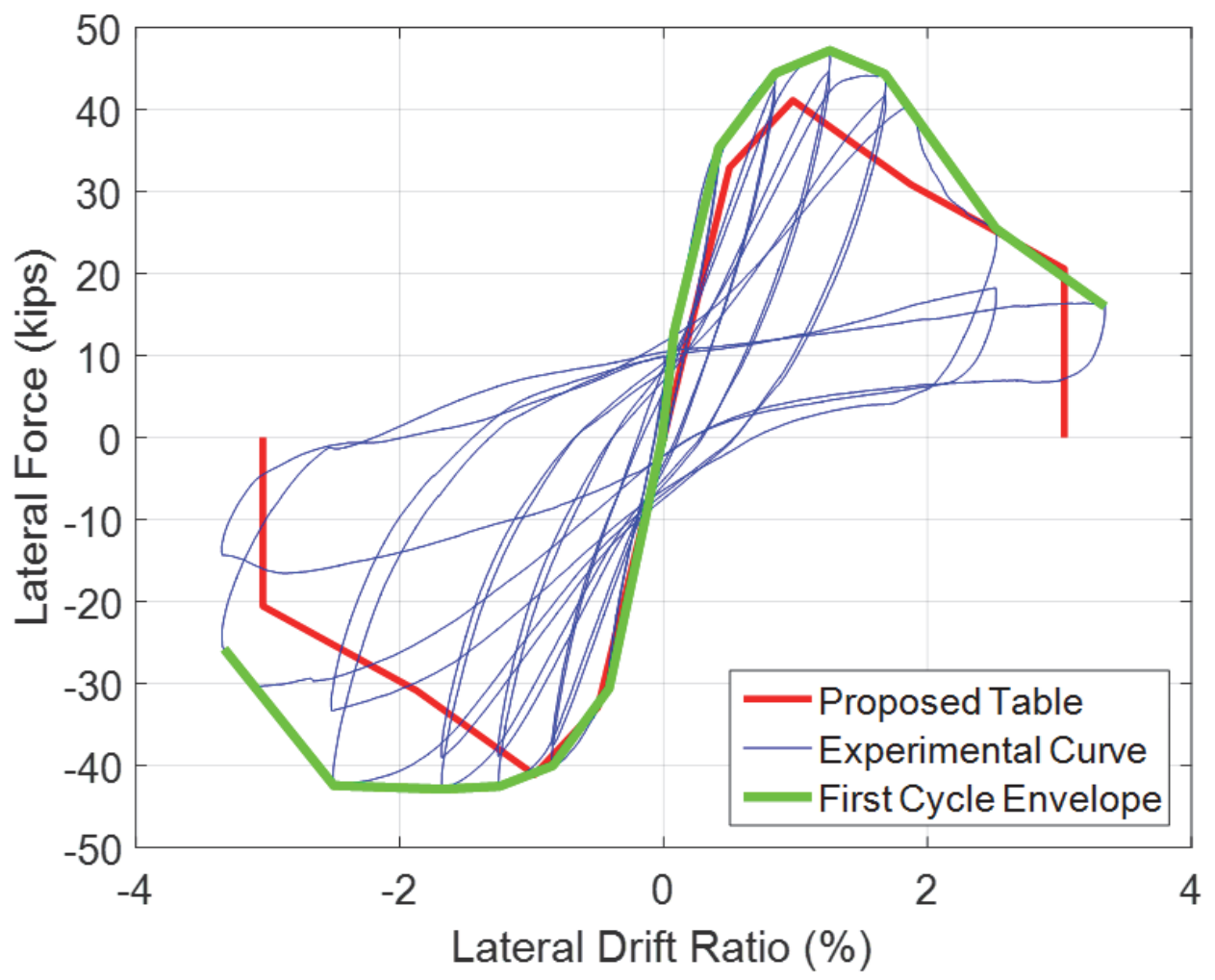

(d) Wall WSU-W-1B

Figure E-2 Backbone load-displacement curves for flexure-dominated walls (continued). 


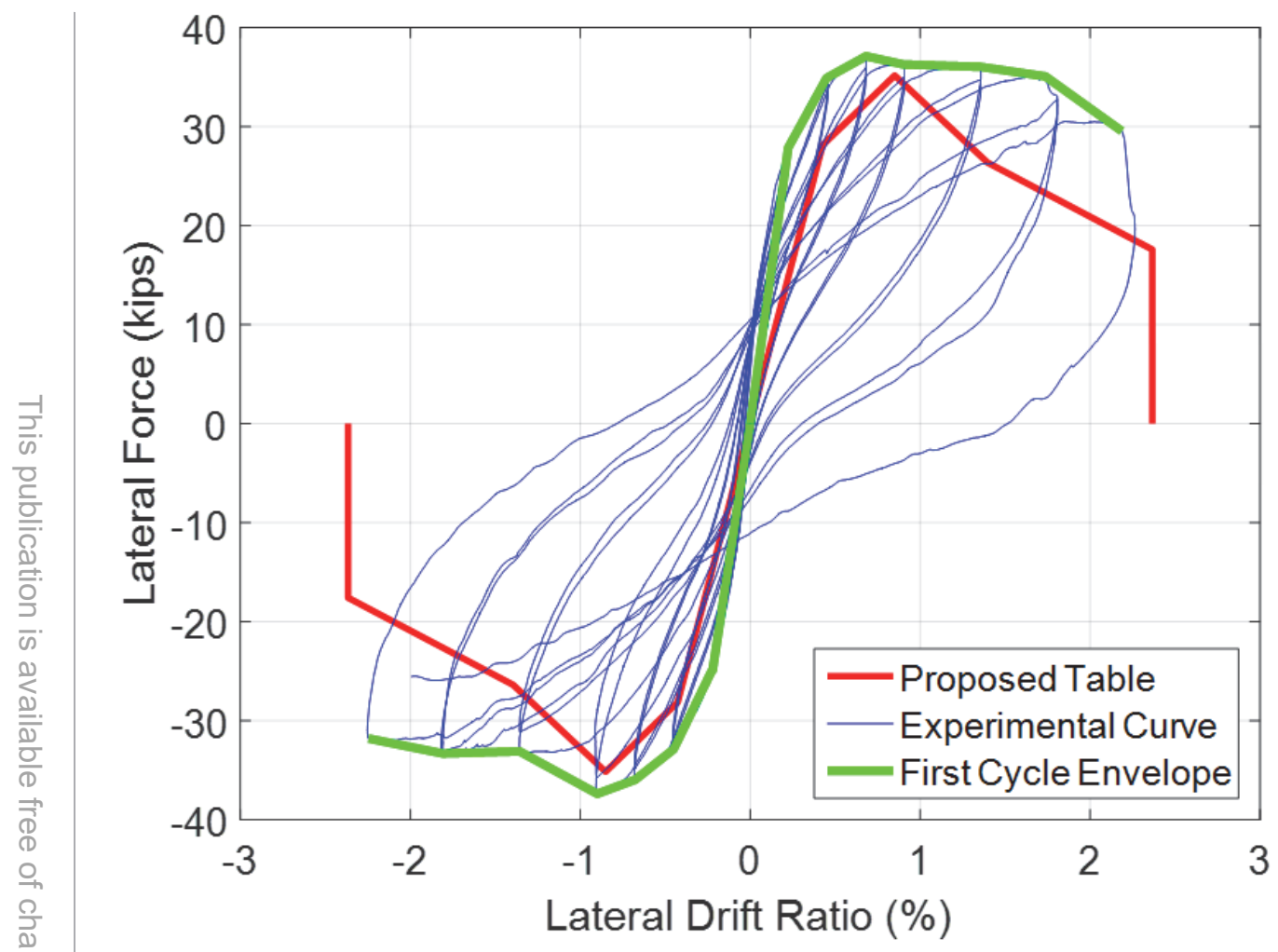

(e) Wall WSU-W-2A

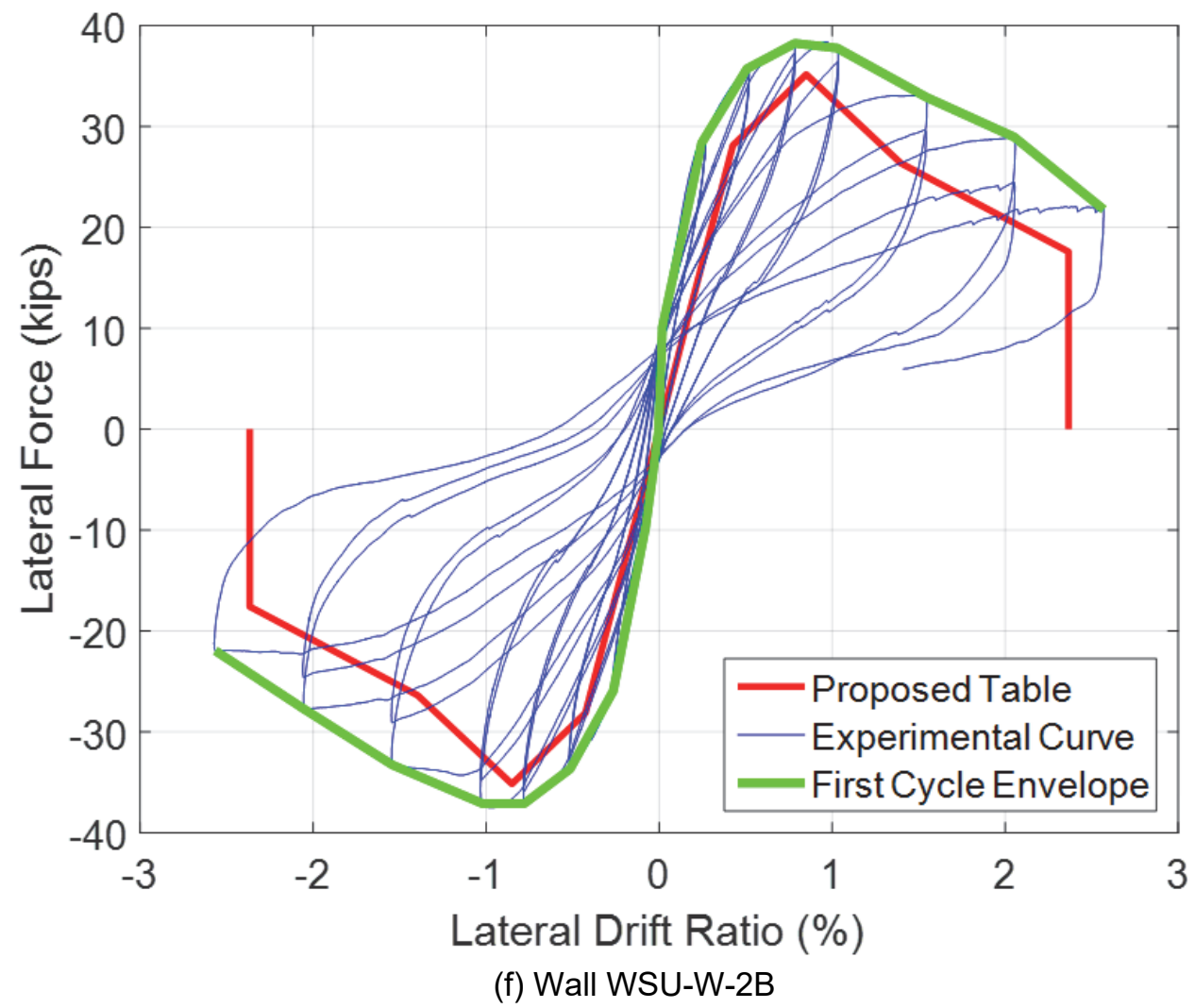

Figure E-2 Backbone load-displacement curves for flexure-dominated walls (continued). 


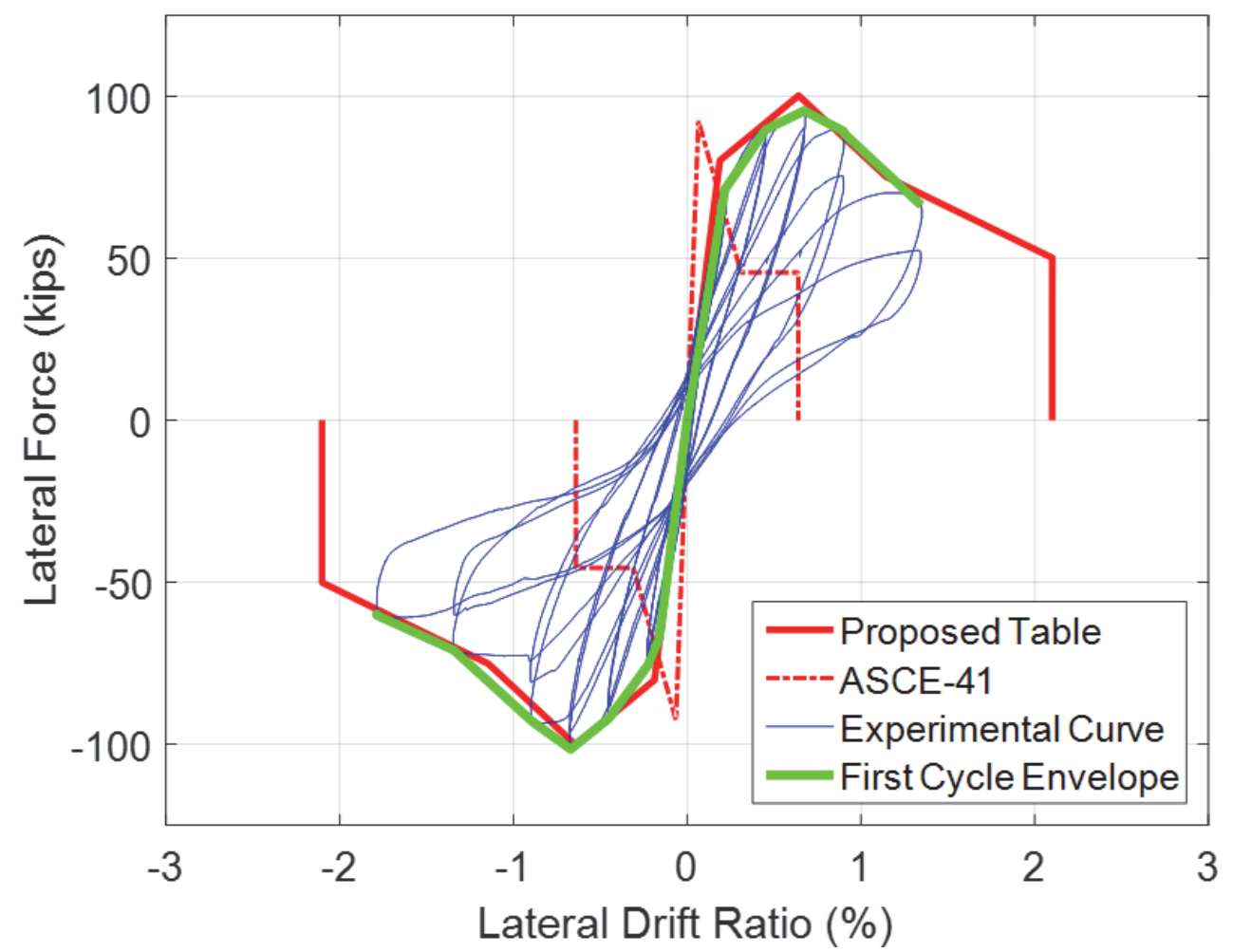

(g) Wall WSU-W-04

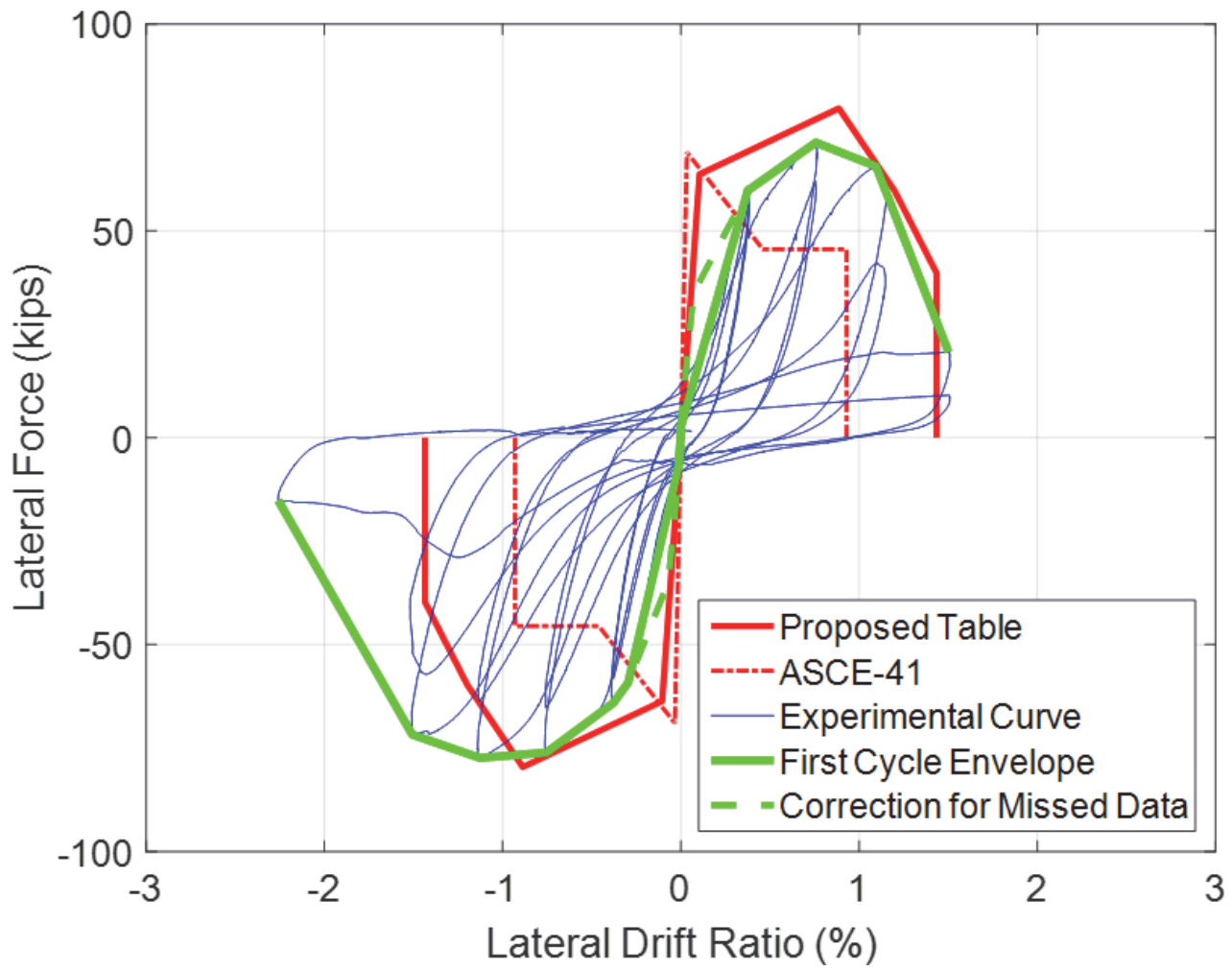

(h) Wall WSU-W-05

Figure E-2 Backbone load-displacement curves for flexure-dominated walls (continued). 

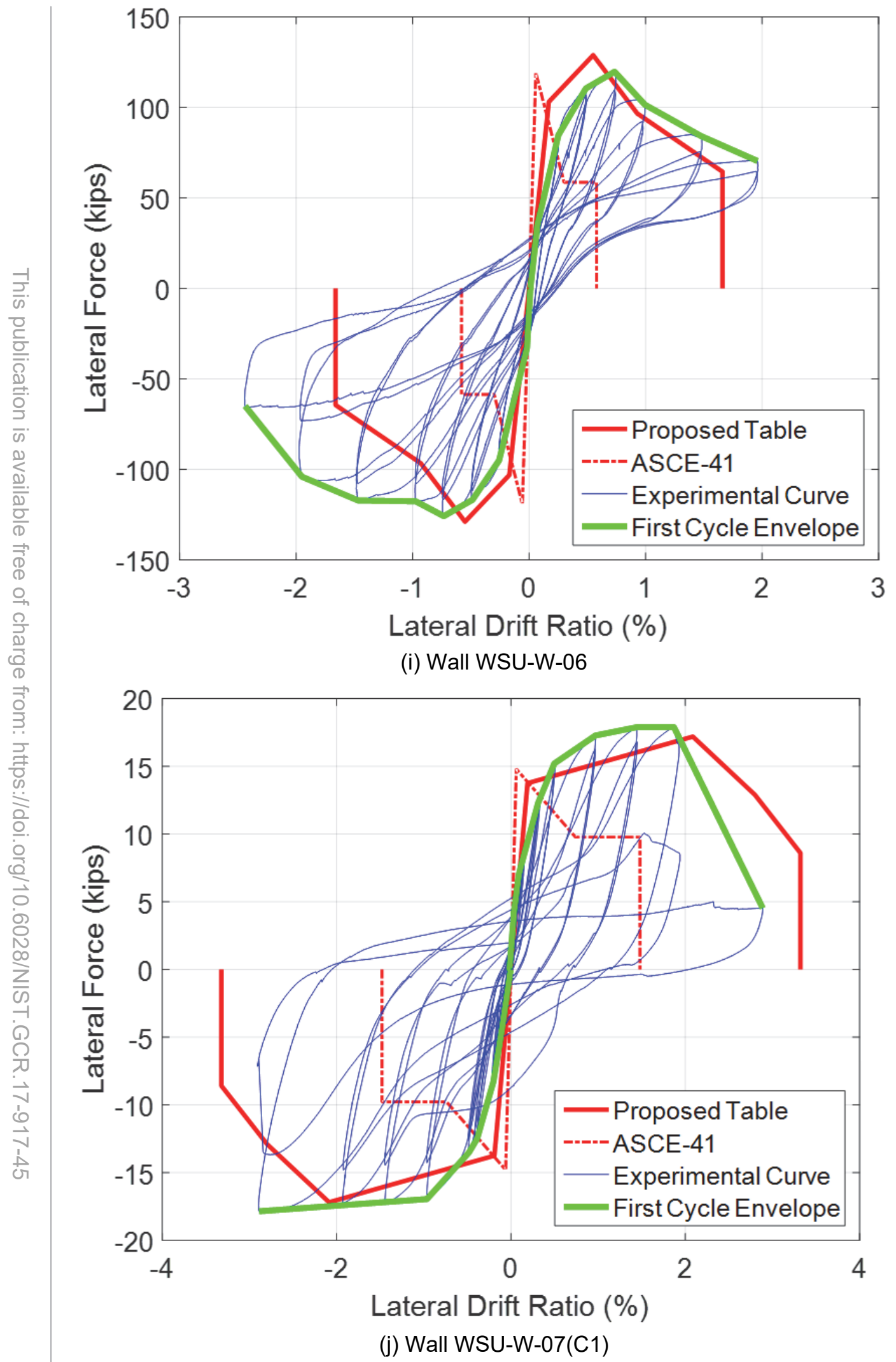

Figure E-2 Backbone load-displacement curves for flexure-dominated walls (continued). 


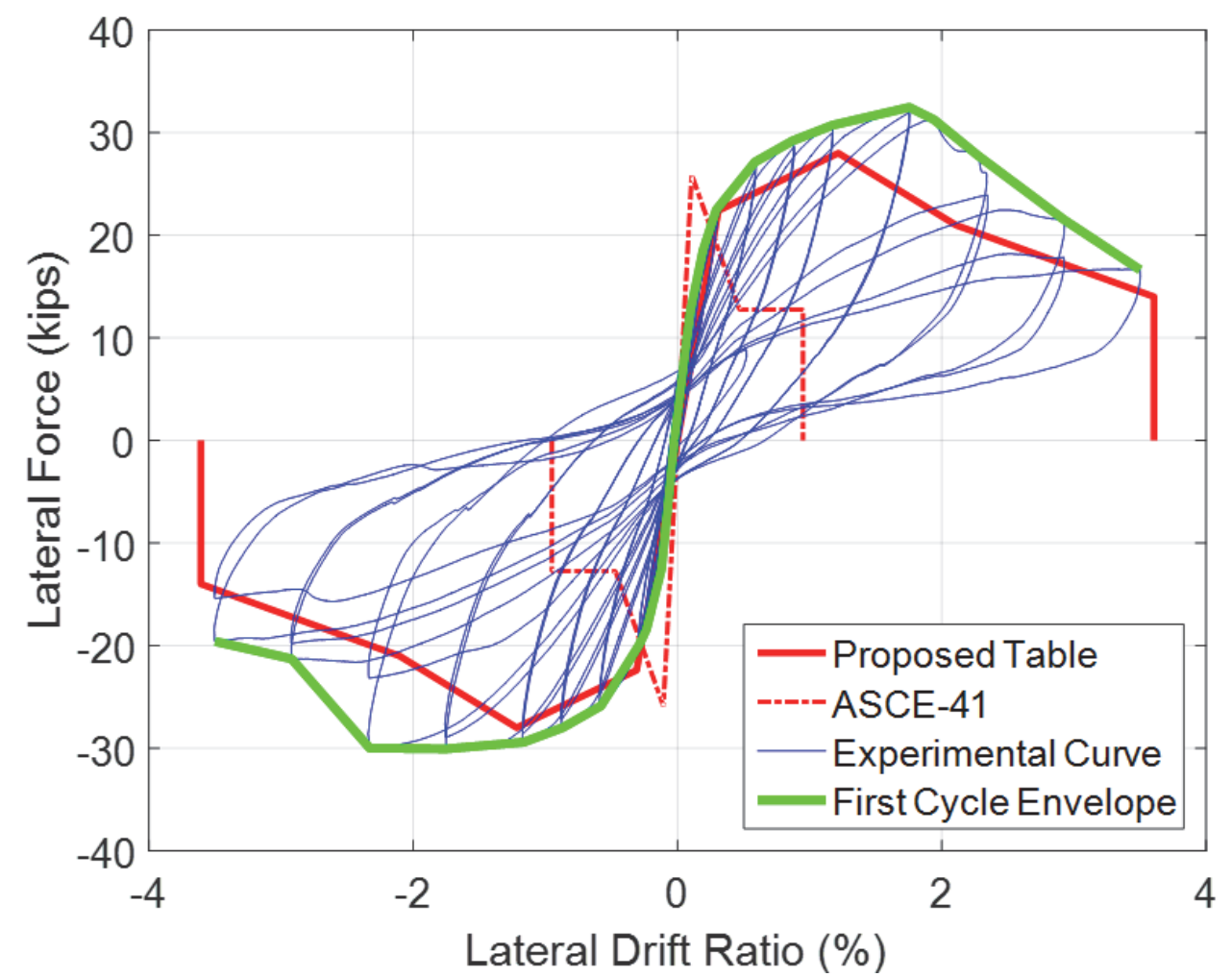

(k) Wall WSU-W-08(C2)

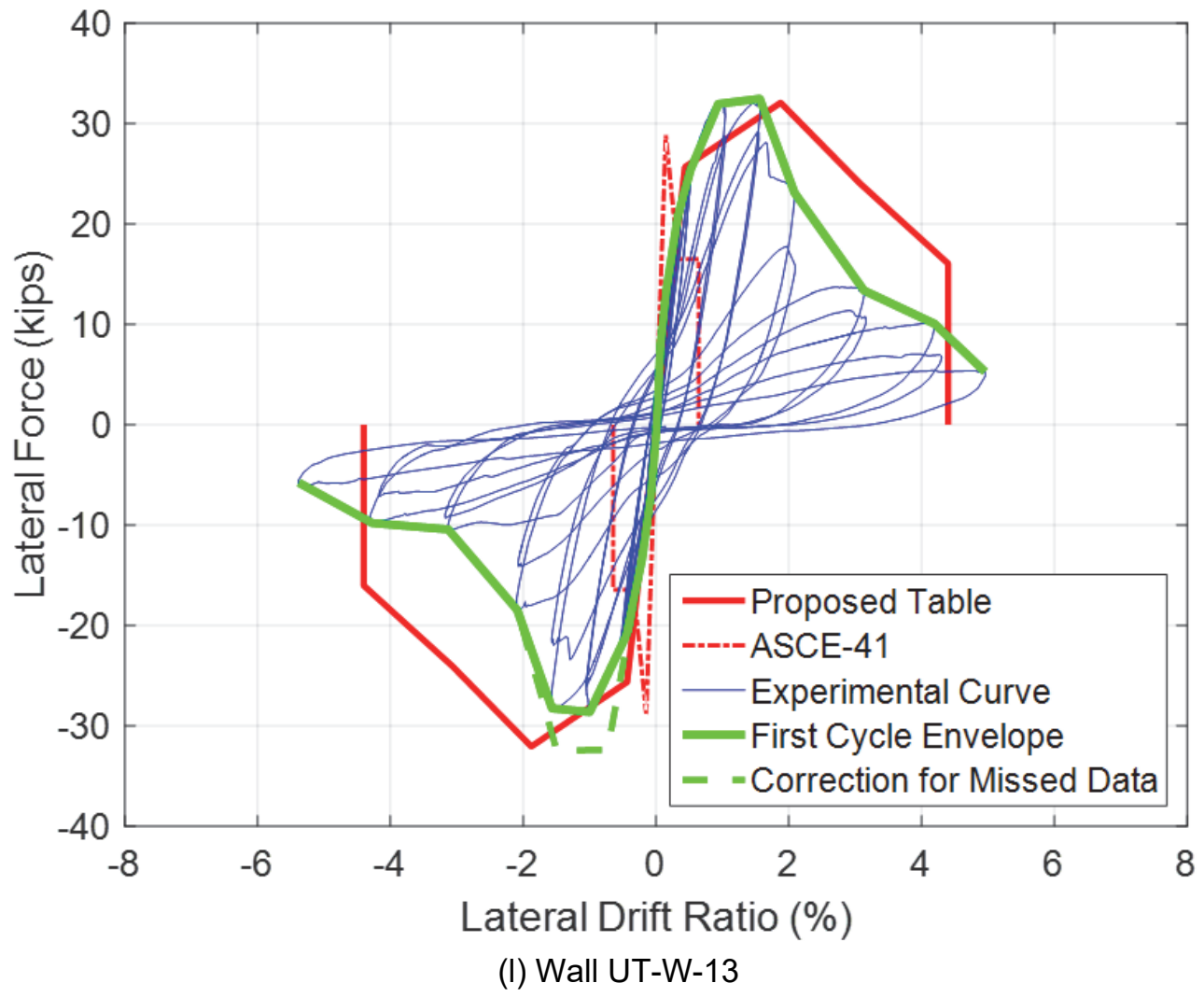

Figure E-2 Backbone load-displacement curves for flexure-dominated walls (continued). 


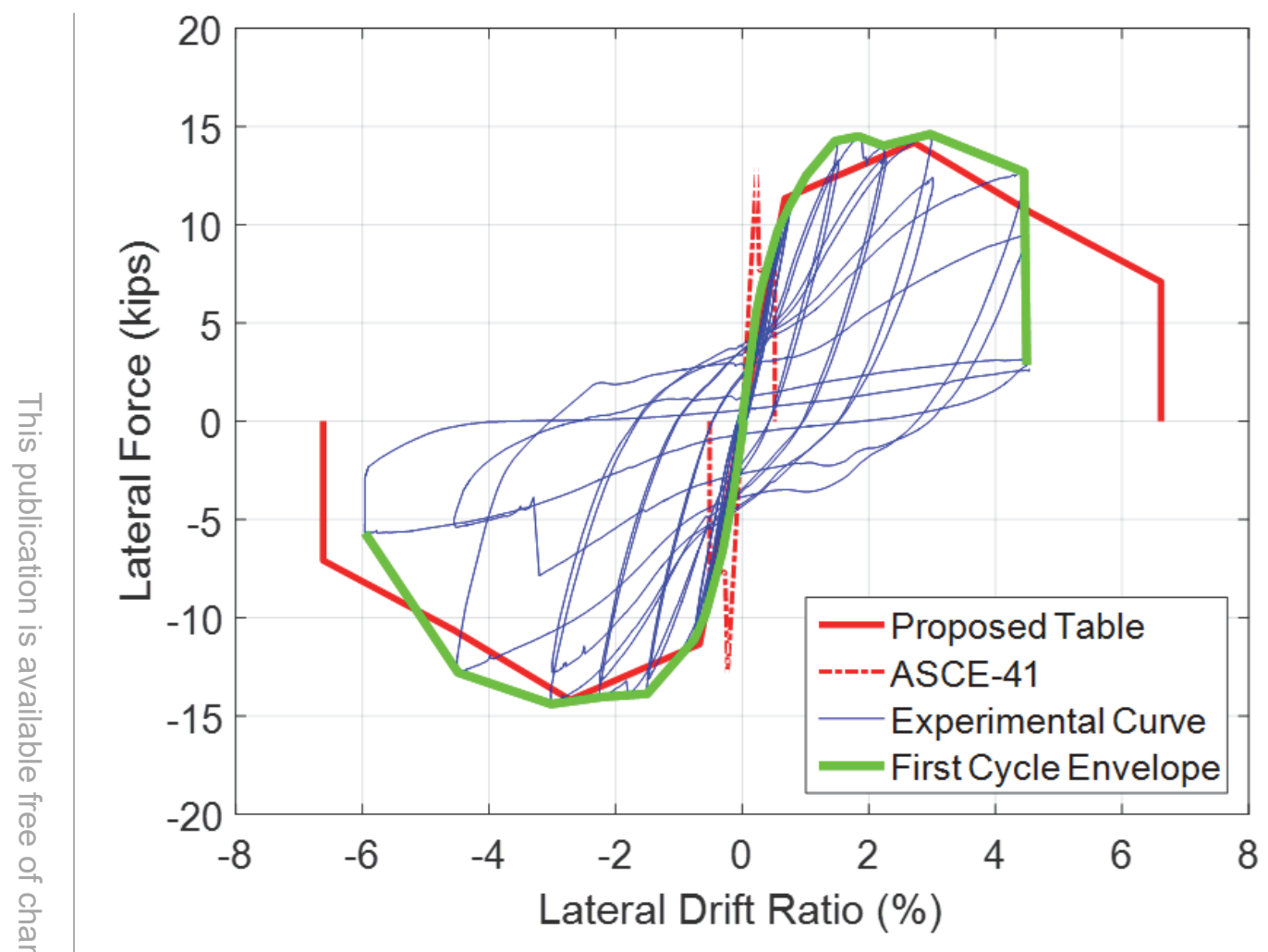

(m) Wall UT-W-17

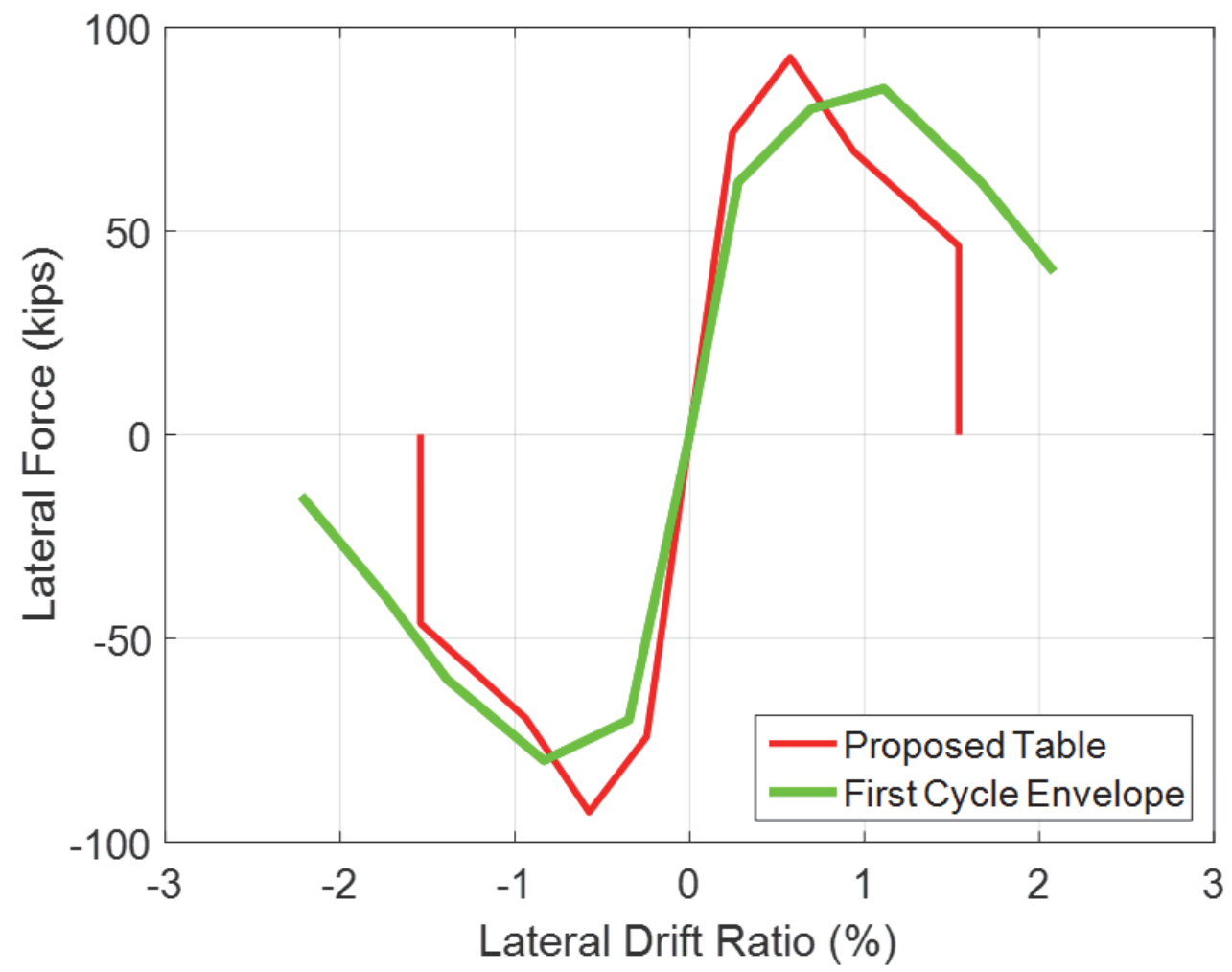

(n) Wall CU-Boulder-1

Figure E-2 Backbone load-displacement curves for flexure-dominated walls (continued). 


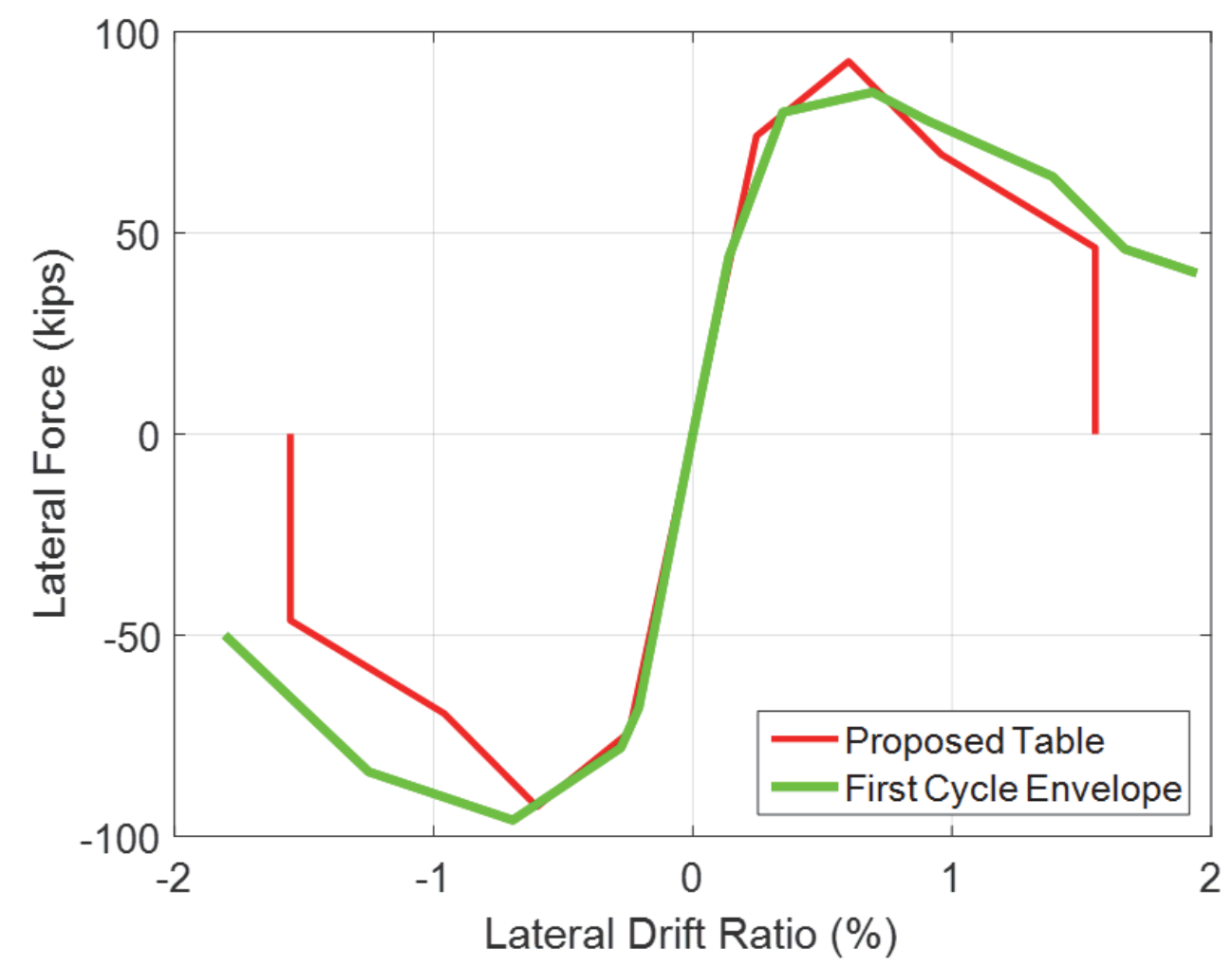

(o) Wall CU-Boulder-2

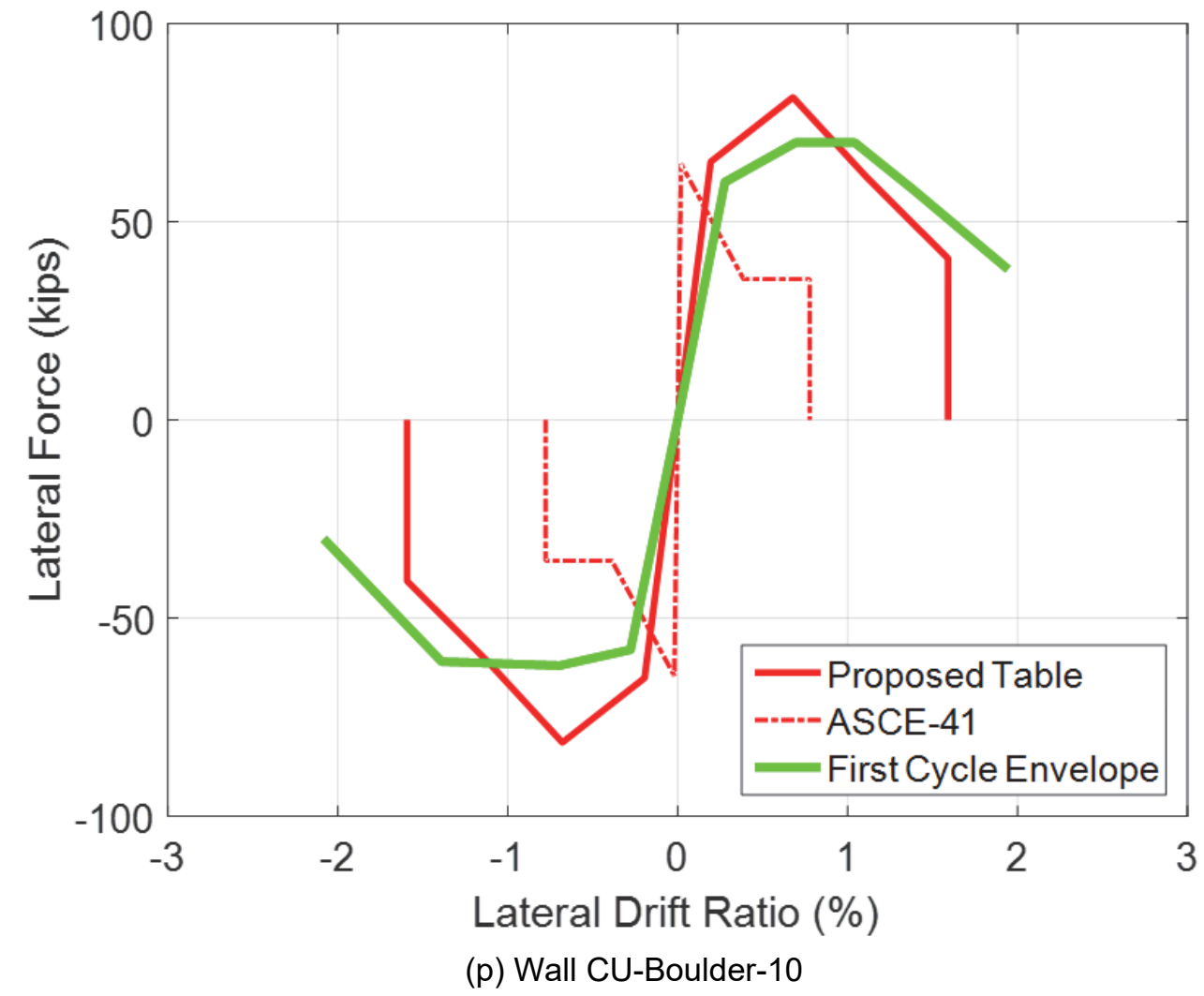

Figure E-2 Backbone load-displacement curves for flexure-dominated walls (continued). 


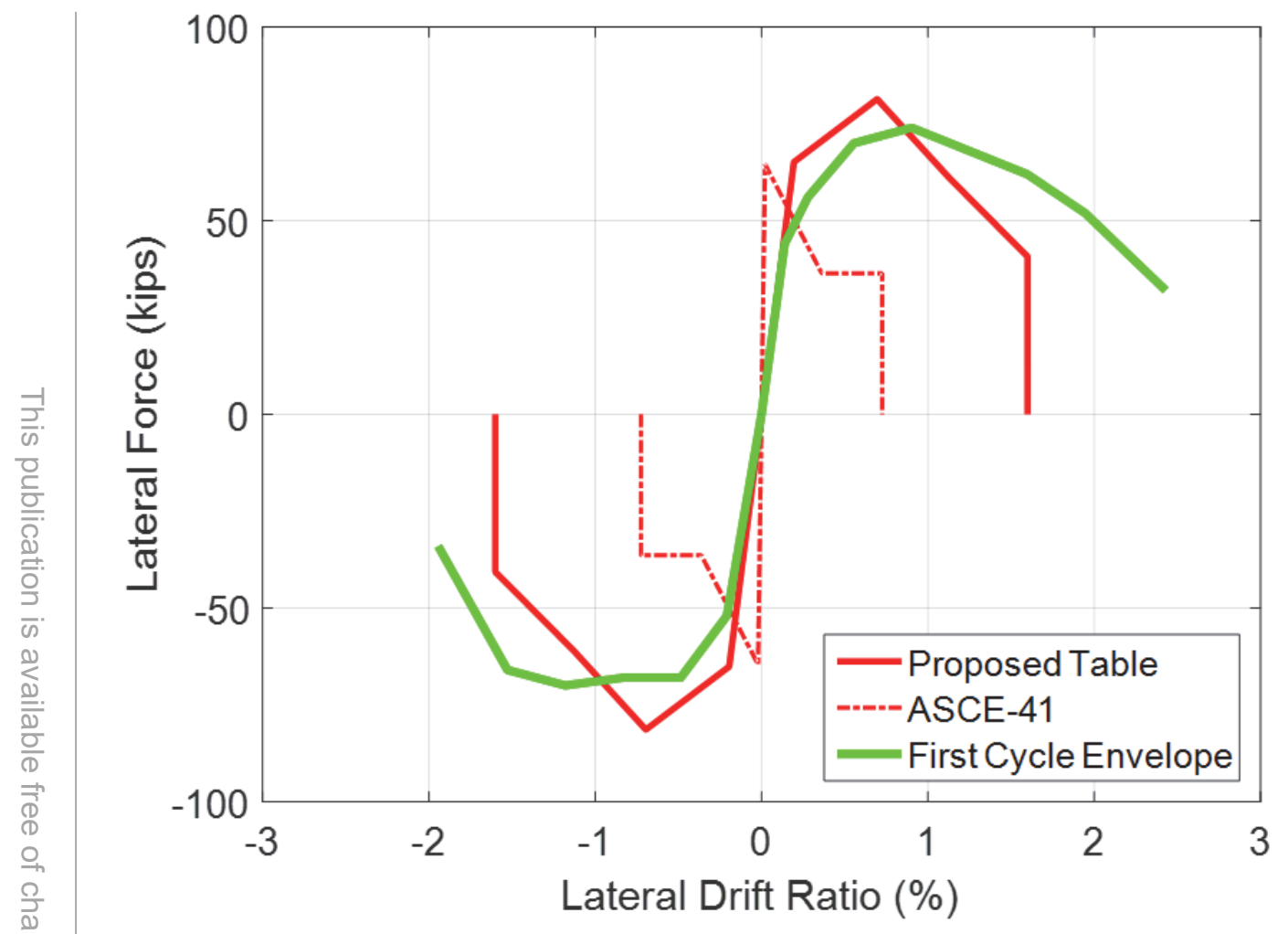

(q) Wall CU-Boulder-12

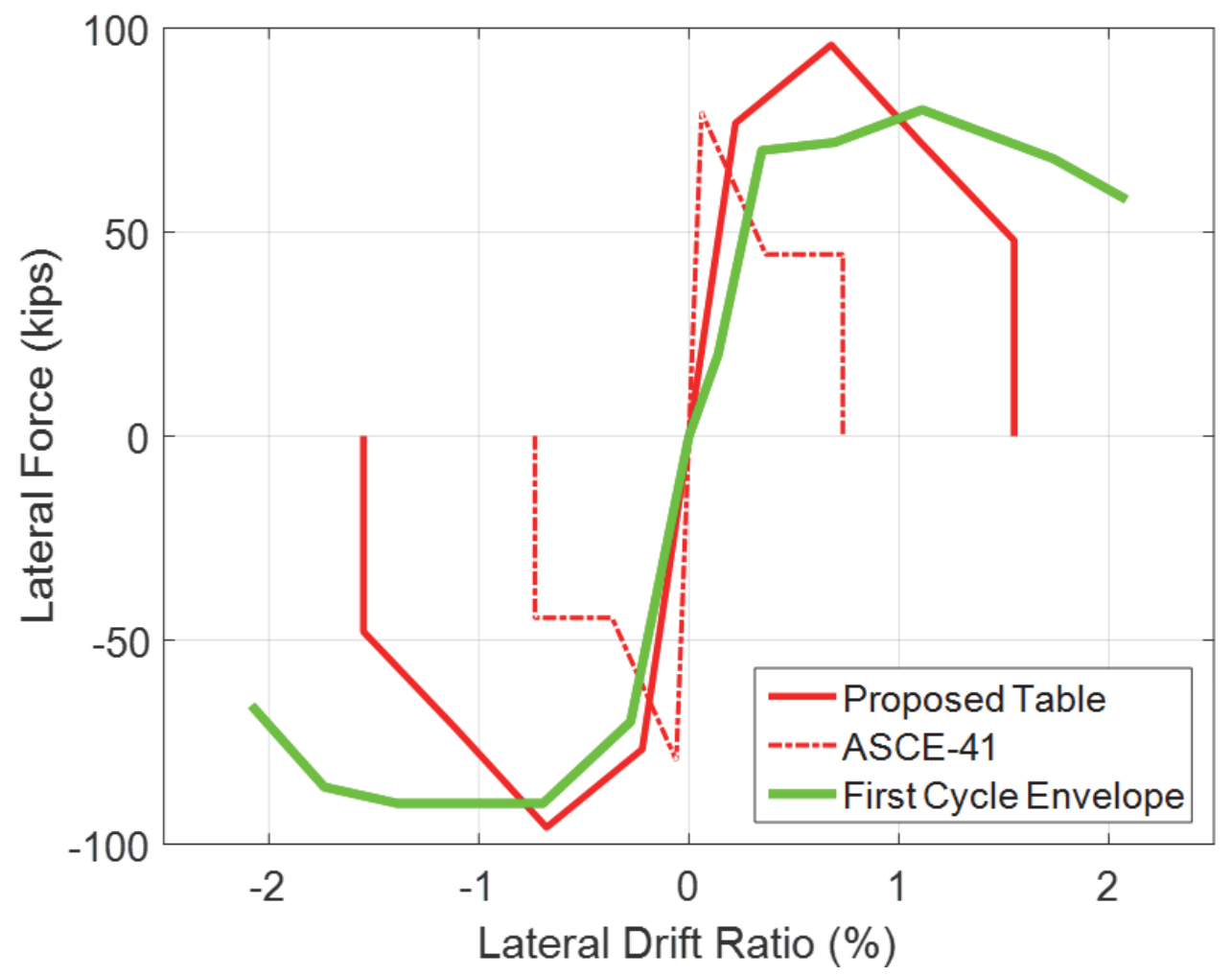

(r) Wall CU-Boulder-15

Figure E-2 Backbone load-displacement curves for flexure-dominated walls (continued). 


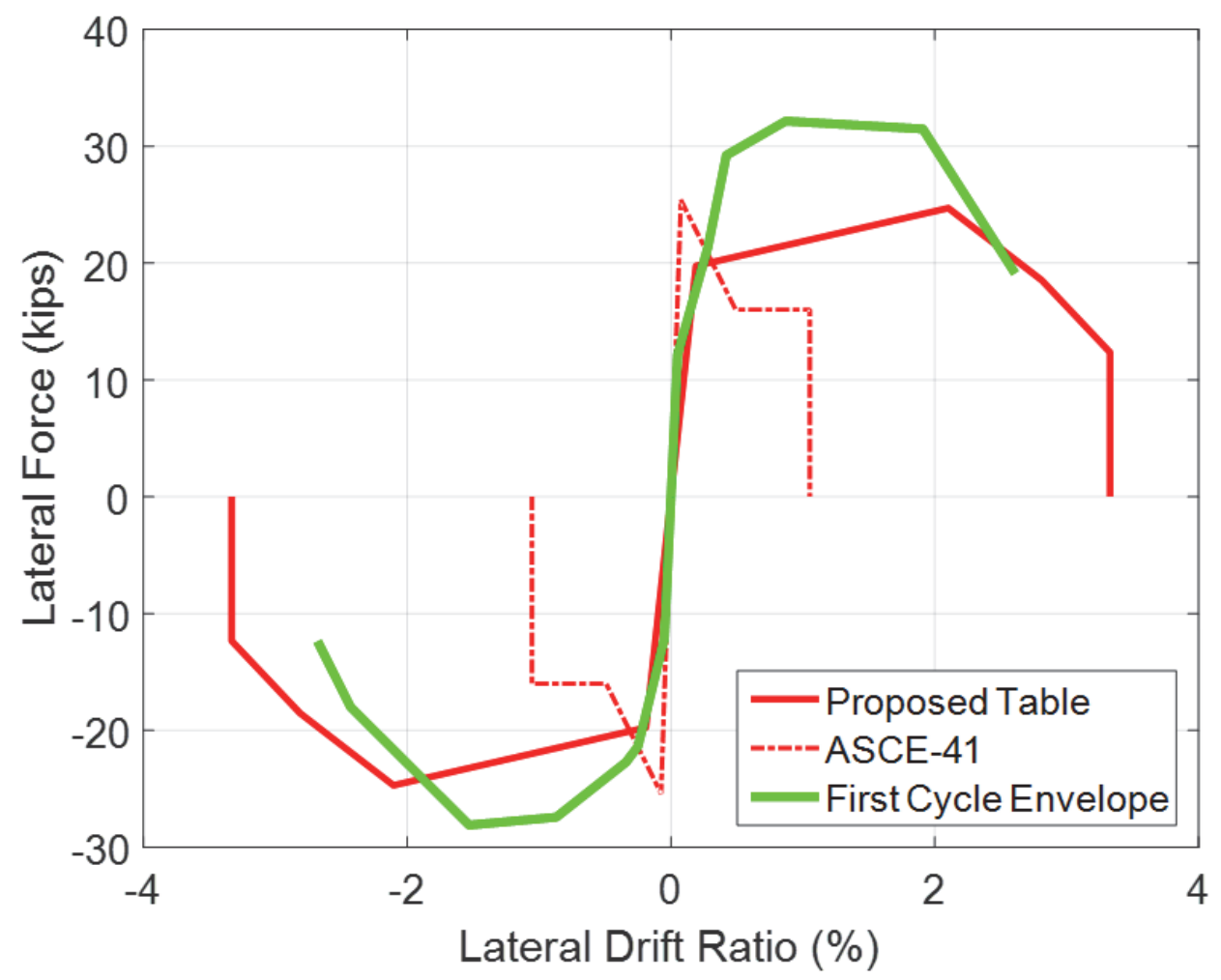

(s) Wall McMaster-1

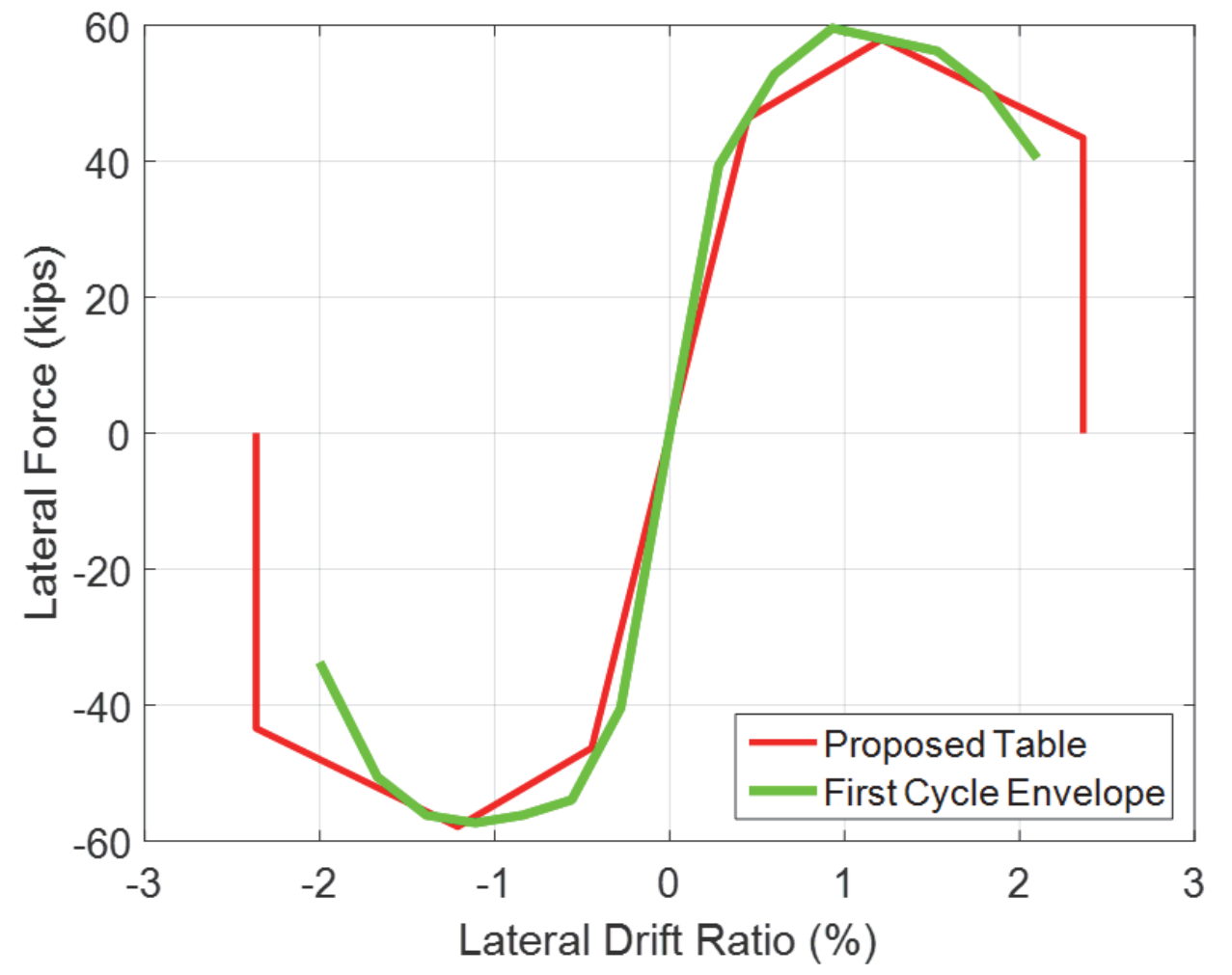

(t) Wall McMaster-2

Figure E-2 Backbone load-displacement curves for flexure-dominated walls (continued). 


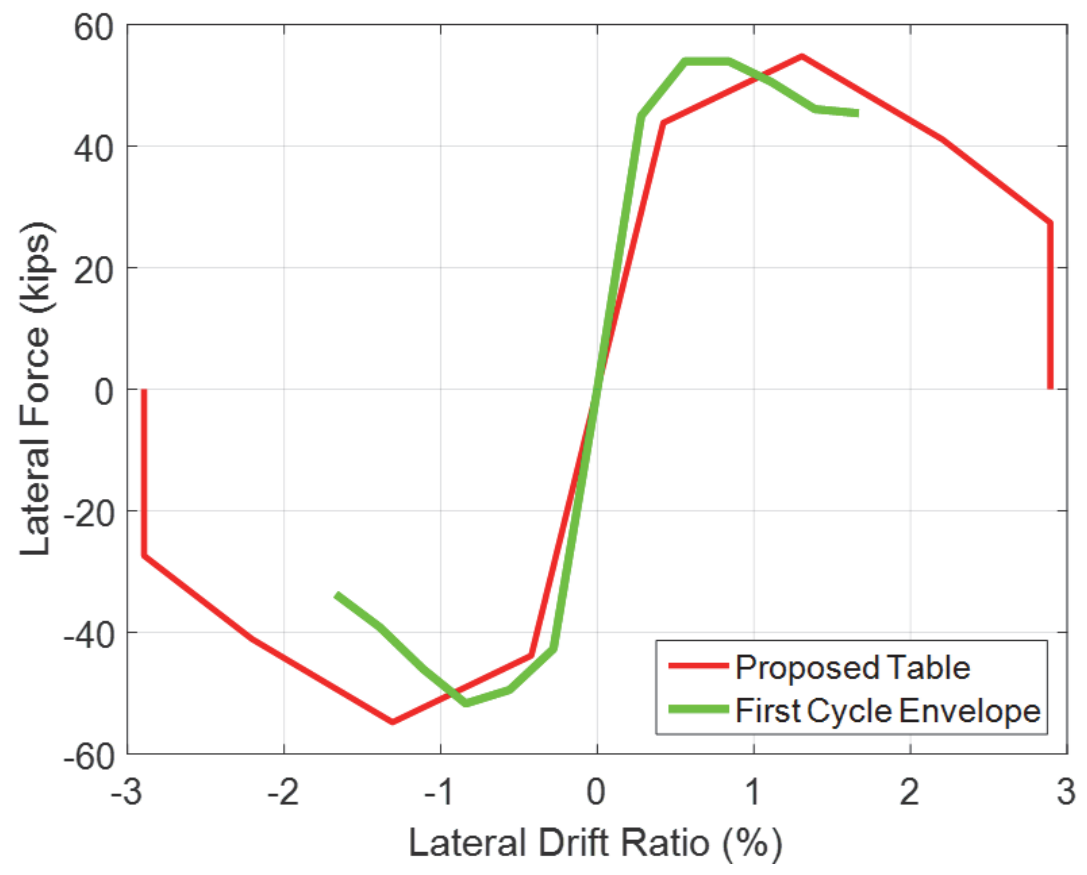

(u) Wall McMaster-3

Figure E-2 Backbone load-displacement curves for flexure-dominated walls (continued).

\section{E.2 Shear-Dominated Walls}

Table E-2 Shear-Dominated Wall Specimens

\begin{tabular}{|c|c|c|c|c|c|c|c|c|c|}
\hline Specimen & $\begin{array}{l}\text { Height } \\
\text { (in) }\end{array}$ & $\begin{array}{l}\text { Length } \\
\text { (in) }\end{array}$ & $\begin{array}{l}\text { Width } \\
\text { (in) }\end{array}$ & $\begin{array}{c}f_{m}^{\prime} \\
(\mathrm{ksi})\end{array}$ & $\begin{array}{c}P_{u} \\
\text { (kips) }\end{array}$ & $\begin{array}{c}M u l \\
\left(V_{u} d_{v}\right) \\
\end{array}$ & $\begin{array}{c}\text { Vertical } \\
\text { Bar }\end{array}$ & $\begin{array}{c}\text { Horizontal } \\
\text { Bar }\end{array}$ & $\begin{array}{r}f_{y} \\
\text { (ksi) }\end{array}$ \\
\hline Auckland-2 & 71 & 71 & 5.512 & 2.553 & 0 & 1 & \#6@16in & \#2@63in & 47.1 \\
\hline Auckland-4 & 71 & 71 & 5.512 & 2.466 & 0 & 1 & \#6@16in & \#3@31in & 46.4 \\
\hline Auckland-7 & 71 & 71 & 5.512 & 2.727 & 28.33 & 1 & \#6@16in & \#2@16in & 47.1 \\
\hline Auckland-8 & 71 & 71 & 5.512 & 2.727 & 14.16 & 1 & \#6@16in & \#2@16in & 47.1 \\
\hline Auckland-9 & 142 & 71 & 5.512 & 3.524 & 14.16 & 2 & \#8@16in & \#2@16in & 47.1 \\
\hline Auckland-10 & 71 & 118 & 5.512 & 3.524 & 23.60 & 0.6 & \#6@16in & \#2@16in & 47.1 \\
\hline UT-PBS-01 & 72 & 72 & 7.625 & 3.113 & 17.09 & 0.5 & \#6@8in & \#4@8in & 62.4 \\
\hline UT-PBS-02 & 72 & 72 & 7.625 & 3.113 & 128.2 & 0.5 & \#6@16in & \#4@16in & 62.4 \\
\hline CU-Boulder-3 & 72 & 72 & 5.625 & 2.958 & 109.4 & 1 & \#7@16in & \#3@16in & 57.0 \\
\hline CU-Boulder-4 & 72 & 72 & 5.625 & 2.637 & 0 & 1 & \#7@16in & \#3@16in & 57.0 \\
\hline CU-Boulder-5 & 72 & 72 & 5.625 & 2.637 & 40.50 & 1 & \#7@16in & \#3@16in & 57.0 \\
\hline CU-Boulder-7 & 72 & 72 & 5.625 & 3.243 & 40.50 & 1 & \#7@16in & \#3@16in & 57.0 \\
\hline CU-Boulder-9 & 72 & 72 & 5.625 & 3.243 & 109.4 & 1 & \#5@16in & \#3@16in & 57.0 \\
\hline CU-Boulder-13 & 72 & 72 & 5.625 & 3.342 & 109.4 & 1 & \#6@16in & \#4@16in & 67.0 \\
\hline CU-Boulder-14 & 72 & 72 & 5.625 & 3.342 & 109.4 & 1 & \#6@16in & \#3@16in & 57.0 \\
\hline CU-Boulder-16 & 72 & 72 & 5.625 & 2.475 & 109.4 & 1 & \#7@16in & \#4@16in & 67.0 \\
\hline
\end{tabular}




\section{E.2.1 Backbone Curves for Shear-Dominated Walls}

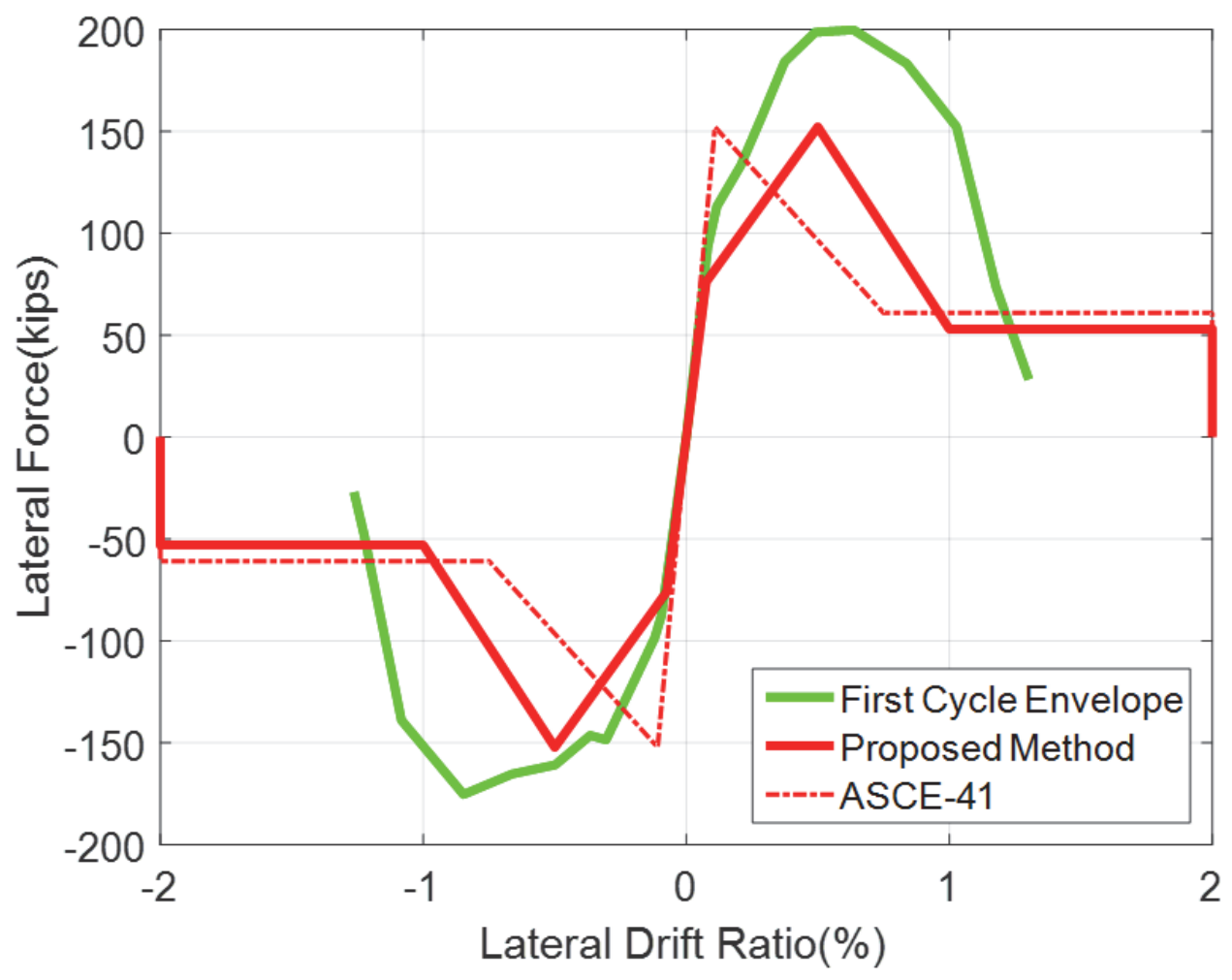

(a) UT-PBS-01

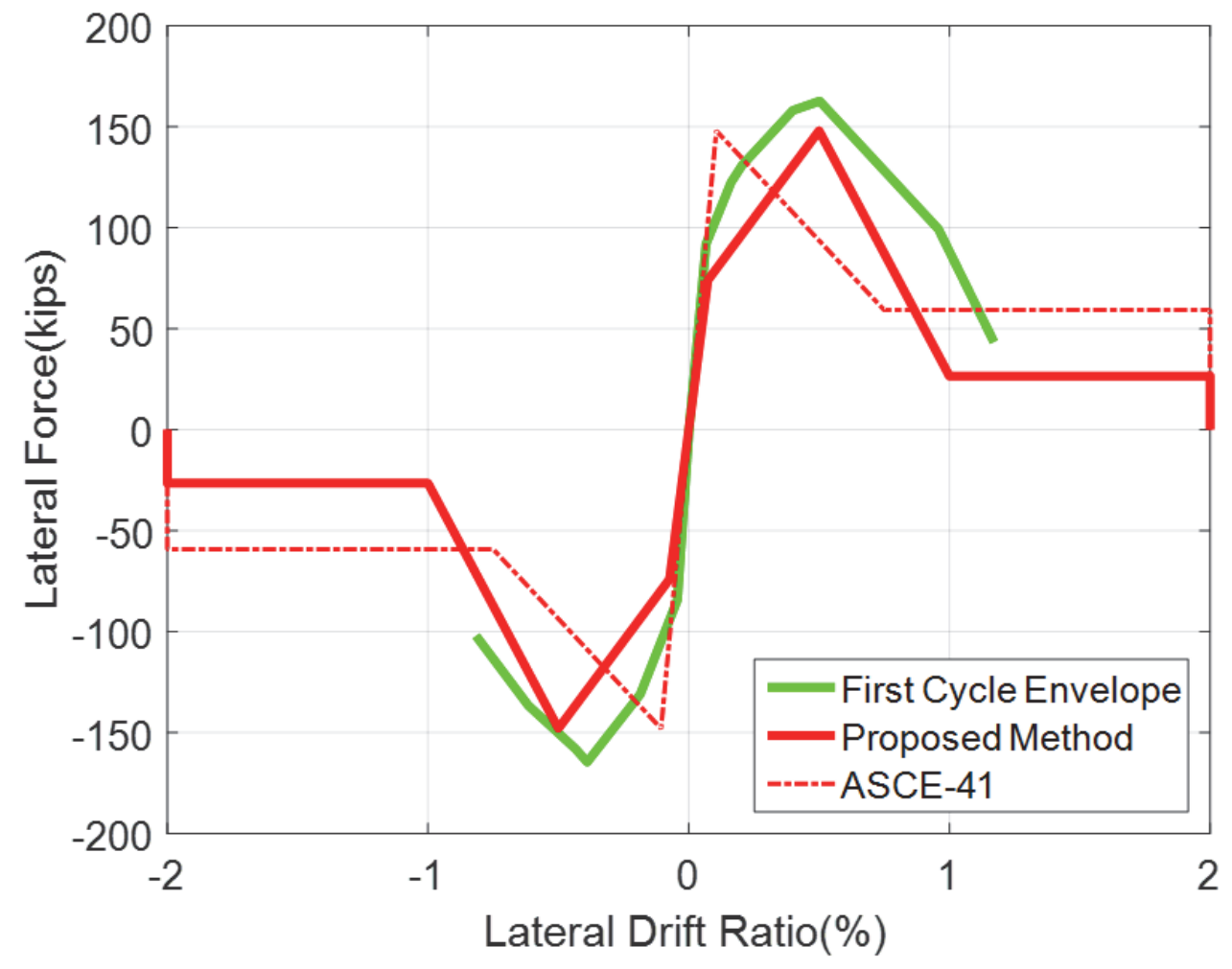

(b) UT-PBS-02

Figure E-3 Backbone lateral load-vs.-story drift ratio curves for shear-dominated walls. 


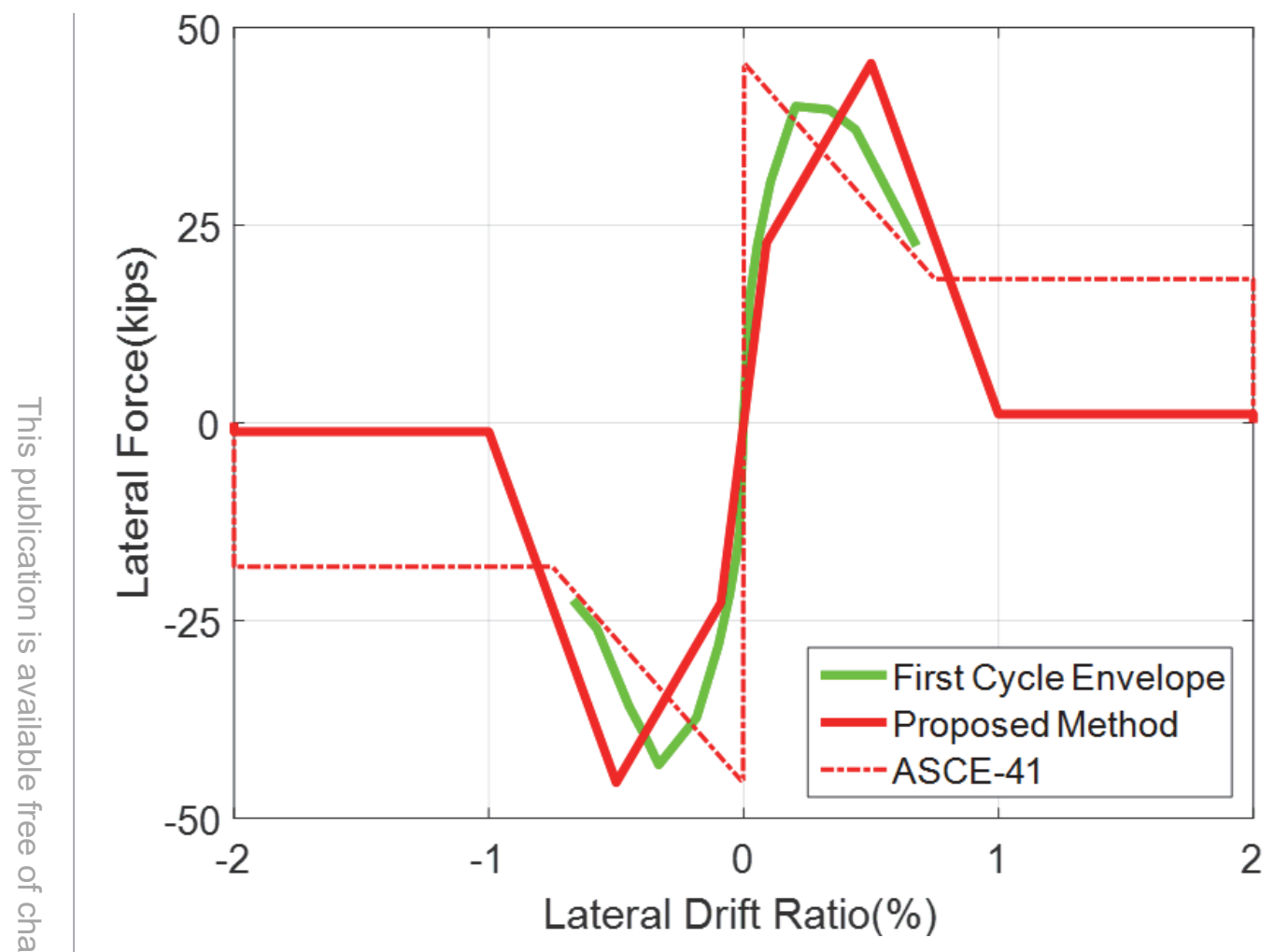

(c) Auckland-2

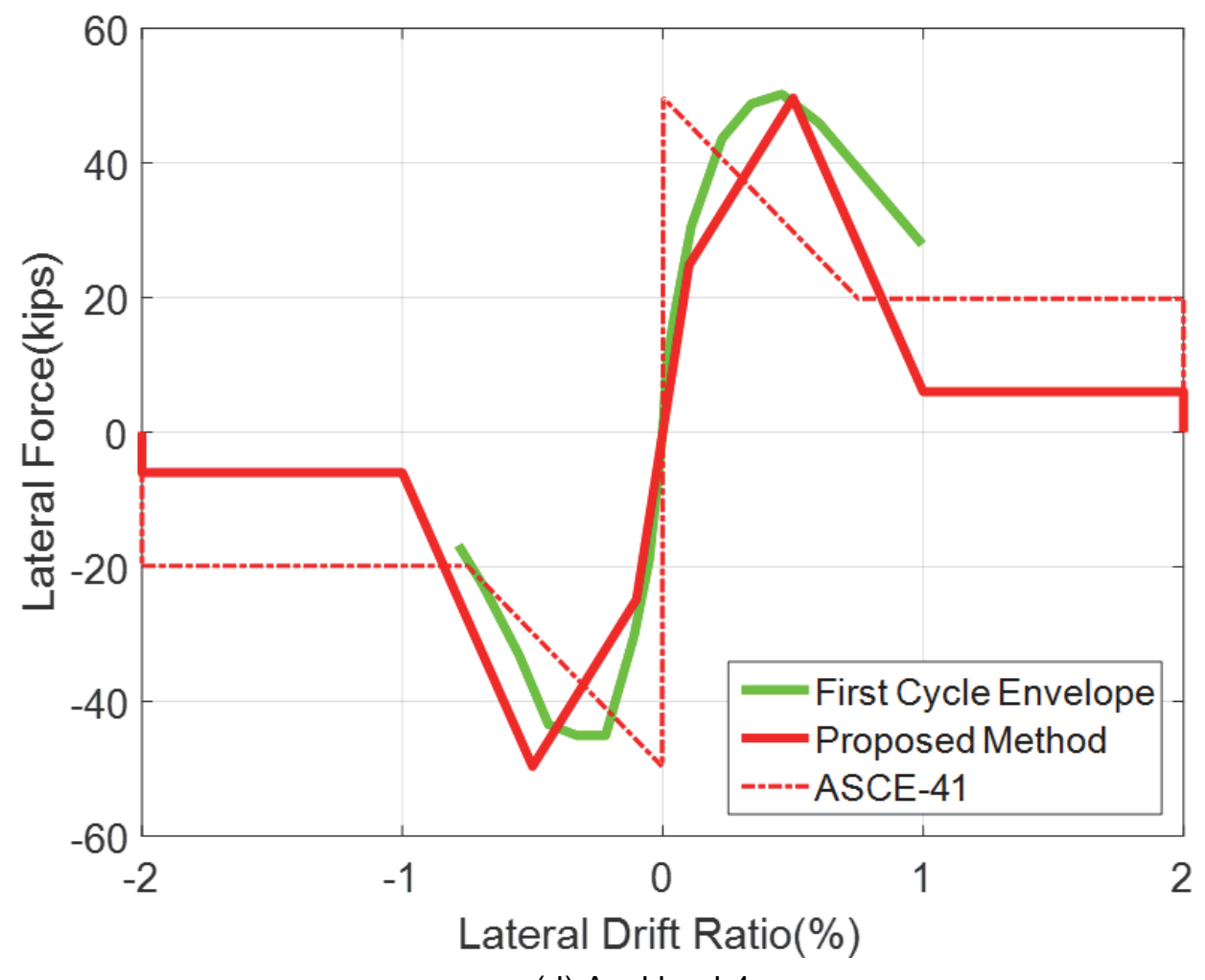

(d) Auckland-4

Figure E-3 Backbone lateral load-vs.-story drift ratio curves for shear-dominated walls (continued). 


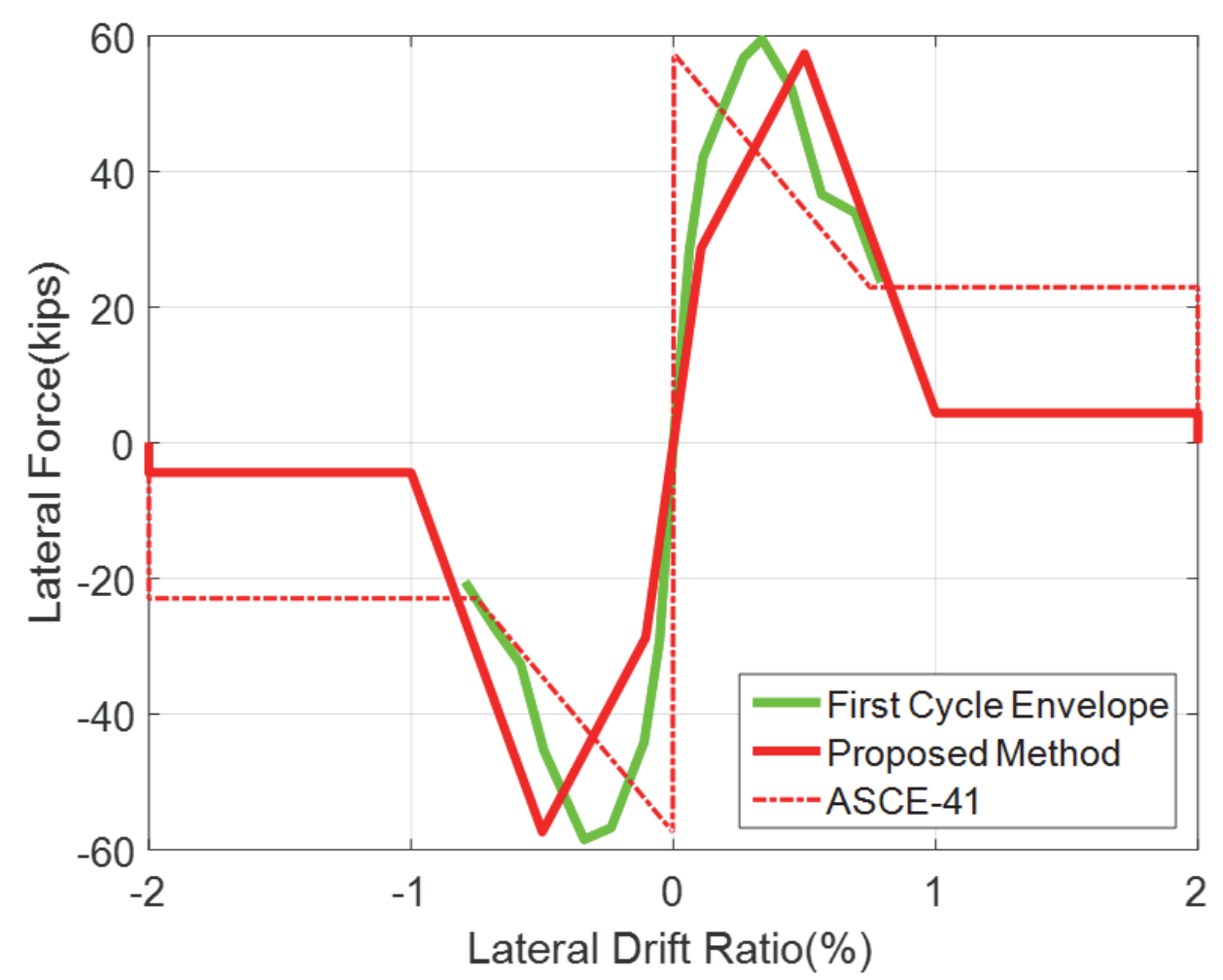

(e) Auckland-7

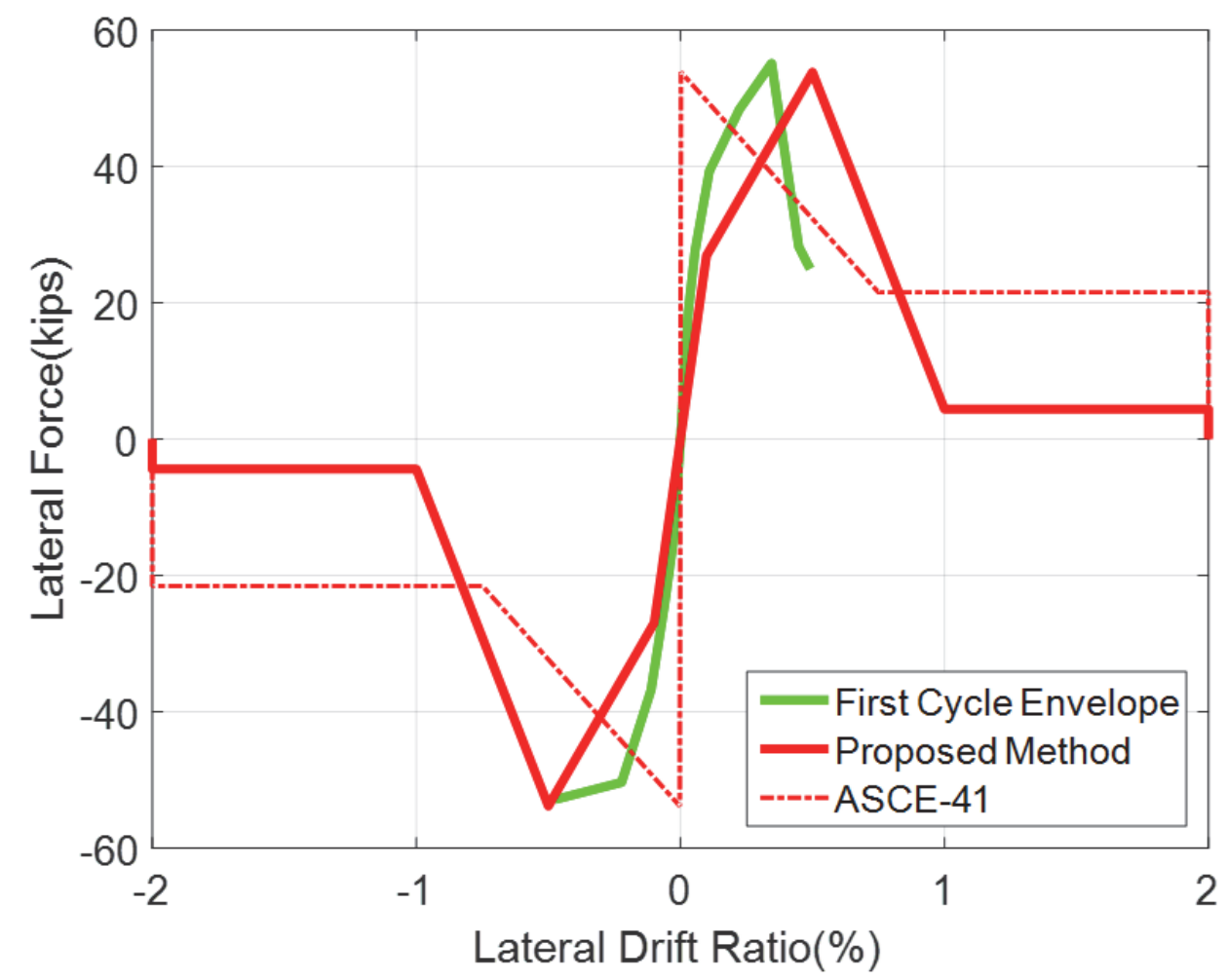

(f) Auckland-8

Figure E-3 Backbone lateral load-vs.-story drift ratio curves for shear-dominated walls (continued). 

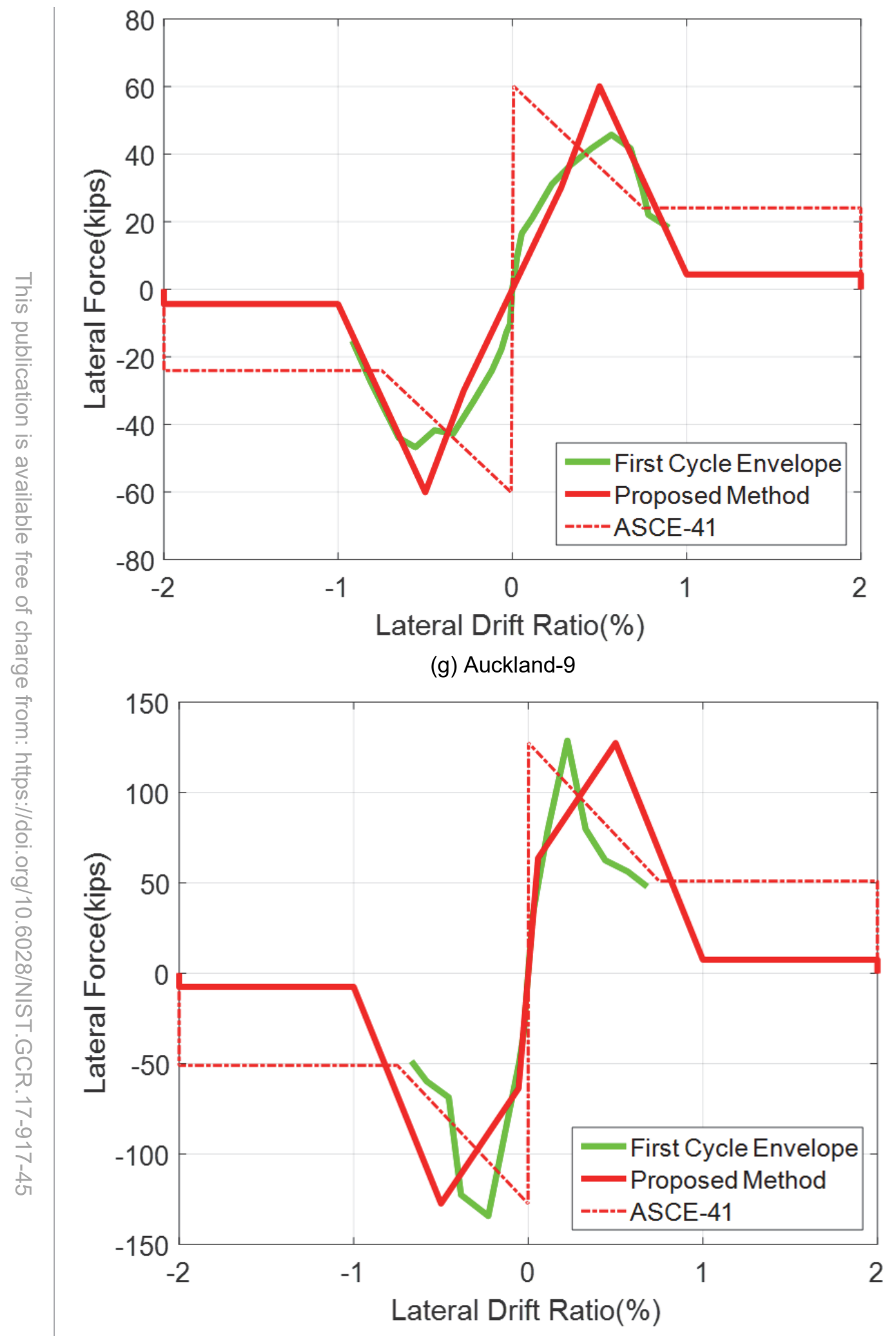

Figure E-3 Backbone lateral load-vs.-story drift ratio curves for shear-dominated walls (continued). 

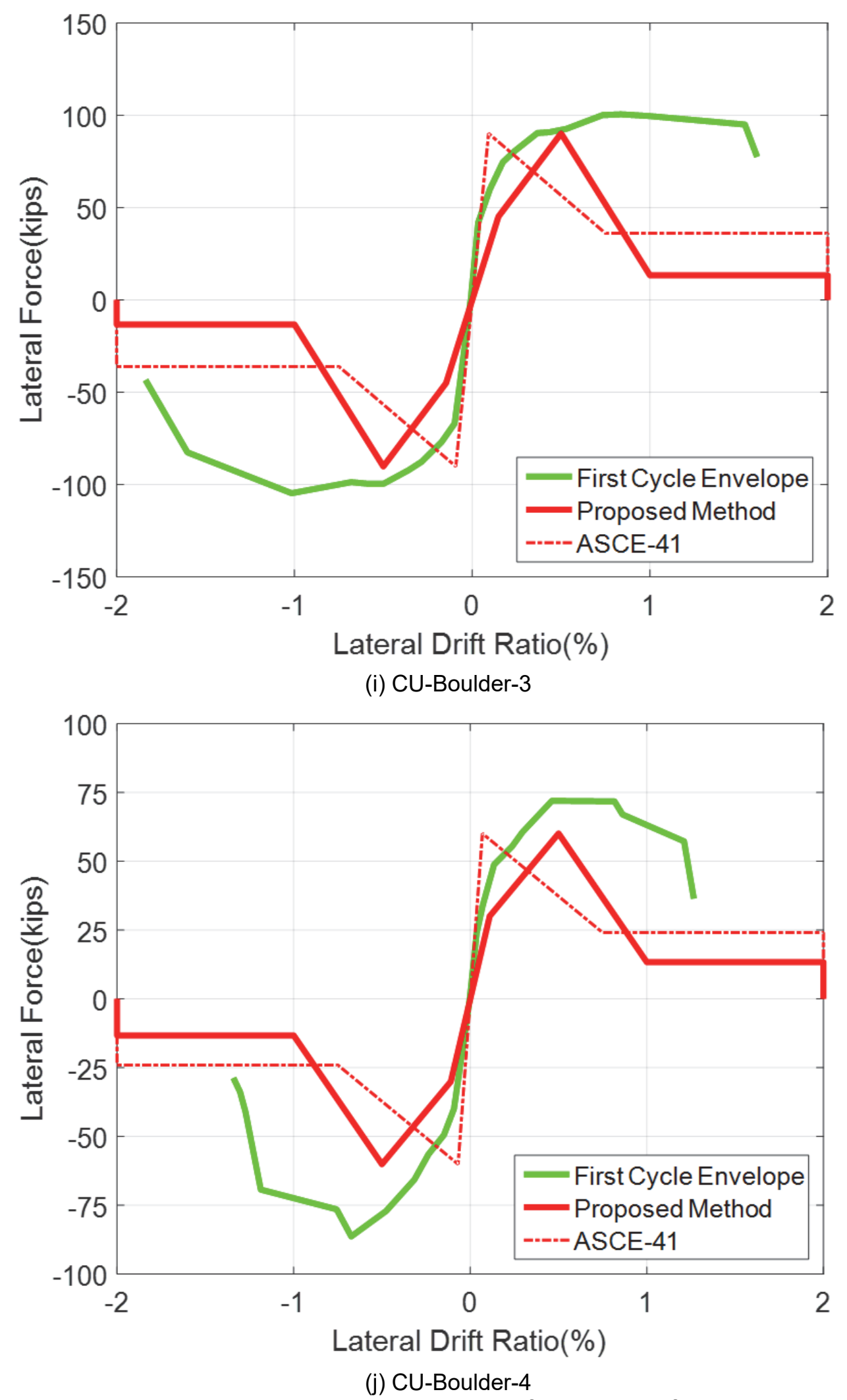

Figure E-3 Backbone lateral load-vs.-story drift ratio curves for shear-dominated walls (continued). 


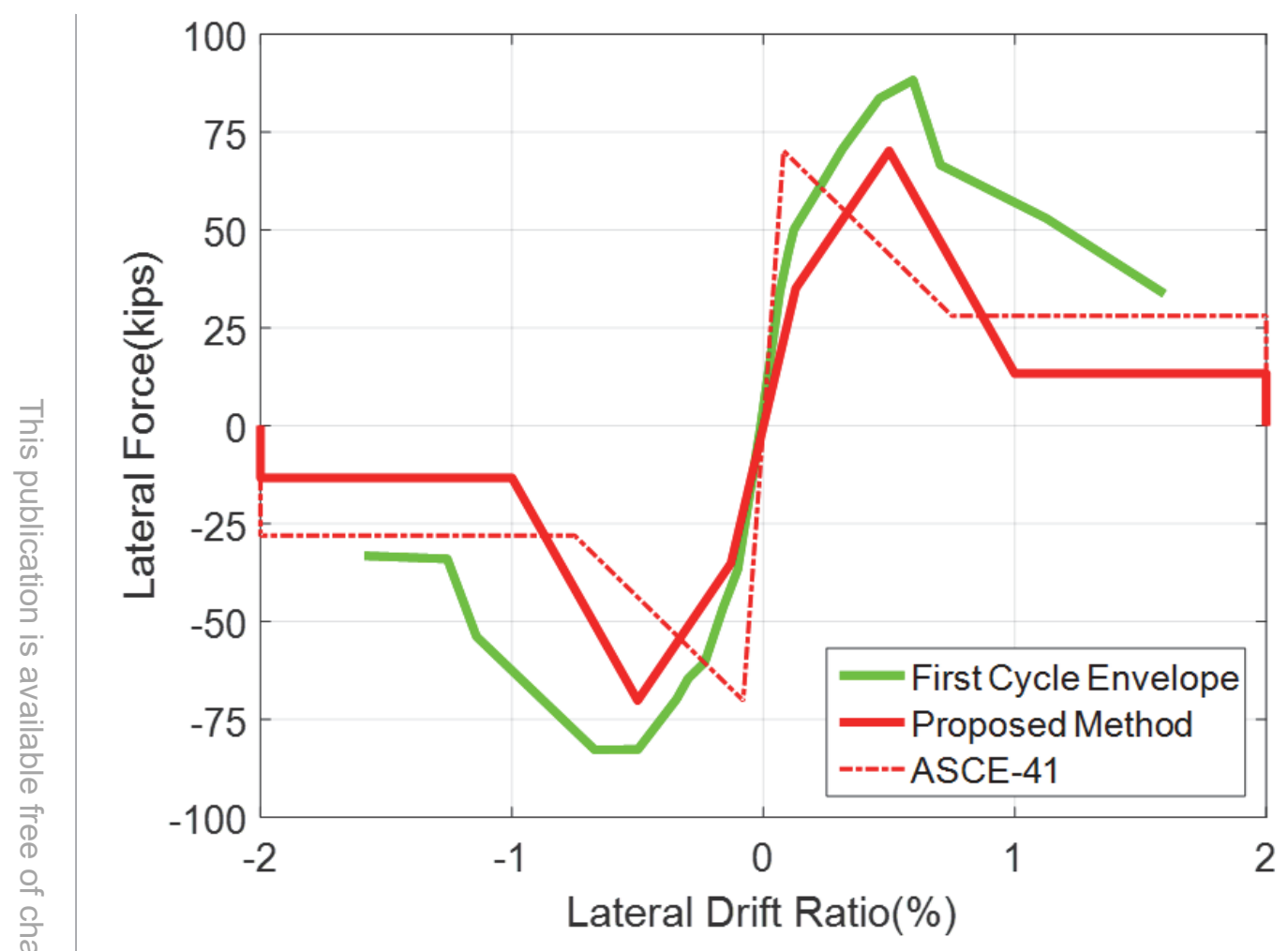

(k) CU-Boulder-5

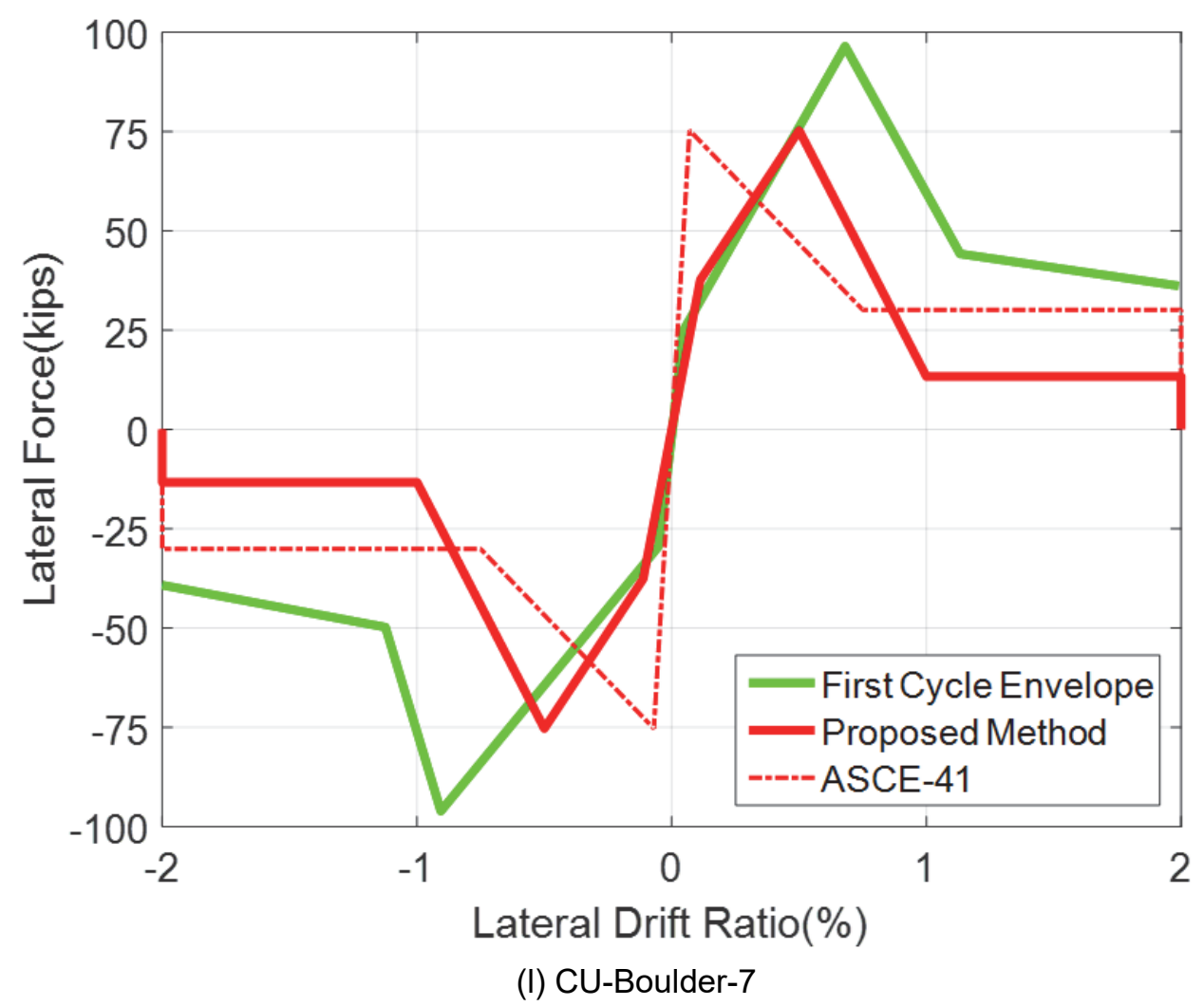

Figure E-3 Backbone lateral load-vs.-story drift ratio curves for shear-dominated walls (continued). 


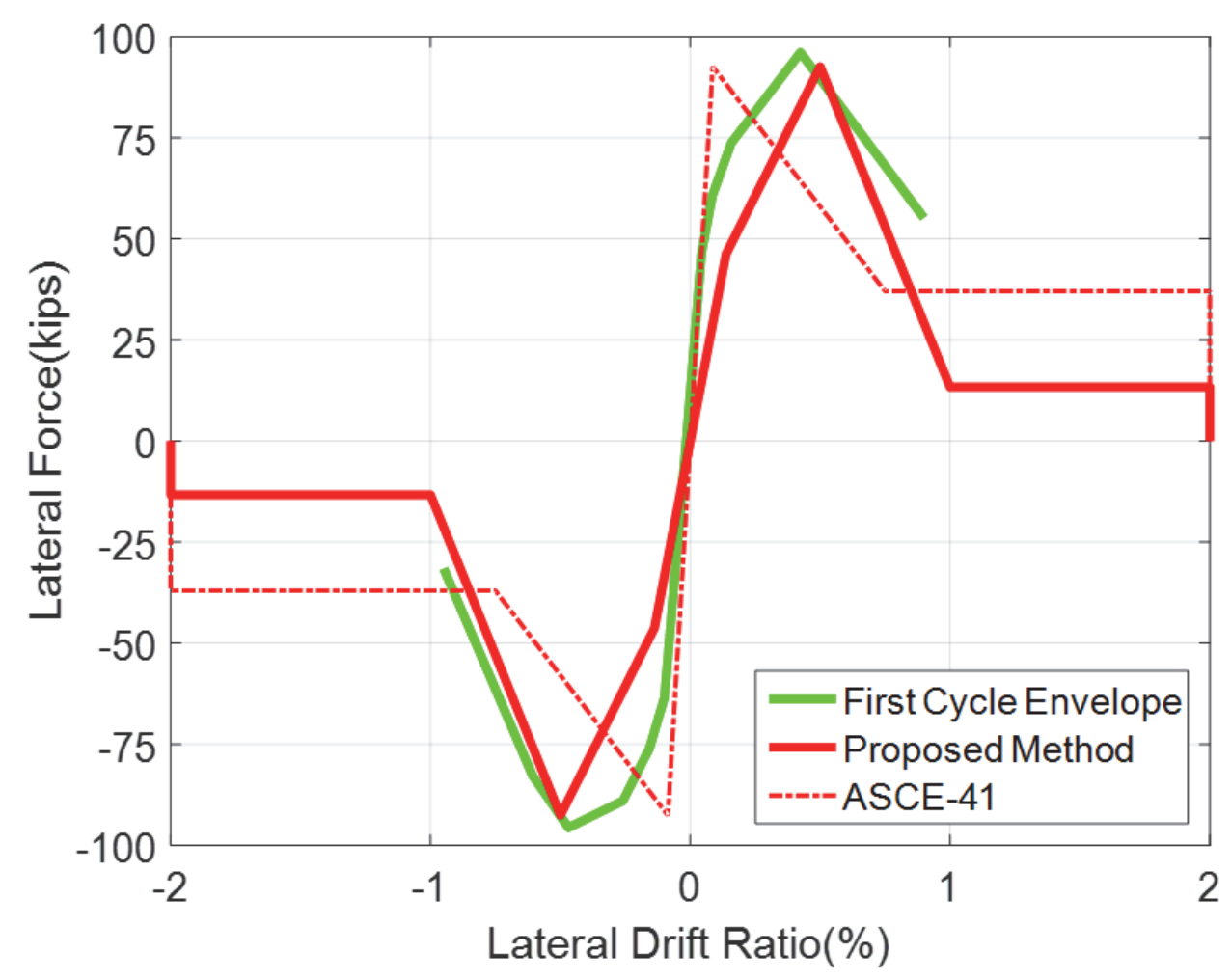

(m) CU-Boulder-9

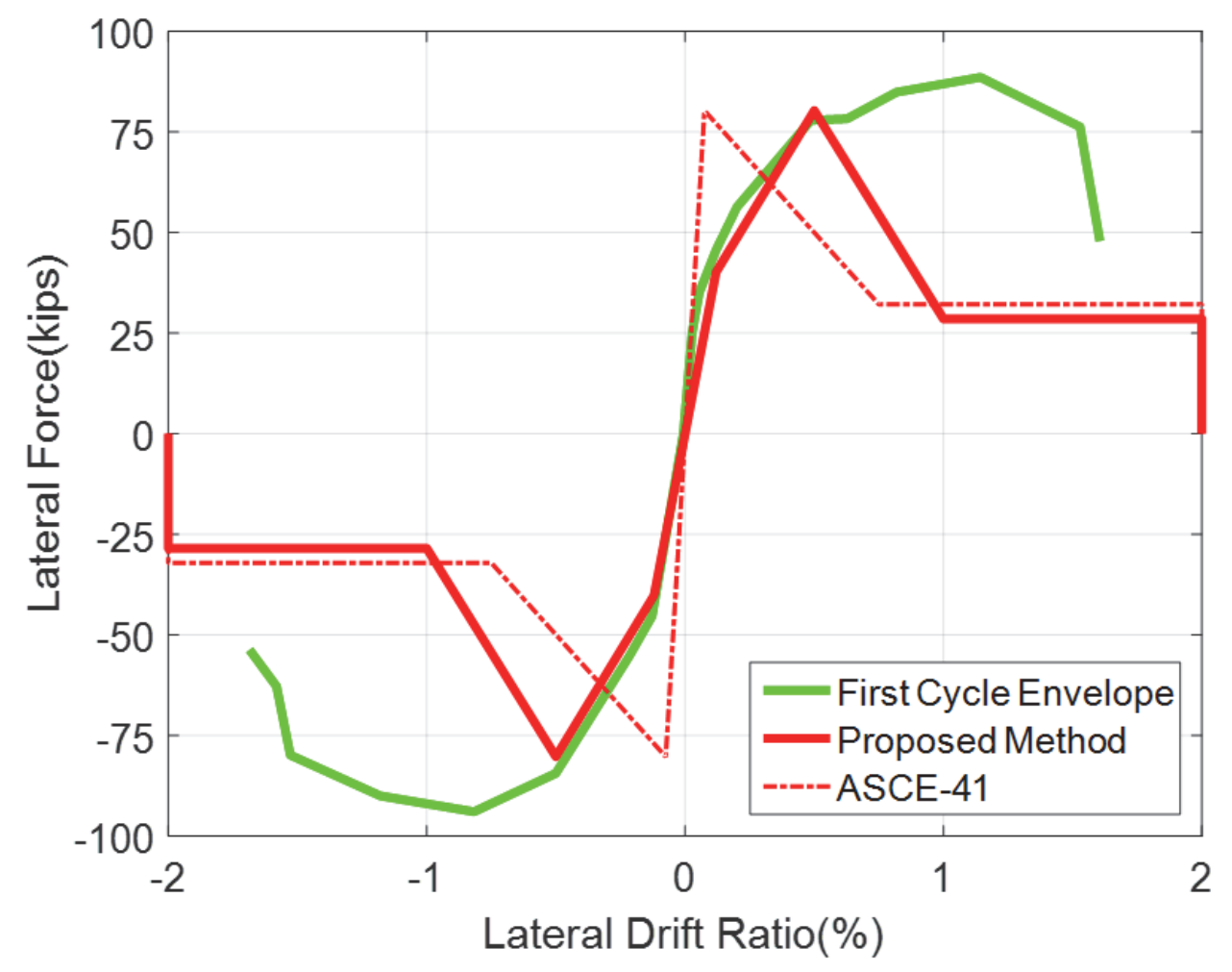

Figure E-3 Backbone lateral load-vs.-story drift ratio curves for shear-dominated walls (continued). 


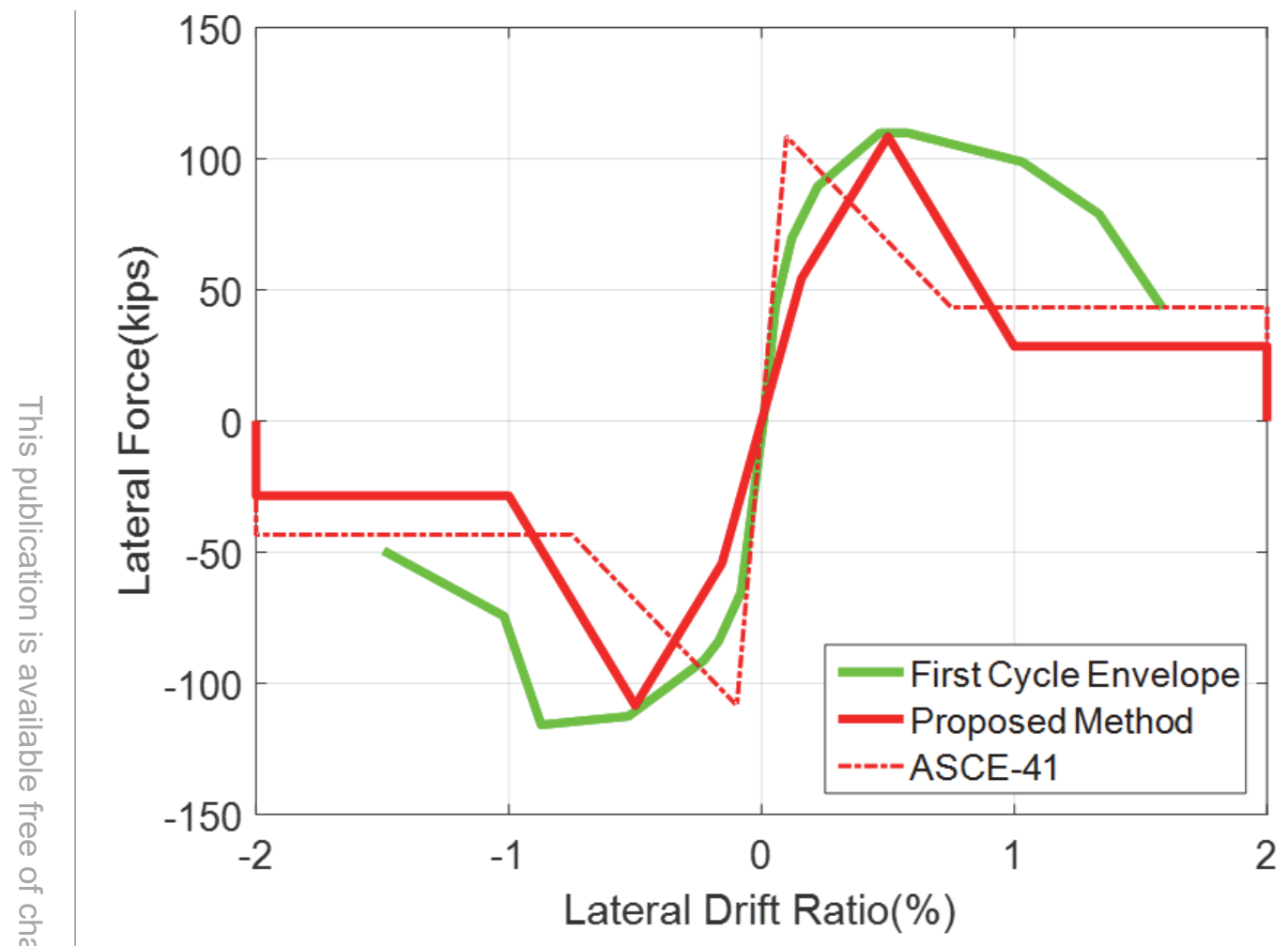

(o) CU-Boulder-13

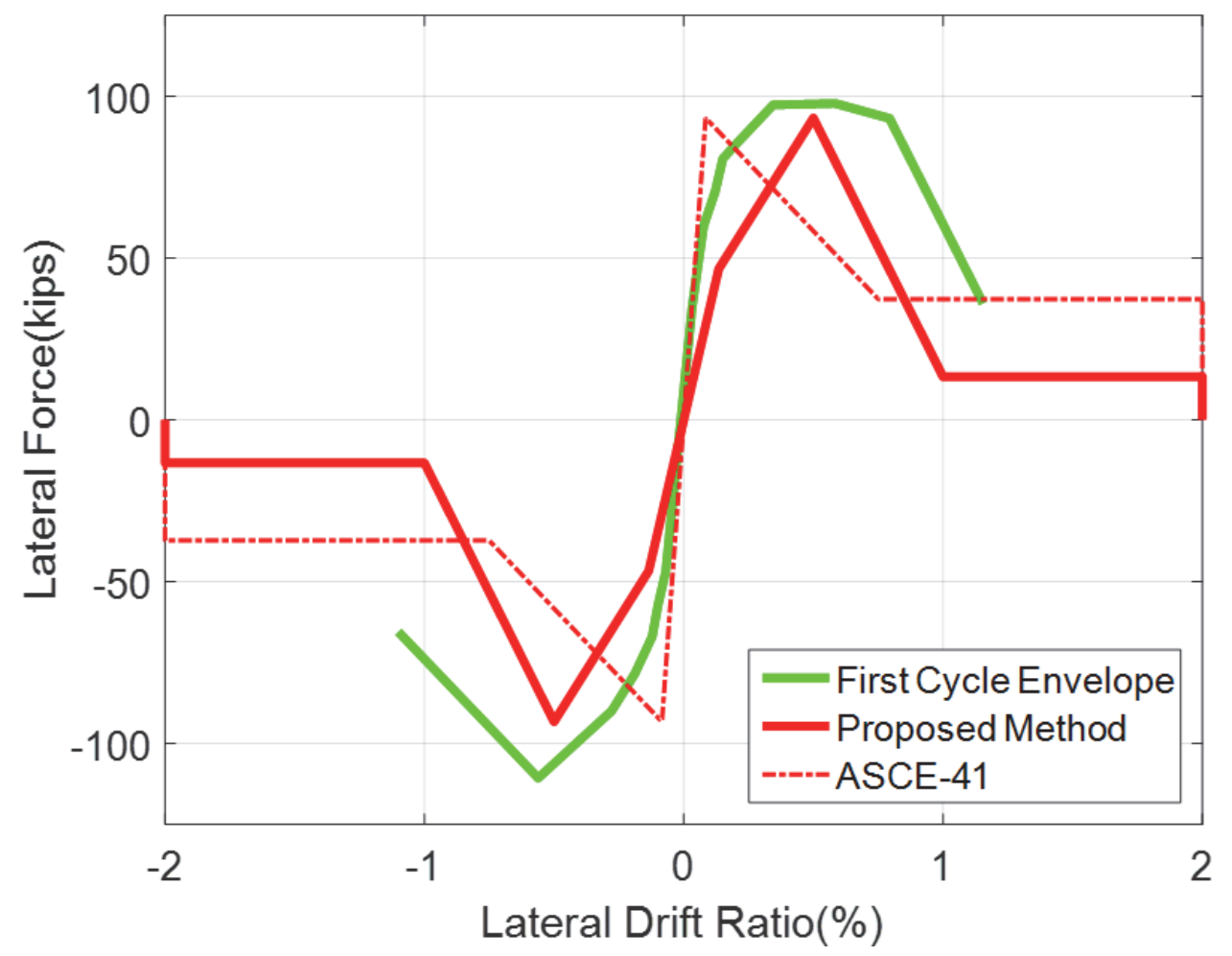

(p) CU-Boulder-14

Figure E-3 Backbone lateral load-vs.-story drift ratio curves for shear-dominated walls (continued). 


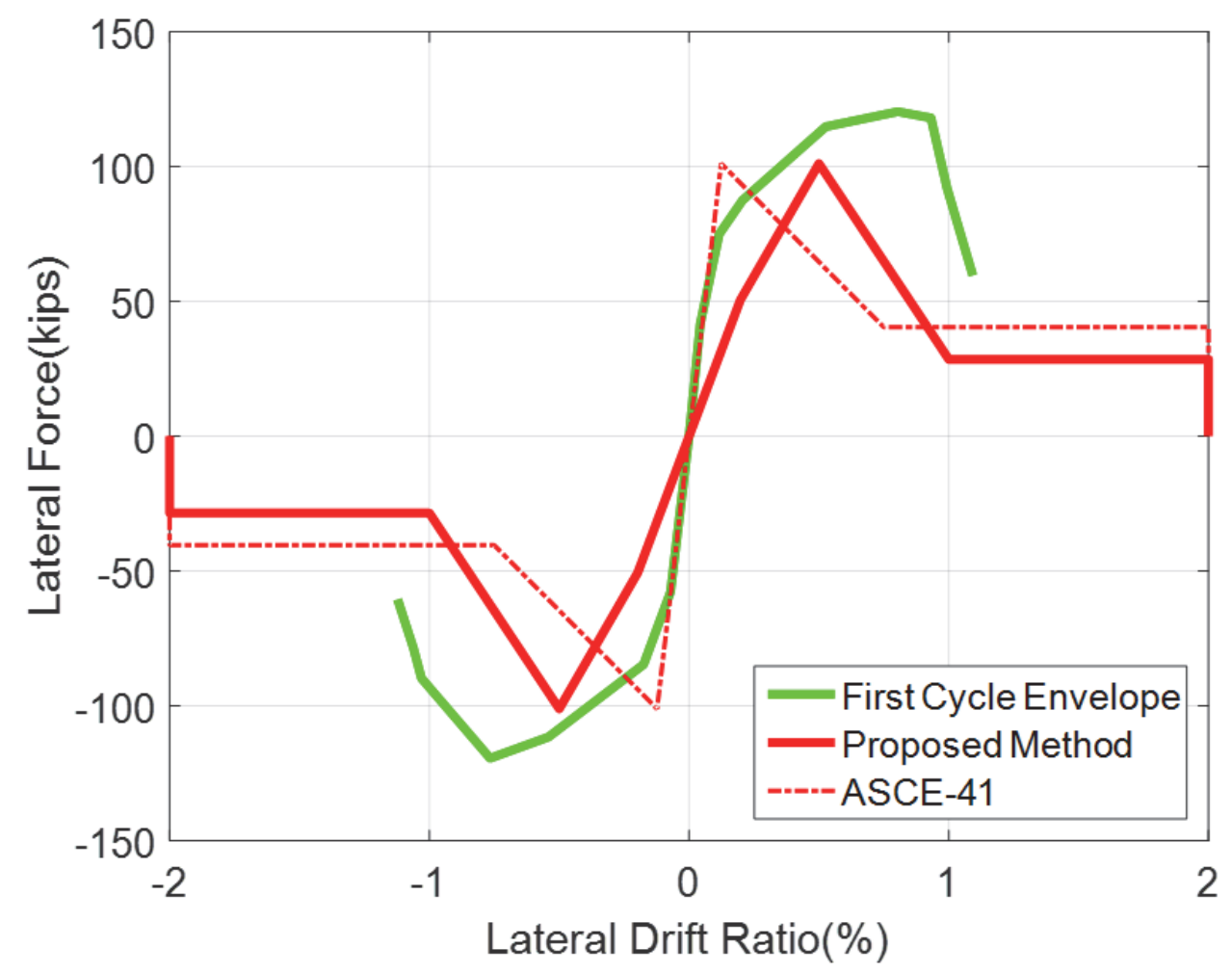

(q) CU-Boulder-16

Figure E-3 Backbone lateral load-vs.-story drift ratio curves for shear-dominated walls (continued) (continued). 


\section{Appendix F}

\section{Wood Shear Wall Systems - Database}

A large amount of wood structural panel shear wall test data are available, but significantly less data are available for walls with finishes such as gypsum wallboard and stucco. Tables F-1 through F-6 present the data from wall test data sets considered for the studies of wood shear walls in Chapter 10. Tables F-7 through F-12 present the statistically computed values (mean/average, maximum, minimum, standard deviation, and coefficient of variation (COV)) of shear resistance at different drift rations for the different wall material combinations considered.

Table F-1 Gypsum Wallboard Sheathing Shear Capacities (plf) (data from FEMA P-807 (FEMA, 2012) and Bahmani and van de Lindt (2014))

\begin{tabular}{|c|c|c|c|c|c|c|c|c|c|c|}
\hline \multirow[b]{2}{*}{ Material } & \multicolumn{10}{|c|}{ Drift Ratio (\%) } \\
\hline & $\underline{0}$ & 0.5 & 0.7 & 1 & 1.5 & 2 & 2.5 & 3 & 4 & 5 \\
\hline McMullin ${ }^{1}$ Test 1 (h-1) & 0 & 235 & 225 & 205 & 176 & 149 & 138 & & & \\
\hline McMullin Test 6 (h-2) & 0 & 190 & 202 & 210 & 209 & 197 & 153 & 141 & 115 & \\
\hline McMullin Test 6 positive quadrant & 0 & 215 & 250 & 282 & 303 & 282 & 215 & 205 & 175 & \\
\hline McMullin Test 6 negative quadrant & 0 & 164 & 154 & 138 & 115 & 113 & 92 & 77 & 56 & \\
\hline McMullin Test 7 (h-2) & 0 & 159 & 169 & 174 & 151 & 146 & 128 & 123 & 77 & \\
\hline McMullin Test 11 (h-3) & 0 & 225 & 254 & 225 & 205 & 197 & 186 & 170 & 128 & \\
\hline Pardoen $^{2}$ 19A (a) & 0 & 131 & 125 & 115 & 102 & 92 & 77 & 73 & 69 & \\
\hline Pardoen 19B (a) & 0 & 121 & 115 & 102 & 88 & 86 & 83 & 78 & 71 & \\
\hline CoLA ${ }^{3}$ Test $8 \mathrm{~A}(\mathrm{e})$ & 0 & 198 & 175 & 139 & 125 & 109 & & & & \\
\hline CoLA Test 8B (e) & 0 & 165 & 131 & 108 & 101 & & & & & \\
\hline Schmid4 Panel 6B (f) & 0 & 230 & 271 & 271 & & & & & & \\
\hline Bahmani \& van de Lindt5 (G-01) & 0 & 80 & 96 & 107 & 111 & 102 & 80 & 65 & 33 & 15 \\
\hline Bahmani \& van de Lindt (G-02) & 0 & 102 & 108 & 110 & 97 & 84 & 71 & 59 & 34 & 16 \\
\hline Gatto \& Uang 6 Test 12 East & 0 & 139 & 139 & 128 & 94 & 86 & 86 & & & \\
\hline Gatto \& Uang 6 Test 12 West & 0 & 129 & 140 & 148 & 136 & 103 & 74 & & & \\
\hline lotes: ${ }^{1}$ McMullin and Merrick (2002) & & & & & & & & & & \\
\hline 2 Pardoen et al., (2003) & & & & $\mathrm{mal}$ & $\mathrm{d} d \mathrm{v}$ & 1. & 10011 & & & \\
\hline${ }^{3}$ CoLA-UCI Light Frame Test & & e $(200$ & & atto an & Uang & 2002) & & & & \\
\hline
\end{tabular}


Table F-2 Stucco Sheathing Shear Capacities (plf) (data from FEMA P-807 (FEMA, 2012) and Bahmani and van de Lindt (2014))

\begin{tabular}{|c|c|c|c|c|c|c|c|c|c|c|}
\hline \multirow[b]{2}{*}{ Material } & \multicolumn{10}{|c|}{ Drift Ratio (\%) } \\
\hline & 0 & 0.5 & 0.7 & 1 & 1.5 & 2 & 2.5 & 3 & 4 & 5 \\
\hline CoLA Test 20A (f-1) & 0 & 344 & 332 & 219 & & & & & & \\
\hline CoLA Test 20B (f-1) & 0 & 379 & 379 & 278 & & & & & & \\
\hline CoLA Test 21A (f-2) & 0 & 325 & 325 & 262 & & & & & & \\
\hline CoLA Test 21B (f-2) & 0 & 325 & 312 & 289 & & & & & & \\
\hline Schmid Panel 7B (b-2) & 0 & 292 & 250 & & & & & & & \\
\hline Schmid Panel 3 (b-1) & 0 & 512 & 443 & 304 & & & & & & \\
\hline Bahmani \& van de Lindt (S-01) & 0 & 350 & 340 & 330 & 310 & 280 & 240 & 200 & 150 & 130 \\
\hline Bahmani \& van de Lindt (S-02) & 0 & 383 & 415 & 389 & 321 & 253 & 185 & 150 & 120 & 100 \\
\hline
\end{tabular}

Table F-3 Stucco and Gypsum Sheathing Shear Capacities (plf) (data from FEMA P-807 (FEMA, 2012))

\begin{tabular}{|c|c|c|c|c|c|c|c|c|c|}
\hline \multirow[b]{2}{*}{ Material } & \multicolumn{9}{|c|}{ Drift Ratio (\%) } \\
\hline & 0.0 & 0.5 & 1.0 & 1.5 & 2.0 & 2.5 & 3 & 4 & 5 \\
\hline CUREE-CEA ${ }^{1}$ top Story Wall 5 & 0 & 988 & 1275 & 1213 & 1063 & 925 & & & \\
\hline CUREE-CEA top Story Wall 6 & 0 & 911 & 1093 & 1093 & 911 & 804 & 718 & & \\
\hline CUREE-CEA top Story Wall 7 & 0 & 1150 & 1275 & 1250 & 1138 & 1038 & 1038 & & \\
\hline CUREE-CEA top Story Wall 8 & 0 & 900 & 1071 & 1072 & 1040 & 943 & 836 & 836 & \\
\hline Pardoen² Test 14A (g-3) & 0 & 457 & 628 & 712 & 750 & 790 & 813 & 790 & 750 \\
\hline Pardoen Test 15A (g-4) & 0 & 635 & 731 & 744 & 756 & 769 & 731 & 635 & 555 \\
\hline
\end{tabular}

${ }^{1}$ Arnold et al. (2002)

2 Pardoen et al. (2003)

Table F-4 Horizontal Wood Siding Sheathing Shear Capacities (plf) (data from Bahmani and van de Lindt (2014)

\begin{tabular}{|c|c|c|c|c|c|c|c|c|c|c|}
\hline \multirow[b]{2}{*}{ Material } & \multicolumn{10}{|c|}{ Drift Ratio (\%) } \\
\hline & 0 & 0.5 & 1 & 2 & 3 & 4 & 5 & 7 & 8 & 9 \\
\hline Bahmani \& van de Lindt $(\mathrm{H}-01)$ & 0 & 40 & 52 & 71 & 86 & 102 & 117 & 149 & 166 & 161 \\
\hline Bahmani \& van de Lindt (H-02) & 0 & 37 & 61 & 83 & 98 & 111 & 124 & 149 & 163 & 159 \\
\hline
\end{tabular}


Table F-5 Wood Structural Panels (8'×8') Sheathing Shear Capacities (plf) and ASD design values (Ibf) (data from Bahmani and van de Lindt (2014) and Line et al. (2008))

\begin{tabular}{|c|c|c|c|c|c|c|c|c|c|c|}
\hline \multirow[b]{2}{*}{ Material } & \multicolumn{9}{|c|}{ Drift Ratio (\%) } & \multirow[b]{2}{*}{ ASD } \\
\hline & 0 & 0.5 & 1 & 2 & 2.5 & 3 & 3.5 & 4 & 5 & \\
\hline Line, Waltz \& Skaggs ${ }^{1}(\mathrm{~A} 1)$ & 0 & 438 & 513 & 625 & 563 & 438 & 375 & 313 & 250 & 1,780 \\
\hline Line, Waltz \& Skaggs (A2) & 0 & 438 & 525 & 500 & 438 & 344 & & & & 1,780 \\
\hline Line, Waltz \& Skaggs (C1) & 0 & 1000 & 1375 & 1625 & 1688 & 1625 & 938 & & & 4,530 \\
\hline Line, Waltz \& Skaggs (C2) & 0 & 900 & 1250 & 1562.5 & 1625 & 375 & & & & 4,530 \\
\hline Line, Waltz \& Skaggs (E1) & 0 & 562.5 & 625 & 750 & 687.5 & 606.25 & 468.75 & 312.5 & & 1,920 \\
\hline Line, Waltz \& Skaggs (E2) & 0 & 612.5 & 700 & 812.5 & 625 & 500 & 437.5 & 362.5 & & 1,920 \\
\hline Line, Waltz \& Skaggs (G1) & 0 & 1187.5 & 1600 & 1987.5 & 1875 & & & & & 4,680 \\
\hline Line, Waltz \& Skaggs (G2) & 0 & 1125 & 1625 & 2000 & 2125 & 2112.5 & 1750 & 1250 & 500 & 4,680 \\
\hline Line, Waltz \& Skaggs (I3) & 0 & 612.5 & 750 & 843.75 & 718.75 & 562.5 & 500 & 375 & & 2,720 \\
\hline Line, Waltz \& Skaggs (15) & 0 & 625 & 750 & 875 & 887.5 & 875 & 750 & 600 & & 2,720 \\
\hline Line, Waltz \& Skaggs (K1) & 0 & 1250 & 1650 & 2000 & 2125 & 1625 & 1000 & & & 6,690 \\
\hline Line, Waltz \& Skaggs (K2) & 0 & 1375 & 1750 & 2243.75 & 2268.75 & 2275 & 2000 & 1625 & 625 & 6,690 \\
\hline Line, Waltz \& Skaggs (M1) & 0 & 562.5 & 643.7 & 750 & 687.5 & 500 & 375 & 250 & & 1,840 \\
\hline Line, Waltz \& Skaggs (M2) & 0 & 612.5 & 643.7 & 750 & 731.25 & 500 & 425 & 375 & & 1,840 \\
\hline Line, Waltz \& Skaggs (01) & 0 & 1000 & 1500 & 1962.5 & 1993.75 & 2000 & & & & 4,880 \\
\hline Line, Waltz \& Skaggs (O2) & 0 & 1062.5 & 1500 & 1775 & 1906.25 & 1975 & 2000 & & & 4,880 \\
\hline
\end{tabular}

1 Line et al. (2008) 
Table F-6 Wood Structural Panels (8'×4') Sheathing Shear Capacities (plf) and ASD design values (Ibf) (data from Line et al. (2008) and AWC (2016))

\begin{tabular}{|c|c|c|c|c|c|c|c|c|c|c|}
\hline \multirow[b]{2}{*}{ Material } & \multicolumn{9}{|c|}{ Drift Ratio (\%) } & \multirow[b]{2}{*}{ ASD } \\
\hline & 0 & 0.5 & 1 & 1.5 & 2 & 2.5 & 3 & 3.5 & 4 & \\
\hline Line, Waltz \& Skaggs (B1) & 0 & 488 & 563 & 625 & 675 & 638 & 625 & 500 & 387.5 & 890 \\
\hline Line, Waltz \& Skaggs (B2) & 0 & 450 & 538 & 625 & 630 & 625 & 625 & 625 & 562.5 & 890 \\
\hline Line, Waltz \& Skaggs (D1) & 0 & 750 & 1125 & 1375 & 1500 & 1562.5 & 1625 & 1750 & 1625 & 2,265 \\
\hline Line, Waltz \& Skaggs (D2) & 0 & 875 & 1125 & 1312.5 & 1450 & 1500 & 1500 & 1500 & 1375 & 2,265 \\
\hline Line, Waltz \& Skaggs (F1) & 0 & 500 & 625 & 725 & 750 & 750 & 737.5 & 625 & 500 & 960 \\
\hline Line, Waltz \& Skaggs (F2) & 0 & 500 & 637.5 & 750 & 812.5 & 812.5 & 775 & 775 & 562.5 & 960 \\
\hline Line, Waltz \& Skaggs (H1) & 0 & 937.5 & 1375 & 1625 & 1775 & 1875 & 2000 & 1000 & & 2,340 \\
\hline Line, Waltz \& Skaggs (H2) & 0 & 975 & 1275 & 1525 & 1625 & 1600 & 1500 & 1250 & 1000 & 2,340 \\
\hline Line, Waltz \& Skaggs (J2) & 0 & 450 & 737.5 & 812.5 & 875 & 875 & 872.5 & 872.5 & 625 & 1,360 \\
\hline Line, Waltz \& Skaggs (J3) & 0 & 525 & 675 & 812.5 & 875 & 875 & 750 & 625 & & 1,360 \\
\hline Line, Waltz \& Skaggs (L1) & 0 & 1000 & 1500 & 1625 & 1787.5 & 1937.5 & 1975 & 2000 & 1650 & 3,480 \\
\hline Line, Waltz \& Skaggs (L2) & 0 & 1025 & 1500 & 1750 & 1875 & 2000 & 2000 & 1950 & 1612.5 & 3,480 \\
\hline Line, Waltz \& Skaggs (N1) & 0 & 500 & 625 & 700 & 737.5 & 637.5 & 600 & 500 & 437.5 & 920 \\
\hline Line, Waltz \& Skaggs (N2) & 0 & 500 & 625 & 775 & 750 & 750 & 712.5 & 700 & 562.5 & 920 \\
\hline Line, Waltz \& Skaggs (P1) & 0 & 1000 & 1500 & 1750 & 1787.5 & 1800 & 1775 & 1250 & & 2,440 \\
\hline Line, Waltz \& Skaggs (P2) & 0 & 1000 & 1375 & 1625 & 1750 & 1875 & 1900 & 750 & & 2,440 \\
\hline $\begin{array}{l}\text { Unpublished (AWC, 2016) } \\
\text { (3/8" Str I, 6d, } 2 \text { in./6 in.) }\end{array}$ & 0 & 812.5 & 1125 & 1487.5 & 1500 & 1562.5 & 1625 & & & 2,265 \\
\hline $\begin{array}{l}\text { Unpublished (AWC, 2016) } \\
\text { (3/8" Str I, 6d, } 6 \text { in./6 in.) -1 }\end{array}$ & 0 & 375 & 500 & 612.5 & 625 & 600 & 587.5 & 312.5 & & 890 \\
\hline $\begin{array}{l}\text { Unpublished (AWC, 2016) } \\
\text { (3/8" Str I, } 6 \mathrm{~d}, 6 \text { in./6 in.) -2 }\end{array}$ & 0 & 375 & 475 & 525 & 587.5 & 625 & 525 & 300 & & 890 \\
\hline $\begin{array}{l}\text { Unpublished (AWC, 2016) } \\
\text { (19/32" sheathing, 10d, } 2 \\
\text { in./12 in.) -1 }\end{array}$ & 0 & 750 & 1300 & 1750 & 1800 & 1962.5 & 2000 & 1700 & 1500 & 3,480 \\
\hline $\begin{array}{l}\text { Unpublished (AWC, 2016) } \\
\text { (19/32" sheathing, 10d, } 2 \\
\text { in./12 in.) -2 }\end{array}$ & 0 & 1000 & 1375 & 1712.5 & 1812.5 & 2000 & 2000 & 2125 & 2025 & 3,480 \\
\hline $\begin{array}{l}\text { Unpublished (AWC, 2016) } \\
\text { (19/32" sheathing, 10d } 6 \\
\text { in./12 in.) }\end{array}$ & 0 & 625 & 750 & 875 & 962.5 & 975 & 950 & 875 & 750 & 1,360 \\
\hline
\end{tabular}


Table F-7 Statistical Values for Varying Drift Ratio Values for Gypsum Wallboards

\begin{tabular}{|c|c|c|c|c|c|}
\hline \multirow[b]{2}{*}{ Drift Ratio (\%) } & \multicolumn{5}{|c|}{ Statistical Parameters of Sheathing Shear Capacities (plf) } \\
\hline & Minimum & Maximum & Average & $\begin{array}{l}\text { Standard } \\
\text { Deviation }\end{array}$ & COV \\
\hline 0 & 0.00 & 0.00 & 0.00 & 0.00 & 0.00 \\
\hline 0.5 & 80.00 & 235.00 & 165.50 & 48.74 & 0.29 \\
\hline 0.7 & 96.00 & 271.00 & 170.22 & 57.21 & 0.34 \\
\hline 1.0 & 102.00 & 282.00 & 164.05 & 60.64 & 0.37 \\
\hline 1.5 & 88.00 & 303.00 & 143.75 & 60.87 & 0.42 \\
\hline 2.0 & 84.21 & 282.00 & 134.30 & 59.24 & 0.44 \\
\hline 2.5 & 71.46 & 215.00 & 115.29 & 48.46 & 0.42 \\
\hline 3.0 & 59.09 & 205.00 & 110.12 & 52.27 & 0.47 \\
\hline 4.0 & 33.00 & 175.00 & 84.22 & 46.78 & 0.56 \\
\hline 5.0 & 15.00 & 16.00 & 15.50 & 0.71 & 0.05 \\
\hline
\end{tabular}

Table F-8 Statistical Values for Varying Drift Ratio Values for Stucco Walls

\begin{tabular}{|c|c|c|c|c|c|}
\hline \multirow[b]{2}{*}{ Drift Ratio (\%) } & \multicolumn{5}{|c|}{ Statistical Parameters of Sheathing Shear Capacities (plf) } \\
\hline & Minimum & Maximum & Average & $\begin{array}{l}\text { Standard } \\
\text { Deviation }\end{array}$ & COV \\
\hline 0 & 0.00 & 0.00 & 0.00 & 0.00 & 0.00 \\
\hline 0.5 & 292.00 & 512.00 & 363.69 & 66.86 & 0.18 \\
\hline 0.7 & 250.00 & 443.00 & 349.53 & 61.22 & 0.18 \\
\hline 1.0 & 219.00 & 389.00 & 295.86 & 53.74 & 0.18 \\
\hline 1.5 & 310.00 & 321.00 & 315.50 & 7.78 & 0.02 \\
\hline 2.0 & 253.10 & 280.00 & 266.55 & 19.02 & 0.07 \\
\hline 2.5 & 185.10 & 240.00 & 212.55 & 38.82 & 0.18 \\
\hline 3.0 & 150.00 & 200.00 & 175.00 & 35.36 & 0.20 \\
\hline 4.0 & 120.00 & 150.00 & 135.00 & 21.21 & 0.16 \\
\hline 5.0 & 100.00 & 130.00 & 115.00 & 21.21 & 0.18 \\
\hline
\end{tabular}


Table F-9 Statistical Values for Varying Drift Ratio Values for Stucco and Gypsum Wallboard Combination

\begin{tabular}{|c|c|c|c|c|c|}
\hline \multirow[b]{2}{*}{ Drift Ratio (\%) } & \multicolumn{5}{|c|}{ Statistical Parameters of Sheathing Shear Capacities (plf) } \\
\hline & Minimum & Maximum & Average & $\begin{array}{l}\text { Standard } \\
\text { Deviation }\end{array}$ & COV \\
\hline 0 & 0.00 & 0.00 & 0.00 & 0.00 & 0.00 \\
\hline 0.5 & 456.67 & 1150.00 & 840.15 & 251.14 & 0.30 \\
\hline 1.0 & 628.33 & 1275.00 & 1012.30 & 273.78 & 0.27 \\
\hline 1.5 & 711.67 & 1250.00 & 1013.85 & 232.00 & 0.23 \\
\hline 2.0 & 750.00 & 1137.50 & 942.81 & 164.09 & 0.17 \\
\hline 2.5 & 769.23 & 1037.50 & 878.13 & 106.74 & 0.12 \\
\hline 3.0 & 718.11 & 1037.50 & 827.15 & 128.15 & 0.15 \\
\hline 4.0 & 635.38 & 836.01 & 753.80 & 105.10 & 0.14 \\
\hline 5.0 & 554.62 & 750.00 & 652.31 & 138.16 & 0.21 \\
\hline
\end{tabular}

Table F-10 Statistical Values for Varying Drift Ratio Values for Wood Horizontal Siding Wall Elements

\begin{tabular}{|c|c|c|c|c|c|}
\hline \multirow[b]{2}{*}{ Drift Ratio (\%) } & \multicolumn{5}{|c|}{ Statistical Parameters of Sheathing Shear Capacities (plf) } \\
\hline & Minimum & Maximum & Average & $\begin{array}{l}\text { Standard } \\
\text { Deviation }\end{array}$ & $\mathrm{COV}$ \\
\hline 0 & 0.00 & 0.00 & 0.00 & 0.00 & 0.00 \\
\hline 0.5 & 37.00 & 40.00 & 38.50 & 2.12 & 0.06 \\
\hline 1.0 & 51.86 & 61.03 & 56.45 & 6.48 & 0.11 \\
\hline 2.0 & 70.83 & 83.44 & 77.14 & 8.92 & 0.12 \\
\hline 3.0 & 86.49 & 98.08 & 92.29 & 8.20 & 0.09 \\
\hline 4.0 & 101.90 & 110.80 & 106.35 & 6.29 & 0.06 \\
\hline 5.0 & 117.00 & 123.50 & 120.25 & 4.60 & 0.04 \\
\hline 7.0 & 148.50 & 148.70 & 148.60 & 0.14 & 0.001 \\
\hline 8.0 & 163.00 & 166.00 & 164.50 & 2.12 & 0.01 \\
\hline 9.0 & 159.00 & 161.20 & 160.10 & 1.56 & 0.01 \\
\hline
\end{tabular}


Table F-11 Statistical Values for Varying Drift Ratio Values for Wood Structural Panels (8'x8')

\begin{tabular}{|c|c|c|c|c|c|}
\hline \multirow[b]{2}{*}{ Drift Ratio (\%) } & \multicolumn{5}{|c|}{ Statistical parameters of sheathing shear capacities (plf) } \\
\hline & Minimum & Maximum & Average & $\begin{array}{l}\text { Standard } \\
\text { Deviation }\end{array}$ & COV \\
\hline 0 & 0.00 & 0.00 & 0.00 & 0.00 & 0.00 \\
\hline 0.5 & 437.50 & 1375.00 & 800.55 & 308.05 & 0.38 \\
\hline 1.0 & 512.50 & 1750.00 & 1042.68 & 465.29 & 0.45 \\
\hline 2.0 & 500.00 & 2243.75 & 1260.47 & 607.04 & 0.48 \\
\hline 2.5 & 437.50 & 2268.75 & 1258.57 & 661.34 & 0.53 \\
\hline 3.0 & 343.75 & 2275.00 & 1049.46 & 703.51 & 0.67 \\
\hline 3.5 & 375.00 & 2000.00 & 883.58 & 595.53 & 0.67 \\
\hline 4.0 & 250.00 & 1625.00 & 602.57 & 438.08 & 0.73 \\
\hline 5.0 & 250.00 & 625.00 & 438.52 & 142.56 & 0.33 \\
\hline
\end{tabular}

Table F-12 Statistical Values for Varying Drift Ratio Values for Wood Structural Panels (8' $\left.\times 4^{\prime}\right)$

\begin{tabular}{|c|c|c|c|c|c|}
\hline \multirow[b]{2}{*}{ Drift Ratio (\%) } & \multicolumn{5}{|c|}{ Statistical Parameters of Sheathing Shear Capacities (plf) } \\
\hline & Minimum & Maximum & Average & $\begin{array}{l}\text { Standard } \\
\text { Deviation }\end{array}$ & COV \\
\hline 0 & 0.00 & 0.00 & 0.00 & 0.00 & 0.00 \\
\hline 0.5 & 375.00 & 1025.00 & 700.57 & 241.38 & 0.34 \\
\hline 1.0 & 475.00 & 1500.00 & 969.32 & 383.28 & 0.40 \\
\hline 1.5 & 525.00 & 1750.00 & 1153.41 & 469.17 & 0.41 \\
\hline 2.0 & 587.50 & 1875.00 & 1224.66 & 500.81 & 0.41 \\
\hline 2.5 & 600.00 & 2000.00 & 1265.34 & 559.19 & 0.44 \\
\hline 3.0 & 525.00 & 2000.00 & 1257.27 & 589.87 & 0.47 \\
\hline 3.5 & 300.00 & 2125.00 & 1046.90 & 577.45 & 0.55 \\
\hline 4.0 & 387.50 & 2025.00 & 1011.67 & 557.19 & 0.55 \\
\hline
\end{tabular}





\section{Appendix G}

\section{Wood Shear Wall Systems - Application Case Study}

\section{G.1 Overview and Introduction}

This appendix presents a case study illustrating the application of the proposed backbone curve for wood frame shear wall systems that accounts for non-trivial residual strength and improved displacement capacity. Nonlinear response analyses were first conducted at the component level by modeling the wood shear wall components incorporating representative design details of a five story multi-family dwelling (MFD) archetype located in the San Francisco, California, while the analyses were then extended to the system level by investigating the global response of the five-story MFD archetype. The results were compared with the nonlinear response of the systems modeled considering the ASCE/SEI 41-13, Seismic Evaluation and Retrofit of Existing Buildings (ASCE, 2014), backbone curve. Based on the results of this analytical case study, the effectiveness of the proposed backbone curve in estimating the various performance levels, e.g., Immediate Occupancy (IO), Life Safety (LS) and CP (collapse prevention) of a typical wood frame structure is illustrated and verified.

\section{G.2 Structural System and Design Considerations}

A typical five-story multi-family dwelling located in San Francisco, California was considered to conduct nonlinear time history analyses accounting for the two different designs for backbone curves (ASCE/SEI 41-13 and the simplified backbone curve presented in Figure 10-12). The building archetype was designed by practicing engineers in California (WoodWorks, 2016) per ASCE/SEI 7-10, Minimum Designs Loads for Buildings and Other Structures (ASCE, 2010), for Seismic Design Category D $\left(S_{D S}=1.206\right.$ and $\left.S_{D I}=0.692\right)$, Risk Category II, a response modification factor (R-factor) of 6.5 for building frame systems with light-frame walls sheathed with structural panels, $\Omega_{0}$ equal to 3.0 and $C_{d}$ equal to 4.0 . The building archetype has a total height of 50 feet to the roof and 12,000 square feet of living area per floor. The elevation and a typical plan view of the building archetype considered in this study are presented in Figure G-1. The initial design was for a five-story building over a podium slab, which was treated as two separate structures (upper flexible and lower rigid) in the design. The design for the upper structure (without the podium structure) was used in this case study. 


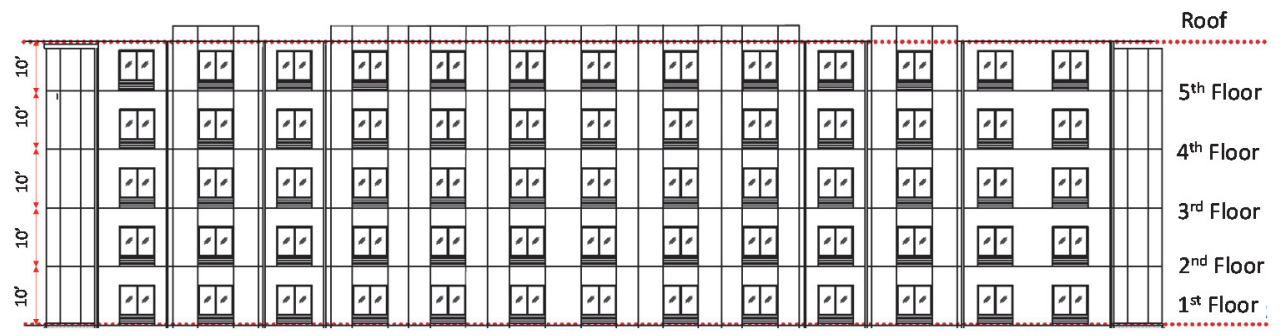

(a)

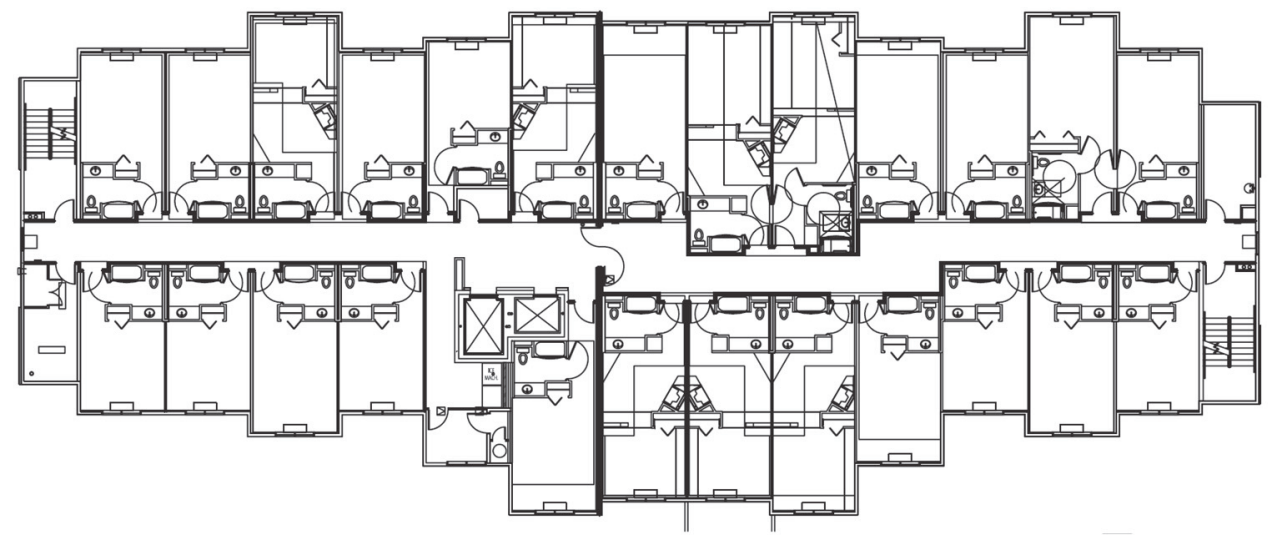

(b)

Figure G-1 (a) Building elevation and (b) typical floor plan (WoodWorks, 2016).

The lateral force-resisting system uses both interior and exterior shear walls. The loading assumptions for computing the dead roof and floor weights accounted for roofing, sheathing, insulation, ceiling, interior and exterior walls, and beam components. The total seismic weight per floor was calculated based on the tributary area of the shear walls and the roof diaphragm. The weight calculations are presented in detail in Table G-1 and Table G-2.

Table G-1 Roof and Floor Dead Load Calculations

\begin{tabular}{|c|c|c|c|}
\hline \multicolumn{2}{|l|}{ Roof } & \multicolumn{2}{|c|}{ Floor } \\
\hline Component & Weight & Component & Weight \\
\hline Roofing material & $5 p s f$ & Flooring material & $1 \mathrm{psf}$ \\
\hline Wood rood sheathing & $3 p s f$ & Wood sheathing & $2.5 p s f$ \\
\hline Roof trusses and blocking & $2 p s f$ & Joists and blocking & $4 p s f$ \\
\hline Insulation and sprinklers & $2 p s f$ & Ceiling and miscellaneous & $7 p s f$ \\
\hline Ceiling and miscellaneous & 15psf & Beams & 1.5psf \\
\hline Beams & $1 \mathrm{psf}$ & -- & -- \\
\hline
\end{tabular}




\begin{tabular}{cc} 
Table G-2 & $\begin{array}{l}\text { Weights Considered in the } \\
\text { Design and Analysis }\end{array}$ \\
\cline { 1 - 2 } Floor/Level & $\frac{\text { Total weight (kips) }}{2}$ \\
\cline { 1 - 2 } 2 & 647 \\
3 & 647 \\
4 & 647 \\
5 & 639 \\
Roof & 587 \\
Total & 3,167 \\
\hline
\end{tabular}

The shear walls used $15 / 32$-inch Structural I sheathing with $10 \mathrm{~d}$ common nails with a minimum penetration of 1.5 inches into the framing members. A $2 \times 4$ sill plate was used at the base of the wood frame shear walls. The nailing schedule of the shear walls per floor is summarized in Table G-3.

Table G-3 Wood Frame Shear Wall Nailing Schedule

\begin{tabular}{|c|c|c|c|}
\hline Floor/Level & $\begin{array}{l}\text { Wall Length } \\
\text { (ft) }\end{array}$ & $\begin{array}{c}\text { Wall Sheathed } \\
1 \text { or } 2 \text { Sides }\end{array}$ & $\begin{array}{c}\text { Fastener Edge } \\
\text { Spacing }\end{array}$ \\
\hline 2 & 29.0 & 2 & 2" o.c. \\
\hline 3 & 29.0 & 2 & 2" o.c. \\
\hline 4 & 29.0 & 2 & 2" o.c. \\
\hline 5 & 29.0 & 1 & 2" o.c. \\
\hline Roof & 29.0 & 1 & 6" o.c. \\
\hline
\end{tabular}

\section{G.3 Case study Description and Results}

The case study described in this section intended to highlight the importance of using a non-trivial envelope backbone curve for nonlinear modeling of wood frame shear walls, in particular how the component and system response can be influenced. The response of a typical light-frame wood shear wall system for the 5-story MDF (wood shear wall stack five stories high) as well as the response of the full building were investigated.

\section{G.3.1 Component Level Nonlinear Model Development and Structural Analysis}

A wall frame model was developed in the SAPWood software (Pei and van de Lindt, 2010) to address a typical exterior wall configuration stack five stories high of the building archetype considered. The exterior wall consisted of wood structural panels, gypsum and stucco. The wood shear wall was modeled following the geometric configuration of the design described above as well as the nailing schedule incorporated. The combination rules for wood shear walls incorporating different 
sheathing materials as proposed by FEMA P-807, Seismic Evaluation and Retrofit of Multi-Unit Wood-Frame Buildings with Weak First Stories (FEMA, 2012b), were applied. According to FEMA P-807, walls sheathed with dissimilar materials can be added with $100 \%$ of the strength of the strongest sheathing type and $50 \%$ of the strength of the other sheathing types considered. Following the FEMA P-807 combination rules, interior walls sheathed with $100 \%$ wood and $50 \%$ gypsum, while exterior walls sheathed with $100 \%$ wood, $50 \%$ gypsum and $50 \%$ stucco were used for the analyses in this study.

The proposed envelope backbone curves as shown in Figure G-2 (introduced in Chapter 10) incorporating the values for wood panels, gypsum, and stucco were used for developing the backbone curve considered for modeling the interior wood shear wall. Similarly the combination of ASCE/SEI 41-13 backbone curves considered was also computed and used for the analyses. The individual force-displacement curves for wood structural panels, gypsum wallboard, and stucco panels used to synthesize the numerical model accounting for both modeling approaches are shown in Figure G-3. Note that the hysteretic properties assigned to the hysteretic elements representing the interior and exterior walls were obtained by adjusting the strength and stiffness values for the length and height of the wall elements considered in this study. Simple mathematical calculations were performed using linear extrapolation to adjust the strength and stiffness values.

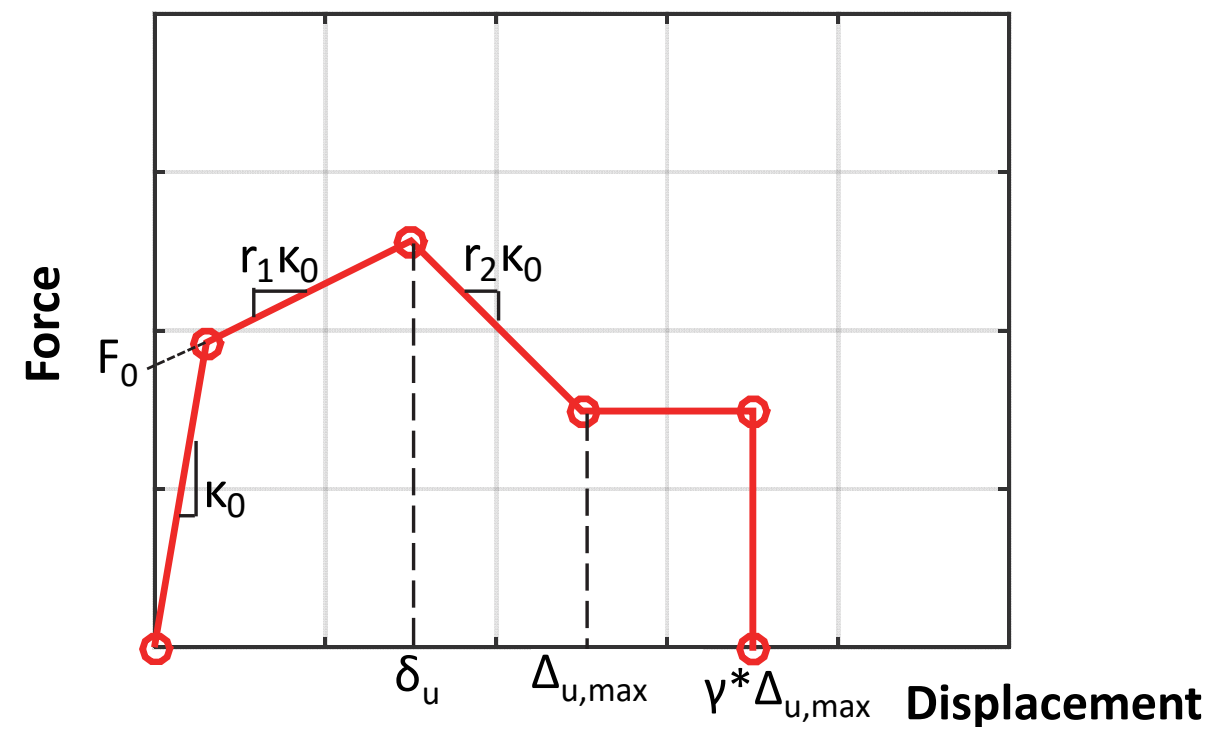

Figure G-2 Proposed backbone curve envelope.

The wood shear wall model was used to conduct nonlinear time history analyses for increasing seismic intensities - incremental dynamic analyses (IDAs) (Vamvatsikos and Cornell, 2002) - under the FEMA P-695 (FEMA, 2009) Far-Field ground motion ensemble. The total seismic weight considered in the analyses of the wood wall system was set equal to a wall dead load of $16 \mathrm{psf}$ accounting for the weight of the 
framing member, the sheathing material, stucco and gypsum. Assuming that the wall system is a typical first story exterior wall of the five-story building archetype, the gravity load was computed and applied to the wall based on tributary area. The wall gravity load was calculated accounting for the roof, wall and floor dead loads.

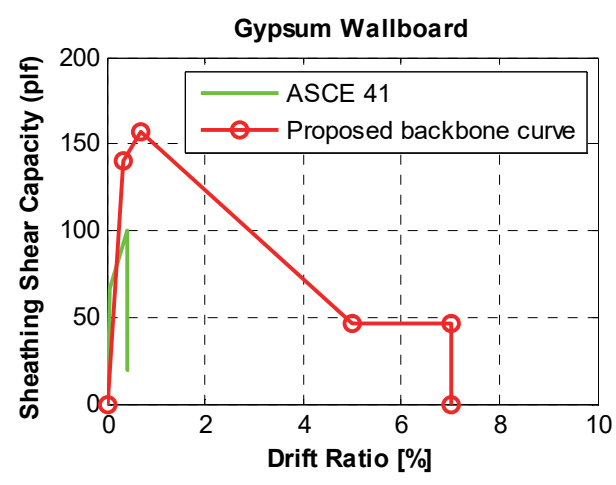

(a)

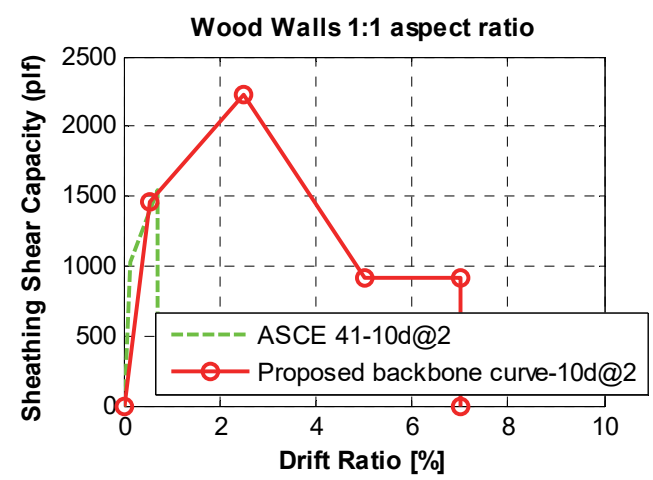

(c)

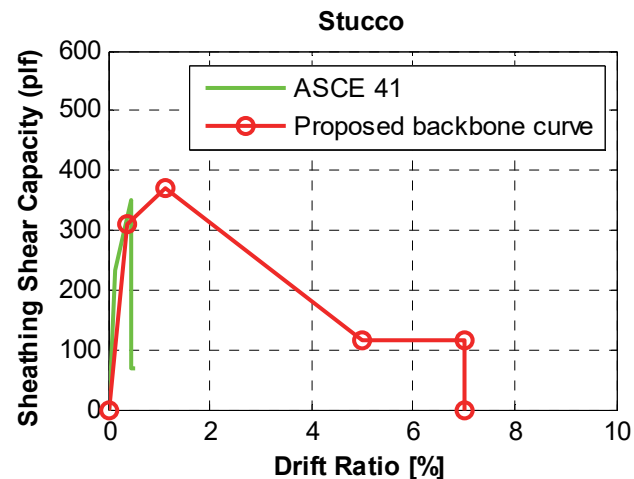

(b)

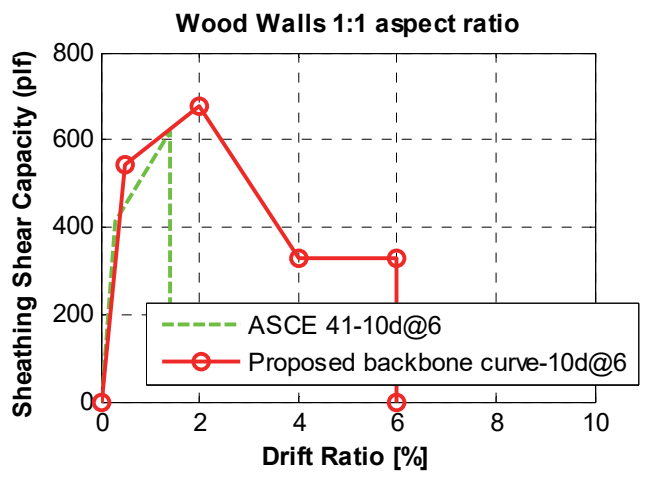

(d)

Figure G-3 Individual force-displacement curves for wood structural panels, gypsum wallboard and stucco panels used to synthesize the numerical model.

Fragility curves for the various levels of performance were considered (IO, LS, and $\mathrm{CP})$ as a function of the spectral acceleration, $S_{a}$, at the fundamental period of the wall system were developed and presented in Figure G-3 for a damping ratio of 3\% of critical. The story drift ratio was used as the Engineering Demand Parameter, while the spectral acceleration, $S_{a}$, at the fundamental period of the building was the Intensity Measure (IM) considered. Limit state values in terms of wall drift ratio were used in the development of the fragility curves. Drift limit state values of $1 \%$, $2 \%$, and $6 \%$ were associated with IO, LS and CP levels, respectively. A lognormal distribution was fitted to the fragility function for both modeling recommendations/assumptions and the results are presented in Figure G-4. The distribution associated parameters of the fragility curves (natural logarithm $\left(m_{R}\right)$ of the spectral acceleration and standard deviation values) for both models are summarized and compared in Table G-4. 


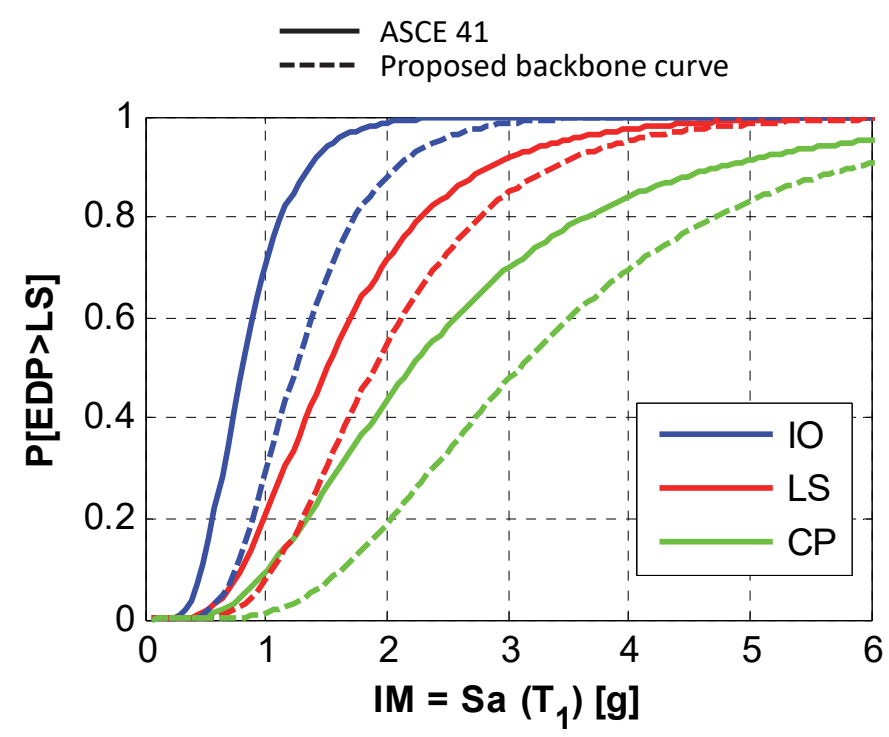

Figure G-4 Fragility curves for a typical exterior wood frame wall system for various performance levels.

Table G-4 Parameters for the Fragility Curves of the Wood-Frame Shear Wall

\begin{tabular}{|c|c|c|c|}
\hline Modeling Assumption & $\begin{array}{c}\text { Performance } \\
\text { Level }\end{array}$ & $\begin{array}{c}\text { Median } \\
\left(m_{R}\right)\end{array}$ & $\begin{array}{c}\text { Stand. Deviation } \\
\left(\xi_{R}\right)\end{array}$ \\
\hline \multirow{3}{*}{ ASCE/SEI 41-13 } & 10 & -0.22 & 0.41 \\
\hline & LS & 0.41 & 0.50 \\
\hline & $\mathrm{CP}$ & 0.79 & 0.60 \\
\hline \multirow{3}{*}{ Proposed backbone curve } & 10 & 0.22 & 0.40 \\
\hline & LS & 0.64 & 0.45 \\
\hline & $\mathrm{CP}$ & 1.13 & 0.50 \\
\hline
\end{tabular}

\section{G.3.2 System Level Nonlinear Model Development and Structural Analysis}

Further extending this study, system level analytical investigations considering the two modeling recommendations (ASCE/SEI 41-13 and the simplified backbone curve proposed in this document) were conducted.

A full building model of the five-story building archetype was developed in the SAPWood software accounting for all interior and exterior wood shear walls, roof diaphragms, the material and geometric properties as described in the design section. The wood roof diaphragms of the building archetype were also modeled with hysteretic elements described by the CUREE-SAWS model. The hysteretic properties of the roof diaphragm elements were identified based on their geometry and nailing configuration considering the roof diaphragm model developed by Koliou et al. (2016). The seismic weight assigned at each shear wall and roof diaphragm 
node was computed based on their tributary area, while a viscous damping ratio equal to $3 \%$ of critical was considered in the analyses.

Similar to the component level study, incremental dynamic analyses were conducted to evaluate and compare the two modeling assumptions for the wood shear walls. The story drift ratio was considered as the EDP in this set of analyses, while the spectral acceleration at the fundamental period of the structure was the IM used.

The results of the nonlinear incremental dynamic analyses are presented in Figure G-5 in the form of fragility curves, while the distribution associated fragility parameters are summarized in Table G-5.

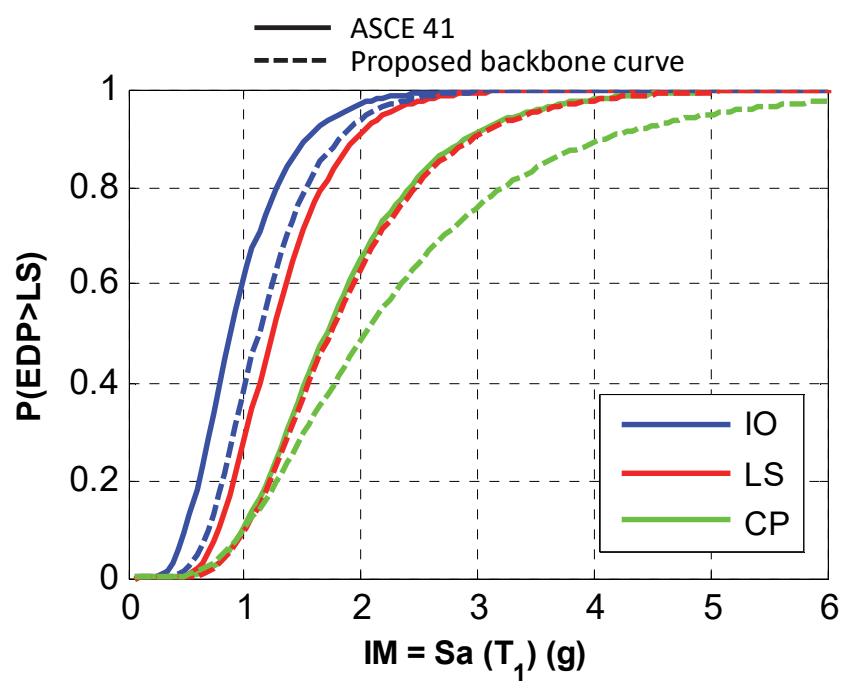

Figure G-5 Fragility curves for the building archetype for various performance levels.

Table G-5 Parameters for the Fragility Curves of the 5-Story Wood-Frame Shear Wall

\begin{tabular}{|c|c|c|c|}
\hline Modeling Assumption & $\begin{array}{c}\text { Performance } \\
\text { Level }\end{array}$ & $\begin{array}{c}\text { Median } \\
\left(m_{R}\right) \\
\end{array}$ & $\begin{array}{c}\text { Stand. Deviation } \\
\left(\xi_{R}\right)\end{array}$ \\
\hline \multirow{3}{*}{ ASCE/SEI 41-13 } & 10 & -0.13 & 0.44 \\
\hline & LS & 0.21 & 0.36 \\
\hline & $\mathrm{CP}$ & 0.53 & 0.42 \\
\hline \multirow{3}{*}{ Proposed backbone curve } & 10 & 0.11 & 0.38 \\
\hline & LS & 0.55 & 0.42 \\
\hline & $\mathrm{CP}$ & 0.71 & 0.55 \\
\hline
\end{tabular}




\section{G.4 Summary}

Based on the results of this limited analytical effort at both the component (wood shear walls) and system level (full building archetype), the proposed backbone curve (Figure G-2) was found to be associated with higher response capacities compared to the ASCE/SEI 41-13 recommendation. Thus, the non-trivial residual strength level and increased displacement capacity accounted for the wall assemblies in the proposed backbone curve may result in a more realistic representation of the response of wood frame wall systems aligning better with observed earthquake performance in-situ and shake table studies. It can be argued that buildings designed to current code are not anticipated to collapse at $2 \mathrm{~g}$ to $3 \mathrm{~g}$ as captured by the building analyzed with the new proposed backbone curve. It is recommended that the proposed backbone curve be used for conducting nonlinear time history analyses of wood frame wall systems since it will lead to more accurate and economic design or retrofit of buildings. 


\section{References}

ACI, 1989, Building Code Requirements for Structural Concrete and Commentary, ACI 318-89, American Concrete Institute, Farmington Hills, Michigan.

ACI, 2005, Acceptance Criteria for Moment Frames Based on Structural Testing and Commentary, ACI 374. 1-05, American Concrete Institute, Farmington Hills, Michigan.

ACI, 2011, Guide for Seismic Rehabilitation of Existing Concrete Frame Buildings and Commentary, ACI 369R-11, American Concrete Institute, Farmington Hills, Michigan.

ACI, 2012, Bond and Development of Straight Reinforcing Bars in Tension (Reapproved 2012), ACI 408R-03, American Concrete Institute, Detroit, Michigan.

ACI, 2014, Building Code Requirements for Structural Concrete and Commentary, ACI 318-14, American Concrete Institute, Farmington Hills, Michigan.

ACI, 2017, Guide for Seismic Rehabilitation of Existing Concrete Frame Buildings and Commentary, ACI 369R-17, American Concrete Institute, Farmington Hills, Michigan.

Ahmadi, F., 2012, "Displacement-based seismic design and tools for reinforced masonry shear-wall structures," Ph.D. Dissertation, Department of Civil Engineering, The University of Texas at Austin.

AIJ, 1985a, "Load-deflection characteristics of nuclear reactor building structures: Parts 8-9-10," Summaries of Technical Papers, Structural Division, Architectural Institute of Japan, Vol. 58.

AIJ, 1985b, "Load-deflection characteristics of nuclear reactor building structures: Parts 37-38-39-40," Summaries of Technical Papers of Annual Meeting, Architectural Institute of Japan.

AIJ, 1986a, "Load-deflection characteristics of nuclear reactor building structures: Parts 21-22," Summaries of Technical Papers, Structural Division, Architectural Institute of Japan, Vol. 59.

AIJ, 1986b, "Load-deflection characteristics of nuclear reactor building structures: Parts 59-60-61," Summaries of Technical Papers of Annual Meeting, Architectural Institute of Japan. 
AIJ, 1986c, "Load-deflection characteristics of nuclear reactor building structures:

Parts 62-63," Summaries of Technical Papers of Annual Meeting,

Architectural Institute of Japan.

AISC, 2005a, Seismic Provisions for Structural Steel Buildings, ANSI/AISC 341-05,

American National Standards Institute/American Institute of Steel

Construction, Chicago, Illinois.

AISC, 2005b, Prequalified Connections for Special and Intermediate Steel Moment

Frames for Seismic Applications, ANSI/AISC 358-05, American National

Standards Institute/American Institute of Steel Construction, Chicago,

Illinois.

AISC, 2010a, Seismic Provisions for Structural Steel Buildings, ANSI/AISC 341-10,

American National Standards Institute/American Institute of Steel

Construction, Chicago, Illinois.

AISC, 2010b, Prequalified Connections for Special and Intermediate Steel Moment

Frames for Seismic Applications, ANSI/AISC 358-10, American National

Standards Institute/American Institute of Steel Construction, Chicago,

Illinois.

AISC, 2010c, Specification for Structural Steel Buildings, ANSI/AISC 360-10,

American National Standards Institute/American Institute of Steel

Construction, Chicago, Illinois.

Alexander, C.M., Heidebrecht, A.C., and Tso, W.K., 1973, "Cyclic load tests on shear wall panels," Proceedings, Fifth World Conference on Earthquake Engineering, Rome, Italy, pp. 1116-1119.

Almeida, J.P., Targuini, D., and Beyer, K., 2016, "Modelling approaches for inelastic behaviour of RC walls: multi-level assessment and dependability of results," Archives of Computational Methods in Engineering, Vol. 23, No. 1 pp. 69-100.

Almeida, J., Prodan, O., Rosso, A., and Beyer, K., 2017, "Tests on thin reinforced concrete walls subjected to in-plane and out-of-plane cyclic loading," Earthquake Spectra, Vol. 33, No. 1., pp. 323-345.

Altoontash, A., 2004, Simulation and Damage Models for Performance Assessment of Reinforced Concrete Beam-Column Joints, Ph.D. dissertation, Stanford University, Stanford, California.

Arnold, A.E., Uang, C.-M., and Filiatrault, A., 2002, Cyclic Behavior and Repair of Stucco and Gypsum Sheathed Woodframe Walls: Phase I, Structural Systems Research Project Report No. SSRP-2002/07, Department of Structural Engineering, University of California, San Diego, La Jolla. 
ASCE, 1994, "Guidelines for design of joints between steel beams and reinforced concrete columns," Journal of Structural Division, Vol. 120, No. 8, pp. 2330-2357.

ASCE, 2006, Minimum Designs Loads for Buildings and Other Structures, ASCE/SEI 7-05, American Society of Civil Engineers, Reston, Virginia.

ASCE, 2007, Seismic Rehabilitation of Existing Buildings, ASCE/SEI 41-06, American Society of Civil Engineers, Reston, Virginia.

ASCE, 2010, Minimum Designs Loads for Buildings and Other Structures, ASCE/SEI 7-10, American Society of Civil Engineers, Reston, Virginia.

ASCE, 2014, Seismic Evaluation and Retrofit of Existing Buildings, ASCE/SEI 41-13, American Society of Civil Engineers, Reston, Virginia.

ASCE, 2017, Minimum Design Loads and Associated Criteria for Buildings and Other Structures, ASCE/SEI 7-16, American Society of Civil Engineers, Reston, Virginia (forthcoming).

ATC, 1992, Guidelines for Cyclic Seismic Testing of Components of Steel Structures, ATC-24, prepared by the Applied Technology Council, Redwood City, California.

ATC, 1996, Seismic Evaluation and Retrofit of Concrete Buildings, ATC-40, prepared by the Applied Technology Council, Redwood City, California.

Athanasopoulou, A., 2010, Shear Strength and Drift Capacity of Reinforced Concrete and High-Performance Fiber Reinforced Concrete Low-Rise Walls Subjected to Displacement Reversals, Ph.D. Dissertation, University of Michigan.

Atkinson, R.H., and Kingsley, G.R., 1985, A Comparison of the Behavior of Clay and Concrete Masonry in Compression, Report No. 1.1-1, U.S.-Japan Coordinated Program for Masonry Building Research, Atkinson-Noland \& Associates, Boulder, Colorado.

AWC, 2008, Special Design Provisions for Wind and Seismic - 2008, ANSI/AWC SDPWS-2008, American Wood Council, Leesburg, Virginia.

AWC, 2016, Personal communication with P. Line.

Bahmani, P., and van de Lindt, J.W., 2014, "Experimental and numerical assessment of woodframe sheathing layer combinations for use in strength-based and performance-based design," Journal of Structural Engineering, Special Issue on Seismic Resistant Timber Structures, Vol. 142, No. 4.

Barda, F., Hanson, J.M., and Corley, W.G., 1977, "Shear strength of low-rise walls with boundary elements," ACI Special Publication, Vol. 53, pp. 149-202. 
Bažant, Z.P., and Oh, B.H., 1983, "Crack band theory for fracture of concrete," Materials and Structures, Vol. 16, pp. 155-177.

Bech, D., Tremayne, B., and Houston, J., 2015, "Proposed changes to steel column evaluation criteria for existing buildings," Proceedings, ATC/SEI $2^{\text {nd }}$ Conference on Improving the Seismic Performance of Existing Buildings and Other Structures, Dec. 10-12, 2015, San Francisco, California.

Behrouzi, A., 2016, Impact of Cross Section, Web Reinforcement, and Load History on the Seismic Performance of Slender Concrete Walls, Ph.D. Dissertation, University of Illinois. Available at: https://www.ideals.illinois.edu /handle/2142/95368, last accessed April 25, 2017.

Behrouzi, A., Mock, A., Lowes, L., Lehman, D., and Kuchma, D., 2015, Summary of Large-Scale Nonplanar Reinforced Concrete Wall Tests, Charles Pankow Foundation.

Berry, M.P., Parrish, M., and Eberhard, M.O., 2004, PEER Structural Performance Database User's Manual, Pacific Earthquake Engineering Research Institute, Berkeley, California. Available at: http://nisee.berkeley.edu/spd/, last accessed April 17, 2017.

Berry, M.P., and Eberhard, M.O., 2008, Performance Modeling Strategies for Modern Reinforced Concrete Bridge Columns, PEER Report 2007/07, Pacific Earthquake Engineering Research Center, University of California, Berkeley, California.

Birely, A.C., Lowes, L.N., and Lehman, D., 2012, "Linear analysis of concrete frames considering joint flexibility," Structural Journal, Vol. 109, No. 3, pp. 381-92.

Birely, A., 2013, Seismic Performance of Slender Reinforced Concrete Structural Walls, Dissertation, University of Washington, Seattle, Washington.

Bohl, A., and Adebar P., 2011, "Plastic hinge lengths in high-rise concrete shear walls," Structural Journal, Vol. 108, No. 2, pp. 148-157.

Bolhassani, M., 2015, Improvement of Seismic Performance of Ordinary Reinforced Partially Grouted Concrete Masonry Shear Walls, Doctoral Dissertation, Drexel University, Philadelphia, Pennsylvania.

Brueggen, B.L., 2009, Performance of T-shaped Reinforced Concrete Structural Walls under Multi-Directional Loading, Ph.D. thesis, University of Minnesota.

Bruneau, M., and Mahin, S.A., 1991, "Full scale tests of butt welded splices in heavy rolled steel sections subjected to primary tensile stresses," Engineering Journal, Vol. 28, No. 1, pp. 1-17. 
Brunaeu, M., Mahin, S.A., and Popov, E.P., 1987, Ultimate Behavior of Butt Welded Splices in Heavy Rolled Steel Sections, Report No. UBC/EERC-87/10, University of California, Berkeley, California.

Caltrans, 2006, Seismic Design Criteria, Version 1.4, California Department of Transportation, Sacramento, California.

CEB, 1996, RC Frames Under Earthquake Loading - State of the Art Report, Comité Euro-International du Béton, Convenor Michael N. Fardis, published by Thomas Telford, London.

Celik, O.C., and Ellingwood, B.R., 2008, "Modeling beam-column joints in fragility assessment of gravity load designed reinforced concrete frames," Journal of Earthquake Engineering, Vol. 12, No. 3, pp. 357-81.

Charney, F.A., and Downs, W.M., 2004, "Modeling procedures for panel zone deformations in moment resisting frames," Proceedings, Connections in Steel Structures V, June 3-4, Amsterdam.

Cheng, F.Y., 1992, Coupling Bending and Shear Hysteretic Models of Low-Rise R.C. Walls, Concrete Shear in Earthquake, University of Houston, Texas.

Cheung, C.K., and Itani, R.Y., 1983, "Analysis of sheathed wood-stud walls," Proceedings, 8th Conference on Electronic Computation, American Society of Civil Engineers, New York, pp. 683-696.

Chi, B., and Uang, C.M., 2002, "Cyclic response and design recommendations of reduced beam section moment connections with deep columns," Journal of Structural Engineering, Vol. 128, No. 4, pp. 464-473.

Chopra, A.K., and Goel, R.K., 2004, "Evaluation of a modified MPA procedure assuming higher modes as elastic to estimate seismic demands," Earthquake Spectra, Vol. 20, No. 3, pp. 757-778.

Christovasilis, I.P., Filiatrault, A., and Wanitkorkul, A., 2009, Seismic Testing of a Full-Scale Two-Story Light-Frame Wood Building: NEESWOOD Benchmark Test, Technical Report MCEER-09-0005, University at Buffalo, New York.

CoLA-UCI Light Frame Test Committee, 2001, Report of a Testing Program of Light-Framed Walls with Wood-Sheathed Shear Panels, Final Report to the City of Los Angeles Department of Building and Safety.

Coleman, J., and Spacone, E., 2001, "Localization issues in forced-based frame elements," Journal of Structural Engineering, Vol. 127, No. 11, pp. 1257-1265.

Cordova, P.P., and Deierlein, G.G., 2005, Validation of the Seismic Performance of Composite RCS Frames: Full-Scale Testing, Analytical Modeling, and 
Seismic Design, Report No. 155, John A. Blume Earthquake Engineering Center, Stanford University, Stanford, California.

Corley, W.G., 1966, "Rotational capacity of reinforced concrete beams," Journal of the Structural Division, Vol. 92, No. 5, pp. 121-46.

CSI, 2016, Nonlinear Analysis and Performance Assessment of 3D Structures, PERFORM-3D, Computers and Structures, Inc., Walnut Creek, California.

Dazio, A., Beyer, K., and Bachmann, H., 2009, "Quasi-static cyclic tests and plastic hinge analysis of RC structural walls," Engineering Structures, Vol. 31, No. 7, pp.1556-71.

Deierlein, G.G., Sheikh, T.M., Yuram, J.A., and Jirsa, J.O., 1989, "Beam-column moment connections for composite frames: part 2," Journal of Structural Engineering, Vol. 115, No. 11, pp. 2877-2896.

Dolan, J.D., and Filiatrault, A., 1990, "A mathematical model to predict the steadystate response of timber shear walls," Proceedings of the International Timber Engineering, pp. 765-772.

Effendy, E., Liao, W., Song, G., Mo, Y., and Loh, C., 2006, "Seismic behavior of low-rise shear walls with SMA bars," Earth \& Space.

Elkady, A., and Lignos, D.G., 2013, "Effect of composite action on the dynamic stability of special steel moment resisting frames designed in seismic regions," Proceedings, ASCE Structures Congress, Pittsburgh, Pennsylvania, pp. 2151-2160.

Elkady, A., and Lignos, D.G., 2014, "Modeling of the composite action in fully restrained beam-to-column connections: implications in the seismic design and collapse capacity of steel special moment frames," Earthquake Engineering and Structural Dynamics, Vol. 43, No. 13, pp. 1935-1954.

Elkady, A., and Lignos, D.G., 2015a, "Effect of gravity framing on the overstrength and collapse capacity of steel frame buildings with perimeter special moment frames," Earthquake Engineering and Structural Dynamics, Vol. 44, No. 8, pp. 1289-1307.

Elkady, A., and Lignos, D.G., 2015b, "Analytical investigation of the cyclic behavior and plastic hinge formation in deep wide-flange steel beam-columns," Bulletin of Earthquake Engineering, Vol. 13, No. 4, pp. 1097-1118.

Elkady, A., and Lignos, D.G., 2016, "Dynamic stability of deep and slender wideflange steel columns - full scale experiments," Proceedings, Annual Stability Conference, Structural Stability Research Council (SSRC), April 12-15, Orlando, Florida. 
El-Sokkary, H., and Galal, K., 2013, "Seismic behavior of RC shear walls strengthened with fiber-reinforced polymer," Journal of Composites for Construction, Vol. 17, pp. 603-613.

Elwood, K.J., and Eberhard, M.O., 2009, "Effective stiffness of reinforced concrete columns," Structural Journal, Vol. 106, No. 4, pp. 476-84.

Elwood, K.J., Matamoros, A.B., Wallace, J.W., Lehman, D.E., Heintz, J.A., Mitchell, A.D., Moore, M.A., Valley, M.T., Lowes, L.N., Comartin, C.D., and Moehle, J.P., 2007, "Update to ASCE/SEI 41 concrete provisions," Earthquake Spectra, Vol. 23, No. 3, pp. 493-523.

Engelhardt, M.D., and Sabol, T.A., 1994, Testing of Welded Steel Moment Connections in Response to the Northridge Earthquake, AISC Progress Report, Chicago, Illinois.

Engelhardt, M.D., Fry, D.T., and Venti, M.J., 2000, Behavior and Design of Radius Cut Reduced Beam Section Connections, SAC Steel Project Document, Report No. SAC/BD-00/17, prepared by the SAC Joint Venture, a partnership of the Structural Engineers Association of California (SEAOC), Applied Technology Council (ATC), and California Universities for Research in Earthquake Engineering (CUREE).

Falk, R.H., and Itani, R.Y., 1989, "Finite element modeling of wood diaphragms," Journal of Structural Engineering, Vol. 115, No. 3, pp. 543-559.

Farvashany, F.E., Foster, S.J., and Rangan, B.V., 2008, "Strength and deformation of high-strength concrete shear walls," Structural Journal, Vol. 105.

Fédération Internationale du Béton (FIB), 2010, "FIB model code for concrete structures," Ernst \& Sohn.

FEMA, 1997a, NEHRP Guidelines for the Seismic Rehabilitation of Buildings, FEMA 273, prepared by Applied Technology Council for Federal Emergency Management Agency, Washington, D.C.

FEMA, 1997b, NEHRP Commentary on the Guidelines for the Seismic Rehabilitation of Buildings, FEMA 274, prepared by Applied Technology Council for Federal Emergency Management Agency, Washington, D.C.

FEMA, 1997c, Connection Test Summaries, FEMA 289, prepared by the SAC Joint Venture, a partnership of the Structural Engineers Association of California (SEAOC), Applied Technology Council (ATC), and California Universities for Research in Earthquake Engineering (CUREE), for the Federal Emergency Management Agency, Washington, D.C. 
FEMA, 1998, Evaluation of Earthquake Damaged Concrete and Masonry Wall Buildings, FEMA 306, prepared by Applied Technology Council for Federal Emergency Management Agency, Washington, D.C.

FEMA, 2000a, Prestandard and Commentary for the Seismic Rehabilitation of Buildings, FEMA 356, prepared by the American Society of Civil Engineers for the Federal Emergency Management Agency, Washington, D.C.

FEMA, 2000b, Recommended Seismic Design Criteria for New Steel Moment Frame Buildings, FEMA 350, prepared by the SAC Joint Venture, a partnership of the Structural Engineers Association of California (SEAOC), Applied Technology Council (ATC), and California Universities for Research in Earthquake Engineering (CUREe), for the Federal Emergency Management Agency, Washington, D.C.

FEMA, 2000c, State of the Art Report on Systems Performance of Moment Steel Frame Buildings in Earthquakes, FEMA 355C, prepared by the SAC Joint Venture, a partnership of the Structural Engineers Association of California (SEAOC), Applied Technology Council (ATC), and California Universities for Research in Earthquake Engineering (CUREe), for the Federal Emergency Management Agency, Washington, D.C.

FEMA, 2000d, State of the Art Report on Connection Performance, FEMA 355D, prepared by the SAC Joint Venture, a partnership of the Structural Engineers Association of California (SEAOC), Applied Technology Council (ATC), and California Universities for Research in Earthquake Engineering (CUREE), for the Federal Emergency Management Agency, Washington, D.C.

FEMA, 2009a, Effects of Strength and Stiffness Degradation on Seismic Response, FEMA P-440A, prepared by the Applied Technology Council for the Federal Emergency Management Agency, Washington, D.C.

FEMA, 2009b, Quantification of Building Seismic Performance Factors, FEMA P-695, prepared by Applied Technology Council for Federal Emergency Management Agency, Washington, D.C.

FEMA, 2012a, Seismic Performance Assessment of Buildings, FEMA P-58, prepared by Applied Technology Council for Federal Emergency Management Agency, Washington, D.C.

FEMA, 2012b, Seismic Evaluation and Retrofit of Multi-Unit Wood-Frame Buildings with Weak First Stories, FEMA P-807, prepared by Applied Technology Council for Federal Emergency Management Agency, Washington, D.C. 
Filiatrault, A., Christovasilis, I., Wanitkorkul, A., and van de Lindt, J.W., 2010, "Experimental seismic response of a full-scale light-frame wood building," Journal of Structural Engineering, Vol. 136, No. 3, pp. 246-254.

Filippou F.C., Popov, E.P., and Bertero, V.V., 1983, Effects of Bond Deterioration on Hysteretic Behavior of Reinforced Concrete Joints, Report EERC 83-19, Earthquake Engineering Research Center, University of California, Berkeley.

Fisher, J.M., and Kloiber, L.A., 2006, Design Guide 1: Base Plate and Anchor Rod Design, American Institute of Steel Construction (AISC), Chicago, Illinois.

Flores, F.X., Charney, F.A., and Lopez-Garcia, D., 2014, "Influence of the gravity framing system on the collapse performance of special steel moment frames," Journal of Constructional Steel Research, Vol. 101, No. 10, pp. 351-362.

Folz, B., and Filiatrault, A., 2001, "Cyclic analysis of wood shear walls," Journal of Structural Engineering, Vol. 127, No. 4, pp. 433-441.

Folz, B., and Filiatrault, A., 2004a, "Seismic analysis of woodframe structures, I: Model formulation," Journal of Structural Engineering, Vol. 130, No. 9, pp. 1353-1360.

Folz, B., and Filiatrault, A., 2004b, "Seismic analysis of woodframe structures, II: Model implementation and verification," Journal of Structural Engineering, Vol. 130, No. 9, pp. 1361-1370.

Ganesh, M., Upadhyay, A., and Kaushik, S.K., 2006, “Assessment of longitudinal shear strength parameters of composite slab by artificial neural network", Asian Journal of Civil Engineering, Vol. 7, No. 3, pp. 287-300.

Gatto, K., and Uang, C.-M., 2002, Cyclic Response of Woodframe Shearwalls: Loading Protocol and Rate of Loading Effects, the CUREE-Caltech Woodframe Project Publication No. W-13, Consortium of Universities for Research in Earthquake Engineering, California.

Ghannoum, W.M., and Moehle, J.P., 2012, "Dynamic collapse analysis of a concrete frame sustaining column axial failures," Structural Journal, Vol. 109, No. 3, pp. 403-12.

Ghannoum, W.M., and Sivaramakrishnan, B., 2012a, ACI 369 Circular Column Database, Network for Earthquake Engineering Simulation (NEES), DOI:10.4231/D39Z90B9T.

Ghannoum, W.M., and Sivaramakrishnan, B., 2012b, ACI 369 Rectangular Column Database, Network for Earthquake Engineering Simulation (NEES), DOI:10.4231/D36688J50. 
Gomez, I.R., 2010, Behavior and Design of Column Base Connections, Ph.D. Dissertation, University of California, Davis, California.

Greifenhagen, C., Papas, D., and Lestuzzi, P., 2005, Static-Cyclic Tests on Reinforced Concrete Shear Walls with Low Reinforcement Ratios, Report No N4, School of Architecture, Civil and Environmental Engineering, Ecole Polytechnique Federale De Lausanne, Switzerland.

Grilli, D.A., and Kanvinde, A.M., 2015, Embedded Column Base Connections Subjected to Flexure and Axial Load: Tests and Strength Models, Report 3-11, Charles Pankow Foundation, Vancouver, Washington.

Gulec, C.K., and Whittaker, A.S., 2011a, "Empirical equations for peak shear strength of low aspect ratio reinforced concrete walls," Structural Journal, Vol. 108, No. 1, pp. 80-89.

Gulec, C.K., and Whittaker, A.S., 2011b, "Hysteretic modeling of squat reinforced concrete shear walls," Proceedings, 21th International Conference on Structural Mechanics in Reactor Technology (SMiRT21), International Association for Structural Mechanics in Reactor Technology (IASMiRT), New Delhi, India.

Gulec, C.K., Whittaker, A.S., and Stojadinovic, B., 2008, "Shear strength of squat rectangular reinforced concrete walls," Structural Journal, Vol. 105, pp. 488-497.

Gulec, C.K., Whittaker, A.S., and Stojadinovic, B., 2009, "Peak shear strength of squat reinforced concrete walls with boundary barbells or flanges," Structural Journal, Vol. 106, pp. 368-377.

Gunnarsson, I.R., 2004, Numerical Performance Evaluation of Braced Frame Systems, a thesis submitted in partial fulfillment of the MSCE, University of Washington, Seattle, Washington.

Gupta, A., and Krawinkler, H., 1999, Seismic Demands for Performance Evaluation of Steel Moment Resisting Frame Structures, Report No. 132, John A. Blume Earthquake Engineering Center, Stanford University, Stanford, California.

Gupta, A., and Krawinkler, H., 2000a, "Behavior of ductile SMRFs at various seismic hazard levels," Journal of Structural Engineering, Vol. 126, No. 1, pp. 98-107.

Gupta, A., and Krawinkler, H., 2000b, "Dynamic P-delta effects for flexible inelastic steel structures," Journal of Structural Engineering, Vol. 126, No. 1, pp. 145-154.

Gupta, A.K., and Kuo, P.-H., 1985, "Behavior of wood-framed shear walls," Journal of Structural Engineering, Vol. 111, No. 8, pp. 1722-1733. 
Hajyalikhani, P., 2015, Experimental Study on Seismic Performance of Reinforced Concrete Coupling Beams and Rectangular Squat Walls with Innovative Reinforcement Configurations, Ph.D. Dissertation, University of Texas at Arlington.

Hartloper, A., and Lignos, D.G., 2016, Updates to the ASCE-41-13 Nonlinear Modeling Provisions for Performance-Based Seismic Assessment of New and Existing Steel Moment Resisting Frames, Masters of Engineering Thesis, McGill University, Montreal, Canada.

Hartloper, A., and Lignos, D.G., 2017, "Updates to the ASCE-41-13 provisions for the nonlinear modeling of steel wide-flange columns for performance-based earthquake engineering," Proceedings Eurosteel, September 13-15, 2017, Copenhagen, Denmark.

Haselton, C.B., Liel, A.B., Lange, S. T., and Deierlein, G.G., 2008, Beam-Column Element Model Calibrated for Predicting Flexural Response Leading to Global Collapse of RC Frame Buildings, PEER Report 2007/03, Pacific Engineering Research Center, University of California, Berkeley, California.

Haselton, C.B., Liel, A.B., Taylor Lange, S., and Deierlein, G.G., 2016, “Calibration of reinforced concrete beam-columns for simulating seismic response to collapse," Structural Journal, American Concrete Institute, Vol. 113, No. 06, pp. 1141-1152.

Hertz, J., Lignos, D.G., and Rogers, C.A., 2015, "Full-scale experimental testing of extended beam-to-column and beam-to-girder shear tab connections subjected to shear," Proceedings, $8^{\text {th }}$ International Conference on Behavior of Steel Structures in Seismic Areas, July 1-3, Shanghai, China.

Hidalgo, P.A., Ledezma, C.A., and Jordan, R.M., 2002, "Seismic behavior of squat reinforced concrete shear walls," Earthquake Spectra, Vol. 18, pp. 287-308.

Hirosawa, M., 1975, Past Experimental Results on Reinforced Concrete Shear Walls and Analysis on Them, Kenchiku Kenkyu Shiryo, No. 6, Building Research Institute, Ministry of Construction, Tokyo, Japan.

Hsiao, P., Lehman, D., and Roeder, C., 2012, "Improved analytical model for special concentrically braced frames," Journal of Constructional Steel Research, Vol. 73, pp. 80-94.

Hsiao, P., Lehman, D., and Roeder, C., 2013, “A model to simulate special concentrically braced frames beyond brace fracture," Journal of Earthquake Engineering and Structural Dynamics, Vol. 42, Issue 2. 
Huang, C.C., and Sheu, M.S., 1988, "Experimental and theoretical study on aseismic behaviors of low-rise RC shear walls," Proceedings, Ninth World Conference on Earthquake Engineering, Tokyo-Kyoto, Japan, pp. 6/35-6/40.

Hwang, S.J., and Moehle, J.P., 2000, "Models for laterally loaded slab-column frames," Structural Journal, Vol. 97, No. 2, pp. 345-52.

Ibarra, L.F., and Krawinkler, H., 2005, Global Collapse of Frame Structures under Seismic Excitations, Report No. 152, John A. Blume Earthquake Engineering Center, Stanford, California.

Ibarra, L.F., Medina, R.A., and Krawinkler, H., 2005, "Hysteretic models that incorporate strength and stiffness deterioration," Earthquake Engineering and Structural Dynamics, Vol. 34, No. 12, pp. 1489-1511.

ICBO, 1949, 1952, 1955, 1958, 1961, 1964, 1967, 1973, 1976, 1982, 1985, 1988, 1994, 1997, Uniform Building Code, International Conference of Building Officials, Whittier, California.

ICC, 2000, 2006, 2012, International Building Code, International Code Council, Washington, D.C.

Jansen, D.C., and Shah, S.P., 1997, "Effect of length on compressive strain softening of concrete," Journal of Engineering Mechanics, Vol. 123, No. 1, pp. 25-35.

Jeon, J-S., Lowes, L.N., and DesRoches, R., 2014, Numerical Models for BeamColumn Joints in Reinforced Concrete Building Frames, Special Publication SP 297, American Concrete Institute, Farmington Hills, Michigan.

Johnston, A.R., Dean, P.K., and Shenton III, H.W., 2006, "Effect of vertical load and hold-down anchors on the cyclic response of wood framed shear walls," Journal of Structural Engineering, Vol. 132, No. 9, pp. 1426-1434.

Kang, T.H., and Wallace, J.W., 2005, "Dynamic responses of flat plate systems with shear reinforcement," Structural Journal, Vol. 102, No. 5, pp. 763.

Kanvinde, A., Grilli, D., and Zareian, F., 2012, "Rotational stiffness of exposed column base connections: experiments and analytical models," Journal of Structural Engineering, Vol. 138, No. 5, pp. 549-560.

Kapoi, C.M., 2012, Experimental Performance of Concrete Masonry Shear Walls under In-Plane Loading, Master Thesis, Washington State University, Pullman, Washington.

Kassem, W., 2015, "Shear strength of squat walls: A strut-and-tie model and closedform design formula," Engineering Structures, Vol. 84, pp. 430-438.

Kazaz, I., 2013, "Analytical study on plastic hinge length of structural walls," Journal of Structural Engineering, Vol. 139, No. 11, pp. 1938-1950. 
Kent, D.C., and Park, R., 1971, "Flexural members with confined concrete," Journal of the Structural Division, Vol. 97, No. 7, pp. 1969-1990.

Kim, K.D., and Engelhardt, M.D., 2002, "Monotonic and cyclic loading models for panel zones in steel moment frames," Journal of Constructional Steel Research, Vol. 58, pp. 605-635.

Koliou, M., 2014, Seismic Analysis and Design of Rigid Wall-Flexible Roof Diaphragm Structures, Ph.D. Thesis, University at Buffalo, State University of New York, Buffalo, New York.

Koliou, M., Filiatrault, A., Kelly, D. J., and Lawson, J., 2016, “Distributed yielding concept for improved seismic collapse performance of rigid wall-flexible diaphragm buildings," Journal of Structural Engineering, Vol. 142, No. 2, pp. 1-16.

Kostic, S., and Filippou, F., 2012, "Section discretization of fiber beam-column elements for cyclic inelastic response," Journal of Structural Engineering, Vol. 138, No. 5, pp. 592-601.

Krawinkler, H., 1978, "Shear in beam-column joints in seismic design of steel frames," Engineering Journal, Vol. 15, No. 3, pp. 82-91.

Krawinkler, H., and Seneviratna, G.D.P.K., 1998, "Pros and cons of a pushover analysis of seismic performance evaluation," Engineering Structures, Vol. 20, No. 4-6, pp. 452-464.

Krawinkler, H., 2009, "Loading histories for cyclic tests in support of performance assessment of structural components," PEER Annual Meeting, San Francisco, California.

Krawinkler, H., Bertero, V.V., and Popov, E.P., 1971, Inelastic Behavior of Steel Beam-to- Column Subassemblages, Report EERC-71-7, University of California, Berkeley, California.

Krawinkler, H., Lignos, D.G., and Putman, C., 2011, "Prediction of nonlinear response - pushover analysis versus simplified nonlinear response history analysis," Proceedings, ASCE Structures Congress, April 14-16, 2011, Las Vegas, Nevada, United States.

Kuang, J.S., and Ho, Y.B., 2008, "Seismic behavior and ductility of squat reinforced concrete shear walls with nonseismic detailing," Structural Journal, Vol. 105, pp. 225-231.

Kwon, J., 2016, Strength, Stiffness, and Damage of Reinforced Concrete Buildings Subjected to Seismic Motions, University of Texas at Austin, Austin, Texas, p. 239. 
Lefas, I.D., and Kotsovos, M.D, 1990, "Strength and deformation characteristics of reinforced concrete walls under load reversals," Structural Journal, Vol. 87, pp. 716-726.

Lehman, D.E., Roeder, C.W., Herman, D., Johnson, S., and Kotulka, B., 2008, "Improved Seismic Performance of Gusset Plate Connections," Journal of Structural Engineering, Vol. 134, No. 6, pp. 890-901.

Lehman, D.E., Turgeon, A.J., Birely, A.C., Hart, C.R., Marley, K.P., Kuchma, D.A., and Lowes, L.N., 2013, "Seismic behavior of a modern concrete coupled wall," Journal of Structural Engineering, Vol. 139, No. 8, pp. 1371-1381.

Li, B., Pan, Z., and Xiang, W., 2015, "Experimental evaluation of seismic performance of squat RC structural walls with limited ductility reinforcing details," Journal of Earthquake Engineering, Vol. 19, pp. 313-331.

Lignos, D.G., and Krawinkler, H., 2010, “A steel database for component deterioration of tubular hollow square steel columns under varying axial load for collapse assessment of steel structures under earthquakes," Proceedings, $7^{\text {th }}$ International Conference on Urban Earthquake Engineering (7CUEE) and $5^{\text {th }}$ International Conference on Earthquake Engineering (5ICEE), March 3-5, Tokyo Institute of Technology, Tokyo, Japan.

Lignos, D.G., and Krawinkler, H., 2011, “Deterioration modeling of steel components in support of collapse prediction of steel moment frames under earthquake loading," Journal of Structural Engineering, Vol. 137, No. 11, pp. 1291-1302.

Lignos, D.G., Krawinkler, H., and Whittaker, A., 2011a, "Prediction and validation of sidesway collapse of two scale models of a 4-story steel moment frame," Earthquake Engineering and Structural Dynamics, Vol. 40, No. 7, pp. 807825.

Lignos, D.G., Eads, L., and Krawinkler, H., 2011b, "Effect of composite action on collapse capacity of steel moment frames under cyclic loading," Proceedings, Eurosteel, Budapest, Hungary.

Lignos, D.G., and Krawinkler, H., 2012a, "Development and utilization of structural component databases for performance-based earthquake engineering," Journal of Structural Engineering, Vol. 139, No. 8, pp. 1382-1394.

Lignos, D.G., and Krawinkler, H., 2012b, Sidesway Collapse of Deteriorating Structural Systems under Seismic Excitations, Report 177, John A. Blume Earthquake Engineering Center, Stanford University, Stanford, California.

Lignos, D.G., Hikino, T., Matsuoka, Y., and Nakashima, M., 2013, “Collapse assessment of steel moment frames based on e-defense full scale shake table 
collapse tests," Journal of Structural Engineering, Vol. 139, No. 1, pp. 120132.

Lignos, D.G., Putman, C., and Krawinkler H., 2015, “Application of simplified analysis procedures for performance-based earthquake evaluation of steel special moment frames," Earthquake Spectra, Vol. 31, No. 4, pp. 1949-1968.

Lignos, D.G., Cravero, J., and Elkady, A., 2016, "Experimental investigation of the hysteretic behavior of wide-flange steel columns under high axial load and lateral drifts demands," Proceedings, the $11^{\text {th }}$ Pacific Structural Steel Conference, October 26-28, Shanghai, China.

Line, P., Waltz, N., and Skaggs, T., 2008, "Seismic equivalence parameters for engineered wood frame wood structural panel shear walls," Wood Design Focus, Vol. 18, No. 2.

Liu, J., 2000, Experimental and Analytical Studies of the Cyclic Behavior of Simple Connections in Steel Frame Buildings, Ph.D. Dissertation, University of California, Berkeley, California.

Liu, J., and Astaneh, A., 2000, "Cyclic testing of simple connections including effects of slab," Journal of Structural Engineering, Vol. 126, No.1, pp. 32-39.

Liu, J., and Astaneh, A., 2004, "Moment-rotation parameters for composite shear tab connections," Journal of Structural Engineering, Vol. 130, No.9, pp. 1371-1380.

Lopes, M.S., and Elnashai, A.S., 1991, Seismic Behaviour of RC Walls with Low Shear Ratio, Report No. 91-9, ESEE Research Report, Civil Engineering Department, Imperial College, London, United Kingdom.

Lu, L.W., Wang, S.J., and Lee, S.J., 1988, "Cyclic behavior of steel and composite joint with panel zone deformation", Proceedings, $9^{\text {th }}$ World Conference on Earthquake Engineering, August 2-9, Tokyo-Kyoto, Japan.

Lu, L.W., Ricles, J.M., Mao, C., and Fisher, J.W., 2000, "Critical issues in achieving ductile behaviour of welded moment connections," Journal of Constructional Steel Research, Vol. 55, No. 1-3, pp. 325-341.

Luna, B.N., Rivera, J.P., and Whittaker, A.S., 2015, "Seismic behavior of lowaspect-ratio reinforced concrete shear walls," Structural Journal, Vol. 112, pp. 593-603.

MacRae, GA., Carr, A.J. and Walpole, W.R., 1990, The Seismic Response of Steel Frames, Report No. 90-6, Department of Civil Engineering, University of Canterbury, Christchurch, New Zealand. 
Mahin, S.A., 1998, "Lessons from damage to steel buildings during the Northridge earthquake," Engineering Structures, Vol. 20, No. 4-6, pp. 261-270.

Maier, J., and Thürlimann, B., 1985, Bruchversuche an Stahlbetonscheiben, Institut für Baustatik und Konstruktion, Eidgenössische Technische Hochschule (ETH), Zürich, Switzerland.

Mander, J., Priestley, M., and Park, R., 1988, “Observed stress-strain behavior of confined concrete," Journal of Structural Engineering, Vol. 114, No. 8, pp. $1827-1849$.

Mander, J.B., Priestley, M.J.N., Park, R., 1998, “Theoretical stress-strain model for confined concrete," Journal of Structural Engineering, Vol. 114, No. 8, pp. 1804-26.

Mansour, M., and Hsu, T.T.C., 2005, "Behavior of reinforced concrete elements under cyclic shear. I: Experiments.” Journal of Structural Engineering, Vol. 131, pp. 44-53.

Marini, A., and Spacone, E., 2006, “Analysis of reinforced concrete elements including shear effects," Structural Journal, Vol. 103, No. 5, pp. 645-655.

Massone, L.M., 2006, RC Wall Shear-Flexure Interaction: Analytical and Experimental Responses, University of California, Los Angeles.

Mattock, A.H., and Gaafar, G.H., 1982, "Strength of embedded steel sections as brackets," Structural Journal, Vol. 79, No. 2, pp. 83-93.

Mavros, M., 2015, Experimental and Numerical Investigation of the Seismic Performance of Reinforced Masonry Structures, Ph.D. Dissertation, University of California at San Diego, La Jolla, California.

Mavros, M., Koutromanos, I., and Shing, P.B., 2015, "Nonlinear finite element analysis of reinforced masonry structures subjected to earthquake forces," Proceedings, $12^{\text {th }}$ North American Masonry Conference, Denver, Colorado.

Mavros, M., Ahmadi, F., Shing, P.B., Klingner, R.E., McLean, D., and Stavridis, A., 2016, "Shake-table tests of a full-scale two-story shear-dominated reinforced masonry wall structure," Journal of Structural Engineering, Vol. 142, No. 10.

McKenna, F., 1997, Object Oriented Finite Element Programming Framework for Analysis, Algorithms and Parallel Computing, Ph.D. Dissertation, University of California, Berkeley.

McKenna, F., Fenves, G.L., Scott, M.H., Jeremie, B., 2000, Open System for Earthquake Engineering Simulation, OpenSEES. 
McKenna, F., and Fenves, G.L., 2001, The Opensees Command Language Manual, University of California, Berkeley.

McMullin, K.M., and Merrick, D., 2002, Seismic Performance of Gypsum Walls: Experimental Test Program, the CUREE-Caltech Woodframe Project Publication No. W-15, Consortium of Universities for Research in Earthquake Engineering, California.

Minaie, E., Mota, M., Moon, F.L., and Hamid, A.A., 2010, "In-plane behavior of partially grouted reinforced concrete masonry shear walls," Journal of Structural Engineering, Vol. 136, No. 9, pp. 1089-1097.

Moehle, J., 2015, Seismic Design of Reinforced Concrete Buildings, McGraw Hill Professional.

Moehle, J.P., Bozorgnia, Y., Jayaram, N., Jones, P., Rahnama, M., Shome, N., Tuna, Z., Wallace, J., Yang, T., and Zareian, F., 2011, Case Studies of the Seismic Performance of Tall Buildings Designed by Alternative Means, PEER Report 2011/05, Pacific Earthquake Engineering Research Center, Berkeley, California.

Mohammadi-Doostdar, H., 1994, Behavior and Design of Earthquake Resistant LowRise Shear Walls, University of Ottawa, Canada.

Nakamura, H., and Higai, T, 2001, "Compressive fracture energy and fracture zone length of concrete," Modeling of Inelastic Behavior of RC Structures under Seismic Loads, American Society of Civil Engineers, Editors, Reston, Virginia.

Nakashima, M., Matsumiya, T., Suita, K., and Zhou, F., 2007, "Full-scale test of composite frame under large cyclic loading," Journal of Structural Engineering, Vol. 133, No. 2, pp. 297-304.

Nakashima, M., Roeder, C., and Maruoka, Y., 2000, "Steel moment frames for earthquakes in United States and Japan," Journal of Structural Engineering, Vol. 126, No. 8, pp. 861-868.

Nam, T.T., and Kasai, K., 2012, "Study on shake table experimental results regarding composite action of a full-scale steel building tested to collapse," Proceedings, $9^{\text {th }}$ International Conference on Urban Earthquake Engineering $/ 4^{\text {th }}$ Asia Conference on Earthquake Engineering, Tokyo Institute of Technology, Tokyo, Japan.

Newell, J.D., and Uang, C.M., 2006a, Cyclic Behavior of Steel Columns with Combined High Axial Load and Drift Demand, Structural Systems Research Project Report No. SSRP-06/22, Department of Structural Engineering, University of California, San Diego, La Jolla. 
Newell, J.D., and Uang, C.M., 2006b, Cyclic Testing of Steel Moment Connections for the Caltrans District 4 Office Building Seismic Upgrade, Structural Systems Research Project Report No. SSRP-05/03 Department of Structural Engineering, University of California, San Diego, La Jolla.

NIST, 2008, NEHRP Seismic Design Technical Brief No. 1: Seismic Design of Reinforced Concrete Special Moment Frames, A Guide for Practicing Engineers, NIST GCR 8-917-1, prepared by the NEHRP Consultants Joint Venture, a partnership of the Applied Technology Council and the Consortium of Universities for Research in Earthquake Engineering for the National Institute of Standards and Technology, Gaithersburg, Maryland.

NIST, 2010a, Evaluation of the FEMA P-695 Methodology for Quantification of Building Seismic Performance Factors, NIST GCR 10-917-8, prepared by the NEHRP Consultants Joint Venture, a partnership of the Applied Technology Council and the Consortium of Universities for Research in Earthquake Engineering for the National Institute of Standards and Technology, Gaithersburg, Maryland.

NIST, 2010b, NEHRP Seismic Design Technical Brief No. 3: Seismic Design of Castin-Place Concrete Diaphragms, Chords, and Collectors, A Guide for Practicing Engineers, NIST GCR 10-917-4, prepared by the NEHRP Consultants Joint Venture, a partnership of the Applied Technology Council and the Consortium of Universities for Research in Earthquake Engineering for the National Institute of Standards and Technology, Gaithersburg, Maryland.

NIST, 2010c, NEHRP Seismic Design Technical Brief No. 4: Nonlinear Structural Analysis for Seismic Design, A Guide for Practicing Engineers, NIST GCR 10-917-5, prepared by the NEHRP Consultants Joint Venture, a partnership of the Applied Technology Council and the Consortium of Universities for Research in Earthquake Engineering for the National Institute of Standards and Technology, Gaithersburg, Maryland.

NIST, 2013a, NEHRP Seismic Design Technical Brief No. 8: Seismic Design of Steel Special Concentrically Braced Frame Systems, A Guide for Practicing Engineers, NIST GCR 13-917-24, prepared by the NEHRP Consultants Joint Venture, a partnership of the Applied Technology Council and the Consortium for Universities for Research in Earthquake Engineering, for the National Institute of Standards and Technology, Gaithersburg, Maryland.

NIST, 2013b, Nonlinear Analysis Research and Development Program for

Performance-Based Engineering, NIST GCR 14-917-27, prepared by the NEHRP Consultants Joint Venture, a partnership of the Applied Technology Council and the Consortium for Universities for Research in Earthquake 
Engineering, for the National Institute of Standards and Technology, Gaithersburg, Maryland.

NIST, 2014, NEHRP Seismic Design Technical Brief No. 9: Seismic Design of Special Reinforced Masonry Shear Walls, A Guide for Practicing Engineers, NIST GCR 14-917-31, prepared by the Applied Technology Council for the National Institute of Standards and Technology, Gaithersburg, MD.

NIST, 2017a, Guidelines for Nonlinear Structural Analysis and Design of Buildings, Part I-General, NIST GCR 17-917-46v1, prepared by the Applied Technology Council for the National Institute of Standards and Technology, Gaithersburg, Maryland.

NIST, 2017b, Guidelines for Nonlinear Structural Analysis and Design of Buildings, Part IIa - Steel Moment Frames, NIST GCR 17-917-46v2, prepared by the Applied Technology Council for the National Institute of Standards and Technology, Gaithersburg, Maryland.

NIST, 2017c, Guidelines for Nonlinear Structural Analysis and Design of Buildings, Part IIb - Reinforced Concrete Moment Frames, NIST GCR 17-917-46v3, prepared by the Applied Technology Council for the National Institute of Standards and Technology, Gaithersburg, Maryland.

Nojavan, A., Schultz, A.E., Chao, S.H., Haselton, C., Simasathien, S., Palacios, G., and Liu, X., 2014, Preliminary Results for Neesr Full-Scale Rc Column Tests Under Collapse-Consistent Loading Protocols, Tenth U.S. National Conference on Earthquake Engineering. Frontiers of Earthquake Engineering, 10NCEE Anchorage, Alaska.

Nojavan, A., Schultz, A.E., Chao, S., and Haselton, C.B., 2016, "Influence of cross sectional size on seismic performance of rc columns," American Concrete Institute Structural Journal (in press).

NZS, 1997, Steel Structures Standard, NZS 3404: Part 1, New Zealand Standards, New Zealand.

Orakcal, K., Massone, L., and Wallace, J., 2009, "Shear strength of lightly reinforced wall piers and spandrels," Structural Journal, Vol. 106, No. 4, pp. 455-465.

Panagiotakos, T.B., and Fardis, M.N., 2001, "Deformations of reinforced concrete members at yielding and ultimate," Structural Journal, Vol. 98, No. 2, pp. 135-48.

Pardoen, G.C., Waltman, A., Kazanjy, R.P., Freund, E., and Hamilton, C.H., 2003, Testing and Analysis of One-Story and Two-Story Shear Walls under Cyclic Loading, the CUREE-Caltech Woodframe Project Publication No. W-25, 
Consortium of Universities for Research in Earthquake Engineering, California.

Park, H-G., Baek, J-W., Lee, J-H., and Shin, H-M., 2015, "Cyclic loading tests for shear strength of low-rise reinforced concrete walls with Grade $550 \mathrm{MPa}$ bars," Structural Journal, Vol. 112, pp. 299-310.

Paulay, T., and Priestley, M.J.M., 1992, Seismic Design of Reinforced Concrete and Masonry Buildings, John Wiley \& Sons, New York.

PEER, 2010, Guidelines for Performance-Based Seismic Design of Tall Buildings, PEER TBI, Pacific Earthquake Engineering Research Center Tall Buildings Initiative, College of Engineering, University of California, Berkeley.

PEER/ATC, 2010, Modeling and Acceptance Criteria for Seismic Design and Analysis of Tall Buildings, PEER/ATC 72-1, prepared by the Pacific Earthquake Engineering Research Center (PEER) and Applied Technology Council (ATC), Redwood City, California.

Pei, S., and van de Lindt, J.W., 2007, SAPWood User's Manual, released with SAPWood program. Available at http://www.engr.colostate.edu /NEESWood/sapwood.html, last accessed April 13, 2017.

Pei, S., and van de Lindt, J.W., 2009, "Coupled shear-bending formulation for seismic analysis of stacked shear wall systems," Earthquake Engineering and Structural Dynamics, Vol. 38, pp. 1631-1647.

Pei, S., van de Lindt, J.W., Wehbe, N., and Liu, H., 2013, "Experimental study of collapse limits for woodframe shear walls," Journal of Structural Engineering, Vol., 139, No. 9, pp. 1489-1497.

Peng, Y., Wu, H., and Zhuge, Y., 2015, "Strength and drift capacity of squat recycled concrete shear walls under cyclic loading," Engineering Structures, Vol. 100, pp. 356-368.

Pilette, F.C., 1987, Behavior of Earthquake Resistant Squat Shear Walls, University of Ottawa, Canada.

Pilakoutas, K., and Elnashai, A.S., 1995, "Cyclic behavior of reinforced concrete cantilever walls, Part I: Experimental results,” Structural Journal, Vol. 92, pp. 271-281.

Popov, E.P., Blondet, J.M., and Stepanov, L., 1997, Cyclic Testing of Four FullScale Steel Beam-Column Connections with "Dogbones, ” Report No. UCB/SEMM-1997/04, Department of Civil and Environmental Engineering, University of California, Berkeley.

Popovics, S., 1973, "A numerical approach to the complete stress-strain curve of concrete," Cement and Concrete Research, Vol 3, No. 5, pp. 553-599. 
Priestley, M. J. N., and Park, R., 1987, "Strength and ductility of concrete bridge columns under seismic loading," Structural Journal, Vol. 84, No. 1.

Priestley, M.J.N., Calvi, G.M., and Kowalsky, M.J., 2007, Displacement-Based Design of Structures, IUSS Press, Pavia, Italy.

Pugh, J.S., 2012, Numerical Simulation of Walls and Seismic Design Recommendations for Walled Buildings, Dissertation, University of Washington, Seattle, Washington. Available at: https://dlib.lib.washington .edu/researchworks/handle/1773/22579, last accessed April 13, 2017.

Pugh, J.S., Lowes, L.N., and Lehman, D.E., 2015, "Nonlinear line-element modeling of flexural reinforced concrete walls," Engineering Structures, Vol. 104 pp. 174-192.

Ramirez, C.M., and Miranda, E., 2012, "Significance of residual drifts in building earthquake loss estimation," Earthquake Engineering and Structural Dynamics, Vol. 41, No. 11, pp. 1477-1493.

Ricles, J.M., Mao, C., Lu, L.W., and Fisher, J.W., 2000a, Development and Evaluation of Improved Ductile Welded Unreinforced Flange Connections, SAC Steel Project Document, Report No. SAC/BD-00/24, prepared by the SAC Joint Venture, a partnership of the Structural Engineers Association of California (SEAOC), Applied Technology Council (ATC), and California Universities for Research in Earthquake Engineering (CUREE).

Ricles, J.M., Mao, C., Lu, L.W., and Fisher, J.W., 2000b, "Ductile details for welded unreinforced moment connections subject to inelastic cyclic loading," Engineering Structures, Vol. 25, No. 5, pp. 667-680.

Ricles, J.M., Fisher, J.W., Lu, L-W., and Kaufmann, E.J., 2002, “Development of improved welded moment connections for earthquake-resistant design," Journal of Constructional Steel Research, Vol. 58, No. 5-8, pp. 565-604.

Ricles, J.M., Zhang, X., Lu, L.W., and Fisher, J., 2004, Development of Seismic Guidelines for Deep-Column Steel Moment Connections, Report No. 04-13, Advanced Technology for Large Structural Systems Engineering Research Center, Lehigh University, Bethlehem, Pennsylvania.

Rodas, P.T., Zareian, F., and Kanvinde, A., 2016, "Hysteretic model for exposed column base connections," Journal of Structural Engineering, Vol. 142, No. 12.

Roeder, C., and Popov, E., 1977, Inelastic Behavior of Eccentrically Braced Steel Frames under Cyclic Loadings, Report No. UBC/EERC-08/1977, University of California, Berkeley, California. 
Roeder, C., Schneider, S., and Carpenter, J., 1993, "Seismic behavior of momentresisting steel frames: analytical study," Journal of Structural Engineering, Vol. 119, No. 6, pp. 1866-1884.

Roeder, C., Lumpkin, E., and Lehman, D., 2011, “A balanced design procedure for special concentrically braced frame connections," Journal of Constructional Steel Research, Vol. 67, No. 11, pp. 1760-1772.

Saatcioglu, M., 1991, "Hysteretic shear response of low-rise walls," International Workshop on Concrete Shear in Earthquake, University of Houston, Texas.

Saatcioglu, M., and Razvi, S.R., 1992, "Strength and ductility of confined concrete," Journal of Structural Engineering, Vol. 118, No. 6, pp. 1590-1607.

Salenikovich, A., and Payeur, M., 2010, "Influence of vertical loads on lateral resistance of light-frame shear walls," World Conference on Timber Engineering, June, Riva Del Garda, Italy.

Salonikios, T.N., Kappos, A.J., Tegos, I.A., and Penelis, G.G., 1999, "Cyclic load behavior of low-slenderness reinforced concrete walls: Design basis and test results," Structural Journal, Vol. 96, pp. 649-660.

Sato, S., Ogata, Y., Yoshizaki, S., Kanata, K., Yamaguchi, T., and Nakayama, T., 1989, "Behavior of shear wall using various yield strength of rebar, Part 1: An experimental study," Tenth International Conference on Structural Mechanics in Reactor Technology, Anaheim, California.

Schmid, B., 1984, Shear Test of Existing Wood Lath and Plaster Walls Relative to Division 88, Ben L. Schmid Consulting Structural Engineer, Pasadena, California.

Scott, M.H., and Fenves, G.L., 2006, "Plastic hinge integration methods for forcebased beam-column elements," Journal of Structural Engineering, Vol. 132, No. 2, pp. 244-252.

Seible, F., Priestley, M.J.N., Kingsley, G.R., and Kürkchübasche, A.G., 1994, "Seismic Response of Full-Scale Five-Story Reinforced Masonry Building," Journal of Structural Engineering, Vol. 120, No. 3, 925-946.

Sen, A., Sloat, D., Ballard, R., Johnson, M., Roeder, C., Lehman, D., and Berman, J., 2016, "Experimental evaluation of the seismic vulnerability of braces and connections in older concentrically braced frames," Journal of Structural Engineering, Vol. 10, No. 1061.

Sezen, H., and Moehle, J.P., 2006, "Seismic tests of concrete columns with light transverse reinforcement," Structural Journal, Vol. 103, No. 6, pp. 842-849. 
Shaw, S.M., 2013, Seismic Performance of Partial Joint Penetration Welds in Steel Moment Resisting Frames, Ph.D. Dissertation, University of California, Davis, California.

Shedid, M.T., Drysdale, R.G., and El-Dakhakhni, W.W., 2008, "Behavior of fullygrouted reinforced concrete masonry shear walls failing in flexure: experimental results," Journal of Structural Engineering, Vol. 134, No. 11, 1754-1767.

Sherman, J.D., 2011, Effects of Key Parameters on the Performance of Concrete Masonry Shear Walls under In-Plane Loading, Master Thesis, Washington State University, Pullman, Washington.

Sherman, D.R., and Ghorbanpoor, A1., 2002, Design of Extended Shear Tabs, Final Report Submitted, American Institute of Steel Construction, Chicago, Illinois.

Sheu, M.S., and Huang, G-S., 1991, "Prediction of hysteretic loops for RC shear walls with 0.65 to 1.90 shear span to wall width ratios," International Workshop on Concrete Shear in Earthquake, University of Houston, Texas.

Shiga, T., Shibata, A., and Takahashi, J., 1973, "Experimental study on dynamic properties of reinforced concrete shear walls," Fifth World Conference on Earthquake Engineering, Rome, Italy, pp. 1157-1166.

Shin, S., and Engelhardt, M.D., 2013, "Cyclic performance of deep column moment frames with weak panel zones," Proceedings, NEES Annual Meeting.

Shing, P.B., Noland, J.L., Spaeh, H.P., Klamerus, E.W., and Schuller, M.P., 1991, Response of Single-Story Reinforced Masonry Shear Walls to In-Plane Lateral Loads, Report No. 3.1(a)-2, U.S.-Japan Coordinated Program for Masonry Building Research, University of Colorado at Boulder.

Sittipunt, C., Wood, S.L., Lukkunaprasit, P., and Pattararattanakul, P., 2001, "Cyclic behavior of reinforced concrete structural walls with diagonal web reinforcement," Structural Journal, Vol. 98, pp. 554-562.

Sokoli, D., and Ghannoum, W.M., 2016, "High-strength reinforcement in columns under high shear stresses," Structural Journal, Vol. 113, No. 3, pp. 605-14.

Stavridis, A., Ahmadi, F., Mavros, M., Shing, P.B., Klingner, R.E., and McLean, D., 2016, "Shake-table tests of a full-scale three-story reinforced masonry shear wall structure," Journal of Structural Engineering, Vol. 142, No. 10.

Suita, K. Yamada, S., Tada, M., Kasai, K., Matsuoka, Y., and Sato, E., 2008, "Results of recent E-defense tests on full-scale steel buildings: Part 1 collapse experiment on 4-story moment frame," Proceedings, ASCE Structures Congress, Vancouver, Canada. 
Suzuki, Y., and Lignos, D.G., 2014, "Development of loading protocols for experimental testing of steel columns subjected to combined lateral drift and high axial load," Proceedings, $10^{\text {th }}$ National Conference on Earthquake Engineering (10 $\left.{ }^{\text {th }} \mathrm{NCEE}\right)$, Anchorage, Alaska, July $21^{\text {st }}-25^{\text {th }}$, Paper No. 280.

Suzuki, Y., and Lignos, D.G., 2015, "Large-scale collapse experiments of wide flange steel beam-columns," Proceedings, $8^{\text {th }}$ International Conference on the Behavior of Steel Structures in Seismic Areas, July 1-3, Shanghai, China.

Synge, A.J., 1980, Ductility of Squat Shear Walls, Report No. 80-8, Department of Civil Engineering, University of Canterbury, Christchurch, New Zealand.

Thomsen, J., and Wallace, J., 1995, Displacement-Based Design of RC Structural Walls: An Experimental Investigation of Walls with Rectangular and TShaped Cross Sections, Technical Report No. CU/CEE-95/06, Clarkson University, Department of Civil Engineering, Potsdam, New York.

TMS, 2011, 2013, 2015, Building Code Requirements for Masonry Structures, TMS 402/ACI 530/ASCE 5, The Masonry Society, Longmont, Colorado.

Tomii, M., Essaki, F., and Mitsuyama, H., 1988, "Experimental study to increase ductility of R/C framed shear walls by preventing slip failure of monolithic infilled wall panel," Ninth World Conference on Earthquake Engineering, Tokyo-Kyoto, Japan.

Tran, T., and Wallace, J., 2012, Experimental and Analytical Studies of Moderate Aspect Ratio Reinforced Concrete Structural Walls, Research Report, University of California, Los Angeles.

Trautner, C.A., Hutchinson, T., Grosser, P.R., and Silva, J.F., 2015, "Effects of detailing on the cyclic behavior of steel baseplate connections designed to promote anchor yielding," Journal of Structural Engineering, Vol. 142, No. 2, pp. 1-10.

Tremblay, R., Tchebotarev, N., and Filiatrault, A., 1997, "Seismic performance of RBS connections for steel moment resisting frames: influence of loading rate and floor slab," Proceedings, Second International Conference on the Behavior of Steel Structures in Seismic Areas, STESSA 1997, Kyoto, Japan.

Tsai, K-C., and Popov, E.P., 1988, Steel Beam-Column Joints in Seismic Moment Resisting Frames, Report No. UCB/EERC-88/19, University of California, Berkeley.

Uang, C.M., and Fan, C.C., 2001, "Cyclic stability criteria for steel moment connections with reduced beam section," Journal of Structural Engineering, Vol. 127, No. 9, pp. 1021-1027. 
Vamvatsikos, D., and Cornell, C., 2002, "Incremental dynamic analysis," Earthquake Engineering and Structural Dynamics, Vol., 31, No. 3, pp. 491-514.

van de Lindt, J.W., 2008, "Experimental investigation of the effect of multiple earthquakes on woodframe structural integrity," Practice Periodical on Structural Design and Construction, Vol. 13, No. 3, pp. 111-117.

van de Lindt, J.W., Pei, S., Pang, W., and Sharazi, S., 2012, "Collapse testing and analysis of a light-frame wood garage wall," Journal of Structural Engineering, Vol. 138, No. 4, pp. 492-501.

van de Lindt, J.W., Bahmani, P., Mochizuki, G., Pryor, S.E., Gershfeld, M., Tian, J., Symans, M.D. and Rammer, D., 2014, "Experimental seismic behavior of a full-scale four-story soft-story wood-frame building with retrofits II: shake table test results," Journal of Structural Engineering, Vol. 142, No. 4.

Voon, K.C., and Ingham, J.M., 2006, "Experimental in-plane shear strength investigation of reinforced concrete masonry walls," Journal of Structural Engineering, Vol. 132, No. 3, 400-408.

Wasiewicz, Z.F., 1988, Sliding Shear in Low-Rise Walls under Lateral Load Reversals, University of Ottawa, Canada.

Welt, T.S., 2015, Detailing for Compression in Reinforced Concrete Wall Boundary Elements: Experiments, Simulations, and Design Recommendations, Dissertation, University of Illinois, Urbana-Champaign, Champaign, Illinois. Available at: https://www.ideals.illinois.edu/handle/2142/88022, last accessed April 13, 2017.

Welt, T., Massone, L., LaFave, J., Lehman, D., McCabe, S. and Polanco, P., 2017, "Confinement behavior of rectangular reinforced concrete prisms simulating wall boundary elements," Journal of Structural Engineering, Vol. 143, No. 4.

Whitman, Z.J., 2015, Investigation of Seismic Failure Modes in Flexural Concrete Walls Using Finite Element Analysis, Thesis, University of Washington, Seattle, Washington. Available at: https://digital.lib.washington.edu /researchworks/handle/1773/33674? show=full, last accessed April 13, 2017.

Wiradinata, S., 1985, Behavior of Squat Walls Subjected to Load Reversals, University of Toronto, Canada.

Wood, S.L., 1990, "Shear strength of low-rise reinforced concrete walls," Structural Journal, Vol. 87, pp. 99-107.

Woods, J.E., Lau, D.T., and Cruz-Noguez, C.A., 2016, "In-plane seismic strengthening of nonductile reinforced concrete shear walls using externally 
bonded CFRP sheets," Journal of Composites for Construction, Vol. 20, No. 6.

WoodWorks, 2016, Five-Story Wood-Frame Structure over Podium Slab, developed by Douglas S. Thompson, STB Structural Enginers, Inc., for Wood Products Council. Available at: http://www.woodworks.org/wp-content/uploads/5 -over-1-Design-Example.pdf, last accessed April 13, 2017.

Xie, L., and Xiao, Y., 2000, Study on Retrofit of Existing Squat Concrete Shear Walls, Report No USC-SERP 2000-5, Department of Civil Engineering, University of Southern California.

Yassin, M., 1994, Nonlinear Analysis of Prestressed Concrete Structures under Monotonic and Cyclic Loads, Ph.D. dissertation, Dept. of Civil and Environmental Engineering, University of California, Berkeley.

Yuan, Y., 2000, “A review of trust region algorithms for optimization," Proceedings, Fourth International Congress on Industrial and Applied Mathematics, JM Ball and JCR Hunt, Oxford University Press, Oxford, pp. 271-282.

Zareian, F., and Kanvinde, A., 2013, "Effect of column-base flexibility on the seismic response and safety of steel moment-resisting frames," Earthquake Spectra, Vol. 29, No. 4, pp. 1537-1559.

Zhang, X., and Ricles, J.M., 2006, "Experimental evaluation of reduced beam section connections to deep columns," Journal of Structural Engineering, Vol. 132, No. 3, pp. 346-357. 


\section{Project Participants}

National Institute of Standards and Technology

Steven L. McCabe

Engineering Laboratory (MS8604)

National Institute of Standards and Technology

100 Bureau Drive

Gaithersburg, Maryland 20899

John (Jay) L. Harris III

Engineering Laboratory (MS8604)

National Institute of Standards and Technology

100 Bureau Drive

Gaithersburg, Maryland 20899

Siamak Sattar

Engineering Laboratory (MS8604)

National Institute of Standards and Technology

100 Bureau Drive

Gaithersburg, Maryland 20899

\section{Applied Technology Council}

Jon A. Heintz (Program Manager)

Applied Technology Council

201 Redwood Shores Parkway, Suite 240

Redwood City, California 94065

Ayse Hortacsu (Associate Program Manager)

Applied Technology Council

201 Redwood Shores Parkway, Suite 240

Redwood City, California 94065

\section{Program Committee on Seismic Engineering}

Jon A. Heintz (Chair)

Applied Technology Council

201 Redwood Shores Parkway, Suite 240

Redwood City, California 94065

Michael Cochran

Thornton Tomasetti

4551 Glencoe Avenue, Suite 350

Marina del Rey, California 90292
Matthew S. Speicher

Engineering Laboratory (MS8604)

National Institute of Standards and Technology

100 Bureau Drive

Gaithersburg, Maryland 20899

Kevin K.F. Wong

Engineering Laboratory (MS8604)

National Institute of Standards and Technology

100 Bureau Drive

Gaithersburg, Maryland 20899

Veronica Cedillos (Associate Project Manager)

Applied Technology Council

201 Redwood Shores Parkway, Suite 240

Redwood City, California 94065

James R. Harris

J.R. Harris \& Company

1775 Sherman Street, Suite 1525

Denver, Colorado 80203

James Jirsa

Department of Civil, Architectural and Environmental

Engineering

University of Texas at Austin

301 E. Dean Keeton Street, Stop C1700

Austin, Texas 78712 
Roberto Leon

Department of Civil and Environmental Engineering

Virginia Tech

102-D Patton Hall

Blacksburg, Virginia 24061

Stephen Mahin

Department of Civil and Environmental

Engineering

University of California, Berkeley

721 Davis Hall

Berkeley, California 94720

James O. Malley

Degenkolb Engineers

375 Beale Street, Suite 500

San Francisco, California 94105

\section{Project Technical Committee}

Ronald O. Hamburger (Project Director)

Simpson Gumpertz \& Heger, Inc.

100 Pine Street, Suite 1600

San Francisco, California 94111

Gregory G. Deierlein

Dept. of Civil and Environmental Engineering

Blume Earthquake Engineering Center

MC 3037

Stanford, California 94305

Dawn E. Lehman

University of Washington

214B Moore Hall, Box 352700

Seattle, Washington 98195

Dimitrios G. Lignos

Swiss Federal Institute of Technology, Lausanne (EPFL)

Department of Architecture, Civil and

Environmental Engineering (ENAC)

GC B3 485 (Bâtiment GC), Station 18

CH-1015 Lausanne, Switzerland

Laura N. Lowes

University of Washington

233C More Hall, Box 352700

Seattle, Washington 98195
Donald Scott

PCS Structural Solutions

811 First Avenue, Suite 620

Seattle, Washington 98104

Andrew Whittaker

Department of Civil, Structural and

Environmental Engineering

University at Buffalo

230 Ketter Hall

Buffalo, New York 14260
Robert Pekelnicky

Degenkolb Engineers

375 Beale Street, Suite 500

San Francisco, California 94105

P. Benson Shing

University of California, San Diego

Jacobs School of Engineering

9500 Gilman Drive

La Jolla, California 92093

Peter Somers

Magnusson Klemencic Associates

1301 Fifth Avenue, Suite 3200

Seattle, Washington 98101

John W. van de Lindt

Colorado State University

College of Engineering

Engineering A201

Fort Collins, Colorado 80523 


\title{
Project Review Panel
}

Martin Button

Button Engineering

4701 Shoal Creek Blvd

Austin, Texas 78756

Charles J. Carter

American Institute of Steel Construction

One East Wacker Drive, Suite 700

Chicago, Illinois 960601

Kelly E. Cobeen

Wiss Janney Elstner Associates, Inc.

2000 Powell Street, Suite 1650

Emeryville, California 94608

Philip Line

American Wood Council

222 Catoctin Circle SE

Suite 201

Leesburg, Virginia 20175

Justin D. Marshall

Civil Engineering

Auburn University

Auburn, Alabama 36849

\section{Working Group Members}

Jianyu Cheng

University of California, San Diego

Jacobs School of Engineering

9500 Gilman Drive

La Jolla, California 92093

Ahmed Elkady

Swiss Federal Institute of Technology, Lausanne

(EPFL), Department of Architecture, Civil and

Environmental Engineering (ENAC)

GC B3 485 (Bâtiment GC), Station 18

CH-1015 Lausanne, Switzerland

Siamak Epackachi

University at Buffalo

Department of Civil, Structural, and

Environmental Engineering

212 Ketter Hall

Buffalo, New York 14260
Lawrence C. Novak

Portland Cement Association

5420 Old Orchard Road

Skokie, Illinois 60077

Graham Powell

Graham Powell Inc.

14710 Wolfgang Rd.

Truckee, California 96161

Michael P. Schuller

Atkinson-Noland \& Associates, Inc.

2619 Spruce Street

Boulder, Colorado 80302

William Tremayne

Holmes Structures

235 Montgomery Street, Suite 1250

San Francisco, California 94104

Kent Yu (ATC Board Contact)

SEFT Consulting Group

4800 SW Griffith Drive, Suite 100

Beaverton, Oregon 97005

\author{
Alexander Hartloper \\ Swiss Federal Institute of Technology, Lausanne \\ (EPFL) \\ Department of Architecture, Civil and \\ Environmental Engineering (ENAC) \\ GC B3 485 (Bâtiment GC), Station 18 \\ CH-1015 Lausanne, Switzerland \\ Maria Koliou \\ Colorado State University \\ College of Engineering \\ Engineering A201 \\ Fort Collins, Colorado 80523 \\ Daniel Sloat \\ Degenkolb Engineers \\ 375 Beale Street, Suite 500 \\ San Francisco, California 94105
}

